\title{
Numerical Modelling of Hollow Concrete Block Masonry Mechanics
}

Bradley Hunt

A thesis submitted to the Faculty of Graduate and Postdoctoral Affairs in partial fulfillment of the requirements for the degree of

Doctor of Philosophy

in

Civil Engineering

Carleton University

Ottawa, Ontario

(C) 2018

Bradley Hunt 
The complex interaction between materials used to make masonry elements causes the mechanics of hollow concrete masonry to not be fully understood. To gain a better understanding of hollow concrete masonry mechanics, this thesis presents three numerical models to describe these mechanics from the micro to the macro level.

The Smeared Area Compression Model is a numerical model for predicting the compression strength of ungrouted and grouted hollow concrete masonry, using equilibrium, compatibility, and constitutive relationships. The model uses a smeared area approach to construct a composite stiffness matrix from each material's secant stiffness matrix accounting for any strength enhancement or degradation. The Simplified Smeared Area Compression Model and Design Smeared Area Compression Equations are extensions of the Smeared Area Compression Model for use in design codes. The models are verified against 247 masonry prism tests.

The Smeared Area Finite Element Model is a numerical model for predicting the stress, strain, displacement, and crack patterns of ungrouted, grouted, and reinforced masonry structures. The model is a micro-macro-model, which uses a smeared rotating crack model and combines the average macroscopic representation of the masonry behavior with the local response of mortar joints. The model uses equilibrium, compatibility, and constitutive relations specific designed for masonry. The model is verified against 89 experimental tests.

The Three Parameter Kinematic Theory captures the complete prepeak and postpeak response of cantilevered reinforced masonry shear walls using three degrees of freedom. The wall is separated into two parts by a diagonal crack, an upper rigid block and a lower fan of struts. The mechanisms of shear resistance across this crack are modeled with nonlinear springs. The base section of the wall is also modeled to account for yielding of the reinforcement and crushing of the masonry. The model is verified against 40 experimental tests.

Each one of these models is shown to be accurate in its predictions. Additionally, each model produces simple equations that can be used to predict key masonry properties, which ultimately helps engineers to design safer structures and more accurately assess the safety of existing construction. 


\section{ACKNOWLEDGEMENTS}

Firstly, I would like to thank my advisor, Professor Edward G. Sherwood, for all of the support that he has provided over the course of my studies at Carleton University. His guidance over the years has not only greatly benefitted the work comprising this thesis, but has made this a truly enjoyable experience and has shaped the way in which I will approach future endeavours.

I thank Professors Heng-Aik Khoo, Rong Liu, Beatriz Martin-Perez, and Shelley Lissel for their thorough review of this thesis. Additionally, their valuable comments and suggestions provided are gratefully acknowledged.

Financial support provided by NSERC, Professor Edward G. Sherwood, and the Department of Civil Engineering of Carleton University made my studies financially feasible, and for that I am grateful.

To my parents, Barry and Deborah. Thank you always for your unending support. I wouldn't be here without you.

Lastly, but most importantly, I would like to thank my wife Patricia for her unconditional support and encouragement. You have been with me since the first day of my undergrad studies to the last day of my $\mathrm{PhD}$, through all the highs and all the lows. And to Emmett, our son, thank you for providing me with an incentive to finish my $\mathrm{PhD}$ in a timely manner. I hope that one day, this thesis will inspire you to greatness. 


\section{TABLE OF CONTENTS}

Abstract. ............................ ii

Acknowledgements ........................ iii

Table of Contents. . . . . . . . . . . . . . . . . iv

List of Figures . . . . . . . . . . . . . . . . . xv

List of Tables . . . . . . . . . . . . . . . . . . xxii

List of Symbols . . . . . . . . . . . . . . . . . . . . . xxiv

List of Abbreviations . . . . . . . . . . . . . . . . . xxxi

Part I Thesis Introduction . . . . . . . . . . . . . . 1

Chapter 1: Introduction . . . . . . . . . . . . . . . 3

1.1. Masonry History . . . . . . . . . . . . . . . . . . 3

1.1.1. Ancient .................... 4

1.1.2. Classical . . . . . . . . . . . . . . . . . 5

1.1.3. Contemporary .................... 6

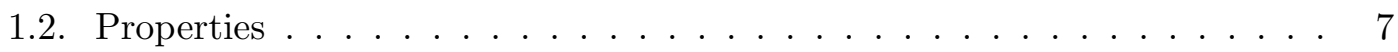

1.2.1. Hollow Concrete Blocks . . . . . . . . . . . . . . . 7

1.2.2. Mortar . . . . . . . . . . . . . . . . 7

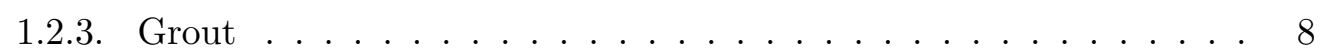

1.2.4. Reinforcement . . . . . . . . . . . . . 8

1.2.5. Assemblages . . . . . . . . . . . . . . . 9

1.2.6. Joints . . . . . . . . . . . . . . . . 9

1.2.7. Prisms . . . . . . . . . . . . . . . 10

1.3. Inspiration for Current Study . . . . . . . . . . . . . . . . . . 11

1.4. Objectives . . . . . . . . . . . . . . . . . . 11

1.5. Organization of Thesis . . . . . . . . . . . . . . 12

Part II Masonry Compression Strength Modeling . . . . . . 15

Chapter 2: Background . . . . . . . . . . . . . . . . . 17

2.1. Mechanics of Ungrouted Masonry . . . . . . . . . . . . . . . . . 17

2.2. Mechanics of Grouted Masonry . . . . . . . . . . . . . . . 20

2.3. Empirical Modeling . . . . . . . . . . . . . . . . . . . . 21

2.3.1. Sarhat and Sherwood (2014) and Sarhat and Sherwood (2015) . . 21 
2.4. Analytical Modeling . . . . . . . . . . . . . . . . . . . . 23

2.4.1. Hamid $(1978) \ldots \ldots \ldots . \ldots \ldots$

2.5. Finite Element Modeling . . . . . . . . . . . . . . . . 25

2.5.1. Cheema and Klingner (1986) _. . . . . . . . . . . 25

2.5.2. Others . . . . . . . . . . . . . . . 27

2.6. Background Summary . . . . . . . . . . . . . . . . . 27

2.7. Model Objectives . . . . . . . . . . . . . . . . . . . . . . . . 28

Chapter 3: Smeared Area Compression Model. . . . . . . . . . . . . . . . 29

3.1. Assumptions . . . . . . . . . . . . . . . . 30

3.2. Dimensions and Areas . . . . . . . . . . . . . . . . . . . . 32

3.3. Equilibrium Conditions . . . . . . . . . . . . . . . 36

3.4. Compatibility Conditions . . . . . . . . . . . . 36

3.5. Poisson's Effects . . . . . . . . . . . . . . . . . . 37

3.6. Stress-Strain Relations . . . . . . . . . . . . . . . . . . . . . . 39

3.7. Proposed Material Models . . . . . . . . . . . . . . . . . . . 40

3.7.1. Constitutive Models for Compression . . . . . . . . . . . . . . . . . 41

3.7.2. Strength Enhancement or Strength Degradation . . . . . . . . . . 44

3.7.3. Constitutive Model for Tension . . . . . . . . . . . . . . . . . . . 46

3.7.4. Lateral Expansion . . . . . . . . . . . . . . . . . . . . 47

3.8. Failure . . . . . . . . . . . . . . . . . . . . . . 48

3.9. Uniaxial Compressive Strength Corrections . . . . . . . . . . . . . . . 49

3.9.1. Block Uniaxial Compressive Strength Corrections . . . . . . . . . . 49

3.9.2. Mortar Uniaxial Compressive Strength Corrections . . . . . . . . . 50

3.9.3. Grout Uniaxial Compressive Strength Corrections . . . . . . . . 51

3.10. Solution Procedure . . . . . . . . . . . . . . . . 51

3.11. Model Inputs and Default Values . . . . . . . . . . . . . . . . . 52

3.12. SACM Program . . . . . . . . . . . . . . . . . . 55

Chapter 4: Comparison of Experimental Data and the Smeared Area

Compression Model . . . . . . . . . . . . . . . . . . . . . 59

4.1. Data Requirements . . . . . . . . . . . . . . . . . . 59

4.2. Ungrouted Experimental Database . . . . . . . . . . . . . . . . . 59

4.2.1. SACM Results . . . . . . . . . . . . . . . 60 60

4.3. Grouted Experimental Database . . . . . . . . . . . . . . . . . . . . 64

Numerical Modelling of Hollow Concrete Block Masonry Mechanics 
4.3.1. SACM Results . . . . . . . . . . . . . . . . . 64

4.4. Discussion . . . . . . . . . . . . . . . . . . . 69

4.4.1. Block Strain at Peak Strength _. . . . . . . . . . . . 69

4.4.2. Initial Block Poisson's Ratio . . . . . . . . . . . . . . . . . 71

4.4.3. Mortar Strain at Peak Strength . . . . . . . . . . . . . . . . 71

4.4.4. Initial Mortar Poisson's Ratio . . . . . . . . . . . . . . . . . 73

4.4.5. Grout Strain at Peak Strength . . . . . . . . . . . . . . . 74

4.4.6. Initial Grout Poisson's Ratio . . . . . . . . . . . . . . . . 75

Chapter 5: Parametric Study of the Smeared Area Compression Model 76

5.1. Parameters of Study . . . . . . . . . . . . . . . . . . 76

5.2. Influence of Mortar Compressive Strength . . . . . . . . . . . . 79

5.3. Influence of Grout Compressive Strength . . . . . . . . . . . . . . 80

5.4. Influence of Mortar Thickness . . . . . . . . . . . . . . . . . . 81

5.5. Influence of Block Height . . . . . . . . . . . . . . . 82

5.6. Influence of Block Percent Solid . . . . . . . . . . . . . . . . 83

5.7. Influence of Block Face Shell Thickness . . . . . . . . . . . . . . . 84

5.8. Influence of Block Tensile Strength . . . . . . . . . . . . . . . . . 85

5.9. Influence of Block Initial Poisson's Ratio . . . . . . . . . . . . . . . . 87

5.10. Influence of Mortar Initial Poisson's Ratio . . . . . . . . . . . . . 88

5.11. Influence of Grout Initial Poisson's Ratio ～. . . . . . . . . . . . 89

5.12. Influence of Block Strain at Peak Stress . . . . . . . . . . . . . . . . . 90

5.13. Influence of Mortar Strain at Peak Stress _ . . . . . . . . . . . . . . . 91

5.14. Influence of Grout Strain at Peak Stress . . . . . . . . . . . . . . . . . . 92

Chapter 6: Simplified Smeared Area Compression Model . . . . . . . . . 93

6.1. Simplified Smeared Area Compression Model . . . . . . . . . . . . . . . 94

6.1.1. Ungrouted Face Shell Bedded Mortar . . . . . . . . . . . . . . 94

6.1.2. Ungrouted Full Bedded Mortar . . . . . . . . . . . . . . . . . 94

6.1.3. Grouted . . . . . . . . . . . . . . . . . 95

6.2. Comparison of Simplified Smeared Area Compression Model to Experimental Data . . . . . . . . . . . . . . . . . . . . 96

6.3. Design Equations . . . . . . . . . . . . . . . . . . . . 104

6.4. Comparison to Current International Codes . . . . . . . . . . . . . . . . . 104

6.4.1. Canadian Masonry Code: CSA S304-14 . . . . . . . . . . . . . 104

6.4.2. United States Masonry Code: 2013 MSJC . . . . . . . . . . . . . . 105 
6.4.3. Australian Masonry Code: AS3700-2011 . . . . . . . . . . . . . 106

6.4.4. United Kingdom Masonry Code: Eurocode 6 . . . . . . . . . 107

6.4.5. Results . . . . . . . . . . . . . . . . . 107

Chapter 7: Compression Parallel to Bed Joints . . . . . . . . . . . . 110

7.1. Background . . . . . . . . . . . . . . . . . . 110

7.1.1. Mechanics of Compression Parallel to Bed Joints . . . . . . . . . 115

7.2. Conceptual Model . . . . . . . . . . . . . . . . 116

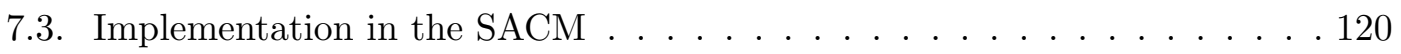

7.3.1. Dimensions and Areas . . . . . . . . . . . . . . . . . . 122

7.3.2. Block Compression Strength _ . . . . . . . . . . . . . . 124

7.4. Results . . . . . . . . . . . . . . . . . . 126

Chapter 8: Part II Concluding Remarks . . . . . . . . . . . . . . . . . . . 129

8.1. Part II Conclusions . . . . . . . . . . . . . . . . . . . . . . 129

8.2. Part II Future Research . . . . . . . . . . . . . . . . . . . . . 131

Part III Masonry Finite Element Modeling . . . . . . . . . . 132

Chapter 9: Background . . . . . . . . . . . . . . . . . . 134

9.1. Micro-Models . . . . . . . . . . . . . . . . . . . . . . . . 134

9.2. Macro-Models . . . . . . . . . . . . . . . . . . . . 137

9.2.1. Masonry Macro-Models . . . . . . . . . . . . . . . . 137

9.2.2. Concrete Macro-Models . . . . . . . . . . . . . . . . . . . . 138

9.3. Micro-Macro-Models . . . . . . . . . . . . . . . . . . . . . . . . . . . . . . 142

9.3.1. Masonry Micro-Macro-Models . . . . . . . . . . . . . . . . . . 142

9.3.2. Concrete Micro-Macro-Models . . . . . . . . . . . . . . . . . . 143

9.4. Background Summary . . . . . . . . . . . . . . . . . . 145

9.5. Model Objectives . . . . . . . . . . . . . . . . . . . . . . 145

Chapter 10: Smeared Area Finite Element Model. . . . . . . . . . . . . . 147

10.1. Assumptions ． . . . . . . . . . . . . . . . . . . . . . 149

10.2. Equilibrium Conditions ． . . . . . . . . . . . . . . . . . 149

10.2.1. Average Stresses . . . . . . . . . . . . . . . . . 151

10.2.2. Local Crack Surface . . . . . . . . . . . . . . . . . 153

10.2.3. Local Mortar Joints . . . . . . . . . . . . . . . . . 155

10.3. Compatibility Conditions . . . . . . . . . . . . . . . 156

Numerical Modelling of Hollow Concrete Block Masonry Mechanics 
10.3.1. Net Strains . . . . . . . . . . . . . . . . . . . 158

10.3.2. Crack Shear Slip Strain . . . . . . . . . . . . . . . 158

10.3.3. Mortar Joint Shear Strains . . . . . . . . . . . . . . . . . 159

10.3.4. Poisson's Strain Effects _ . . . . . . . . . . . . . . . . . 160

10.4. Stress-Strain Relations . . . . . . . . . . . . . . . . . . . . . 162

10.5. Proposed Material Models . . . . . . . . . . . . . . . . . . 162

10.5.1. Masonry Anisotropic Nature . . . . . . . . . . . . . . . . . 162

10.5.2. Constitutive Models for Masonry Pre-Peak Compression . . . . . . 170

10.5.3. Constitutive Models for Masonry Post-Peak Compression . . . . . 173

10.5.4. Compression Softening Modeling . . . . . . . . . . . . . 174

10.5.5. Constitutive Models for Tension . . . . . . . . . . . . . 177

10.5.6. Lateral Expansion Model . . . . . . . . . . . . . . . . . 180

10.5.7. Cracking Criteria . . . . . . . . . . . . . . . 182

10.5.8. Crack Slip . . . . . . . . . . . . . . . . . . . 183

10.5.9. Shear Slip Model for Masonry Joints . . . . . . . . . . . . . . 185

10.5.10.Steel Reinforcement Response . . . . . . . . . . . . . . . . 188

Chapter 11: Implementation of Models into Masonry Finite Element . . 189

11.1. Element Description . . . . . . . . . . . . . . . . . . . . 189

11.1.1. Partial Derivative Computation . . . . . . . . . . . . . . . . 191

11.1.2. Computing the Jacobian Matrix . . . . . . . . . . . . . . . 191

11.1.3. The Strain-Displacement Matrix . . . . . . . . . . . . . . 193

11.2. Element Stiffness Matrix . . . . . . . . . . . . . . . . . . . . . . 193

11.3. Composite Material Stiffness Matrix . . . . . . . . . . . . . . . . . . 194

11.4. Element and Material Stresses _ . . . . . . . . . . . . . . . . . 196

11.5. Material Prestrains . . . . . . . . . . . . . . . . . . 197

11.6. Total Load Iterative Secant Stiffness SAFEM Solution Procedure . . . . . 198

11.7. Applying Support Conditions . . . . . . . . . . . . . . . . . . 199

11.8. External Support Reactions . . . . . . . . . . . . . . . . . . . . 199

11.9. Finite Element Implementation . . . . . . . . . . . . . . . 200

11.10Finite Element Mesh Size . . . . . . . . . . . . . . . . . . 200

11.11Model Inputs and Default Values . . . . . . . . . . . . . . . . . 200

11.12SAFEM Program . . . . . . . . . . . . . . . . 200 
Chapter 12: Smeared Area Finite Element Model Verification . . . . . . 206

12.1. Element Verification . . . . . . . . . . . . . . . 207

12.1.1. Symmetry of Stiffness Matrix . . . . . . . . . . . . . 208

12.1.2. Eigenvalues and Rank Sufficiency of Stiffness Matrix . . . . . . . 209

12.1.3. Element Jacobian Values . . . . . . . . . . . . . . . . . . 210

12.1.4. Equivalent Nodal Loads . . . . . . . . . . . . . . . . . . . 210

12.2. Numerical Tests . . . . . . . . . . . . . . . . . . . . . . . . 212

12.2.1. Element Force Patch Test . . . . . . . . . . . . . . . . . . . . 212

12.2.2. Straight Cantilever Beam _ . . . . . . . . . . . . . 215

12.2.3. Thin Arched Beam . . . . . . . . . . . . . . . . . . . . 217

12.2.4. Pure Bending of a Cantilever Beam . . . . . . . . . . . 218

12.2.5. Cook's Skew Beam . . . . . . . . . . . . . . . . 218

12.3. VecTor2 . . . . . . . . . . . . . . . . . . . . . 222

12.3.1. Panel Test . . . . . . . . . . . . . . . . . . . . . . 222

12.3.2. Shear Wall Test . . . . . . . . . . . . . . . . . 225

Chapter 13: Comparison of Single Pier Grouted Shear Wall Data and the Smeared Area Finite Element Model . . . . . . . . . . . . . . . . . 230

13.1. Data Requirements . . . . . . . . . . . . . . . . . . 230

13.2. Database Literature Review . . . . . . . . . . . . . . . . . . 231

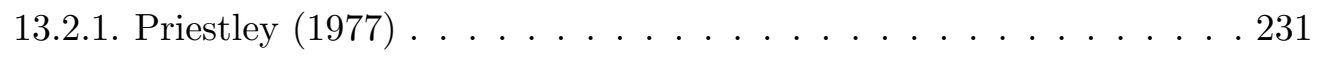

13.2.2. Chen et al. (1978) f . . . . . . . . . . . . . . 232

13.2.3. Hidalgo et al. (1979) . . . . . . . . . . . . . . 233

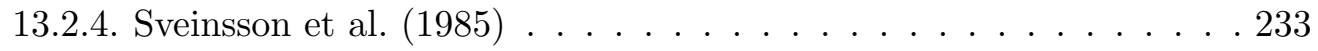

13.2.5. Shing et al. (1991) . . . . . . . . . . . . . . . . 234

13.2.6. Ibrahim $(1995) \ldots \ldots . \ldots \ldots \ldots$

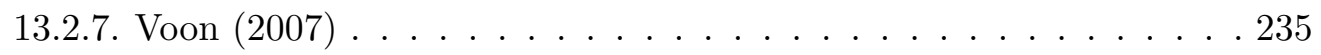

13.2.8. Shedid et al. (2008) _ . . . . . . . . . . . . . 236

13.2.9. Seif ElDin (2016) f . . . . . . . . . . . . . 237

13.3. Distribution of Specimen Parameters . . . . . . . . . . . . . . . 237

13.3.1. Masonry Compression Strength . . . . . . . . . . . . . 238

13.3.2. Block Compression Strength _. . . . . . . . . . . . . 238

13.3.3. Mortar Compression Strength . . . . . . . . . . . . . . . 239

13.3.4. Grout Compression Strength . . . . . . . . . . . . . . . 240 
13.3.5. Vertical Reinforcement Ratio . . . . . . . . . . . . . . . . . 240

13.3.6. Horizontal Reinforcement Ratio . . . . . . . . . . . . . . . . 241

13.3.7. Aspect Ratio . . . . . . . . . . . . . . . . . . . . . . 241

13.3.8. Axial Load Ratio . . . . . . . . . . . . . . . . . . . . . . . . . . 242

13.4. SAFEM Results . . . . . . . . . . . . . . . . . . . 243

13.5. Discussion . . . . . . . . . . . . . . . . . . . . . 247

13.5.1. Masonry Strain at Peak Strength . . . . . . . . . . . . . . . 247

13.5.2. Modulus of Elasticity for Grouted Masonry . . . . . . . . . . . . . 247

13.5.3. Initial Masonry Poisson's Ratio . . . . . . . . . . . . . . . . . . 249

13.5.4. Block Tensile Strength . . . . . . . . . . . . . . . . . . 250

13.5.5. Mortar Bond Strength . . . . . . . . . . . . . . . . . . 250

13.5.6. Mortar Cohesion Strength . . . . . . . . . . . . . . . . 251

13.5.7. Grout Tensile Strength . . . . . . . . . . . . . . . . . 252

13.5.8. Effect of Masonry Compression Strength . . . . . . . . . . . . 253

13.5.9. Effect of Reinforcement . . . . . . . . . . . . . . 253

13.5.10Effect of Axial Load Ratio . . . . . . . . . . . . . . . . . 253

13.5.11Effect of Boundary Conditions . . . . . . . . . . . . 253

Chapter 14: Comparison of Single Pier Ungrouted Shear Wall Data and the Smeared Area Finite Element Model . . . . . . . . . . . . . . . . 255

14.1. Data Requirement . . . . . . . . . . . . . . . . . . . 255

14.2. Database Literature Review . . . . . . . . . . . . . . . . . 256

14.3. Distribution of Specimen Parameters . . . . . . . . . . . . 256

14.4. SAFEM Results . . . . . . . . . . . . . . . . . . 256

14.5. Discussion . . . . . . . . . . . . . . . . . . . . . 258

14.5.1. Masonry Strain at Peak Strength . . . . . . . . . . . . . 258

14.5.2. Modulus of Elasticity for Ungrouted Masonry . . . . . . . . . . . 259

14.5.3. Initial Masonry Poisson's Ratio . . . . . . . . . . . . . . . . . 260

14.5.4. Block Tensile Strength . . . . . . . . . . . . . . . . . . . 260

14.5.5. Mortar Bond Strength . . . . . . . . . . . . . . . . . 260

14.5.6. Mortar Cohesion Strength . . . . . . . . . . . . . . . . . 261

14.5.7. Effect of Masonry Material Properties . . . . . . . . . . . . . 262

14.5.8. Effect of Aspect Ratio and Axial Load . . . . . . . . . . . . 262

Chapter 15: Comparison of Reinforced Beam Data and the Smeared

Area Finite Element Model . . . . . . . . . . . . . . . . . . . . . . . . . . . 263

15.1. Data Requirements . . . . . . . . . . . . . . . . . . . . 263

Numerical Modelling of Hollow Concrete Block Masonry Mechanics 
15.2. Literature Review . . . . . . . . . . . . . . . . . . . 264

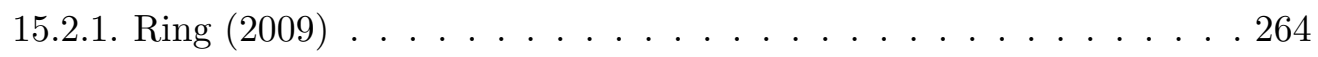

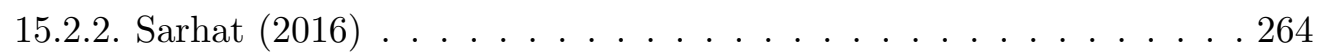

15.3. Distribution of Specimen Parameters ～. . . . . . . . . . . . . . 267

15.3.1. Masonry Compression Strength . . . . . . . . . . . . . . 267

15.3.2. Block Compression Strength _ . . . . . . . . . . . . . 268

15.3.3. Mortar Compression Strength . . . . . . . . . . . . . . 268

15.3.4. Grout Compression Strength _ . . . . . . . . . . . . . . . 269

15.3.5. Longitudinal Reinforcement Ratio . . . . . . . . . . . . . 270

15.3.6. Shear Reinforcement Ratio . . . . . . . . . . . . . . . . . 270

15.3.7. Shear Span-to-Depth Ratio . . . . . . . . . . . . . . . . . . 271

15.4. SAFEM Results . . . . . . . . . . . . . . . . . . . 271

15.5. Discussion . . . . . . . . . . . . . . . . . . 273

15.5.1. Masonry Strain at Peak Strength . . . . . . . . . . . . . 273

15.5.2. Modulus of Elasticity for Masonry . . . . . . . . . . . . . 274

15.5.3. Initial Masonry Poisson's Ratio . . . . . . . . . . . . . . . . 274

15.5.4. Block Tensile Strength . . . . . . . . . . . . . . . . 275

15.5.5. Mortar Bond Strength . . . . . . . . . . . . . . . 276

15.5.6. Mortar Cohesion Strength . . . . . . . . . . . . . . 276

15.5.7. Grout Tensile Strength . . . . . . . . . . . . . . . . 277

15.5.8. Block Web Interruption . . . . . . . . . . . . . . 278

15.5.9. Size Effect . . . . . . . . . . . . . . . . 278

15.5.10.Effect of Stirrups . . . . . . . . . . . . . . . 279

15.5.11.Effect of Crack Control Reinforcement . . . . . . . . . . . . 279

15.5.12.Strain Effect . . . . . . . . . . . . . . . . 280

Chapter 16: Part III Concluding Remarks . . . . . . . . . . . . . . . . . . 281

16.1. Part III Conclusions . . . . . . . . . . . . . . . . . . . . . . . 282

16.2. Part III Future Research . . . . . . . . . . . . . . . . . . . . . . . . . 284 
Part IV Masonry Displacement Based Modeling . . . . . . . 286

Chapter 17: Background . . . . . . . . . . . . . . . . . 288

17.1. Shear Wall Failure Modes . . . . . . . . . . . . . . . . . . . . 288

17.1.1. Studies of Shear Dominated Masonry Wall Behaviour . . . . . . 290

17.1.2. Studies of Flexure Dominated Masonry Wall Behaviour . . . . . . 292

17.1.3. Shear Span Ratio . . . . . . . . . . . . . . . . . . . . 293

17.2. Prediction of Nonlinear Response . . . . . . . . . . . . . . . . . 294

17.2.1. Beam-Column Models . . . . . . . . . . . . . . . . . . 294

17.2.2. Fiber Element Models . . . . . . . . . . . . . . . . . . 295

17.2.3. Strut-and-tie Models . . . . . . . . . . . . . . . . . . 296

17.2.4. Finite Element Models . . . . . . . . . . . . . . . . . 296

17.3. Background Summary _. . . . . . . . . . . . . . . . 297

17.4. Model Objectives . . . . . . . . . . . . . . . . . . . . 297

Chapter 18: 3PKT for Cantilevered Reinforced Masonry Shear Walls. . 298

18.1. Three-Parameter Kinematic Theory Applicability . . . . . . . . . . . . 298

18.2. Kinematics of Shear Walls . . . . . . . . . . . . . . . . . . . . . . 299

18.3. Geometry of Kinematic Model . . . . . . . . . . . . . . . . . . 302

18.4. Nine Spring Model . . . . . . . . . . . . . . . . . 306

18.4.1. Forces Transferred across the Critical Diagonal Crack . . . . . . 307

18.4.2. Forces in the Critical Loading Zone . . . . . . . . . . . . . . . 314

18.5. Calculations within the Fan . . . . . . . . . . . . . . . . . 319

Chapter 19: Implementation of 3PKT for Reinforced Masonry Shear

Walls . . . . . . . . . . . . . . . . . . . . . . . . . 322

19.1. Spring Stiffness's . . . . . . . . . . . . . . . . . . . . . 322

19.1.1. Aggregate Interlock Spring Stiffness _ . . . . . . . . . . . . 322

19.1.2. Transverse Reinforcement Spring Stiffness . . . . . . . . . . . . . . 323

19.1.3. Longitudinal Dowel Action Spring Stiffness . . . . . . . . . . . . 323

19.1.4. Compression in the Masonry Spring Stiffness's . . . . . . . . . . 323

19.1.5. Contact Spring Stiffness's . . . . . . . . . . . . . . . . . 324

19.1.6. Longitudinal Reinforcement in CLZ Spring Stiffness . . . . . . . . 324

19.1.7. Longitudinal Reinforcement Tension Spring Stiffness . . . . . . . . 325

19.1.8. Stiffness Matrix . . . . . . . . . . . . . . . . . 325

19.2. Three Degrees of Freedom . . . . . . . . . . . . . . . . 326

Numerical Modelling of Hollow Concrete Block Masonry Mechanics 
19.3. Transformation Matrix . . . . . . . . . . . . . . . . . . . 327

19.4. Converged Load Step Calculations . . . . . . . . . . . . . . . . 327

19.4.1. Shear Components . . . . . . . . . . . . . . . . 327

19.4.2. Moment . . . . . . . . . . . . . . . . . . . . . 328

19.5. Solution Procedure . . . . . . . . . . . . . . . . . . . . 328

19.6. 3PKT Procedure . . . . . . . . . . . . . . . . . . . . . 330

Chapter 20: 3PKM Verification. . . . . . . . . . . . . . . . 331

20.1. Shear Response . . . . . . . . . . . . . . . . . . . . . . . 332

20.1.1. Shear Carried by Aggregate Interlock . . . . . . . . . . . . . 332

20.1.2. Shear Carried by the CLZ . . . . . . . . . . . . . . . 334

20.1.3. Shear Carried by the Stirrups . . . . . . . . . . . . . . 335

20.1.4. Shear Carried by the Longitudinal Reinforcement . . . . . . . . . . 336

20.1.5. Shear Carried by the Contact Springs . . . . . . . . . . . . . . 337

20.1.6. Total Shear . . . . . . . . . . . . . . . . . . . . . . 337

20.2. Longitudinal Steel Reinforcement Response . . . . . . . . . . . . . . 338

20.3. Displacement Response ． . . . . . . . . . . . . . . . . . 341

Chapter 21: Comparison of Single Pier Grouted Shear Wall Data and

3PKM . . . . . . . . . . . . . . . . . . . . . . . . . 342

21.1. 3PKM for Masonry Results . . . . . . . . . . . . . . . . . . 342

21.2. Discussion . . . . . . . . . . . . . . . . . . 346

21.2.1. Pre-Peak Behaviour . . . . . . . . . . . . . . . 346

21.2.2. Post-Peak Behaviour . . . . . . . . . . . . . . . . . 346

Chapter 22: Part IV Concluding Remarks . . . . . . . . . . . . . . 347

22.1. Part IV Conclusions ． . . . . . . . . . . . . . . . . . . 348

22.2. Part IV Future Research . . . . . . . . . . . . . . . . . . . . . 348

Part V Thesis Conclusion. . . . . . . . . . . 350

Chapter 23: Summary, Conclusions and Future Research . . . . . . . . 352

23.1. Summary . . . . . . . . . . . . . . . . . . . . . . . . . . . . . . . . . .

23.2. Conclusions . . . . . . . . . . . . . . . . . . . 353

23.3. Thesis Contributions . . . . . . . . . . . . . . . . . . . 354

23.4. Future Research . . . . . . . . . . . . . . . . . 355

References. . . . . . . . . . . . . . . . . . 357

Appendix A: Ungrouted Prism Experimental Data . . . . . . . . . . . 369

Numerical Modelling of Hollow Concrete Block Masonry Mechanics 
Appendix B: SACM Input for Grouted Prism Experimental Data . . . . 374

Appendix C: Parallel Prism Experimental Data . . . . . . . . . . . . . . 379

Appendix D: Single Piear Grouted Shear Wall Smeared Area Finite

Element Model Results . . . . . . . . . . . . . . . . . . . . . . . . . 381

Appendix E: Single Piear Ungrouted Shear Wall Smeared Area Finite

Element Model Results . . . . . . . . . . . . . . . . . . . . . . . 577

Appendix F: Reinforced Beam Smeared Area Finite Element Model

Results . . . . . . . . . . . . . . . . . . . . . . . . . . 619

Appendix G: Three Parameter Kinematic Model Results . . . . . . . . . 669 


\section{LIST OF FIGURES}

Figure 2.1. Local State of Stresses in Masonry Prisms under Uniaxial Vertical Compression . . . . . . . . . . . . . . . 18

Figure 2.2. Predictive Ability of Sarhat and Sherwood Ungrouted Equation

(Sarhat and Sherwood, 2014) . . . . . . . . . . . . 22

Figure 2.3. Predictive Ability of Sarhat and Sherwood Grouted Equation (Sarhat and Sherwood, 2015) . . . . . . . . . . . . 23

Figure 2.4. Predictive Ability of Hamid Equations (Hamid, 1978) . . . . . . . 24

Figure 2.5. Cheema and Klingner FEM Model (Cheema and Klingner, 1986) . 25

Figure 2.6. Cheema and Klinger Failure Curves for Grouted Prisms (Cheema and Klingner, 1986) . . . . . . . . . . . . . . . 27

Figure 3.1. Area of Prism Being Modeled . . . . . . . . . . . . . . . . 30

Figure 3.2. Model Stress Assumptions . . . . . . . . . . . . . . . . . . 31

Figure 3.3. Model Strain Assumptions . . . . . . . . . . . . . . . . . 31

Figure 3.4. Dimensions of the Block, Mortar, and Grout fir a Two Cell Block 33

Figure 3.5. Hognestad Parabola . . . . . . . . . . . . . . . . . . . 41

Figure 3.6. Popovics Compression Model . . . . . . . . . . . . . . . . . 42

Figure 3.7. Smith-Young Model . . . . . . . . . . . . . . . . . . 43

Figure 3.8. Linear Compression Model . . . . . . . . . . . . . . . . . 44

Figure 3.9. Hognestad Parabola Compression Response with Strength Enhancement or Strength Degradation . . . . . . . . . . . . . 45

Figure 3.10. Tension Response . . . . . . . . . . . . . . . . . . . 46

Figure 3.11. Kupfer Variable Poisson's Ratio Model . . . . . . . . . . . . . . . 47

Figure 3.12. Failure Criteron ． . . . . . . . . . . . . . . . . . . 48

Figure 3.13. SACM Solution Algorithm . . . . . . . . . . . . . . 53

Figure 3.14. SACM Program Main Window . . . . . . . . . . . . . 56

Figure 3.15. SACM Program Model Running Window . . . . . . . . . . . . . 57

Figure 3.16. SACM Program Output . . . . . . . . . . . . 58

Figure 4.1. SACM Ungrouted Predictions . . . . . . . . . . . . . . . 63

Figure 4.2. SACM Grouted Predictions . . . . . . . . . . . . . . . . 69

Figure 4.3. SACM Inputs for Block Strain at Peak Strength . . . . . . . . . 70

Figure 4.4. SACM Inputs for Initial Block Poisson's Ratio . . . . . . . . . . . 71

Figure 4.5. SACM Inputs for Mortar Strain at Peak Strength . . . . . . . . . 72 
Figure 4.6. SACM Inputs for Initial Mortar Poisson's Ratio . . . . . . . . . . 73

Figure 4.7. SACM Inputs for Grout Strain at Peak Strength . . . . . . . . . . 74

Figure 4.8. SACM Inputs for Initial Grout Poisson's Ratio . . . . . . . . . . . 75

Figure 5.1. Mortar Compressive Strength Influence on Masonry Compressive Strength . . . . . . . . . . . . . . . . . . 79

Figure 5.2. Grout Compressive Strength Influence on Masonry Compressive Strength . . . . . . . . . . . . . . . . 80

Figure 5.3. Mortar Thickness Influence on Masonry Compressive Strength . . 81

Figure 5.4. Block Height Influence on Masonry Compressive Strength . . . . . 82

Figure 5.5. Block Percent Solid Influence on Masonry Compressive Strength . 83

Figure 5.6. Block Face Shell Thickness Influence on Masonry Compressive

Strength . . . . . . . . . . . . . . . . . . 84

Figure 5.7. Block Cracking Stress . . . . . . . . . . . . . . . 85

Figure 5.8. Block Tensile Strength Influence on Masonry Compressive Strength 86

Figure 5.9. Block Poisson's Ratio Influence on Masonry Compressive Strength 87

Figure 5.10. Mortar Poisson's Ratio Influence on Masonry Compressive Strength 88

Figure 5.11. Grout Poisson's Ratio Influence on Masonry Compressive Strength 89

Figure 5.12. Block Strain at Peak Stress Influence on Masonry Compressive Strength . . . . . . . . . . . . . . . . . 9 90

Figure 5.13. Mortar Strain at Peak Stress Influence on Masonry Compressive Strength . . . . . . . . . . . . . . . . . . . . 91

Figure 5.14. Grout Strain at Peak Stress Influence on Masonry Compressive Strength . . . . . . . . . . . . . . . . . . 92

Figure 6.1. SACM Ungrouted Full Bedded Mortar Predictions . . . . . . . . . 95

Figure 6.2. Comparison of Predictive Ability for the International Masonry Codes, SACM, and Proposed Formula for the Entire Database . . . . . . 109

Figure 7.1. Prism Configurations Used by Lee et al. (1984) . . . . . . . . . . . 111

Figure 7.2. Prism Configurations Used by Wong and Drysdale (1985) . . . . . 111

Figure 7.3. Prism Configurations Used by Khalaf (1997) . . . . . . . . . . . 112

Figure 7.4. Prism Configurations Used by Ring (2009) . . . . . . . . . . . . . 113

Figure 7.5. Web Interruption Effect of Compression Strength Parallel to Bed Joints $($ Ring, 2009) . . . . . . . . . . . . . . . . . 113

Figure 7.6. Typical Stress-Strain Curves of the Masonry Prisms Tested Normal and Parallel to the Bed Joint Grouted Predictions (Sarhat, 2016) . . 114 
Figure 7.7. Prism Configurations . . . . . . . . . . . . . . . . 115

Figure 7.8. Frame Equivalent System of the Block . . . . . . . . . . . . 116

Figure 7.9. Effect of End Restraint on Effective Length Factor . . . . . . . . . 117

Figure 7.10. Buckling of a Column in a Sway Frame (For a Stiff Beam Relative to Columns) . . . . . . . . . . . . . . . . . 118

Figure 7.11. Buckling of a Column in a Non-Sway Frame (For a Stiff Beam Relative to Columns) . . . . . . . . . . . . . . . . . . . 119

Figure 7.12. Wood's Method for Non-Sway Frame . . . . . . . . . . . . . . . 121

Figure 7.13. Wood's Method for Sway Frame . . . . . . . . . . . . . . . . . . . 121

Figure 7.14. Loading Parallel to Bed Joints . . . . . . . . . . . . . . . . . . 122

Figure 7.15. Strain Assumptions . . . . . . . . . . . . . . . . . . . . 123

Figure 7.16. SACM Parallel Results . . . . . . . . . . . . . . . . . . . 128

Figure 9.1. Joint Failure Criterion $($ Page, 1979) … . . . . . . . . . . 135

Figure 9.2. Experimental and Analytical Load Displacement Curves (Arya

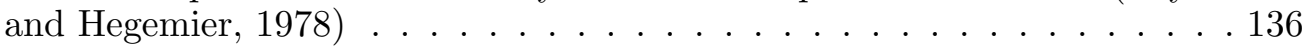

Figure 9.3. Failure Surface for Brickwork Under Biaxial Stress (Samarashinghe and Hendry, 1980) . . . . . . . . . . . . . . 138

Figure 9.4. Predicted and Experimental Crack Patterns (Samarashinghe and Hendry, 1980) . . . . . . . . . . . . . . . . . . . . 139

Figure 9.5. Experimental and Analytical Load Displacement Curves (Shing

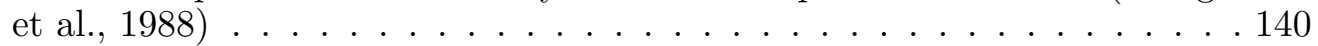

Figure 9.6. Facconi et al. (2014) Experimental and Analytical Load Displacement Curves . . . . . . . . . . . . . . . . . . 144

Figure 10.1. Global and Principal Stresses within a Region of Wall . . . . . . . 148

Figure 10.2. General Masonry Element Global Stresses . . . . . . . . . . . 150

Figure 10.3. Element Equilibrium Planes ． . . . . . . . . . . . . . . . 151

Figure 10.4. Element Average Stress Conditions . . . . . . . . . . . . . . . . 152

Figure 10.5. Mohr's Circle of Stress for the Element . . . . . . . . . . . . . 153

Figure 10.6. Reinforced Masonry Stress Conditions at Crack (Vecchio and Collins, 1986) . . . . . . . . . . . . . . . . . . 154

Figure 10.7. Masonry Aggregate Interlock (Vecchio and Collins, 1986) . . . . 155

Figure 10.8. Masonry Joint Stresses _ . . . . . . . . . . . . . . . . 156

Figure 10.9. Masonry Compatibility Conditions . . . . . . . . . . . . . . 157

Figure 10.10Poisson's Strain Effects (Selby and Vecchio, 1997) . . . . . . . 161

Numerical Modelling of Hollow Concrete Block Masonry Mechanics 
Figure 10.11Element Stresses at Angle $\theta$. . . . . . . . . . . . . . . 164

Figure 10.12Proposed Failure Envelope For Hamid and Drysdale (1982) Failure

Model . . . . . . . . . . . . . . . . . . 165

Figure 10.13Reinforced Masonry Stress Conditions (Hamid, 1978) . . . . . 167

Figure 10.14Property Value at $\theta_{\sigma} \ldots \ldots \ldots \ldots$. . . . . . . . . . . . .

Figure 10.15Hognestad Parabola . . . . . . . . . . . . . . . . . 171

Figure 10.16Popovics Compression Model . . . . . . . . . . . . . . . . . . 172

Figure 10.17Linear Compression Model . . . . . . . . . . . . . . . . . . 172

Figure 10.18Modified Park-Kent Post-Peak Compression Model . . . . . . . . 174

Figure 10.19Strength and Strain-softened Compression Response . . . . . . . . 175

Figure 10.20.Vecchio 1992-A Model Compression Softening Model (Vecchio and Collins, 1993) $\ldots \ldots \ldots \ldots \ldots \ldots$

Figure 10.21Hordjik Tension Softening Model . . . . . . . . . . . . . . . . 179

Figure 10.22.Vecchio Tension Stiffening Model _ . . . . . . . . . . . . . . 179

Figure 10.23Principal Poisson's Ratios . . . . . . . . . . . . . . . . . . . . 180

Figure 10.24Kupfer Variable Poisson's Ratio Model . . . . . . . . . . . . . . . 181

Figure 10.25Mohr-Coulomb Cracking Stress . . . . . . . . . . . . . . . . . 183

Figure 10.26Shear Stress-Strain Relationship for Bed and Head Joints . . . . . 186

Figure 10.27Hyperbolic Yield Criterion for Bed and Head Joints . . . . . . . . 187

Figure 10.28Steel Reinforcement Stress-Strain Response . . . . . . . . . . . . . 188

Figure 11.1. Natural Coordinates of Eight Node Isoparametric Quadrilateral Element . . . . . . . . . . . . . . . . . . . . . . . . 189

Figure 11.2. Guass Integration Points for Eight Node Isoparametric Quadrilateral Element . . . . . . . . . . . . . . . . . . . . . . 194

Figure 11.3. Definition of Secant Moduli (Wong et al., 2013) . . . . . . . . . 196

Figure 11.4. SAFEM Analysis Algorithm . . . . . . . . . . . . . . . . 201

Figure 11.5. SAFEM Program Material Input . . . . . . . . . . . . . . . . 204

Figure 11.6. SAFEM Program Mesh Generation . . . . . . . . . . . . . . . 204

Figure 11.7. SAFEM Program Model Running Window . . . . . . . . . . . . 205

Figure 12.1. Element Verification Mesh _. . . . . . . . . . . . 207

Figure 12.3. Equivalent Nodal Loads for the Mesh of Elements in Figure 12.1 . 211

Figure 12.2. Equivalent Nodal Loads for Eight Node Isoparametric Quadrilateral211

Figure 12.4. Straight Cantilever Element Layouts . . . . . . . . . . . . . . 216 
Figure 12.5. Thin Arched Beam (MacNeal and Harder, 1985) . . . . . . . . . . 218

Figure 12.6. Pure Bending of Cantilever Beam (Cen et al., 2015) . . . . . . . . 219

Figure 12.7. Cook's Skew Beam Problem . . . . . . . . . . . . . . . . . 220

Figure 12.8. Vertical Displacement Convergence . . . . . . . . . . . . . . . . . 221

Figure 12.9. Panel Specimen . . . . . . . . . . . . . . . . . . . . . . . 223

Figure 12.10Model Run Results . . . . . . . . . . . . . . . . . . . . . . 225

Figure 12.11Details of Wall SW22 (Lefas et al., 1990) . . . . . . . . . . . . 226

Figure 12.12 Model of SW22 f . . . . . . . . . . . . . . . 227

Figure 12.13Horizontal Force vs Tip Displacement Results . . . . . . . . . . 229

Figure 13.1. Priestley (1977) Experimental Wall . . . . . . . . . . . . . 231

Figure 13.2. Chen et al. (1978) Test Setup . . . . . . . . . . . . . . . . . . 232

Figure 13.3. Sveinsson et al. (1985) Experiment Setup . . . . . . . . . . . . . 234

Figure 13.4. Shedid et al. (2008) Experimental Wall . . . . . . . . . . . . . 236

Figure 13.5. Grouted Wall Masonry Compression Strength Histogram . . . . . 238

Figure 13.6. Grouted Wall Block Compression Strength Histogram . . . . . . . 238

Figure 13.7. Grouted Wall Mortar Compression Strength Histogram . . . . . . 239

Figure 13.8. Grouted Wall Grout Compression Strength Histogram . . . . . . . 240

Figure 13.9. Grouted Wall Vertical Reinforcement Ratio Histogram . . . . . . 240

Figure 13.10Grouted Wall Horizontal Reinforcement Ratio Histogram . . . . . 241

Figure 13.11Aspect Ratio Histogram ． . . . . . . . . . . . . . . . . . . . . 242

Figure 13.12Axial Load Ratio Histogram . . . . . . . . . . . . . . . . . . . . 242

Figure 13.13Shear Force $(V, \mathrm{kN})$ Verses Lateral Drift $(\delta=\Delta / a, \%)$ Response of Grouted Walls; Experimental and the SAFEM Predictions . . . . . . . 244

Figure 13.13Continued from Previous Page . . . . . . . . . . . . . . . . 245

Figure 13.13Continued from Previous Page . . . . . . . . . . . . . . 246

Figure 13.14SAFEM Inputs for Grouted Wall Masonry Strains . . . . . . . . . 247

Figure 13.15SAFEM Inputs for Grouted Wall Masonry Modulus of Elasticity . 249

Figure 13.16SAFEM Inputs for Grouted Wall Initial Masonry Poisson's Ratio 249

Figure 13.17SAFEM Inputs for Grouted Wall Block Tensile Strength . . . . . 250

Figure 13.18SAFEM Inputs for Grouted Wall Mortar Bond Strength . . . . . 251

Figure 13.19SAFEM Inputs for Grouted Wall Mortar Cohesion Strength . . . 252

Figure 13.20SAFEM Inputs for Grouted Wall Grout Tensile Strength . . . . . 252 
Figure 14.1. Shear Force $(V, \mathrm{kN})$ Verses Lateral Drift $(\delta=\Delta / a, \%)$ Response of Ungrouted Walls; Experimental and the SAFEM Predictions . . . . . . 257

Figure 14.2. SAFEM Inputs for Ungrouted Masonry Strains . . . . . . . . . 258

Figure 14.3. SAFEM Inputs for Ungrouted Masonry Modulus of Elasticity . 259

Figure 14.4. SAFEM Inputs for Ungrouted Initial Masonry Poisson's Ratio . . 260

Figure 14.5. SAFEM Inputs for Ungrouted Wall Mortar Bond Strength . . . . 261

Figure 14.6. SAFEM Inputs for Ungrouted Wall Mortar Cohesion Strength . . 262

Figure 15.1. Sarhat (2016) Beam Specimens . . . . . . . . . . . . . 266

Figure 15.2. Grouted Beam Masonry Compression Strength Histogram . . . 267

Figure 15.3. Grouted Beam Block Compression Strength Histogram . . . . . . 268

Figure 15.4. Grouted Beam Mortar Compression Strength Histogram . . . . . 269

Figure 15.5. Grouted Beam Grout Compression Strength Histogram . . . . . . 269

Figure 15.6. Grouted Beam Longitudinal Reinforcement Ratio Histogram . . 270

Figure 15.7. Grouted Beam Shear Reinforcement Ratio Histogram . . . . . . . 270

Figure 15.8. Grouted Beam Shear Span-to-Depth Ratio Histogram . . . . . . . 271

Figure 15.9. Load $(P, \mathrm{kN})$ Verses Relative Mid-Span Deflection $(\delta=\Delta / a, \%)$

Response of Grouted Beams; Experimental and the SAFEM Predictions . 272

Figure 15.10SAFEM Inputs for Grouted Beam Masonry Strains . . . . . . . . 273

Figure 15.11SAFEM Inputs for Grouted Beam Masonry Modulus of Elasticity 274

Figure 15.12SAFEM Inputs for Grouted Beam Initial Masonry Poisson's Ratio 275

Figure 15.13SAFEM Inputs for Grouted Beam Block Tensile Strength . . . . . 275

Figure 15.14SAFEM Inputs for Grouted Beam Mortar Bond Strength . . . . . 276

Figure 15.15SAFEM Inputs for Grouted Beam Mortar Cohesion Strength . . . 277

Figure 15.16SAFEM Inputs for Grouted Beam Grout Tensile Strength . . . . 277

Figure 15.17SM6 Experimental Beam Sarhat $(2016)$. . . . . . . . . . . . 279

Figure 17.1. Fiber Element Model . . . . . . . . . . . . . . . . . . 295

Figure 18.1. Kinematics of 3PKT (Hannewald, 2013) _ . . . . . . . . . 300

Figure 18.2. Three-Parameter Kinamatic Model for Shear Dominated Walls

(Hannewald, 2013) . . . . . . . . . . . . . . . . . . 303

Figure 18.3. Geometry of the Kinematic Model (Mihaylov et al., 2016) . . . . . 305

Figure 18.4. Spring Model Representing Load Bearing Mechanisms of the Rigid

Block (Mihaylov et al., 2016) . . . . . . . . . . . . . 306 
Figure 18.5. Spring Model Representing Load Bearing Mechanisms of the Fan (Mihaylov et al., 2016) . . . . . . . . . . . . . . . . 307

Figure 18.6. Definitions and Notations for Contact Density Model Li et al.

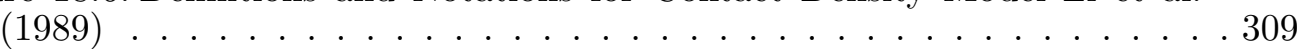

Figure 18.7. Elasto-Plastic Model for Contact Compressive Stress . . . . . . 310

Figure 18.8. Load-Deformation Response for the Aggregate Interlock Spring . 310

Figure 18.9. Transverse Reinforcement Stress Strain Relationship . . . . . . . . 312

Figure 18.10Longitudinal Reinforcement Stress Strain Relationship . . . . . . 313

Figure 18.11Dimensions of Confinement in the CLZ (Mander et al., 1988) . . . 316

Figure 18.12Angles in the CLZ . . . . . . . . . . . . . . . . . . 317

Figure 18.13Fan Calculation Steps . . . . . . . . . . . . . . . . . 321

Figure 19.1. 3PKT Model Solution Algorithm . . . . . . . . . . . . . . 329

Figure 19.2. 3PKT Program Main Window . . . . . . . . . . . . . . 330

Figure 20.1. Model Inputs Properties _ . . . . . . . . . . . . . . . . 331

Figure 20.2. Diagonal Crack Width . . . . . . . . . . . . . . . . 332

Figure 20.3. Crack Slip . . . . . . . . . . . . . . . . . . . 333

Figure 20.4. Shear Stress on Diagonal Crack . . . . . . . . . . . . . . . 333

Figure 20.5. Shear in Aggregate Interlock . . . . . . . . . . . . . . . 334

Figure 20.6. CLZ Force in Direction 1 . . . . . . . . . . . . . . 334

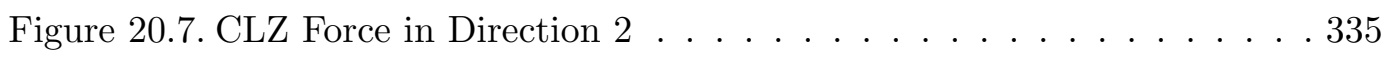

Figure 20.8. Shear in CLZ . . . . . . . . . . . . . . . . . 335

Figure 20.9. Shear in Stirrups . . . . . . . . . . . . . . . . 336

Figure 20.10Shear in Dowel Action . . . . . . . . . . . . . 336

Figure 20.11Shear in Contact Springs . . . . . . . . . . . . . . 337

Figure 20.12Total Shear . . . . . . . . . . . . . . . . . . 338

Figure 20.13Longitudinal Steel Strain Comparisons . . . . . . . . . . . . . 339

Figure 20.14Longitudinal Steel Stress Comparisons . . . . . . . . . . . . . 340

Figure 20.15Model Displacements $(\times 15) \ldots \ldots 44$

Figure 21.1. Shear Force $(V, \mathrm{kN})$ Verses Lateral Drift $(\delta=\Delta / a, \%)$ Response of Grouted Walls; Experimental and 3PKM Predictions . . . . . . . . . . 344

Figure 21.1. Continued from Previous Page . . . . . . . . . . . . . . 345 


\section{LIST OF TABLES}

Table 2.1. Parameters Influencing Ungrouted Masonry Compression Strength . 19

Table 2.2. Parameters Influencing Grouted Masonry Compression Strength . . 21

Table 3.1. Modified Material Configurations . . . . . . . . . . . . . 34

Table 3.2. SACM Input for Block Dimensions . . . . . . . . . . . . . . 52

Table 3.3. SACM Input for Block Material Properties . . . . . . . . . . . . 54

Table 3.4. SACM Input for Mortar . . . . . . . . . . . . . . . 55

Table 3.5. SACM Input for Grout . . . . . . . . . . . . . . . 55

Table 4.1. Ungrouted Experimental Range of Parameters . . . . . . . . . . . 60

Table 4.2. Ungrouted SACM Results . . . . . . . . . . . . . . . . . 61

Table 4.3. Grouted Experimental Range of Parameters . . . . . . . . . . . . . 64

Table 4.4. Grouted SACM Results . . . . . . . . . . . . . . . . . . 65

Table 5.1. Parametric Study Default Values _. . . . . . . . . . . . . 77

Table 5.2. The Significance of Parameters Studied and Their Value of Inves-

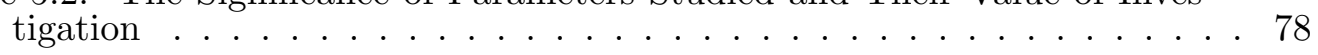

Table 6.1. Ungrouted SSACM Results . . . . . . . . . . . . . . . . . . . 97

Table 6.2. Grouted SSACM Results . . . . . . . . . . . . . . . . . . . 99

Table 6.3. CSA304-14 Unit Strength Values . . . . . . . . . . . . . . . 105

Table 6.4. 2013 MSJC Unit Strength Values . . . . . . . . . . . . . . . 105

Table 6.5. Results of International Masonry Codes, SACM, and Proposed

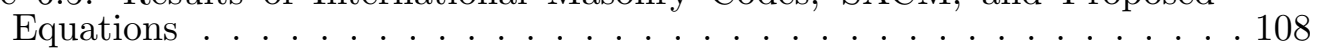

Table 7.1. Effective Stiffness of Beam . . . . . . . . . . . . . . . . 120

Table 7.2. Ungrouted Parallel Results . . . . . . . . . . . . . . . . . . 127

Table 7.3. Grouted Parallel Results . . . . . . . . . . . . . . . . 127

Table 11.1. 3 x 3 Gauss Integration Points and Weights . . . . . . . . . . . 194

Table 11.2. Ungrouted SAFEM Inputs . . . . . . . . . . . . . . . . 202

Table 11.3. Reinforcement SAFEM Inputs . . . . . . . . . . . . . . . 203

Table 11.4. Constitutive Model Options . . . . . . . . . . . . . . . . . . 203

Table 12.1. Coordinates of Nodes . . . . . . . . . . . . . . . . . 208

Table 12.2. Determinants of the Jacobian Matrices . . . . . . . . . . . . . . 210 
Table 12.3. Nodal Displacements and Forces for $\sigma_{y}=10 \mathrm{~N} / \mathrm{mm} \ldots . . .213$

Table 12.4. Element Stresses for $\sigma_{y}=10 \mathrm{~N} / \mathrm{mm} \ldots \ldots$. . . . . . . . . . 214

Table 12.5. Nodal Displacements and Forces for $\sigma_{x}=10 \mathrm{~N} / \mathrm{mm}$. . . . . . . . . 215

Table 12.6. Element Stresses for $\sigma_{x}=10 \mathrm{~N} / \mathrm{mm} \ldots \ldots \ldots$. . . . . . . 216

Table 12.8. Pure Bending Cantilever Beam Displacement at Tip . . . . . . . . . 217

Table 12.7. Straight Cantilever Beam Displacement in Direction of Load . . . . 217

Table 12.9. Cook's Beam Displacement at Point A . . . . . . . . . . . . . . 221

Table 12.10Panel Test Material Properties . . . . . . . . . . . . . . . . . 224

Table 12.11Constitutive Models Included in Model Runs . . . . . . . . . . . . . 224

Table 12.12Material Properties in Each Zone . . . . . . . . . . . . . . . . . 228

Table 13.1. Effect of Aspect Ratio on Deflection Due to Shear . . . . . . . . . . 254 


\section{LIST OF SYMBOLS}

$a \quad=$ maximum aggregate size, $\mathrm{mm}$

$=$ shear span, $\mathrm{mm}$

$A_{\text {cell }}^{b l} \quad=$ area of hollow cell within the block, $\mathrm{mm}^{2}$

$A_{s} \quad=$ one-half of total area of longitudinal reinforcement, $\mathrm{mm}^{2}$

$A_{v} \quad=$ area of transverse reinforcement resisting shear, $\mathrm{mm}^{2}$

$A_{x}^{b l}=$ block area in the $x$-direction, $\mathrm{mm}^{2}$

$A_{x}^{g r b l}=$ grout area within block in the $x$-direction, $\mathrm{mm}^{2}$

$A_{x}^{g r m r}=$ grout area within mortar in the $x$-direction, $\mathrm{mm}^{2}$

$A_{x}^{m r} \quad=$ mortar area in the $x$-direction, $\mathrm{mm}^{2}$

$A_{y}^{b l}=$ block area in the $y$-direction, $\mathrm{mm}^{2}$

$A_{y}^{g r b l}=$ grout area within block in the $y$-direction, $\mathrm{mm}^{2}$

$A_{y}^{g r m r}=$ grout area within mortar in the $y$-direction, $\mathrm{mm}^{2}$

$A_{y}^{m r} \quad=$ mortar area in the $y$-direction, $\mathrm{mm}^{2}$

$A_{z}^{b l} \quad=$ block area in the $z$-direction, $\mathrm{mm}^{2}$

$A_{z}^{g r b l}=$ grout area within block in the $z$-direction, $\mathrm{mm}^{2}$

$A_{z}^{g r m r}=$ grout area within mortar in the $z$-direction, $\mathrm{mm}^{2}$

$A_{z}^{m r} \quad=$ mortar area in the $z$-direction, $\mathrm{mm}^{2}$

$[B]=$ strain-displacement matrix

$c=$ cohesive strength, $\mathrm{MPa}$

$C_{d} \quad=$ compression softening factor

$[D] \quad=$ composite material stiffness matrix, $\mathrm{MPa}$

$\left[D^{b l}\right] \quad=$ block material stiffness matrix, $\mathrm{MPa}$

$\left[D^{g r b l}\right]=$ grout within block material stiffness matrix, $\mathrm{MPa}$

$\left[D^{g r m r}\right]=$ grout within mortar material stiffness matrix, $\mathrm{MPa}$

$\left[D_{m}\right] \quad=$ masonry material stiffness matrix, $\mathrm{MPa}$

$\left[D^{m r}\right] \quad=$ mortar material stiffness matrix, $\mathrm{MPa}$

$\left[D_{s}\right] \quad=$ reinforcement material stiffness matrix, $\mathrm{MPa}$

$d_{1}=$ distance from compressive edge of section to furthest tension longitudinal bar, $\mathrm{mm}$

$d \quad=$ effective depth, $\mathrm{mm}$

$d_{b} \quad=$ diameter of reinforcing bar, $\mathrm{mm}$

$\bar{E}_{1} \quad=$ secant modulus of elasticity in first principal direction, $\mathrm{MPa}$

$\bar{E}_{2} \quad=$ secant modulus of elasticity in second principal direction, $\mathrm{MPa}$

$\bar{E}_{b l} \quad=$ block secant modulus of elasticity, $\mathrm{MPa}$

$\bar{E}_{g r} \quad=$ grout secant modulus of elasticity, MPa

$E_{m} \quad=$ masonry modulus of elasticity, $\mathrm{MPa}$

$\bar{E}_{m 1} \quad=$ masonry secant modulus of elasticity in the first principle direction, $\mathrm{MPa}$

$\bar{E}_{m 2} \quad=$ masonry secant modulus of elasticity in the second principle direction, $\mathrm{MPa}$

$\bar{E}_{m r} \quad=$ mortar secant modulus of elasticity, $\mathrm{MPa}$

$E_{0} \quad=$ initial modulus of elasticity, $\mathrm{MPa}$ 


\begin{tabular}{|c|c|c|}
\hline$E_{r}^{b l}$ & $=$ & block reduced modulus of elasticity, $\mathrm{MPa}$ \\
\hline $\bar{E}_{s x}$ & . & $\begin{array}{l}\text { reinforcement secant modulus of elasticity in the } x \text { - } \\
\text { direction, MPa }\end{array}$ \\
\hline sy & $=$ & $\begin{array}{l}\text { reinforcement secant modulus of elasticity in the } y \text { - } \\
\text { direction, MPa }\end{array}$ \\
\hline$E_{t}^{b l}$ & $=$ & block tangent modulus of elasticity, $\mathrm{MPa}$ \\
\hline$(\Gamma)$ & & total nodal force vector, $\mathrm{N}$ \\
\hline$\left\{F^{E A}\right\}$ & $=$ & externally applied force vector, $\mathrm{N}$ \\
\hline$\left\{F^{o}\right\}$ & $=$ & pseduo load vector, $\mathrm{N}$ \\
\hline$F_{b}$ & $=$ & compression forces at the base of fan, $\mathrm{N}$ \\
\hline$F_{C L Z}$ & $=$ & compression force in the masonry of the CLZ, $\mathrm{N}$ \\
\hline$F_{c n}$ & $=$ & $\begin{array}{l}\text { normal contact force at the bottom of the critical } \\
\text { diagonal crack, } N\end{array}$ \\
\hline$F_{c t}$ & $=$ & $\begin{array}{l}\text { tangential contact force at the bottom of the critical } \\
\text { diagonal crack, } \mathrm{N}\end{array}$ \\
\hline$F_{m i}$ & $=$ & aggregate interlock force, $\mathrm{N}$ \\
\hline$F_{d}$ & $=$ & dowel action force, $\mathrm{N}$ \\
\hline$F_{l, C L Z}$ & $=$ & force in longitudinal reinforcement in the CLZ, N \\
\hline$F_{s}$ & $=$ & force in the stirrups, $\mathrm{N}$ \\
\hline$F_{t}$ & $=$ & n longitudinal tension reinforcement $A_{s}, \mathrm{~N}$ \\
\hline$f_{b}$ & $=$ & section, $\mathrm{MPa}$ \\
\hline$f_{b l}^{\prime}$ & $=$ & block compression strength, MPa \\
\hline & $=$ & buckling strength of the block's face shell, MPa \\
\hline$f_{c, r e p}^{\prime}, b l$ & $=$ & reported block compression strength, $\mathrm{MPa}$ \\
\hline$f_{m, C L Z}$ & $=$ & ive stress in CLZ, MPa \\
\hline$f_{c}^{\prime}$ & $=$ & ength, MPa \\
\hline$f_{c r}^{\prime}$ & $=$ & cracking strength, $\mathrm{MPa}$ \\
\hline$f_{g r}^{\prime}$ & $=$ & grout compression strength, $\mathrm{MPa}$ \\
\hline$f_{c, r e p}^{\prime}$ & $=$ & reported grout compression strength, $\mathrm{MPa}$ \\
\hline$f_{m}^{\prime}$ & 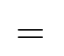 & trength, $\mathrm{MPa}$ \\
\hline$f_{m 1}$ & $=$ & first principal maso \\
\hline$f_{m 2}$ & $=$ & second principal masonry stress, $\mathrm{MPa}$ \\
\hline$f_{m x}^{\prime}$ & $=$ & average masonry axial stress in the $x$-direction, $\mathrm{MPa}$ \\
\hline$f_{m y}^{\prime}$ & $=$ & average masonry axial stress in the $y$-direction, $\mathrm{MPa}$ \\
\hline$f_{m r}^{\prime}$ & $=$ & mortar compression strength, $\mathrm{MPa}$ \\
\hline$f_{c, r e p}^{\prime}, m r$ & $=$ & reported $n$ \\
\hline$f_{n b j}$ & $=$ & normal to the mortar bed joint, $\mathrm{MPa}$ \\
\hline$f_{n h j}$ & . & average stress normal to the mortar head joint, $\mathrm{MPa}$ \\
\hline$f_{p}$ & $=$ & peak compression strength, MPa \\
\hline$f_{s c}$ & $=$ & $\begin{array}{l}\text { local stress at a crack of reinforcement parallel to the } \\
x \text {-direction, } \mathrm{MPa}\end{array}$ \\
\hline$f_{s c r_{y}}$ & $=$ & $\begin{array}{l}\text { local stress at a crack of reinforcement parallel to the } \\
y \text {-direction, } \mathrm{MPa}\end{array}$ \\
\hline$f_{s x}$ & $=$ & $\begin{array}{l}\text { average stress of reinforcement parallel to the } x \text { - } \\
\text { direction, } \mathrm{MPa}\end{array}$ \\
\hline
\end{tabular}




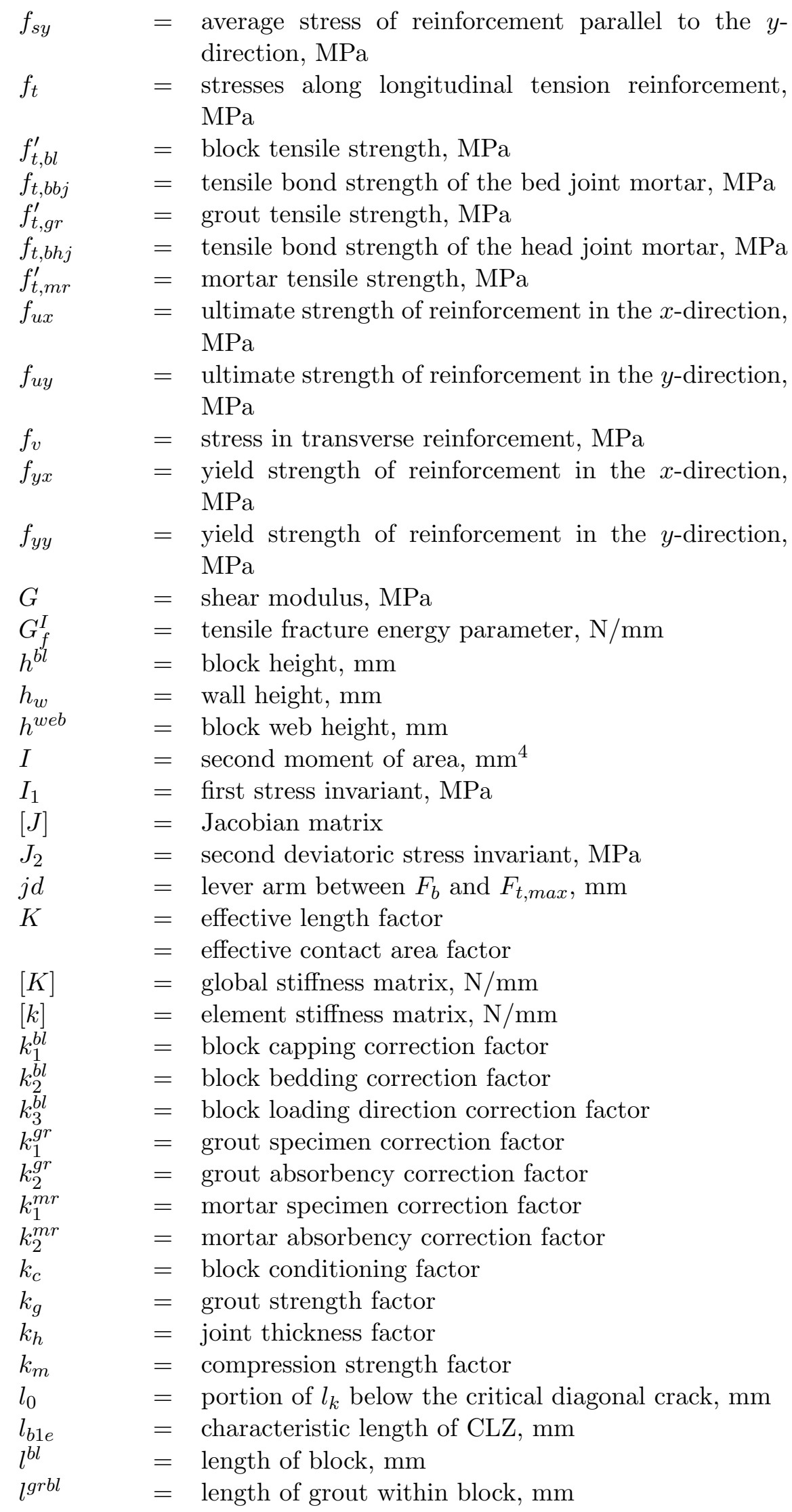




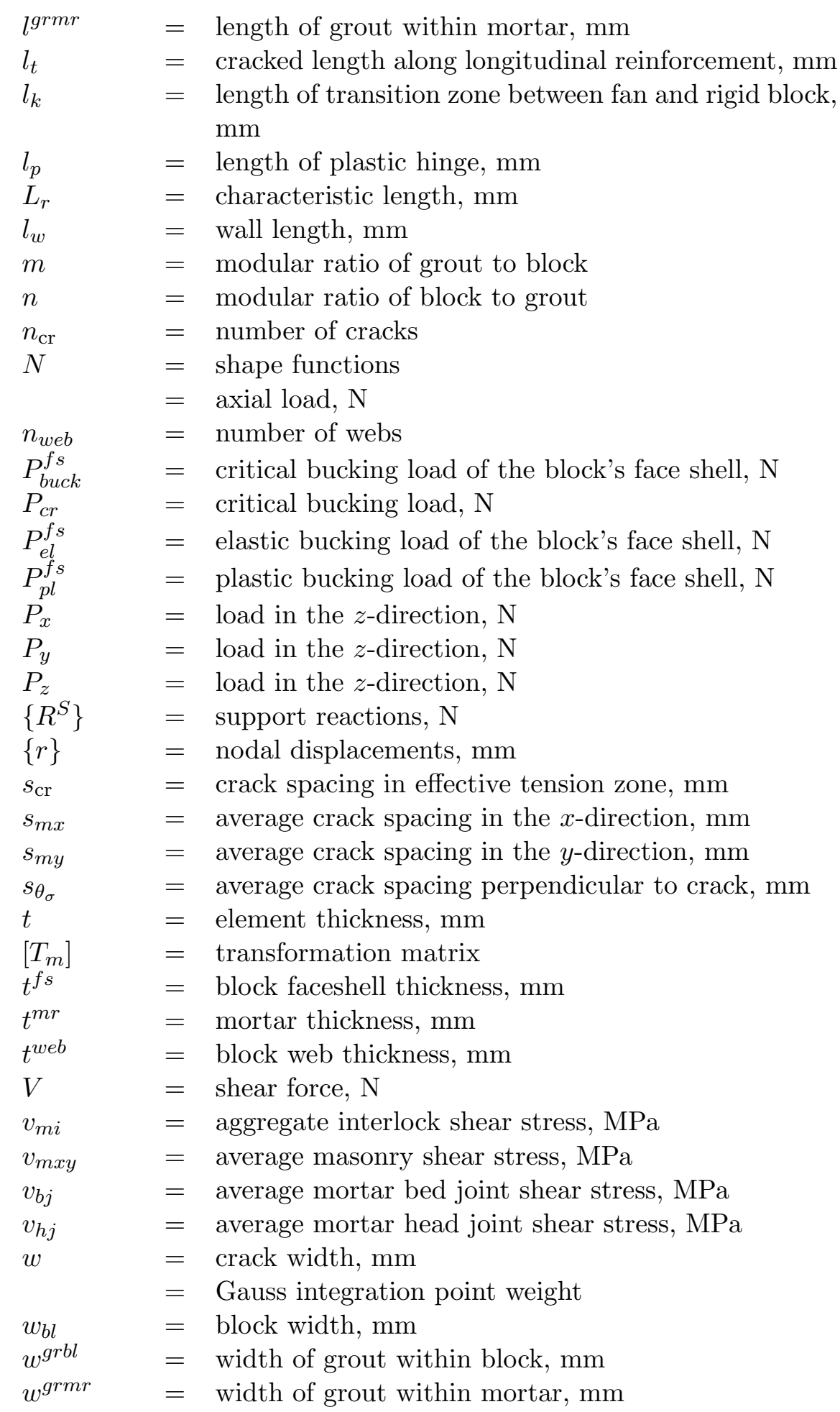


$\alpha$

$\alpha_{1}$

$\beta_{c s}$

$\beta_{a b}$

$\gamma$

$\gamma_{12}$

$\gamma_{b l}$

$\gamma_{g r}$

$\gamma_{s}$

$\Delta$

$\Delta_{c}$

$\Delta_{c y}$

$\delta_{s}$

$\delta_{x}$

$\delta_{y}$

$\{\epsilon\}$

$\epsilon_{b}$

$\epsilon_{b j}^{s}$

$\epsilon_{C L Z}$

$\epsilon_{\mathrm{cr}}$

$\epsilon_{\mathrm{cr}}^{s}$

$\epsilon_{h j}^{s}$

$\epsilon_{m 1}$

$\epsilon_{m 2}$

$\left\{\epsilon_{m}\right\}$

$\left\{\epsilon_{m}^{s}\right\}$

$\left\{\epsilon_{m}^{v}\right\}$

$\epsilon_{O}$

$\epsilon_{O x}$

$\epsilon_{\text {oy }}$

$\epsilon_{p}$

$\epsilon_{s h}$

$\left\{\epsilon_{s}^{p}\right\}$

$\epsilon_{t}$

$\epsilon_{t, a v g}$

$\epsilon_{u}$

$\epsilon_{v}$

$\epsilon_{x}$

$\epsilon_{x}^{b l}$

$\epsilon_{x}^{g r b l}$

$\epsilon_{x}^{g r m r}$

$\epsilon_{x}^{m r}$

$\epsilon_{y}$
$=$ ratio of mortar thickness to block height

$=$ angle of critical crack

$=$ strength enhancement or degradation factor

$=$ compression softening factor

$=$ anisotropic behavior factor

$=$ ratio of web height to block height

$=$ shear strain

$=$ block unit weight

$=$ grout unit weight

$=$ shear slip strain

$=$ applied lateral displacement, $\mathrm{mm}$

$=$ horizontal displacement of the CLZ, mm

$=$ vertical displacement of the CLZ, $\mathrm{mm}$

$=$ slip along crack, mm

$=x$ displacement of points in the wall, $\mathrm{mm}$

$=y$ displacement of points in the wall, $\mathrm{mm}$

$=$ total strain vector

$=$ strains along the base section

$=$ strain due to slip along mortar bed joint

$=$ average stain in CLZ

$=$ cracking strain

$=$ strain due to slip along crack

$=$ strain due to slip along mortar head joint

$=$ net masonry strain in first principal direction

$=$ net masonry strain in second principal direction

$=$ net masonry strains

$=$ masonry slip strains

$=$ masonry Poisson's strains

$=$ masonry compressive strain corresponding to $f_{m}^{\prime}$

$=$ masonry compressive strain corresponding to $f_{m x}^{\prime}$

$=$ masonry compressive strain corresponding to $f_{m y}^{\prime}$

$=$ strain at peak compression strength

$=$ reinforcement strain hardening strain

$=$ prestress strain in the reinforcement vector

$=$ strains along longitudinal tension reinforcement

$=$ average longitudinal reinforcement strain

$=$ reinforcement ultimate strain

$=$ strain in transverse reinforcement

$=$ strain distribution in the $x$-direction

$=$ block strain distribution in the $x$-direction

$=$ grout within block strain distribution in the $x$ direction

$=$ grout within mortar strain distribution in the $x$ direction

$=$ mortar strain distribution in the $x$-direction

$=$ strain distribution in the $y$-direction

$=$ reinforcement yield strain 


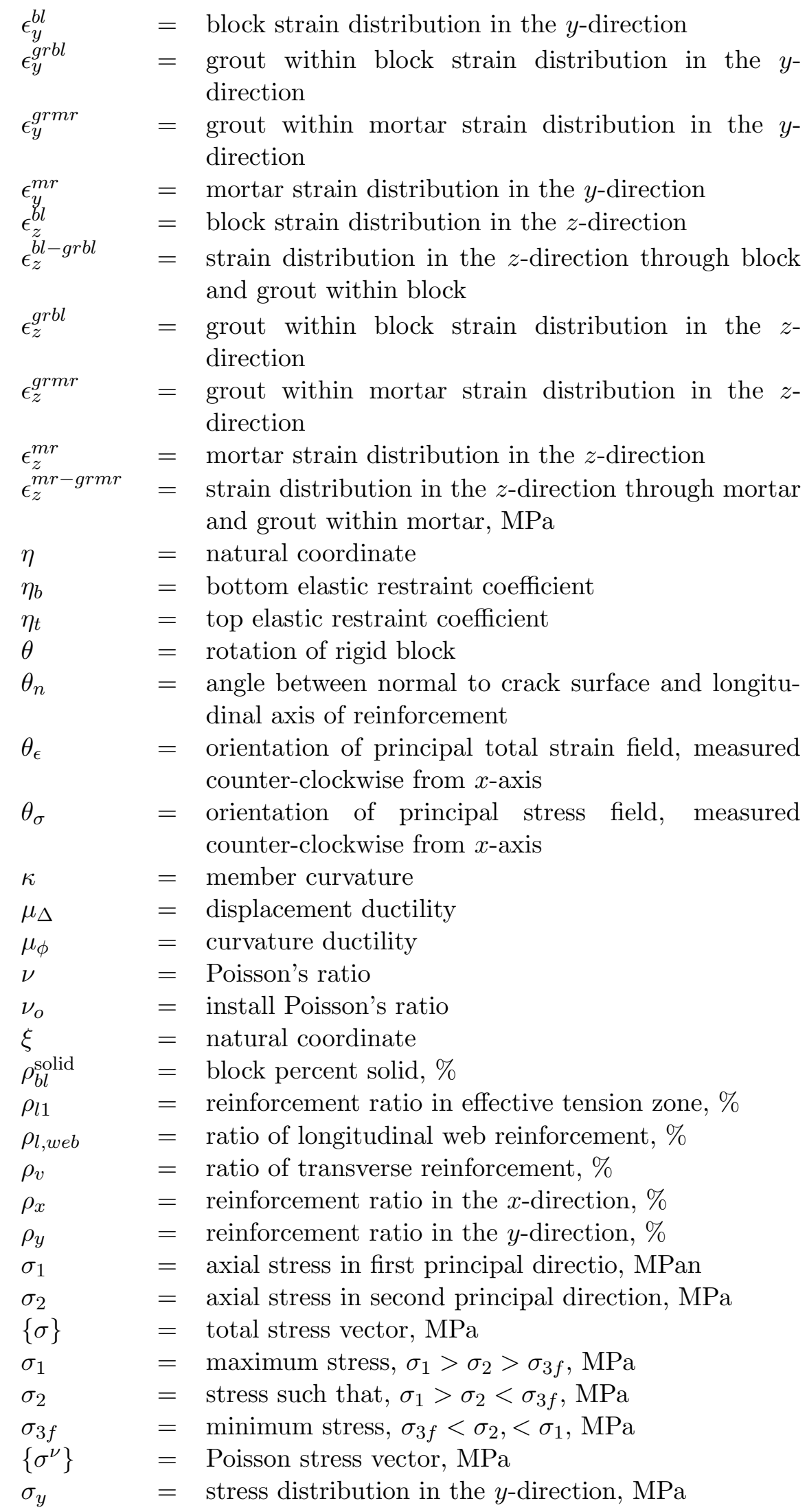




$\begin{aligned} \sigma_{x} & \text { stress distribution in the } x \text {-direction, } \mathrm{MPa} \\ \sigma_{z}^{b l-g r b l}= & \text { stress distribution in the } z \text {-direction through block } \\ & \text { and grout within block, } \mathrm{MPa} \\ \sigma_{z}^{m r-g r m r} & \text { stress distribution in the } z \text {-direction through mortar } \\ & \text { and grout within mortar, } \mathrm{MPa} \\ \tau_{12} & \text { shear stress, } \mathrm{MPa} \\ \phi & =\end{aligned}$




\section{LIST OF ABBREVIATIONS}

3PKM Three-Parameter Kinematic Model

3PKT Three-Parameter Kinematic Theory

CDM Contact Density Model

CLZ Critical Loading Zone

DOF Degree of Freedom

DSACE Design Smeared Area Compression Equations

DSFT Disturbed Stress Field Theory

MCFT Modified Compression Field Theory

SACM Smeared Area Compression Model

SAFEM Smeared Area Finite Element Model

SSACM Simplified Smeared Area Compression Model 


\section{PART I}

\section{THESIS INTRODUCTION}


Part I presents an overall context into which this thesis can be placed. In contains a general introduction, inspiration for the current study and the overall goals of the thesis.

Part I: Thesis Introduction 


\section{INTRODUCTION}

To show the long use of masonry, a historical background is presented. Despite its long use, the mechanics of hollow concrete block masonry are not fully understood. Current design codes are typically based "rules-of-thumb" rather then rational material model. There is a need for a further understanding of hollow concrete block masonry mechanics to allow for the proposal of new design methods. One way to do this is through numerical modelling. This thesis presents three numerical models to describe hollow concrete block masonry mechanics from the micro to the macro level. Additionally, a number of equations are presented to estimate key masonry parameters.

Masonry is the oldest documented construction material. The longevity of masonry as a building material has permitted ancient masonry structures to outlast other ancient structures built from organic materials such as wood.

Although masonry structures have been built since the earliest days of human civilization, it has only been in relatively recent times that masonry structures have been designed using engineering principles. Stone and brick masonry structures, designed and built without steel reinforcing to support only gravity loads, have given way to reinforced masonry with sufficient deformation capabilities necessary to resist the lateral dynamic forces of wind and major earthquakes. Lately, the engineering community has demonstrated great interest in the development of sophisticated numerical tools for the analysis and design of masonry structures to replace traditional rules-of-thumb or empirical design approaches. Adopting existing numerical tools from more advanced research fields, such as concrete and composite materials, is hindered by the particular characteristics of masonry. The major difficulty in modeling masonry is how to take into account the highly anisotropic nature of masonry resulting from blocks, being connected together by mortar joints with the possibility of grout and reinforcement in the block's hollow cells.

\subsection{Masonry History}

A brief history of masonry, to demonstrate the long use of masonry as a building material and a number of the "rules-of-thumb" used to construct masonry in the past. 


\subsubsection{Ancient}

The first documented masonry structures were built in pre-historic times in the Mediterranean basin. In this rocky area with little timber for construction, these primitive structures were dome-shaped and constructed by stacking stones one upon another. This method was made easier by using smaller, lighter blocks in the higher courses. Eventually, they would expand this technology to building half-domes and corbelled arches (Escrig, 2006).

The first use of clay masonry occurred in the Mesopotamian Plains and Nile River Valley. In these areas the scarcity of stone and timber compelled the inhabitants to search for other construction materials. Mesopotamians were the first to use unfired mud-bricks and the practice later spread to Egypt. These early Mesopotamian mud-brick buildings continued the earlier tradition of constructing either round or oval-shaped structures. Eventually rectangular structures with arched roofs and stone foundations took over as normal practice (Escrig, 2006).

The use of mud bricks facilitated the development and wide-spread use of the arch within the Fertile Crescent region. The bricks were light enough for a single worker to carry and could be easily formed or shaped into voussoirs for use in arches. Each arch was constructed from concentric, inclined rings. As each ring was constructed the voussoirs partially rested on the previously completed ring. The friction between the adjacent rings was sufficient to keep the partially-built ring standing until the key stone could be placed (Fletcher, 1996).

In Egypt, mud-brick masonry became the predominant form of construction. Walls of buildings were typically tapered from the bottom to the top for added stability since the roof provided little lateral support. This practice was a distinction of Egyptian architecture that would influence the design of future structures (Fletcher, 1996).

In the twenty-seventh century BC, in the Third Dynasty, Egyptians begin to use limestone hewn from nearby outcroppings. The royal tombs steadily increased in size with each pharaoh trying to out-do the accomplishments of the previous. Gradually, the Egyptian tombs grew in height and the walls became steeper. This practice continued such that the walls met at an apex and a pyramid was formed (Fletcher, 1996).

During the New Kingdom, Egyptian focus shifted away from building pyramids and towards building smaller, yet more elaborate tombs and temples. The new designs did not provide the same amount of lateral stability that was inherent in the pyramids.

Part I: Thesis Introduction 
Similarly, the new structures contained more usable space. The new challenge was to find a stable way of transferring all gravity loads downward. The Egyptians met this challenge by making another advance in masonry construction through the use of vertical stone columns (Fletcher, 1996).

While the Egyptians were using hewn limestone for their monumental structures, early Mesopotamians discovered that firing their mud bricks in a fire allowed them to became substantially stronger and weathered much better. Together with bitumen-based mortar they constructed numerous large edifices and ziggurats, using the fired brick in areas subject to higher stresses. Later, the Babylonians developed this technology further by creating glazed bricks, which they used in some of their most important buildings (Fletcher, 1996).

\subsubsection{Classical}

Through their trade empire, the Minoans came into contact with the Egyptian masonry technologies and architecture and spread this knowledge to the Mycenaeans in Greece. The Greeks continued the column, lintel, and triangular arch components of the Egyptian stonework and also incorporated the use of corbelled arches which they had previously developed. This knowledge blossomed into new architectural styles during the Classical and Hellenistic Greek periods. Additionally, the Greeks made stone columns more slender and used keys between column sections to align them and allowing them to resist lateral shear forces (Fletcher, 1996). The discovery of pozzolanic cement created a new, stronger mortar to be used in masonry construction (Hegemier et al., 2003). Much of the Greek architecture and practice was adopted by the Romans at the outset of Roman rule.

The Romans had a large influence on the further development and spreading of masonry knowledge. They were skilled at incorporating existing technology from other cultures, innovating new ones, and spreading them throughout their broad reach. The vastness of the Roman Empire put them in contact with the masonry technologies of Mesopotamia, Egypt, and Greece (Fletcher, 1996). The first innovation of the Romans was to combine the arch technology from Mesopotamia with the stonework and concrete technology from Greece to form large stone arches (Malhotra and Mehta, 2003).

Through Roman masonry technology stone masonry walls became more slender, creating more usable space within the structure. The next step was to construct walls in an annular manner to form hollow towers. The cross-section of a tower is efficient because the material at the extremities of the structure work as a couple to resist lateral

Part I: Thesis Introduction 
and overturning forces. The efficiency of the shape permitted the exterior walls to be relatively thinner compared to free-standing walls of comparable height. The Romans constructed many notable masonry walls, cities, and towers throughout Europe and the Near East during their rule (Drysdale and Hamid, 2005).

The Romans also made new developments in the use of the arch. Roman engineers understood that a semi-circular arch was not as efficient as a parabolic or catenary arch, but determined that the semi-circular shape was simplest to lay out and construct. Semi-circular arches use a single, constant radius with voussoirs that are all identically shaped. As Roman construction technology improved parabolic and catenary shapes were used (Drysdale and Hamid, 2005).

European masonry took on a new form in the Medieval period with the advent of Gothic architecture. The Europeans took the concept of the ribbed dome and generalized it for use in vaults (Escrig, 2006). By using ribbed vaults, the ribs could be constructed initially and the other portions of the vault could be constructed in sections. Another characteristic was the use of pointed arches, originally developed by the Byzantines, which decreased the amount of outward thrust at the base of the arch. The combination of ribbed vaults and pointed arches led to the creation of the Gothic vault, a characteristic feature of Gothic architecture (Fletcher, 1996).

With the development of flying buttresses, Gothic builders were able to reach astounding heights with their structures. The height and use of ribbed vaults enabled Gothic structures to include many more windows than had previously been used in masonry structures (Escrig, 2006).

\subsubsection{Contemporary}

The earliest appearance of modern reinforced masonry technology dates back to the early nineteenth century when iron hoops were placed in the mortar joints of two brick shafts in London. This construction technique was investigated further by several engineers throughout England. Another advance in masonry technology that occurred soon afterward was the discovery and use of portland cement as a cementitious agent in mortar. Both of these technologies were highly publicized in 1851 at the Great Exposition of London. The use of reinforced masonry did not spread to the United States and other nations until a century after its initial discovery in England (Schneider and Dickey, 1994).

Part I: Thesis Introduction 
The deficiencies of unreinforced masonry were highly publicized after the 1933 Long Beach earthquake in which 70 schools built of unreinforced masonry were completely destroyed. The State of California subsequently passed laws and new building codes that prohibited the use of unreinforced masonry (State of California Seismic Safety Commission, 2009). Over the next four decades, the vast majority of research on reinforced masonry was conducted within the State of California. The regulations in the Uniform Building Code (UBC) are mostly based on the findings from these research programs.

The understanding of reinforced masonry during the last half century has increased through the efforts of research institutions, both domestically and internationally. This research has been aided by more sophisticated testing and data collection systems, the advent of Finite Element Analysis, greater computational power, and the formation of research coalitions dedicated to the subject.

\section{$1.2 \quad$ Properties}

A brief introduction to the materials used to construct masonry and some of their basic mechanics.

\subsubsection{Hollow Concrete Blocks}

Hollow concrete blocks are manufactured from portland cement, aggregate, and water, but can also include fly ash and other additives such as air en-training agents and workability agents. In the manufacturing process, a very dry zero-slump concrete is delivered to a block machine. The concrete is pressed and vibrated into steel moulds having the desire shape of the block. The block percent solid is typically in the range from 50 to $60 \%$ for hollow blocks. Stress-strain curves for hollow concrete blocks under axial compression are similar to those of concrete, with the shape of the curve, particularly at higher stress levels, sensitive to the type of capping used and the resulting platen restraint. Flexural tensile strengths typically range between 10 and $20 \%$ of the block compression strength. Direct tension test result in tension splitting across the block's face shells typically in the range of 8 to $16 \%$ of the compression strength.

\subsubsection{Mortar}

In North America, mortars are grouped into types based on their proportioning of lime and are designated as M, S, N, O, and K. Compressive strength and ductility are related to the mortar type, with the first type being the strongest and least ductile mortar 
and the last type being the weakest but most ductile. Increasing the proportion of lime within mortar decreases the compressive strength of the mortar but increases its ductility. The use of lime in mortar greatly increases the workability and longevity of wet mortar by increasing its ability to retain moisture. The ease of use helps to decrease errors in construction and reduce variability within masonry walls. Another benefit of using lime in mortar is the ability over time for cracks in the mortar to heal through autogenous healing.

\subsubsection{Grout}

Masonry grout is a mixture of portland cement concrete wherein the aggregate sizes are limited to sizes smaller than those typically used in normal concrete. Grout must have a high slump for it to flow down the masonry cores and fill the voids around reinforcement. High-slump grout is created through the use of plasticizers within the grout mix. There are several purposes of grouting masonry. The primary purpose is to transfer loads from the masonry to the reinforcement, so that the two act as a single unit. The grout also serves to cover and protect the reinforcement from corrosion, permits the full development of strength in the reinforcing bars at splices and hooks, and adds to the net shear area, thereby increasing shear capacity.

\subsubsection{Reinforcement}

Reinforcement used in masonry is generally made from steel, though carbon fiber and other fibrous media are sometimes employed. The deformed bars used in masonry are the same as those used in reinforced concrete construction. Deformed bars are placed vertically within the cores of the masonry blocks and horizontally within depressed webs of blocks or open lintel blocks and are grouted in place.

Reinforcement can be placed in masonry shear walls in two orthogonal directions, horizontally and vertically. In the case of shear walls the horizontal reinforcement is also called shear reinforcement because it is laid parallel to and is most effective in resisting the lateral in-plane shear forces. As wall panels develop diagonal shear cracks from increasing shear forces the shear reinforcement spans these cracks and provides means of resisting the lateral tensile forces from one side of the crack to the other. For this reason, the two terms are used interchangeably within the literature. The vertical reinforcement is sometimes called the flexural reinforcement because it resists the tensile forces created by the overturning moment within the wall. This term can be somewhat of a misnomer because it is the vertical reinforcement nearest the ends of the wall that is must effective in resisting in-plane moment within the wall. The vertical reinforcement

Part I: Thesis Introduction 
in the interior of the wall panel is less effective in resisting the in-plane moment due to its proximity to the centroidal axis of the wall. The interior reinforcement acts in resisting any out-of-plane moment forces and reduce crack width, as well as sliding at the base of the wall.

A second type of reinforcement used in masonry walls is welded wire reinforcement. It consists of two parallel wires that are attached together in one of two ways. In laddertype reinforcement, short bars that run perpendicular to the two longer parallel wires and are welded at regular intervals. In truss-type reinforcement, a third continuous wire is bent into a zigzag shape and welded onto the other two bars at its corners. The purposes of the central wires in each type are to maintain the two primary wires at a uniform distance apart, to keep the primary wires straight, and to provide some lateral anchorage to the wires when they are embedded within the wall. Both truss- and ladder-type reinforcement are used primarily as horizontal reinforcement and are placed within the bed joints of the masonry. This reduces labor and materials by avoiding the use of special blocks and grouting necessary in forming fully reinforced and grouted masonry. However, the limited size of the bed joints constrains the amount of welded wire reinforcement that can be included in the wall.

\subsubsection{Assemblages}

An overview of how mortar joints are formed and a brief introduction to how masonry compression strength is determined.

\subsubsection{Joints}

Joints are a major source of the anisotropy within masonry assemblages. Bed joints run horizontally and are generally continuous from one end of an element to the other. Head joints run vertically and may or may not run continuously from the bottom to top of a masonry element, depending on the bond pattern used in laying the blocks.

Running bond, the most commonly used bond pattern, is defined as a pattern in which the head joints of adjacent courses are offset by a distance of at least a fourth the length of a masonry block. Stack bond is defined as a bond pattern in which the head joints of each consecutive course are aligned so that each head joint runs continuously from the bottom to top of the masonry element.

Bed joints are mortared in one of two ways; full bedding or face shell bedding. A fully bedded joint describes the case when the tops of both face shells and webs of the masonry

Part I: Thesis Introduction 
blocks are mortared. A face shell-bedded joint describes the case when only the tops of the face shells of the masonry blocks are mortared.

When mortar is placed on a masonry block, some of the wet cement paste is wicked into the drier masonry block by capillary action. To achieve optimum bond strength between the mortar and the block, the mortar and masonry blocks must be within an acceptable range of moisture content. If the masonry block is too wet, the mortar will not wick into the masonry. If the masonry block is too dry, then too much of the moisture in the mortar will be sucked out and the cement in the mortar will not have enough water to properly hydrate, forming a weak mortar joint.

\subsubsection{Prisms}

Compressive strength parameters are often measured by testing a series of masonry prisms. Masonry prisms are assemblages that are typically a whole block long and multiple courses high. Prisms may be left hollow or be fully-grouted, and are meant to represent the actual building element.

Masonry prisms tested under vertical compression loading will demonstrate an ultimate net compression stress less than that of the masonry blocks. The ultimate stress for the prism is generally greater than that of the mortar. This phenomenon is related to the three-dimensional stress states within the masonry prism. Masonry blocks, mortar, and grout each have a different Poisson's ratio. The masonry blocks tend to have a Poisson's ratio less than that of the mortar or grout. This disparity in ratios results in each material expanding laterally at different rates as they are strained in the vertical direction. These differences in lateral expansion rates induce lateral stresses in the materials because they are all interconnected together. Since the mortar has a higher Poisson's ratio than the masonry blocks, the mortar seeks to expand laterally more than the adjacent masonry blocks. This causes the mortar to be confined in the two lateral directions, placing it into compression in all three coordinate directions. This tridimensional compression state allows the mortar to resist greater compressive loads than it could in a typical uniaxial compression state. This correlates to research which has determined that masonry prism strength is not influenced by deviations in mortar strength. While the masonry blocks are confining the mortar, the mortar is inducing outward forces on the masonry blocks themselves. This is further compounded by the inclusion of grout within the block cells which induces further outward strains on the masonry. Masonry prisms typically fail by cracking and spalling of the face shells initiated by the lateral

Part I: Thesis Introduction 
tension induced within it. The lateral tension results in prisms having lower compression strength than the blocks alone.

For prism tests to be truly representative of actual conditions they should be at least three courses high because the central course is laterally unconfined from its top to its bottom. Taller prisms may be tested as well, but this is generally avoided because costs outweigh the additional benefits. Additionally, the results from taller prisms are adjusted to account for slenderness effects.

\subsection{Inspiration for Current Study}

Despite the long use of hollow concrete masonry, the mechanics of hollow concrete masonry are still not fully understood. This is because of the complex interaction between the materials used to make masonry elements. This thesis is motivated by the need for a deeper understanding of the mechanical behaviour of hollow concrete block masonry, which could in turn help engineers design masonry buildings more effectively. Engineers produce designs and perform inspections based on prescribed code clauses and equations in international codes. While history has shown these equations to be safe, they are often based on "rules-of-thumb" rather then rational material models. This tends to lead to very over conservative values with large variabilities and inconsistencies between international design codes. This causes hollow concrete masonry to be at a large disadvantage compared to other building materials such as steel or concrete.

\subsection{Objectives}

The overall goal of this thesis is to provide a better understanding of the relationship between hollow concrete masonry properties and the structural response of hollow concrete masonry building elements through the numerical modelling of masonry mechanics. An understanding of mechanical behaviour is essential for the design of safe structures. This will allow for the proposal of new design methods that are based on rational material models, that reduce variabilities, but still produce safe results. This will allow hollow concrete masonry to become more competitive, as lower variabilities allow for larger material reduction factors.

Part I: Thesis Introduction 
A series of three numerical models is proposed to describe hollow concrete masonry mechanics from the micro to the macro level:

1. A micro model to describe the behaviour of hollow concrete block masonry loaded in compression;

2. A micro-macro model to describe the behaviour of hollow concrete block masonry at the macro level when subject to a general loading condition of compression, tension, and in-plane shear; and,

3. A kinematic model to describe the behaviour of reinforced single pier hollow concrete block masonry shear walls in a timely manner.

The objective of each model is develop an accurate, yet easily understood, numerical model based on the mechanical and geometric properties of hollow concrete block masonry and its components. The principle reason for using mechanics of materials in the numerical models is to determine the stresses, strains, and displacements in hollow concrete block masonry structures due to the loads acting on them. Additionally, as numerical models typically involve the input of a large amount of data, the goal of each model is to reduce the amount amount of inputs required by providing default values and the proposal of new equations for the inputs required for each model.

Ultimately, it is hoped that a further understanding of hollow concrete block masonry mechanics will allow for practicing engineers to better design masonry structures.

\subsection{Organization of Thesis}

As each model examines hollow concrete block masonry at a different level (micro or macro) it was decided that the most effective way to approach the numerical models and present the information was to fully investigate each model separately. Therefore, each model presented in this thesis will have its own individual part with a review of relevant literature, details of the model, an evaluation of the model, and model conclusions.

Part I, provides an introduction to the thesis, including an overview of masonry history, to show masonry long use, but limit design methods based on rational methods; a brief summary of masonry materials properties; the inspiration for the current study; the overall objects of this thesis and each model; and the organization of this thesis.

Part II, presents the Smeared Area Compression Model, a micro model for predicting the compression strength of masonry. Chapter 2 presents the current understanding of

Part I: Thesis Introduction 
hollow concrete block masonry when subject to compression, as well as a number of empirical, analytical, and finite element models that have been developed in the past to determine the compression strength of hollow concrete block masonry. This chapter concludes with objectives specifically for the compression model. Chapter 3 presents the theoretical background for the Smeared Area Compression Model including the equilibrium conditions, compatibility conditions, and constitutive relations. Chapter 4 compares the numerical model results from the model presented in Chapter 3 to experimental data found in literature. Chapter 5 presents the results of parametric study on the model inputs and compares the results to observed hollow concrete block masonry mechanics. Chapter 6 provides simplified equations for predicting the compression strength of hollow concrete block masonry that can be used for "back-of-envelope" calculations and implementations in international design codes. The model presented in Chapter 3 is further extended to compression parallel to the bed joint in Chapter 7, with comparison to experimental data found in literature. Chapter 8 is the conclusion to Part II and presents the model conclusions and future research possibilities.

Part III, outlines the Smeared Area Finite Element Model, a micro-macro model that can be used to predicted the structural response of masonry elements that are either ungrouted or grouted. Additionally, the crack patterns and the masonry and reinforcement strains and stresses can also be predicted. Chapter 9 presents an overview of finite element models and techniques for hollow concrete block masonry, as well as, the specific objects for the finite element model developed in this thesis. Chapter 10 presents the equilibrium conditions, compatibility conditions, and the material constitutive models for use in the finite element model. Chapter 11 presents the finite element formulation used to develop the finite element program. The finite element code is verified through a series of tests, with the results presented in Chapter 12. Chapter 13, 14, and 15 present the comparison between the finite element model and experimental tests from literature. The conclusion of the finite element model are presented in Chapter 16, in addition to future research.

Part IV, provides the details of the Three Parameter Kinematic Model for reinforced masonry shear walls. This is a macro model that uses three displacements to predict the full load-displacement response of reinforced cantilevered masonry shear walls. The background of different methods for displacement based modelling and the objectives of the model presented in this thesis are given in Chapter 17. Chapter 18 provides an overview of the kinematic theory for reinforced hollow concrete block masonry. The implementation of this theory into a computer program is given in Chapter 19. The 
model implementation is verified in Chapter 20 and Chapter 21 compares model results to experimental results from literature. Chapter 22 provides concluding remarks for Part IV of this thesis.

Part V, provides the overall thesis conclusions, an overview of the contributions made throughout this thesis, and possible future research to further support the findings of this thesis.

Part I: Thesis Introduction 


\section{PART II}

\section{MASONRY COMPRESSION STRENGTH MODELING}


Compressive strength is often of prime importance in masonry structures, as it is often the basis of all designs due to the fact that allowable compressive, flexural, bearing, and shearing stresses; and, the modulus of elasticity are typically expressed as functions of the compressive strength. The importance of masonry compressive strength has made it a major research focus allowing for significant advances in masonry mechanics, both with respect to experimental testing and numerical modeling. But despite this, the composite behaviour of hollow concrete block masonry represents a true challenge due to the complex interaction of individual materials used to make masonry structures. Though each material (concrete masonry blocks, mortar, and possibly grout) is cementitous in nature, they each have different material properties and each responds in a non-linear manner when subject to compression.

During the past decades numerous experimental and numerical investigations have been carried out on masonry prisms to examine their behaviour and strength under compressive loads. This has resulted in numerous empirical equations. These equations are typically derived from a small subset of masonry prisms with different equations needed depending upon the block, mortar, or grout; or complex finite element models just to estimate the compression strength of a prism. In addition, actual prism testing has many complications including the height-to-thickness ratios, weight of specimens, capping, bearing plate thickness, machine dimensions and capacity, and construction methods.

The current Canadian masonry design standard (Canadian Standards Association, 2014a) provides two approaches to determine the compressive strength for the design of masonry members: testing prisms with site representative materials or using prescribed values based on block compressive strength and mortar type. Design engineers typically use the latter method since it is a more convenient approach. However, there is ample evidence that prescribed values are significantly lower than strength values measured from prism testing. The research used to develop the current prescribed values was carried out in the 1970 s and 1980 s, using a $20 \%$ reduction to a best-fit linear regression between average concrete block compressive strength values and average prism compressive strength values (Gayed et al., 2012). Much has changed since then such as the quality of block manufacturing, properties of blocks and mortar, and construction practices have advanced. The recalibration of the correlation between block strength and prism strength is necessary to realize the full potential strength of masonry, and to deliver cost-effective and economically competitive masonry designs.

Part II: Masonry Compression Strength Modeling 


\section{BACKGROUND}

The mechanics of hollow concrete block masonry subject to compression have been studied through experiments, that have resulted in numerous empirical, analytical, and finite element models that are typically limited to a small subset of masonry materiel combinations. The goal of Part II of this thesis is to develop a model that is applicable to all masonry material properties and combinations.

Historically, research on the compression strength of masonry has focused on three categories: ungrouted prisms, grouted prisms, and modeling (empirical, analytical, or finite element). This chapter will review past research and discuss different factors that are known to influence the compression strength of masonry, as well as, provide the objectives for the proposed numerical model.

\subsection{Mechanics of Ungrouted Masonry}

A review of existing ungrouted masonry experimental programs indicated that the generally accepted failure mode of ungrouted masonry under compression is that blocks fail under a state of biaxial tension stresses when the vertical compressive load is transferred through the weaker and less stiff mortar thereby producing a greater tendency for the mortar to expand laterally. This lateral expansion of the mortar is resisted by the block, thereby creating triaxial confinement of the mortar and lateral tensile stresses in the block, see Figure 2.1 .

This review has revealed a number of parameters that influence the compression strength of ungrouted masonry. These parameters include block strength, mortar strength, bedding type, and mortar thickness.

Copeland and Timms (1932) tested walletes that were six blocks high and either two blocks in running bond or one block wide. It was observed that as the compression strength of the block increased so did the compression strength of the wallette. Similar results were obtained by Richart et al. (1932) and by Gaynor et al. (1987) for prisms. Roman and Romagna (2002) also observed that masonry compressive strength increases 


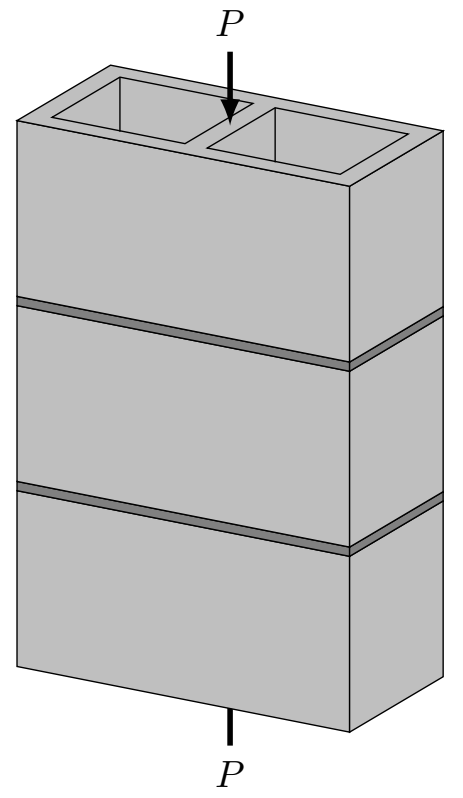

(a) Prism Under Compression

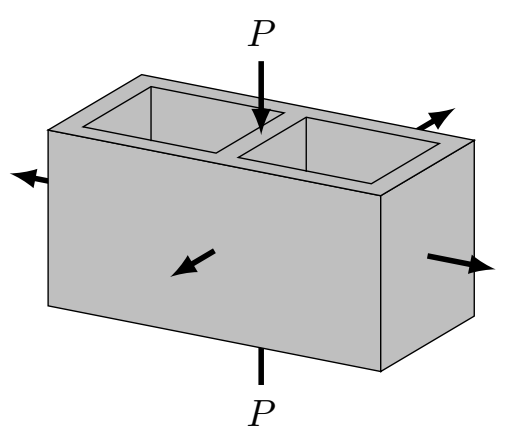

(b) Block Stresses

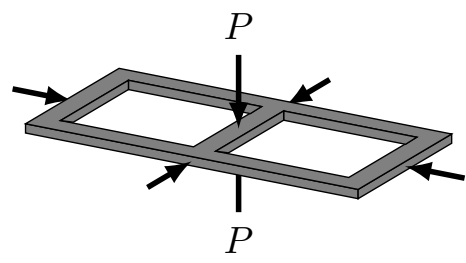

(c) Mortar Stresses

Figure 2.1: Local State of Stresses in Masonry Prisms under Uniaxial Vertical Compression

with increasing block strength, but that the increase was not proportional to the increasing compressive strength of the block. Given that masonry compressive failure is associated with tensile cracking in the block, it is not surprising a slightly non-linear relationship between masonry compressive strength and block strength is observed, as the tensile strength of concrete is related in a non-linear fashion to compressive strength.

Richart et al. (1932) observed that variations in mortar strength had a negligible effect on a prism's compressive strength. Gaynor et al. (1987) also obtained the same results. Ramamurthy et al. (2000) observed that for a given block strength, a reduction in mortar strength leads to a reduction in masonry prism strength. It was also observed that mortar strength had less of an influence on the strength of face shell bedding as compared to that of full mortar bedding. Roman and Romagna (2002) found similar results to Ramamurthy et al. (2000).

Gaynor et al. (1987) noted that the mode of failure of ungrouted masonry was strongly dependent on the type of mortar bedding used; with full mortar bedding exhibiting compressive shear failures with diagonal cracking through the thickness of a face shell or web and face shell bedding exhibited cracking along the webs. Wong and Drysdale (1985) noted that failure of the face shell mortared prisms was always initial splitting of the webs followed by instability and collapse of the face shells at higher loads. Ramamurthy 
et al. (2000) stated that face shell mortar causes only face shells to effectively carry the load and that splitting is attributed to the very high lateral tensile stresses developed in the webs due to deep beam action. Though there are different failure modes, Gaynor et al. (1987) and Das et al. (2013) noted that strength difference between full bedding and face shell bedding is statistically insignificant, based on mortar bedding area.

Prism tests completed by Drysdale and Hamid (1979) demonstrated that increasing joint thickness from $9.5 \mathrm{~mm}$ to $19 \mathrm{~mm}$ resulted in a $16 \%$ decreases in prism capacity. Ramamurthy et al. (2000) found similar results, stating that as the thickness of mortar joints increased, the lateral confinement effect offered by the blocks reduces considerably, thus bringing the crushing strength of mortar in the prism closer to its uniaxial compressive strength established by mortar cube tests. In some cases the failure occurred at stress levels much lower than the uniaxial compressive strength of mortar.

Additional observations of ungrouted masonry mechanics are that there is no difference in full or half blocks on the compressive strengh (Drysdale and Hamid, 1979) and that stack or running bond has no significant effect on prism strength (Das et al., 2013).

\section{Summary}

There are various parameters that influence the compression behaviour of ungrouted masonry compression strength. Among these factors, the most important are: the block strength, the mortar strength, the bedding type, and mortar thickness. See Table 2.1 for how these parameters influence ungrouted masonry compression strength.

Table 2.1: Parameters Influencing Ungrouted Masonry Compression Strength

\begin{tabular}{ll}
\hline Parameter & Influence \\
\hline Block Strength & $\begin{array}{l}\text { Increasing block strength, more specifically increasing block } \\
\text { tensile strength, increases ungrouted masonry compression } \\
\text { strength } \\
\text { Mortar Strength } \\
\text { Increasing mortar strength increases ungrouted masonry } \\
\text { compression strength, though this increase is less pro- } \\
\text { nounced due to the triaxial compression of the mortar } \\
\text { The bedding type has a significant impact of the failure } \\
\text { mode of ungrouted masonry strength, but little impact on } \\
\text { compression strength based on minimum mortar area } \\
\text { Mortar Thickness } \\
\text { Increasing mortar thickness decreases ungrouted masonry } \\
\text { compression strength }\end{array}$ \\
\hline
\end{tabular}

Part II: Masonry Compression Strength Modeling 


\subsection{Mechanics of Grouted Masonry}

The failure mode of grouted masonry under compression is typically characterized by tensile splitting of the block's face shells, with the grout core remaining largely intact. Drysdale and Hamid (1979) suggest that the incompatibility of the deformation characterics of the grout and the block contributes to this result. At high vertical compression strain levels the grout undergoes inelastic deformations in the horizontal direction caused by extensive microcracking and a greatly increased Poisson's ratio. This produces high bilateral tensile stresses in the outer shell of the block, as the block tends to confine the expansion of the grout. These stresses in combination with the vertical stresses cause a splitting failure of the block under a state of biaxial tension stress. This typically results in an average compressive strength for grouted prisms less than that for similar ungrouted prisms indicating that the concept of superposition of grout strength and ungrouted prism strength is not valid. Also, grouted masonry tends to show a more gradual failure when compared to ungrouted masonry, and also may exhibit some post-peak response.

A review of existing grouted masonry experimental programs indicated that there are a number of parameters that influence the compression strength of grouted masonry. These parameters include block strength, mortar strength, grout strength, and mortar thickness.

Block strength is an influential factor in determining the strength of grouted masonry (Boult, 1979), but that often it is the tensile strength rather than the compressive strength of the block that is the most strongly correlated with prism strength (Ross et al., 2013).

Khalaf et al. (1994) and Roman and Romagna (2002) observed that increasing mortar strength had little impact on grouted masonry compressive strength.

Khalaf et al. (1994) and Roman and Romagna (2002) found that the masonry strength increased with increasing grout strength, though grout strengths exceeding the block strengths did not provide additional capacity beyond the compressive strength of the block (Steadman et al., 1995).

Drysdale and Hamid (1979) indicated that increasing joint thickness from $9 \mathrm{~mm}$ to 19 $\mathrm{mm}$ resulted in a slight $(3 \%)$ deceased prism compression capacity. Additionally, the compressive strength of grouted masonry could be increased by matching the deformational characteristics of the grout and block (Drysdale and Hamid, 1979; Khalaf et al., 
1994; Roman and Romagna, 2002). Also, Drysdale and Hamid (1979) found that testing full or half blocks and the bond pattern did not change the failure mode or capacity.

\section{Summary}

There are various parameters that affect the compression behaviour of grouted masonry compression strength. Among these factors, the most important are: the block strength, the mortar strength, the grout strength, and mortar thickness (see Table 2.2).

Table 2.2: Parameters Influencing Grouted Masonry Compression Strength

\begin{tabular}{ll}
\hline Parameter & Influence \\
\hline Block Strength & $\begin{array}{l}\text { Increasing block strength, more specifically increasing block } \\
\text { tensile strength, increases grouted masonry compression } \\
\text { strength }\end{array}$ \\
Mortar Strength & $\begin{array}{l}\text { Increasing mortar strength increases grouted masonry com- } \\
\text { pression strength, though the increase is less then the un- } \\
\text { grouted compression strength }\end{array}$ \\
Increasing grout strength increases grouted masonry com- \\
pression strength, however this is limited by the block \\
strength as failure is typically characterized as tensile split- \\
ting or crushing of the block \\
Increasing mortar thickness decreases grouted masonry com- \\
pression strength, though the increase is less then the un- \\
grouted compression strength
\end{tabular}

\subsection{Empirical Modeling}

\subsubsection{Sarhat and Sherwood (2014) and Sarhat and Sherwood (2015)}

Sarhat and Sherwood $(2014 ; 2015)$ conducted regression analyses to obtain an accurate empirical formula to predict the compressive strength of ungrouted and grouted hollow concrete block masonry. Based on a total of 248 average masonry compressive strength data points, representing the test results of 1092 individual prisms, the following equation was proposed for the prediction of ungrouted masonry strength:

$$
f_{m}^{\prime}=1.107\left(f_{b l}^{\prime}\right)^{0.75}\left(f_{m r}^{\prime}\right)^{0.18}
$$

This equation gives an average ratio of experimental $f_{m}^{\prime}$ to predicted $f_{m}^{\prime}$ of 1.0 for the entire database with a coefficient of variation of $12.5 \%$, see Figure 2.2 .

Part II: Masonry Compression Strength Modeling 


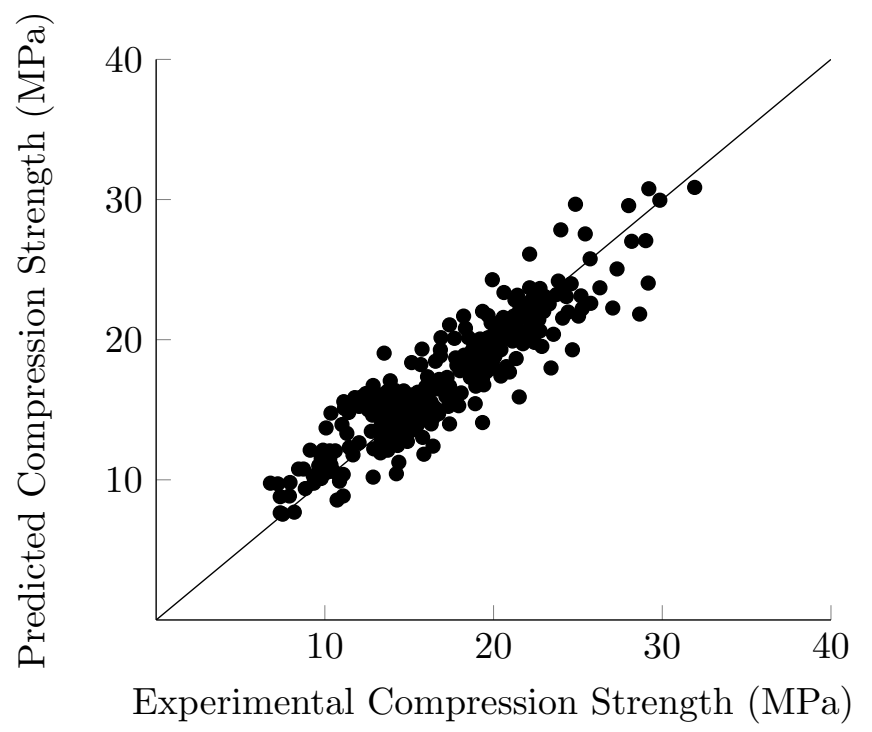

Figure 2.2: Predictive Ability of Sarhat and Sherwood Ungrouted Equation (Sarhat and Sherwood, 2014)

In the case of grouted masonry, a total of 156 average masonry compressive strength data points, representing the test results of 555 individual prisms were collected and analyzed. The following equation was proposed for the prediction of grouted masonry strength:

$$
f_{m}^{\prime}=0.295 f_{b l}^{\prime}+0.125 f_{m r}^{\prime}+0.239 f_{g r}^{\prime}+0.604
$$

This model gives an average ratio of experimentally determined $f_{m}^{\prime}$ to predicted $f_{m}^{\prime}$ of 1.0 with a coefficient of variation of $15 \%$, see Figure 2.3 .

Part II: Masonry Compression Strength Modeling 


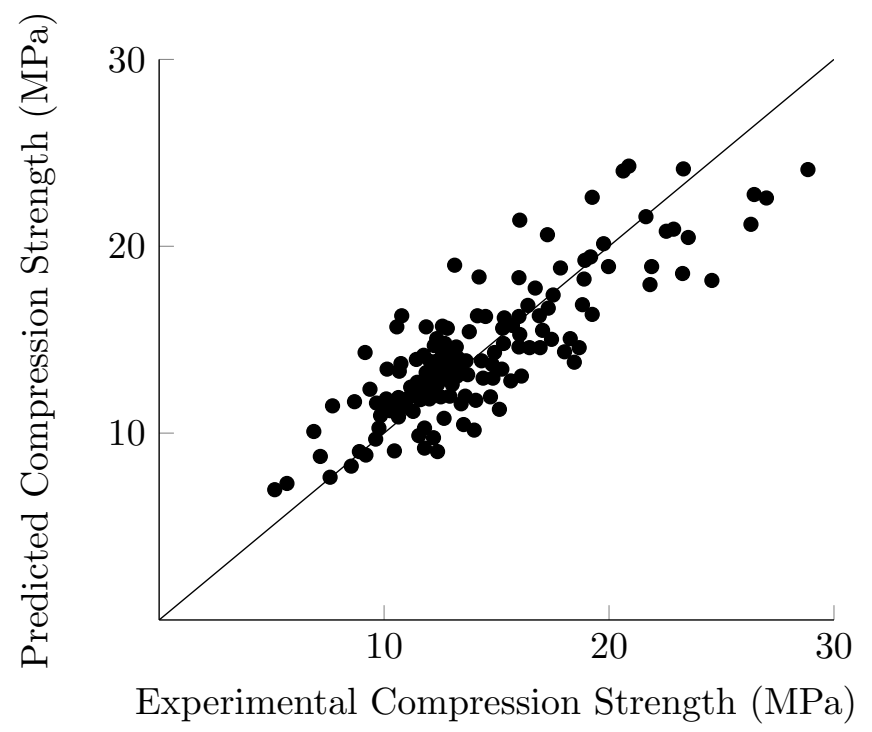

Figure 2.3: Predictive Ability of Sarhat and Sherwood Grouted Equation (Sarhat and Sherwood, 2015)

\subsection{Analytical Modeling}

\subsubsection{Hamid (1978)}

Hamid (1978) tested 150 ungrouted and grouted prism tests to study the effect of individual material properties and geometric parameters on masonry compression strength. In this investigation an analytical strength formula is proposed to express the compression strength of both ungrouted and grouted masonry.

Hamid (1978) suggests that two failure conditions are possible for grouted masonry under axial compression depending on which material, the block shell or the grouted core, reaches its unconfined compressive strength first. In the first case, the grout is stiffer than the shell and has a lower strain level at maximum stress. Hence, the grout's unconfined compressive strength would be reached first and the shell would tend to confine the grout. Failure will occur in the shell which is no longer capable of confining the grout. The compressive strength masonry can be expressed in terms of the strength and geometric characteristics of its components as follows:

$$
f_{m}^{\prime}=\left(\frac{4.1 f_{t, b l}^{\prime}+1.14 \alpha f_{m r}^{\prime}+\beta f_{g r}^{\prime}}{4.1 f_{t, b l}^{\prime}+\left(1.14 \alpha+\frac{\beta}{n}\right) f_{b l}^{\prime}}\right)\left(\frac{f_{b l}^{\prime}}{n \gamma K}\right)
$$

for

Part II: Masonry Compression Strength Modeling 


$$
\begin{gathered}
\alpha=\frac{t_{m r}}{h_{b l}}, \\
\beta=\frac{\sqrt{1-\rho_{b l}^{\text {solid }}}}{1-\sqrt{1-\rho_{b l}^{\text {solid }}}}, \\
n=\frac{E_{b l}}{E_{g r}}, \\
\gamma=\frac{1}{1+(n-1) \rho_{b l}^{\text {solid }}}, \text { and } \\
K=1.04+0.19 \frac{1}{n} .
\end{gathered}
$$

In the second case, the shell stiffness is equal to or greater than the grout and failure would be controlled by the unconfined strength of either the shell or the grout; hence,

$$
f_{m}^{\prime}=\max \left\{\begin{array}{l}
\frac{3.6 f_{t, b l}^{\prime}+\alpha f_{m r}^{\prime}}{3.6 f_{t, b l}^{\prime}+\alpha f_{b l}^{\prime}} \frac{f_{b l}^{\prime}}{n \gamma \gamma K} \\
\left(1-\rho_{b l}^{\text {solid }}\right) f_{g r}^{\prime}
\end{array}\right.
$$

For the ungrouted case Hamid (1978) gave the following equation for the compression strength:

$$
f_{m}^{\prime}=\left(\frac{3.6 f_{t, b l}^{\prime}+\alpha f_{m r}^{\prime}}{3.6 f_{t, b l}^{\prime}+\alpha f_{b l}^{\prime}}\right)\left(\frac{\rho_{b l}^{\text {solid }} f_{b l}^{\prime}}{K}\right)
$$

The results of these equations are shown in Figure 2.4.

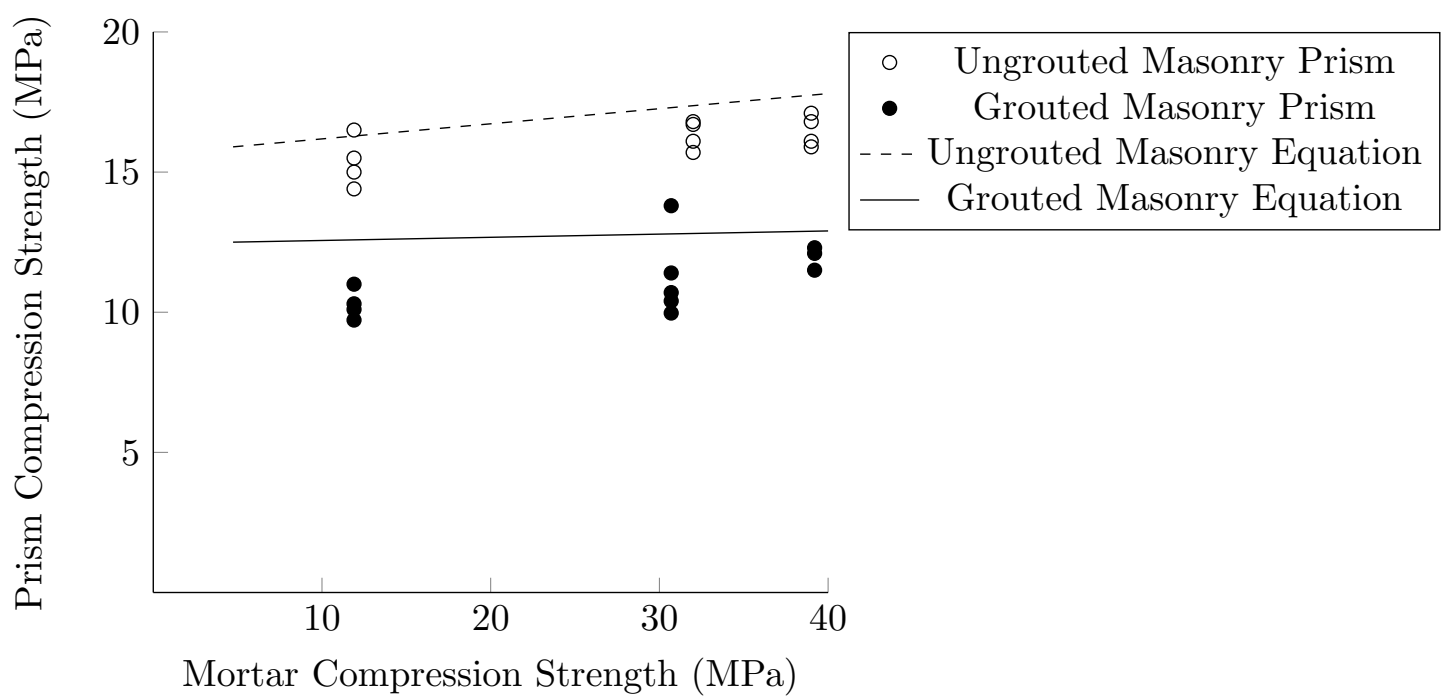

Figure 2.4: Predictive Ability of Hamid Equations (Hamid, 1978)

Part II: Masonry Compression Strength Modeling 


\subsection{Finite Element Modeling}

\subsubsection{Cheema and Klingner (1986)}

Cheema and Klingner (1986) performed experimental tests on prisms and consitituent materials to calibrate a linear elastic finite element model for grouted masonry prisms. The finite element model was then used to develop simplified relationships to predict the compressive strength and failure modes of masonry prisms.

During testing the grouted prisms failed by vertical splitting through the block shell, starting at the bed joint; neither the grout nor the mortar suffered much damage. Grout and mortar, which had Poisson ratios greater than those of blocks at large strains, expended near the mortar joints under axial compression and split the block shell at the bed joint. Axial capacity depended on the splitting resistance of the shell and the crushing resistance of the grout.

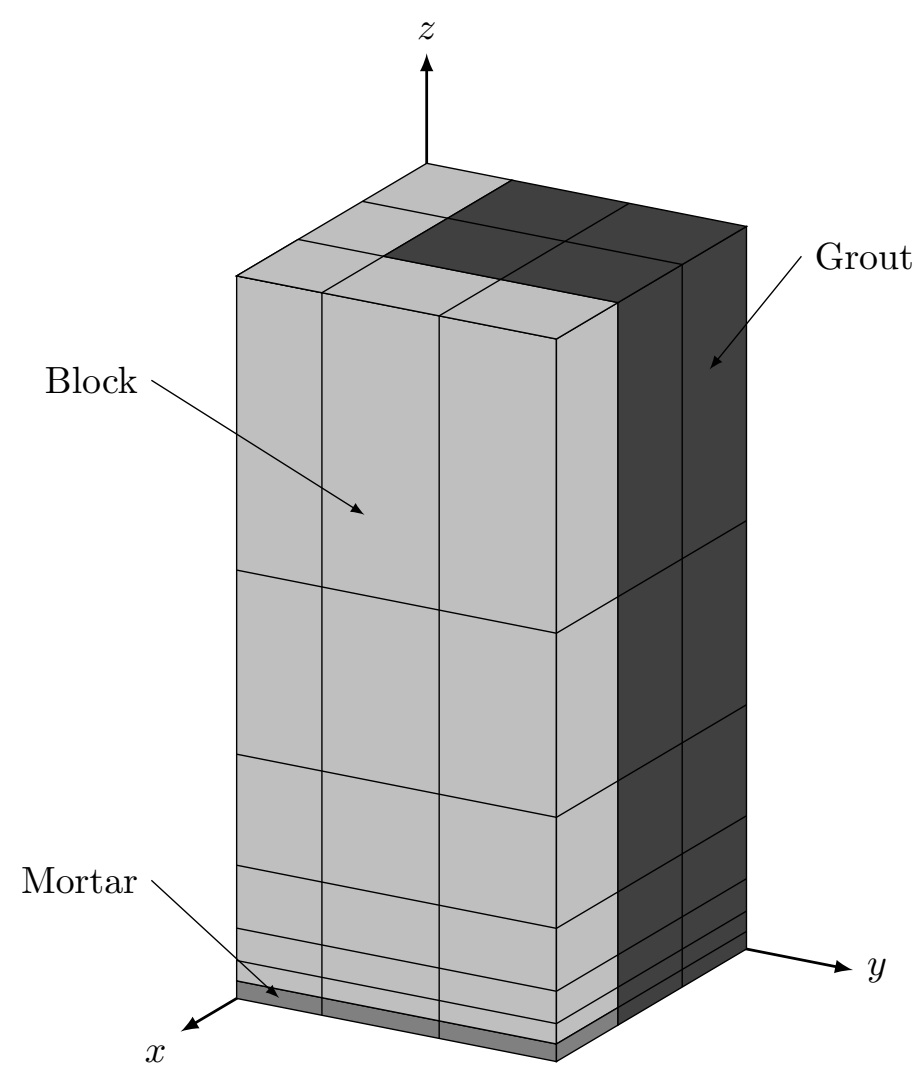

Figure 2.5: Cheema and Klingner FEM Model (Cheema and Klingner, 1986)

A three-dimensional finite element was constructed using a one-sixteenth model of the block and mortar, see Figure 2.5. The upper face was subjected to uniform vertical

Part II: Masonry Compression Strength Modeling 
displacement and zero lateral restraint. The block secant modulus of elasticity was given by:

$$
E_{b l}=22 \gamma_{b l}^{1.5} \sqrt{f_{b l}^{\prime}} \text { in psi }
$$

where, $\gamma_{b l}$ is the unit weight of the block and block tensile strength was predicted from:

$$
f_{t, b l}^{\prime}=5 \sqrt{f_{b l}^{\prime}} \text { in psi }
$$

A Poisson's ratio of 0.28 was used for the blocks. For the mortar a Poisson's ratio of 0.28 was used and a secant modulus of:

$$
E_{m r}=500 f_{m r}^{\prime} \text { in psi }
$$

was used. The mortar tensile strength was estimated from:

$$
f_{t, m r}^{\prime}=7 \sqrt{f_{m r}^{\prime}} \text { in psi }
$$

For the grout a Poisson's ratio of 0.37 was used, with a secant modulus of

$$
E_{g r}=16.5 \gamma_{g r}^{1.5} \sqrt{f_{g r}^{\prime}} \text { in psi }
$$

From the finite element model the failure modes predicted were block splitting, block crushing, mortar crushing, mortar splitting, and grout crushing. These failure modes are then used to develop simplified relationships to predict the compression strength of grouted masonry. The process of determining the grouted compressive strength using the simplified method can be summarized as follows:

1. Find modular ratios $E_{m r} / E_{b l}$ and $E_{g r} / E_{b l}$ using secant moduli.

2. Determine the block splitting strength, $f_{b l}=f_{t, b l}^{\prime} / \sigma_{1 b l}$ using Figure 2.6a.

3. Determine the block crushing strength, $f_{b 2}=f_{b l}^{\prime}\left[\rho_{b l}^{\text {solid }}+E_{g r} / E_{b l}\left(1-\rho_{b l}^{\text {solid }}\right)\right]$.

4. Determine the mortar crushing strength, $f_{m 1}=\lambda f_{m r}^{\prime}$ using Figure 2.6b.

5. Determine the mortar splitting strength, $f_{m 2}=f_{t, m r}^{\prime} / \sigma_{1 m r}$ using Figure 2.6c.

6. Determine the grout crushing strength, $f_{g}=\delta f_{g r}^{\prime}$ using Figure 2.6d.

7. $f_{m}^{\prime}=\min \left\{f_{b l}, f_{b 2}, f_{m 1}, f_{m 2}, f_{g}\right\}$.

The results obtained from the simplified relationships agreed well with prism test results. This model is limited, however, to prisms with $9.5 \mathrm{~mm}$ bed joints and new curves would have to be developed for other joint thicknesses.

Part II: Masonry Compression Strength Modeling 


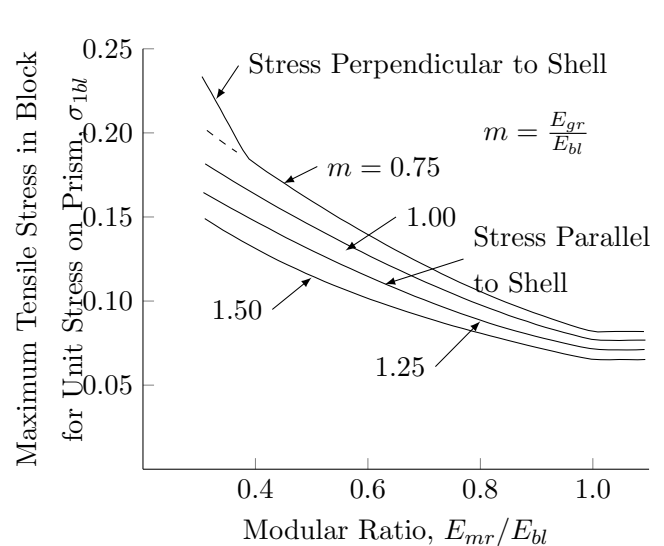

(a) Block Splitting

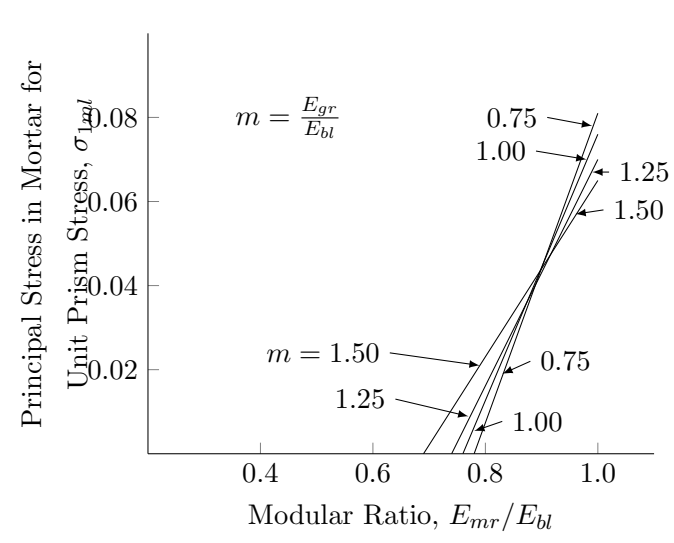

(c) Mortar Splitting

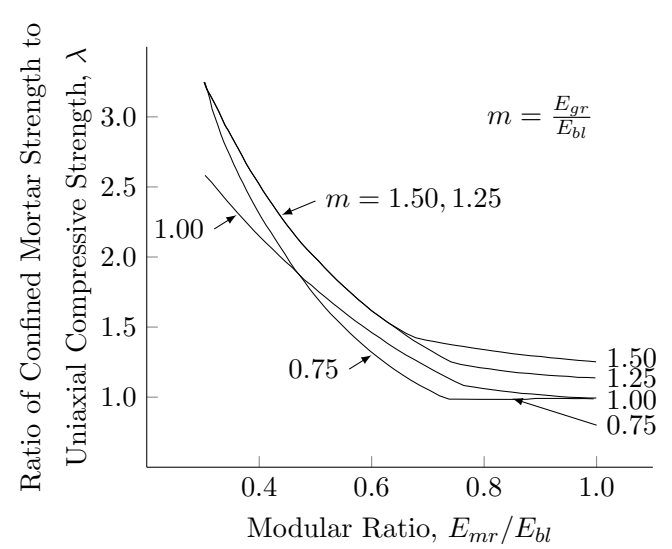

(b) Mortar Crushing

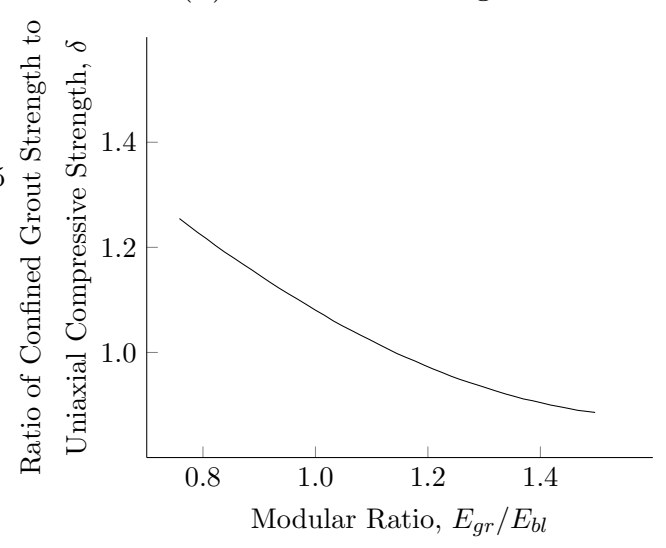

(d) Grout Crushing

Figure 2.6: Cheema and Klinger Failure Curves for Grouted Prisms (Cheema and Klingner, 1986)

\subsubsection{Others}

Hamid and Chukwunenye (1986), Ganesan and Ramamurthy (1992), Fahmy and Ghoneim (1995), and Köksal et al. (2005) used three-dimensional finite element analysis to study the effects of block strength, block geometry, mortar strength, bedding type, and grout strength. Analysis of the finite element models had similar results to those discussed in Table 2.1 and Table 2.2.

\subsection{Background Summary}

There are a number of factors that are known to influence the compression strength of masonry including the block strength, mortar strength, grout strength, mortar bedding type, and mortar thickness. There are an infinite number of combinations for these known influences; thus, empirical and analytical tools that only consider a few of these 
influences or are based only on a few experimental tests fail to capture all the possibilities. Finite element models provide a way of capturing all of the possibilities, but they are unpractical for practicing engineers due to the complexities of setting and running a finite element model. There is a need for a simple model that is able to capture all the complexities of masonry at the micro level, but which produces accurate results. Additional, the model should produce low variability to allow for the possibility of increased material reduction factors in international design codes.

\subsection{Model Objectives}

In addition to the objectives listed in Section 1.4, the specific objectives of a numerical model to determine the compression strength of hollow concrete block masonry include:

1. Determine the stresses and strains acting in individual material components used to make masonry when subject to compression;

2. Implement a model that accounts for confinement and biaxial tension;

3. Introduce a model that accounts for the anisotropic of hollow concrete block masonry, but maintains the symmetry of the composite stiffness matrix to allow for fast solution times;

4. Account for non-linearity and the Poisson's ratio of each component material;

5. Verify the model by comparing the results of analyses with the experimentally observed behaviour of both ungrouted and grouted hollow concrete block masonry prisms; and,

6. Use the numerical results to produce simplified design equations.

Part II: Masonry Compression Strength Modeling 


\section{SMEARED AREA COMPRESSION MODEL}

A numerical model is developed using equilibrium, compatibility, and material constitutive relations to determine the masonry compression strength at the micro level based on the block, mortar, and grout properties.

The Smeared Area Compression Model (SACM) is an numerical model for predicting the compression strength of ungrouted and grouted hollow concrete masonry. The model has been developed to predict the compression strength of hollow concrete block masonry accounting for block, mortar, and grout material properties.

The model consists of a hollow concrete masonry block, mortar (either full bedding or face shell bedding), and can be ungrouted or grouted. To eliminate the platen restraining effect caused in the end blocks of a prism, the model considers the central block in a prism of three or more courses (see Figure 3.1). It is important to note that even though there are only three materials, the grout (if present) is assumed to act differently inside the block when compared to inside the mortar. This is a result of the different material properties of the block and mortar influencing the response of the grout. Thus, there are four material responses that need to be considered by the model: the block (superscript $b l$ ); the mortar (superscript $m r$ ); the grout inside the block (superscript $g r b l$ ); and, the grout inside the mortar (superscript $g r m r$ ). In addition to this, the block can be considered as an anisotropic material in the model with different material properties in the $x, y$, and $z$ directions. The remaining materials, mortar and grout, are considered to be isotropic in nature, though their response in the $x, y$, and $z$ directions will be considered independently.

Each material stiffness matrix is formed using a secant stiffness approach, in which secant moduli are defined and progressively refined according to current material net stressstrain state and the material's stress-strain relation. In the formation of the material stiffness matrix it is assumed that the Poisson's effects are negligible and all off-diagonal terms in the stiffness matrix become zero. As Poisson's effects are important material properties that differ between the masonry materials it is necessary to include them in the model to obtain an accurate material response. Also, in a triaxial compressive stress condition the lateral expansion effects cannot be neglected. To account for Poisson's 


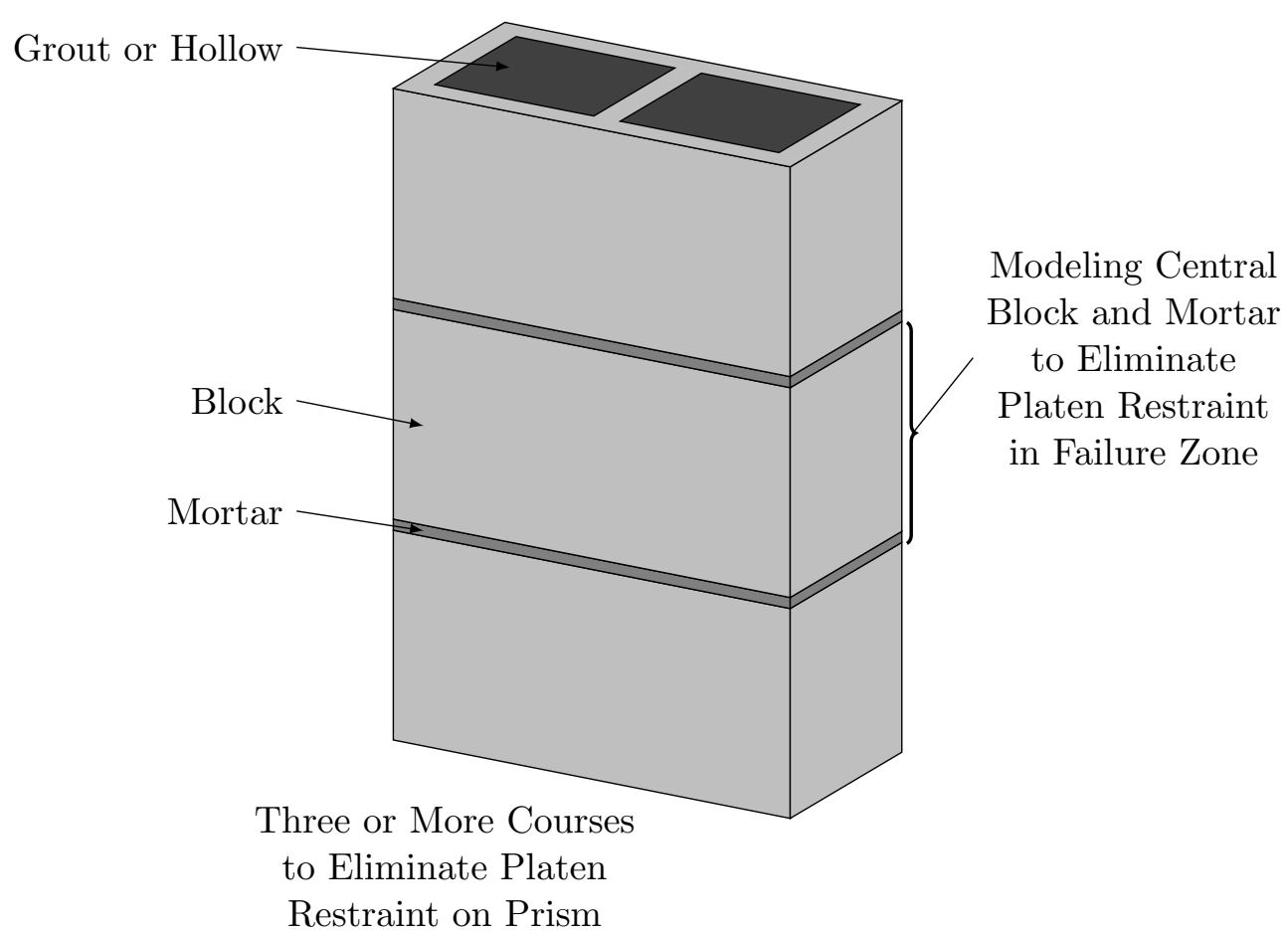

Figure 3.1: Area of Prism Being Modeled

effects, but keep the material stiffness matrix symmetric, a material prestrain concept is used (Vecchio, 1990).

The composite stiffness matrix is formed by combining the contribution from each materials stiffness matrix based on a smeared area approach. This approach allows each material to contribute to the composite stiffness depending on the ratio of material area in direction $i$ to the total composite net area in direction $i$.

Failure of the prism is defined by failure of any of the materials using a Mohr-Coulomb failure surface with a tension cut-off.

\subsection{Assumptions}

A concentric vertical load, $P_{z}$, is applied and for equilibrium must be the same through Section A (block and grout, superscript $b l-g r b l$ ) and Section B (mortar and grout, superscript $m r-g r m r$ ), see Figure 3.2. This load is assumed to cause uniform axial stress distribution at both sections, $\sigma_{z}^{b l-g r b l}$ for Section A and $\sigma_{z}^{m r-g r m r}$ for Section B. Deformation of the element is assumed to occur such that the edges remain straight and parallel, see Figure 3.3. The deformed shape is defined by four normal strains, $\epsilon_{x}, \epsilon_{y}$, $\epsilon_{z}^{b l-g r b l}$, and $\epsilon_{z}^{m r-g r m r}$. This allows for different strains to occur in Section A and B.

Part II: Masonry Compression Strength Modeling 


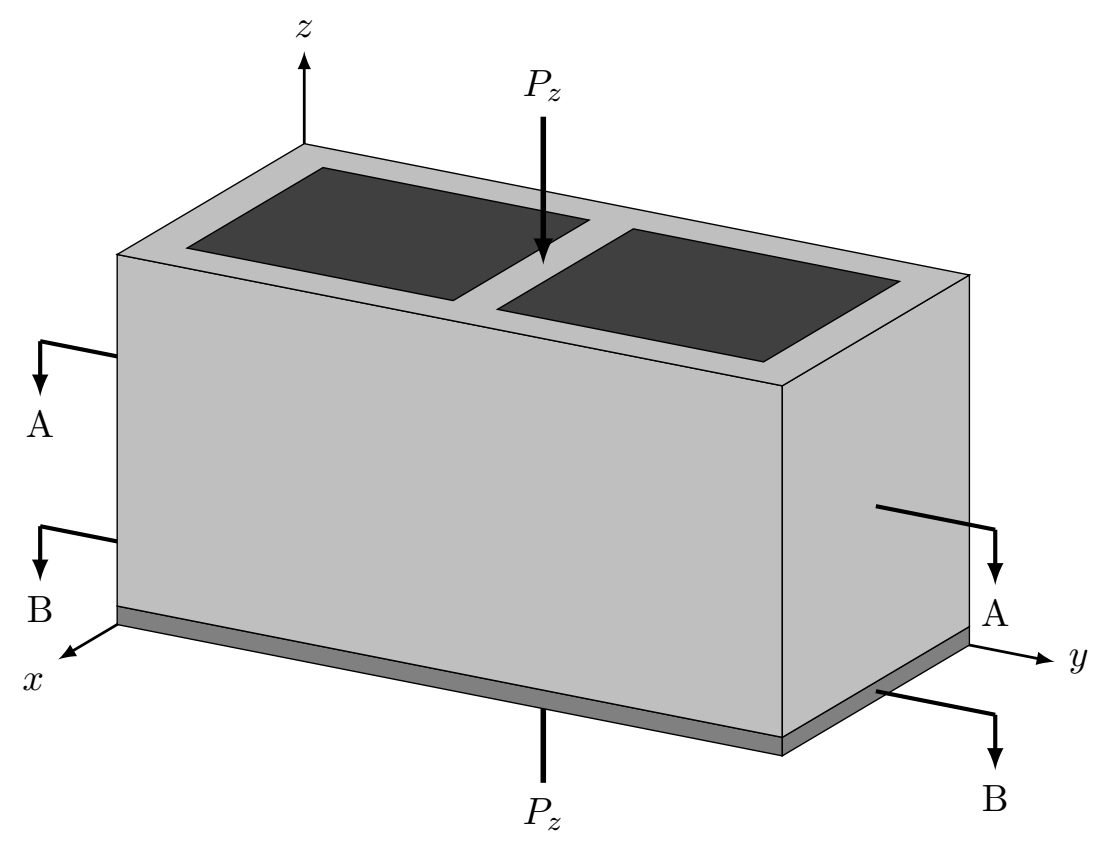

Figure 3.2: Model Stress Assumptions

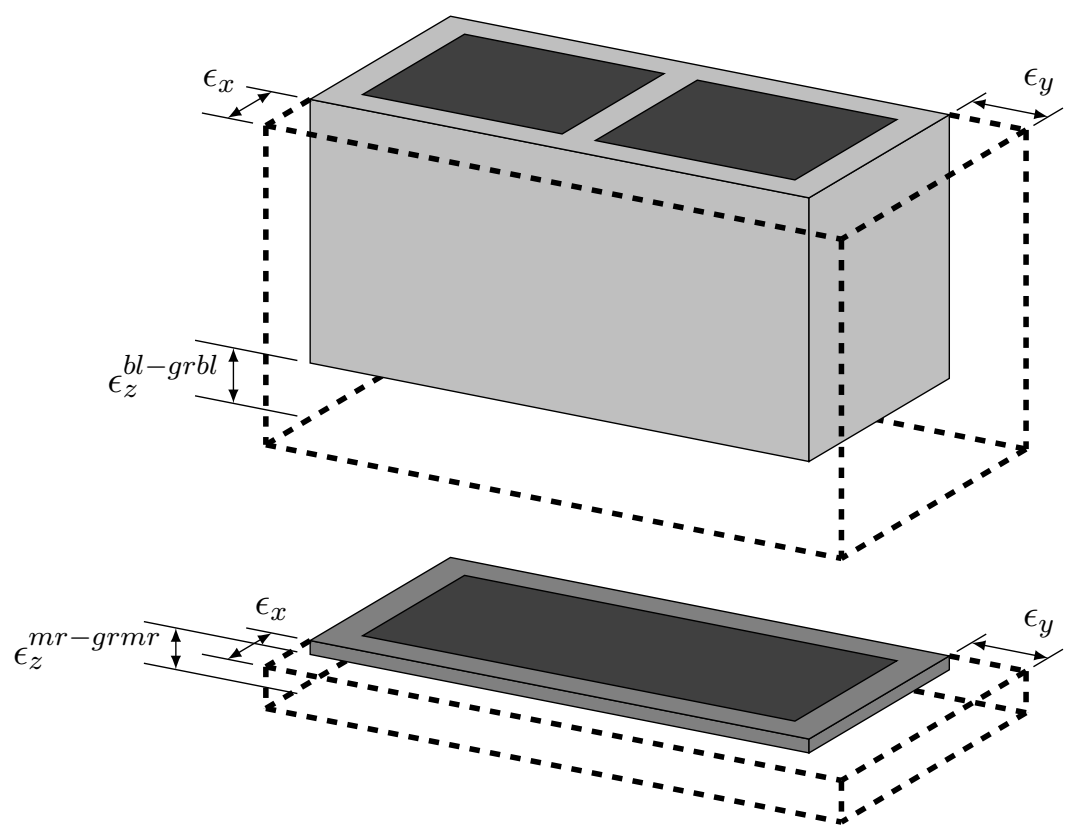

Figure 3.3: Model Strain Assumptions 
The SACM also makes the following assumptions:

1. Equilibrium, compatibility, and stress-strain relationships are formulated in terms of average stress and average strain.

2. Stresses and strains are averaged over the total area and length of the material.

3. For each strain state there exists only one corresponding stress state.

4. Equal strain acts through each material in each direction.

5. The block has uniform face shell and web thickness.

6. Perfect bond exists between the block, mortar, and grout.

7. Shear stresses at the block-mortar interface are ignored.

8. There are independent constitutive relations for each material.

9. The mortar width is equal to the face shell thickness.

10. Strengthening or softening of a material is only considered in the primary loading direction, $z$.

Additionally, tensile stresses and tensile strains will be treated as positive quantities and compressive stresses and strains will be taken as negative.

\subsection{Dimensions and Areas}

A number of important dimensions are shown in Figure 3.4, though a two cell block is shown the model is capable of working on three cell blocks as well. Thus in general, the length of grout in the mortar for face shell bedding is:

$$
l^{g r m r}=l^{b l}-2 t^{w e b}
$$

the width of the grout in the block and mortar is:

$$
w^{g r b l}=w^{g r m r}=w^{b l}-2 t^{f s} ;
$$

the block cell area is:

$$
A_{\text {cell }}^{b l}=l^{b l} w^{b l}\left(1-\rho_{\mathrm{solid}}^{b l}\right)
$$

length of grout in the block is given by

$$
l^{g r b l}=\frac{A_{\mathrm{cell}}^{b l}}{w^{g r b l}}
$$

and, the thickness of the block webs is given by

Part II: Masonry Compression Strength Modeling 


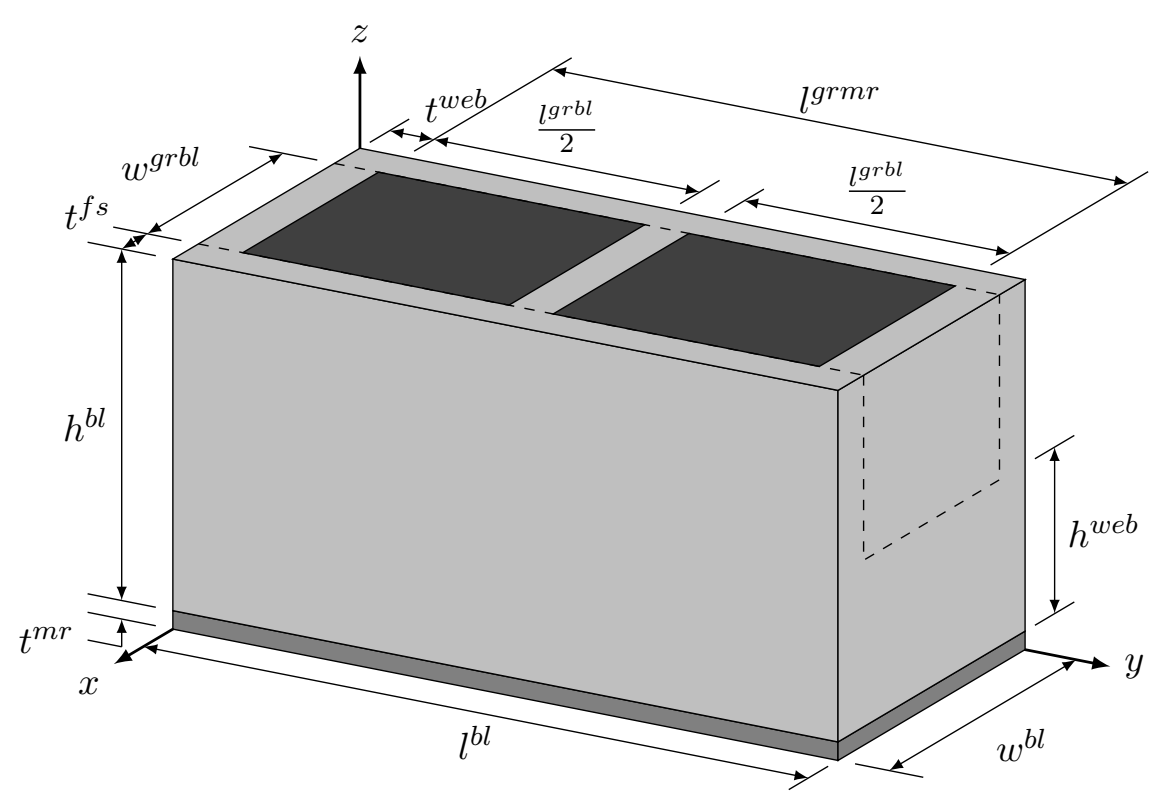

Figure 3.4: Dimensions of the Block, Mortar, and Grout fir a Two Cell Block

$$
t^{w e b}=\frac{l^{b l}-l^{g r b l}}{n_{w e b}}
$$

where $n_{w e b}$ is the number of webs.

To achieve equal strain in each material in each direction all the materials must act in parallel; thus, a modified material configuration in $x$ and $y$ direction must be used.

The modified material configuration in the $x$ direction is shown in Table 3.1. This configuration removes the two face shells of the block, which are more flexible about their $y$ axes when compared to the stiffer block webs; therefore, the face shells provide negligible stiffness in the $x$ direction. With the two face shells removed each material is subjected to equal strain $\epsilon_{x}$. The areas of each material in the $x$ direction are determined from the following equations:

$$
\begin{gathered}
A_{x}^{b l}=\left(l^{b l}-l^{g r b l}\right) h^{b l} \frac{h^{w e b}}{h^{b l}} ; \\
A_{x}^{m r}=\left\{\begin{array}{ll}
\left(l^{b l}-l^{g r b l}\right) t^{m r} & \text { if full bed mortar (grouted and ungrouted) } \\
l^{b l} t^{m r} & \text { if face shell mortar and ungrouted } \\
2 t^{w e b} t^{m r} & \text { if face shell mortar and grouted }
\end{array} ;\right. \\
A_{x}^{g r b l}=\left\{\begin{array}{ll}
l^{b l} h^{b l}-A_{x}^{b l} & \text { if grouted } \\
0 & \text { if ungrouted }
\end{array} ; \text { and },\right.
\end{gathered}
$$


Table 3.1: Modified Material Configurations

(G) Direction

${ }^{\dagger}$ Grout can be removed and left hollow

$$
A_{x}^{g r m r}= \begin{cases}l^{g r b l} t^{m r} & \text { if full mortar and grouted } \\ l^{g r m r} t^{m r} & \text { if face shell mortar and grouted } \\ 0 & \text { if ungrouted. }\end{cases}
$$

The percentage of each material, $j$, in the $x$ direction is given by:

$$
\rho_{x}^{j}=\frac{A_{x}^{j}}{\sum A_{x}^{j}} .
$$

In the $y$ direction the modified material configuration, shown in Table 3.1, removes the block webs; thus, each material is subjected to the equal strain, $\epsilon_{y}$. Similar to the $x$ direction, the block webs provide negligible stiffness in the $y$ direction due to their higher flexibility. The areas of each material in the $y$ direction are determined from the following equations:

$$
\begin{gathered}
A_{y}^{b l}=2 t^{f s} h^{b l} ; \\
A_{y}^{m r}=2 t^{f s} t^{m r} ; \\
A_{y}^{g r b l}=\left\{\begin{array}{ll}
w^{g r b l} h^{b l} & \text { if grouted } \\
0 & \text { if ungrouted }
\end{array} ;\right. \text { and, } \\
A_{y}^{g r m r}= \begin{cases}w^{g r m r} t^{m r} & \text { if grouted } \\
0 & \text { if ungrouted }\end{cases}
\end{gathered}
$$

Part II: Masonry Compression Strength Modeling 
The percentage of each material, $j$, in the $y$ direction is given by:

$$
\rho_{y}^{j}=\frac{A_{y}^{j}}{\sum A_{y}^{j}} .
$$

In the $z$ direction there is no modification to the material configuration as the materials in Section A and Section B are already in parallel and subjected to equal strains, $\epsilon_{z}^{b l-g r b l}$ and $\epsilon_{z}^{m r-g r m r}$ through each section. The areas of each material in the $z$ direction are determined from the following equations:

$$
A_{z}^{b l}=\rho_{\text {solid }}^{b l} l^{b l} w^{b l}
$$

$$
\begin{gathered}
A_{z}^{m r}=\left\{\begin{array}{ll}
\rho_{\text {solid }}^{b l} l^{b l} w^{b l} & \text { if full bed mortar (grouted and ungrouted) } \\
2 t^{f s} l^{b l} & \text { if face shell mortar and ungrouted } \\
2 t^{f s} l^{b l}+2 t^{w e b} w^{g r b l} & \text { if face shell mortar and grouted }
\end{array} ;\right. \\
A_{z}^{g r b l}=\left\{\begin{array}{ll}
A_{\text {cell }}^{b l} & \text { if grouted } \\
0 & \text { if ungrouted }
\end{array} ; \text { and },\right. \\
A_{z}^{g r m r}= \begin{cases}A_{\text {cell }}^{b l} & \text { if full mortar and grouted } \\
w^{g r b l} l^{g r m r} & \text { if face shell mortar and grouted } \\
0 & \text { if ungrouted }\end{cases}
\end{gathered}
$$

The percentage of each material in the $z$ direction for Section A is given by:

$$
\begin{gathered}
\rho_{z}^{b l}=\frac{A_{z}^{b l}}{A_{z}^{b l}+A_{z}^{g r b l}} ; \text { and } \\
\rho_{z}^{g r b l}=\frac{A_{z}^{g r b l}}{A_{z}^{b l}+A_{z}^{g r b l}} .
\end{gathered}
$$

The percentage of each material in the $z$ direction for Section B is given by:

$$
\begin{gathered}
\rho_{z}^{m r}=\frac{A_{z}^{m}}{A_{z}^{m r}+A_{z}^{g r m r}} ; \text { and } \\
\rho_{z}^{g r m r}=\frac{A_{z}^{g r m r}}{A_{z}^{m r}+A_{z}^{g r m r}} .
\end{gathered}
$$

Part II: Masonry Compression Strength Modeling 


\subsection{Equilibrium Conditions}

The applied forces and average stresses in each direction must be in equilibrium. This results in uniformly distributed normal stress, $\sigma_{i}$, in the $i^{\text {th }}$ direction of the modified material configurations. In the $z$ direction equilibrium must be satisfied at Section $\mathrm{A}$ and Section B, see Figure 3.2, resulting in two normal stresses in the $z$ direction. Using equilibrium, the average normal stress in each direction can be defined as:

$$
\begin{gathered}
\sigma_{x}=\frac{P_{x}}{\sum A_{x}^{j}} ; \\
\sigma_{y}=\frac{P_{y}}{\sum A_{y}^{j}} ; \\
\sigma_{z}^{b l-g r b l}=\frac{P_{z}}{A_{z}^{b l}+A_{z}^{g r b l}} ; \text { and }, \\
\sigma_{z}^{m r-g r m r}=\frac{P_{z}}{A_{z}^{m r}+A_{z}^{g r m r}} .
\end{gathered}
$$

Where $P_{i}$ is the load in the $i^{\text {th }}$ direction. For a prism compression test $P_{x}=P_{y}=0$.

In matrix form the above equations become:

$$
\{\sigma\}=\left\{\begin{array}{c}
\sigma_{x} \\
\sigma_{y} \\
\sigma_{z}^{b l-g r b l} \\
\sigma_{z}^{m r-g r m r}
\end{array}\right\}=\left\{\begin{array}{c}
\frac{P_{x}}{\sum_{P_{x}^{j}}} \\
\frac{P_{y}}{\sum A_{y}^{j}} \\
\frac{P_{z}}{A_{z}^{b l}+A_{z}^{g r b l}} \\
\frac{P_{z}}{A_{z}^{m r}+A_{z}^{g r m r}}
\end{array}\right\} .
$$

\subsection{Compatibility Conditions}

Strains and compatibility requirements are considered in terms of average values. For compatibility and the assumption of perfect bond, the total strain each material is subject to in the modified material configuration for the $x$ and $y$ directions must be equal. In the $z$ direction the block and grout at Section A (see Figure 3.2) will have equal strains, $\epsilon_{z}^{b l-g r b l}$, and the mortar and the grout at Section B (see Figure 3.2) will have equal strains, $\epsilon_{z}^{m r-g r m r}$, but these two strains do not have to equal each other. This results in the following compatibility conditions:

$$
\begin{gathered}
\epsilon_{x}^{b l}=\epsilon_{x}^{m r}=\epsilon_{x}^{g r b l}=\epsilon_{x}^{g r m r}=\epsilon_{x} ; \\
\epsilon_{y}^{b l}=\epsilon_{y}^{m r}=\epsilon_{y}^{g r b l}=\epsilon_{y}^{g r m r}=\epsilon_{y} ;
\end{gathered}
$$

Part II: Masonry Compression Strength Modeling 


$$
\begin{gathered}
\epsilon_{z}^{b l}=\epsilon_{z}^{g r b l}=\epsilon_{z}^{b l-g r b l} ; \text { and, } \\
\epsilon_{z}^{m r}=\epsilon_{z}^{g r m r}=\epsilon_{z}^{m r-g r m r} .
\end{gathered}
$$

In matrix form:

$$
\{\epsilon\}=\left\{\begin{array}{c}
\epsilon_{x} \\
\epsilon_{y} \\
\epsilon_{z}^{b l-g r b l} \\
\epsilon_{z}^{m r-g r m r}
\end{array}\right\} .
$$

\subsection{Poisson's Effects}

The differences in material Poisson's ratios are important to consider in the interaction between the different materials used to make masonry. The differences in each materials Poisson's ratio causes them to expand in the $x$ and $y$ directions at different rates causing individual materials to be confined or softened. This results in a change in the material peak strength and strain, which is an important factor in the ultimate prism strength. The approach used is to decompose, for each material, the total strain into elastic (superscript $n$ ) and Poisson's strains (superscript $\nu$ ). In the following equations if a material is not present in Section A, then $\epsilon_{z}^{b l-g r b l}$ is zero. Likewise, for Section B and $\epsilon_{z}^{m r-g r m r}$. The decomposition of strains in the block is given by:

$$
\begin{gathered}
\epsilon_{x}=\epsilon_{x}^{b l, n}+\epsilon_{x}^{b l, \nu} ; \\
\epsilon_{y}=\epsilon_{y}^{b l, n}+\epsilon_{y}^{b l, \nu} ; \\
\epsilon_{z}^{b l-g r b l}=\epsilon_{z}^{b l, n}+\epsilon_{z}^{b l, \nu} ; \text { and }, \\
\epsilon_{z}^{m r-g r m r}=0 .
\end{gathered}
$$

For the mortar the decomposition is:

$$
\begin{gathered}
\epsilon_{x}=\epsilon_{x}^{m r, n}+\epsilon_{x}^{m r, \nu} ; \\
\epsilon_{y}=\epsilon_{y}^{m r, n}+\epsilon_{y}^{m r, \nu} ; \\
\epsilon_{z}^{b l-g r b l}=0 ; \text { and, } \\
\epsilon_{z}^{m r=g r m r}=\epsilon_{z}^{m r, n}+\epsilon_{z}^{m r, \nu} .
\end{gathered}
$$

Part II: Masonry Compression Strength Modeling 
For the grout in the block the decomposition is:

$$
\begin{gathered}
\epsilon_{x}=\epsilon_{x}^{g r b l, n}+\epsilon_{x}^{g r b l, \nu} ; \\
\epsilon_{y}=\epsilon_{y}^{g r b l, n}+\epsilon_{y}^{g r b l, \nu} ; \\
\epsilon_{z}^{b l-g r b l}=\epsilon_{z}^{g r b l, n}+\epsilon_{z}^{g r b l, \nu} ; \text { and }, \\
\epsilon_{z}^{m r-g r m r}=0 .
\end{gathered}
$$

Finally for the grout in the mortar:

$$
\begin{gathered}
\epsilon_{x}=\epsilon_{x}^{g r m r, n}+\epsilon_{x}^{g r m r, \nu} ; \\
\epsilon_{y}=\epsilon_{y}^{g r m r, n}+\epsilon_{y}^{g r m r, \nu} ; \\
\epsilon_{z}^{b l-g r b l}=0 ; \text { and, } \\
\epsilon_{z}^{m r-g r m r}=\epsilon_{z}^{g r m r, n}+\epsilon_{z}^{g r m r, \nu} .
\end{gathered}
$$

In general for material $j$, the decomposition in matrix form is:

$$
\{\epsilon\}=\left\{\begin{array}{c}
\epsilon_{x} \\
\epsilon_{y} \\
\epsilon_{z}^{b l-g r b l} \\
\epsilon_{z}^{m r-g r m r}
\end{array}\right\}=\left\{\begin{array}{c}
\epsilon_{x}^{j, n}+\epsilon_{x}^{j, \nu} \\
\epsilon_{y}^{j, n}+\epsilon_{y}^{j, \nu} \\
\epsilon_{z}^{j, n}+\epsilon_{z}^{j, \nu} \\
\epsilon_{z}^{j, n}+\epsilon_{z}^{j, \nu}
\end{array}\right\}=\left\{\epsilon^{j, n}\right\}+\left\{\epsilon^{j, \nu}\right\}
$$

where $\epsilon_{i}$ is the total strain, $\epsilon_{i}^{j, n}$ is the net elastic strains (used for stress calculations), and $\epsilon_{i}^{j, \nu}$ is the Poisson's strain effect for material $j$ in direction $i$. The Poisson's strain for material $j$ can be determined as follows:

$$
\begin{aligned}
\epsilon_{x}^{j, \nu} & =-\nu_{x y} \frac{\sigma_{y}^{j, n}}{\bar{E}_{y}^{j}}-\nu_{x z} \frac{\sigma_{z}^{j, n}}{\bar{E}_{z}^{j}} ; \\
\epsilon_{y}^{j, \nu} & =-\nu_{y x} \frac{\sigma_{x}^{j, n}}{\bar{E}_{x}^{j}}-\nu_{y z} \frac{\sigma_{z}^{j, n}}{\bar{E}_{z}^{j}} ; \\
\epsilon_{z}^{j, \nu} & =-\nu_{z x} \frac{\sigma_{x}^{j, n}}{\bar{E}_{x}^{j}}-\nu_{z y} \frac{\sigma_{y}^{j, n}}{\bar{E}_{y}^{j}} ;
\end{aligned}
$$

where $\nu_{i k}$ is the Poisson's ratio, $\sigma_{i}^{j, n}$ is the net elastic stress, and $\bar{E}_{i}^{j}$ is the secant stiffness in the $i^{\text {th }}$ direction.

Part II: Masonry Compression Strength Modeling 


\subsection{Stress-Strain Relations}

Using a total load secant stiffness model the stress-strain relation accounting for Poisson's effects for the system becomes:

$$
\{\sigma\}=[D]\{\epsilon\}-\left\{\sigma^{\nu}\right\}
$$

where $\{\sigma\}$ is the total stress vector, $[D]$ is the composite secant stiffness matrix, $\{\epsilon\}$ is the total strain vector, and $\left\{\sigma^{\nu}\right\}$ is the Poisson's stress vector. The composite material stiffness matrix is defined as:

$$
[D]=\left[D^{b l}\right]+\left[D^{m r}\right]+\left[D^{g r b l}\right]+\left[D^{g r m r}\right]
$$

where

$$
\begin{gathered}
{\left[D^{b l}\right]=\left[\begin{array}{cccc}
\rho_{x}^{b l} \bar{E}_{x}^{b l} & 0 & 0 & 0 \\
0 & \rho_{y}^{b l} \bar{E}_{y}^{b l} & 0 & 0 \\
0 & 0 & \rho_{z}^{b l} \bar{E}_{z}^{b l} & 0 \\
0 & 0 & 0 & 0
\end{array}\right] ;} \\
{\left[D^{m r}\right]=\left[\begin{array}{cccc}
\rho_{x}^{m r} \bar{E}_{x}^{m r} & 0 & 0 & 0 \\
0 & \rho_{y}^{m r} \bar{E}_{y}^{m r} & 0 & 0 \\
0 & 0 & 0 & 0 \\
0 & 0 & 0 & \rho_{z}^{m r} \bar{E}_{z}^{m r}
\end{array}\right] ;} \\
{\left[D^{g r b l}\right]=\left[\begin{array}{cccc}
\rho_{x}^{g r b l} \bar{E}_{x}^{g r b l} & 0 & 0 & 0 \\
0 & \rho_{y}^{g r b l} \bar{E}_{y}^{g r b l} & 0 & 0 \\
0 & 0 & \rho_{z}^{g r b l} \bar{E}_{z}^{g r b l} & 0 \\
0 & 0 & 0 & 0
\end{array}\right] ; \text { and, }} \\
{\left[\begin{array}{cccc}
\rho_{x}^{g r m r} \bar{E}_{x}^{g r m r} & 0 & 0 & 0 \\
0 & \rho_{y}^{g r m r} \bar{E}_{y}^{g r m r} & 0 & 0 \\
0 & 0 & 0 & 0 \\
0 & 0 & 0 & \rho_{z}^{g r m r} \\
\bar{E}_{z}^{g r m r}
\end{array}\right] .}
\end{gathered}
$$

In the material stiffness matrices secant moduli for material $j$ in direction $i$ is defined as:

$$
\bar{E}_{i}^{j}=\frac{\sigma_{i}^{j, n}}{\epsilon_{i}^{j, n}},
$$

Part II: Masonry Compression Strength Modeling 
determined using net strains, not total strain. Notice the off-diagonal terms in the material stiffness matrices are zero as all the expansion strains are modelled through the prestrain concept (Vecchio, 1990). The Poisson's stress vector is given by:

$$
\begin{aligned}
\left\{\sigma^{\nu}\right\} & =\left\{\begin{array}{c}
\sigma_{x}^{\nu} \\
\sigma_{y}^{\nu} \\
\sigma_{z}^{b l-g r b l, \nu} \\
\sigma_{z}^{m r-g r m r, \nu}
\end{array}\right\} \\
& =\left[D^{b l}\right]\left\{\epsilon^{b l, \nu}\right\}+\left[D^{m r}\right]\left\{\epsilon^{m r, \nu}\right\}+\left[D^{g r b l}\right]\left\{\epsilon^{g r b l, \nu}\right\}+\left[D^{g r m r}\right]\left\{\epsilon^{g r m r, \nu}\right\} .
\end{aligned}
$$

The stress in the block can be determined from:

$$
\left\{\sigma^{b l}\right\}=\left\{\rho^{b l}\right\}^{-1}\left[D^{b l}\right]\left\{\{\epsilon\}-\left\{\epsilon^{b l, \nu}\right\}\right\} \text { and }
$$

the stress in the mortar:

$$
\left\{\sigma^{m r}\right\}=\left\{\rho^{m r}\right\}^{-1}\left[D^{m r}\right]\left\{\{\epsilon\}-\left\{\epsilon^{m r, \nu}\right\}\right\} .
$$

If grouted, the grout material stresses can be determined by:

$$
\begin{aligned}
& \left\{\sigma^{g r b l}\right\}=\left\{\rho^{g r b l}\right\}^{-1}\left[D^{g r b l}\right]\left\{\{\epsilon\}-\left\{\epsilon^{g r b l, \nu}\right\}\right\} \text { and } \\
& \left\{\sigma^{g r m r}\right\}=\left\{\rho^{g r m r}\right\}^{-1}\left[D^{g r m r}\right]\left\{\{\epsilon\}-\left\{\epsilon^{g r m r}, \nu\right\}\right\} .
\end{aligned}
$$

\subsection{Proposed Material Models}

Constitutive models are necessary to relate the strains in the compatibility relationships with the stresses in the equilibrium relationships. To describe the response of each material, constitutive relations are required for the base strength, which can then be modified to account for strength enhancement due to confinement or strength degradation due to bi-axial tension. To reflect the nonlinear behavior of the block, mortar, and grout $\left[D^{b l}\right]$, $\left[D^{m r}\right],\left[D^{g r b l}\right]$, and $\left[D^{g r m r}\right]$ are modified according to constitutive relations. The form of these stiffness matrices is based on a secant stiffness approach.

Part II: Masonry Compression Strength Modeling 


\subsubsection{Constitutive Models for Compression}

Compression response models compute the compressive stress $\sigma_{i}^{j, n}$ from the compressive strain $\epsilon_{i}^{j, n}$. The peak stress, $f_{p, i}^{j}$, and peak strain, $\epsilon_{p, i}^{j}$, are determined by adjusting the unconfined uniaxial material strength $f_{c, i}^{\prime j}$ and the corresponding strain $\epsilon_{o, i}^{j}$ for strength enhancement due to confinement or strength degradation due to bi-axial tension.

The stress-strain response of blocks, mortar, and grout are all generally non-linear, but their response can all be slightly different; thus, SACM incorporates four compression models: Hognestad Model (1951), Popovics Model (1970), Smith and Young Model (1956), and a linear model.

\section{Hognestad Model}

The Hognestad parabola (1951), as shown Figure 3.5, is a simple compression response curve. The stress-strain curve is described by the following relationship:

$$
\sigma_{i}^{j, n}=-f_{p, i}^{j}\left\{2\left(\frac{\epsilon_{i}^{j, n}}{\epsilon_{p, i}^{j}}\right)-\left(\frac{\epsilon_{i}^{j, n}}{\epsilon_{p, i}^{j}}\right)^{2}\right\} \text { for } 2 \epsilon_{p, i}^{j}<\epsilon_{i}^{j, n}<0 .
$$

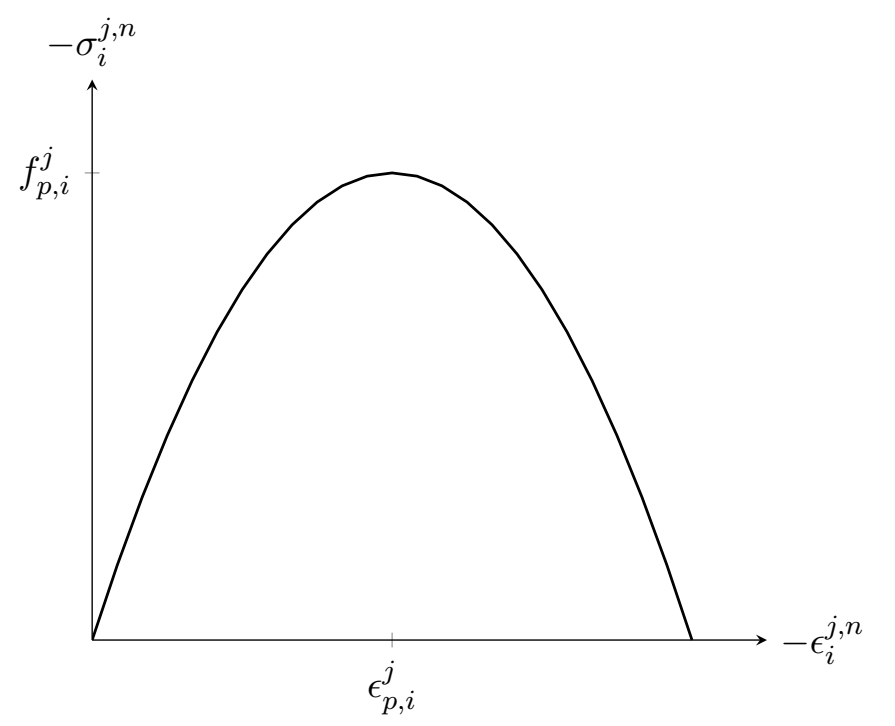

Figure 3.5: Hognestad Parabola

Part II: Masonry Compression Strength Modeling 


\section{Popovics Model}

Popovics (1970) presented stress-strain curves, as shown in Figure 3.6. These curves reflect the greater stiffness and linearity of the ascending branch and the reduced ductility as peak compressive stress increases. The stress-strain curve is given by the following equation:

$$
\sigma_{i}^{j, n}=-\left(\frac{\epsilon_{i}^{j, n}}{\epsilon_{p, i}^{j}}\right) f_{p, i}^{j} \frac{n}{n-1+\left(\frac{\epsilon_{i}^{j, n}}{\epsilon_{p, i}^{j}}\right)^{n}} \text { for } \epsilon_{i}^{j, n}<0
$$

The long fraction represents the deviation from linear-elastic response. The curve fitting parameter, $n$, captures the greater linearity of higher strength blocks, mortar, and grout through the diminishing difference between the initial tangent stiffness $E_{i, o}^{j}$, and secant stiffness, $E_{i, s e c}^{j}$. These values are computed as follows:

$$
\begin{gathered}
n=\frac{E_{i, o}^{j}}{E_{i, o}^{j}-E_{i, s e c}^{j}} \text { and } \\
E_{i, s e c}^{j}=\frac{f_{p, i}^{j}}{\epsilon_{p, i}^{j}} .
\end{gathered}
$$

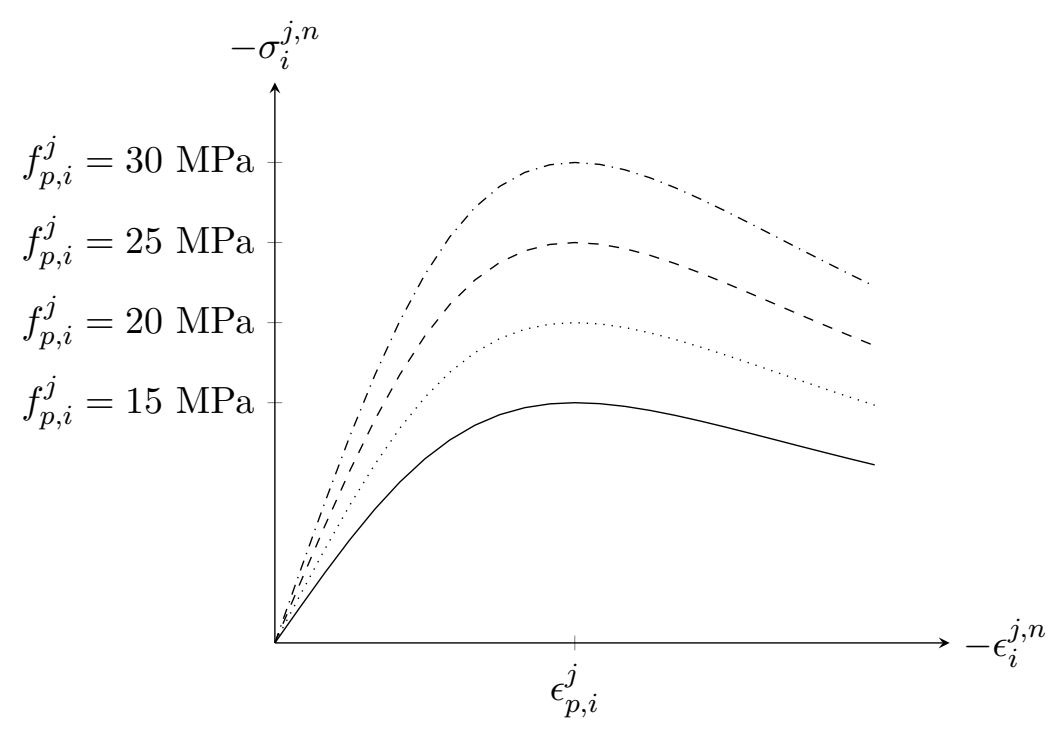

Figure 3.6: Popovics Compression Model

Part II: Masonry Compression Strength Modeling 


\section{Smith-Young Model}

Smith and Young (1956) determined the following equation for the compression stressstrain behaviour:

$$
\sigma_{i}^{j, n}=-f_{p, i}^{j}\left(\frac{\epsilon_{i}^{j, n}}{\epsilon_{p, i}^{j}}\right) \exp \left[1-\left(\frac{\epsilon_{i}^{j, n}}{\epsilon_{p, i}^{j}}\right)\right] \text { for } \epsilon_{i}^{j, n}<0 .
$$

The Smith-Young curve is illustrated in Figure 3.7.

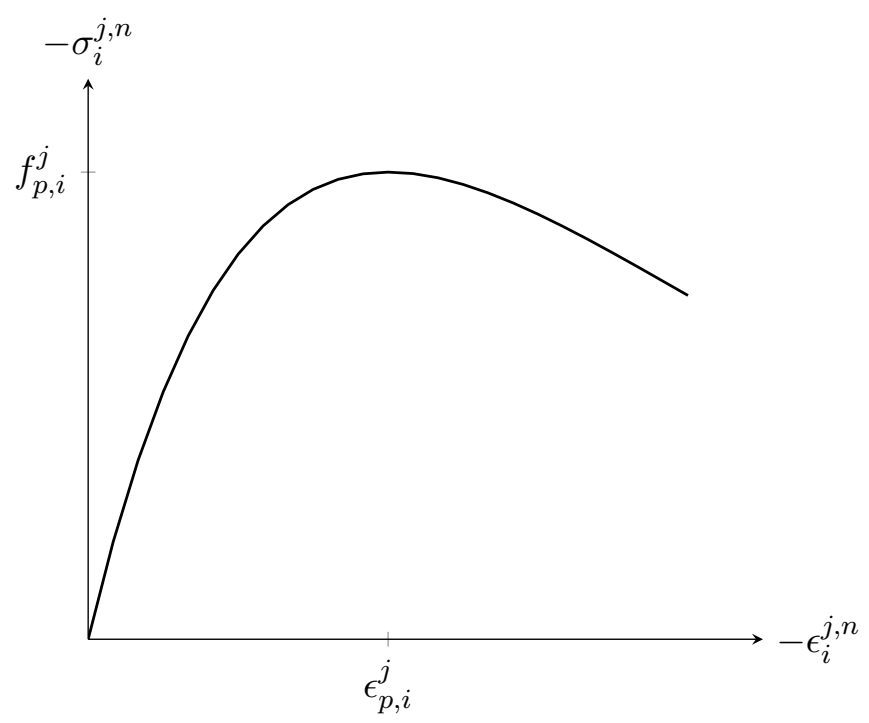

Figure 3.7: Smith-Young Model

Part II: Masonry Compression Strength Modeling 


\section{Linear Model}

The linear model, shown in Figure 3.8, is an elastic-plastic compression response curve defined as:

$$
\sigma_{i}^{j, n}= \begin{cases}-\left(\frac{\epsilon_{i}^{j, n}}{\epsilon_{p, i}^{j}}\right) f_{p, i}^{j} & \text { for } \epsilon_{p, i}^{j}<\epsilon_{i}^{j, n}<0 \\ -f_{p, i}^{j} & \text { for } \epsilon_{i}^{j, n}<\epsilon_{p, i}^{j}<0\end{cases}
$$

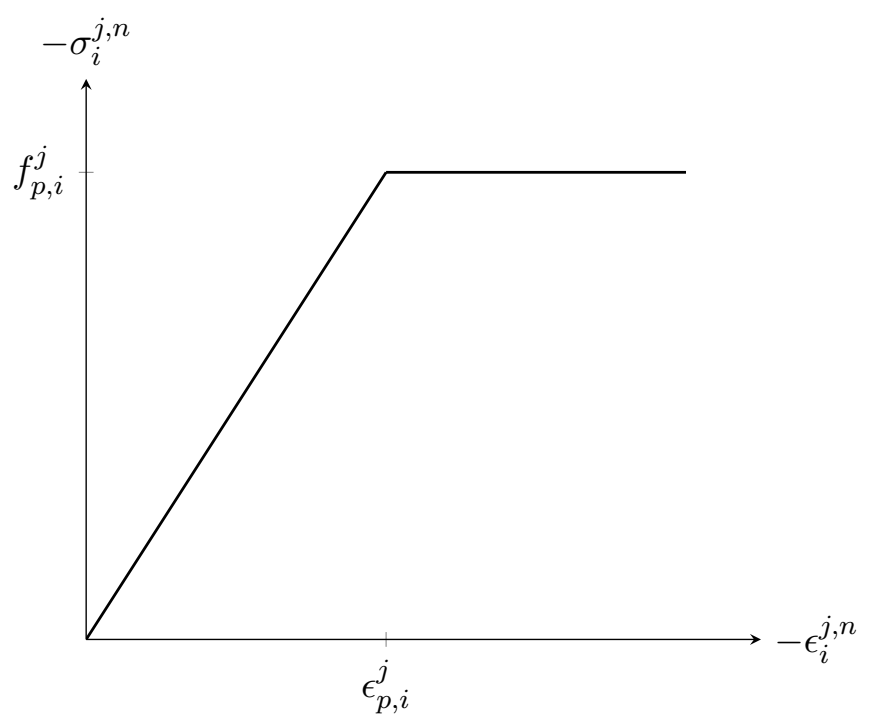

Figure 3.8: Linear Compression Model

\subsubsection{Strength Enhancement or Strength Degradation}

The peak stress and strain in compression are determined by modifying the uniaxial compressive strength, $f_{c, i}^{\prime}$, and the corresponding strain, $\epsilon_{o, i}^{j}$, by $\beta_{i}^{j}$ to account for any strength enhancement or strength degradation in the material. The strength enhancement can be caused by confinement of the material, while bilateral tension on a material can case strength degradation. Thus,

$$
\begin{gathered}
f_{p, i}^{j}=\beta_{i}^{j} f_{c, i}^{\prime j} \text { and } \\
\epsilon_{p, i}^{j}=\beta_{i}^{j} \epsilon_{o, i}^{j} .
\end{gathered}
$$

As the block is typically in biaxial tension in both ungrouted masonry and grouted masonry $\beta_{z}^{b l}$ will normally be less than one. On the other hand, the mortar and grout are typically confined thus $\beta_{z}^{m r}, \beta_{z}^{g r b l}$, and $\beta_{z}^{g r m r}$ will usually be greater than one (see Figure 3.9).

Part II: Masonry Compression Strength Modeling 


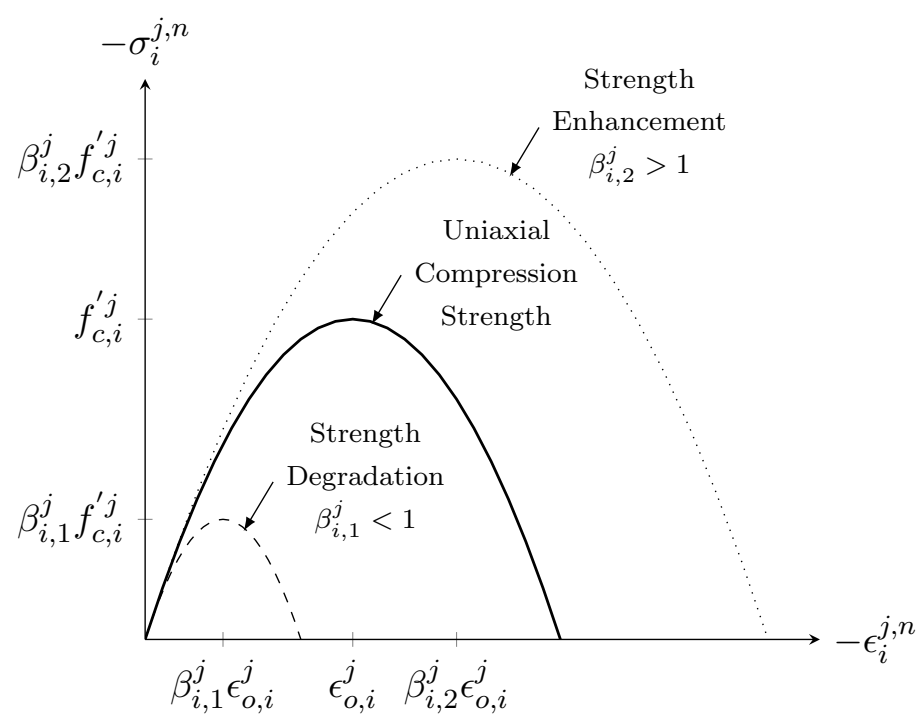

Figure 3.9: Hognestad Parabola Compression Response with Strength Enhancement or Strength Degradation

The strength enhancement or degradation factor $\beta_{z}^{j}$, in the direction of major compression load ( $z$-direction), is the ratio of the failure stress $\sigma_{3 f}^{j}$ to the uniaxial compressive strength in the $z$-direction:

$$
\beta_{z}^{j}=\frac{\sigma_{3 f}^{j}}{f_{c, z}^{\prime j}}
$$

The failure stress $\sigma_{3 f}^{j}$ is the stress required to cause failure in the direction of maximum compressive stress in the presence of stresses $\sigma_{1}^{j}$ and $\sigma_{2}^{j}$ and is determined by numerically solving the failure surface of Hsieh et al. (1979):

$$
2.0108 \frac{J_{2}}{\left(f_{c, z}^{\prime j}\right)^{2}}+0.9714 \frac{\sqrt{J_{2}}}{f_{c, z}^{\prime j}}+9.1412 \frac{\sigma_{1}^{j}}{f_{c, z}^{\prime j}}+0.2312 \frac{I_{1}}{f_{c, z}^{\prime j}}-1=0
$$

where, $I_{1}$ is the first stress invariant determined from:

$$
I_{1}=\sigma_{1}^{j}+\sigma_{2}^{j}+\sigma_{3 f}^{j}
$$

and $J_{2}$ is the second deviatoric stress invariant defined as:

$$
J_{2}=\frac{1}{6}\left[\left(\sigma_{1}^{j}-\sigma_{2}^{j}\right)^{2}+\left(\sigma_{2}^{j}-\sigma_{3 f}^{j}\right)^{2}+\left(\sigma_{3 f}^{j}-\sigma_{1}^{j}\right)^{2}\right] .
$$

Part II: Masonry Compression Strength Modeling 
In the $x$ and $y$ direction the effect of strength enhancement or strength degradation is assumed not to impact the overall strength of the prism, giving

$$
\beta_{x}^{j}=\beta_{y}^{j}=1 .
$$

\subsubsection{Constitutive Model for Tension}

In tension cementitious materials tend to be brittle and their response can be differentiated into uncracked and cracked responses. The response of the materials in tension is given by:

$$
\sigma_{i}^{j, n}= \begin{cases}E_{o, i}^{j} \epsilon_{i}^{j, n} & 0<\epsilon_{i}^{j, n} \leq \epsilon_{\mathrm{cr}, i}^{j} \\ 0 & \epsilon_{i}^{j, n}>\epsilon_{\mathrm{cr}, i}^{j}\end{cases}
$$

where,

$$
\epsilon_{\mathrm{cr}, i}^{j}=\frac{f_{t, i}^{j}}{E_{o, i}^{j}}
$$

as shown in Figure 3.10.

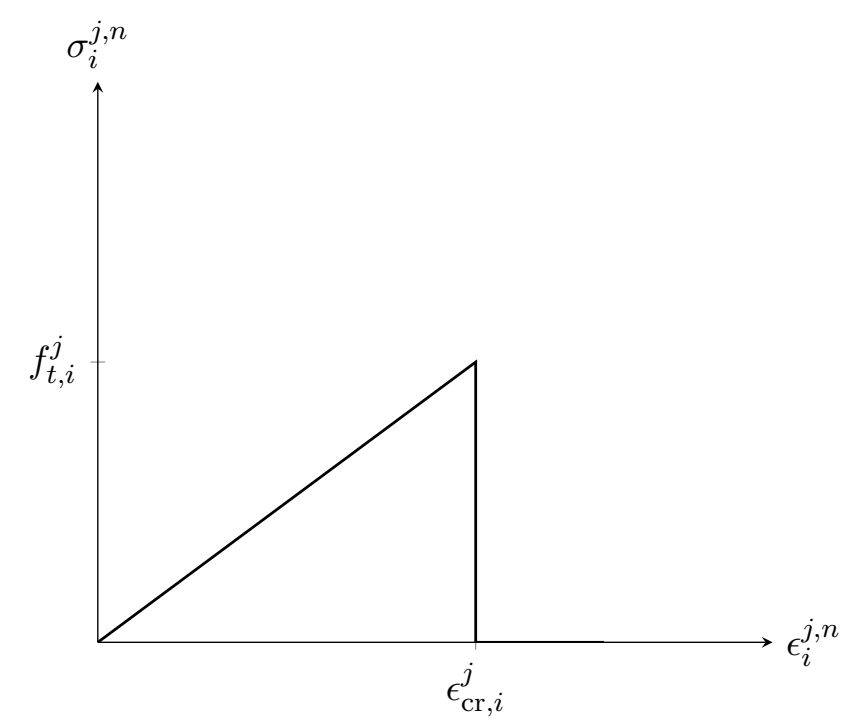

Figure 3.10: Tension Response

Part II: Masonry Compression Strength Modeling 


\subsubsection{Lateral Expansion}

When the block, mortar, or grout is uncracked or experiences relatively small tensile strains, lateral expansion may account for a significant portion of the total strains. If these strains are incorrectly accounted for strength enhancement or strength degradation may be overestimated or underestimated.

Also, due to internal microcracking, the rate of lateral expansion increases as the compressive stresses increase. Near the peak compressive stress the lateral expansion increases rapidly. This behaviour is modelled by a fit to the uniaxial Poisson's ratio data of Kupfer et al. (1969), shown in Figure 3.11, and is computed as follows:

$$
\nu_{i k}^{j}=\left\{\begin{array}{ll}
\nu_{o}^{j} & 0>\epsilon_{k}^{j} \leq \frac{\beta_{i}^{j} \epsilon_{o, k}^{j}}{2} \\
\nu_{o}^{j}\left[1+1.5\left(\frac{2 \epsilon_{k}^{j}}{\beta_{i}^{j} \epsilon_{o, k}^{j}}-1\right)^{2}\right] \leq 0.50 & \epsilon_{k}^{j}>\frac{\beta_{i}^{j} \epsilon_{o, k}^{j}}{2}
\end{array},\right.
$$

where $\nu_{o}^{j}$ is the initial Poisson's ratio of the material. This relationship implies that only three Poisson's ratios are independent, since

$$
\begin{gathered}
\nu_{y x}^{j}=\nu_{z x}^{j} ; \\
\nu_{x y}^{j}=\nu_{z y}^{j} ; \text { and, } \\
\nu_{x z}^{j}=\nu_{y z}^{j} .
\end{gathered}
$$

For materials in tension, the initial Poisson's ratio is used.

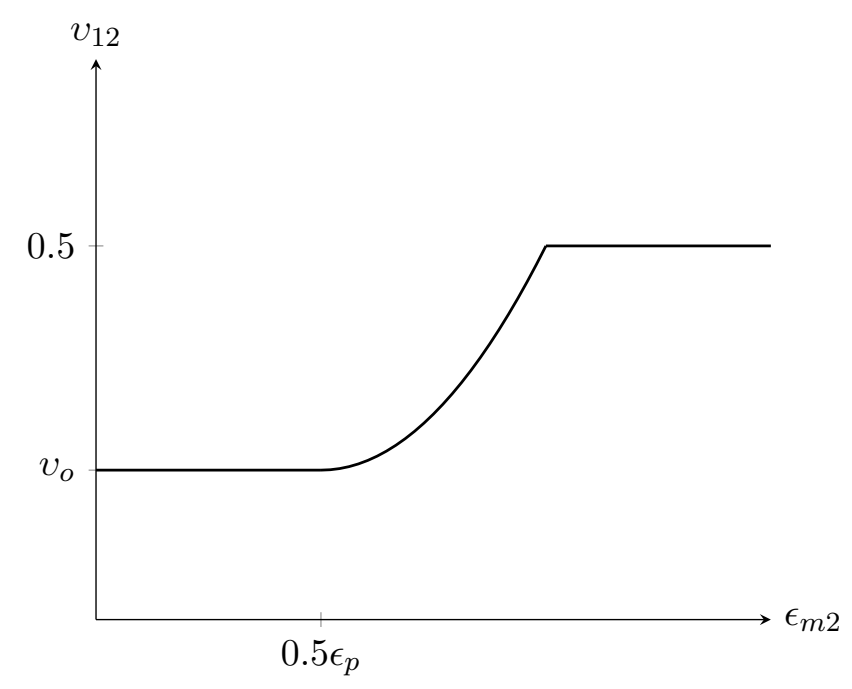

Figure 3.11: Kupfer Variable Poisson's Ratio Model

Part II: Masonry Compression Strength Modeling 


\subsection{Failure}

Failure of the block, mortar, or grout is defined by a Mohr-Coulomb failure surface with a tension cut-off. The Mohr-Coulomb stress failure surface, see Figure 3.12a, is defined as:

$$
\sigma_{1}^{j}\left(\frac{1+\sin \phi}{2 c_{i} \cos \phi}\right)-\sigma_{3}^{j}\left(\frac{1-\sin \phi}{2 c_{i} \cos \phi}\right)-1=0
$$

for $\sigma_{1}^{j}>\sigma_{2}^{j}>\sigma_{3}^{j}$, where

$$
c_{i}=\frac{f_{p, i}^{j}}{4}
$$

and

$$
\phi=35^{\circ} \text {. }
$$

The values of $c_{i}$ and $\phi$ have been successfully used by Chen (1982).

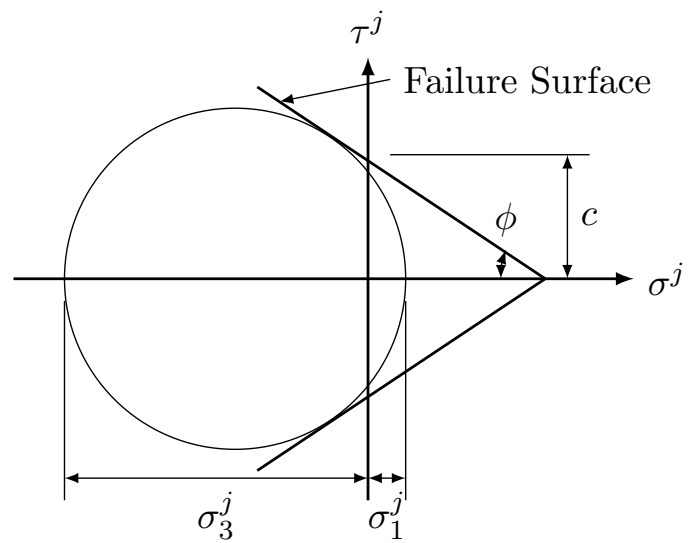

(a) Compression Failure

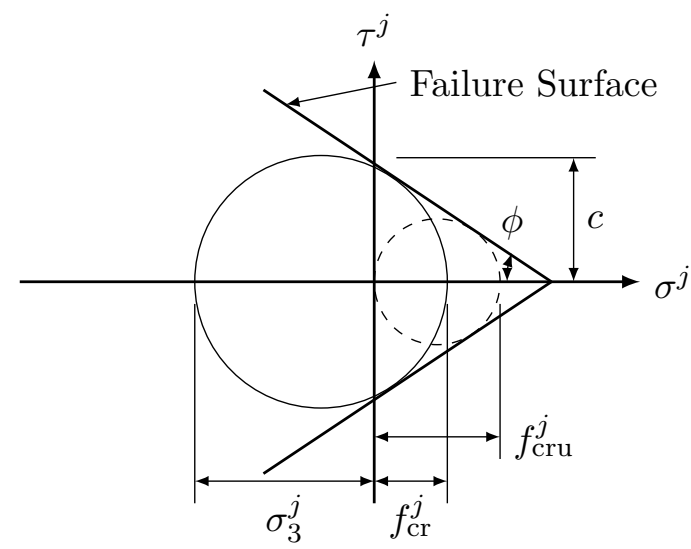

(b) Tension Failure

Figure 3.12: Failure Criteron

The tension cut-off, Figure $3.12 \mathrm{~b}$, is defined by the cracking strength of the material, which can vary with different stress states. The tension cut-off surface is defined by:

$$
\sigma_{1}^{j}=f_{\text {cr }}^{j} \text { or } \sigma_{2}^{j}=f_{\text {cr }}^{j} \text { or } \sigma_{3}^{j}=f_{\text {cr }}^{j}
$$

where

$$
f_{\mathrm{cr}}^{j}=f_{\mathrm{cru}}^{j}\left(1+\frac{\sigma_{3}^{j}}{f_{c, z}^{\prime j}}\right), 0.20 f_{t, z}^{\prime j} \leq f_{\mathrm{cr}}^{j} \leq f_{t, z}^{\prime j} .
$$

Part II: Masonry Compression Strength Modeling 
The failure stress $\sigma_{1}^{j}$ is equal to $f_{\text {cru }}^{j}$ when $\sigma_{3}^{j}$ is zero, which is computed from the envelope as:

$$
f_{\text {cru }}^{j}=\frac{2 c \cos \phi}{1+\sin \phi}
$$

The cohesion, $c$, which is determined by noting that at failure in uniaxial compression, the maximum compressive stress, $\sigma_{3}^{j}$, is equal to the compressive strength of the material $f_{c, z}^{\prime, j}$ and $\sigma_{1}^{j}$ is zero is; thus:

$$
c=f_{c, z}^{\prime j}\left(\frac{1-\sin \phi}{2 \cos \phi}\right) .
$$

Failure of any of the component materials, in either compression or tension, represents failure of the prism. The load, $P_{z}$, at failure of the prism allows for the failure stress, $f_{m}^{\prime}$, to be determined by dividing this load by an appropriate area depending on mortar bedding and grouting.

\subsection{Uniaxial Compressive Strength Corrections}

Since there is no universally accepted method for determining or reporting the uniaxial compressive strength of any of the materials used to construct masonry, this thesis presents conversion factors to unify the reported values for use in the model.

\subsubsection{Block Uniaxial Compressive Strength Corrections}

Blocks are typically tested as single units that are fully hard capped with cement paste or sulfur. This type of capping is meant to reduce the platen restraint of the loading machine, but the capping itself also produces a platen restraint effect which will increase the reported uniaxial compressive strength. To reduce capping restraint soft capping, such as fibreboard, can be used. Chahine (1989) reported full soft capping results in a $19 \%$ decrease in block compressive strength, compared to full hard capping. In addition, only face shell capping instead of full capping a block resulted in a $20 \%$ and $25 \%$ increases the block's compressive strength for soft capping and hard capping respectively (Chahine, 1989). To unify the methods of determining the block compressive strength for the model, full soft capping will be used in the model, thus:

$$
f_{c, i}^{\prime b l}=k_{1}^{b l} k_{2}^{b l} f_{c, r e p}^{\prime b l},
$$

Part II: Masonry Compression Strength Modeling 
where $f_{c, r e p}^{\prime}$ is the reported block net area compressive strength, $k_{1}^{b l}$ is a block capping correction factor equal to:

$$
k_{1}^{b l}= \begin{cases}1.00 & \text { if soft capped } \\ 0.81 & \text { if hard capped }\end{cases}
$$

and $k_{2}^{b l}$ is a block bedding correction factor equal to:

$$
k_{2}^{b l}= \begin{cases}1.00 & \text { if full capped } \\ 0.80 & \text { if faceshell soft capped } \\ 0.75 & \text { if faceshell hard capped }\end{cases}
$$

\subsubsection{Mortar Uniaxial Compressive Strength Corrections}

Mortar uniaxial compressive strengths are typically reported and measured by testing either cubes or cylinders. To unify the measurement methods a cube compressive strength will be used for the model. In addition, neither of these testing methods represent the insitu strength of the mortar, as each testing specimen is formed in a non-absorbent mold. In actual masonry assemblages water is removed from the mortar due to the suction exerted by the masonry blocks, thereby increasing the mortar strength. Hamid (1978) reported a $17 \%$ increase in cube compression strength when some of the water was allowed to be absorbed by the block before placing the mortar in the nonabsorbent molds. The conversion for model mortar compressive strength can therefore be calculated as:

$$
f_{c}^{\prime m r}=k_{1}^{m r} k_{2}^{m r} f_{c, r e p}^{\prime m r}
$$

where $f_{c, r e p}^{\prime m r}$ is the reported mortar compressive strength, $k_{1}^{m r}$ is a mortar specimen correction factor equal to:

$$
k_{1}^{m r}= \begin{cases}1.00 & \text { if cube specimen } \\ 1.17 & \text { if cyclinder specimen (Singh, 1988) }\end{cases}
$$

and $k_{2}^{m r}$ is a mortar absorbency correction factor equal to:

$$
k_{2}^{m r}=\left\{\begin{array}{ll}
1.00 & \text { if absorbent mold } \\
1.17 & \text { if non-absorbent mold }
\end{array} .\right.
$$

Part II: Masonry Compression Strength Modeling 


\subsubsection{Grout Uniaxial Compressive Strength Corrections}

Grout strengths are determined by testing different types of specimens: cubes, cylinders or absorbent block moulded prisms. The grout compressive strength for use in the SACM is unified based on the cylinder strength. Similar to mortar, there is also a grout absorbency correction factor, based on research done by Hamid (1978). Thus, the grout unaxial compressive strength for the model is:

$$
f_{c}^{\prime g r}=k_{1}^{g r} k_{2}^{g r} f_{c r e p}^{\prime}, g r
$$

where $f_{c, r e p}^{\prime}$ is the reported grout compressive strength, $k_{1}^{g r}$ is a grout specimen correction factor equal to:

$$
k_{1}^{g r}= \begin{cases}1.00 & \begin{array}{l}
\text { if cyclinder specimen } \\
\text { if cube specimen }
\end{array} \\
0.85\left[0.56+\frac{0.697}{\left.\frac{V}{152 h_{g} d}+\frac{h_{g}}{d}\right]}\right. & \text { if block moulded prism specimen (Neville, 1966) }\end{cases}
$$

and $k_{2}^{g r}$ is a grout absorbency correction factor equal to:

$$
k_{2}^{g r}=\left\{\begin{array}{ll}
1.00 & \text { if absorbent mold } \\
1.53-0.01 k_{1}^{g} f_{c, r e p}^{\prime g} & \text { if non-absorbent mold (Hamid, 1978) }
\end{array} .\right.
$$

\subsection{Solution Procedure}

As the composite material stiffness matrix depends on the current strain and the current strain level is dependent on the composite material stiffness an iterative solution is required. A flowchart of the procedure is shown in Figure 3.13.

An analysis begins with the modeller correcting the material compression properties, then inputting these values and the material dimensions. From there the SACM calculates the areas, calculates the material stiffness matrices and forms the composite stiffness matrix. The material Poisson's strains and stresses are then determined. The total material strains can then be determined, followed by the net material strains and an evaluation of each materials stress in each direction using the constitutive models. This permits determination of new secant moduli and if they have converged to the

Part II: Masonry Compression Strength Modeling 
specified limit, the load stage is complete. Otherwise, the new secant moduli are averaged with secant moduli from the start of the load step and are used to perform another iteration.

\subsection{Model Inputs and Default Values}

The model requires a number of inputs, including block dimensions and material properties, before an analysis can proceed. The user can get these inputs from compression tests preformed on the masonry material components, but a number of the material properties can be set to default values as described in Table 3.2, Table 3.3, Table 3.4, and Table 3.5. Additionally, the default compression constitutive relationship is the Hognestad parabola, but the modeller is able to change this to either the Popovics model, Smith-Young model or linear model as discussed in Subsection 3.7.1.

Table 3.2: SACM Input for Block Dimensions

\begin{tabular}{llll}
\hline Variable Name & Symbol & Units & Default Value \\
\hline Length & $l^{b l}$ & $\mathrm{~mm}$ & None \\
Width & $w^{b l}$ & $\mathrm{~mm}$ & None \\
Height & $h^{b l}$ & $\mathrm{~mm}$ & None \\
Percent Solid & $\rho_{\text {solid }}^{b l}$ & $\%$ & None \\
Face Shell Thickness & $t^{f s}$ & $\mathrm{~mm}$ & None \\
Number of Webs & $n^{\text {web }}$ & - & None \\
\hline
\end{tabular}

Part II: Masonry Compression Strength Modeling 


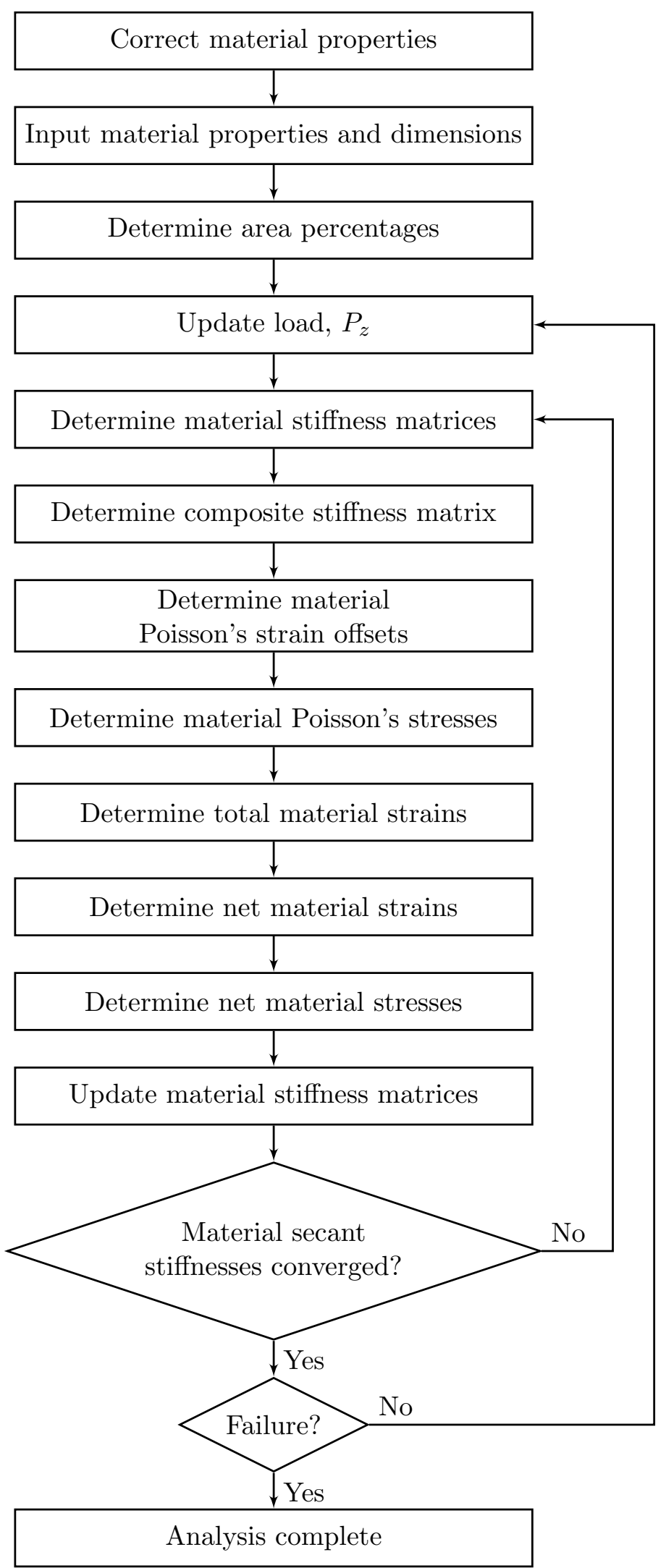

Figure 3.13: SACM Solution Algorithm

Part II: Masonry Compression Strength Modeling 
Table 3.3: SACM Input for Block Material Properties

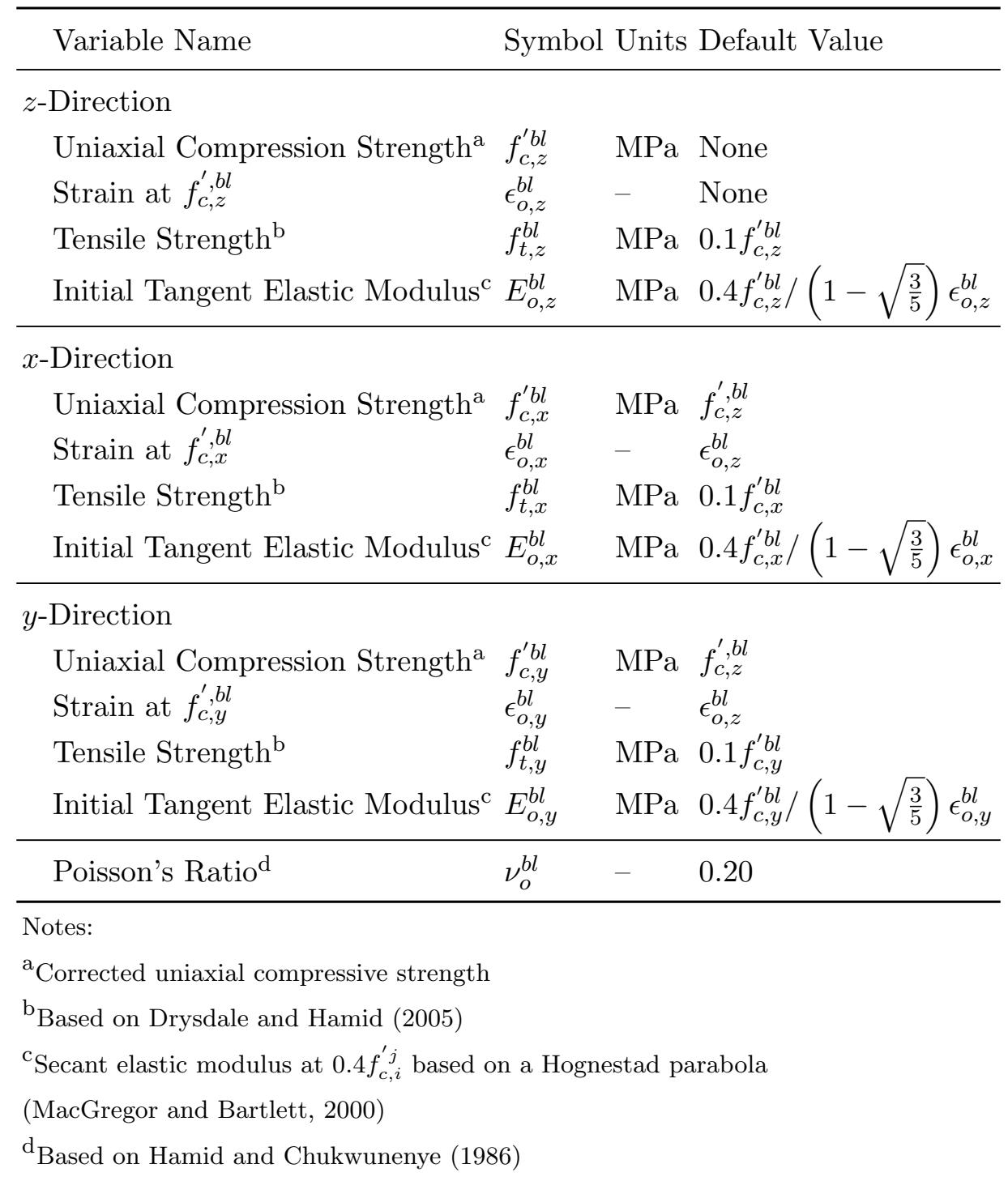


Table 3.4: SACM Input for Mortar

\begin{tabular}{llll}
\hline Variable Name & \multicolumn{3}{l}{ Symbol Units Default Value } \\
\hline Uniaxial Compression Strength $^{\mathrm{a}}$ & $f_{c}^{\prime m r}$ & $\mathrm{MPa}$ & None \\
${\text { Strain at } f_{c}^{\prime}, m r}^{{ }^{m r}}$ & $\epsilon_{o}^{m r}$ & - & None \\
Tensile Strength $^{\mathrm{b}}$ & $f_{t}^{m r}$ & $\mathrm{MPa}$ & $0.33 \sqrt{f_{c}^{\prime m r}}$ \\
Initial Tangent Elastic Modulus $^{\mathrm{c}}$ & $E_{o}^{m r}$ & $\mathrm{MPa}$ & $0.4 f_{c}^{\prime m r} /\left(1-\sqrt{\frac{3}{5}}\right) \epsilon_{o}^{m r}$ \\
Poisson's Ratio $^{\mathrm{d}}$ & $\nu_{o}^{m r}$ & - & 0.30 \\
Bedding Thickness $^{m r}$ & $t^{m r}$ & $\mathrm{~mm}$ & 10 \\
Bedding Type & \multicolumn{3}{l}{ Face shell or Full } \\
\hline
\end{tabular}

Notes:

${ }^{\mathrm{a} C o r r e c t e d ~ u n i a x i a l ~ c o m p r e s s i v e ~ s t r e n g t h ~}$

${ }^{\mathrm{b}}$ Similar to concrete

${ }^{\mathrm{c}}$ Secant elastic modulus at $0.4 f_{c, i}^{\prime j}$ based on a Hognestad parabola

(MacGregor and Bartlett, 2000)

${ }^{d}$ Based on Cheema and Klingner (1986)

Table 3.5: SACM Input for Grout

\begin{tabular}{|c|c|c|c|}
\hline Variable Name & Symbol & Units & Default Value \\
\hline Uniaxial Compression Strength ${ }^{\mathrm{a}}$ & $f_{c}^{\prime} g r$ & $\mathrm{MPa}$ & None \\
\hline Strain at $f_{c}^{\prime}, g r$ & $\epsilon_{o}^{g r}$ & - & None \\
\hline Tensile Strength ${ }^{\mathrm{b}}$ & $f_{t}^{g r}$ & $\mathrm{MPa}$ & $0.33 \sqrt{f_{c}^{\prime} g r}$ \\
\hline Initial Tangent Elastic Modulus ${ }^{\mathrm{c}}$ & $E_{o}^{g r}$ & $\mathrm{MPa}$ & $0.4 f_{c}^{\prime} g r /\left(1-\sqrt{\frac{3}{5}}\right) \epsilon_{o}^{g r}$ \\
\hline Poisson's Ratio ${ }^{d}$ & $\nu_{o}^{g r}$ & - & 0.35 \\
\hline
\end{tabular}

Notes:

${ }^{\mathrm{a}}$ Corrected uniaxial compressive strength

${ }^{\mathrm{b}}$ Similar to concrete

${ }^{\mathrm{c}}$ Secant elastic modulus at $0.4 f_{c, i}^{\prime}{ }_{\text {i }}$ based on a Hognestad parabola

(MacGregor and Bartlett, 2000)

$\mathrm{d}_{\text {Based on Cheema and Klingner (1986) }}$

\subsection{SACM Program}

The SACM program is programmed in C with a graphical user interface (GUI) to allow for easy entry of model inputs. When the program starts the main window, Figure 3.14, is shown with the default values loaded. The user is able to easily change these values 


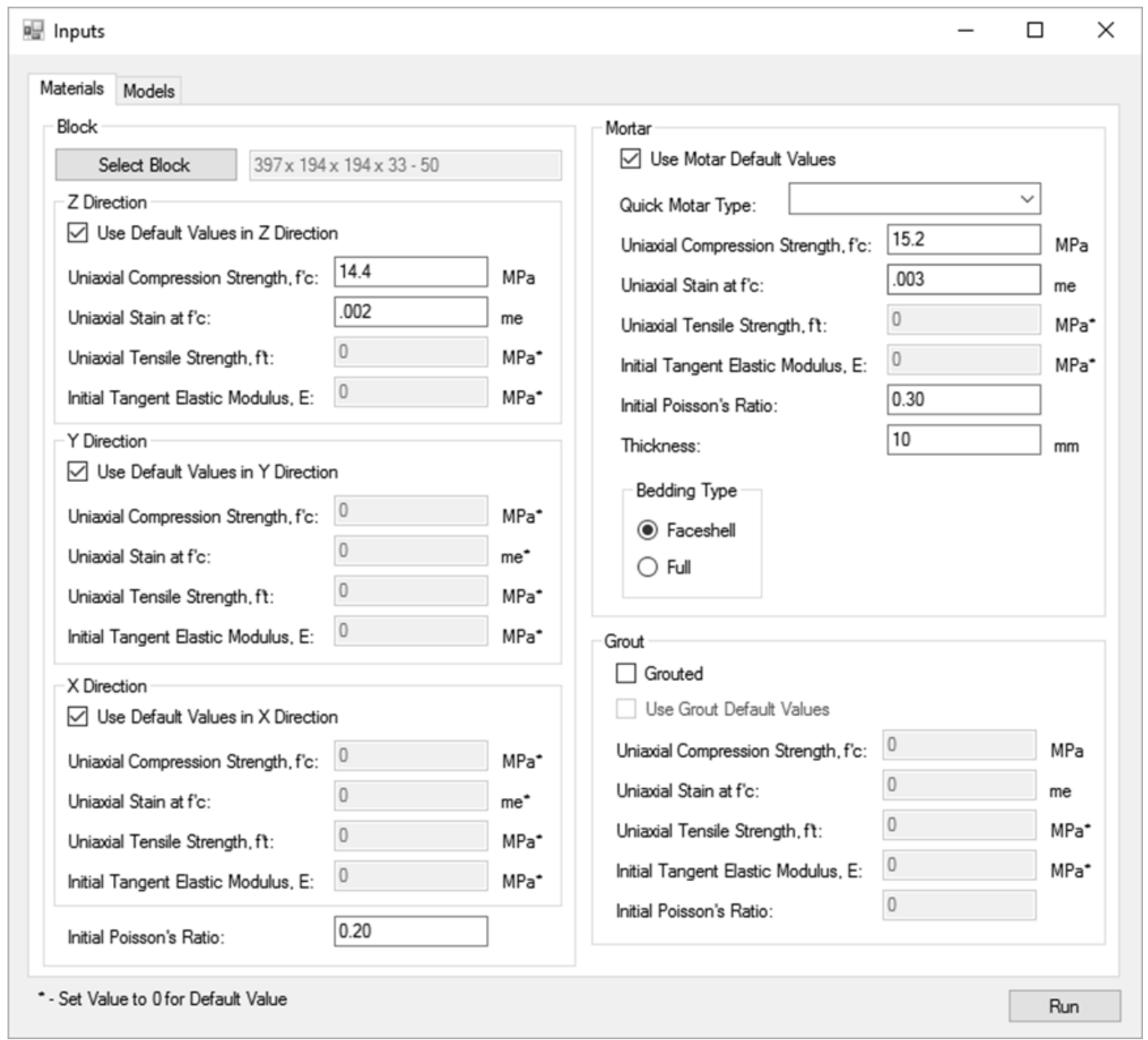

Figure 3.14: SACM Program Main Window

and select a block size and properties. After the material properties are entered the user is able to run the model in a new window, shown in Figure 3.15, which displays the load step, convergence, and converged load step values. These converged load step values are also stored in a data file, Figure 3.16, as comma separated values that can be easy imported into other software for further analysis.

Part II: Masonry Compression Strength Modeling 


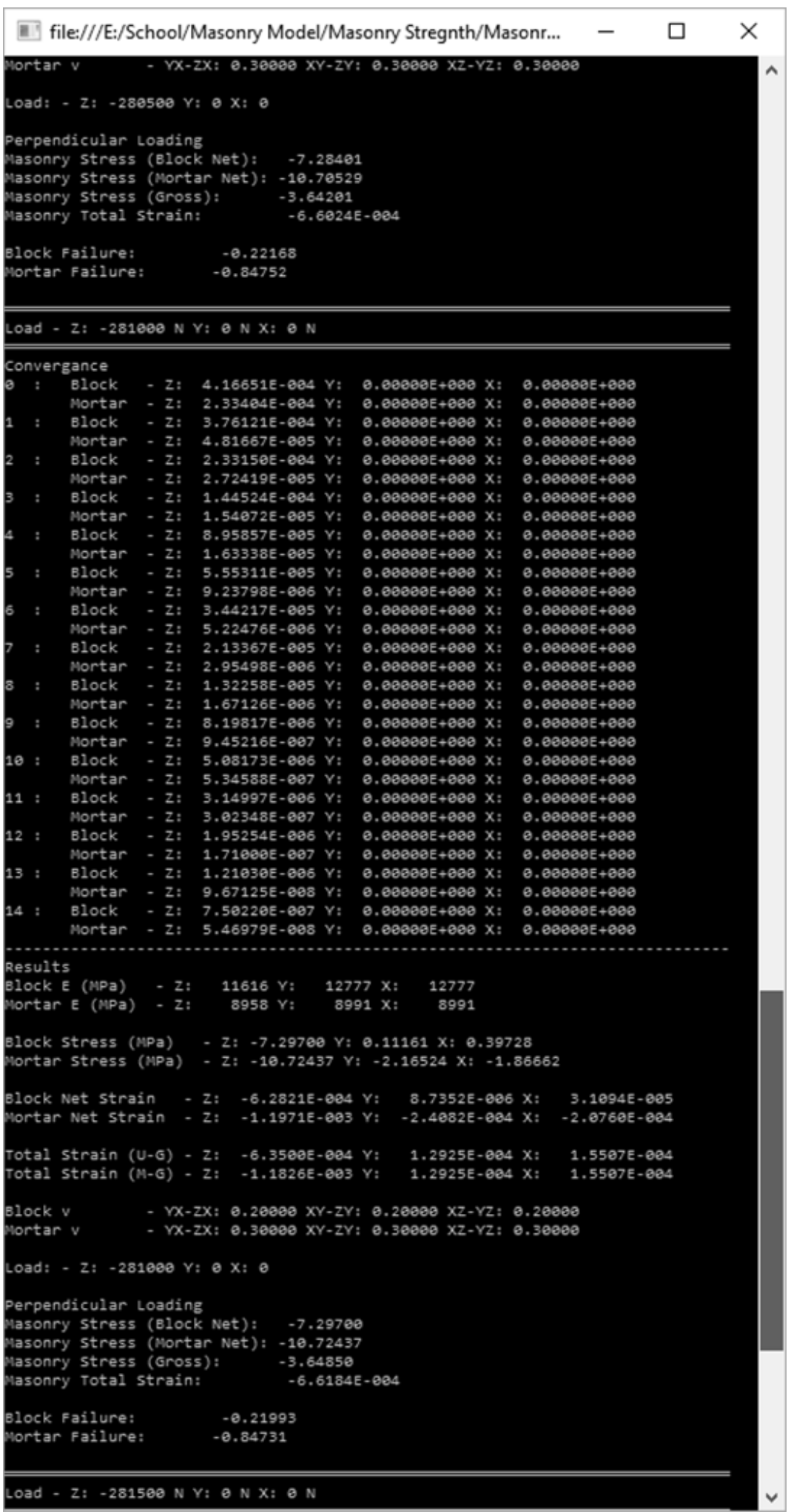

Figure 3.15: SACM Program Model Running Window

Part II: Masonry Compression Strength Modeling 


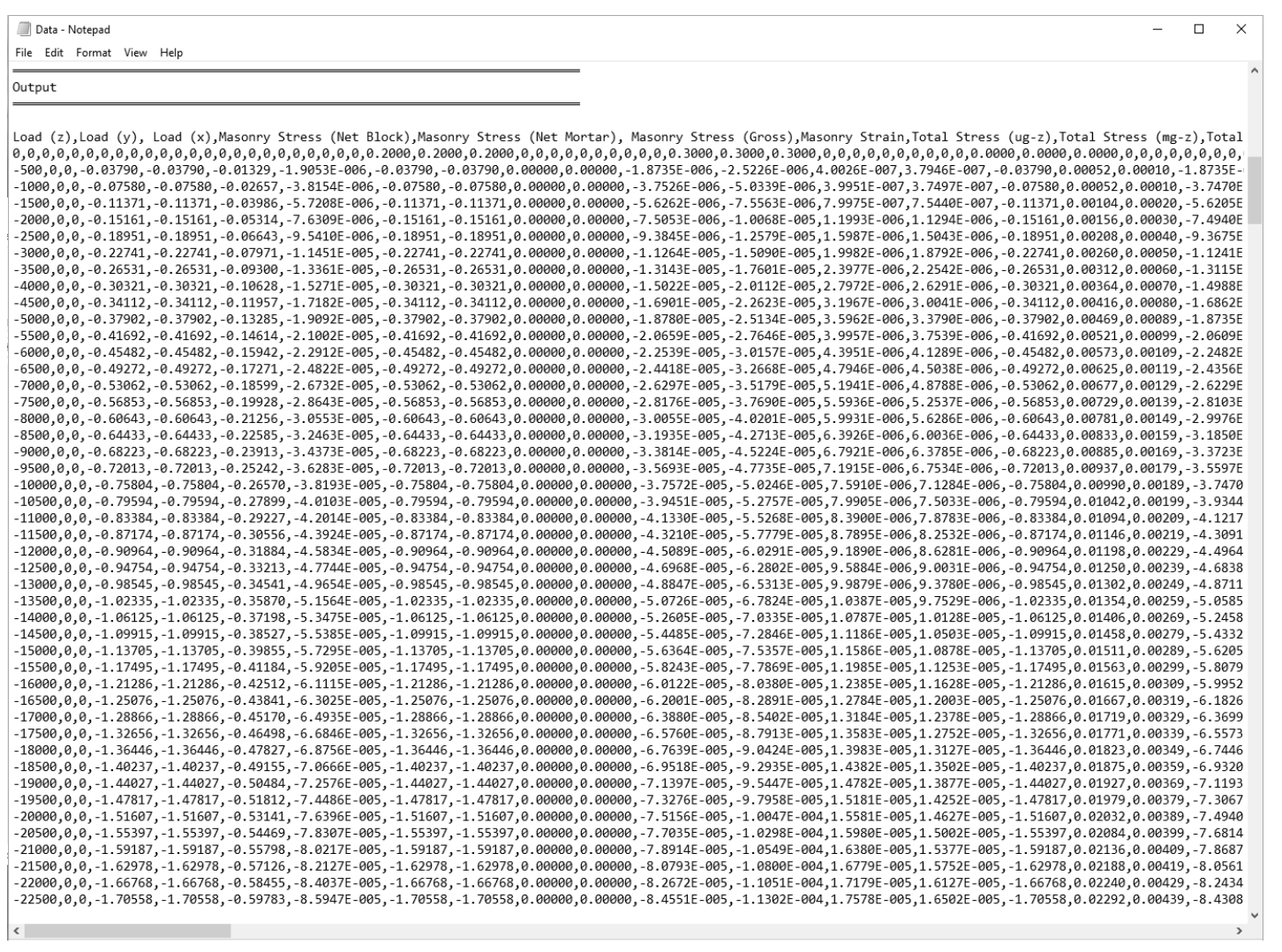

Figure 3.16: SACM Program Output

Part II: Masonry Compression Strength Modeling 


\section{COMPARISON OF EXPERIMENTAL DATA AND THE SMEARED AREA COMPRESSION MODEL}

The model presented in Chapter 3 is used to determine the masonry compression strength of a database of average masonry compression strengths gathered from literature. The ungrouted and grouted compression strengths are compared based on the experimental-to-predicted strength ratios. Additionally equations are produced for key masonry material properties.

Over the past century hundreds of prism tests have been conducted. This chapter assembles experimental databases of compressive tests for both ungrouted and grouted hollow concrete block masonry prisms to evaluate the Smeared Area Compression Model (SACM).

\subsection{Data Requirements}

The selection of compression test results for the databases is based on the following criteria:

1. The prism is constructed of three or more courses in either stacked or running bond;

2. The compression strength of all materials used to construct the prism is given;

3. The dimensions of the block are given;

4. The block has two or three vertical voids; and,

5. The mortar bedding type is given.

If a value required to run the model was not given an assumed value was used as listed in Appendix A or Appendix B.

\subsection{Ungrouted Experimental Database}

A large number of results of compressive tests on ungrouted hollow concrete block masonry prisms were assembled from published literature (Andolfato et al., 2007; Barbosa et al., 2010; Chahine, 1989; Copeland and Timms, 1932; Das et al., 2013; Drysdale and 
Hamid, 1979, 1983; Gayed et al., 2012; Gaynor et al., 1987; Khalaf, 1997; Khalaf et al., 1994; Maurenbrecher, 1980, 1985; Ramamurthy et al., 2000; Richart et al., 1932; Ring, 2009; Roman and Romagna, 2002; Steadman et al., 1995; Wong and Drysdale, 1985). A total of 126 average masonry compressive strength data points were gathered (see Appendix A). Table 4.1 shows the ranges of values for different parameters covered in the database.

Table 4.1: Ungrouted Experimental Range of Parameters

\begin{tabular}{lll}
\hline Parameter & $\begin{array}{l}\text { Face Shell } \\
\text { Bedded } \\
\text { Prisms }\end{array}$ & $\begin{array}{l}\text { Full } \\
\text { Bedded } \\
\text { Prisms }\end{array}$ \\
\hline${\text { Unified Block Compressive Strength }(\mathrm{MPa})^{\mathrm{a}}}^{\mathrm{a}}$ & $6.70-33.05$ & $7.40-31.51$ \\
Block Thickness (mm) & $140-240$ & $140-220$ \\
Block Height (mm) & $188-200$ & $189-203$ \\
Block Percent Solid (\%) & $50-75$ & $52-73$ \\
Faceshell Thickness (mm) & $24-58$ & $25-50$ \\
Unified Mortar Compressive Strength (MPa) & $5.00-24.57$ & $4.20-31.01$ \\
Prism h/t Ratio & $2.97-9.98$ & $2.97-6.24$ \\
\hline Notes: & & \\
${ }^{\mathrm{a}}$ Unified to soft full capping strength & & \\
${ }^{\mathrm{b}}$ Unified to absorbent cube strength & &
\end{tabular}

\subsubsection{SACM Results}

The reported data for each prism test was entered into the SACM with unreported values assumed as listed in Appendix A. The experimental-to-predicted ratios for the masonry compression strength are listed in Table 4.2. The average experimental-to-predicted ratio is 1.00 with a coefficient of variation $(\mathrm{COV})$ of $4.36 \%$ and 1.01 with a COV of $4.89 \%$ for face shell mortar bedded prisms and full mortar bedded prisms respectively. The overall average of experimental-to-predicted ratio is 1.01 with a COV of $4.63 \%$ for the entire ungrouted database. This low COV is further demonstrated in Figure 4.1, as all experimental-to-predicted ratios are closely grouped around the average of 1.00.

Part II: Masonry Compression Strength Modeling 
Chapter 4: Comparison of Experimental Data and the Smeared Area Compression Model

Table 4.2: Ungrouted SACM Results

\begin{tabular}{|c|c|c|c|c|c|c|c|}
\hline \multicolumn{4}{|c|}{ Face Shell Bedded Prisms } & \multicolumn{4}{|c|}{ Full Bedded Prisms } \\
\hline No. $^{a}$ & $\begin{array}{l}f_{m, r e p}^{\prime} \\
(\mathrm{MPa})^{\mathrm{b}}\end{array}$ & $\begin{array}{l}f_{m, p r e d}^{\prime} \\
(\mathrm{MPa})^{\mathrm{b}}\end{array}$ & $\begin{array}{l}f_{m, \text { rep }}^{\prime} / \\
f_{m, \text { pred }}^{\prime}\end{array}$ & No. $^{a}$ & $\begin{array}{l}f_{m, r e p}^{\prime} \\
(\mathrm{MPa})^{\mathrm{b}}\end{array}$ & $\begin{array}{l}f_{m, \text { pred }}^{\prime} \\
(\mathrm{MPa})^{\mathrm{b}}\end{array}$ & $\begin{array}{l}f_{m, \text { rep }}^{\prime} / \\
f_{m, \text { pred }}^{\prime}\end{array}$ \\
\hline 15 & 7.52 & 7.37 & 1.02 & 1 & 15.32 & 15.29 & 1.00 \\
\hline 30 & 14.30 & 14.90 & 0.96 & 2 & 15.32 & 15.45 & 0.99 \\
\hline 31 & 13.90 & 13.18 & 1.05 & 3 & 13.68 & 14.73 & 0.93 \\
\hline 32 & 16.30 & 17.12 & 0.95 & 4 & 13.68 & 14.40 & 0.95 \\
\hline 33 & 24.90 & 23.23 & 1.07 & 5 & 7.88 & 7.59 & 1.04 \\
\hline 34 & 18.00 & 17.36 & 1.04 & 6 & 6.78 & 6.68 & 1.01 \\
\hline 35 & 20.80 & 19.59 & 1.06 & 7 & 7.11 & 7.62 & 0.93 \\
\hline 36 & 23.80 & 23.75 & 1.00 & 8 & 6.67 & 7.05 & 0.95 \\
\hline 37 & 29.90 & 29.33 & 1.02 & 9 & 10.18 & 10.44 & 0.98 \\
\hline 38 & 21.60 & 20.88 & 1.03 & 10 & 10.61 & 10.00 & 1.06 \\
\hline 39 & 13.90 & 12.89 & 1.08 & 11 & 9.41 & 9.06 & 1.04 \\
\hline 40 & 22.40 & 21.88 & 1.02 & 12 & 8.00 & 8.86 & 0.90 \\
\hline 41 & 18.77 & 18.53 & 1.01 & 13 & 17.49 & 17.90 & 0.98 \\
\hline 43 & 18.63 & 18.24 & 1.02 & 14 & 7.17 & 7.71 & 0.93 \\
\hline 44 & 14.38 & 14.69 & 0.98 & 16 & 6.51 & 7.06 & 0.92 \\
\hline 46 & 14.00 & 14.24 & 0.98 & 17 & 10.30 & 11.09 & 0.93 \\
\hline 48 & 19.00 & 19.05 & 1.00 & 18 & 15.80 & 15.15 & 1.04 \\
\hline 49 & 19.00 & 17.99 & 1.06 & 19 & 16.00 & 15.20 & 1.05 \\
\hline 50 & 19.60 & 18.65 & 1.05 & 20 & 15.93 & 15.17 & 1.05 \\
\hline 54 & 23.60 & 23.34 & 1.01 & 21 & 15.03 & 14.00 & 1.07 \\
\hline 55 & 31.00 & 30.05 & 1.03 & 22 & 16.30 & 15.36 & 1.06 \\
\hline 56 & 29.30 & 27.89 & 1.05 & 23 & 25.92 & 23.91 & 1.08 \\
\hline 60 & 19.60 & 19.78 & 0.99 & 24 & 23.24 & 22.84 & 1.02 \\
\hline 61 & 18.40 & 18.96 & 0.97 & 25 & 18.00 & 16.76 & 1.07 \\
\hline 62 & 17.40 & 18.32 & 0.95 & 26 & 17.78 & 16.70 & 1.06 \\
\hline 63 & 15.28 & 14.46 & 1.06 & 27 & 16.95 & 15.92 & 1.06 \\
\hline 64 & 16.61 & 17.05 & 0.97 & 28 & 16.14 & 15.91 & 1.01 \\
\hline 65 & 15.41 & 16.11 & 0.96 & 29 & 12.76 & 13.05 & 0.98 \\
\hline 66 & 14.41 & 14.95 & 0.96 & 42 & 18.64 & 18.37 & 1.01 \\
\hline 67 & 13.78 & 13.80 & 1.00 & 45 & 14.29 & 13.95 & 1.02 \\
\hline 68 & 11.85 & 12.15 & 0.98 & 47 & 13.58 & 13.29 & 1.02 \\
\hline 69 & 11.21 & 11.53 & 0.97 & 51 & 17.80 & 17.28 & 1.03 \\
\hline
\end{tabular}

Continued on next page 
Chapter 4: Comparison of Experimental Data and the Smeared Area Compression Model

Table 4.2 Continued from Previous Page

\begin{tabular}{|c|c|c|c|c|c|c|c|}
\hline \multicolumn{4}{|c|}{ Face Shell Bedded Prisms } & \multicolumn{4}{|c|}{ Full Bedded Prisms } \\
\hline No. ${ }^{a}$ & $\begin{array}{l}f_{m, r e p}^{\prime} \\
(\mathrm{MPa})^{\mathrm{b}}\end{array}$ & $\begin{array}{l}f_{m, p r e d}^{\prime} \\
(\mathrm{MPa})^{\mathrm{b}}\end{array}$ & $\begin{array}{l}f_{m, \text { rep }}^{\prime} / \\
f_{m, \text { pred }}^{\prime}\end{array}$ & No. ${ }^{a}$ & $\begin{array}{l}f_{m, r e p}^{\prime} \\
(\mathrm{MPa})^{\mathrm{b}}\end{array}$ & $\begin{array}{l}f_{m, \text { pred }}^{\prime} \\
(\mathrm{MPa})^{\mathrm{b}}\end{array}$ & $\begin{array}{l}f_{m, \text { rep }}^{\prime} / \\
f_{m, \text { pred }}^{\prime}\end{array}$ \\
\hline 70 & 11.26 & 11.47 & 0.98 & 52 & 17.40 & 18.24 & 0.95 \\
\hline 71 & 8.80 & 9.48 & 0.93 & 53 & 21.40 & 19.42 & 1.10 \\
\hline 72 & 7.77 & 8.37 & 0.93 & 57 & 17.80 & 17.08 & 1.04 \\
\hline 73 & 6.29 & 6.09 & 1.03 & 58 & 17.40 & 18.06 & 0.96 \\
\hline 94 & 10.08 & 10.06 & 1.00 & 59 & 21.40 & 21.01 & 1.02 \\
\hline 95 & 15.88 & 16.71 & 0.95 & 74 & 19.49 & 19.85 & 0.98 \\
\hline 96 & 20.55 & 22.68 & 0.91 & 75 & 18.41 & 19.03 & 0.97 \\
\hline 97 & 12.20 & 11.65 & 1.05 & 76 & 14.42 & 14.80 & 0.97 \\
\hline 98 & 17.23 & 18.35 & 0.94 & 77 & 14.11 & 14.77 & 0.96 \\
\hline 99 & 23.56 & 23.21 & 1.02 & 78 & 17.36 & 17.47 & 0.99 \\
\hline 108 & 24.44 & 23.49 & 1.04 & 79 & 16.90 & 16.86 & 1.00 \\
\hline 109 & 22.01 & 23.13 & 0.95 & 80 & 14.39 & 13.74 & 1.05 \\
\hline 110 & 28.09 & 27.79 & 1.01 & 81 & 13.31 & 13.70 & 0.97 \\
\hline 111 & 26.31 & 25.28 & 1.04 & 82 & 13.48 & 12.63 & 1.07 \\
\hline 112 & 23.30 & 23.13 & 1.01 & 83 & 11.21 & 11.52 & 0.97 \\
\hline 113 & 20.17 & 20.8 & 0.97 & 84 & 11.00 & 11.45 & 0.96 \\
\hline 114 & 21.67 & 20.36 & 1.06 & 85 & 11.14 & 10.47 & 1.06 \\
\hline 115 & 21.56 & 20.02 & 1.08 & 86 & 10.86 & 10.38 & 1.05 \\
\hline 116 & 19.23 & 19.46 & 0.99 & 87 & 6.43 & 6.52 & 0.99 \\
\hline 117 & 16.00 & 16.67 & 0.96 & 88 & 12.47 & 12.33 & 1.01 \\
\hline 118 & 21.38 & 20.89 & 1.02 & 89 & 15.73 & 15.47 & 1.02 \\
\hline 119 & 17.42 & 16.74 & 1.04 & 90 & 17.06 & 16.03 & 1.06 \\
\hline 120 & 4.54 & 4.43 & 1.02 & 91 & 8.00 & 7.29 & 1.10 \\
\hline 121 & 4.77 & 5.13 & 0.93 & 92 & 17.89 & 17.24 & 1.04 \\
\hline 122 & 4.99 & 5.02 & 0.99 & 93 & 22.04 & 23.52 & 0.94 \\
\hline 123 & 4.96 & 5.16 & 0.96 & 100 & 7.73 & 7.88 & 0.98 \\
\hline 124 & 25.10 & 24.72 & 1.02 & 101 & 7.11 & 7.65 & 0.93 \\
\hline
\end{tabular}

Continued on next page 
Chapter 4: Comparison of Experimental Data and the Smeared Area Compression Model

Table 4.2 Continued from Previous Page

\begin{tabular}{|c|c|c|c|c|c|c|c|c|}
\hline \multicolumn{4}{|c|}{ Face Shell Bedded Prisms } & & \multicolumn{4}{|c|}{ Full Bedded Prisms } \\
\hline No. ${ }^{a}$ & $\begin{array}{l}f_{m, r e p}^{\prime} \\
(\mathrm{MPa})^{\mathrm{b}}\end{array}$ & $\begin{array}{l}f_{m, p r e d}^{\prime} \\
(\mathrm{MPa})^{\mathrm{b}}\end{array}$ & $\begin{array}{l}f_{m, \text { rep }}^{\prime} / \\
f_{m, \text { pred }}^{\prime}\end{array}$ & & No. $^{\mathrm{a}}$ & $\begin{array}{l}f_{m, r e p}^{\prime} \\
(\mathrm{MPa})^{\mathrm{b}}\end{array}$ & $\begin{array}{l}f_{m, \text { pred }}^{\prime} \\
(\mathrm{MPa})^{\mathrm{b}}\end{array}$ & $\begin{array}{l}f_{m, \text { rep }}^{\prime} / \\
f_{m, \text { pred }}^{\prime}\end{array}$ \\
\hline \multirow[t]{7}{*}{126} & 23.89 & 21.93 & 1.09 & & 102 & 6.71 & 6.90 & 0.97 \\
\hline & & & & & 103 & 9.24 & 9.63 & 0.96 \\
\hline & & & & & 104 & 18.81 & 17.69 & 1.06 \\
\hline & & & & & 105 & 17.86 & 17.71 & 1.01 \\
\hline & & & & & 106 & 21.43 & 20.41 & 1.05 \\
\hline & & & & & 107 & 30.18 & 29.65 & 1.02 \\
\hline & & & & & 125 & 24.10 & 24.75 & 0.97 \\
\hline \multicolumn{2}{|c|}{ Average } & & 1.00 & & \multicolumn{2}{|c|}{ Average } & & 1.01 \\
\hline \multicolumn{2}{|c|}{ STDV } & & 0.04 & & \multicolumn{2}{|c|}{ STDV } & & 0.05 \\
\hline \multicolumn{2}{|c|}{$\mathrm{COV}$} & & $4.36 \%$ & & $\mathrm{COV}$ & & & $4.89 \%$ \\
\hline \multicolumn{4}{|c|}{ Average } & 1.01 & & & & \\
\hline \multicolumn{4}{|c|}{ STDV } & 0.05 & & & & \\
\hline \multicolumn{4}{|c|}{$\mathrm{COV}$} & $4.63 \%$ & & & & \\
\hline
\end{tabular}

Concluded

Notes:

${ }^{a}$ For Input Values the Number Corresponds to the Number in Appendix A

${ }^{\mathrm{b}}$ Based on minimum net area of the prism

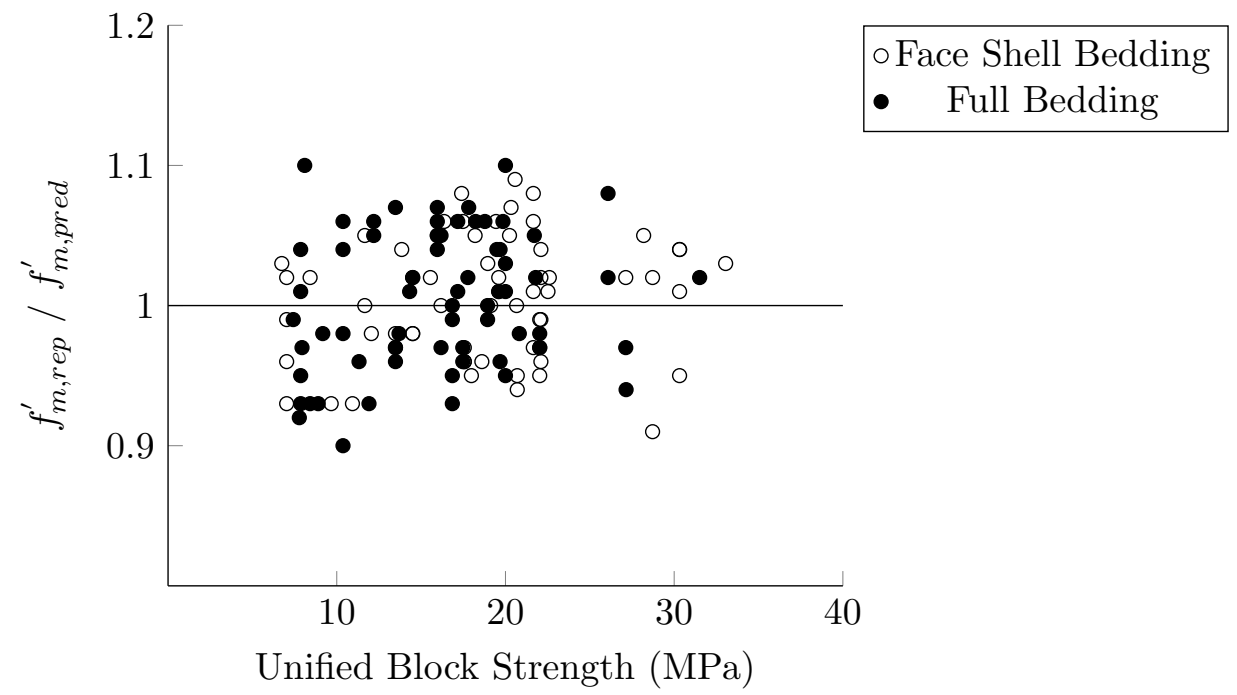

Figure 4.1: SACM Ungrouted Predictions 


\subsection{Grouted Experimental Database}

A total of 121 average masonry compressive strength data points were gathered (see Appendix B) from from published literature (Boult, 1979; Drysdale and Hamid, 1979, 1983; Hamid et al., 1978; Khalaf, 1997; Khalaf et al., 1994; Priestley, 1977; Ring, 2009; Roman and Romagna, 2002; Ross et al., 2013; Sarhat, 2016; Seif ElDin, 2016; Shedid et al., 2008; Steadman et al., 1995; Wong and Drysdale, 1985). Table 4.3 shows the ranges of values for different parameters covered in the database.

Table 4.3: Grouted Experimental Range of Parameters

\begin{tabular}{|c|c|}
\hline Parameter & Range \\
\hline Unified Block Compressive Strength $(\mathrm{MPa})^{\mathrm{a}}$ & $6.17-49.75$ \\
\hline Block Thickness (mm) & $140-240$ \\
\hline Block Height (mm) & $189-203$ \\
\hline Block Percent Solid (\%) & $51-75$ \\
\hline Faceshell Thickness (mm) & $17-58$ \\
\hline Unified Mortar Compressive Strength $(\mathrm{MPa})^{\mathrm{b}}$ & $4.50-50.19$ \\
\hline Unified Grout Compressive Strength $(\mathrm{MPa})^{\mathrm{c}}$ & $8.57-45.65$ \\
\hline Prism $\mathrm{h} / \mathrm{t}$ Ratio & $2.46-5.21$ \\
\hline \multicolumn{2}{|l|}{ Notes: } \\
\hline \multicolumn{2}{|l|}{${ }^{\mathrm{a}}$ Unified to soft full capping strength } \\
\hline${ }^{\mathrm{b}}$ Unified to absorbent cube strength & \\
\hline${ }^{\mathrm{C}}$ Unified to absorbent cylinder strength & \\
\hline
\end{tabular}

\subsubsection{SACM Results}

The reported data for each prism test was entered into the SACM with unreported values assumed as litsted in Appendix B. The experimental-to-predicted ratios for the masonry compression strength are listed in Table 4.4. The average of experimental-to-predicted ratio is 1.00 with a COV of $5.32 \%$ for the entire grouted database. The experimentalto-predicted ratios are illustrated in Figure 4.2. As can be seen the ratios are tightly grouped around unity. 
Chapter 4: Comparison of Experimental Data and the Smeared Area Compression Model

Table 4.4: Grouted SACM Results

\begin{tabular}{llll}
\hline No. $^{\mathrm{a}}$ & $f_{m, r e p}^{\prime}$ & $f_{m, \text { pred }}^{\prime}$ & $f_{m, r e p}^{\prime} /$ \\
$(\mathrm{MPa})^{\mathrm{b}}$ & $(\mathrm{MPa})^{\mathrm{b}}$ & $f_{m, \text { pred }}^{\prime}$ \\
\hline 1 & 18.30 & 18.43 & 0.99 \\
2 & 18.30 & 18.30 & 1.00 \\
3 & 10.84 & 11.56 & 0.94 \\
4 & 11.18 & 11.35 & 0.99 \\
5 & 11.32 & 11.38 & 0.99 \\
6 & 10.41 & 11.06 & 0.94 \\
7 & 11.32 & 11.11 & 1.02 \\
8 & 12.07 & 11.76 & 1.03 \\
9 & 12.82 & 12.86 & 1.00 \\
10 & 14.35 & 14.33 & 1.00 \\
11 & 12.07 & 11.72 & 1.03 \\
12 & 16.91 & 16.52 & 1.02 \\
13 & 16.41 & 16.27 & 1.01 \\
14 & 13.17 & 13.84 & 0.95 \\
15 & 14.13 & 13.88 & 1.02 \\
16 & 12.13 & 12.88 & 0.94 \\
17 & 13.33 & 13.39 & 1.00 \\
18 & 9.54 & 10.34 & 0.92 \\
19 & 15.18 & 15.33 & 0.99 \\
20 & 15.17 & 16.18 & 0.94 \\
21 & 14.60 & 14.24 & 1.03 \\
22 & 13.20 & 13.15 & 1.00 \\
23 & 7.38 & 8.07 & 0.91 \\
24 & 7.25 & 7.88 & 0.92 \\
25 & 5.61 & 5.96 & 0.94 \\
26 & 7.71 & 8.33 & 0.93 \\
27 & 7.22 & 6.77 & 1.07 \\
28 & 7.51 & 7.97 & 0.94 \\
29 & 7.87 & 8.37 & 0.94 \\
30 & 6.50 & 6.96 & 0.93 \\
31 & 13.00 & 12.09 & 1.08 \\
32 & 15.80 & 14.69 & 1.08 \\
33 & 17.90 & 18.15 & 0.99 \\
34 & 13.80 & 12.84 & 1.07 \\
\hline & & &
\end{tabular}

Continued on next page

Part II: Masonry Compression Strength Modeling 
Chapter 4: Comparison of Experimental Data and the Smeared Area Compression Model

Table 4.4 Continued from Previous Page

\begin{tabular}{llll}
\hline No. $^{\mathrm{a}}$ & $\begin{array}{l}f_{m, r e p}^{\prime} \\
(\mathrm{MPa})^{\mathrm{b}}\end{array}$ & $\begin{array}{l}f_{m, \text { pred }}^{\prime} \\
(\mathrm{MPa})^{\mathrm{b}}\end{array}$ & $\begin{array}{l}f_{m, \text { rep }}^{\prime} / \\
f_{m, \text { pred }}^{\prime}\end{array}$ \\
\hline 35 & 11.40 & 12.68 & 0.90 \\
36 & 13.40 & 13.86 & 0.97 \\
37 & 13.90 & 13.31 & 1.04 \\
38 & 14.50 & 14.91 & 0.97 \\
39 & 19.30 & 19.12 & 1.01 \\
40 & 17.40 & 17.20 & 1.01 \\
41 & 20.10 & 19.40 & 1.04 \\
42 & 17.40 & 16.90 & 1.03 \\
43 & 20.20 & 20.44 & 0.99 \\
44 & 23.00 & 22.61 & 1.02 \\
45 & 23.50 & 21.53 & 1.09 \\
46 & 27.10 & 25.62 & 1.06 \\
47 & 19.40 & 19.54 & 0.99 \\
48 & 15.80 & 14.48 & 1.09 \\
49 & 17.90 & 17.90 & 1.00 \\
50 & 13.40 & 13.91 & 0.96 \\
51 & 13.90 & 13.06 & 1.06 \\
52 & 14.50 & 14.97 & 0.97 \\
53 & 19.30 & 18.82 & 1.03 \\
54 & 9.17 & 9.92 & 0.92 \\
55 & 12.92 & 12.62 & 1.02 \\
56 & 15.50 & 14.89 & 1.04 \\
57 & 18.20 & 17.12 & 1.06 \\
58 & 11.01 & 11.99 & 0.92 \\
59 & 14.40 & 14.49 & 0.99 \\
60 & 16.48 & 16.70 & 0.99 \\
61 & 19.09 & 18.89 & 1.01 \\
62 & 11.61 & 12.42 & 0.93 \\
63 & 15.77 & 14.26 & 1.11 \\
64 & 17.12 & 17.10 & 1.00 \\
65 & 20.27 & 19.51 & 1.04 \\
66 & 5.56 & 6.15 & 0.90 \\
68 & 11.15 & 10.46 & 1.07 \\
& 12.90 & 12.01 & 1.07 \\
\hline & & $C o n t i n e$ \\
5
\end{tabular}

Continued on next page 
Chapter 4: Comparison of Experimental Data and the Smeared Area Compression Model

Table 4.4 Continued from Previous Page

\begin{tabular}{|c|c|c|c|}
\hline No. $^{a}$ & $\begin{array}{l}f_{m, r e p}^{\prime} \\
(\mathrm{MPa})^{\mathrm{b}}\end{array}$ & $\begin{array}{l}f_{m, p r e d}^{\prime} \\
(\mathrm{MPa})^{\mathrm{b}}\end{array}$ & $\begin{array}{l}f_{m, \text { rep }}^{\prime} / \\
f_{m, \text { pred }}^{\prime}\end{array}$ \\
\hline 69 & 15.76 & 14.68 & 1.07 \\
\hline 70 & 10.92 & 12.29 & 0.89 \\
\hline 71 & 17.05 & 15.42 & 1.11 \\
\hline 72 & 17.84 & 17.18 & 1.04 \\
\hline 73 & 21.11 & 20.53 & 1.03 \\
\hline 74 & 6.05 & 6.37 & 0.95 \\
\hline 75 & 12.65 & 11.90 & 1.06 \\
\hline 76 & 15.00 & 14.25 & 1.05 \\
\hline 77 & 15.81 & 15.06 & 1.05 \\
\hline 78 & 9.62 & 9.84 & 0.98 \\
\hline 79 & 14.46 & 14.97 & 0.97 \\
\hline 80 & 17.11 & 17.13 & 1.00 \\
\hline 81 & 19.53 & 18.88 & 1.03 \\
\hline 82 & 13.04 & 13.27 & 0.98 \\
\hline 83 & 17.08 & 18.02 & 0.95 \\
\hline 84 & 20.12 & 20.61 & 0.98 \\
\hline 85 & 20.44 & 22.80 & 0.90 \\
\hline 86 & 8.16 & 8.42 & 0.97 \\
\hline 87 & 13.10 & 12.37 & 1.06 \\
\hline 88 & 16.69 & 16.20 & 1.03 \\
\hline 89 & 17.34 & 18.02 & 0.96 \\
\hline 90 & 10.29 & 10.39 & 0.99 \\
\hline 91 & 16.10 & 15.26 & 1.06 \\
\hline 92 & 18.51 & 18.48 & 1.00 \\
\hline 93 & 20.52 & 19.59 & 1.05 \\
\hline 94 & 13.54 & 13.71 & 0.99 \\
\hline 95 & 18.18 & 19.00 & 0.96 \\
\hline 96 & 20.13 & 21.58 & 0.93 \\
\hline 97 & 25.12 & 23.53 & 1.07 \\
\hline 98 & 14.80 & 14.16 & 1.05 \\
\hline 99 & 19.53 & 20.61 & 0.95 \\
\hline 100 & 21.30 & 20.37 & 1.05 \\
\hline 101 & 21.28 & 19.96 & 1.07 \\
\hline 102 & 17.84 & 16.03 & 1.11 \\
\hline
\end{tabular}

Continued on next page 
Chapter 4: Comparison of Experimental Data and the Smeared Area Compression Model

Table 4.4 Continued from Previous Page

\begin{tabular}{llll}
\hline No. $^{\mathrm{a}}$ & $\begin{array}{l}f_{m, \text { rep }}^{\prime} \\
(\mathrm{MPa})^{\mathrm{b}}\end{array}$ & $\begin{array}{l}f_{m, \text { pred }}^{\prime} \\
(\mathrm{MPa})^{\mathrm{b}}\end{array}$ & $\begin{array}{l}f_{m, \text { rep }}^{\prime} / \\
f_{m, \text { pred }}^{\prime}\end{array}$ \\
\hline 103 & 16.40 & 15.52 & 1.06 \\
104 & 14.16 & 14.62 & 0.97 \\
105 & 15.42 & 14.54 & 1.06 \\
106 & 7.85 & 8.14 & 0.96 \\
107 & 8.14 & 8.02 & 1.01 \\
108 & 8.12 & 8.13 & 1.00 \\
109 & 8.27 & 8.06 & 1.03 \\
110 & 14.80 & 15.57 & 0.95 \\
111 & 14.80 & 14.15 & 1.05 \\
112 & 17.50 & 17.41 & 1.01 \\
113 & 18.00 & 17.77 & 1.01 \\
114 & 17.90 & 17.87 & 1.00 \\
115 & 20.00 & 17.70 & 1.13 \\
116 & 19.10 & 17.79 & 1.07 \\
117 & 17.30 & 18.70 & 0.93 \\
118 & 18.50 & 17.36 & 1.07 \\
119 & 19.50 & 17.76 & 1.10 \\
120 & 18.90 & 18.40 & 1.03 \\
121 & 18.90 & 18.83 & 1.00 \\
\hline Average & & 1.00 \\
STDV & & 0.05 \\
COV & & & $5.32 \%$ \\
\hline & & & Concluded \\
& & & \\
110
\end{tabular}

Notes:

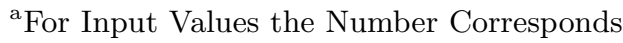

to the Number in Appendix B

${ }^{\mathrm{b}}$ Based on gross area of the prism 


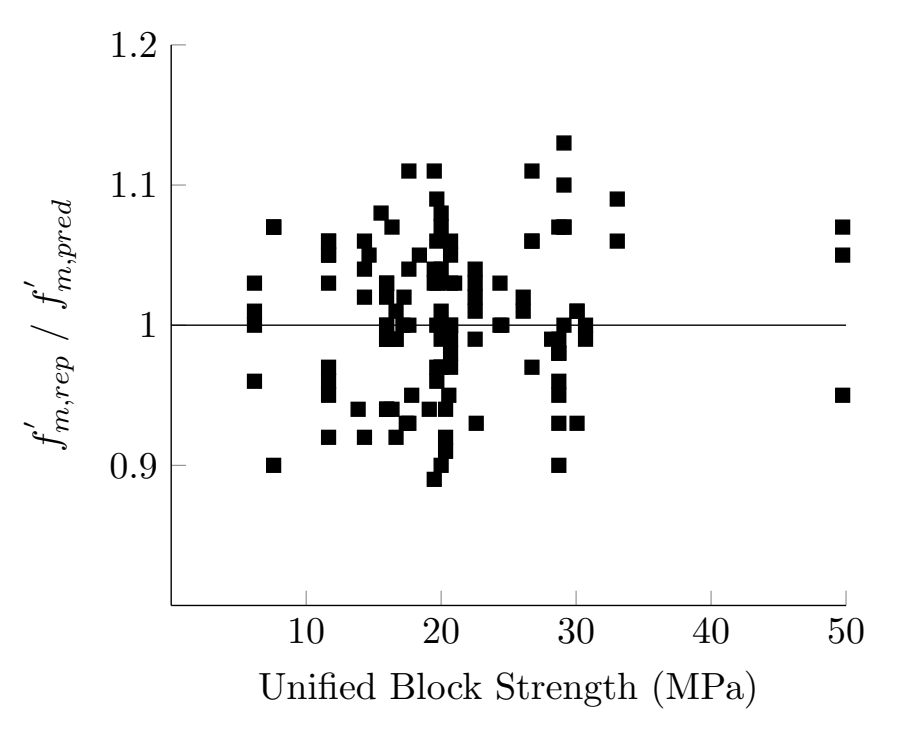

Figure 4.2: SACM Grouted Predictions

\subsection{Discussion}

The following discussion examines the SACM inputs used to produce the results presented in Section 4.3.1. This discussion will focus on verifying the SACM default values, as well as proposed equations to estimate required SACM inputs.

\subsubsection{Block Strain at Peak Strength}

The strain's at peak block strength, $\epsilon_{o, z}^{b l}$, from the ungrouted and grouted databases are plotted in Figure 4.3. As can be seen a majority (74\% of the points) of the strains are 0.002. This strain is a commonly assumed strain at peak strength in normal strength concrete (ie. $f_{c}^{\prime} \leq 50 \mathrm{MPa}$ ) when the strain is unknown (Kent and Park, 1971; Roy and Sozen, 1964; Saatcioglu and Razvi, 1992). Other expressions for the strain at peak strength of normal strength concrete include:

$$
\epsilon_{o}=0.0018+0.0000075 f_{c}^{\prime} \text { (Wong et al., 2013) }
$$

or from Popovics (1970):

$$
\epsilon_{o}=\frac{f_{c}^{\prime}}{E_{c}} \frac{n}{n-1}
$$


where for normal weight concrete the modulus of elasticity, $E_{c}$, is assumed to be:

$$
E_{c}=3320 \sqrt{f_{c}^{\prime}}+6900 \text { (Carrasquillo et al., 1981) }
$$

and

$$
n=0.8+\frac{f_{c}^{\prime} b l}{17}(\text { Collins and Mitchell, 1997) }
$$

As shown in Figure 4.3 these two equations for $\epsilon_{o, z}^{b l}$ are approximately equal to the basic assumption of 0.002 over the unified block strengths presented in the databases. Cheema and Klingner (1986) also experimental observed an average strain at maximum block stress of 0.0021 , further confirming the use of 0.002 as the block strain at peak strength.

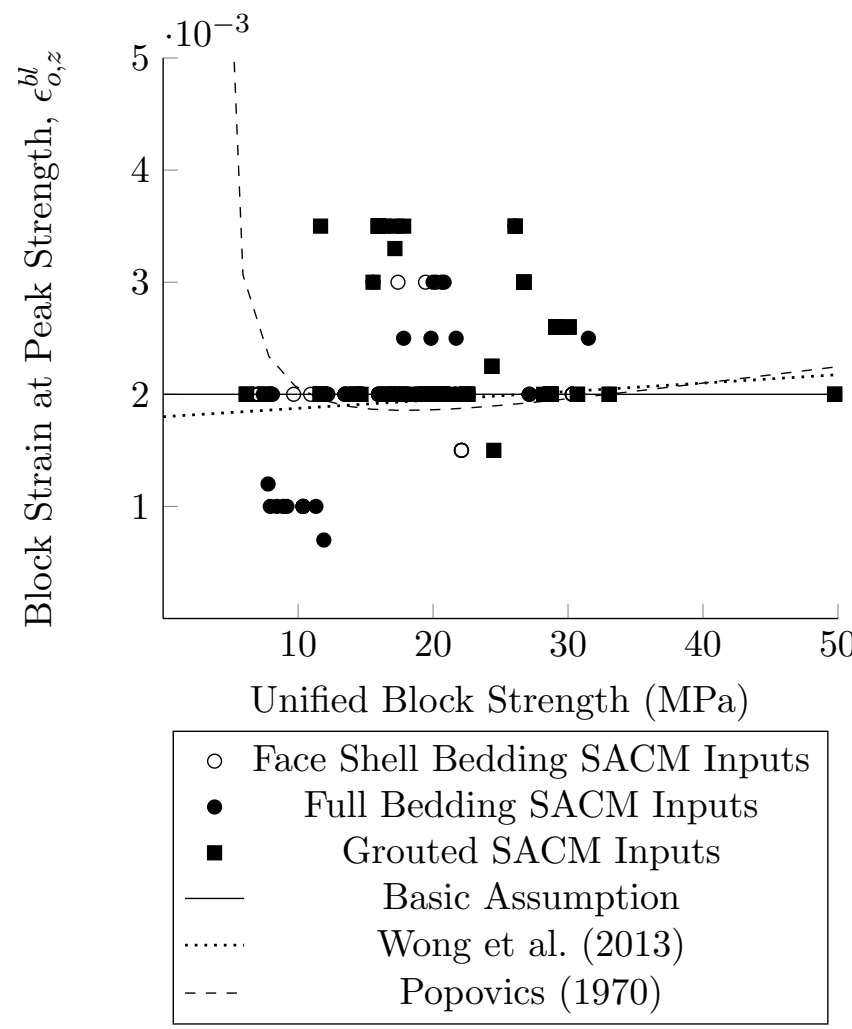

Figure 4.3: SACM Inputs for Block Strain at Peak Strength 


\subsubsection{Initial Block Poisson's Ratio}

The initial block Poisson's ratios, $\nu_{o, z}^{b l}$, from the ungrouted and grouted databases are plotted against the unified block strength, $k_{1}^{b l} k_{2}^{b l} f_{c, \text { rep }}^{\prime}$, in Figure 4.4. Most initial block Poisson's ratios were 0.20 as used by Hamid and Chukwunenye (1986) and implemented in the SACM as the default value. There are a few lower block Poisson's ratios from the grouted database that are similar to the experimentally measured value of 0.17 made by Cheema and Klingner (1986). Cheema and Klingner (1986) also experimentally measured linearly increasing block Poisson ratio up 0.34 just before failure and used 0.28 as an initial block Poisson's ratio in their finite element model. This shows that the default value of 0.20 should be used in no other data is available.

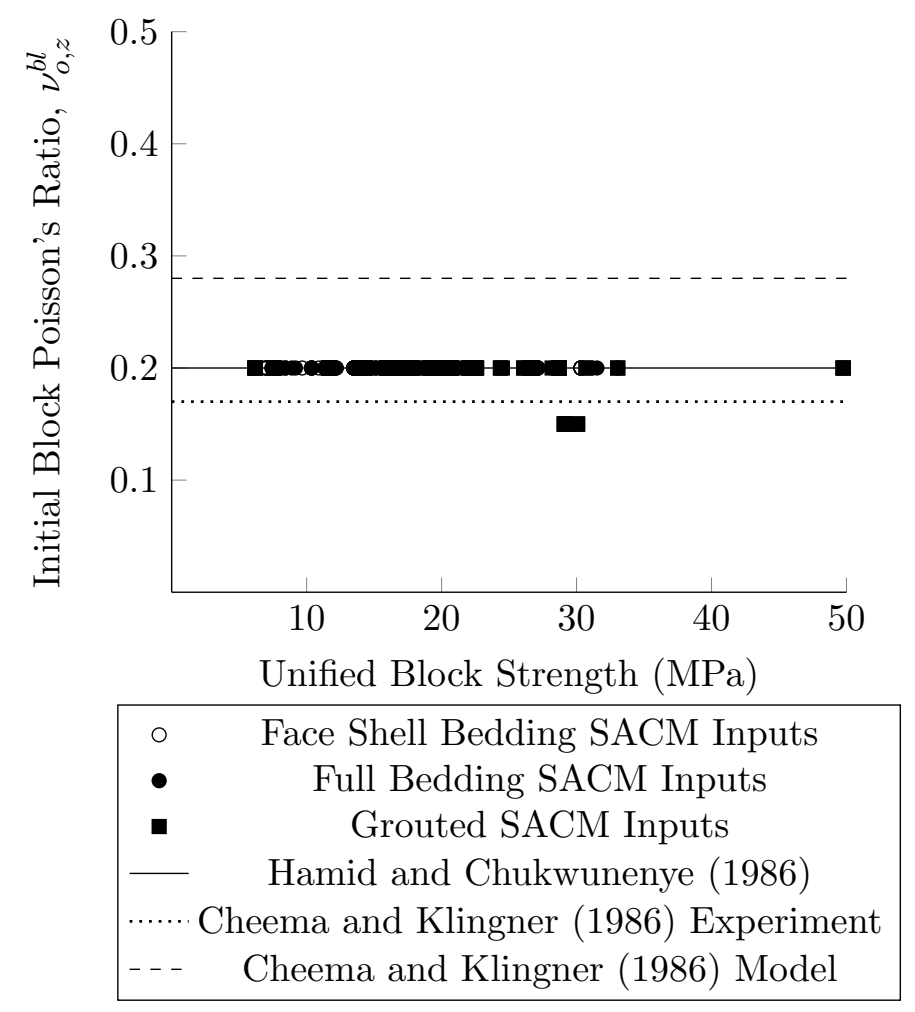

Figure 4.4: SACM Inputs for Initial Block Poisson's Ratio

\subsubsection{Mortar Strain at Peak Strength}

Mortar is typically less stiff than the hollow concrete block; thus, if the mortar and block have the same peak strength the mortar will typically have a larger strain at peak strength. The strain's at peak mortar strength, $\epsilon_{o, z}^{m r}$, from the ungrouted and grouted databases are plotted in Figure 4.5. For the ungrouted database $5 \%$ of the strains are $0.001,22 \%$ are 0.002 , and $63 \%$ are 0.003 . There are $2 \%$ above the strain of 0.003 with 
the reminaing $8 \%$ between 0.001 and 0.0022 . The grouted database has less scatter with $39 \%$ of the strains at $0.002,60 \%$ at 0.003 , and $1 \%$ above 0.003 . Thus, the majority of the strains are 0.002 and 0.003 . Popovics (1970) estimated the mortar strain at peak strength with:

$$
\epsilon_{o}=\frac{f_{c}^{\prime}, m r}{E^{m r}} \frac{n}{n-1}
$$

where

$$
n=0.02176 f_{c}^{\prime}, m r+1.5
$$

The modulus of elasticity, $E^{m r}$, can be estimated according to Cheema and Klingner (1986) and Fahmy and Ghoneim (1995) as:

$$
E^{m r}=1000 f_{c}^{\prime}, m r
$$

As shown in Figure 4.5 these equations fail to accurately predict the strains in the databases. Cheema and Klingner (1986) simply assumed a strain at mortar peak strength of 0.002 , which closely represent $30 \%$ of the databases; while the majority of the strains from the databases are 0.003 and tends to produce good results.

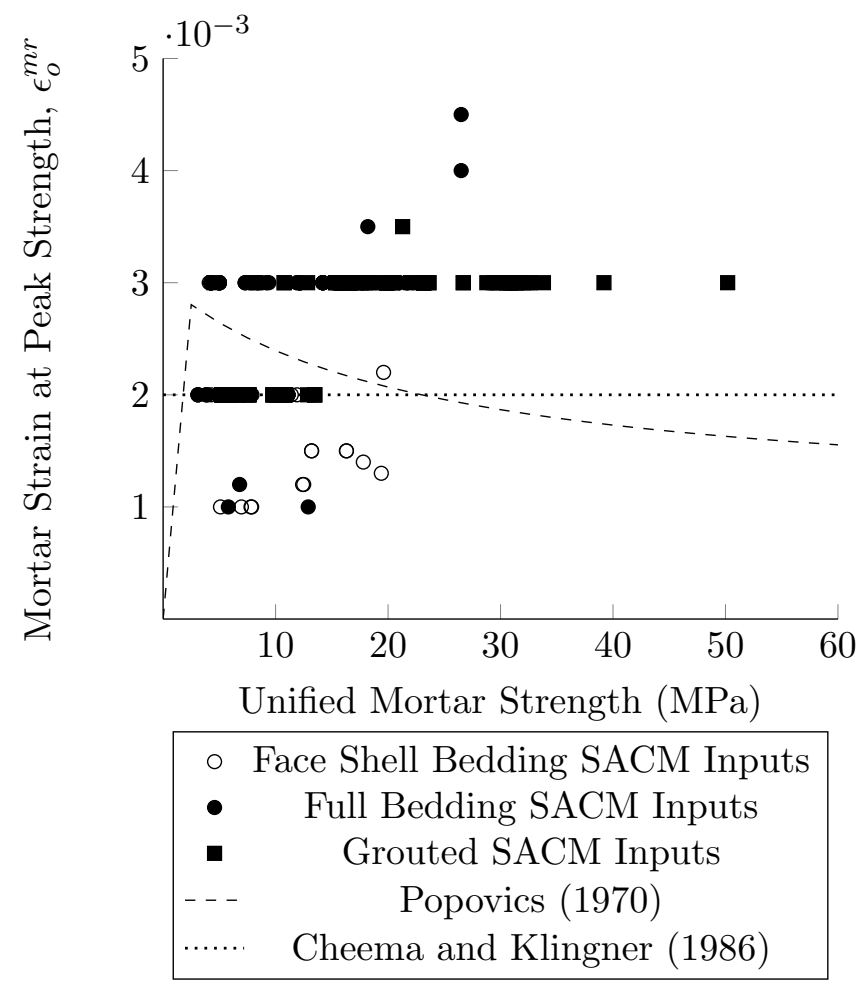

Figure 4.5: SACM Inputs for Mortar Strain at Peak Strength 


\subsubsection{Initial Mortar Poisson's Ratio}

Hilsdorf (1972) reports intial Poisson's ratios for mortar of 0.20 and approaching 0.48 near crushing. Maher and Darwin (1982) observed an initial Poisson's ratio of 0.20 and at an axial strain of 0.002 the ratio became 0.30. The initial mortar Poisson's ratios, $\nu_{o}^{m r}$, from the ungrouted and grouted databases are plotted against the unified mortar compressive strength in Figure 4.6. The default value of 0.30, similar to the value used by Cheema and Klingner (1986), produced good prodictions of the masonry compression strength.

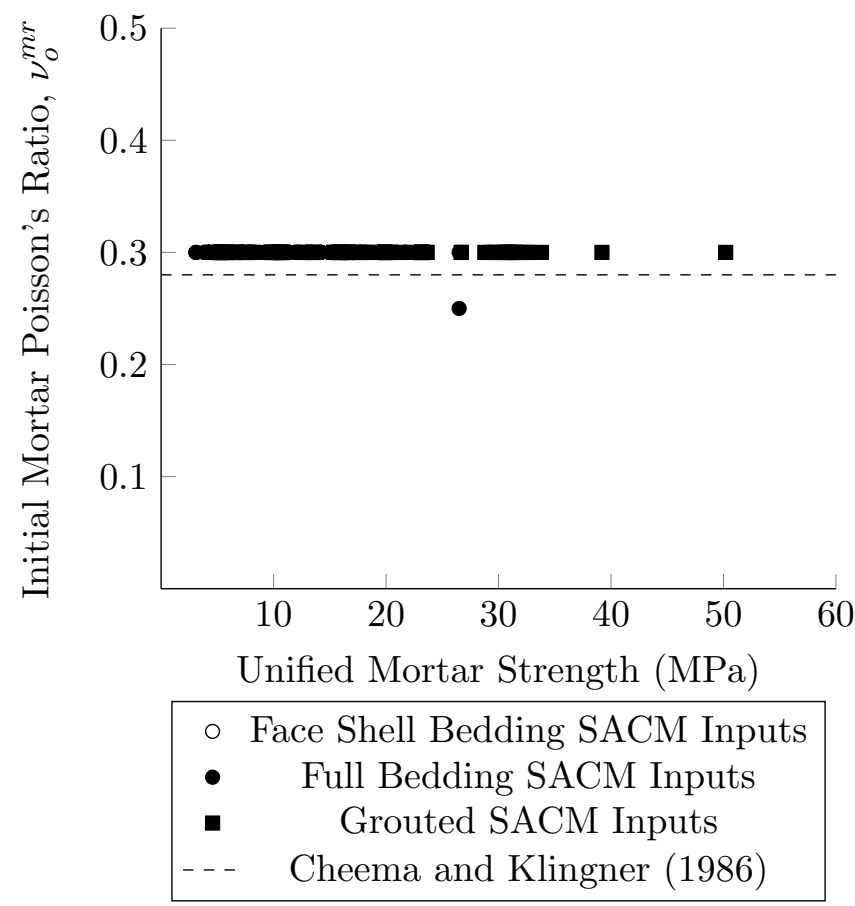

Figure 4.6: SACM Inputs for Initial Mortar Poisson's Ratio 


\subsubsection{Grout Strain at Peak Strength}

The values for the grout strain's at peak strength from the database are plotted in Figure 4.7 against their unified grout strengths. These strains range from 0.002 to 0.004. Of the plotted values $36 \%$ are at a strain of $0.002,36 \%$ at 0.003 , and $11 \%$ at 0.004. Cheema and Klingner (1986) observed and used in their model a grout strain at peak strength of 0.002. As grout is typically less stiff it is expected to have a strain a peak strength equal to or greater than the block. All the grout strains used to produce the results presented in Section 4.3.1 have a strain a peak strength equal to or greater than the block.

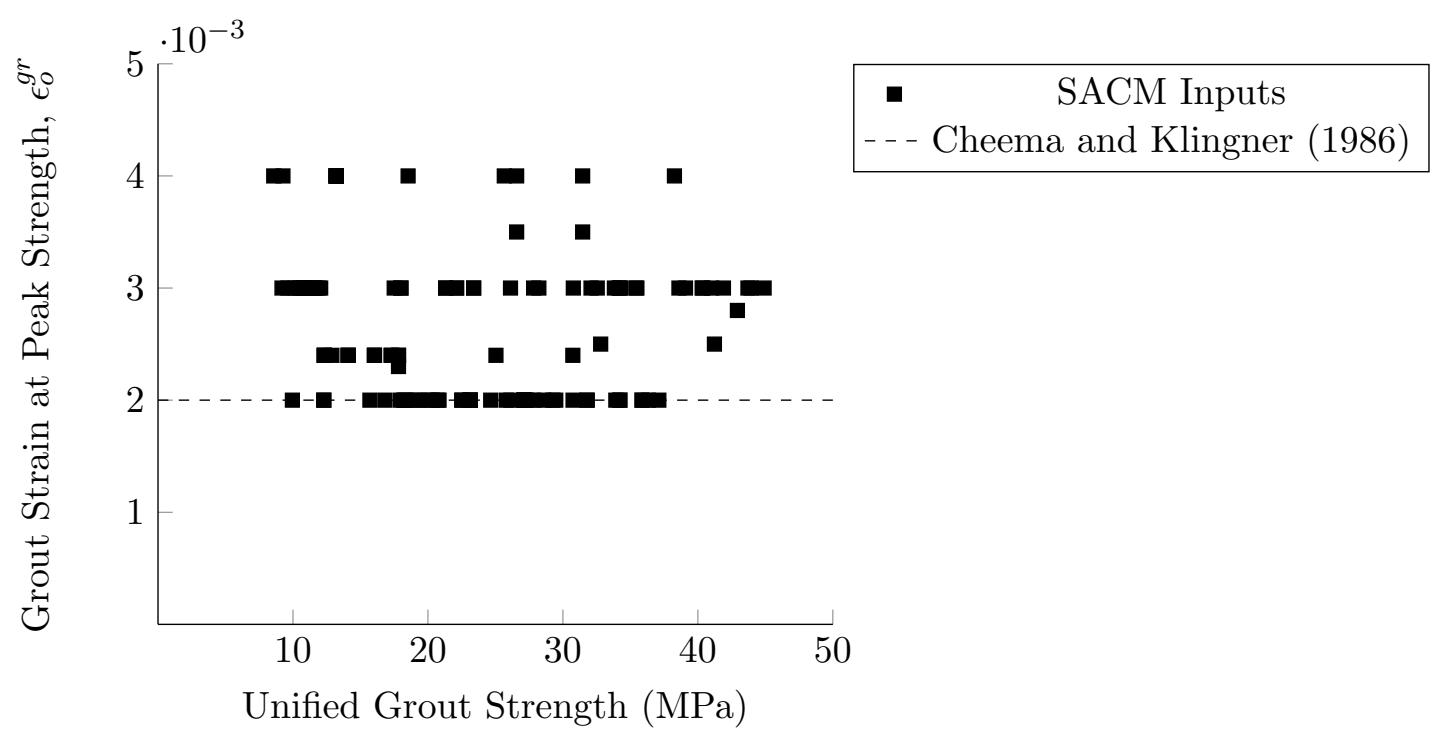

Figure 4.7: SACM Inputs for Grout Strain at Peak Strength 


\subsubsection{Initial Grout Poisson's Ratio}

Cheema and Klingner (1986) observed initial grout Poisson's ratios of 0.17 that increased rapidly near failure. The finite element model developed by Cheema and Klingner (1986) used an initial grout Poisson's ratio of 0.37. Fahmy and Ghoneim (1995) assumed an initial value of 0.21 . These values along with the SACM grouted database values are plotted in Figure 4.8. As can be seen, the SCAM grout stains from the database are mostly the default value of 0.35 and closely match the general accepted values; thus, if the grout Poisson's ratio is unavailable the default value should be used.

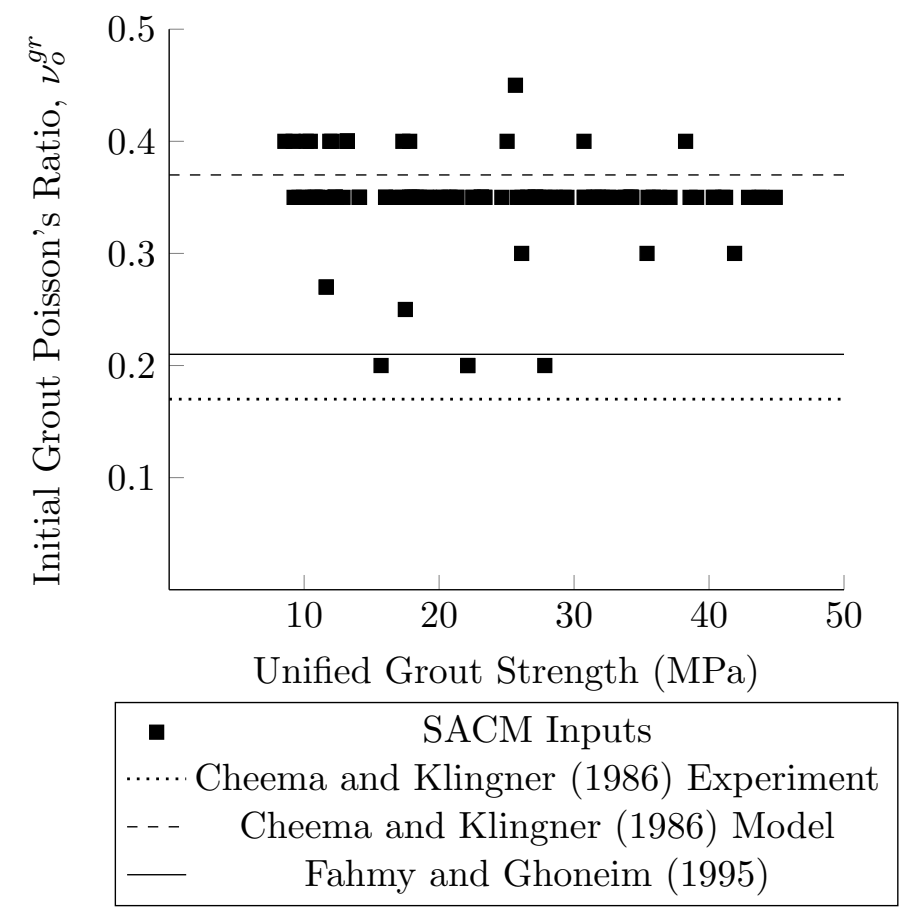

Figure 4.8: SACM Inputs for Initial Grout Poisson's Ratio 


\section{PARAMETRIC STUDY OF THE SMEARED AREA COM- PRESSION MODEL}

The model presented in Chapter 3 requires a number of inputs. This chapter looks at how varying these input parameters impacts the predicted compression strength.

In assessing the results of the Smeared Area Compression Model (SACM) developed in Chapter 3, a number of the inputs can be varied to determined their impact on masonry compression strength. Additionally, the SACM results can be compared to experimental observed behaviour.

\subsection{Parameters of Study}

Ungrouted masonry compressive strength is known to be influenced by: block strength, mortar strength, bedding type, and mortar thickness (see Section 2.1 and Table 2.1). The compression strength of grouted masonry is also known to be influenced by: block strength, mortar strength, grout strength, and mortar thickness (see Section 2.2 and Table 2.2). Additionally, all other inputs can be easily varied over a range of values to determine their influence on ungrouted and grouted masonry compressive strength. Table 5.2 lists each parameters, its significance to the compression strength of masonry and the range of values being investigated in this study. The range of values being studied is within the limits seen in typical construction practice.

The compression strength of ungrouted masonry is based on minimum net area, which is determined by the net mortar area. The compression strength of grouted masonry is based on gross area of the block.

In the parametric study the masonry compression strength is the dependent variable and the independent variables are the parameter being studied and the block compression strength. The typical failure mode for masonry is tensile splitting of the block, which is typically a function of block compression strength; thus, it is important to see how the block strength also affects the parameter being studied. Additionally, all nonindependent inputs will be left to the default values as outlined in Table 3.3, Table 3.4, Table 3.5, and Table 5.1. 
Table 5.1: Parametric Study Default Values

\begin{tabular}{lll}
\hline Variable Name & Symbol & Parametric Study Default Value \\
\hline Block Length & $l^{b l}$ & $390 \mathrm{~mm}$ \\
Block Width & $w^{b l}$ & $140 \mathrm{~mm}$ \\
Block Height & $h^{b l}$ & $190 \mathrm{~mm}$ \\
Block Percent Solid & $\rho_{\text {solid }}^{b l}$ & $58 \%$ \\
Block Face Shell Thickness & $t^{f s}$ & $26 \mathrm{~mm}$ \\
Number of Webs in Block & $n^{w e b}$ & 3 \\
Strain at $f_{c, z}^{\prime b l}$ & $\epsilon_{o, z}^{b l}$ & 0.002 \\
Uniaxial Mortar Compression Strength & $f_{c}^{\prime m r}$ & $14.6 \mathrm{MPa}$ \\
Strain at $f_{c}^{\prime m r}$ & $\epsilon_{o}^{m r}$ & 0.003 \\
Uniaxial Grout Compression Strength & $f_{c}^{\prime} g r$ & $16.9 \mathrm{MPa}$ \\
Strain at $f_{c}^{\prime} g r$ & $\epsilon_{o}^{g r}$ & 0.002 \\
\hline
\end{tabular}

Each parametric study will be conducted on the two bedding types, face shell and full bedding, and on ungrouted and grouted prisms. 
Table 5.2: The Significance of Parameters Studied and Their Value of Investigation

\begin{tabular}{|c|c|c|}
\hline Parameter & Significance & Value Range \\
\hline Block Compressive Strength & $\begin{array}{l}\text { Influences the tensile } \\
\text { strength of the block }\end{array}$ & $5 \mathrm{MPa}$ to $50 \mathrm{MPa}$ \\
\hline Mortar Compressive Strength & $\begin{array}{l}\text { Influences the strength } \\
\text { degradation of the block }\end{array}$ & $3 \mathrm{MPa}$ to $30 \mathrm{MPa}$ \\
\hline Grout Compressive Strength & $\begin{array}{l}\text { Influences the strength } \\
\text { degradation of the block }\end{array}$ & $5 \mathrm{MPa}$ to $50 \mathrm{MPa}$ \\
\hline Bedding Type & Influences the failure mode & Full or Face shell \\
\hline Mortar Thickness & $\begin{array}{l}\text { Influences the strength en- } \\
\text { hancement or degradation }\end{array}$ & $5 \mathrm{~mm}$ to $25 \mathrm{~mm}$ \\
\hline Block Height & $\begin{array}{l}\text { Influences the strength en- } \\
\text { hancement or degradation }\end{array}$ & $95 \mathrm{~mm}$ or $190 \mathrm{~mm}$ \\
\hline Block Percent Solid & $\begin{array}{l}\text { Influences the width of } \\
\text { webs in the SACM }\end{array}$ & $40 \%$ to $70 \%$ \\
\hline Block Faceshell Thickness & $\begin{array}{l}\text { Influences the mortar bed- } \\
\text { ding area in the SACM }\end{array}$ & $20 \mathrm{~mm}$ to $40 \mathrm{~mm}$ \\
\hline Block Tensile Strength & $\begin{array}{l}\text { Influence masonry com- } \\
\text { pressive strength as most } \\
\text { blocks fail under bi-axial } \\
\text { tension }\end{array}$ & $0.5 \mathrm{MPa}$ to $5 \mathrm{MPa}$ \\
\hline Block Poisson's Ratio & $\begin{array}{l}\text { Influences the strength en- } \\
\text { hancement or degradation }\end{array}$ & 0.1 to 0.3 \\
\hline Mortar Poisson's Ratio & $\begin{array}{l}\text { Influences the strength en- } \\
\text { hancement or degradation }\end{array}$ & 0.1 to 0.4 \\
\hline Grout Poisson's Ratio & $\begin{array}{l}\text { Influences the strength en- } \\
\text { hancement or degradation }\end{array}$ & 0.1 to 0.4 \\
\hline Block Strain at Peak Stress & $\begin{array}{l}\text { Influences the strength en- } \\
\text { hancement or degradation }\end{array}$ & 0.001 to 0.003 \\
\hline Mortar Strain at Peak Stress & $\begin{array}{l}\text { Influences the strength en- } \\
\text { hancement or degradation }\end{array}$ & 0.001 to 0.004 \\
\hline Grout Strain at Peak Stress & $\begin{array}{l}\text { Influences the strength en- } \\
\text { hancement or degradation }\end{array}$ & 0.001 to 0.003 \\
\hline
\end{tabular}

Part II: Masonry Compression Strength Modeling 


\subsection{Influence of Mortar Compressive Strength}

Figure 5.1 depicts the predictions of the SACM as influenced by mortar compressive strength. As observed by Richart et al. (1932), Gaynor et al. (1987), Khalaf et al. (1994), Ramamurthy et al. (2000), and Roman and Romagna (2002) the mortar strength has little effect on the ungrouted masonry compressive strength and negligible effect on grouted masonry compressive strength. The mortar bedding type also has little effect other then changing the area used to determine the compressive strength for ungrouted masonry. The main reason for there being little impact on the hollow concrete compression strength is due to the fact that the mortar is in triaxial confinement, thus, the compression strength is increased.

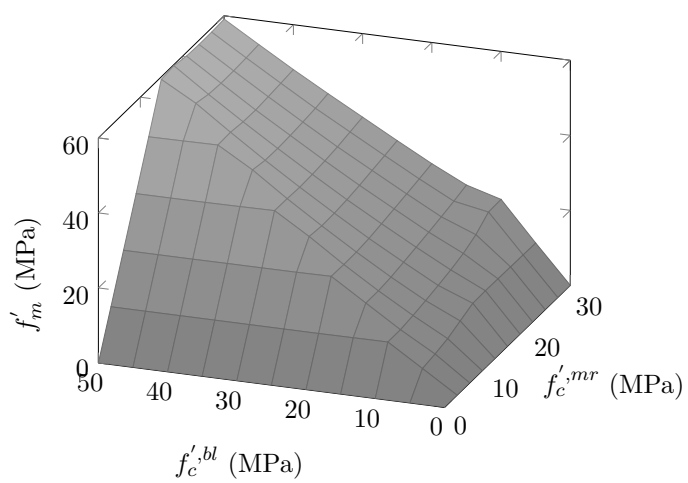

(a) Ungrouted Face Shell Bedding Mortar

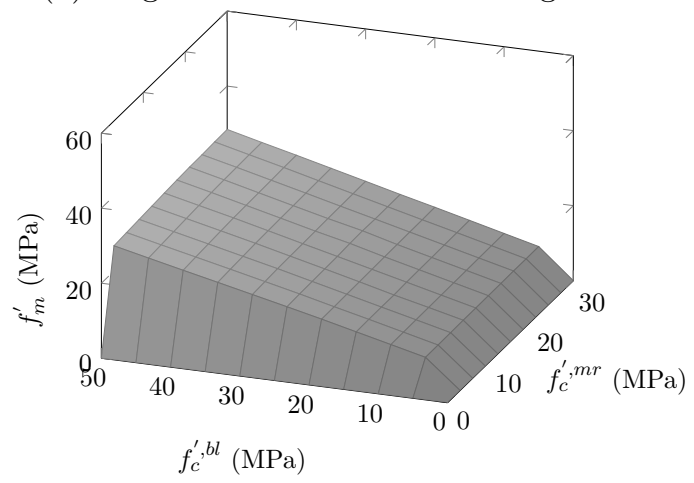

(c) Grouted Face Shell Bedding Mortar

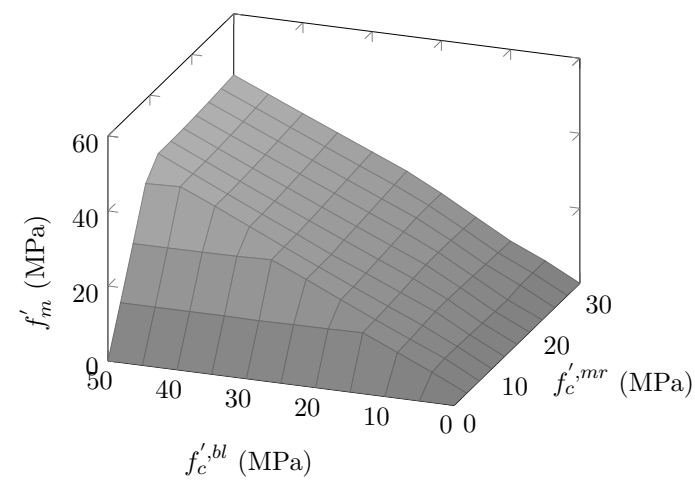

(b) Ungrouted Full Bedding Mortar

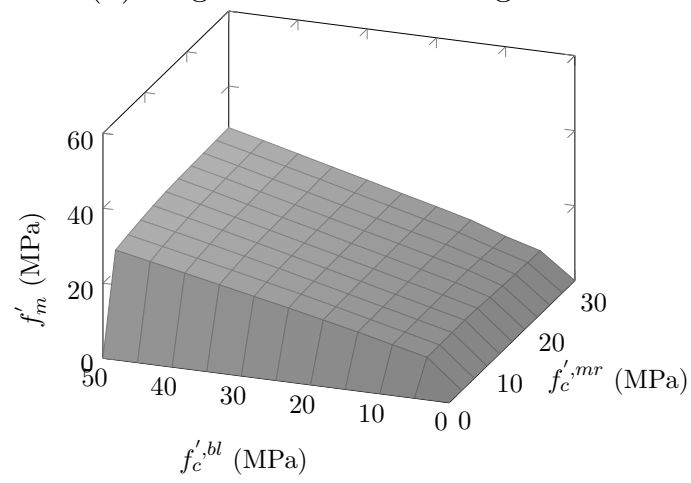

(d) Grouted Full Bedding Mortar

Figure 5.1: Mortar Compressive Strength Influence on Masonry Compressive Strength 


\subsection{Influence of Grout Compressive Strength}

The influence of grout compressive strength is illustrated in Figure 5.2. As can be seen the bedding type has negligible effect, but as the grout strength increases so does the masonry compression strength. This matches the experimental results of Khalaf et al. (1994) and Roman and Romagna (2002). The SACM also agrees with Hamid (1978) that the compression strength of grouted masonry is lower than the superposition of the capacity of the grouted area and the capacity of the block.

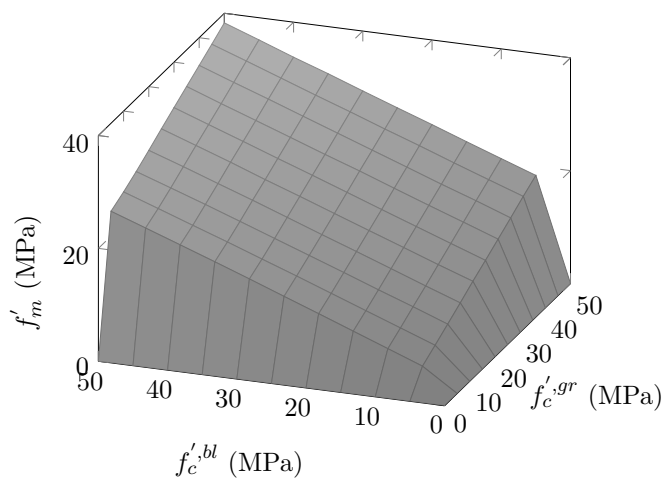

(a) Grouted Face Shell Bedding Mortar

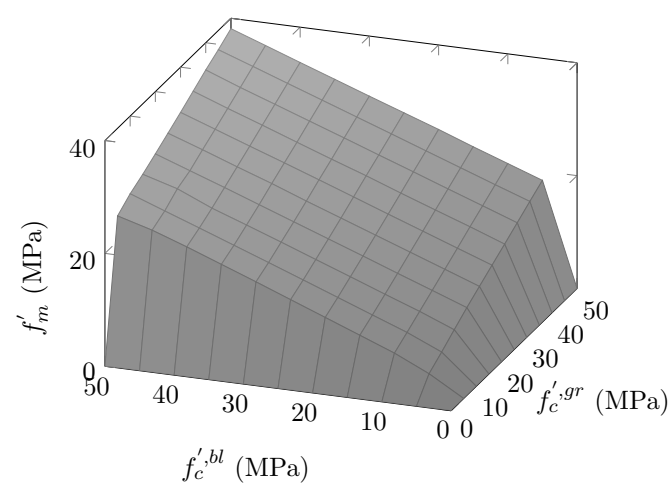

(b) Grouted Full Bedding Mortar

Figure 5.2: Grout Compressive Strength Influence on Masonry Compressive Strength 


\subsection{Influence of Mortar Thickness}

As can be seen in Figure 5.3a and Figure 5.3b as the mortar thickness increases there is a substantial decrease in the ungrouted compressive strength of masonry. This decrease is more noticeable in ungrouted face shell bedding mortar strength predictions. At a block strength of $50 \mathrm{MPa}$ increasing the joint thickness from $10 \mathrm{~mm}$ to $25 \mathrm{~mm}$ results in a $41 \%$ decrease in compression strength, compared to a $34 \%$ reduction for $15 \mathrm{MPa}$ blocks. Both Drysdale and Hamid (1979) and Ramamurthy et al. (2000) experimentally observed a decease in prism capacity as the joint thickness increased. The SACM results also show that as the mortar joints increase in thickness the lateral confinement effect offered by the block reduces considerably, as stated by Ramamurthy et al. (2000). The mortar joint thickness has a much less impact on the compression strength of grouted masonry (Figure 5.3c and Figure 5.3d), similar results were obtained experimental by Drysdale and Hamid (1979), this is a result of the grout providing continuity.

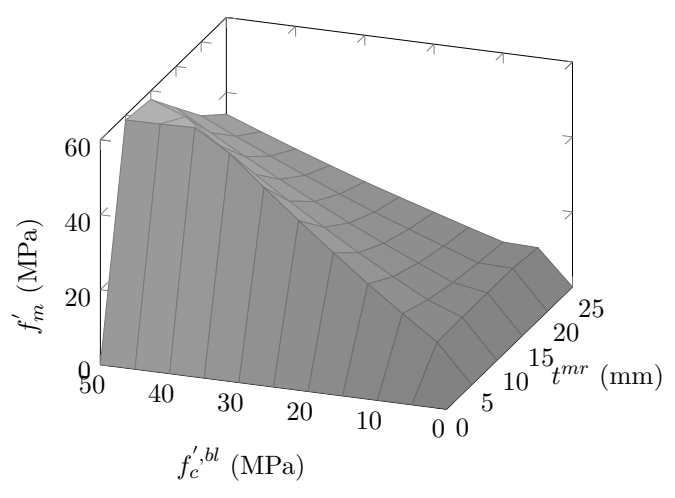

(a) Ungrouted Face Shell Bedding Mortar

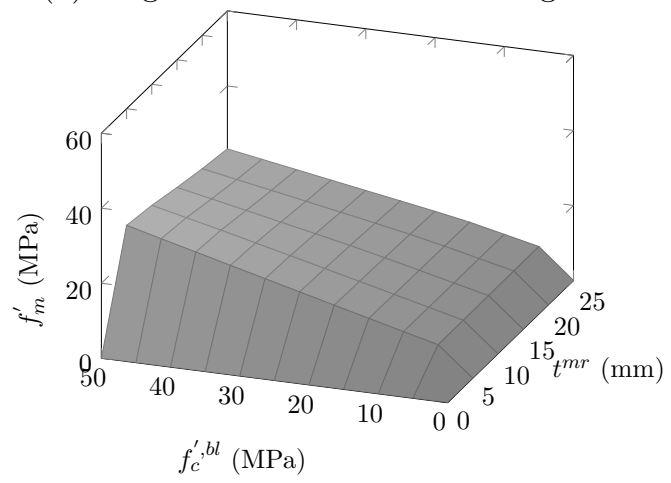

(c) Grouted Face Shell Bedding Mortar

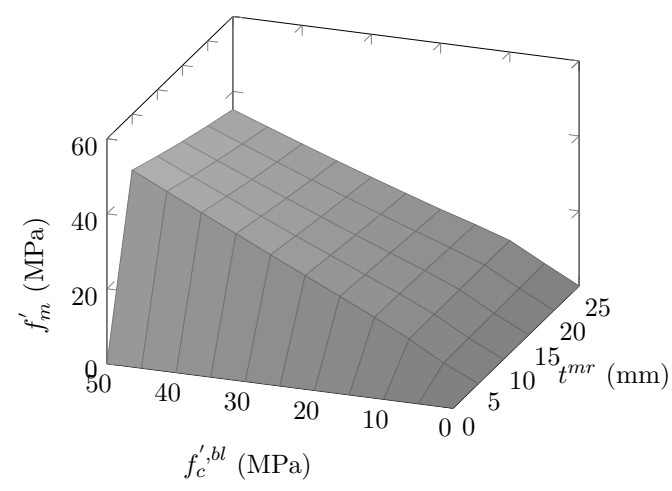

(b) Ungrouted Full Bedding Mortar

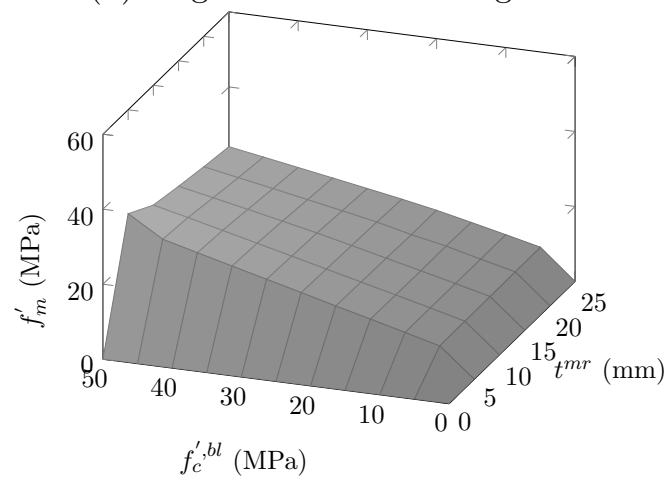

(d) Grouted Full Bedding Mortar

Figure 5.3: Mortar Thickness Influence on Masonry Compressive Strength 


\subsection{Influence of Block Height}

Figure 5.4 illustrates the effects of block height on compression strength. As can be seen as the block height increases the masonry compression strength tends to increase. As failure of the model is typically web tensile splitting for face shell bedded mortar or face shell tensile splitting for full mortar bedding or grouted masonry the area of the webs and face shells has a large impact on the masonry compression strength. As the block height increases so does the area of the webs and face shells. This increase in area causes the average tensile stresses in the webs and face shells to decrease. This causes a smaller block strength degradation factor, $\beta_{z}^{b l}$, to be calculated resulting in a larger block peak strength and strain. The effect of block height is most noticeable in ungrouted masonry, as grout provides continuity.

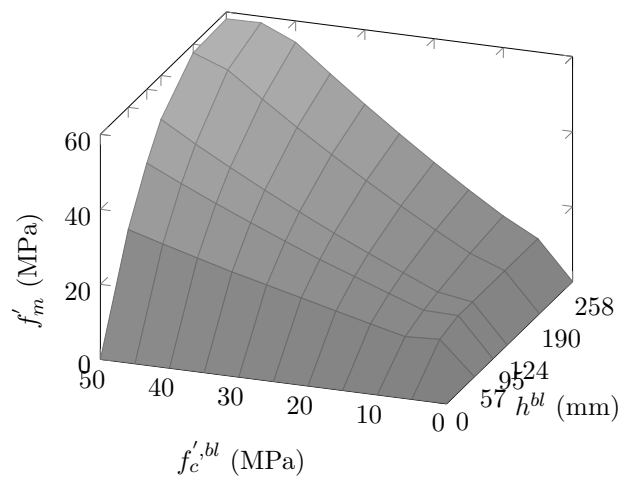

(a) Ungrouted Face Shell Bedding Mortar

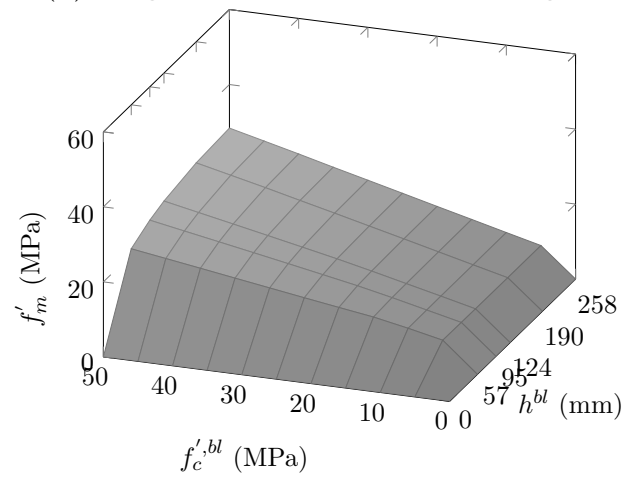

(c) Grouted Face Shell Bedding Mortar

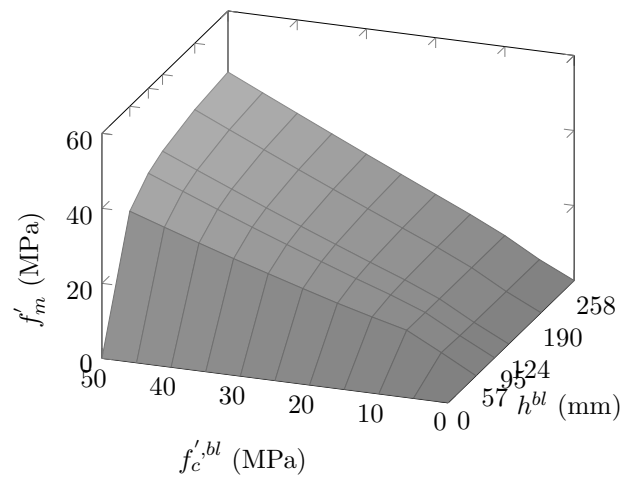

(b) Ungrouted Full Bedding Mortar

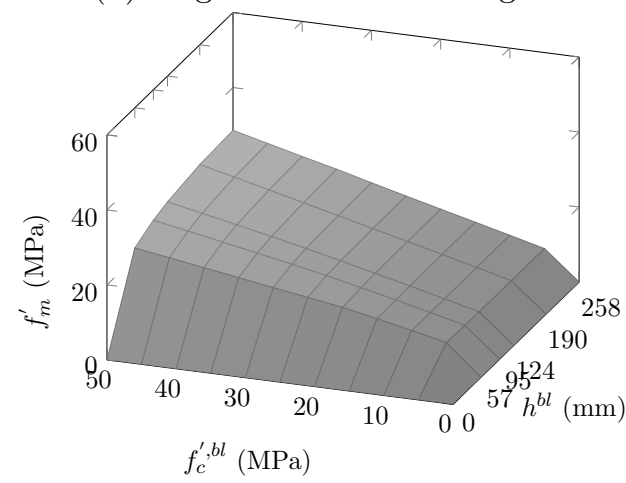

(d) Grouted Full Bedding Mortar

Figure 5.4: Block Height Influence on Masonry Compressive Strength 


\subsection{Influence of Block Percent Solid}

The influence of the percent solid of the block is shown in Figure 5.5. As the face shell thickness is constant at $26 \mathrm{~mm}$ increasing the percentage solid results in increasing web thickness. Ungrouted face shell bedded mortar typically fails by web tensile splitting; thus, the thickness of the webs has a large influence on the predicted masonry compression strength as shown in Figure 5.5a. As the thickness of the web increases so does the total area of the webs resulting in a lower average tensile stress in the webs. This causes a smaller block strength degradation factor, $\beta_{z}^{b l}$, to be calculated resulting in a larger block peak strength and strain. This same effect is seen in the grouted compression strengths. As the web area of the block increases there is less area for grout and there is more web area to resist the lateral expansion of the grout. This results in lower average tensile stresses in the block webs. The block percent solid has negligible impact on the minimum net area compression strength of masonry for full bedded ungroued masonry.

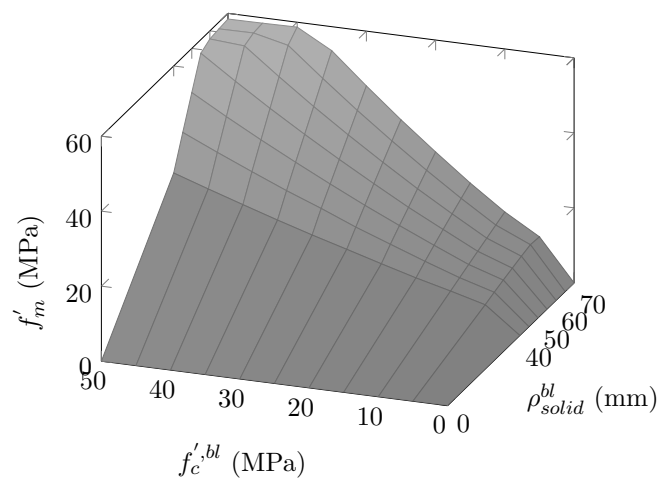

(a) Ungrouted Face Shell Bedding Mortar

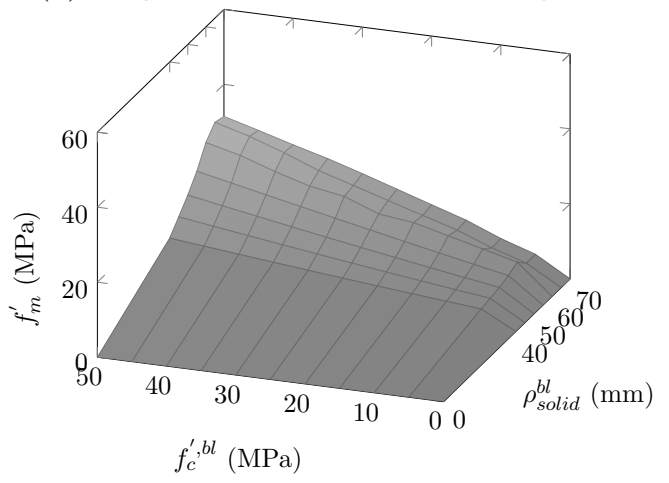

(c) Grouted Face Shell Bedding Mortar

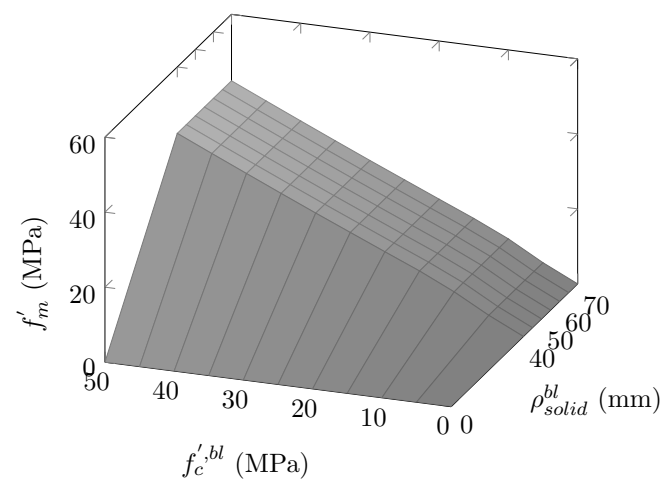

(b) Ungrouted Full Bedding Mortar

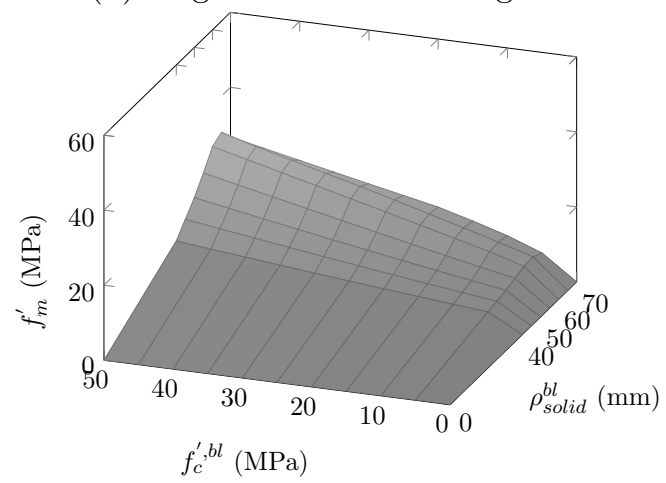

(d) Grouted Full Bedding Mortar

Figure 5.5: Block Percent Solid Influence on Masonry Compressive Strength 


\subsection{Influence of Block Face Shell Thickness}

The block face shell thickness has little impact on the compression strength of masonry as shown in Figure 5.6, except for ungrouted face shell bedded mortar. As can be seen in Figure 5.6a as the face shell thickness increases the masonry compression strength deceases. Though the thicker face shell block is able to resist a larger load (64\% increase for the $50 \mathrm{MPa}$ block from $15 \mathrm{~mm}$ to $45 \mathrm{~mm}$ face shell thickness), the larger minimum net area (200\% increase from a face shell thickness of $15 \mathrm{~mm}$ to $45 \mathrm{~mm}$ ) of the thicker face shell results in much lower masonry compressive stress. Additionally, as the face shell thickness increases the mortar bedding width increase allowing for a larger compression struct to form from deep beam action (Ramamurthy et al., 2000); thus, the face shell thickness and resulting mortar width have a large impact on the failure load of face shell mortared prisms.

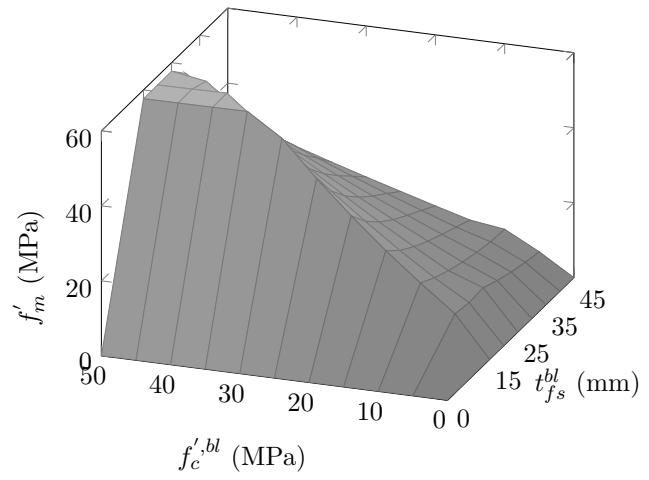

(a) Ungrouted Face Shell Bedding Mortar

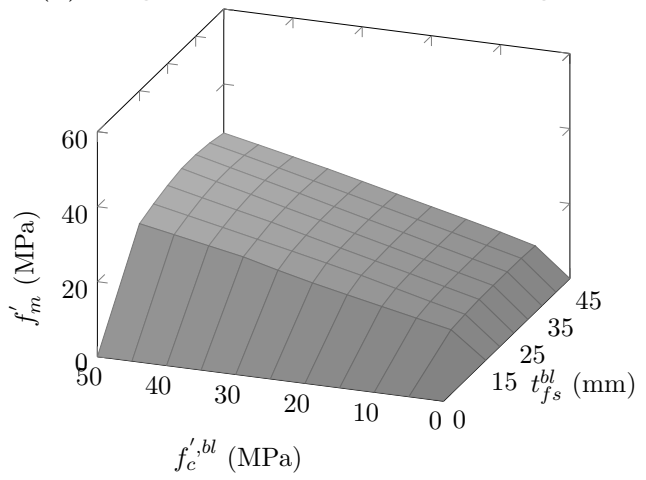

(c) Grouted Face Shell Bedding Mortar

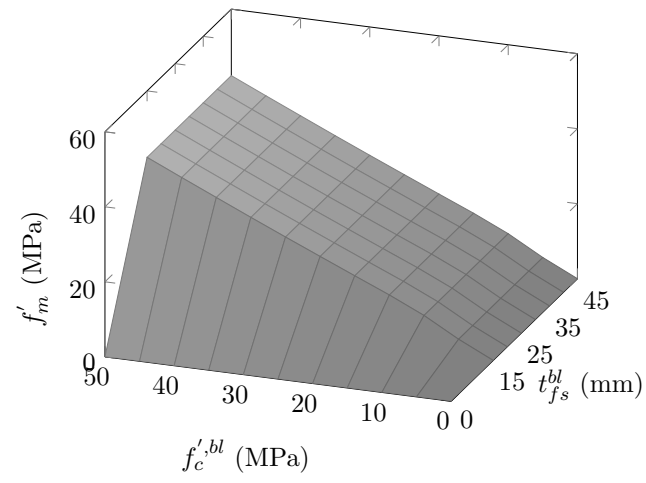

(b) Ungrouted Full Bedding Mortar

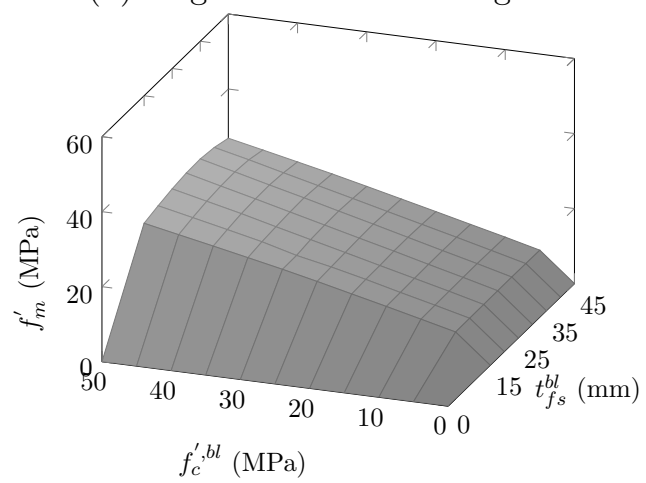

(d) Grouted Full Bedding Mortar

Figure 5.6: Block Face Shell Thickness Influence on Masonry Compressive Strength 


\subsection{Influence of Block Tensile Strength}

For the range of parameters studied in the parametric study the cracking strength of the block is never reached, but instead the additional tension stress in the biaxial direction reduces the compression capacity of the block according to the Mohr-Coulomb failure criteria as discussed in Section 3.8. As the initial modulus of elasticity of the block does not change, for a given block strength, increasing the tensile strength of the block also increases the strain required to cause cracking. This causes the stress in each direction of the block to follow the same path (see Figure 5.7); thus, the failure strength is approximately the same for a given block strength and mortar bedding as shown in Figure 5.8.

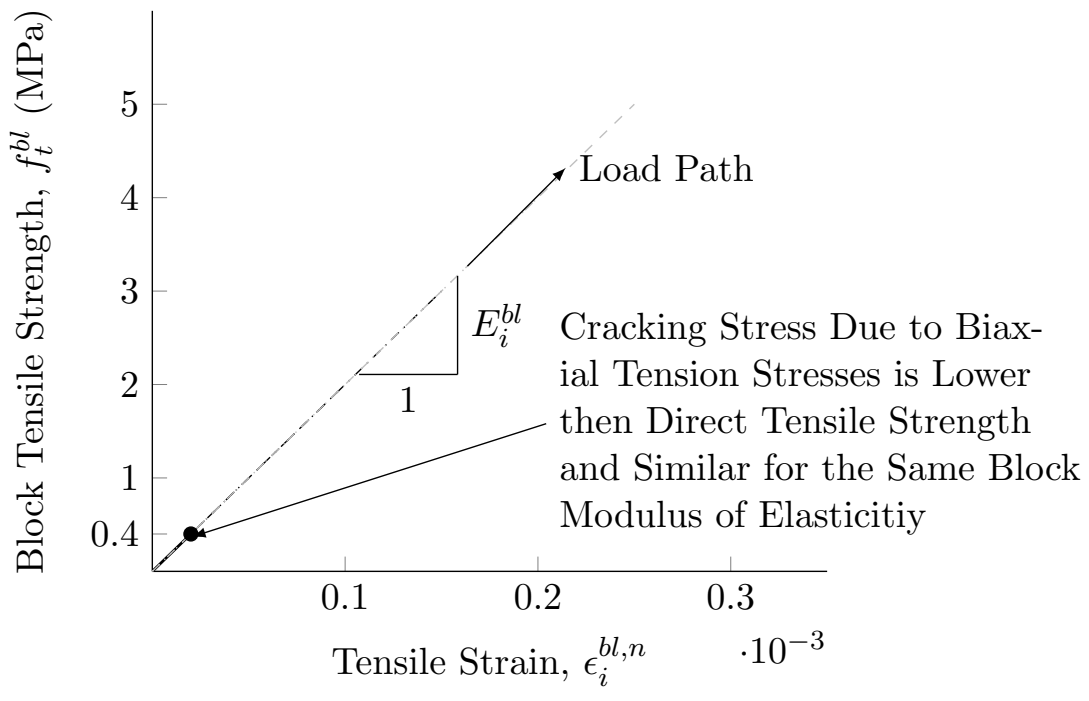

Figure 5.7: Block Cracking Stress 


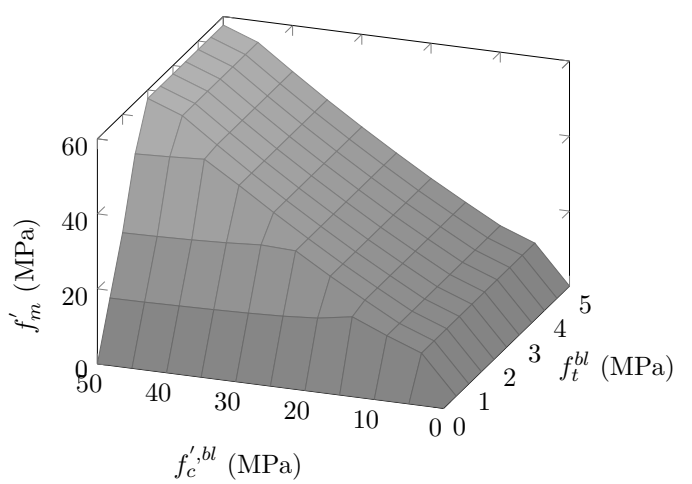

(a) Ungrouted Face Shell Bedding Mortar

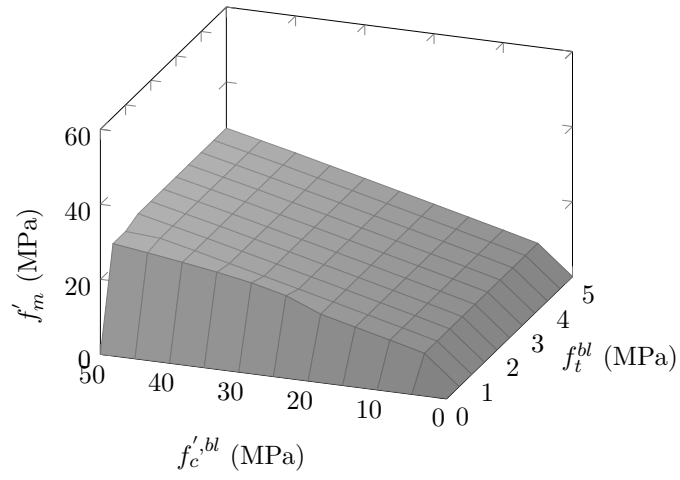

(c) Grouted Face Shell Bedding Mortar

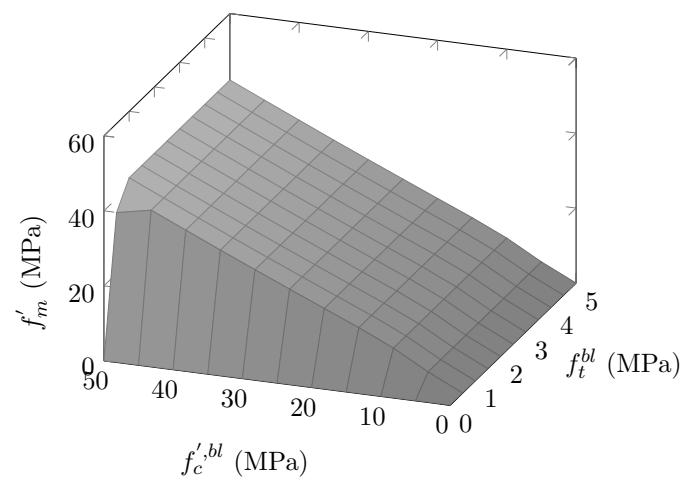

(b) Ungrouted Full Bedding Mortar

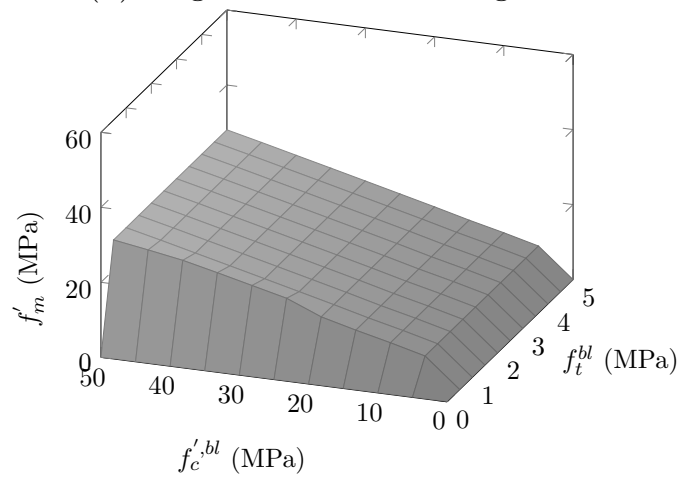

(d) Grouted Full Bedding Mortar

Figure 5.8: Block Tensile Strength Influence on Masonry Compressive Strength 


\subsection{Influence of Block Initial Poisson's Ratio}

The initial Poisson's ratio block has little impact on the compression strength of ungrouted masonry as shown in Figure 5.9a and Figure 5.9b. In the case of the grouted masonry the compression strength reaches a maximum value when the block Poisson's ratio is 0.25 to 0.30 , which is approximately equal to the mortar Poisson's ratio of 0.30 and 0.35 for the grout. Drysdale and Hamid (1979), Khalaf et al. (1994), and Roman and Romagna (2002) also concluded that the compressive strength of grouted masonry could be increased by matching the deformational characteristics of the grout and block.

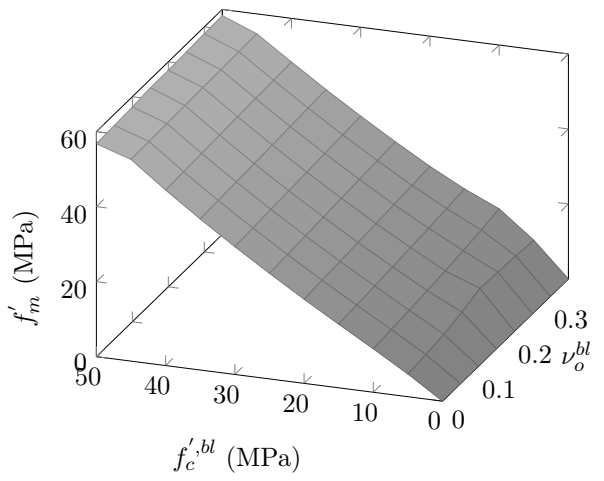

(a) Ungrouted Face Shell Bedding Mortar

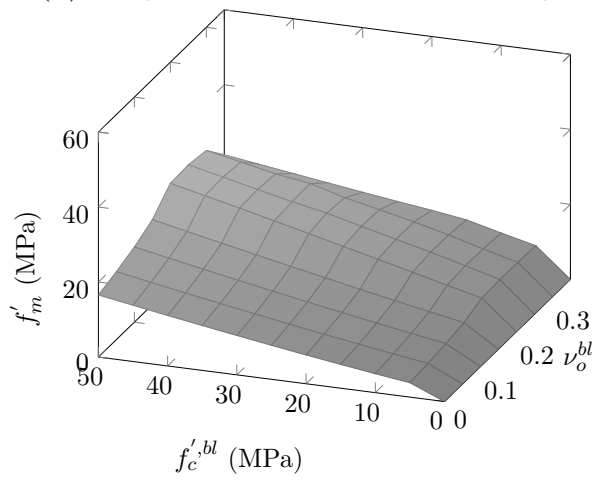

(c) Grouted Face Shell Bedding Mortar

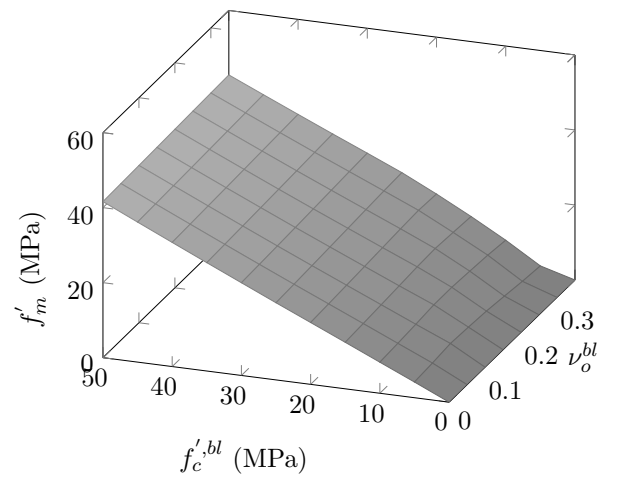

(b) Ungrouted Full Bedding Mortar

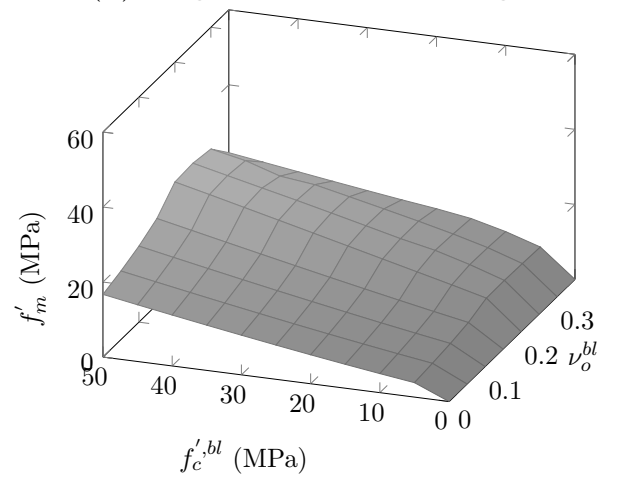

(d) Grouted Full Bedding Mortar

Figure 5.9: Block Poisson's Ratio Influence on Masonry Compressive Strength 


\subsection{Influence of Mortar Initial Poisson's Ratio}

Figure 5.10 shows the influence of the mortar initial Poisson's ratio. For both the ungrouted and grouted masonry the maximum compression strength is reached when the mortar Poisson's ratio is approximately 0.20, similar to the assumed block Poisson's ratio of 0.20 . Further confirming that matching the deformation properties (Drysdale and Hamid, 1979; Khalaf et al., 1994; Roman and Romagna, 2002) is important to achieve maximum masonry compressive strength.

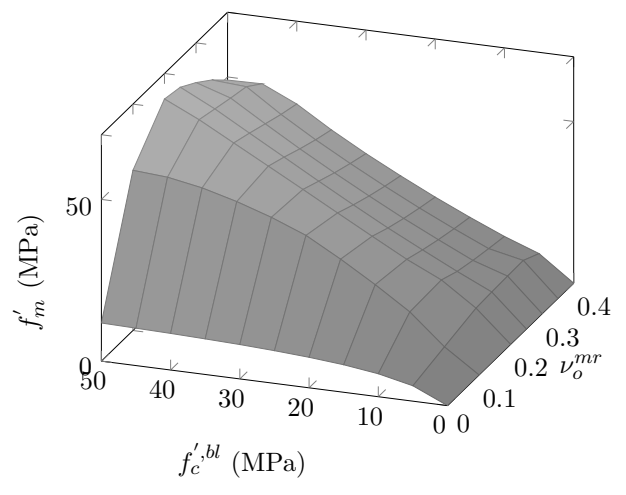

(a) Ungrouted Face Shell Bedding Mortar

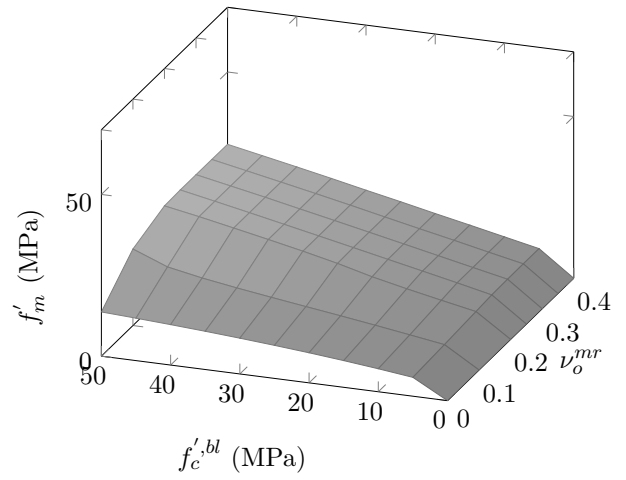

(c) Grouted Face Shell Bedding Mortar

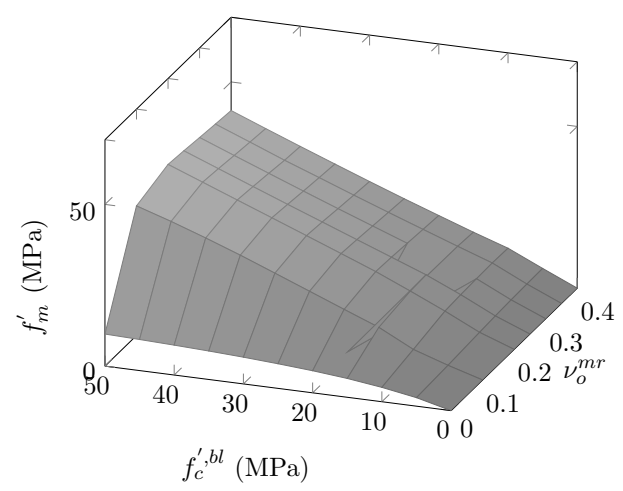

(b) Ungrouted Full Bedding Mortar

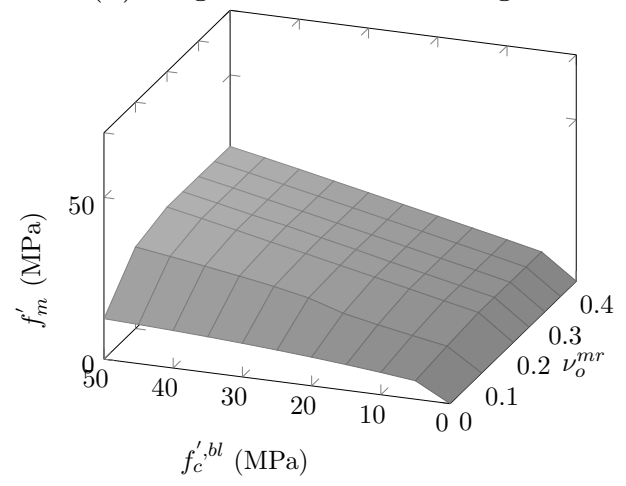

(d) Grouted Full Bedding Mortar

Figure 5.10: Mortar Poisson's Ratio Influence on Masonry Compressive Strength 


\subsection{Influence of Grout Initial Poisson's Ratio}

The influence grout initial Poisson's ratio is shown in Figure 5.11. The grout Poisson's ratio influences both the failure mode and the masonry compression strength. From a grout Poisson's ratio of 0 to about 0.25 the SACM predicts failure of the grout under biaxial tension to cause failure of the prism. This is due to the grout being unconfined by the block as the lateral expansion of the block is larger than that of the grout. Around a grout Poisson's ratio of 0.25 to 0.30 the maximum masonry compressive strength is achieved and failure mode changes to block face shell tensile splitting.

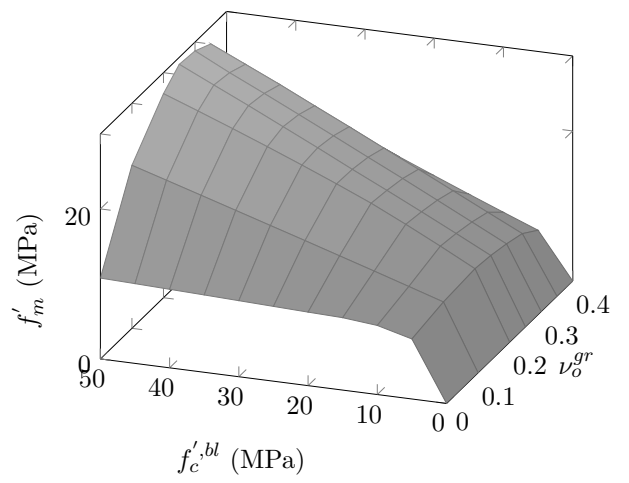

(a) Grouted Face Shell Bedding Mortar

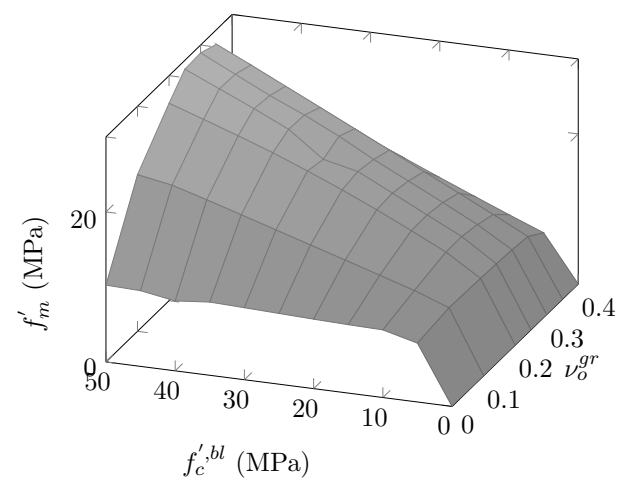

(b) Grouted Full Bedding Mortar

Figure 5.11: Grout Poisson's Ratio Influence on Masonry Compressive Strength 


\subsection{Influence of Block Strain at Peak Stress}

The block strain at peak strength has negligible effects on the masonry compression strength as shown in Figure 5.12. The block strain at peak stress has a larger impact on the deformation characteristics of the prisms. As the strain at peak stress increases the stiffness of the block decreases; thus, there are larger strains at a given stress.

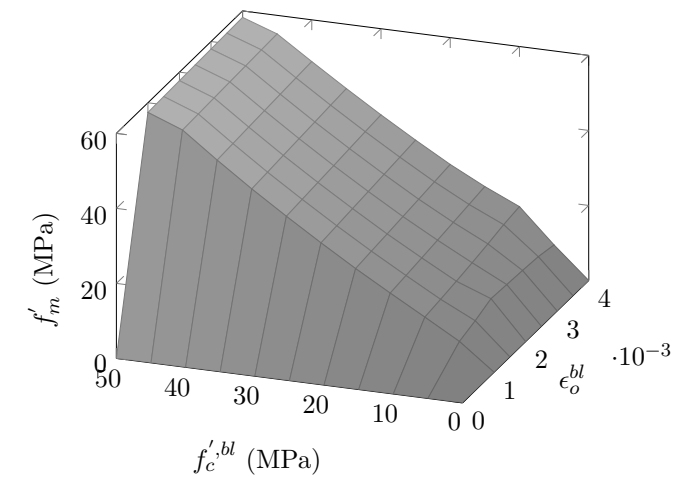

(a) Ungrouted Face Shell Bedding Mortar

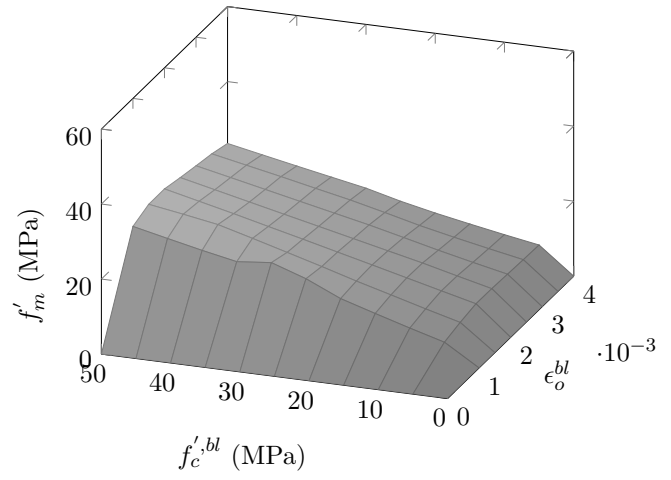

(c) Grouted Face Shell Bedding Mortar

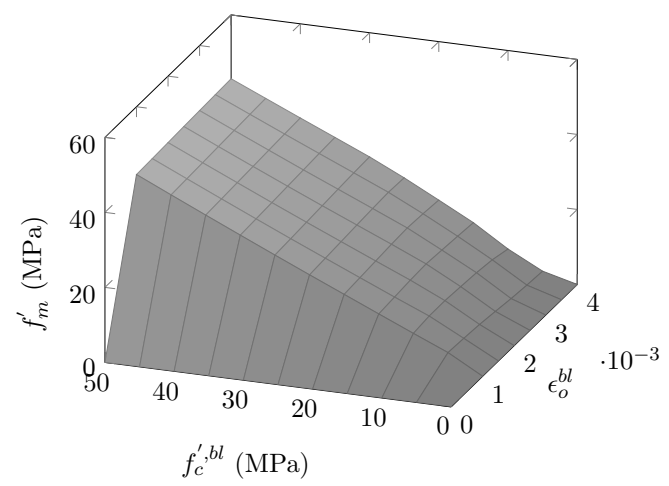

(b) Ungrouted Full Bedding Mortar

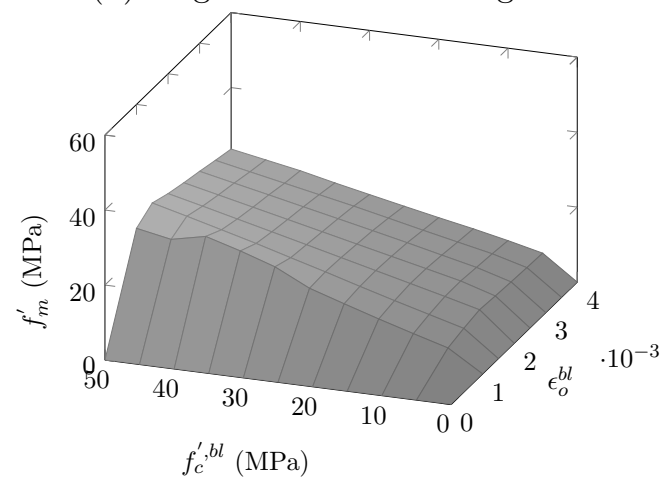

(d) Grouted Full Bedding Mortar

Figure 5.12: Block Strain at Peak Stress Influence on Masonry Compressive Strength 


\subsection{Influence of Mortar Strain at Peak Stress}

The mortar strain at peak strength has negligible effects on the masonry compression strength as shown in Figure 5.13. This is due to the fact that the mortar is typically under triaxial confinement (see Figure 2.1); thus, the peak stress and strain and peak stress are typically increased due to strength enhancement factor, $\beta_{z}^{m r}$.

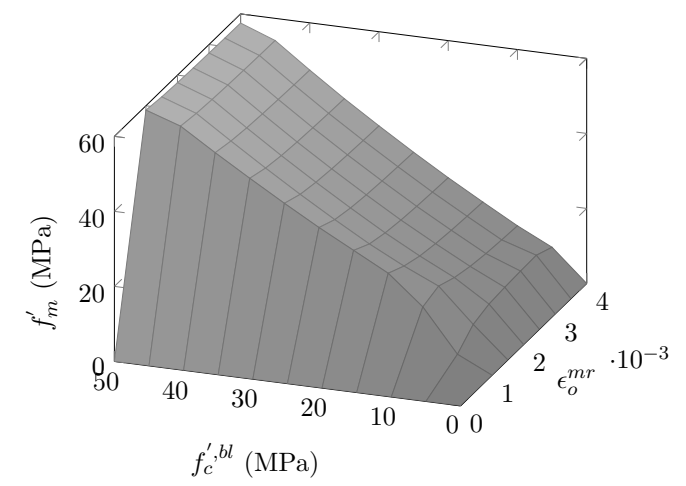

(a) Ungrouted Face Shell Bedding Mortar

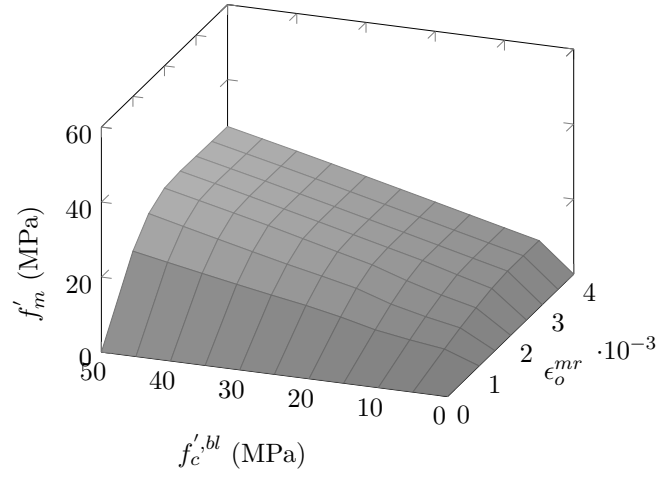

(c) Grouted Face Shell Bedding Mortar

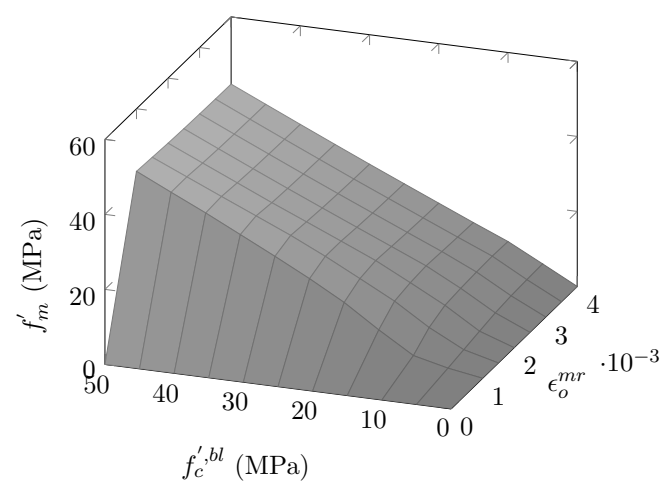

(b) Ungrouted Full Bedding Mortar

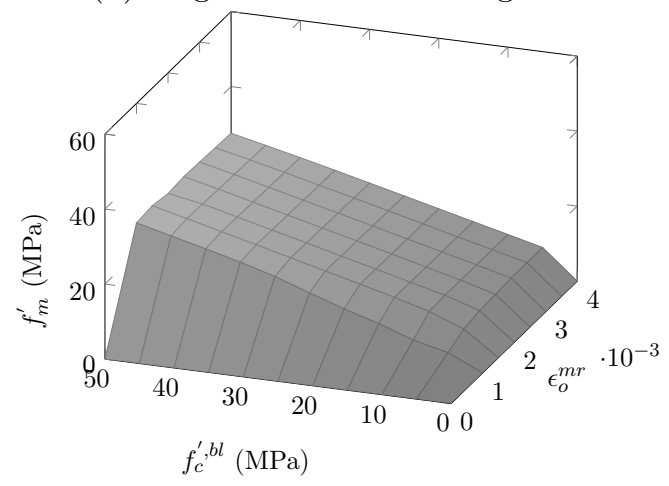

(d) Grouted Full Bedding Mortar

Figure 5.13: Mortar Strain at Peak Stress Influence on Masonry Compressive Strength 


\subsection{Influence of Grout Strain at Peak Stress}

The effect of grout strain at peak stress is shown in Figure 5.14. For small values of grout peak strain the grout tends to be stiffer than the block. This causes the grout to attract more of the vertical load; thus, reducing the vertical confinement of the block. The reduction in confinement causes the block's degradation factor, $\beta_{z}^{b l}$, to be less than one resulting in an earlier failure and lower masonry compression strength. As the peak strain of the grout increases the stiffness of the grout decreases. The softer grout expands more in the lateral directions then stiff grout. This results in more confinement in the grout from the block and additional lateral tensile stresses in the block. This additional lateral tensile stress in the block causes more internal microcracking resulting in a larger percentage of the total stain to be obtained from Poisson effects. Though the resulting total strain may be larger, the net strain in the block may be similar or less resulting in a block degradation factor, $\beta_{z}^{b l}$, closer to one which allows for an increased masonry compression strength to be determined.

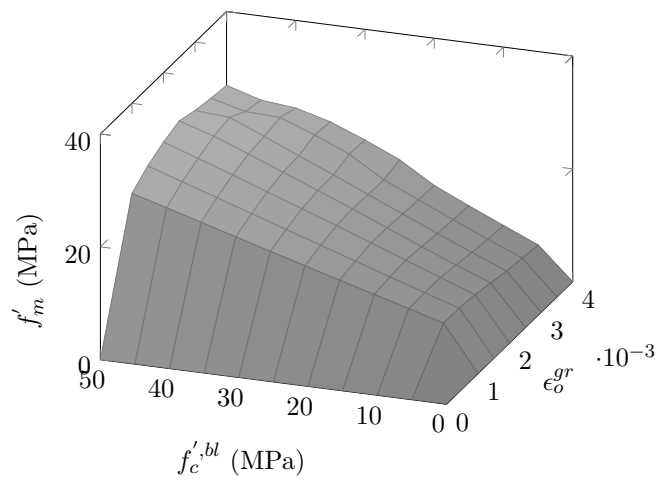

(a) Grouted Face Shell Bedding Mortar

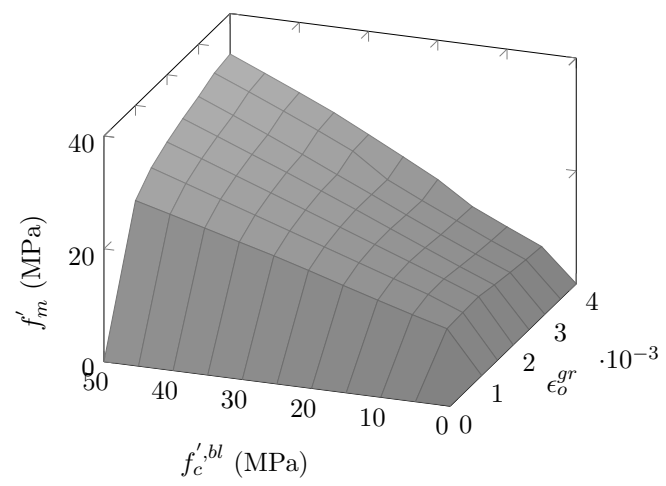

(b) Grouted Full Bedding Mortar

Figure 5.14: Grout Strain at Peak Stress Influence on Masonry Compressive Strength 


\section{SIMPLIFIED SMEARED AREA COMPRESSION MODEL}

The model developed in Chapter 3 is complex and requires a computer due to iterative nature of the solution. The model can however be used to produce simplified equations that can be used for "back-of-envelope" calculations and implementation into design codes. These equations are developed in this chapter and comparisons made to current international design codes.

Masonry design codes generally suggest two methods to determine masonry compression strength: masonry prisms or unit strength. The first method, masonry prisms, consists of constructing three to five masonry prisms with site representative materials; testing the prisms in a universal testing machine under compressive loading; and correcting the average compression strength determined from testing by a factor that accounts for the height to thickness ratio of the tested prisms. This method has certain practical limitations in terms of the complexities of transporting samples and the capacity and size of available testing machines. Furthermore, there is disagreements on the values of the height to thickness correction factors. The second method, known as the unit strength method, involves testing individual samples of masonry component materials. In this approach, the compressive strength of masonry is estimated from either an equation or through tabulated values based on block strength, mortar type or strength, and possible grout strength.

The unit strength method is more widely used by engineers due to its simplicity when compared to the masonry prism method. In addition, in design applications the unit strength method provides savings in both time and cost. However, the data used to develop these equations and tables has been limited, and not based on a full dataset of all available compressive tests. Furthermore, it is widely acknowledged that values of $f_{m}^{\prime}$ in design codes are conservative (Fortes et al., 2015; Korany and Glanville, 2005).

This chapter will review the unit strength methods from four different design codes, review the results of the smeared area compression model (SACM), and propose and evaluate new equations for predicting the compression strength of masonry. 


\subsection{Simplified Smeared Area Compression Model}

Due to the complexities of SACM, a simpler model was desired with similar accuracy; thus, the Simplified Smeared Area Compression Model (SSACM) was derived.

\subsubsection{Ungrouted Face Shell Bedded Mortar}

Face shell bedded masonry typically fail by splitting of the block webs (Gayed et al., 2012; Gaynor et al., 1987; Ramamurthy et al., 2000; Steadman et al., 1995; Wong and Drysdale, 1985). This behaviour can be accurately captured through a Mohr-Coulomb failure criteria cast in form of principal stresses:

$$
\sigma_{1} \frac{1+\sin \phi}{2 c \cos \phi}-\sigma_{3} \frac{1-\sin \phi}{2 c \cos \phi}=1
$$

By setting the first principal stress, $\sigma_{1}$, equal to the block tensile strength, $f_{t}^{b l}$ and solving for the third principal stress, $\sigma_{3}$, or the masonry compression strength $f_{m}^{\prime}$ :

$$
f_{m}^{\prime}=\frac{2 c^{b l} \cos \phi^{b l}-f_{t}^{b l}\left(1+\sin \phi^{b l}\right)}{1-\sin \phi^{b l}} .
$$

The ungrouted face shell bedded mortar masonry compression strength, based on minimum cross sectional area (ie. mortar bedding area), is:

$$
f_{m}^{\prime}=\frac{2 c^{b l} \cos \phi^{b l}-f_{t}^{b l}\left(1+\sin \phi^{b l}\right)}{1-\sin \phi^{b l}} \frac{t^{b l} \rho_{\text {solid }}^{b l}}{2 t^{f s}},
$$

where the block tensile strength can be taken as $0.1 k_{1}^{b l} k_{2}^{b l} f_{c, \text { rep }}^{\prime}$, the block angle of internal friction, $\phi^{b l}$, can be taken as $35^{\circ}$, and the block cohesion, $c^{b l}$, can be taken as $k_{1}^{b l} k_{2}^{b l} f_{c, r e p}^{\prime}$, $/ 4$. This makes the compression strength equal to:

$$
f_{m}^{\prime}=0.300 \frac{t^{b l} \rho_{\text {solid }}^{b l} k_{1}^{b l} k_{2}^{b l} f_{c, r e p}^{\prime}, b l}{t^{f s}} .
$$

where $k_{1}^{b l}$ and $k_{2}^{b l}$ are defined as discussed in Subsection 3.9.1.

\subsubsection{Ungrouted Full Bedded Mortar}

Through the use of the SACM in predicting the strength of ungrouted fully bedded prisms, it was observed that a factor of 0.9 applied to the corrected block strength would accurately predict the compression strength (Figure 6.1). This would imply a net area masonry compression strength of:

$$
f_{m}^{\prime}=0.9 k_{1}^{b l} k_{2}^{b l} f_{c, r e p}^{\prime, b l} .
$$

Part II: Masonry Compression Strength Modeling 
To account for the reduction in compression strength due to increasing mortar thickness, the masonry compression strength can be determined by:

$$
f_{m}^{\prime}=\min \left(\frac{h^{b l}-t^{m r}}{h^{b l}}, 0.9\right) k_{1}^{b l} k_{2}^{b l} f_{c, r e p}^{\prime} \text {. }
$$

where $k_{1}^{b l}$ and $k_{2}^{b l}$ are defined as discussed in Subsection 3.9.1.

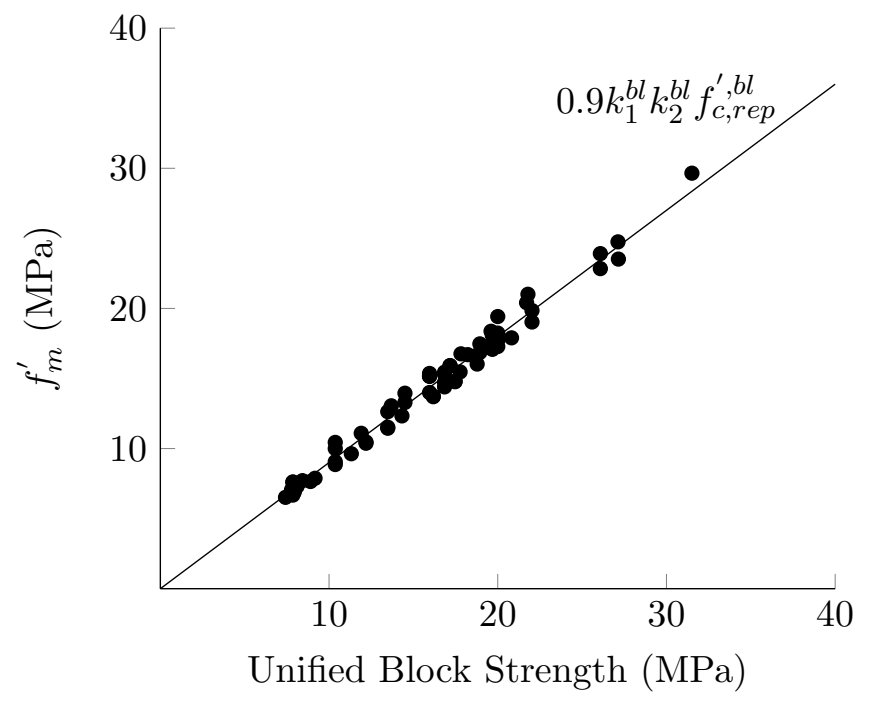

Figure 6.1: SACM Ungrouted Full Bedded Mortar Predictions

\subsubsection{Grouted}

Grouted masonry has a much more complex behaviour relative to ungrouted masonry. It is well known that the compression strength of grouted masonry is not the superposition of the capacities of the hollow prism and the columns of grout in the cells (Drysdale and Hamid, 1979). Suggested causes for this include incomplete grout compaction, plastic and drying shrinkage of the grout, or geometric factors (Drysdale and Hamid, 2005). But one of the primary causes, as often suggested in literature (Chahine, 1989; Drysdale and Hamid, 1979, 2005) and confirmed through the use of the SACM, is incompatibility between the stress-strain properties of the block and grout. The block and grout do not reach peak strength at the same strain, typically the block reaches its peak strain first. Thus, the grout has not yet reached its peak strength when failure occurs. In addition, the lateral expansion of the block and grout are normally different due to different Poisson's ratios which causes a reduction in compression strength of the block. Thus it is suggested that the grouted compression strength can be determined by:

$$
f_{m}^{\prime}=\left[f^{b l} \rho_{\text {solid }}^{b l}+f^{g r}\left(1-\rho_{\text {solid }}^{b l}\right)\right] \sqrt{\frac{\nu^{b l}}{\nu^{g r}}},
$$

Part II: Masonry Compression Strength Modeling 
where $\nu^{b l}$ and $\nu^{g r}$ are the Poisson's ratio of the block and grout respectively and $\rho_{\text {solid }}^{b l}$ is the percent solid of the block. The stress in the block, $f^{b l}$, is defined by a Hognestad parabola; thus,

$$
f^{b l}=k_{1}^{b l} k_{2}^{b l} f_{c, \text { rep }}^{\prime}\left[2\left(\frac{\epsilon}{\epsilon_{o, \text { rep }}^{b l}}\right)-\left(\frac{\epsilon}{\epsilon_{o, \text { rep }}^{b l}}\right)^{2}\right]
$$

where $\epsilon_{o, r e p}^{b l}$ is the strain at the reported block net area compression strength, $f_{c, r e p}^{\prime}$, , and the stress is determined at the strain, $\epsilon$, defined as:

$$
\epsilon= \begin{cases}0.8 \epsilon_{\min } & \text { if } \epsilon_{\min }=\epsilon_{o, r e p}^{b l}, \\ \epsilon_{\min } & \text { otherwise }\end{cases}
$$

where the 0.8 factor comes from the average degradation factor, $\beta_{z}^{b l}$, as determined from the use of the SACM,

$$
\epsilon_{\min }=\min \left(\epsilon_{o, r e p}^{b l}, \epsilon_{o, r e p}^{g r}\right)
$$

and $\epsilon_{o, r e p}^{g r}$ is the strain at the reported grout compression strength, $f_{c, r e p}^{\prime}$. The factors $k_{1}^{b l}$ and $k_{2}^{b l}$ are defined as discussed in Subsection 3.9.1. Similarly, the stress in the grout is defined as:

$$
f^{g r}=k_{1}^{g r} k_{2}^{g r} f_{c, r e p}^{\prime, g r}\left[2\left(\frac{\epsilon}{\epsilon_{o, r e p}^{g r}}\right)-\left(\frac{\epsilon}{\epsilon_{o, r e p}^{g r}}\right)^{2}\right]
$$

where $k_{1}^{g r}$ and $k_{2}^{g r}$ are defined as discussed in Subsection 3.9.3.

If either $\nu^{b l}$ or $\nu^{g r}$, or both Poisson's ratios are unknown the $\sqrt{\nu^{b l} / \nu^{g r}}$ term can be replaced with 0.75 ; from the default values of the SACM. If $\epsilon_{o, r e p}^{b l}$ is unknown 0.0022 can be assumed, while if $\epsilon_{o, \text { rep }}^{g r}$ is unknown it can be assumed to be equal to $0.0011\left(k_{1}^{g r} k_{2}^{g r} f_{c, r e p}^{\prime}\right)^{0.306}$, both from the analysis of the SACM input values.

\subsection{Comparison of Simplified Smeared Area Compression Model to Experimental Data}

Using the same databases presented in Chapter 4, the results of the SSACM are presented in Table 6.1 and Table 6.2 for the ungrouted and grouted data respectively. The results presented in Table 6.2 are for the materiel properties as presented in Appendix B and the simplifing assumptions as stated in Section 6.1.3, i.e. $\sqrt{\nu^{b l} / \nu^{g r}}=0.75, \epsilon_{o, \text { rep }}^{b l}=0.0022$, and $\epsilon_{o, \text { rep }}^{g r}=0.0011\left(k_{1}^{g r} k_{2}^{g r} f_{c, \text { rep }}^{\prime}\right)^{0.306}$. 
Table 6.1: Ungrouted SSACM Results

\begin{tabular}{|c|c|c|c|c|c|c|c|}
\hline \multicolumn{4}{|c|}{ Face Shell Bedded Prisms } & \multicolumn{4}{|c|}{ Full Bedded Prisms } \\
\hline No. ${ }^{\mathrm{a}}$ & $\begin{array}{l}f_{m, r e p}^{\prime} \\
(\mathrm{MPa})^{\mathrm{b}}\end{array}$ & $\begin{array}{l}f_{m, \text { pred }}^{\prime} \\
(\mathrm{MPa})^{\mathrm{b}}\end{array}$ & $\begin{array}{l}f_{m, r e p}^{\prime} / \\
f_{m, \text { pred }}^{\prime}\end{array}$ & No. $^{\mathrm{a}}$ & $\begin{array}{l}f_{m, r e p}^{\prime} \\
(\mathrm{MPa})^{\mathrm{b}}\end{array}$ & $\begin{array}{l}f_{m, \text { pred }}^{\prime} \\
(\mathrm{MPa})^{\mathrm{b}}\end{array}$ & $\begin{array}{l}f_{m, \text { rep }}^{\prime} / \\
f_{m, \text { pred }}^{\prime}\end{array}$ \\
\hline 15 & 7.52 & 6.09 & 1.23 & 1 & 15.32 & 15.16 & 1.01 \\
\hline 30 & 14.30 & 15.45 & 0.93 & 2 & 15.32 & 15.16 & 1.01 \\
\hline 31 & 13.90 & 13.86 & 1.00 & 3 & 13.68 & 15.16 & 0.90 \\
\hline 32 & 16.30 & 13.07 & 1.25 & 4 & 13.68 & 15.16 & 0.90 \\
\hline 33 & 24.90 & 24.22 & 1.03 & 5 & 7.88 & 7.07 & 1.11 \\
\hline 34 & 18.00 & 16.50 & 1.09 & 6 & 6.78 & 7.07 & 0.96 \\
\hline 35 & 20.80 & 19.49 & 1.07 & 7 & 7.11 & 7.07 & 1.01 \\
\hline 36 & 23.80 & 25.14 & 0.95 & 8 & 6.67 & 7.07 & 0.94 \\
\hline 37 & 29.90 & 33.65 & 0.89 & 9 & 10.18 & 9.33 & 1.09 \\
\hline 38 & 21.60 & 21.20 & 1.02 & 10 & 10.61 & 9.33 & 1.14 \\
\hline 39 & 13.90 & 13.69 & 1.02 & 11 & 9.41 & 9.33 & 1.01 \\
\hline 40 & 22.40 & 18.57 & 1.21 & 12 & 8.00 & 9.33 & 0.86 \\
\hline 41 & 18.77 & 17.72 & 1.06 & 13 & 17.49 & 18.74 & 0.93 \\
\hline 43 & 18.63 & 17.72 & 1.05 & 14 & 7.17 & 7.58 & 0.95 \\
\hline 44 & 14.38 & 12.60 & 1.14 & 16 & 6.51 & 7.00 & 0.93 \\
\hline 46 & 14.00 & 12.60 & 1.11 & 17 & 10.30 & 10.72 & 0.96 \\
\hline 48 & 19.00 & 20.12 & 0.94 & 18 & 15.80 & 14.36 & 1.10 \\
\hline 49 & 19.00 & 18.93 & 1.00 & 19 & 16.00 & 14.36 & 1.11 \\
\hline 50 & 19.60 & 19.71 & 0.99 & 20 & 15.93 & 14.36 & 1.11 \\
\hline 54 & 23.60 & 22.14 & 1.07 & 21 & 15.03 & 14.36 & 1.05 \\
\hline 55 & 31.00 & 32.50 & 0.95 & 22 & 16.30 & 14.36 & 1.13 \\
\hline 56 & 29.30 & 27.72 & 1.06 & 23 & 25.92 & 23.47 & 1.10 \\
\hline 60 & 19.60 & 19.22 & 1.02 & 24 & 23.24 & 23.47 & 0.99 \\
\hline 61 & 18.40 & 19.22 & 0.96 & 25 & 18.00 & 16.04 & 1.12 \\
\hline 62 & 17.40 & 19.22 & 0.91 & 26 & 17.78 & 16.40 & 1.08 \\
\hline 63 & 15.28 & 15.24 & 1.00 & 27 & 16.95 & 15.45 & 1.10 \\
\hline 64 & 16.61 & 15.32 & 1.08 & 28 & 16.14 & 15.45 & 1.04 \\
\hline 65 & 15.41 & 15.32 & 1.01 & 29 & 12.76 & 12.32 & 1.04 \\
\hline 66 & 14.41 & 15.32 & 0.94 & 42 & 18.64 & 17.64 & 1.06 \\
\hline 67 & 13.78 & 14.12 & 0.98 & 45 & 14.29 & 13.05 & 1.10 \\
\hline 68 & 11.85 & 10.51 & 1.13 & 47 & 13.58 & 13.05 & 1.04 \\
\hline 69 & 11.21 & 11.76 & 0.95 & 51 & 17.80 & 18.00 & 0.99 \\
\hline
\end{tabular}

Continued on next page 
Table 6.1 Continued from Previous Page

\begin{tabular}{|c|c|c|c|c|c|c|c|}
\hline \multicolumn{4}{|c|}{ Face Shell Bedded Prisms } & \multicolumn{4}{|c|}{ Full Bedded Prisms } \\
\hline No. ${ }^{a}$ & $\begin{array}{l}f_{m, r e p}^{\prime} \\
(\mathrm{MPa})^{\mathrm{b}}\end{array}$ & $\begin{array}{l}f_{m, \text { pred }}^{\prime} \\
(\mathrm{MPa})^{\mathrm{b}}\end{array}$ & $\begin{array}{l}f_{m, r e p}^{\prime} / \\
f_{m, \text { pred }}^{\prime}\end{array}$ & No. $^{a}$ & $\begin{array}{l}f_{m, r e p}^{\prime} \\
(\mathrm{MPa})^{\mathrm{b}}\end{array}$ & $\begin{array}{l}f_{m, p r e d}^{\prime} \\
(\mathrm{MPa})^{\mathrm{b}}\end{array}$ & $\begin{array}{l}f_{m, \text { rep }}^{\prime} / \\
f_{m, \text { pred }}^{\prime}\end{array}$ \\
\hline 70 & 11.26 & 11.76 & 0.96 & 52 & 17.40 & 18.00 & 0.97 \\
\hline 71 & 8.80 & 9.54 & 0.92 & 53 & 21.40 & 18.00 & 1.19 \\
\hline 72 & 7.77 & 8.44 & 0.92 & 57 & 17.80 & 17.71 & 1.00 \\
\hline 73 & 6.29 & 5.88 & 1.07 & 58 & 17.40 & 17.71 & 0.98 \\
\hline 94 & 10.08 & 9.65 & 1.04 & 59 & 21.40 & 19.61 & 1.09 \\
\hline 95 & 15.88 & 17.14 & 0.93 & 74 & 19.49 & 19.83 & 0.98 \\
\hline 96 & 20.55 & 23.78 & 0.86 & 75 & 18.41 & 19.83 & 0.93 \\
\hline 97 & 12.20 & 9.65 & 1.26 & 76 & 14.42 & 15.72 & 0.92 \\
\hline 98 & 17.23 & 17.14 & 1.01 & 77 & 14.11 & 15.72 & 0.90 \\
\hline 99 & 23.56 & 23.78 & 0.99 & 78 & 17.36 & 17.05 & 1.02 \\
\hline 108 & 24.44 & 26.05 & 0.94 & 79 & 16.90 & 17.05 & 0.99 \\
\hline 109 & 22.01 & 26.05 & 0.84 & 80 & 14.39 & 14.56 & 0.99 \\
\hline 110 & 28.09 & 26.05 & 1.08 & 81 & 13.31 & 14.56 & 0.91 \\
\hline 111 & 26.31 & 26.05 & 1.01 & 82 & 13.48 & 12.13 & 1.11 \\
\hline 112 & 23.30 & 18.61 & 1.25 & 83 & 11.21 & 12.13 & 0.92 \\
\hline 113 & 20.17 & 18.61 & 1.08 & 84 & 11.00 & 12.13 & 0.91 \\
\hline 114 & 21.67 & 18.61 & 1.16 & 85 & 11.14 & 10.97 & 1.02 \\
\hline 115 & 21.56 & 18.61 & 1.16 & 86 & 10.86 & 10.97 & 0.99 \\
\hline 116 & 19.23 & 19.00 & 1.01 & 87 & 6.43 & 6.68 & 0.96 \\
\hline 117 & 16.00 & 19.00 & 0.84 & 88 & 12.47 & 12.89 & 0.97 \\
\hline 118 & 21.38 & 19.00 & 1.13 & 89 & 15.73 & 15.99 & 0.98 \\
\hline 119 & 17.42 & 19.00 & 0.92 & 90 & 17.06 & 16.90 & 1.01 \\
\hline 120 & 4.54 & 6.04 & 0.75 & 91 & 8.00 & 7.29 & 1.1 \\
\hline 121 & 4.77 & 6.04 & 0.79 & 92 & 17.89 & 17.54 & 1.02 \\
\hline 122 & 4.99 & 6.04 & 0.83 & 93 & 22.04 & 24.44 & 0.9 \\
\hline 123 & 4.96 & 6.04 & 0.82 & 100 & 7.73 & 8.25 & 0.94 \\
\hline 124 & 25.10 & 24.77 & 1.01 & 101 & 7.11 & 8.01 & 0.89 \\
\hline
\end{tabular}

Continued on next page 
Table 6.1 Continued from Previous Page

\begin{tabular}{|c|c|c|c|c|c|c|c|c|}
\hline \multicolumn{4}{|c|}{ Face Shell Bedded Prisms } & & \multicolumn{4}{|c|}{ Full Bedded Prisms } \\
\hline No. ${ }^{a}$ & $\begin{array}{l}f_{m, r e p}^{\prime} \\
(\mathrm{MPa})^{\mathrm{b}}\end{array}$ & $\begin{array}{l}f_{m, \text { pred }}^{\prime} \\
(\mathrm{MPa})^{\mathrm{b}}\end{array}$ & $\begin{array}{l}f_{m, \text { rep }}^{\prime} / \\
f_{m, \text { pred }}^{\prime}\end{array}$ & & No. ${ }^{a}$ & $\begin{array}{l}f_{m, r e p}^{\prime} \\
(\mathrm{MPa})^{\mathrm{b}}\end{array}$ & $\begin{array}{l}f_{m, p r e d}^{\prime} \\
(\mathrm{MPa})^{\mathrm{b}}\end{array}$ & $\begin{array}{l}f_{m, \text { rep }}^{\prime} / \\
f_{m, \text { pred }}^{\prime}\end{array}$ \\
\hline \multirow[t]{7}{*}{126} & 23.89 & 18.05 & 1.32 & & 102 & 6.71 & 7.15 & 0.94 \\
\hline & & & & & 103 & 9.24 & 10.19 & 0.91 \\
\hline & & & & & 104 & 18.81 & 17.86 & 1.05 \\
\hline & & & & & 105 & 17.86 & 18.00 & 0.99 \\
\hline & & & & & 106 & 21.43 & 19.54 & 1.10 \\
\hline & & & & & 107 & 30.18 & 28.36 & 1.06 \\
\hline & & & & & 125 & 24.10 & 24.41 & 0.99 \\
\hline \multicolumn{2}{|c|}{ Average } & & 1.01 & & \multicolumn{2}{|c|}{ Average } & & 1.01 \\
\hline \multicolumn{2}{|c|}{ STDV } & & 0.12 & & \multicolumn{2}{|c|}{ STDV } & & 0.08 \\
\hline \multicolumn{2}{|c|}{$\mathrm{COV}$} & & $11.38 \%$ & & $\mathrm{COV}$ & & & $7.60 \%$ \\
\hline \multicolumn{4}{|c|}{ Average } & 1.01 & & & & \\
\hline \multicolumn{4}{|c|}{ STDV } & 0.10 & & & & \\
\hline \multicolumn{4}{|c|}{$\mathrm{COV}$} & $9.54 \%$ & & & & \\
\hline
\end{tabular}

Concluded

Notes:

* For Input Values the Number Corresponds to the Number in Appendix A

${ }^{\mathrm{a}}$ Based on minimum net area of the prism

Table 6.2: Grouted SSACM Results

\begin{tabular}{|c|c|c|c|c|c|}
\hline \multirow[b]{2}{*}{ No. ${ }^{b}$} & \multirow[b]{2}{*}{$\begin{array}{l}f_{m, r e p}^{\prime} \\
(\mathrm{MPa})^{\mathrm{c}}\end{array}$} & \multicolumn{2}{|c|}{$\begin{array}{l}\text { Based Material } \\
\text { Properties }\end{array}$} & \multicolumn{2}{|c|}{$\begin{array}{l}\text { Based on Simplifying } \\
\text { Assumptions }^{\mathrm{a}}\end{array}$} \\
\hline & & $\begin{array}{l}f_{m, p r e d}^{\prime} \\
(\mathrm{MPa})^{\mathrm{c}}\end{array}$ & $\begin{array}{l}f_{m, \text { rep }}^{\prime} / \\
f_{m, \text { pred }}^{\prime}\end{array}$ & $\begin{array}{l}f_{m, p r e d}^{\prime} \\
(\mathrm{MPa})^{\mathrm{c}}\end{array}$ & $\begin{array}{l}f_{m, \text { rep }}^{\prime} / \\
f_{m, \text { pred }}^{\prime}\end{array}$ \\
\hline 1 & 18.30 & 22.66 & 0.81 & 20.67 & 0.89 \\
\hline 2 & 18.30 & 22.66 & 0.81 & 20.67 & 0.89 \\
\hline 3 & 10.84 & 10.41 & 1.04 & 10.46 & 1.04 \\
\hline 4 & 11.18 & 10.23 & 1.09 & 10.31 & 1.08 \\
\hline 5 & 11.32 & 10.23 & 1.11 & 10.31 & 1.10 \\
\hline 6 & 10.41 & 10.78 & 0.97 & 10.75 & 0.97 \\
\hline 7 & 11.32 & 10.23 & 1.11 & 10.31 & 1.10 \\
\hline 8 & 12.07 & 10.78 & 1.12 & 10.75 & 1.12 \\
\hline
\end{tabular}

Continued on next page

Part II: Masonry Compression Strength Modeling 
Table 6.2 Continued from Previous Page

\begin{tabular}{|c|c|c|c|c|c|}
\hline \multirow[b]{2}{*}{ No. ${ }^{b}$} & \multirow[b]{2}{*}{$\begin{array}{l}f_{m, r e p}^{\prime} \\
(\mathrm{MPa})^{\mathrm{c}}\end{array}$} & \multicolumn{2}{|c|}{$\begin{array}{l}\text { Based Material } \\
\text { Properties }\end{array}$} & \multicolumn{2}{|c|}{$\begin{array}{l}\text { Based on Simplifying } \\
\text { Assumptions }^{\mathrm{a}}\end{array}$} \\
\hline & & $\begin{array}{l}f_{m, p r e d}^{\prime} \\
(\mathrm{MPa})^{\mathrm{c}}\end{array}$ & $\begin{array}{l}f_{m, \text { rep }}^{\prime} / \\
f_{m, \text { pred }}^{\prime}\end{array}$ & $\begin{array}{l}f_{m, p r e d}^{\prime} \\
(\mathrm{MPa})^{\mathrm{c}}\end{array}$ & $\begin{array}{l}f_{m, \text { rep }}^{\prime} / \\
f_{m, \text { pred }}^{\prime}\end{array}$ \\
\hline 9 & 12.82 & 11.76 & 1.09 & 11.51 & 1.11 \\
\hline 10 & 14.35 & 13.26 & 1.08 & 13.23 & 1.08 \\
\hline 11 & 12.07 & 10.78 & 1.12 & 10.75 & 1.12 \\
\hline 12 & 16.91 & 15.45 & 1.09 & 15.52 & 1.09 \\
\hline 13 & 16.41 & 15.45 & 1.06 & 15.52 & 1.06 \\
\hline 14 & 13.17 & 12.54 & 1.05 & 12.53 & 1.05 \\
\hline 15 & 14.13 & 12.29 & 1.15 & 12.24 & 1.15 \\
\hline 16 & 12.13 & 11.51 & 1.05 & 11.43 & 1.06 \\
\hline 17 & 13.33 & 11.45 & 1.16 & 12.22 & 1.09 \\
\hline 18 & 9.54 & 9.31 & 1.02 & 9.63 & 0.99 \\
\hline 19 & 15.18 & 16.49 & 0.92 & 15.88 & 0.96 \\
\hline 20 & 15.17 & 14.91 & 1.02 & 14.41 & 1.05 \\
\hline 21 & 14.60 & 14.08 & 1.04 & 14.52 & 1.01 \\
\hline 22 & 13.20 & 14.49 & 0.91 & 15.94 & 0.83 \\
\hline 23 & 7.38 & 10.12 & 0.73 & 12.06 & 0.61 \\
\hline 24 & 7.25 & 9.14 & 0.79 & 11.09 & 0.65 \\
\hline 25 & 5.61 & 7.79 & 0.72 & 9.59 & 0.58 \\
\hline 26 & 7.71 & 10.94 & 0.70 & 12.93 & 0.60 \\
\hline 27 & 7.22 & 8.69 & 0.83 & 10.55 & 0.68 \\
\hline 28 & 7.51 & 9.76 & 0.77 & 11.65 & 0.64 \\
\hline 29 & 7.87 & 12.04 & 0.65 & 15.30 & 0.51 \\
\hline 30 & 6.50 & 9.02 & 0.72 & 10.92 & 0.60 \\
\hline 31 & 13.00 & 15.92 & 0.82 & 14.31 & 0.91 \\
\hline 32 & 15.80 & 15.32 & 1.03 & 14.40 & 1.10 \\
\hline 33 & 17.90 & 19.05 & 0.94 & 17.00 & 1.05 \\
\hline 34 & 13.80 & 10.90 & 1.27 & 11.40 & 1.21 \\
\hline 35 & 11.40 & 12.07 & 0.94 & 13.45 & 0.85 \\
\hline 36 & 13.40 & 13.78 & 0.97 & 15.33 & 0.87 \\
\hline 37 & 13.90 & 12.05 & 1.15 & 11.85 & 1.17 \\
\hline 38 & 14.50 & 14.82 & 0.98 & 16.41 & 0.88 \\
\hline 39 & 19.30 & 19.58 & 0.99 & 17.34 & 1.11 \\
\hline 40 & 17.40 & 17.81 & 0.98 & 16.47 & 1.06 \\
\hline
\end{tabular}

Continued on next page

Part II: Masonry Compression Strength Modeling 
Table 6.2 Continued from Previous Page

\begin{tabular}{|c|c|c|c|c|c|}
\hline \multirow[b]{2}{*}{ No. ${ }^{b}$} & \multirow[b]{2}{*}{$\begin{array}{l}f_{m, r e p}^{\prime} \\
(\mathrm{MPa})^{\mathrm{c}}\end{array}$} & \multicolumn{2}{|c|}{$\begin{array}{l}\text { Based Material } \\
\text { Properties }\end{array}$} & \multicolumn{2}{|c|}{$\begin{array}{l}\text { Based on Simplifying } \\
\text { Assumptions }^{\mathrm{a}}\end{array}$} \\
\hline & & $\begin{array}{l}f_{m, p r e d}^{\prime} \\
(\mathrm{MPa})^{\mathrm{c}}\end{array}$ & $\begin{array}{l}f_{m, \text { rep }}^{\prime} / \\
f_{m, \text { pred }}^{\prime}\end{array}$ & $\begin{array}{l}f_{m, \text { pred }}^{\prime} \\
(\mathrm{MPa})^{\mathrm{c}}\end{array}$ & $\begin{array}{l}f_{m, \text { rep }}^{\prime} / \\
f_{m, \text { pred }}^{\prime}\end{array}$ \\
\hline 41 & 20.10 & 21.00 & 0.96 & 18.61 & 1.08 \\
\hline 42 & 17.40 & 18.74 & 0.93 & 13.76 & 1.26 \\
\hline 43 & 20.20 & 19.72 & 1.02 & 15.33 & 1.32 \\
\hline 44 & 23.00 & 21.68 & 1.06 & 16.63 & 1.38 \\
\hline 45 & 23.50 & 22.09 & 1.06 & 20.72 & 1.13 \\
\hline 46 & 27.10 & 25.38 & 1.07 & 19.57 & 1.38 \\
\hline 47 & 19.40 & 20.11 & 0.96 & 18.76 & 1.03 \\
\hline 48 & 15.80 & 15.19 & 1.04 & 14.28 & 1.11 \\
\hline 49 & 17.90 & 18.92 & 0.95 & 16.87 & 1.06 \\
\hline 50 & 13.40 & 14.23 & 0.94 & 15.21 & 0.88 \\
\hline 51 & 13.90 & 11.92 & 1.17 & 11.72 & 1.19 \\
\hline 52 & 14.50 & 15.38 & 0.94 & 16.28 & 0.89 \\
\hline 53 & 19.30 & 19.45 & 0.99 & 17.21 & 1.12 \\
\hline 54 & 9.17 & 8.75 & 1.05 & 9.25 & 0.99 \\
\hline 55 & 12.92 & 11.91 & 1.09 & 11.30 & 1.14 \\
\hline 56 & 15.50 & 14.51 & 1.07 & 14.49 & 1.07 \\
\hline 57 & 18.20 & 16.88 & 1.08 & 16.36 & 1.11 \\
\hline 58 & 11.01 & 9.83 & 1.12 & 10.32 & 1.07 \\
\hline 59 & 14.40 & 13.14 & 1.10 & 12.46 & 1.16 \\
\hline 60 & 16.48 & 15.51 & 1.06 & 15.49 & 1.06 \\
\hline 61 & 19.09 & 17.92 & 1.07 & 17.37 & 1.10 \\
\hline 62 & 11.61 & 10.09 & 1.15 & 10.57 & 1.10 \\
\hline 63 & 15.77 & 13.00 & 1.21 & 12.45 & 1.27 \\
\hline 64 & 17.12 & 15.93 & 1.08 & 15.89 & 1.08 \\
\hline 65 & 20.27 & 18.56 & 1.09 & 17.95 & 1.13 \\
\hline 66 & 5.56 & 5.08 & 1.10 & 6.22 & 0.89 \\
\hline 67 & 11.15 & 9.54 & 1.17 & 8.84 & 1.26 \\
\hline 68 & 12.90 & 11.59 & 1.11 & 11.61 & 1.11 \\
\hline 69 & 15.76 & 14.32 & 1.10 & 13.40 & 1.18 \\
\hline 70 & 10.92 & 11.24 & 0.97 & 11.14 & 0.98 \\
\hline 71 & 17.05 & 14.40 & 1.18 & 13.70 & 1.24 \\
\hline 72 & 17.84 & 16.17 & 1.10 & 16.23 & 1.10 \\
\hline
\end{tabular}

Continued on next page

Part II: Masonry Compression Strength Modeling 
Table 6.2 Continued from Previous Page

\begin{tabular}{|c|c|c|c|c|c|}
\hline \multirow[b]{2}{*}{ No. ${ }^{b}$} & \multirow[b]{2}{*}{$\begin{array}{l}f_{m, r e p}^{\prime} \\
(\mathrm{MPa})^{\mathrm{c}}\end{array}$} & \multicolumn{2}{|c|}{$\begin{array}{l}\text { Based Material } \\
\text { Properties }\end{array}$} & \multicolumn{2}{|c|}{$\begin{array}{l}\text { Based on Simplifying } \\
\text { Assumptions }^{\text {a }}\end{array}$} \\
\hline & & $\begin{array}{l}f_{m, \text { pred }}^{\prime} \\
(\mathrm{MPa})^{\mathrm{c}}\end{array}$ & $\begin{array}{l}f_{m, \text { rep }}^{\prime} / \\
f_{m, \text { pred }}^{\prime}\end{array}$ & $\begin{array}{l}f_{m, \text { pred }}^{\prime} \\
(\mathrm{MPa})^{\mathrm{c}}\end{array}$ & $\begin{array}{l}f_{m, \text { rep }}^{\prime} / \\
f_{m, \text { pred }}^{\prime} \\
\end{array}$ \\
\hline 73 & 21.11 & 19.60 & 1.08 & 18.04 & 1.17 \\
\hline 74 & 6.05 & 7.02 & 0.86 & 8.00 & 0.76 \\
\hline 75 & 12.65 & 11.22 & 1.13 & 10.52 & 1.20 \\
\hline 76 & 15.00 & 13.97 & 1.07 & 13.11 & 1.14 \\
\hline 77 & 15.81 & 14.93 & 1.06 & 14.61 & 1.08 \\
\hline 78 & 9.62 & 10.51 & 0.92 & 11.70 & 0.82 \\
\hline 79 & 14.46 & 14.26 & 1.01 & 13.71 & 1.05 \\
\hline 80 & 17.11 & 16.35 & 1.05 & 16.47 & 1.04 \\
\hline 81 & 19.53 & 18.42 & 1.06 & 18.13 & 1.08 \\
\hline 82 & 13.04 & 14.07 & 0.93 & 15.51 & 0.84 \\
\hline 83 & 17.08 & 17.21 & 0.99 & 16.73 & 1.02 \\
\hline 84 & 20.12 & 19.10 & 1.05 & 19.28 & 1.04 \\
\hline 85 & 20.44 & 21.67 & 0.94 & 21.39 & 0.96 \\
\hline 86 & 8.16 & 7.30 & 1.12 & 8.10 & 1.01 \\
\hline 87 & 13.10 & 10.95 & 1.20 & 9.77 & 1.34 \\
\hline 88 & 16.69 & 14.80 & 1.13 & 13.63 & 1.22 \\
\hline 89 & 17.34 & 16.54 & 1.05 & 14.90 & 1.16 \\
\hline 90 & 10.29 & 10.28 & 1.00 & 11.90 & 0.86 \\
\hline 91 & 16.10 & 14.19 & 1.13 & 13.65 & 1.18 \\
\hline 92 & 18.51 & 17.52 & 1.06 & 17.42 & 1.06 \\
\hline 93 & 20.52 & 18.72 & 1.10 & 18.37 & 1.12 \\
\hline 94 & 13.54 & 14.11 & 0.96 & 15.55 & 0.87 \\
\hline 95 & 18.18 & 17.90 & 1.02 & 17.26 & 1.05 \\
\hline 96 & 20.13 & 20.17 & 1.00 & 20.18 & 1.00 \\
\hline 97 & 25.12 & 22.28 & 1.13 & 21.86 & 1.15 \\
\hline 98 & 14.80 & 15.50 & 0.95 & 14.70 & 1.01 \\
\hline 99 & 19.53 & 21.85 & 0.89 & 22.32 & 0.87 \\
\hline 100 & 21.30 & 23.88 & 0.89 & 24.36 & 0.87 \\
\hline 101 & 21.28 & 23.88 & 0.89 & 24.36 & 0.87 \\
\hline 102 & 17.84 & 16.10 & 1.11 & 13.95 & 1.28 \\
\hline 103 & 16.40 & 16.10 & 1.02 & 13.95 & 1.18 \\
\hline 104 & 14.16 & 18.23 & 0.78 & 17.14 & 0.83 \\
\hline
\end{tabular}

Continued on next page

Part II: Masonry Compression Strength Modeling 
Table 6.2 Continued from Previous Page

\begin{tabular}{|c|c|c|c|c|c|}
\hline \multirow[b]{2}{*}{ No. ${ }^{b}$} & \multirow[b]{2}{*}{$\begin{array}{l}f_{m, r e p}^{\prime} \\
(\mathrm{MPa})^{\mathrm{c}}\end{array}$} & \multicolumn{2}{|c|}{$\begin{array}{l}\text { Based Material } \\
\text { Properties }\end{array}$} & \multicolumn{2}{|c|}{$\begin{array}{l}\text { Based on Simplifying } \\
\text { Assumptions }^{\mathrm{a}}\end{array}$} \\
\hline & & $\begin{array}{l}f_{m, p r e d}^{\prime} \\
(\mathrm{MPa})^{\mathrm{c}}\end{array}$ & $\begin{array}{l}f_{m, \text { rep }}^{\prime} / \\
f_{m, \text { pred }}^{\prime}\end{array}$ & $\begin{array}{l}f_{m, p r e d}^{\prime} \\
(\mathrm{MPa})^{\mathrm{c}}\end{array}$ & $\begin{array}{l}f_{m, \text { rep }}^{\prime} / \\
f_{m, \text { pred }}^{\prime}\end{array}$ \\
\hline 105 & 15.42 & 18.23 & 0.85 & 17.14 & 0.90 \\
\hline 106 & 7.85 & 10.39 & 0.76 & 9.38 & 0.84 \\
\hline 107 & 8.14 & 10.39 & 0.78 & 9.38 & 0.87 \\
\hline 108 & 8.12 & 10.39 & 0.78 & 9.38 & 0.87 \\
\hline 109 & 8.27 & 10.39 & 0.80 & 9.38 & 0.88 \\
\hline 110 & 14.80 & 19.58 & 0.76 & 17.38 & 0.85 \\
\hline 111 & 14.80 & 18.19 & 0.81 & 15.72 & 0.94 \\
\hline 112 & 17.50 & 17.54 & 1.00 & 18.96 & 0.92 \\
\hline 113 & 18.00 & 17.97 & 1.00 & 19.30 & 0.93 \\
\hline 114 & 17.90 & 18.12 & 0.99 & 19.28 & 0.93 \\
\hline 115 & 20.00 & 17.92 & 1.12 & 19.13 & 1.05 \\
\hline 116 & 19.10 & 17.99 & 1.06 & 19.18 & 1.00 \\
\hline 117 & 17.30 & 18.93 & 0.91 & 20.04 & 0.86 \\
\hline 118 & 18.50 & 17.53 & 1.06 & 18.83 & 0.98 \\
\hline 119 & 19.50 & 17.92 & 1.09 & 19.13 & 1.02 \\
\hline 120 & 18.90 & 19.95 & 0.95 & 17.69 & 1.07 \\
\hline \multirow[t]{5}{*}{121} & 18.90 & 20.46 & 0.92 & 18.02 & 1.05 \\
\hline & & Average & 1.00 & Average & 1.02 \\
\hline & & STDV & 0.13 & STDV & 0.17 \\
\hline & & $\mathrm{COV}$ & $12.54 \%$ & $\mathrm{COV}$ & $16.54 \%$ \\
\hline & & & & & Concluded \\
\hline \multicolumn{6}{|l|}{ Notes: } \\
\hline \multicolumn{6}{|c|}{$\begin{array}{l}\text { a Based on } \sqrt{\nu^{b l} / \nu^{g r}}=0.75, \epsilon_{o r \text { rep }}^{b l}=0.0022 \\
\text { and } \epsilon_{o, \text { rep }}^{g r}=0.0011\left(k_{1}^{g r} k_{2}^{g r} f_{c, \text { rep }}^{\prime}\right)^{\text {gr }}\end{array}$} \\
\hline \multicolumn{6}{|c|}{${ }^{b}$ For Input Values the Number Corresponds to the Number } \\
\hline
\end{tabular}

Part II: Masonry Compression Strength Modeling 


\subsection{Design Equations}

When used for design purposes, SSACM needs to be associated with a margin of safety through setting a confidence lower limit. Assuming the $f_{m, \text { exp }}^{\prime} / f_{m, p r e d}^{\prime}$ ratios are normally distributed, the $95 \%$ confidence lower limit can be calculated by subtracting $1.96 \sigma$ from the arithmetic mean, where $\sigma$ is the standard deviation. For example, consider the SSACM for the full bedded ungrouted prisms. The lower confidence limit for an average of 1.01 and a standard deviation of 0.08 is 0.85 ; thus, with this limit we can be $95 \%$ confident that the $f_{m, \text { exp }}^{\prime} / f_{m, p r e d}^{\prime}$ ratio will be equal to or greater than 1.0. Applying the same idea to the face shell bedded ungrouted prisms and grouted prisms results in a lower confidence limit of 0.77 and 0.67 respectably. Thus, the proposed SSACM formulas can be rewritten as the Design Smeared Area Compression Equations (DSACE) based on minimum net area:

$$
f_{m}^{\prime}=\left\{\begin{array}{ll}
0.23 \frac{t^{b l} \rho_{\text {solid }}^{b l} k_{1}^{b l} k_{2}^{b l} f_{c, r e p}^{\prime}, b l}{t^{f s}} & \text { if ungrouted face shell bedded mortar } \\
\min \left(\frac{h^{b l}-t^{m r}}{h^{b l}}, 0.9\right) 0.85 k_{1}^{b l} k_{2}^{b l} f_{c, r e p}^{\prime}, b l & \text { if ungrouted full bedded mortar } \\
0.50\left[f^{b l} \rho_{\text {solid }}^{b l}+f^{g r}\left(1-\rho_{\text {solid }}^{b l}\right)\right] & \text { if grouted }
\end{array} .\right.
$$

\subsection{Comparison to Current International Codes}

\subsubsection{Canadian Masonry Code: CSA S304-14}

The current Canadian masonry design standard CSA S304-14 (Canadian Standards Association, 2014a) prescribes strengths using the unit strength method through a table format, based on block strength and mortar type. This table (Table 6.3) has been modified from past versions, with a decrease on the upper limit of block strengths from 40 $\mathrm{MPa}$ to $30 \mathrm{MPa}$. Currently, the values listed are based on research that was completed in the 1970s and 1980s (Gayed et al., 2012; Maurenbrecher, 1986) and were developed from a linear regression analysis between average concrete block compressive strength values and average prism compressive strength values. A $20 \%$ reduction was then applied to obtain the values listed in CSA S304-14 (Canadian Standards Association, 2014a; Gayed et al., 2012). 
Table 6.3: CSA304-14 Unit Strength Values

\begin{tabular}{|c|c|c|c|c|}
\hline \multirow{2}{*}{$\begin{array}{l}\text { Net Area } \\
\text { Specified } \\
\text { Compressive } \\
\text { Strength of } \\
\text { Block }(\mathrm{MPa})\end{array}$} & \multicolumn{2}{|c|}{ Type S Mortar } & \multicolumn{2}{|c|}{ Type N Mortar } \\
\hline & $\begin{array}{l}\text { Ungrouted } \\
\text { Hollow Blocks } \\
(\mathrm{MPa})\end{array}$ & $\begin{array}{l}\text { Solid or } \\
\text { Grouted } \\
\text { Hollow Blocks } \\
(\mathrm{MPa})\end{array}$ & $\begin{array}{l}\text { Ungrouted } \\
\text { Hollow Blocks } \\
(\mathrm{MPa})\end{array}$ & $\begin{array}{l}\text { Solid or } \\
\text { Grouted } \\
\text { Hollow Blocks } \\
(\mathrm{MPa})\end{array}$ \\
\hline 30 or more & 17.5 & 13.5 & 12.0 & 9.0 \\
\hline 20 & 13.0 & 10.0 & 10.0 & 7.5 \\
\hline 15 & 10.0 & 7.5 & 8.0 & 6.0 \\
\hline 10 & 6.5 & 5.0 & 6.0 & 4.5 \\
\hline
\end{tabular}

\subsubsection{United States Masonry Code: 2013 MSJC}

Prior to the 2013 MSJC code (The Masonry Society, 2013), masonry compression strength values were developed using prism test results collected from the 1950s through the 1980s (The Masonry Society, 2013). These compression strengths had high variability, which caused design values to be conservative. In 2010, the National Concrete Masonry Association (National Concrete Masonry Association, 2012; The Masonry Society, 2013) began research that would permit the development of a new unit strength table for hollow concrete masonry. Based on this research, a new unit strength table was included in the 2013 code, as presented in Table 6.4.

Table 6.4: 2013 MSJC Unit Strength Values

\begin{tabular}{|c|c|c|}
\hline \multirow{2}{*}{$\begin{array}{l}\text { Net Area Compressive } \\
\text { Strength of Concrete } \\
\text { Masonry (MPa) }\end{array}$} & \multicolumn{2}{|c|}{$\begin{array}{l}\text { Net Area Compressive Strength of } \\
\text { Concrete Masonry Blocks (MPa) }\end{array}$} \\
\hline & Type M or S Mortar & $\begin{array}{l}\text { Type N Mor- } \\
\text { tar }\end{array}$ \\
\hline 11.72 & - & 13.10 \\
\hline 13.10 & 13.10 & 14.84 \\
\hline 13.79 & 13.79 & 18.27 \\
\hline 15.51 & 17.93 & 23.44 \\
\hline 17.24 & 22.41 & 28.96 \\
\hline 18.96 & 26.89 & - \\
\hline 20.69 & 31.03 & - \\
\hline
\end{tabular}

Part II: Masonry Compression Strength Modeling 


\subsubsection{Australian Masonry Code: AS3700-2011}

The Australian standard AS3700-2011 (Australian Standard, 2011) provides an equation to determine masonry compressive strength from the block strength modified by the block height to mortar thickness ratio. For ungrouted masonry the characteristic compressive strength of hollow concrete blocks, is:

$$
f_{m}^{\prime}=k_{h} f_{m b}^{\prime}
$$

where $k_{h}$ is a joint thickness factor equal to:

$$
k_{h}=\min \left(1.3\left(\frac{h^{b l}}{19 t^{m r}}\right)^{0.29}, 1.3\right)
$$

and $f_{m b}^{\prime}$ is the characteristic compressive strength of the masonry defined as:

$$
f_{m b}^{\prime}=k_{m} \sqrt{f_{u c}^{\prime}}
$$

where $k_{m}$ is a compression strength factor equal to 1.4 or 1.6 for full beding mortar and face shell bedding mortar respectively and $f_{u c}^{\prime}$ is the characteristic unconfined compressive strength of the block.

For grouted masonry, the compressive strength is:

$$
f_{m}^{\prime}=f_{u g}^{\prime} \rho_{\text {solid }}^{b l}+k_{g} \sqrt{\frac{f_{c g}^{\prime}}{1.3}}\left(1-\rho_{\text {solid }}^{b l}\right)
$$

where $f_{u g}^{\prime}$ is the ungrouted compression strength determined in Equation 6.13, $k_{g}$ is a strength factor for grout in compression equal to 1.4 for hollow concrete masonry blocks of density greater than $2000 \mathrm{~kg} / \mathrm{m}^{3}$, and $f_{c g}^{\prime}$ is the design characteristic compressive strength of grout equal to the lesser of the cylinder compression strength of grout or $1.3 f_{u g}^{\prime}$. 


\subsubsection{United Kingdom Masonry Code: Eurocode 6}

The United Kingdom Eurocode 6 code (British Standards Institution, 2006) suggests using the following formula to determine the compressive strength of masonry:

$$
f_{m}^{\prime}=K f_{b l, n o r}^{\alpha} f_{m r, p a r t s}^{\beta}
$$

where $K, \alpha$, and $\beta$ are constants given in the national Annex for a particular country, $f_{m r, p a r t s}$ is the compression strength of the mortar in accordance with BS EN1015-11 (British Standards Institution, 1999), and $f_{b l, n o r}$ is the normalised mean compressive strength of the blocks, defined as:

$$
f_{b l, n o r}=k_{c} \delta f_{b l}^{\prime}
$$

were $k_{c}$ is a conditioning factor equal to 1.0 for air-dried blocks, $\delta$ is a shape factor to account for block thickness and height from EN 772-1 (British Standards Institution, 2011), and $f_{b l}^{\prime}$ is the declared average compressive strength of the block. In the UK (British Standards Institution, 2007) the values of the constants are 0.52 (for hollow concrete block which have more than $25 \%$ but less than $60 \%$ of formed vertical voids or cavities, which pass completely through the block), 0.7 and 0.3 for $K, \alpha$ and $\beta$ respectively. If the prism is grouted the compression strength is determined with $K=$ 0.55 (Group 1) with $f_{b l, n o r}$ set to the normalised compressive strength of the blocks or of the concrete infill, whichever is the lesser.

The method of measurement for mortar in Eurocode 6 (British Standards Institution, 2006) is done in accordance with BS EN1015-11 (British Standards Institution, 1999), where the compressive strength is determined on the broken parts of a flexural strength specimen. Ferguson (Ferguson, 1995; Sarhat and Sherwood, 2014) demonstrated that on average the strength of such specimens is 1.28 times that of mortar cubes. As such, the $f_{m r, p a r t s}$ values used to predict $f_{m}^{\prime}$ are the mortar cube strength increased by a factor of 1.28 .

\subsubsection{Results}

Table 6.5 summarizes the predictive abilities for the various codes and models in terms of experimental-over-predicted compression strength ratio $\left(f_{m, \text { exp }}^{\prime} / f_{m, \text { pred }}^{\prime}\right)$ for the gathered databases. All reported compression strengths were corrected by each codes height to thickness factors, no correction is needed for the SACM, the SSACM, or the DSACE before the $f_{m, \text { exp }}^{\prime} / f_{m, p r e d}^{\prime}$ ratio is determined. The SACM gives the best average $f_{m, \text { exp }}^{\prime} /$ 
$f_{m, p r e d}^{\prime}$ ratio and the lowest coefficient of variation $(\mathrm{COV})$ for the entire database or any individual subset of the data. However, due to the complex nature of the SACM it is not suited for direct implementation into any design code, but the SSACM produces the second best average $f_{m \text {,exp }}^{\prime} / f_{m \text {,pred }}^{\prime}$ ratio and the second lowest COV for the entire database or any individual subset of the data. The DSACE result in a slight increase in the average $f_{m, \text { exp }}^{\prime} / f_{m, \text { pred }}^{\prime}$ ratio and coefficient of variation, but result in only $2 \%$ of the database having non-conservative predictions. CSA S304-14, AS3700-2011, and UK Eurocode 6 have a high COV and as illustrated in Figure 6.2 they tend to considerable underestimate $f_{m}^{\prime}$. It can also be seen that although the MSJC 2013 exhibited a low average $f_{m, \text { exp }}^{\prime} / f_{m, \text { pred }}^{\prime}$ ratio, it has a high COV and it gives non-conservative predictions $\left(f_{m, \text { exp }}^{\prime} / f_{m, \text { pred }}^{\prime}\right.$ of less than 1.0) for more than $35 \%$ of the entire database. This is because the unit strength table used in MSJC 2013 is derived based on two block high prisms, which tend to overestimate the compression strength due to platen restraint.

Table 6.5: Results of International Masonry Codes, SACM, and Proposed Equations

\begin{tabular}{|c|c|c|c|c|c|c|c|c|c|c|c|c|c|c|c|}
\hline \multirow{3}{*}{ Provision } & \multirow{2}{*}{\multicolumn{3}{|c|}{$\begin{array}{l}\text { Entire } \\
\text { Database }\end{array}$}} & \multicolumn{9}{|c|}{ Ungrouted Prisms } & \multirow{2}{*}{\multicolumn{3}{|c|}{$\begin{array}{l}\text { All } \\
\text { - Grouted } \\
\text { Prisms }\end{array}$}} \\
\hline & & & & \multicolumn{3}{|c|}{$\begin{array}{l}\text { All Un- } \\
\text { grouted } \\
\text { Prisms }\end{array}$} & \multicolumn{3}{|c|}{$\begin{array}{l}\text { Face Shell } \\
\text { Bedded } \\
\text { Prisms }\end{array}$} & \multicolumn{3}{|c|}{$\begin{array}{l}\text { Full } \\
\text { Bedded } \\
\text { Prisms }\end{array}$} & & & \\
\hline & 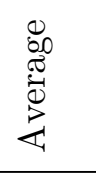 & 尝 & $\begin{array}{l}a \\
0 \\
0 \\
0\end{array}$ & 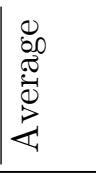 & 尝 & $\begin{array}{l}3 \\
8 \\
8 \\
0\end{array}$ & 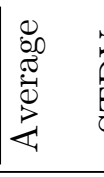 & 点 & $\begin{array}{l}0 \\
0 \\
2 \\
0 \\
0\end{array}$ & 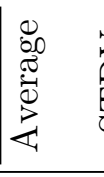 & 党 & $\begin{array}{l}20 \\
2 \\
0 \\
0\end{array}$ & 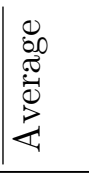 & 盆 & $\begin{array}{l}20 \\
0 \\
0\end{array}$ \\
\hline CSA S304-14 & 1.42 & 0.41 & 29 & 1.35 & 0.25 & 18 & 1.39 & 0.26 & 19 & 1.31 & 0.22 & 17 & 1.47 & 0.51 & 35 \\
\hline 2013 MSJC & 1.08 & 0.27 & 25 & 1.12 & 0.23 & 21 & 1.16 & 0.27 & 23 & 1.09 & 0.20 & 18 & 1.03 & 0.28 & 28 \\
\hline AS 3700-2011 & 1.79 & 0.42 & 23 & 1.74 & 0.41 & 23 & 1.71 & 0.44 & 26 & 1.77 & 0.37 & 21 & 1.84 & 0.41 & 22 \\
\hline UK Eurocode 6 & 1.50 & 0.42 & 28 & 1.37 & 0.21 & 16 & 1.41 & 0.26 & 18 & 1.34 & 0.16 & 12 & 1.59 & 0.53 & 33 \\
\hline SACM & 1.00 & 0.05 & 5 & 1.01 & 0.05 & 5 & 1.00 & 0.04 & 4 & 1.01 & 0.05 & 5 & 1.00 & 0.05 & 5 \\
\hline $\mathrm{SSACM}^{\mathrm{a}}$ & 1.01 & 0.11 & 11 & 1.01 & 0.10 & 10 & 1.01 & 0.12 & 11 & 1.01 & 0.08 & 8 & 1.00 & 0.13 & 13 \\
\hline DSACE & 1.38 & 0.25 & 18 & 1.24 & 0.13 & 11 & 1.30 & 0.15 & 11 & 1.18 & 0.09 & 8 & 1.53 & 0.27 & 17 \\
\hline
\end{tabular}

It is worth while to point out that all of the code methods are based on curve fits to experimental data. The SACM, SSACM, and the DSACE are based solely on fundamental application of mechanics of materials. As such, strength predictions generated by these models and equations are true predictions.

Part II: Masonry Compression Strength Modeling 


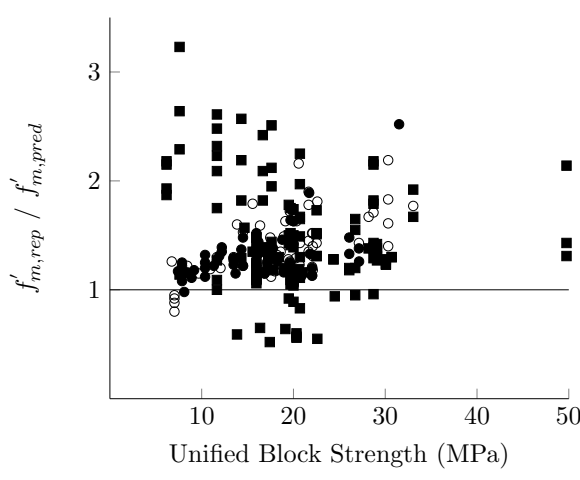

(a) CSA S304-14

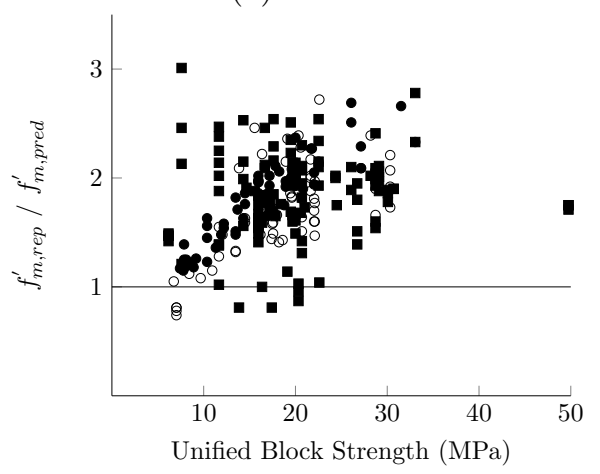

(c) AS 3700-2011

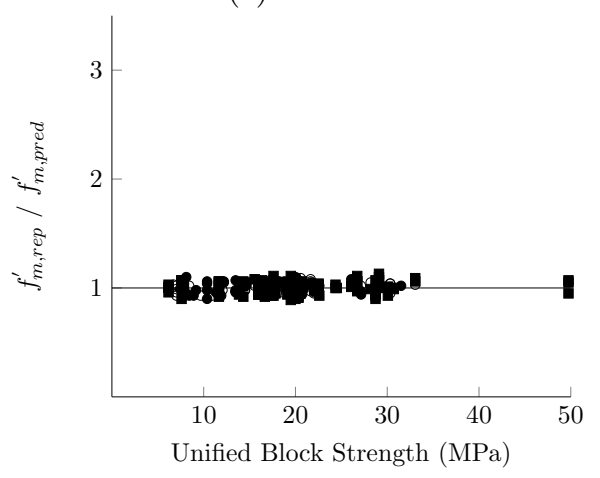

(e) SACM

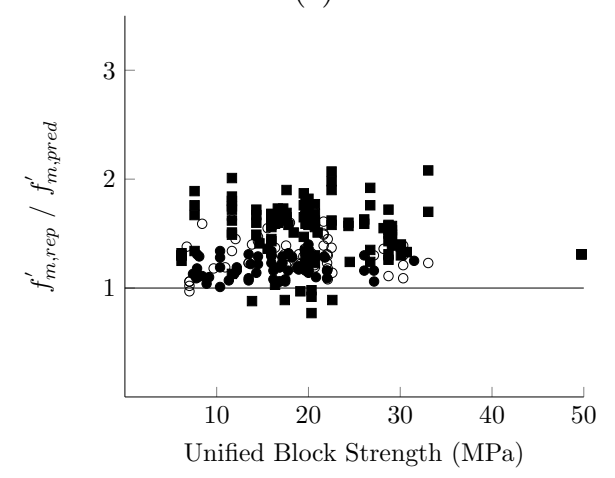

(g) DSACE

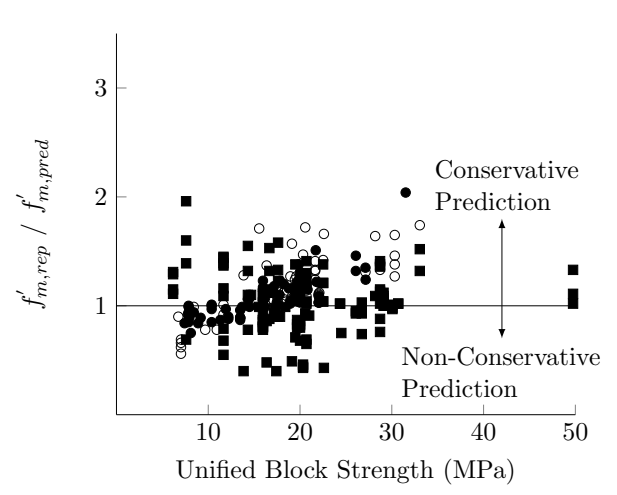

(b) 2013 MSJC

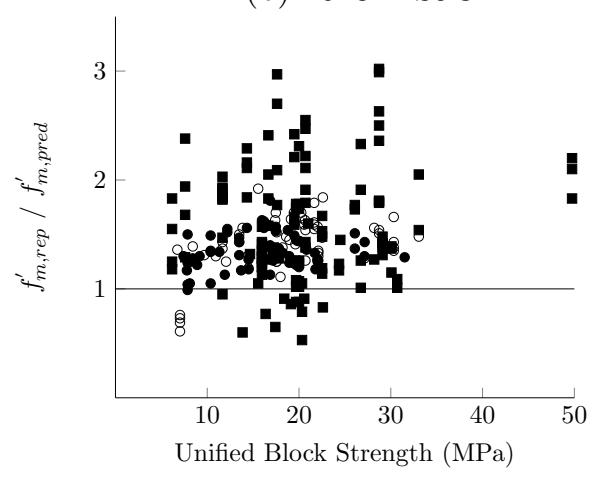

(d) UK Eurocode 6

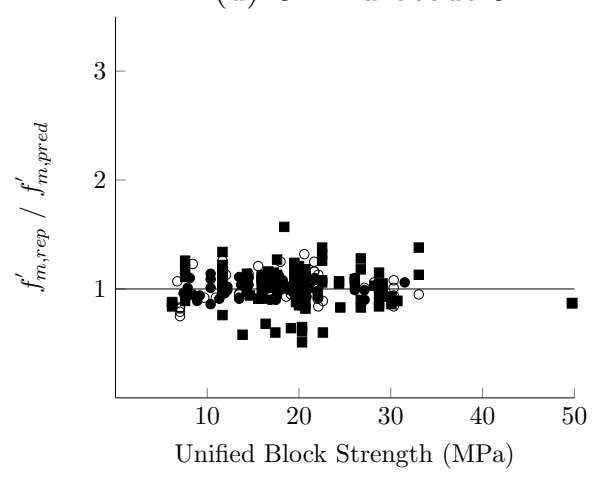

(f) SSACM

Figure 6.2: Comparison of Predictive Ability for the International Masonry Codes, SACM, and Proposed Formula for the Entire Database

Part II: Masonry Compression Strength Modeling 


\section{COMPRESSION PARALLEL TO BED JOINTS}

In masonry beams the compression force above the neutral axis acts parallel to the mortar bed joints; thus, determining the compression strength in this direction is important to fully understanding hollow concrete block masonry mechanics in compression. This chapter takes the model presented in Chapter 3 and extends the theory to compression parallel to the mortar bed joints. The results of this extension are compared to experimental results found in literature.

In a masonry beam, the compressive force acts parallel to the mortar bed joint. However, there is limited research on this loading direction in compression. Additionally, there is no standard experimental procedure to determine the compressive strength of masonry parallel to the bed joint. As masonry is an anisotropic material the compression strength of masonry parallel to the bed joint is an importatnt material property that needs to be considered.

\subsection{Background}

The first experimental work that was undertaken to look specifically into the effect that load orientation (parallel versus normal to the bed joint) had on prism strength was conducted by Lee et al. (1984). This investigation used $190 \mathrm{~mm} \times 190 \mathrm{~mm} \times 390 \mathrm{~mm}$ standard stretcher blocks, two types of mortar (average compressive strength of $10 \mathrm{MPa}$ and $23 \mathrm{MPa}$ ), and two types of coarse grout (average compressive strength of $6.9 \mathrm{MPa}$ and 24.6 MPa). A prism width of $590 \mathrm{~mm}$ and thickness of $190 \mathrm{~mm}$ were kept constant in all the specimens. Three different prism heights were used (see Figure 7.1): $390 \mathrm{~mm}$ (height to thickness ratio of 2:1), $590 \mathrm{~mm}$ (height to thickness ratio of 3:1), and $790 \mathrm{~mm}$ (height to thickness ratio of 4:1). There were a total of nine prisms (three at each height to thickness ratio) loaded parallel to the bed joint with unfilled frogs on the blocks. In addition, there were three prisms loaded normal to the bed joint for each mortar and grout combination. This study indicated that the loading direction did have a large effect on compression strength. The prisms loaded parallel to the bed joint resulted in a decrease in strength of 55 to $66 \%$ for ungrouted and 50 to $55 \%$ for grouted specimens. As the mortar strength was increase from $10 \mathrm{MPa}$ to $23 \mathrm{MPa}$ the compression strength 


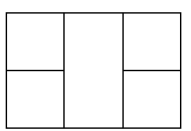

(a) $h / t=2: 1$

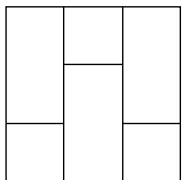

(b) $\mathrm{h} / \mathrm{t}=3: 1$

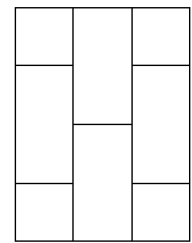

(c) $h / t=4: 1$

Figure 7.1: Prism Configurations Used by Lee et al. (1984)

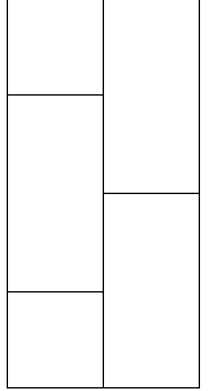

(a) Compression Parallel to Bed Joints

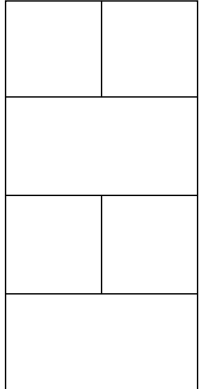

(b) Compression Normal to Bed Joints

Figure 7.2: Prism Configurations Used by Wong and Drysdale (1985)

inceased by 15 to $50 \%$. This study also found that a large increase in grout strength only resulted in a small increase in prism compressive strength.

Wong and Drysdale (1985) studied the effect of loading direction on prism compression strength using prisms of $390 \mathrm{~mm}$ wide $\times 190 \mathrm{~mm}$ thick $\times 790 \mathrm{~mm}$ high, see Figure $7.2 \mathrm{a}$, for compression strength parallel to the bed joints and prisms that were $2,3,4$, or 5 courses in height in running bond, see Figure 7.2b, for compression strength normal to the bed joint. This study found that compressive strength for both hollow and grouted prisms compressed parallel to the bed joint was approximately $25 \%$ lower than that compressed normal to the bed joint. It was also observed that the grouted prisms exhibited about a $35 \%$ reduction in strength compared to that of the hollow prisms when loaded in both normal and parallel to the bed joint.

Khalaf (1997) studied the difference in compression strength produced from loading normal to the bed joint versus parallel to the bed joint using both hollow and grouted prisms with varying grout and mortar strengths. Prisms for compression in parallel were constructed from two blocks stacked on end (see Figure 7.3a), while prisms of three courses were used for compression normal to the bed joint. Hollow two-block prisms loaded parallel to the bed joint were found to be $23 \%$ stronger than hollow threeblock prisms loaded normal to the bed joint. The potential explanation given for this 


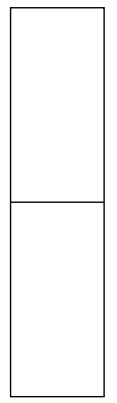

(a) Compression Parallel to Bed Joints

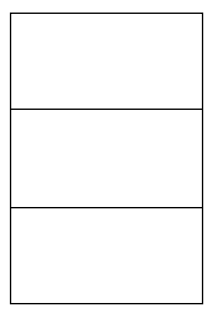

(b) Compression Normal to Bed Joints

Figure 7.3: Prism Configurations Used by Khalaf (1997)

was that the prisms loaded normal to the bed joint had a much larger net area which gives a higher statistical chance for failure. This is in disagreement with previous work (Lee et al., 1984; Wong and Drysdale, 1985) where the hollow prisms loaded parallel to the bed joint were found to be weaker. When the block cells were grouted there was a reduction in strength for those specimens loaded parallel to the bed joint of 16 to $42 \%$. The largest difference occurred at the highest grout strength. Khalaf (1997) stated this could be because the grout acts as a short column for loading normal to the bed joint, but the block webs interrupt this grout column when loaded parallel to the bed joint. The mortar strength was found to have little effect on the compression strength for compression parallel and normal to the bed joint. For both the loading parallel and normal to the bed joint it has found that a large increase in grout strength did not result in a large increase in prism strength.

Ring (2009) studied the effect of compression direction in addition to the effect the web interruption has on compression strength parallel to the bed joints. For compression parallel to the bed joints prisms were $390 \mathrm{~mm}$ wide $\times 190 \mathrm{~mm}$ thick $\times 790 \mathrm{~mm}$ high (see Figure 7.4a) and had 38\%, 68\% and 100\% percent of the area interrupted by webs. Prisms constructed for compression normal to the bed joints were four courses high and in running bond. Hollow prisms were found to have a decrease of $19 \%$ in strength when loaded parallel to the bed joint versus normal to the bed joint. For grouted prisms, an increase in strength of $26 \%$ was found in prisms loaded parallel to the bed joint when compared to that loading normal to bed joints. Ungrouted prisms loaded normal to the bed joint were determined to be $35 \%$ stronger then grouted prisms, based on minimum net area. This study also found that as the amount of web interruption increased, the average compressive strength decreased for loading parallel to the bed joints (see Figure 7.5). 


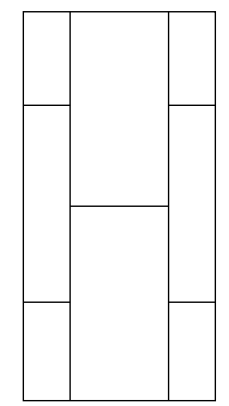

(a) Compression Parallel to Bed Joints

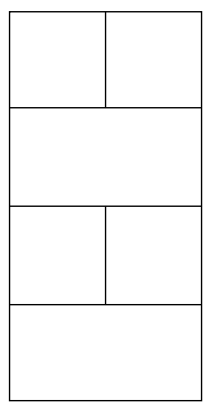

(b) Compression Normal to Bed Joints

Figure 7.4: Prism Configurations Used by Ring (2009)

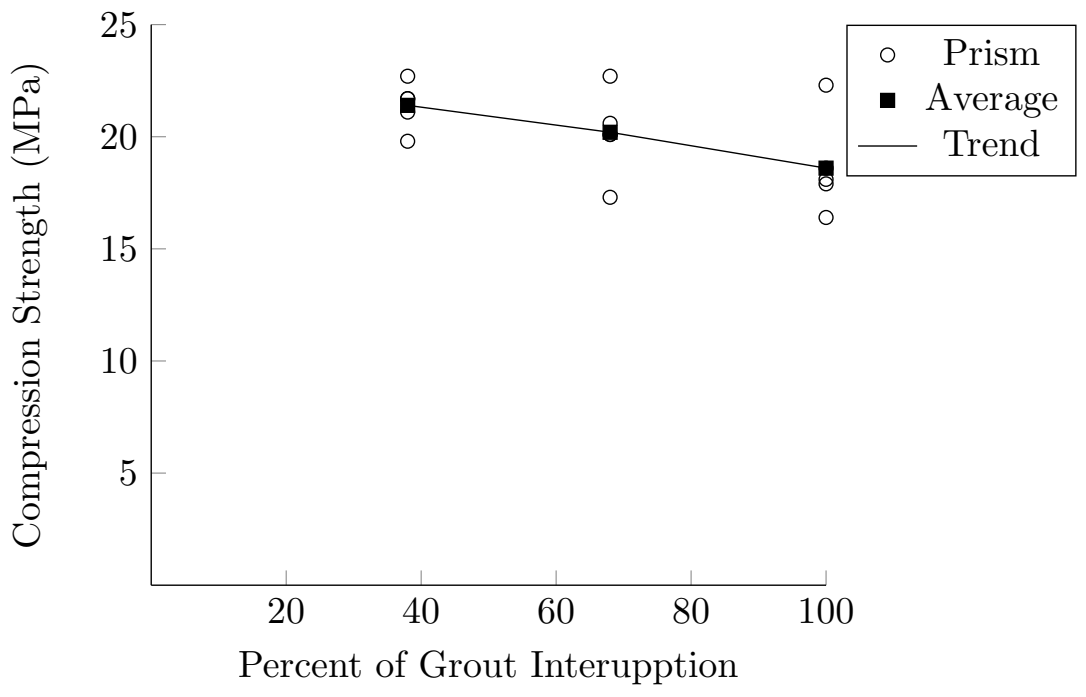

Figure 7.5: Web Interruption Effect of Compression Strength Parallel to Bed Joints (Ring, 2009)

Sarhat (2016) constructed grouted prisms with depressed webs in the same configuration as Ring (2009) and found that compressive strengths parallel to the bed joints ranged from 84 to $113 \%$ of the compressive strength normal to bed joints with an average of 98\%, see Figure 7.6.

The Canadian masonry standard, CSA S304-14, (Canadian Standards Association, 2014a) recommends a relationship between compressive strength parallel to the bed joint with the strength normal to the bed joint $\left(f_{m}^{\prime}\right)$. The compressive strength parallel to the bed joint is found by multiplying the compressive strength normal to the bed joint by a reduction factor $\chi$. This factor is taken as equal to 0.5 where compressive forces are applied normal to the head joint and the grout is not horizontally continuous in the zone of compression. The reduction factor increases to 0.7 for compressive forces applied normal to the head joint and the grout is continuous horizontally in the compression zone. The 


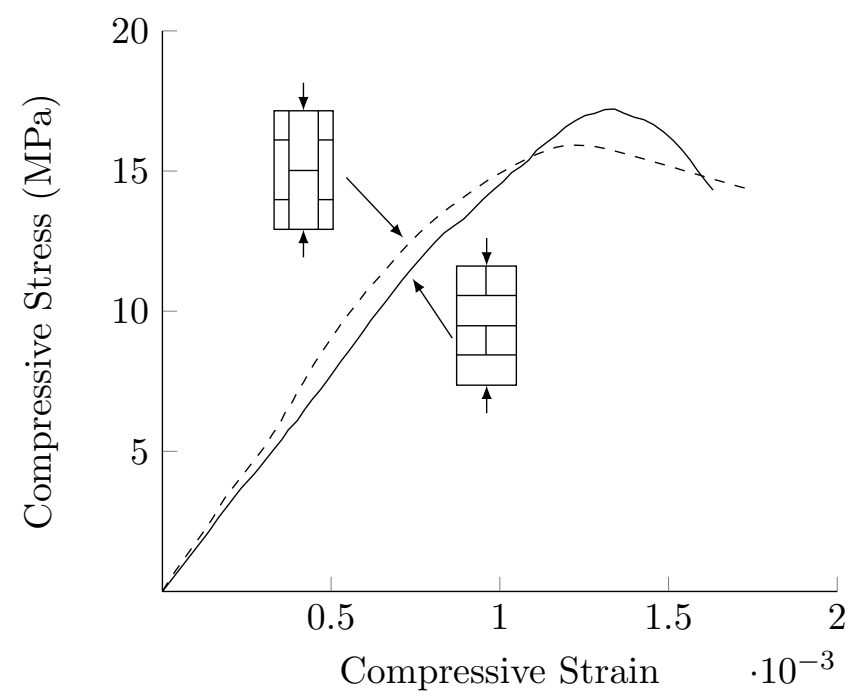

Figure 7.6: Typical Stress-Strain Curves of the Masonry Prisms Tested Normal and Parallel to the Bed Joint Grouted Predictions (Sarhat, 2016)

issue of grout continuity refers to the interruption by the web of the blocks. Standard blocks have the web in place resulting in a $\chi$ value of 0.5 . When using knockout blocks the webs may be removed completely, resulting in a $\chi$ value of 0.7 . A lintel block may also have a $\chi$ value of 0.7 . This is often argued to be a very conservative measure (Ring, 2009).

In both the Australian masonry standard (Australian Standard, 2011) and the MSJC (The Masonry Society, 2013) the direction of the force in relation to the orientation of the block in not considered. However, in UK Eurocode 6 (British Standards Institution, 2006) when calculating the compressive strength of the masonry in the compression zone of a beam, the orientation of the block is mentioned. UK Eurocode 6 (British Standards Institution, 2006) states that a block must be tested in the same manner as the force that will be applied in order to determine the strength. Hence, according to UK Eurocode 6 (British Standards Institution, 2006) the compressive strength parallel to the bed joint is directly related to the strength in that direction. In contrast, Eurocode 6 (British Standards Institution, 2006), the Australian msaony standard AS 3700 (Australian Standard, 2011), and the MSJC (The Masonry Society, 2013) do not specifically mention the effect of grout interruption on the compressive strength of masonry parallel to the bed joint. They do not consider a difference in strength if the grout is interrupted by the web of a block or not. 


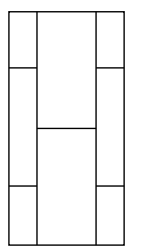

(a) Symmetric

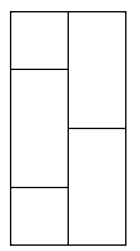

(b) Asymmetric

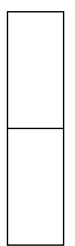

(c) Stacked

Figure 7.7: Prism Configurations

\subsubsection{Mechanics of Compression Parallel to Bed Joints}

As there is no standard experimental procedure to determine the compressive strength of masonry parallel to the bed joint Ring (2009) suggest that the prism configuration could have an impact on the compression strength, see Figure 7.7. Prisms constructed by Ring (2009) and Sarhat (2016) were symmetric in geometry and mortar joints about the line of load application, whereas prisms constructed by Wong and Drysdale (1985) used an asymmetric construction and in the study completed by Khalaf (1997) prisms were made of two block stack with no bed joints. The symmetry of the Ring (2009) and Sarhat (2016) prisms allows for the head joint of the central blocks to be reinforced on either side by the two blocks. In the asymmetric configuration the head joints are only reinforced on one side and in the stacked blocks no reinforcing is provided. Reinforcing this head joint allows for larger strengths to be estimated.

For both ungrouted and grouted prisms initial cracking occurs along the bed joints between courses and crushing of the head joint mortar. For hollow prisms the final failure is typically caused by the face shells detaching from the webs followed by buckling of the face shells (Ring, 2009; Wong and Drysdale, 1985). For grouted prisms failure is initiated by the growth of cracks between the face shells and grout with final failure occurring by web cracking followed by either buckling of the face shells or shearing of the block face shells (Ring, 2009; Sarhat, 2016; Wong and Drysdale, 1985). The grout cores tend to remain mostly intact, as the block tends to confine the grout (Ring, 2009; Sarhat, 2016). At high strains grout undergoes high lateral expansions due to Poisson's effect. These large lateral expansions create tension in the block shells and compression in the grout. 


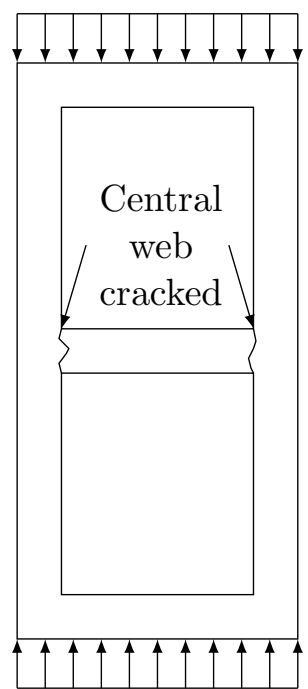

(a) Compression Loading of a Block

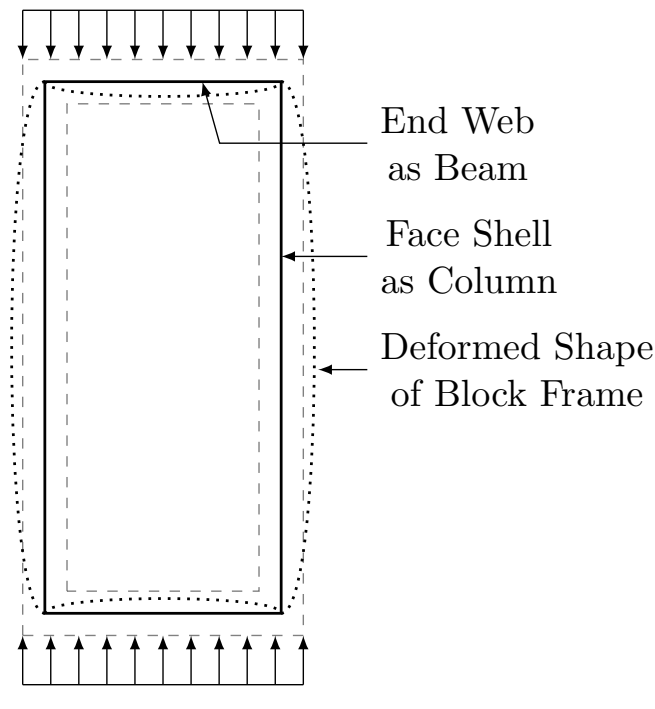

(b) Block Frame System

Figure 7.8: Frame Equivalent System of the Block

\subsection{Conceptual Model}

Khalaf (1997) tested single block specimens in compression oriented parallel to the bed joints. Failure was started by splitting of the central web from the face shells, followed by lateral deformation of the block side shells. Final failure was crushing at the corners between the block end webs and face shells followed by complete disintegration of the specimen. Wong and Drysdale (1985) observed that the final failure of hollow prisms compressed parallel to the bed joints was buckling of the face shells. In consideration of these observed failure modes the block can be viewed as a frame system, in which the webs can be viewed as beams and the face shells as columns. This allows for the face shell to be analyzed using column buckling theory taking into account the different end restraints provided by the end webs as shown in Figure 7.8 .

Buckling is an instability phenomenon in structural systems subjected to compression loads. In columns it is associated with the transition from a straight configuration to a laterally deformed state. The critical load describes the load at which this transition occurs. In order to determine the critical load the equilibrium of a laterally deformed column must be solved. Assuming deflections and rotations are small, the curvature of a member, $\kappa$, can be defined by:

$$
\kappa=\frac{d^{2} v}{d x^{2}}
$$




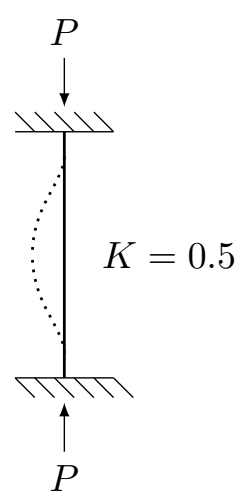

(a) Fixed-Fixed

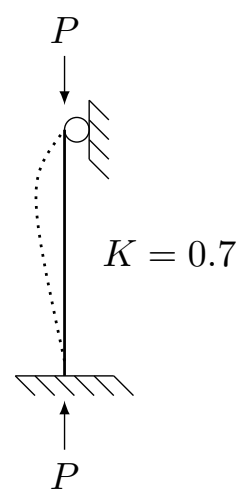

(b) Fixed-Pinned

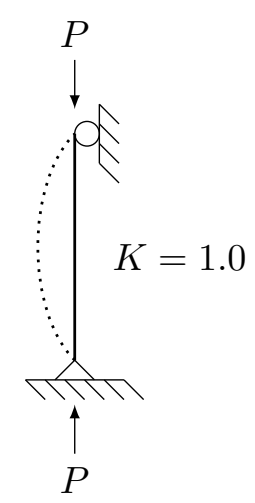

(c) Pinned-Pinned

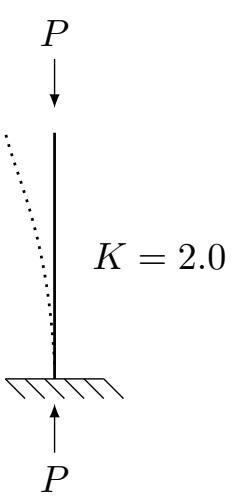

(d) Fixed-Free

Figure 7.9: Effect of End Restraint on Effective Length Factor

where $v$ is the deflection. If the member is perfectly elastic and the material obeys Hookes Law, deflection theory states that the bending moment is proportional to the curvature, with the member's flexural stiffness as the constant of proportionality:

$$
M=-E I \frac{d^{2} v}{d x^{2}}
$$

The solution for the critical buckling load, $P_{c r}$, is:

$$
P_{c r}=\frac{\pi^{2} E I}{(K L)^{2}}
$$

where $L$ is the length of the column and $K$ is the effective length factor, which depends on the end restraints. Theoretical analysis uses idealised end restraints, whose translational and rotational stiffnesses are set to either zero or infinity as shown in Figure 7.9.

More generally for the columns in a sway frame, a frame in which the top of the columns moves relative to the bottom, $K$ can range from one to infinity (see Figure 7.10). A column in a non-sway frame would have no sideways movement at the top relative to the bottom. The buckling of a non-sway frame would result in a buckled column shape having at least one point of contraflexure between the ends of the member as shown in Figure 7.11.

The above considerations concerning single storey frames can be generalized to extend them to frames of more than one story. In most real structures, the rotational and translational stiffness of the end restraints is somewhere between rigid and free. In the case of partial end-restraint the effective length factor $K$ can be determined either by a generalized second order rotation method or by using stability functions. The solution to the problem of partial end-restraint is expressed in the form: 


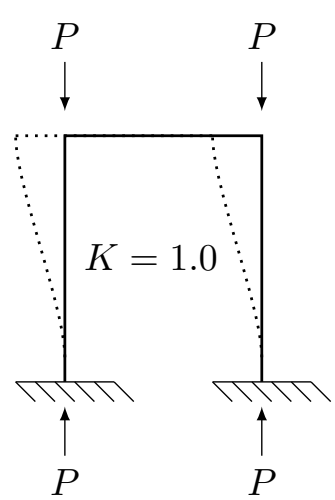

(a) Fixed-Rigid

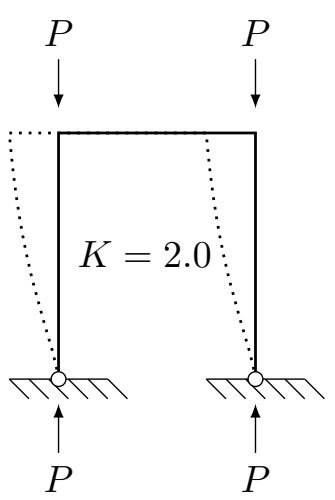

(c) Pinned-Rigid

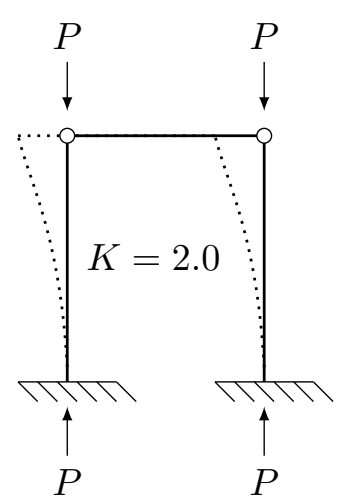

(b) Fixed-Pinned

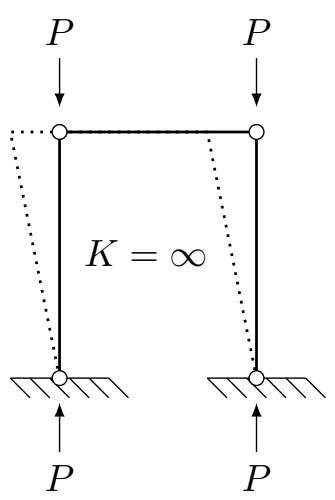

(d) Pinned-Pinned

Figure 7.10: Buckling of a Column in a Sway Frame (For a Stiff Beam Relative to Columns)

$$
K=f\left(\eta_{t}, \eta_{b}\right)
$$

where $\eta_{t}$ and $\eta_{b}$ are elastic restraint coefficients at the top and bottom of the column under consideration. A number of methods have been proposed to evaluate this function including Wood's method (Wood, 1974), Bridge and Fraser (1987), Cheong-Siat-Moy (1991), and Hellesland and Bjorhovde (1996). 


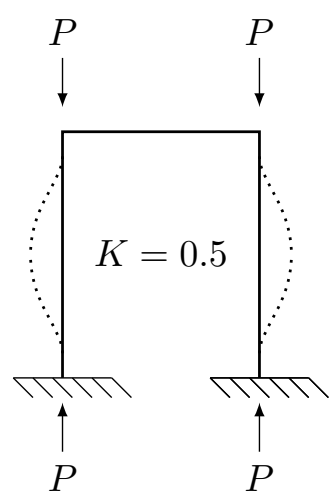

(a) Fixed-Rigid

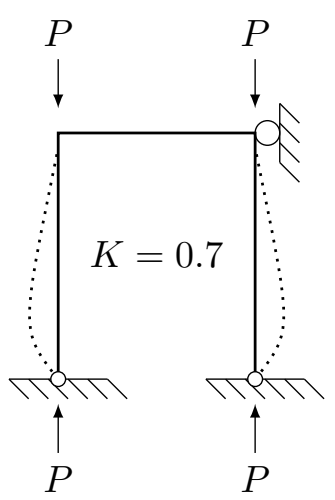

(c) Pinned-Rigid

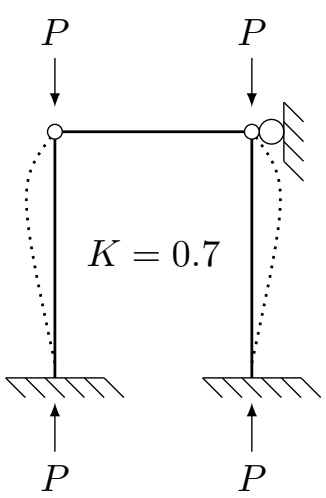

(b) Fixed-Pinned

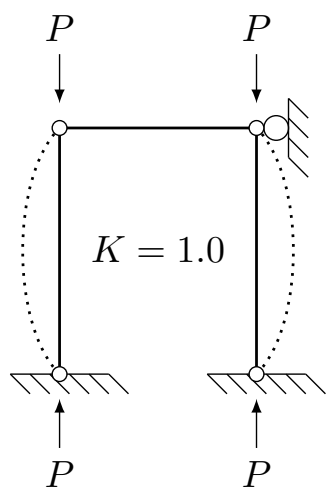

(d) Pinned-Pinned

Figure 7.11: Buckling of a Column in a Non-Sway Frame (For a Stiff Beam Relative to Columns)

\section{Wood's Method}

Wood's method (Wood, 1974) considers a sub-element, for example column EH, in a multistory frame as illustrated in Figure 7.12 or Figure 7.13. The top and bottom elastic restraint coefficients $\eta_{t}$ and $\eta_{b}$ are calculated using the following formulae:

$$
\eta_{t}=\frac{\sum k_{c, t}}{\sum k_{c, t}+\sum k_{b, t}}
$$

and

$$
\eta_{b}=\frac{\sum k_{c, b}}{\sum k_{c, b}+\sum k_{b, b}}
$$

where $\sum k_{c, t}$ is the total column stiffness at the top of the column under consideration, $\sum k_{b, t}$ is the total beam stiffness at the top of the column, $\sum k_{c, b}$ is the total column stiffness at the bottom of the column, and $\sum k_{b, b}$ is the total stiffness due to the beams at the bottom of the column under consideration. Where the beams are not subject to 
Table 7.1: Effective Stiffness of Beam

\begin{tabular}{ll}
\hline Rotational Restraint at Far End of Beam & Beam Stiffness $\left(k_{b, i}\right)$ \\
\hline Fixed & $1.0 I_{b} / L_{b}$ \\
Pinned & $0.75 I_{b} / L_{b}$ \\
Rotation that causes single curvature & $0.50 I_{b} / L_{b}$ \\
Rotation that causes double curvature & $1.50 I_{b} / L_{b}$ \\
\hline
\end{tabular}

axial forces and remain in the elastic region, their effective stiffness's can be determined in accordance with Table 7.1 .

Thus for column EH the elastic restraint coefficients become:

$$
\eta_{t}=\frac{k_{E H}+k_{B E}}{k_{E H}+k_{B E}+k_{D E}+k_{E F}}
$$

and

$$
\eta_{t}=\frac{k_{E H}+k_{H K}}{k_{E H}+k_{H K}+k_{G H}+k_{H I}} .
$$

With the elastic restraint coefficients determined the effective length of the column under consideration can be determined depending on if the system is a non-sway frame or a sway frame. For a non-sway frame:

$$
K=\frac{1+0.145\left(\eta_{t}+\eta_{b}\right)-0.256 \eta_{t} \eta_{b}}{2-0.364\left(\eta_{t}+\eta_{b}\right)-0.247 \eta_{t} \eta_{b}}
$$

and for a sway frame:

$$
K=\sqrt{\frac{1-0.200\left(\eta_{t}+\eta_{b}\right)-0.120 \eta_{t} \eta_{b}}{1-0.800\left(\eta_{t}+\eta_{b}\right)+0.600 \eta_{t} \eta_{b}}}
$$

\subsection{Implementation in the SACM}

Similar to implementation of the SACM for compression normal to the bed joint described in Chapter 3, the SACM can be extended to predict the compression strength parallel to the bed joint with a few modifications. The first modification is to areas and the second modification is to the block compression strength.

Part II: Masonry Compression Strength Modeling 


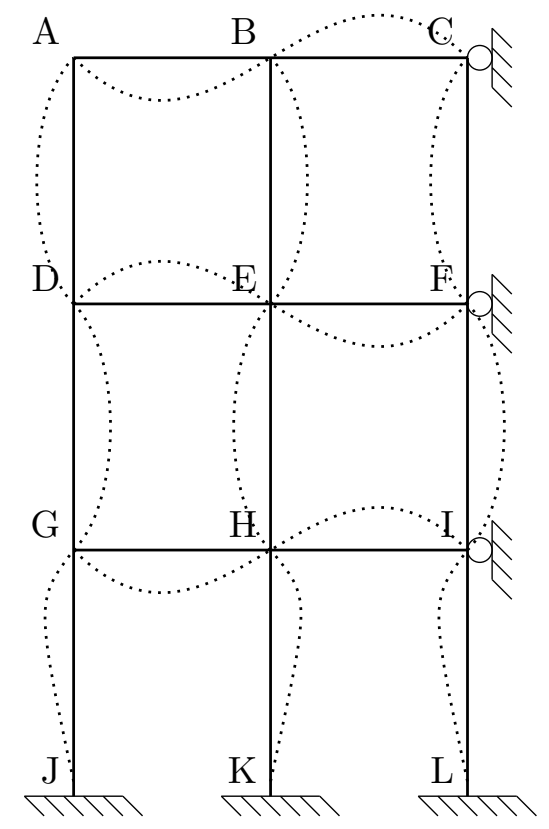

Figure 7.12: Wood's Method for Non-Sway Frame

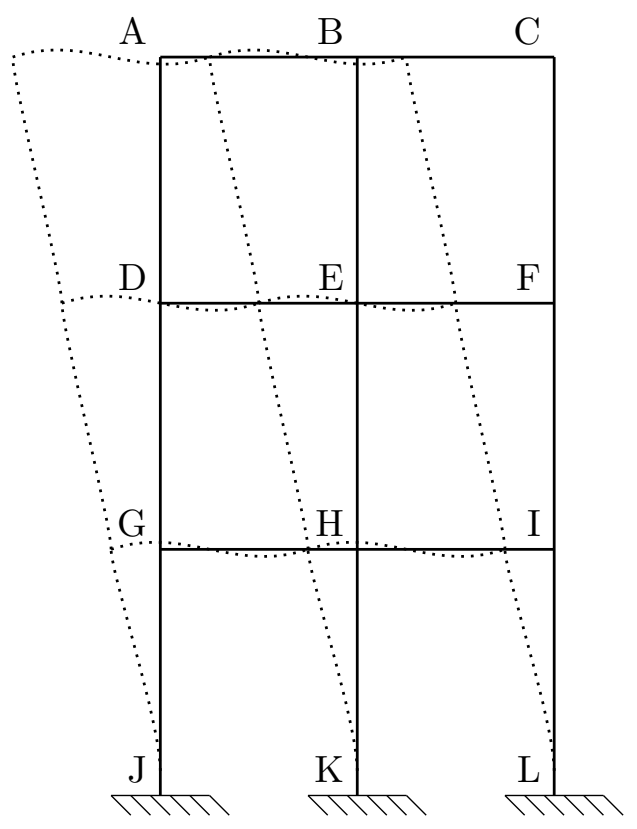

Figure 7.13: Wood's Method for Sway Frame 


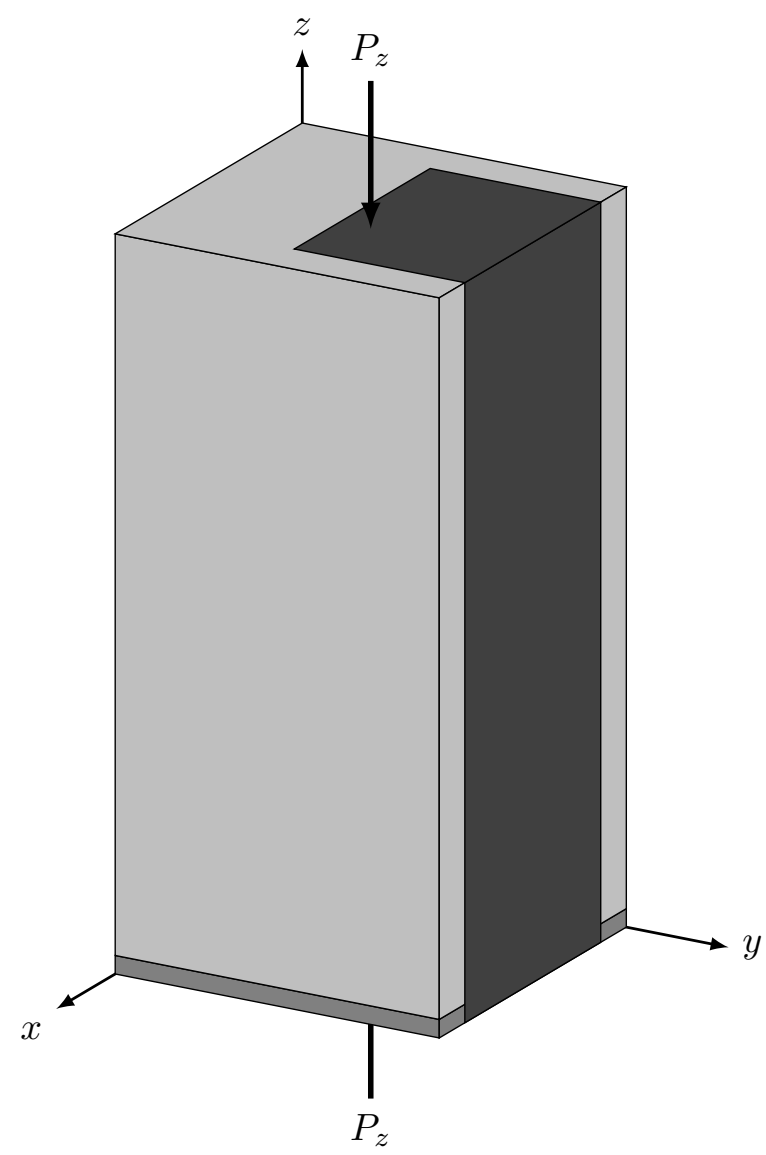

Figure 7.14: Loading Parallel to Bed Joints

\subsubsection{Dimensions and Areas}

Once again to achieve equal strain in each material in each direction all the materials must act in parallel; thus, a modified material configuration in $x$ and $z$ direction must be used.

The areas of each material in the $x$ direction are determined from the following equations:

$$
\begin{gathered}
A_{x}^{b l}=\left(l^{b l}-l^{g r b l}\right) h^{b l} \frac{h^{w e b}}{h^{b l}} ; \\
A_{x}^{m r}=\left\{\begin{array}{ll}
h^{b l} t^{m r} & \text { if full bed mortar or face shell mortar and ungrouted } \\
h^{b l} t^{m r} \frac{h^{w e b}}{h^{b l}} & \text { if face shell mortar and grouted }
\end{array} ;\right. \\
A_{x}^{g r b l}=\left\{\begin{array}{ll}
l^{b l} h^{b l}-A_{x}^{b l} & \text { if grouted } \\
0 & \text { if ungrouted }
\end{array} ;\right. \text { and, } \\
A_{x}^{g r m r}=\left\{\begin{array}{ll}
h^{b l} t^{m r}-A_{x}^{m r} & \text { if grouted } \\
0 & \text { if ungrouted }
\end{array} .\right.
\end{gathered}
$$

Part II: Masonry Compression Strength Modeling 


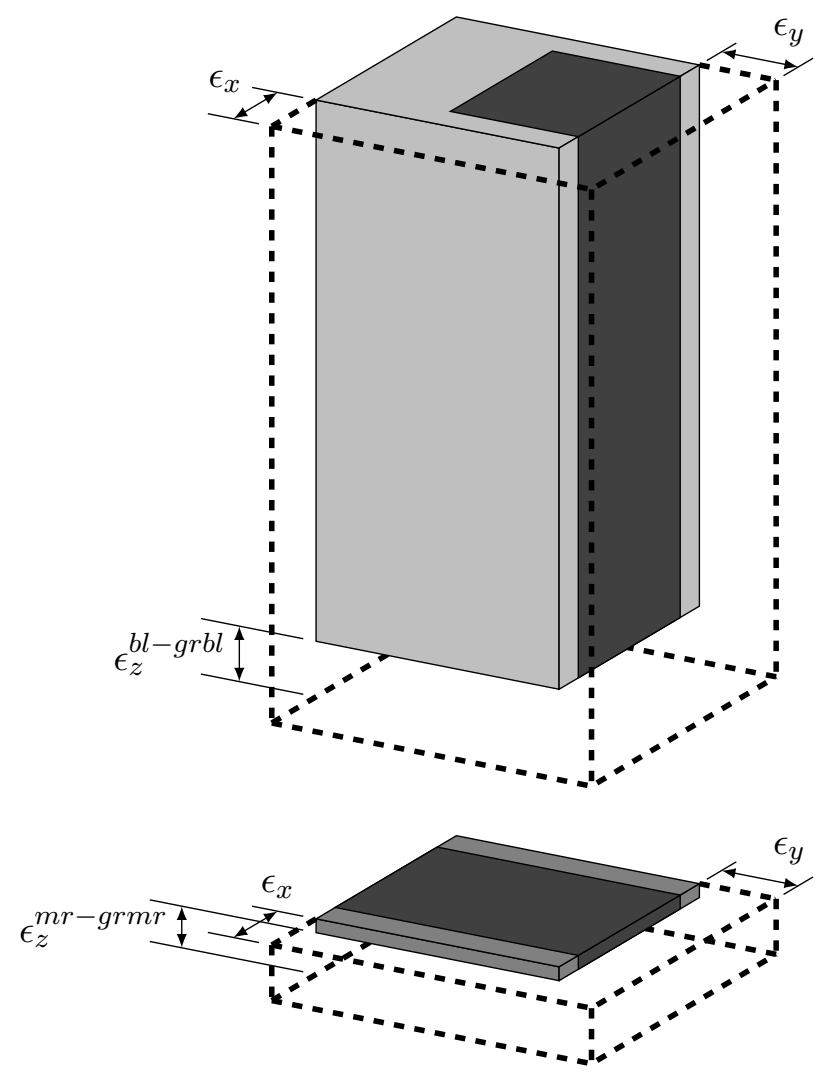

Figure 7.15: Strain Assumptions

The areas of each material in the $y$ direction are determined from the following equations:

$$
\begin{gathered}
A_{y}^{b l}=\rho_{\text {solid }}^{b l} l^{b l} w^{b l} ; \\
A_{y}^{m r}=\left\{\begin{array}{ll}
w^{b l} t^{m r} & \text { if full bed mortar } \\
2 t^{f s} t^{m r} & \text { if face shell mortar }
\end{array} ;\right. \\
A_{y}^{g r b l}=\left\{\begin{array}{ll}
A_{\text {cell }}^{b l} & \text { if grouted } \\
0 & \text { if ungrouted }
\end{array} ; \text { and },\right. \\
A_{y}^{g r m r}= \begin{cases}w^{b l} t^{m r}-A_{y}^{m r} & \text { if grouted } \\
0 & \text { if ungrouted }\end{cases}
\end{gathered}
$$

The areas of each material in the $z$ direction are determined from the following equations:

$$
\begin{gathered}
A_{z}^{b l}=2 t^{f s} h^{b l} ; \\
A_{z}^{m r}=\left\{\begin{array}{ll}
2 t^{f s} h^{b l}+w^{g r b l} h^{w e b} & \text { if full bed mortar } \\
2 t^{f s} h^{b l} & \text { if face shell mortar }
\end{array} ;\right.
\end{gathered}
$$




$$
\begin{aligned}
A_{z}^{g r b l} & =\left\{\begin{array}{ll}
w^{g r b l} h^{b l} & \text { if grouted } \\
0 & \text { if ungrouted }
\end{array} ;\right. \text { and, } \\
A_{z}^{g r m r} & = \begin{cases}w^{b l} h^{b l}-A_{z}^{m r} & \text { if grouted } \\
0 & \text { if ungrouted }\end{cases}
\end{aligned}
$$

The percentage of each material is determined in a similar manner to compression normal to the bed joints.

\subsubsection{Block Compression Strength}

The block compression strength, $f_{c}^{\prime b l}$, is modified according to the block's face shell buckling load, $P_{\text {buck }}^{\text {fs }}$; thus:

$$
f_{c}^{\prime b l}= \begin{cases}\min \left(f_{c, b u c k}^{\prime b l}, 2 k_{1}^{b l} k_{2}^{b l} k_{3}^{b l} f_{c, r e p}^{\prime b l}\right) & \text { if } h^{b l}=h^{w e b} \\ f_{c, b u c k}^{\prime b l} & \text { if } \frac{h^{w e b}}{h^{b l}} \leq 0.62 \\ \min \left(\frac{h^{w e b}}{h^{b l}} f_{c, b u c k}^{\prime b l}, 2 k_{1}^{b l} k_{2}^{b l} k_{3}^{b l} f_{c, r e p}^{\prime b l}\right) & \text { otherwise }\end{cases}
$$

where $k_{3}^{b l}$ is a block loading direction corection factor equal to 1.11 (Khalaf, 1997) and the buckling strength of the face shell, $f_{c, b u c k}^{\prime}$, is determined from:

$$
f_{c, b u c k}^{\prime}=\frac{P_{b u c k}^{f s}}{A^{f s}}
$$

where the area of the face shell, $A^{f s}$, is equal to $t^{f s} h^{b l}$. The critical buckling load of the face shell, $P_{b u c k}^{f s}$, is determined from the Rankine Gordon formula:

$$
P_{b u c k}^{f s}=\frac{P_{e l}^{f s} P_{p l}^{f s}}{P_{e l}^{f s}+P_{p l}^{f s}}
$$

where $P_{e l}^{f s}$ is the elastic buckling load of the face shell and $P_{p l}^{f s}$ is the plastic buckling load of the face shell.

The elastic buckling load is given by:

$$
P_{e l}^{f s}=\frac{\pi^{2} E_{z}^{b l} I^{f s}}{\left(K L^{f s}\right)^{2}}
$$

and the plastic buckling load is determined from:

$$
P_{p l}^{f s}=\frac{\pi^{2} E_{r}^{b l} I^{f s}}{\left(K L^{f s}\right)^{2}}
$$


where $E_{z}^{b l}$ is the initial tangent modulus of elasticity of the block in the $z$ direction, $E_{r}^{b l}$ is the reduced modulus of elasticity, $I^{f s}$ is the second moment of area about the weak axis of the face shell, $K$ is the effective length factor for the face shell based on Wood's method (Wood, 1974), and $L^{f s}$ is the length of the face shell based on the center line dimensions of the external webs.

The second moment of area about the weak axis of the face shell is determined from:

$$
I^{f s}=\frac{h^{b l} t^{f s^{3}}}{12}
$$

and the length of the face shell is:

$$
L^{f s}=l^{b l}-t^{w e b}
$$

The modulus of elasticity of the block in the $z$ direction is determined from user input or the default value. The reduced modulus of elasticity is determined from the reduced modulus theory for a rectangular cross section (Chen and Atsuta, 2008):

$$
E_{r}^{b l}=\frac{4 E_{t}^{b l} E_{z}^{b l}}{\left(\sqrt{E_{t}^{b l}}+\sqrt{E_{z}^{b l}}\right)^{2}} .
$$

The tangent modulus $E_{t}^{b l}$ is given directly from stress-strain curve of the material. For the Hognestad parabola the tangent modulus becomes:

$$
E_{t}^{b l}=2 f_{c, z}^{\prime}, b l\left(\frac{\epsilon_{o, z}^{b l}-\epsilon}{\epsilon_{o, z}^{b l}{ }^{2}}\right) ;
$$

thus, the tangent modulus is dependent on the strain, $\epsilon$, at which it is evaluated at. As the stain increases from zero to $\epsilon_{o, z}^{b l}$ the tangent modulus decreases from $2 f_{c}^{\prime}, b l / \epsilon_{o, z}^{b l}$ to zero. As the tangent modulus decreases, the reduced modulus also decreases, which ultimately causes the buckling strength of the face shell to decrease. Relating this to the web interruption effect on compression strength parallel to bed joints observed by Ring (2009), the strain, $\epsilon$, used to the tangent modulus is defined as:

$$
\epsilon=\epsilon_{o, z}^{b l}-\sqrt{(1-\gamma) \epsilon_{o, z}^{b l}}
$$

where $\gamma=h^{w e b} / h^{b}$. To ensure that $E_{t}^{b}$ is neither to large or to small, $\gamma=h^{w e b} / h^{b}$ is subject to the following limits:

$$
0.40 \geq \gamma \leq 0.95
$$


If the prism is grouted, the grout acts as a brace; thus, the effective length factor, $K$, as defined in Equation 7.9 for a non-sway frame is used. If the prism is ungrouted, Equation 7.10 for a sway frame can be used to determine $K$. The top elastic restraint coefficient, $\eta_{t}$, for the face shell and external web is

$$
\eta_{t}=\frac{k^{f s}}{k^{f s}+k^{t w}}
$$

and the bottom elastic restraint coefficient becomes

$$
\eta_{b}=\frac{k^{f s}}{k^{f s}+k^{b w}}
$$

where $k^{f s}$ is the stiffness of the face shell determined as:

$$
k^{f s}=\frac{I^{f s}}{L^{f s}}
$$

and the stiffness of the top web $k^{t w}$ and bottom web $k^{b w}$ are given in Table 7.1 as

$$
k^{t w}=k^{b w}=0.5 \frac{I^{w e b}}{L^{w e b}} .
$$

The second moment of area of the web about its weak axis is

$$
I^{w e b}=\frac{h^{w e b} t^{w e b^{3}}}{12}
$$

and the length of the web is determined from

$$
L^{w e b}=w^{b l}-t^{f s}
$$

\section{$7.4 \quad$ Results}

A total of 11 average masonry compressive strength data points were gathered from literature (Ring, 2009; Sarhat, 2016; Wong and Drysdale, 1985) as shown in Appendix C. The experimental-to-predicted ratios for the masonry compression strength parallel to the bed joints are listed in Table 7.2 and Table 7.3 for ungrouted and grouted masonry respectively. The average ratio for ungrouted masonry is 0.96 with a COV of $10.60 \%$ and for grouted masonry the ratio is 1.04 with a COV 10.26\%. Figure 7.16 shows the results are close to unity. 
Table 7.2: Ungrouted Parallel Results

\begin{tabular}{llll}
\hline \multicolumn{4}{c}{ Face Shell Bedded Prisms } \\
\hline No. $^{\mathrm{a}}$ & $\begin{array}{l}f_{m, \text { rep }}^{\prime} \\
(\mathrm{MPa})^{\mathrm{b}}\end{array}$ & $\begin{array}{l}f_{m, \text { pred }}^{\prime} \\
(\mathrm{MPa})^{\mathrm{b}}\end{array}$ & $\begin{array}{l}f_{m, \text { rep }}^{\prime} / \\
f_{m, \text { pred }}^{\prime}\end{array}$ \\
\hline 1 & 17.30 & 16.69 & 1.04 \\
2 & 19.00 & 21.30 & 0.89 \\
\hline Average & 0.96 \\
STDV & 0.10 \\
COV & $10.60 \%$ \\
\hline a For Input Values the Number Corresponds \\
to the Number in Appendix C \\
b Based on net area
\end{tabular}

Table 7.3: Grouted Parallel Results

\begin{tabular}{llll}
\hline No. $^{\mathrm{a}}$ & $\begin{array}{l}f_{m, r e p}^{\prime} \\
(\mathrm{MPa})^{\mathrm{b}}\end{array}$ & $\begin{array}{l}f_{m, p r e d}^{\prime} \\
(\mathrm{MPa})^{\mathrm{b}}\end{array}$ & $\begin{array}{l}f_{m, \text { rep }}^{\prime} \\
f_{m, \text { pred }}^{\prime}\end{array}$ \\
\hline 1 & 10.6 & 11.86 & 0.89 \\
2 & 21.4 & 19.78 & 1.08 \\
3 & 20.2 & 22.29 & 0.91 \\
4 & 18.6 & 16.14 & 1.15 \\
5 & 17.5 & 17.67 & 0.99 \\
6 & 19.32 & 17.56 & 1.10 \\
7 & 16.66 & 17.55 & 0.95 \\
8 & 17.4 & 14.82 & 1.17 \\
9 & 20.35 & 18.25 & 1.12 \\
\hline Average & & 1.04 \\
STDV & & 0.11 \\
COV & & $10.29 \%$ \\
\hline
\end{tabular}

\footnotetext{
${ }^{\mathrm{a}}$ For Input Values the Number Corresponds

to the Number in Appendix C

${ }^{\mathrm{b}}$ Based on gross area
} 


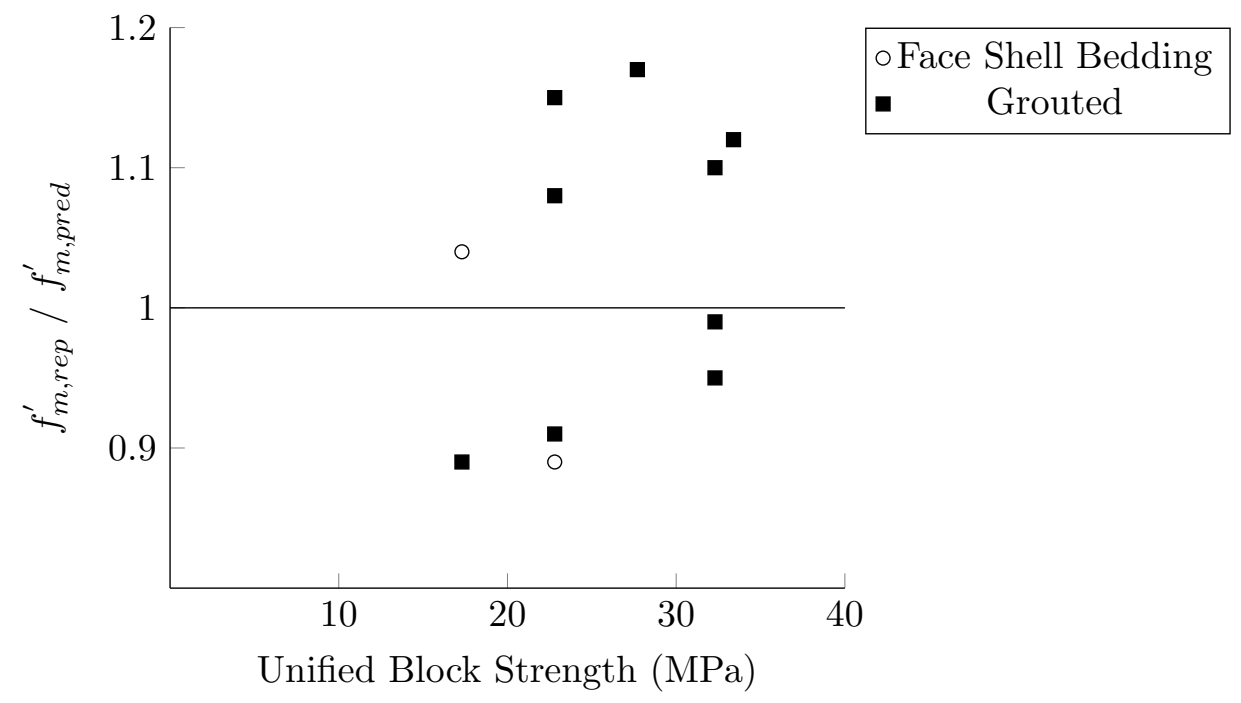

Figure 7.16: SACM Parallel Results 


\section{CHAPTER 8}

\section{PART II CONCLUDING REMARKS}

Part II of this thesis develops an innovative micro model to predict the compression strength of hollow concrete block masonry loaded in compression both perpendicular and parallel to the mortar bed joint. Results of the numerical model are compared to experimental data and simplified equations are proposed. This chapter makes a number of concluding remarks about Part II of this thesis and presents a number of items for future research.

The goal of Part II of this thesis was to develop a model to predict the compression strength of masonry, $f_{m}^{\prime}$. The compression strength is often of prime importance in designing masonry structures, as it is often the basis of all designs due to the fact that allowable compressive, flexural, bearing, and shearing stresses; and, the modulus of elasticity are typically expressed as functions of the compressive strength.

Two models are presented to predict the compression strength of masonry, $f_{m}^{\prime}$. The first model, the Smeared Area Compression Model (SACM), is a complex model that uses equilibrium, compatibility, and material constitutive models to determine the compression strength. The second method, the Simplified Smeared Area Compression Model (SSACM), uses three simple equations to determined the masonry compression strength. Both models are shown to be accurate with the SACM producing an average experimentto-predicted ratio of 1.00 (COV of 5\%) and 1.01 (COV of 11\%) for the SSACM from a database of 247 masonry prism tests. The application of the SSACM is further demonstrated through the proposed Design Smeared Area Compression Equations (DSACE) which produces the best results compared to other international design codes.

\subsection{Part II Conclusions}

The following statements can be made based on Part II of this thesis:

1. Each material used in masonry has a different peak strength, strain at peak strength, modulus of elasticity, and Poisson's ratio, which leads to a complex interaction between individual material components used to construct masonry.

2. An numerical model that uses equilibrium, compatibility, and material constitutive models that accounts for different material properties is able to predict the 
compression strength of masonry. Additional, the stresses and strains can be determined in each material component accounting for both the effects of confinement and biaxial tension.

3. The Smeared Area Compression Model requires 23 material properties to act as inputs for the model, but 17 (74\%) of these can be set to default values. Additionally, the model requires 8 geometric properties of the block and mortar.

4. Modifying the material configurations, leaving only the elements resisting load in a given direction allows for the anisotropic nature of hollow concrete block masonry to be included in the compression model.

5. Accounting for Poisson's ratios of each material using a prestrain concept allows for the composite material stiffness matrix to remain symmetric, but allows for the inclusion of the different Poisson's ratios causing confinement or softening.

6. Using a secant stiffness for each material component with an iterative solution till convergence of the secant stiffness is achieved allows for the non-linear response of hollow concrete block masonry to be included in the compression model.

7. Unifying the reported uniaxial compressive strength of the masonry components allows for different testing methods to be compared. It also allows for a single unified value to used within the model that accounts for the in-situ strength of material components.

8. The Smeared Area Compression Model is verified against a database of 224 average prism strength data points and produces an average experimental-to-predicted of 1.00 with a coefficient of variation of $5 \%$.

9. The mortar compression strength has little impact on the compression strength of hollow concrete block masonry. This is typically due to the fact that the uniaxial compression strength is increases due to tiaxial confinement of the mortar.

10. The mortar joint thickness has a large impact on the compression strength of ungrouted face shell bedded hollow concrete block masonry. This is due to the fact that as the mortar joint thickness increases the lateral confinement effect offered by the block reduces.

11. Matching the deformation properties of individual material components allows for each material to deform together and tends to produce higher hollow concrete block compression strength and optimal use of materials.

12. The development of simple equations to predict the masonry compression strength allows for "back-of-the-envelope" calculations. The proposed Simplified Smeared Area Compression Model formula predict the compression strength of hollow concrete block masonry with an average experimental-to-predicted ratio of 1.01 with 
a coefficient of variation of $11 \%$ from a database of 224 average prism strength data points.

13. The compression strength of ungrouted hollow concrete block masonry can be determined based on bedding type.

14. The compression strength of grouted hollow concrete block can be determined based on which material, the block or the grout, reaches its strain at peak strength first.

15. The proposed Design Smeared Area Compression Equations predict the compression strength of hollow concrete block masonry with an average experimental-topredicted ratio of 1.38 with a coefficient of variation of $18 \%$. The design equations present the best conservative predictions with the lowest coefficient of variation of major international masonry design code.

16. The compression strength parallel to the bed joints can be estimated considering the buckling load of the face shells.

\subsection{Part II Future Research}

Part II performed a comprehensive analysis of masonry compression strength; however, the topic is complex and merits considerable further investigation. To begin with, further experimental tests are necessary to strengthen the conclusions of the models presented in Part II and ensure that they are valid. In particular, the strain in each direction in each material needs to be experimentally determined and compared to the numerical model.

Additionally, the SACM should be able to predict the strain level at a given stress level. This may be accomplished directly from the model strains or with the addition of springs to determine strains of the given materials.

Further experimental and numerical work is required for compression strength parallel to the bed joint. There is currently only a few experimental results for compression strength parallel to the bed joints and the previous work has resulted in contradicting results as to if the compression strength parallel to the bed joint is weaker than normal to the bed joint. More experimental work in this area would allow for more defined material relationships and improved numerical modeling.

With further research there is a possibility of completing the coding of the SACM in C to allow for a version to be released to the public for use.

Part II: Masonry Compression Strength Modeling 
MASONRY FINITE ELEMENT

MODELING 
The development of adequate stress analyses for masonry structures represents an important task not only for verifying the stability of older masonry construction, but also to effectively design new masonry structures. The analysis of masonry structures is not simple for at least two reasons. For one, the masonry material presents a strong nonlinear behaviour, so that linear elastic analyses cannot be considered. And two, the compatibility of individual materials used in masonry structures, can cause complex interactions. As a consequence, the behaviour and analysis of masonry structures still represents an important research field in civil engineering.

Nonlinear models implemented in suitable finite elements formulations currently represent the most common advanced strategy to simulate the structural behaviour of masonry structures. The main problem in the development of accurate stress analyses for masonry structures is the definition and the use of suitable material constitutive laws that take into account the heterogeneity of the masonry material as a result of the composition of blocks connected together by mortar joints and possible grout and reinforcement. Three modeling approaches have been proposed in the literature: micro, micro-macro, and macro modeling.

Micro-models consider the blocks, mortar joints, and grout separately, characterized by different constitutive laws for each material. The mechanical properties that define the models used for blocks, mortar, and grout, are obtained through experimental tests conducted on the individual material components. This approach requires a lot of computational effort in a finite element formulation framework as the blocks, mortar, and possible grout have to be discretized individually resulting in a high number of nodal unknowns.

Micro-macro models consider different constitutive laws for the blocks, mortar joints and grout; then, a homogenization procedure is performed obtaining a macro-model for masonry which is used to develop the analysis. The mechanical properties of individual masonry materials are obtained through individual material tests. Micro-macro models are very appealing, as they are derived in a rational way that accounts for the mechanical properties of each material component, but also consider the overall masonry behaviour. Moreover, this approach can lead to effective models as larger elements can be used in the finite element model leading to less nodes.

Macro-models are based on the use of homogeneous constitutive laws for the masonry material; the stress-strain relationships adopted for the structural analysis are derived performing tests on masonry assemblages, without distinguishing the individual material behaviour. A macro-model might be unable to describe in a detailed manner some micro mechanisms occurring in the damage evolution of masonry, but it is very effective from a computational point of view when finite element analysis is performed.

One modeling strategy cannot be promoted over the other because different applications exist for micro and macro models. Micro-modelling studies are necessary to give a better understanding about local behaviours of masonry structures, particularly in structural details. Macro-models are applicable when the structure is sufficiently large so that the stresses across a large area will be essentially uniform.

Part III: Masonry Finite Element Modeling 


\section{CHAPTER 9}

\section{BACKGROUND}

The finite element modelling of hollow concrete block masonry is typically divided into three categories, micro-model, macro-modeling, and micro-macromodeling. Each on of these methods is suitable for modelling different aspects of masonry depending on the detail required. The goal of Part III of this thesis is to develop a micro-macro model that can be used to predict the load-deflection, cracking patterns, stresses, and strains of ungrouted and grouted, and reinforced hollow concrete masonry elements.

Many different models have been proposed for simulating the behaviour of masonry using a finite element framework. Three main approaches have been considered: micromodelling, macro-modeling, and micro-macro-modeling. The first aims primary at representing the actual texture of masonry with blocks and mortar joints being considered separately as homogeneous sub-regions each characterized by distinct properties. The second attempts to define an equivalent continuum, the characteristics of which permit the description of the global behaviour of masonry. The third method considers different constitutive laws for the blocks, mortar joints and grout; then, a homogenization procedure is performed obtaining a macro-model for masonry which is used to develop the analysis.

\subsection{Micro-Models}

In micro-modeling particular attention needs to be placed on the mortar joints since they constitute a major source of nonlinearity and failure. Strictly speaking, in a planestress micro-model, both mortar joints and blocks should be represented by dimensional elements for consideration at the same micro-level. Though mortar joints, because of numerical problems related to their low thickness, have been generally modeled by onedimensional linkage elements and blocks discretized using conventional plane stress elements.

In the model proposed by Page (1979), bricks were assumed isotropic elastic, with elastic constants derived from uniaxial compressive tests. Mortar joints were modeled by linkage elements that could deform both in the normal and shear directions according to two non-linear stress-strain relationships determined from uniaxial compression tests 


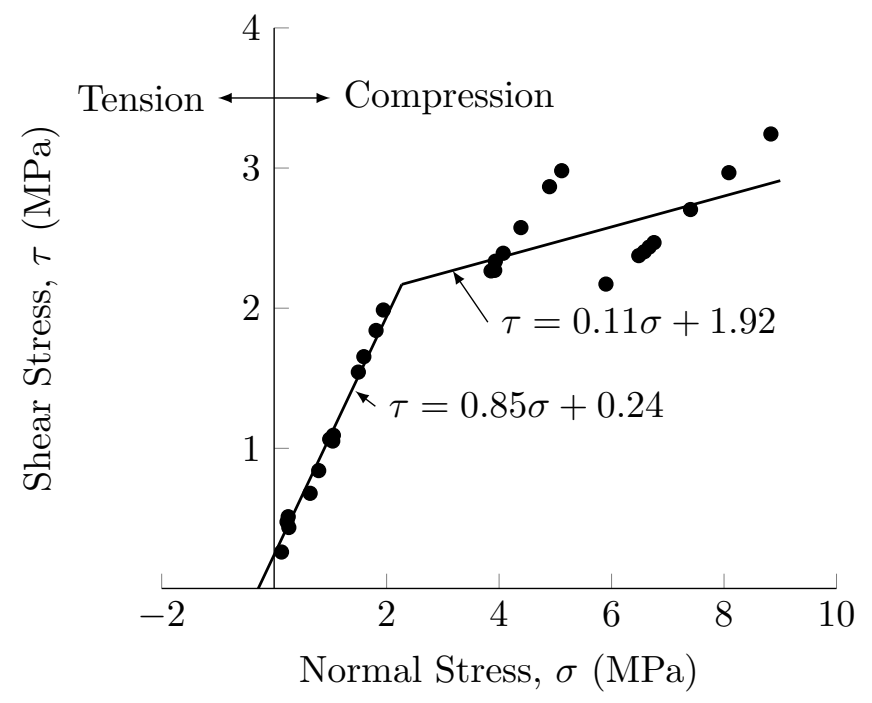

Figure 9.1: Joint Failure Criterion (Page, 1979)

performed on masonry panels with varying bed joint orientation. The failure criterion, which considered the joint only, consisted of two linear best-fit lines, produced from experimental data, see Figure 9.1. Beyond failure, residual properties were assigned only to those joint elements which had failed under a normal compressive stress. The normal stiffness remained unchanged while the shear stiffness was reduced according the magnitude of the compressive stress present at failure. The model was used to reproduce an in-plane bending test on a deep masonry beam under vertical load. Stress distributions have been reproduced to a reasonable degree of accuracy, even for higher loads when substantial stress redistribution had occurred. However, as the criterion for block failure was not included, the ultimate load could not be predicted since the final collapse involved both bricks and joints.

The possibility of both block or mortar failure were included in the micro-model proposed by Arya and Hegemier (1978) for both ungrouted and reinforced grouted concrete masonry. Blocks were modeled as plane-stress elements with the steel reinforcement providing additional stiffness and mortar joints were modeled as linkage elements. In compression the grouted concrete blocks were assumed to have a strain-softening effect and ware isotropic elastic before the yield curve was reached as defined by a Von Mises yield criterion. Loading beyond the yield curve was assumed to follow a homothetic yield curve, shrinking to the origin with the increasing value of the equivalent strain until collapse. Once the initial yield curve has been reached, an element was also declared collapsed as soon as it was subjected to tensile stresses. In tension the grouted concrete blocks were assumed linear-elastic until cracking. The cracks were assumed 


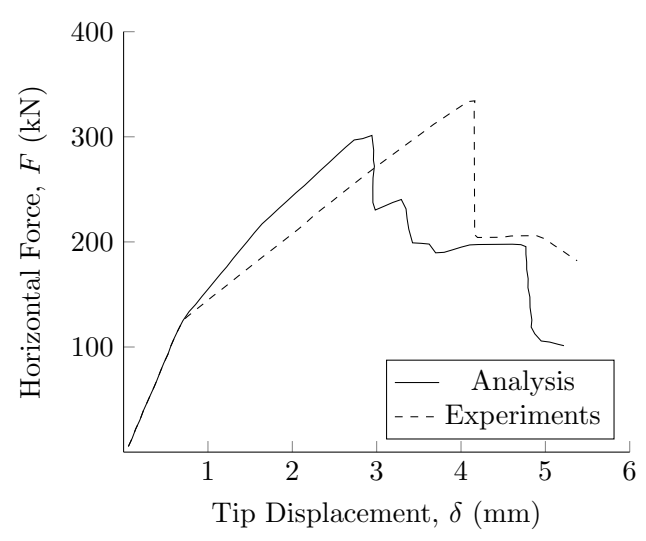

(a) Reinforced Specimen

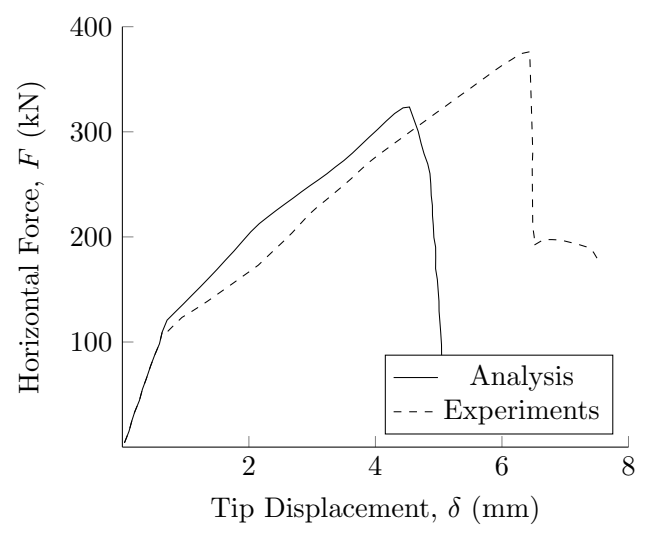

(b) Unreinforced Specimen

Figure 9.2: Experimental and Analytical Load Displacement Curves (Arya and Hegemier, 1978)

smeared across the element and normal to the first principle stress just prior to cracking. The Poisson's ratio and the stiffness normal to the cracks were set to zero and the shear stress modulus was reduced by a factor depending on the opening of the cracks. When the cracks closed, the element was considered to regain its entire elastic stiffness except for the shear modulus which was only partially recovered. The reinforcing steel was assumed elastic perfectly plastic in both tension and compression. The mortar and gout interfaces were assumed to have perfect bond as long as the strength capacities of the interface given by a Mohr-Coulomb's condition were not exceeded. The cohesion and the coefficient of friction were assumed to be decreasing functions of the relative tangential displacements at the interface. When implemented into a finite element code, the progressive change in stiffness and strength characteristics of the structure required an iterative approach. The iterative process was terminated if either the incremental displacements or the nodal forces converged. The behaviour of reinforced and unreinforced masonry walls under constant vertical pressure and monotically increasing shear deformation was simulated. The correlation between analytical and experimental results was good, the predicted ultimate strength of the specimens was within $15 \%$ of the experimental values, see Figure 9.2. 
As already mentioned, micro-modelling of masonry requires a refined knowledge of each constituent. Sophisticated constitutive laws have thus been used to describe complex mechanical events such as cracking and crushing of the blocks and debonding, sliding or recontact of the joints. Unfortunately, such refined idealizations may cause a number of issues including:

1. The introduced constitutive parameters are often numerous and difficult to identify and the experimental tests to determine the parameters suffer from high variability; and,

2. The computer capability is easily exhausted by the high number of degrees of freedom required to discretize even small structures.

Consequently, modeling research has moved towards macro-models even for dealing with fairly simple structures.

\subsection{Macro-Models}

The existing macro-models may be divided in two main groups:

1. Those which have been specifically developed for masonry (masonry macro-models); and,

2. Those which are similar to the ones used for reinforced concrete, with slight modifications for characteristics of masonry (concrete macro-models).

Concrete macro-models are in fact quite suitable for fully grouted reinforced concrete masonry, which is expected to behave similarly to reinforced concrete (Anthoine, 1992). However, they may fail in representing the behaviour of ungrouted block masonry since they do not take into account the marked anisotropic effects due to the mortar joints, whereas masonry macro-models have been designed specially to account for this particular feature (Anthoine, 1992).

\subsubsection{Masonry Macro-Models}

Samarashinghe and Hendry (1980) proposed a simple failure surface in the tensioncompression region, see Figure 9.3. As long as the failure surface is not violated, masonry is assumed to be linear isotropic elastic. Once the failure surface is reached, the residual stiffness and strength of the corresponding element is taken as zero. The constitutive models were implemented in an incremental finite element program where, at each load 


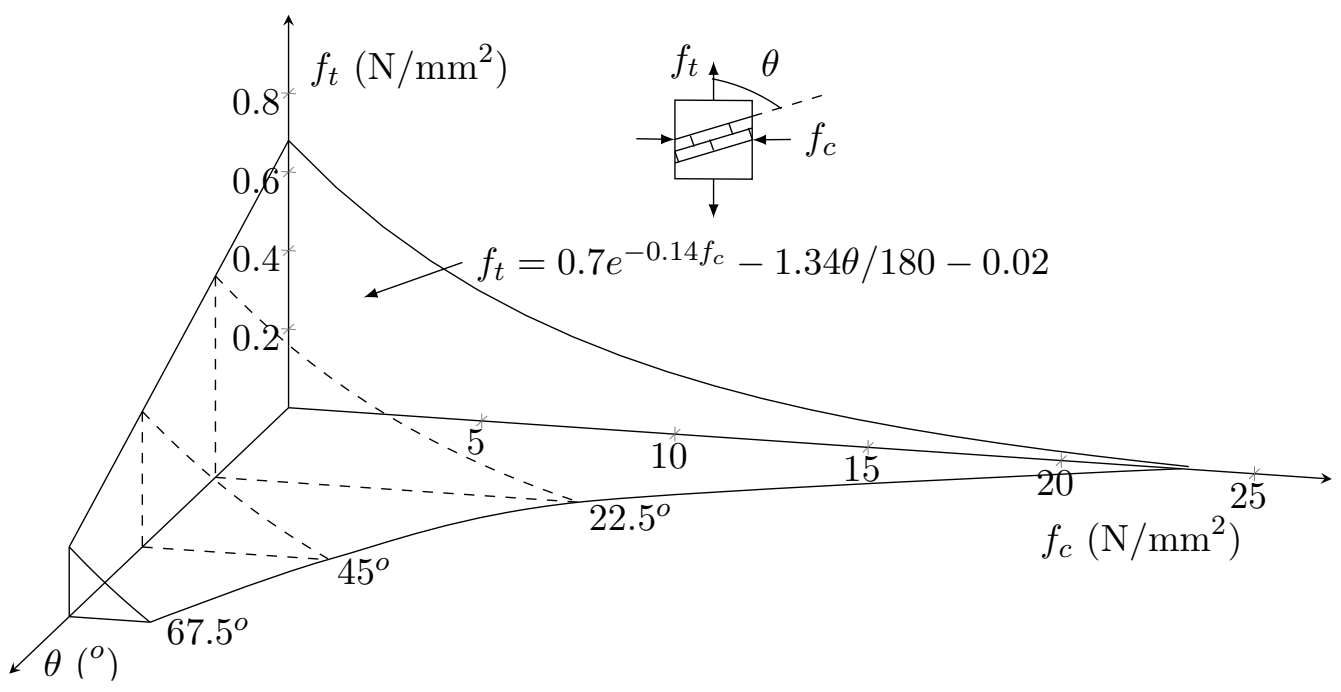

Figure 9.3: Failure Surface for Brickwork Under Biaxial Stress (Samarashinghe and Hendry, 1980)

increment, iterations are carried out to find a state of stress satisfying the equilibrium equations and compatible with the strength and stiffness properties. Shear walls subjected to vertical precompression and monotonie shear loads applied at varies heights, have been used as a basis for comparison between theory and experiments. Since all the shear walls failed suddenly in a brittle manner, it was found reasonable to identify the ultimate load with the first cracking load. Both location of the cracks and subsequent crack propagation were well predicted in the tension-compression region (Figure 9.4).

\subsubsection{Concrete Macro-Models}

Using finite element models to predict the response of concrete elements has been the object of intensive research for a long time. Some of these models have been used to simulate the non-linear behaviour of masonry. In most cases, the original model was used without any fundamental modification, the various parameters being merely adjusted to fit with the characteristics of masonry.

Shing et al. (1988) modelled reinforced masonry using a concrete model with a DruckerPrager yield surface. Crushing or cracking was assumed depending on whether the ultimate compressive or tensile strain was reached. In the post cracking-failure range, the smeared crack approach was used and the tension-stiffening effect was simulated by allowing a gradual drop of tensile stress. No softening regime was assumed in the post crushing failure range. Testing the model resulted in a monotonic load-displacement 


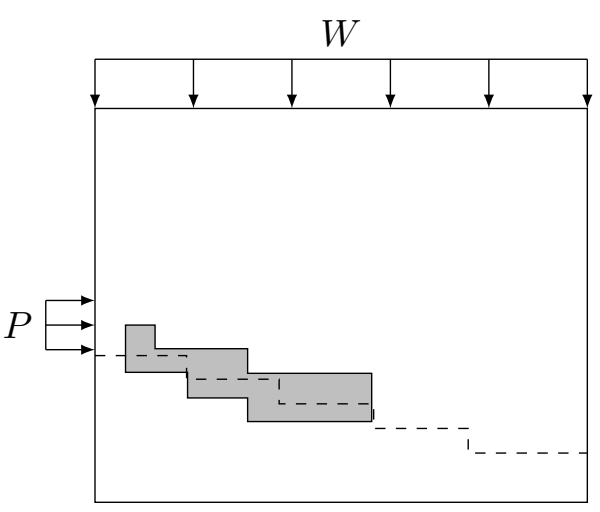

Shear Wall F $\left(\alpha=36^{\circ}\right)$

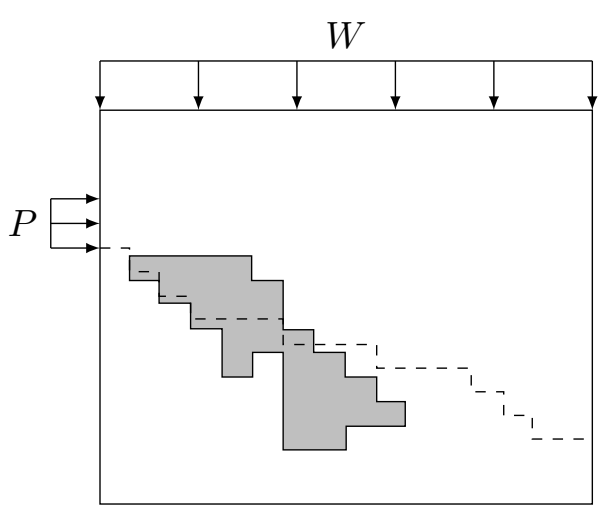

Shear Wall C $\left(\alpha=50^{\circ}\right)$

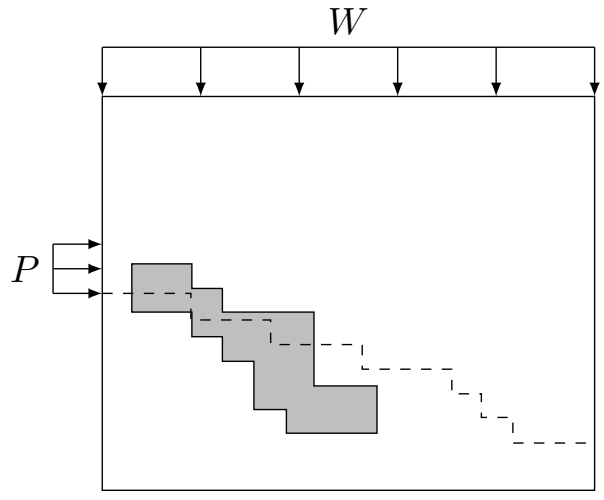

Shear Wall D $\left(\alpha=43^{\circ}\right)$

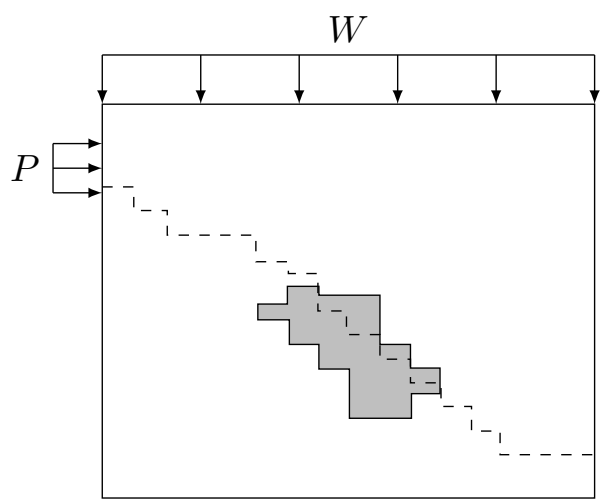

Shear Wall A $\left(\alpha=56^{\circ}\right)$

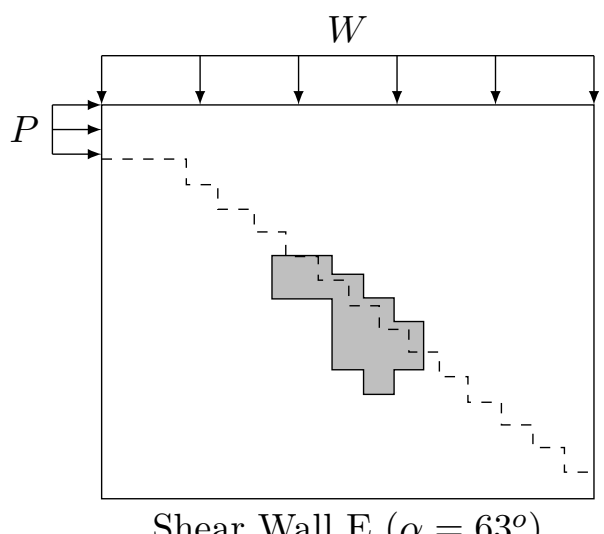

Shear Wall E $\left(\alpha=63^{\circ}\right)$

$W / P=\tan \alpha$

- - - - Experimental Failure

Predicted Zone of Failure Under Biaxial Tension-Compression

Figure 9.4: Predicted and Experimental Crack Patterns (Samarashinghe and Hendry, 1980) 


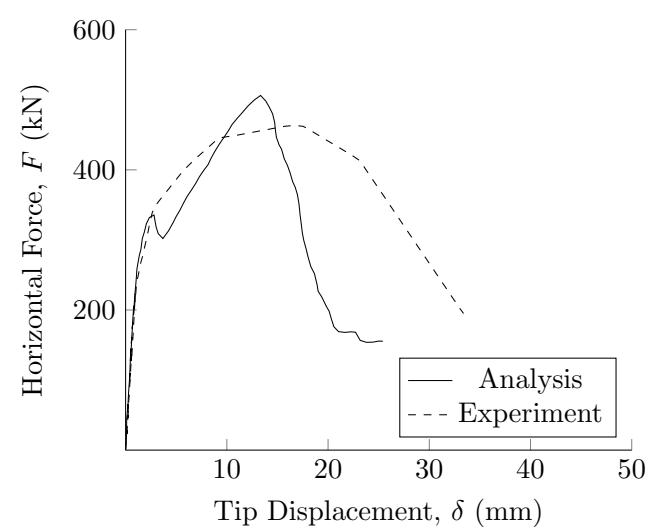

(a) Shear Failure

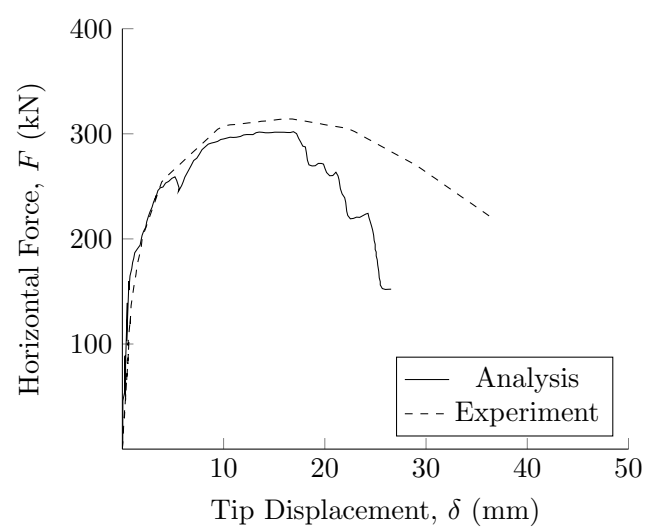

(b) Flexure Failure

Figure 9.5: Experimental and Analytical Load Displacement Curves (Shing et al., 1988)

curve very close to the envelope of the experimentally obtained hysteresis curve, especially for panels exhibiting flexural behaviour (Figure 9.5b). For panels exhibiting shear behaviour, the diagonal crack opening could not be satisfactorily modeled by the smeared crack approach. The shear cracking load appeared to be underestimated while the ultimate shear strength was overestimated (Figure 9.5a). According to Shing et al. (1988), this was due to the residual strength attributed to the cracked element; the diagonal crack opening would have been probably better simulated by a discrete crack modelling.

Calvi et al. (1983) used a concrete model in which the nonlinear behaviour of unreinforced masonry was described by a linear elastic stress-strain relationship with an isotropic tangential stiffness dependent on the current state of stress. This stress dependency was expressed through variable tangential bulk and shear moduli, so that stress and strain increments could be easily decomposed into hydrostatic and deviatoric components. Nine input parameters were required for the definition of the model: three for the shear modulus, two for the bulk modulus, and four for the failure criterion. They were identified by two experimental tests on masonry panels: a vertical compression test and a diagonal compression test. Good agreement between the experimental and analytical load-displacement curves was achieved. 
In the case of reinforced concrete masonry, Seible et al. (1990) used a monotonic model where nonlinear behaviour of reinforced masonry was described by relating total stresses to total strains. Masonry was considered to be an orthotropic material with reference to the principal stress axes which in turn were assumed to coincide with the principal strain axes. The stress-strain relationship expressed in the principal stress axes was:

$$
\left\{\begin{array}{c}
\sigma_{1} \\
\sigma_{2} \\
\tau_{12}
\end{array}\right\}=\left[\begin{array}{ccc}
E_{1} & 0 & 0 \\
0 & E_{2} & 0 \\
0 & 0 & \frac{E_{1}+E_{2}}{4}
\end{array}\right]\left\{\begin{array}{c}
\epsilon_{1} \\
\epsilon_{2} \\
\gamma_{12}
\end{array}\right\}
$$

where $E_{1}$ and $E_{2}$ were dependent on the principal strains $\epsilon_{1}$ and $\epsilon_{2}$. The modified compression field theory (MCFT) (Vecchio and Collins, 1986) was used as the bases of the model with a few modifications. The principal tensile stress-strain law $\left(\epsilon_{i}>0\right)$ before cracking was given by:

$$
\sigma_{i}=E_{m} \epsilon_{i} \text { if } \epsilon_{i} \leq \epsilon_{c r}
$$

where $\epsilon_{c r}$, was the cracking strain. After cracking $\left(\epsilon_{i}>\epsilon_{c r}\right)$, different tension-stiffening models were considered:

$$
\sigma_{i}=\left\{\begin{array}{l}
E_{m} \frac{\epsilon_{\mathrm{cr}}}{1+\sqrt{200 \epsilon_{i}}} \\
\frac{E_{m}}{48}\left(25 \epsilon_{\mathrm{cr}}-\epsilon_{i}\right) \\
E_{m} \epsilon_{\mathrm{cr}} e^{-\frac{\alpha\left(\epsilon_{\mathrm{cr}}-\epsilon_{i}\right)}{\epsilon_{c r}}}
\end{array}\right.
$$

where the coefficient $\alpha$ was increased with increasing reinforcement The principal compressive stress strain law $\left(\epsilon_{i} \leq 0\right)$ was given by:

$$
\sigma_{i}= \begin{cases}-\frac{f_{m}^{\prime}}{\beta}\left(2 \frac{\epsilon_{i}}{\epsilon_{o}}-\left(\frac{\epsilon_{i}}{\epsilon_{o}}\right)^{2}\right) & \text { if } 2 \epsilon_{o} \leq \epsilon_{i} \leq 0 \\ 0 & \text { if } \epsilon_{i} \leq 2 \epsilon_{o} \leq 0\end{cases}
$$

where

$$
\beta=\max \left(0.8+0.34 \frac{\epsilon_{i}}{\epsilon_{o}}, 1\right), \text { and }
$$

$f_{m}^{\prime}$ and $\epsilon_{o}$ were the peak compression stress and corresponding strain.

The reinforcement stress was determined by:

$$
\left\{\begin{array}{c}
\sigma_{x} \\
\sigma_{x} \\
\tau_{x y}
\end{array}\right\}=\left[\begin{array}{ccc}
\rho_{x} E_{s x} & 0 & 0 \\
0 & \rho_{y} E_{s y} & 0 \\
0 & 0 & 0
\end{array}\right]\left\{\begin{array}{c}
\epsilon_{x} \\
\epsilon_{y} \\
\gamma_{x y}
\end{array}\right\}
$$


where $\rho_{x}, \rho_{y}$ were the steel ratios in the $x, y$ directions and $E_{s x}, E_{s y}$ the corresponding moduli. Each modulus was dependent on the corresponding strain since a bilinear stressstrain relation was assumed for steel. Both materials, masonry and steel, were assumed perfectly bonded and therefore subjected to the same strain field. From a numerical point of view, tangent or initial stiffness methods were used to solve the nonlinear equilibrium equations under prescribed load and/or displacements. Convergence was based on the ratio of the norm of residual forces to total forces. Excellent agreement was obtained in the case of ductile behaviour, but the lateral strength of specimens exhibiting non-ductile behaviour were overestimated. However, in both cases, the sequential crack pattern and yield developments were well predicted.

\subsection{Micro-Macro-Models}

For similar reasons to the macro-models discussed in Section 9.2, micro-macro-models can be divided into masonry and concrete models.

\subsubsection{Masonry Micro-Macro-Models}

The damage model proposed by Maier et al. (1991) combines two levels of analysis. On the one hand, the material characteristics were defined at the micro-level, the bricks were assumed isotropic and mortar was considered as an isotropic elastic material susceptible to isotropic damage; perfect bond was assumed at the interface. On the other hand, equilibrium equations and compatibility conditions were expressed at the macro-level, in terms of the average stresses and strains. The passage from the micro-level to the macrolevel is based on a homogenization procedure which is completed using the moduli of the bricks and mortar. The passage from the macro-level to the micro-level was completed by converting the total stresses to the local stresses using the stress-strain law of each constituent. According to the chosen damage law, eleven or twelve parameters were required (four for the bricks and seven or eight for the mortar). They could be identified by uniaxial tension and compression tests performed on each constituent. In the model, failure occurred when either a strain limit was reached in the bricks or a critical damage value was reached in the mortar. The model was implemented with several variants of the damage law, in a finite element program with good results.

Facconi et al. (2014) proposed a modification to the disturbed stress field model (DSFM), see next section, for unreinforced masonry. The DFSM has the ability to combine average behavior of the composite material with the local shear-slip response of masonry joints. By modeling the joints behavior separately, the proposed model is able to capture the 
local shear response of the joints in both the elastic and inelastic stages. Equilibrium, compatibility, and stress-strain relationships are formulated in terms of average principal stresses and strain. An elastic-plastic shear stress-slip relationship associated with a hyperbolic yield criterion is used to represent rigid body slip occurring along joints plane. A verification of the proposed formulation was undertaken through comparisons with the results of two full-scale shear wall tests reported in the literature, see Figure 9.6. The simulations provided reasonably accurate predictions of the walls structural response and demonstrated the ability of the model to represent well both ductile and brittle failure modes in masonry structures. Further work is required to improve the joints shear-slip model and allow the head joints to be staggered instead of stacked.

\subsubsection{Concrete Micro-Macro-Models}

Originally published in the 1980s by Vecchio and Collins (1986), the modified compression field theory (MCFT), serves as a generalized approach for modelling the behaviour of reinforced concrete elements subjected to biaxial loading conditions. Central to the theory is the treatment of cracked reinforced concrete as a new orthotropic material with its own set of governing constitutive equations. The application of a smeared, rotating crack model treats stresses and strains in an average sense, and allows stresses and strains to gradually reorient as a result of changing load and/or material response. The composite element, which consists of cracked concrete and in-plane reinforcement, is governed by equations of equilibrium, compatibility, and constitutive material models. Though cracks are considered smeared, and stresses and strains are averaged, a key feature of the model is the examination of local behaviour at crack locations.

Since its inception the MCFT has been applied in a wide range of reinforced concrete analysis applications spanning various structure types and loading configurations. Its implementation in various analytical tools has shown that in most cases it provides reasonable estimates of load capacities and failure modes, and can be used to identify critical factors influencing the performance of reinforced concrete structures (Vecchio et al., 2004). Although the use of the MCFT has generally resulted in high accuracy and reliability, some deficiencies pertaining to specific situations have been noted (Vecchio, 2000). For panel elements with high amounts of reinforcement, the MCFT has exhibited the tendency to underestimate the shear strength and stiffness; for panels with low amounts of reinforcement, shear strength and stiffness can be overestimated.

The disturbed stress field model (DSFM) (Vecchio, 2000) is essentially an extension of the formulations of the MCFT, with the removal of the restriction that the orientation of 


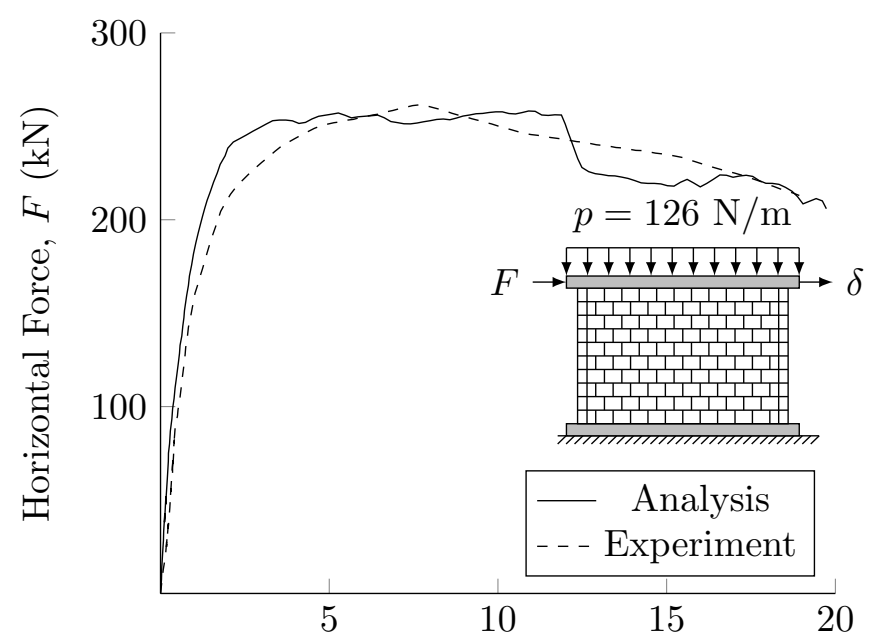

Tip Displacement, $\delta(\mathrm{mm})$

(a) Ganz and Thrlimann (1984) Hollow Brick Results

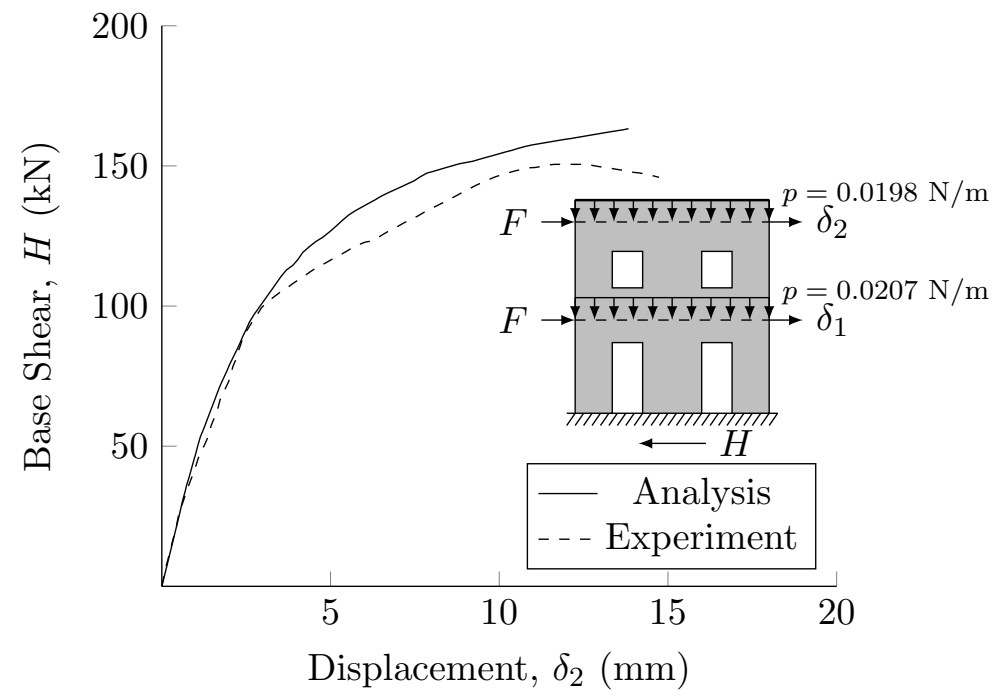

(b) Magenes et al (1995) Two-Strory Two-Wythe Solid Brick Results

Figure 9.6: Facconi et al. (2014) Experimental and Analytical Load Displacement Curves

the stresses and strains remain coincident. Additionally, in contrast to the original presentation of the MCFT which was formulated on the basis of reinforcement components being orthogonally aligned in the planar $x$ and $y$ directions, the equilibrium relations of the DSFM are cast such that any number of reinforcement components, oriented in any planar direction, can be considered.

To accommodate the rotational lag of the stress field observed from the experimental data, the DSFM incorporates deformations due to crack slip in the element compatibility 
relationships. As an added benefit of the explicit inclusion of the slip deformation on the crack surface, the crack slip check required in the MCFT need not be performed.

\subsection{Background Summary}

The finite element modeling of masonry has been grouped into three different approaches: micro, micro-macro, and macro modeling. Micro-models consider the blocks, mortar joints, and grout separately, characterized by different constitutive laws for each material. This approach requires a lot of computational effort as the blocks, mortar, and possible grout have to be discretized individually resulting in a high number of nodal unknowns. Macro-models are based on masonry material constitutive laws without distinguishing between the individual material behaviours. A macro-model might be unable to describe in a detailed manner some micro mechanisms occurring in the damage evolution of masonry, but it is very effective from a computational point of view when finite element analysis is performed. Micro-macro models consider different constitutive laws for the blocks, mortar joints and grout; then, a homogenization procedure is performed obtaining a macro-model for masonry which is used to develop the analysis. Micromacro models are very appealing, as they are derived in a rational way that accounts for the mechanical properties of each material component, but also consider the overall masonry behaviour. Currently there is no micro-macro models specifically designed for hollow concrete block masonry.

\subsection{Model Objectives}

In addition to the objectives listed in Section 1.4, the specific objectives of a micro-macro finite element for both ungrouted and grouted hollow concrete block masonry include:

1. Determination of the stresses, strains, and displacements of masonry elements such as shear walls and beams due to the loads acting on it;

2. To expand the formulation and applicability of the disturbed stress field model for the analysis of ungrouted, grouted, and reinforced hollow concrete block masonry;

3. To ease the preparation and interpretation of data a graphical user interface is desired to allow for easy user input of material properties and setting up the finite element options;

4. Verify the finite element model by comparing the results of analyses with the experimentally observed behaviour of several types of structural elements, including shear walls and beams constructed from hollow concrete block masonry. These results will illustrate the ability of the constitutive models to capture the response

Part III: Masonry Finite Element Modeling 
of structural problems involving different characteristics of hollow, grouted, and reinforced hollow concrete block masonry;

5. Use the finite element results to produce simplified design equations to allow for the estimation key masonry properties such as the modulus of elasticity. 


\section{SMEARED AREA FINITE ELEMENT MODEL}

A numerical model to be implemented in a finite element software is developed using equilibrium, compatibility, and constitutive relationships presented in this chapter. The constitutive relationships are designed specifically considering the anisotropic nature of hollow concrete block masonry.

The Smeared Area Finite Element Model (SAFEM) is an numerical model for predicting the stress, strain, displacement, and crack patterns of ungrouted, grouted, and reinforced masonry structures.

The model is a micro-macro-model for masonry that is based on the disturbed stress field model (DSFM) developed by Vecchio (2000; 2001) for reinforced concrete. Unlike conventional smeared crack models, the DSFM is able to combine the average macroscopic representation of the material behaviour with the local response of mortar joints. In addition to the typical advantage of macro-models (low computational costs), the proposed formulation attempts to enable the prediction of structural response even in cases in which the damage mechanism is governed by the local behaviour of masonry joints.

The formulation of SAFEM presented in this thesis can be used only for the analysis of masonry structures subjected to monotonic loading conditions.

For the purposes of the following discussion depicted in Figure 10.1 is a masonry shear wall. Assuming that such a structure is reasonably larger than the a few blocks, the joints and blocks can be considered smeared over the entire wall. The wall is taken to be of uniform thickness and can be grouted and reinforced with longitudinal and transverse bars. Consider a small region of the wall that is large enough to span a number of joints and blocks, but at the same time is sufficiently contained such that the sectional forces can be considered uniform. The loads acting on this region's (or element's) edges are assumed to consist of uniform axial stresses $f_{x}$ and $f_{y}$ and the uniform shear stress $v_{x y}$ (see Figure 10.1b). Thus, a field of internal average stresses and strains sustaining the applied load may be defined. Within this element, the material is subjected to a field of average principal tensile stress, $f_{m 1}$, and compressive stress, $f_{m 2}$, (see Figure 10.1c) that are related to the corresponding average strains by a Mohr's 
circle. As a consequence of the principal tensile stresses, the composite material can experience smeared cracks oriented in the principal average compressive stress direction. Due to the stress conditions rigid body slip can occur between the two sides of the crack. There can also be rigid body slip between the block-mortar interfaces. The block-mortar slip has to be combined with the average strains and crack slip deformations to obtain the total strains that represent the deformation of the whole masonry element.

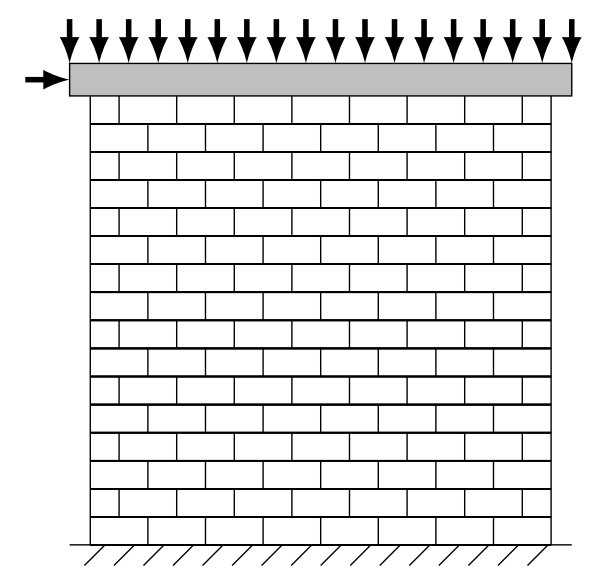

(a) Masonry Wall

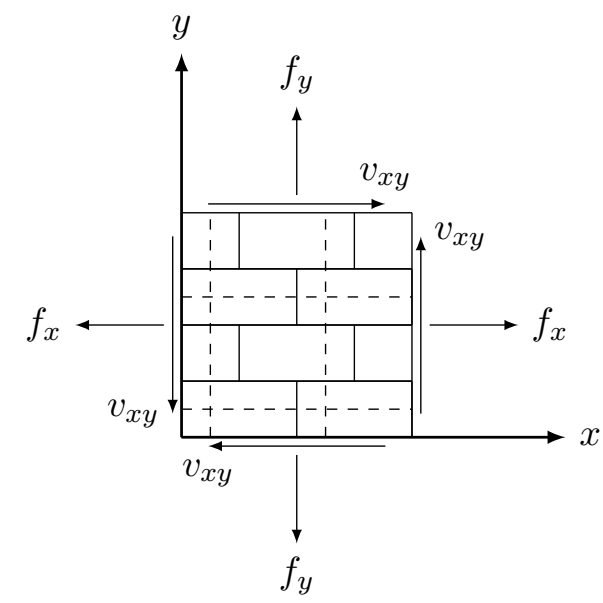

(b) Global Stresses

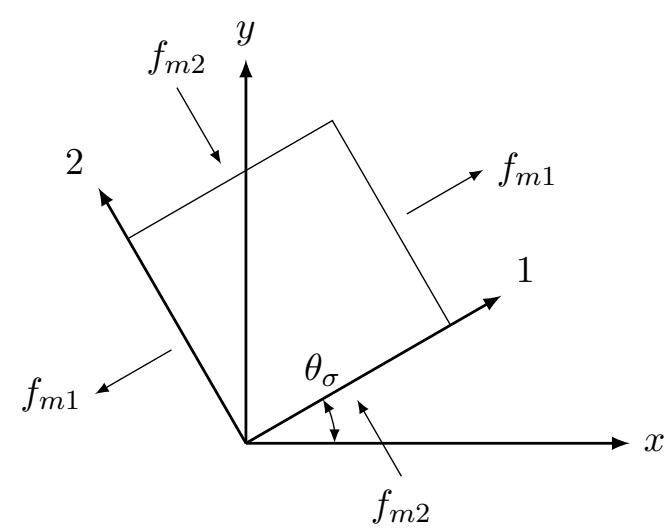

(c) Principal Stresses

Figure 10.1: Global and Principal Stresses within a Region of Wall 


\section{$10.1 \quad$ Assumptions}

The SAFEM utilizes the following assumptions:

1. Blocks are placed in a half-running bond pattern.

2. The masonry structure consists of rectangular blocks that are mutually connected by head and bed mortar joints having a constant thickness and spacing.

3. There is an unique stress state for each strain state, without consideration of strain history.

4. Strains and stresses are averaged over a distances including several cracks.

5. Orientations of principal strain, $\theta_{\epsilon}$, and orientations of principal stress, $\theta_{\sigma}$, can be different.

6. Cracks are uniformly distributed and rotating.

7. There are uniformly applied shear and normal stresses.

8. Constitutive relationships are independent for masonry and reinforcement.

9. Any reinforcement is uniformly distributed.

10. Perfect bond exists between reinforcement and masonry.

11. Shear stresses in reinforcement are negligible.

Tensile stresses and tensile strains will be treated as positive quantities and compressive stresses and strains will be taken as negative.

\subsection{Equilibrium Conditions}

Consider the reinforced masonry element shown in Figure 10.2, subjected to subjected to uniform stresses, $\{\sigma\}=\left\{f_{x}, f_{y}, v_{x y}\right\}^{T}$, applied along the membrane edges. The masonry element consists of several blocks connected by mortar joints that are aligned with reference axes, $x$ and $y$. As is typically the case in practice, the head and bed joints are considered perpendicular to each other. The element is orthogonally reinforced and the reinforcement is aligned with the reference axes to allow placement in the hollow cells or knockout webs. 


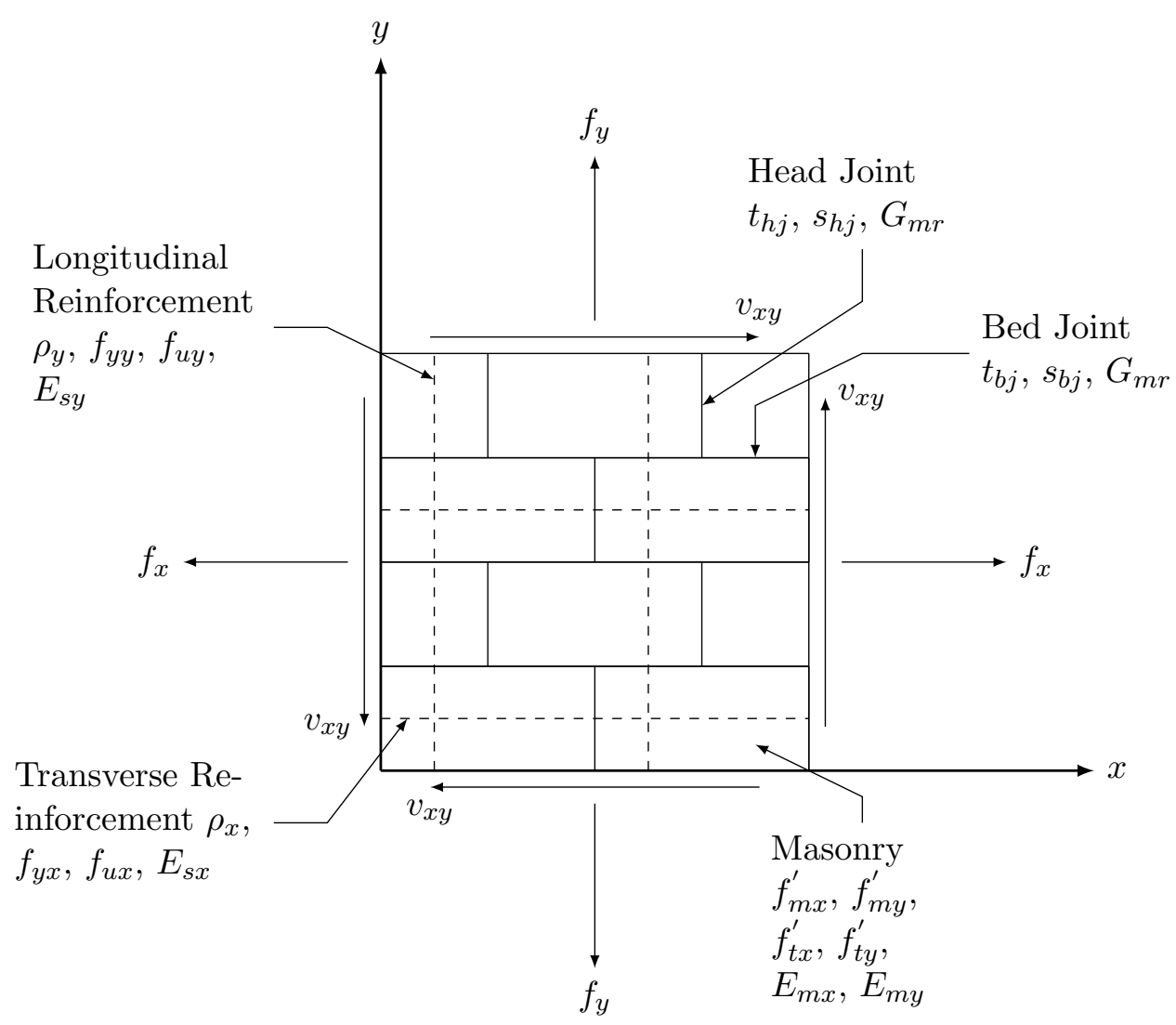

Figure 10.2: General Masonry Element Global Stresses

The forces applied to the element are resisted by internal stresses in the masonry and in the reinforcement. In this context, it is necessary to examine element equilibrium on three levels: in terms of average stresses smeared over the area of the element, in terms of local conditions along the crack surfaces, and in terms of the stresses acting along the mortar joints (see Figure 10.3). 


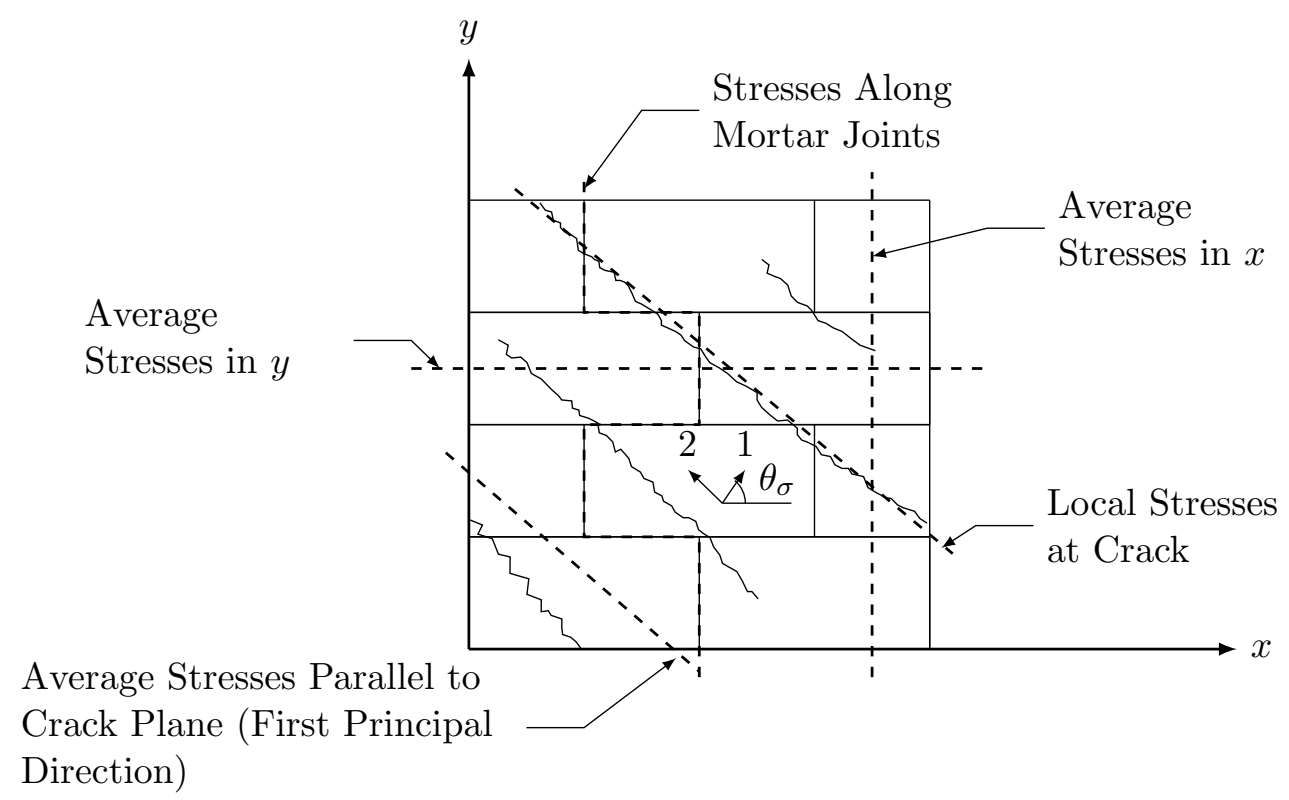

Figure 10.3: Element Equilibrium Planes

SAFEM considers masonry as an orthotropic material that may experience the formation of rotating smeared cracks; thus, the average principal masonry stresses $f_{m 2}$ and $f_{m 1}$ act parallel and perpendicular to the crack plane whose direction is defined by the angle $\theta_{\sigma}$. In order to take into account the tension-softening or tension-stiffening behavior of masonry, the principal stress $f_{m 1}$ is considered active even after cracking.

\subsubsection{Average Stresses}

Average stresses are considered acting in the reinforcement, $f_{s x}$ and $f_{s y}$ for the $x$ and $y$ reinforcement respectively. These average stress conditions are represented in Figure 10.4. However, it should be noted that they do not represent conditions at any one point, but rather average smeared conditions. Hence the element equilibrium for average stress conditions becomes:

$$
\begin{gathered}
f_{x}=f_{m x}+\rho_{x} f_{s x}, \\
f_{y}=f_{m y}+\rho_{y} f_{s y}, \text { and } \\
v_{x y}=v_{m x y}
\end{gathered}
$$

where $f_{m x}$ is the average masonry stress in the $x$-direction, $f_{m y}$ is the average masonry stress in the $y$-direction, and $\rho_{x}$ and $\rho_{y}$ are the reinforcement ratios in the $x$ and $y$ 
directions, respectively. The masonry stresses $f_{m x}, f_{m y}$, and $v_{m x y}$ can be conveniently determined from the principal stresses using the Mohr's circle of stress shown in Figure 10.5 as:

$$
\begin{gathered}
f_{m x}=f_{m 1}-v_{m x y} \cot \left(90-\theta_{\sigma}\right) \text { and } \\
f_{m y}=f_{m 1}-v_{m x y} \tan \left(90-\theta_{\sigma}\right) .
\end{gathered}
$$

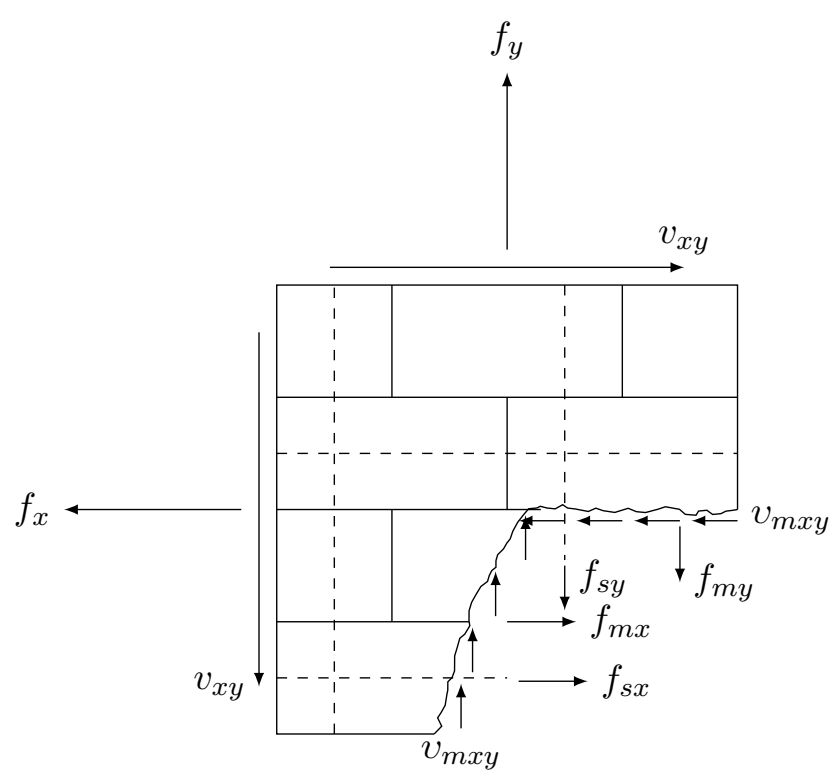

Figure 10.4: Element Average Stress Conditions 


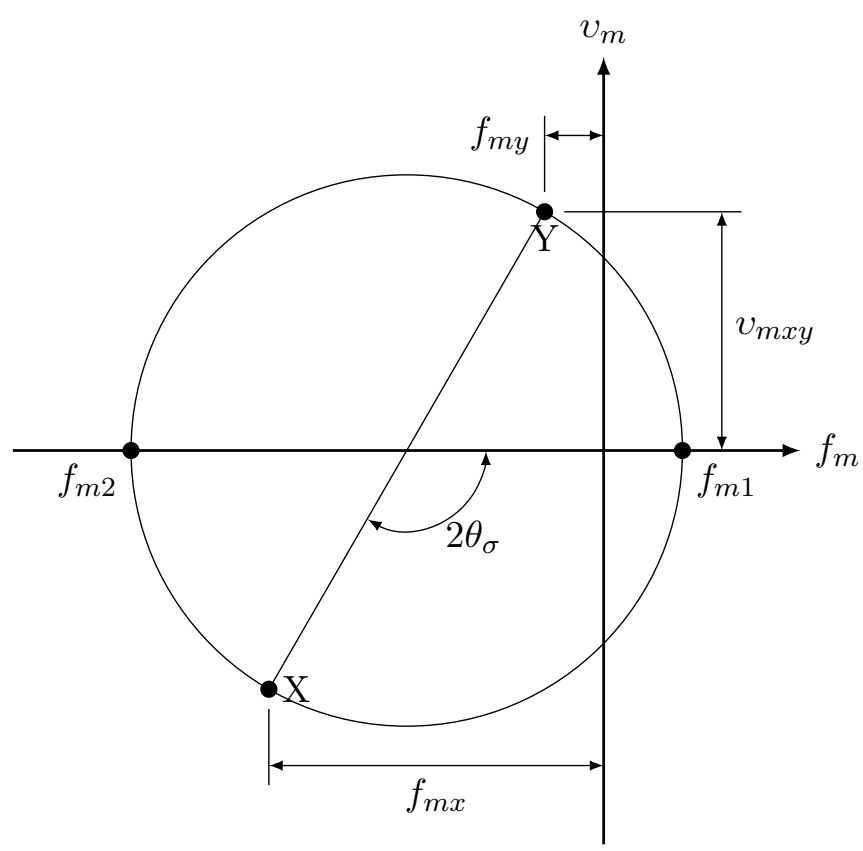

Figure 10.5: Mohr's Circle of Stress for the Element

\subsubsection{Local Crack Surface}

Crack interfaces can be considered planes of weakness in the continuum, and it is necessary to check that the average stresses can be transmitted across the cracks. To transmit the average stress $f_{m 1}$ (Figure 10.6a) local increases in the reinforcement stresses are necessary. These local stresses are denoted by $f_{s c r_{x}}$ and $f_{s c r_{y}}$ for the $x$ and $y$ reinforcement respectively, as shown in Figure 10.6b. The magnitude of $f_{m 1}$ that can be transmitted via this mechanism is limited by the reserve capacity of the reinforcement, which is given by the difference between the average stresses and the yield stresses. Static equivalency of the average and local stresses in the direction normal to the crack surface results in the following equation:

$$
\begin{aligned}
f_{m 1} \leq \rho_{x}\left(f_{s c r_{x}}-f_{s x}\right) \cos ^{2} \theta_{n x} & \\
& +\rho_{y}\left(f_{s c r_{y}}-f_{s y}\right) \cos ^{2} \theta_{n y} \text { (Vecchio and Collins, 1986) }
\end{aligned}
$$

where $\theta_{n x}$ and $\theta_{n y}$ are the angles between the normal to the crack and the reinforcement $\left(\theta_{\sigma}-\alpha\right.$, where $\alpha$ is the angle from the x-axis to the reinforcement). Local reinforcement stresses, $f_{s c r_{x}}$ and $f_{s c r_{y}}$, are determined from the local reinforcement strains $\epsilon_{s c r_{x}}$ and $\epsilon_{s c r_{y}}$.

Part III: Masonry Finite Element Modeling 
The local increases in reinforcement stresses, at the crack locations, lead to the development of shear stresses along the crack surface, $v_{m i}$. Static equivalency of average and local stresses in the direction tangential to the crack determines the local shear stresses as follows:

$$
\begin{aligned}
v_{m i}=\rho_{x}\left(f_{s c r_{x}}-\right. & \left.f_{s x}\right) \cos \theta_{n x} \sin \theta_{n x} \\
& +\rho_{y}\left(f_{s c r_{y}}-f_{s y}\right) \cos \theta_{n y} \sin \theta_{n y}(\text { Vecchio and Collins, 1986) }
\end{aligned}
$$

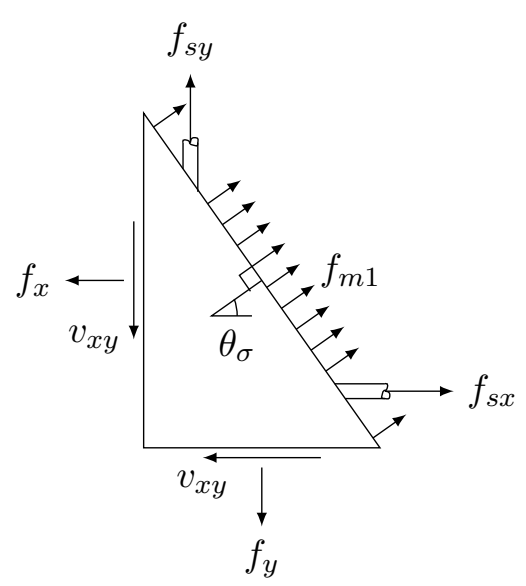

(a) Average Stresses Parallel to Crack

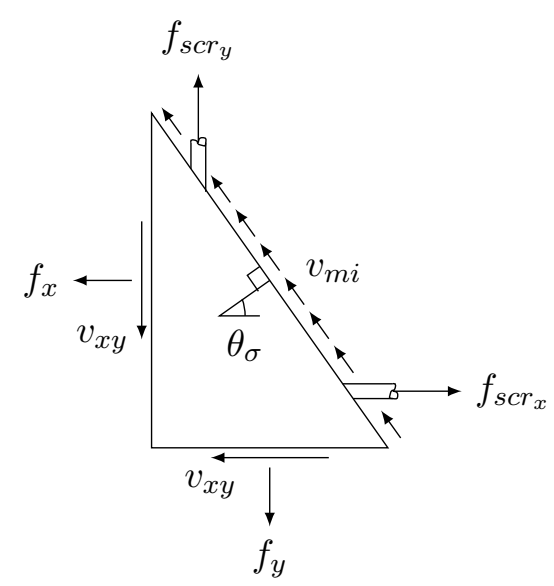

(b) Local Stresses at a Crack

Figure 10.6: Reinforced Masonry Stress Conditions at Crack (Vecchio and Collins, 1986)

However, the above approach is problematic as the equilibrium equations predict that the shear stress at a crack are always zero for unreinforced masonry. This is equivalent to ignoring crack shear stresses arising from aggregate interlock (see Figure 10.7). To address this deficiency a constant rotation lag, $\theta_{l}$, between the inclination of the principal total strain axis, $\theta_{\epsilon}$ and the inclination of the principal stress axis, $\theta_{\sigma}$, can be specified as discussed in the material relation section. 


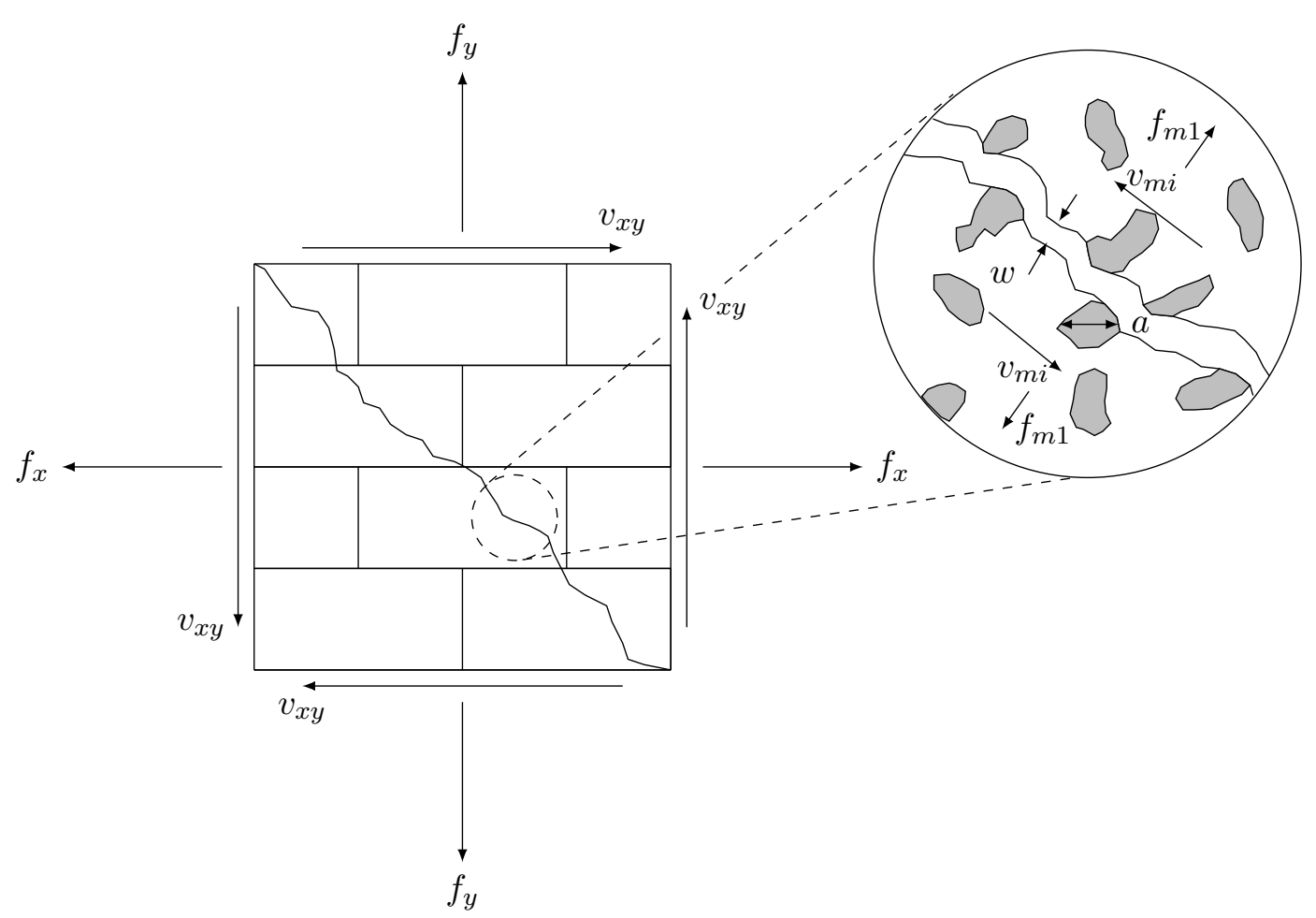

Figure 10.7: Masonry Aggregate Interlock (Vecchio and Collins, 1986)

\subsubsection{Local Mortar Joints}

The local internal stresses acting at the mortar joint locations may be derived from the equilibrium conditions of the masonry element; therefore, with reference to the Mohrs circle of stress shown in Figure 10.5 and to the equilibrium conditions depicted in Figure 10.8, the joints stresses result from standard relations:

$$
\begin{gathered}
f_{m x}=f_{n h j}=\frac{f_{m 1}+f_{m 2}+\left(f_{m 1}-f_{m 2}\right) \cos 2 \theta_{\sigma}}{2}, \\
f_{m y}=f_{n b j}=\frac{f_{m 1}+f_{m 2}-\left(f_{m 1}-f_{m 2}\right) \cos 2 \theta_{\sigma}}{2}, \text { and } \\
v_{m x y}=v_{b j}=v_{h j}=\frac{\left(f_{m 1}-f_{m 2}\right) \sin 2 \theta_{\sigma}}{2} .
\end{gathered}
$$

According to the equilibrium condition represented above, the head joints shown in Figure 10.8 are assumed to be all aligned and not staggered as is typically the case in hollow concrete block masonry, the staggered head joints will be included in the masonry tensile strength constitutive models. Additionally there is no contribution due to reinforcement as it is considered in the average stress and it is assumed that there is no shear stress in the reinforcement. 


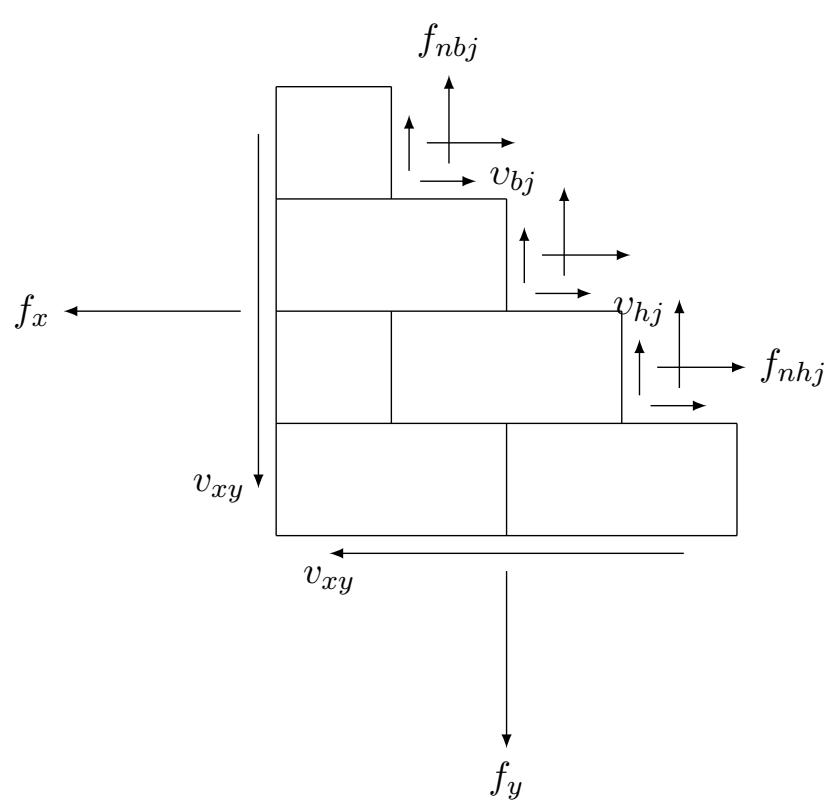

Figure 10.8: Masonry Joint Stresses

\subsection{Compatibility Conditions}

The compatibility conditions that characterize the response of the masonry elements are represented by the illustrations shown in Figure 10.9. It is assumed that total deformations exhibited by the masonry element is from the superposition of four components:

1. The strain resulting from the deformation of the continuum due to the applied stresses, with the cracks considered smeared within the element area;

2. The strain resulting from the deformations due to local rigid body slip along a crack;

3. The strain that results from the rigid body slip occurring along bed and head joints; and,

4. The strain due to Poisson's effects. 

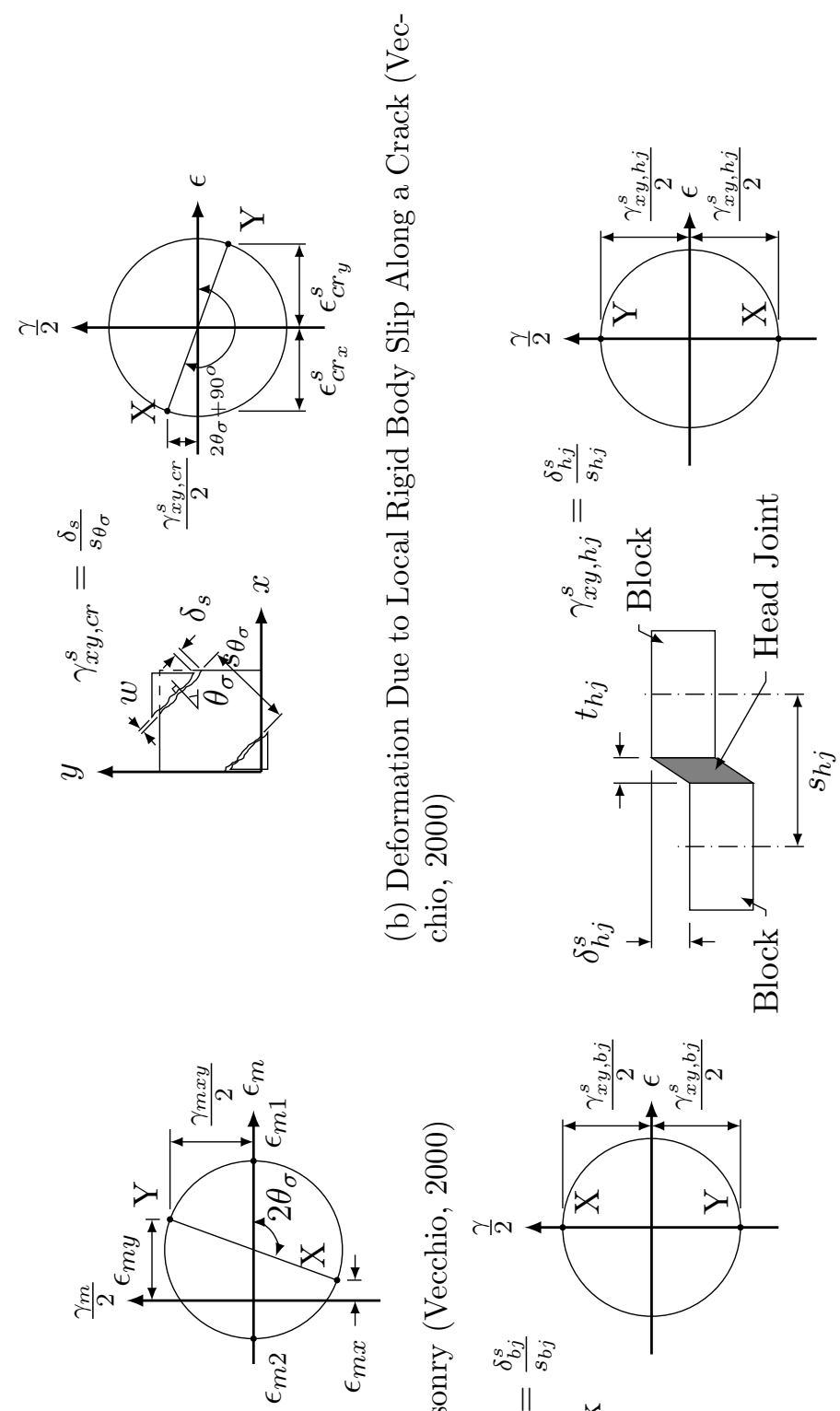

$$
\text { ॠ }
$$

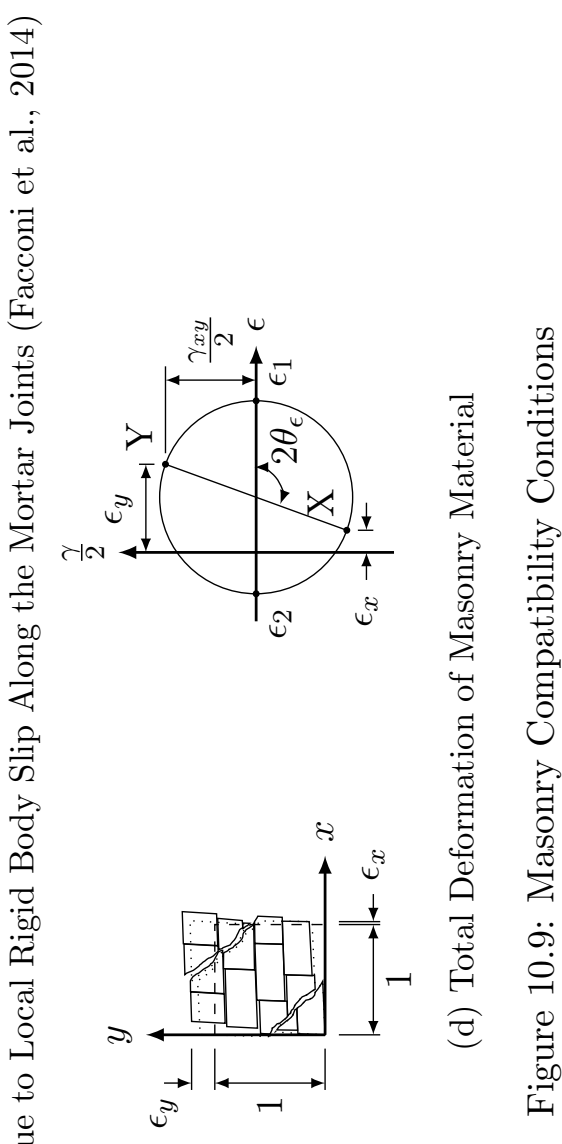

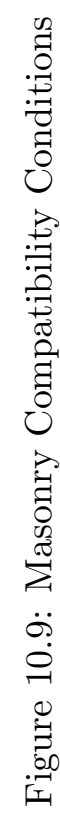
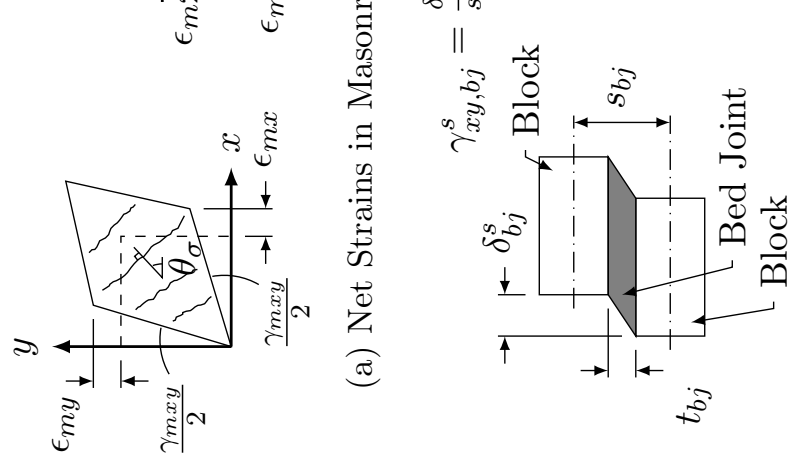
Additionally, having assumed that the reinforcement is perfectly bonded to the masonry, compatibility requites that the total strain in both the masonry and the reinforcement must be the same. Hence the total element strains $\{\epsilon\}$, for the masonry:

$$
\{\epsilon\}=\left\{\epsilon_{m}\right\}+\left\{\epsilon_{m}^{v}\right\}+\left\{\epsilon_{m}^{s}\right\}
$$

where $\left\{\epsilon_{m}\right\}$ is the masonry net strain used in stress calculations, $\left\{\epsilon_{m}^{v}\right\}$ is the strain due to Possion effects, and $\left\{\epsilon_{m}^{s}\right\}$ is the strain due to slip equal to:

$$
\left\{\epsilon_{m}^{s}\right\}=\left\{\epsilon_{\mathrm{cr}}^{s}\right\}+\left\{\epsilon_{b j}^{s}\right\}+\left\{\epsilon_{h j}^{s}\right\} .
$$

For the reinforcement:

$$
\{\epsilon\}=\left\{\epsilon_{s}\right\}
$$

where $\left\{\epsilon_{s}\right\}$ is the reinforcement net strain used in stress calculations.

Additional. the angle $\theta_{\epsilon}$, which denotes the inclination of the total principal strains, can be derived as follows:

$$
\theta_{\epsilon}=\frac{1}{2} \tan ^{-1}\left[\frac{\gamma_{x y}}{\epsilon_{x}-\epsilon_{y}}\right]
$$

\subsubsection{Net Strains}

The Mohrs circle of strain shown in Figure 10.9a can be used to determine the principal strains from the net strains as:

$$
\epsilon_{m 1}, \epsilon_{m 2}=\frac{\epsilon_{m x}+\epsilon_{m y}}{2} \pm \frac{1}{2} \sqrt{\left(\epsilon_{m x}-\epsilon_{m y}\right)^{2}+\tau_{m x y}^{2}}
$$

The inclination of the cracks within the continuum is assumed to be coincident with the inclination of the net principal strains and the inclination of the principal stresses, $\theta_{\sigma}$. Using a rotating crack concept, $\theta_{\sigma}$ becomes:

$$
\theta_{\sigma}=\frac{1}{2} \tan ^{-1}\left[\frac{\gamma_{m x y}}{\epsilon_{m x}-\epsilon_{m y}}\right]
$$

\subsubsection{Crack Shear Slip Strain}

The crack shear slip strain, $\gamma_{s}$, is computed by:

$$
\gamma_{s}=\frac{\delta_{s}}{s_{\theta_{\sigma}}}
$$


where $s_{\theta_{\sigma}}$ is the crack spacing, defined as:

$$
s_{\theta_{\sigma}}=\frac{1}{\frac{\sin \theta_{\sigma}}{s_{m x}}+\frac{\cos \theta_{\sigma}}{s_{m y}}} \text { (Wong et al., 2013) }
$$

where $s_{m x}$ and $s_{m y}$ are the crack spacing in the $x$ and $y$ directions respectively defined by the block length and height. Now a slip strain vector can be defined as:

$$
\left\{\epsilon_{c r}^{s}\right\}=\left\{\begin{array}{c}
\epsilon_{x}^{s} \\
\epsilon_{y}^{s} \\
v_{x y}^{s}
\end{array}\right\}=\left\{\begin{array}{c}
-\frac{\gamma_{s}}{2} \sin 2 \theta_{\sigma} \\
\frac{\gamma_{s}}{2} \sin 2 \theta_{\sigma} \\
\gamma_{s} \cos 2 \theta_{\sigma}
\end{array}\right\}
$$

where the components of the vector can be resolved using a Mohr's circle, see Figure $10.9 \mathrm{~b}$.

\subsubsection{Mortar Joint Shear Strains}

It is assumed that the masonry element consists of rectangular blocks that are mutually connected by bed and head mortar joints having a constant thickness $\left(t_{b j}, t_{h j}\right)$ and spacing $\left(s_{b j}, s_{h j}\right)$, see Figure 10.9c. The shear stress acting parallel to the joint causes a local slip displacement along the joint plane whose magnitude is $\delta_{b j}^{s}$ for the bed joints and $\delta_{h j}^{s}$ for the head joints. Thus, the average shear strain due to the slip of bed and head joints may be respectively defined as follows:

$$
\begin{gathered}
\gamma_{b j}^{s}=\frac{\delta_{b j}^{s}}{s_{b j}} \text { and } \\
\gamma_{h j}^{s}=\frac{\delta_{h j}^{s}}{s_{h j}} .
\end{gathered}
$$

The total slip strain can be decomposed into orthogonal components relative to the $x-y$ reference system, which can be computed respectively for bed $\left\{\epsilon_{b j}^{s}\right\}$ and head $\left\{\epsilon_{b j}^{s}\right\}$ by Mohrs circle construction (Figure 10.9c).

$$
\begin{gathered}
\left\{\epsilon_{b j}^{s}\right\}=\left\{\begin{array}{c}
\epsilon_{x, b j}^{s} \\
\epsilon_{y, b j}^{s} \\
\gamma_{x y, b j}^{s}
\end{array}\right\}=\left\{\begin{array}{c}
0 \\
0 \\
-\gamma_{b j}^{s}
\end{array}\right\} \text { and } \\
\left\{\epsilon_{h j}^{s}\right\}=\left\{\begin{array}{c}
\epsilon_{x, h j}^{s} \\
\epsilon_{y, h j}^{s} \\
\gamma_{x y, h j}^{s}
\end{array}\right\}=\left\{\begin{array}{c}
0 \\
0 \\
\gamma_{h j}^{s}
\end{array}\right\} .
\end{gathered}
$$

The sum of the resultant vectors provides the equivalent average strain slip vector $\left\{\epsilon_{m}^{s}\right\}$. 


\subsubsection{Poisson's Strain Effects}

When masonry is uncracked or experiences relatively small tensile strains, lateral expansion may account for a significant portion of the total strains in the principal maximum strain direction. If these strains are incorrectly attributed to tensile straining due to stress, the compression softening effect may be overestimated. To address this potential inaccuracy, SAFEM implements masonry lateral expansion effects by modifying the formulation of the stress-strain relationship.

For a linear-elastic material that is orthotropic in the principal directions, plane stresses $\left[\sigma_{1}, \sigma_{2}, \tau_{12}\right]^{T}$ are related to plane strains $\left[\epsilon_{1}, \epsilon_{2}, \gamma_{12}\right]^{T}$ by the material stiffness matrix, $[D]$, where

$$
[D]=\frac{1}{1-v_{12} v_{21}}\left[\begin{array}{ccc}
E_{1} & v_{12} E_{1} & 0 \\
v_{21} E_{2} & E_{2} & 0 \\
0 & 0 & \left(1-v_{12} v_{21}\right) G_{12}
\end{array}\right]
$$

where $v_{12}$ is the Poisson's ratio relating $\epsilon_{1}$ to $\sigma_{2}, v_{21}$ is the Poisson's ratio relating $\epsilon_{2}$ to $\sigma_{1}$, and the shear modulus is approximated as:

$$
G_{12}=\frac{E_{1} E_{2}}{E_{1}\left(1+v_{12}\right)+E_{2}\left(1+v_{21}\right)} .
$$

For the symmetry of $[D]$ the off-diagonal elements must be equal; thus,

$$
v_{12} E_{1}=v_{21} E_{2}
$$

The response of masonry violates the above condition, such that the material stiffness matrix becomes unsymmetrical (Selby and Vecchio, 1997). Consider a two-dimensional element under biaxial compression, see Figure 10.10a, such that $f_{m 2}<f_{m 1}<0$. The constitutive response of masonry in compression is characterized by a progressively softening stress-stain curve Figure 10.10b. Also, masonry exhibits a progressively higher proportion of lateral expansion as compression is increased, see Figure 10.10c. Thus, it will general be the case that $E_{m 1}>E_{m 2}, v_{12}>v_{21}$, and $v_{12} E_{1} \neq v_{21} E_{2}$. In SAFEM, the symmetry conditions are enforced in the material stiffness matrix, all off-diagonal are zero, and the asymmetry of the masonry lateral expansion response is handled through the material prestrain concept (Vecchio, 1990); thus,

$$
\left\{\epsilon_{m}^{v}\right\}=\left\{\begin{array}{c}
\epsilon_{m x}^{v} \\
\epsilon_{m y}^{v} \\
v_{m x y}^{v}
\end{array}\right\}=\left\{\begin{array}{c}
\frac{1}{2} \epsilon_{m 1}^{v}\left(1+\cos 2 \theta_{\sigma}\right)+\frac{1}{2} \epsilon_{m 2}^{v}\left(1-\cos 2 \theta_{\sigma}\right) \\
\frac{1}{2} \epsilon_{m 1}^{v}\left(1-\cos 2 \theta_{\sigma}\right)+\frac{1}{2} \epsilon_{m 2}^{v}\left(1+\cos 2 \theta_{\sigma}\right) \\
\epsilon_{m 1}^{v} \sin 2 \theta_{\sigma}-\epsilon_{m 2}^{v} \sin 2 \theta_{\sigma}
\end{array}\right\}
$$

Part III: Masonry Finite Element Modeling 
where

$$
\epsilon_{m 1}^{v}=-v_{12} \epsilon_{m 2}
$$

and

$$
\epsilon_{m 2}^{v}=-v_{21} \epsilon_{m 1}
$$
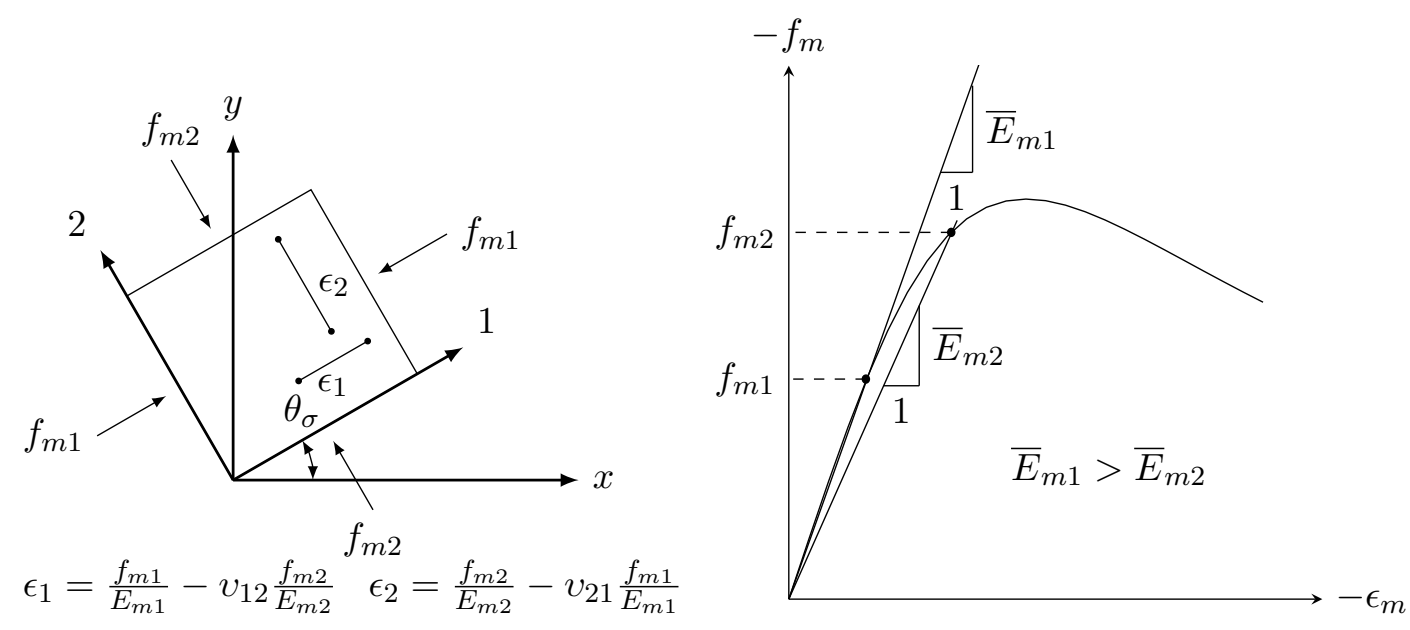

(a) Masonry Element Stresses and Strains

(b) Principal Masonry Modulus of Elasticity

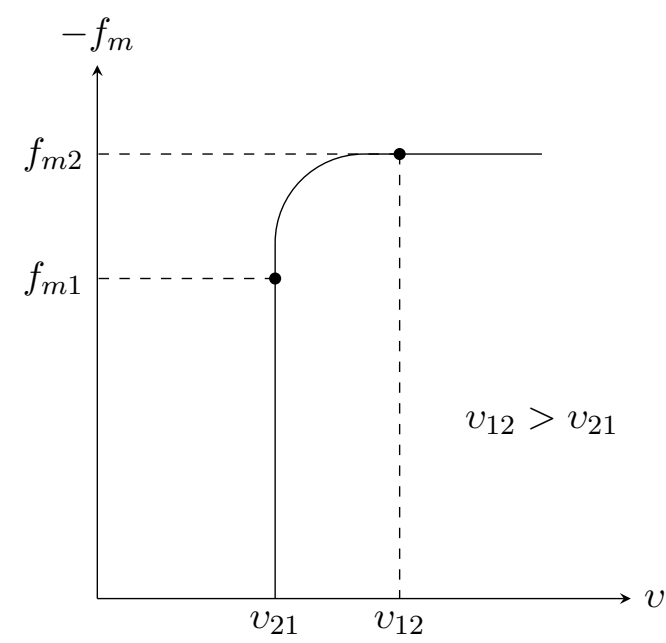

(c) Principal Poisson's Ratios

Figure 10.10: Poisson's Strain Effects (Selby and Vecchio, 1997) 


\subsection{Stress-Strain Relations}

Constitutive relationships are required to link average stress to average strain for both the masonry and the reinforcement. Compression stress-strain relationships for grouted masonry are similar to those for concrete and may be represented by similar equations (Paulay and Priestley, 1992). However, ungrouted masonry under compression exhibits different directional properties due to mortar joints acting as planes of weakness. Hence,the material failure cannot be simply defined in terms of principal stresses but needs an additional parameter, i.e. the bed joint orientation relative to the principal stress direction. Under tension ungrouted and grouted masonry exhibits anisotropic behaviour due to the mortar joints and the running bond pattern used in general construction. Once this behaviour is considered tension stress-strain relationships similar to concrete may be used to model the behaviour of masonry under tension.

The axial stress in the reinforcement will be assumed to depend on only one strain parameter, the axial strain in the reinforcement. It will further be assumed that average shear stress on the plane normal to the reinforcement resisted by the reinforcement is zero.

\subsection{Proposed Material Models}

To describe the response of ungrouted, grouted, and reinforced masonry, models are required. First, models to convert the anisotropic nature of masonry into an orthotropic material for use in a smeared area model are presented. Secondly, the masonry compressive stress-strain response is discussed followed by an associated model for compression softening. Thirdly, the masonry tensile stress-strain response is discussed followed by the associated models of tension stiffening and softening. Fourthly, a lateral expansion model for masonry is described. Fifthly, cracking criterion, crack stress calculations, and slip distortion models for masonry are considered. Then a model for the masonry joint slip is presented. Lastly, a model for the response of steel reinforcement is discussed.

\subsubsection{Masonry Anisotropic Nature}

As masonry is considered an orthotropic material in the SAFEM, a number of material properties that are direction dependent need to be converted to a single value for use in the model.

Part III: Masonry Finite Element Modeling 


\section{Ungrouted Masonry Compression Strength Under Combined Loading}

Ungrouted masonry exhibits different directional properties due to the mortar joints acting as planes of weakness. Hence, the material failure cannot be simply defined in terms of principal stresses but needs an additional parameter, $\beta_{a b}$, to account for the bed joint orientation relative to the principal stresses direction. Hamid and Drysdale (1982) proposed a simple analytical failure criterion for masonry subjected to in-plane forces that accounts for the anisotropic nature of ungrouted masonry. The model considers two modes of failure: a shear-slip failure along the bed or head joint or tensile splitting failure due to incompatible deformational characteristics of stronger blocks and weaker mortar joints.

\section{Shear-Slip Failure}

Shear failure of mortar joints is governed by a Mohr-Coulomb failure surface, that is:

$$
\tau=c+\sigma_{n} \tan \phi
$$

where $\tau$ is the average shear strength along the slip plane, $c$ is the cohesion, $\sigma_{n}$ is the compressive stress normal to the slip plane and $\phi$ is the angle of internal friction.

Under combined stresses, the normal, $\sigma_{\theta}$, and shear, $\tau_{\theta}$, stresses acting along an arbitrary plane having an angle $\theta$ from the x-axis, see Figure 10.11, can be expressed in terms of the stress $\sigma_{x}$ and $\sigma_{y}$ as follows:

$$
\sigma_{\theta}=\frac{\sigma_{x}+\sigma_{y}}{2}+\frac{\sigma_{y}-\sigma_{x}}{2} \cos 2 \theta_{\sigma}
$$

and

$$
\tau_{\theta}=\frac{\sigma_{y}-\sigma_{x}}{2} \sin 2 \theta_{\sigma}
$$




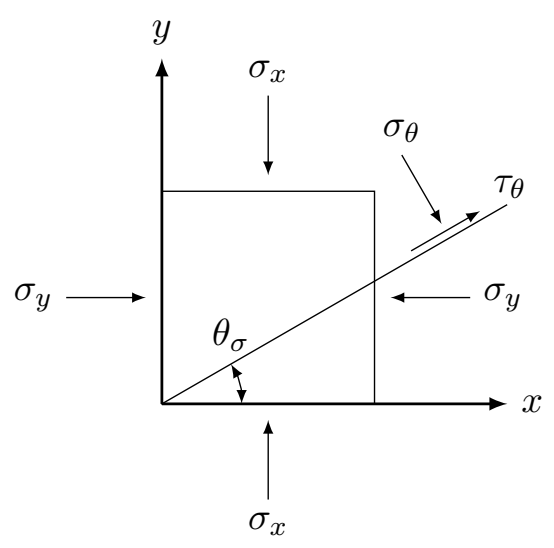

Figure 10.11: Element Stresses at Angle $\theta$

The condition for slip on a joint, $j$ is governed by:

$$
\tau_{\theta}=c_{j}+\sigma_{\theta} \tan \phi_{j} .
$$

Thus Equation 10.31 and Equation 10.32 into Equation 10.33, an expression for the stress difference required to produce slip can be written as:

$$
\sigma_{y}-\sigma_{x}=\frac{2\left(c_{j}+\sigma_{x} \tan \phi_{j}\right)}{\left(1-\tan \phi_{j} \cot \theta\right) \sin 2 \theta_{\sigma}} .
$$

Since the bed joint is continuous single plane of weakness, this equation can be applied directly $(j=b j)$ (Hamid and Drysdale, 1982). For shear along the head joint, it is possible that either just the mortar head joint or both the block and the mortar head joint would contribute to the strength. Assuming that the angle of internal friction for the mortar and the block are the same, Equation 10.34 can also be used for the head joints $(j=h j$ ) (Hamid and Drysdale, 1982).

\section{Tensile Splitting Failure}

If the compression component of the stress normal to the weak plane, $\sigma_{\theta}$, is high, the slip failure may be suppressed, thereby forcing failure to occur through the block material in the form of tensile splitting. This is due to the incompatible material properties of the strong block and weaker mortar (Hamid and Drysdale, 1982).

Due to the anisotropic nature of unreinforced masonry, the compressive strength varies with orientation of the stress with respect to the bed joint. Adopting the maximum stress theory, the strength for compression perpendicular to the bed joint $(\theta=0)$ is given by $f_{m y}^{\prime}$ and parallel to the bed joint $\left(\theta=90^{\circ}\right), f_{m x}^{\prime}$. These stresses are assumed to be constant to the point of transition to the shear-slip failures.

Part III: Masonry Finite Element Modeling 


\section{Failure Envelope}

The single plane of weakness theory has been applied to shear-slip failure along the weak bed and head joints and the maximum stress theory has been applied to splitting tension failure. When the results are superimposed, as shown in Figure 10.12. The minimum values within each of the three regions $\left(0^{\circ}\right.$ to $45-\phi_{h j} / 2,45-\phi_{h j} / 2$ to $45+\phi_{b j} / 2$, and $45+\phi_{b j} / 2$ to $\left.90^{\circ}\right)$ defines the failure envelope.

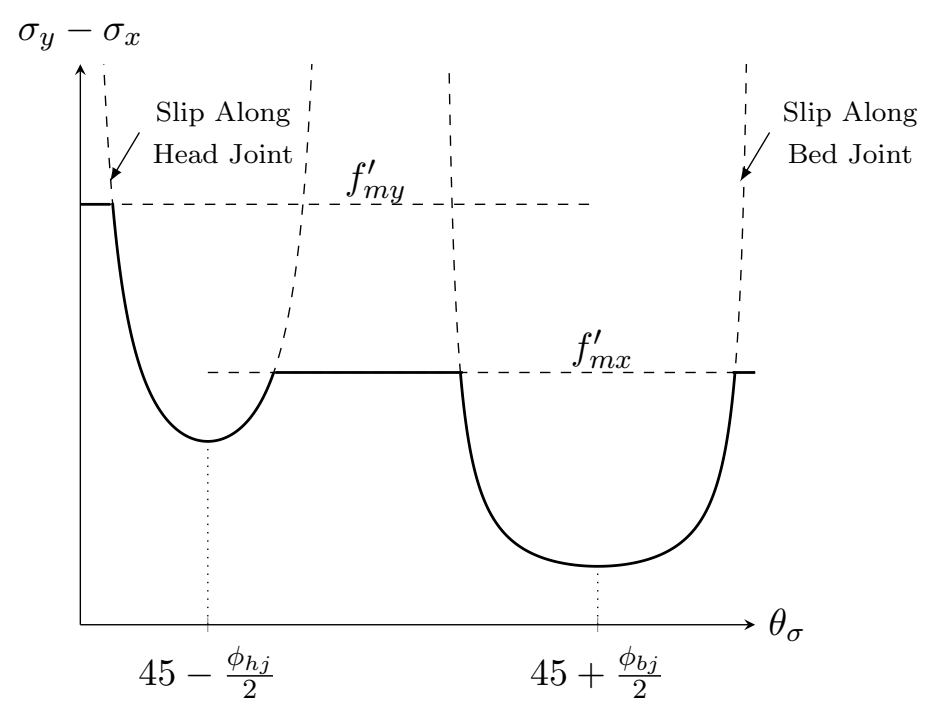

— Failure Envelope

Figure 10.12: Proposed Failure Envelope For Hamid and Drysdale (1982) Failure Model

\section{Hamid and Drysdale Model}

Taking the above failure criteria and ignoring any benefits of confinement (compression softening is taken into account through $\left.\beta_{c s}\right), \beta_{a b}$ becomes:

$$
\beta_{a b}= \begin{cases}\frac{f\left(\theta_{\theta}\right)}{f_{m y}^{\prime}} & \text { if ungrouted } \\ 1 & \text { if grouted }\end{cases}
$$

where for the ungrouted case the failure criteria is a function of $\theta_{\sigma}$. Slipping stress along the head joint is given by:

$$
f_{h j}^{s}=\frac{2 c_{h j}}{\left(1-\tan \phi_{h j} \cot \left(\theta_{\sigma}+\phi_{h j}\right)\right) \sin \left(2\left(\theta_{\sigma}+\phi_{h j}\right)\right)}
$$

and the slipping along the bed joint is given by:

$$
f_{b j}^{s}=\frac{2 c_{b j}}{\left(1-\tan \phi_{b j} \cot \theta_{\sigma}\right) \sin 2 \theta_{\sigma}}
$$


where $C_{h j}$ and $C_{b j}$ are the cohession, $\phi_{h j}$ and $\phi_{b j}$ are the angle of internal friction for the head joint (combination of head joint mortar and block) and bed joint mortar respectively, and $\theta_{\sigma}$ is the principal stress direction. Thus, the failure criteria becomes:

$$
f\left(\theta_{\sigma}\right)= \begin{cases}\min \left(f_{m y}^{\prime}, f_{h j}^{s}\right) & \text { if } 0 \leq \theta_{\sigma} \leq 45-\frac{\phi_{h j}}{2} \\ \min \left(f_{m y}^{\prime}, f_{h j}^{s}, f_{m x}^{\prime}, f_{b j}^{s}\right) & \text { if } 45-\frac{\phi_{h j}}{2} \leq \theta_{\sigma} \leq 45+\frac{\phi_{b j}}{2} \\ \min \left(f_{b j}^{s}, f_{m x}^{\prime}\right) & \text { if } 45+\frac{\phi_{b j}}{2} \leq \theta_{\sigma} \leq 90\end{cases}
$$

where $f_{m y}^{\prime}$ is the strength for compression perpendicular to the bed joints and $f_{m x}^{\prime}$ is the compression strength parallel to the bed joints.

\section{Masonry Under Tension}

The inherent anisotropy of masonry as a composite of materials leads to mechanical behaviour characteristics quite different from those of conventional isotropic material and consequently, there is no single value of tensile strength, but different values for different directions of the tensile stresses (Hamid, 1978).

\section{Masonry Tensile Strength Normal to the Bed Joints}

Under tensile stresses normal to the bed joints, separation occurs at the block-mortar interfaces including tensile failure of the cores for the cases of grouted masonry. For ungrouted masonry the capacity is governed by the tensile bond strength of the mortar acting along the block-mortar contact area, while for grouted masonry the grout will contribute to the tensile strength by its tensile capacity which is a function of the core area and the grout tensile strength (Hamid, 1978). The tensile strength of the masonry can be described by incorporating the resistance of the component materials as follows:

$$
f_{t y}= \begin{cases}f_{t, b b j} & \text { if ungrouted } \\ \rho_{\text {solid }}^{b l} f_{t, b b j}+\left(1-\rho_{\text {solid }}^{b l}\right) f_{t, g r}^{\prime} & \text { if grouted }\end{cases}
$$

where $f_{t, b b j}$ is the tensile bond strength of the mortar, $\rho_{\text {solid }}^{b l}$ is the percent solid of the block, and $f_{t, g r}^{\prime}$ is the tensile strength of the grout. 


\section{Masonry Tensile Strength Parallel to the Bed Joints}

Under tensile stresses parallel to the bed joints, there are two possible modes of failure:

- Mode I: Splitting failure along a plane passing through the head joints and the block's face shells even for grouted masonry as shown in Figure 10.13a; or,

- Mode II: A stepped failure surface passing through the head and bed joints as shown in Figure Figure 10.13b. It is a joint failure as the blocks suffer no damage. For ungrouted masonry, failure occurs at the block-mortar interfaces, whereas the failure of grouted masonry is similar but with shearing of the cores at the horizontal section of minimum grout area. (Hamid, 1978)

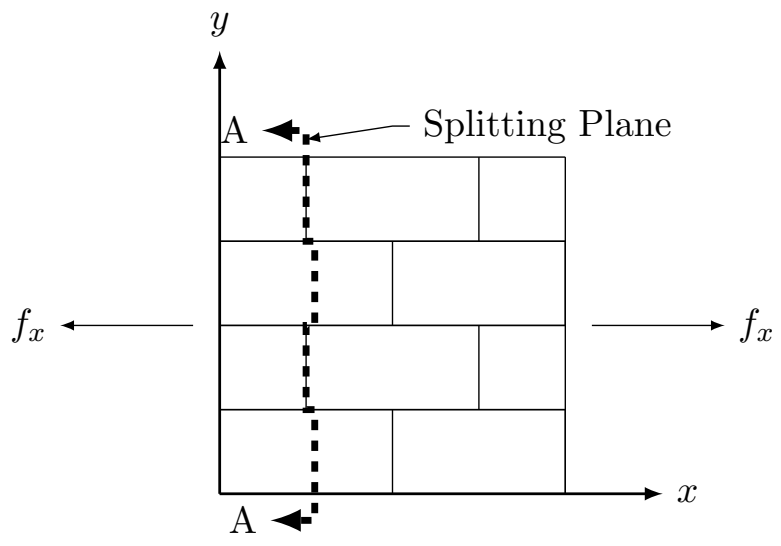

(a) Mode I Failure

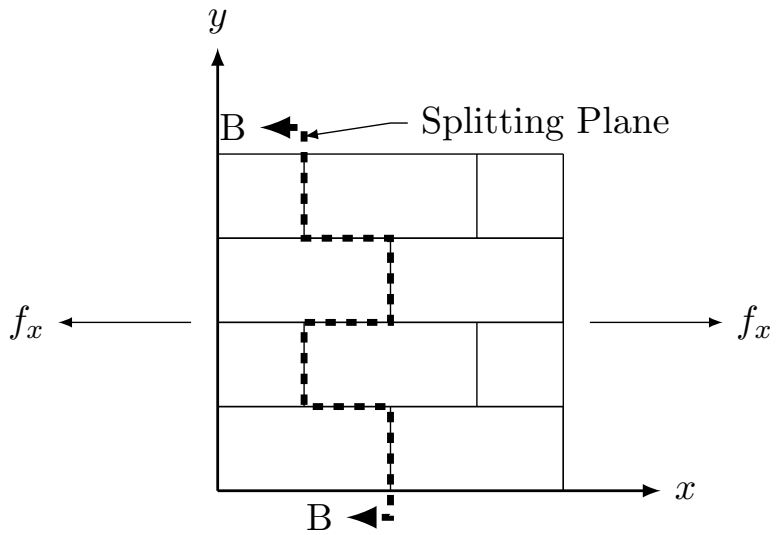

(b) Mode II Failure

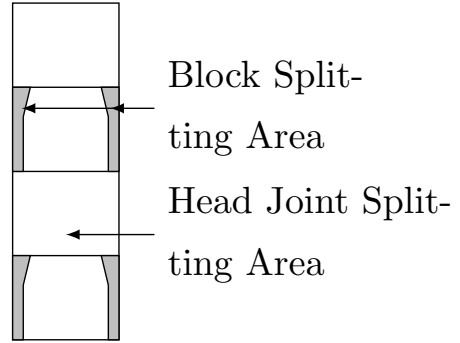

Section A-A

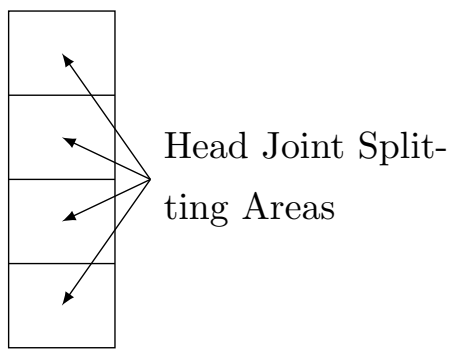

Section B-B

Figure 10.13: Reinforced Masonry Stress Conditions (Hamid, 1978) 
The governing mode is the one giving the lower capacity:

$$
f_{t x}=\min \left(f_{t p-I}, f_{t p-I I}\right)
$$

The tensile stress associated with failure is described by:

$$
f_{t p-I}=\frac{1}{2}\left(f_{t, h j}+n_{v} f_{t, b l}^{\prime}\right)
$$

where $f_{t, h j}$ is the tensile bond strength of the head joint, $f_{t, b l}^{\prime}$ is the tensile strength of the block, and $n_{v}$ is the ratio of net to gross area for the vertical section of the block:

$$
n_{v}=\frac{2 t^{f s}}{w^{b l}}
$$

For mode II failure the tensile strength can be determined by:

$$
f_{t p-I I}=f_{t, h j}+\frac{1.3 l^{b l}}{2 h^{b l}} \rho_{\text {solid }}^{b} f_{t, h j}+\frac{l^{b l}}{2 h^{b l}}\left(1-\rho_{\text {solid }}^{b l}\right) f_{t, g r}^{\prime}
$$

where $l^{b}$ is the length of the block and $h^{b}$ is the height of the block; ungrouted masonry causes $f_{t, g r}^{\prime}$ to be zero (Hamid, 1978). 


\section{Masonry Properties at an Arbitrary Angle}

The initial elastic modulus $\left(E_{m}\right)$, peak strain $\left(\epsilon_{o}\right)$, initial Poisson's ratio $\left(v_{o}\right)$, peak tensile stress $\left(f_{t}\right)$, and peak tensile strain $\left(\epsilon_{t}\right)$ are all dependent on the direction of loading. In direction $\theta_{\sigma}$ the property, $P$, becomes:

$$
P\left(\theta_{\sigma}\right)=P_{x} \cos ^{2} \theta_{\sigma}+P_{y} \sin ^{2} \theta_{\sigma}
$$

where $P_{x}$ and $P_{y}$ are the property in the $x$ and $y$-direction respectively (Ghiassi et al., 2012). Equation 10.44 is illustrated in Figure 10.14.

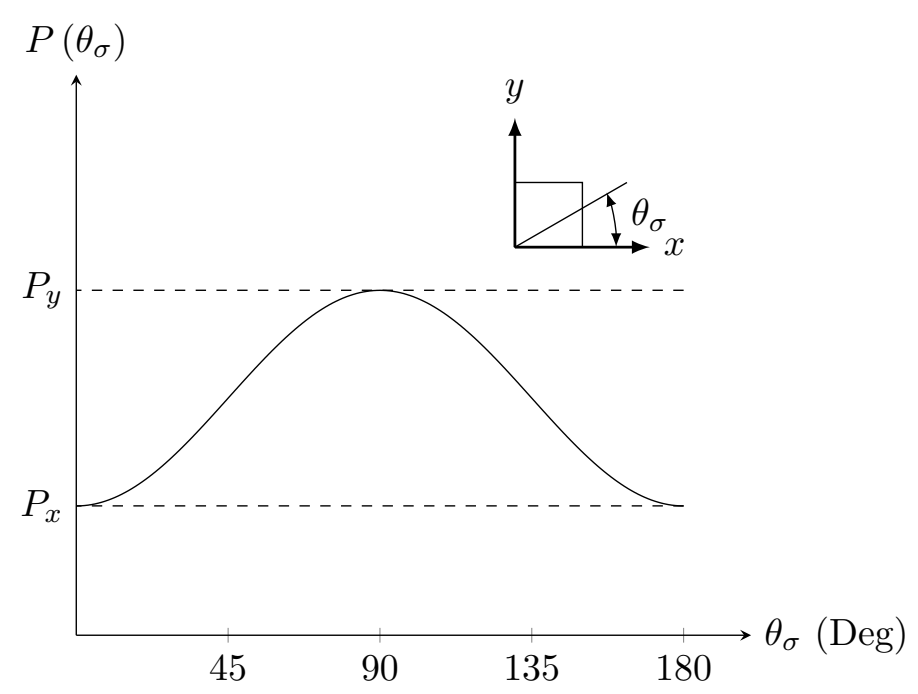

Figure 10.14: Property Value at $\theta_{\sigma}$

Additional the masonry peak compression strength, $f_{m}^{\prime}$, is determined from:

$$
f_{m}^{\prime}= \begin{cases}f_{m x}^{\prime} \cos ^{2} \theta_{\sigma}+f_{m y}^{\prime} \sin ^{2} \theta_{\sigma} & \text { if grouted } \\ f_{m y}^{\prime} & \text { if ungrouted }\end{cases}
$$

where the masonry peak compression strength for ungrouted masonry is modified to account for the direction of loading by anisotropic behaviour factor, $\beta_{a b}$. 


\subsubsection{Constitutive Models for Masonry Pre-Peak Compression}

Compression pre-peak response models compute the principal compressive stress, $f_{m i}$, if the compressive principal strain $\epsilon_{m i}$ is greater than the strain $\epsilon_{p}$ corresponding to the peak compressive stress, $f_{p}$. The peak compression strength is determine by adjusting the masonry compression strength $f_{m}^{\prime}$ for compression softening, $\beta_{c s}$, and anisotropic behavior, $\beta_{a b}$; hence,

$$
f_{p}=\beta_{c s} \beta_{a b} f_{m}^{\prime}
$$

where $f_{m}^{\prime}$ is determined as a function of $f_{m x}^{\prime}, f_{m y}^{\prime}$, and $\theta_{\sigma}$.

The peak strain is determined by adjusting the strain $\epsilon_{o}$; which is a function of the peak strain under uniaxial compression perpendicular to the bed joints $\epsilon_{\text {oy }}$, the peak strain under uniaxial compression parallel to the bed joints $\epsilon_{o x}$, and the inclination of the principal stresses, $\theta_{\sigma}$, for compression softening.

$$
\epsilon_{p}=\beta_{c s} \epsilon_{o}
$$

where

$$
\epsilon_{o}=f\left(\epsilon_{o x}, \epsilon_{o y}, \theta_{\sigma}\right)
$$

As there are a number of stress-strain responses that masonry can experience due to block, mortar, and grout combinations, the SAFEM incorporates three compression models: Hognestad Model (1951), Popovics Model (1970), and a linear model.

\section{Hognestad Model}

The Hognestad parabola (1951), as shown Figure 10.15, is a simple compression response curve. The stress-strain curve is described by the following relationship:

$$
f_{m i}=-f_{p}\left\{2\left(\frac{\epsilon_{m i}}{\epsilon_{p}}\right)-\left(\frac{\epsilon_{m i}}{\epsilon_{p}}\right)^{2}\right\} \text { for } 2 \epsilon_{p}<\epsilon_{m i}<0 .
$$




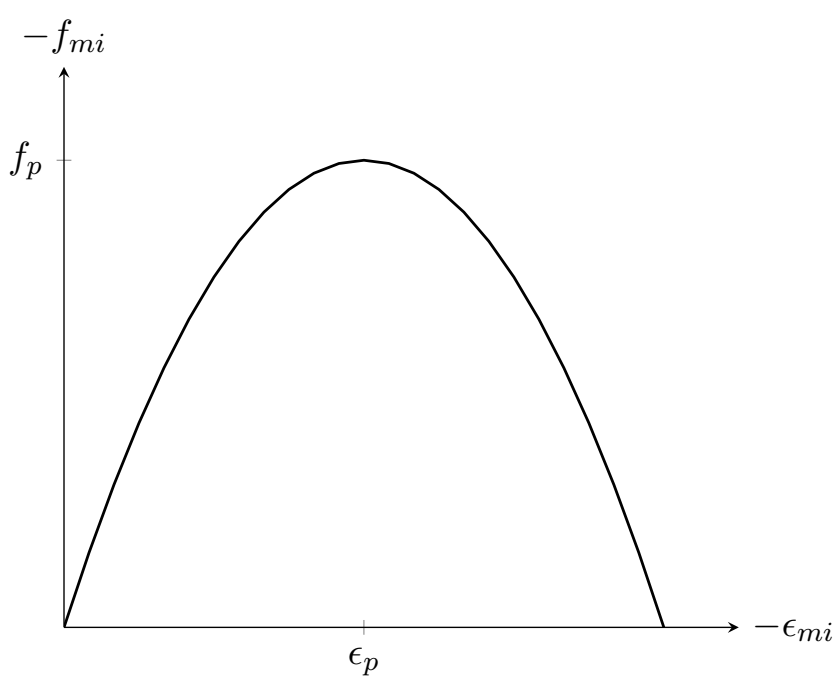

Figure 10.15: Hognestad Parabola

\section{Popovics Model}

Popovics (1970) presented stress-strain curves, as shown in Figure 10.16. These curves reflect the greater stiffness and linearity of the ascending branch and the reduced ductility as peak compressive stress increases. The stress-strain curve is given by the following equation:

$$
f_{m i}=-\left(\frac{\epsilon_{m i}}{\epsilon_{p}}\right) f_{p} \frac{n}{n-1+\left(\frac{\epsilon_{m i}}{\epsilon_{p}}\right)^{n}} \text { for } \epsilon_{m i}<0
$$

The long fraction represents the deviation from linear-elastic response. The curve fitting parameter, $n$, captures the greater linearity of higher strength masonry through the diminishing difference between the initial stiffness $E_{m}$, and secant stiffness, $E_{s e c}$. These values are computed as follows:

$$
n=\frac{E_{m}}{E_{m}-E_{\text {sec }}}
$$

where

$$
E_{\text {sec }}=\frac{f_{p}}{\epsilon_{p}}
$$




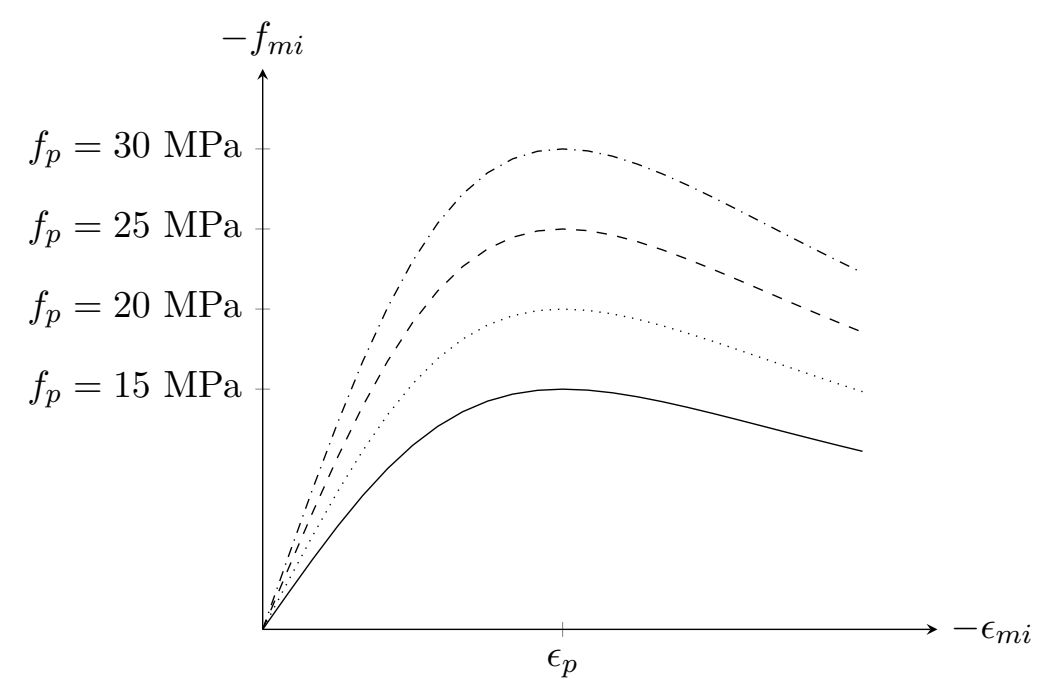

Figure 10.16: Popovics Compression Model

\section{Linear Model}

The linear model, shown in Figure 10.17, is an elastic-plastic compression response curve, defined by:

$$
f_{m i}=\left\{\begin{array}{ll}
-\left(\frac{\epsilon_{m i}}{\epsilon_{p}}\right) f_{p} & \text { for } \epsilon_{p}<\epsilon_{m i}<0 \\
-f_{p} & \text { for } \epsilon_{m i}<\epsilon_{p}<0
\end{array}\right. \text {. }
$$

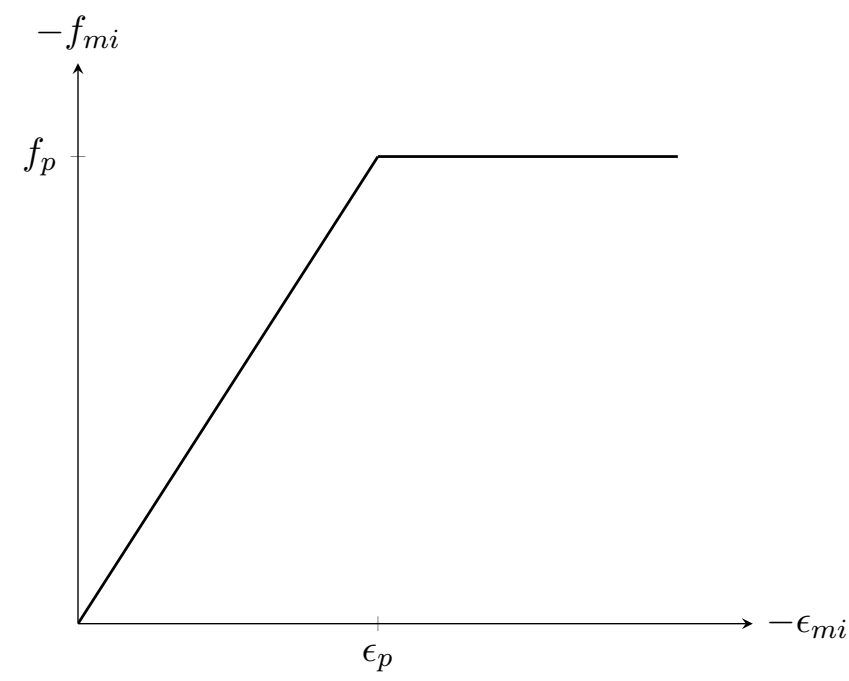

Figure 10.17: Linear Compression Model 


\subsubsection{Constitutive Models for Masonry Post-Peak Compression}

Beyond the peak compressive stress, $f_{p}$, and corresponding strain, $\epsilon_{p}$, masonry can continue to resists compressive stress under continued compressive straining. This compressive strength and ductility may allow localized regions of a masonry structure to fail, but gradually unload to allow a redistribution of internal stresses and prevent total failure of the structure until additional deformation occurs.

Compression post-peak response models compute the principal compressive stress, $f_{m i}$, if the compressive principal compressice strain, $\epsilon_{m i}$ is less than the peak compressive strain $\epsilon_{p}$. Once again,

$$
f_{p}=\beta_{c s} \beta_{a b} f_{m}^{\prime}
$$

where

$$
f_{m}^{\prime}=f\left(f_{m x}, f_{m y}, \theta_{\sigma}\right)
$$

and

$$
\epsilon_{p}=\beta_{c s} \epsilon_{o}
$$

where

$$
\epsilon_{o}=f\left(\epsilon_{o x}, \epsilon_{o y}, \theta_{\sigma}\right)
$$

The SAFEM has two options for the post-peak curve, either the pre-peak curve can be used or the Modified Park-Kent Model can be used.

\section{Pre-Peak Base Curve}

The compression stress post-peak is computed using the equations presented in the previous section, limited to $20 \%$ of the peak strength:

$$
f_{m i}=\min \left\{\begin{array}{l}
\text { Base Curve } f_{m i} \\
0.2 f_{p}
\end{array} .\right.
$$




\section{Modified Park-Kent Model}

Priestley and Elder (1983) modified a stress-strain curve, shown in Figure 10.18, proposed by Kent and Park (1971) for masonry. A linearly descending branch describes the post-peak masonry compression stress-strain curve. This branch is described as:

$$
f_{m i}=\min \left\{\begin{array}{l}
f_{p}\left[1+Z_{m}\left(\epsilon_{m i}-\epsilon_{p}\right)\right] \quad \text { for } \epsilon_{m i}<\epsilon_{p}<0 \\
0.2 f_{p}
\end{array}\right.
$$

where the slope $Z_{m}$ of the linear descending branch is given by:

$$
Z_{m}=\left[\frac{3+0.29 f_{m}^{\prime}}{145 f_{m}^{\prime}-1000}-0.002\right]^{-1} \leq 1
$$

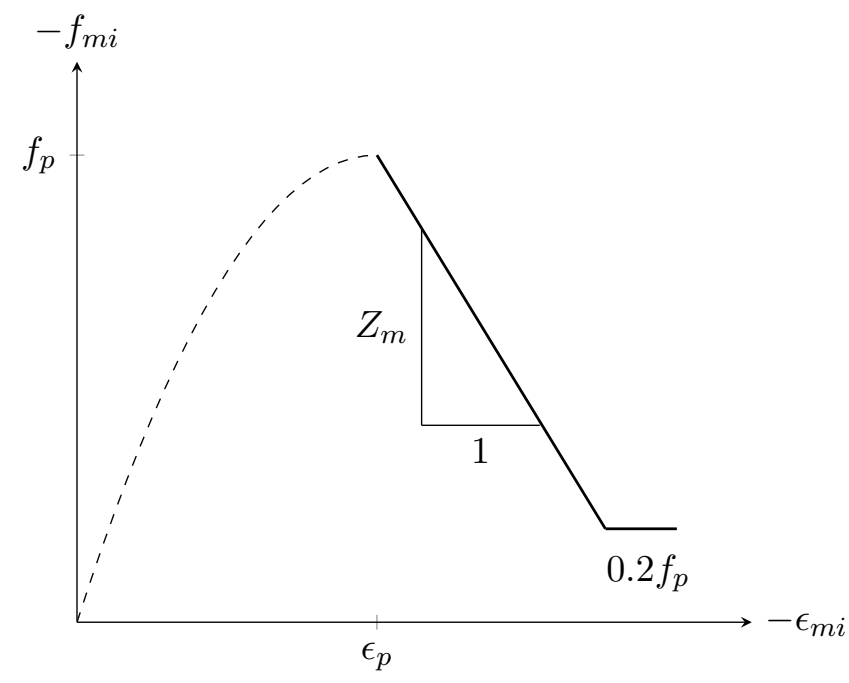

Figure 10.18: Modified Park-Kent Post-Peak Compression Model

\subsubsection{Compression Softening Modeling}

As with concrete, cracked masonry may exhibit a compression softening effect due to tensile strain acting in the transverse direction (Lotfi and Shing, 1991), which can significantly reduce the compressive strength of the composite material. This reduction can be substantial and have considerable effects on the load-deformation response of masonry structures, in terms of stiffness, ultimate strength capacity, and ductility.

The compression softening is considered by a softening parameter, $\beta_{c s}$, with a value between zero and one, which is calculated by the compression softening model. Strength and strain softening as shown in Figure 10.19 use $\beta_{c s}$ to reduce both the masonry compression strength $f_{m}^{\prime}$ and corresponding strain $\epsilon_{o}$ to determine the peak compressice 
strength, $f_{p}$, and corresponding strain, $\epsilon_{p}$, used in the compression response models. The value of $\beta_{c s}$ is a function of the ratio of principal tensile strain to the principal compressive strain $\epsilon_{m 1} / \epsilon_{m 2}$. Thus,

$$
f_{p}=\beta_{c s} \beta_{a b} f_{m}^{\prime}
$$

and

$$
\epsilon_{p}=\beta_{c s} \epsilon_{o}
$$

where

$$
\begin{gathered}
\beta_{c s}=f\left(\epsilon_{m 1} / \epsilon_{m 2}\right), \\
f_{m}^{\prime}=f\left(f_{m x}^{\prime}, f_{m y}^{\prime}, \theta_{\sigma}\right), \\
\epsilon_{o}=f\left(\epsilon_{o x}, \epsilon_{o y}, \theta_{\sigma}\right),
\end{gathered}
$$

and $\beta_{a b}$ is a parameter that accounts for anisotropic behavior.

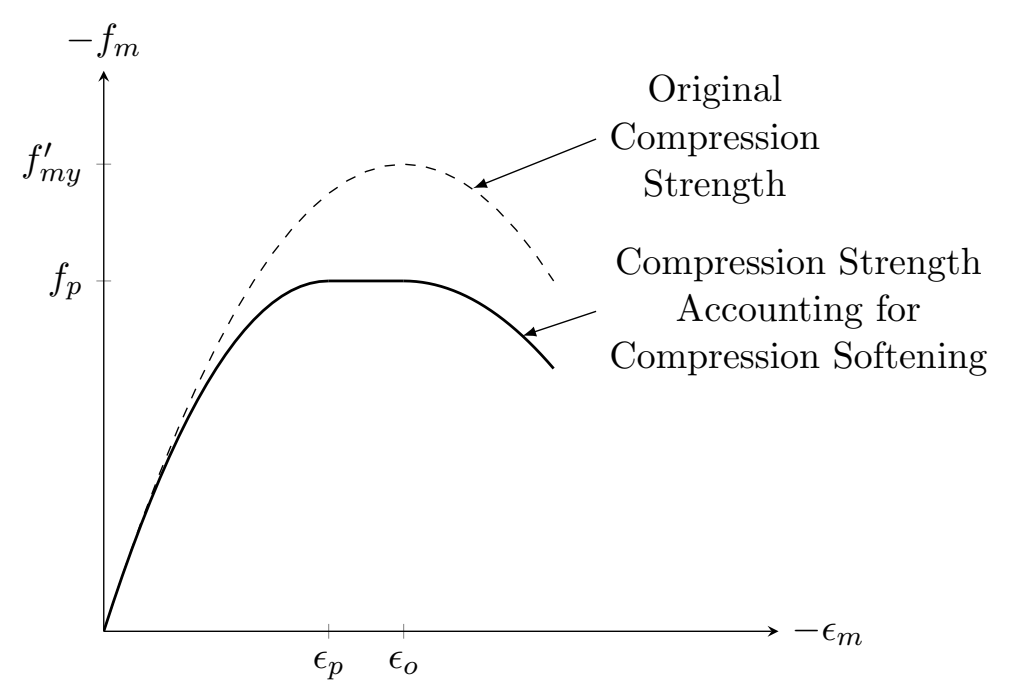

Figure 10.19: Strength and Strain-softened Compression Response

The SAFEM has the option to either include compression softening or ignore it. In the following it is assumed that the principal compressive strain $\epsilon_{m 2}$ and the strain corresponding to peak stress, $\epsilon_{o}$, are negative values while the principal tensile strain, $\epsilon_{m 1}$, is a positive value.

Part III: Masonry Finite Element Modeling 


\section{No Compression Softening}

The compression response is independent of the coexisting tensile strains, thus

$$
\beta_{c s}=1 \text {. }
$$

\section{Vecchio 1992-A Model}

The compression softening model, shown in Figure 10.20, is based on the results of 116 concrete panel and concrete shell element tests (Vecchio and Collins, 1993):

$$
\beta_{c s}=\frac{1}{1+0.55 C_{d}} \leq 1
$$

where

$$
C_{d}=\left\{\begin{array}{ll}
0 & \text { if } r \leq 0.28 \\
0.35(r-0.28)^{0.8} & \text { if } r>0.28
\end{array},\right.
$$

where

$$
r=\frac{-\epsilon_{m 1}}{\epsilon_{m 2}} \leq 400
$$

The ratio of the principal tensile strain to principal compressive stress is limited to 400 to avoid overestimation of the softening effect when the principal tensile strains are very large (e.g. when the reinforcement has yielded).

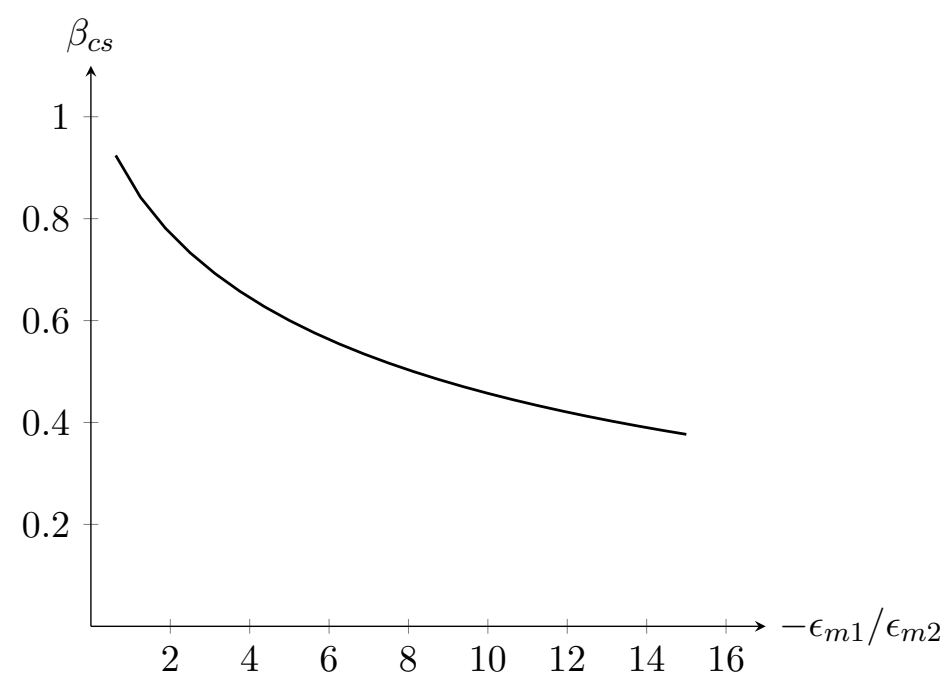

Figure 10.20: Vecchio 1992-A Model Compression Softening Model (Vecchio and Collins, 1993) 
However, if the compression softening is significant enough that $\epsilon_{m 2}$ is more compressive than $\beta_{c s} \epsilon_{o}$ a modification must be made to the calculation of $\epsilon_{p}$ so that the compression response ascends for strains up to $\epsilon_{o}$ and descends thereafter. If this is the case, $\epsilon_{p}$ is calculated as follows:

$$
\epsilon_{p}=\left\{\begin{array}{ll}
\epsilon_{m 2} & \text { if } \epsilon_{o}<\epsilon_{m 2}<\beta_{c s} \epsilon_{o}<0 \\
\epsilon_{o} & \text { if } \epsilon_{m 2}<\epsilon_{o}<\beta_{c s} \epsilon_{o}<0
\end{array}\right. \text { (Wong et al., 2013). }
$$

Furthermore, if $\epsilon_{m i}$ is between the peak strain $\epsilon_{p}$ and $\epsilon_{o}$ the compression strength is limited to $f_{p}=\beta_{c s} \beta_{a b} f_{m}^{\prime}$.

\subsubsection{Constitutive Models for Tension}

Similarly to compression, masonry in tension is characterized by an orthotropic behavior both in the elastic and in the inelastic stage. In the elastic stage, masonry is assumed to be linear elastic until the principal tensile stress, $f_{m 1}$, reaches the maximum tensile strength, $f_{c r}$, and as a consequence, the cracking process begins. Hence, before cracking, the following linear relation is used:

$$
f_{m 1}=E_{m}\left(E_{m x}, E_{m y}, \theta_{\sigma}\right) \epsilon_{m 1} \text { if } 0 \leq \epsilon_{m 1} \leq \epsilon_{c r}
$$

where $E_{m}\left(E_{m x}, E_{m y}, \theta_{\sigma}\right)$ is the elastic modulus evaluated to account for the anisotropic nature of masonry, and $\epsilon_{c r}$ is the cracking strain defined as:

$$
\epsilon_{c r}=\frac{f_{c r}}{E_{m}\left(E_{m x}, E_{m y}, \theta_{\sigma}\right)}
$$

After the cracking process has begun, the tensile stress in unreinforced masonry does not abruptly drop to zero but decreases gradually, exhibiting a so-called tension-softening response. According to Van Der Pluijm and Vermeltfoort (1991), the tensile response of unreinforced masonry may be represented by an exponential function to indicate the relationship between the tensile stress and the crack opening.

For reinforced masonry, average masonry tensile stresses continue to exist in the masonry between the cracks in the vicinity of the reinforcement. With additional tensile straining, cracks widen, the bond action degrades near the cracks and the average masonry tensile stresses gradually diminish to zero. As such the stiffness of the reinforced masonry in tension is greater than that of the reinforcement alone. For this reason, the phenomenon is known as tension-stiffening (Wong et al., 2013). 
Both tension-softening and tension-stiffening cause a gradually decreasing average stressstrain response of masonry in tension, as presented in the following discussion. The tensile stress after cracking is determined from:

$$
f_{m 1}=\min \left(f_{m 1}^{a v g}, f_{m 1}^{t s o f t}, f_{m 1}^{t s t i f f}\right) \text { if } \epsilon_{m 1}>\epsilon_{c r}
$$

where the average concrete tensile stress, $f_{m 1}^{a v g}$, and required for equilibrium at a crack is determined by

$$
\begin{aligned}
f_{m 1}^{a v g}=\rho_{x} & \left(f_{s c r_{x}}-f_{s x}\right) \cos ^{2} \theta_{n x} \\
& +\rho_{y}\left(f_{s c r_{y}}-f_{s y}\right) \cos ^{2} \theta_{n y} \text { if } \epsilon_{m 1} \geq \epsilon_{c r} \text { (Vecchio and Collins, 1986) }
\end{aligned}
$$

and $f_{m 1}^{t s o f t}$ is the average concrete tensile stress due to tension-softening and $f_{m 1}^{\text {tstiff }}$ is the average concrete tensile stress due to tension-stiffening.

\section{Hordjik Tension Softening Model}

For unreinforced masonry tension softening is molded by the exponential decay curve suggested by Hordijk et al. (1987) is used. The formulation is as follows:

$$
f_{m 1}^{t s o f t}=f_{c r}\left\{\left[1+\left(3 \frac{\epsilon_{m 1}}{\epsilon_{t u}}\right)^{3}\right] e^{-6.93 \frac{\epsilon_{m 1}}{\epsilon_{t u}}}-0.027 \frac{\epsilon_{m 1}}{\epsilon_{t u}}\right\} \text { if } \epsilon_{m 1} \geq \epsilon_{c r}
$$

where the ultimate tensile strain, $\epsilon_{t u}$, is based on the value of the Mode-I fracture energy, $G_{f}^{I}$ and may be determined as follows:

$$
\epsilon_{t u}=5.136 \frac{G_{f}^{I}}{f_{c r} L_{r}}
$$

where $L_{r}$ is the characteristic length. Reasonable values of the tensile fracture energy can be chosen in the range of 0.005 to $0.02 \mathrm{~N} / \mathrm{mm}$; thus, the SAFEM assumes $G_{f}^{I}=$ $0.01 \mathrm{~N} / \mathrm{mm}$ and $L_{r}=\min \left(s_{m x}, s_{m y}\right)$. 


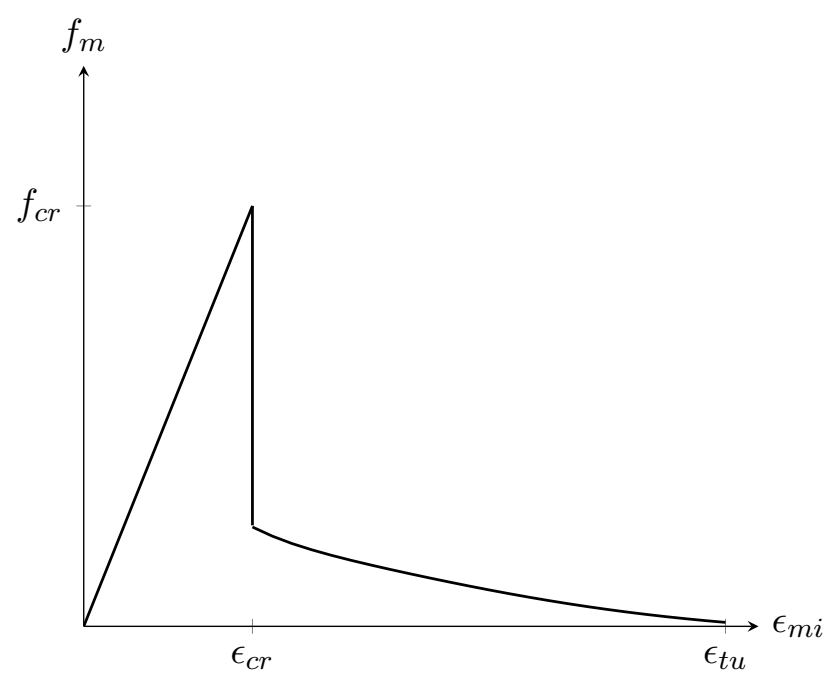

Figure 10.21: Hordjik Tension Softening Model

\section{Vecchio Tension Stiffening Model}

The model used for tension stiffening is originally used in the Modified Compression Field Theory (Vecchio and Collins, 1986), is suitable for reinforced masonry structures. The average masonry tensile stress-strain response is shown in Figure 10.22 and described as:

$$
f_{m 1}^{t s t i f f}=\frac{f_{c r}}{1+\sqrt{200 \epsilon_{m 1}}} \text { if } \epsilon_{m 1} \geq \epsilon_{c r}
$$

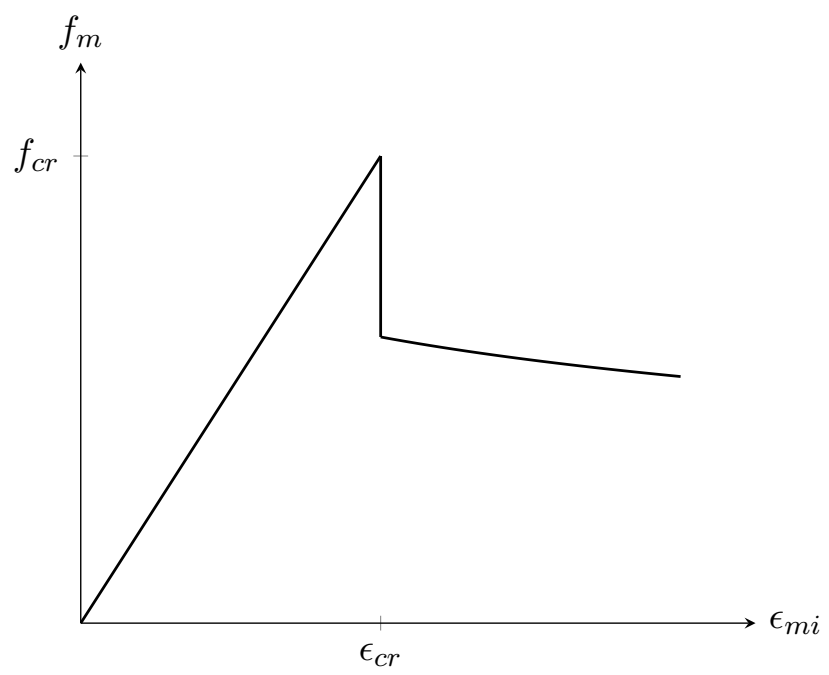

Figure 10.22: Vecchio Tension Stiffening Model 


\subsubsection{Lateral Expansion Model}

Masonry, similar to concrete, will typically expand after cracking in the direction perpendicular to an applied tensile load, due to microcracking. This phenomenon is known as tension splitting, and causes $v_{21}$ to become negative, see Figure 10.23a. Compared this to Figure 10.23b, which produces $v_{12}>0$; thus, SAFEM assumes the following:

$$
v_{12}=f\left(v_{o}, \epsilon_{m 2} / \epsilon_{p}\right)
$$

and

$$
v_{21}=0
$$

where $v_{12}$ is a function of the initial Poisson's ratio and the ratio of the principal compression strain to the peak strain. The assumption of $v_{21}=0$ has been shown in concrete to predict increasing compression strain in the principal direction due to Poisson's effects which in fact may be tensile strains due to tension splitting. However, it has also been shown that this assumption has no measurable impact on the load capacity, stress in the masonry and stress in the reinforcement (Vecchio and De Roo, 1995).

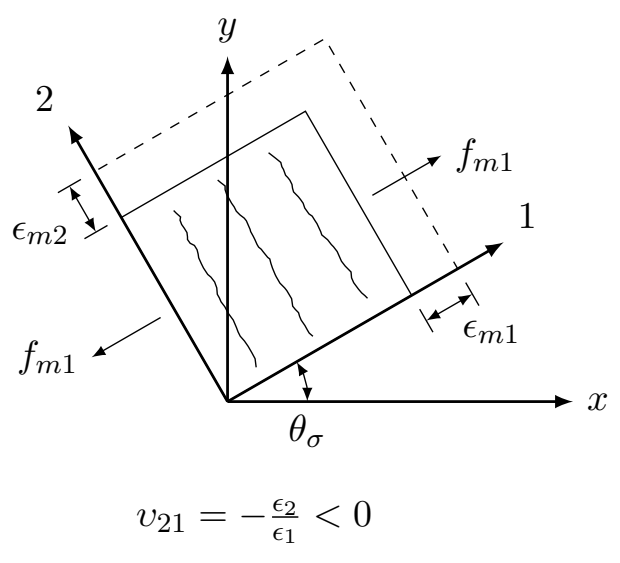

(a) $v_{21}$

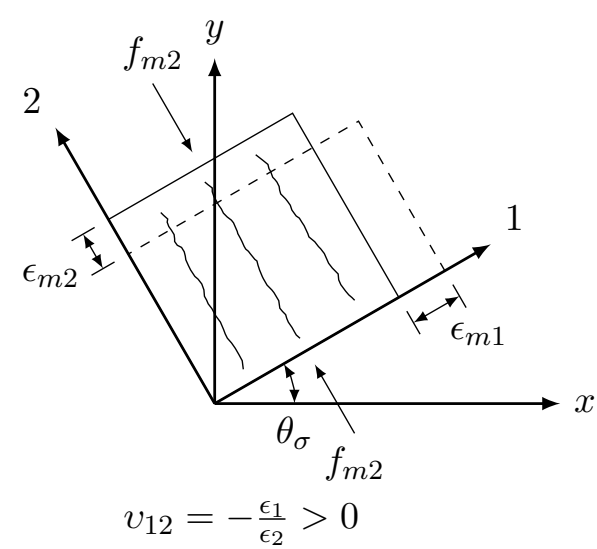

(b) $v_{12}$

Figure 10.23: Principal Poisson's Ratios

The initial masonry Poisson's ratio is a function of the masonry Poisson's ratio in the $x-$ and $y$-directions and the principal strain direction; thus,

$$
v_{o}=f\left(v_{o x}, v_{o y}, \theta_{\sigma}\right) .
$$

The second principal strain could be tensional under low or biaxial tension loading. For uncracked masonry in tension, $v_{12}$ is equal to the initial Poisson's ratio, $v_{o}$. If the 
masonry is cracked, then the Poisson's ratio decreases linearly from $v_{o}$ at the cracking strain, $\epsilon_{c r}$, to zero at two times the cracking strain (Wong et al., 2013). Thus $v_{12}$ when $\epsilon_{m 2}$ is greater than zero is defined as:

$$
v_{12}=\left\{\begin{array}{ll}
v_{o} & \text { for } 0 \leq \epsilon_{m 2} \leq \epsilon_{c r} \\
\max \left(v_{o}\left(1-\frac{\epsilon_{m 2}}{2 \epsilon_{c r}}\right), 0\right) & \text { for } \epsilon_{m 2}>\epsilon_{c r}
\end{array} .\right.
$$

Under compressive loading the rate of masonry lateral expansion increases as the compressive stress increases because of internal microcracking. Near the peak compressive stress, the volume of masonry expands as cracking becomes extensive. Based on strain data of Kupfer et al. (1969), the Poisson's ratio $v_{12}$ increases nonlinearly as compressive strain increases. The Poisson's ratio, shown in Figure 10.24, is computed as follows:

$$
v_{12}=\left\{\begin{array}{ll}
v_{o} & \text { for }-0.5 \epsilon_{p} \leq \epsilon_{m 2} \leq 0 \\
v_{o}\left[1+1.5\left(\frac{-2 \epsilon_{m 2}}{\epsilon_{p}}-1\right)^{2}\right] \leq 0.5 & \text { for } \epsilon_{m 2}<-0.5 \epsilon_{p}
\end{array} .\right.
$$

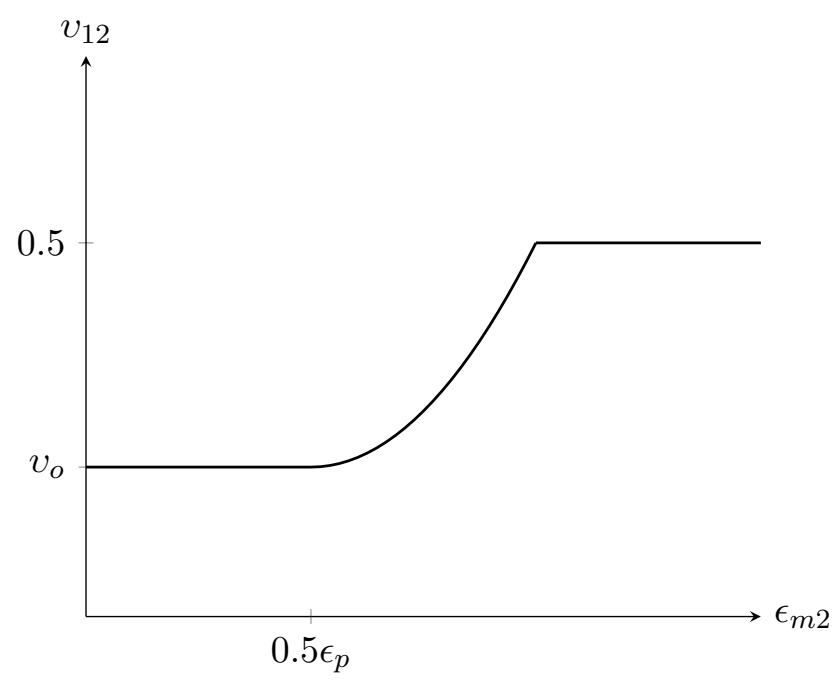

Figure 10.24: Kupfer Variable Poisson's Ratio Model 


\subsubsection{Cracking Criteria}

The cracking strength of masonry, $f_{c r}$, is affected by the compressive strength and varies with different stress states. The SAFEM includes two methods for determining the cracking stress. The first method sets the cracking strength equal to the masonry tensile strength. While the second method allows the cracking criterion to account for the coexisting stresses. Therefore, $f_{c r}$ is generally different from the input value of masonry tensile strength, $f_{t}$. Having determined $f_{c r}$, the cracking strain, $\epsilon_{c r}$, is computed by assuming a linear-elastic relationship before cracking. The values of $f_{c r}$ and $\epsilon_{c r}$ are then used in the tension stress-strain response.

\section{Uniaxial Cracking Stress}

The cracking strength is taken as the specified tensile strength; thus,

$$
f_{c r}=f_{t}^{\prime}
$$

where

$$
f_{t}^{\prime}=f\left(f_{t x}^{\prime}, f_{t y}^{\prime}, \theta_{\sigma}\right)
$$

\section{Mohr-Coulomb Cracking Stress}

The Mohr-Coulomb criterion is often used to determine the failure shear stress and failure plane for a given set of coexisting normal stresses in a frictional material. It is implicitly assumed that the masonry is sufficiently ductile to redistribute stresses along the failure plane. The failure envelope is tangent to the Mohrs circles, see Figure 10.25, defining combinations of shear stress, $\tau$, and normal stresses, $f_{m 1}$ and $f_{m 2}$, resulting in shear failure.

The shear strength is comprised of a stress-independent component and a stress-dependent component. The latter is the internal angle of friction, $\phi$, which the SAFEM assumes is $35^{\circ}$. The former is the cohesion, $c$, which is determined by noting that at failure in uniaxial compression, the maximum compressive stress, is equal to the masonry strength, $f_{m}^{\prime}$, and $f_{m 1}$ is zero; therefore,

$$
c=f_{m}^{\prime} \frac{1-\sin \phi}{2 \cos \phi} .
$$


Further, when $f_{m 2}=0$, the failure tensile stress $f_{m 1}$ is equal to $f_{c r u}$, which is computed from the envelope as:

$$
f_{c r u}=\frac{2 c \cos \phi}{1-\sin \phi}
$$

The value of $f_{c r}$ is computed as follows:

$$
f_{c r}=f_{c r u}\left(1+\frac{f_{m 2}}{f_{m}^{\prime}}\right), 0.20 f_{t} \leq f_{c r}^{j} \leq f_{t}
$$

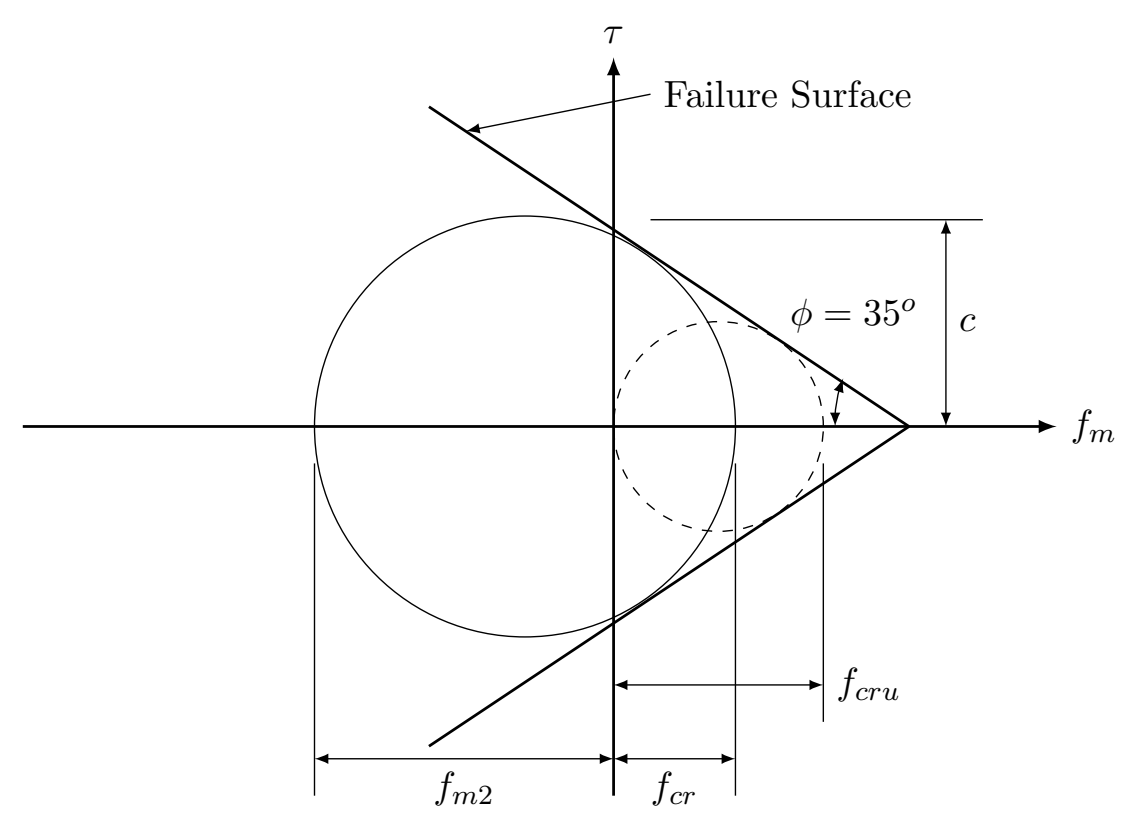

Figure 10.25: Mohr-Coulomb Cracking Stress

\subsubsection{Crack Slip}

The crack slip models allow the SAFEM to explicitly account for strains due to shear slip along the crack. The shear slip, $\delta_{s}$, along the crack is related to the local shear stress, $v_{m i}$, along the crack.

\section{No Slip}

The analysis does not account for shear slip along the crack; thus,

$$
\gamma_{s}=0
$$


It must be checked that the shear stress on a crack, $v_{m i}$,

$$
\begin{aligned}
v_{m i}=\rho_{x}\left(f_{s c r_{x}}-\right. & \left.f_{s x}\right) \cos \theta_{n x} \sin \theta_{n x} \\
& +\rho_{y}\left(f_{s c r_{y}}-f_{s y}\right) \cos \theta_{n y} \sin \theta_{n y}(\text { Vecchio and Collins, 1986) }
\end{aligned}
$$

does not exceed $v_{\text {mimax }}$, otherwise slip on the crack will ensue. Vecchio and Collins (1986) suggested that in the absence of compressive stresses on the crack, the limiting shear stress on the crack can be estimated from:

$$
v_{\operatorname{mimax}}=\frac{0.18 \sqrt{-f_{m}^{\prime}}}{0.31+\frac{24 w}{a+16}}
$$

where $a$ is the maximum aggregate size and $w$ is the average crack width over the crack surface. It can be taken as the product of the principal tensile strain and the crack spacing $s_{\theta_{\sigma}}$; that is

$$
w=\epsilon_{m 1} s_{\theta_{\sigma}}
$$

where

$$
s_{\theta_{\sigma}}=\frac{1}{\frac{\sin \theta_{\sigma}}{s_{m x}}+\frac{\cos \theta_{\sigma}}{s_{m y}}}(\text { Wong et al., 2013) }
$$

and where $s_{m x}$ and $s_{m y}$ are the crack spacing in the $x$ and $y$ directions respectively defined by the block length and height.

If the limited shear stress is exceeded, then the average masonry tensile stress, $f_{m 1}$, is reduced to $f_{m 1}^{*}$ according to

$$
f_{m 1}^{*}=\frac{v_{\operatorname{mimax}}}{v_{m i}} f_{m 1}
$$

\section{Walraven Reinforced Model}

This model is an adaptation of the formulations proposed by Walraven and Reinhardt (1981), based on an analysis of crack structure and contact area of crack faces. The slip along the crack, $\delta_{s}$, is computed as follows:

$$
\delta_{s}=\frac{v_{m i}}{1.8 w^{-0.8}+\left(0.234 w^{-0.707}-0.20\right) f_{m c}} \leq 2 w
$$

where $v_{m i}$ is the local shear stress on the crack, $w$ is the crack width, and $f_{m c}=$ $1.2 f_{m}^{\prime}\left(f_{m x}^{\prime}, f_{m y}^{\prime}, \theta_{\sigma}\right)$ is the masonry cube strength. 
The local shear stress on the crack is determined by:

$$
\begin{aligned}
v_{m i}=\rho_{x}\left(f_{s c r_{x}}-\right. & \left.f_{s x}\right) \cos \theta_{n x} \sin \theta_{n x} \\
& +\rho_{y}\left(f_{s c r_{y}}-f_{s y}\right) \cos \theta_{n y} \sin \theta_{n y}(\text { Vecchio and Collins, 1986). }
\end{aligned}
$$

\section{Constant Rotation Lag}

The approach used to determine crack slip for reinforced masonry is problematic for ungrouted masonry as the shear stress and crack slip will be zero. This is equivalent to ignoring the aggregate interlock. To address this deficiency a constant rotation lag, $\theta^{l}$ between the inclination of the principal total strain axis and the inclination of the principal stress axis is specified. A constant rotation lag of $10^{\circ}$ is used, similar to unreinforced concrete (Wong et al., 2013).

\subsubsection{Shear Slip Model for Masonry Joints}

The SAFEM considers masonry as a continuum that may slip along the head and bed joints, even when the material is uncracked. Based on the calculated shear strain along the joints, the shear slip, $\delta_{j}^{s}$, can be determined:

$$
\delta_{j}^{s}=\gamma_{j}^{s} t_{j}
$$

where the strain parallel to the joints, $\gamma_{j}^{s}$, is determined from:

$$
\gamma_{j}^{s}=\frac{v_{j}}{\bar{G}_{j}}
$$

where $v_{j}$ is the joint shear stress and the joint secant shear stiffness $\bar{G}_{j}$ is determined from an elastic-plastic shear stressstrain relationship as shown in Figure 10.26. Where the slope of the linear branch is given by:

$$
G_{j}=\frac{E_{m y}}{2\left(1+v_{m y}\right)}
$$




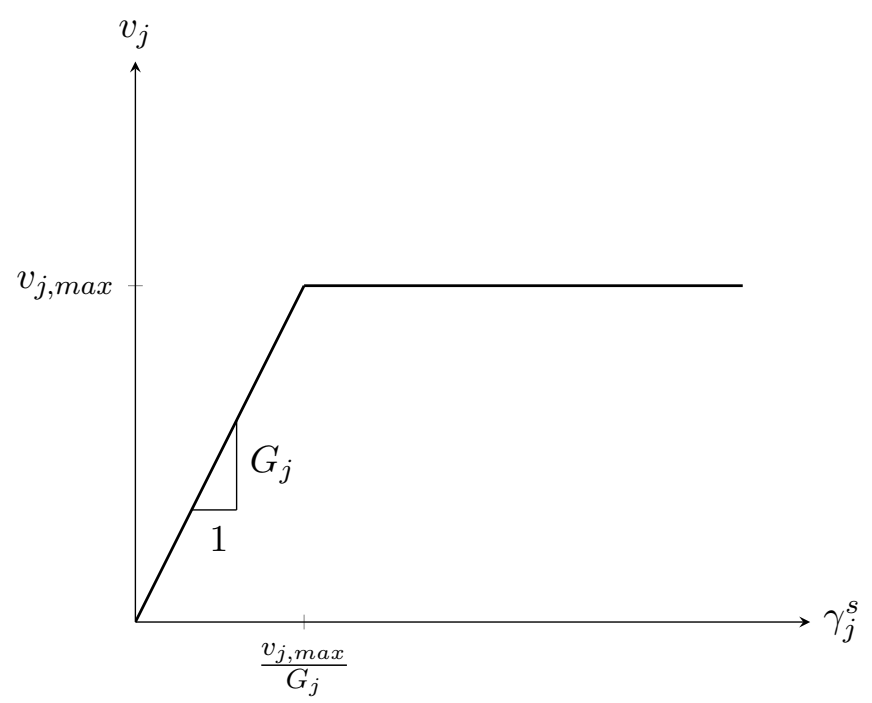

Figure 10.26: Shear Stress-Strain Relationship for Bed and Head Joints

To determine the level of the horizontal plastic plateau, $v_{j, \max }$, a hyperbolic MohrCoulomb type yield criterion is used (Figure 10.27). Similar to the yield criterion proposed by Lotfi and Shing (1991), it consists of a three-parameter hyperbola that provides a smooth transition between the Mohr-Coulomb friction law and the tension cutoff yield criterion. The failure criterion is determined by numerical solving the following for $v_{j}=v_{j, \max }$ :

$$
v_{j}^{2}-\mu^{2}\left(f_{n j}-f_{t}^{\prime}\right)^{2}+2 \rho\left(f_{n j}-f_{t}^{\prime}\right)=0
$$

where $f_{n j}$ is the stress normal to head or bed joint, $f_{t}^{\prime}$ is the bond tensile strength of the joint, $\mu$ is the slope of the hyperbola asymptotes (i.e $\tan \phi$ ), and $\rho$ is the radius of curvature of the yield curve at the vertex of the hyperbola and is equal to:

$$
\rho=\frac{c^{2}-2 \mu f_{t}^{\prime}}{2 f_{t}^{\prime}}
$$

where $c$ is the cohesion. The slope of the hyperbola asymptote and the cohesion are kept constant and equal to their initial values both in the elastic and during the tensionsoftening stage. Moreover, dilatancy phenomena are not considered in the model and, as a consequence, joints cannot be subjected to normal displacement under shear stresses acting along the joint plane. 


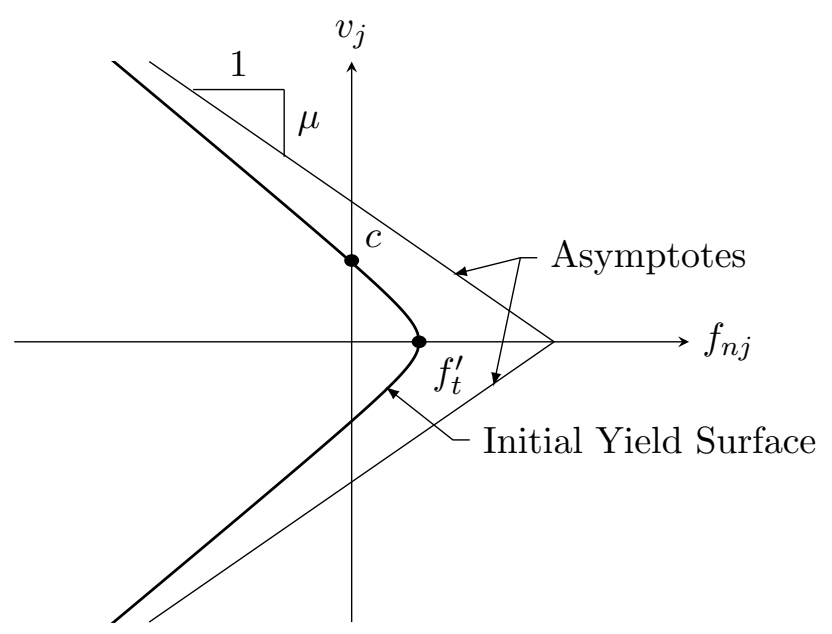

Figure 10.27: Hyperbolic Yield Criterion for Bed and Head Joints

If the following relationship is satisfied:

$$
\left|v_{j}\right| \leq\left|v_{j, \max }\right|
$$

then the shear slip is controlled by the linear elastic branch of the shear stress-strain relationship as follows:

$$
\bar{G}_{j}=G_{j}
$$

As soon as the yield condition is reached according to

$$
\left|v_{j}\right|>\left|v_{j, \max }\right|
$$

then a friction plastic slip will occur and

$$
\bar{G}_{j}=\frac{v_{j, \max }}{\gamma_{j}^{s(h-1)}}
$$

where $\gamma_{j}^{s(h-1)}$ is the strain parallel to the joints for the previous iteration $h-1$. 


\subsubsection{Steel Reinforcement Response}

The reinforcement stress-strain response is composed mainly of four parts, as shown in Figure 10.28, including an initial linear-elastic response, a yield plateau, a linear strain hardening phase until ultimate strength, and an ultimate plateau. The reinforcement stress, $f_{s}$, in tension and compression is determined as follows:

$$
f_{s}= \begin{cases}E_{s} \epsilon_{s} & \text { if } \epsilon_{s} \leq \epsilon_{y} \\ f_{y} & \text { if } \epsilon_{y}<\epsilon_{s} \leq \epsilon_{s h} \\ f_{y}+\left(\frac{f_{u}-f_{y}}{\epsilon_{u}-\epsilon_{s h}}\right)\left(\epsilon_{s}-\epsilon_{s h}\right) & \text { if } \epsilon_{s h}<\epsilon_{s} \leq \epsilon_{u} \\ f_{u} & \text { if } \epsilon_{s}>\epsilon_{u}\end{cases}
$$

where $\epsilon_{s}=\left|\epsilon_{s}\right|$ is the reinforcement stain, $\epsilon_{y}$ is the yield strain, $\epsilon_{s h}$ is the strain at the onset of the strain hardening, $\epsilon_{u}$ is the ultimate strain, $E_{s}$ is the elastic modulus, $f_{y}$ is the yield strength, and $f_{u}$ is the ultimate strength.

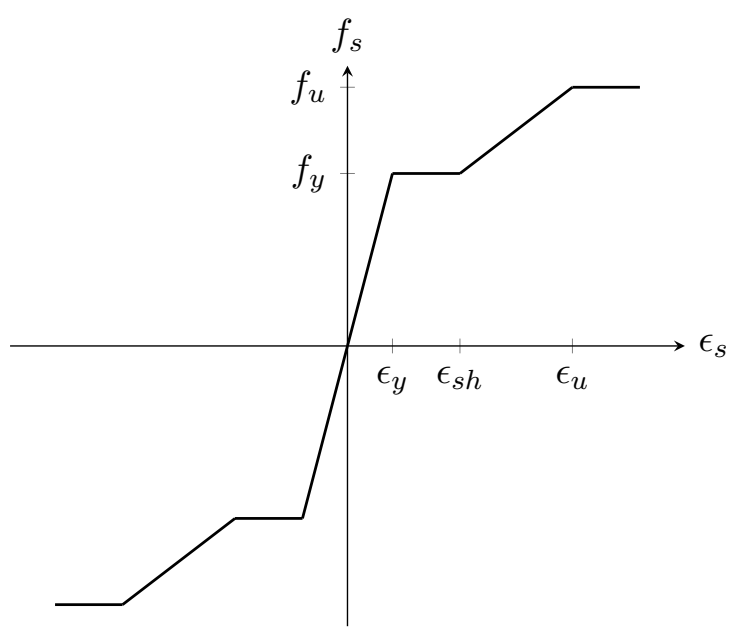

Figure 10.28: Steel Reinforcement Stress-Strain Response 


\section{IMPLEMENTATION OF MODELS INTO MASONRY FI-} NITE ELEMENT

The models presented in Chapter 10 are implemented into a two-dimensional finite element formulation using a fully integrated eight node isoparametric quadrilateral element. The finite element model is programmed in $\mathrm{C}++$.

\subsection{Element Description}

The formulation for the eight node isoparametric quadrilateral element shown in Figure 11.1 will be presented.

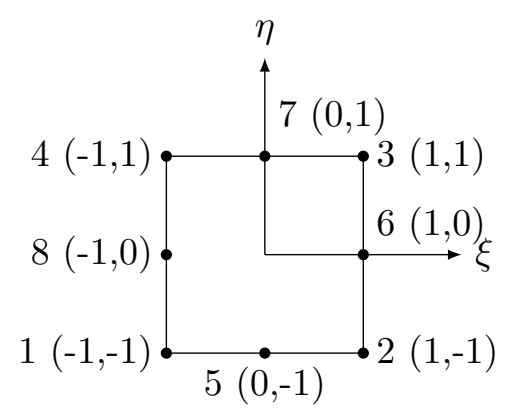

Figure 11.1: Natural Coordinates of Eight Node Isoparametric Quadrilateral Element 
In finite element modeling the basic concept is to assume an approximate solution that satisfies the governing differential equation and boundary conditions. To do this it is assumed the approximate solution is a combination of simpler functions. These simpler functions are called shape functions. The shape functions for the eight node isoparametric quadrilateral element in local natural coordinates are given by:

$$
\begin{aligned}
& N_{1}=\frac{1}{4}(1-\xi)(1-\eta)(-1-\xi-\eta), \\
& N_{2}=\frac{1}{4}(1+\xi)(1-\eta)(-1+\xi-\eta), \\
& N_{3}=\frac{1}{4}(1+\xi)(1+\eta)(-1+\xi+\eta), \\
& N_{4}=\frac{1}{4}(1-\xi)(1+\eta)(-1-\xi+\eta), \\
& N_{5}=\frac{1}{2}\left(1-\xi^{2}\right)(1-\eta), \\
& N_{6}=\frac{1}{2}(1+\xi)\left(1-\eta^{2}\right), \\
& N_{7}=\frac{1}{2}\left(1-\xi^{2}\right)(1+\eta), \text { and } \\
& N_{8}=\frac{1}{2}(1-\xi)\left(1-\eta^{2}\right) \text { (Cook et al., 1989). }
\end{aligned}
$$

As the element is isoparametric, the same shape functions are used to interpolate both coordinates and displacements of a point within the element from coordinates and displacements of nodes; thus:

$$
\left\{\begin{array}{l}
x \\
y
\end{array}\right\}=[N]\{c\}
$$

and

$$
\left\{\begin{array}{l}
u \\
v
\end{array}\right\}=[N]\{d\}
$$

where

$$
\begin{aligned}
& \{c\}=\left[\begin{array}{llllllllllllllll}
x_{1} & y_{1} & x_{2} & y_{2} & x_{3} & y_{3} & x_{4} & y_{4} & x_{5} & y_{5} & x_{6} & y_{6} & x_{7} & y_{7} & x_{8} & y_{8}
\end{array}\right]^{T}
\end{aligned}
$$

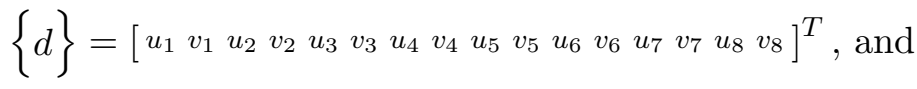

$$
\begin{aligned}
& {[N]=\left[\begin{array}{cccccccccccccccc}
N_{1} & 0 & N_{2} & 0 & N_{3} & 0 & N_{4} & 0 & N_{5} & 0 & N_{6} & 0 & N_{7} & 0 & N_{8} & 0 \\
0 & N_{1} & 0 & N_{2} & 0 & N_{3} & 0 & N_{4} & 0 & N_{5} & 0 & N_{6} & 0 & N_{7} & 0 & N_{8}
\end{array}\right] \text { (Cook et al., 1989). }}
\end{aligned}
$$




\subsubsection{Partial Derivative Computation}

Partial derivatives of shape functions with respect to the Cartesian coordinates $x$ and $y$ are required for strain and stress calculations, but as the shape functions are derived in terms of the natural coordinates $\xi$ and $\eta$; thus, determination of Cartesian partial derivatives is not trivial. The chain rule of partial differentiation will be employed to calculate the shape function derivatives with respect to the Cartesian coordinate system; thus:

$$
\begin{gathered}
\frac{\partial N_{i}}{\partial x}=\frac{\partial N_{i}}{\partial \xi} \frac{\partial \xi}{\partial x}+\frac{\partial N_{i}}{\partial \eta} \frac{\partial \eta}{\partial x} \text { and } \\
\frac{\partial N_{i}}{\partial y}=\frac{\partial N_{i}}{\partial \xi} \frac{\partial \xi}{\partial y}+\frac{\partial N_{i}}{\partial \eta} \frac{\partial \eta}{\partial y}
\end{gathered}
$$

This can be put in matrix form as:

$$
\left[\begin{array}{l}
\frac{\partial N_{i}}{\partial x} \\
\frac{\partial N_{i}}{\partial y}
\end{array}\right]=\left[\begin{array}{ll}
\frac{\partial \xi}{\partial x} & \frac{\partial \eta}{\partial x} \\
\frac{\partial \xi}{\partial y} & \frac{\partial \eta}{\partial y}
\end{array}\right]\left[\begin{array}{c}
\frac{\partial N_{i}}{\partial \xi} \\
\frac{\partial N_{i}}{\partial \eta}
\end{array}\right]=\frac{\partial(\xi, \eta)}{\partial(x, y)}\left[\begin{array}{c}
\frac{\partial N_{i}}{\partial \xi} \\
\frac{\partial N_{i}}{\partial \eta}
\end{array}\right]=[J]^{-1}\left[\begin{array}{c}
\frac{\partial N_{i}}{\partial \xi} \\
\frac{\partial N_{i}}{\partial \eta}
\end{array}\right]
$$

where $[J]$ and $[J]^{-1}$ are are called simply the Jacobian and inverse Jacobian, respectively (Cook et al., 1989).

\subsubsection{Computing the Jacobian Matrix}

To compute the entries of the Jacobian matrix, $[J]$, it is necessary to start with the summation of the result of each Cartesian coordinate $x_{i}$ and $y_{i}$ times its respective shape function over all eight nodes:

$$
\begin{gathered}
x=\sum_{i=1}^{8} x_{i} N_{i} \text { and } \\
y=\sum_{i=1}^{8} y_{i} N_{i} .
\end{gathered}
$$

Then differentiate with respect to the natural coordinates,

$$
\begin{gathered}
\frac{\partial x}{\partial \xi}=\sum_{i=1}^{8} x_{i} \frac{\partial N_{i}}{\partial \xi}, \\
\frac{\partial y}{\partial \xi}=\sum_{i=1}^{8} y_{i} \frac{\partial N_{i}}{\partial \xi}, \\
\frac{\partial x}{\partial \eta}=\sum_{i=1}^{8} x_{i} \frac{\partial N_{i}}{\partial \eta}, \text { and }
\end{gathered}
$$

Part III: Masonry Finite Element Modeling 


$$
\frac{\partial y}{\partial \eta}=\sum_{i=1}^{8} y_{i} \frac{\partial N_{i}}{\partial \eta}
$$

as $x_{i}$ and $y_{i}$ do not depend on $\xi$ and $\eta$. In matrix form these partial derivatives become:

$$
[J]=\left[\begin{array}{ll}
J_{11} & J_{12} \\
J_{21} & J_{22}
\end{array}\right]=\left[\begin{array}{ll}
\frac{\partial x}{\partial \xi} & \frac{\partial y}{\partial \xi} \\
\frac{\partial x}{\partial \eta} & \frac{\partial y}{\partial \eta}
\end{array}\right]
$$

or

$$
[J]=\left[\begin{array}{llllllll}
\frac{\partial N_{1}}{\partial \xi} & \frac{\partial N_{2}}{\partial \xi} & \frac{\partial N_{3}}{\partial \xi} & \frac{\partial N_{4}}{\partial \xi} & \frac{\partial N_{5}}{\partial \xi} & \frac{\partial N_{6}}{\partial \xi} & \frac{\partial N_{7}}{\partial \xi} & \frac{\partial N_{8}}{\partial \xi} \\
\frac{\partial N_{1}}{\partial \eta} & \frac{\partial N_{2}}{\partial \eta} & \frac{\partial N_{3}}{\partial \eta} & \frac{\partial N_{4}}{\partial \eta} & \frac{\partial N_{5}}{\partial \eta} & \frac{\partial N_{6}}{\partial \eta} & \frac{\partial N_{7}}{\partial \eta} & \frac{\partial N_{8}}{\partial \eta}
\end{array}\right]\left[\begin{array}{ll}
x_{1} & y_{1} \\
x_{2} & y_{2} \\
x_{3} & y_{3} \\
x_{4} & y_{4} \\
x_{5} & y_{5} \\
x_{6} & y_{6} \\
x_{7} & y_{7} \\
x_{8} & y_{8}
\end{array}\right]
$$

where

$$
\begin{aligned}
\frac{\partial N_{1}}{\partial \xi} & =\frac{1}{4}(1-\eta)(2 \xi+\eta) & \frac{\partial N_{1}}{\partial \eta} & =\frac{1}{4}(1-\xi)(2 \eta+\xi) \\
\frac{\partial N_{2}}{\partial \xi} & =\frac{1}{4}(1-\eta)(2 \xi-\eta) & \frac{\partial N_{2}}{\partial \eta} & =\frac{1}{4}(1+\xi)(2 \eta-\xi) \\
\frac{\partial N_{3}}{\partial \xi} & =\frac{1}{4}(1+\eta)(2 \xi+\eta) & \frac{\partial N_{3}}{\partial \eta} & =\frac{1}{4}(1+\xi)(2 \eta+\xi) \\
\frac{\partial N_{4}}{\partial \xi} & =\frac{1}{4}(1+\eta)(2 \xi-\eta) & \frac{\partial N_{4}}{\partial \eta} & =\frac{1}{4}(1-\xi)(2 \eta-\xi) \\
\frac{\partial N_{5}}{\partial \xi} & =-\xi(1-\eta) & \frac{\partial N_{5}}{\partial \eta} & =-\frac{1}{2}\left(1-\xi^{2}\right) \\
\frac{\partial N_{6}}{\partial \xi} & =\frac{1}{2}\left(1-\eta^{2}\right) & \frac{\partial N_{6}}{\partial \eta} & =-\eta(1+\xi) \\
\frac{\partial N_{7}}{\partial \xi} & =-\xi(1+\eta) & \frac{\partial N_{7}}{\partial \eta} & =\frac{1}{2}\left(1-\xi^{2}\right) \\
\frac{\partial N_{8}}{\partial \xi} & =-\frac{1}{2}\left(1-\eta^{2}\right) & \frac{\partial N_{8}}{\partial \eta} & =-\eta(1-\xi)
\end{aligned}
$$

The inverse Jacobian $[J]^{-1}$ is then obtained by inverting this $2 \times 2$ matrix. Additional, the determinant, $|J|$, of $[J]$ can be determined. 


\subsubsection{The Strain-Displacement Matrix}

The strain-displacement matrix $[B]$ that is used in the computation of the element stiffness matrix is given by:

$$
[B]=\left[\begin{array}{cccccccccccccccc}
\frac{\partial N_{1}}{\partial x} & 0 & \frac{\partial N_{2}}{\partial x} & 0 & \frac{\partial N_{3}}{\partial x} & 0 & \frac{\partial N_{4}}{\partial x} & 0 & \frac{\partial N_{5}}{\partial x} & 0 & \frac{\partial N_{6}}{\partial x} & 0 & \frac{\partial N_{7}}{\partial x} & 0 & \frac{\partial N_{8}}{\partial x} & 0 \\
0 & \frac{\partial N_{1}}{\partial y} & 0 & \frac{\partial N_{2}}{\partial y} & 0 & \frac{\partial N_{3}}{\partial y} & 0 & \frac{\partial N_{4}}{\partial y} & 0 & \frac{\partial N_{5}}{\partial y} & 0 & \frac{\partial N_{6}}{\partial y} & 0 & \frac{\partial N_{7}}{\partial y} & 0 & \frac{\partial N_{8}}{\partial y} \\
\frac{\partial N_{1}}{\partial y} & \frac{\partial N_{1}}{\partial x} & \frac{\partial N_{2}}{\partial y} & \frac{\partial N_{2}}{\partial x} & \frac{\partial N_{3}}{\partial y} & \frac{\partial N_{3}}{\partial x} & \frac{\partial N_{4}}{\partial y} & \frac{\partial N_{4}}{\partial x} & \frac{\partial N_{5}}{\partial y} & \frac{\partial N_{5}}{\partial x} & \frac{\partial N_{6}}{\partial y} & \frac{\partial N_{6}}{\partial x} & \frac{\partial N_{7}}{\partial y} & \frac{\partial N_{7}}{\partial x} & \frac{\partial N_{8}}{\partial y} & \frac{\partial N_{8}}{\partial x}
\end{array}\right]
$$

The nonzero entries of $[B]$ are partial derivatives of the shape functions with respect to $x$ and $y$ (Cook et al., 1989). The calculation of those partial derivatives is done by computing the Jacobian, $[J]$, via Equation 11.17, inverting the Jaobian, $[J]^{-1}$, and using the chain rule, Equation 11.9 .

\subsection{Element Stiffness Matrix}

The stiffness matrix of an element of thickness $t$, is

$$
[k]=\iint[B]^{T}[D][B] t d x d y=\int_{-1}^{1} \int_{-1}^{1}[B]^{T}[D][B] t|J| d \xi d \eta
$$

Performing the matrix multiplication and subsequent integration indicated above by manual methods is impractical, as with nearly all isoparametric elements (Cook et al., 1989). Since for the general case closed-form integration cannot be used, the element stiffness matrices are computed using numerical integration. Gauss-Legendre integration is performed in the $\xi-\eta$ domain, as suggested by the limits on the integral above. Thus, the stiffness matrix becomes:

$$
[k]=\int_{-1}^{1} \int_{-1}^{1}[B]^{T}[D][B] t|J| d \xi d \eta=\sum_{i=1}^{3} \sum_{j=1}^{3}[B]^{T}[D][B] t|J| w_{i} w_{j}
$$

for $3 \times 3$ Gauss-Legendre integration (which is for full integrate of the eight node isoparametric quadrilateral element), where $w_{i}$ and $w_{j}$ are the wights given to each Gauss integration point, shown in Figure 11.2 and Table 11.1. Thus, the element stiffness matrix consists of nine partial matrices, with each partial matrix representing the stiffness associated with its respective Gauss point (Cook et al., 1989). 


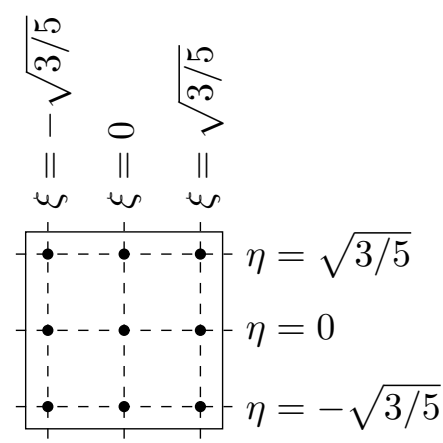

Figure 11.2: Guass Integration Points for Eight Node Isoparametric Quadrilateral Element

Table 11.1: 3 x 3 Gauss Integration Points and Weights

\begin{tabular}{lllll}
\hline Point & $\xi_{i}$ & $\eta_{j}$ & $w_{i}$ & $w_{j}$ \\
\hline 1 & $-\sqrt{3 / 5}$ & $-\sqrt{3 / 5}$ & $5 / 9$ & $5 / 9$ \\
2 & 0 & $-\sqrt{3 / 5}$ & $8 / 9$ & $5 / 9$ \\
3 & $\sqrt{3 / 5}$ & $-\sqrt{3 / 5}$ & $5 / 9$ & $5 / 9$ \\
4 & $-\sqrt{3 / 5}$ & 0 & $5 / 9$ & $8 / 9$ \\
5 & 0 & 0 & $8 / 9$ & $8 / 9$ \\
6 & $\sqrt{3 / 5}$ & 0 & $5 / 9$ & $8 / 9$ \\
7 & $-\sqrt{3 / 5}$ & $\sqrt{3 / 5}$ & $5 / 9$ & $5 / 9$ \\
8 & 0 & $\sqrt{3 / 5}$ & $8 / 9$ & $5 / 9$ \\
9 & $\sqrt{3 / 5}$ & $\sqrt{3 / 5}$ & $5 / 9$ & $5 / 9$ \\
\hline
\end{tabular}

\subsection{Composite Material Stiffness Matrix}

The composite material stiffness matrix, $[D]$, is the sum of the masonry stiffness matrix, $\left[D_{m}\right]$, and the steel reinforcement material stiffness matrix, $\left[D_{s}\right]$, as follows:

$$
[D]=\left[D_{m}\right]+\left[D_{s}\right]
$$

where the masonry stiffness matrix in the principal directions, $\left[D_{m}\right]^{\prime}$, is transformed to the global axes by:

$$
\left[D_{m}\right]=\left[T_{m}\right]^{T}\left[D_{m}\right]^{\prime}\left[T_{m}\right]
$$


As the SAFEM models masonry as an orthotropic material in the principal stress directions, it is necessary to formulate the masonry material stiffness matrix, $\left[D_{m}\right]^{\prime}$, relative to these directions. If it assumed that the Poisson's effect is negligible $\left[D_{m}\right]^{\prime}$ is computed as follows:

$$
\left[D_{m}\right]^{\prime}=\left[\begin{array}{ccc}
\bar{E}_{m 1} & 0 & 0 \\
0 & \bar{E}_{m 2} & 0 \\
0 & 0 & \bar{G}_{m}
\end{array}\right]
$$

The secant moduli, $\bar{E}_{m 1}, \bar{E}_{m 2}, \bar{G}_{m}$, are computed from the current values of the principal stresses, $f_{m 1}$ and $f_{m 2}$ and the corresponding principal net masonry strains, $\epsilon_{m 1}$ and $\epsilon_{m 2}$ (see Figure 11.3a) as follows:

$$
\begin{gathered}
\bar{E}_{m 1}=\frac{f_{m 1}}{\epsilon_{m 1}}, \\
\bar{E}_{m 2}=\frac{f_{m 2}}{\epsilon_{m 2}}, \text { and } \\
\bar{G}_{m}=\frac{\bar{E}_{m 1} \bar{E}_{m 2}}{\bar{E}_{m 1}+\bar{E}_{m 2}} .
\end{gathered}
$$

The transformation matrix $\left[T_{m}\right]^{\prime}$ is given by:

$$
\left[T_{m}\right]=\left[\begin{array}{ccc}
\cos ^{2} \theta_{\sigma} & \sin ^{2} \theta_{\sigma} & \cos \theta_{\sigma} \sin \theta_{\sigma} \\
\sin ^{2} \theta_{\sigma} & \cos ^{2} \theta_{\sigma} & -\cos \theta_{\sigma} \sin \theta_{\sigma} \\
-2 \cos \theta_{\sigma} \sin \theta_{\sigma} & 2 \cos \theta_{\sigma} \sin \theta_{\sigma} & \cos ^{2} \theta_{\sigma}-\sin ^{2} \theta_{\sigma}
\end{array}\right] \text { (Cook et al., 1989). }
$$




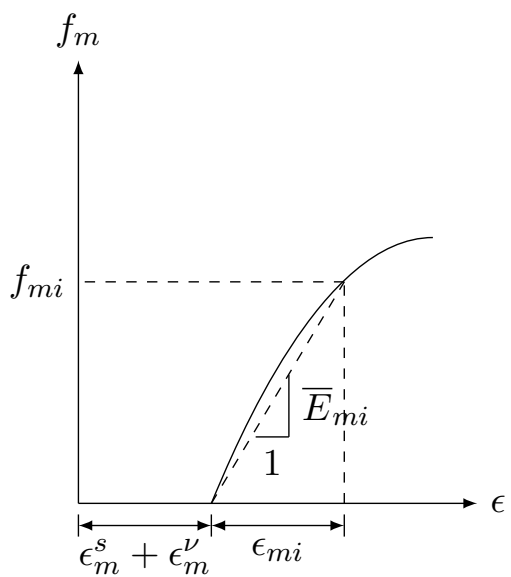

(a) Masonry Secant Moduli

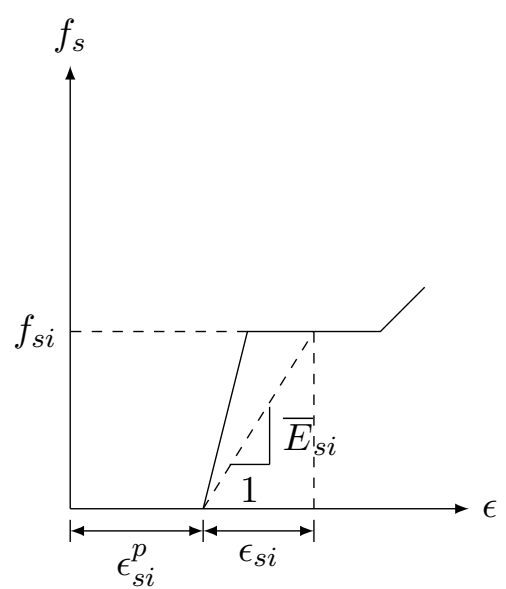

(b) Reinforcement Secant Moduli

Figure 11.3: Definition of Secant Moduli (Wong et al., 2013)

The stiffness matrix for the smeared reinforcement component is

$$
\left[D_{s}\right]=\left[\begin{array}{ccc}
\rho_{x} \bar{E}_{s x} & 0 & 0 \\
0 & \rho_{y} \bar{E}_{s y} & 0 \\
0 & 0 & 0
\end{array}\right]
$$

where $\rho_{x}$ and $\rho_{y}$ are the reinforcement ratios in the $x$ and $y$ directions respectively. The secant modulus, $\bar{E}_{s x}$ and $\bar{E}_{s y}$ are computed from the current value of stresses $f_{s x}$ or $f_{s y}$ and the corresponding net steel strains $\epsilon_{s x}$ or $\epsilon_{s y}$ (see Figure 11.3b) as follows:

$$
\bar{E}_{s x}=\frac{f_{s x}}{\epsilon_{s x}}
$$

and

$$
\bar{E}_{s y}=\frac{f_{s y}}{\epsilon_{s y}}
$$

The composite material stiffness matrix will by fully populated and symmetric.

\subsection{Element and Material Stresses}

At any point within the masonry continuum, the total strains are related to stresses by the composite material stiffness matrix, $[D]$ :

$$
\{\sigma\}=[D]\{\epsilon\}-\left\{\sigma^{o}\right\} \text { (Wong et al., 2013). }
$$

While the composite material stiffness matrix operates on total strains, material element stresses can be directly related only to net strains of the masonry and reinforcement. 
Therefore, it is necessary to subtract the stress contribution of masonry Poisson's effects and shear slip strains and steel reinforcement prestress by the use of a pseduo stress vector $\left\{\sigma^{o}\right\}$ calculated as:

$$
\left\{\sigma^{o}\right\}=\left[D_{m}\right]\left\{\left\{\epsilon_{m}^{s}\right\}+\left\{\epsilon_{m}^{\nu}\right\}\right\}+\left[D_{s}\right]\left\{\epsilon_{s}^{p}\right\} \text { (Wong et al., 2013). }
$$

The net stresses within the masonry is determined form:

$$
\left\{\sigma_{m}\right\}=\left[D_{m}\right]\left\{\{\epsilon\}-\left\{\epsilon_{m}^{s}\right\}-\left\{\epsilon_{m}^{\nu}\right\}\right\}
$$

and within the reinforcement:

$$
\left\{\sigma_{s}\right\}=\left[D_{s}\right]\left\{\{\epsilon\}-\left\{\epsilon_{s}^{p}\right\}\right\} \text { (Wong et al., 2013). }
$$

\subsection{Material Prestrains}

The SAFEM includes provisions to model prestrains arising from strain offset effects such as prestressing of reinforcement, Poisson's effects and slip within the masonry. For masonry, a prestrain matrix is defined relative to the global $x$ and $y$ system:

$$
\left\{\epsilon_{m}^{o}\right\}=\left\{\begin{array}{c}
\epsilon_{m x}^{o} \\
\epsilon_{m y}^{o} \\
\gamma_{m x y}^{o}
\end{array}\right\}=\left\{\epsilon_{m}^{\nu}\right\}+\left\{\epsilon_{m}^{s}\right\} .
$$

A similar matrix is developed for the reinforcement prestrains. An equivalent nodal force is then used to incorporate the prestrain effects. From the known prestrains, the nodal forces can be calculated:

$$
\left\{F^{o}\right\}=\sum_{i=1}^{3} \sum_{j=1}^{3}[B]^{T}\left\{\sigma^{o}\right\} t|J| w_{i} w_{j}
$$

where

$$
\left\{\sigma^{o}\right\}=\left[D_{m}\right]\left\{\epsilon_{m}^{o}\right\}+\left[D_{s}\right]\left\{\epsilon_{s}^{o}\right\} \text { (Wong et al., 2013) }
$$

These equivalent nodal forces due to the prestrains are added to the externally applied nodal forces, $\left\{F^{E A}\right\}$, to form the total nodal forces, $\{F\}$ :

$$
\{F\}=\left\{F^{E A}\right\}+\left\{F^{o}\right\}
$$

The total nodal load vector is updated on each iteration since both the externally applied forces and the pseduo load forces change.

Part III: Masonry Finite Element Modeling 


\subsection{Total Load Iterative Secant Stiffness SAFEM Solu- tion Procedure}

The global stiffness matrix, $[K]$, of the entire structure is assembled by the summation of all, $n$, element stiffness matrices, $[k]$ :

$$
[K]=\sum_{i=1}^{n}[k]_{i} .
$$

Having determined the global stiffness matrix and the total nodal vector, corrected for boundary conditions, the following system of equations results involving the unknown nodal displacements, $\{r\}$ :

$$
\{F\}=[K]\{r\}
$$

This can be solved by using the direct sparse LDLT Cholesky factorizations without square root function provided in the Eigen $\mathrm{C}++$ template library for linear algebra (Eigen).

The total element strains are determined from the nodal displacements, utilizing the strain displacement matrix. The strain is calculated at the nine Gauss points and then a weighted average is calculated based on the volume associated with each Gauss point to determine the total element strains (ElMohandes, 2013); thus,

$$
\{\epsilon\}=\sum_{i=1}^{3} \sum_{j=1}^{3} \frac{|J| w_{i} w_{j}}{V}[B]\{r\}
$$

where

$$
V=\sum_{i=1}^{3} \sum_{j=1}^{3}|J| w_{i} w_{j}
$$

The shear and normal stresses acting upon the element can then be determined according to Section 11.4. However, the secant moduli used to determine the structure stiffness matrix are only estimates that are initially based on the stress-strain state of the previous load step. Owing to the finite size of each load step, the stress-strain state corresponding to the current load step differs from that of the previous load step by a finite amount, resulting in an error in the computed nodal displacements. The error can be made arbitarily small by an iterative refining of the secant moduli. The masonry net strains 
are recomputed as $\left\{\epsilon_{m}^{n}\right\}^{\prime}$ at the end of the iteration and then averaged with the assumed values $\left\{\epsilon_{m}^{n}\right\}$ from the beginning of the iteration as follows:

$$
\left\{\epsilon_{m}^{n}\right\}_{(i+1)}=(1-c)\left\{\epsilon_{m}^{n}\right\}_{i}+c\left\{\epsilon_{m}^{n}\right\}_{i}^{\prime}
$$

where $c$ is a specified convergence averaging factor and $i$ is the iteration number. When the displacements have acceptably converged or a specified number of iterations have been performed, the SAFEM concludes the analysis for the load step, stores the analysis results and proceeds to the next load step. The convergence factor, C.F., is determined from:

$$
C . F .=\sqrt{\frac{1}{n} \sum_{i=1}^{n}\left\{\frac{\left|r_{i}^{\prime}\right|}{\left|r_{i}\right|}\right\}^{2}}
$$

with $n$ equal to the number of degrees of freedom. The optimal convergence is when C.F. $=1$ (Wong et al., 2013).

\subsection{Applying Support Conditions}

To eliminate rigid body motions and render the system non-singular physical support conditions as displacement boundary conditions must be applied. When solving the overall stiffness equations by hand, the simplest way to account for support conditions is to remove equations associated with known zero joint displacements from the master system. Reduction is convenient for hand computations because it cuts down on the number of equations to solve, but it has a serious flaw for computer implementation: the equations must be rearranged. Rearrangement can be more computational expensive than solving the equations, particularly if the stiffness matrix is stored in sparse form. To apply support conditions without rearranging the equations, set the rows and columns corresponding to prescribed zero displacements as well as the corresponding force components to zero, and place ones on the diagonal to maintain non-singularity. The resulting system is called the modified set of master stiffness equations.

\subsection{External Support Reactions}

The externally applied support reactions, $\left\{R^{S}\right\}$, are determined for converged load steps by:

$$
\left\{R^{S}\right\}=[K]\{r\}-\{F\}
$$




\subsection{Finite Element Implementation}

The SAFEM uses a displacement-based finite element method in which a system of equations is derived relating unknown nodal displacements to specified forces by the structure stiffness matrix. The SAFEM algorithm for nonlinear finite element analysis is summarized by the flow chart in Figure 11.4.

\subsection{Finite Element Mesh Size}

The SAFEM includes an automatic mesh generator that will determine the node location and numbers, as well as assign the element a number and material type. The element size should be based on the block size and mortar thickness. Minaie et al. (2014) determined that an element size of half the nominal block size was optimal in terms of computational cost and model accuracy, but SAFEM allows for a user defined mesh size. The results presented in this thesis use Minaie et al. (2014) recommendation.

\subsection{Model Inputs and Default Values}

The SAFEM requires a number of inputs before an analysis can proceed. A number of the material properties can be set to default values as described in Table 11.2 and Table 11.3. Additional, the model options for constitutive relationships are give in Table 11.4.

\subsection{SAFEM Program}

The SAFEM program is programmed in $\mathrm{C}++$, using the Eigen Library (Eigen) for matrix manipulation, and OpenGL (OpenGL) with Microsoft Foundation Class Library for a graphical user interface (GUI). The program allows for easy material input (Figure 11.5), mesh generation (Figure 11.6), support generation, and load generation. When the model is running a window shows convergence factors (Figure 11.7) and converged load load stage data, including element properties, stresses, and strains; node displacements; crack directions and widths; composite matrix elements; and reinforcement stresses and strains, is stored in text files for further analysis.

Part III: Masonry Finite Element Modeling 


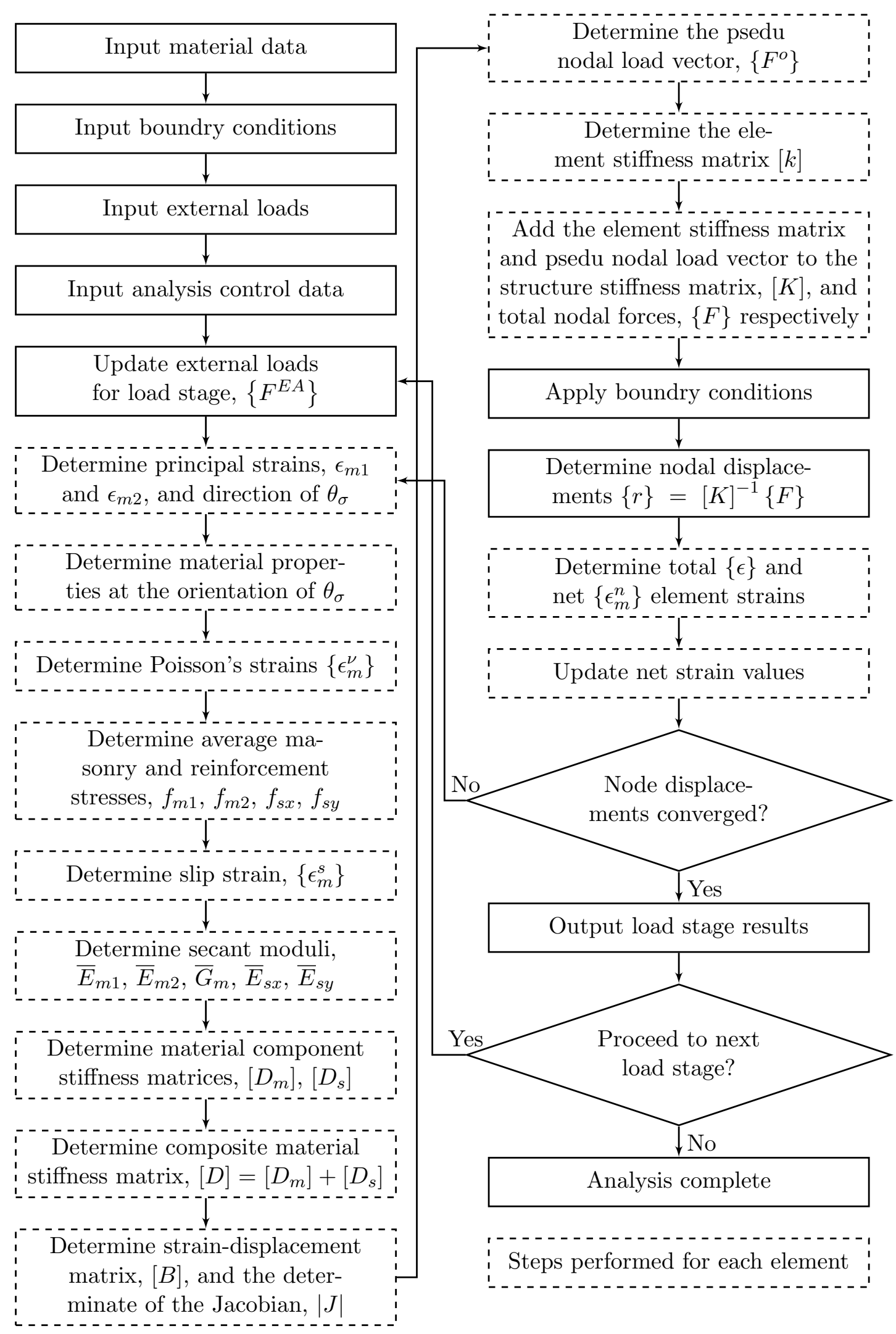

Figure 11.4: SAFEM Analysis Algorithm 
Table 11.2: Ungrouted SAFEM Inputs

\begin{tabular}{|c|c|c|c|}
\hline Variable Name & Symbol & Units & Default Value \\
\hline \multicolumn{4}{|l|}{$y$-Direction Masonry } \\
\hline Gross Peak Compressive Strength & $f_{m y}^{\prime}$ & $\mathrm{MPa}$ & None \\
\hline Strain at $f_{m y}^{\prime}$ & $\epsilon_{o y}$ & - & None \\
\hline Tensile Strength & $f_{c r, y}$ & $\mathrm{MPa}$ & See Equation 10.39 \\
\hline Tensile Strain & $\epsilon_{c r, y}$ & - & $f_{c r, y} / E_{m y}$ \\
\hline Initial Tangent Elastic Modulus ${ }^{\mathrm{a}}$ & $E_{m y}$ & $\mathrm{MPa}$ & $0.4 f_{m y}^{\prime} /\left(1-\sqrt{\frac{3}{5}}\right) \epsilon_{o y}$ \\
\hline Poisson's Ratiob & $v_{m y}$ & - & 0.20 \\
\hline \multicolumn{4}{|l|}{$x$-Direction Masonry } \\
\hline Gross Peak Compressive Strength & $f_{m x}^{\prime}$ & $\mathrm{MPa}$ & None \\
\hline Strain at $f_{m x}^{\prime}$ & $\epsilon_{o x}$ & - & None \\
\hline Tensile Strength & $f_{c r, x}$ & $\mathrm{MPa}$ & See Equation 10.40 \\
\hline Tensile Strain & $\epsilon_{c r, x}$ & - & $f_{c r, x} / E_{m x}$ \\
\hline Initial Tangent Elastic Modulus ${ }^{\mathrm{a}}$ & $E_{m x}$ & $\mathrm{MPa}$ & $0.4 f_{m x}^{\prime} /\left(1-\sqrt{\frac{3}{5}}\right) \epsilon_{o x}$ \\
\hline Poisson's Ratiob & $v_{m x}$ & - & 0.20 \\
\hline \multicolumn{4}{|l|}{ Masonry Options } \\
\hline Block Length & $l^{b l}$ & $\mathrm{~mm}$ & None \\
\hline Block Height & $h^{b l}$ & $\mathrm{~mm}$ & None \\
\hline Block Face Shell Thickness & $t^{f s}$ & $\mathrm{~mm}$ & None \\
\hline Block Percent Solid & $\rho_{\text {solid }}^{b l}$ & $\mathrm{~mm}$ & None \\
\hline Block Tensile Strength & $f_{t, b l}^{\prime}$ & $\mathrm{mm}$ & None \\
\hline Head Joint Thickness & $t_{h j}$ & $\mathrm{~mm}$ & 10 \\
\hline Head Joint Tensile Bond Strength & $f_{t, h j}$ & $\mathrm{MPa}$ & None \\
\hline Head Joint Cohesion & $c_{h j}$ & $\mathrm{MPa}$ & None \\
\hline Head Joint Angle of Internal Friction & $\phi_{h j}$ & Degrees & 42 \\
\hline Bed Joint Thickness & $t_{b j}$ & $\mathrm{~mm}$ & 10 \\
\hline Bed Joint Tensile Bond Strength & $f_{t, b j}$ & $\mathrm{MPa}$ & None \\
\hline Bed Joint Cohesion & $c_{b j}$ & $\mathrm{MPa}$ & None \\
\hline Bed Joint Angle of Internal Friction & $\phi_{b j}$ & Degrees & 42 \\
\hline Grout Tensile Strength & $f_{t, g r}^{\prime}$ & $\mathrm{MPa}$ & None \\
\hline Thickness & $t$ & $\mathrm{~mm}$ & None \\
\hline \multicolumn{4}{|l|}{ Notes: } \\
\hline \multicolumn{4}{|c|}{${ }^{\mathrm{a} S e c a n t}$ elastic modulus at $0.4 f_{c, i}^{\prime j}$ based on a Hognestad parabola } \\
\hline \multicolumn{4}{|l|}{ (MacGregor and Bartlett, 2000) } \\
\hline bBased on Ip (1999) & & & \\
\hline
\end{tabular}

Part III: Masonry Finite Element Modeling 
Table 11.3: Reinforcement SAFEM Inputs

\begin{tabular}{|c|c|c|c|}
\hline Variable Name & Symbol & Units & Default Value \\
\hline \multicolumn{4}{|l|}{$y$-Direction Reinforcement } \\
\hline Reinforcement Ratio & $\rho_{y}$ & $\%$ & None \\
\hline Yield Strength & $f_{y y}$ & $\mathrm{MPa}$ & None \\
\hline Yield Strain & $\epsilon_{y y}$ & - & None \\
\hline Strain Hardening Strain & $\epsilon_{\text {shy }}$ & - & None \\
\hline Ultimate Strength & $f_{u y}$ & $\mathrm{MPa}$ & None \\
\hline Ultimate Strain & $\epsilon_{u y}$ & - & None \\
\hline \multicolumn{4}{|l|}{$x$-Direction Reinforcement } \\
\hline Reinforcement Ratio & $\rho_{x}$ & $\%$ & None \\
\hline Yield Strength & $f_{y x}$ & $\mathrm{MPa}$ & None \\
\hline Yield Strain & $\epsilon_{y x}$ & - & None \\
\hline Strain Hardening Strain & $\epsilon_{\operatorname{sh} x}$ & - & None \\
\hline Ultimate Strength & $f_{u x}$ & $\mathrm{MPa}$ & None \\
\hline Ultimate Strain & $\epsilon_{u x}$ & - & None \\
\hline
\end{tabular}

Table 11.4: Constitutive Model Options

\begin{tabular}{ll}
\hline Constitutive Relationship & Model Options \\
\hline Compression Pre-Peak & Hognestad \\
& Linear \\
& Popovics \\
\hline Compression Post-Peak & Modified Park-Kent \\
& Pre-Peak Base Curve \\
\hline Compression Softening & No Modification \\
& Vecchio \\
\hline Tension Post-Peak & Hordijk \\
& Vecchio \\
\hline Cracking Criterion & Mohr-Coulomb \\
& Uniaxial Cracking Stress \\
\hline Slip & No Slip \\
& Constant Rotation Lag \\
& Walraven Reinforced \\
\hline
\end{tabular}

Part III: Masonry Finite Element Modeling 


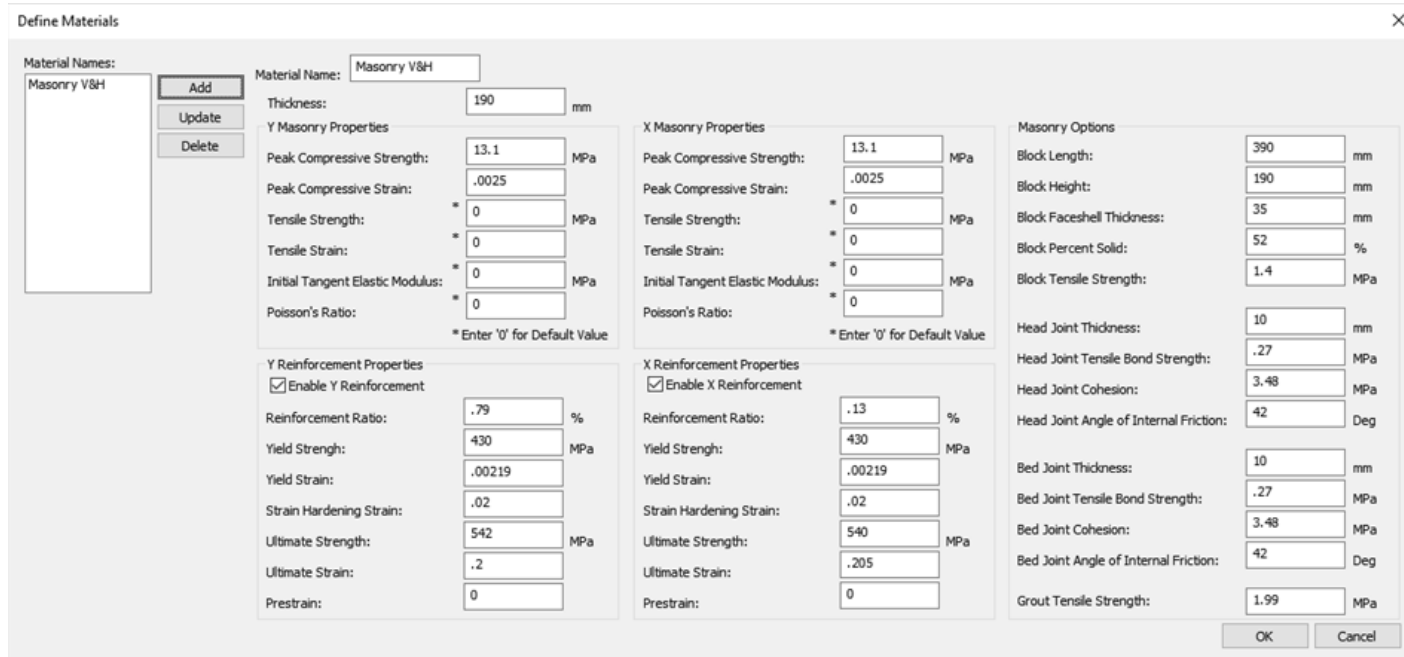

Figure 11.5: SAFEM Program Material Input

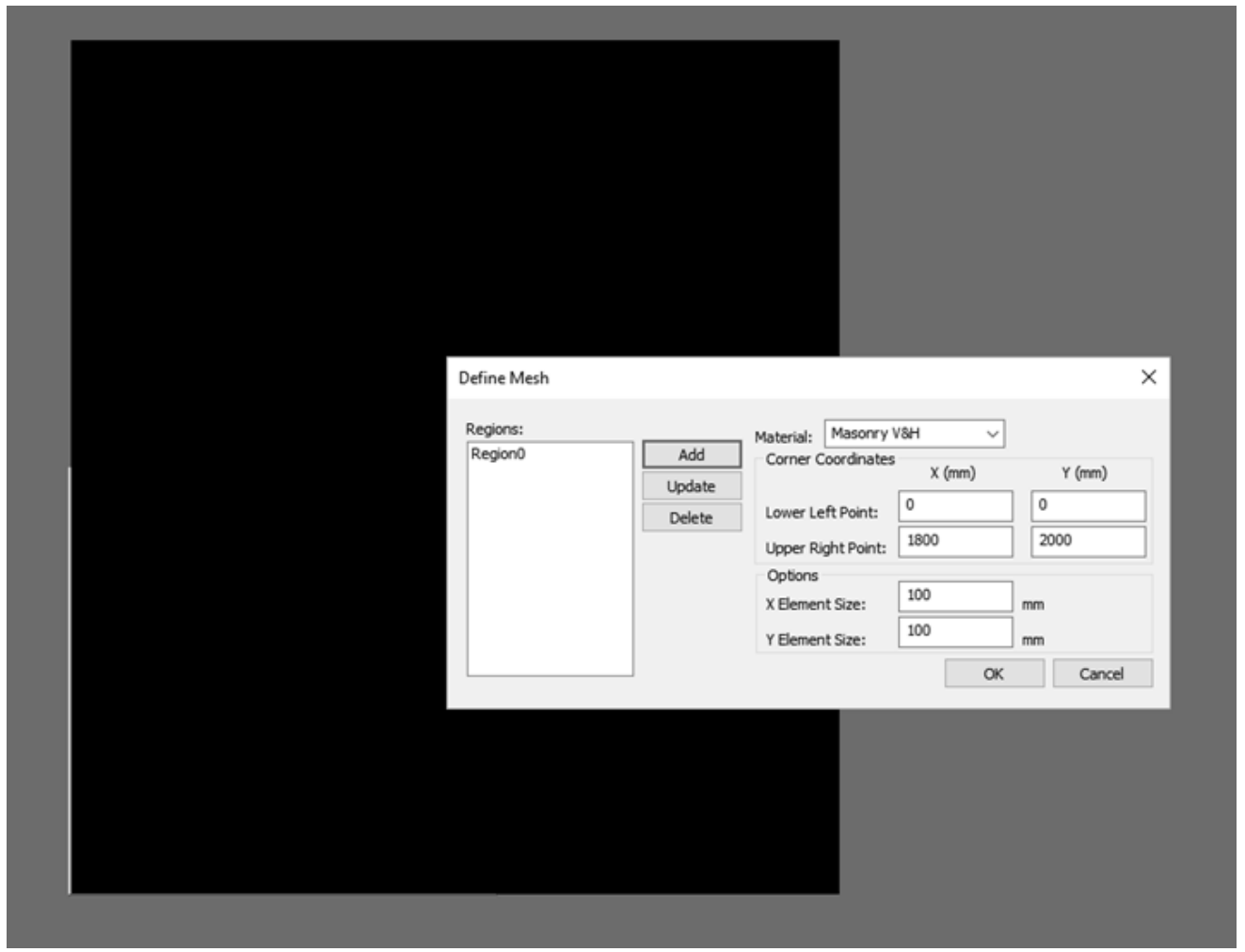

Figure 11.6: SAFEM Program Mesh Generation 


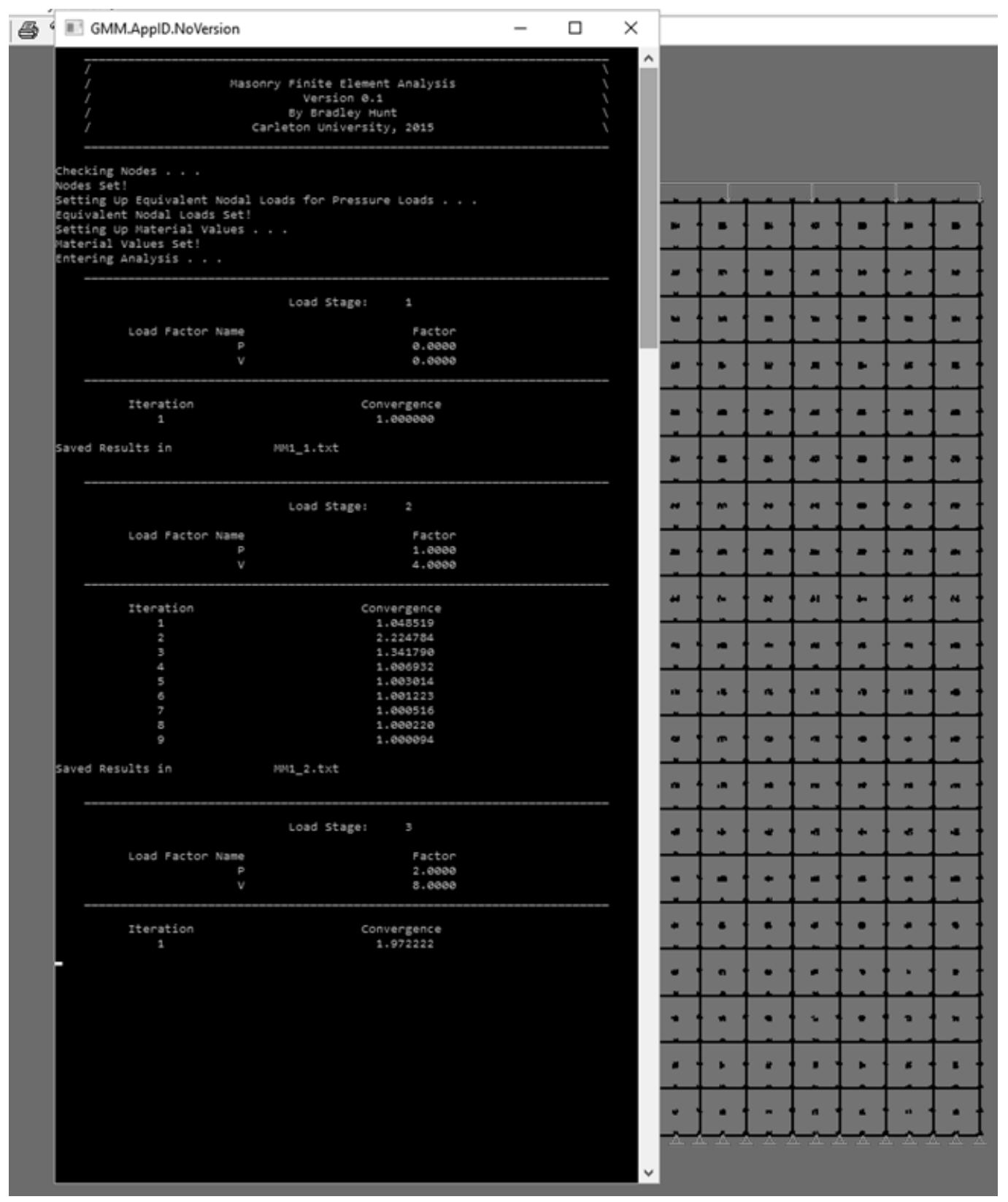

Figure 11.7: SAFEM Program Model Running Window 


\section{SMEARED AREA FINITE ELEMENT MODEL VERIFI- CATION}

The finite element implementation presented in Chapter 11 is verified through element verification, analytic verification and constitutive relationship and non-linear verification.

Verification is the process of determining that a mathematical model implementation accurately represents the conceptual description and solution. Mathematical models usually consist of a set of partial differential equations and the associated boundary conditions, initial conditions, and constitutive equations. Implementing the mathematical model in a computational code requires numerical discretization, solution algorithms, and convergence criteria. Numerical code verification assesses the mathematical accuracy of the code and the implementation of the discrete algorithms for solving the partial differential equations. Numerical code verification involves comparing solutions produced by the code's algorithms to test problems for which the answer is known. The goal is to verify that numerical algorithms are implemented correctly.

Nothing is as important to the success of a finite element analysis as the accuracy of the elements. The finite elements embody all of the discretizing assumptions; while, the rest of the calculations are exact except for their lack of precision. Thus the accuracy of the finite elements is of primary concern in finite element analyses and needs to be verified before any reasonable conclusions can be drawn from analysis.

The verification of the Smeared Area Finite Element Model (SAFEM) will be completed in three parts. First the element code will be examined through the stiffness matrix of each element and the global stiffness matrix of a simple mesh of elements. Secondly, the finite element code will be verified by comparing the results of the SAFEM to known analytic results. Finally, the results of the SAFEM will be compared to a similar finite element program, to test the constitutive relationships. 


\subsection{Element Verification}

The mesh shown in Figure 12.1, with nodal coordinates given in Table 12.1, will be used for element verification. The elements are of unit thickness with isotropic properties. The modulus of elasticity is equal to $1000 \mathrm{MPa}$ and the Poisson's ratio is 0.20 .

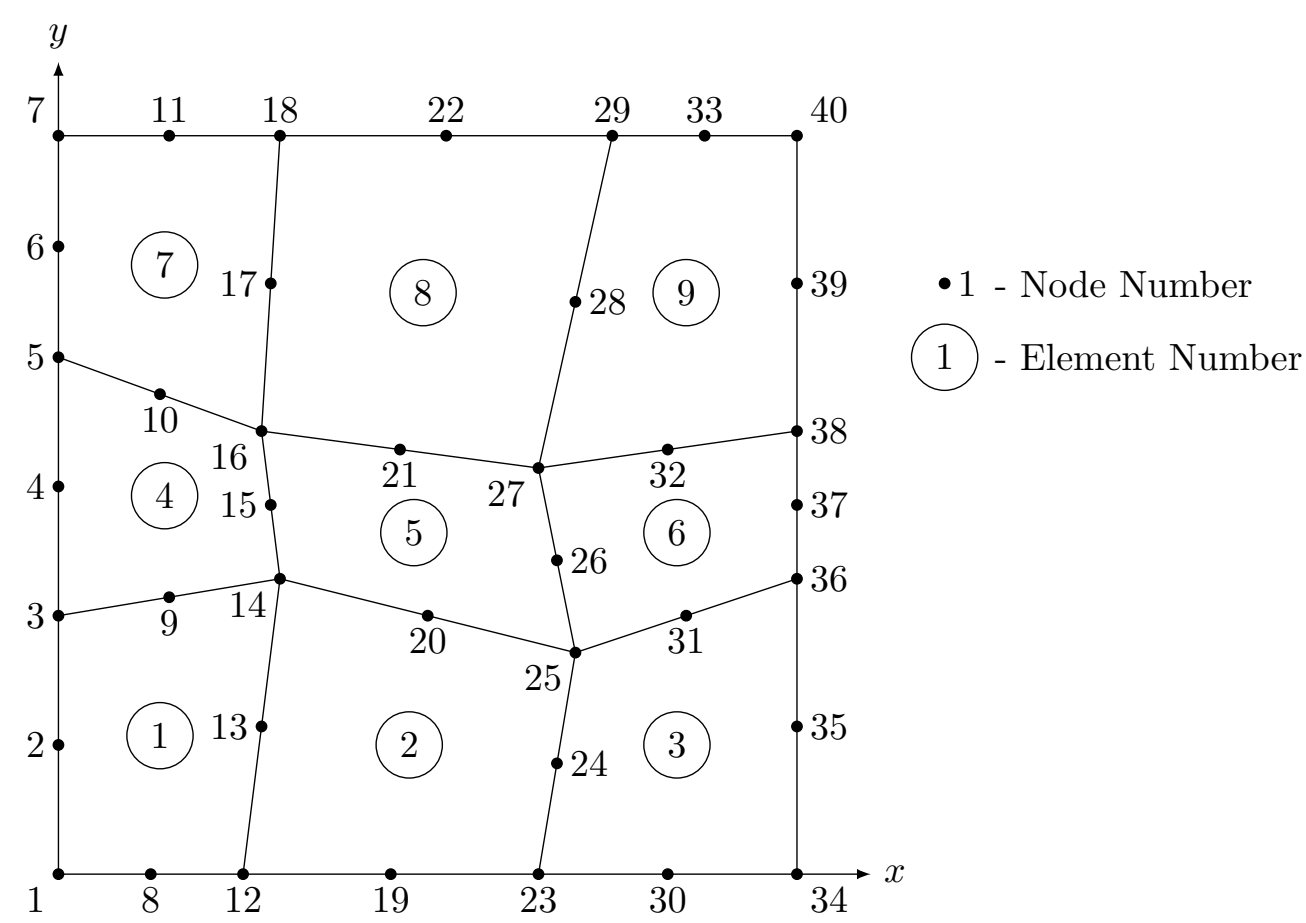

Figure 12.1: Element Verification Mesh 
Table 12.1: Coordinates of Nodes

\begin{tabular}{llllll}
\hline Node & $x$-Coordinate & $y$-Coordinate & Node & $x$-Coordinate & $y$-Coordinate \\
\hline 1 & 0 & 0 & 21 & 4.625 & 5.75 \\
2 & 0 & 1.75 & 22 & 5.25 & 10 \\
3 & 0 & 3.5 & 23 & 6.5 & 0 \\
4 & 0 & 5.25 & 24 & 6.75 & 1.5 \\
5 & 0 & 7 & 25 & 7 & 3 \\
6 & 0 & 8.5 & 26 & 6.75 & 4.25 \\
7 & 0 & 10 & 27 & 6.5 & 5.5 \\
8 & 1.25 & 0 & 28 & 7 & 7.75 \\
9 & 1.5 & 3.75 & 29 & 7.5 & 10 \\
10 & 1.375 & 6.5 & 30 & 8.25 & 0 \\
11 & 1.5 & 10 & 31 & 8.5 & 3.5 \\
12 & 2.5 & 0 & 32 & 8.25 & 5.75 \\
13 & 2.75 & 2 & 33 & 8.75 & 10 \\
14 & 3 & 4 & 34 & 10 & 0 \\
15 & 2.875 & 5 & 35 & 10 & 2 \\
16 & 2.75 & 6 & 36 & 10 & 4 \\
17 & 2.875 & 8 & 37 & 10 & 5 \\
18 & 3 & 10 & 38 & 10 & 6 \\
19 & 4.5 & 0 & 39 & 10 & 8 \\
20 & 5 & 3.5 & 40 & 10 & 10 \\
\hline & & & & & \\
\hline
\end{tabular}

\subsubsection{Symmetry of Stiffness Matrix}

Symmetry of the element and global stiffness matrix is a requirement, as proven by Maxwell-Betti Reciprocal theorem, if all the strain energy is to be positive. If the stiffness matrix is symmetric, subtracting its transpose from itself should result in a matrix of the same size of all zeros. Completing this for all elements, $i$, in Figure 12.1 results in all element stiffness matrices being symmetric, i.e.

$$
[k]_{i, 16 \times 16}-[k]_{i, 16 \times 16}^{T}=\left[\begin{array}{ccc}
0 & \cdots & 0 \\
\vdots & \ddots & \vdots \\
0 & \cdots & 0
\end{array}\right]_{i, 16 \times 16} .
$$

The resulting global stiffness matrix, $[K]$, for the mesh shown in Figure 12.1, is $80 \times 80$. To ensure the global stiffness matrix is also symmetric, a similar process as the element 
stiffness matrix symmetric check can be applied. SAFEM also has a symmetric global stiffness matrix, by:

$$
[K]_{80 \times 80}-[K]_{80 \times 80}^{T}=\left[\begin{array}{ccc}
0 & \cdots & 0 \\
\vdots & \ddots & \vdots \\
0 & \cdots & 0
\end{array}\right]_{80 \times 80}
$$

\subsubsection{Eigenvalues and Rank Sufficiency of Stiffness Matrix}

The element stiffness matrix must not possess any zero-energy kinematic mode other than rigid body modes, this can be be tested by the rank of the stiffness matrix (Cook et al., 1989). A stiffness matrix that has proper rank is called rank sufficient. For a positive semi-definite matrix, i.e. all positive eigenvalues (which the element stiffness matrix should be), the rank is simply the total of non-zero eigenvalues. The rank, $r$, of the stiffness matrix can be determined from:

$$
r=\min \left(n_{F}-n_{R}, n_{G} n_{E}\right)
$$

where $n_{F}$ is the number of degrees of freedom in the element, $n_{R}$ is the number of independent rigid body modes, $n_{G}$ is the number of Gauss points, and $n_{E}$ is the order of the stress-strain matrix. The stiffness matrix of an eight node isoparametric quadrilateral element with full integration should have a rank of 13 and 3 independent rigid body modes (Cook et al., 1989). As can be seen by Equation 12.4, each element has a positive semi-definite stiffness matrix, that is rank sufficient, and 3 independent rigid body modes.

$$
\begin{aligned}
& {\left[\lambda^{1}\right]=[5806,3915,2518,2004,1939,1549,1485,1184,547,449,406,210,162,0,0,0]} \\
& {\left[\lambda^{2}\right]=[4942,4117,2146,2028,1914,1674,1455,1125,509,489,361,252,159,0,0,0]} \\
& {\left[\lambda^{3}\right]=[5030,4286,2540,1942,1861,1635,1535,1084,624,414,384,215,158,0,0,0]} \\
& {\left[\lambda^{4}\right]=[4999,4458,2378,2039,1882,1690,1472,1096,574,448,381,243,143,0,0,0]} \\
& {\left[\lambda^{5}\right]=[7693,4372,3340,2129,1981,1734,1400,1288,572,548,340,225,110,0,0,0]} \\
& {\left[\lambda^{6}\right]=[6234,3955,2508,2126,1956,1491,1463,1246,524,478,417,196,165,0,0,0]} \\
& {\left[\lambda^{7}\right]=[5229,4063,2378,1990,1861,1687,1476,1128,559,443,378,241,152,0,0,0]} \\
& {\left[\lambda^{8}\right]=[4321,4295,2229,1872,1851,1776,1476,1100,544,466,330,274,157,0,0,0]} \\
& {\left[\lambda^{9}\right]=[6167,3996,2676,2040,1929,1482,1549,1196,559,474,390,153,208,0,0,0]}
\end{aligned}
$$




\subsubsection{Element Jacobian Values}

The determinant of the Jacobian matrix, $|J|$, that relates Cartesian and natural coordinates must be positive everywhere within the element (Cook et al., 1989). The determinant of the Jacobian matrix for each Gauss integration point within each element is given in Table 12.2. As can seen all the determinants of the Jacobian matrices are positive.

Table 12.2: Determinants of the Jacobian Matrices

\begin{tabular}{llllllllll}
\hline \multirow{2}{*}{ Element } & \multicolumn{10}{c}{ Gauss Point } \\
\cline { 2 - 10 } & 1 & 2 & 3 & 4 & 5 & 6 & 7 & 8 & 9 \\
\hline 1 & 2.27 & 2.39 & 2.51 & 2.44 & 2.56 & 2.68 & 2.61 & 2.73 & 2.85 \\
2 & 3.90 & 3.51 & 3.13 & 3.95 & 3.56 & 3.18 & 4.00 & 3.61 & 3.22 \\
3 & 2.67 & 3.01 & 3.35 & 2.47 & 2.81 & 3.15 & 2.28 & 2.62 & 2.96 \\
4 & 2.48 & 2.05 & 1.63 & 2.39 & 1.97 & 1.55 & 2.31 & 1.88 & 1.46 \\
5 & 1.98 & 2.15 & 2.32 & 1.94 & 2.11 & 2.28 & 1.90 & 2.07 & 2.24 \\
6 & 1.97 & 1.78 & 1.58 & 2.07 & 1.88 & 1.68 & 2.17 & 1.97 & 1.78 \\
7 & 2.17 & 2.46 & 2.75 & 2.24 & 2.53 & 2.82 & 2.31 & 2.60 & 2.89 \\
8 & 3.93 & 4.14 & 4.36 & 4.20 & 4.42 & 4.64 & 4.48 & 4.70 & 4.92 \\
9 & 3.66 & 3.54 & 3.42 & 3.28 & 3.16 & 3.04 & 2.89 & 2.77 & 2.65 \\
\hline
\end{tabular}

\subsubsection{Equivalent Nodal Loads}

In finite element models, loads can only be applied to the nodes; hence, distributed loads must be converted to equivalent nodal loads. Equivalent loads must produce same strain energy within the element as the distributed load (Cook et al., 1989). The equivalent nodal loads for the eight node isoparametric quadrilateral element are shown in Figure 12.2 resulting in the equivalent nodal loads for the nine element mesh shown in Figure 12.3. The finite element code produces the same equivalent nodal loads as required by strain energy.

Part III: Masonry Finite Element Modeling 


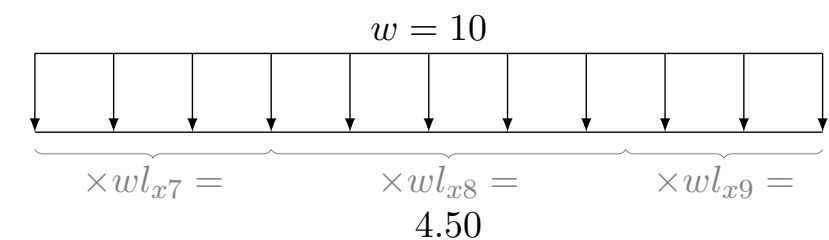

Uniformly Distributed Load

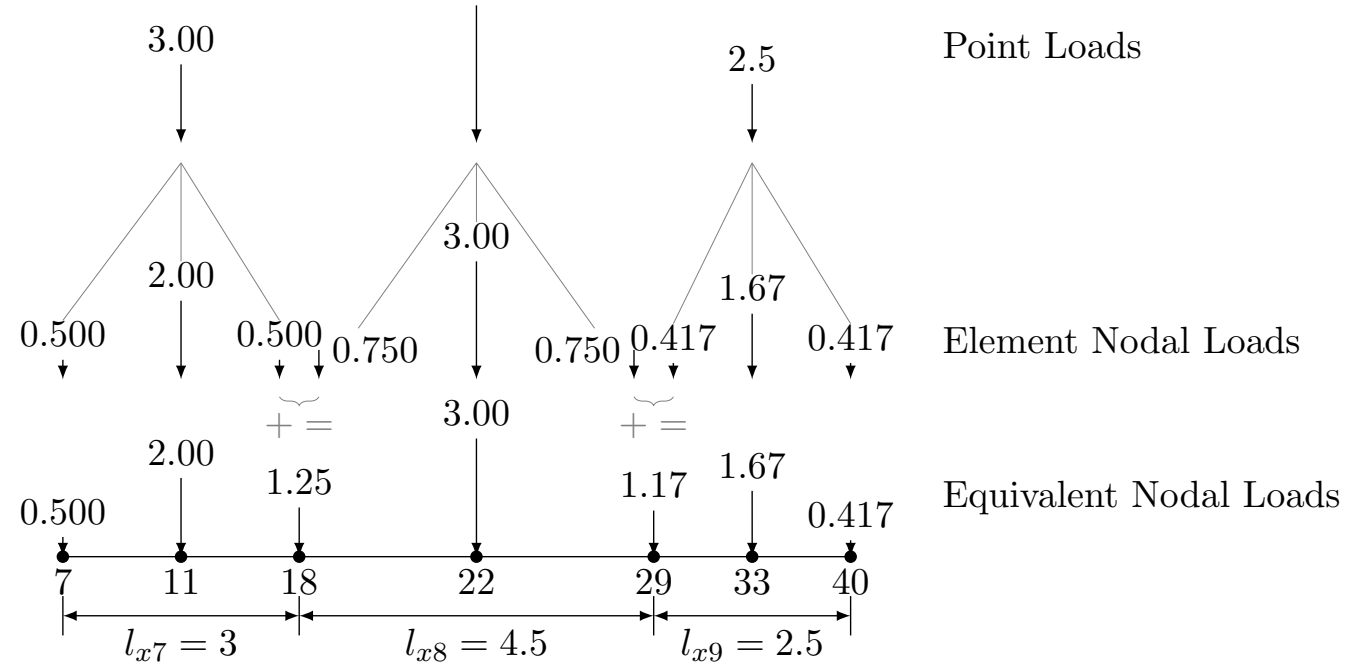

Figure 12.3: Equivalent Nodal Loads for the Mesh of Elements in Figure 12.1

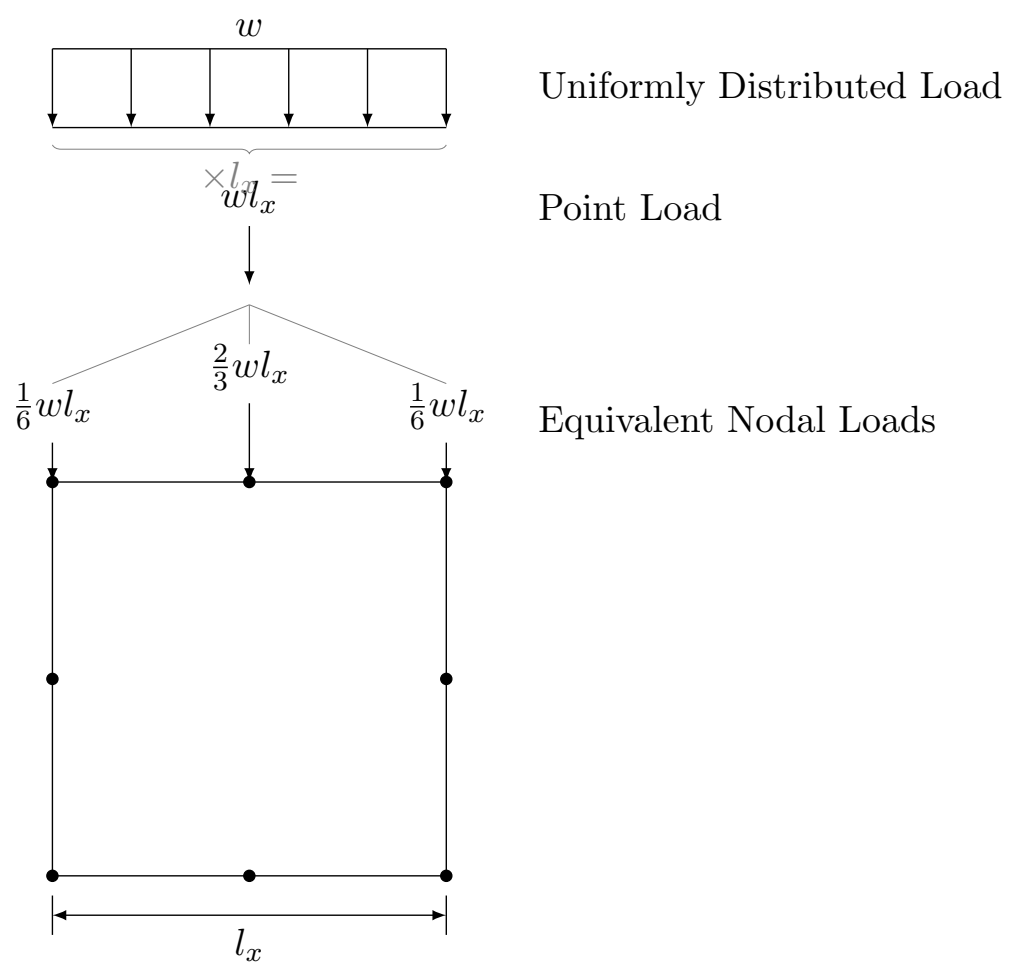

Figure 12.2: Equivalent Nodal Loads for Eight Node Isoparametric Quadrilateral 


\subsection{Numerical Tests}

In the second stage of verification, the proposed finite element code is employed to solve linear numerical models. The obtained results are compared with the known responses generally published in the open literature or in FEM program documentation. The design of a comprehensive set of element test problems should, of course, take into account the parameters which affect accuracy. These parameters can be classified under the headings of loading, element geometry, and problem geometry (MacNeal and Harder, 1985). With regard to loading, the problem set should, as a whole, provide significant loading for each of the types of deformation which the elements can exhibit. For an eight node quadrilateral this includes extension, in-plane shear, and in-plane bending. Care should also be taken to test nonstandard shapes, such as parallelograms and trapezoids for eight node quadrilateral elements.

\subsubsection{Element Force Patch Test}

The patch test is a simple test which should be performed when first using any finite element program. The patch test both ensures that the theory for the finite element formulation has been correctly implemented and that installation of the analysis system is also correct. The computed stresses should recover exactly the test state.

The principal virtue of a patch test is that, if an element produces correct results for the test, the results for any problem solved with the element will converge toward the correct solution as the elements are subdivided. The reason is that the stress within each element tends to a uniform value at the limit.

First, apply $1 \mathrm{MPa}$ in the y-direction along the top side of the mesh shown in Figure 12.1 with node 1 fully restrained and node 8, 12, 19, 23, 30, and 34 restrained only in the $\mathrm{y}$-direction. This results in the nodal displacements and forces in Table 12.3 and element stresses shown in Table 12.4. The applied load causes a stress of $1 \mathrm{MPa}$ in the y-direction as confirmed by the element stresses. Furthermore, the stress causes a strain of 0.001 $\mathrm{mm} / \mathrm{mm}$ in the $\mathrm{y}$-direction which is confirmed by both the nodal displacements and element strains. 
Table 12.3: Nodal Displacements and Forces for $\sigma_{y}=10 \mathrm{~N} / \mathrm{mm}$

\begin{tabular}{|c|c|c|c|c|c|c|c|c|c|}
\hline \multirow[t]{2}{*}{ Nodes } & \multicolumn{2}{|c|}{$\begin{array}{c}\text { Displacements } \\
(\mathrm{mm})\end{array}$} & \multicolumn{2}{|c|}{$\begin{array}{c}\text { Forces } \\
(\mathrm{N})\end{array}$} & \multirow[t]{2}{*}{ Nodes } & \multicolumn{2}{|c|}{$\begin{array}{l}\text { Displacements } \\
\qquad(\mathrm{mm})\end{array}$} & \multicolumn{2}{|c|}{$\begin{array}{l}\text { Forces } \\
(\mathrm{N})\end{array}$} \\
\hline & $u$ & $v$ & $F_{x}$ & $F_{y}$ & & $u$ & $v$ & $F_{x}$ & $F_{y}$ \\
\hline 1 & 0 & 0 & 0 & -0.417 & 21 & 0 & 0.00575 & 0 & 0 \\
\hline 2 & 0 & 0.00175 & 0 & 0 & 22 & 0 & 0.010 & 0 & 3 \\
\hline 3 & 0 & 0.0035 & 0 & 0 & 23 & 0 & 0 & 0 & -1.25 \\
\hline 4 & 0 & 0.00525 & 0 & 0 & 24 & 0 & 0.0015 & 0 & 0 \\
\hline 5 & 0 & 0.007 & 0 & 0 & 25 & 0 & 0.003 & 0 & 0 \\
\hline 6 & 0 & 0.0085 & 0 & 0 & 26 & 0 & 0.00425 & 0 & 0 \\
\hline 7 & 0 & 0.01 & 0 & 0.5 & 27 & 0 & 0.0055 & 0 & 0 \\
\hline 8 & 0 & 0 & 0 & -1.667 & 28 & 0 & 0.00775 & 0 & 0 \\
\hline 9 & 0 & 0.00375 & 0 & 0 & 29 & 0 & 0.010 & 0 & 1.17 \\
\hline 10 & 0 & 0.0065 & 0 & 0 & 30 & 0 & 0 & 0 & -2.333 \\
\hline 11 & 0 & 0.01 & 0 & 2 & 31 & 0 & 0.0035 & 0 & 0 \\
\hline 12 & 0 & 0 & 0 & -1.083 & 32 & 0 & 0.00575 & 0 & 0 \\
\hline 13 & 0 & 0.002 & 0 & 0 & 33 & 0 & 0.010 & 0 & 1.67 \\
\hline 14 & 0 & 0.004 & 0 & 0 & 34 & 0 & 0 & 0 & -0.583 \\
\hline 15 & 0 & 0.005 & 0 & 0 & 35 & 0 & 0.002 & 0 & 0 \\
\hline 16 & 0 & 0.006 & 0 & 0 & 36 & 0 & 0.004 & 0 & 0 \\
\hline 17 & 0 & 0.008 & 0 & 0 & 37 & 0 & 0.005 & 0 & 0 \\
\hline 18 & 0 & 0.010 & 0 & 0 & 38 & 0 & 0.006 & 0 & 0 \\
\hline 19 & 0 & 0 & 0 & -2.667 & 39 & 0 & 0.008 & 0 & 0 \\
\hline 20 & 0 & 0.0035 & 0 & 0 & 40 & 0 & 0.0010 & 0 & 0.417 \\
\hline
\end{tabular}


Table 12.4: Element Stresses for $\sigma_{y}=10 \mathrm{~N} / \mathrm{mm}$

\begin{tabular}{llllllll}
\hline \multirow{2}{*}{ Element } & \multicolumn{3}{c}{ Stain $(\mathrm{mm} / \mathrm{mm})$} & & \multicolumn{3}{c}{ Stress $(\mathrm{MPa})$} \\
\cline { 2 - 5 } \cline { 7 - 8 } & $\epsilon_{x}$ & $\epsilon_{y}$ & $\gamma_{x y}$ & & $\sigma_{x}$ & $\sigma_{y}$ & $\tau_{x y}$ \\
\hline 1 & 0 & 0.001 & 0 & & 0 & 1 & 0 \\
2 & 0 & 0.001 & 0 & & 0 & 1 & 0 \\
3 & 0 & 0.001 & 0 & & 0 & 1 & 0 \\
4 & 0 & 0.001 & 0 & & 0 & 1 & 0 \\
5 & 0 & 0.001 & 0 & & 0 & 1 & 0 \\
6 & 0 & 0.001 & 0 & & 0 & 1 & 0 \\
7 & 0 & 0.001 & 0 & & 0 & 1 & 0 \\
8 & 0 & 0.001 & 0 & & 0 & 1 & 0 \\
9 & 0 & 0.001 & 0 & & 0 & 1 & 0 \\
\hline
\end{tabular}

Secondly, applying $1 \mathrm{MPa}$ in the x-direction along the right side of the mesh shown in Figure 12.1 with node 1 fully restrained and node 2, 3, 4, 5, 6, and 7 restrained only in the $\mathrm{x}$-direction. The recovered displacements, strains, and stresses shown in Table 12.5 and Table 12.6 confirm the applied load of $1 \mathrm{MPa}$ in each element. 
Table 12.5: Nodal Displacements and Forces for $\sigma_{x}=10 \mathrm{~N} / \mathrm{mm}$

\begin{tabular}{|c|c|c|c|c|c|c|c|c|c|}
\hline \multirow[t]{2}{*}{ Nodes } & \multicolumn{2}{|c|}{$\begin{array}{l}\text { Displacements } \\
\quad(\mathrm{mm})\end{array}$} & \multicolumn{2}{|c|}{$\begin{array}{l}\text { Forces } \\
(\mathrm{N})\end{array}$} & \multirow[t]{2}{*}{ Nodes } & \multicolumn{2}{|c|}{$\begin{array}{l}\text { Displacements } \\
\quad(\mathrm{mm})\end{array}$} & \multicolumn{2}{|c|}{$\begin{array}{c}\text { Forces } \\
(\mathrm{N})\end{array}$} \\
\hline & $u$ & $v$ & $F_{x}$ & $F_{y}$ & & $u$ & $v$ & $F_{x}$ & $F_{y}$ \\
\hline 1 & 0 & 0 & -0.583 & 0 & 21 & 0.004625 & 0 & 0 & 0 \\
\hline 2 & 0 & 0 & -2.333 & 0 & 22 & 0.00525 & 0 & 0 & 0 \\
\hline 3 & 0 & 0 & -1.167 & 0 & 23 & 0.0065 & 0 & 0 & 0 \\
\hline 4 & 0 & 0 & -2.333 & 0 & 24 & 0.00675 & 0 & 0 & 0 \\
\hline 5 & 0 & 0 & -1.083 & 0 & 25 & 0.007 & 0 & 0 & 0 \\
\hline 6 & 0 & 0 & -2 & 0 & 26 & 0.00675 & 0 & 0 & 0 \\
\hline 7 & 0 & 0 & -0.5 & 0 & 27 & 0.0065 & 0 & 0 & 0 \\
\hline 8 & 0.00125 & 0 & 0 & 0 & 28 & 0.007 & 0 & 0 & 0 \\
\hline 9 & 0.0015 & 0 & 0 & 0 & 29 & 0.0075 & 0 & 0 & 0 \\
\hline 10 & 0.001375 & 0 & 0 & 0 & 30 & 0.00825 & 0 & 0 & 0 \\
\hline 11 & 0.0015 & 0 & 0 & 0 & 31 & 0.0085 & 0 & 0 & 0 \\
\hline 12 & 0.0025 & 0 & 0 & 0 & 32 & 0.00825 & 0 & 0 & 0 \\
\hline 13 & 0.00275 & 0 & 0 & 0 & 33 & 0.0075 & 0 & 0 & 0 \\
\hline 14 & 0.003 & 0 & 0 & 0 & 34 & 0.01 & 0 & 0.667 & 0 \\
\hline 15 & 0.002875 & 0 & 0 & 0 & 35 & 0.01 & 0 & 2.667 & 0 \\
\hline 16 & 0.00275 & 0 & 0 & 0 & 36 & 0.01 & 0 & 1 & 0 \\
\hline 17 & 0.002875 & 0 & 0 & 0 & 37 & 0.01 & 0 & 1.333 & 0 \\
\hline 18 & 0.003 & 0 & 0 & 0 & 38 & 0.01 & 0 & 1 & 0 \\
\hline 19 & 0.0045 & 0 & 0 & 0 & 39 & 0.01 & 0 & 2.667 & 0 \\
\hline 20 & 0.005 & 0 & 0 & 0 & 40 & 0.01 & 0 & 0.667 & 0 \\
\hline
\end{tabular}

\subsubsection{Straight Cantilever Beam}

The straight cantilever beam is frequently used as a test problem which can be easily applied to quadrilateral elements (MacNeal and Harder, 1985). Its simplicity and the fact that all the principal element deformation modes can be evoked by loads applied to the free end make it an ideal problem to verify finite element codes. Consider a cantilever beam of $6000 \mathrm{~mm}$ in length, $100 \mathrm{~mm}$ deep, and $200 \mathrm{~mm}$ thick with a modulus of elesticity of $1.0 \times 10^{7} \mathrm{MPa}$ and a Poisson's ratio of 0.3 . The theoretical displacement of a horizontal tensile unit load applied to the free end of the beam is $3.0 \times 10^{-5} \mathrm{~mm}$ of extension. For a downward vertical unit load at the free end of the beam the theoretical downward vertical tip displacement is $0.432 \mathrm{~mm}$. To test the effects of element irregularities, in addition to rectangular elements, two different non-rectangular element layouts are 
Table 12.6: Element Stresses for $\sigma_{x}=10 \mathrm{~N} / \mathrm{mm}$

\begin{tabular}{lllllllll}
\hline \multirow{2}{*}{ Element } & \multicolumn{3}{l}{ Stain $(\mathrm{mm} / \mathrm{mm})$} & & \multicolumn{3}{c}{ Stress $(\mathrm{MPa})$} \\
\cline { 2 - 5 } \cline { 7 - 8 } & $\epsilon_{x}$ & $\epsilon_{y}$ & $\gamma_{x y}$ & & $\sigma_{x}$ & $\sigma_{y}$ & $\tau_{x y}$ \\
\hline 1 & 0.001 & 0 & 0 & & 1 & 0 & 0 \\
2 & 0.001 & 0 & 0 & & 1 & 0 & 0 \\
3 & 0.001 & 0 & 0 & & 1 & 0 & 0 \\
4 & 0.001 & 0 & 0 & & 1 & 0 & 0 \\
5 & 0.001 & 0 & 0 & & 1 & 0 & 0 \\
6 & 0.001 & 0 & 0 & & 1 & 0 & 0 \\
7 & 0.001 & 0 & 0 & & 1 & 0 & 0 \\
8 & 0.001 & 0 & 0 & & 1 & 0 & 0 \\
9 & 0.001 & 0 & 0 & & 1 & 0 & 0 \\
\hline
\end{tabular}

used (see Figure 12.4). The finite element code results are compared to the theoretical solutions in Table 12.7. The rectangular and parallelogram shaped elements perform very well in both the extension and in-plane shear test, while the trapezoidal shaded elements perform well in the extension test, but failed to capture the in-place shear displacement because of the element distortion, a well documented issue of quadrilateral elements (MacNeal and Harder, 1985).

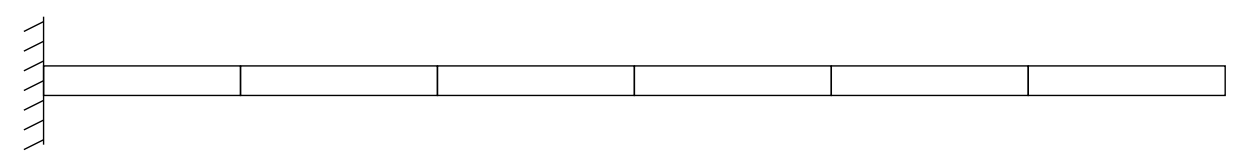

(a) Rectangular Shaped Elements (MacNeal and Harder, 1985)

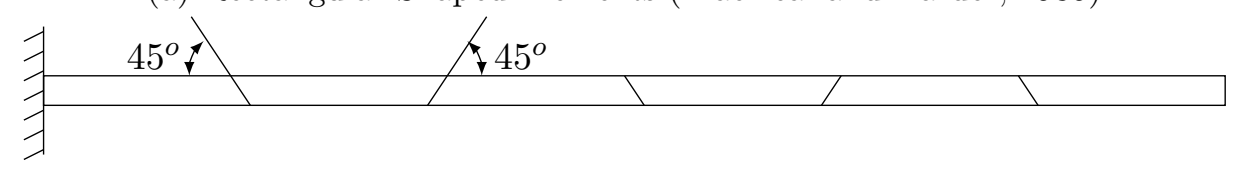

(b) Trapezoidal Shaped Elements

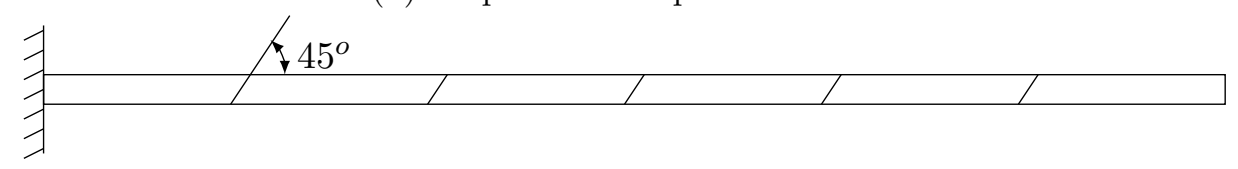

(c) Parallelogram Shaped Elements

Figure 12.4: Straight Cantilever Element Layouts 
Table 12.8: Pure Bending Cantilever Beam Displacement at Tip

\begin{tabular}{llllllll}
\hline Mesh Number & 1 & 2 & 3 & 4 & 5 & 6 & 7 \\
\hline Model $\left(\times 10^{-3} \mathrm{~mm}\right)$ & 12.0 & 1.78 & 0.450 & 0.480 & 0.424 & 0.060 & 0.059 \\
\hline \hline Theoretical $^{\mathrm{a}}\left(\times 10^{-3} \mathrm{~mm}\right)$ & 12.0 & 12.0 & 12.0 & 0.480 & 0.480 & 0.060 & 0.060 \\
\hline
\end{tabular}

Notes:

${ }^{a}$ Based on Timoshenko and Goodier (1934)

Table 12.7: Straight Cantilever Beam Displacement in Direction of Load

\begin{tabular}{lll}
\hline Mesh Type & Extension $(\mathrm{mm})$ & In-Plane Shear $(\mathrm{mm})$ \\
\hline Rectangular Shaped Elements & $3.0 \times 10^{-5}$ & 0.429 \\
Trapezoidal Shaped Elements & $3.0 \times 10^{-5}$ & 0.391 \\
Parallelogram Shaped Elements & $3.0 \times 10^{-5}$ & 0.421 \\
\hline \hline Theoretical & $3.0 \times 10^{-5}$ & 0.432 \\
\hline
\end{tabular}

\subsubsection{Thin Arched Beam}

In the thin arched beam (see Figure 12.5), combinations of principal deformation modes are evoked by a single in-plane shear load at the tip. Also, the element shape is not quite rectangular, which will test the effect of slight irregularity. One end of the structure is fixed, and the other one is under a shear force equivalent to $1000 \mathrm{~N}$. The elasticity modulus, the Poisson's ratio and the beam thickness are equal to $1.0 \times 10^{7} \mathrm{MPa}, 0.25$ and $100 \mathrm{~mm}$, respectively. The near exact vertical displacement under the load is available and equals to $0.0901 \mathrm{~mm}$ (Cen et al., 2007). The beam was analyzed using the $1 \times 6$ mesh shown in Figure 12.5b, and the resulting tip vertical displacement was $0.0880 \mathrm{~mm}$. 


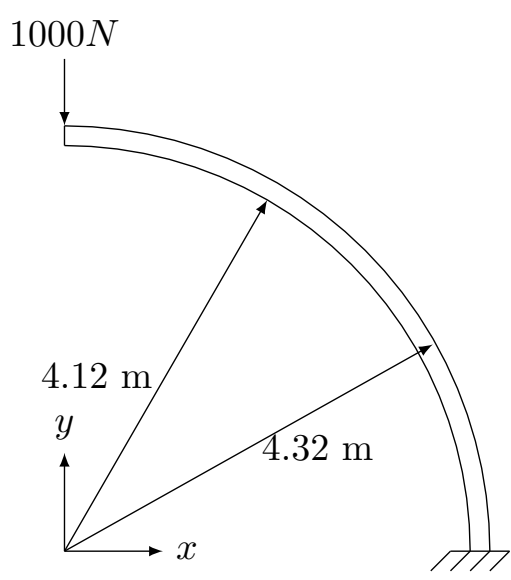

(a) Structure

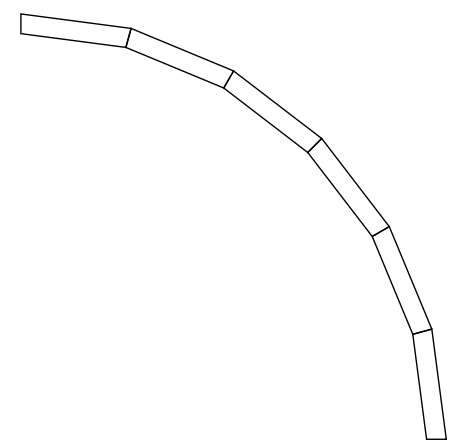

(b) Modeled Elements

Figure 12.5: Thin Arched Beam (MacNeal and Harder, 1985)

\subsubsection{Pure Bending of a Cantilever Beam}

This example of a single moment applied at the free end of a cantilever beam is often used to test eight node element models (Cen et al., 2015). As shown in Figure 12.6, seven meshes are tested with varies beam lengths, beam depths, and element configurations. The elasticity modulus, the Poisson's ratio and the beam thickness are equal to $1.0 \times 10^{7}$ $\mathrm{MPa}, 0.3$ and $1 \mathrm{~mm}$ respectively. The results listed in Table 12.8 show that distortion of the element from a rectangle tends to lead to numerical errors for pure bending as element distortion increases.

\subsubsection{Cook's Skew Beam}

The structure, shown in Figure 12.7a, is often employed to investigate the performance of quadrilateral elements (Cook et al., 1989). In this test, a skew cantilever beam is subjected to a shear distributed load at the free edge and distorted quadrilateral elements are deployed for meshing. The modulus of elasticity, Poisson's ratio and beam's thickness are $1 \mathrm{MPa}, 0.33$, and $1000 \mathrm{~mm}$ respectively. Six different mesh sizes were analyzed to study convergence with decreasing mesh spacing as shown in Figure 12.7b to Figure 12.7g. The vertical displacement of Point A is listed in Table 12.9 and displayed in Figure 12.8 for each mesh as well as the excepted theoretical value. As expected as the number elements increases the tip displacement converges to the theoretical excepted value. 


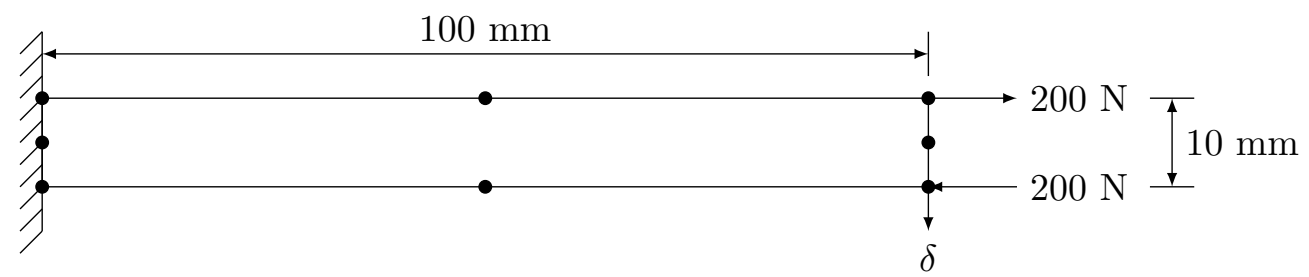

(a) Mesh 1

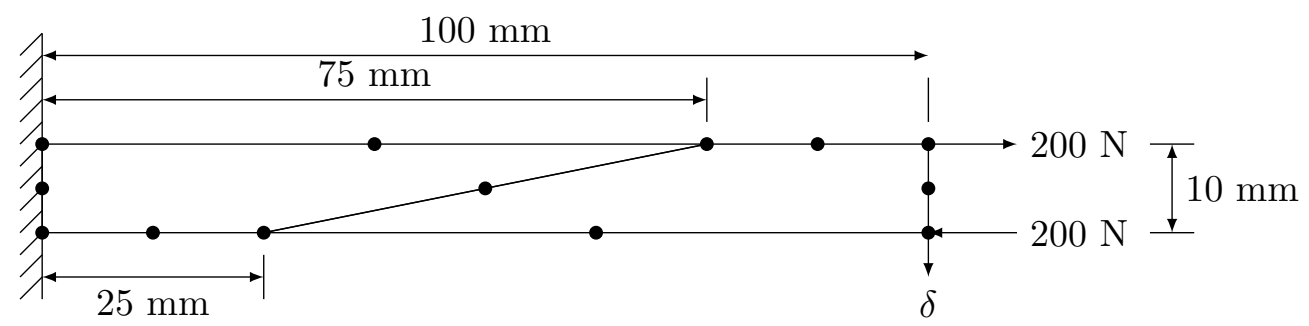

(b) Mesh 2

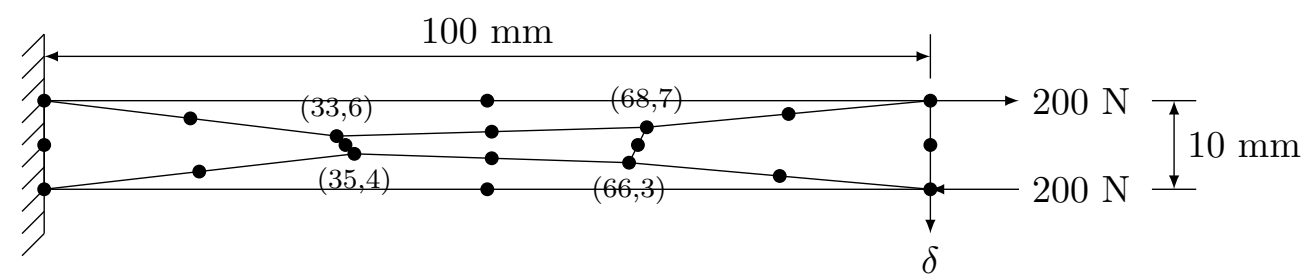

(c) Mesh 3

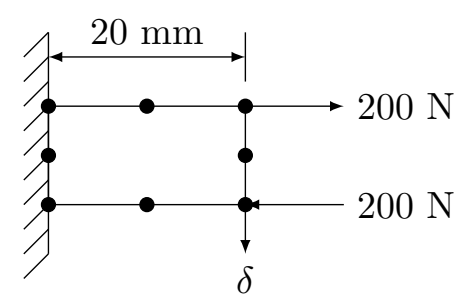

(d) Mesh 4

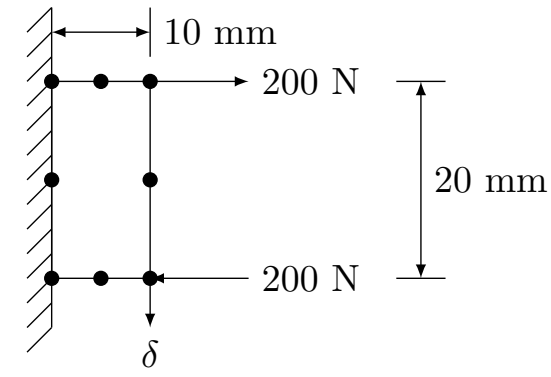

(f) Mesh 6

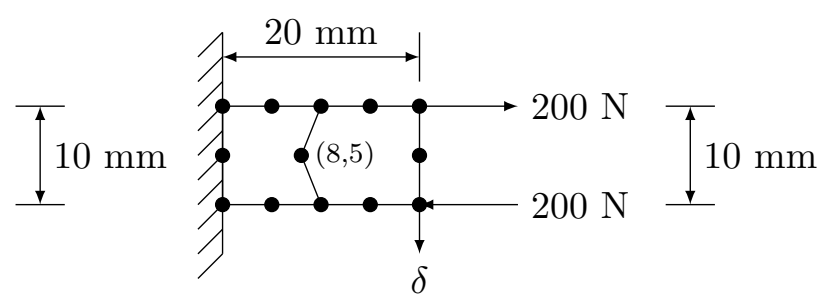

(e) Mesh 5

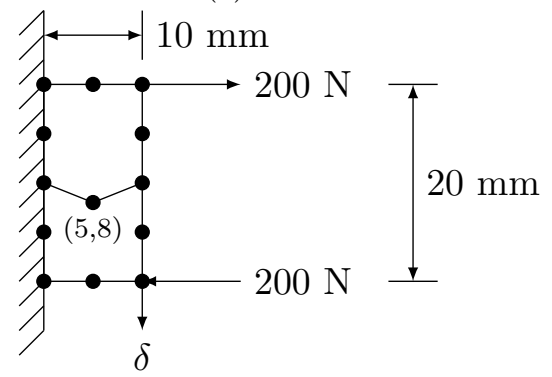

(g) Mesh 7

Figure 12.6: Pure Bending of Cantilever Beam (Cen et al., 2015) 


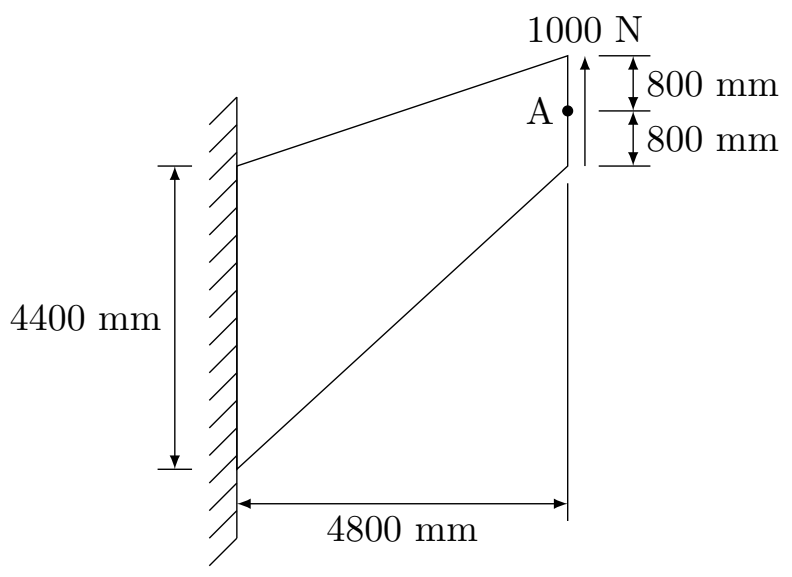

(a) Structure

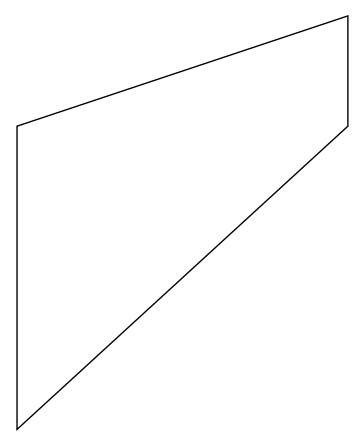

(b) $1 \times 1$ Mesh

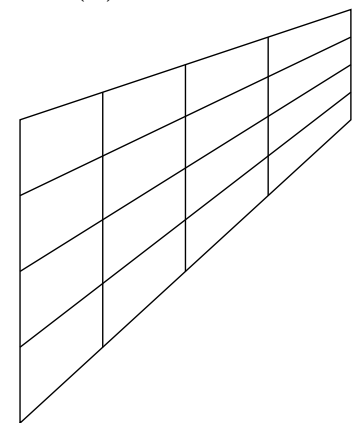

(d) $4 \times 4$ Mesh

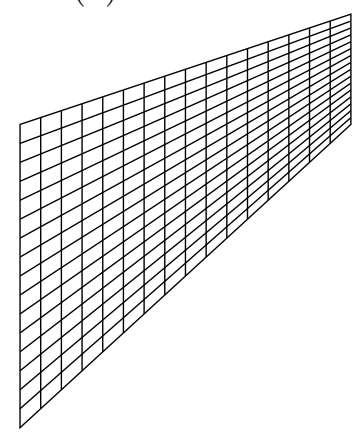

(f) $16 \times 16 \mathrm{Mesh}$

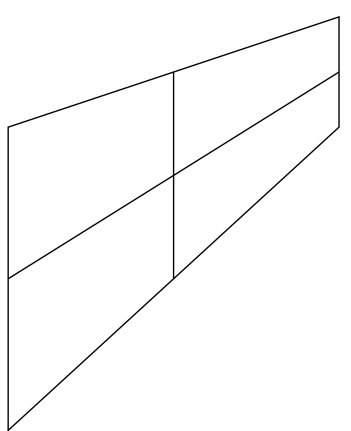

(c) $2 \times 2$ Mesh

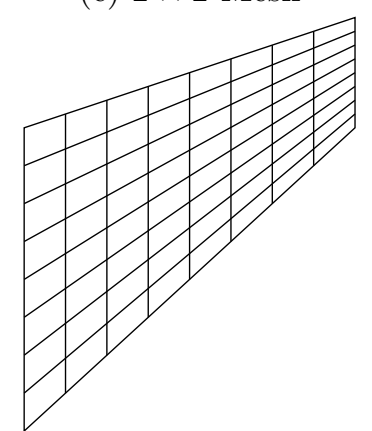

(e) $8 \times 8$ Mesh

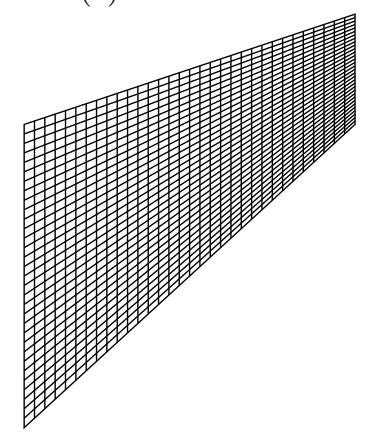

(g) $32 \times 32$ Mesh

Figure 12.7: Cook's Skew Beam Problem 
Table 12.9: Cook's Beam Displacement at Point A

\begin{tabular}{lll}
\hline Mesh Size & $\begin{array}{l}\text { Number of } \\
\text { Degrees of } \\
\text { Freedom }\end{array}$ & $\begin{array}{l}\text { Vertical Displace- } \\
\text { ment of Point A } \\
(\mathrm{mm})\end{array}$ \\
\hline $1 \times 1$ & 16 & 17.85 \\
$2 \times 2$ & 42 & 22.76 \\
$4 \times 4$ & 130 & 23.55 \\
$8 \times 8$ & 450 & 23.67 \\
$16 \times 16$ & 1666 & 23.70 \\
$32 \times 32$ & 6420 & 23.71 \\
\hline \hline Theoretical & \\
\hline
\end{tabular}

Notes:

${ }^{\mathrm{a}}$ Based on Long and Xu (1994)

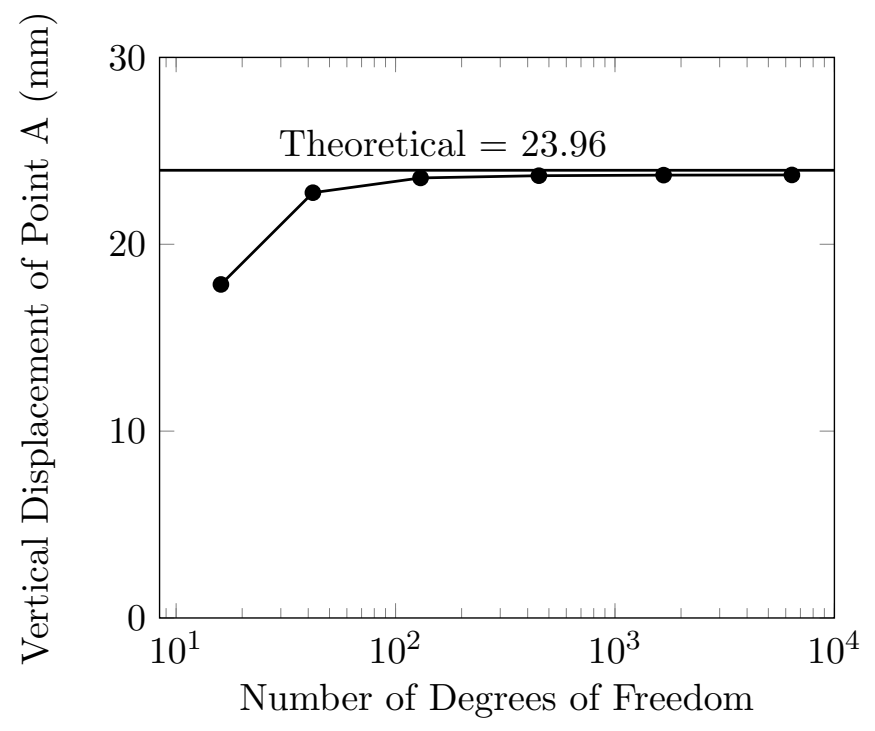

Figure 12.8: Vertical Displacement Convergence 


\subsection{VecTor2}

VecTor2 (Vecchio, 2001) was developed at the University of Toronto to analyze the response of two-dimensional reinforced concrete membrane structures subjected to inplane monotonic, cyclic, and reverse cyclic loading using the Modified Compression Field Theory (MCFT) (Vecchio and Collins, 1986) and the Disturbed Stress Field Model (DSFM) (Vecchio, 2000) as the theoretical bases.

The MCFT incorporates a rotating smeared crack approach along with average stresses and strains, and consideration of local crack conditions to analytically predict the load deformation response of reinforced concrete membrane elements. The DSFM was developed to enhance the MCFT. Two examples of such enhancements are: 1) an inclusion of crack shear slip deformations; and 2) a decoupling of the orientations of the principal stress and strain fields. Although VecTor2 was originally based on the MCFT and the constitutive relations formulated with it, the user is free to select alternative relations.

As the SAFEM and VecTor2 are based on similar theoretical bases, the computer code for the SAFEM can be manually modified to simulate VecTor2. This will allow the constitutive models and non-linearity of the SAFEM to be verified. This will be completed through a two step process; first, a single element subjected to in-plane shear will be analyzed, followed by a full scale shear wall.

\subsubsection{Panel Test}

Consider the panel specimen shown in Figure 12.9a, subjected to pure shear loading. The finite element for VecTor2 is shown in Figure 12.9b and for the SAFEM in Figure 12.9c, both are comprised of a single rectangular element with properties listed in Table 12.10. 


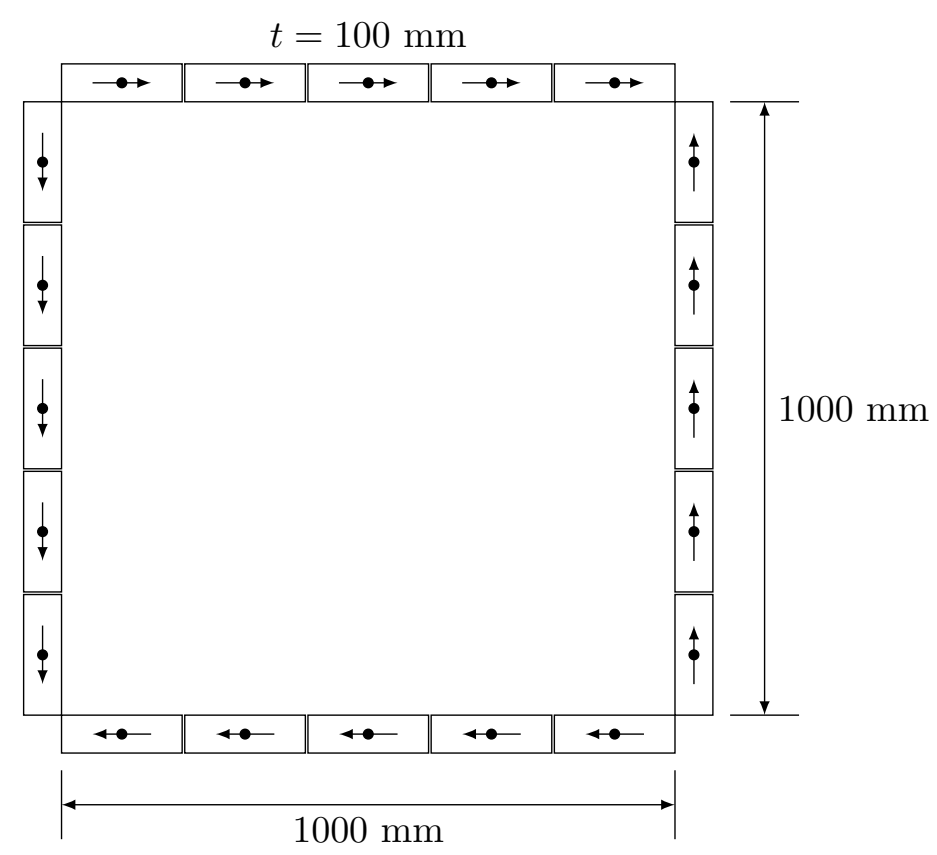

(a) Specimen

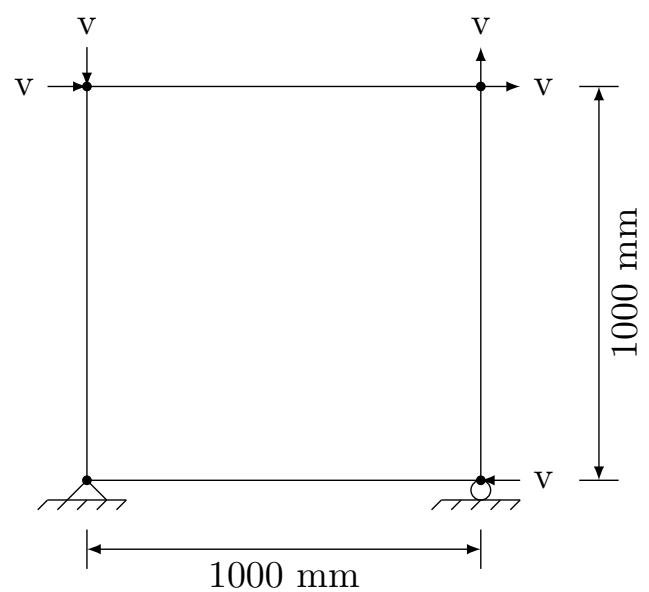

(b) VecTor2 Model

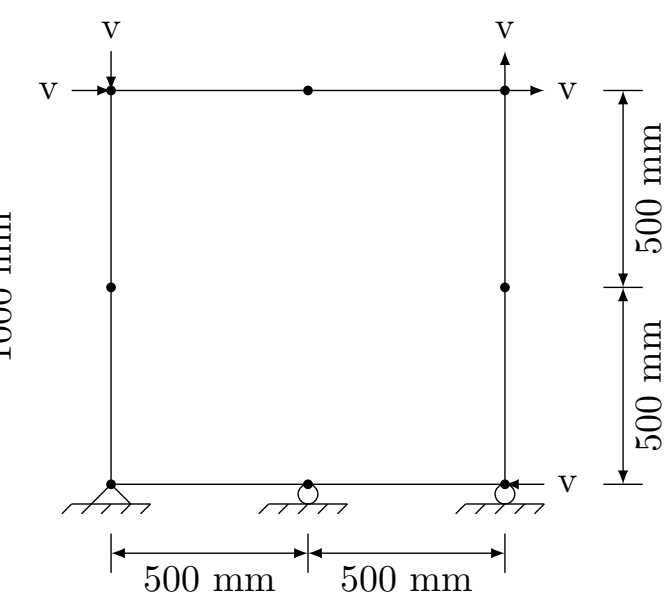

(c) SAFEM Model

Figure 12.9: Panel Specimen 
Table 12.10: Panel Test Material Properties

\begin{tabular}{lll}
\hline Concrete/Masonry & $x$-Dir. Reinforcement & $y$-Dir. Reinforcement \\
\hline$f_{c}^{\prime}=f_{m, x}^{\prime}=f_{m, y}^{\prime}=19 \mathrm{MPa}$ & $\rho_{x}=0.01785$ & $\rho_{y}=0.00713$ \\
$f_{t}^{\prime}=f_{t, x}^{\prime}=f_{t, y}^{\prime}=1.44 \mathrm{MPa}$ & $f_{y, x}=458 \mathrm{MPa}$ & $f_{y, y}=300 \mathrm{MPa}$ \\
$E_{c}=E_{m, x}=E_{m, y}=19000 \mathrm{MPa}$ & $E_{s, x}=200000 \mathrm{MPa}$ & $E_{s, y}=200000 \mathrm{MPa}$ \\
$\epsilon_{o}=\epsilon_{o, x}=\epsilon_{o, y}=0.002$ & $\epsilon_{s h, x}=0.01$ & $\epsilon_{s h, y}=0.01$ \\
$\nu_{o}=\nu_{o m, x}=\nu_{o m, y}=0.15$ & $f_{u, x}=600 \mathrm{MPa}$ & $f_{u, y}=450 \mathrm{MPa}$ \\
$a_{\max }=10 \mathrm{~mm}$ & $\epsilon_{u, x}=0.19$ & $\epsilon_{u, y}=0.18$ \\
$S_{x}=S_{y}=1000 \mathrm{~mm}$ & & \\
\hline
\end{tabular}

To verify individual constitutive models, four different model runs were conducted including different constitutive models in each run as listed in Table 12.11. Model run 1 only considers only the most basic constitutive models that the SAFEM and VecTor2 have to offer well run 4 is the most complicated the SAFEM can currently handle. As can be seen in Figure 12.10, the SAFEM produces almost identical results for the shear panel as VecTor2 for all model runs, showing that the constitutive models of the SAFEM are programmed correctly.

Table 12.11: Constitutive Models Included in Model Runs

\begin{tabular}{llll}
\hline Run Number & Slip & Compression Softening & Cracking Stress \\
\hline 1 & Not Considered & Not Considered & Uniaxial \\
2 & Not Considered & Considered & Uniaxial \\
3 & Not Considered & Considered & Mohr-Coulomb \\
4 & Considered & Considered & Mohr-Coulomb \\
\hline
\end{tabular}




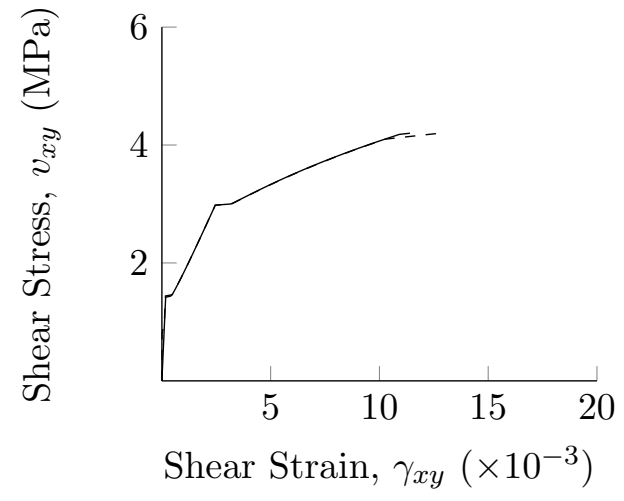

(a) Model Run 1

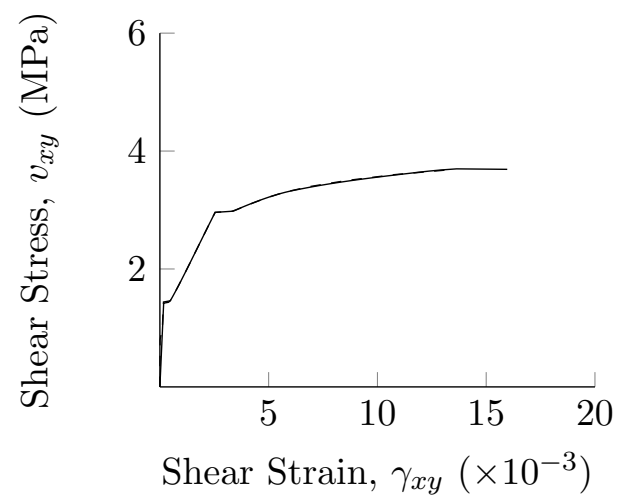

(c) Model Run 3

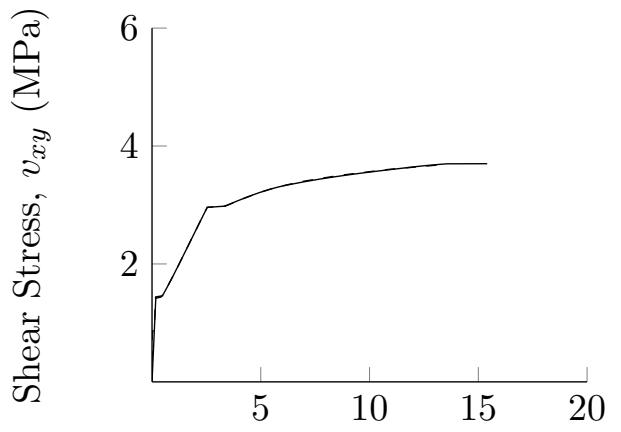

Shear Strain, $\gamma_{x y}\left(\times 10^{-3}\right)$

(b) Model Run 2

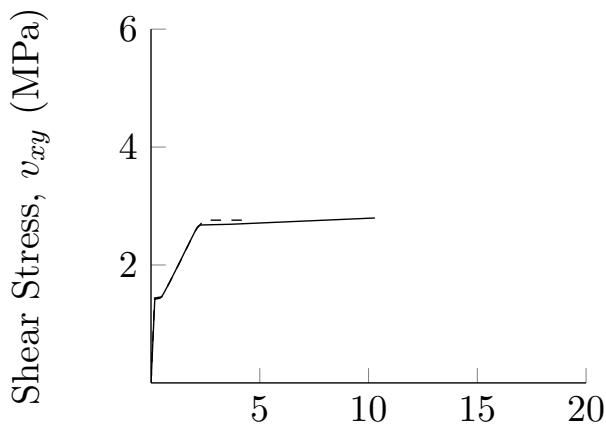

Shear Strain, $\gamma_{x y}\left(\times 10^{-3}\right)$

(d) Model Run 4

- - - SAFEM — VecTor2

Figure 12.10: Model Run Results

\subsubsection{Shear Wall Test}

To verify that the constitutive models and non-linearity of the SAFEM are functioning as expected for larger structures, a shear wall is modeled in the SAFEM and VecTor2. Consider a wall simliar to SW22, one of a series of walls tested by Lefas et al. (1990), shown in Figure 12.11. The wall measures $650 \mathrm{~mm}$ wide x $1300 \mathrm{~mm}$ high x $65 \mathrm{~mm}$ thick, with two layers of $8 \mathrm{~mm}$ diameter vertical bars at $62 \mathrm{~mm} \mathrm{c} / \mathrm{c}$, two layers of $6.25 \mathrm{~mm}$ diameter horizontal bars at $115 \mathrm{~mm} \mathrm{c} / \mathrm{c}$, and concealed columns at each end of the wall that are $140 \mathrm{~mm}$ wide with $4 \mathrm{~mm}$ diameter hops at $115 \mathrm{~mm} \mathrm{c} / \mathrm{c}$. A horizontal lateral load applied to the top spreader beam was monotonically increased until failure. There was also an axial compressive load of $182 \mathrm{kN}$. 

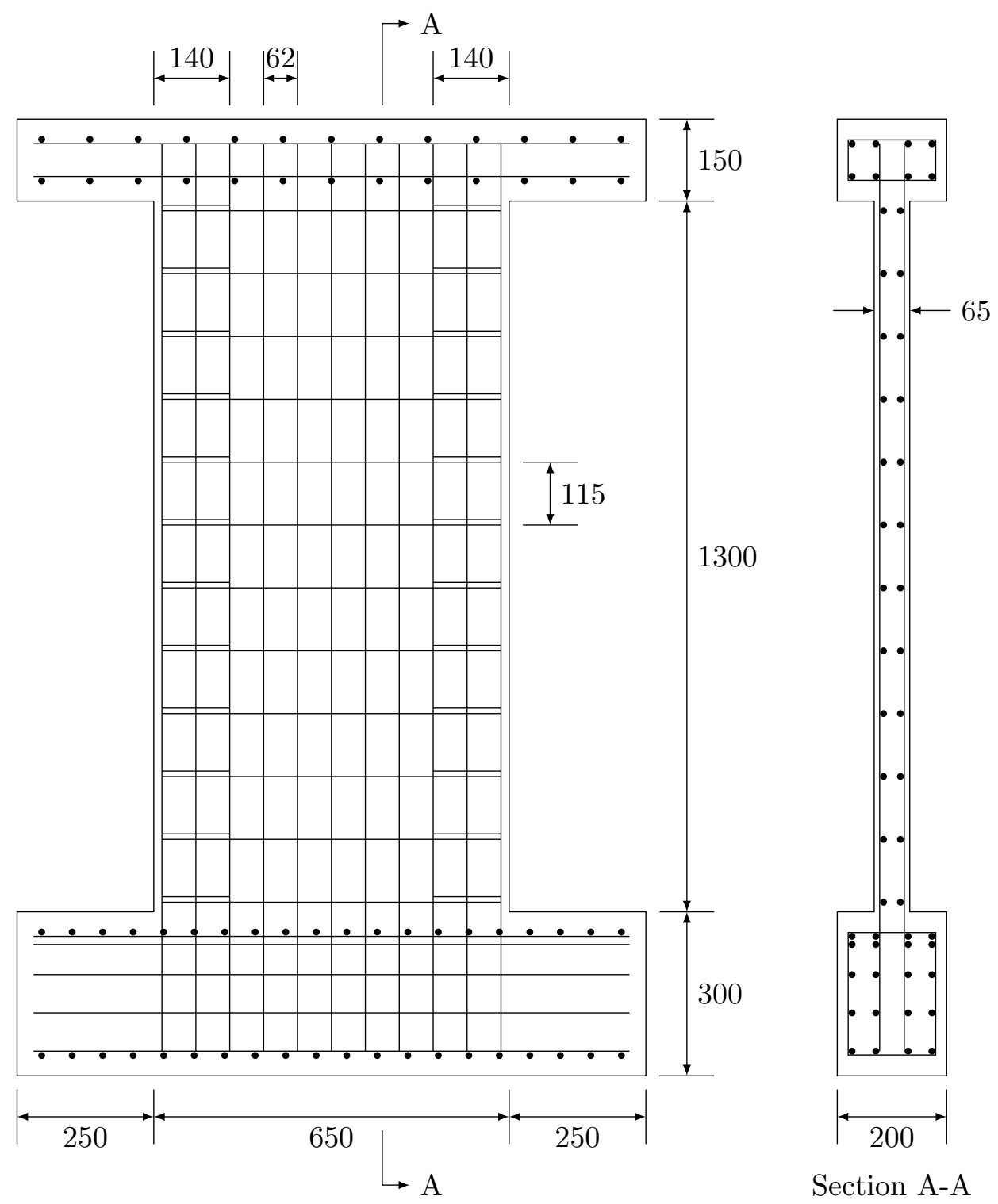

Figure 12.11: Details of Wall SW22 (Lefas et al., 1990)

For the analysis the structure is divided into three material zones with different thicknesses and percentages of reinforcement. These zones are shown in Figure 12.12a and the properties of each zone listed in Table 12.12. Both SAFEM and VecTor2 have all the nodes at the base of each wall fixed in $x$ and $y$-direction and an axial compression load of $182 \mathrm{kN}$. The SAFEM model contains 420 elements as shown in Figure 12.12b with 1373 nodes, while the VecTor 2 model contains 496 elements with 559 nodes. The SAFEM uses a force at the end of the top spreader beam, while VecTor2 uses a displacement. The results of the load-deflection responses from both the SAFEM and VecTor2 are shown in Figure 12.13 and correspond well to each other up to the peak load. After the Part III: Masonry Finite Element Modeling 
peak load the SAFEM can no longer predict the response due to being a forced based model, while VecTo2 uses a displacement based model that can predict the post-peak response. Additionally, the crack pattern, crack widths, and reinforcement stresses are all similar between the two finite element models.

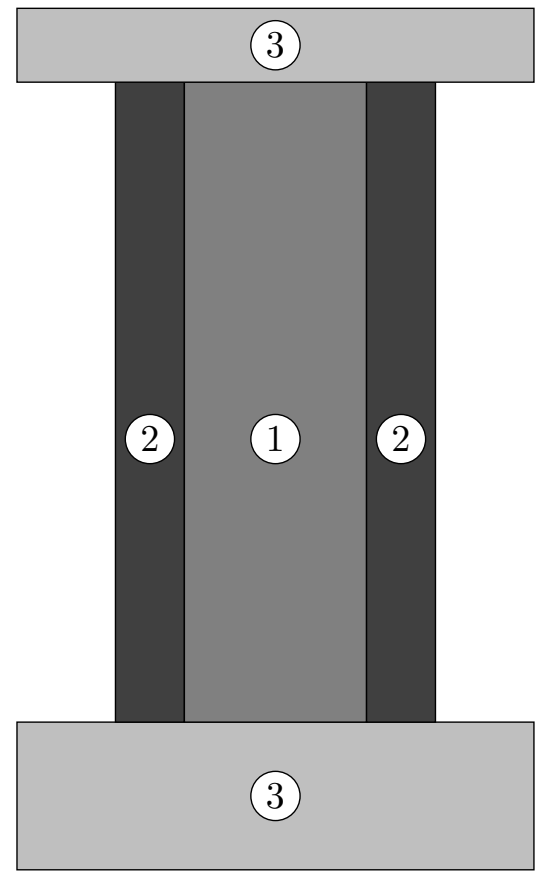

(a) Material Zones

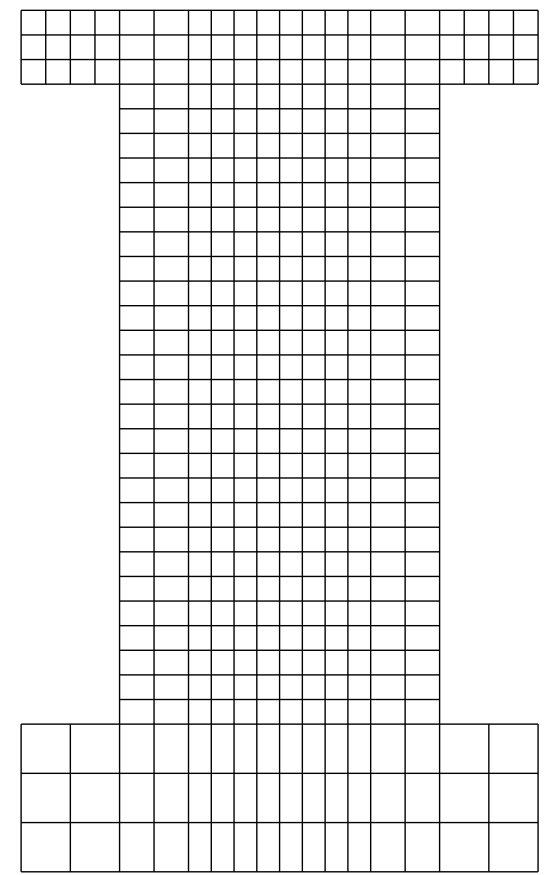

(b) SAFEM Elements

Figure 12.12: Model of SW22 
Table 12.12: Material Properties in Each Zone

\begin{tabular}{|c|c|c|c|}
\hline Zone Number & 1 & 2 & 3 \\
\hline \multicolumn{4}{|l|}{ Element } \\
\hline Thickness (mm) & 65 & 65 & 200 \\
\hline \multicolumn{4}{|l|}{ Concrete/Masonry } \\
\hline$f_{c}^{\prime}=f_{m, x}^{\prime}=f_{m, y}^{\prime}(\mathrm{MPa})$ & 36.6 & 36.6 & 36.6 \\
\hline$f_{t}^{\prime}=f_{t, x}^{\prime}=f_{t, y}^{\prime}(\mathrm{MPa})$ & 2.16 & 2.16 & 2.16 \\
\hline$E_{c}=E_{m, x}=E_{m, y}(\mathrm{MPa})$ & 32800 & 32800 & 32800 \\
\hline$\epsilon_{o}=\epsilon_{o, x}=\epsilon_{o, y}$ & 0.002 & 0.002 & 0.002 \\
\hline$\nu_{o}=\nu_{o m, x}=\nu_{o m, y}$ & 0.15 & 0.15 & 0.15 \\
\hline$a_{\max }(\mathrm{mm})$ & 10 & 10 & 10 \\
\hline$S_{x}=S_{y}(\mathrm{~mm})$ & 100 & 100 & 100 \\
\hline \multicolumn{4}{|l|}{$x$-Dir. Reinforcement } \\
\hline$\rho_{x}$ & 0.0082 & 0.03312 & 0.025 \\
\hline$f_{y, x}(\mathrm{MPa})$ & 520 & 520 & 420 \\
\hline$E_{s, x}(\mathrm{MPa})$ & 200000 & 200000 & 200000 \\
\hline$\epsilon_{s h, x}$ & 0.01 & 0.01 & 0.01 \\
\hline$f_{u, x}(\mathrm{MPa})$ & 650 & 650 & 650 \\
\hline$\epsilon_{u, x}$ & 0.075 & 0.10 & 0.125 \\
\hline \multicolumn{4}{|l|}{$y$-Dir. Reinforcement } \\
\hline$\rho_{y}$ & 0.0209 & 0.01156 & 0.025 \\
\hline$f_{y, y}(\mathrm{MPa})$ & 470 & 470 & 420 \\
\hline$E_{s, y}(\mathrm{MPa})$ & 200000 & 200000 & 200000 \\
\hline$\epsilon_{s h, y}$ & 0.01 & 0.01 & 0.01 \\
\hline$f_{u, y}(\mathrm{MPa})$ & 650 & 650 & 650 \\
\hline$\epsilon_{u, y}$ & 0.10 & 0.125 & 0.125 \\
\hline
\end{tabular}




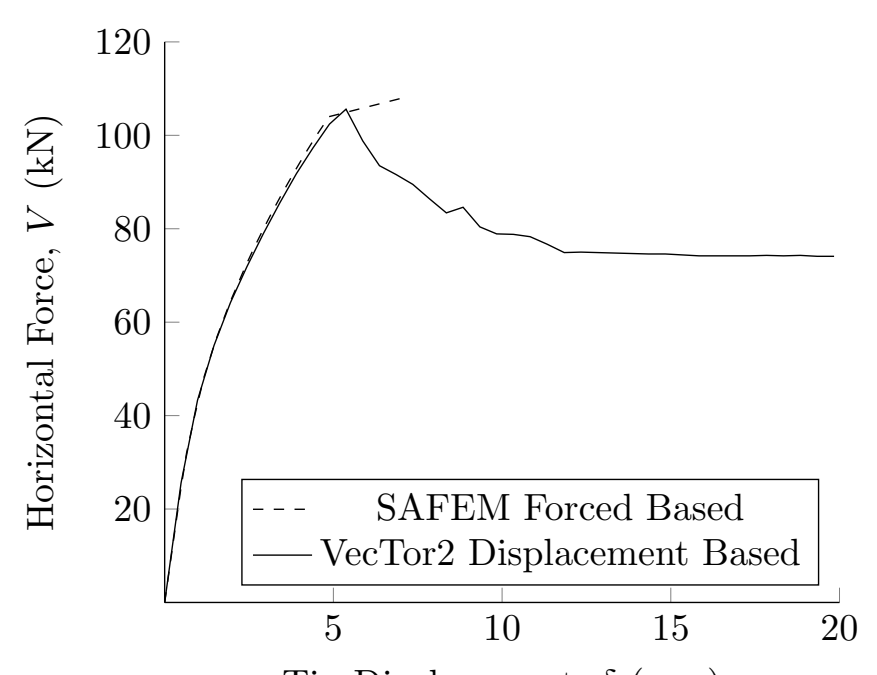

Tip Displacement, $\delta(\mathrm{mm})$

Figure 12.13: Horizontal Force vs Tip Displacement Results 


\title{
COMPARISON OF SINGLE PIER GROUTED SHEAR WALL DATA AND THE SMEARED AREA FINITE EL- EMENT MODEL
}

\begin{abstract}
The model presented in Chapter 10, implemented in Chapter 11, and verified in Chapter 12 is used to determine the structural response of grouted and reinforced single per shear walls constructed from hollow concrete block masonry. The results of the finite element model are compared to the experimental results found in literature. The results of model inputs are used to produce equations to predict hollow concrete block masonry mechanics.
\end{abstract}

Over the past 70 years there have been numerous experiments on single pier grouted shear walls. This chapter assembles an experimental database of single pier grouted shear wall tests made from hollow concrete blocks to allow comparison of experimental and the SAFEM results.

\subsection{Data Requirements}

To use the SAFEM a number of material properties are required as inputs; thus, to be included in the database the following had to be reported for the test:

1. The masonry compression strength as determined from a prism test of three or more courses;

2. The block length, height, faceshell thickness, percent solid, and either tensile or compression strength;

3. The bed and head joint thickness and mortar compression strength;

4. The grout tensile or compression strength; and,

5. The reinforcement details, including bar size and placement, yield strength, and ultimate strength. 
Further requires are:

1. The dimensions of the wall had to be given;

2. The experimental test setup had to be reported to ensure that the correct boundary and loading conditions were represented in the finite element analysis;

3. The specimen had to be fully grouted with no opens within the masonry continuum;

4. The specimen had to be constructed from full scale blocks with in joint reinforcement;

5. To facilitate the comparison of the SAFEM results and the experiment a load deflection graph had to be reported, with the reported deflection corresponding to the specimen only (i.e. the relative displace of the experimental setup is removed).

In total, 62 single pier grouted shear wall experiments were used to validate the SAFEM. If a value required to run the model was not given an assumed value was used as listed in Appendix D.

\subsection{Database Literature Review}

\subsubsection{Priestley (1977)}

Priestley (1977) tested three single pier grouted shear walls without confining plates at the ends of the walls. Figure 13.1 shows the wall dimensions used, resulting in an aspect ratio of 0.75 . The test program was designed to investigate the low maximum shear stress specified in masonry codes.

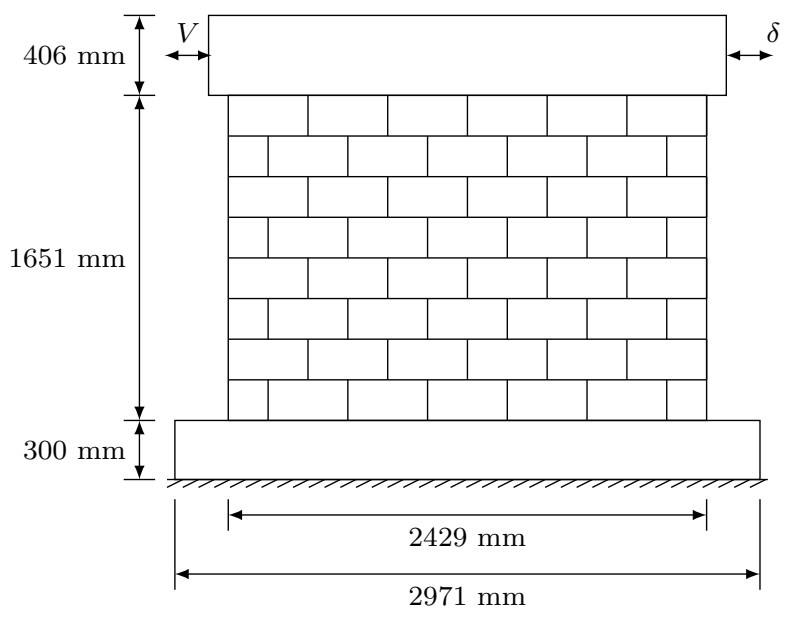

Figure 13.1: Priestley (1977) Experimental Wall 
Results indicate that the maximum current code allowance for ultimate shear stress is unreasonably low. No walls suffered diagonal shear failure despite exceeding the maximum code allowable shear stress by four times. All walls displayed stable hysteresis loops at a displacement ductility factor of two, and the less heavily reinforced walls (designed to approximately twice code levels) were satisfactory at ductility factor of four. Failure of the walls was never catastrophic and occurred due to slip of the entire wall along the foundation beam.

Walls subjected to moderate vertical loading (0.69 MPa) behaved better than walls without vertical load. Base-course slip was reduced, and degradation at ductility factor of four was less severe.

\subsubsection{Chen et al. (1978)}

Chen et al. (1978) tested 11 single pier grouted shear walls with an aspect ratio of 1.0. All the walls were tested using in-plane cyclic shear loading with increasing peak displacements. All walls were tested in the frame shown in Figure 13.2, in which the top and bottom of the walls remained parallel during the test. The frame subjected each wall to double-flexure bending, locating the inflection point at the mid-height of the wall, producing a shear span ratio of 0.5. The amount of vertical and horizontal reinforcement varied between each specimen.

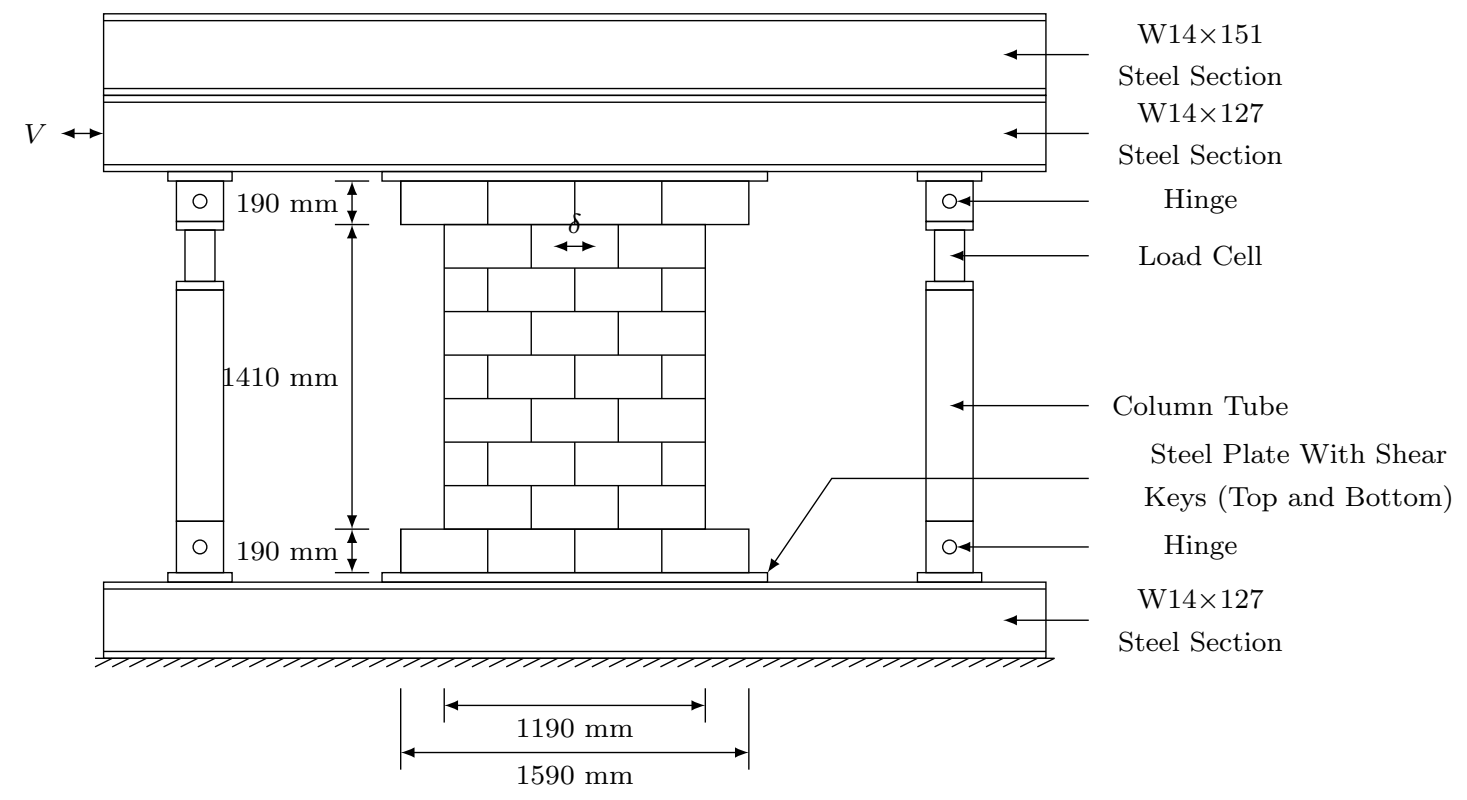

Figure 13.2: Chen et al. (1978) Test Setup 
Chen et al. (1978) observed that all of the piers showed a shear mode of failure and in some specimens this was accompanied by flexural failure as well. Part of the reason for this was due to the additional compressive load on the piers at higher drift levels. As the walls failed in shear, there was no significant difference between walls with more vertical reinforcement. Though the higher vertical reinforcement ratio allowed for more energy dissipation due to increasing cracking and aggregate interlock.

\subsubsection{Hidalgo et al. (1979)}

Hidalgo et al. (1979) tested six full-scale fully-grouted masonry walls with a geometric aspect ratio of 0.5 . All of the walls were tested using in-plane cyclic shear loading with increasing peak displacements. The frame subjected each wall to double-flexure bending, locating the inflection point at the mid-height of the wall, producing a shear span ratio of 0.25. All of the walls were tested using the same setup as Chen et al. (1978) (see Figure 13.2) and were subject to similar downward force induced by the hinged columns at either end of the specimen. It was observed that in the case of walls with low aspect ratio, the axial force contribution from the hinged columns did not affect the failure mode of the specimens.

All walls had three \#7 bars placed vertical, one in each end cell and one in the center of the wall. The quantity of horizontal reinforcement varied in each wall from none to four \#6 bars. All of the other variables were kept constant between the tests.

Two of the specimens displayed a combined shear and sliding failure mechanism and the remaining four displayed typical shear failure characterized by diagonal cracking. There was a positive correlation between the amount of horizontal reinforcement and the ultimate strength. The shear deformation in the squat piers had a greater importance to the overall behavior of the specimens than for those with large aspect ratios.

\subsubsection{Sveinsson et al. (1985)}

Sveinsson et al. (1985) tested two single pier shear walls with $190 \times 190 \times 390 \mathrm{~mm}$ blocks. The walls both had an aspect ratio of unity and a shear span ratio of 0.5 , but had two different azial loads. They used a test setup, shown in Figure 13.3, similar to that of Chen et al. (1978) with a modification such that the columns were replaced with actuators that were force-controlled. This modification enabled the specimens to be tested under double-curvature conditions while maintaining constant axial load for all lateral drifts. The increase in axial load allowed allowed for a larger shear load to be resisted by the wall. 


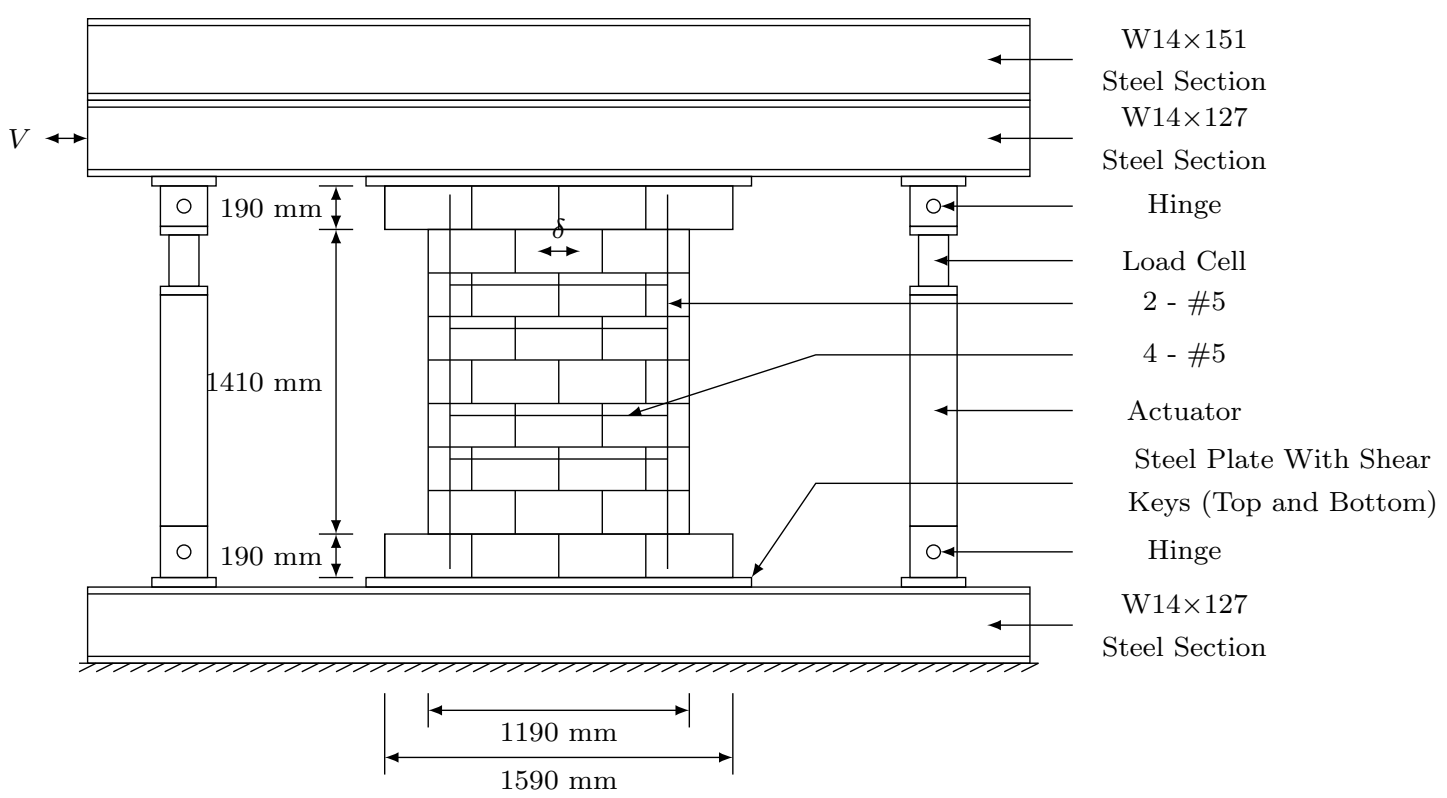

Figure 13.3: Sveinsson et al. (1985) Experiment Setup

\subsubsection{Shing et al. (1991)}

Shing et al. (1991) tested 18 concrete block specimens under in-plane cyclic loading at the University of Colorado-Boulder as part of the U.S.-Japan Coordinated Program on Masonry Building Research. All specimens were constructed at full scale and were fully grouted.

The main objective of their study was to investigate the effects of the amount of vertical and horizontal reinforcement and the applied axial load on the capacity of masonry shear walls. The aim of the experiments was to evaluate the validity of the Universal Building Code (UBC) design equations in determining reinforced masonry shear wall strength. They evaluated the forumal for both flexure and shear strength, determining that the existing UBC provision were to simplistic, and proposed a new model for calculating shear strength.

From the experiments, it was observed four of the masonry walls where governed by flexural yielding whereas the remaining specimens were governed by diagonal shear cracking. The full grouting of the walls caused the diagonal cracks to propagate through the blocks rather than being confined to the joints. It was also observed that diagonal shear cracking caused a more brittle failure when compaired to the walls that failed with flexural toe crushing. The contribution of the horizontal reinforcement given by $A_{v} \rho_{v} f_{y}$ to shear resistance is overestimated by the UBC and proposed that the factor be reduced. There 
was no correlation between the amount of reinforcement and the stress at the onset of diagonal cracking and Shing et al. (1991) concluded that they are unrelated. It was also concluded that the amount of vertical reinforcement and the axial load have an influence on the shear strength by increasing the post-cracking strength of the masonry.

\subsubsection{Ibrahim (1995)}

Ibrahim (1995) tested five $1.4 \mathrm{~m}$ high reinforced masonry shear walls that were subjected to lateral displacement patterns to simulate earthquake excitation while a constant compressive vertical load was applied to simulate the dead load of the structure. Each wall was built with $140 \times 190 \times 390 \mathrm{~mm}$ hollow concrete blocks. All the walls were designed to experience a shear failure. The walls had different aspect ratios, vertical reinforcement, and applied vertical stress; while, the horizontal reinforcement was held constant to ensure a shear dominated failure.

Four of the walls experienced a shear failure that was explained by three mechanisms. First, a set of diagonal cracks formed. Then a strut mechanism was formed that consisted of one or more struts depending on the wall geometry. Under the cyclic loading, major diagonal cracks started opening and extended diagonally from the top to the bottom of the wall. At this time, the strut mechanism became ineffective and the shear resistance was taken over by aggregate interlock along the diagonal crack surfaces and by horizontal steel in tension at the cracks. Failure happened with the breakdown of aggregate interlock. The wall with the largest aspect ratio experienced a mixed flexure/shear failure. There was considerable yilding of the extreme vertical steel and the wall finally failed by toe crushing.

\subsubsection{Voon (2007)}

Voon (2007) tested eight fully grouted single pier shear walls made from hollow concrete blocks at the University of Auckland, New Zealand. Axially loads of 0, 0.25, and 0.50 $\mathrm{MPa}$ were used. The purpose of the study was to investigate concrete masonry shear strength to assess the validity of the new shear equation in the New Zealand code (Standard Association of New Zealand, 2004). All walls were tested using stepwiseincreasing cyclic loading.

A comparison of experimental wall shear strengths to those predicted by the New Zealand code (Standard Association of New Zealand, 2004) and Building Seismic Safety Council (1997) was completed. Voon observed that the Building Seismic Safety Council (1997)

Part III: Masonry Finite Element Modeling 
equation did a better job predicting the experimental shear strengths than the New Zealand code (Standard Association of New Zealand, 2004) equation.

\subsubsection{Shedid et al. (2008)}

Six full-scale walls were tested by Shedid et al. (2008) to failure under reversed cyclic lateral loading to investigate the effects of the amount and distribution of vertical reinforcement and the level of axial compressive stress on the inelastic behavior and wall ductility. To achieve flexure dominated behavior with a distinct region of plastic hinging, it was decided that the aspect ratio of the test walls should be 2. Standard two-cell hollow $20 \mathrm{~cm}$ concrete masonry block were selected, with each course of the wall being constructed using 4.5 masonry blocks as shown in Figure 13.4.

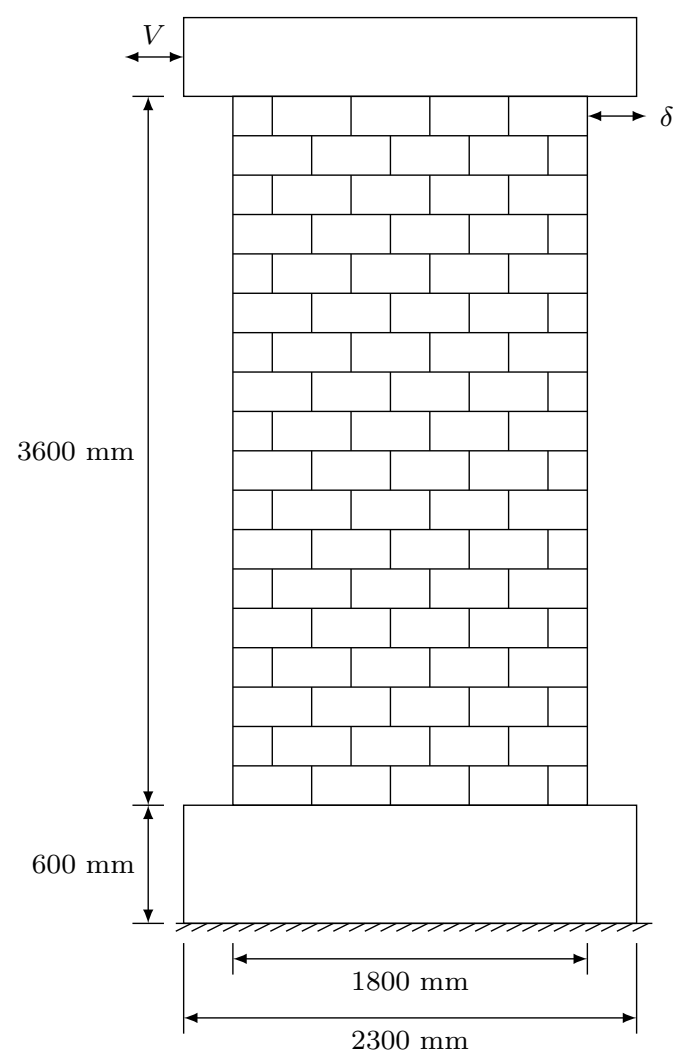

Figure 13.4: Shedid et al. (2008) Experimental Wall

The behavior of the walls was characterized by rotation over the lower part of the wall and rigid body deformation for the upper part of the wall. The extent of yielding reached a height, above the foundation, approximately equal to half the wall length, whereas, it only penetrated to a depth of less than $150 \mathrm{~mm}$ inside the foundation. Also, the top wall drift at the onset of yielding of the vertical reinforcement was highly dependent on the 
amount of reinforcement, but only minimally affected by the level of axial compressive load. However, at maximum loads, the displacements were less sensitive to either the amount of vertical reinforcement or the level of axial compression. Correspondingly, the displacement ductility was found to be very sensitive to the amount of vertical reinforcement, but was not dependent on the level of axial compression.

\subsubsection{Seif ElDin (2016)}

Seif ElDin (2016) tested nine full-scale fully grouted shear walls. They were subjected to an axial compressive stress, cyclic lateral loading, and top moment. The main variables considered were the level of axial compressive stress, shear span to depth ratio, horizontal reinforcement ratio, anchorage end detail, and the spacing of horizontal and vertical reinforcement.

Seif ElDin (2016) observed that providing horizontal reinforcement to masonry shear walls enhanced their in-plane shear behaviour in terms of shear strength and displacement ductility; however, the horizontal reinforcement does not have a significant influence on the pre-crack behaviour. Increasing the axial compressive stress, resulted in a higher shear strength accompanied by a more brittle failure. The spacing of the reinforcement has a considerable effect on the stress flow, and thereby on the behaviour of the shear walls. Walls that were constructed with closely spaced reinforcement were able to reach higher strength and ductility compared to similar walls with large spacing when using the same reinforcement ratio.

\subsection{Distribution of Specimen Parameters}

In this section, properties of the wall specimens are plotted as histograms. The quantitative attributes make up some of the important input parameters used in the SAFEM and show the wide range of inputs used to verify the SAFEM.

Part III: Masonry Finite Element Modeling 


\subsubsection{Masonry Compression Strength}

The masonry compression strength, based on gross area, ranged from 9.17 to $28.3 \mathrm{MPa}$ with the majority of the strengths in the 12 to $24 \mathrm{MPa}$ range (see Figure 13.5). These strengths are typical for fully grouted masonry used in today's construction.

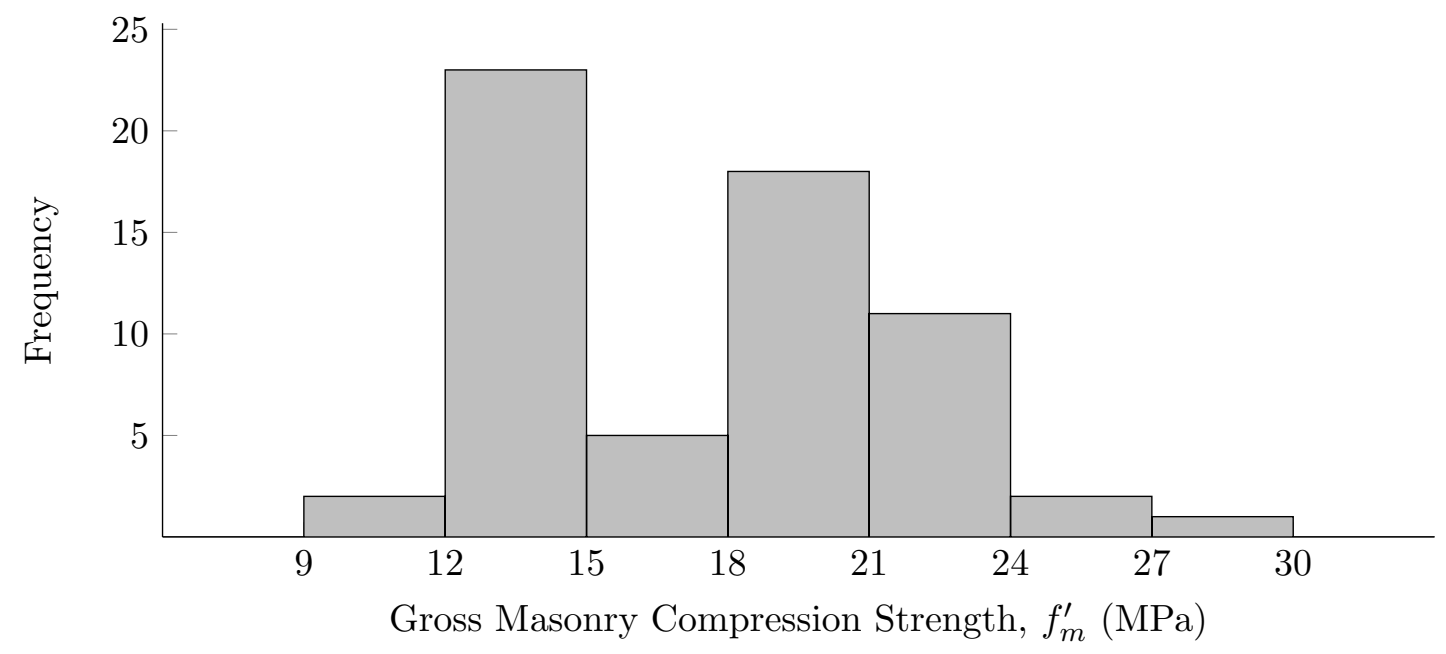

Figure 13.5: Grouted Wall Masonry Compression Strength Histogram

\subsubsection{Block Compression Strength}

The block compression strengths for the 62 walls ranged from 16.5 to $30.7 \mathrm{MPa}$, see Figure 13.6 based on the net area of the block tested with hard capping. The majority of the strengths ranged from 16 to $24 \mathrm{MPa}$ which is typical for today's hollow concrete blocks.

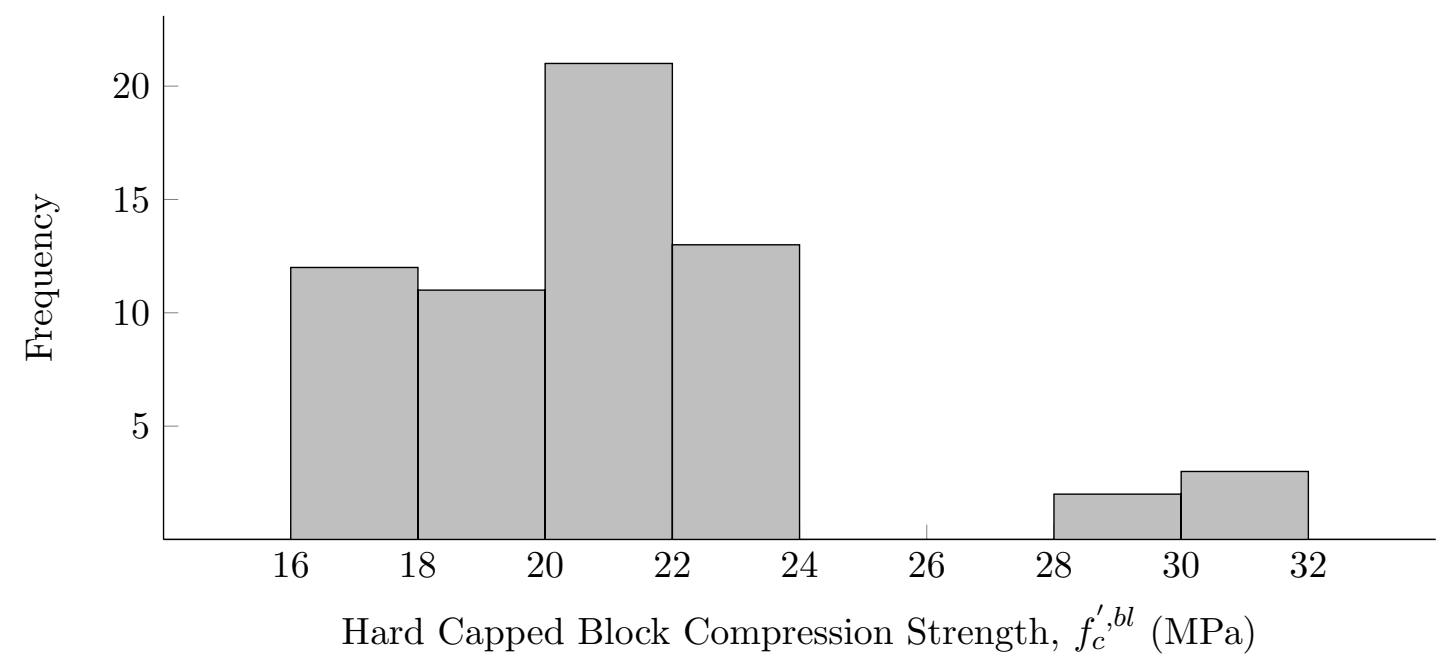

Figure 13.6: Grouted Wall Block Compression Strength Histogram 


\subsubsection{Mortar Compression Strength}

The mortar compression strengths shown in Figure 13.7 are unified to absorbent cube strength (see subsection 3.9.2). This unification attempts to represent the in-situ strength of the mortar used in the walls. The unified mortar strengths represented in the single pier grouted wall database ranged from 16 to $52 \mathrm{MPa}$, with the majority of the strengths between 16 and $29 \mathrm{MPa}$.

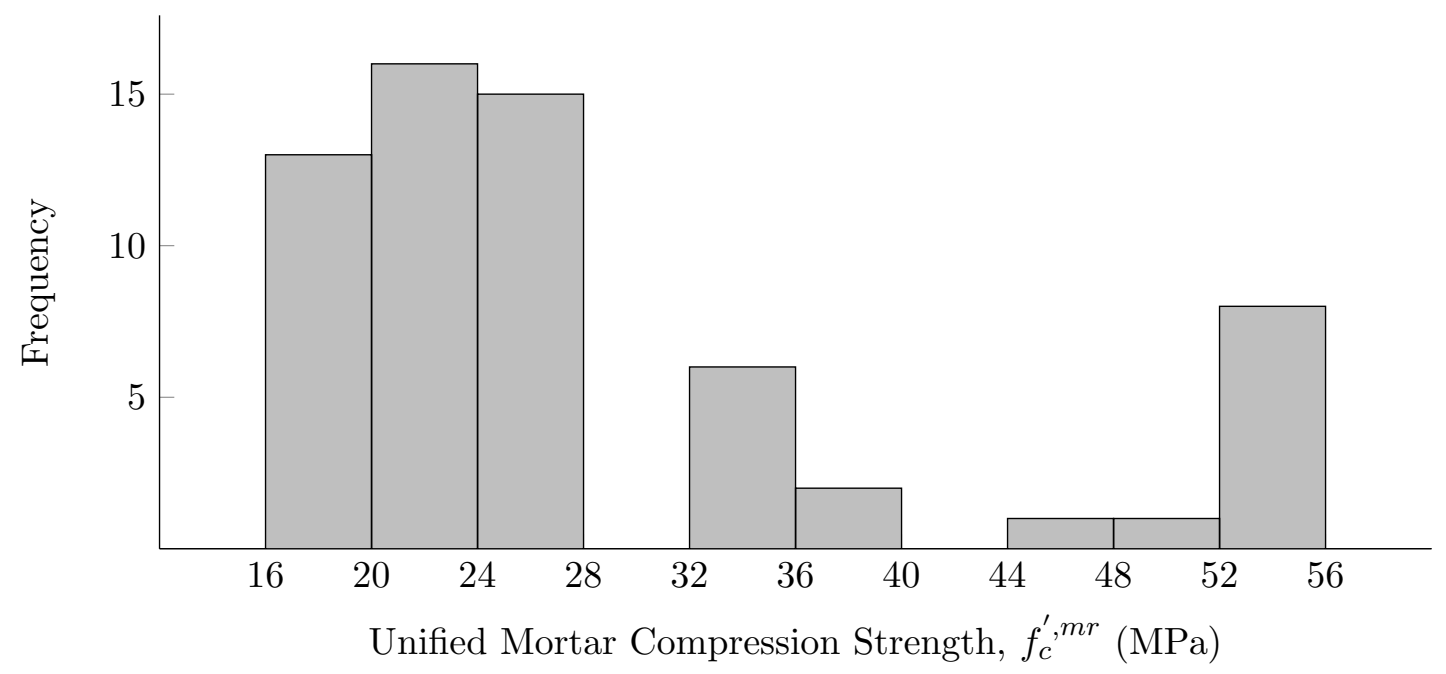

Figure 13.7: Grouted Wall Mortar Compression Strength Histogram 


\subsubsection{Grout Compression Strength}

To represent the in-situ strength of the grout the grout compression strengths presented in Figure 13.8 are unified to absorbent cylinder strength (see Subsection 3.9.3). The unified grout compression strengths from the single pier grouted shear wall database ranged from 17.4 to $40.1 \mathrm{MPa}$ with almost equal distribution.

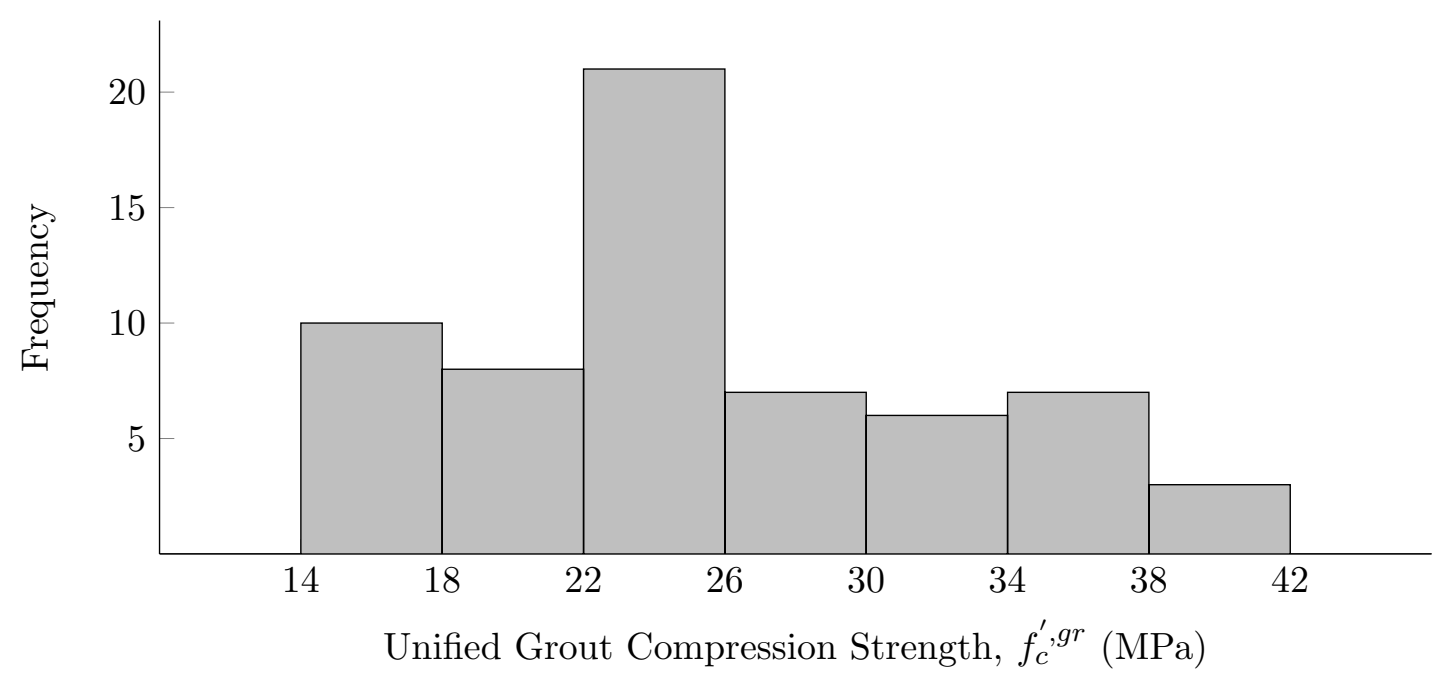

Figure 13.8: Grouted Wall Grout Compression Strength Histogram

\subsubsection{Vertical Reinforcement Ratio}

The vertical reinforcement ratio for the single pier grouted shear wall database had values that ranged from 0 to $0.97 \%$ as shown in Figure 13.9.

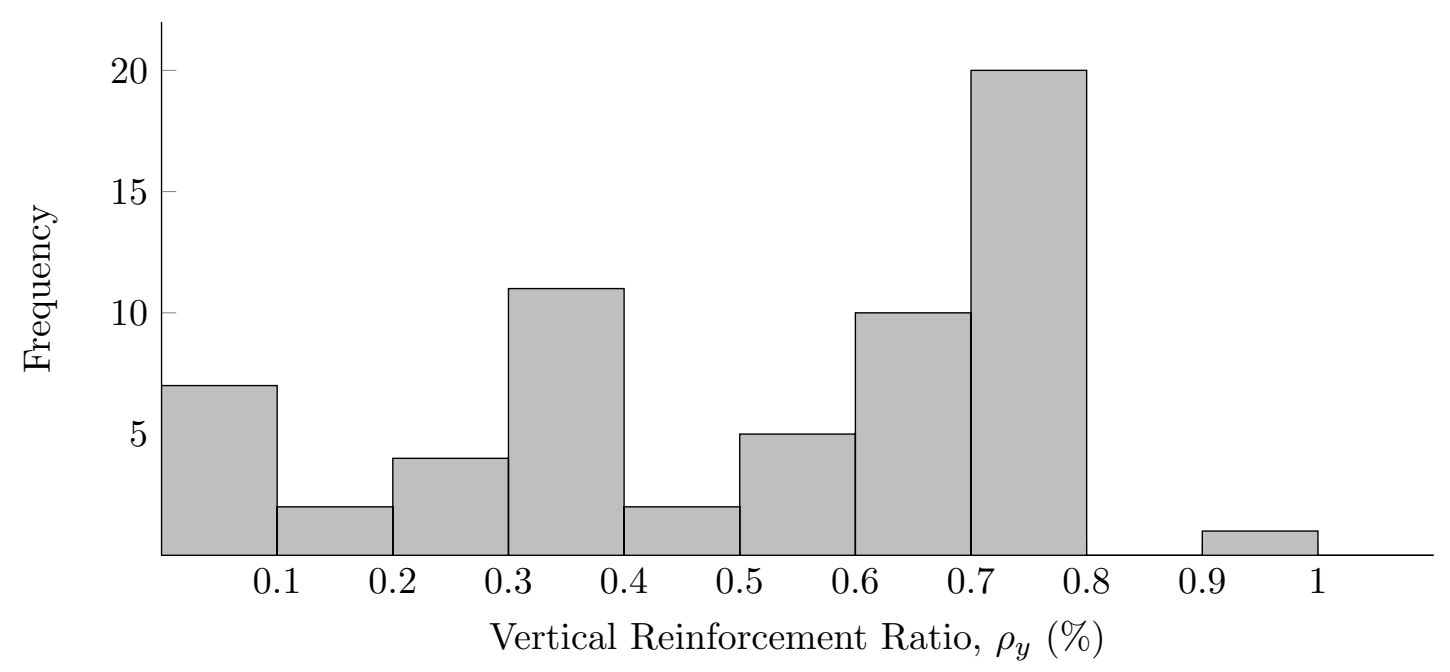

Figure 13.9: Grouted Wall Vertical Reinforcement Ratio Histogram 


\subsubsection{Horizontal Reinforcement Ratio}

The horizontal reinforcement ratio for the single pier grouted shear wall database had values that ranged from 0 to $1.02 \%$ as shown in Figure 13.10, with the majority of ratios between 0 and $0.3 \%$.

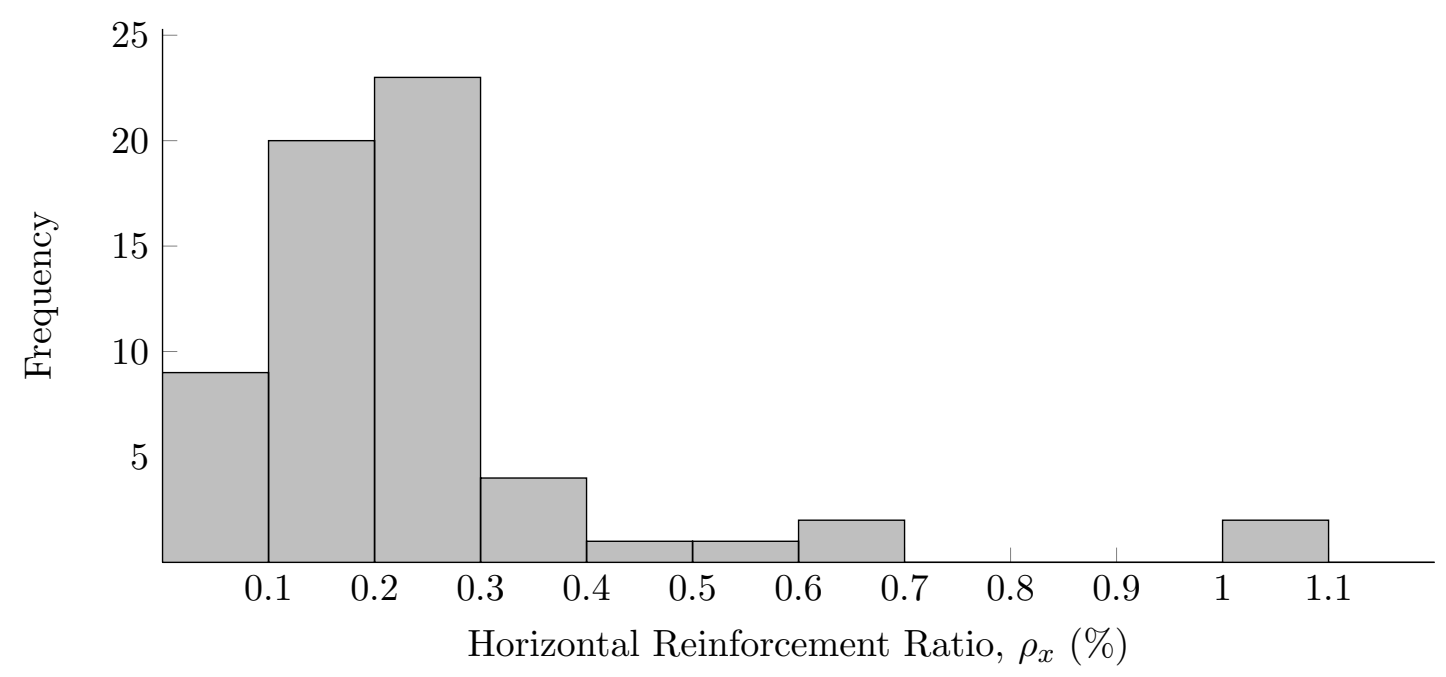

Figure 13.10: Grouted Wall Horizontal Reinforcement Ratio Histogram

\subsubsection{Aspect Ratio}

The histogram of aspect ratios of the single pier grouted shear walls, $a / l_{w}$, is shown in Figure 13.11. The aspect ratios ranged from 0.68 to $2.11 \%$, with just over half of the walls in the range of 1 to $1.25 \%$. Walls with an aspect ratio of less than 1 are considered squat walls and tend to fail in shear. Walls with aspect ratios between 1 and 2 are intermediate walls that could fail through ether shear or flexure. While, walls with an aspect ratios greater than 2 are slender and tend to fail in flexure. Thus the database contains specimens that cover all possible shear wall failure modes. 


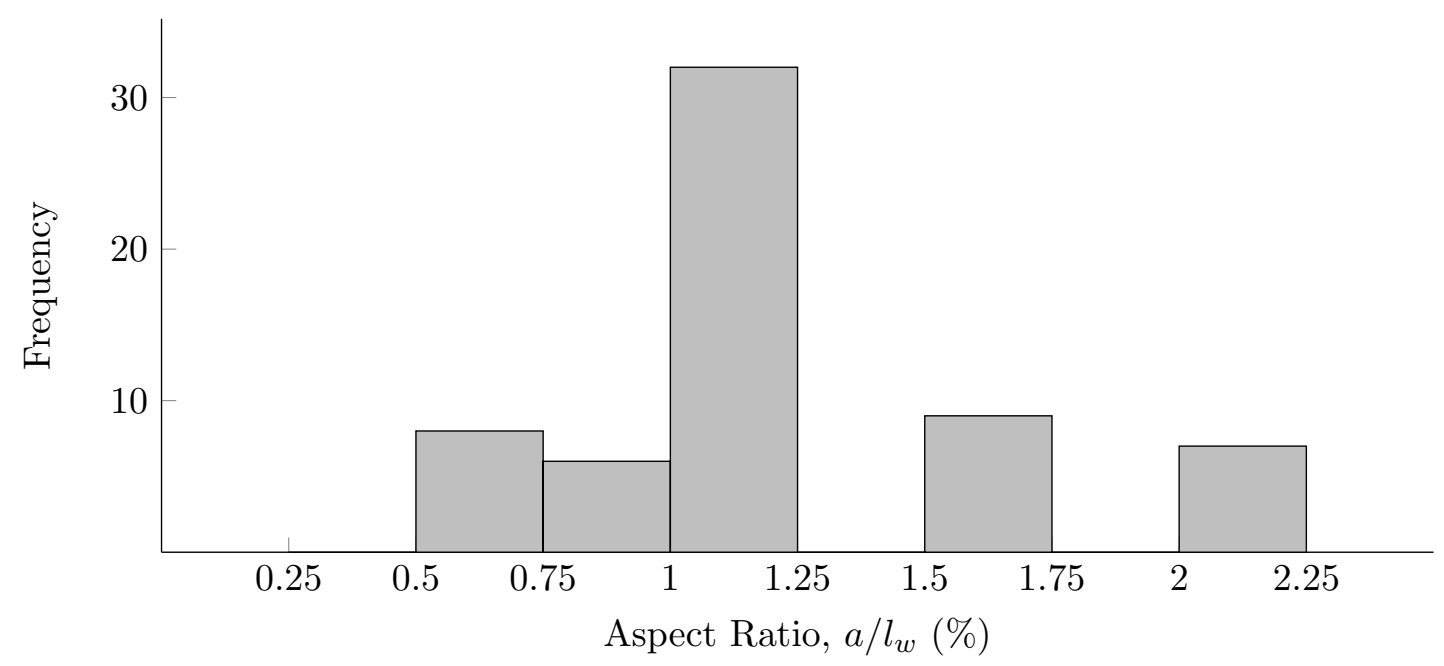

Figure 13.11: Aspect Ratio Histogram

\subsubsection{Axial Load Ratio}

The axial load ratio, $n=N /\left(t l_{w} f_{m}^{\prime}\right)$, defined by the ratio of axial demand to axial capacity has a significant influence on the failure characteristics and the deformation capability of walls. An axial compression load puts the wall under an initial axial compression stress that must be overcome before the wall exceeds the cracking stress of the masonry. The range of axial load ratio for the 62 single grouted shear walls is from 0 to 0.1573 with 17 walls in the range of 0 to 0.02 and 0.06 to 0.08 as shown in Figure 13.12.

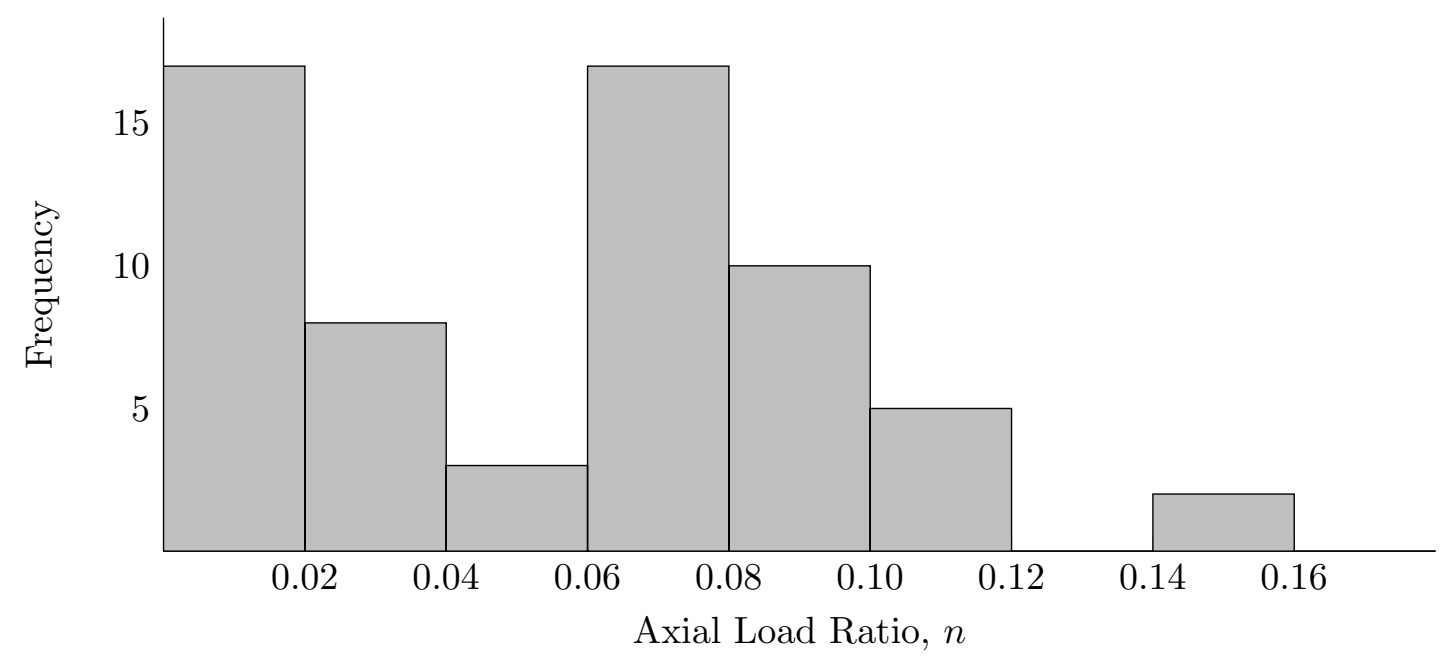

Figure 13.12: Axial Load Ratio Histogram 


\subsection{SAFEM Results}

The SAFEM was used to predict the load-displacement response of the 62 walls from the database. These responses are shown in Figure 13.13, where the experiment included cyclic loading the backbone curve is given in both the positive and negative directions. As can be seen the SAFEM produces accurate results across the entire database in terms of stiffness, peak load, and displacement. Appendix D provides more detail on each model including the layout of elements, material properties, model options, load displacement diagrams, node displacements, and crack patterns. Where possible comparisons are made between experimental and SAFEM results. The results presented in Figure 13.13 and Appendix D are produced by keeping model inputs and parameters constant between walls in the same series of experiments. 

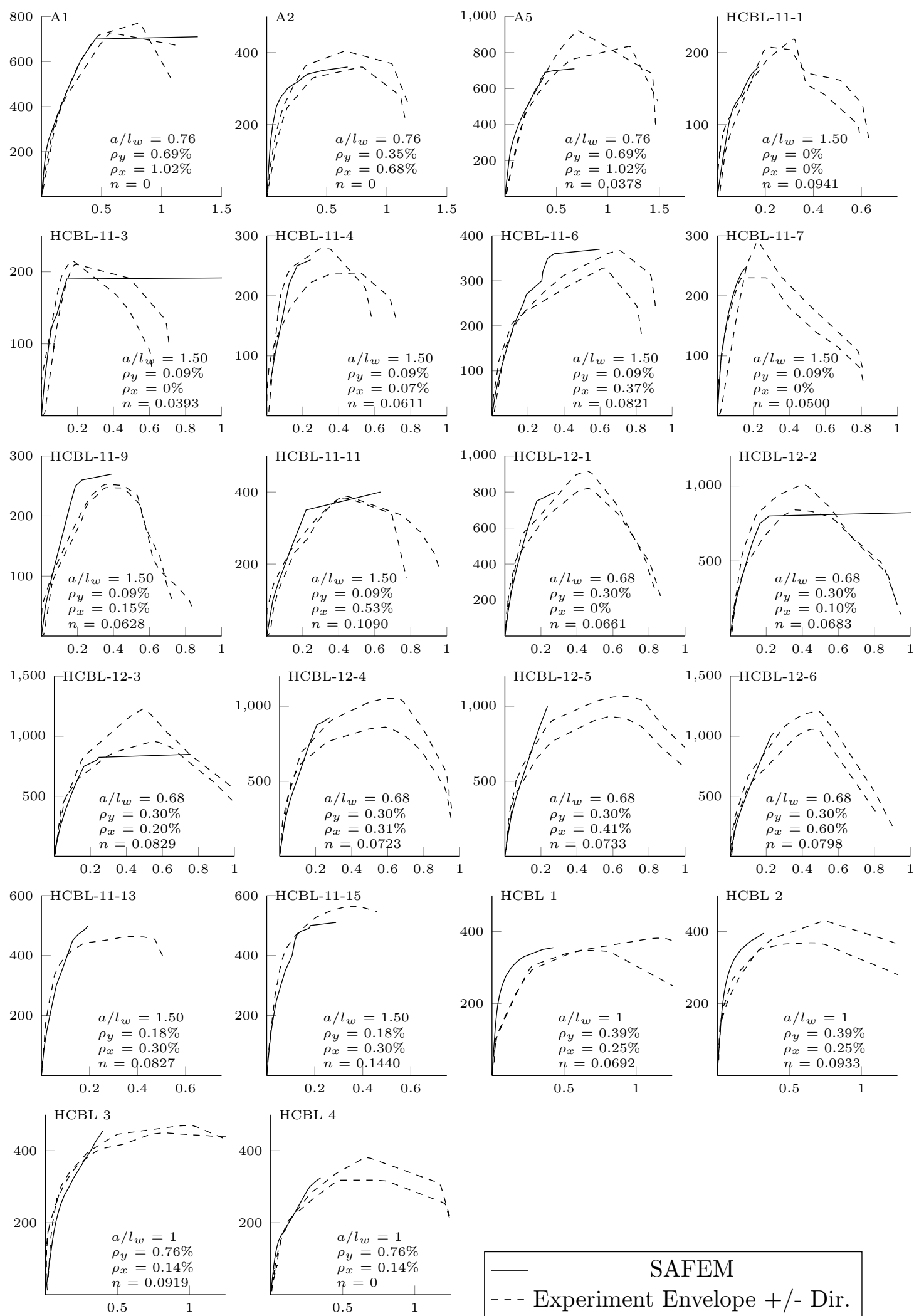

Figure 13.13: Shear Force $(V, \mathrm{kN})$ Verses Lateral Drift $(\delta=\Delta / a, \%)$ Response of Grouted Walls; Experimental and the SAFEM Predictions 

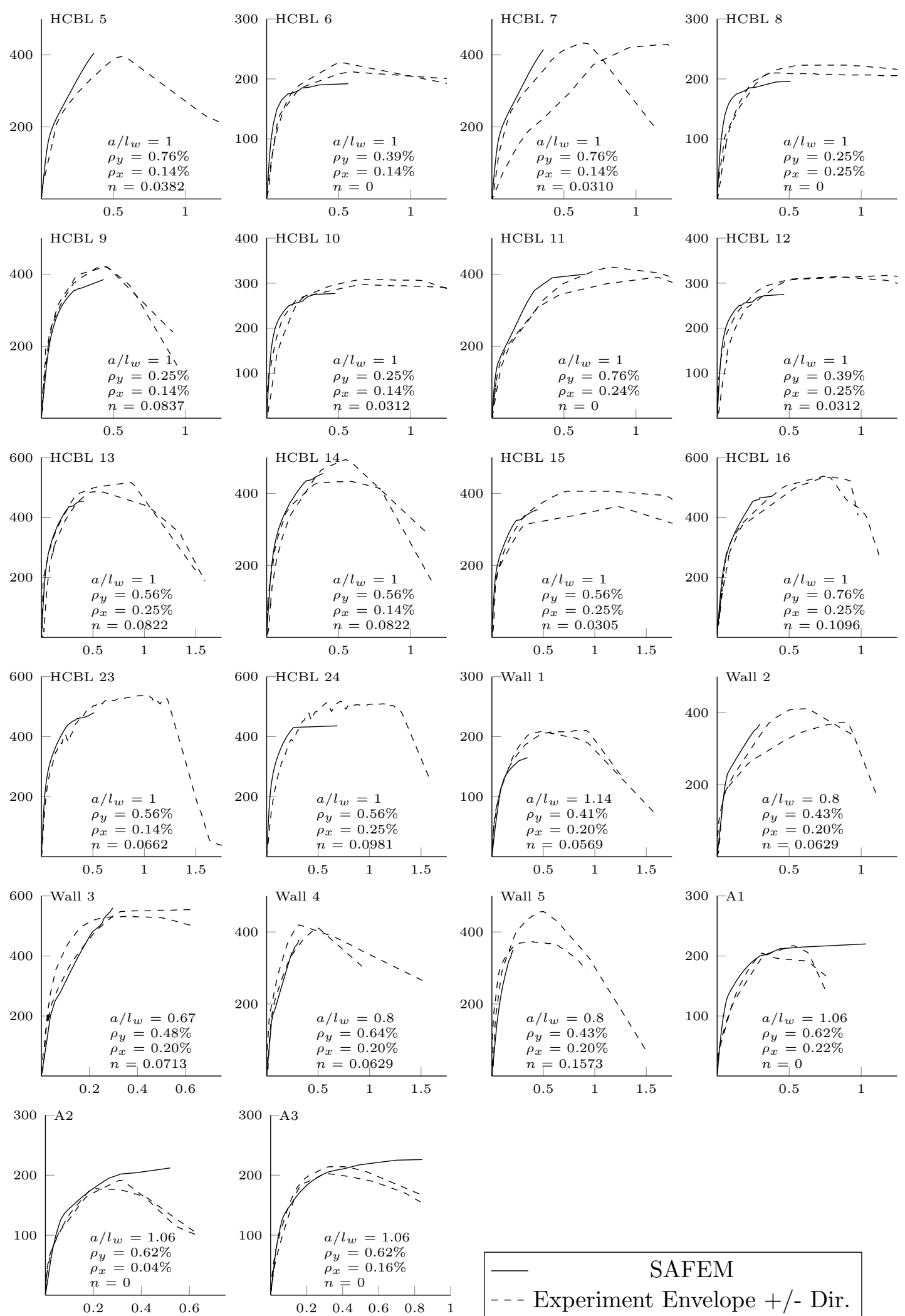

Figure 13.13: Continued from Previous Page 

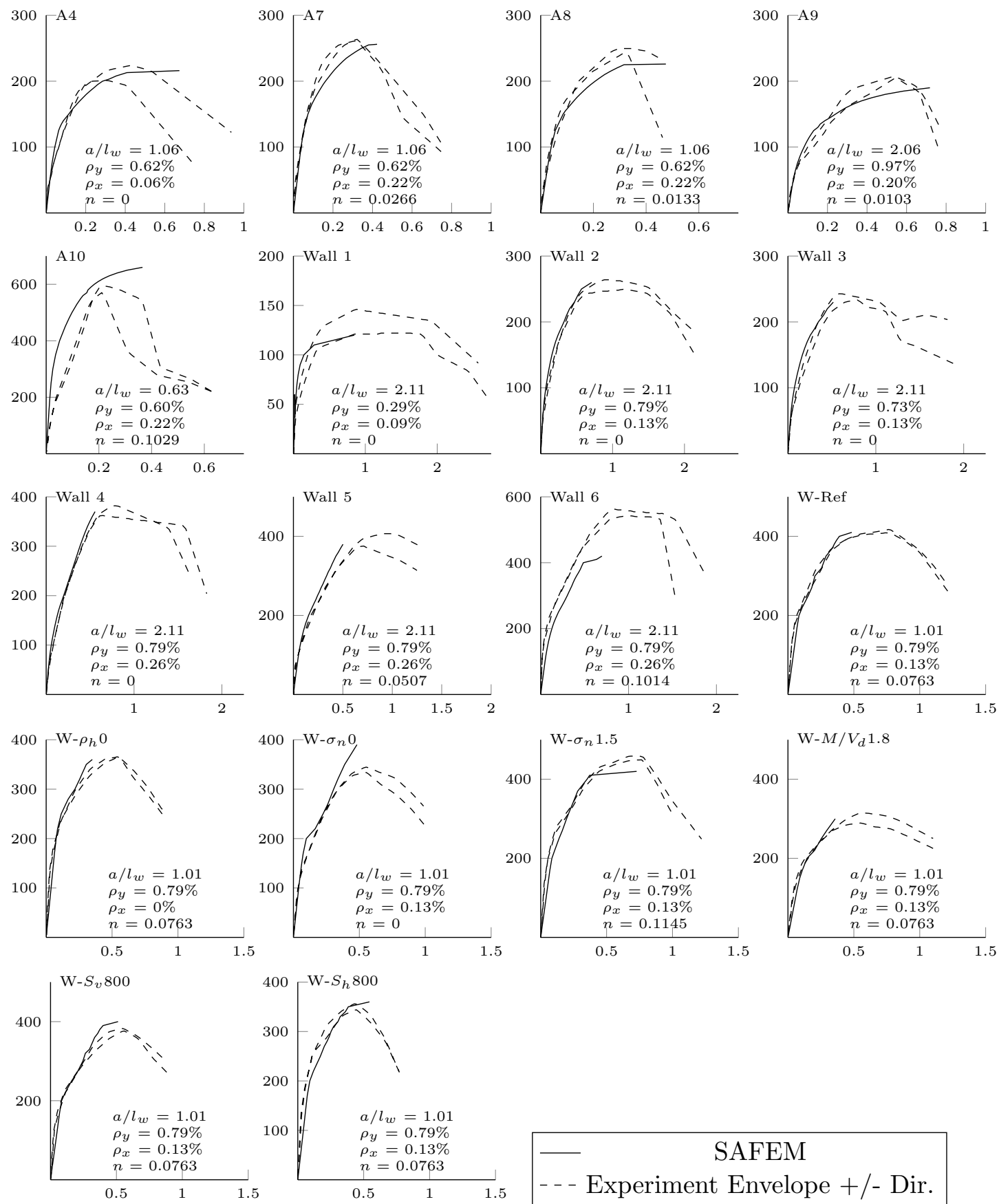

Figure 13.13: Continued from Previous Page 


\subsection{Discussion}

The following discussion examines the SAFEM inputs used to produce the results presented in Figure 13.13 and Appendix D. This discussion will focus on verifying the SAFEM default values, as well as proposing equations to estimate required SAFEM inputs.

\subsubsection{Masonry Strain at Peak Strength}

The masonry strains at peak strength, $\epsilon_{o}$, used in the SAFEM to produce the results presented in Figure 13.13 and Appendix D are plotted in Figure 13.14. These strains are underestimated by the expression proposed by Wong et al. (2013) for normal strength concrete. To better estimate the strain, $\epsilon_{o}$, it is proposed to use:

$$
\epsilon_{o}=0.0009\left(f_{m}^{\prime}\right)^{0.37} \text {. }
$$

As shown in Figure 13.14, this equation produces larger peak strains as the masonry strength increases. Additional, it produces zero strain when the peak strength is zero.

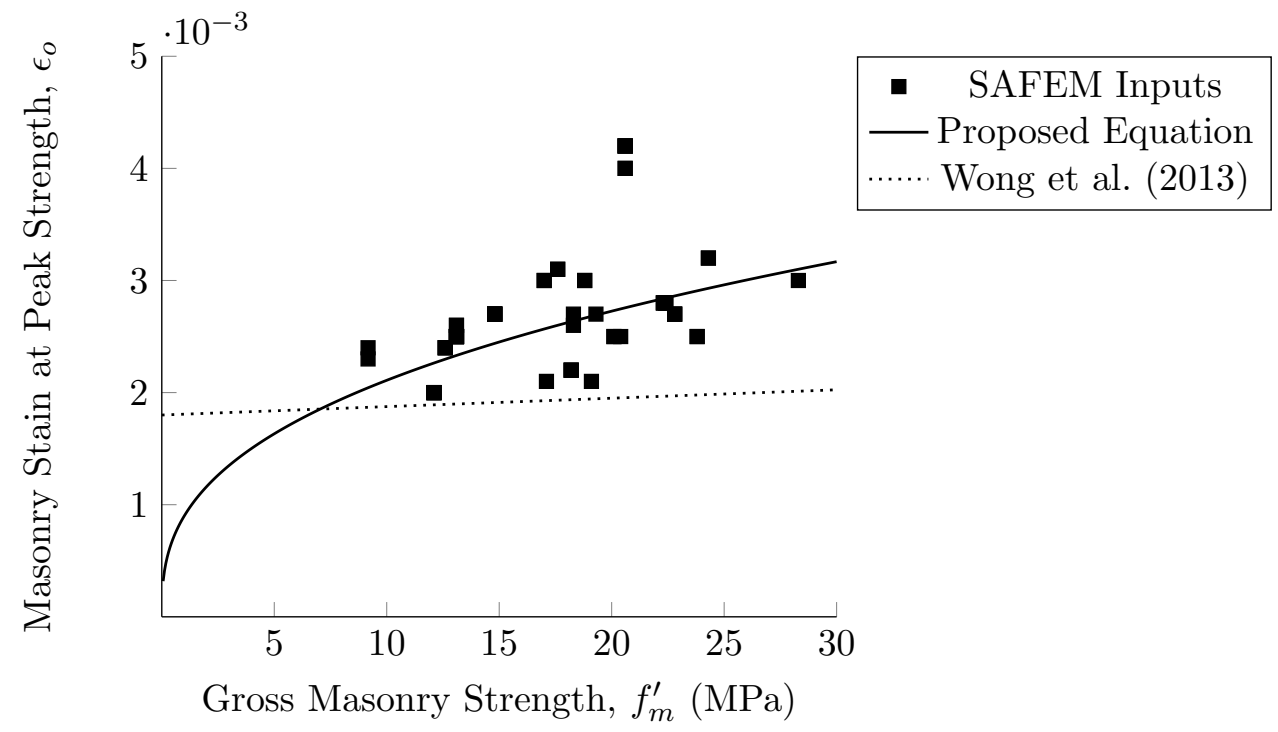

Figure 13.14: SAFEM Inputs for Grouted Wall Masonry Strains

\subsubsection{Modulus of Elasticity for Grouted Masonry}

Figure 13.15 shows the variation of the modulus of elasticity compared to the masonry compression strength. Hamid (1978) noted that there is no direct correlation between the modulus of elasticity and the compressive strength of masonry as the parameters 
affecting these two characteristics are not the same. The reason for this is because at failure the interaction between the blocks, mortar, and grout associated with inelastic deformations creates a complex state of stresses which are different from those at an early stage of loading. Hamid (1978) did provide a range of modulus of elasticities that was later modified by Hamid et al. (1987) as:

$$
E_{m}=400 \text { to } 1290 f_{m}^{\prime} \text {. }
$$

The current Canadian masonry design standard CSA S304-14 (Canadian Standards Association, 2014a) suggests the formula:

$$
E_{m}=850 f_{m}^{\prime} \leq 20000 \mathrm{MPa}
$$

which is the average of the range suggected by Hamid et al. (1987). As shown in Figure 13.15 the CSA S304-14 formula overestimates the elastic modulus used in the SAFEM.

The current Canadian concrete design standard CSA A23.3-14 (Canadian Standards Association, 2014b) notes that modulus of elasticity of concrete varis depending on the concrete strength, concrete density, and type of coarse aggregate. A23.3-14 suggests using:

$$
E_{c}=4500 \sqrt{f^{\prime} c}
$$

for the secant modulus of concrete. This is shown in Figure 13.15 to significantly overestimate the elastic modulus used in the SAFEM. This is largely due to the fact that the strain a peak strength of concrete tends to be around 0.002, while as shown in Figure 13.14 grouted hollow concrete block masonry has a larger strain of 0.0025 to 0.003 at peak strength.

The SAFEM modulus of elasticity data points suggest using a non-linear exponential relationship with an upper limit. From the data used to produce the results presented in Figure 13.13 and Appendix D it is proposed that the modulus of elasticity of grouted concrete block masonry be:

$$
E_{m}= \begin{cases}475 f_{m}^{\prime} & \text { if } f_{m}^{\prime} \leq 8 \mathrm{MPa} \\ \min \left(1850 e^{0.09 f_{m}^{\prime}}, 20000\right) & \text { otherwise }\end{cases}
$$




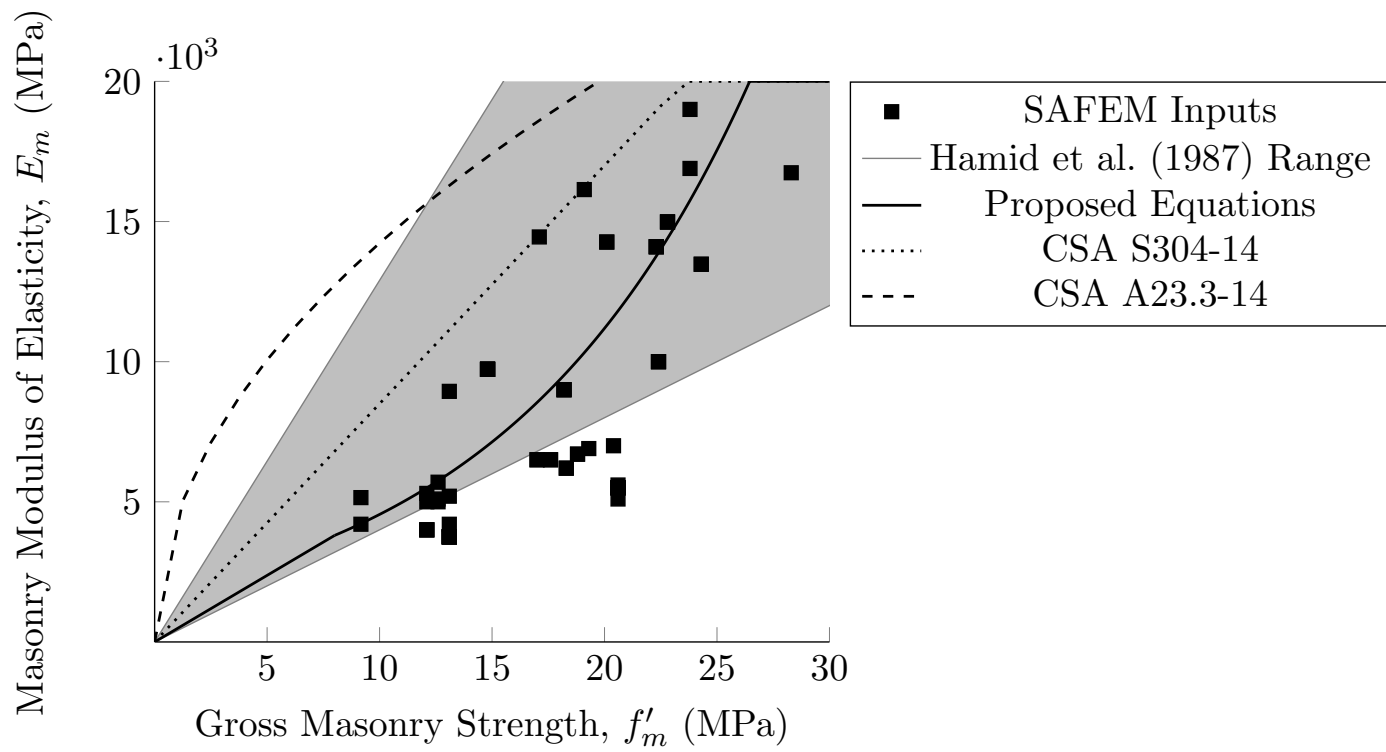

Figure 13.15: SAFEM Inputs for Grouted Wall Masonry Modulus of Elasticity

\subsubsection{Initial Masonry Poisson's Ratio}

The initial masonry Poisson's ratios used in the SAFEM are shown in Figure 13.16. As can be seen the default value of 0.20 for the initial masonry Poisson's ratio does a good good at producing the structural response of grouted hollow concrete block masonry. This value is a typically used in modeling masonry (Ip, 1999).

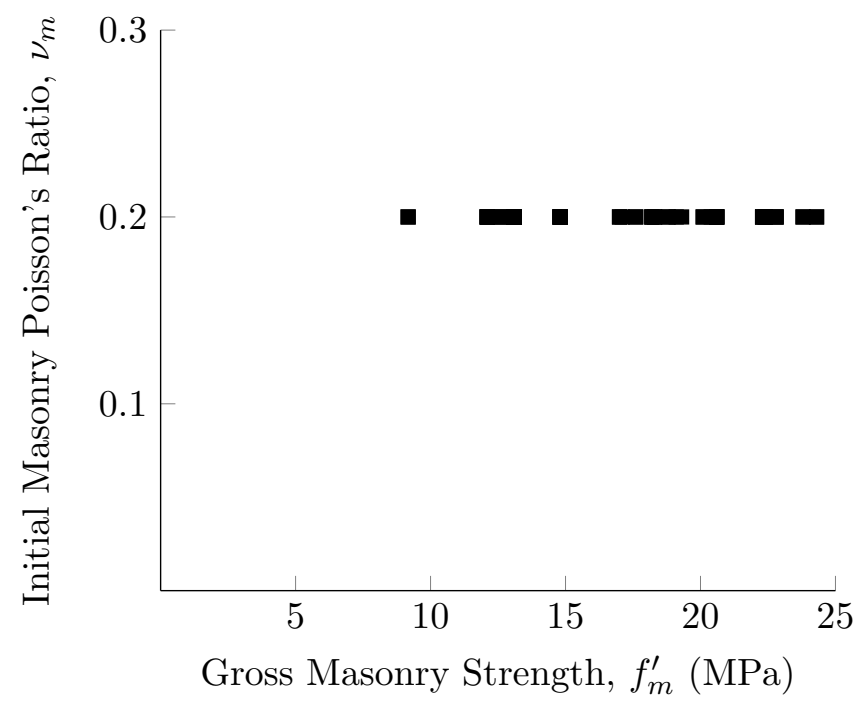

Figure 13.16: SAFEM Inputs for Grouted Wall Initial Masonry Poisson's Ratio 


\subsubsection{Block Tensile Strength}

The block tensile strengths from the grouted wall database are plotted in Figure 13.17 versus the block compression strength (based on net hard capping). Hard capping for the block compression strength is used because as can be seen the equation

$$
f_{t, b l}=0.33 \sqrt{f_{c}^{\prime}, b l},
$$

which is typically used for determining the tensile strength on concrete based on the cylinder strength, predicts the block tensile strength used in the results of the SAFEM as shown in Figure 13.17.

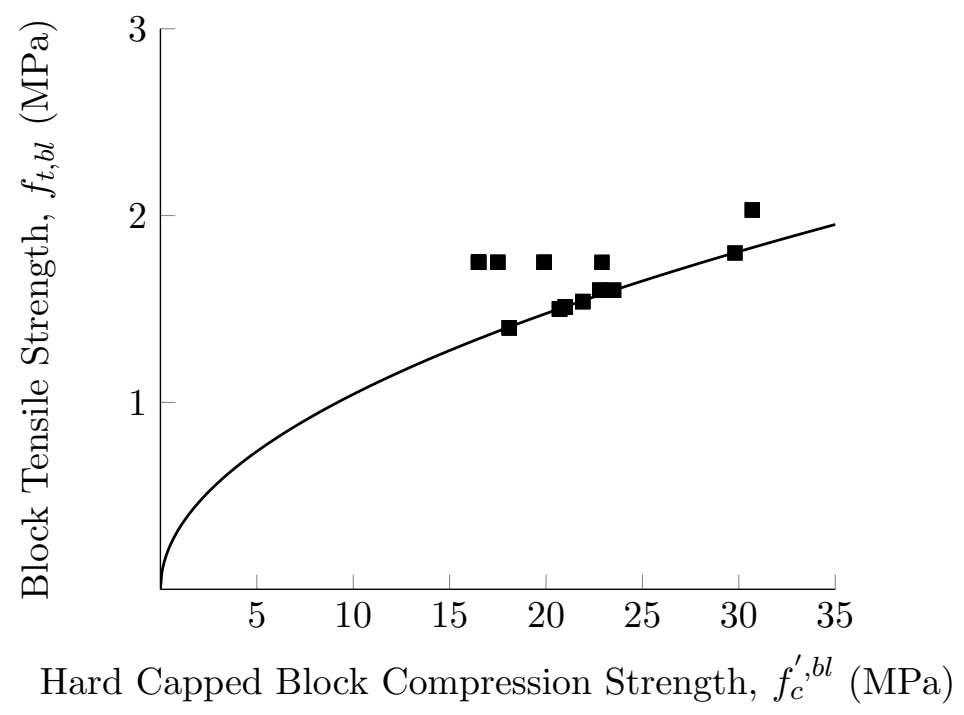

Figure 13.17: SAFEM Inputs for Grouted Wall Block Tensile Strength

\subsubsection{Mortar Bond Strength}

Figure 13.18 shows the mortar bond strength compared to the unified mortar compression strength for the input strengths used to produce the results presented in Figure 13.13 and Appendix D. Mortar compression strength are unified to absorbent cube strength as discussed in subsection 3.9.2. The bond strength to the hollow concrete block can be estimated from the following proposed equation:

$$
f_{\text {bond }, m r}=0.0802\left(f_{c}^{\prime}, m r\right)^{0.4387} .
$$

As can be seen in Figure 13.18 this equation predicts the mortar bond strength accurately. 


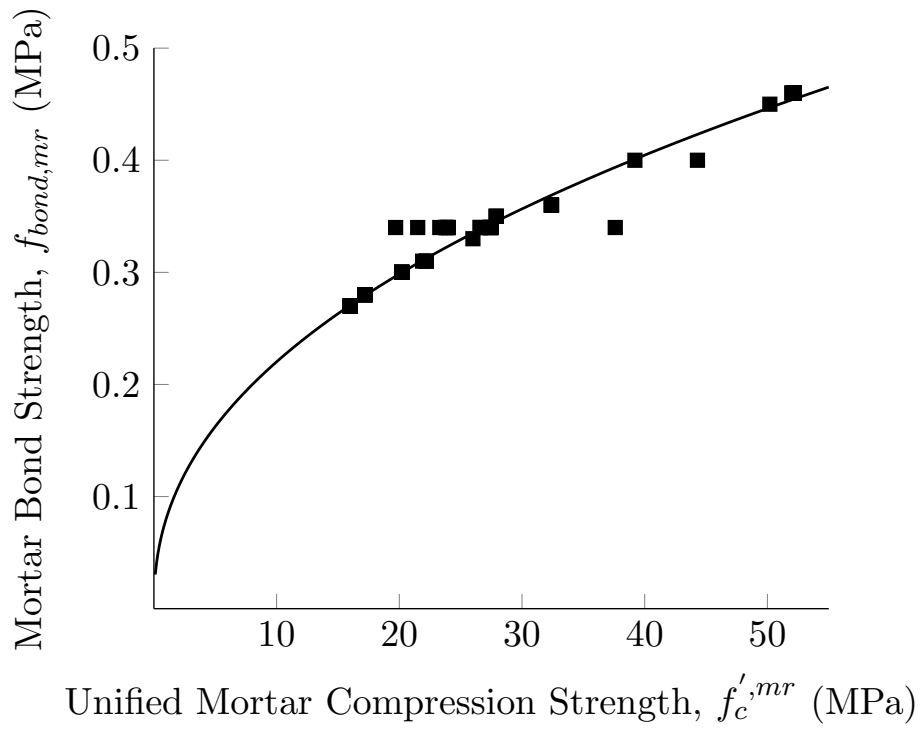

Figure 13.18: SAFEM Inputs for Grouted Wall Mortar Bond Strength

\subsubsection{Mortar Cohesion Strength}

The cohesion of the mortar, $c_{m r}$, can be determined through a Mohr-Coulomb cast in the form of principal stresses. This is done by noting that at failure in uniaxial compression, the maximum compressive stress, $\sigma_{3}^{j}$ is equal to the compressive strength of the mortar $f_{c}^{\prime}, m r$ and $\sigma_{1}^{j}$ is zero is, thus:

$$
c_{m r}=f_{c}^{\prime}, m r\left(\frac{1-\sin \phi}{2 \cos \phi}\right)
$$

where $f_{c}^{\prime}, m r$ is the uniformed mortar compression strength (subsection 3.9.2) and $\phi$ is the angle of internal friction of the mortar. Taking $\phi$ as $42^{\circ}$, results in

$$
c_{m r}=0.223 f_{c}^{\prime}, m r
$$

which is shown in Figure 13.19 to correctly predict the mortar cohesion strengths used as inputs in the SAFEM to produce the results shown in Figure 13.13. 


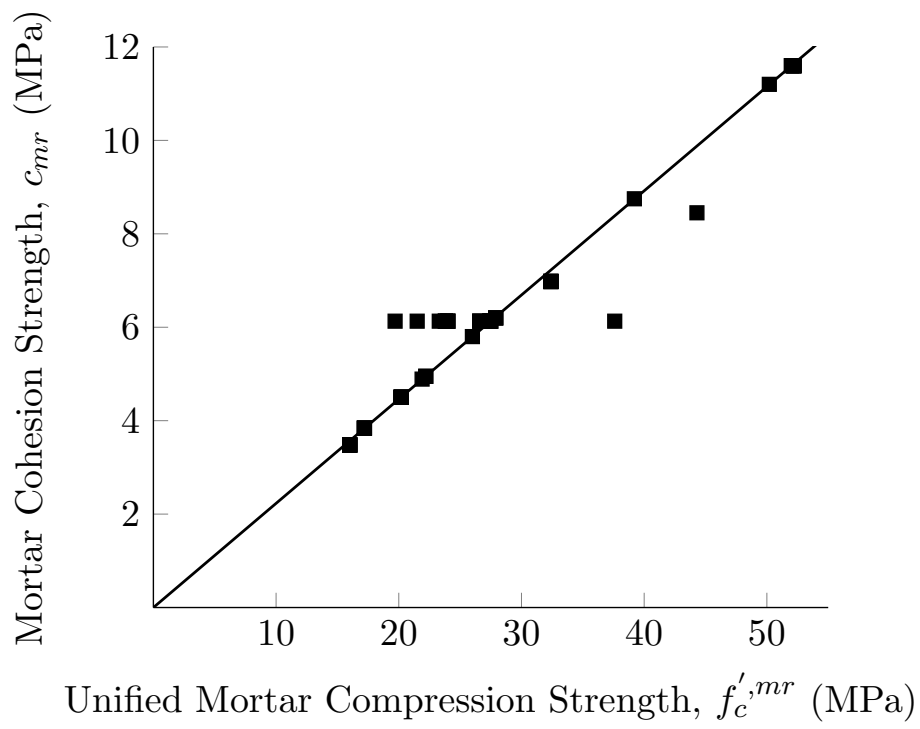

Figure 13.19: SAFEM Inputs for Grouted Wall Mortar Cohesion Strength

\subsubsection{Grout Tensile Strength}

The grout tensile strength used to produce the results presented in Figure 13.13 and Appendix D are plotted in Figure 13.20. The grout tensile strength can be predicted using the unified grout compression strength (see subsection 3.9.3) and a typical equation used to predict the tensile strength of concrete, i.e.

$$
f_{t, g r}=0.33 \sqrt{f_{c}^{\prime}, g r} .
$$

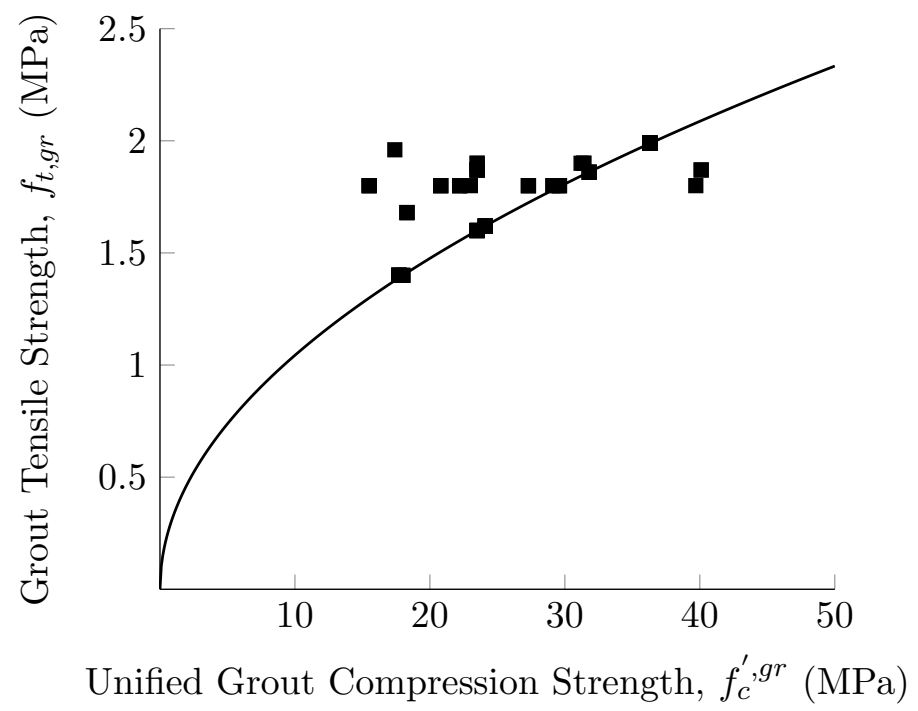

Figure 13.20: SAFEM Inputs for Grouted Wall Grout Tensile Strength 


\subsubsection{Effect of Masonry Compression Strength}

Increasing the compression strength of masonry wall increases both the bearing capacity and shear strength of the wall. This effect can be seen when comparing wall HCBL 13 to HCBL 24 and HCBL 14 to HCBL 23 each tested by Shing et al. (1991). Each one of these series of walls had the same wall dimensions, reinforcing bars, and axial load. The only difference was the loading (cyclic vs monotonic) and the masonry strength (22.8 MPa for HCBL 13 compared to 19.1 MPa for HCBL 24 and 22.8 MPa for wall HCBL 14 vs $28.3 \mathrm{MPa}$ for HCBL 23). Both the experimental and SAFEM results agree and show an increase in shear capacity for walls with higher masonry compression strength.

\subsubsection{Effect of Reinforcement}

Both vertical and horizontal reinforcement improve the performance of grouted shear walls by allowing for higher axial loads and increased shear capacity. Vertical tensile reinforcement uniformly distributed along the length of the wall results in improvements to the behaviour of the shear wall including better resistance to sliding, higher compressionsplitting resistance at the toe of the wall, and more effective control of diagonal cracking. Horizontal reinforcement allows for larger shear capacity as the reinforcement is able to transfer the shear forces across the cracks. There is a wide range of vertical and horizontal ratios presented in the database and the SAFEM is able to accruatly predicted the load deflection response of each wall as shown in Figure 13.13.

\subsubsection{Effect of Axial Load Ratio}

Higher axial compression load affects wall behaviour by delaying initial cracking of the masonry and tensile yielding of the reinforcement. This results in increased shear capacity and can increase flexural capacity, except for compression-controlled failures. The experimental results in the database cover a wide range of axial compressive stress and the SAFEM is able to predict the response of all these walls.

\subsubsection{Effect of Boundary Conditions}

In the database of single pier grouted shear wall there are 40 walls that are cantilevered and 22 wall with fixed ends. The SAFEM is able to predict the response of both cantilevered and fixed-end walls as shown in Figure 13.13.

\section{Effect of Aspect Ratio}

In addition, the relative contributions of the bending and shear deformations depend on the wall aspect ratio, $h_{w} / l_{w}$, see Table 13.1. For walls with relatively large aspect ratios, 
Chapter 13: Comparison of Single Pier Grouted Shear Wall Data and the Smeared Area Finite Element Model

Table 13.1: Effect of Aspect Ratio on Deflection Due to Shear

\begin{tabular}{lll}
\hline \multirow{2}{*}{$\begin{array}{l}\text { Aspect Ratio } \\
\left(h_{w} / l_{w}\right)\end{array}$} & \multicolumn{2}{l}{ Percentage of Deflection Due to Shear } \\
\cline { 2 - 3 } & Cantilever Wall & Fixed-End Wall \\
\hline 0.25 & 92 & 98 \\
1.00 & 43 & 75 \\
2.00 & 16 & 43 \\
4.00 & 5 & 16 \\
8.00 & 1 & 4.5 \\
\hline
\end{tabular}

the effect of shear deformation is very small and the walls tend to fail due to flexure. For very squat walls, deformation is largely from shear and shear fails are predominate. For intermediate cantilevers walls with aspect ratios ranging between 1 to 4 , it is important to consider both shear and bending deformations as the wall could fail due to shear or bending. As shown in Figure 13.13 and Appendix D the SAFEM is able to predict the results of walls over all aspect ratios. 


\title{
COMPARISON OF SINGLE PIER UNGROUTED SHEAR WALL DATA AND THE SMEARED AREA FINITE EL- EMENT MODEL
}

\begin{abstract}
The model presented in Chapter 10, implemented in Chapter 11, and verified in Chapter 12 is used to determine the structural response of ungrouted single per shear walls constructed from hollow concrete block masonry. The results of the finite element model are compared to the experimental results found in literature. The results of model inputs are used to produce equations to predict hollow concrete block masonry mechanics.
\end{abstract}

This chapter assembles an experimental database of single pier ungrouted shear wall tests made from hollow concrete blocks to allow for the comparison of experimental and the SAFEM results.

\subsection{Data Requirement}

To use the SAFEM a number of material properties are required as inputs; thus, to be included in the database the following had to be reported for the test:

1. The masonry compression strength as determined from a prism test of three or more courses;

2. The block length, height, faceshell thickness, percent solid, and either tensile or compression strength; and,

3. The bed and head joint thickness and mortar compression strength.

Further requires are:

1. The dimensions of the wall had to be given;

2. The experimental test setup had to be reported to ensure that the correct boundary and loading conditions were represented in the finite element analysis;

3. The specimen had no opens within the masonry continuum;

4. The specimen had to be constructed from full scale blocks with in joint reinforcement; 
5. To facilitate the comparison of the SAFEM results and the experiment a load deflection graph had to be reported, with the reported deflection corresponding to the specimen only (i.e. the relative displace of the experimental setup is removed).

In total, 14 single pier ungrouted shear wall experiments were used to validate the SAFEM. If a value required to run the model was not given an assumed value was used as listed in Appendix E.

\subsection{Database Literature Review}

Woodward and Rankin (1985) tested seventeen ungrouted, unreinforced shear walls constructed with hollow concrete blocks (only fourteen of these walls had all required inputs reported). The primary variables in the test series are block strength, mortar strength, applied vertical compressive load, and wall aspect ratio. The walls are tested using fixed-ended boundary conditions at the top and bottom. A vertical compressive load is applied and maintained while in-plane lateral displacements are imposed at the top of the wall. The test results indicate that for the lower levels of vertical compressive load the effect of block and mortar strength on maximum in-plane shear resistance is relatively small, while with increasing vertical compressive loads the effect of increasing material strength becomes significant and results in increased shear resistance.

\subsection{Distribution of Specimen Parameters}

Woodward and Rankin (1985) built walls with either a high or low strength block having a net compression strength of 23.9 $\mathrm{MPa}$ and 17.9 MPa respectively. The mortar is either Type S or Type N and ranged from 13.1 MPa to $30.1 \mathrm{MPa}$ based on absorbent cube strength. Ten of the walls were $1626 \mathrm{~mm}$ long $\times 1626 \mathrm{~mm}$ tall $\times 194 \mathrm{~mm}$ thick, two were $1219 \mathrm{~mm}$ long $\times 1626 \mathrm{~mm}$ tall $\times 194 \mathrm{~mm}$ thick and two were $2438 \mathrm{~mm}$ long $\times$ $1626 \mathrm{~mm}$ tall $\times 194 \mathrm{~mm}$ thick. The applied axial load is constant for a given test, but varied between 0.69 $\mathrm{MPa}$ to $2.75 \mathrm{MPa}$ (based on net area).

\subsection{SAFEM Results}

The SAFEM was used to predict the experimental response of the 14 walls from the database. The load-displacement response of each wall is shown in Figure 14.1. As can be seen the SAFEM produces correct results across the entire database in terms of stiffness, peak load, and displacement. Appendix E provides more detail on each model including the layout of elements, material properties, model options, load displacement 
Chapter 14: Comparison of Single Pier Ungrouted Shear Wall Data and the Smeared Area Finite Element Model
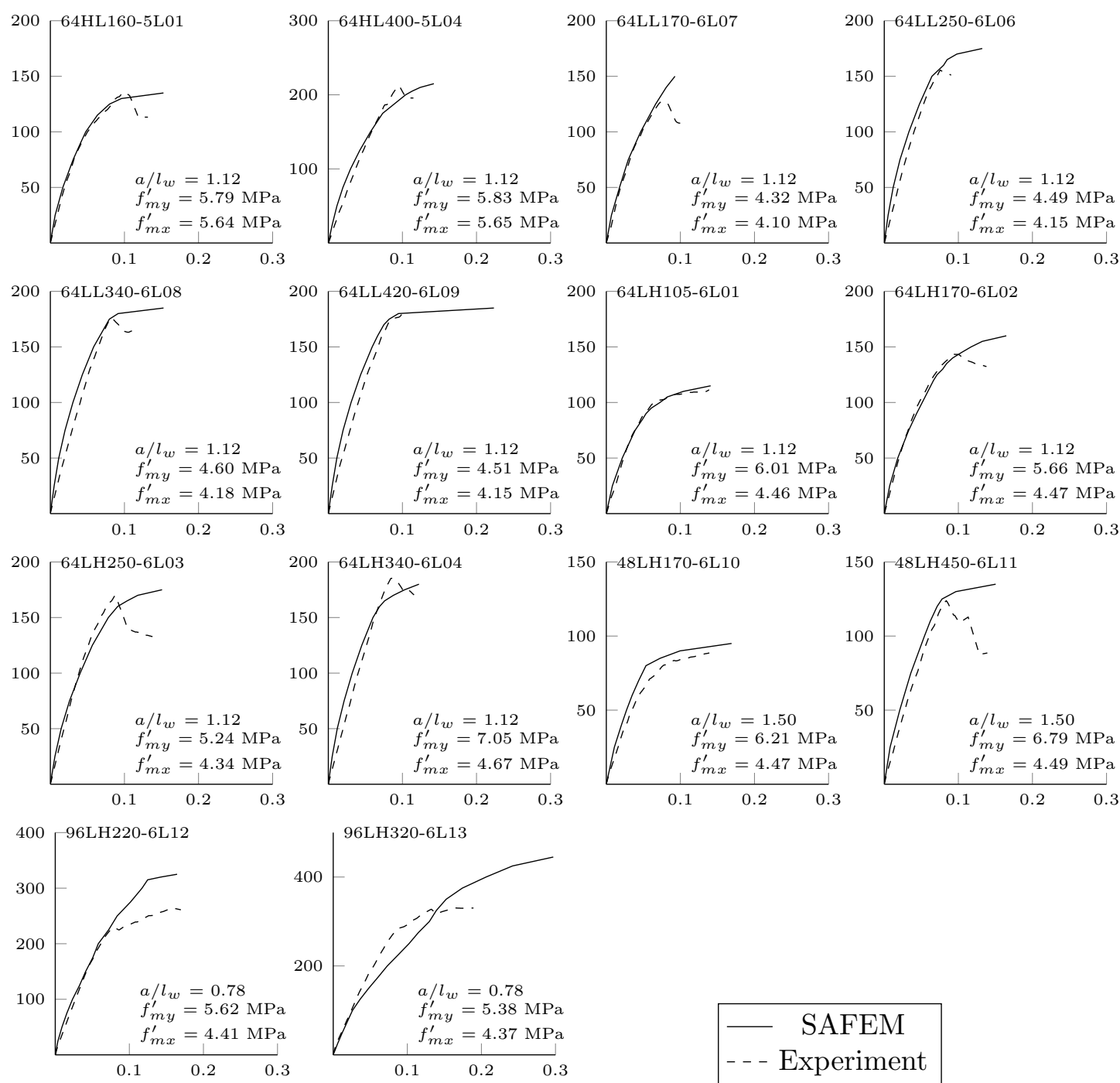

Figure 14.1: Shear Force $(V, \mathrm{kN})$ Verses Lateral Drift $(\delta=\Delta / a, \%)$ Response of Ungrouted Walls; Experimental and the SAFEM Predictions

diagrams, node displacements, and crack patterns. Where possible comparisons are made between experimental and the SAFEM results. 
Chapter 14: Comparison of Single Pier Ungrouted Shear Wall Data and the Smeared Area Finite Element Model

\subsection{Discussion}

The following discussion examines the SAFEM inputs used to produce the results presented in Figure 14.2 and Appendix E. This discussion will focus on verifying the SAFEM default values, as well as proposing equations to estimate required SAFEM inputs.

\subsubsection{Masonry Strain at Peak Strength}

The ungrouted masonry strains at peak strength used to produced the results in Figure 14.1 and Appendix E are compared to masonry compression strength (based on gross area) is shown in Figure 14.2. The strains range from 0.0015 to 0.003 , but do not show any real trend except that the majority $(50 \%)$ of the strains are 0.002 . As discussed in Subsection 4.4.1 this is a commonly assumed strain at peak strength in normal strength concrete (ie. $f_{c}^{\prime} \leq 50 \mathrm{MPa}$ ) when the strain is unknown (Kent and Park, 1971; Roy and Sozen, 1964; Saatcioglu and Razvi, 1992).

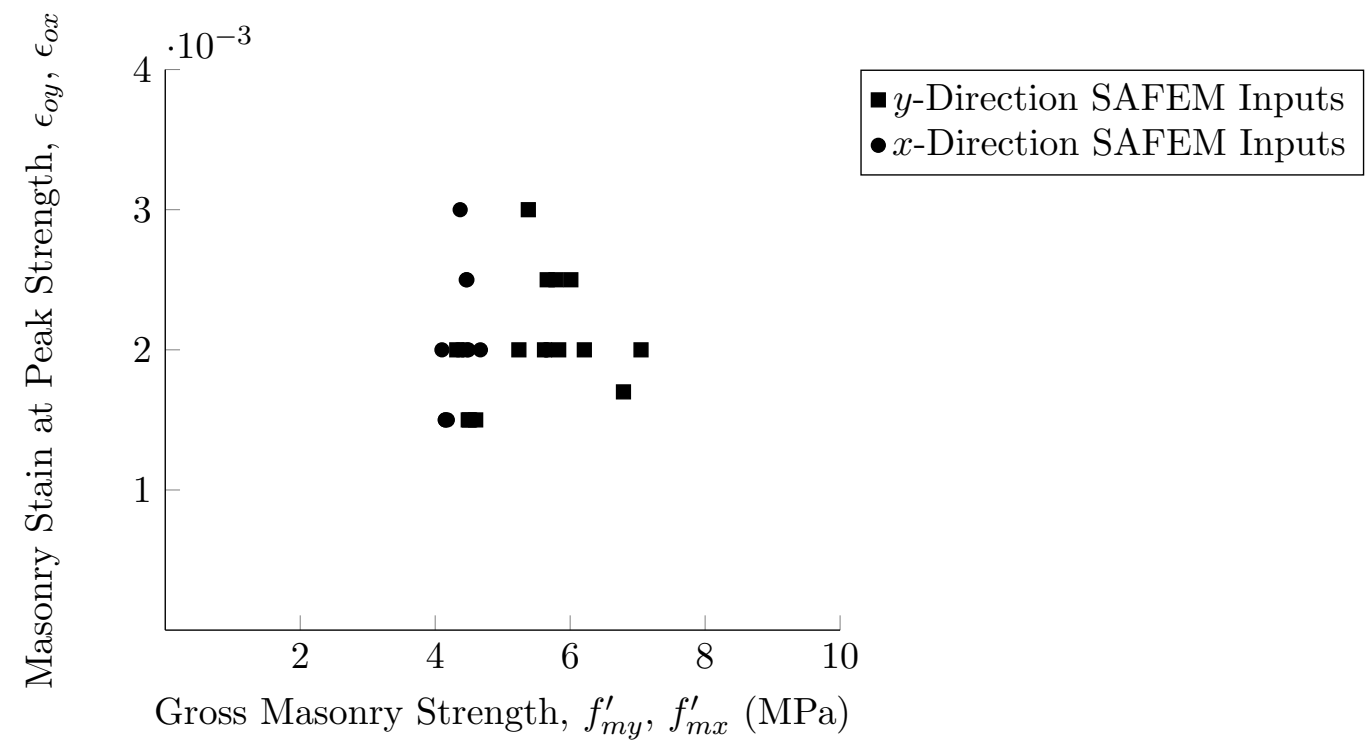

Figure 14.2: SAFEM Inputs for Ungrouted Masonry Strains 
Chapter 14: Comparison of Single Pier Ungrouted Shear Wall Data and the Smeared Area Finite Element Model

\subsubsection{Modulus of Elasticity for Ungrouted Masonry}

Figure 14.3 shows the modulus of elasticity's used to produce the results shown in Figure 14.1 and Appendix E. The masonry compression strengths are based on gross area for use in the finite element model. The modulus of elasticity values were based on the default value within the SAFEM. This modulus of elasticity is determined from:

$$
E_{m}=\frac{0.4 f_{c}^{\prime}, m}{\left(1-\sqrt{\frac{3}{5}}\right) \epsilon_{o}^{m}},
$$

which is the secant modulus at $40 \%$ of the peak strength based on a Hognestad parabola. As can be seen in Figure 14.3 these values are within the modulus of elasticity range suggested by Hamid et al. (1987) and are closely predicted by the current Canadian masonry design standard CSA S304-14 (Canadian Standards Association, 2014a).

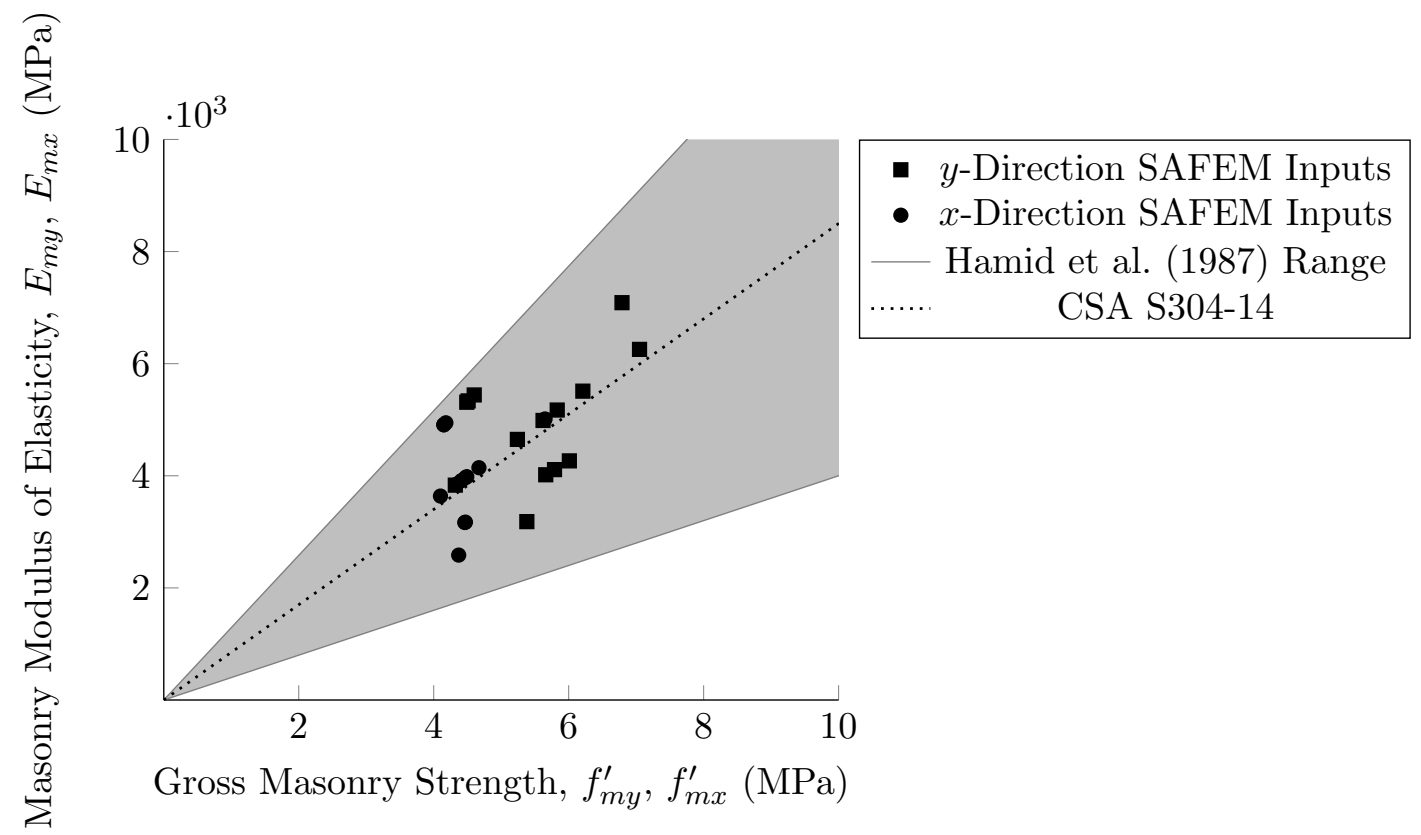

Figure 14.3: SAFEM Inputs for Ungrouted Masonry Modulus of Elasticity 
Chapter 14: Comparison of Single Pier Ungrouted Shear Wall Data and the Smeared Area Finite Element Model

\subsubsection{Initial Masonry Poisson's Ratio}

The initial masonry Poisson's ratios used as inputs for the SAFEM are shown in Figure 14.4. As can be seen the Poisson's ratio is constant at 0.20 (the default value for the SAFEM) in both the $y$ and $x$ directions, which is a typical value for masonry (Ip, 1999).

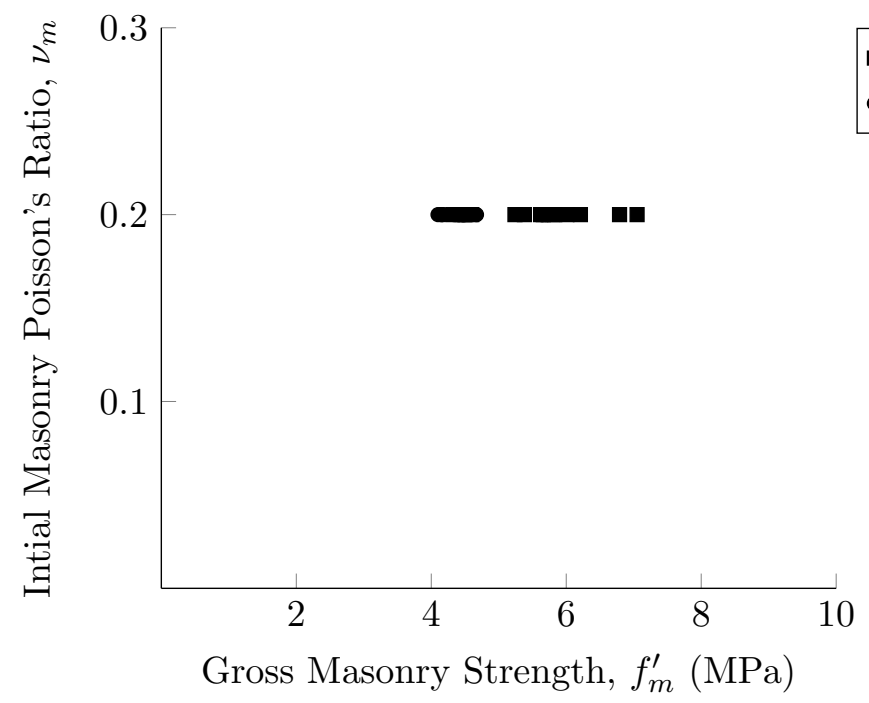

- $y$-Direction SAFEM Inputs - $x$-Direction SAFEM Inputs

Figure 14.4: SAFEM Inputs for Ungrouted Initial Masonry Poisson's Ratio

\subsubsection{Block Tensile Strength}

The block tensile strength is determined from:

$$
f_{t, b l}=0.33 \sqrt{f_{c}^{\prime}, b l}
$$

where $f_{c, b l}^{\prime}$ is based on the net area block compression strength determined with hard capping. This results in a tensile strength of $1.62 \mathrm{MPa}$ and $1.39 \mathrm{MPa}$ for the 23.9 $\mathrm{MPa}$ and 17.9 MPa block respectively.

\subsubsection{Mortar Bond Strength}

The mortar-block bond strength values used to produce the structural responses shown in Figure 14.1 are plotted in Figure 14.5. This bond strength is shown to be highly variable, but can be estimated by:

$$
f_{\text {bond }, m r}=0.0802\left(f_{c}^{\prime}, m r\right)^{0.4387 \frac{A_{n, \min }}{A_{g}}} .
$$


Chapter 14: Comparison of Single Pier Ungrouted Shear Wall Data and the Smeared Area Finite Element Model

This equation is similar to the equation proposed for estimating grouted mortar bond strength, but has a factor to account for the minimum area within the wall section.

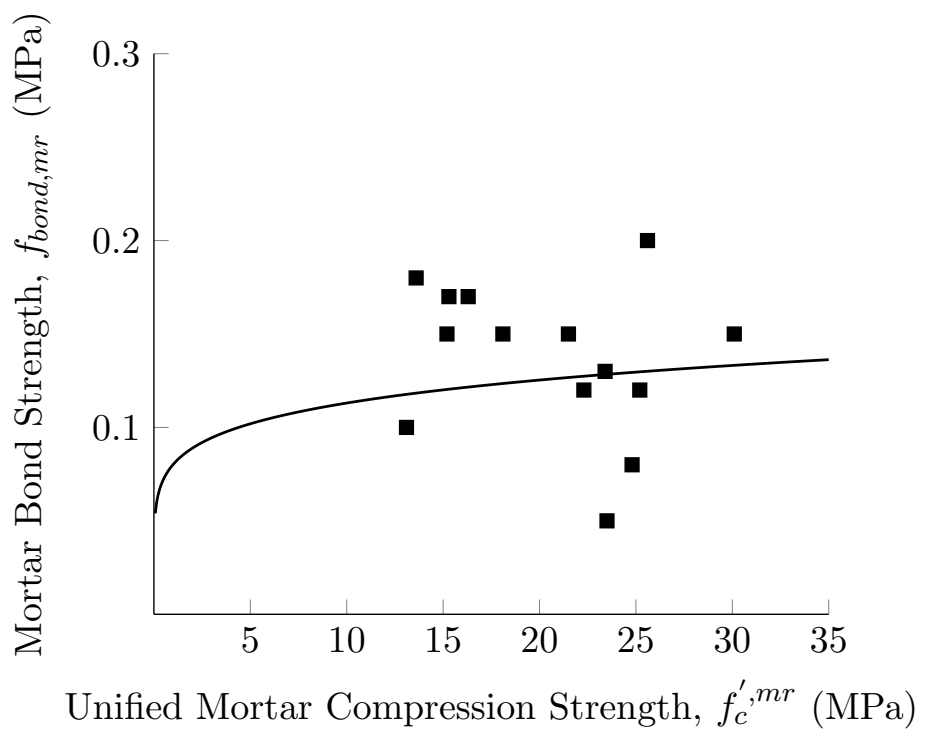

Figure 14.5: SAFEM Inputs for Ungrouted Wall Mortar Bond Strength

\subsubsection{Mortar Cohesion Strength}

The mortar cohesion strengths used as inputs in the SAFEM are shown in Figure 14.6. The mortar cohesion strength has a range of $1 \mathrm{MPa}$ to $2.5 \mathrm{MPa}$ with no trend, but can be estimated an equation similar to the grouted masonry, thus:

$$
c_{m r}=0.223 \frac{A_{\min }}{A_{g}} f_{m r}^{\prime} .
$$

Part III: Masonry Finite Element Modeling 


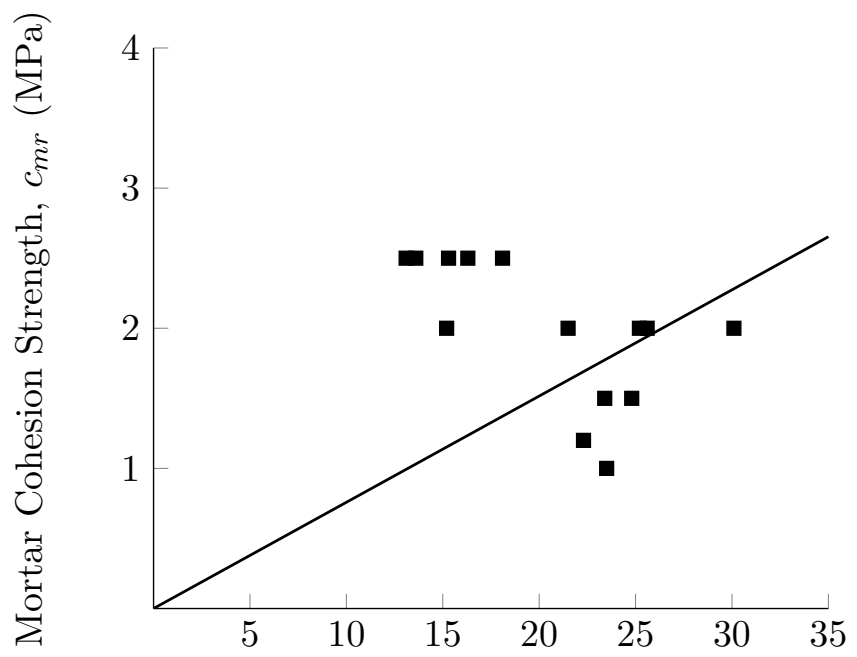

Unified Mortar Compression Strength, $f_{c, m r}^{\prime}(\mathrm{MPa})$

Figure 14.6: SAFEM Inputs for Ungrouted Wall Mortar Cohesion Strength

\subsubsection{Effect of Masonry Material Properties}

The masonry compression strength based on gross area ranged from 4.32 $\mathrm{MPa}$ to 7.05 $\mathrm{MPa}$ in the $y$-direction and $4.10 \mathrm{MPa}$ to $5.65 \mathrm{MPa}$ in the $x$-direction as determined from the SACM presection in Part I. Increasing the block strength allowed for increased shear capacity, but had similar deflections as shown by comparing wall 64LL340-6L08 to 64LH340-6L04 and 64LL170-6L07 with 64LH170-6L02. Comparing wall 64LL340-6L08 to 64LH340-6L04 and 64LL170-6L07 with 64LH170-6L02 shows that increasing mortar strength had no effect on wall strength and deflection. The SAFEM results shown in Figure 14.1 agree with the experimental results.

\subsubsection{Effect of Aspect Ratio and Axial Load}

The behaviour of the walls after cracking is affected by both the aspect ratio and the axial compressive stress. The resistance due to aggregate interlock was increased for higher compressive stresses and lower aspect ratios as there is longer diagonal cracks, thus a larger area to transfer shear. As the aspect ratio decreases from 1.50 to 0.78 the shear capacity of the wall increases from approximately $90 \mathrm{kN}$ to $280 \mathrm{kN}$, as shown in walls 48LH170-6L10, 64LH170-6102, and 96LH220-6L12. Comparing wall 64LL1706L07, 64LL250-6L06, 64LL340-6L08, and 64LL420-6L09 it is shown that as the axial compressive load increases so does the shear capacity of the wall. This is further confirmed with the remaining walls. The SAFEM results agree with these experimental results. 


\section{COMPARISON OF REINFORCED BEAM DATA AND THE SMEARED AREA FINITE ELEMENT MODEL}

The model presented in Chapter 10, implemented in Chapter 11, and verified in Chapter 12 is used to determine the structural response of reinforced beams constructed from hollow concrete block masonry. The results of the finite element model are compared to the experimental results found in literature. The results of model inputs are compared to the equations produced in Chapter 13 to predict hollow concrete block masonry mechanics.

Reinforced concrete masonry beams are commonly used to span various openings such as doors, windows, and passages. Beams are also used as coupling beams, to connect shear walls to provide a lateral load-resisting system for buildings. Despite the popularity of beams in masonry buildings only a few experimental investigations have been reported in literature (Dhanasekar and Wong, 2001; Ferieg, 1994; Li et al., 1995; Ring, 2009; Sarhat, 2016; Suter and Keller, 1980).

\subsection{Data Requirements}

To use the SAFEM a number of material properties are required as inputs; thus, to be included in the database the following had to be reported for the test:

1. The masonry compression strength as determined from a prism test of three or more courses;

2. The block length, height, faceshell thickness, percent solid, and either tensile or compression strength;

3. The bed and head joint thickness and mortar compression strength;

4. The grout tensile or compression strength; and,

5. The reinforcement details, including bar size and placement, yield strength, and ultimate strength. 
Further requires are:

1. The dimensions of the beam had to be given;

2. The experimental test setup had to be reported to ensure that the correct boundary and loading conditions were represented in the finite element analysis;

3. The specimen had to be fully grouted with no opens within the masonry continuum;

4. The specimen had to be constructed from full scale blocks with in joint reinforcement;

5. To facilitate the comparison of the SAFEM results and the experiment a load deflection graph had to be reported, with the reported deflection corresponding to the specimen only (i.e. the relative displace of the experimental setup is removed).

In total, 13 grouted masonry beam experiments were used to validate the SAFEM. If a value required to run the model was not given an assumed value was used as listed in Appendix F.

\subsection{Literature Review}

\subsubsection{Ring (2009)}

Ring (2009) tested 12 beams to investigate the effect of web interruption and of a strain gradient on masonry compressive strength parallel to the bed joint. The beams were four courses high, $7.7 \mathrm{~m}$ long, with a clear span of $7.3 \mathrm{~m}$. The first set of beams tested (B1$3(100)$ ) are not included in the analysis due to suspected arching effect that may have existed in these tests since the roller support was seated in a depression. No clear trend between web interruption and compressive strength parallel to the bed joint was found from the remaining beam tests. The prism test data shows a reasonably acceptable linear fit and based on the linear trend line, the reduction in strength parallel to the bed joint reduces as web interruption increases. This agrees with the recommendations in current Canadian standard CSA S304-14 (Canadian Standards Association, 2014a). However, the amount of reduction in strength when going from $100 \%$ to $0 \%$ web interruption (based on the linear trend line) is not the same as what is recommended in CSA S304-14 (Canadian Standards Association, 2014a) (see Section 7.1 for more details).

\subsubsection{Sarhat (2016)}

Sarhat (2016) performed an extensive experimental program consisting of fifteen largescale reinforced masonry beams, see Figure 15.1. The results show that principles that 
govern the shear design of reinforced concrete beams in Canada can be applied to reinforced masonry beams. Various phenomena that have been observed in reinforced concrete subjected to shear were also observed in these tests, includes "the size effect" and "the strain effect". It is shown that design codes that do not account for these effects exhibit higher variability and in the case of size effect, may produce unsafe designs. Detailed instrumentation of the test specimens showed that aggregate interlock is the major mechanism of shear transfer in masonry beams, and as such this mechanism can explain much of the observed experimental behaviour. The size effect, wherein the failure shear stress decreases as the effective depth increases, can be explained by the reduced aggregate interlock capacity. Likewise, the strain effect, wherein the failure shear stress decrease as longitudinal strains increase, can be explained based on reduced aggregate interlock. It is also found that stirrups and longitudinal reinforcement distributed over the height of the beam have highly beneficial effects on shear behaviour of reinforced masonry. 
Chapter 15: Comparison of Reinforced Beam Data and the Smeared Area Finite Element Model

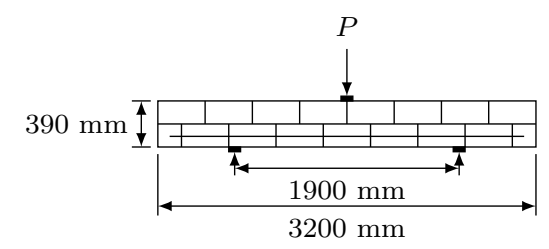

(a) Small Beam with No Stirrups

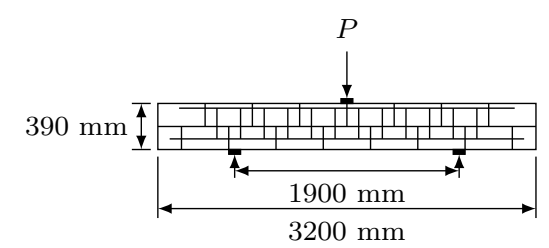

(b) Small Beam with Stirrups

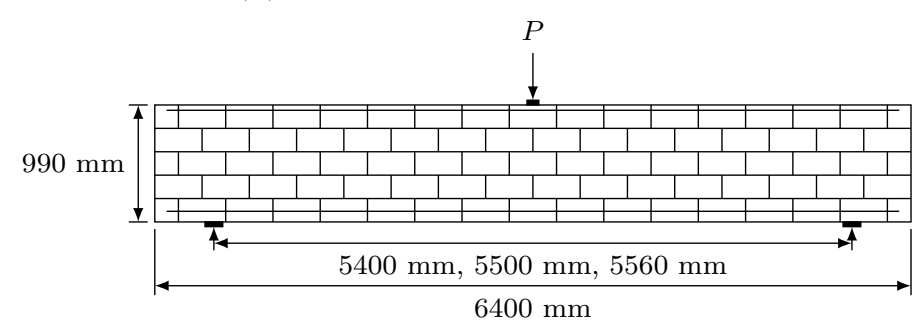

(c) Medium Beam with No Stirrups

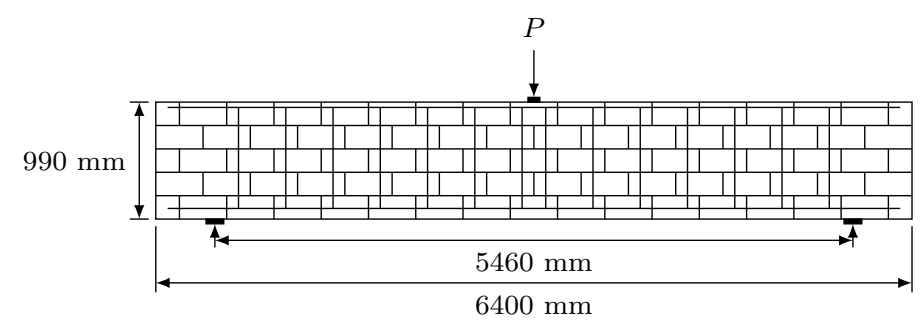

(d) Medium Beam with Stirrups

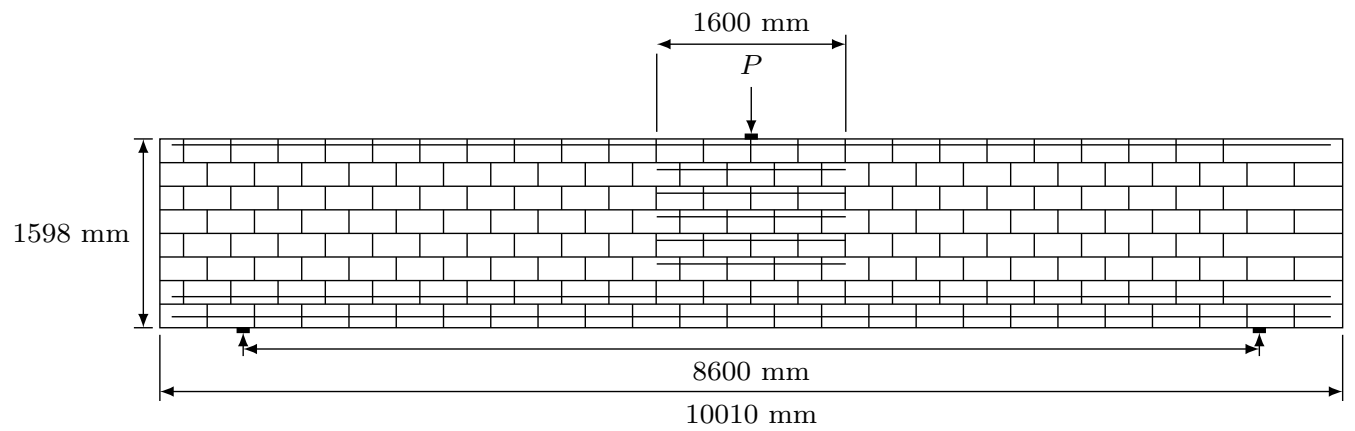

(e) Large Beam with No Stirrups

Figure 15.1: Sarhat (2016) Beam Specimens 


\subsection{Distribution of Specimen Parameters}

In this section, properties of the beam specimens are plotted as histograms. The quantitative attributes make up some of the important input parameters used in the SAFEM and show the range of inputs used to verify the SAFEM.

\subsubsection{Masonry Compression Strength}

The masonry compression strength, based on gross area, ranged from 17.5 to $25.7 \mathrm{MPa}$, see Figure 15.2. These strengths are typical for fully grouted masonry used in today's construction.

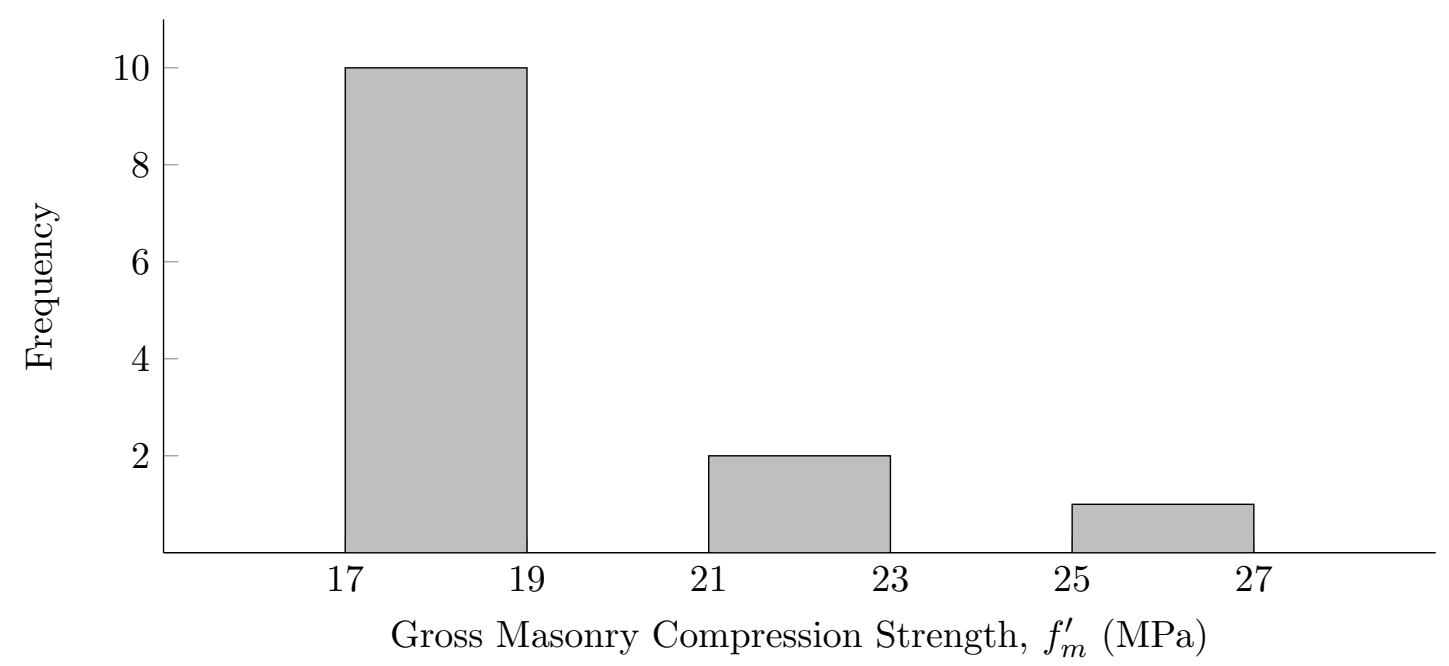

Figure 15.2: Grouted Beam Masonry Compression Strength Histogram 


\subsubsection{Block Compression Strength}

The block compression strengths for the 13 beams ranged from 25.4 to $37.5 \mathrm{MPa}$, see Figure 15.3 based on the net area of the block tested with hard capping.

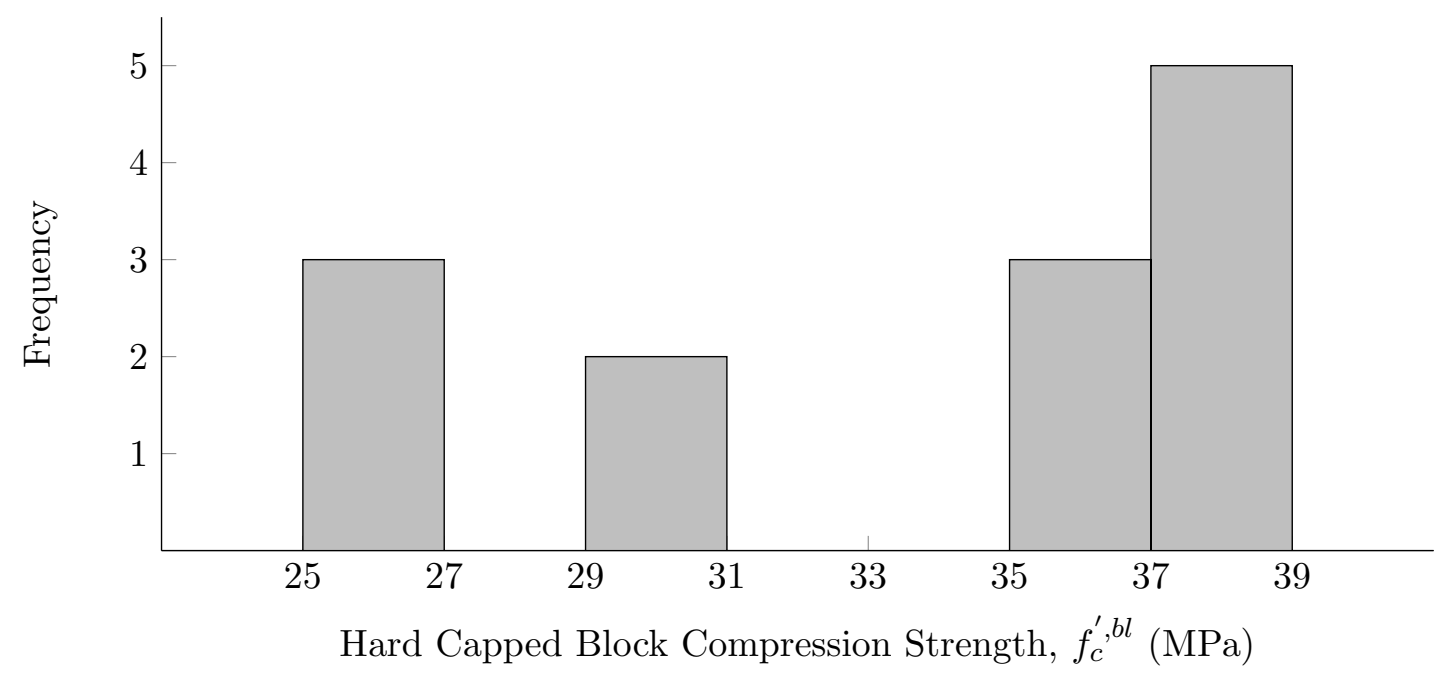

Figure 15.3: Grouted Beam Block Compression Strength Histogram

\subsubsection{Mortar Compression Strength}

The mortar compression strengths shown in Figure 15.4 are unified to absorbent cube strength (see subsection 3.9.2). This unification attempts to represent the in-situ strength of the mortar used in the beams. The unified mortar strengths represented in the reinforced beam database ranged from 14.1 to $33.8 \mathrm{MPa}$, with the majority of strengths between 29 and $32 \mathrm{MPa}$.

Part III: Masonry Finite Element Modeling 


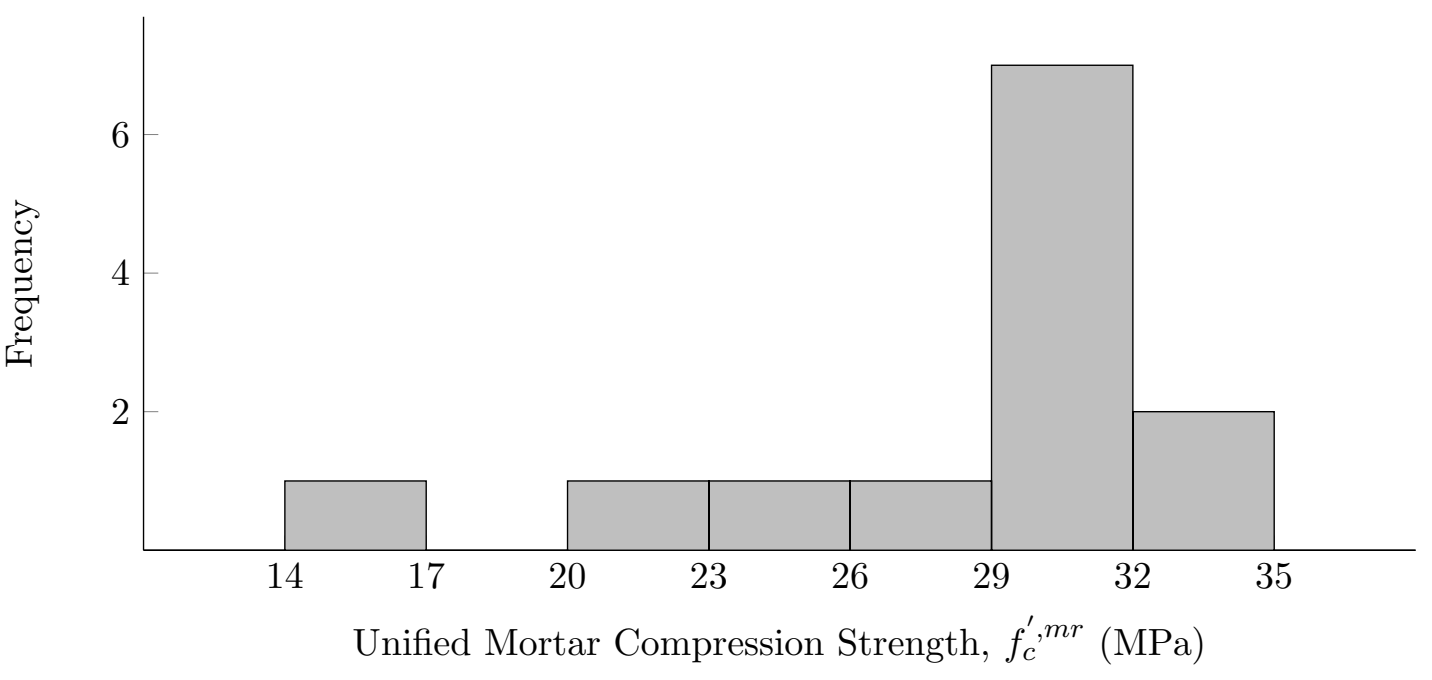

Figure 15.4: Grouted Beam Mortar Compression Strength Histogram

\subsubsection{Grout Compression Strength}

To represent the in-situ strength of the grout the grout compression strengths presented in Figure 15.5 are unified to absorbent cylinder strength (see Subsection 3.9.3). The unified grout compression strengths from the reinforced beam database ranged from 25.8 to $30.7 \mathrm{MPa}$.

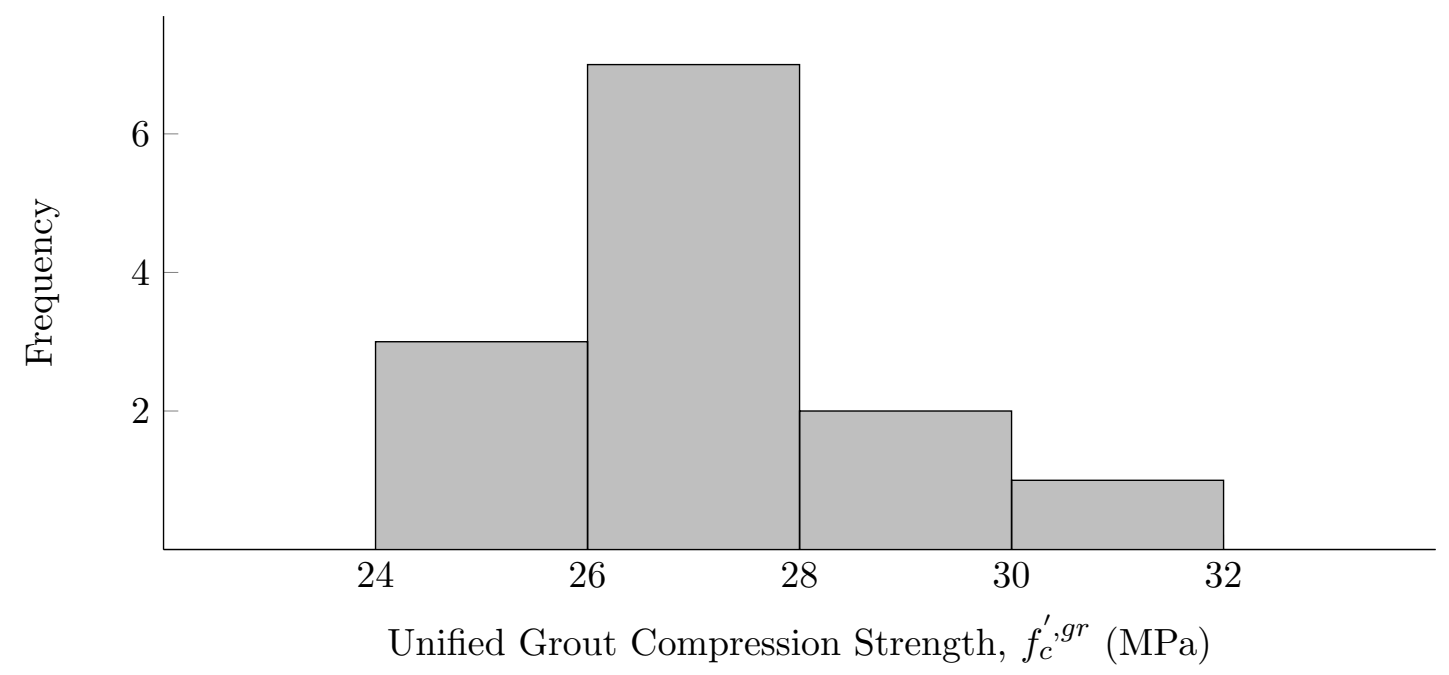

Figure 15.5: Grouted Beam Grout Compression Strength Histogram 


\subsubsection{Longitudinal Reinforcement Ratio}

The longitudinal reinforcement ratio, $\rho=A_{s} / b d$, for the grouted beam database is shown in Figure 15.6. The longitudinal reinforcement ratio ranged from 0.82 to $2.4 \%$.

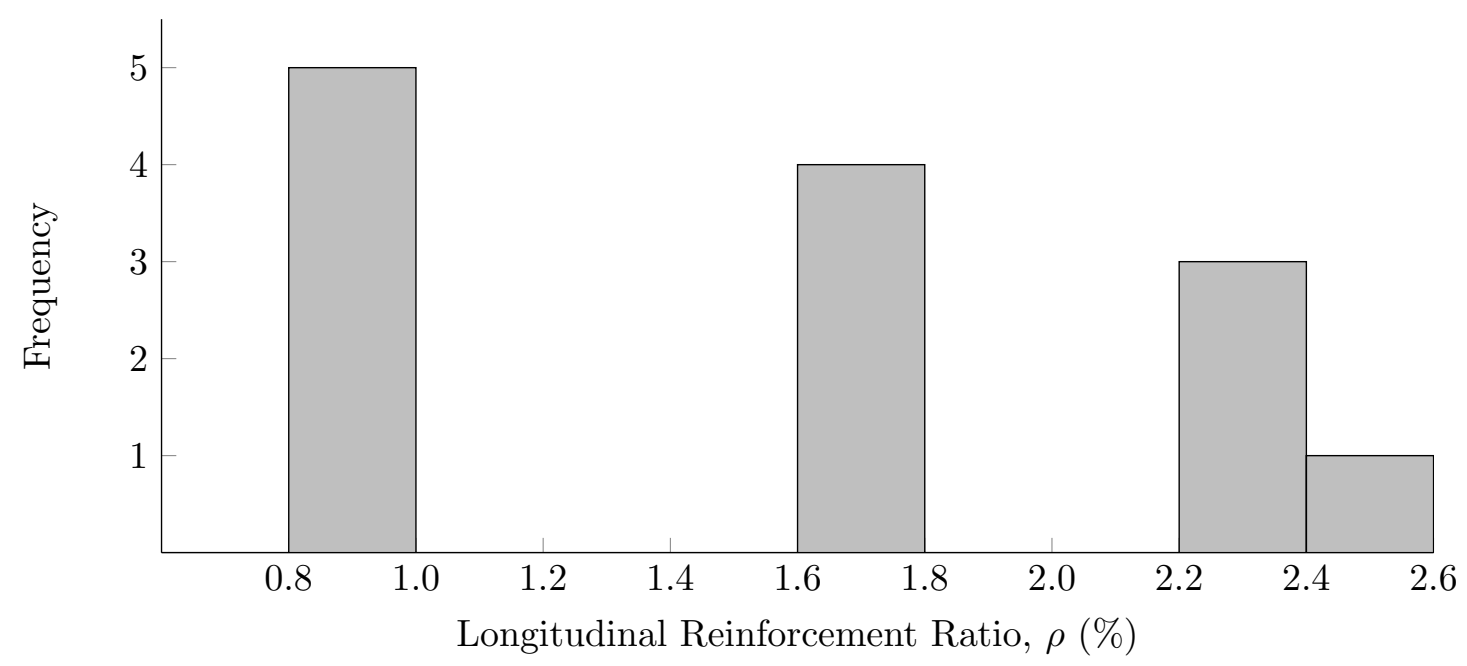

Figure 15.6: Grouted Beam Longitudinal Reinforcement Ratio Histogram

\subsubsection{Shear Reinforcement Ratio}

The shear reinforcement ratio, $\rho_{v}=A_{v} / b s$, for the grouted beam database is shown in Figure 15.7 and ranges from 0 to $0.26 \%$.

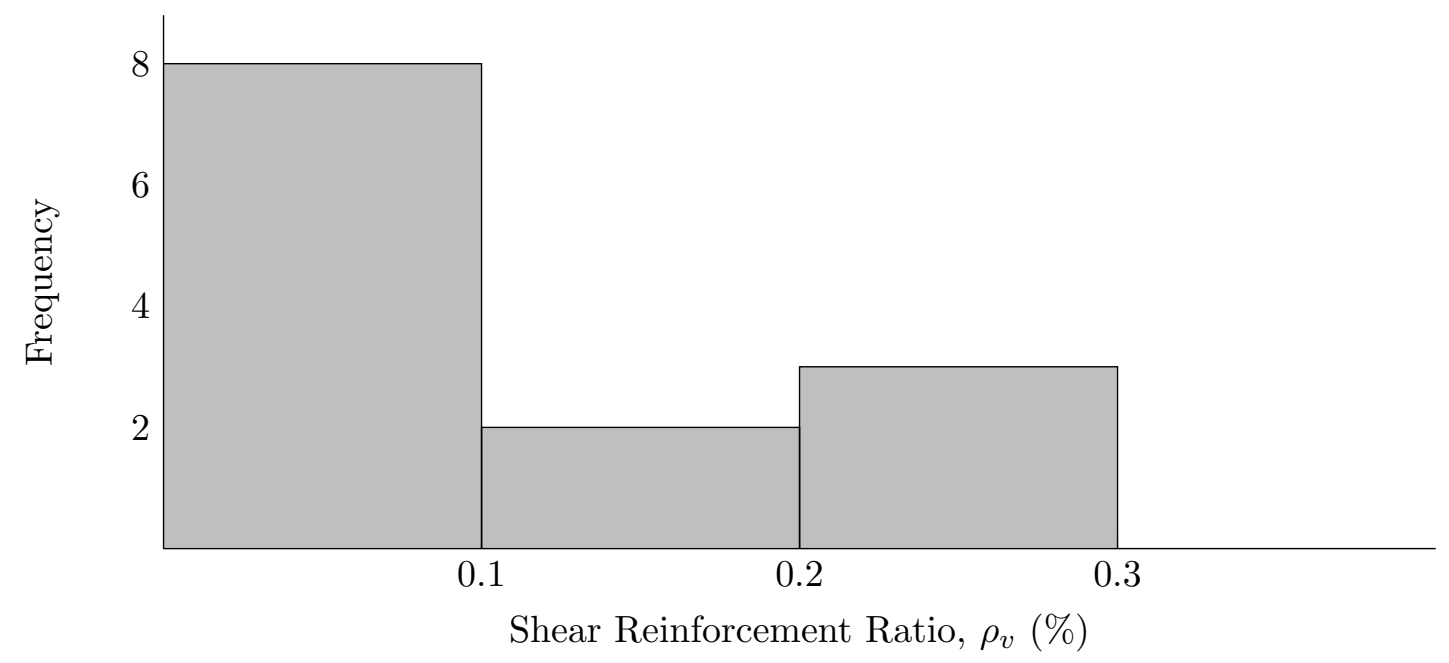

Figure 15.7: Grouted Beam Shear Reinforcement Ratio Histogram 


\subsubsection{Shear Span-to-Depth Ratio}

The shear span-to-depth ratio, $a / d$, of the grouted beam database is shown in Figure 15.8. The beams in the database are all considered slender beams with a span-todepth ratio between 3.02 to 4.50 .

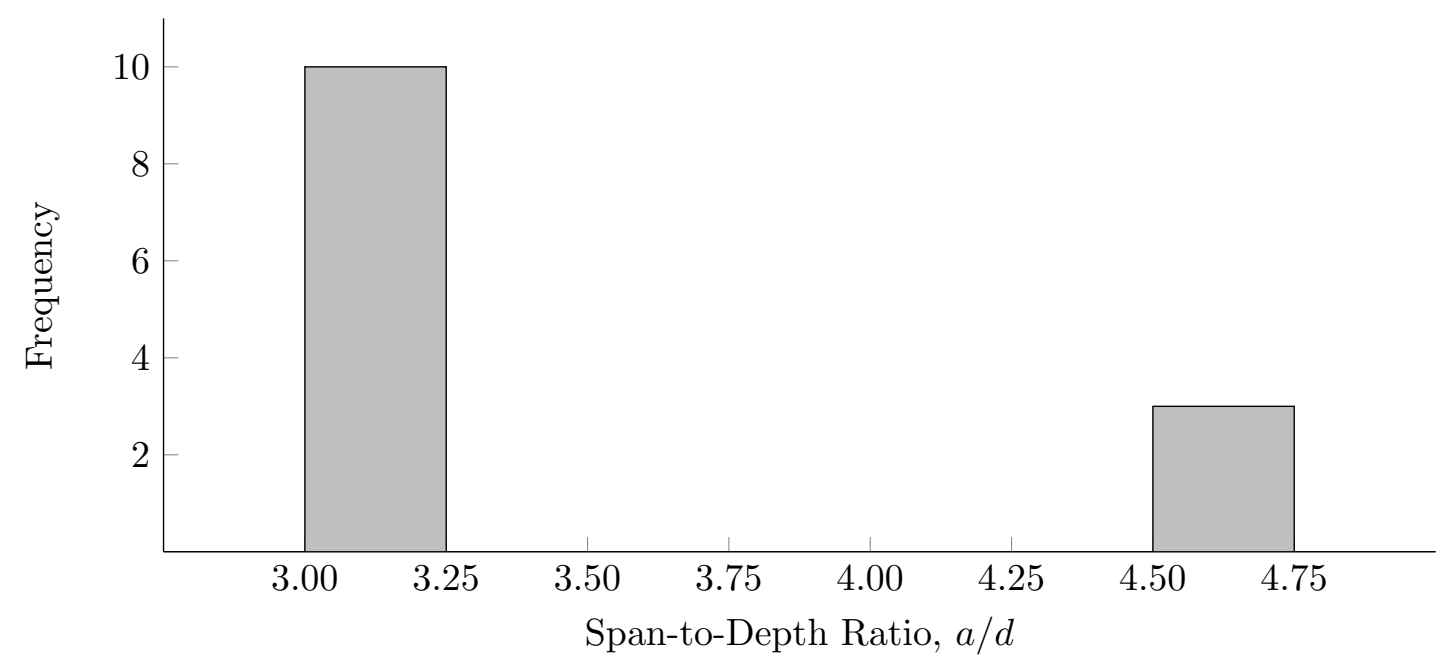

Figure 15.8: Grouted Beam Shear Span-to-Depth Ratio Histogram

\subsection{SAFEM Results}

The SAFEM was used to predict the response of the 13 grouted beams from the database, with each response shown in Figure 15.9 with more details provided in Appendix F. The SAFEM produces correct results across the entire database in terms of stiffness, peak load, and displacement.

The SAFEM results are produced by smearing the longitudinal reinforcement over an area of $\left(h-d+7 d_{b}\right) w$ along the bottom of the beams (Hrynyk, 2013) where $h$ is the height of the beam, $d$ is the effective depth, $d_{b}$ is the diameter of the longitudinal bars, and $w$ is the width of the beam. 

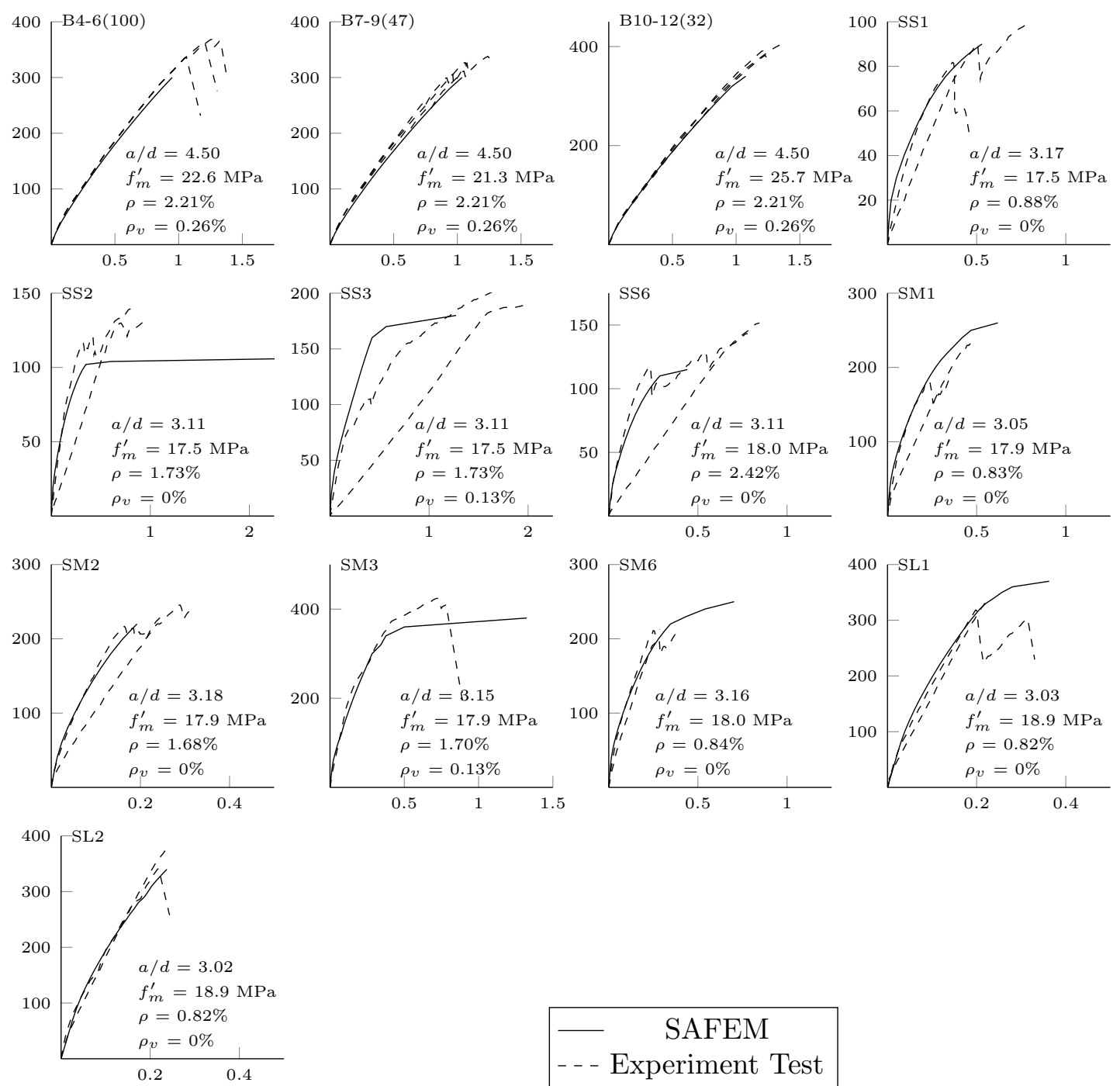

Figure 15.9: Load $(P, \mathrm{kN})$ Verses Relative Mid-Span Deflection $(\delta=\Delta / a, \%)$ Response of Grouted Beams; Experimental and the SAFEM Predictions 


\subsection{Discussion}

Similar to the discussion in Section 13.5 the same proposed equations developed for grouted shear walls can be used to predict the grouted beam values. The following discussion examines the SAFEM inputs used to produce the results presented in Figure 15.9 and Appendix F. This discussion will focus on verifying the SAFEM default values, as well as proposing equations to estimate required SAFEM inputs.

\subsubsection{Masonry Strain at Peak Strength}

The masonry strain's at peak strength, $\epsilon_{o}$, used in the SAFEM to produce the results presented in Figure 15.9 are plotted in Figure 15.10. Neither the equation proposed by Wong et al. (2013) for normal strength concrete or the equation presented in Subsection 13.5.1 accurate predict the strains; though, the equation presented in Subsection 13.5.1 closely predicts the strains for the Ring (2009) tests.

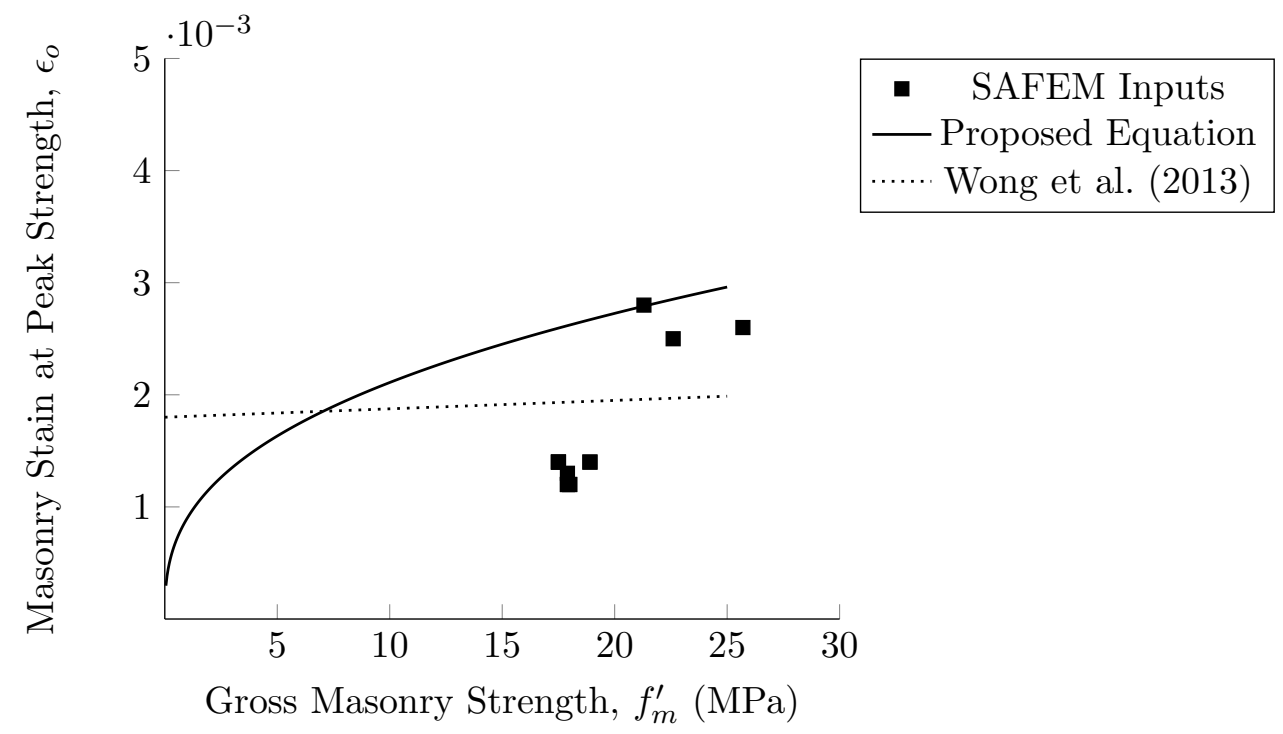

Figure 15.10: SAFEM Inputs for Grouted Beam Masonry Strains 


\subsubsection{Modulus of Elasticity for Masonry}

The masonry modulus of elasticity for the database of beam tests presented in Appendix $\mathrm{F}$ are shown in Figure 15.11. These modulus of elasticities are all within the range proposed by Hamid et al. (1987) and are predicted by the proposed equation given in Subsection 13.5.2 for grouted walls.

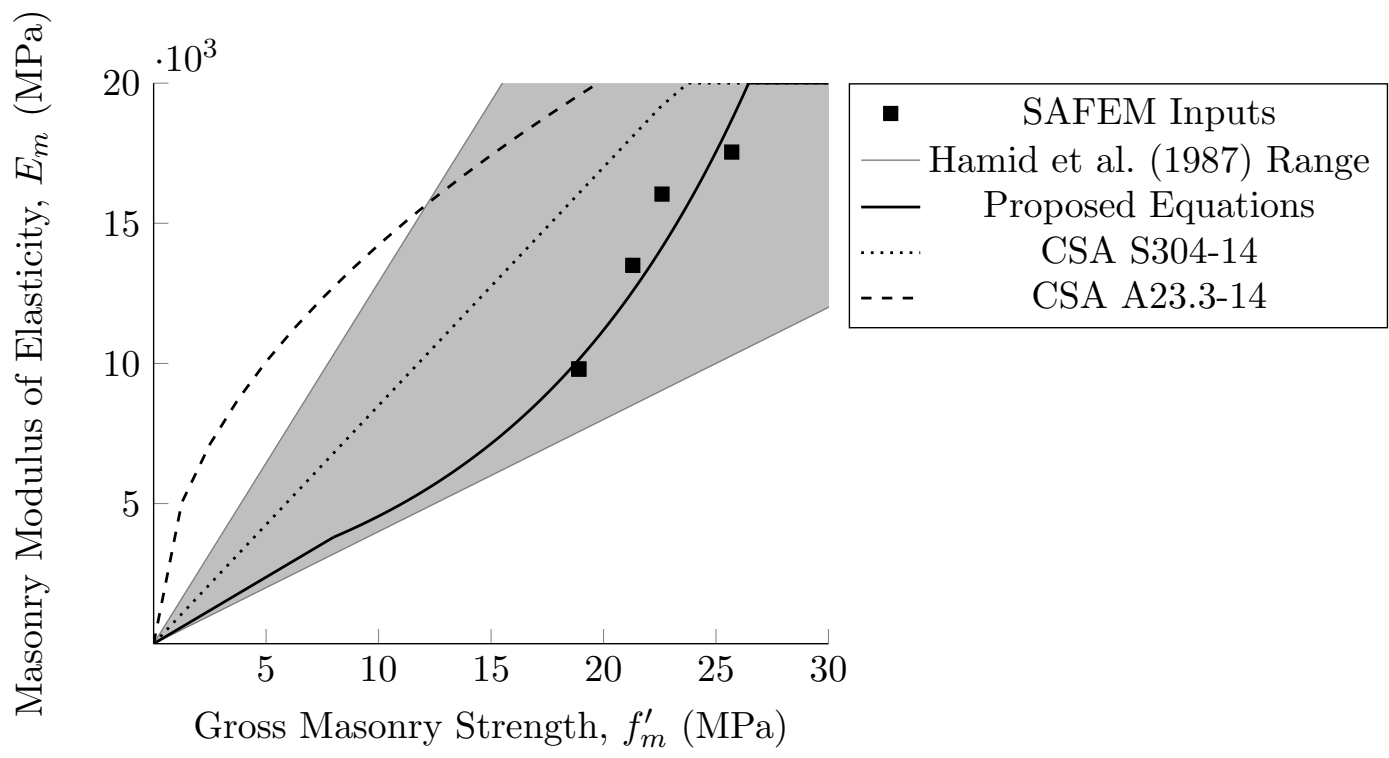

Figure 15.11: SAFEM Inputs for Grouted Beam Masonry Modulus of Elasticity

\subsubsection{Initial Masonry Poisson's Ratio}

The initial masonry Poisson's ratios used as inputs in the SAFEM are shown in Figure 15.12. As can be seen the Poisson's ratio is constant at 0.20, the SAFEM default value, which is a typical value for masonry (Ip, 1999). 


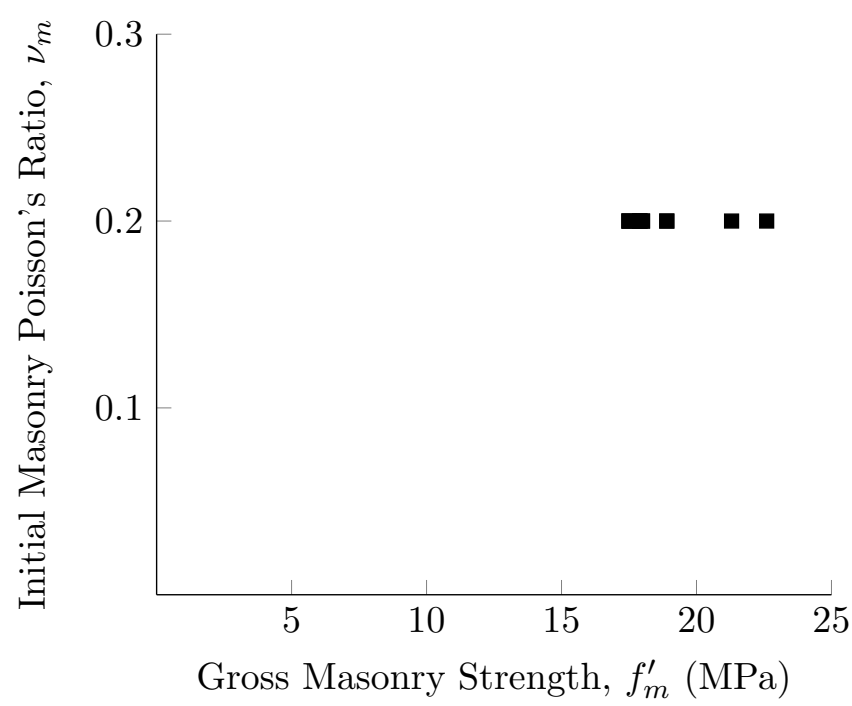

Figure 15.12: SAFEM Inputs for Grouted Beam Initial Masonry Poisson's Ratio

\subsubsection{Block Tensile Strength}

The block tensile strengths used to produce the results presented in Figure 15.9 are plotted in Figure 15.13 and are accurately predicted by:

$$
f_{t, b l}=0.33 \sqrt{f_{c}^{\prime}, b l}
$$

the same equation used to predict the block tensile strength in both the grouted and ungrouted shear walls.

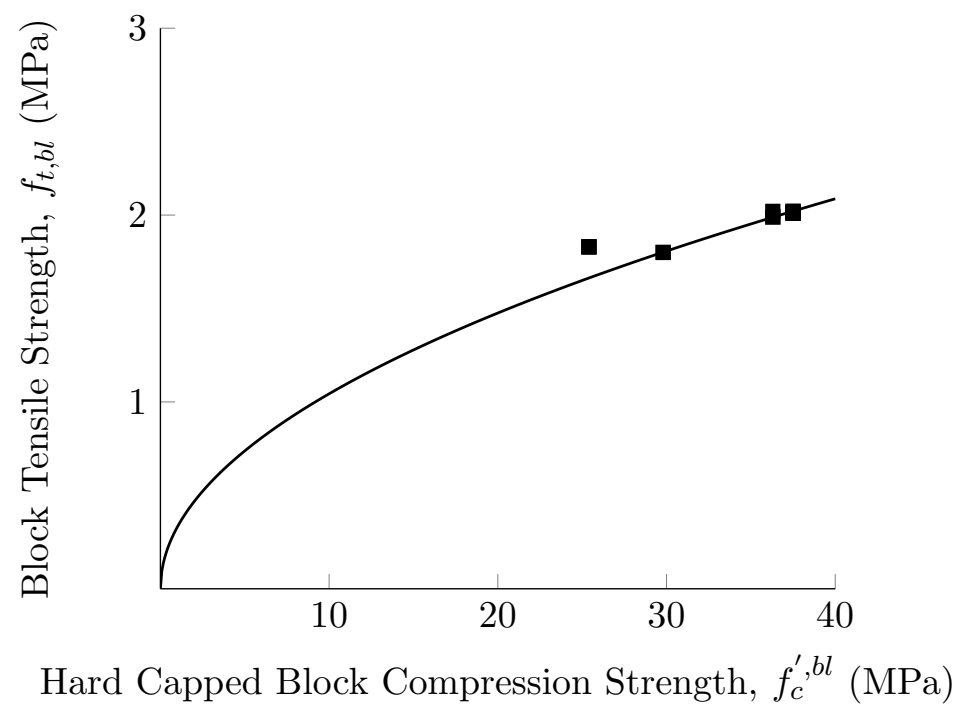

Figure 15.13: SAFEM Inputs for Grouted Beam Block Tensile Strength 


\subsubsection{Mortar Bond Strength}

Figure 15.14 shows the mortar bond strength compared to the uniformed mortar compression strength, $f_{c}^{\prime}, m r$, used to produce the results shown in Figure 15.9. Mortar compression strength are unified to absorbent cube strength as discussed in subsection 3.9.2. The bond strength to the hollow concrete block can be predicted from the following the following equation:

$$
f_{\text {bond }, m r}=0.0802\left(f_{c}^{\prime}, m r\right)^{0.4387}
$$

the same equation used for grouted walls.

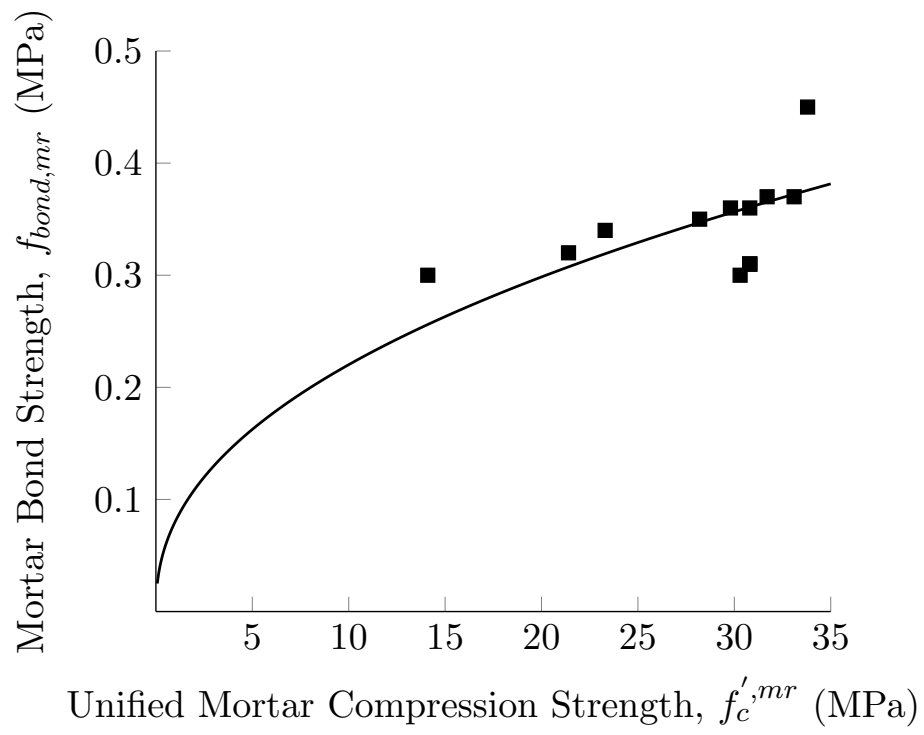

Figure 15.14: SAFEM Inputs for Grouted Beam Mortar Bond Strength

\subsubsection{Mortar Cohesion Strength}

Similar to the grouted walls the mortar cohesion for reinforced hollow concrete masonry beams can be estimated by:

$$
c_{m r}=0.223 f_{c}^{\prime}, m r
$$

This equation and the mortar cohesion strengths, used as inputs to produce the structural responses shown in Figure 15.9, are plotted in Figure 15.15. 


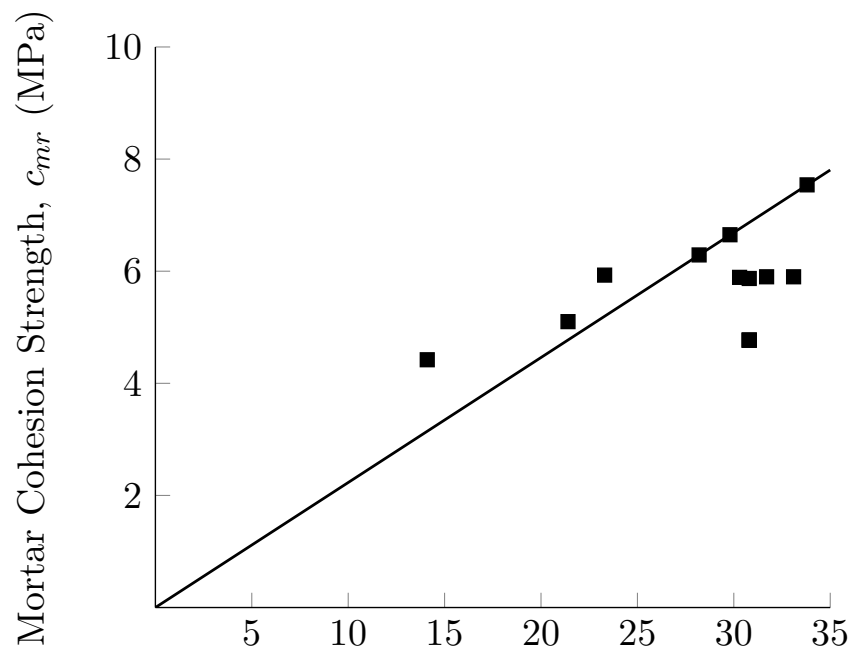

Unified Mortar Compression Strength, $f_{c}^{\prime}, m r(\mathrm{MPa})$

Figure 15.15: SAFEM Inputs for Grouted Beam Mortar Cohesion Strength

\subsubsection{Grout Tensile Strength}

The grout tensile strengths used to produce the results presented in Figure 15.9 and Appendix F are plotted in Figure 15.16. The grout tensile strength can be predicted using the unified grout compression strength (see subsection 3.9.3) and a typical equation used to predict the tensile strength of concrete, i.e.

$$
f_{t, g r}=0.33 \sqrt{f_{c}^{\prime}, g r} .
$$

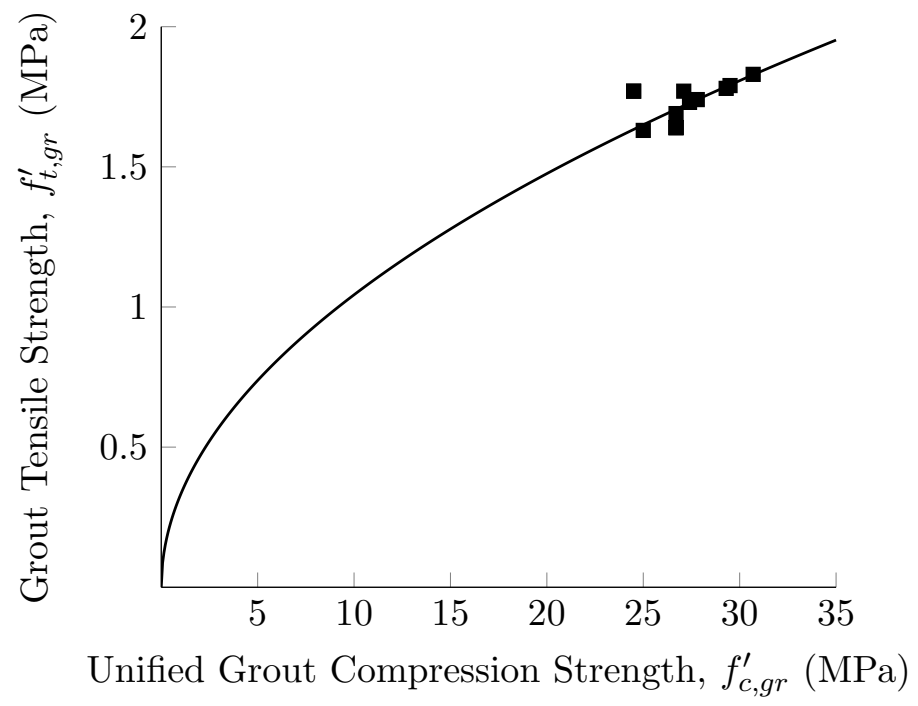

Figure 15.16: SAFEM Inputs for Grouted Beam Grout Tensile Strength 


\subsubsection{Block Web Interruption}

Ring (2009) studied the effect of block web interruption on masonry compressive strength parallel to the bed joint by using the beam's ultimate moment capacity and employing an equivalent stress block method. No clear trend between web interruption and compressive strength parallel to the bed joint was found from the beam tests. Samy et al. (2015) also concluded that the $\chi$ factor used in the current Canadian standard CSA S304-14 (Canadian Standards Association, 2014a) to account for differences in masonry compressive strength measured normal and parallel to the head joint and for web interruption should be set equal to 1.0 for grouted masonry beams; thus, removing any differences in loading direction. The SAFEM agrees with these studies, as the results presented in Figure 15.9 were determined with $f_{m x}^{\prime}=f_{m y}^{\prime}=f_{m}^{\prime}$, i.e. $\chi=1$.

\subsubsection{Size Effect}

The size effect is a phenomenon in which the shear stress at failure of concrete or grouted masonry beams decreases as the effective depth increases with all other properties remaining the same. Numerous experimental investigations have been conducted on the size effect in concrete beams (Collins and Kuchma, 1999; Ghannoum, 1998; Kani, 1967; ShenCao, 2001; Sherwood, 2008; Yoshida, 2000) with Sarhat (2016) recently completing an investigation confirming the size effect in grouted masonry beams.

The existence of the size effect in reinforced masonry can be investigated by studying specimens SS1, SM1, SL1 and SL2. These beams have similar values of $a / d, \rho$ and $f_{m}^{\prime}$, but they have different depths. To show the size effect in reinforced masonry at different reinforcement ratios, a comparison of SS2 with SM2 can be made.

Figure 15.9 shows that both the large (SL1 and SL2) and the small (SS1) specimens exhibited very brittle behaviour, though the large specimens were more brittle than the small specimen. The relative displacements at failure in large specimens were less than half of those in the small specimen. Specimen SM2 failed at a deflection of $1 / 830$ th of the span compared to specimen SS2 which failed at a deflection of $1 / 246$ th of the span. The SAFEM is able to capture the effect that as the effective depth of masonry beams without stirrups increases, the shear failures became more brittle. Additionally, the SAFEM captures the size effect of decreasing shear stress at failure with increasing effective depth. 


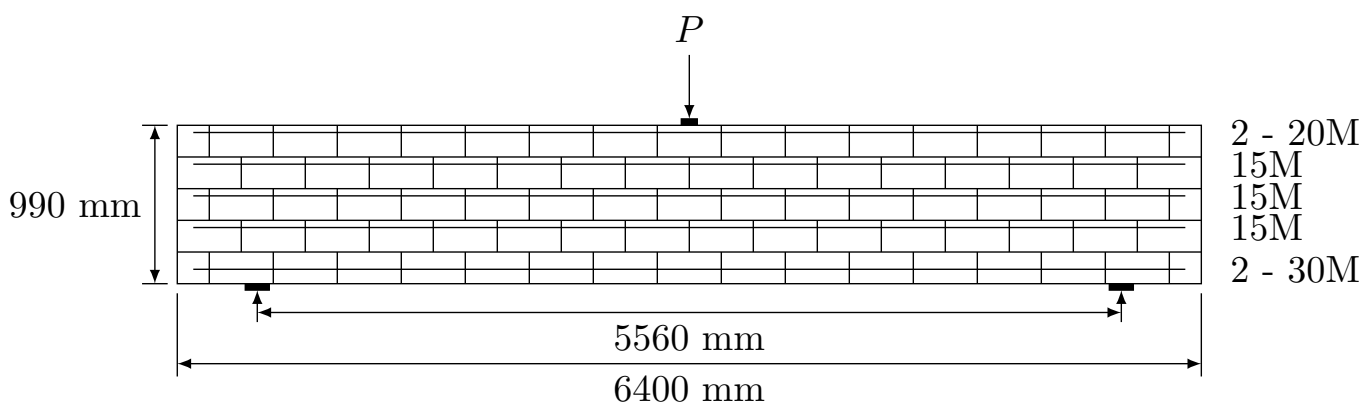

Figure 15.17: SM6 Experimental Beam Sarhat (2016)

\subsubsection{Effect of Stirrups}

The presence of stirrups provides an additional vertical force, $V_{S}$, which contributes to the shear resistance in addition to the contribution provided by the masonry. In designing beams with stirrups, it is common to assume that stirrups are yielding at the crack and that the stirrups do not prevent the formation of diagonal cracks, but rather become effective after the formation of the diagonal cracks.

Figure 15.9 shows that SM3, with a shear reinforcement ratio of $1.70 \%$, exhibited more ductility than SM2, with no shear reinforcement. Furthermore, the relative displacement of SM3 was 3 times greater than that of SM2. The SAFEM results agree with the experimental results of Sarhat (2016).

\subsubsection{Effect of Crack Control Reinforcement}

It has been shown that the use of horizontally distributed steel (also known as crack control reinforcement) along the height of reinforced concrete beams increases the shear strength of these beams (Collins and Kuchma, 1999; Sherwood, 2008). It has also been shown that if this steel is provided in a sufficiently large quantity that the failure shear stress is related to the vertical spacing between the layers of steel, rather than the effective depth. Specimen SM6 of Sarhat (2016) is reinforced with a single No. 15 bar in every course (200 $\mathrm{mm}$ between the layers) from the second to the forth course (see Figure 15.17). The presence of crack control steel had a major effect on shear strength allowing for larger shear stress to be carried, smaller deflections, smaller crack spacing, and narrower cracks. These results represent the first experimental evidence showing that crack control reinforcement is beneficial in masonry. The SAFEM was able to reproduce these results. 


\subsubsection{Strain Effect}

Actions that reduce aggregate interlock will decrease shear strength. These actions can include:

1. Axial tension;

2. Decreasing the reinforcement ratio;

3. Increasing the ratio of moment to shear at the section; or,

4. The use of lower stiffness reinforcement.

Similarly, prestressing and axial compression will lead to narrower cracks, higher aggregate interlock capacity and enhanced shear strengths. These actions are collectively termed the "strain effect" as each affects the longitudinal strain at the section.

\section{Effect of Longitudinal Reinforcement Ratio}

In this section two series of beams will be utilized to study the effect of main reinforcement ratio on shear strength of reinforced masonry beams. The first series consists of three two-course beams (SS1, SS2 and SS6). These beams are approximately identical (average values of $d=300 \mathrm{~mm}, a / d=3.1$ and $f_{m}^{\prime}=17.9 \mathrm{MPa}$ ) except for the main reinforcement ratio that ranges from $0.88 \%$ to $2.4 \%$. The second series encompasses two five-course beams (SM1 and SM2). These beams have approximately constant values of $d=880 \mathrm{~mm}, a / d=3.1$ and $f_{m}^{\prime}=17.9 \mathrm{MPa}$, but with different main reinforcement ratios that ranges from $0.83 \%$ to $1.68 \%$.

Sarhat (2016) test results showed that the shear strength increased considerably as the reinforcement ratio increased. Figure 15.9 shows that SAFEM is able to accurately predict the strain effect. 


\title{
PART III CONCLUDING REMARKS
}

\begin{abstract}
Part III of this thesis develops a two-dimensional micro-macro finite element model to predicted the structural response of hollow concrete block masonry structures including stress and stains of the materials, deformations, and crack patterns. Results of the model are compared to experiments found in literature. A number of simplified equations are proposed to estimate key hollow concrete block masonry properties. This chapter makes a number of concluding remarks about Part III of this thesis and presents a number of items for future research.
\end{abstract}

Part III of this thesis presents a finite element model for hollow concrete block structural elements that are either ungrouted or grouted and possibly reinforced. The model considers masonry as a composite material based on a smeared area approach, where the block, mortar joints, and possible grout and reinforcement are smeared across the element. Cracks in the composite material are considered smeared and rotate based on the inclination of the net principal strains. The model considers non-linearity through the use of a secant stiffness and accounts for directional properties due to mortar joints acting as planes of weakness. The success of a nonlinear finite element analysis model of a masonry structure depends almost entirely on the material modeling; thus, the model includes a number of material constitutive models. The model considers the anisotropic nature of masonry; models for compression pre and post peak; compression softening; models for tension; lateral expansion; inclusion of slip along the cracks and mortar joints; and a steel reinforcement stress-strain response. The models are implemented through a two-dimensional eight node isoparametric quadrilateral elements in the smeared area finite element model.

The finite element model is programmed in $\mathrm{C}++$, using the Microsoft Foundation Class Library for the graphical user interface, and the Eigen Library (Eigen) for matrix manipulation. The programming and constitutive models are verified through the examination of stiffness matrices, comparing the results to known analytical results, and comparing the results to similar finite element programs.

Three databases were compiled to compare experimental and finite element results. In total 62 single pier grouted shear walls, 14 ungrouted single pier shear walls, and 
13 reinforced masonry beams were examined. These databases cover a wide range of material and structural properties including:

- Masonry compression strengths ranging from $12 \mathrm{MPa}$ to $24 \mathrm{MPa}$ for the grouted shear wall database, 4.32 $\mathrm{MPa}$ to $7.05 \mathrm{MPa}$ in the $y$-direction and 4.10 $\mathrm{MPa}$ to $5.65 \mathrm{MPa}$ in the $x$-direction for the ungrouted shear wall database, and $17.5 \mathrm{MPa}$ to $25.7 \mathrm{MPa}$ for the reinforced masonry beams.

- Mortar compression strength based on absorbent cube strength ranged from 16 to 52.2 MPa for all three sets of databases. The absorbent cube strength attempts to represent the in-situ of the mortar as some of the water is absorbed by the block when it is placed.

- Grout compression strength based on absorbent cylinder strength, to represent the in-situ strength of the grout, ranged from 17.7 to $40.1 \mathrm{MPa}$. This is the typical range of strengths of grout used in the construction of grouted masonry.

- Vertical wall reinforcement within the grouted walls ranged from 0 to $0.97 \%$.

- Horizontal wall reinforcement within the grouted walls ranged from 0 to $1.02 \%$.

- Wall aspect ratio, $a / l_{w}$, ranged from 0.68 to $2.11 \%$ for grouted walls and 0.78 to $1.50 \%$ for ungrouted walls.

- Wall axial load ratio, $N /\left(t l_{w} f_{m}^{\prime}\right)$, ranged from 0 to 0.157 for grouted walls. For the ungrouted walls the axial load varied between 0.69 and $2.75 \mathrm{MPa}$ (based on net area).

- Beam longitudinal reinforcement, $A_{s} / b d$, ranged from 0.82 to $2.4 \%$.

- Beam shear reinforcement, $A_{v} / b s$, ranged from 0 to $0.26 \%$.

- Beam shear span-to-depth ratio, $a / d$, ranged from 3.02 to 4.50 ; thus, the beams in the database are considered slender beams.

\subsection{Part III Conclusions}

The Smeared Area Finite Element Model is capable of predicting the response of ungrouted, grouted, and reinforced masonry elements subject to in-plane shear and axial stresses by considering equilibrium conditions, compatibility requirements, and material constitutive relationships.

Part III: Masonry Finite Element Modeling 
The following statements can be made about Part III of this thesis:

1. The Smeared Area Finite Element Model considers average stresses and average strains, with particular attention given to compression softening, tension stiffening, and tension softening mechanics.

2. Crack shear slip, mortar joint slip, and Poisson's effect are handled using a prestrain nodal force.

3. The finite element code is verified through a series of element, numerical, and finite element comparison tests.

4. The homogenized method presented in the Smeared Area Finite Element Model permits the simplified treatment of hollow concrete block masonry.

5. The treatment of the geometry through structured meshes with quadrilateral elements reflects the real arrangement of joints and blocks in masonry. This type of mesh leads to simple mesh generation.

6. The micro-macro modelling technique considered by the Smeared Area Finite Element Model reduces the process of mesh generation and computation time.

7. Disturbances in the stress fields caused by cracks and mortar joints can influence hollow concrete masonry behaviour.

8. The finite element model presented is capable of capturing the failure even when it is governed at the mortar level.

9. In ungrouted masonry a lag-based approach for $10^{\circ}$ allows for crack slip to be determined.

10. The Smeared Area Finite Element Model is programmed with a graphical user interface to allow easy entry of model inputs, mesh generation, boundary conditions, and applied loads. This helps reduce the possibility of input errors.

11. The Smeared Area Finite Element Model is verified against 62 single pier grouted and reinforced shear walls, 14 ungrouted single pier shear walls, and 13 reinforced hollow concrete beams and is shown to have good correlation in terms of loaddisplacement response and crack patterns.

12. The Smeared Area Finite Element Model can determine the total stresses and strains acting on an element, as well as the net stresses and strains acting in the masonry and the reinforcement. Additional the model can estimate the shear acting along the crack of reinforced elements.

13. The Smeared Area Finite Element Model allows for the expansion of the disturbed stress field model for the analysis of ungrouted, grouted, and reinforced hollow concrete block masonry. 
In addition, a number of simplified equations were developed to predict masonry material properties for use in the finite element model and international codes. These equations allow for an estimation of the important masonry properties including the:

1. Grouted masonry modulus of elasticity:

$$
E_{m}= \begin{cases}475 f_{m}^{\prime} & \text { if } f_{m}^{\prime} \leq 8 \mathrm{MPa} \\ \min \left(1850 e^{0.09 f_{m}^{\prime}}, 20000\right) & \text { otherwise }\end{cases}
$$

where $f_{m}^{\prime}$ is based on gross area.

2. Ungrouted masonry modulus of elasticity:

$$
E_{m}=\frac{0.4 f_{c}^{\prime}, m}{\left(1-\sqrt{\frac{3}{5}}\right) \epsilon_{o}^{m}} .
$$

where $f_{m}^{\prime}$ is based on gross area.

3. Masonry Poisson's ratio:

$$
\nu_{m}=0.2
$$

4. Block tensile strength:

$$
f_{t, b l}=0.33 \sqrt{f_{c}^{\prime}, b l}
$$

where $f_{c}^{\prime}, b l$ is based on hard capping of the block.

5. Mortar bond strength for grouted masonry:

$$
f_{\text {bond }, m r}=0.0802\left(f_{c}^{\prime}, m r\right)^{0.4387}
$$

where $f_{c}^{\prime}, m r$ is determined from the absorbent cube strength.

6. Mortar cohesion for grouted masonry:

$$
c_{m r}=0.223 f_{c}^{\prime}{ }_{c} m r
$$

where $f_{c}^{\prime}, m r$ is determined from the absorbent cube strength.

7. Grout tensile strength:

$$
f_{t, g r}=0.33 \sqrt{f_{c}^{\prime}, g r}
$$

where $f_{c}^{\prime}, g r$ is determined from absorbent cylinder strength.

\subsection{Part III Future Research}

Part III of this thesis explained in depth the Smeared Area Finite Element Model and compared its results to that of 89 structural members found in literature with accurate results; however, there are a number of additions that can be made to both the finite element model and the constitutive models.

Part III: Masonry Finite Element Modeling 
To begin the model needs to be extended to include displacement based modeling which will allow for postpeak behaviour of the modeled elements to be determined. This also leads to including cyclic loading or time series loading from an earthquake. This will allow for the response of masonry structural elements to be analysed under an earthquake. Additional loading could also be added due to thermal loading from temperature change or a fire. Furthermore, prestressing effect can also be incorporated into the model to allow for prestressed or poststressed masonry to be studied. The model also requires the option of adding additional materials such as concrete, steel, prestressing wires, and fiber reinforced polymers. The model also can be extended to include truss elements for reinforcing and springs to model slip of the reinforcement relative to the masonry.

To assist in processing the finite element model output a post processor needs to be developed. This post processor would be able to visually display the output data so users would be able to easily interpret results of the model.

Further experimental tests are necessary with a specific concentration on factors that affect the constitutive models, such as mortar joint and crack slippage. Additionally, the effects of masonry confinement and reinforcement dowel action need to be examined. Also, tests of larger structures are required to ensure the model can predict the crack patterns around door and window opens that are common in concrete block walls. Additional experimental tests on ungrouted masonry walls will allow for a larger database to be collected and the model results compared to the experimental results allowing for more accurate simplified equations to be determined for important masonry properties. An additional database of partially grouted shear walls need to be created examining how the model predicts the structural response if such walls.

Finally, the theory presented in Part III of this thesis is for two-dimensional finite element modeling, but with further work on constitutive models and experimental tests the theories should be able to be transformed for three-dimensional analysis of full masonry buildings.

Part III: Masonry Finite Element Modeling 


\section{PART IV}

\section{MASONRY DISPLACEMENT BASED MODELING}


Many unreinforced and reinforced masonry wall structures were built before the introduction of modern seismic design guidelines and as a result feature smaller lateral resistance than today's seismic codes require. Many of these structures also do not meet the current capacity design requirements and are susceptible to shear failures that limit their deformation capacity. To determine if a retrofit is required to improve the lateral resistance or ductility, the seismic behaviour of the structure must be assessed using methods that account for all deformations of the wall. This assessment, may be done according to either a force-based or a displacement-based approach. In recent years, there is a strong tendency towards displacement-based approaches, as these often prove to be the more economical, especially for the assessment of existing structures. While the force-based approach may almost inevitably lead to the conclusion that costly retrofitting measures are necessary to increase the resistance of a structure, the displacement-based approach may lead to the conclusion that the deformation capacity of a structure is actually sufficient.

In the last decade, a number of seismic design approaches have been developed whose goal is to achieve a specified local deformation level under design earthquakes. These approaches provide more rational design methods than current force-based methods, because damage can be directly related to local inelastic deformations. For this reason, designing structures to achieve a specified global displacement limit or specified local deformation limit implies designing for a specified risk of damage (Priestley et al., 2007). 


\title{
BACKGROUND
}

\begin{abstract}
A background of hollow concrete block masonry shear wall failure modes and various displacement based modelling techniques is presented. The goal of Part IV of this thesis is to develop a model that will predict the full loaddisplacement response of reinforced hollow concrete block masonry shear walls in a timely manner.
\end{abstract}

Early investigations of masonry shear walls started by the late 1950's (Benjamin and Williams, 1958), but it was not until the beginning of the 1970's that available research facilities enabled studying the seismic behaviour of masonry shear walls under reversed cyclic loading (Scrivener and Williams, 1971). Most of the recent studies on seismic behaviour of masonry shear walls include the effect of wall aspect ratio, amount of horizontal and vertical reinforcement, and level of applied axial load. Knowledge of the behavior of shear walls has increased significantly due to these studies.

\subsection{Shear Wall Failure Modes}

The increased use of masonry shear walls has brought about numerous experimental investigations into the performance and failure modes of these walls. Several different failure mechanisms have been identified under in-plane shear loading, including flexural, shear, rocking, and sliding mechanisms. However, if adequate anchorage is provided, shear and flexure failure mechanisms become dominant and are much more likely to control wall behaviour.

In seismic design, a ductile flexural behaviour is generally preferred. Flexural failure is characterized by the tensile yielding of vertical reinforcement, the formation of one or more plastic hinge zones, and crushing of masonry at critical wall sections (Paulay and Priestley, 1992; Shedid et al., 2008; Shing et al., 1991). This is followed by block crushing, face shell spalling, grout crumbling, and buckling of vertical reinforcements at the ends of the wall. Research has indicated that flexural theory and the assumption that plane sections remain plane is a satisfactory representation of walls dominated by flexural behaviour (Shing et al., 1990). The ductility of a flexural failure provides greater energy dissipation, due to yielding of reinforcement. However, brittle failures can occur, 
if large amounts of flexural reinforcement are used as the flexural reinforcement might not yield prior to the extreme masonry compression fiber reaching its critical strain (Eikanas, 2003). As a result, it is important to limit the amount of flexural reinforcement to ensure proper ductility in masonry walls controlled by flexural behaviour.

Shear failure is undesirable in seismic events, as failure or collapse of structures without adequate warning becomes much more likely. A shear failure is characterized by diagonal tensile cracking or shear slip along bed joints. Diagonal tensile cracking happens when the principal diagonal stress exceeds the masonry tensile strength (Voon, 2007). The diagonal cracking strength is mainly dependent on the level of axial stress, the strength of the masonry, and the aspect ratio of the wall. Before diagonal cracking begins, horizontal and vertical reinforcement in the wall carries little load. Shear strength after cracking, however, is dependent upon the horizontal steel, aggregate interlock, the uncracked masonry zone at the compression toe of the wall, and dowel action of the vertical steel (Shing et al., 1990). The ductility of a wall controlled by a shear failure can be enhanced if sufficient amounts of horizontal reinforcement are used and the reinforcement is appropriately anchored (Shing et al., 1990).

Failures due to sliding are characterized by a sliding plane along either a continuous horizontal flexural crack or between two diagonal cracks. A lack of friction between the wall base and foundation also contributes to sliding. Under in-plane loading, the slip plane is typically found at the base of the wall due to the non-continuous construction between the footing and the wall base. Sliding is generally initiated by flexural reinforcement yielding along the wall base joint. Large displacements initiate dowel action from the flexural reinforcement and a significant clamping force is developed. Sliding resistance is enhanced as the clamping force increases friction and aggregate interlock forces become effective. Adequate amounts of uniformly distributed flexural reinforcement provide dowel action and a clamping force and can effectively eliminate sliding behavior (Priestley, 1986).

The wall aspect ratio, quantity and distribution of horizontal and vertical reinforcement, and magnitude of axial load all factor into the type of failure mechanism that will dominate. In low aspect ratio walls, shear failure is much more likely to govern behavior. The flexural mechanism is more likely to control failure in high aspect ratio walls (Shedid, 2006).

Part IV: Masonry Displacement Based Modeling 


\subsubsection{Studies of Shear Dominated Masonry Wall Behaviour}

The first sign of shear failure under lateral loading occurs as a 45 degree diagonal crack starts to form and spreads from the center of the wall. The diagonal cracking load is dependent on the tensile strength of the masonry and of the orientation of stresses within the wall. This can be observed by using Mohrs circle to represent the biaxial stress pattern within the wall (Mayes et al., 1976). Axial load also increases the aggregate interlock force, increasing the mechanical bond resistance and prolonging the breaking of the chemical bonds in the cementitious material (Ghanem et al., 1992, 1993; Shing et al., 1990). Since the reinforcement is not engaged until the masonry cracks, it is not effective in increasing the cracking strength of the wall panel (Ghanem et al., 1992, 1993; Haach et al., 2010; Matsumura, 1987; Schultz et al., 1998).

Once initial diagonal cracking has taken place within the wall, the horizontal reinforcement begins resisting the lateral forces and helps to keep the crack from opening. Though not directly operative in resisting the lateral load, it has been shown that distributed vertical reinforcement is also effective in keeping the shear cracks from opening (Ghanem et al., 1992). By keeping the diagonal crack closed, the masonry along both sides of the crack are able to transfer shear stresses via aggregate interlock, resulting in an increased lateral capacity beyond the diagonal cracking load. Without adequate confinement, the diagonal crack will open and the masonry will no longer provide resistance to the lateral load.

Shear failure initiates in one of two ways. A brittle shear failure occurs in walls that are heavily reinforced or which are subjected to large axial loads (Ghanem et al., 1992). Brittle failure occur with a sudden crushing of the masonry between adjacent horizontal reinforcement. A more ductile shear failure occurs in walls that are lightly reinforced with distributed reinforcement and subjected to axial load less than 5 percent of the compressive strength (Ghanem et al., 1992). Ductile failure initiates as yielding of the horizontal reinforcement and is followed by sliding along the cracked planes and ultimately by crushing of the masonry.

Shing et al. (1989) indicated that the applied axial load significantly increases aggregate interlock which in turns increases the shear resistance of the wall. It was also indicated that a shear wall with an increased amount of vertical or horizontal reinforcement exhibits better ductility and energy dissipation capabilities. It was concluded that reinforced masonry shear walls can exhibit a certain extent of ductility and energy dissipation

Part IV: Masonry Displacement Based Modeling 
capabilities and are suitable for seismic resistance provided that proper reinforcement guidelines are followed.

Shing et al. (1990) studied the sequence of major diagonal crack formation with a monotonic test on a shear wall with an aspect ratio of one, where horizontal steel was instrumented with strain gauges in the vicinity of the diagonal crack. With the horizontal steel in compression, a diagonal strut was formed by two diagonal cracks. After the formation of the third major crack, extending from one comer of the square wall to the other comer along the main diagonal, the strut is no longer effective and at this stage the shear resistance is taken by the residual strength of masonry and by the horizontal steel that is subjected to high tension in the vicinity of the crack. Shing et al. (1990) concluded that the ductility of shear-dominated walls is relatively low unless a large amount of horizontal reinforcement is introduced.

Sucuoglu, H. and McNiven, H. (1991) studied the effect of the amount of horizontal reinforcement on the behaviour of reinforced masonry walls having an aspect ratio of one. The amount of horizontal steel had a significant influence on the hysteretic shear behaviour while the distribution of vertical reinforcement had a negligible effect. The horizontal reinforcement was effective in reducing the opening of the diagonal shear cracks. When the horizontal steel yields, a ductile shear failure can be obtained where diagonal cracks do not open excessively but are distributed evenly over the wall height. On the other hand, higher amounts of horizontal steel restrain the post-cracking deformation capacity, because the steel remains in the elastic range.

The effect of the aspect ratio on shear behaviour of masonry walls was investigated by Brunner and Shing (1996). They tested three squat masonry shear walls, with aspect ratio $\left(h_{w} / l_{w}\right)$ varying from 0.96 to 0.60 ; the walls were heavily vertically reinforced in order to promote shear failure. The tests showed that walls with lower aspect ratios had higher stiffness and reached their maximum resistance at smaller displacements.

Voon (2007) tested ten single storey concrete masonry shear walls to investigate the effects of the amount and distribution of shear reinforcement, level of axial compressive stress, and wall aspect ratio have on the shear strength of masonry walls under inplane loading. Eight walls were $1.8 \mathrm{~m}$ long having an aspect ratio of one, one wall with aspect ratio of two had the same length, and the final wall was $3 \mathrm{~m}$ long with an aspect ratio of 0.6 . The results of the tests indicated a benefit of distributing shear reinforcement throughout the height of the wall, as well as the beneficial effect of axial compressive load on the behaviour of masonry walls. The study also illustrated that 
masonry shear strength increases with a decrease of the aspect ratio. The foregoing conclusions confirmed the previous research work conducted on shear walls.

\subsubsection{Studies of Flexure Dominated Masonry Wall Behaviour}

The flexural mode of failure is characterized by horizontal bed joint cracking in the bottom courses, yielding of the vertical reinforcement, and ultimately by crushing of the masonry at the toe of the wall. Flexure failures have been observed to be more ductile in large part because the failure requires yielding of the reinforcement. The amount that the reinforcement yields before crushing commences in the masonry toe is dependent on the quantity of vertical reinforcement. For large areas of vertical reinforcement, the reinforcement may just reach yield strength or not at all before the masonry begins to crush at the toe of the wall. For walls with small areas of reinforcement, toe crushing is delayed and the reinforcement will undergo large amounts of plastic yielding before toe crushing begins. In the extreme case of plastic yielding, a rocking failure will occur when tested cyclically.

Shing et al. (1989) indicated that the flexural capacity and stiffness of reinforced masonry shear walls increases with increasing axial compression load which may be attributed to the fact that the opening of the flexural cracks is significantly reduced by application of axial load. On the other hand, the increase of flexure capacity and stiffness due to the increase of axial load may not be a desirable factor in seismic resistance design as this was associated with a reduction of the flexural ductility. The delayed yielding of the vertical tensile reinforcement as well as reaching compression failure at lower curvatures tend to reduce the flexural ductility of reinforced masonry shear walls subjected to axial load. Also, higher axial load causes accelerated toe spalling along with the higher tendency of the reinforcing bars to buckle leading to reduce the flexure ductility.

Based on the experimental results of testing reinforced masonry shear walls, having aspect ratio of one, under in-plane loading, Shing et al. (1990) indicated that the strain profile measured at the base of a typical wall tends to be linear. However, for walls subjected to axial load, this profile deviates slightly from the plane section assumption of simple flexure theory. They indicated that this slight deviation will result in only minor redistribution of the tensile stresses in the flexure steel, and therefore will not lead to a significant change of the flexure strength. Shing et al. (1990) indicated that the stiffness of the test walls tended to increase with increasing axial load, whereas, the ultimate curvature and displacements tended to decrease. The plastic hinge length was measured for the flexural dominated walls in this study, having an aspect ratio of one. 
Based on the measured curvature profile over the wall height, the plastic hinge length was estimated to be equal to 0.15 times the wall length.

Cantilever masonry shear walls tend to concentrate large inelastic deformations to the base of the wall, to satisfy displacement demand. In order to idealize the actual curvature profile, Paulay and Priestley (1992) suggested representing it by an elastic region and a plastic region. They considered the plastic rotation to act at mid-height of the equivalent plastic hinge length and presented relationships between curvature ductility, $\mu_{\phi}$, equivalent plastic hinge length, $l_{p}$, and wall height, $h_{w}$ to determine displacement ductility, $\mu_{\Delta}$, as defined by:

$$
\mu_{\Delta}=1+3\left(\mu_{\phi}-1\right) \frac{l_{p}}{h_{w}}\left(1-0.5 \frac{l_{p}}{h_{w}}\right) .
$$

Based on integration of the curvature distribution along the wall height, theoretical values for the equivalent plastic hinge length, $l_{p}$, would be proportional to the wall height. Paulay and Priestley (1992) suggested that the plastic hinge length for reinforced masonry shear walls varies between 0.3 to 0.8 times the wall length. The theoretical values for estimating the plastic hinge length did not match well with experimental measured lengths as described by Paulay and Priestley (1992) and this discrepancy was justified as the elongation of tensile flexural bars into the footing, produces additional rotation and deflection. An estimation of the plastic hinge length was suggested based on the wall height, $h_{w}$, the size of the vertical reinforcement, $d_{b}$, and the yield strength of the vertical reinforcement, $f_{y}$, as:

$$
l_{p}=0.08 h_{w}+0.022 d_{b} f_{y} .
$$

\subsubsection{Shear Span Ratio}

The shear span ratio is a value that has a significant influence on the failure mode of masonry walls (Maleki, 2008; Matsumura, 1987; Schultz, 1996) and is mentioned by nearly every author in the literature either directly or indirectly. The definition of the shear span ratio is the height of the inflection point divided by the horizontal length of the wall. It is frequently represented in the code and literature by:

$$
\frac{M_{u}}{V_{u} l_{w}}
$$

where $M_{u}$ is the ultimate moment demand, $V_{u}$ is the ultimate shear demand, and $l_{w}$ is the length of the wall panel. It is more representative to think of the shear span ratio as being represented by: 


$$
\frac{M_{u}}{V_{u} l_{w}}=\frac{h_{e}}{l_{w}}
$$

where $\frac{M_{u}}{V_{u}}$ and $h_{e}$ are the effective wall height or the height of the inflection point. For walls tested under cantilever-type loading, the effective height $h_{e}$ and the geometric height of the wall are the same. For wall panels tested with both their top and bottom edges fixed against rotation (i.e., fixed-fixed or double-curvature loading), the inflection point is located at the mid-height of the wall and the effective height is half the geometric height. This definition of shear span ratio permits the same equations to be used with walls which have other boundary conditions or loading (e.g., when the top surface is only partially restrained against rotation).

\subsection{Prediction of Nonlinear Response}

Displacement based design calculates a building's response under a specified displacement and allows for the design of a building so that its inelastic deformation capacity equals or exceeds the inelastic deformation demand.

Prediction of the nonlinear response of reinforced masonry structures is fundamental to their displacement based design. Various analytical models have been proposed for this purpose. Each has advantages and disadvantages in terms of level of sophistication, suitability for specific wall configurations, and computational efficiency.

\subsubsection{Beam-Column Models}

A common approach for modeling the nonlinear hysteretic behavior of masonry wall elements uses beam-column elements aligned with the centroidal axes of horizontal and vertical wall elements, and connected with rigid links. A single component beam-column element is most often used, consisting of an elastic flexural element with a nonlinear rotational spring at each end to account for the inelastic behavior of critical regions. To more realistically model walls, beam-column models have been refined with multiple springs (Takayanagi and Schnobrich, 1976) and varying inelastic zones (Keshavarzian and Schnobrich, 1984). However, inelastic response of structural walls subjected to horizontal loads is dominated by large tensile strains and fixed-end rotations due to bond slip, both of which are associated with shifting of the neutral axis. This behavior cannot be modeled directly by beam-column models, which assume that rotations occur along the centroidal axis of the element. Therefore, the beam column element disregards important features of experimentally observed behavior, including shifting of the neutral 
axis of the wall cross-section, rocking of the wall, and interaction with the frame members connected to the wall (Kabeyasawa et al., 1983).

One of the main challenges in using beam-column models is that no reliable method is currently available to estimate the effective plastic hinge length for reinforced masonry shear walls. The empirical formula proposed by Paulay and Priestley (1992) for reinforced concrete members does not correlate well with experimental observations for reinforced masonry shear walls (Shedid et al., 2008; Shing et al., 1990). However, beam column models can be useful if calibrated against experimental data for plastic hinge lengths for wall segments.

\subsubsection{Fiber Element Models}

In fiber-based analysis, a member is represented by unidirectional fibers. The member stiffness and forces are obtained by numerically integrating the stiffness and forces of sections along the member length. The section deformation (displacement and rotation) is used to obtain the strain in each fiber using a plane sections remain plane assumption. The fiber stress and stiffness are updated according to the material models, followed by upgrading of the section force resultant and the corresponding stiffness.

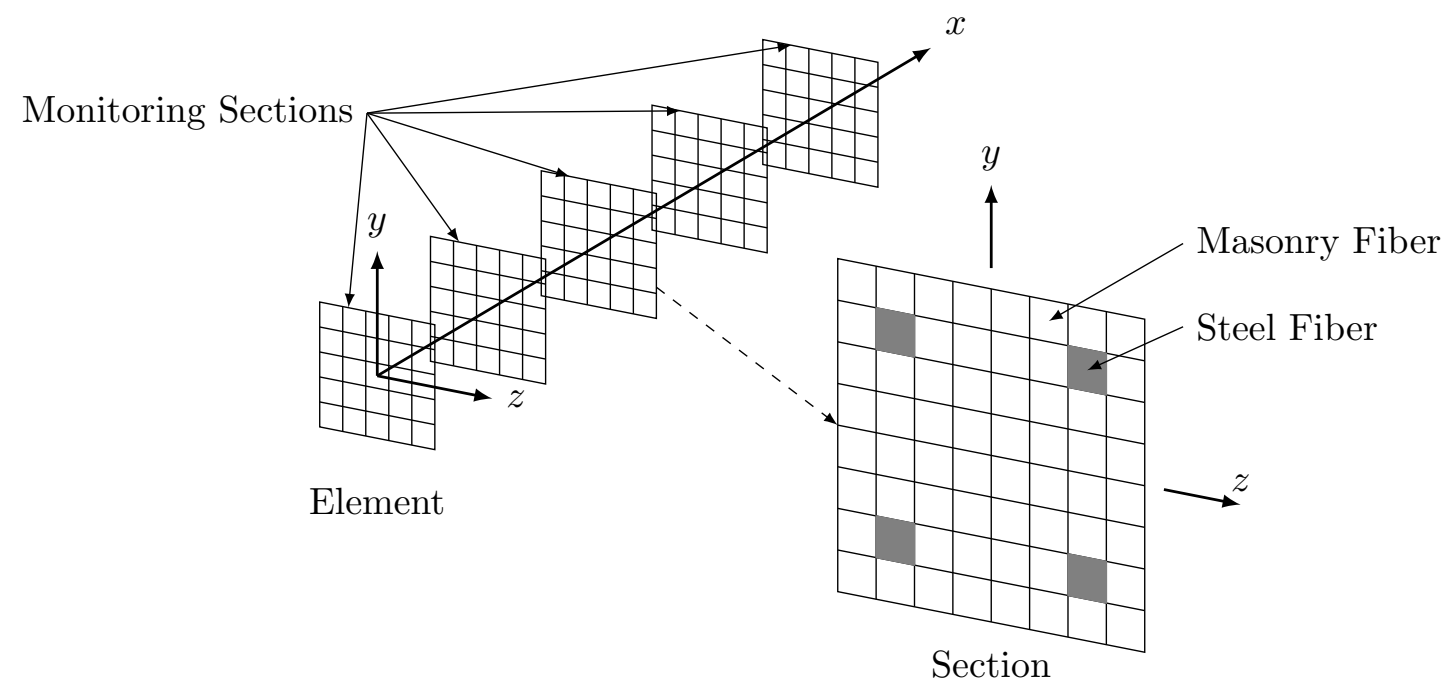

Figure 17.1: Fiber Element Model

Fiber-element models are computationally efficient and well suited for flexure dominated walls with regular openings. As shown in Figure 17.1, in these models, the stress-strain relation of each fiber is governed by a uniaxial constitutive law. They naturally account for the axial load-moment interaction phenomenon of a wall section, and can therefore 
closely simulate the flexure dominated behavior of a reinforced concrete and masonry shear wall (Koutromanos and Shing, 2012). These models have also been extended by replacing the uniaxial stresstrain relation of a fiber with a multi-axial constitutive law to capture the linear and nonlinear shear behavior of a reinforced concrete sections (Petrangeli et al., 1999). While these models can capture axial-flexural-shear interaction, they idealize shear failure as developing at a section level, and do not represent a diagonal shear crack realistically. More often, shear behavior can be modeled simplistically by incorporating a nonlinear empirical shear behavior (Marini and Spacone, 2006).

One challenge for fiber-element models is their tendency to localize plastic deformation in a single element in the case of a displacement-based element formulation, or at a single Gauss point in the case of a force-based element formulation, as the moment-curvature relation enters the strain-softening regime. This causes numerical results to depend on element size and leads to erroneous post-peak behavior if the elements in the plastic zone are not properly sized. To circumvent this problem, Coleman and Spacone (2001) propose a fracture-energy-based regularization method, in which the post-peak stressstrain or moment-curvature relation are adjusted based on the relationship between element size and the expected effective plastic hinge length in an element.

\subsubsection{Strut-and-tie Models}

Strut-and-tie models have generally been used for walls with irregular openings and shear-dominated behavior. They have been used to analyze reinforced masonry shear walls with openings (Voon and Ingham, 2008), and reinforced concrete shear walls with irregularly located openings (Yanez et al., 1991). Their main challenge is the assumptions necessary to determine the location, orientation, and size of the equivalent compression struts.

\subsubsection{Finite Element Models}

Nonlinear finite-element models based on smeared and discrete crack formulations are the most general analysis method for any wall type, but may require much more computational effort than the models discussed above. A main challenge in finite element is stress-locking, which is an over-stiff response in the softening of the plastic zone, not allowing for the full release of the stress. This stress-locking can lead to a significant over-estimation of the shear capacity of an element. These challenges can be addressed by supplementing smeared-crack models with cohesive crack interface models, to capture cracks in a discrete manner. The introduction of strong discontinuities represented by discrete cracks will remove the stress-locking problem and to a certain extent the 
mesh-size dependence, by concentrating the release of fracture energy in line interface elements. Discrete and smeared-crack modeling approaches have been successfully applied in combination to the modeling of non-ductile reinforced-concrete frames with clay masonry infills (Stavridis and Shing, 2008).

\subsection{Background Summary}

Various modeling techniques have been proposed for the purpose of determining the nonlinear response of reinforced masonry structures. Beam-column models suffer from a number of behaviours that cannot be captured including the shifting of the neutral axis and the determination of the plastic hinge length. While fiber element models are a much improved over beam-columns, they have a tendency to localize plastic deformations in a single element causing results to depend on the element size used on the sections. Strutand-tie models are useful for modeling walls with irregular openings, but tend to be conservative in the assumptions used to produce the models. Finite element models are very capable of the nonlinear response of masonry structures, including loading caused by displacements, but the models are computationally heavy. There is a need for a simple model to predict the full load-deflection response of masonry structures that is both accurate and not computationally demanding.

\subsection{Model Objectives}

In addition to the objectives listed in Section 1.4, the specific objectives of a displacement based model for grouted and reinforced hollow concrete block masonry shear walls are:

1. To produce a load-displacement response of a single pier cantilevered masonry shear wall;

2. To predict both pre- and post-peak response of the shear walls in a timely manner; and,

3. Verify the model by comparing the results of the model with the experimentally observed behaviour.

Part IV: Masonry Displacement Based Modeling 


\section{PKT FOR CANTILEVERED REINFORCED MASONRY SHEAR WALLS}

A theory is presented to estimate the pre-peak and post-peak response of reinforced cantilevered hollow concrete block masonry shear walls by modifying a newly developed model for concrete shear walls.

The Three Parameter Kinematic Theory (3PKT) describes the deformation patterns of cantilevered reinforced masonry shear walls using three degrees of freedom (DOFs). The 3PKT captures the complete prepeak and postpeak response of shear dominated walls in a time-efficient way. The model predicts the response of shear dominated walls with major diagonal cracks that fail either in shear along the diagonal cracks or at the base section under the combined action of flexure and shear. The failure along the diagonal cracks can occur either in a brittle manner prior to yielding of the flexural reinforcement or in a more ductile manner after flexure yielding. The base section of the wall is also modeled to account for yielding of the reinforcement and crushing of the masonry.

\subsection{Three-Parameter Kinematic Theory Applicability}

The theory is suitable for walls with rectangular sections subjected to single curvature (cantilever walls) that develop major shear cracks; therefore, flexural failures or sliding failures are not adequately captured by the kinematic model. The other limits of applicability of the model are as follows:

1. The axial load ratio $N / t l_{w} f_{m}^{\prime}$ should be smaller than 0.2 , to avoid crushing failures;

2. The aspect ratio, $a / l_{w}$, should be less than or equal to 3 , to take into account that in slender walls the assumption made for a rigid block above the critical crack is not valid;

3. A wall-height-to-thickness ratio, $a / t$ of 30 or less, to ensure out-of-plane stability;

4. The masonry compressive strength must be less then $60 \mathrm{MPa}$; and,

5. No lap-splices in the base section.

To set clear criteria for the applicability of the model, it will be assumed that pure flexural failures and sliding shear failures would not likely occur if the 3PKT approach predicts that the transverse reinforcement yields before the flexural reinforcement. 
In its range of applicability, the $3 \mathrm{PKT}$ forms the basis of the three-parameter kinematic model for predicting the load-displacement response of reinforced masonry shear walls. The DOFs of the model will be predicted by combining the kinematic equations with equilibrium equations and constitutive relationships for the load-bearing mechanisms in the wall. Two potential failure planes will be considered: a diagonal crack and the horizontal crack at the base.

\subsection{Kinematics of Shear Walls}

The Three Parameter Kinematic Theory for the behaviour of walls was developed by Mihaylov et al. (2016) as an extension of a Two Parameter Kinematic Theory for deep beams (Mihaylov et al., 2013). It is a kinematic theory because it is based on an idealized representation of the deformation pattern of walls. The theory is developed for shear critical walls, that means walls which develop diagonal cracks and eventually fail along such a crack. Hence, the 3 PKT assumes that such a shear crack develops which separates the upper nearly uncracked part of the wall, which is regarded as a rigid body, from the radially cracked fan below. In wall tests, it has been observed that failure along a shear crack is accompanied by severe damage of the wall around the crack tip. The critical loading zone (CLZ) accounts for this damaged area in describing the failure mechanism.

Figure 18.1 visualizes the kinematics and the resulting deformation pattern assumed in the 3PKT. The wall is divided in three parts: a radially cracked fan under the shear crack, a rigid body above the shear crack, and the previously mentioned critical loading zone at the bottom tip of the rigid body. The deformation of these parts is described with the three parameters also indicated in Figure 18.1. The elongation of the longitudinal reinforcement is described by the average strain $\epsilon_{t, a v g}$, the horizontal displacement of the CLZ $\Delta_{c}$ and the shortening of the CLZ $\Delta_{c y}$. All points below the crack are assumed to rotate around the crack tip, which means that any deformation is assumed perpendicular to the radial cracks. The magnitude of this rotation is determined by the average strain in the longitudinal reinforcement $\epsilon_{t, a v g}$. The rigid body itself is assumed to rotate around the tip of the shear crack and translate according to displacements $\Delta_{c}$ and $\Delta_{c y}$. The $\Delta_{c y}$ causes downward sliding of the rigid body along the shear crack while the elongation of the longitudinal reinforcement and $\Delta_{c}$ causes the opening of the shear crack (Hannewald, 2013). 


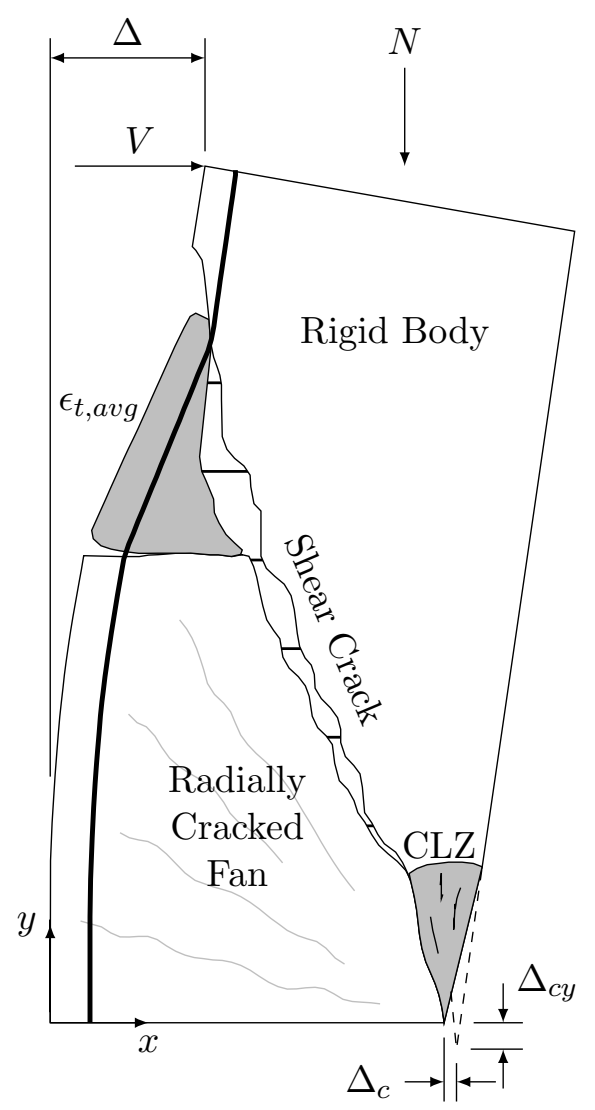

Figure 18.1: Kinematics of 3PKT (Hannewald, 2013)

Each DOF used to describe the deformation of a shear wall is shown individually in Figure 18.2. The first DOF of the model is the average tensile strain in the flexural reinforcement $\left(\epsilon_{t, a v g}\right)$ within the cracked zone below the critical crack (Figure 18.2a). The length over which the strains are averaged is denoted as $l_{t}$. The critical shear crack is approximated with a straight line at an angle $\alpha_{1}$ with respect to the vertical axis. It can be seen that strain $\epsilon_{t, \text { avg }}$ is associated with rotation of the rigid block about point $\mathrm{B}$ by an angle $\epsilon_{t, \text { avg }} l_{t} / d$, and with deformations in the cracked masonry below the critical crack. This bottom part of the wall is modeled with a series of rigid radial struts pinned at point $\mathrm{B}$ and connected to the tension reinforcement. The rotation of each strut about point $\mathrm{B}$ is proportional to the elongation of the reinforcement between the base of the wall and the point where the strut connects to the reinforcement. This deformation pattern can be associated with flexural deformations.

The second DOF of the kinematic model is the horizontal displacement $\Delta_{c}$ in the CLZ (Figure 18.2b). In this deformation pattern, the rigid block translates laterally while the fan remains stationary. At the top of the critical diagonal crack, displacement 
$\Delta_{c}$ develops over a length $l_{k}$ in which the flexural reinforcement is subjected to double curvature. The kinematics related to DOF $\Delta_{c}$ can be associated with shear deformations.

The third DOF of the kinematic model is the downward displacement $\Delta_{c y}$ in the CLZ (Figure 18.2c), which is associated with rotation of the rigid block about point $\mathrm{A}$ by an angle $\Delta_{c y} / d$. This rotation can result in a contact between the rigid block and the masonry below the critical diagonal crack in the vicinity of the CLZ. It is apparent that DOF $\Delta_{c y}$ is related to the action of the axial load $N$, which drives the rigid block downwards.

When the deformation patterns associated with the three DOFs are superimposed, they produce the complete deformed shape of the wall shown in (Figure 18.2d). The displacement of each point from the wall with respect to the coordinate system $x-y$ is expressed as a sum of the displacements associated with each deformation pattern. Based on small-displacement kinematics, it can be shown that the displacements of the points of the rigid block are given by:

$$
\begin{gathered}
\delta_{x}(x, y)=\left(\frac{\epsilon_{t, a v g} l_{t}}{d}+\frac{\Delta_{c y}}{d}\right) y+\Delta_{c} \text { and } \\
\delta_{y}(x, y)=\frac{\epsilon_{t, a v g} l_{t}}{d}\left(l_{w}-x\right)+\frac{\Delta_{c y}}{d}\left(l_{w}-d-x\right)
\end{gathered}
$$

(Mihaylov et al., 2016) while the displacements in the fan below the critical diagonal crack are dependent only on DOF $\epsilon_{t, \text { avg }}$ (Mihaylov et al., 2013) and are given by:

$$
\begin{gathered}
\delta_{x}(x, y)=\frac{\epsilon_{t, a v g} y^{2}}{l_{w}-x} \text { and } \\
\delta_{y}(x, y)=\epsilon_{t, a v g} y
\end{gathered}
$$

In addition to the displacement field, the kinematic model can also be used to estimate the width of the critical diagonal crack, $w$, half-way along the crack. This width is obtained as the sum of the widths associated with the three DOFs of the model:

$$
w=\left[\frac{\epsilon_{t, \min } l_{k}}{2 \sin \alpha_{1}} \frac{h}{d}+\Delta_{c} \cos \alpha_{1}+\frac{\Delta_{c y}}{d}\left(\frac{h}{2 \sin \alpha_{1}}-d \sin \alpha_{1}\right)\right] \frac{a}{n_{\mathrm{cr}}}
$$

(Mihaylov et al., 2016) where $\epsilon_{t, \min }$ is minimum strain in the flexural reinforcement. The expression in the brackets is derived directly from the deformation patterns in Figure 18.2a to Figure 18.2c assuming a single dominant diagonal crack. In members with sufficient web reinforcement, however, this width will be distributed among several major cracks. The number of these cracks $n_{\text {cr }}$ can be estimated as; 


$$
n_{\mathrm{cr}}=\left\{\begin{array}{ll}
\max \left(\frac{l_{k}}{s_{\mathrm{cr}}}, 1\right) & \text { if } \rho_{l, w e b} \geq 0.2 \% \\
1 & \text { otherwise }
\end{array} .\right.
$$

The ratio $l_{k} / s_{c r}$ is the number of cracks within the transition zone between the fan and the rigid block on the flexural tension side of the section. These cracks tend to merge into a single wide crack in the web if they are not controlled by web reinforcement.

The DOFs $\epsilon_{t, a v g}, \Delta_{c}$, and $\Delta_{c y}$ are suitable for the formulation of the 3PKT; however, they are not convenient for the numerical solution as they do not include the imposed displacement $\Delta$. Therefore, for the numerical solution, it is suggested to use DOFs $\Delta$, $\Delta_{c y}$, and $\theta$, where $\theta$ is the rotation of the rigid block about the point of the intersection of the lateral load and the compressive edge of the wall (Mihaylov et al., 2016). The DOFs used for the formulation of the $3 \mathrm{PKT}$ can be expressed with $\Delta, \Delta_{c y}$, and $\theta$ :

$$
\begin{gathered}
\epsilon_{t, a v g}=\frac{\theta d-\Delta_{c y}}{l_{t}} \text { and } \\
\Delta_{c}=\Delta-\theta a .
\end{gathered}
$$

\subsection{Geometry of Kinematic Model}

To complete the formulation of the kinematic model, it is necessary to define the geometry of the wall. The critical crack angle, $\alpha_{1}$, is obtained from a shear strength calculation according to the CSA S304-14 masonry code provisions (Canadian Standards Association, 2014a). Since the steepest cracks in short walls typically propagate along the diagonal of the wall, angle $\alpha_{1}$ should remain larger than or equal to the angle $\alpha$ of the wall diagonal; thus,

$$
\alpha_{1}=\max \left(\theta_{\mathrm{CSA}}, \alpha\right)
$$

where

$$
\theta_{\mathrm{CSA}}=29^{\circ}+7000 \epsilon_{y}(\text { Canadian Standards Association, 2014a })
$$

and

$$
\alpha=\arctan \frac{l_{w}}{h_{w}} .
$$




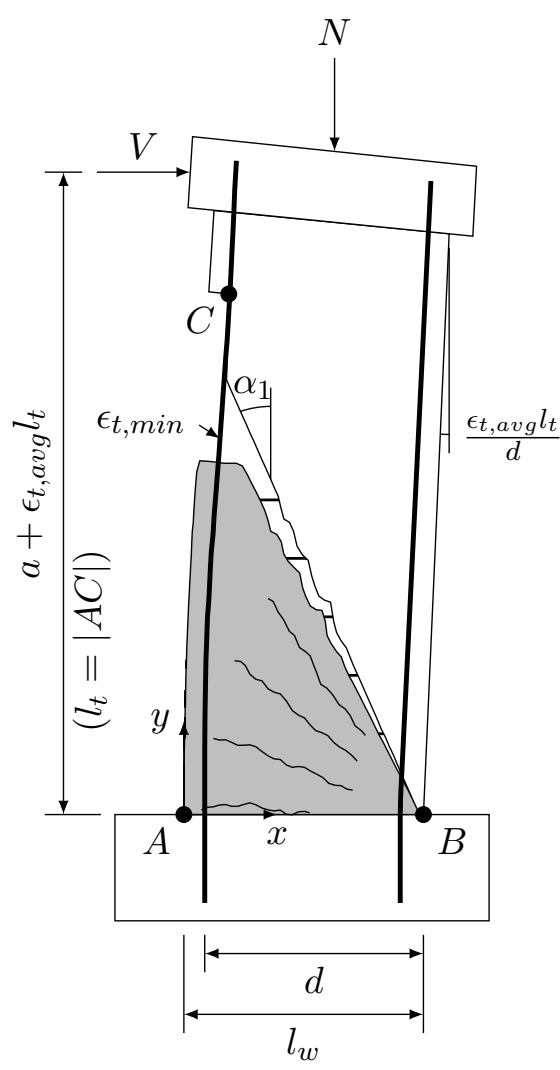

(a) DOF $\epsilon_{t, a v g}$

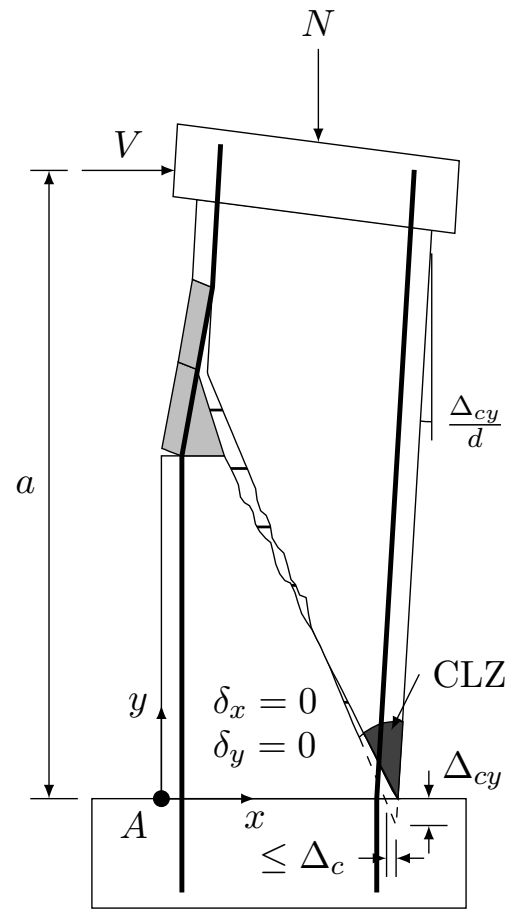

(c) DOF $\Delta_{c y}$

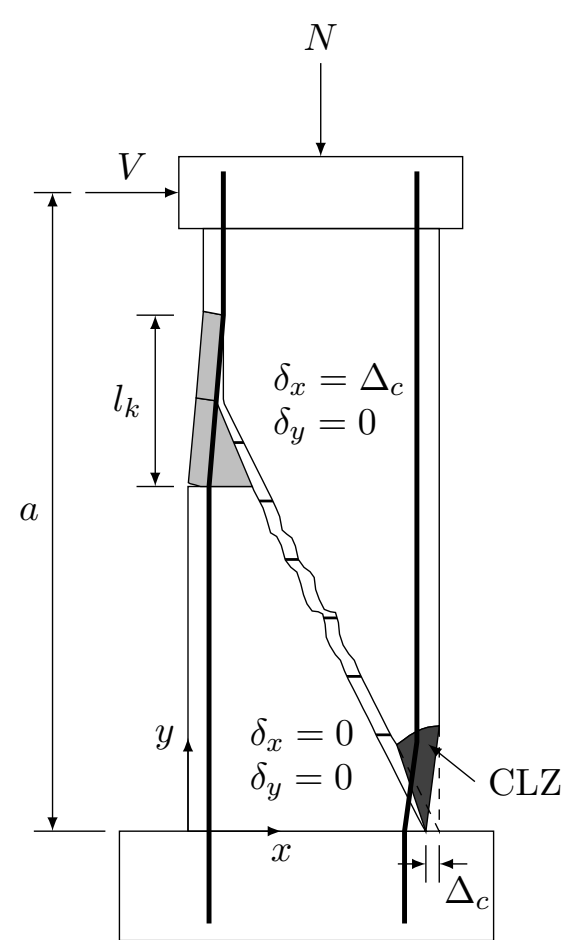

(b) DOF $\Delta_{c}$

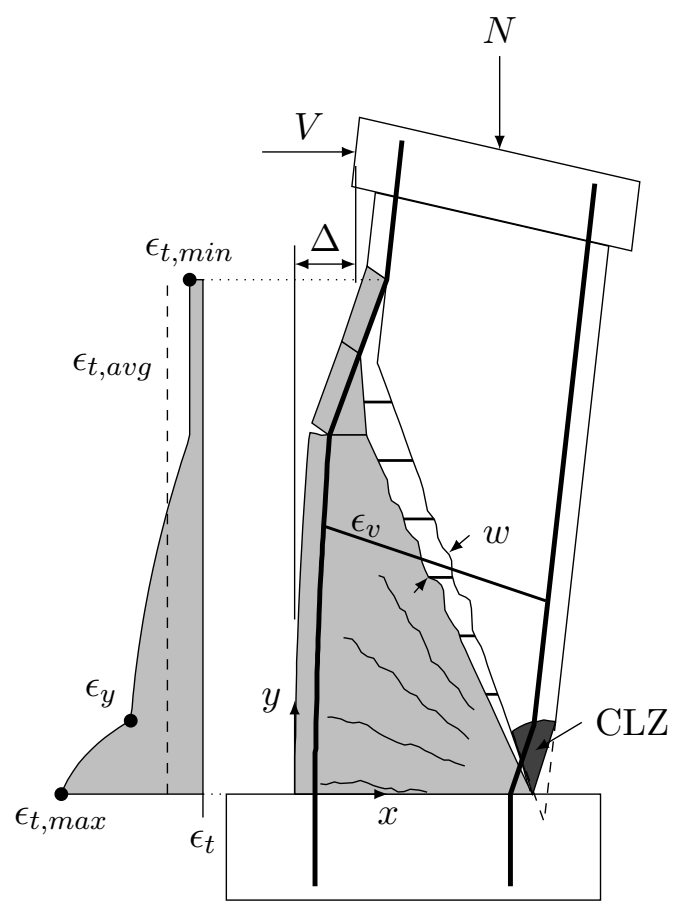

(d) Combined Deformed Shape

Figure 18.2: Three-Parameter Kinamatic Model for Shear Dominated Walls (Hannewald, 2013)

Part IV: Masonry Displacement Based Modeling 
The crack spacing along the flexural reinforcement $s_{c} r$ is estimated based on Model Code 90 (Comite Euro-International Du Beton, 1990) by considering the reinforcement ratio $\rho_{l 1}$ in the effective tension zone:

$$
s_{c r}=\frac{0.28 d_{b}}{\rho_{l 1}}
$$

where $d_{b}$ is the diameter of the flexural reinforcement.

The expressions for $l_{0}$ and $l_{k}$ are derived by considering one crack below and one above the critical diagonal crack, taking also into account limits imposed by the wall height and the width of an effective tension zone, i.e. $\min \left[1.5\left(l_{w}-d\right), d-l_{w} / 2\right]$. The portion of the transition between the fan and rigid block below the critical diagonal crack is defined as:

$l_{0}=\min \left\{\max \left[\min \left(1.5\left(l_{w}-d\right), d-\frac{l_{w}}{2}\right) \cot \alpha_{1}, s_{c r}\right], d \cot \alpha_{1}\right\}$ (Mihaylov et al., 2016).

The length of the transition zone between the fan and ridge, $l_{k}$, is

$$
l_{k}=l_{0}+\min \left[d\left(\cot \alpha-\cot \alpha_{1}\right), s_{c r}\right] \text { (Mihaylov et al., 2016). }
$$

The expression for $l_{t}$ stems directly from the variables given in Figure 18.3:

$$
l_{t}=d \cot \alpha_{1}+\left(l_{k}-l_{0}\right) \text { (Mihaylov et al., 2016). }
$$

The effective depth, $d$ of the wall section is calculated by considering the centroid of the longitudinal reinforcement in one-half the section. The area of flexural reinforcement, $A_{s}$, is also the area of the reinforcement in one-half of the section. Figure 18.3 also shows the geometry of the CLZ. 


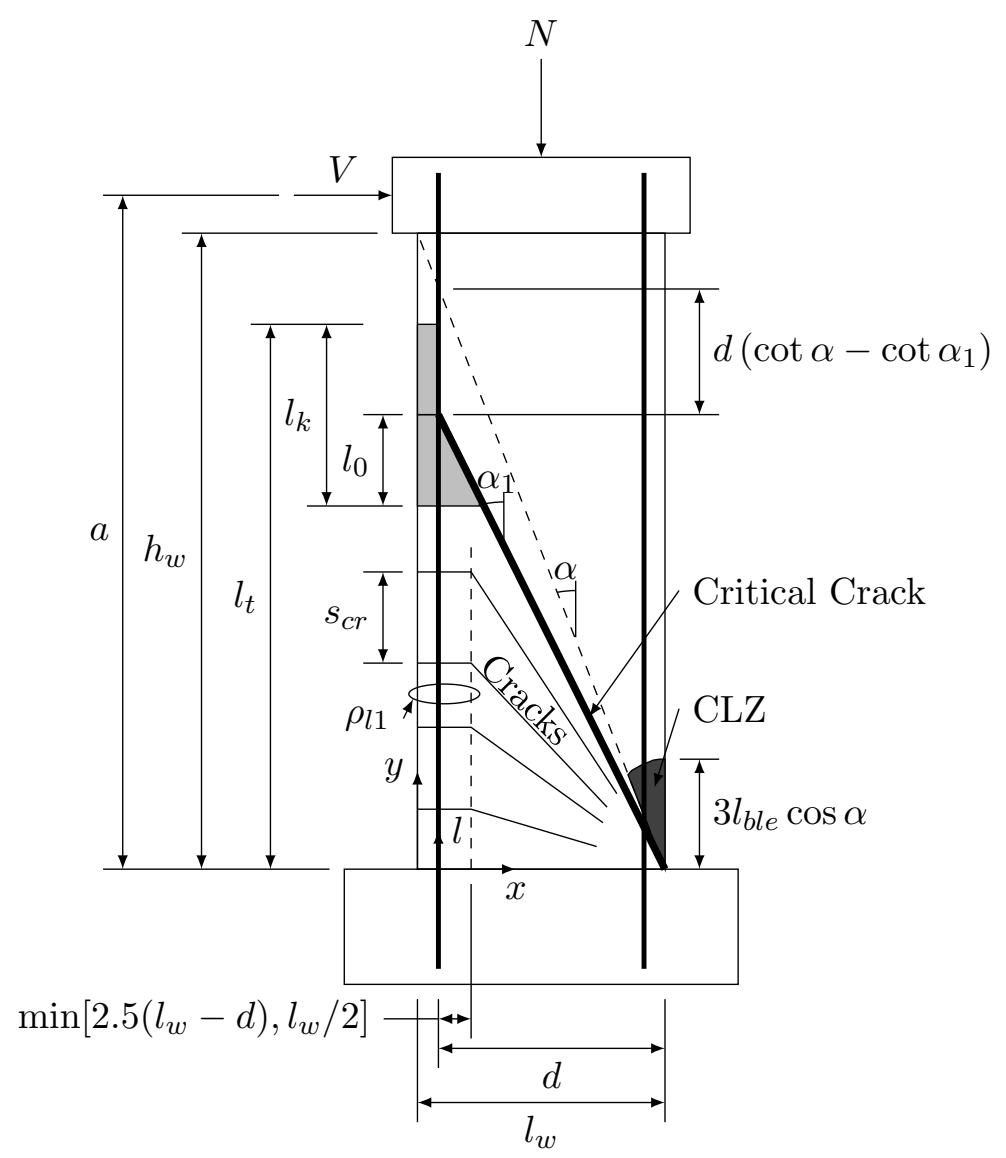

Figure 18.3: Geometry of the Kinematic Model (Mihaylov et al., 2016) 


\subsection{Nine Spring Model}

In the 3PKT, load-bearing mechanisms are modeled with nonlinear springs. The locations of the springs are shown in Figure 18.4 for the rigid block and Figure 18.5 for the fan. The springs are connected on both sides of the crack at the solid dots. The deformations in the springs is derived from the kinematic model as a function of the three DOFs of the model. The block interacts with the foundation through the forces depicted at the tip of the CLZ and with the fan through the forces across the critical diagonal crack.

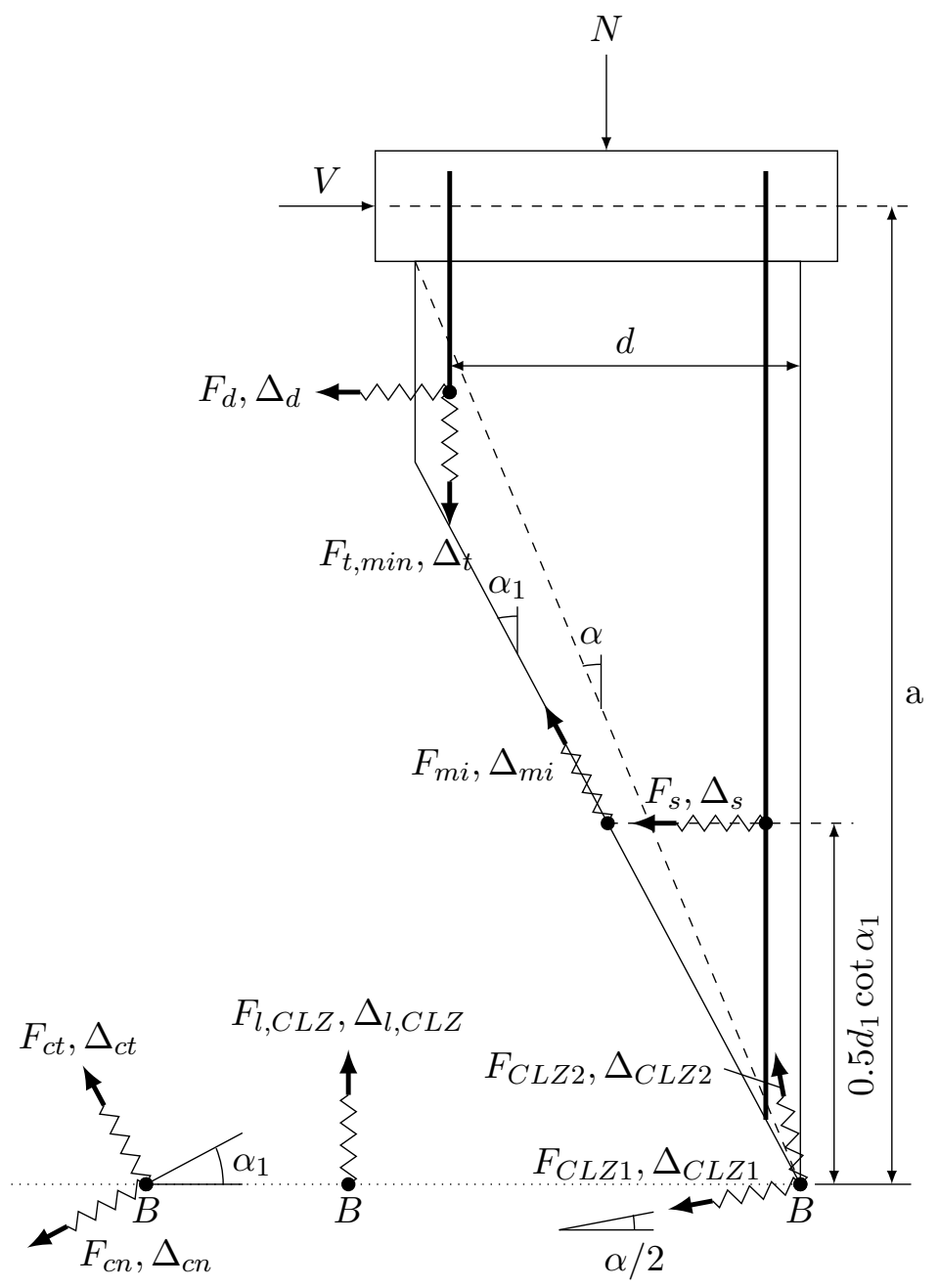

Figure 18.4: Spring Model Representing Load Bearing Mechanisms of the Rigid Block (Mihaylov et al., 2016) 

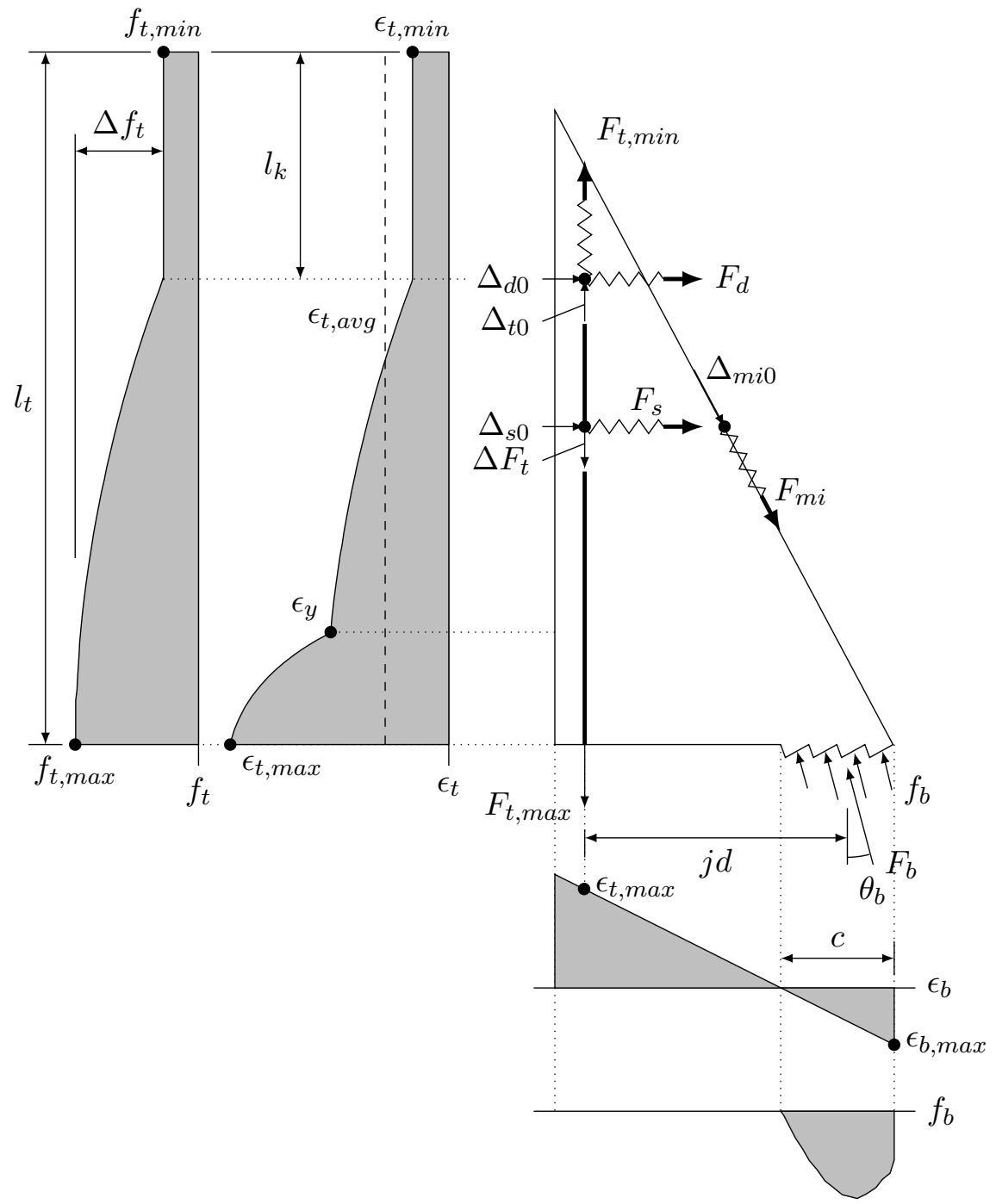

Figure 18.5: Spring Model Representing Load Bearing Mechanisms of the Fan (Mihaylov et al., 2016)

\subsubsection{Forces Transferred across the Critical Diagonal Crack}

The forces across the critical diagonal crack include the aggregate interlock force $F_{m i}$, the tension in the stirrups $F_{s}$, the tension in the flexural reinforcement $F_{t, \min }$, and the force due to dowel action of the flexural reinforcement $F_{d}$. 


\section{Constitutive Relationship for Aggregate Interlock Spring}

The aggregate interlock spring $F_{m i}$ is located half-way along the critical diagonal crack and is parallel to the crack. The deformation of this spring $\Delta_{m i}$ corresponds to the slip displacement between the crack faces and can be expressed as:

$$
\Delta_{m i}=\Delta \sin \alpha_{1}+\Delta_{c y} \cos \alpha_{1}-a \theta \sin \alpha_{1}-\Delta_{m i 0} \geq 0 \text { (Mihaylov et al., 2016). }
$$

The fourth term of the equation is the displacement of the end of spring $F_{m i}$ attached to the fan (Figure 18.4). More precisely, $\Delta_{m i 0}$ is the shortening of the bottom half of the top strut from the fan, which reduces the crack slip. This offset displacement is determined as:

$$
\Delta_{m i 0}=\frac{\epsilon_{b, \max } 0.5 d_{1}}{\sin \alpha_{1}} \text { (Mihaylov et al., 2016). }
$$

The aggregate interlock force, $F_{m i}$, is evaluated as:

$$
F_{m i}=\frac{0.18 v_{m i}\left(f_{m}^{\prime}, w, s, a_{g}\right) t d_{1}}{\sin \alpha_{1}}
$$

where the quantity $t d_{1} / \sin \alpha_{1}$ is the surface of the the critical crack from the base of the wall to the furthest layer of flexural reinforcement, $d_{1}$. The shear stress on the crack $v_{m i}\left(f_{m}^{\prime}, w, s, a_{g}\right)$ is determined according to a contact density model ${ }^{1}$ (CDM) proposed by Li et al. (1989), in which a single rough crack surface is idealized as a set of numerous contact units with various inclinations. The distribution of their directions is expressed by the contact density function, $\Omega\left(\theta_{s}\right)$. In each direction, a contact unit can transfer normal stress and shear stress. The stress, $\sigma_{c o n}\left(\theta_{s}, w, s\right)$, is always assumed perpendicular to the surface. To obtain the shear stress, the horizontal components of $\sigma_{c o n}\left(\theta_{s}, w, s\right)$ are integrated for all inclinations occurring along the crack surface based on the contact density function and the surface area per unit crack plane. Thus, the shear stress transferred is

\footnotetext{
${ }^{1}$ In the CDM the crack surfaces are represented by a series of planes at different angles. Planes at the same angle on each side of the crack are connected by contact springs that have an elastic perfectly plastic behaviour in compression and zero resistance in tension. Depending on the crack width and the direction of the movement at the crack, some of the springs are active (planes are in contact) while others are at zero stress (no contact). The forces in the springs are projected on axes perpendicular and parallel to the crack direction. In this way the CDM accounts for compressive stresses on the crack surfaces (clamping stresses) which enhance the shear resistance $v_{m i}$. As the clamping stresses are neglected in the $3 \mathrm{PKT}$, the shear resistance obtained from the CDM is reduced by a factor of 0.18 adopted from Vecchio and Collins (1986).
} 


$$
v_{m i}\left(f_{m}^{\prime}, w, s, a_{g}\right)=\int_{-\pi / 2}^{\pi / 2} A_{t} \sigma_{c o n}\left(\theta_{s}, w, s\right) \sin \theta_{s} K\left(a_{g}, w\right) \Omega\left(\theta_{s}\right) d \theta_{s}
$$

where $A_{t}$ is the whole surface area per unit crack plane, $\sigma_{c o n}\left(\theta_{s}, w, s\right)$ is the contact stress generated when the positive and negative planes met at $\theta_{s}$, the directional angle of contact stress acting on the negative side as defined in Figure 18.6, $K$ is a reduction factor to account for the reduction in contact area as the crack opens, and $\Omega\left(\theta_{s}\right)$ is the stochastic density function representing the distribution of crack surface direction.

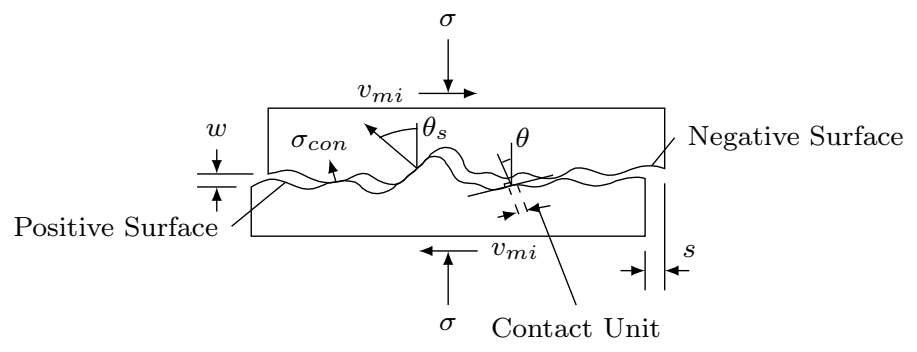

Figure 18.6: Definitions and Notations for Contact Density Model Li et al. (1989)

To determine the contact density function, Li et al. (1989) measured the shapes of rugged crack surfaces and found that:

$$
\Omega\left(\theta_{s}\right)=0.5 \cos \theta_{s}
$$

was a good representation of the surface profile. Bases on this contact density function the surface area of the crack is:

$$
A_{t}=\frac{4}{\pi}=1.27
$$

Meaning that the surface area of the crack is 1.27 times the sectional area of the crack plane, due to surface being rough rather than smooth. The concrete density function only includes the effects of the crack surface shape, but no information concerning the size. To address this issue an effective contact area factor is included and defined as:

$$
K\left(a_{g}, w\right)=\max \left(1-\exp \left(1-0.5 \frac{a_{g}}{w}\right), 0\right)
$$

where $a_{g}$ is the maximum aggregate size. The contact stress, $\sigma_{c o n}\left(\theta_{s}, w, s\right)$, is modeled as a perfectly elasto-plastic relationship, see Figure 18.7, based on the local displacement width, $w_{\theta_{s}}$. 


$$
\sigma_{\text {con }}\left(\theta_{s}, w, s\right)=\max \left[\min \left(\frac{f_{y, \text { con }}^{\prime}}{w_{\text {lim }}} w_{\theta_{s}}, f_{y, \text { con }}^{\prime}\right), 0\right]
$$

where $w_{\text {lim }}$ is $0.04 \mathrm{~mm}$, the yield compressive stress of the masonry, $f_{y, c o n}^{\prime}$ is $13.7 \sqrt[3]{f_{m}^{\prime}\left(f_{m x}^{\prime}, f_{m y}^{\prime}, \alpha_{1}\right)}$, where $f_{m}^{\prime}$ is determined at angle $\alpha_{1}$ (see Equation10.45) and

$$
w_{\theta_{s}}=s \sin \theta_{s}-w \cos \theta_{s}
$$

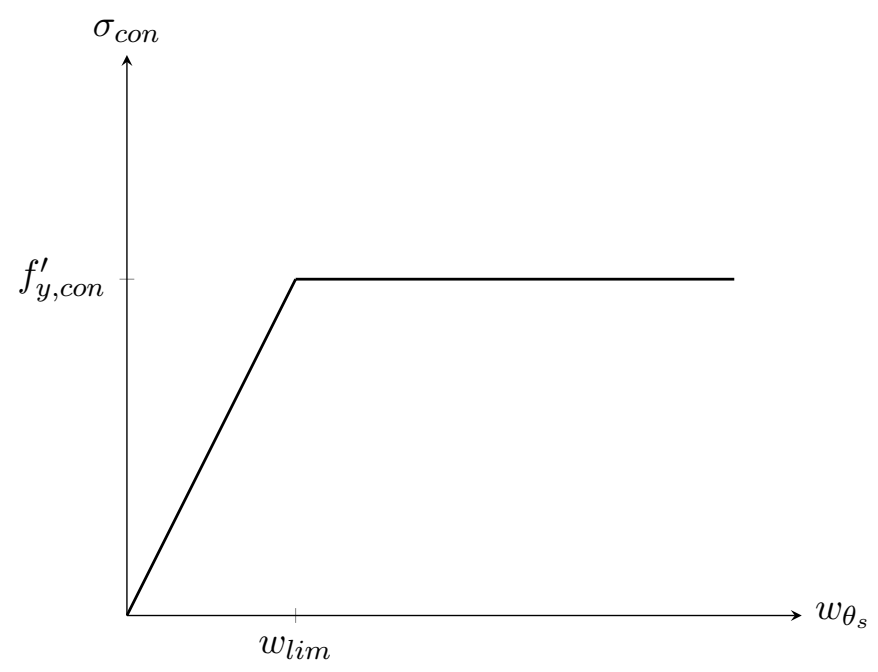

Figure 18.7: Elasto-Plastic Model for Contact Compressive Stress

This results in a load-deformation response for the aggregate interlock spring as shown in Figure 18.8.

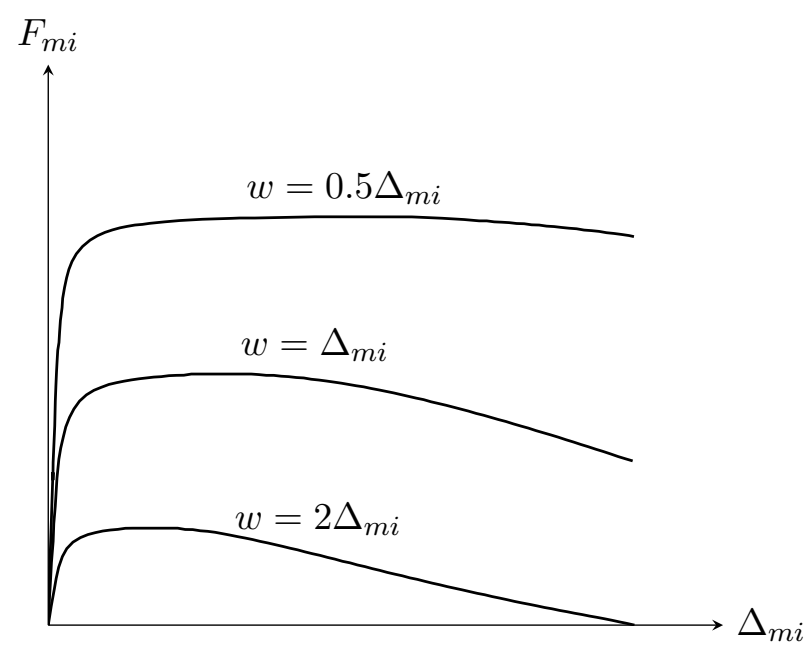

Figure 18.8: Load-Deformation Response for the Aggregate Interlock Spring 


\section{Constitutive Relationship for Transverse Reinforcement}

The second spring located half-way along the critical crack is the spring representing the transverse reinforcement. The deformation of this spring is equal to the horizontal expansion of the web of the wall at distance $0.5 d_{1} \cot \alpha_{1}$ from the base:

$$
\Delta_{s}=\Delta-\theta \frac{a-d_{1}}{\tan \alpha_{3}}-\Delta_{s 0} \text { (Mihaylov et al., 2016) }
$$

where $\alpha_{3}$ is the angle of the strut connected to the stirrup spring:

$$
\alpha_{3}=\arctan \frac{d_{1}}{2 \tan \alpha_{1}} .
$$

The last term in the expression is the offset displacement due to the deformations of the fan and is taken as:

$$
\Delta_{s 0}=\frac{0.5 d_{1} \cot \alpha_{1}}{d} \int_{0}^{0.5 d_{1} \cot \alpha_{1}} \epsilon_{t} d l \text { (Mihaylov et al., 2016). }
$$

The average strain in the transverse reinforcement, over a length of $0.9 d_{1}$, across the web is estimated as:

$$
\epsilon_{v}=\frac{\Delta_{s}}{0.9 d_{1}}
$$

and the stress in the transverse reinforcement, $f_{v}$, is calculated from this strain based on a bilinear stress-strain relationship shown in Figure 18.9. Having obtained $f_{v}$, the force in the stirrups is calculated from:

$$
F_{s}=A_{v} f_{v}=\rho_{v} t \cdot \max \left[d_{1} \cot \alpha_{1}-1.5 l_{b 1 e}-l_{0}\left(\frac{d}{d_{1}}\right), 0.5 d_{1} \cot \alpha_{1}\right] f_{v}
$$

where the expression in the brackets is the height over which the stirrups are activated by the expansion of the web. The distance $l_{b l e}$ is the characteristic length of the CLZ. 


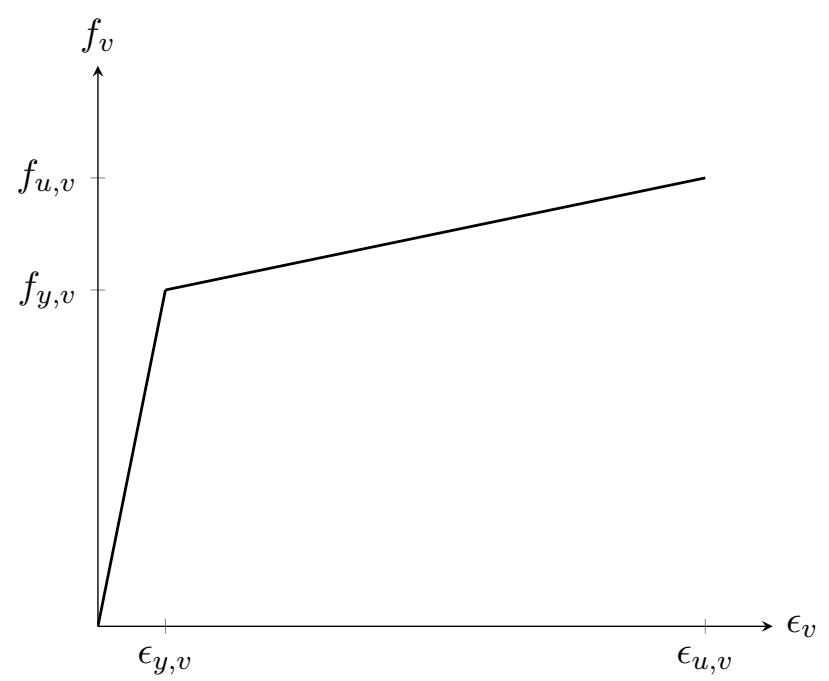

Figure 18.9: Transverse Reinforcement Stress Strain Relationship

\section{Constitutive Relationship for Dowel Action of the Longitudinal Reinforce- ment}

In addition to the transverse reinforcement force, the other horizontal force acting across the critical crack is the dowel action force, $F_{d}$. This force develops in the zone of transition between the fan and the rigid block where the flexural reinforcement is subjected to double curvature. Therefore, each of the bars in this zone works as a dowel of length $l_{k}$. The deformation of the spring that models this effect is equal to the relative horizontal displacement between the ends of the dowels:

$$
\Delta_{d}=\Delta+\theta\left(l_{t}-a\right)-\Delta_{d 0} \text { (Mihaylov et al., 2016). }
$$

The dowel offset displacement is defined as:

$$
\Delta_{d 0}=\frac{l_{t}-l_{k}}{d} \int_{0}^{l_{t}-l_{k}} \epsilon_{t} d l \text { (Mihaylov et al., 2016) }
$$

and the dowel force, $F_{d}$, is obtained by modeling the dowels as fixed-fixed beams subjected to $\Delta_{d}$

$$
F_{d}=n_{b} \frac{12 E_{s} \pi d_{b}^{4}}{64 l_{k}^{3}} \Delta_{d} \leq n_{b} f_{y e} \frac{d_{b}^{3}}{3 l_{k}}
$$

where $n_{b}$ is the number of longitudinal reinforcement bars, $d_{b}$ their diameter and $f_{y e}$ is the effective steel yield stress defining the upper limit of the stress accounting for the 
reduction of the plastic moment capacity of the bar-dowels due to the tension force in the dowels $F_{t, \text { min }}$, definedas :

$$
f_{y e}=f_{y}\left\{1-\min \left[\left(\frac{F_{t, \min }}{f_{y} A_{s t}}\right)^{2}, 1\right]\right\} .
$$

\section{Constitutive Relationship for Longitudinal Reinforcement Tension}

This tension in the longitudinal reinforcement is modeled with the last spring across the critical crack. The elongation of this spring is defined as:

$$
\Delta_{t}=\epsilon_{t, a v g} l_{t}-\Delta_{t 0}
$$

and the corresponding stain in the reinforcement is

$$
\epsilon_{t, \min }=\frac{\Delta_{t}}{l_{k}}
$$

The stress in the reinforcement $f_{t, \min }$ is obtained from strain $\epsilon_{t, \min }$ by using a bilinear stress-strain relationship for the steel as shown in Figure 18.10, and force $F_{t, \min }$ is calculated as:

$$
F_{t, \min }=f_{t, \min } A_{s t}
$$

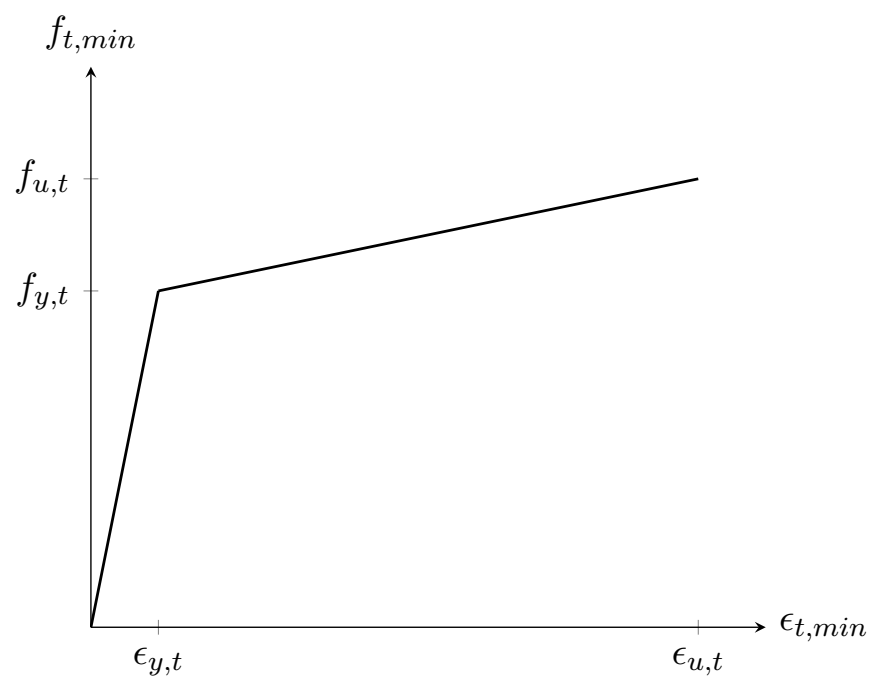

Figure 18.10: Longitudinal Reinforcement Stress Strain Relationship 


\subsubsection{Forces in the Critical Loading Zone}

An important component of the 3PKT approach is the modeling of the critical loading zone (CLZ). In Figure 18.4, this zone is represented by five nonlinear springs that connect the rigid block to the foundation. Springs $F_{C L Z 1}$ and $F_{C L Z 2}$ represent the compression in the masonry in the CLZ, springs $F_{c n}$ and $F_{c t}$ model a potential contact between the rigid block and the fan, and spring $F_{s c}$ models the longitudinal reinforcement in the CLZ.

The CLZ is bound between the vertical edge of the wall and the critical diagonal crack that extends to the base of the wall. The failure of the CLZ occurs in a complex manner, resulting in the crushing of the masonry under the combined action of normal and shear stresses. In addition longitudinal reinforcement can buckled in this zone as failure occurs. To model this complex behavior, it is first suggested to approximate the shape of the CLZ with a circular section with a radius of $3 l_{b 1 e} \cos \alpha$ centered at the corner of the wall (see Figure 18.3). The expression for the radius is adopted from the $2 \mathrm{PKT}$ for deep beams (Mihaylov et al., 2013) where the characteristic length $l_{b 1 e}$ is proportional to the width of the column loading the beam. In walls, the forces from the CLZ are diffused into the foundation; therefore, the characteristic length is less clearly defined than in deep beams (Mihaylov et al., 2016). For this reason, a simple new expression for $l_{b 1 e}$ is proposed based on comparisons with masonry wall tests:

$$
l_{b 1 e}=0.11 \sqrt{a^{2}+l_{w}^{2}} \leq 2 h^{b l}+1.5 t^{b j}
$$

where $\sqrt{a^{2}+l_{w}^{2}}$ is the length of the diagonal of the wall, $h^{b l}$ is the height of the block, and $t^{b j}$ is the thickness of the bed joint.

\section{Constitutive Relationship for Compression in the Masonry}

The displacement of the tip of the critical loading zone $\Delta_{C L Z}$ relative to the rigid block has horizontal and vertical components equal to DOFs $\Delta_{c}$ and $\Delta_{c y}$, respectively. This displacement is associated with compressive strains and stresses in the masonry, as well as a reaction force $F_{C L Z}$. The reaction force, $F_{C L Z}$, is determined accounting for the nonuniform distribution of stress in the CLZ by integrating the stress, $f_{c, C L Z}$, from zero strain to $\epsilon_{C L Z}$ :

$$
F_{C L Z}=\alpha l_{b 1 e} t f_{m, C L Z}
$$


where

$$
f_{m, C L Z}=\int_{0}^{\epsilon_{C L Z}} f_{m, \text { conf }}\left(\epsilon_{m}\right) d \epsilon_{m}
$$

The confined stress, $f_{m, c o n f}$, is determined using Popovics (1970) stress-strain relationship:

$$
f_{m, \text { conf }}=f_{p, C L Z}\left(\frac{\epsilon_{m}}{\epsilon_{p, C L Z}}\right) \frac{n_{C L Z}}{n_{C L Z}-1+\left(\frac{\epsilon_{m}}{\epsilon_{p, C L Z}}\right)^{n_{C L Z} k_{C L Z}}}
$$

where $n_{C L Z}$ is a curve fitting parameter defined as:

$$
n_{C L Z}= \begin{cases}n & \text { if } f_{l}^{\prime}=0 \\ \frac{5000 \sqrt{f_{m}^{\prime}\left(f_{m x}^{\prime}, f_{m y}^{\prime}, \alpha_{3}\right)}}{5000 \sqrt{f_{m}^{\prime}\left(f_{m x}^{\prime}, f_{m y}^{\prime}, \alpha_{3}\right)}-\frac{f_{p, C L Z}}{\epsilon_{p, C L Z}}} & \text { otherwise }\end{cases}
$$

$k_{C L Z}$ is a parameter for post-peak decay determined by:

$$
k_{C L Z}=\left\{\begin{array}{ll}
k & \text { if } f_{l}^{\prime}=0 \\
1 & \text { otherwise }
\end{array},\right.
$$

and the peak strength of the masonry, $f_{p, C L Z}$, is determined according to Mander et al. (1988) accounting for any confinement.

$$
f_{p, C L Z}=f_{m}^{\prime}\left(f_{m x}^{\prime}, f_{m y}^{\prime}, \alpha_{3}\right)\left(-1.254+2.254 \sqrt{1+\frac{7.94 f_{l}^{\prime}}{f_{m}^{\prime}}}-2 \frac{f_{l}^{\prime}}{f_{m}^{\prime}}\right)
$$

where $f_{m}^{\prime}$ is the unconfined masonry compressive strength at angle $\alpha_{1}$ (see Equation10.45), and $f_{l}^{\prime}$ is the lateral confining stress on the masonry:

$$
f_{l}^{\prime}=k_{e} \rho_{t, C L Z} f_{y, t}
$$

where $\rho_{t, C L Z}$ is the reinforcement ratio of the ties within the CLZ, $f_{y, t}$ is the yield strength of the ties, and $k_{e}$ is the confinement effectiveness coefficient. For rectangular hoops, $k_{e}$, is:

$$
k_{e}=\frac{\left(1-\frac{1}{6 b_{c} d_{c}} \sum_{i=1}^{n}\left(w_{i}^{\prime}\right)^{2}\right)\left(1-\frac{s^{\prime}}{2 b_{c}}\right)\left(1-\frac{s^{\prime}}{2 d_{c}}\right)}{1-\rho_{l, C L Z}} \text { (Mander et al., 1988) }
$$

where $w_{i}^{\prime}$ is the $i$ th clear distance between adjacent longitudinal bars, $b_{c}$ is the core dimensions to the centerline of the ties in the $x$-direction, $d_{c}$ is the core dimensions 
to the centerline of the ties through the thickness of the wall, $s^{\prime}$ is the clear vertical spacing between the ties (see Figure 18.11), and $\rho_{l, C L Z}$ is the ratio of the longitudinal reinforcement in the CLZ.

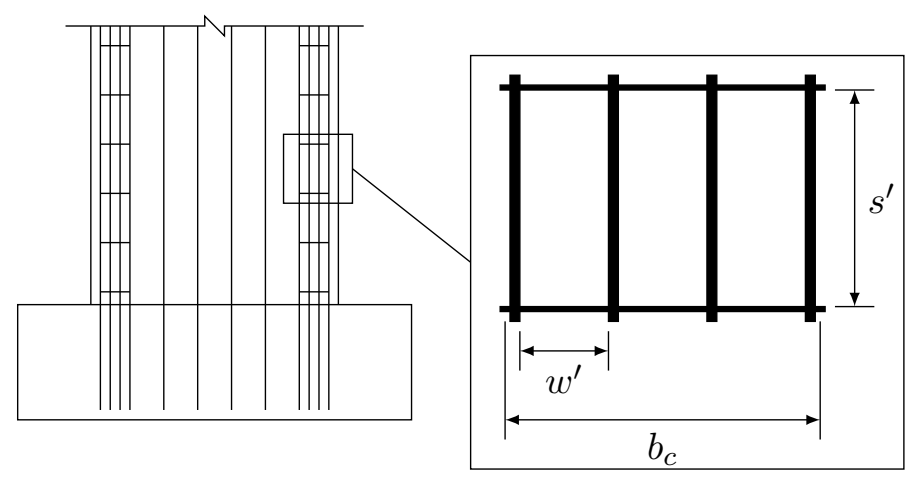

Figure 18.11: Dimensions of Confinement in the CLZ (Mander et al., 1988)

The peak strain in the CLZ, $\epsilon_{p, C L Z}$, is determined from:

$$
\epsilon_{p, C L Z}=\epsilon_{m}^{\prime}\left[1+5\left(\frac{f_{p, C L Z}}{f_{m}^{\prime}}-1\right)\right] \text { (Mander et al., 1988) }
$$

where the strain, $\epsilon_{m}^{\prime}$, at peak stress, $f_{m}^{\prime}\left(f_{m x}^{\prime}, f_{m y}^{\prime}, \alpha_{3}\right)$, is:

$$
\epsilon_{m}^{\prime}=0.0009 f_{m}^{\prime}\left(f_{m x}^{\prime}, f_{m y}^{\prime}, \alpha_{3}\right)^{0.37} \text { (Mander et al., 1988). }
$$

The initial elastic modulus, $E_{m}$, is taken as the proposed Equation from Subsection 13.5.2:

$$
E_{m}= \begin{cases}475 f_{m}^{\prime} & \text { if } f_{m}^{\prime}\left(f_{m x}^{\prime}, f_{m y}^{\prime}, \alpha_{3}\right) \leq 8 \mathrm{MPa} \\ \min \left(1850 e^{0.09 f_{m}^{\prime}}, 20000\right) & \text { otherwise }\end{cases}
$$

The curve fitting parameter, $n$, is:

$$
n=0.8+\frac{f_{m}^{\prime}\left(f_{m x}^{\prime}, f_{m y}^{\prime}, \alpha_{3}\right)}{17}(\text { Collins and Mitchell, 1997) }
$$

and $k$ is:

$$
k=\max \left(0.67+\frac{f_{m}^{\prime}\left(f_{m x}^{\prime}, f_{m y}^{\prime}, \alpha_{3}\right)}{62}, 1\right) \text { (Collins and Mitchell, 1997) }
$$

The angle $\alpha_{3}$ is determined from:

$$
\alpha_{3}=90^{\circ}-\frac{\alpha}{2}-\alpha_{2}
$$


where

$$
\alpha_{2}=\frac{\alpha}{2}-\arctan \left\{\tan \frac{\alpha}{2}-2 \sin ^{2} \frac{\alpha}{2}\left[\tan \frac{\alpha}{2}+\tan \left(\gamma-\frac{\alpha}{2}\right)\right]\right\}
$$

The angle $\gamma$ is determined from:

$$
\gamma=\arctan \frac{\Delta_{C L Z 1}}{\Delta_{C L Z 2}}
$$

where the displacements, $\Delta_{C L Z 1}$ and $\Delta_{C L Z 2}$, are determined from:

$$
\begin{gathered}
\Delta_{C L Z 1}=\Delta \cos \frac{\alpha}{2}-\Delta_{c y} \sin \frac{\alpha}{2}-a \theta \cos \frac{\alpha}{2}, \text { and } \\
\Delta_{C L Z 2}=\Delta \sin \frac{\alpha}{2}+\Delta_{c y} \cos \frac{\alpha}{2}-a \theta \sin \frac{\alpha}{2} .
\end{gathered}
$$

The force $F_{C L Z}$ is projected on axes 1 and 2 as shown in Figure 18.12 to generate the forces in springs $F_{C L Z 1}$ and $F_{C L Z 2}$.

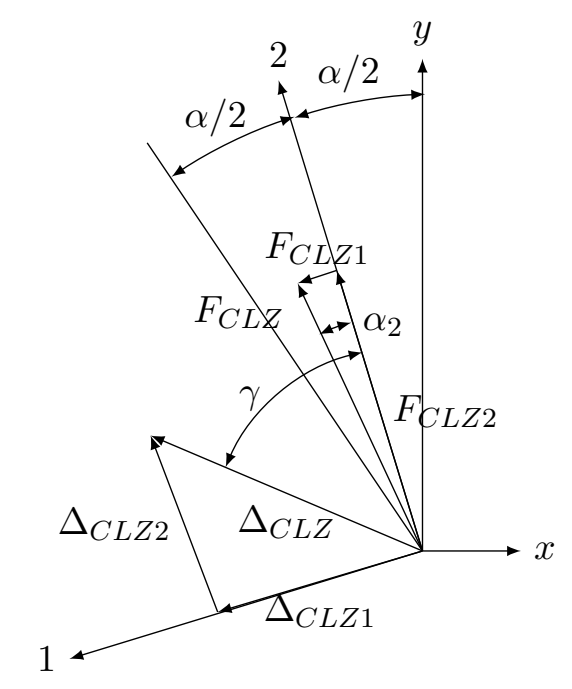

Figure 18.12: Angles in the CLZ

\section{Constitutive Relationship for Contact Between the Rigid Block and Fan}

Springs $F_{c n}$ and $F_{c t}$ model a potential contact between the rigid block and the fan in the vicinity of the CLZ. This contact occurs at some distance from the base of the wall, but for the sake of simplicity springs $F_{c n}$ and $F_{c t}$ are placed at the tip of the CLZ. Spring $F_{c n}$, which is normal to the critical shear crack, is assigned a large constant stiffness in compression and zero stiffness in tension. The stiffness in compression is chosen to prevent any meaningful penetration of the rigid block into the fan. The displacement of this spring, $\Delta_{c n}$, is determined from: 


$$
\Delta_{c n}=\Delta \cos \alpha_{1}-\Delta_{c y} \sin \alpha_{1}-a \theta \cos \alpha_{1} \text { (Mihaylov et al., 2016). }
$$

The force, $F_{c n}$, in this spring is:

$$
F_{c n}=k_{c n} \Delta_{c n}
$$

where $k_{c n}$ is the stiffness of the spring, defined as:

$$
k_{c n}=\left\{\begin{array}{ll}
0 & \text { if } \Delta_{c n} \geq 0 \\
E_{m} t \tan \alpha_{1} & \text { otherwise }
\end{array} .\right.
$$

The initial elastic modulus, $E_{m}$, is taken as the proposed Equation from Subsection 13.5.2:

$$
E_{m}= \begin{cases}475 f_{m}^{\prime} & \text { if } f_{m}^{\prime}\left(f_{m x}^{\prime}, f_{m y}^{\prime}, \alpha_{1}\right) \leq 8 \mathrm{MPa} \\ \min \left(1850 e^{0.09 f_{m}^{\prime}}, 20000\right) & \text { otherwise }\end{cases}
$$

Spring $F_{c t}$ models the friction force between the rigid block and the fan, which is estimated as 0.7 times force $F_{c n}$ (Mihaylov et al., 2016).

\section{Constitutive Relationship for Longitudinal Reinforcement in the CLZ}

The longitudinal reinforcing bars within the CLZ contribute force, $F_{l, C L Z}$. The loaddisplacement response of this spring is obtained on the basis of bars with length $l_{b 1 e}$. Therefore, the strain in the bars is estimated as:

$$
\epsilon_{l, C L Z}=\frac{\Delta_{c y}}{l_{b 1 e}}
$$

and the stress in the bars is calculated from a bilinear stress-strain relationship for the steel, thus:

$$
F_{l, C L Z}=A_{l, C L Z} \min \left(E_{l, C L Z} \epsilon_{l, C L Z}, F_{y l, C L Z}\right)
$$

where $A_{l, C L Z}$ is the cross-sectional area of the bars, $E_{l, C L Z}$ is the modulus of elasticity of the steel bars, and $F_{y l, C L Z}$ is the yield strength of the bars. To account for buckling of the bars, the force $F_{l, C L Z}$ becomes zero if the compressive strain in the CLZ, $\epsilon_{C L Z}$, exceeds the maximum of 0.004 or the strain at peak stress of the concrete in the CLZ, $\epsilon_{p, C L Z}$ (Mihaylov et al., 2016). 


\subsection{Calculations within the Fan}

To obtain offset displacements $\Delta_{m i 0}, \Delta_{s 0}, \Delta_{d 0}$, and $\Delta_{t 0}$ the forces $F_{b}$ and $F_{t, \max }$ at the base of the wall must be determined using equilibrium of the free-body diagram shown in Figure 18.5. This is an iterative procedure as the strain profile in the longitudinal steel and the corresponding forces $F_{t, \text { min }}$ and $F_{t, \max }$, the strain profile in the masonry at the base and the corresponding force $F_{b}$, and the distance $j d$ are all unknown. The following solution procedure is presented from Mihaylov et al. (2016).

Assuming a distance for the lever arm, $j d$, between the forces $F_{b}$ and $F_{t, \text { max }}$ such as $0.9 d$ and using moment equilibrium of the fan about the point of application of the compression force at the base, $F_{b}$, the force difference in the longitudinal steel $\Delta F_{t}=$ $F_{t, \max }-F_{t, \min }$ can be determined from:

$$
\Delta F_{t}=\frac{\frac{d_{1}}{\tan \alpha_{3}} F_{s}+(1-j) d \cos \alpha_{1} F_{m i}+\left(l_{t}-l_{k}\right) F_{d}}{j d}
$$

and the corresponding stress difference, $\Delta f_{t}$ :

$$
\Delta f_{t}=\frac{\Delta F_{t}}{A_{s t}} .
$$

With the difference in stress along the flexural reinforcement known a stress at the base of the wall, $f_{t, \max }$, can be assumed. To obtain the complete stress profile $f_{t}$ along the flexural reinforcement, it is assumed that $f_{t}$ follows a parabolic variation along length $l_{t}-l_{k}$ with a vertical tangent to the parabola at the base and remains constant within $l_{k} ;$ thus,

$$
f_{t}(y)=f_{t, \max }-\Delta f_{t} \frac{\left(l_{t}-l_{k}\right)^{2}}{y^{2}} .
$$

From the stress profile, $f_{t}(y)$, the strain profile can be calculated by using a bilinear stress-strain relationship for the reinforcement with unloading parallel to the initial loading branch. Integrating this strain along the length, $l_{t}$, gives a displacement that can be compared to the displacement produced by:

$$
\Delta_{t}=-\Delta_{c y}+d \theta
$$

If the two displacement differ a new $f_{t, \max }$ can be assumed and the process repeated until convergence. This convergence is efficiently performed using the method of bisection. 
The compression force at the base of the of the fan, $F_{b}$, can be determined from horizontal and vertical equilibrium of the fan:

$$
F_{b}=\sqrt{\left(\Delta F_{t} A_{s}+F_{m i} \cos \alpha_{1}\right)^{2}+\left(F_{m i} \sin \alpha_{1}+F_{d}+F_{s}\right)^{2}} .
$$

This forces acts at angle:

$$
\theta_{b}=\arctan \frac{\Delta F_{t} A_{s t}+F_{m i} \cos \alpha_{1}}{F_{m i} \sin \alpha_{1}+F_{d}+F_{s}}
$$

A linear strain profile across the base section with a compression zone of depth $c$ is defined by $\epsilon_{t, \max }$, determined from the flexural reinforcement analysis of the fan, and an assumed $\epsilon_{b, \max }$. Since significant shear force is transferred through the compression zone, strains $\epsilon_{b}$ in this zone are assumed inclined at the same angle $\theta_{b}$ as force $F_{b}$. From the assumed strain profile the stresses $f_{b}$ in the compression zone can be determined by using Popovics (1970) stress-strain relationship for unconfined masonry taking into account the angle $\theta_{b}$ effects on masonry peak compression strength and a bilinear stressstrain curve for the reinforcement, with the assumption that the reinforcement in the compression is uniformly distributed based on the average ratio $\rho_{l}=A_{s} /\left(0.5 t l_{w}\right)$. If the strain $\epsilon_{b, \max }$ exceeds 0.004 , the stresses in the reinforcement are neglected due to the likelihood of spalling of the block and bucking of the reinforcing bars. The compression force. $F_{b}^{\prime}$ is obtained by integrating the stresses $f_{b}$ long the length of $c$. If $F_{b}^{\prime}$ is different then $F_{b}$ determined from equilibrium assume a new $\epsilon_{b, \max }$ value and repeat until $F_{b}^{\prime}$ and $F_{b}$ have converged using the method of bisection for efficient convergence.

Based on the converged stress profile $f_{b}$ an updated value for the lever arm $j d^{\prime}$ can be determined, and the above calculations are all performed until $j d^{\prime}$ and $j d$ have converged. This process is shown in Figure 18.13.

Finally, the solution procedure for the fan provides criteria for failure of the base section of the wall due to excessive compressive or tensile stresses. If force $F_{b}$ is too large to be balanced by stresses $f_{b}$, the wall is predicted to fail with crushing of the concrete in the compression zone under the combined action of flexure and shear. Flexural failure due to rupture of the reinforcement is predicted if tensile strain $\epsilon_{t, \max }$ reaches $0.6 \epsilon_{u}$, where $\epsilon_{u}$ is the strain at peak stress of the longitudinal steel. 


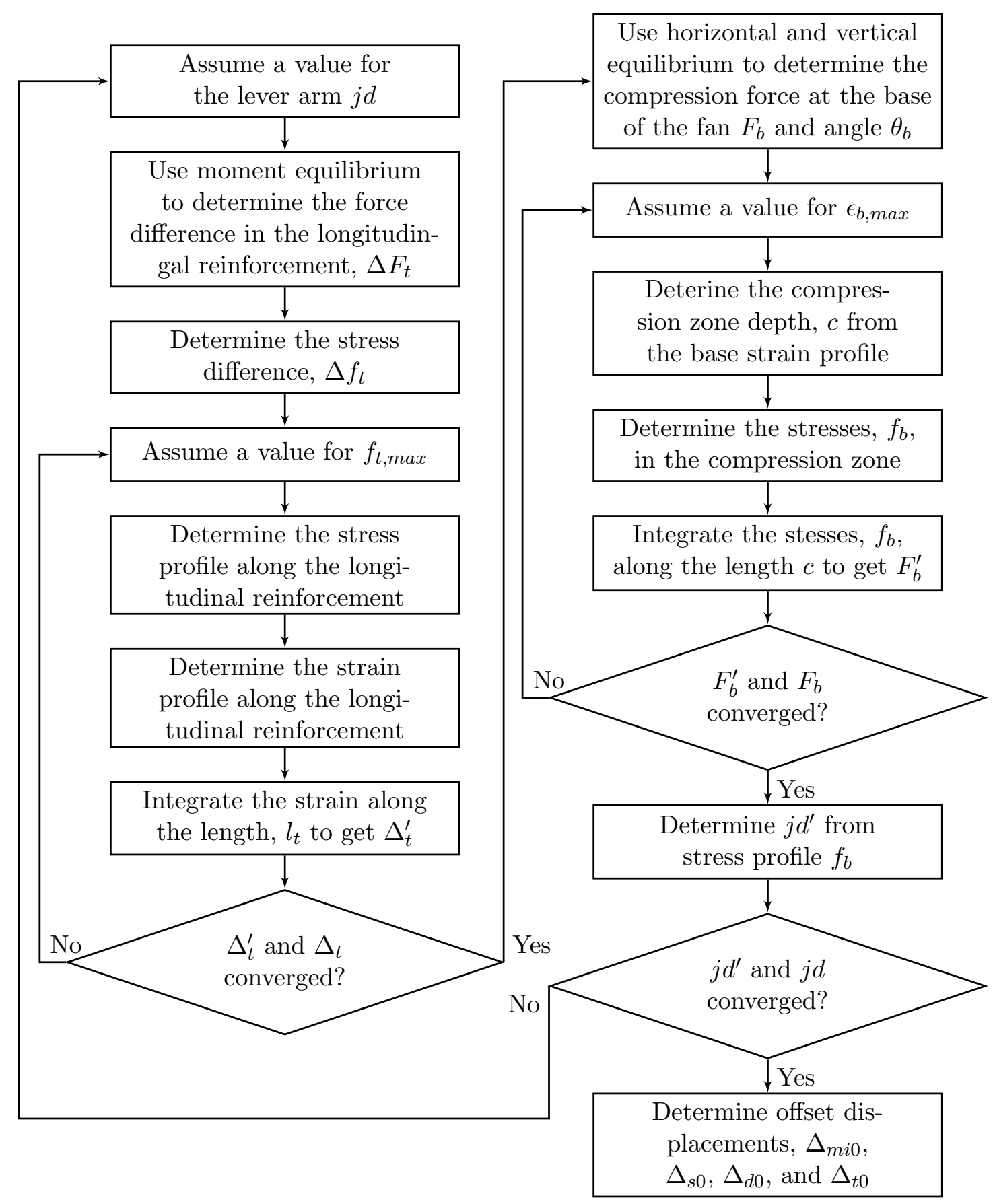

Figure 18.13: Fan Calculation Steps 


\section{IMPLEMENTATION OF 3PKT FOR REINFORCED MA- SONRY SHEAR WALLS}

The theory developed in Chapter 18 is implemented in $\mathrm{C}++$ allowing for the spring forces and shear components to be determined.

The nine spring model is solved under increasing deflection $\Delta$ to obtain the complete shear force versus deflection response for the cantilevered masonry shear wall. At a given $\Delta$ an iterative solution is used to determine the corresponding shear force. A progressively refined spring stiffness for each spring is determined at each iteration, $j$, until convergence of the spring stiffness is achieved and the corresponding shear force determined.

\subsection{Spring Stiffness's}

Each spring is given an initial stiffness that is continually updated according to the given material models.

\subsubsection{Aggregate Interlock Spring Stiffness}

The initial stiffness of the aggregate interlock spring is given by:

$$
k_{m i 0}=\frac{F_{m i 0}}{0.1}
$$

where the initial force, $F_{m i 0}$ is defined as:

$$
F_{m i 0}=\frac{0.18 v_{m i 0} t d_{1}}{\sin \alpha_{1}}
$$

and the initial shear stress, $v_{m i 0}$, is determined according to Equation 18.19 with $w=$ $s=0.1 \mathrm{~mm}$. During the loading of the wall, the stiffness is given by:

$$
k_{m i j}=\left\{\begin{array}{ll}
\frac{F_{m i j}}{\Delta_{m i j}} & \text { if }\left|\Delta_{m i j}\right| \geq 0 \\
0 & \text { otherwise }
\end{array} .\right.
$$




\subsubsection{Transverse Reinforcement Spring Stiffness}

The initial transverse reinforcement spring stiffness is:

$$
k_{s 0}=\frac{E_{s v} A_{v}}{l_{v}}
$$

where $E_{s v}$ is the modulus of elasticity of the transverse reinforcement and $l_{v}$ is equal to $0.9 d_{1}$. During the loading of the wall, the stiffness is given by:

$$
k_{s j}= \begin{cases}\frac{F_{s j}}{\Delta_{s j}} & \text { if } \Delta_{s j} \geq 0 \\ 0 & \text { otherwise }\end{cases}
$$

\subsubsection{Longitudinal Dowel Action Spring Stiffness}

The initial stiffness of the dowel action spring is given as:

$$
k_{d 0}=\frac{12 E_{s l} I_{d}}{l_{k}^{3}}
$$

where $E_{s l}$ is the modulus of elasticity of the longitudinal reinforcement and $I_{d}$ is the moment of inertia of longitudinal bars in tension equal to:

$$
I_{d}=\frac{n_{b} \pi d_{b}^{4}}{64} .
$$

The stiffness of the longitudinal dowel action spring during the loading of the wall is:

$$
k_{d j}=\left\{\begin{array}{ll}
\frac{F_{d j}}{\Delta_{d j}} & \text { if } \Delta_{d j} \geq 0 \\
0 & \text { otherwise }
\end{array} .\right.
$$

\subsubsection{Compression in the Masonry Spring Stiffness's}

The initial stiffness of $k_{C L Z 1}$ and $k_{C L Z 2}$ is defined as:

$$
k_{C L Z 10}=k_{C L Z 20}=E_{m} t \tan \alpha .
$$

The stiffness of these two springs during loading is:

$$
k_{C L Z 1 j}= \begin{cases}\frac{F_{C L Z 1 j}}{\Delta_{C L Z 1 j}} & \text { if } \Delta_{C L Z 2 j} \geq \Delta_{C L Z 1 j} \tan \frac{\alpha}{2} \text { and } \Delta_{C L Z 2 j} \geq-\Delta_{C L Z 1 j} \tan \frac{\alpha}{2} \\ k_{C L Z 10} & \text { otherwise }\end{cases}
$$


and

$$
k_{C L Z 2 j}= \begin{cases}\frac{F_{C L Z 2 j}}{\Delta_{C L Z 2 j}} & \text { if } \Delta_{C L Z 2 j} \geq \Delta_{C L Z 1 j} \tan \frac{\alpha}{2} \text { and } \Delta_{C L Z 2 j} \geq-\Delta_{C L Z 1 j} \tan \frac{\alpha}{2} \\ k_{C L Z 20} & \text { otherwise }\end{cases}
$$

\subsubsection{Contact Spring Stiffness's}

The initial stiffness of the contact spring normal to the direction of the critical crack is:

$$
k_{c n 0}=E_{m} t \tan \alpha_{1}
$$

and the tangential spring:

$$
k_{c t 0}=0
$$

While loading the stiffness's become:

$$
k_{c n j}= \begin{cases}0 & \text { if } \Delta_{c n j}>0 \\ k_{c n 0} & \text { otherwise }\end{cases}
$$

and

$$
k_{c t j}=\frac{0.7}{\max \left(\left|\Delta_{c t j}\right|, 0.1\right)}
$$

\subsubsection{Longitudinal Reinforcement in CLZ Spring Stiffness}

The longitudinal reinforcement within the CLZ is given an initial stiffness of:

$$
k_{l, C L Z 0}=\frac{E_{l, C L Z} A_{l, C L Z}}{l_{b 1 e}}
$$

and during loading:

$$
k_{l, C L Z_{j}}=\frac{F_{l, C L Z_{j}}}{\Delta_{l, C L Z_{j}}}
$$

where

$$
\Delta_{l, C L Z j}=\Delta_{c y}
$$




\subsubsection{Longitudinal Reinforcement Tension Spring Stiffness}

The initial stiffness of the longitudinal reinforcement tension spring is:

$$
k_{l 0}=\frac{E_{s l} A_{s}}{l_{t}}
$$

and during loading:

$$
k_{l j}= \begin{cases}\frac{F_{t, \min }}{\Delta_{t}-\Delta_{t 0}} & \text { if } \Delta_{t}>\Delta_{t 0} \\ 0 & \text { otherwise }\end{cases}
$$

\subsubsection{Stiffness Matrix}

The stiffness of each springs can be combined to give the total spring stiffness in for each of the DOFs $\Delta, \Delta_{c x}$, and $\theta$. The stiffness matrix $[k]$ is defined as:

$$
[k]=\left[\begin{array}{lll}
k_{11} & k_{12} & k_{13} \\
k_{21} & k_{22} & k_{23} \\
k_{31} & k_{32} & k_{33}
\end{array}\right]
$$

where

$$
\begin{gathered}
k_{11=} k_{C L Z 1 j} \cos ^{2}\left(\frac{\alpha}{2}\right)+k_{C L Z 2 j} \sin ^{2}\left(\frac{\alpha}{2}\right)+ \\
+k_{m i j} \sin ^{2}\left(\alpha_{1}\right)+k_{d j}+k_{s j} \\
+k_{c n j} \cos ^{2}\left(\alpha_{1}\right)+k_{c t j} \sin ^{2}\left(\alpha_{1}\right), \\
k_{12}=-k_{C L Z 1 j} \cos \left(\frac{\alpha}{2}\right) \sin \left(\frac{\alpha}{2}\right)+k_{C L Z 2 j} \sin \left(\frac{\alpha}{2}\right) \cos \left(\frac{\alpha}{2}\right) \\
+k_{m i j} \sin \left(\alpha_{1}\right) \cos \left(\alpha_{1}\right)-k_{c n j} \cos \left(\alpha_{1}\right) \sin \left(\alpha_{1}\right)+k_{c t j} \sin \left(\alpha_{1}\right) \cos \left(\alpha_{1}\right), \\
k_{13=-} k_{C L Z 1 j} a \cos ^{2}\left(\frac{\alpha}{2}\right)-k_{C L Z 2 j} a \sin ^{2}\left(\frac{\alpha}{2}\right) \\
-k_{m i j} a \sin ^{2}\left(\alpha_{1}\right)-k_{d j}\left(a-l_{t}\right)-k_{s j}\left(a-\frac{d_{1}}{\tan \alpha_{3}}\right) \\
-k_{c n j} a \cos ^{2}\left(\alpha_{1}\right)-k_{c t j} a \sin ^{2}\left(\alpha_{1}\right), \\
k_{21}=k_{12}, \\
k_{22}=k_{C L Z 1 j} \sin ^{2}\left(\frac{\alpha}{2}\right)+k_{C L Z 2 j} \cos ^{2}\left(\frac{\alpha}{2}\right)+k_{m i j} \cos ^{2}\left(\alpha_{1}\right)+k_{l j} \\
+k_{c n j} \sin ^{2}\left(\alpha_{1}\right)+k_{c t j} \cos ^{2}\left(\alpha_{1}\right)+k_{l, C L Z},
\end{gathered}
$$




$$
\begin{gathered}
k_{23}=k_{C L Z 1 j} a \sin \left(\frac{\alpha}{2}\right) \cos \left(\frac{\alpha}{2}\right)-k_{C L Z 2 j} a \cos \left(\frac{\alpha}{2}\right) \sin \left(\frac{\alpha}{2}\right) \\
-k_{m i j} a \cos \left(\alpha_{1}\right) \sin \left(\alpha_{1}\right)-k_{l j} d \\
+k_{c n j} a \sin \left(\alpha_{1}\right) \cos \left(\alpha_{1}\right)-k_{c t j} a \cos \left(\alpha_{1}\right) \sin \left(\alpha_{1}\right), \\
k_{31}=k_{13}, \\
k_{32}=k_{23}, \text { and } \\
k_{33}=k_{C L Z 1 j}\left[a \cos \left(\frac{\alpha}{2}\right)\right]^{2}+k_{C L Z 2 j}\left[a \sin \left(\frac{\alpha}{2}\right)\right]^{2}+k_{m i j}\left[a \sin \left(\alpha_{1}\right)\right]^{2} \\
+k_{d j}\left(a-l_{t}\right)^{2}+k_{s j}\left(a-\frac{d_{1}}{\tan \alpha_{3}}\right)^{2}+k_{l j} d^{2} \\
+k_{c n j}\left[a \cos \left(\alpha_{1}\right)\right]^{2}+k_{c t j}\left[a \sin \left(\alpha_{1}\right)\right]^{2} .
\end{gathered}
$$

\subsection{Three Degrees of Freedom}

As the displacement $\Delta$ is imposed at each load step a reduced stiffness matrix, $\left[k_{r}\right]$ can be used to solve for the two remaining DOFs. The solution is based on the stiffness of the springs and takes into account the offset displacements due to deformations in the fan; thus,

$$
\left\{\begin{array}{c}
\Delta_{c x} \\
\theta
\end{array}\right\}=\left[k_{r}\right]^{-1}\left\{\begin{array}{c}
F_{\Delta c x} \\
F_{\theta}
\end{array}\right\}
$$

where

$$
\begin{gathered}
{\left[k_{r}\right]=\left[\begin{array}{ll}
k_{22} & k_{23} \\
k_{32} & k_{33}
\end{array}\right]} \\
F_{\Delta c x}=N-k_{21} \Delta+k_{m i j} \Delta_{m i 0} \cos \left(\alpha_{1}\right)-k_{l j} \Delta_{t 0}, \text { and } \\
F_{\theta}=-N \frac{l_{w}}{2}-k_{31} \Delta-k_{m i j} \Delta_{m i 0} a \sin \left(\alpha_{1}\right)-k_{d j} \Delta_{d 0}\left(a-l_{t}\right) \\
-k_{s j} \Delta_{s 0}\left(a-\frac{d_{1}}{\tan \alpha_{3}}\right)+k_{l j} \Delta_{t 0} d .
\end{gathered}
$$




\subsection{Transformation Matrix}

The transformation matrix links the displacements in the springs to the three DOFs $\Delta$, $\Delta_{c x}$, and $\theta$; though:

$$
\left\{\begin{array}{c}
\Delta_{C L Z 1 j} \\
\Delta_{C L Z 2 j} \\
\Delta_{m i j}+\Delta_{m i 0 j} \\
\Delta_{d j}+\Delta_{d 0 j} \\
\Delta_{s j}+\Delta_{s 0 j} \\
\Delta_{t j} \\
\Delta_{c n j} \\
\Delta_{c t j} \\
\Delta_{l C L Z}
\end{array}\right\}=\left[\begin{array}{ccc}
\cos \frac{\alpha}{2} & -\sin \frac{\alpha}{2} & -a \cos \frac{\alpha}{2} \\
\sin \frac{\alpha}{2} & \cos \frac{\alpha}{2} & -a \sin \frac{\alpha}{2} \\
\sin \alpha_{1} & \cos \alpha_{1} & -a \sin \alpha_{1} \\
1 & 0 & l_{t}-a \\
1 & 0 & -\left(a-\frac{d_{1}}{\tan \alpha_{3}}\right) \\
0 & -1 & d \\
\cos \alpha_{1} & -\sin \alpha_{1} & -a \cos \alpha_{1} \\
\sin \alpha_{1} & \cos \alpha_{1} & -a \sin \alpha_{1} \\
0 & 1 & 0
\end{array}\right]\left\{\begin{array}{c}
\Delta \\
\Delta_{c x} \\
\theta
\end{array}\right\} .
$$

\subsection{Converged Load Step Calculations}

For each iteration, $j$, the convergence of the spring's stiffness is used to determine if the load stage has converged for the given displacement $\Delta$. The convergence error is determined from:

$$
\text { C.E. }=\sum_{n=1}^{9}\left(k_{n, j-1}-k_{n, j}\right)^{2}
$$

where $n$ represents each of the nine springs and $j-1$ is the stiffness from the previous iteration. Convergence can be achieved when the convergence error is less than or equal to a given limit, i.e. 0.01 or less.

\subsubsection{Shear Components}

Upon convergence of the spring stiffness's the components of shear resistance and total shear resistance can be determined. The shear forces are the horizontal components of the spring forces. The total shear resistance is determined from:

$$
V=V_{m i}+V_{C L Z}+V_{s}+V_{d}+V_{c}
$$

where the total shear is the summation of the shear carried by aggregate interlock, $V_{m i}$, within the CLZ, $V_{C L Z}$, carried by the stirrups, $V_{s}$, in the dowel action of the longitudinal reinforcement, $V_{d}$, and by the contact springs, $V_{c}$. These shears are defined as follows:

$$
V_{m i}=F_{m i} \sin \alpha_{1}
$$




$$
\begin{gathered}
V_{C L Z}=F_{C L Z 1} \cos \left(\frac{\alpha}{2}\right)+F_{C L Z 2} \sin \left(\frac{\alpha}{2}\right), \\
V_{s}=F_{s}, \\
V_{d}=F_{d}, \text { and } \\
V_{c}=F_{c n} \cos \alpha_{1}+F_{c t} \sin \alpha_{1} .
\end{gathered}
$$

\subsubsection{Moment}

The moment at the base of the wall can be determined from:

$$
M=V a-N \frac{l_{w}}{2}-F_{d} \frac{d}{\tan \alpha_{1}}-F_{s} \frac{d_{1}}{2} \frac{1}{\tan \alpha_{1}}-F_{t, \min } d .
$$

\subsection{Solution Procedure}

The 3PKT is implemented in the three-parameter kinematic model for grouted masonry shear walls with the solution procedure summarized by the flow chart in Figure 19.1. 

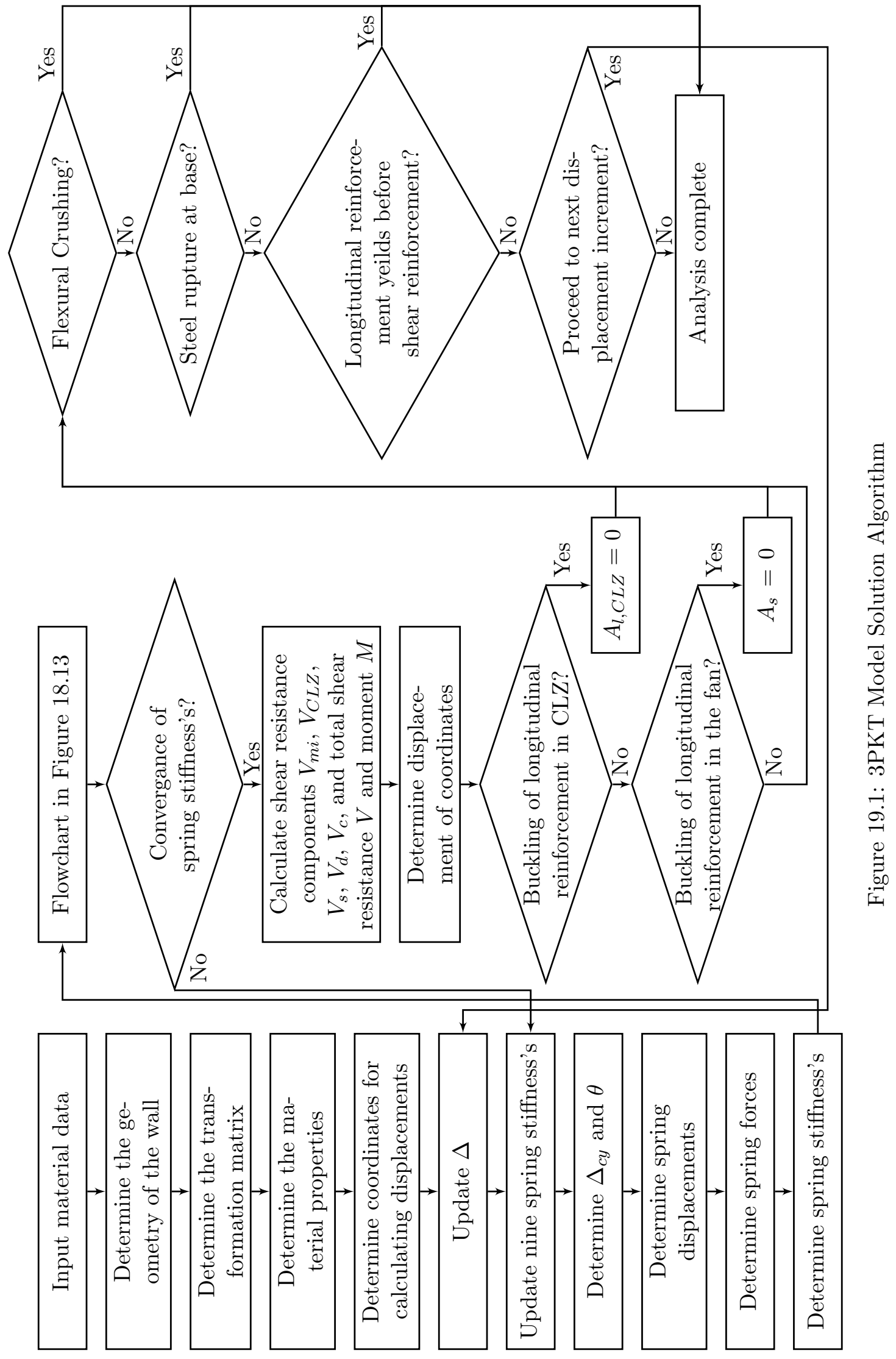

Part IV: Masonry Displacement Based Modeling 


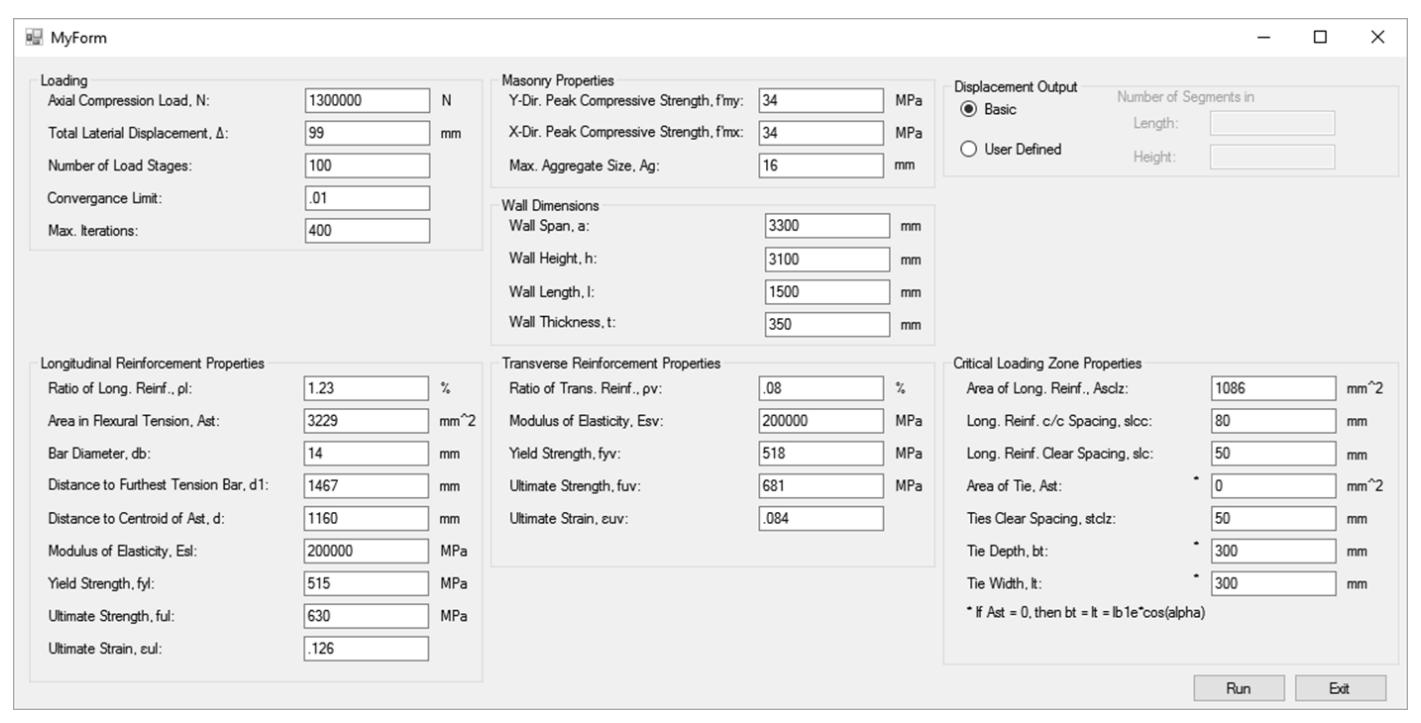

Figure 19.2: 3PKT Program Main Window

\subsection{PKT Procedure}

The 3PKT for masonry reinforced shear walls is programmed in $\mathrm{C}++$, using the Eigen Library (Eigen) for matrix manipulation, with a simple input form for input model properties (Figure 19.2). When running the program displaces convergence values and converged load stage data is stored in three separate files including model data in comma separated values, displacement data, and longitudinal reinforcement data for further analysis. 


\section{PKM VERIFICATION}

The theory developed in Chapter 18 and implemented in Chapter 19 is verified by comparison to a similar model for concrete.

The 3PKM for single pier reinforced masonry shear walls is programmed in $\mathrm{C}++$ using the Eigen Library for matrix manipulation (Eigen). This code implementation, with a few modifications, can be verified by comparing its results to that produced by the 3PKT for shear-dominated reinforced concrete walls (Mihaylov et al., 2016). The wall shown in Figure 20.1 will be modeled in both implementations and the results compared in the following sections.

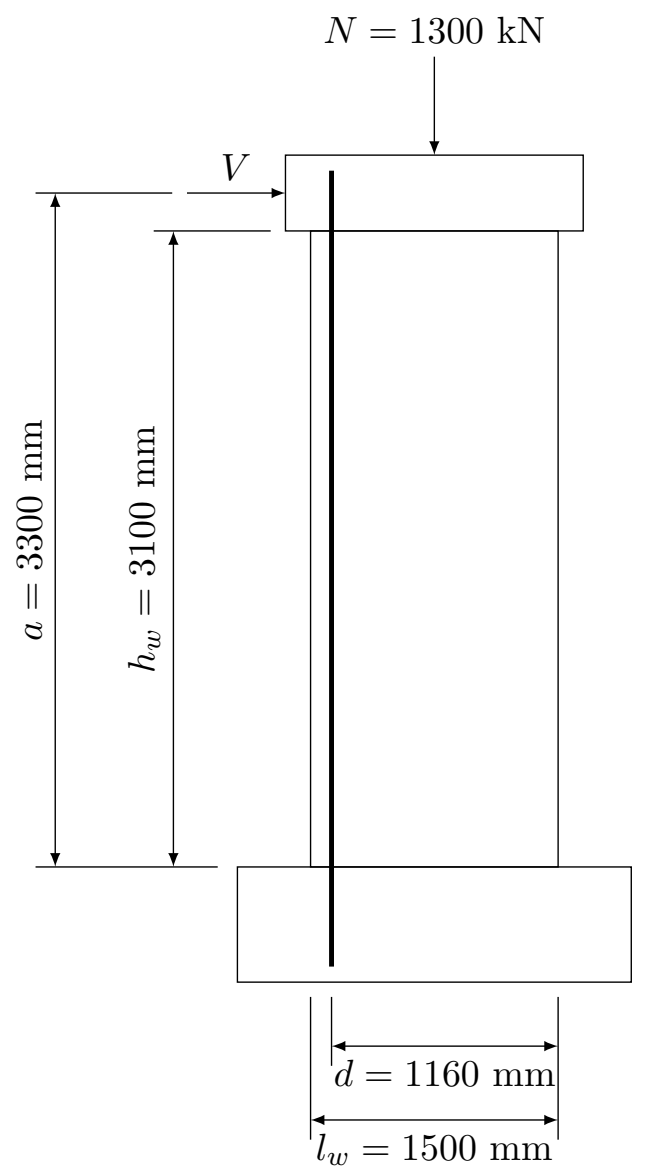

Masonry/Concrete Properties

$t_{w}=350 \mathrm{~mm}$

$f_{m}^{\prime}=34 \mathrm{MPa}$

$a_{g, \max }=16 \mathrm{~mm}$

Longitudinal Reinforcement Properties $\rho=1.23 \%$

$A_{s}=3229 \mathrm{~mm}^{2}$

$d_{b}=14 \mathrm{~mm}$

$d_{1}=1467 \mathrm{~mm}$

$E_{s}=200000 \mathrm{MPa}$

$f_{y}=515 \mathrm{MPa}$

$f_{u}=630 \mathrm{MPa}$

$\epsilon_{u}=0.126$

Transverse Reinforcement Properties $\rho_{v}=0.08 \%$

$E_{s}=200000 \mathrm{MPa}$

$f_{y}=515 \mathrm{MPa}$

$f_{u}=681 \mathrm{MPa}$

$\epsilon_{u}=0.084$

Critical Loading Zone Properties

$A_{s, C L Z}=3229 \mathrm{~mm}^{2}$

$w^{\prime}=50 \mathrm{~mm}$

$s^{\prime}=50 \mathrm{~mm}$

$b_{c}=300 \mathrm{~mm}$

$d_{c}=300 \mathrm{~mm}$

$A_{t, C L Z}=0 \mathrm{~mm}^{2}$

Figure 20.1: Model Inputs Properties 


\subsection{Shear Response}

The shear resistance of the wall is determined by the summation of the horizontal components of the spring forces. As detailed in Section 19.4.1 this includes the shear carried by aggregate interlock, $V_{m i}$, within the CLZ, $V_{C L Z}$, carried by the stirrups, $V_{s}$, in the longitudinal reinforcement, $V_{d}$, and by the contact springs, $V_{c}$.

\subsubsection{Shear Carried by Aggregate Interlock}

The shear carried by aggregate interlock is determined by the shear stress along the crack, which is a function of the crack width and crack slip, in addition to the material compression strength and aggregate size. As the horizontal displacement at the top of the wall increases, both the crack width and crack slip should increase, as shown in Figure 20.2 and Figure 20.3 for crack width and crack slip respectively. Both the 3PTM for masonry and the $3 \mathrm{PKM}$ for concrete produced the same crack widths and crack slip over the entire loading of the wall.

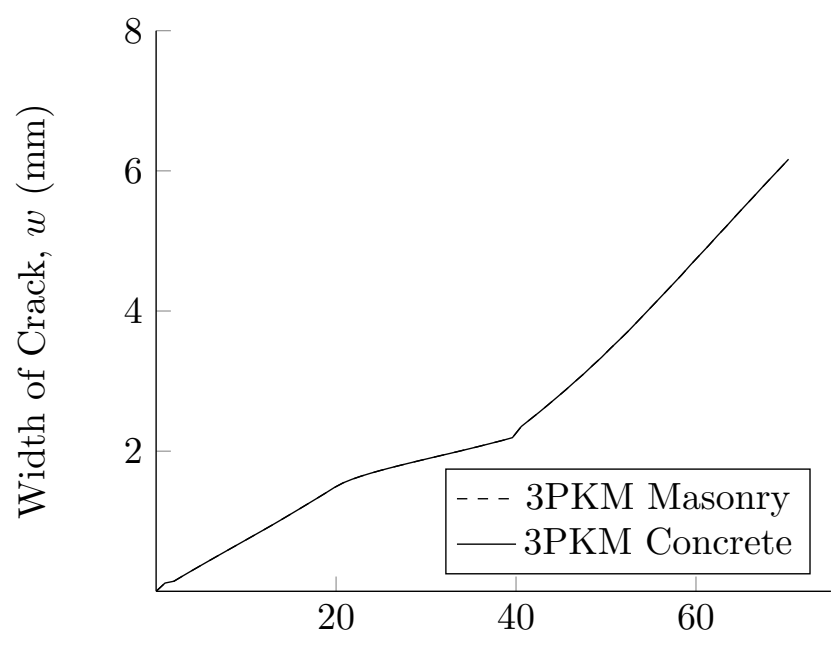

Tip Displacement, $\Delta(\mathrm{mm})$

Figure 20.2: Diagonal Crack Width 


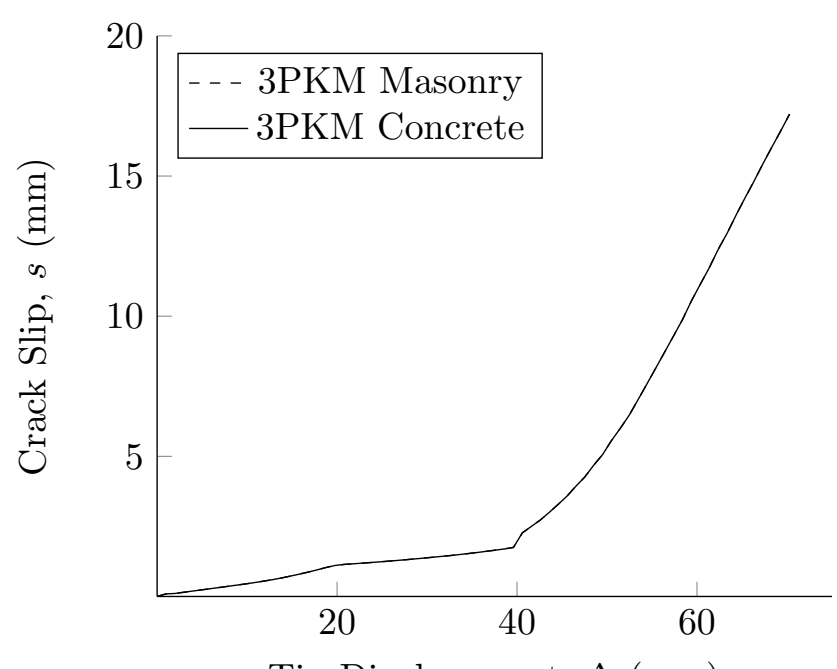

Tip Displacement, $\Delta(\mathrm{mm})$

Figure 20.3: Crack Slip

The next step is to ensure that the contact density model (CDM) proposed by Li et al. (1989) is implemented correctly. This is accomplised by plotting the shear stress at each load step, as shown in Figure 20.4. Both the 3PKM for masonry and concrete produce the same shear stress along the crack for each load step, verifying that the CDM is programmed correctly.

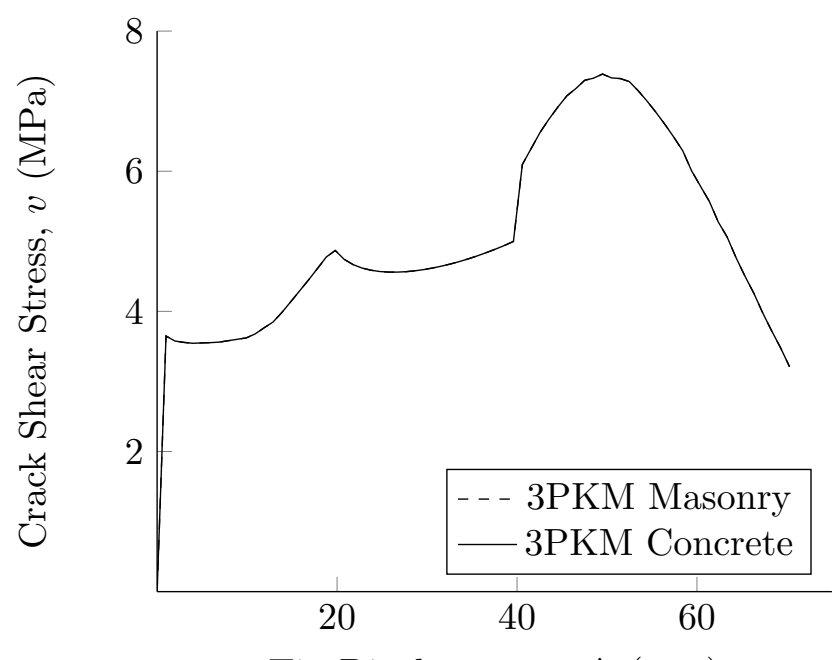

Tip Displacement, $\Delta(\mathrm{mm})$

Figure 20.4: Shear Stress on Diagonal Crack

Finally, the shear carried by aggregate interlock is compared in Figure 20.5 and shown to be the same for both models.

Part IV: Masonry Displacement Based Modeling 


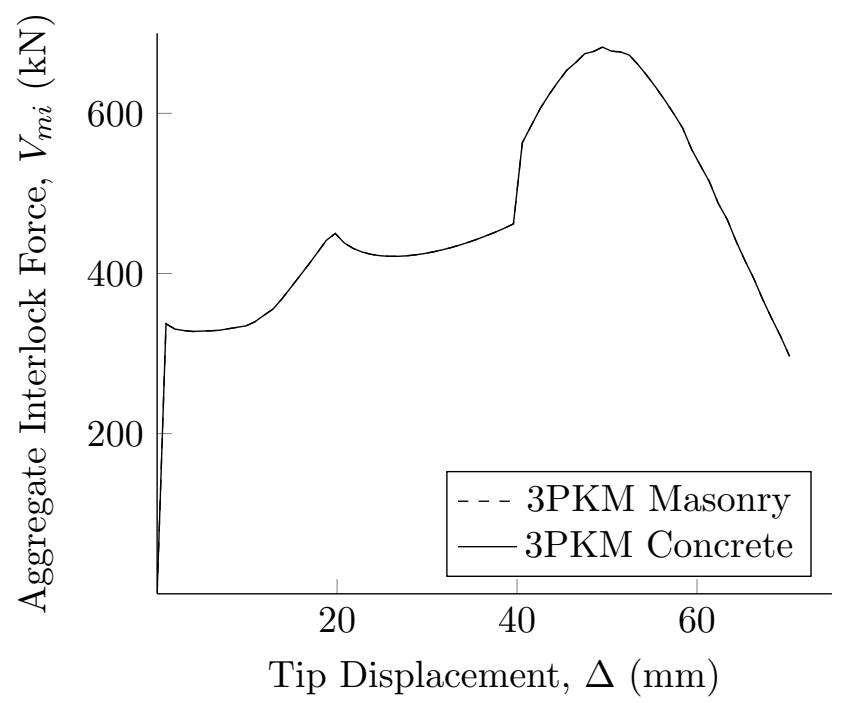

Figure 20.5: Shear in Aggregate Interlock

\subsubsection{Shear Carried by the CLZ}

The shear carried by the critical loading zone (CLZ) is determined by the summation of the horizontal components of the forces $F_{C L Z 1}$ and $F_{C L Z 2}$. Forces $F_{C L Z 1}$ and $F_{C L Z 2}$ are shown in Figure 20.6 and Figure 20.7 respectively and are shown to be the same for both models.

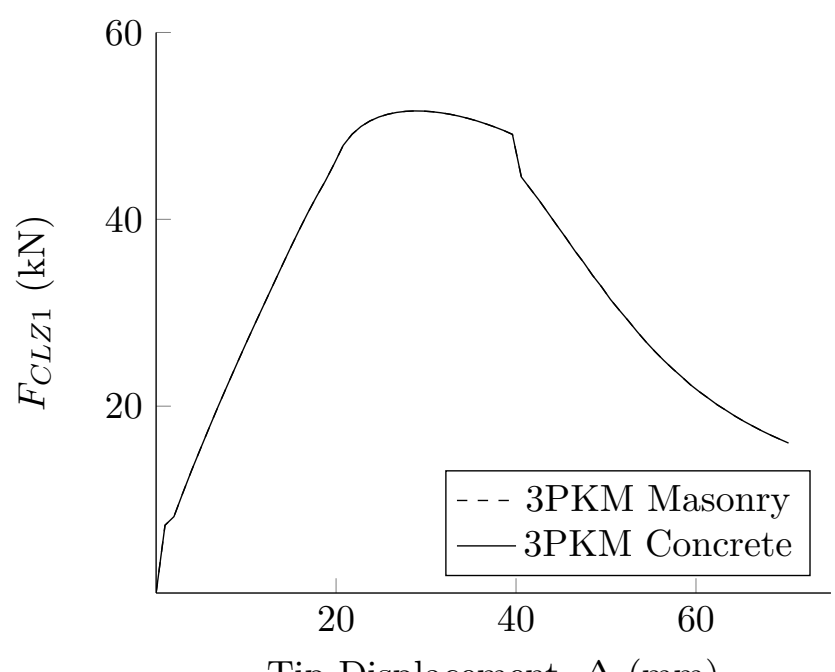

Tip Displacement, $\Delta(\mathrm{mm})$

Figure 20.6: CLZ Force in Direction 1 


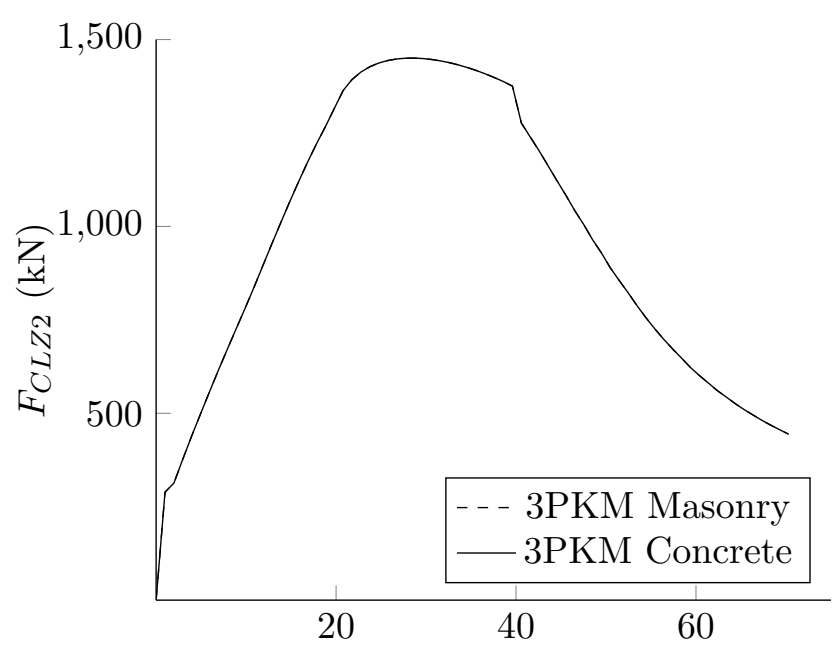

Tip Displacement, $\Delta(\mathrm{mm})$

Figure 20.7: CLZ Force in Direction 2

The 3PKM for masonry and 3PKM for concrete produces the same CLZ shear force as shown in Figure 20.8, meaning that the angle $\alpha$ is accurately calculated in the 3PKM for masonry.

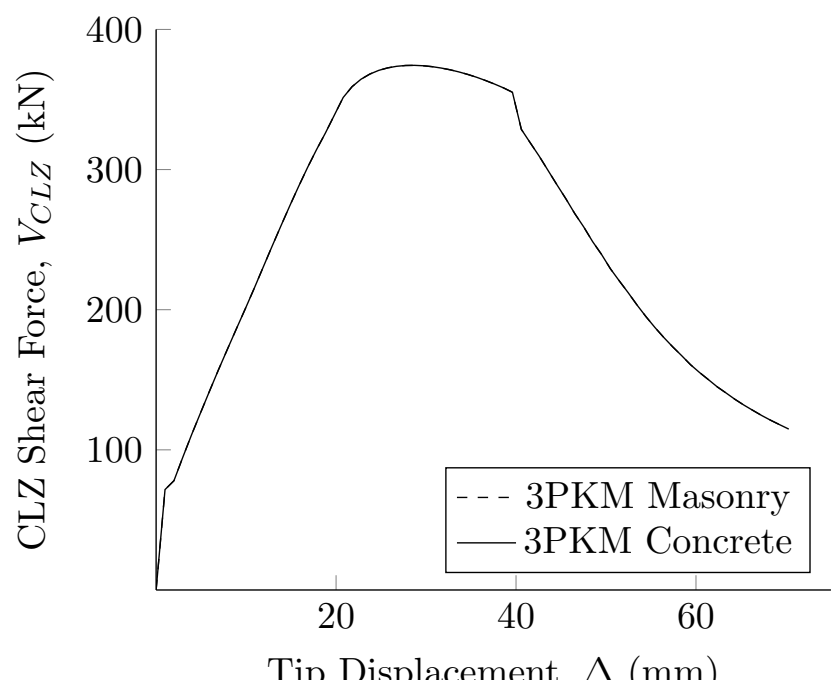

Figure 20.8: Shear in CLZ

\subsubsection{Shear Carried by the Stirrups}

The shear carried by the stirrups is directly related to the force in the spring representing the stirrup force in the 3PKT. As shown in Figure 20.9 the results for the shear carried by the stirrups are the same for both the 3PKM for masonry and concrete. 


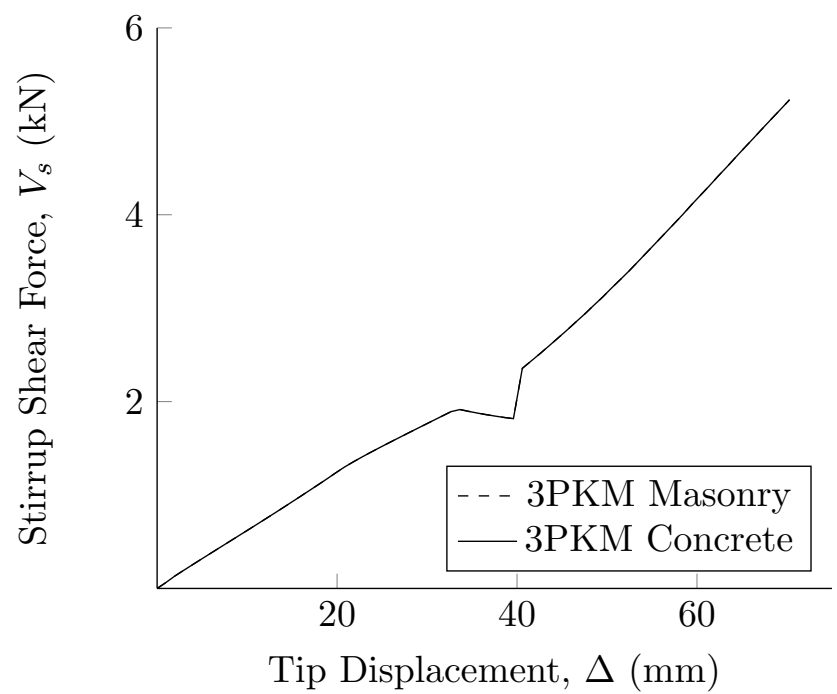

Figure 20.9: Shear in Stirrups

\subsubsection{Shear Carried by the Longitudinal Reinforcement}

There is also shear carried through dowel action in the longitudinal reinforcement. This shear is directly related to the longitudinal dowel force and as shown in Figure 20.10 is the same for both 3PKM for masonry and concrete.

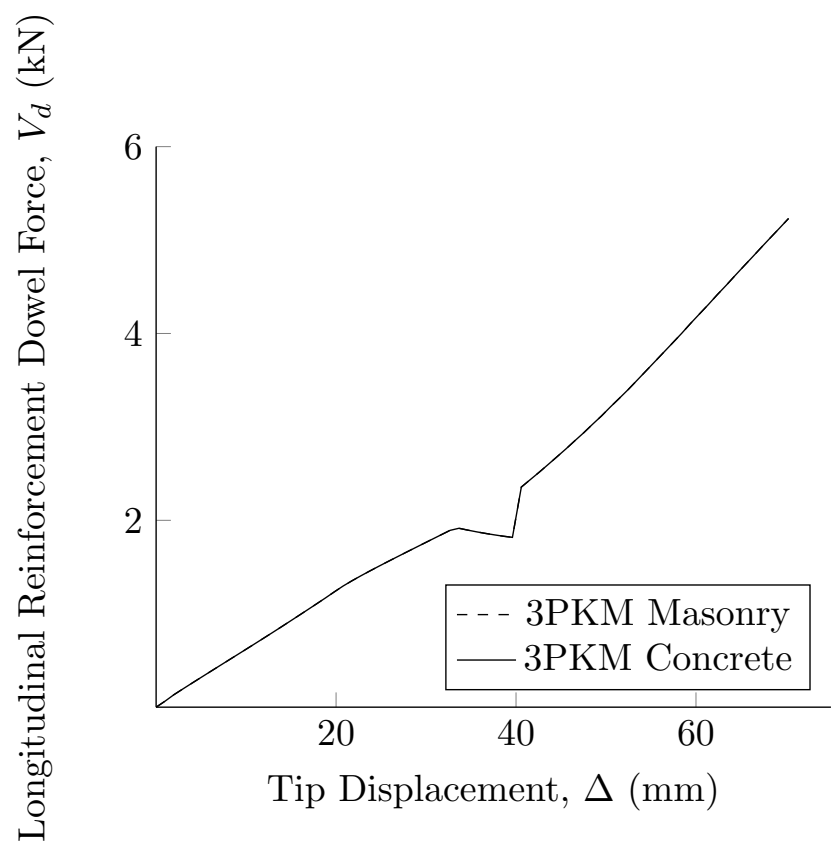

Figure 20.10: Shear in Dowel Action 


\subsubsection{Shear Carried by the Contact Springs}

The shear carried by the contact springs is determined the summation of the horizontal components of the forces $F_{c n}$ and $F_{c t}$. Both 3PKM for masonry and concrete produce the same contact springs shear force as shown in Figure 20.11.

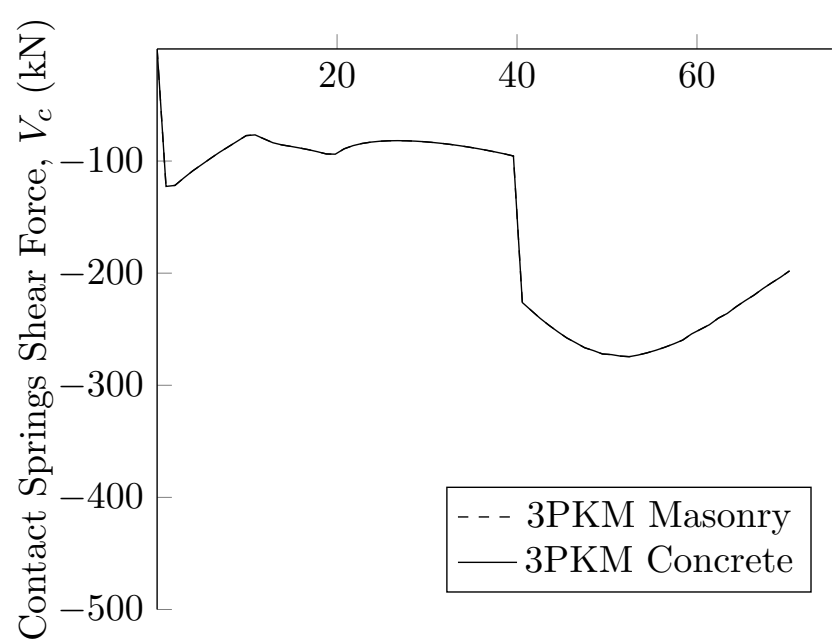

Tip Displacement, $\Delta(\mathrm{mm})$

Figure 20.11: Shear in Contact Springs

\subsubsection{Total Shear}

The total shear resistance is determined from the summation of all the above shear forces:

$$
V=V_{m i}+V_{C L Z}+V_{s}+V_{d}+V_{c}
$$

With all the components of the total shear correctly predicted by the 3PKM for masonry the total shear resistance is also correctly predicted as shown in Figure 20.12. 


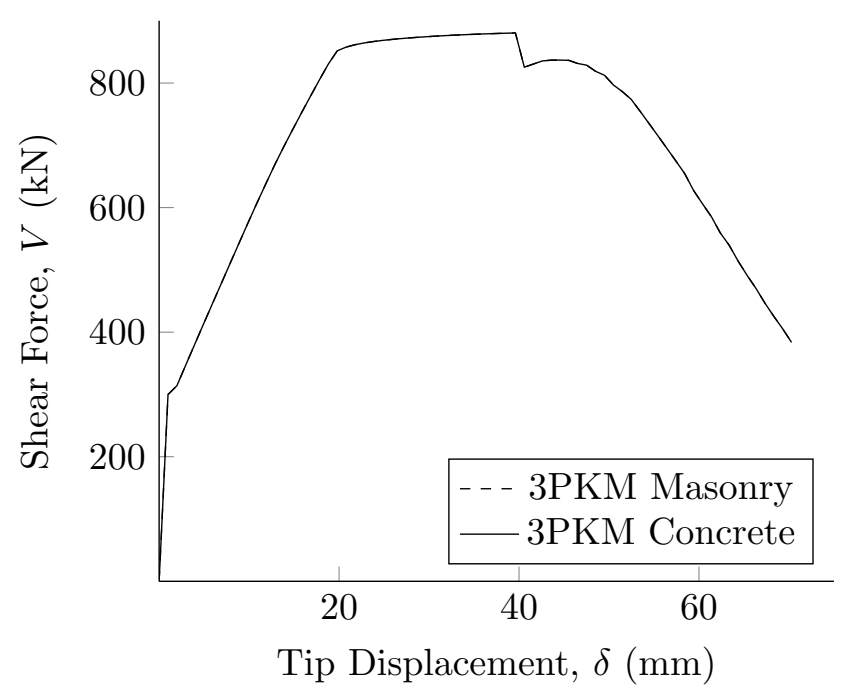

Figure 20.12: Total Shear

\subsection{Longitudinal Steel Reinforcement Response}

The strain profile in the longitudinal reinforcement is important to overall model behaviour as it is the bases for $\epsilon_{t, a v g}$, one of the degrees of freedom used in the 3PKT. The strain profile, as shown in Figure 20.13, is the same for both 3PKM for masonry and concrete. The longitudinal steel stress profile is also important in determining the equilibrium condition of the wall. As can be seen in Figure 20.14 the stress profile for $3 \mathrm{PKM}$ masonry is the same as for 3PKM concrete.

Part IV: Masonry Displacement Based Modeling 


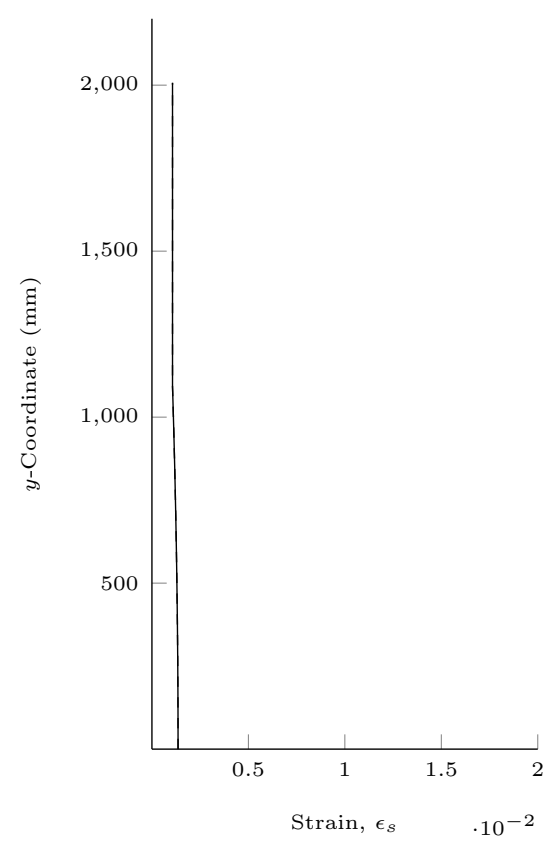

(a) $\Delta=9.9 \mathrm{~mm}$

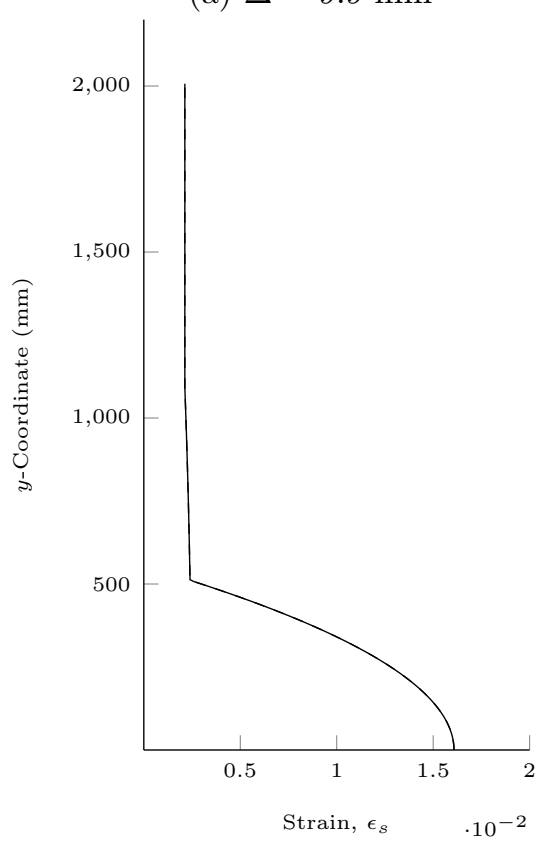

(c) $\Delta=46.53 \mathrm{~mm}$

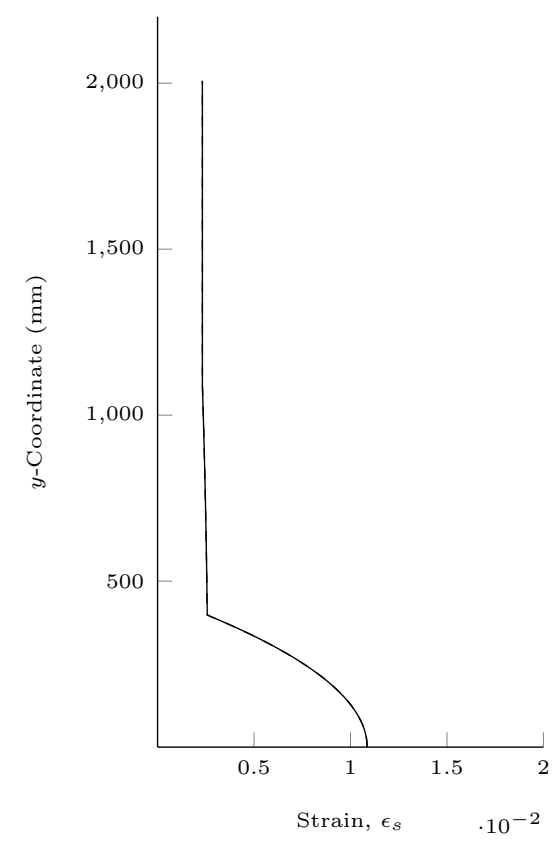

(b) $\Delta=29.7 \mathrm{~mm}$

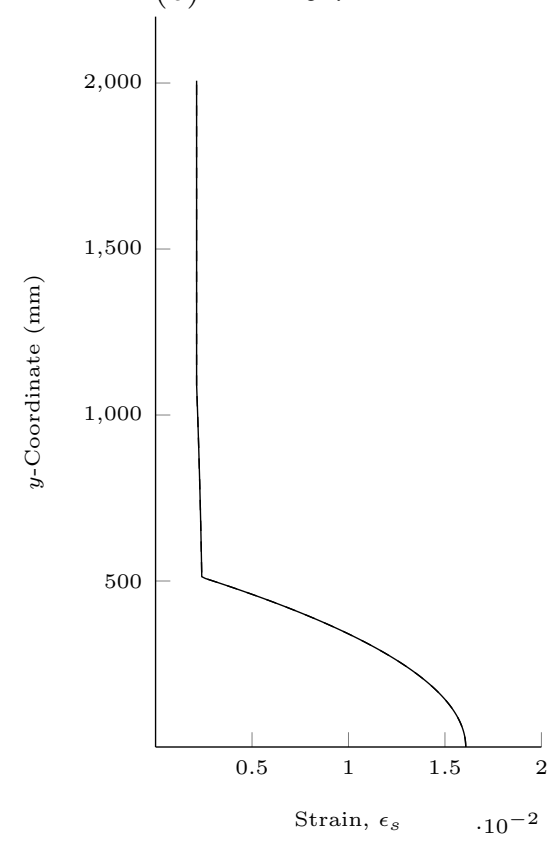

(d) $\Delta=69.3 \mathrm{~mm}$

- - 3PKM Masonry — 3PKM Concrete

Figure 20.13: Longitudinal Steel Strain Comparisons 


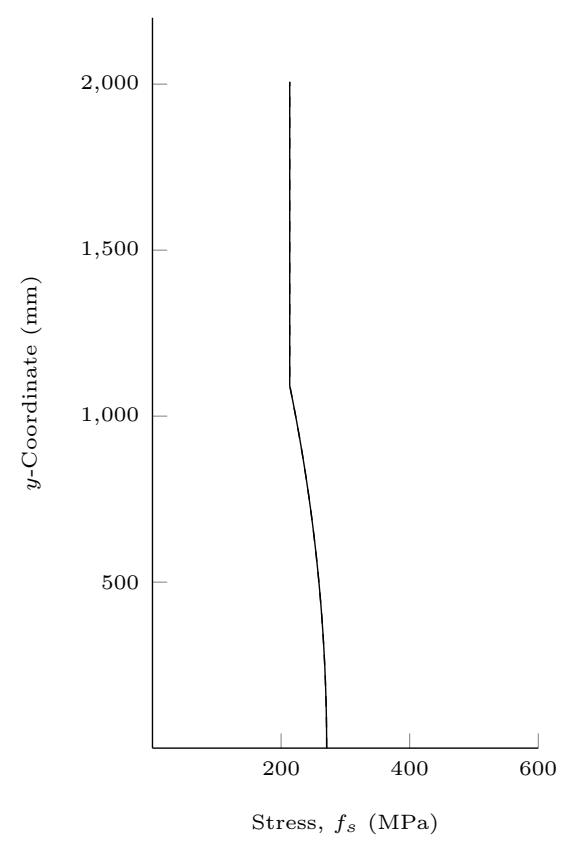

(a) $\Delta=9.9 \mathrm{~mm}$

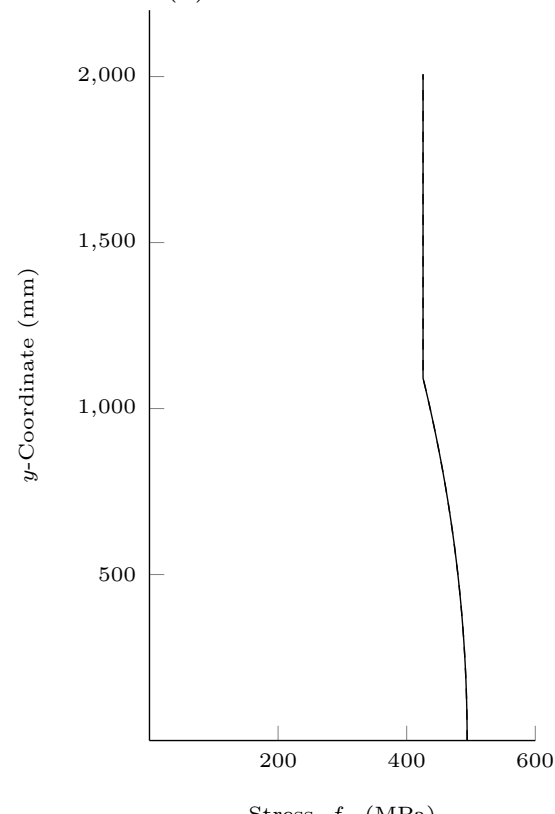

(c) $\Delta=46.53 \mathrm{~mm}$

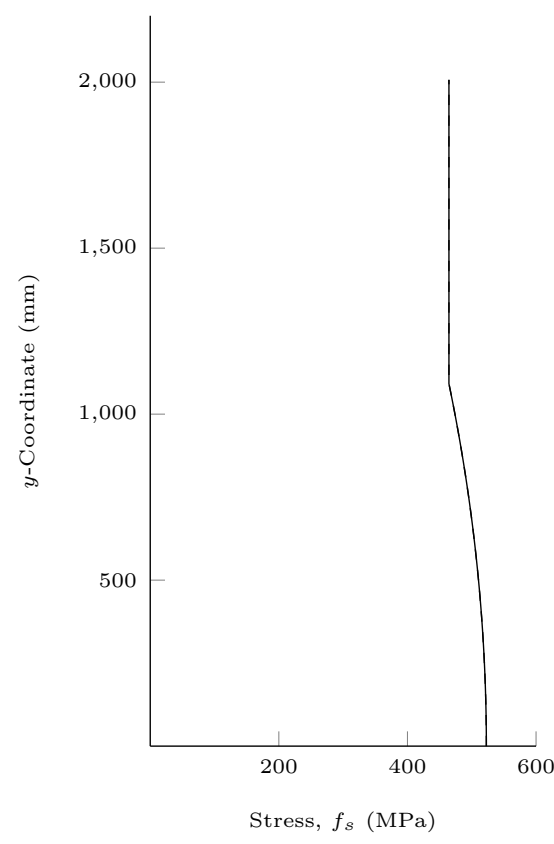

(b) $\Delta=29.7 \mathrm{~mm}$

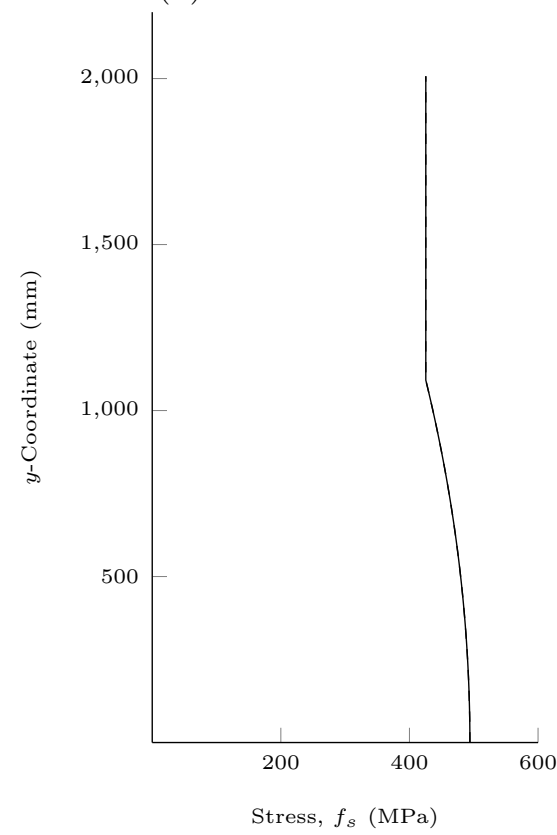

(d) $\Delta=69.3 \mathrm{~mm}$ - - - 3PKM Masonry — 3PKM Concrete

Figure 20.14: Longitudinal Steel Stress Comparisons 


\subsection{Displacement Response}

The displacement at any point within the wall can be determined with the 3PKT as discussed in Section 18.2. The displacements of each wall as determined through both $3 \mathrm{PKM}$ for masonry and 3PKM for concrete were the same. Figure 20.15 shows the displacement of the wall through the rigid block and the fan at various load steps relative to its original position.

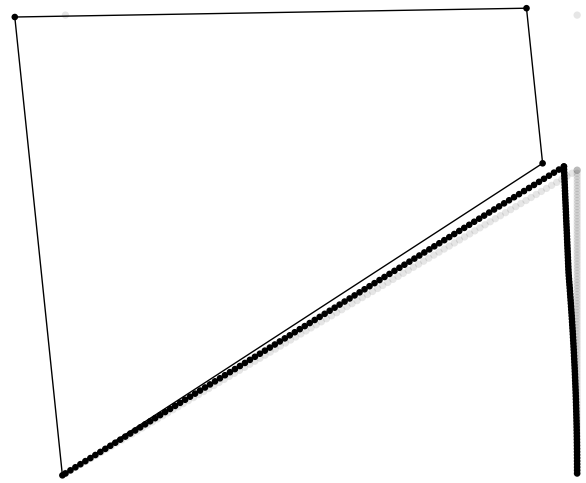

(a) $\Delta=9.9 \mathrm{~mm}$

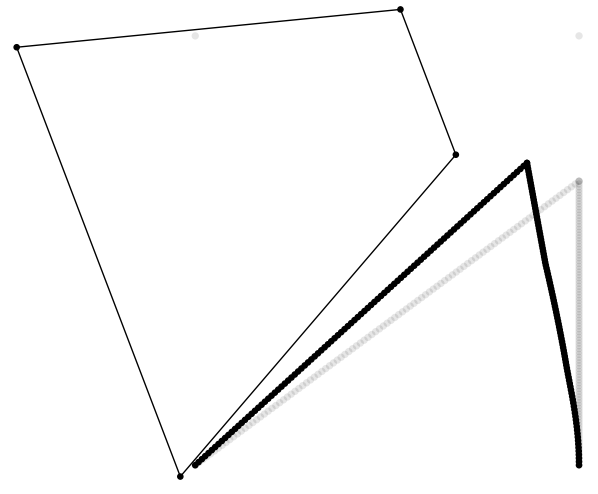

(c) $\Delta=46.53 \mathrm{~mm}$

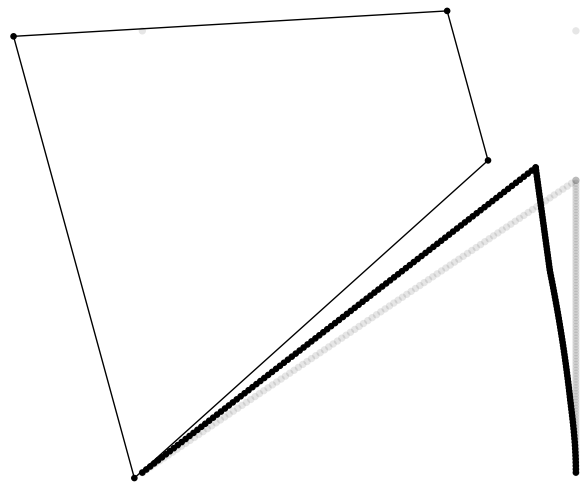

(b) $\Delta=29.7 \mathrm{~mm}$

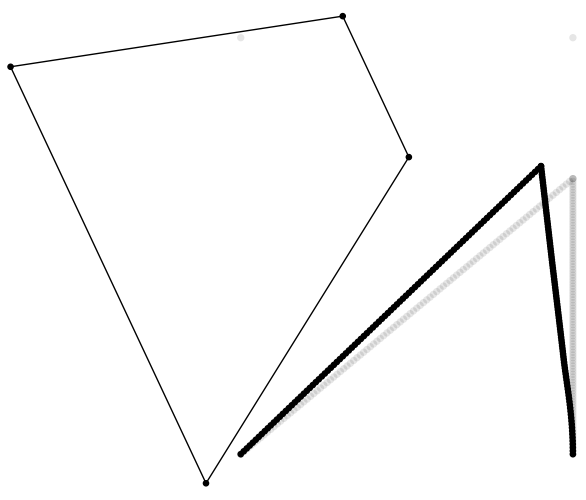

(d) $\Delta=69.3 \mathrm{~mm}$

Figure 20.15: Model Displacements $(\times 15)$ 


\section{COMPARISON OF SINGLE PIER GROUTED SHEAR WALL DATA AND 3PKM}

Results the the theory developed in Chapter 18, implemented in Chapter 19, and verified in Chapter 20 are compared to experimental observed responses from literature.

The database created in Chapter 13 for single pier grouted shear walls can be used to compare the results of the $3 \mathrm{PKM}$ for masonry. For this chapter the database is further filter to include only cantilevered wall specimens.

\subsection{PKM for Masonry Results}

The 3PKT and 3PKM presented in Chapter 18 and 19 is used to compute the complete load displacement response of the walls from the filtered database. The results for all 40 tests to which the model is applicable are shown in Figure 21.1. On the horizontal axis of the plots is the lateral drift $\delta=\Delta / a(\%)$ and on the vertical axis is the lateral load $V(\mathrm{kN})$. The main properties of the walls are also summarized in the plots.

It can be seen from Figure 21.1 that the 3PKT for masonry captures reasonably well the response of most of the walls, except for walls A2, HCBL 3, HCBL 9, HBCL 14, HBCL 16, HBCL 23, HBCL 24, and Wall 6. Significant deviations in terms of strength and/or drift capacity are observed for walls A2, HCBL 3, HCBL 9, HBCL 14, HBCL 16, HBCL 23, HBCL 24, and Wall 6.

Wall A2 is predicted to exhibit a sudden loss of lateral resistance triggered by rupture of the longitudinal steel at the base. Wall A2 failed with half of the total wall displacement resulting from base-course slip; thus, the $3 \mathrm{PKT}$ predicts the failure mode, but at a much smaller displacement.

HCBL 3, HCBL 9, HBCL 14, HBCL 16, HBCL 23, HBCL 24, and Wall 6 failure was predicted to be caused by buckling of the longitudinal bars in the CLZ. While this effect is modeled in a simple and conservative manner in the 3PKT, a more complex model for bar buckling may be necessary to improve the predictions. 
For some of the tests, the model predicted an almost bilinear behaviour while the experiments showed a more gradual loss of stiffness. This difference is explained with the fact that in the model the flexural reinforcement, $A_{s}$, is lumped at its centroid; therefore, all bars are predicted to yield at the same time. In reality, the reinforcement is distributed and yields gradually from the tension edge of the section inwards.

Part IV: Masonry Displacement Based Modeling 

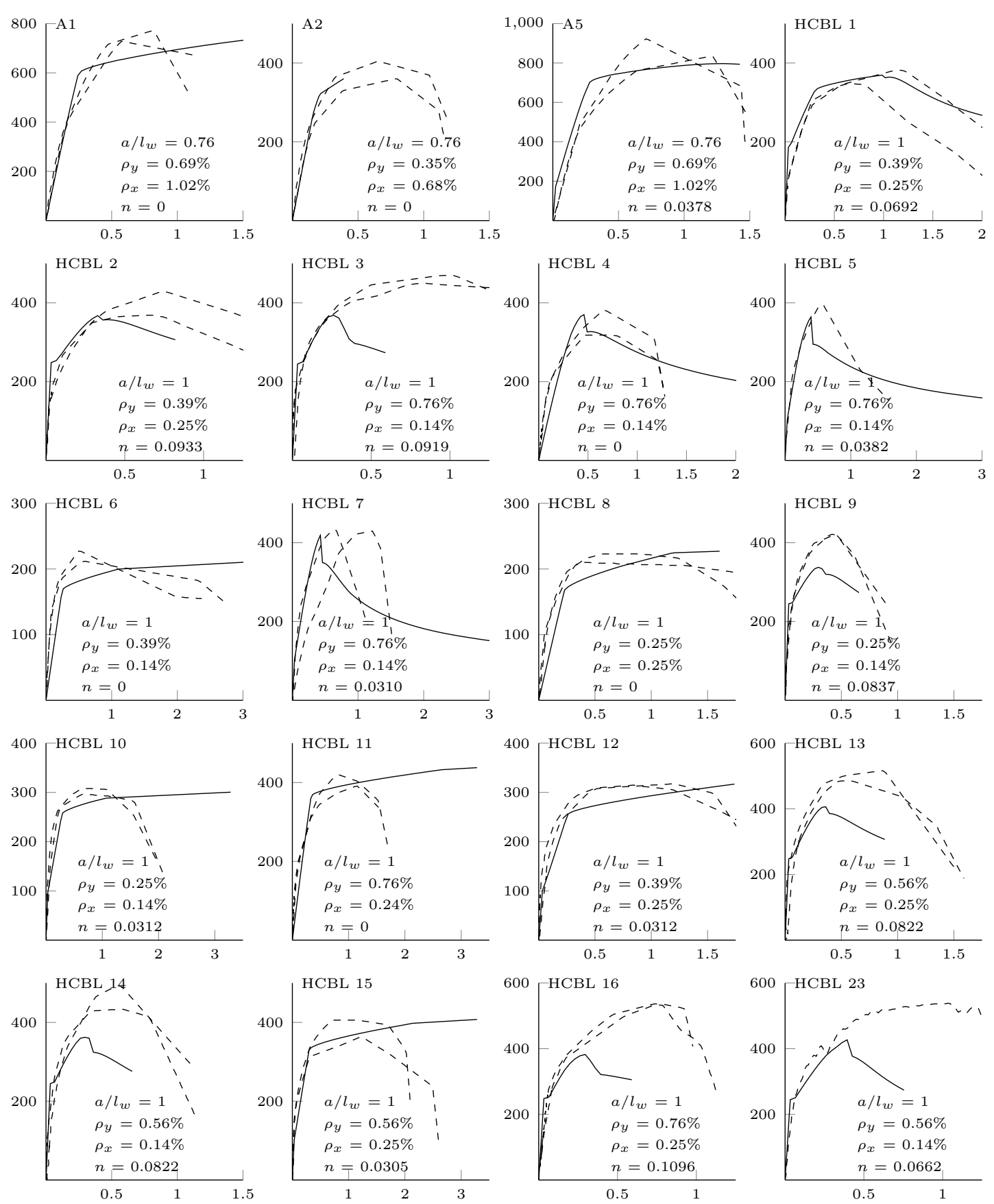

3PKM - - Experiment Envelope +/- Dir.

Figure 21.1: Shear Force $(V, \mathrm{kN})$ Verses Lateral Drift $(\delta=\Delta / a, \%)$ Response of Grouted Walls; Experimental and 3PKM Predictions 

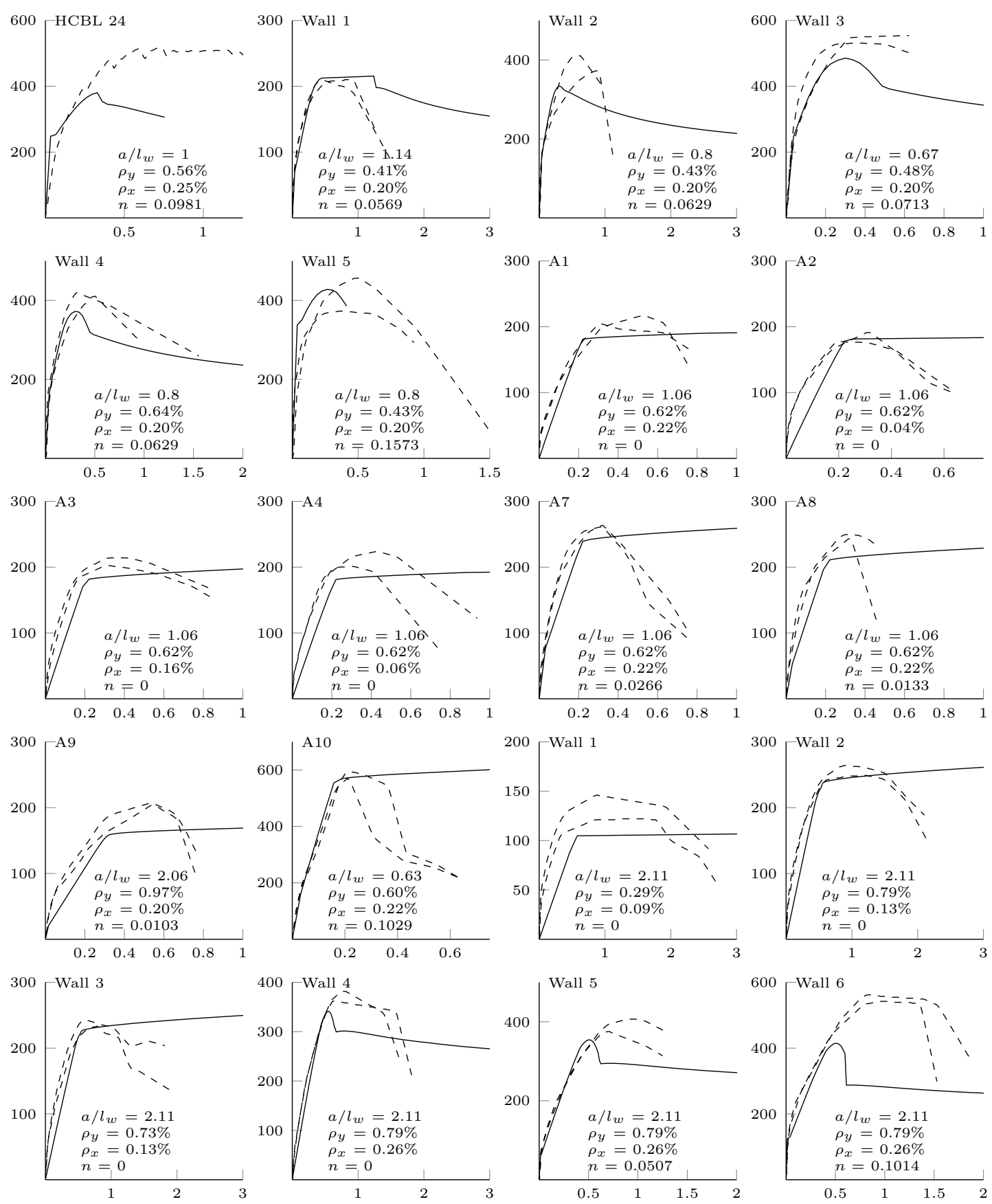

3PKM - - Experiment Envelope +/- Dir.

Figure 21.1: Continued from Previous Page 


\subsection{Discussion}

The 3PKM discussion is divided into pre-peak behaviour and post-peak behaviour in the following subsections.

\subsubsection{Pre-Peak Behaviour}

The 3PKM is able to predict the initial loading for all the walls in the database. This is evident through the initial stiffness of each model prediction. Additionally the 3PKT can predict the load-deflection response up until the peak load with great accuracy.

\subsubsection{Post-Peak Behaviour}

The 3PKM for masonry tends to over estimate the maximum inelastic lateral displacement reached by the wall, $\delta_{\max }$, thus the displacement ductility ratio, $\mu_{\Delta}$, defined as:

$$
\mu_{\Delta}=\frac{\delta_{\max }}{\delta_{y}}
$$

where $\delta_{y}$ is the lateral displacement of the wall at first yielding of the longitudinal reinforcement, is also over estimated. The ductility required of the wall during the response to a major earthquake needs to be matched by the available ductility of the wall; thus, an accurate prediction of the displacement ductility is required to enable designers to ensure that structures have adequate available ductility.

This over estimation in maximum inelastic lateral displacement by the 3PKT for masonry is likely due to a number of reasons:

1. The use of a Popovics (1970) curve to model the compression response of the masonry. Possible a better compression model would be a Hognestad parabola (1951) with a linear descending branch;

2. Masonry walls tend to fail in a more brittle manner when compared to concrete walls because of material compatibility; thus, different material constitutive models may be required;

3. Masonry walls can fail along weaker bed joints, a failure mode not accounted in the 3PKT for masonry; and,

4. Ignoring friction loss caused by sliding along the mortar joint. 


\section{PART IV CONCLUDING REMARKS}

Part IV of thesis presents a three-parameter kinematic model to predict the full load-displacement response of reinforced hollow concrete block masonry shear walls in a timely manner. This chapter presents concluding remarked of Part IV of this thesis, as well as presents a number of items for future research.

Part IV presents a simple model to predict the full load-deflection response of cantilevered reinforced masonry shear walls. The model describes the deformation patterns of diagonally cracked walls with rectangular sections using three degrees of freedom. The three parameter kinematic theory (3PKT) captures in a time-efficient way the complete prepeak and postpeak response of shear dominated walls, including the loss of axial load capacity. The model predicts the response of shear dominated walls with major diagonal cracks that fail either in shear along the diagonal cracks or at the base section under the combined action of flexure and shear. The failure along the diagonal cracks can occur either in a brittle manner prior to yielding of the flexural reinforcement or in a more ductile manner after flexure yielding. The base section of the wall is also modeled to account for yielding of the reinforcement and crushing of the masonry.

The model uses nine nonlinear springs with simple constitutive models to estimate the load-deflection response of the wall. The 3PKT assumes that a shear crack develops which separates the upper nearly uncracked part of the wall, which is regarded as a rigid body, from the radially cracked fan below. The model also includes an area called the critical loading zone (CLZ), which is an area that carries a significant amount of load at the toe of the wall. The wall interacts with the foundation through the forces depicted at the tip of the CLZ.

The three parameter kinematic model is programmed in $\mathrm{C}$ and uses the Eigen Library for matrix manipulation (Eigen). The programming and constitutive models are verified through comparing the results to the concrete three parameter kinematic model (Mihaylov et al., 2016). 


\subsection{Part IV Conclusions}

The three parameter kinematic theory predicts the prepeak response of 40 shear walls with good correlation. The following statements can be made on Part IV of this thesis:

1. Only a few inputs of geometrical wall dimensions and masonry material properties are required to run the model.

2. The model takes only about 5 to 10 seconds to completely run on a typical computer.

3. A model that combines the three degrees of freedom with equations for equilibrium and constitutive relationships can predict the response of grouted shear walls.

4. Nine nonlinear springs can be used to to estimate the load carrying capacity of hollow concrete block masonry shear walls that are reinforced.

5. A simple empirical expression that relates the size of the critical loading zone, a parameter that primarily influences the drift capacity at the onset of shear degradation, to the block height and mortar joint thickness of the wall is proposed.

6. The model implementation is verified by comparing its output to known numerical solutions.

7. The Three Parameter Kinematic Model predicts the total shear load as well as the components of shear resistance and how they vary with increasing vertical deflection at the top of the reinforced hollow concrete block masonry shear wall.

8. The Three Parameter Kinematic Model was generally found to provide close predictions in-terms of the initial stiffness when compared to experimental results. However the failure load and displacement resulted in significant scatter of the results. This load is highly dependent on the displacement field predicted by the model, which needs to include the possibility of slip along the mortar joints.

9. The Three Parameter Kinematic Model is capable of predicting the displacement of the wall, in addition to the stress and strains along longitudinal reinforcement and the nine nonlinear springs.

\subsection{Part IV Future Research}

The 3PKT for masonry tends to over estimate the maximum inelastic lateral displacement. This is likely due to some of the constitutive relationships used within the model, including a constitutive model that incorporates the energy loss due to sliding of the mortar joints. Further research is needed to produce this relationship through experimental tests. 
The theory presented in Part IV of this thesis is applicable to grouted cantilevered masonry shear walls, but there is a possibility of extending the model to different boundary conditions and ungrouted walls. This will involve modifying the constitutive relationships for ungrouted walls and the inclusion of modeling the double curvature for modeling fixed ended walls.

Part IV: Masonry Displacement Based Modeling 


\section{THESIS CONCLUSION}


Part V presents a summary of the thesis, overall conclusions, and an outlook on future research. 


\title{
SUMMARY, CONCLUSIONS AND FUTURE RESEARCH
}

\begin{abstract}
An summary of the three numerical models is given with a discussion on how these models could improve masonry design codes. Overall thesis conclusions are given, as well as the major contributions of the thesis are highlighted. Additionally, potential future work items are given to strength the findings of this thesis.
\end{abstract}

\subsection{Summary}

The analysis of masonry can take many forms, but of most familiarity to engineers is one that uses equilibrium, compatibility, and constitutive relations. This thesis explains the background and theory underpinning a series of three models that use equilibrium, compatibility, and constitution relations to allow for the non linear analysis of ungrouted, grouted, and reinforced masonry prisms, walls, and beams.

The models presented in the thesis are: the Smeared Area Compression Model for determining the compression strength of masonry, the Smeared Area Finite Element Model for determining the load-deflection, stresses and strains, crack widths and patterns of masonry elements, and the Three Parameter Kinematic Model for reinforced masonry shear walls.

Each model is verified against a series of experiments. The experimental data covered a wide range of material properties and specimen sizes used in construction today. These comparisons highlight the ability of each model to predict the response of masonry elements. 
With computers being as fast as they are today, it seems reasonable to suggest that the time to allow engineers to use experimentally verified tools more integrally in the design and analysis of structures has come. It is suggested that international design codes could be changed to directly allow the use of numerical tools that meet a number of requirements. These requirements would be that models:

1. Satisfy equilibrium requirements;

2. Satisfy compatibility requirements;

3. Are based on experimentally verified constitutive relations; and,

4. Have been verified against a large set of data.

It is suggested that the models presented in this thesis represent a good start toward meeting these goals for concrete block masonry design.

\subsection{Conclusions}

The aim of this thesis was to identify and develop models that can be used by practicing engineers for the analysis of hollow concrete block masonry structures. This resulted in three rational material models. A number of simplified equations were presented from these models to replace "rules-of-thumb" equations used in most international masonry design codes.

Part II presents a micro model and simplified equations for predicting compression strength of both ungrouted and grouted masonry. Conclusions of this model are given in Chapter 8.

Part III presents a micro-macro model for implementation in a finite element program. The model is applicable to both ungrouted and reinforced masonry elements. Conclusions of this model are given in Chapter 16 .

Part IV presents a displacement model for predicting the prepeak and postpeak response of cantilevered reinforced masonry shear walls. Conclusions of this model are given in Chapter 22.

Each one of these models is verified against databases of experimental data points gathered from literature and each model shows good correlation to experimental observed responses in-terms of stresses, strains, and displacements. 
Based on the three models presented in this thesis, the following overall conclusions can be made:

1. The anisotropic nature of masonry can be modeled by smearing the area of each component material used to constructed masonry elements, including concrete block, mortar, grout, and reinforcement based on the materials area.

2. Numerical models that uses equilibrium, compatibility, and material constitutive models can predict the response of hollow concrete block masonry and allow for the determination of stresses, strains, and displacements.

3. Unifying the reported uniaxial compressive strength of the concrete block, mortar, and grout allows for comparison of different testing methods.

4. It is possible to predict key masonry properties by simple equations suitable for "back of the envelope" calculations.

Each one of the models furthers the understanding the mechanics of hollow concrete block masonry from the mirco level to the macro level. This ultimately helps engineers to design safer structures and more accurately evaluate the safety of existing construction.

\subsection{Thesis Contributions}

This thesis presents an in-depth look into the mechanics of hollow concrete block behaviour from the micro to the macro level. First, a micro model to predict the compression strength of hollow concrete block masonry is presented; then a micro-macro is presented that allows for the stresses, strains, and displacements of hollow concrete block masonry elements to be determined; finally a displacement-based model is presented using kinematics which allows for the prediction of the full load-displacement response of reinforced hollow concrete block cantilever shear walls to be determined.

This thesis offers an innovate numerical approach to determining the compression strength of hollow concrete block masonry at the micro level. The model uses equilibrium, compatibility, and material constitutive relations to describe the mechanics of hollow concrete block masonry that are either ungrouted or grouted under compression. The model considers modified material configurations to predict the material component stresses and strains accounting for any confinement or softening of the materials due to Poisson effects and differences in stiffnesses. The model accounts for non-linearity using a secant stiffness model. From this model, simplified equations are presented that can predict the compression strength of hollow concrete block masonry and are suitable for implementation in international design codes. 
The success of the compression model, led to the design of a finite element program, that was programmed in $\mathrm{C}++$ with a graphical user interface, for the more general loading of tension, compression and in-plane shear on hollow concrete block masonry that is either ungrouted, grouted, or reinforced. The Smeared Area Finite Element Model is a micro-macro-model that extends the disturbed stress field model (DSFM) developed by Vecchio (2000; 2001) for reinforced concrete to use for ungrouted, grouted, or reinforced hollow concrete block masonry. The model combines material constitutive relations specifically designed to predict the response of hollow concrete block masonry, taking into account the anisotropic nature of masonry caused by mortar joints. The model accounts for the non-linear response of hollow concrete block masonry, in addition to cracking, Poisson effects, and slip along the mortar joints.

Due to the length of time it takes to complete a finite element model a simpler approach was desired to predict the load-deflection response of reinforced masonry shear walls. This lead to the use of the Three Parameter Kinematic Theory (Mihaylov et al., 2016) which was modified in this thesis to account for the specific properties of hollow concrete block masonry mechanics.

Throughout each part a number of equations are proposed to determine parameters that are important to understanding the mechanics of hollow concrete block masonry, such as the modulus of elasticity and tensile strength of material components and the composite material; and the bond strength and cohesion of mortar. Additionally, equations are presented to unify reported compression strength of material components to the in-situ strengths.

\subsection{Future Research}

The future research for the compression model, finite element model, and displacement based model are presented in Chapter 8, 16, and 22 respectively.

While the three models presented in this thesis are seen as being a good start on the provision of a widely available set of non-linear tools to analyses masonry, there are a number of items that can be done to improve them. These are summarized here:

1. Usability improvements to the software developed within this thesis. This mainly includes post processing software to easily interpret the results produced by the models.

Part V: Thesis Conclusion 
2. More numerical verification of models through expansion of the databases and more experimental work. The experimental work can focus on the variables and constitutive relations used within each model.

3. A test program for masonry panels under different combinations of axial and shear loading what will allow for the development of improved constitutive relations. 


\section{REFERENCES}

Andolfato, R. P., Camacho, J. S., and Ramalho, M. A. Brazilian results on structural masonry concrete blocks. ACI Matherials Journal, 104(1):33-39, 2007.

Anthoine, A. In-plane behaviour of masonry: A literature review. Technical report, Commission of the European Communities Joint Research Centre, 1992.

Arya, S. K. and Hegemier, G. A. On non-linear response predicitions of concrete masonry assemblies. In Proceedings of the North American Masonry Conference, pages 19.1$29.24,1978$.

Australian Standard. AS 3700-2011: Masonry structures. Sydney, Australia, 2011.

Barbosa, C. S., Lourehço, P. B., and Hanal, J. B. On the compressive strength prediction for concrete masonry prisms. Materials and Structures, 43(3):331-344, 2010.

Benjamin, R. and Williams, H. A. The behaviour of one-story brick shear walls. Journal of Structural Engineering Division, 84:1-30, 1958.

Boult, B. F. Concrete masonry prism testing. ACI Journal, 76(4):513-536, 1979.

Bridge, R. and Fraser, D. Improved G-factor method for evaluating effective lengths of columns. Journal of Structural Engineering, 113(6):1341-1356, 1987.

British Standards Institution. BS EN 1015-11:1999: Methods of Test for Mortar for Masonry. Determination of Flexural and Compressive Strength of Hardened Mortar. BSI, London, United Kingdom, 1999.

British Standards Institution. BS EN 1996-2:2006: Eurocode 6 - Design of Masonry Structures. Design Considerations, Selection of Materials and Execution of Masonry. BSI, London, United Kingdom, 2006.

British Standards Institution. BS NA EN 1996-1-1:2005: UK National Annex to Eurocode 6. Design of Masonry Structures. General Rules for Reinforced and Unreinforced Masonry Structures. BSI, London, United Kingdom, 2007.

British Standards Institution. BS EN 772-1-2011: Methods of test for Masonry Units. Part 1: Determination of Compressive Strength. BSI, London, United Kingdom, 2011.

Brunner, J. D. and Shing, P. B. Shear strength of reinforced masonry walls. TMS Journal, 14(1):65-77, 1996.

Building Seismic Safety Council. Recommended provisions for seismic regulations for new buildings and other structures, part-1 provisions. Technical report, Washington, D.C., 1997.

Calvi, G. M., Gobetti, A., and Macchi, G. Non conventional finite element model for strengthening of masonry. IABSE Reports, 46:179-186, 1983.

Canadian Standards Association. S304-14: Design of Masonry Structures. CSA, Mississauga, Ontario, Canada, 2014a. 
Canadian Standards Association. A23.3-14: Design of Concrete Structures. CSA, Mississauga, Ontario, Canada, 2014b.

Carrasquillo, R. L., Nilson, A. H., and O., S. F. Properties of high strength concrete subject to short-term loads. ACI Journal, 78(3):195-211, 1981.

Cen, S., Chen, X. M., and Fu, X. R. Quadrilateral membrane element family formulated by the quadrilateral area coordinate method. Computer Methods in Applied Mechanics and Engineering, 196(41-44):4337-4353, 2007.

Cen, S., Zhou, P., Li, C., and Wu, C. An unsymmetric 4-node, 8-dof plane membrane element perfectly breaking through MacNeal's theorem. International Journal for Numerical Methods in Engineering, 103(7):469-500, 2015.

Chahine, G. N. Behaviour characteristics of face shell mortared block masonry under axial compression. MEng thesis, McMaster University, Hamilton, Ontario, Canada, 1989.

Cheema, T. S. and Klingner, R. E. Compressive strength of concrete masonry prisms. ACI Journal, 83(1):88-97, 1986.

Chen, S. J., Hidalgo, P. A., Mayes, R. L., Clough, R. W., and McNiven, H. D. Cyclic loading tests of masonry single piers: Volume 2 - height to width ratio of 1 . Technical Report UCB/EERC-78/28, Earthquake Engineering Research Center, 1978.

Chen, W. and Atsuta, T. Theory of Beam Columns, Volume 1 - In-Plane Behavior and Design. J. Ross Publishing, Inc., Fort Lauderdal, Florida, 2008.

Chen, W. F. Plasticity in Reinforced Concrete. McGrawHill, New York, 1982.

Cheong-Siat-Moy, F. Column design in gravity-loaded frames. Journal of Structural Engineering, 117(5):1448-1461, 1991.

Coleman, J. and Spacone, E. Localization issues in nonlinear force-based frame elements. Journal of Structural Engineering, 127(11):1257-1265, 2001.

Collins, M. P. and Kuchma, D. How safe are our large, lightly reinforced concrete beams, slabs, and footings. ACI Structural Journal, 96(4):482-490, 1999.

Collins, M. P. and Mitchell, D. Prestressed Concrete Structures. Response Publications, Toronto, 1997.

Comite Euro-International Du Beton. CEB-FIP Model Code 1990 - Design Code. CEB, 1990.

Cook, R. D., Malkus, D. S., and Kania, E. Concepts and Applications of Finite Element Analysis. John Wiley \& Sons, Inc., New York, 3rd. edition, 1989.

Copeland, R. E. and Timms, A. G. Effect of mortar strength and strength of unit on the strength of concrete masonry walls. ACI Journal, 28(4):551-562, 1932. 
Das, S., Liu, J., El Sayed, M., and Sturgeon. Effect of height-to-thickness ratio on compressive strength of hollow concrete masonry. In Proceedings of the 12th Canadian Masonry Symposium, Vancouver, British Columbia, 2013.

Dhanasekar, M. and Wong, K. Evaluation of shear capacity equation for masonry beams without web reinforcement. In Proceedings of the 6th Australasian Masonry Conference, pages 115-124, 2001.

Drysdale, R. G. and Hamid, A. A. Behaviour of concrete block masonry under axial compression. ACI Journal, 76(6):707-721, 1979.

Drysdale, R. G. and Hamid, A. A. Capacity of concrete block masonry prisms under eccentric compressive loading. ACI Journal, 80(2):102-108, 1983.

Drysdale, R. G. and Hamid, A. A. Masonry Strusutres Behavior and Design Canadian Edition. Canadian Masonry Design Centre, Mississauga, Ontario, Canada, 2005.

Eigen. Eigen 3.2.2, 2014. URL www.eigen.tuxfamily.org.

Eikanas, I. K. Behavior of concrete masonry shear walls with varying aspect ratio and flexural reinforcement. MASc thesis, Washington State University, Pullman, Washington, 2003.

ElMohandes, F. Advanced Three-Dimensional Nonlinear Analysis of Reinforced Concrete Structures Subjected to Fire and Extreme Loads. PhD thesis, University of Toronto, Toronto, Ontario, Canada, 2013.

Escrig, F. The Great Structures in ArchitrtureL Antiquity to Baroque. WIT Press, Boston, 2006.

Facconi, L., Plizzari, G., and Vecchio, F. J. Disturbed stress field model for unreinforced masonry. Journal of Structural Engineering, 140(4), April 2014.

Fahmy, E. H. and Ghoneim, T. M. Behaviour of concrete block masonry prisms under axial compression. Canadian Journal of Civil Engineering, 22(5):898-915, 1995.

Ferguson, W. A. A comparison between the compressive strength of mortar obtained from $70.7 \mathrm{~mm}$ mortar cubes and $40 \mathrm{~mm} \times 40 \mathrm{~mm} \times 160 \mathrm{~mm}$ prisms. Technical report, Report to Masonry Research Advisory Committee, 1995.

Ferieg, S. M. Shear strength of reinforced concrete masonry beams without web reinforcement. TMS Journal, 12(2):8-15, 1994.

Fletcher, B. Sir Banister Fletcher's a History of Architecture. C Scribner's Sons, New York, 1996.

Fortes, E., Parsekian, G., and Fonseca, F. Relationship between the compressive strength of concrete masonry and the compressive strength of concrete masonry units. Journal of Materials in Civil Engineering, 27(9), 2015.

Ganesan, T. P. and Ramamurthy, K. Behaviour of concrete hollow block masonry prisms under axial compression. Journal of Structural Engineering, 118(7):1751-1769, 1992.

Numerical Modelling of Hollow Concrete Block Masonry Mechanics 
Gayed, M., Yasser, K., and Gary, S. Examination of the prescribed concrete block masonry compressive strengths in the canadian masonry design standard, csa s304.12004. In Proceedings of the 15th International Brick and Block Masonry Conference, Florianopólis, Brazil, 2012.

Gaynor, P., Woodward, K., and Scribner, C. Influence of mortar bedding on masonry prism behavior. Technical Report Report NBSIR 86-3467, U.S. Department of Commerce, National Bureau of Standards, 1987.

Ghanem, G. M., Essawy, A. S., and Hamid, A. A. Effect of steel distribution on the behavior of partially reinforced masonry walls. In Proceedings of the 6th Canadian Masonry Symposium, Saskatoon, Saskatchewan, Canada, 1992.

Ghanem, G. M., Salama, A. E., Elmagd, S. A., and Hamid, A. A. Effect of axial compression on the behavior of partially reinforced masonry shear walls. In Proceedings of the 6th North American Masonry Conference, Philadelphia, Pennsylvania, 1993.

Ghannoum, W. M. Size effect on shear strength of reinforced concrete beams. MEng thesis, McGill University, Montreal, Canada, 1998.

Ghiassi, B., Soltani, M., and Tasnimi, A. A simplified model for analysis of unreinforced masonry shear walls under combined axial, shear and flexural loading. Engineering Structures, 42:396-409, 2012.

Haach, V. G., Vasconcelos, G., and Lourenço, P. B. Experimental analysis of reinforced concrete block masonry walls subjected to inplane cyclic loading. ournal of Structural Engineering, 136(4):452-462, 2010.

Hamid, A. A. Behaviour Characteristics of Concrete Masonry. PhD thesis, McMaster University, Hamilton, Ontario, Canada, 1978.

Hamid, A. A. and Chukwunenye, A. O. Compressive behavior of concrete masonry prisms. Journal of Structural Engineering, 112(3):605-613, 1986.

Hamid, A. A. and Drysdale, R. G. Proposed failure criteria for brick masonry under combined stresses. In Proceedings of the Second North American Masonry Conference, Maryland, 1982.

Hamid, A. A., Drysdale, R. G., and Heidebrecht, A. C. Effect of grouting on the strength characteristics of concrete block masonry. In Proceedings of the North American Masonry Conference, Boulder, Colorado, 1978.

Hamid, A. A., Ziab, G., and Nawany, O. Modulus of elasticity of concrete block masonry. In Proceedings of the Fourth North American Masonry Conference, Los Angeles, CA, 1987.

Hannewald, P. Seismic Behavior of Poorly Detailed RC Bridge Piers. PhD thesis, École Polytechnique Fédérale de Lausanne, Lausanne, Switzerland, 2013.

Hegemier, G. A., Krishnamoorthy, G., Nunn, R. O., and Moorthy, T. Leas Chemistry of Cement and Concrete. ButterworthHeinemann, Oxford, 2003. 
Hellesland, J. and Bjorhovde, R. Impoved frame stability analysis with effective lengths. Journal of Structural Engineering, 122(11):1275-1283, 1996.

Hidalgo, P. A., Mayes, R. L., McNiven, H. D., and Clough, R. W. Cyclic loading tests of masonry single piers: Volume 3 - height to width ratio of 0.5. Technical Report UCB/EERC-79/12, Earthquake Engineering Research Center, 1979.

Hilsdorf, H. K. Masonry materials and their physical properties. In Proceedings of ASCE-IABSE International Conference on Planning and Design of Tall Buildings, pages 981-999, New York, 1972.

Hognestad, E. Study of combined bending and axial load in reinforced concrete members. Technical Report Bulletin 399, University of Illinois, 1951.

Hordijk, D. A., Reinhardt, H. W., and Cornelissen, H. A. W. Fracture mechanics parameters of concrete from uniaxial tests as influenced by specimen length. In Proceedings of International Conference on Fracture of Concrete and Rock, Houston, Texas, 1987.

Hrynyk, T. Behaviour and Modeling of Reinforced Concrete Slabs and Shells under Static and Dynamic Loads. PhD thesis, University of Toronto, Toronto, Ontario, Canada, 2013.

Hsieh, S. S., Ting, E. C., and Chen, W. F. An elastic-fracture model for concrete. In Proceedings of the 3rd Engineering Mechanics Division Special Conference, pages 437-440, Austin, Texas, 1979. ASCE.

Ibrahim, K. S. Inelastic Behaviour of Reinforced Masonry Shear Walls: An Experimental and Analytical Study. PhD thesis, Carleton University, Ottawa, Ontario, Canada, 1995.

Ip, F. Compression strength and modulus of elasticity of masonry prisms. MEng thesis, Carleton University, Ottawa, Ontario, Canada, 1999.

Kabeyasawa, T., Shiohara, H., Otani, S., and Hiroyuki, A. Analysis of the full-scale seven-story reinforced concrete test structure. Journal of Faculty of Engineering, XXXVII(2):432-478, 1983.

Kani, G. N. J. How safe are our large reinforced concrete beams. ACI Journal, 64(3): 128-141, 1967.

Kent, D. C. and Park, R. Flexural members with confined concrete. Journal of the Structural Division, 97(7):1969-1990, 1971.

Keshavarzian, M. and Schnobrich, W. C. Computed seismic response of reinforced concrete wall frame structures. Technical Report Structural Research Series No. 515, University of Illinois, 1984.

Khalaf, F. M. Blockwork masonry compressed in two orthogonal directions. Journal of Structural Engineering, 123(5):591-596, 1997.

Khalaf, F. M., Hendry, A. W., and Fairbairn, D. R. Study of the compressive strength of blockwork masonry. ACI Structural Journal, 91(4):369-375, 1994.

Numerical Modelling of Hollow Concrete Block Masonry Mechanics 
Köksal, H. O., Karakoç, C., and Yildirim, H. Compression behaviour and failure mechanisms of concrete masonry prisms. Journal of Materials in Civil Engineering, 17(1): 107-115, 2005.

Korany, Y. and Glanville, J. Comparing masonry compressive strength in various codes. Concrete International, 27(7):35-39, 2005.

Koutromanos, J. and Shing, P. Cohesive crack model to simulate cyclic response of concrete and masonry structures. ACI Structural Journal, 109(3):349-358, 2012.

Kupfer, H., Hilsdorf, H. K., and Rusch, H. Behavior of concrete under biaxial stress. ACI Journal, 87(2):656-666, 1969.

Lee, R., Longworth, J., and Warwaruk, J. Concrete Masonry Prism Response Due to Loads Parallel and Perpendicular to Bed Joints. Department of Civil Engineering, University of Alberta, Edmonton, Alberta, Canada, 1984.

Lefas, I. D., Kotsovos, M. D., and Ambraseys, N. N. Behavior of reinforced concrete structural walls: Strength, deformation characteristics, and failure mechanisms. Structural Journal, pages 23-31, January 1990.

Li, B., Maekawa, K., and Okamura, H. Contact density model for stress transfer across cracks in concrete. Journal of Faculty of Engineering, 40(2):9-52, 1989.

Li, S., Fried, A. N., and Roberts, J. J. Analysis of shear strength for reinforced concrete blockwork beams. In Proceedings of 10th International Brick and Masonry Conference, pages 1021-1035, Calgary Alberta, Canada, 1995.

Long, Y. Q. and Xu, Y. Generalized conforming quadrilateral membrane element with vertex rigid rotational freedom. Computers and Structures, 52(4):749-755, 1994.

Lotfi, H. R. and Shing, P. B. An appraisal of smeared crack models for shear wall analysis. Computers and Structures, 41(3):413-425, 1991.

MacGregor, J. G. and Bartlett, F. M. Reinforced Concrete Mechanics and Design. Prentice Hall Canada Inc., Scarborough, Ontario, Canada, first canadian edition edition, 2000 .

MacNeal, R. H. and Harder, R. L. A proposed standard set of problems to test finite element accuracy. Finite Elements in Analysis and Design, 1(1):3-20, 1985.

Maher, A. and Darwin, D. Mortar constituent of concrete in compression. ACI Journal, 79(2):100-109, 1982.

Maier, G., Nappi, A., and Papa, E. Damage models for masonry as a composite material a numerical and experimental analysis. Constitutive Laws for Engineering Materials, pages 427-432, 1991.

Maleki, M. Behaviour of Partially Grouted Reinforced Masonry Shear Walls under Cyclic Reversed Loading. PhD thesis, McMaster University, Hamilton, Ontario, Canada, 2008.

Numerical Modelling of Hollow Concrete Block Masonry Mechanics 
Malhotra, V. M. and Mehta, P. K. Pozzolanic and Cementitious Materials. Taylor \& Francis, Oxford, 2003.

Mander, J., Priestley, M. J. N., and Park, R. Theoretical stress strain model for confined concrete. Journal of Structural Engineering, 114(8):1804-1826, 1988.

Marini, A. and Spacone, E. Analysis of reinforced concrete elements including shear effects. ACI Journal of Structural Engineering, 103(5):645-655, 2006.

Matsumura, A. Shear strength of reinforced hollow unit masonry walls - differences between partially grouted walls and fully grouted walls. In Proceedings of the 4 th North American Masonry Conference, Los Angeles, California, 1987.

Maurenbrecher, A. H. P. Effect of test procedures on compressive strength of masonry prisms. Technical Report DBR Paper No. 9229, National Research Council Canada, 1980.

Maurenbrecher, A. H. P. Axial compression tests on masonry walls and prisms. Technical Report DBR Paper No. 1303, National Research Council Canada, 1985.

Maurenbrecher, A. H. P. Compressive strength of hollow concrete block. In Proceedings of the 4th Canadian Masonry Symposium, Fredericton, New Brunswick, Canada, 1986.

Mayes, R. L., Omote, Y., and Clough, R. W. Cyclic shear tests of masonry piers, volume 2: Analysis of test results. Technical Report Tech. Rep. UCB/EERC76/16, University of California, 1976.

Mihaylov, B., Bentz, E., and Collins, M. P. Two-parameter kinematic theory for shear behavior of deep beams. ACI Structural Journal, 110(3):447-456, 2013.

Mihaylov, B., Hannewald, P., and Beyer, K. Three-parameter kinematic theory for shear-dominated reinforced concrete walls. ASCE, 142(7), July 2016.

Minaie, E., Moon, F. L., and A., H. A. Nonlinear finite element modeling of reinforced masonry shear walls for bidirectional loading response. Finite Elements in Analysis and Design, 84(1), July 2014.

National Concrete Masonry Association. Recalibration of the unit strength method for verifying compliance with the specified compressive strength of concrete masonry. Technical report, 2012.

Neville, A. M. A general relation for strengths of concrete specimens of different shapes and sizes. ACI Journal, 63(10):1095-1110, 1966.

OpenGL. Opengl 4.5, 2014. URL www.opengl.org.

Page, A. W. A model for the in-plane behaviour of masonry and a sensitivity analysis of critical parameters. In Proceedings of the 5th International Brick Masonry Conference, pages 262-267, 1979.

Paulay, T. and Priestley, M. J. N. Seismic Design of Reinforced Concrete and Masonry Buildings. John Wiley \& Sons, Inc., Toronto, 1992.

Numerical Modelling of Hollow Concrete Block Masonry Mechanics 
Petrangeli, M., Pinto, P. E., and Ciampi, V. Fibre element for cyclic bending and shear of reinfoced concrete structures. i: Theory. Journal of Engineering Mechanics, 125 (9):994-1001, 1999.

Popovics, S. A numerical approach to complete stress-strain curves of concrete. Cement and Concrete Research, 3(5):583-599, 1970.

Priestley, M. J. N. Seismic resistance of reinforced concrete masonry shear walls with high steel percentages. Bulletin of the New Zealand National Society for Earthquake Engineering, 10(1):1-16, March 1977.

Priestley, M. J. N. Seismic design of concrete masonry shear walls. ACI Journal, 86(8): 58-68, 1986.

Priestley, M. J. N. and Elder, D. M. Stress-strain curves for unconfined and confined concrete masonry. Journal of the American Concrete Institute, 80(3):192-201, 1983.

Priestley, M. J. N., Calvi, G. M., and Kowalsky, M. J. Displacement-Based Seismic Design of Structures. IUSS Press, Pavia, Italy, 2007.

Ramamurthy, K., Sathish, V., and Ambalavanan, R. Compressive strength prediction of hollow concrete block masonry prisms. ACI Structural Journal, 97(1):61-67, 2000.

Richart, F. E., Moorman, R. B., and Woodworth, P. M. Strength and stability of concrete masonry walls. Technical Report Bulletin 251, University of Illinois, 1932.

Ring, T. The influence of horizontal grout continuity on the compressive stength of concrete block masonry. MASc thesis, University of Windsor, Windsor, Ontario, Canada, 2009.

Roman, H. R. and Romagna, R. H. Compressive strength of grouted and ungrouted concrete block masonry. In 9, V., editor, Proceedings of the British Masonry Society, pages 399-404, 2002.

Ross, M., Korany, Y., and Sturgeon, G. Recalibration of the unit strength method in the canadian masonry design standard, csa s304.1-04 for grouted masonry. In Proceedings of 12th Canadian Masonry Symposium, Vancouver, British Columbia, 2013.

Roy, H. and Sozen, M. A. Ductility of concrete. In Proceedings of the International Symposium on Flexural Mechanics of Reinforced Concrete, pages 213-224, Miami, Florida, 1964. ASCE-ACI.

Saatcioglu, M. and Razvi, S. Strength and ductility of confined concrete. Journal of Structural Engineering, 118(6):1590-1607, 1992.

Samarashinghe, W. and Hendry, A. W. The strength of brickwork under biaxial tensile and compressive stress. In Proceedings of the 7th International Symposium on Load Bearing Brickwork, 1980.

Samy, B. S., Sarhat, S. R., and Sherwood, E. G. Comparing flexural capacity of reinforced masonry beams using different codes. In Proceedings of the 12th North American Masonry Conference, 2015.

Numerical Modelling of Hollow Concrete Block Masonry Mechanics 
Sarhat, S. The Size Effect and Strain Effect in Reinforced Masonry. PhD thesis, Carleton University, Ottawa, Ontario, Canada, 2016.

Sarhat, S. and Sherwood, E. G. The prediction of compressive strength of ungrouted hollow concrete block masonry. Construction and Building Materials, 58:111-121, 2014.

Sarhat, S. and Sherwood, E. G. Determination of compressive strength of grouted hollow concrete block masonry from strengths of its constituents. Submitted, 2015.

Schneider, R. R. and Dickey, W. Reinforced Masonry Design. Prentice Hall, Toronto, 3rd. edition, 1994.

Schultz, A. E. Seismic resistance of partially grouted masonry shear walls. Worldwide Advances in Structural Concrete and Masonry, pages 211-22, 1996.

Schultz, A. E., Hutchinson, R. S., and Cheok, G. C. Seismic performance of masonry walls with bed joint reinforcement. In Structural Engineers World Congress Proceedings, San Francisco, California, 1998.

Scrivener, J. C. and Williams, D. Behaviour of reinforced masonry shear walls under cyclic loading. Bulletin of New Zealand Society for Earthquake Engineering, 4(2), 1971.

Seible, F., La Rovere, H., and R., K. G. Non-linear analysis of reinforced concrete masonry sub-assemblages. In Abrams, D. P., editor, Proceedings of the 5th North American Masonry Conference. The Masonry Society, Masonry Society, 1990.

Seif ElDin, H. M. In-Plane Shear Behaviour of Fully Grouted Reinforced Masonry Shear Walls. PhD thesis, Concordia University, Montreal, Qubec, Canada, 2016.

Selby, R. and Vecchio, F. A constitutive model for analysis of reinforced concrete solids. Canadian Journal of Civil Engineering, 24(3):460-470, 1997.

Shedid, M. T. Ductility of reinforced concrete masonry shear walls. MASc thesis, McMaster University, Hamilton, Ontario, Canada, 2006.

Shedid, M. T., Drysdale, R. G., and El-Dakhakhni, W. W. Behavior of fully grouted reinforced concrete masonry shear walls failing in flexure: Experimental results. Journal of Structural Engineering, 134(11):1754-1767, November 2008.

ShenCao. Size effect and the influence of longitudinal reinforcement on the shear response of large reinforced concrete members. MASc thesis, University of Toronto, Toronto, Ontario, Canada, 2001.

Sherwood, E. G. One-Way Shear Behavior of Large, Lightly-Reinforced Concrete Beams and Slabs. PhD thesis, University of Toronto, Toronto, Ontario, Canada, 2008.

Shing, P. B., Klamerus, E., Spaeh, H., and Noland, J. L. Seismic performance of reinforced masonry shear walls. In Proceedings of the 9th World Conference on Earthquake Engineering, 1988.

Numerical Modelling of Hollow Concrete Block Masonry Mechanics 
Shing, P. B., Noland, J. L., Klamerus, E., and Spaeh, H. Inelastic behavior of concrete masonry walls. Journal of Structural Engineering, 115(9):2204-2225, 1989.

Shing, P. B., Schuller, M., and Hoskere, V. S. In-plane resistance of reinforced masonry shear walls. Journal of Structural Engineering, 116(3):619-640, November 1990.

Shing, P. B., Noland, J. L., Spaech, H., Klamerus, E., and Schuller, M. Response of single-storey reinforced masonry shear walls to in-plane lateral loads. Technical Report TCCMAR Report 3.1(a)-2, 1991.

Singh, R. Evaluation of masonry mortar properties and relationship between compressive strength of 2 inch cube and 2 inch $\mathrm{x} 3$ inch cylinder. MASc thesis, University of Texas, Austin, Texas, 1988.

Smith, G. M. and Young, L. E. Ultimate flexural analysis based on stress-strain curves of cylinders. ACI Journal, 53(12):597-609, December 1956.

Standard Association of New Zealand. Design of reinforced concrete masonry structures. Technical report, Standards Associationof New Zealand, 2004.

State of California Seismic Safety Commission. The field act and its relative effectiveness in reducing earthquake damage in california public schools. Technical report, CSSC, Sacramento, California, 2009.

Stavridis, A. and Shing, P. Calibration of a numerical model for masonry infilled reinforced frames. In Proceedings of the 14th World Conference on Earthquake Engineering, Beijing, China, 2008.

Steadman, M., Drysdale, R. G., and Khattab, M. M. Influenace of block geomwtry and grout type on compressive strength of block masonry. In Proceedings of 7th Canadian Masonry Symposium, pages 1116-1127, Hamilton, Canada, 1995.

Sucuoglu, H. and McNiven, H. Seismic shear capacity of reinforced masonry piers. Journal of Structural Engineering, 117(7), 1991.

Suter, G. T. and Keller, H. Carleton university concrete masonry beam test - shear. Technical Report Research Report, Carleton University, 1980.

Sveinsson, B. I., McNiven, H. D., and Sucuoglu, H. Cyclic loading tests of masonry single piers: Volume 4 - additional tests with height to width ratio of 1 . Technical Report UCB/EERC-85/15, Earthquake Engineering Research Center, 1985.

Takayanagi, T. and Schnobrich, W. C. Computed behaviour of reinforced concrete coupled shear walls. Technical Report Structural Research Series No. 434, University of Illinois, 1976.

The Masonry Society. 2013 MSJC Building Code Requirements and Specification for Masonry Structures. The Masonry Society, Longmont, Colorado, United States, 2013.

Timoshenko, S. P. and Goodier, J. N. Theory of Elasticity. McGrawHill, New York, 3rd. edition, 1934.

Numerical Modelling of Hollow Concrete Block Masonry Mechanics 
Van Der Pluijm, R. and Vermeltfoort, A. T. Deformation controlled tension and compression tests in units, mortar and masonry. Technical Report Rep. B-91-0561, ETNOBouw, Delft, The Netherlands, 1991.

Vecchio, F. J. Reinforced concrete membrane element formulation. Journal of Structural Engineering, 116(3):730-750, 1990.

Vecchio, F. J. Disturbed stress field model for reinforced concrete: Formulation. Journal of Structural Engineering, 126(8):1070-1077, 2000.

Vecchio, F. J. Disturbed stress field model for reinforced concrete: Implementation. Journal of Structural Engineering, 127(1):12-20, 2001.

Vecchio, F. J. and Collins, M. P. The modified compression-field theory for reinforced concrete elements subjected to shear. ACI Journal, 83(2):219-231, 1986.

Vecchio, F. J. and Collins, M. P. Compression response of cracked reinforced concrete. Journal of Structural Engineering, 119(12):3590-3610, 1993.

Vecchio, F. J. and De Roo, A. Smeared crack modelling of concrete tension splitting. Journal of Engineering Mechanics, 121(6):702-708, 1995.

Vecchio, F. J., Bentz, E. C., and Collins, M. P. Tools for forensic analysis of concrete structures. Computers and Concrete, 1(1):1-14, 2004.

Voon, K. C. In-Plane Seismic Design Concrete Masonry Structures. PhD thesis, University of Auckland, Auckland, New Zealand, 2007.

Voon, K. C. and Ingham, J. Experimental in-plane strength investigation of concrete masonry walls with openings. Journal of Structural Engineering, 134(5):758-768, 2008.

Walraven, J. C. and Reinhardt, H. W. Btheory and experiments on the mechanical behaviour of cracks in plain and reinforced concrete subjected to shear loading. HERON, 26(1A), 1981.

Wong, H. E. and Drysdale, R. G. Compressive characteristics of concrete block masonry prisms. Masonry: Research, Application, and Problems, ASTM STP 871:167-177, 1985 .

Wong, P. S., Veccjio, F. J., and Trommels, H. VecTor2 83 FormWorks User's Manual. Department of Civil Engineering, University of Toronto, second edition, 2013.

Wood, R. H. Effective lengths of columns in multi-storey buildings. Structural Engineer, 52(7):235-244, 1974.

Woodward, K. and Rankin, F. Influence of block and mortar strength on shear resistance of concrete block masonry walls. Technical Report NBSIR 85-3143, U.S. Department of Commerce, National Bureau of Standards, National Engineering Laboratory, 1985.

Yanez, F., Park, R., and Paulay, T. Seismic behaviour of reinforced concrete structural walls with irregular openings. In Pacific Conference on Earthquake Engineering, pages 3303-3308, New Zealand, 1991.

Numerical Modelling of Hollow Concrete Block Masonry Mechanics 
Yoshida, Y. Shear reinforcement for large, lightly-reinforced concrete members. MASc thesis, University of Toronto, Toronto, Ontario, Canada, 2000. 
APPEndix A

UNGROUTED PRISM EXPERIMENTAL DATA 


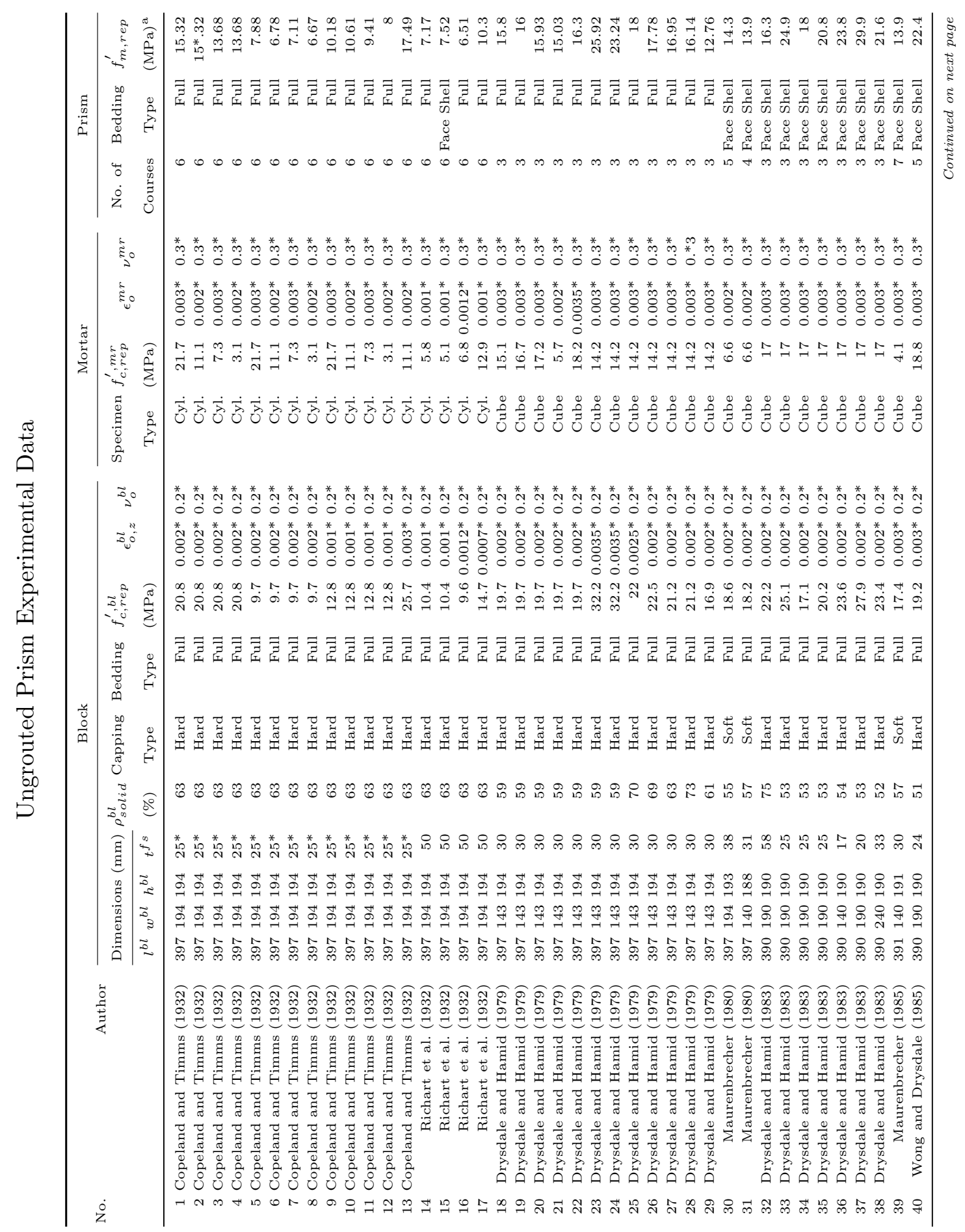




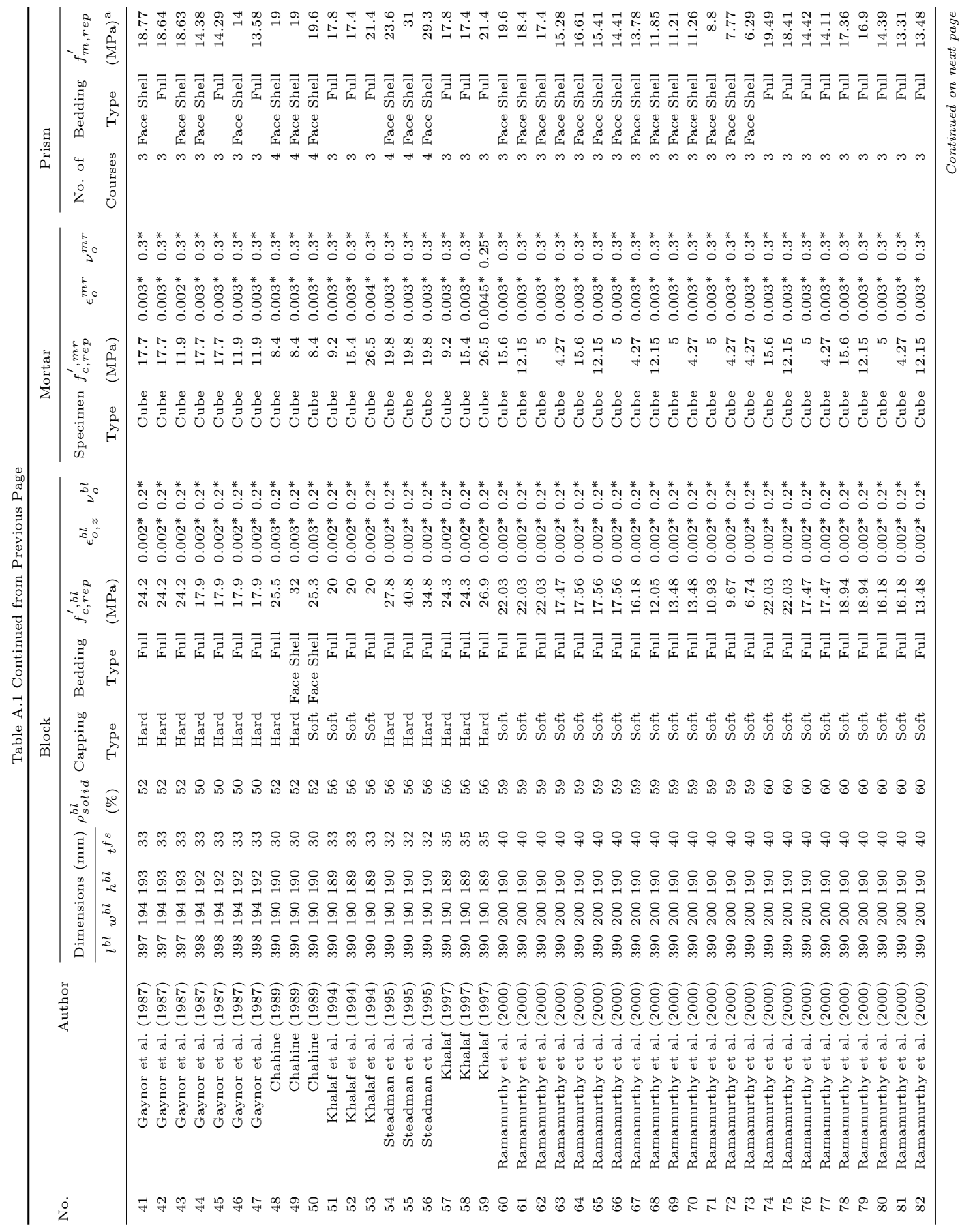




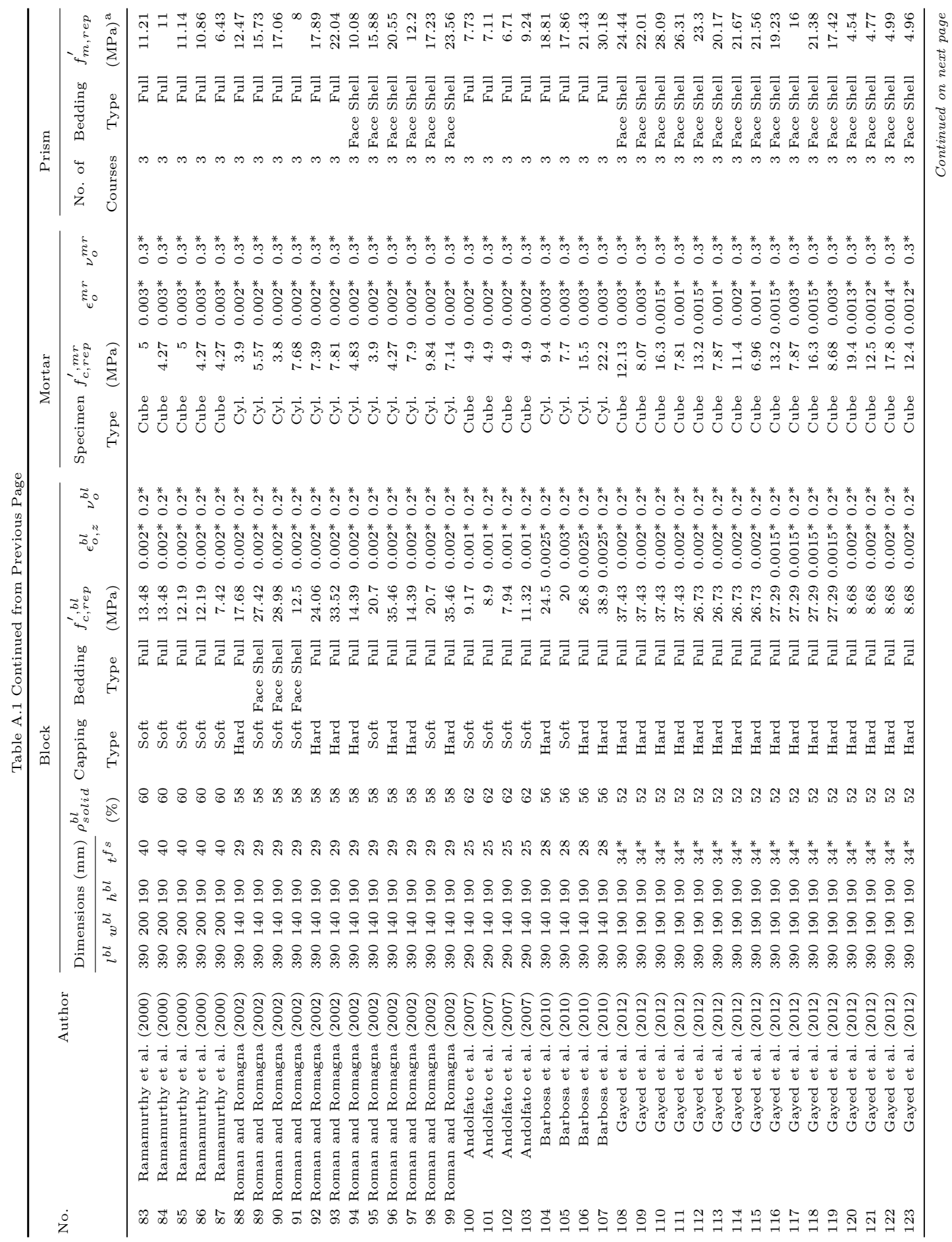




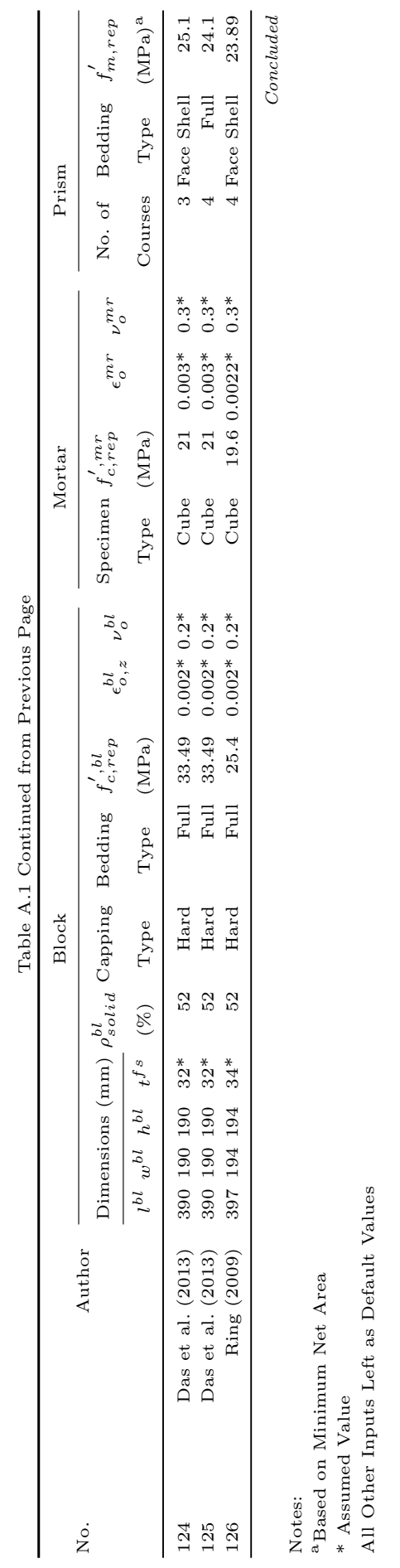


APPENDIX B

SACM INPUT FOR GROUTED PRISM EXPERIMENTAL DATA 


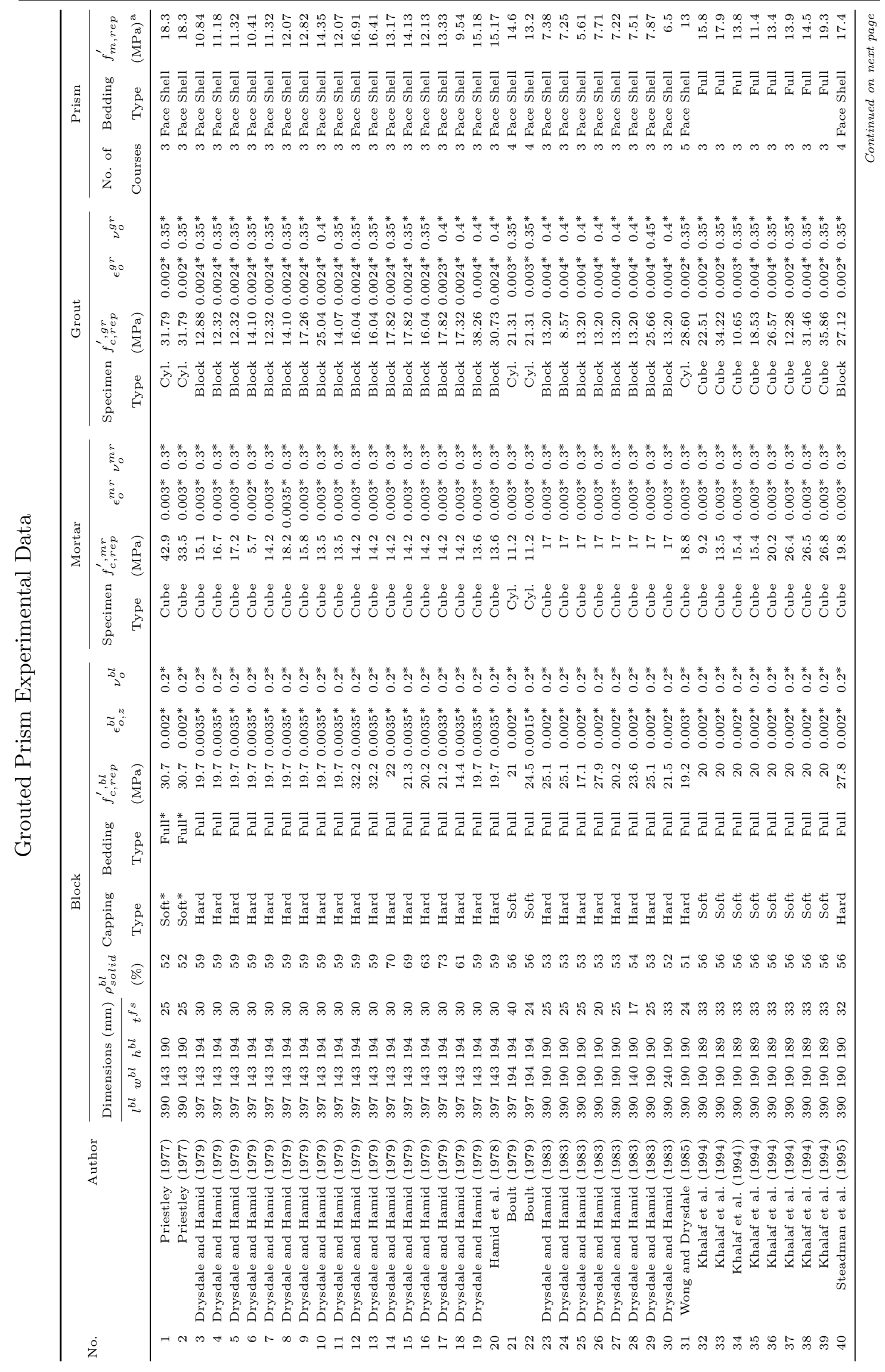




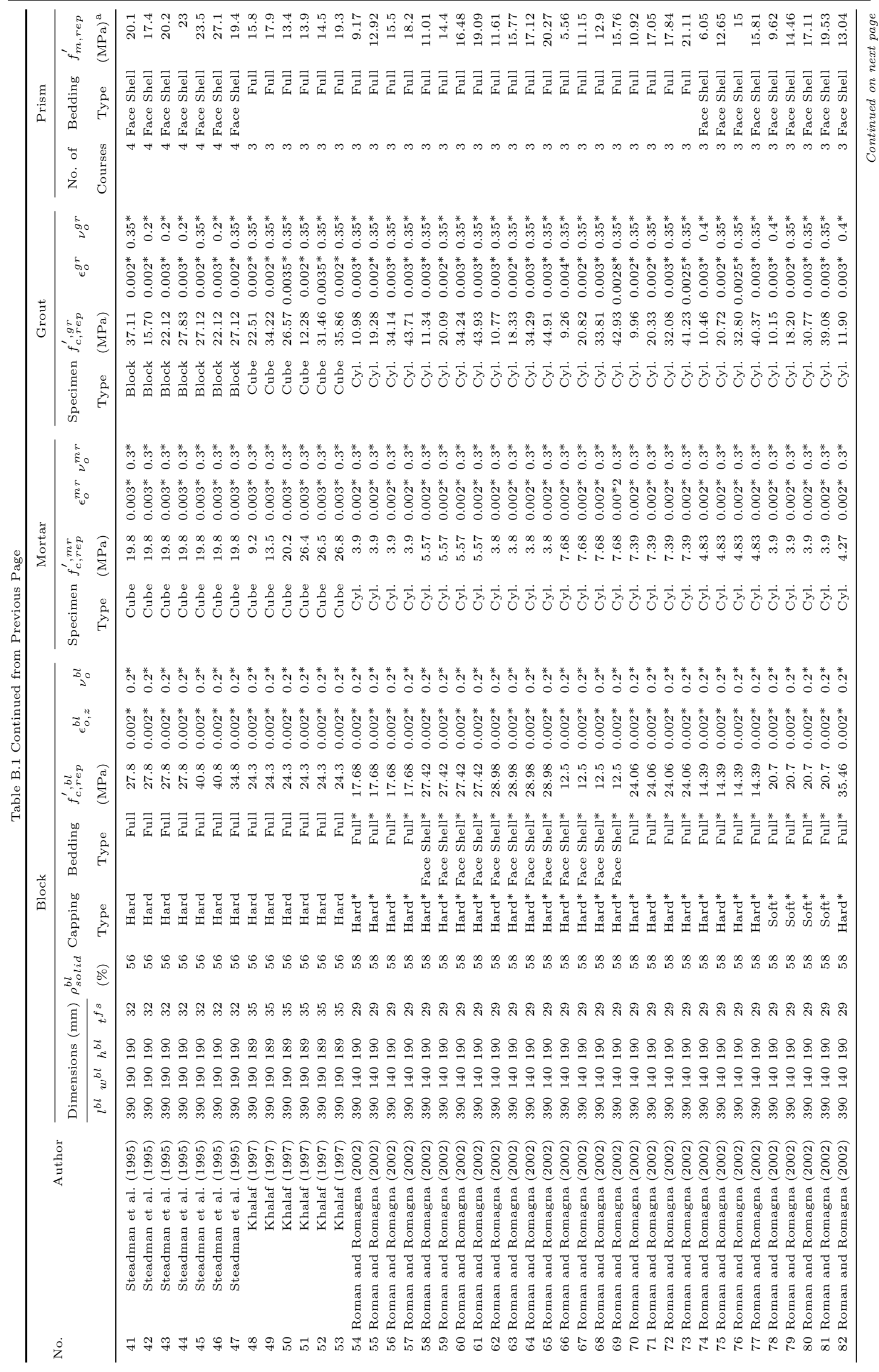

Numerical Modelling of Hollow Concrete Block Masonry Mechanics 


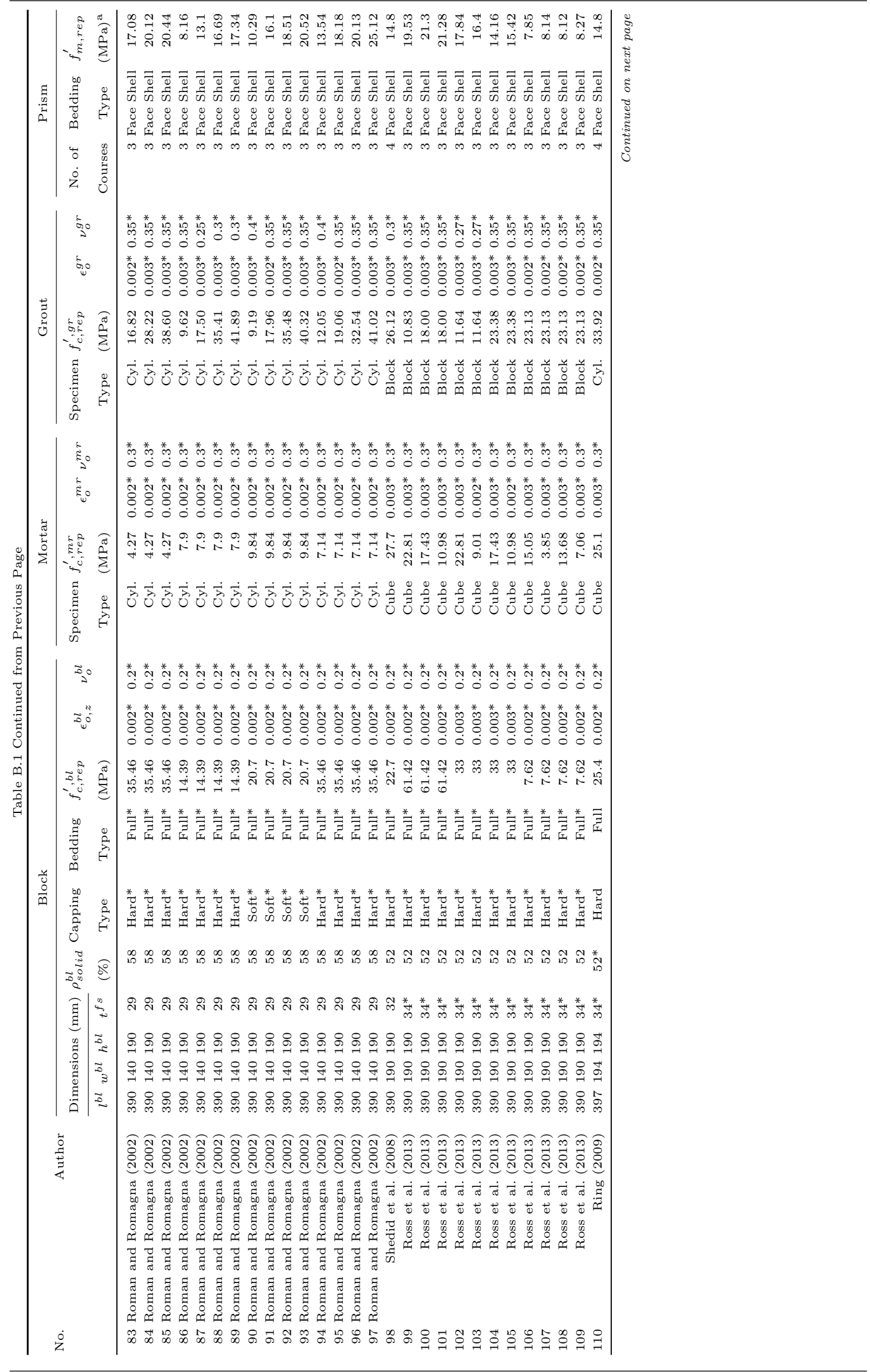




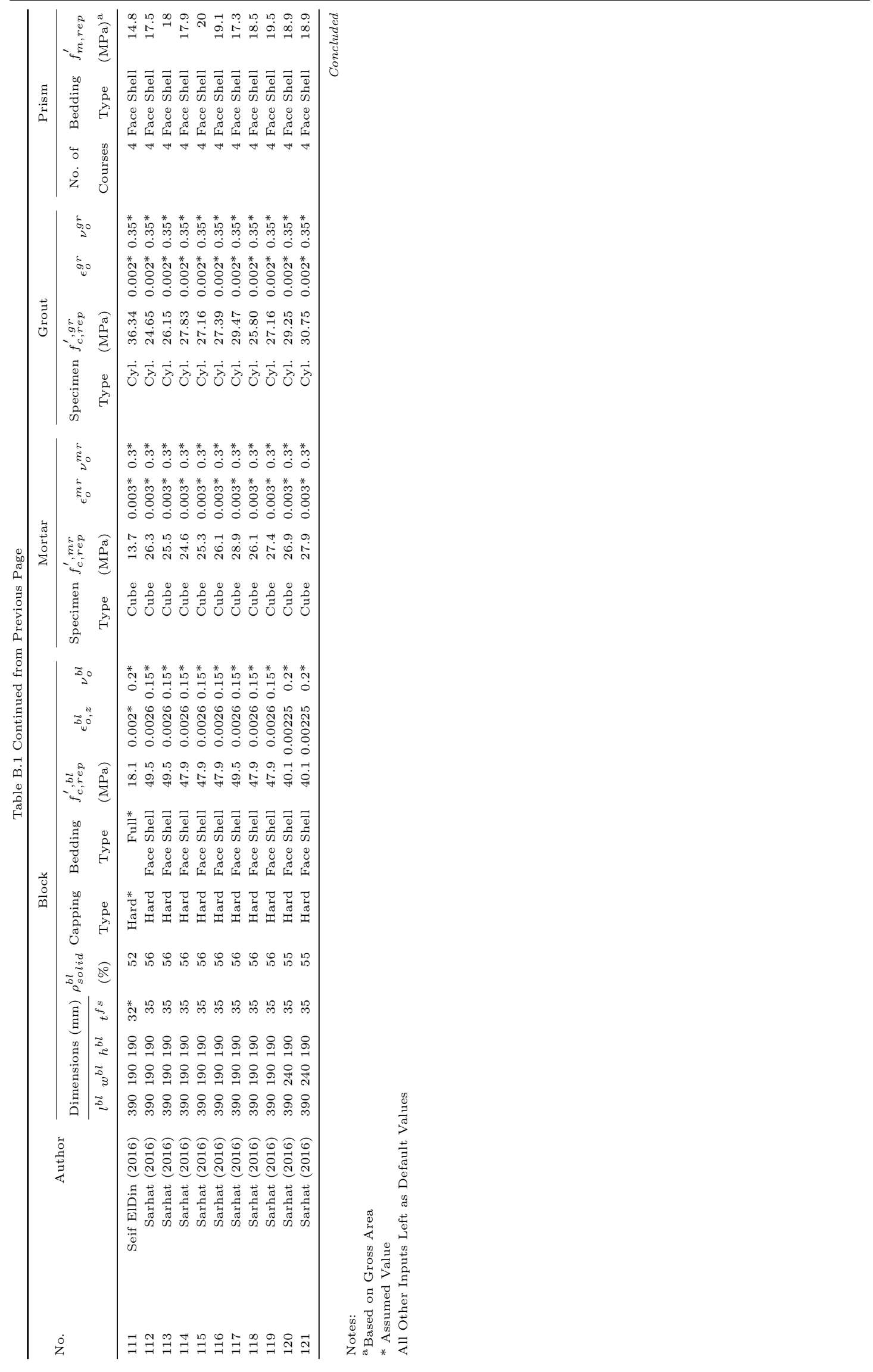




\section{APPENDIX $\mathbf{C}$}

PARALLEL PRISM EXPERIMENTAL DATA 

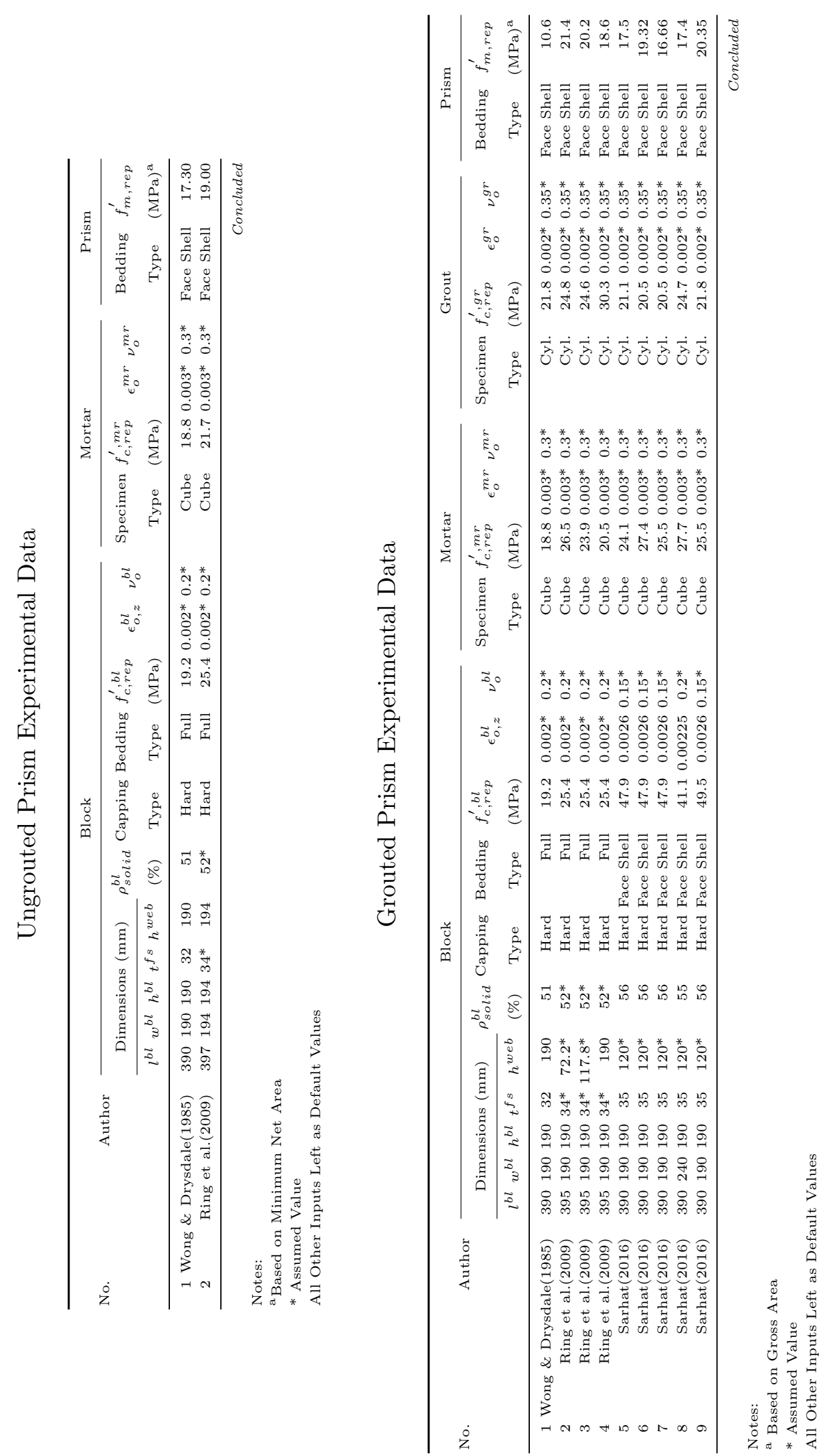
SINGLE PIEAR GROUTED SHEAR WALL SMEARED AREA FINITE ELEMENT MODEL RESULTS

\section{Priestley (1977)}

\section{A1 Shear Wall}

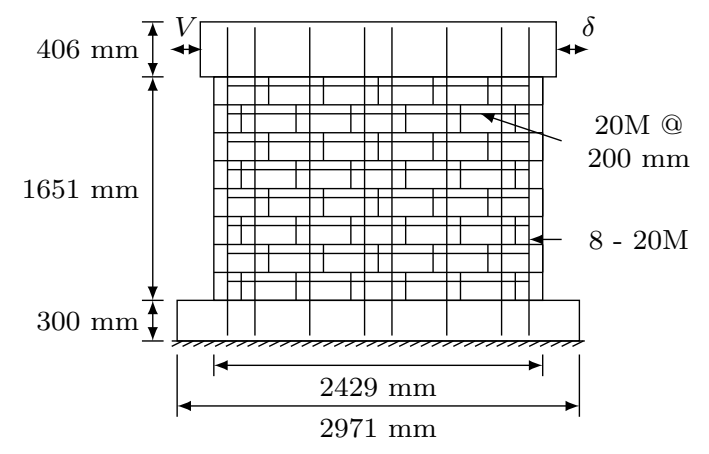

Experimental Wall (Priestley, 1977)

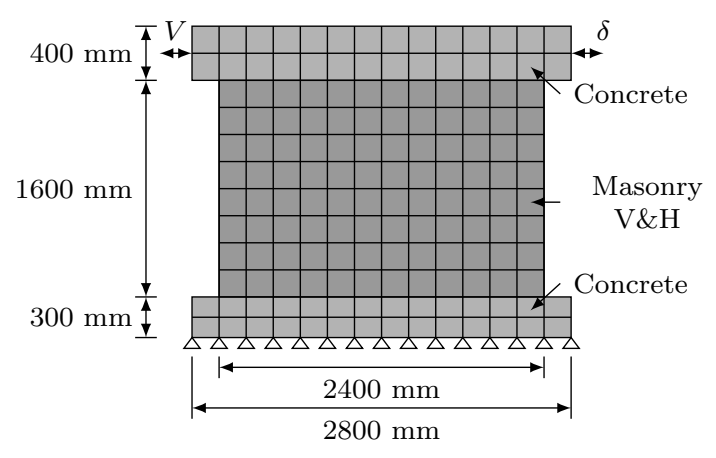

Finite Element Model Wall

A1 Shear Wall

A1 Shear Wall (Priestley, 1977) Model Options

\begin{tabular}{ll}
\hline Model & Selected Option \\
\hline Compression Pre-Peak & Hognestad \\
Compression Post-Peak & Pre-Peak Base Curve \\
Compression Softening & Vecchio \\
Tension Post-Peak & Vecchio \\
Cracking Criterion & Mohr-Coulomb \\
Slip & Walraven Reinforced \\
\hline
\end{tabular}


A1 Shear Wall (Priestley, 1977) Material Properties

\begin{tabular}{|c|c|c|}
\hline \multirow{2}{*}{ Property } & \multicolumn{2}{|c|}{ Material } \\
\hline & Masonry V\&H & Concrete \\
\hline Thickness (mm) & 143 & 560 \\
\hline Grout Tensile Strength $(\mathrm{MPa})$ & 1.86 & 2.09 \\
\hline \multicolumn{3}{|c|}{ Masonry Properties (Y-Direction) } \\
\hline Gross Peak Compression Strength (MPa) & 19.3 & 40 \\
\hline Peak Compressive Strain & 0.0027 & 0.0021 \\
\hline Tensile Strength & 0 & 2.09 \\
\hline Tensile Strain & 0 & 0 \\
\hline Initial Tangent Elastic Modulus (MPa) & 6900 & 34800 \\
\hline Poisson's Ratio & 0 & 0.15 \\
\hline \multicolumn{3}{|c|}{ Masonry Properties (X-Direction) } \\
\hline Gross Peak Compression Strength (MPa) & 19.3 & 40 \\
\hline Peak Compressive Strain & 0.0027 & 0.0021 \\
\hline Tensile Strength & 0 & 2.09 \\
\hline Tensile Strain & 0 & 0 \\
\hline Initial Tangent Elastic Modulus (MPa) & 6900 & 34800 \\
\hline Poisson's Ratio & 0 & 0.15 \\
\hline \multicolumn{3}{|c|}{ Masonry Properties (Block) } \\
\hline Length (mm) & 390 & 2400 \\
\hline Height $(\mathrm{mm})$ & 190 & 400 \\
\hline Face Shell Thickness (mm) & 25 & 280 \\
\hline Percent Solid (\%) & 52 & 100 \\
\hline Tensile Strength & 2.03 & 2.09 \\
\hline \multicolumn{3}{|l|}{ Head Joint } \\
\hline Thickness (mm) & 10 & 10 \\
\hline Tensile Bond Strength (MPa) & 0.40 & 2.09 \\
\hline Joint Cohesion (MPa) & 8.45 & 2.09 \\
\hline Angle of Internal Friction (Deg) & 42 & 42 \\
\hline \multicolumn{3}{|l|}{ Bed Joint } \\
\hline Thickness (mm) & 10 & 10 \\
\hline Tensile Bond Strength (MPa) & 0.40 & 2.09 \\
\hline Joint Cohesion (MPa) & 8.45 & 2.09 \\
\hline Angle of Internal Friction (Deg) & 42 & 42 \\
\hline \multicolumn{3}{|c|}{ Reinforcement (Y-Direction) } \\
\hline Ratio (\%) & 0.66 & 1.00 \\
\hline Yield Strength (MPa) & 528 & 400 \\
\hline Yield Strain & 0.00264 & 0.002 \\
\hline Strain Hardening Strain & 0.02 & 0.02 \\
\hline Ultimate Strength (MPa) & 871 & 600 \\
\hline Ultimate Strain & 0.2 & 0.2 \\
\hline Prestrain & 0 & 0 \\
\hline \multicolumn{3}{|c|}{ Reinforcement (X-Direction) } \\
\hline Ratio (\%) & 0.97 & 1.00 \\
\hline Yield Strength (MPa) & 505 & 400 \\
\hline Yield Strain & 0.00253 & 0.002 \\
\hline Strain Hardening Strain & 0.02 & 0.02 \\
\hline Ultimate Strength (MPa) & 841 & 600 \\
\hline Ultimate Strain & 0.2 & 0.2 \\
\hline Prestrain & 0 & 0 \\
\hline
\end{tabular}

Numerical Modelling of Hollow Concrete Block Masonry Mechanics 


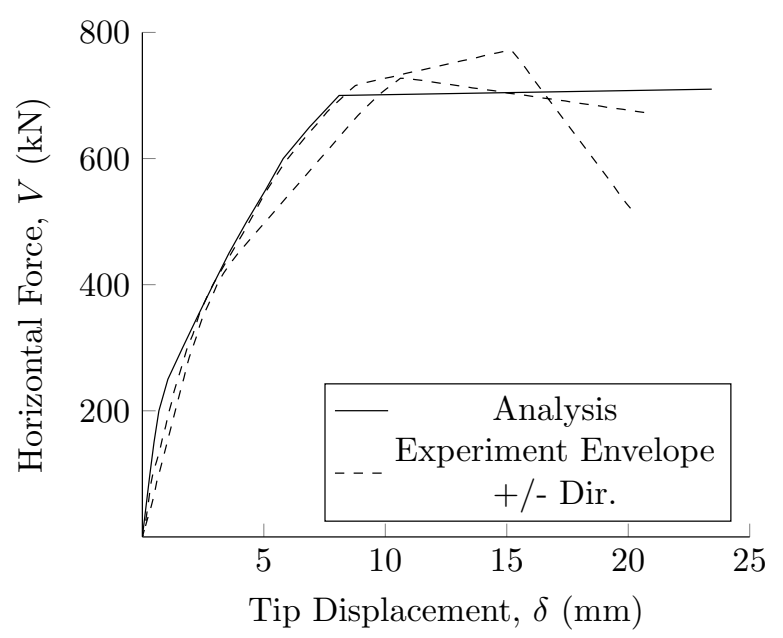

A1 Shear Wall (Priestley, 1977) Horizontal Force vs Tip Displacement
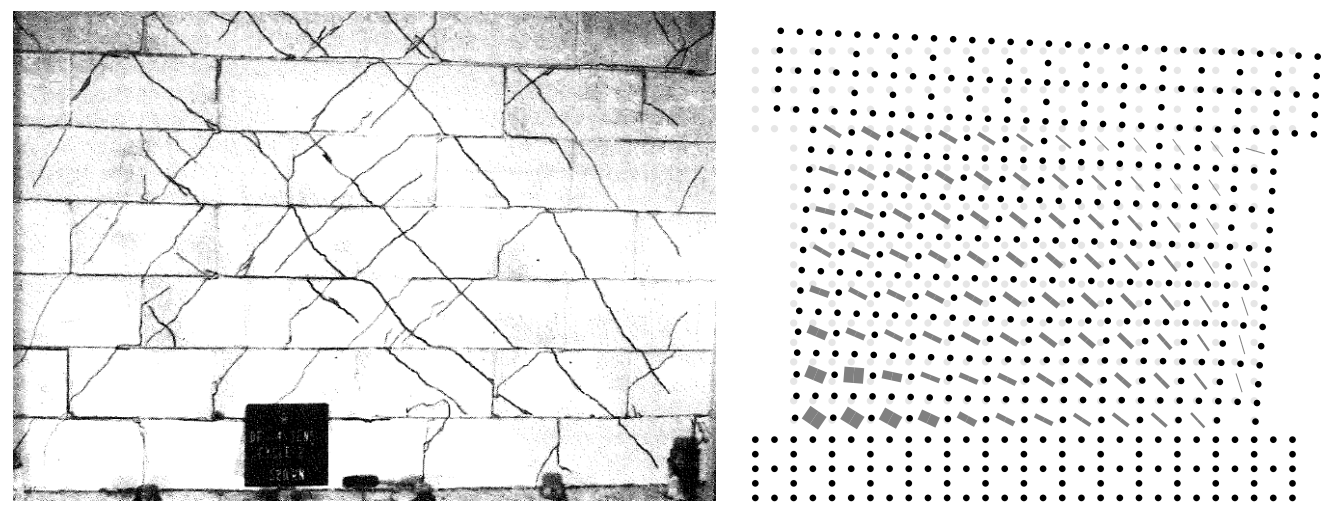

Experiment at $20 \mathrm{~mm}$ Tip Displacement (Priest- Finite Element Model at Peak Horizontal Force ley, 1977) $\times 15$

A1 Shear Wall Displacement, Crack Pattern, and Crack Widths 


\section{A2 Shear Wall}

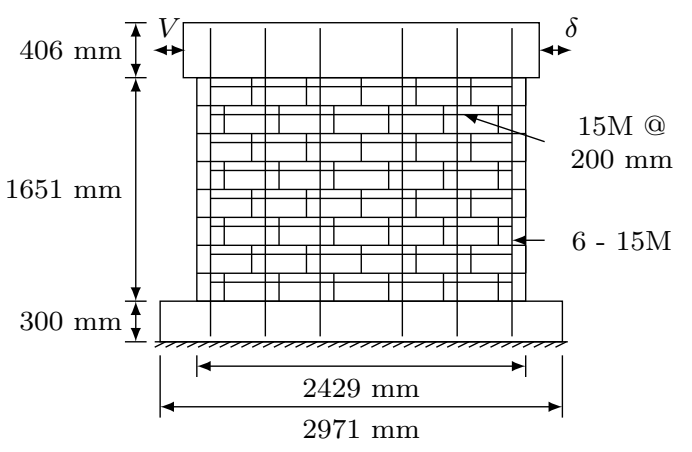

Experimental Wall (Priestley, 1977)

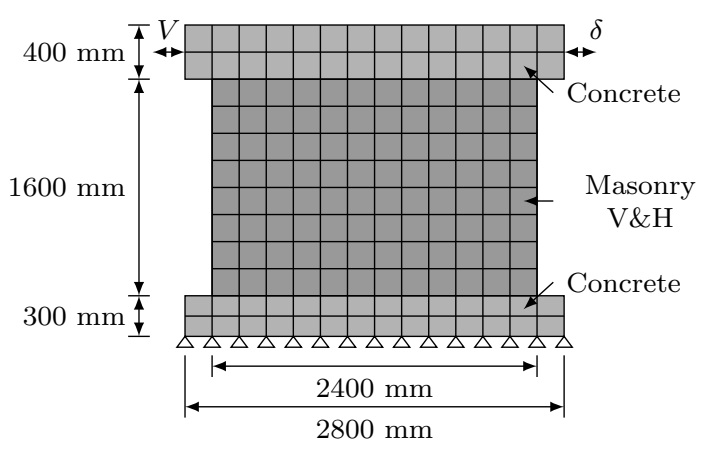

Finite Element Model Wall

A2 Shear Wall

A2 Shear Wall (Priestley, 1977) Model Options

\begin{tabular}{ll}
\hline Model & Selected Option \\
\hline Compression Pre-Peak & Hognestad \\
Compression Post-Peak & Pre-Peak Base Curve \\
Compression Softening & Vecchio \\
Tension Post-Peak & Vecchio \\
Cracking Criterion & Mohr-Coulomb \\
Slip & Walraven Reinforced \\
\hline
\end{tabular}

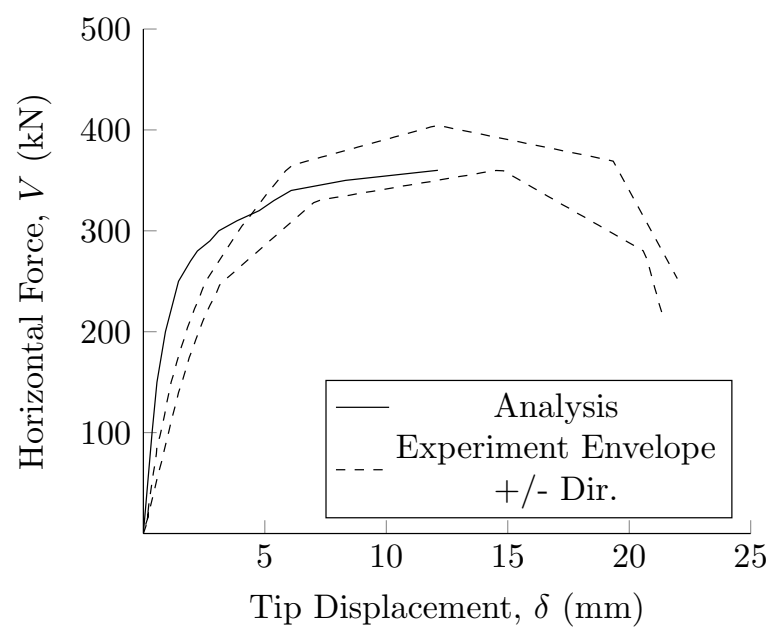

A2 Shear Wall (Priestley, 1977) Horizontal Force vs Tip Displacement 
A2 Shear Wall (Priestley, 1977) Material Properties

\begin{tabular}{|c|c|c|}
\hline \multirow{2}{*}{ Property } & \multicolumn{2}{|c|}{ Material } \\
\hline & Masonry V\&H & Concrete \\
\hline Thickness (mm) & 143 & 560 \\
\hline Grout Tensile Strength (MPa) & 1.86 & 2.09 \\
\hline \multicolumn{3}{|c|}{ Masonry Properties (Y-Direction) } \\
\hline Gross Peak Compression Strength (MPa) & 18.3 & 40 \\
\hline Peak Compressive Strain & 0.0027 & 0.0021 \\
\hline Tensile Strength & 0 & 2.09 \\
\hline Tensile Strain & 0 & 0 \\
\hline Initial Tangent Elastic Modulus (MPa) & 6200 & 34800 \\
\hline Poisson's Ratio & 0 & 0.15 \\
\hline \multicolumn{3}{|c|}{ Masonry Properties (X-Direction) } \\
\hline Gross Peak Compression Strength (MPa) & 18.3 & 40 \\
\hline Peak Compressive Strain & 0.0027 & 0.0021 \\
\hline Tensile Strength & 0 & 2.09 \\
\hline Tensile Strain & 0 & 0 \\
\hline Initial Tangent Elastic Modulus (MPa) & 6200 & 34800 \\
\hline Poisson's Ratio & 0 & 0.15 \\
\hline \multicolumn{3}{|c|}{ Masonry Properties (Block) } \\
\hline Length (mm) & 390 & 2400 \\
\hline Height (mm) & 190 & 400 \\
\hline Face Shell Thickness (mm) & 25 & 280 \\
\hline Percent Solid (\%) & 52 & 100 \\
\hline Tensile Strength & 2.03 & 2.09 \\
\hline \multicolumn{3}{|l|}{ Head Joint } \\
\hline Thickness (mm) & 10 & 10 \\
\hline Tensile Bond Strength (MPa) & 0.45 & 2.09 \\
\hline Joint Cohesion (MPa) & 11.20 & 2.09 \\
\hline Angle of Internal Friction (Deg) & 42 & 42 \\
\hline \multicolumn{3}{|l|}{ Bed Joint } \\
\hline Thickness (mm) & 10 & 10 \\
\hline Tensile Bond Strength (MPa) & 0.45 & 2.09 \\
\hline Joint Cohesion (MPa) & 11.20 & 2.09 \\
\hline Angle of Internal Friction (Deg) & 42 & 42 \\
\hline \multicolumn{3}{|c|}{ Reinforcement (Y-Direction) } \\
\hline Ratio (\%) & 0.34 & 1.00 \\
\hline Yield Strength (MPa) & 432 & 400 \\
\hline Yield Strain & 0.00216 & 0.002 \\
\hline Strain Hardening Strain & 0.02 & 0.02 \\
\hline Ultimate Strength (MPa) & 711 & 600 \\
\hline Ultimate Strain & 0.2 & 0.2 \\
\hline Prestrain & 0 & 0 \\
\hline \multicolumn{3}{|c|}{ Reinforcement (X-Direction) } \\
\hline Ratio (\%) & 0.67 & 1.00 \\
\hline Yield Strength (MPa) & 421 & 400 \\
\hline Yield Strain & 0.00211 & 0.002 \\
\hline Strain Hardening Strain & 0.02 & 0.02 \\
\hline Ultimate Strength (MPa) & 664 & 600 \\
\hline Ultimate Strain & 0.2 & 0.2 \\
\hline Prestrain & 0 & 0 \\
\hline
\end{tabular}

Numerical Modelling of Hollow Concrete Block Masonry Mechanics 


\section{A5 Shear Wall}

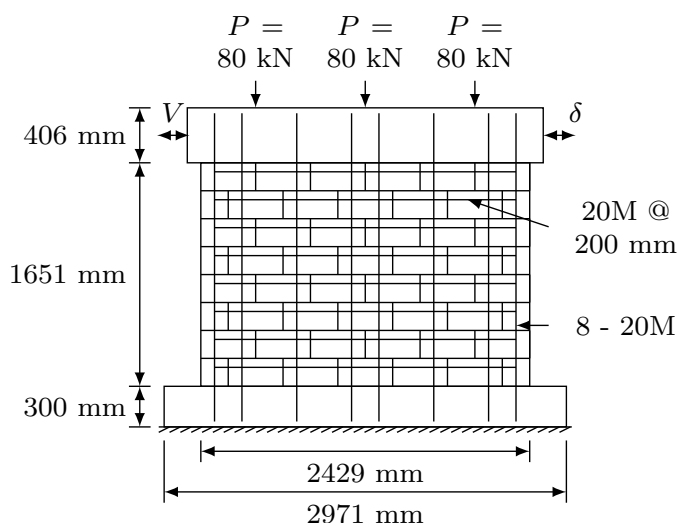

Experimental Wall (Priestley, 1977)

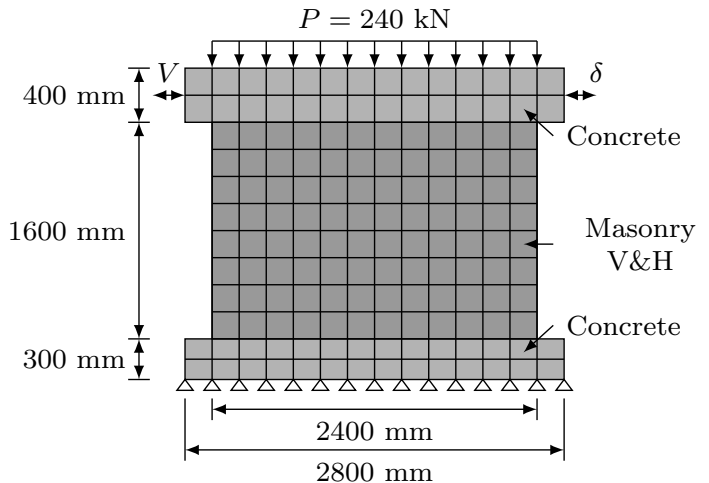

Finite Element Model Wall

A5 Shear Wall

A5 Shear Wall (Priestley, 1977) Model Options

\begin{tabular}{ll}
\hline Model & Selected Option \\
\hline Compression Pre-Peak & Hognestad \\
Compression Post-Peak & Pre-Peak Base Curve \\
Compression Softening & Vecchio \\
Tension Post-Peak & Vecchio \\
Cracking Criterion & Mohr-Coulomb \\
Slip & Walraven Reinforced \\
\hline
\end{tabular}

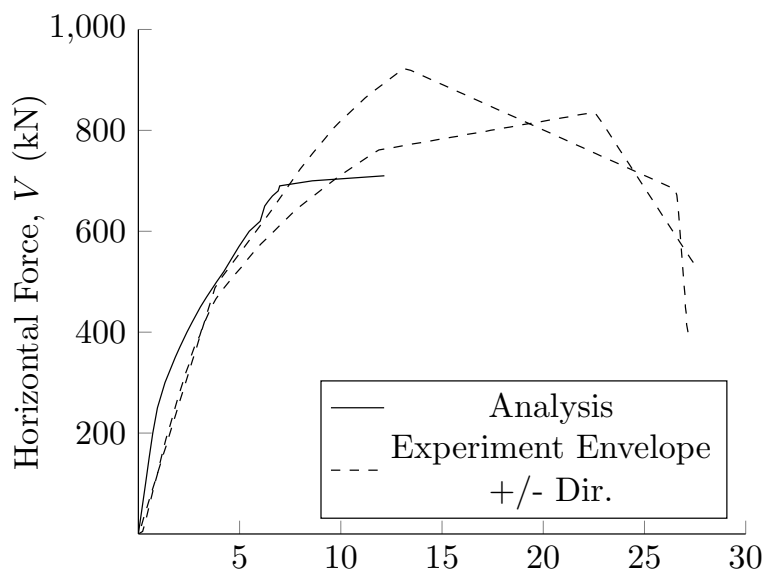

Tip Displacement, $\delta(\mathrm{mm})$

A5 Shear Wall (Priestley, 1977) Horizontal Force vs Tip Displacement 
A5 Shear Wall (Priestley, 1977) Material Properties

\begin{tabular}{|c|c|c|}
\hline \multirow{2}{*}{ Property } & \multicolumn{2}{|c|}{ Material } \\
\hline & Masonry V\&H & Concrete \\
\hline Thickness (mm) & 143 & 560 \\
\hline Grout Tensile Strength $(\mathrm{MPa})$ & 1.86 & 2.09 \\
\hline \multicolumn{3}{|c|}{ Masonry Properties (Y-Direction) } \\
\hline Gross Peak Compression Strength (MPa) & 18.3 & 40 \\
\hline Peak Compressive Strain & 0.0026 & 0.0021 \\
\hline Tensile Strength & 0 & 2.09 \\
\hline Tensile Strain & 0 & 0 \\
\hline Initial Tangent Elastic Modulus (MPa) & 6200 & 34800 \\
\hline Poisson's Ratio & 0 & 0.15 \\
\hline \multicolumn{3}{|c|}{ Masonry Properties (X-Direction) } \\
\hline Gross Peak Compression Strength (MPa) & 18.3 & 40 \\
\hline Peak Compressive Strain & 0.0026 & 0.0021 \\
\hline Tensile Strength & 0 & 2.09 \\
\hline Tensile Strain & 0 & 0 \\
\hline Initial Tangent Elastic Modulus (MPa) & 6200 & 34800 \\
\hline Poisson's Ratio & 0 & 0.15 \\
\hline \multicolumn{3}{|c|}{ Masonry Properties (Block) } \\
\hline Length (mm) & 390 & 2400 \\
\hline Height (mm) & 190 & 400 \\
\hline Face Shell Thickness (mm) & 25 & 280 \\
\hline Percent Solid (\%) & 52 & 100 \\
\hline Tensile Strength & 2.03 & 2.09 \\
\hline \multicolumn{3}{|l|}{ Head Joint } \\
\hline Thickness (mm) & 10 & 10 \\
\hline Tensile Bond Strength (MPa) & 0.40 & 2.09 \\
\hline Joint Cohesion (MPa) & 8.75 & 2.09 \\
\hline Angle of Internal Friction (Deg) & 42 & 42 \\
\hline \multicolumn{3}{|l|}{ Bed Joint } \\
\hline Thickness (mm) & 10 & 10 \\
\hline Tensile Bond Strength (MPa) & 0.40 & 2.09 \\
\hline Joint Cohesion (MPa) & 8.75 & 2.09 \\
\hline Angle of Internal Friction (Deg) & 42 & 42 \\
\hline \multicolumn{3}{|c|}{ Reinforcement (Y-Direction) } \\
\hline Ratio (\%) & 0.67 & 1.00 \\
\hline Yield Strength (MPa) & 488 & 400 \\
\hline Yield Strain & 0.00244 & 0.002 \\
\hline Strain Hardening Strain & 0.02 & 0.02 \\
\hline Ultimate Strength $(\mathrm{MPa})$ & 802 & 600 \\
\hline Ultimate Strain & 0.2 & 0.2 \\
\hline Prestrain & 0 & 0 \\
\hline \multicolumn{3}{|c|}{ Reinforcement (X-Direction) } \\
\hline Ratio (\%) & 0.97 & 1.00 \\
\hline Yield Strength (MPa) & 489 & 400 \\
\hline Yield Strain & 0.00245 & 0.002 \\
\hline Strain Hardening Strain & 0.02 & 0.02 \\
\hline Ultimate Strength (MPa) & 664 & 600 \\
\hline Ultimate Strain & 0.2 & 0.2 \\
\hline Prestrain & 0 & 0 \\
\hline
\end{tabular}

Numerical Modelling of Hollow Concrete Block Masonry Mechanics 


\section{Chen et al. (1978)}

\section{HCBL-11-1 Shear Wall}

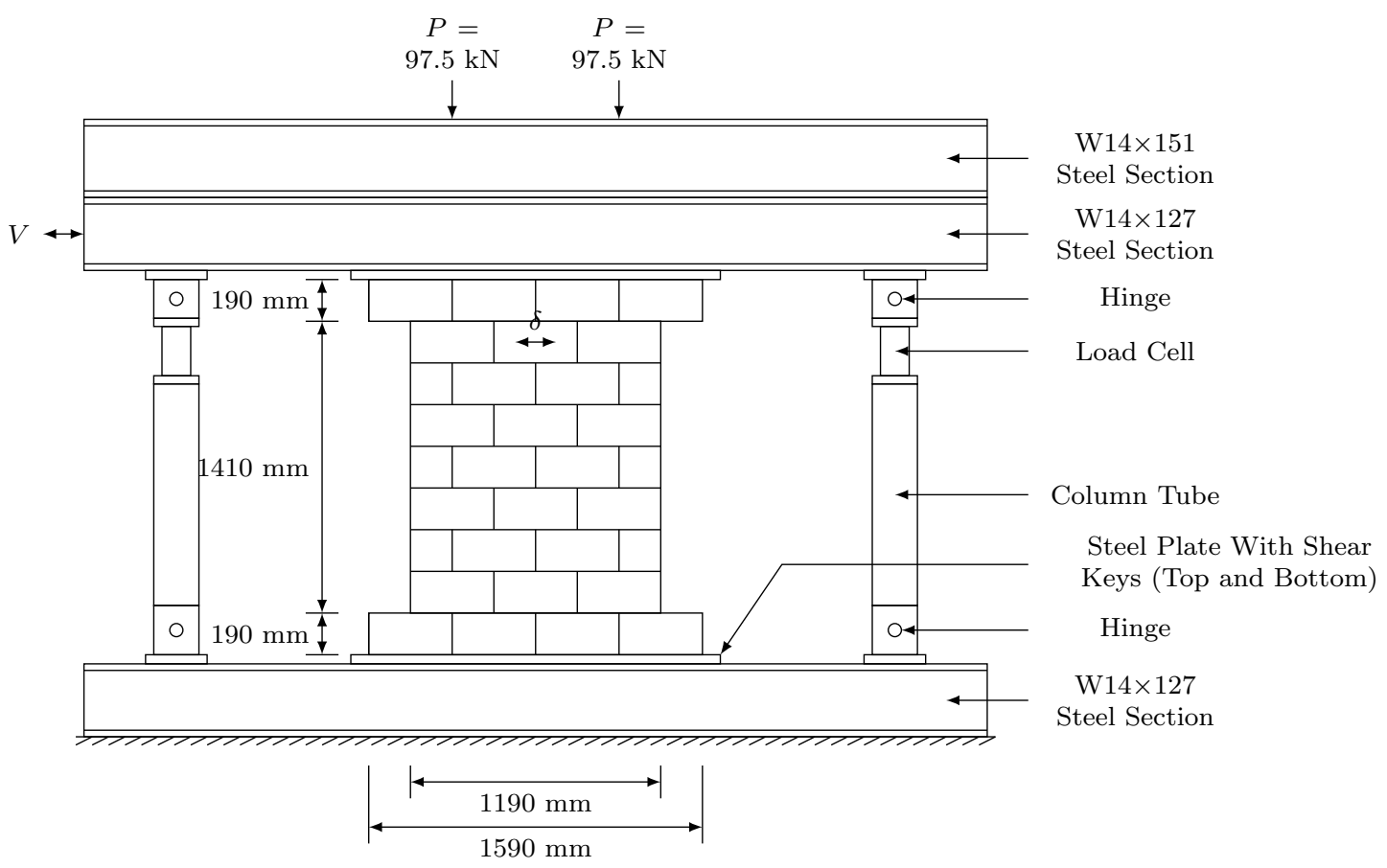

Experimental Wall (Chen et al., 1978)

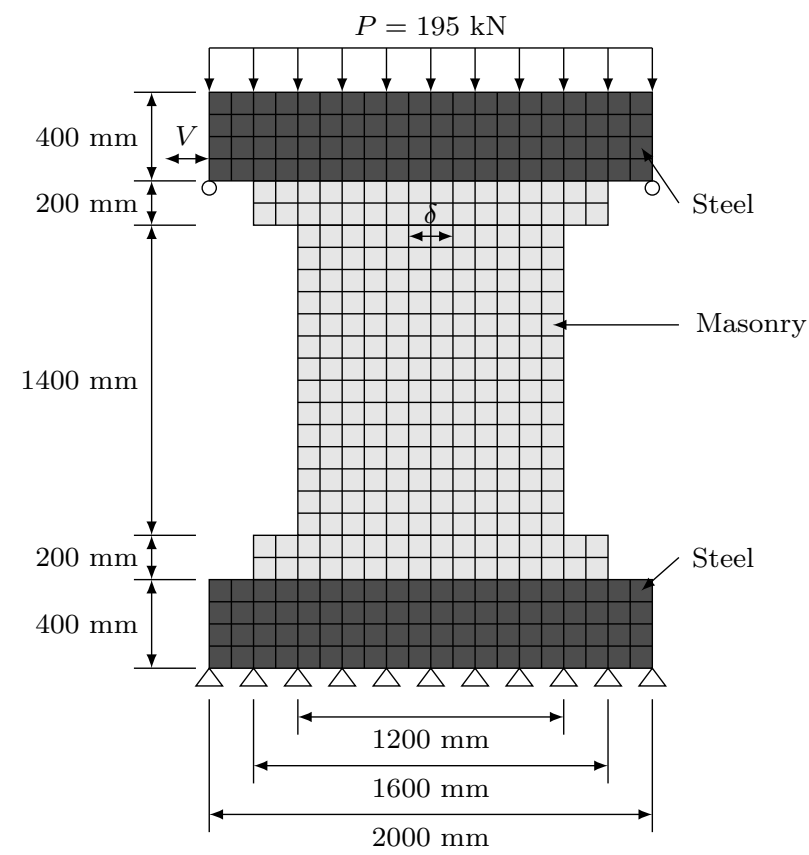

Finite Element Model Wall

HCBL-11-1 Shear Wall 
HCBL-11-1 Shear Wall (Chen et al., 1978) Model Options

\begin{tabular}{ll}
\hline Model & Selected Option \\
\hline Compression Pre-Peak & Hognestad \\
Compression Post-Peak & Pre-Peak Base Curve \\
Compression Softening & Vecchio \\
Tension Post-Peak & Hordijk \\
Cracking Criterion & Mohr-Coulomb \\
Slip & Constant Rotation Lag \\
\hline
\end{tabular}

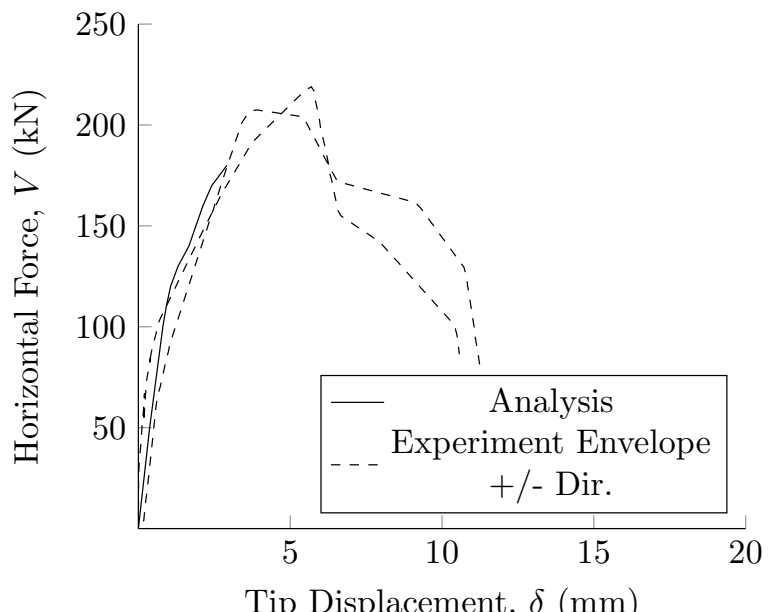

HCBL-11-1 Shear Wall (Chen et al., 1978) Horizontal Force vs Tip Displacement 
HCBL-11-1 Shear Wall (Chen et al., 1978) Material Properties

\begin{tabular}{|c|c|c|}
\hline \multirow{2}{*}{ Property } & \multicolumn{2}{|c|}{ Material } \\
\hline & Masonry & Steel \\
\hline Thickness (mm) & 190 & 200 \\
\hline Grout Tensile Strength (MPa) & 1.96 & 350 \\
\hline \multicolumn{3}{|c|}{ Masonry Properties (Y-Direction) } \\
\hline Gross Peak Compression Strength (MPa) & 9.17 & 350 \\
\hline Peak Compressive Strain & 0.0026 & 0.00175 \\
\hline Tensile Strength & 0 & 350 \\
\hline Tensile Strain & 0 & 0.00175 \\
\hline Initial Tangent Elastic Modulus (MPa) & 4200 & 200000 \\
\hline Poisson's Ratio & 0 & 0 \\
\hline \multicolumn{3}{|c|}{ Masonry Properties (X-Direction) } \\
\hline Gross Peak Compression Strength (MPa) & 9.17 & 350 \\
\hline Peak Compressive Strain & 0.0026 & 0.00175 \\
\hline Tensile Strength & 0 & 350 \\
\hline Tensile Strain & 0 & 0.00175 \\
\hline Initial Tangent Elastic Modulus (MPa) & 4200 & 200000 \\
\hline Poisson's Ratio & 0 & 0 \\
\hline \multicolumn{3}{|l|}{ Masonry Properties (Block) } \\
\hline Length $(\mathrm{mm})$ & 390 & 2000 \\
\hline Height $(\mathrm{mm})$ & 190 & 400 \\
\hline Face Shell Thickness (mm) & 41 & 100 \\
\hline Percent Solid (\%) & 59 & 100 \\
\hline Tensile Strength & 1.51 & 350 \\
\hline \multicolumn{3}{|l|}{ Head Joint } \\
\hline Thickness (mm) & 10 & 10 \\
\hline Tensile Bond Strength (MPa) & 0.33 & 350 \\
\hline Joint Cohesion $(\mathrm{MPa})$ & 5.80 & 350 \\
\hline Angle of Internal Friction (Deg) & 42 & 42 \\
\hline \multicolumn{3}{|l|}{ Bed Joint } \\
\hline Thickness (mm) & 10 & 10 \\
\hline Tensile Bond Strength (MPa) & 0.33 & 350 \\
\hline Joint Cohesion (MPa) & 5.80 & 350 \\
\hline Angle of Internal Friction (Deg) & 42 & 42 \\
\hline \multicolumn{3}{|c|}{ Reinforcement (Y-Direction) } \\
\hline Ratio (\%) & 0 & 100 \\
\hline Yield Strength (MPa) & 0 & 350 \\
\hline Yield Strain & 0 & 0.00175 \\
\hline Strain Hardening Strain & 0 & 0.02 \\
\hline Ultimate Strength (MPa) & 0 & 600 \\
\hline Ultimate Strain & 0 & 0.2 \\
\hline Prestrain & 0 & 0 \\
\hline \multicolumn{3}{|c|}{ Reinforcement (X-Direction) } \\
\hline Ratio (\%) & 0 & 100 \\
\hline Yield Strength (MPa) & 0 & 350 \\
\hline Yield Strain & 0 & 0.00175 \\
\hline Strain Hardening Strain & 0 & 0.02 \\
\hline Ultimate Strength (MPa) & 0 & 600 \\
\hline Ultimate Strain & 0 & 0.2 \\
\hline Prestrain & 0 & 0 \\
\hline
\end{tabular}

Numerical Modelling of Hollow Concrete Block Masonry Mechanics 

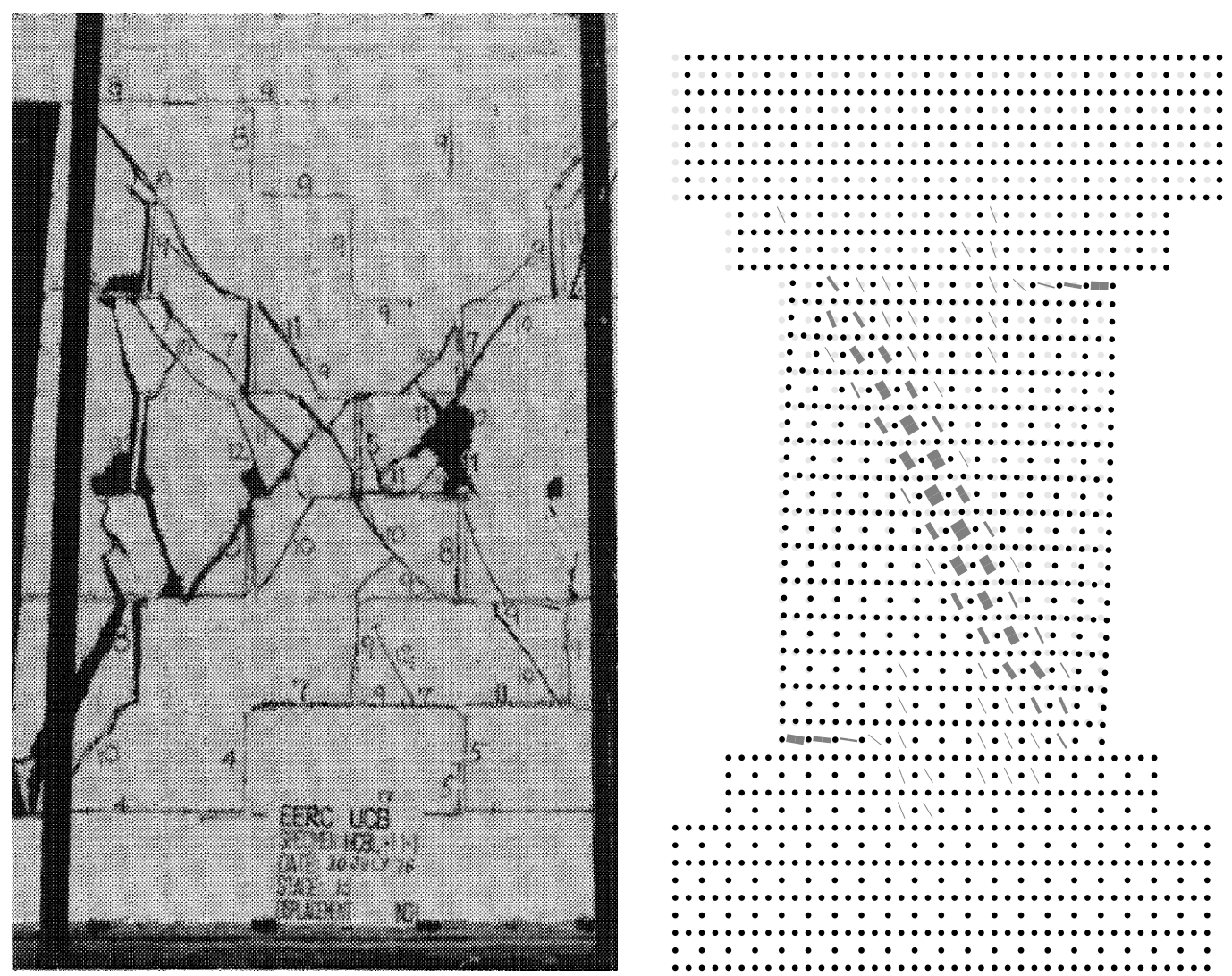

Experiment at Peak Horizontal Force (Chen Finite Element Model at Peak Horizontal Force et al., 1978) $\times 15$

HCBL-11-1 Shear Wall Displacement, Crack Pattern, and Crack Widths 


\section{HCBL-11-3 Shear Wall}

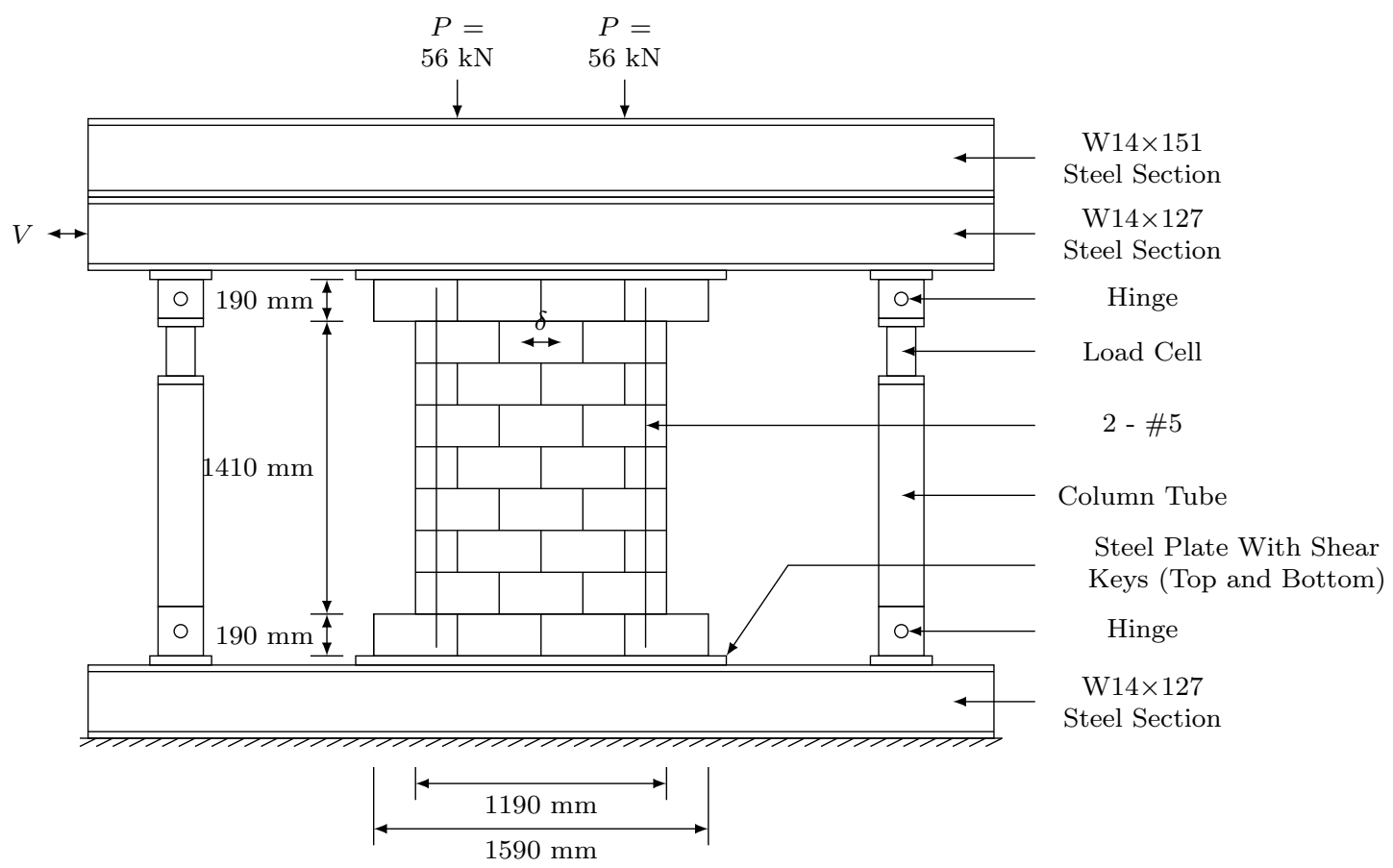

Experimental Wall (Chen et al., 1978)

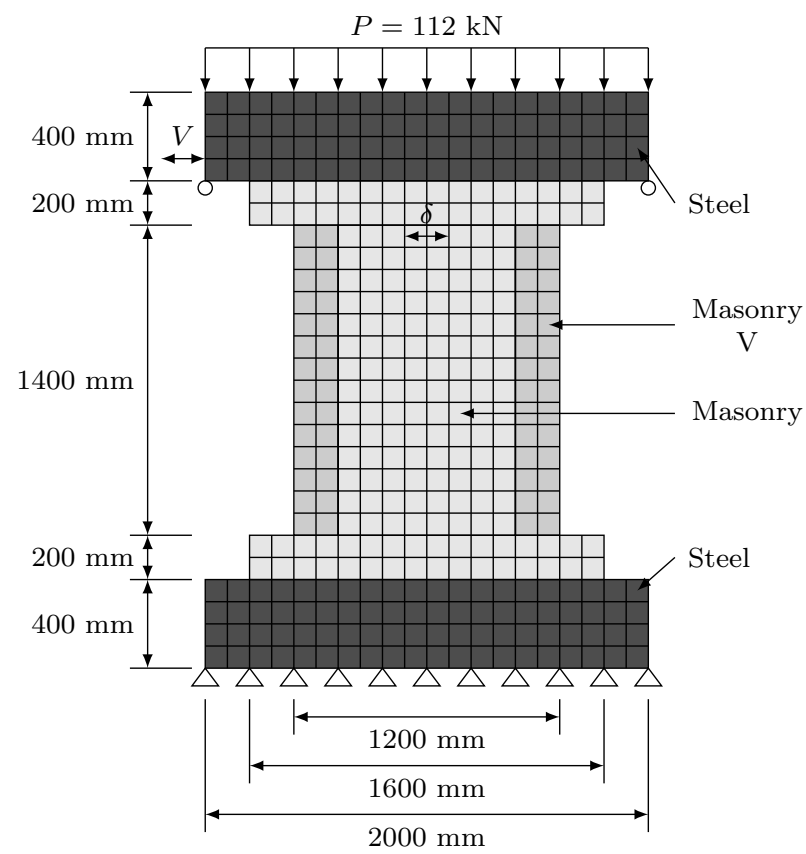

Finite Element Model Wall

HCBL-11-3 Shear Wall 
HCBL-11-3 Shear Wall (Chen et al., 1978) Model Options

\begin{tabular}{ll}
\hline Model & Selected Option \\
\hline Compression Pre-Peak & Hognestad \\
Compression Post-Peak & Pre-Peak Base Curve \\
Compression Softening & Vecchio \\
Tension Post-Peak & Vecchio \\
Cracking Criterion & Mohr-Coulomb \\
Slip & Walraven Reinforced \\
\hline
\end{tabular}

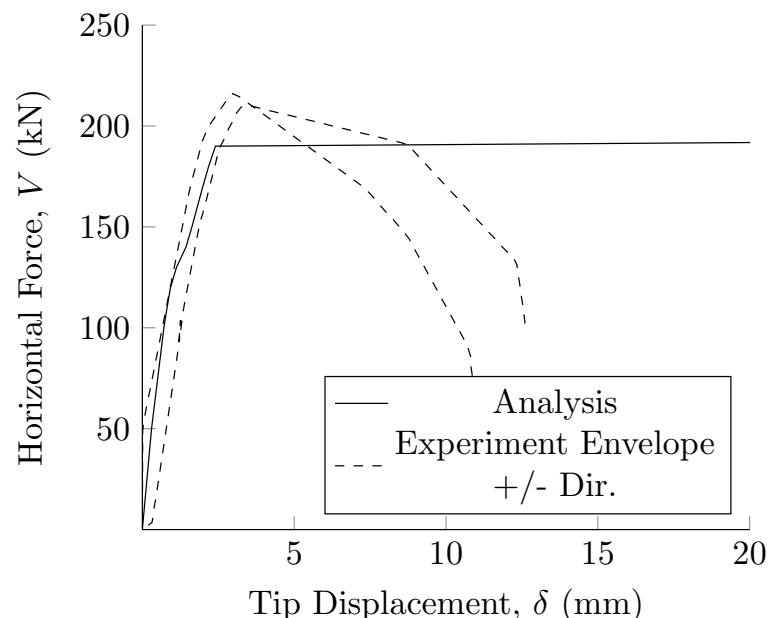

HCBL-11-3 Shear Wall (Chen et al., 1978) Horizontal Force vs Tip Displacement 
HCBL-11-3 Shear Wall (Chen et al., 1978) Material Properties

\begin{tabular}{|c|c|c|c|}
\hline \multirow{2}{*}{ Property } & \multicolumn{3}{|c|}{ Material } \\
\hline & Masonry & Masonry V & Steel \\
\hline Thickness (mm) & 190 & 190 & 200 \\
\hline Grout Tensile Strength (MPa) & 1.68 & 1.68 & 350 \\
\hline \multicolumn{4}{|c|}{ Masonry Properties (Y-Direction) } \\
\hline Gross Peak Compression Strength (MPa) & 12.6 & 12.6 & 350 \\
\hline Peak Compressive Strain & 0.0024 & 0.0024 & 0.00175 \\
\hline Tensile Strength & 0 & 0 & 350 \\
\hline Tensile Strain & 0 & 0 & 0.00175 \\
\hline Initial Tangent Elastic Modulus (MPa) & 5100 & 5100 & 200000 \\
\hline Poisson's Ratio & 0 & 0 & 0 \\
\hline \multicolumn{4}{|c|}{ Masonry Properties (X-Direction) } \\
\hline Gross Peak Compression Strength (MPa) & 12.6 & 12.6 & 350 \\
\hline Peak Compressive Strain & 0.0024 & 0.0024 & 0.00175 \\
\hline Tensile Strength & 0 & 0 & 350 \\
\hline Tensile Strain & 0 & 0 & 0.00175 \\
\hline Initial Tangent Elastic Modulus (MPa) & 5100 & 5100 & 200000 \\
\hline Poisson's Ratio & 0 & 0 & 0 \\
\hline \multicolumn{4}{|c|}{ Masonry Properties (Block) } \\
\hline Length (mm) & 390 & 390 & 2000 \\
\hline Height (mm) & 190 & 190 & 400 \\
\hline Face Shell Thickness (mm) & 41 & 41 & 100 \\
\hline Percent Solid (\%) & 59 & 59 & 100 \\
\hline Tensile Strength & 1.51 & 1.51 & 350 \\
\hline \multicolumn{4}{|c|}{ Head Joint } \\
\hline Thickness (mm) & 10 & 10 & 10 \\
\hline Tensile Bond Strength (MPa) & 0.35 & 0.35 & 350 \\
\hline Joint Cohesion (MPa) & 6.20 & 6.20 & 350 \\
\hline Angle of Internal Friction (Deg) & 42 & 42 & 42 \\
\hline \multicolumn{4}{|c|}{ Bed Joint } \\
\hline Thickness (mm) & 10 & 10 & 10 \\
\hline Tensile Bond Strength (MPa) & 0.35 & 0.35 & 350 \\
\hline Joint Cohesion (MPa) & 6.20 & 6.20 & 350 \\
\hline Angle of Internal Friction (Deg) & 42 & 42 & 42 \\
\hline \multicolumn{4}{|c|}{ Reinforcement (Y-Direction) } \\
\hline Ratio (\%) & 0 & 0.17 & 100 \\
\hline Yield Strength (MPa) & 0 & 488 & 350 \\
\hline Yield Strain & 0 & 0.00244 & 0.00175 \\
\hline Strain Hardening Strain & 0 & 0.02 & 0.02 \\
\hline Ultimate Strength (MPa) & 0 & 750 & 600 \\
\hline Ultimate Strain & 0 & 0.2 & 0.2 \\
\hline Prestrain & 0 & 0 & 0 \\
\hline \multicolumn{4}{|c|}{ Reinforcement (X-Direction) } \\
\hline Ratio (\%) & 0 & 0 & 100 \\
\hline Yield Strength (MPa) & 0 & 0 & 350 \\
\hline Yield Strain & 0 & 0 & 0.00175 \\
\hline Strain Hardening Strain & 0 & 0 & 0.02 \\
\hline Ultimate Strength (MPa) & 0 & 0 & 600 \\
\hline Ultimate Strain & 0 & 0 & 0.2 \\
\hline Prestrain & 0 & 0 & 0 \\
\hline
\end{tabular}

Numerical Modelling of Hollow Concrete Block Masonry Mechanics 

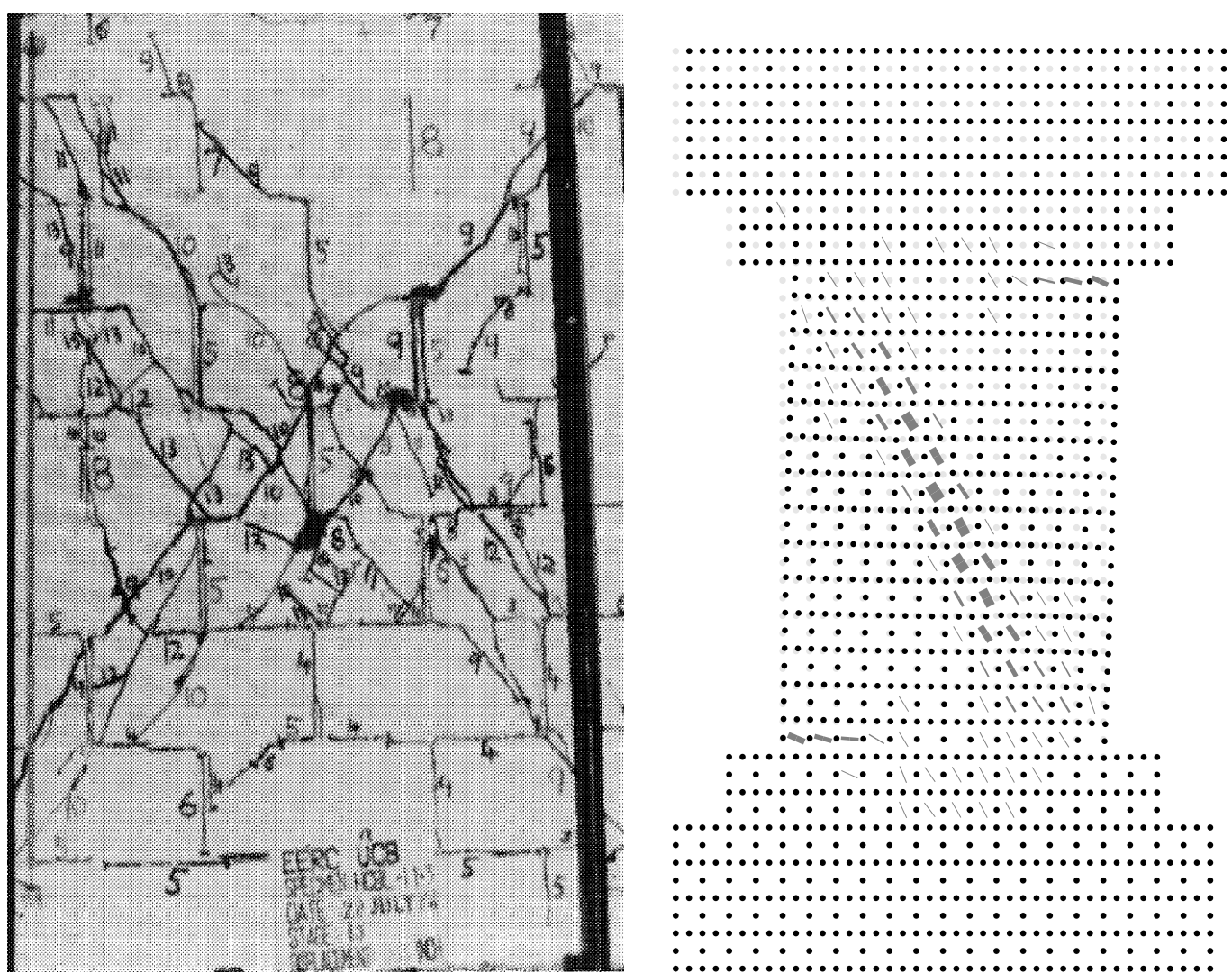

Experiment at Peak Horizontal Force (Chen Finite Element Model at Peak Horizontal Force et al., 1978) $\times 20$

HCBL-11-3 Shear Wall Displacement, Crack Pattern, and Crack Widths 


\section{HCBL-11-4 Shear Wall}

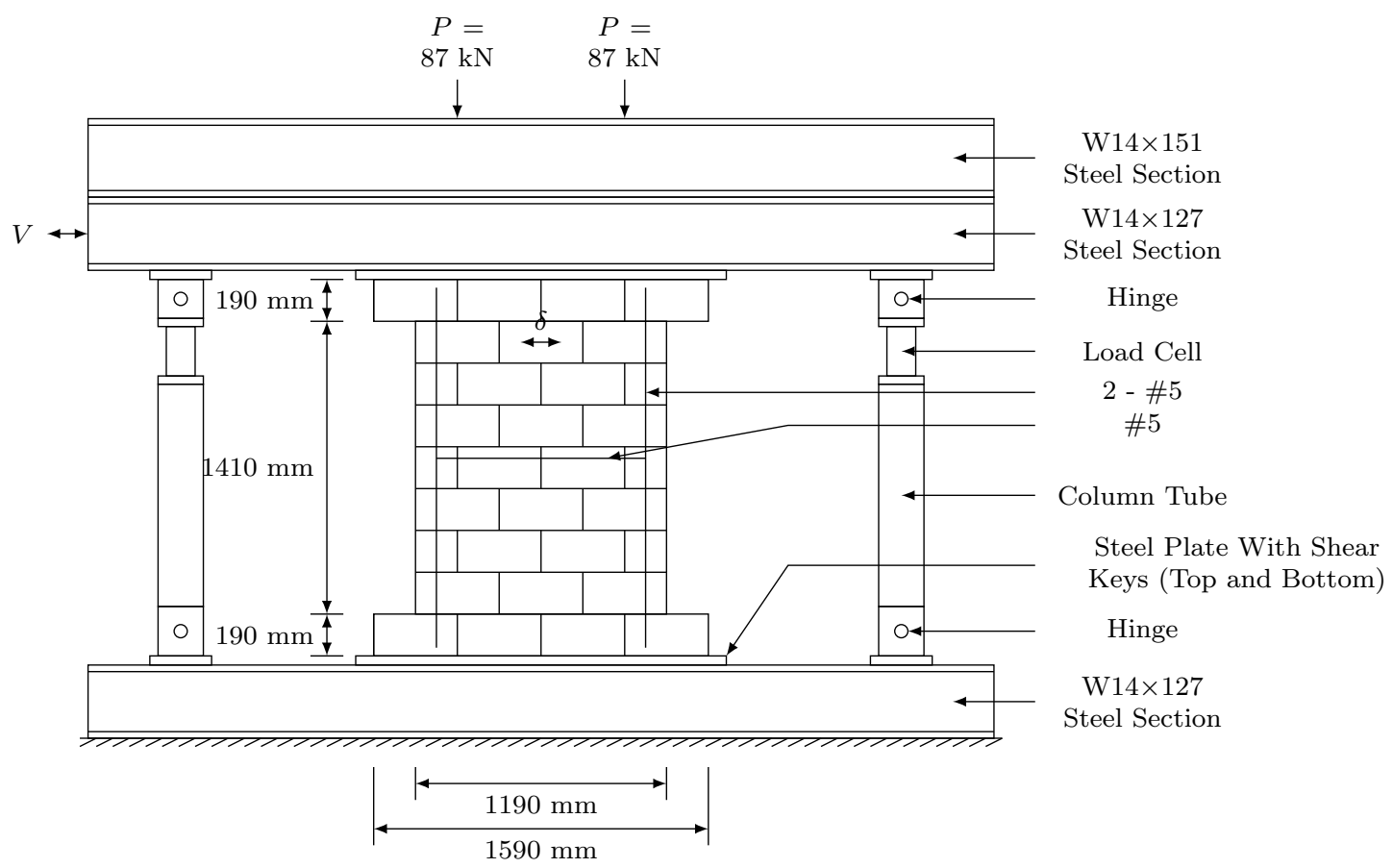

Experimental Wall (Chen et al., 1978)

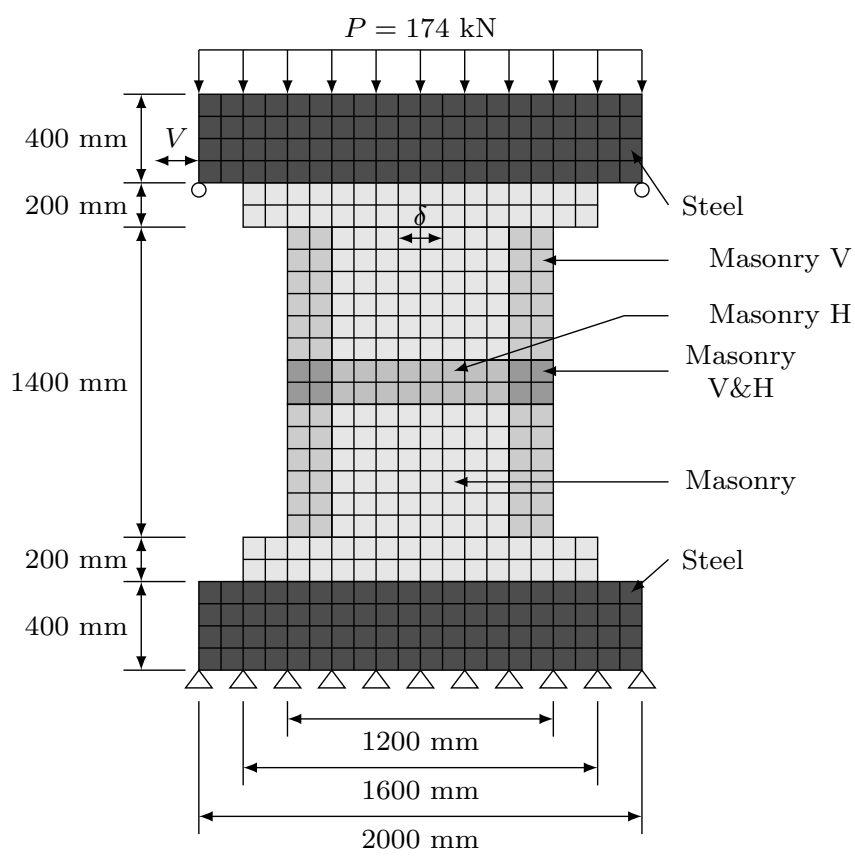

Finite Element Model Wall

HCBL-11-4 Shear Wall 
HCBL-11-4 Shear Wall (Chen et al., 1978) Model Options

\begin{tabular}{ll}
\hline Model & Selected Option \\
\hline Compression Pre-Peak & Hognestad \\
Compression Post-Peak & Pre-Peak Base Curve \\
Compression Softening & Vecchio \\
Tension Post-Peak & Vecchio \\
Cracking Criterion & Mohr-Coulomb \\
Slip & Walraven Reinforced \\
\hline
\end{tabular}

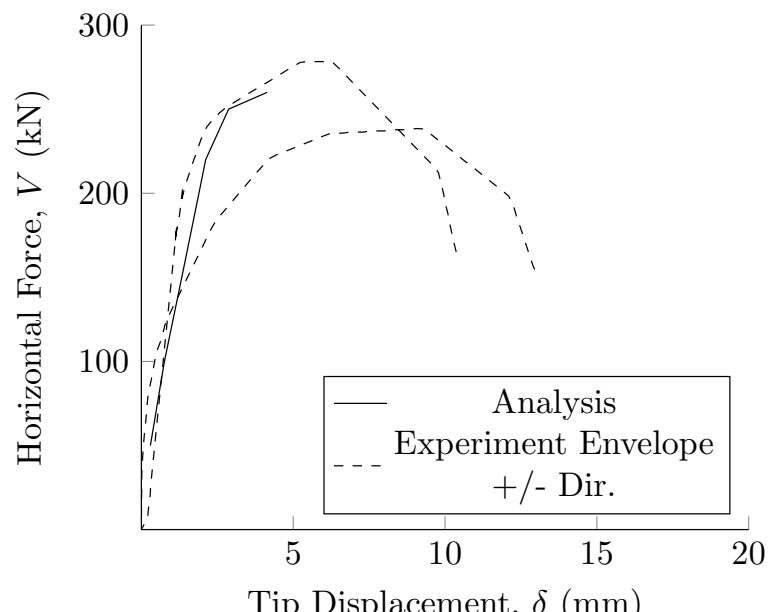

HCBL-11-4 Shear Wall (Chen et al., 1978) Horizontal Force vs Tip Displacement 
HCBL-11-4 Shear Wall (Chen et al., 1978) Material Properties

\begin{tabular}{|c|c|c|c|c|c|}
\hline \multirow{2}{*}{ Property } & \multicolumn{5}{|c|}{ Material } \\
\hline & Masonry & Masonry V & Masonry $\mathrm{H}$ & Masonry V\&H & Steel \\
\hline Thickness (mm) & 190 & 190 & 190 & 190 & 200 \\
\hline Grout Tensile Strength (MPa) & 1.68 & 1.68 & 1.68 & 1.68 & 350 \\
\hline \multicolumn{6}{|c|}{ Masonry Properties (Y-Direction) } \\
\hline Gross Peak Compression Strength (MPa) & 12.6 & 12.6 & 12.6 & 12.6 & 350 \\
\hline Peak Compressive Strain & 0.0024 & 0.0024 & 0.0024 & 0.0024 & 0.00175 \\
\hline Tensile Strength & 0 & 0 & 0 & 0 & 350 \\
\hline Tensile Strain & 0 & 0 & 0 & 0 & 0.00175 \\
\hline Initial Tangent Elastic Modulus (MPa) & 5700 & 5700 & 5700 & 5700 & 200000 \\
\hline Poisson's Ratio & 0 & 0 & 0 & 0 & 0 \\
\hline \multicolumn{6}{|c|}{ Masonry Properties (X-Direction) } \\
\hline Gross Peak Compression Strength (MPa) & 12.6 & 12.6 & 12.6 & 12.6 & 350 \\
\hline Peak Compressive Strain & 0.0024 & 0.0024 & 0.0024 & 0.0024 & 0.00175 \\
\hline Tensile Strength & 0 & 0 & 0 & 0 & 350 \\
\hline Tensile Strain & 0 & 0 & 0 & 0 & 0.00175 \\
\hline Initial Tangent Elastic Modulus (MPa) & 5700 & 5700 & 5700 & 5700 & 200000 \\
\hline Poisson's Ratio & 0 & 0 & 0 & 0 & 0 \\
\hline \multicolumn{6}{|c|}{ Masonry Properties (Block) } \\
\hline Length (mm) & 390 & 390 & 390 & 390 & 2000 \\
\hline Height (mm) & 190 & 190 & 190 & 190 & 400 \\
\hline Face Shell Thickness (mm) & 41 & 41 & 41 & 41 & 100 \\
\hline Percent Solid (\%) & 59 & 59 & 59 & 59 & 100 \\
\hline Tensile Strength & 1.51 & 1.51 & 1.51 & 1.51 & 350 \\
\hline \multicolumn{6}{|c|}{ Head Joint } \\
\hline Thickness (mm) & 10 & 10 & 10 & 10 & 10 \\
\hline Tensile Bond Strength (MPa) & 0.35 & 0.35 & 0.35 & 0.35 & 350 \\
\hline Joint Cohesion (MPa) & 6.20 & 6.20 & 6.20 & 6.20 & 350 \\
\hline Angle of Internal Friction (Deg) & 42 & 42 & 42 & 42 & 42 \\
\hline \multicolumn{6}{|c|}{ Bed Joint } \\
\hline Thickness (mm) & 10 & 10 & 10 & 10 & 10 \\
\hline Tensile Bond Strength (MPa) & 0.35 & 0.35 & 0.35 & 0.35 & 350 \\
\hline Joint Cohesion (MPa) & 6.20 & 6.20 & 6.20 & 6.20 & 350 \\
\hline Angle of Internal Friction (Deg) & 42 & 42 & 42 & 42 & 42 \\
\hline \multicolumn{6}{|c|}{ Reinforcement (Y-Direction) } \\
\hline Ratio (\%) & 0 & 0.17 & 0 & 0.17 & 100 \\
\hline Yield Strength (MPa) & 0 & 488 & 0 & 488 & 350 \\
\hline Yield Strain & 0 & 0.00244 & 0 & 0.00244 & 0.00175 \\
\hline Strain Hardening Strain & 0 & 0.02 & 0 & 0.02 & 0.02 \\
\hline Ultimate Strength (MPa) & 0 & 750 & 0 & 750 & 600 \\
\hline Ultimate Strain & 0 & 0.2 & 0 & 0.2 & 0.2 \\
\hline Prestrain & 0 & 0 & 0 & 0 & 0 \\
\hline \multicolumn{6}{|c|}{ Reinforcement (X-Direction) } \\
\hline Ratio (\%) & 0 & 0 & 0.08 & 0.08 & 100 \\
\hline Yield Strength (MPa) & 0 & 0 & 330 & 330 & 350 \\
\hline Yield Strain & 0 & 0 & 0.00165 & 0.00165 & 0.00175 \\
\hline Strain Hardening Strain & 0 & 0 & 0.02 & 0.02 & 0.02 \\
\hline Ultimate Strength (MPa) & 0 & 0 & 522 & 522 & 600 \\
\hline Ultimate Strain & 0 & 0 & 0.2 & 0.2 & 0.2 \\
\hline Prestrain & 0 & 0 & 0 & 0 & 0 \\
\hline
\end{tabular}

Numerical Modelling of Hollow Concrete Block Masonry Mechanics 

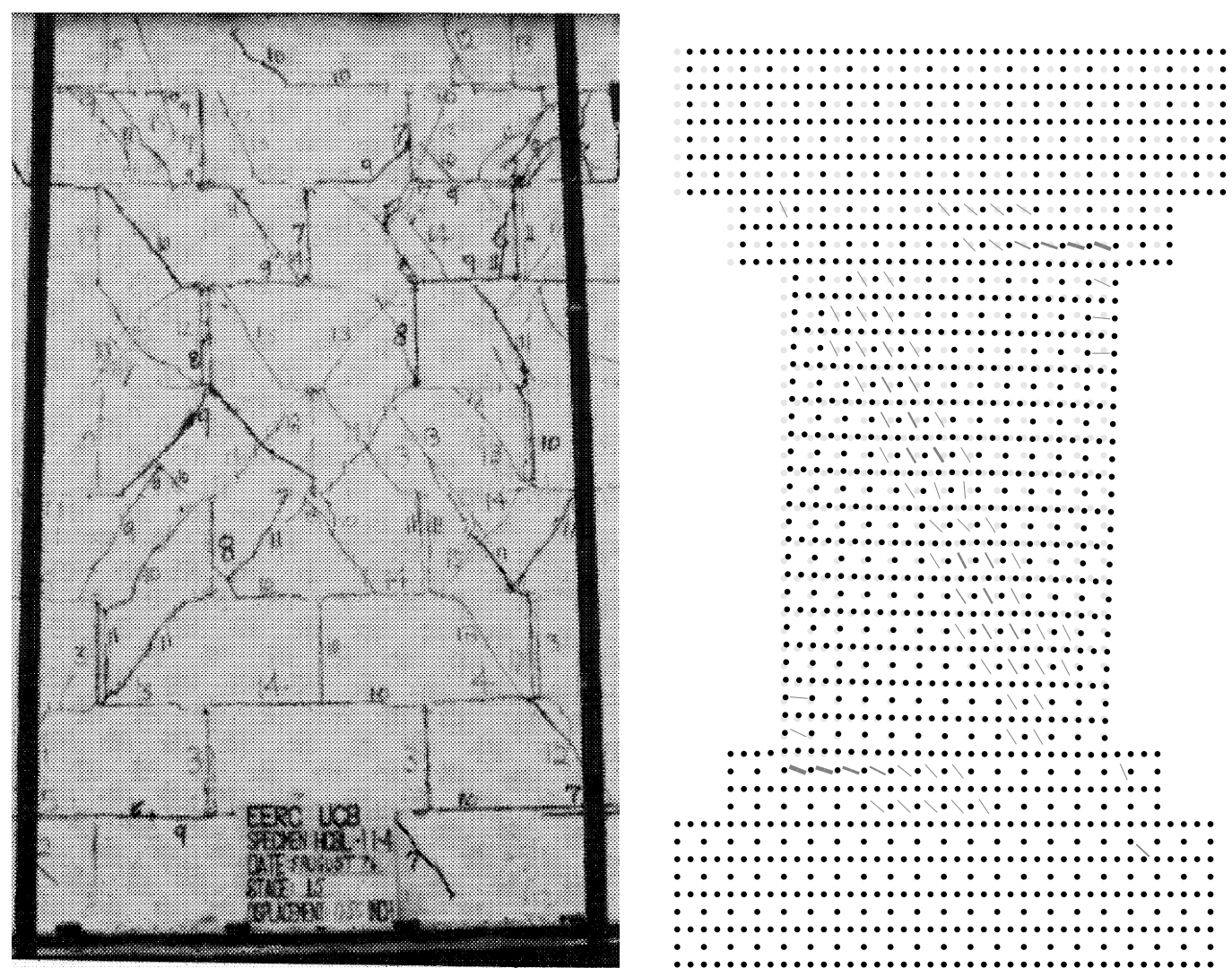

Experiment at Peak Horizontal Force (Chen Finite Element Model at Peak Horizontal Force et al., 1978) $\times 15$

HCBL-11-4 Shear Wall Displacement, Crack Pattern, and Crack Widths 


\section{HCBL-11-6 Shear Wall}

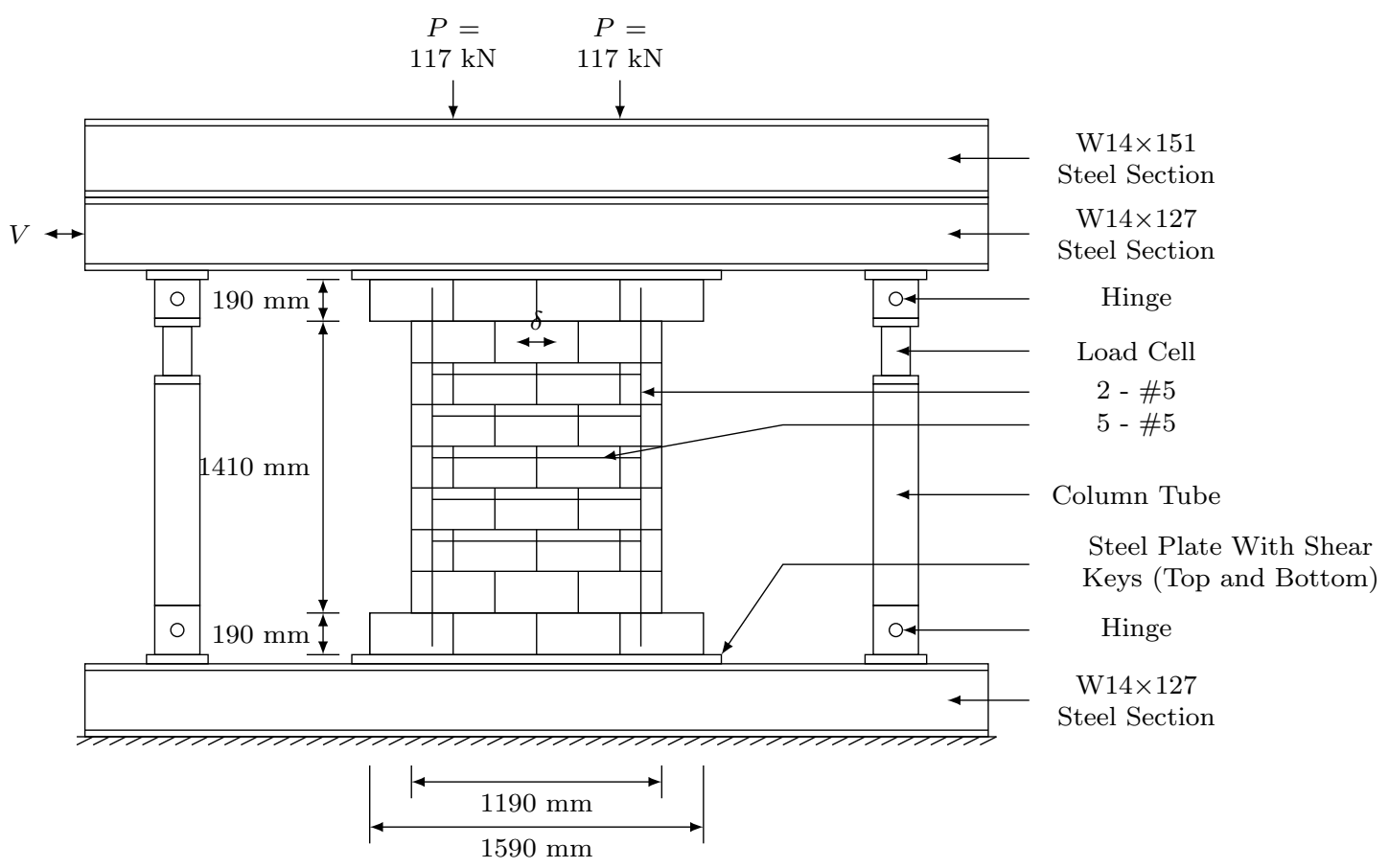

Experimental Wall (Chen et al., 1978)

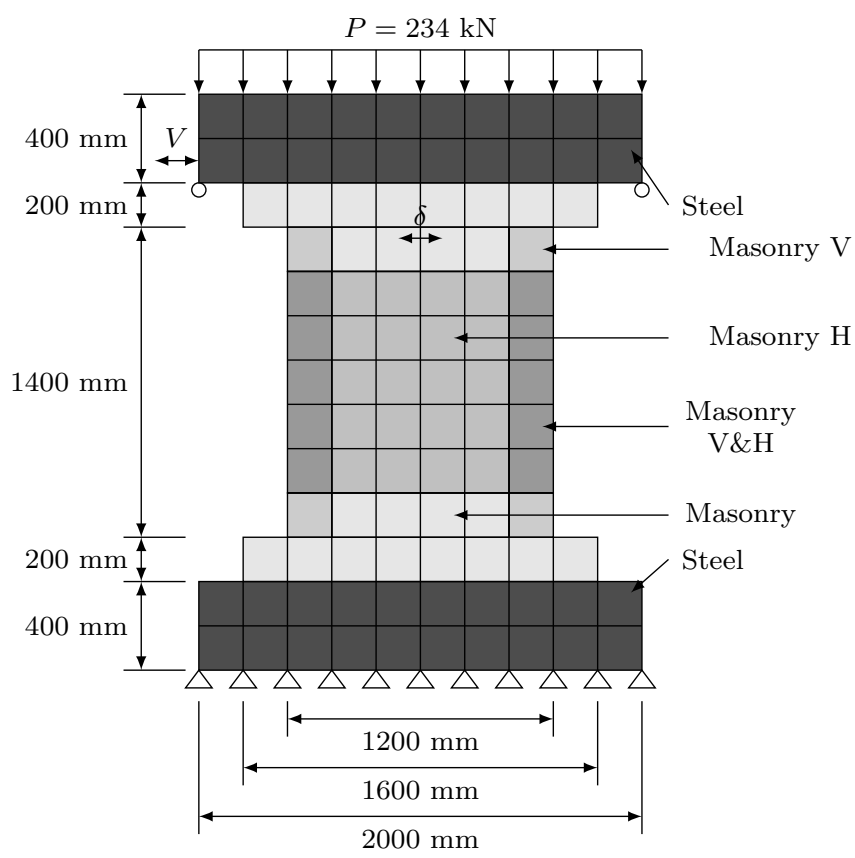

Finite Element Model Wall

HCBL-11-6 Shear Wall 
HCBL-11-6 Shear Wall (Chen et al., 1978) Model Options

\begin{tabular}{ll}
\hline Model & Selected Option \\
\hline Compression Pre-Peak & Hognestad \\
Compression Post-Peak & Pre-Peak Base Curve \\
Compression Softening & Vecchio \\
Tension Post-Peak & Vecchio \\
Cracking Criterion & Mohr-Coulomb \\
Slip & Walraven Reinforced \\
\hline
\end{tabular}

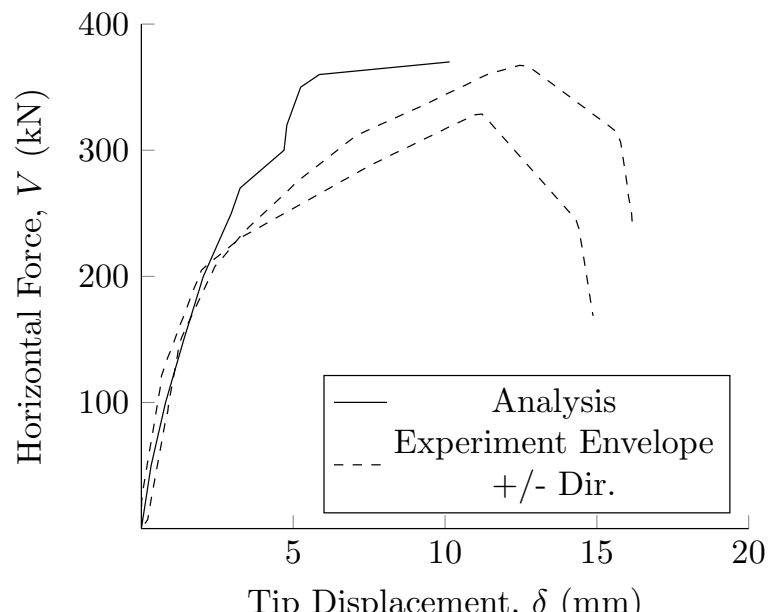

HCBL-11-6 Shear Wall (Chen et al., 1978) Horizontal Force vs Tip Displacement 
HCBL-11-6 Shear Wall (Chen et al., 1978) Material Properties

\begin{tabular}{|c|c|c|c|c|c|}
\hline \multirow{2}{*}{ Property } & \multicolumn{5}{|c|}{ Material } \\
\hline & Masonry & Masonry V & Masonry H & Masonry V\&H & Steel \\
\hline Thickness (mm) & 190 & 190 & 190 & 190 & 200 \\
\hline Grout Tensile Strength (MPa) & 1.68 & 1.68 & 1.68 & 1.68 & 350 \\
\hline \multicolumn{6}{|c|}{ Masonry Properties (Y-Direction) } \\
\hline Gross Peak Compression Strength (MPa) & 12.6 & 12.6 & 12.6 & 12.6 & 350 \\
\hline Peak Compressive Strain & 0.0024 & 0.0024 & 0.0024 & 0.0024 & 0.00175 \\
\hline Tensile Strength & 0 & 0 & 0 & 0 & 350 \\
\hline Tensile Strain & 0 & 0 & 0 & 0 & 0.00175 \\
\hline Initial Tangent Elastic Modulus (MPa) & 5000 & 5000 & 5000 & 5000 & 200000 \\
\hline Poisson's Ratio & 0 & 0 & 0 & 0 & 0 \\
\hline \multicolumn{6}{|c|}{ Masonry Properties (X-Direction) } \\
\hline Gross Peak Compression Strength (MPa) & 12.6 & 12.6 & 12.6 & 12.6 & 350 \\
\hline Peak Compressive Strain & 0.0024 & 0.0024 & 0.0024 & 0.0024 & 0.00175 \\
\hline Tensile Strength & 0 & 0 & 0 & 0 & 350 \\
\hline Tensile Strain & 0 & 0 & 0 & 0 & 0.00175 \\
\hline Initial Tangent Elastic Modulus (MPa) & 5000 & 5000 & 5000 & 5000 & 200000 \\
\hline Poisson's Ratio & 0 & 0 & 0 & 0 & 0 \\
\hline \multicolumn{6}{|c|}{ Masonry Properties (Block) } \\
\hline Length (mm) & 390 & 390 & 390 & 390 & 2000 \\
\hline Height (mm) & 190 & 190 & 190 & 190 & 400 \\
\hline Face Shell Thickness (mm) & 41 & 41 & 41 & 41 & 100 \\
\hline Percent Solid (\%) & 59 & 59 & 59 & 59 & 100 \\
\hline Tensile Strength & 1.51 & 1.51 & 1.51 & 1.51 & 350 \\
\hline \multicolumn{6}{|c|}{ Head Joint } \\
\hline Thickness (mm) & 10 & 10 & 10 & 10 & 10 \\
\hline Tensile Bond Strength (MPa) & 0.35 & 0.35 & 0.35 & 0.35 & 350 \\
\hline Joint Cohesion (MPa) & 6.20 & 6.20 & 6.20 & 6.20 & 350 \\
\hline Angle of Internal Friction (Deg) & 42 & 42 & 42 & 42 & 42 \\
\hline \multicolumn{6}{|c|}{ Bed Joint } \\
\hline Thickness (mm) & 10 & 10 & 10 & 10 & 10 \\
\hline Tensile Bond Strength (MPa) & 0.35 & 0.35 & 0.35 & 0.35 & 350 \\
\hline Joint Cohesion (MPa) & 6.20 & 6.20 & 6.20 & 6.20 & 350 \\
\hline Angle of Internal Friction (Deg) & 42 & 42 & 42 & 42 & 42 \\
\hline \multicolumn{6}{|c|}{ Reinforcement (Y-Direction) } \\
\hline Ratio (\%) & 0 & 0.17 & 0 & 0.17 & 100 \\
\hline Yield Strength (MPa) & 0 & 488 & 0 & 488 & 350 \\
\hline Yield Strain & 0 & 0.00244 & 0 & 0.00244 & 0.00175 \\
\hline Strain Hardening Strain & 0 & 0.02 & 0 & 0.02 & 0.02 \\
\hline Ultimate Strength (MPa) & 0 & 750 & 0 & 750 & 600 \\
\hline Ultimate Strain & 0 & 0.2 & 0 & 0.2 & 0.2 \\
\hline Prestrain & 0 & 0 & 0 & 0 & 0 \\
\hline \multicolumn{6}{|c|}{ Reinforcement (X-Direction) } \\
\hline Ratio (\%) & 0 & 0 & 0.30 & 0.30 & 100 \\
\hline Yield Strength (MPa) & 0 & 0 & 330 & 330 & 350 \\
\hline Yield Strain & 0 & 0 & 0.00165 & 0.00165 & 0.00175 \\
\hline Strain Hardening Strain & 0 & 0 & 0.02 & 0.02 & 0.02 \\
\hline Ultimate Strength (MPa) & 0 & 0 & 522 & 522 & 600 \\
\hline Ultimate Strain & 0 & 0 & 0.2 & 0.2 & 0.2 \\
\hline Prestrain & 0 & 0 & 0 & 0 & 0 \\
\hline
\end{tabular}

Numerical Modelling of Hollow Concrete Block Masonry Mechanics 

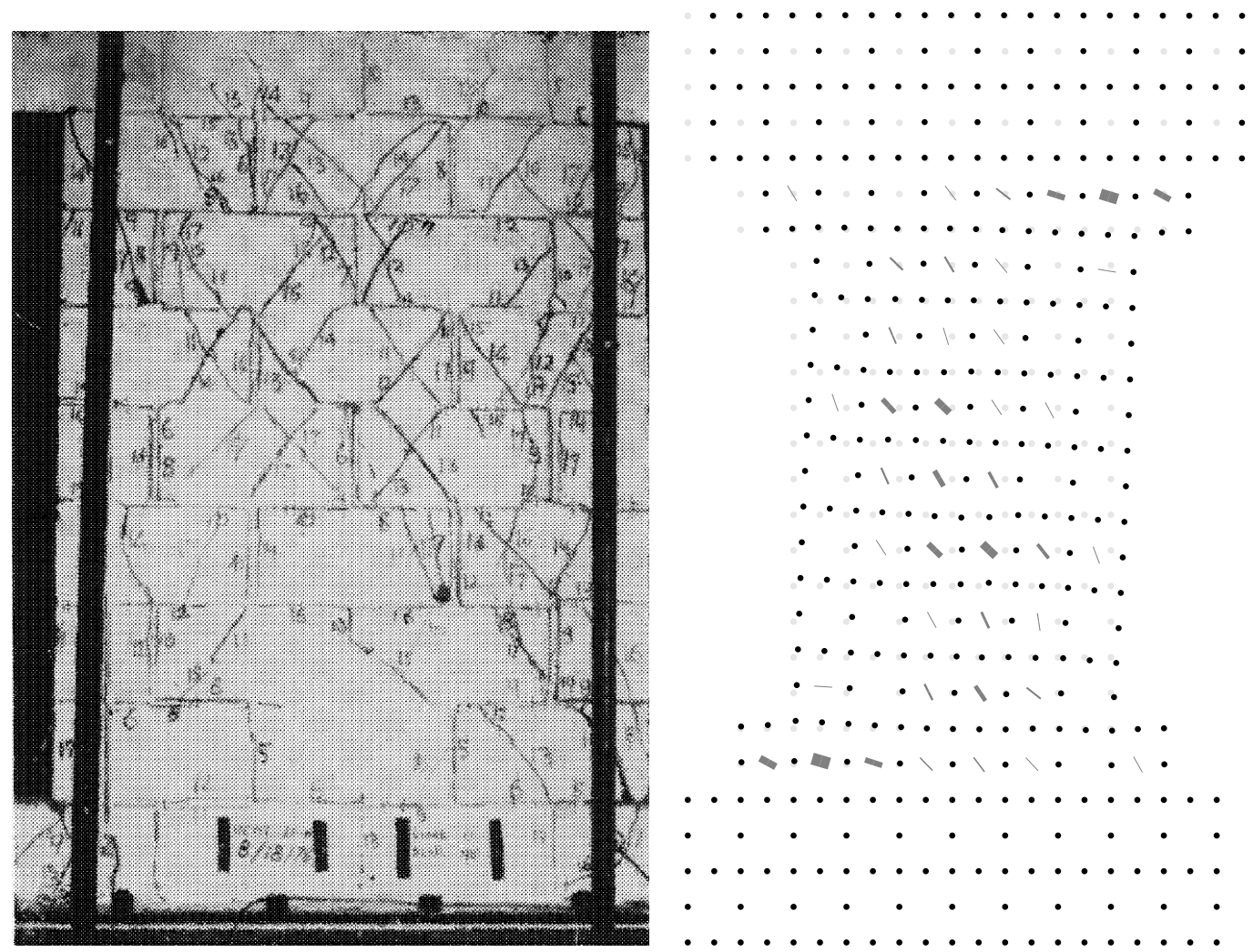

Experiment at Peak Horizontal Force (Chen Finite Element Model at Peak Horizontal Force et al., 1978) $\times 15$

HCBL-11-6 Shear Wall Displacement, Crack Pattern, and Crack Widths 


\section{HCBL-11-7 Shear Wall}

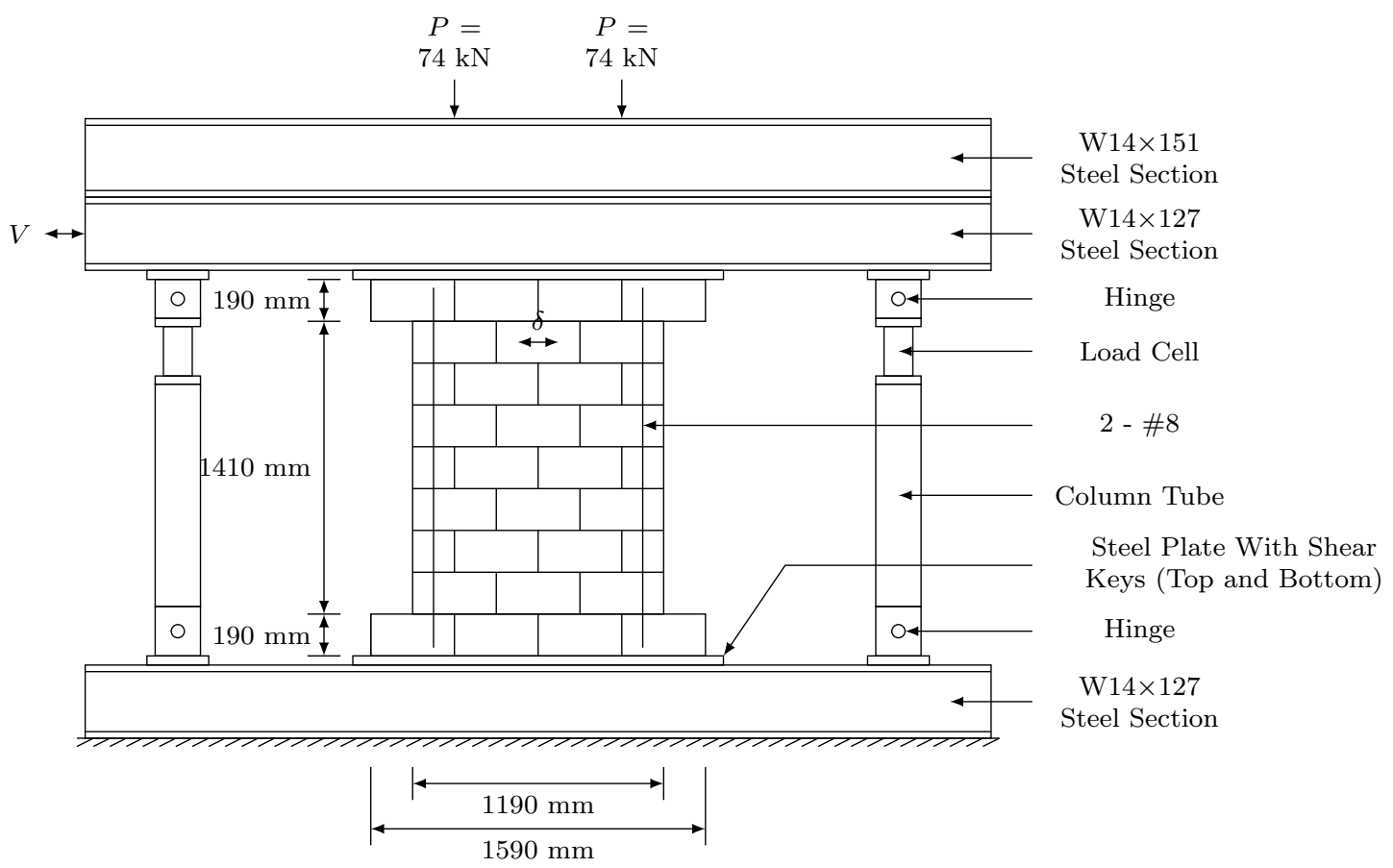

Experimental Wall (Chen et al., 1978)

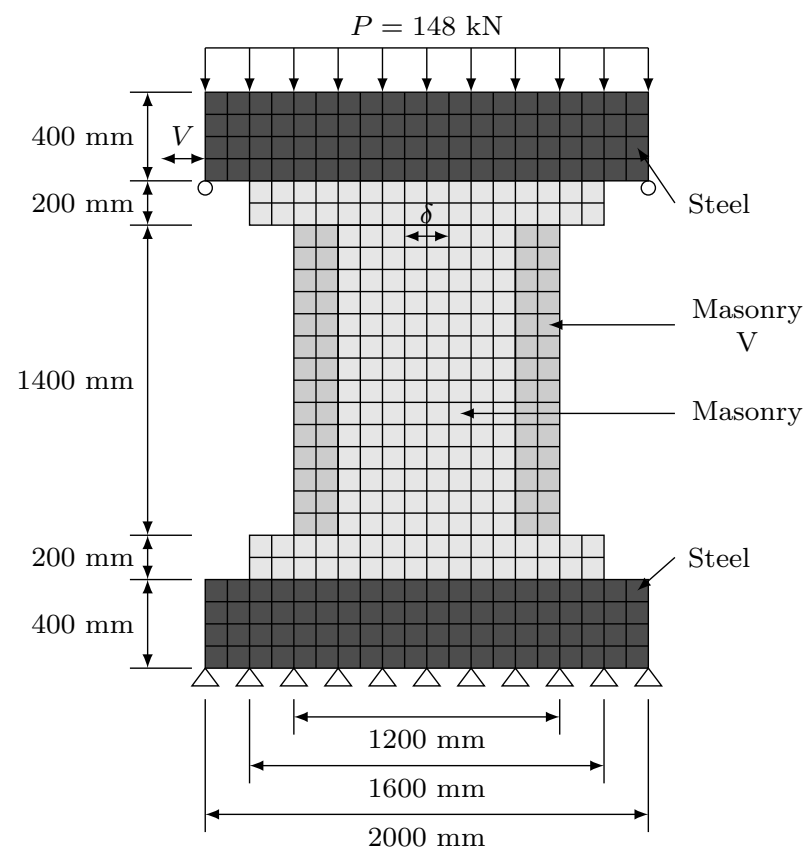

Finite Element Model Wall

HCBL-11-7 Shear Wall 
HCBL-11-7 Shear Wall (Chen et al., 1978) Model Options

\begin{tabular}{ll}
\hline Model & Selected Option \\
\hline Compression Pre-Peak & Hognestad \\
Compression Post-Peak & Pre-Peak Base Curve \\
Compression Softening & Vecchio \\
Tension Post-Peak & Vecchio \\
Cracking Criterion & Mohr-Coulomb \\
Slip & Walraven Reinforced \\
\hline
\end{tabular}

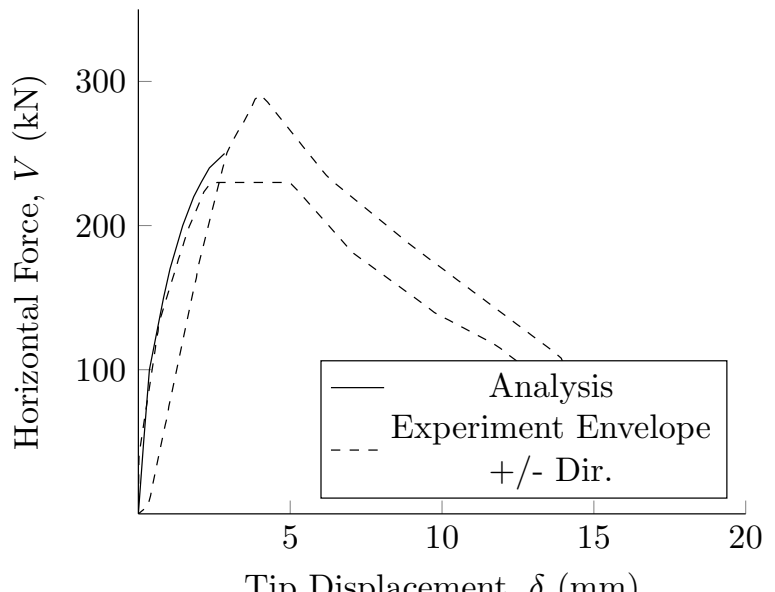

HCBL-11-7 Shear Wall (Chen et al., 1978) Horizontal Force vs Tip Displacement 
HCBL-11-7 Shear Wall (Chen et al., 1978) Material Properties

\begin{tabular}{|c|c|c|c|}
\hline \multirow{2}{*}{ Property } & \multicolumn{3}{|c|}{ Material } \\
\hline & Masonry & Masonry V & Steel \\
\hline Thickness (mm) & 190 & 190 & 200 \\
\hline Grout Tensile Strength (MPa) & 1.90 & 1.90 & 350 \\
\hline \multicolumn{4}{|c|}{ Masonry Properties (Y-Direction) } \\
\hline Gross Peak Compression Strength (MPa) & 13.1 & 13.1 & 350 \\
\hline Peak Compressive Strain & 0.0026 & 0.0026 & 0.00175 \\
\hline Tensile Strength & 0 & 0 & 350 \\
\hline Tensile Strain & 0 & 0 & 0.00175 \\
\hline Initial Tangent Elastic Modulus (MPa) & 0 & 0 & 200000 \\
\hline Poisson's Ratio & 0 & 0 & 0 \\
\hline \multicolumn{4}{|c|}{ Masonry Properties (X-Direction) } \\
\hline Gross Peak Compression Strength (MPa) & 13.1 & 13.1 & 350 \\
\hline Peak Compressive Strain & 0.0026 & 0.0026 & 0.00175 \\
\hline Tensile Strength & 0 & 0 & 350 \\
\hline Tensile Strain & 0 & 0 & 0.00175 \\
\hline Initial Tangent Elastic Modulus (MPa) & 0 & 0 & 200000 \\
\hline Poisson's Ratio & 0 & 0 & 0 \\
\hline \multicolumn{4}{|c|}{ Masonry Properties (Block) } \\
\hline Length (mm) & 390 & 390 & 2000 \\
\hline Height (mm) & 190 & 190 & 400 \\
\hline Face Shell Thickness (mm) & 41 & 41 & 100 \\
\hline Percent Solid (\%) & 59 & 59 & 100 \\
\hline Tensile Strength & 1.51 & 1.51 & 350 \\
\hline \multicolumn{4}{|c|}{ Head Joint } \\
\hline Thickness (mm) & 10 & 10 & 10 \\
\hline Tensile Bond Strength (MPa) & 0.31 & 0.31 & 350 \\
\hline Joint Cohesion (MPa) & 4.95 & 4.95 & 350 \\
\hline Angle of Internal Friction (Deg) & 42 & 42 & 42 \\
\hline \multicolumn{4}{|c|}{ Bed Joint } \\
\hline Thickness (mm) & 10 & 10 & 10 \\
\hline Tensile Bond Strength (MPa) & 0.31 & 0.31 & 350 \\
\hline Joint Cohesion (MPa) & 4.95 & 4.95 & 350 \\
\hline Angle of Internal Friction (Deg) & 42 & 42 & 42 \\
\hline \multicolumn{4}{|c|}{ Reinforcement (Y-Direction) } \\
\hline Ratio (\%) & 0 & 0.43 & 100 \\
\hline Yield Strength (MPa) & 0 & 477 & 350 \\
\hline Yield Strain & 0 & 0.00239 & 0.00175 \\
\hline Strain Hardening Strain & 0 & 0.02 & 0.02 \\
\hline Ultimate Strength (MPa) & 0 & 730 & 600 \\
\hline Ultimate Strain & 0 & 0.2 & 0.2 \\
\hline Prestrain & 0 & 0 & 0 \\
\hline \multicolumn{4}{|c|}{ Reinforcement (X-Direction) } \\
\hline Ratio (\%) & 0 & 0 & 100 \\
\hline Yield Strength (MPa) & 0 & 0 & 350 \\
\hline Yield Strain & 0 & 0 & 0.00175 \\
\hline Strain Hardening Strain & 0 & 0 & 0.02 \\
\hline Ultimate Strength (MPa) & 0 & 0 & 600 \\
\hline Ultimate Strain & 0 & 0 & 0.2 \\
\hline Prestrain & 0 & 0 & 0 \\
\hline
\end{tabular}

Numerical Modelling of Hollow Concrete Block Masonry Mechanics 

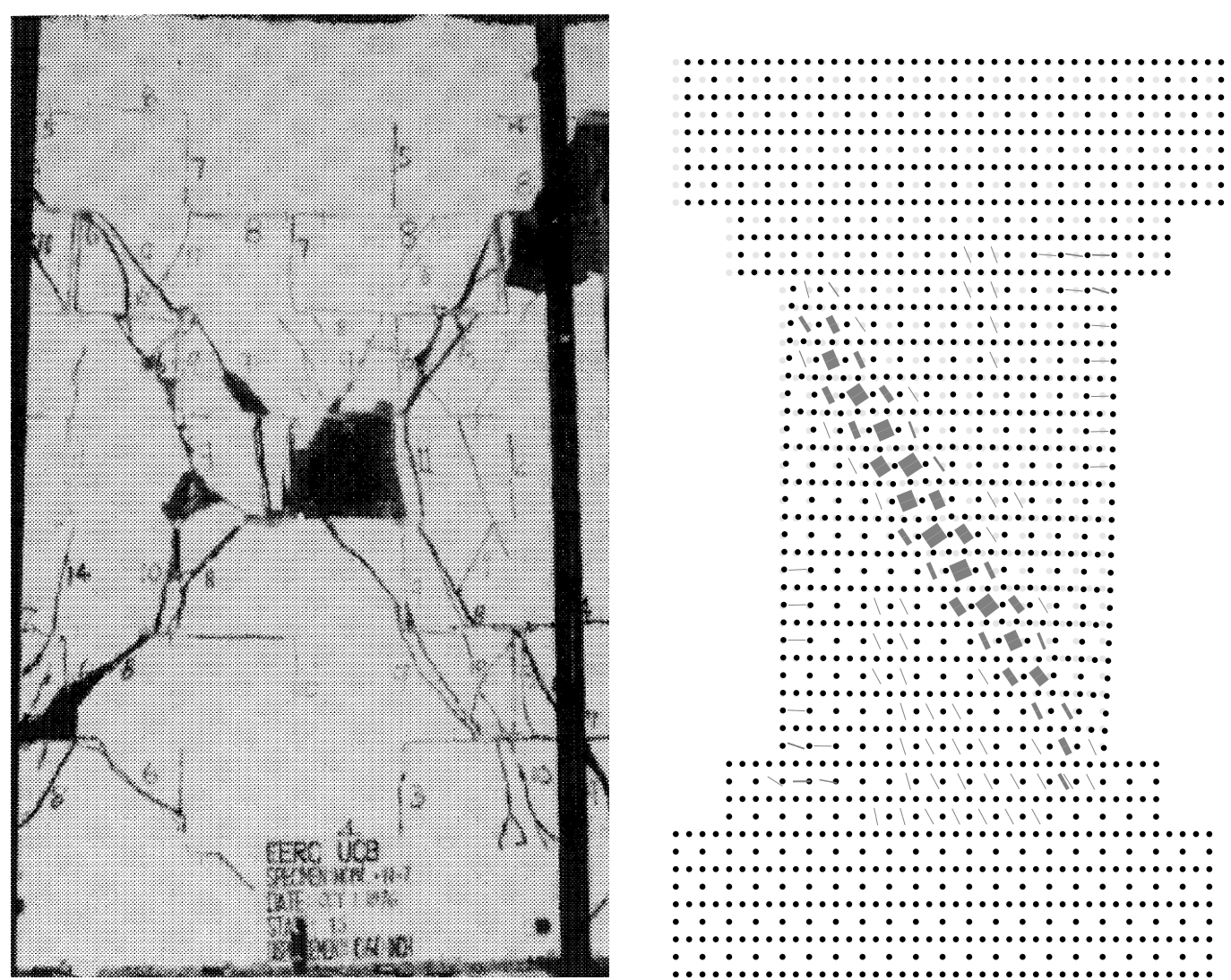

Experiment at Peak Horizontal Force (Chen Finite Element Model at Peak Horizontal Force et al., 1978) $\times 15$

HCBL-11-7 Shear Wall Displacement, Crack Pattern, and Crack Widths 


\section{HCBL-11-9 Shear Wall}

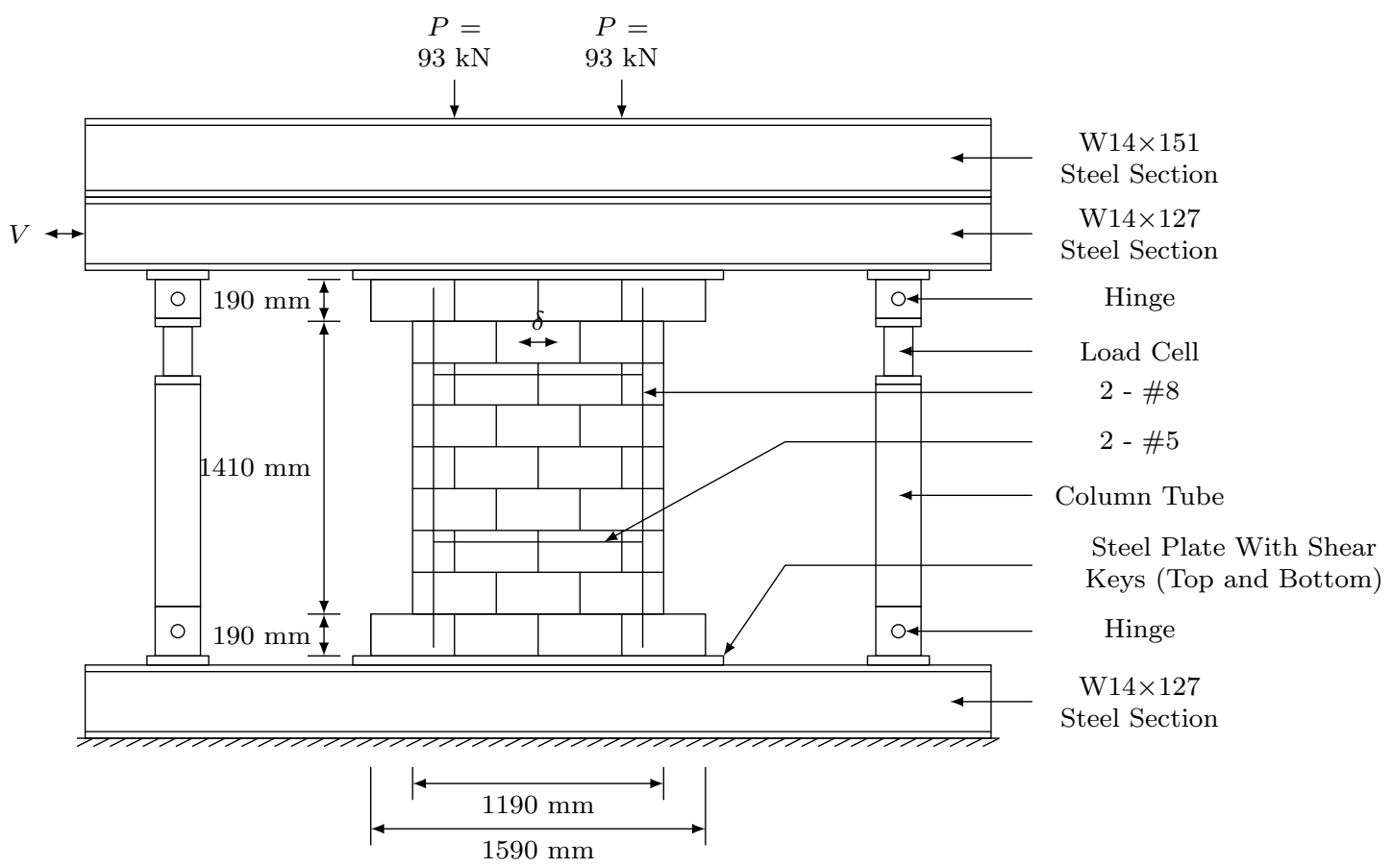

Experimental Wall (Chen et al., 1978)

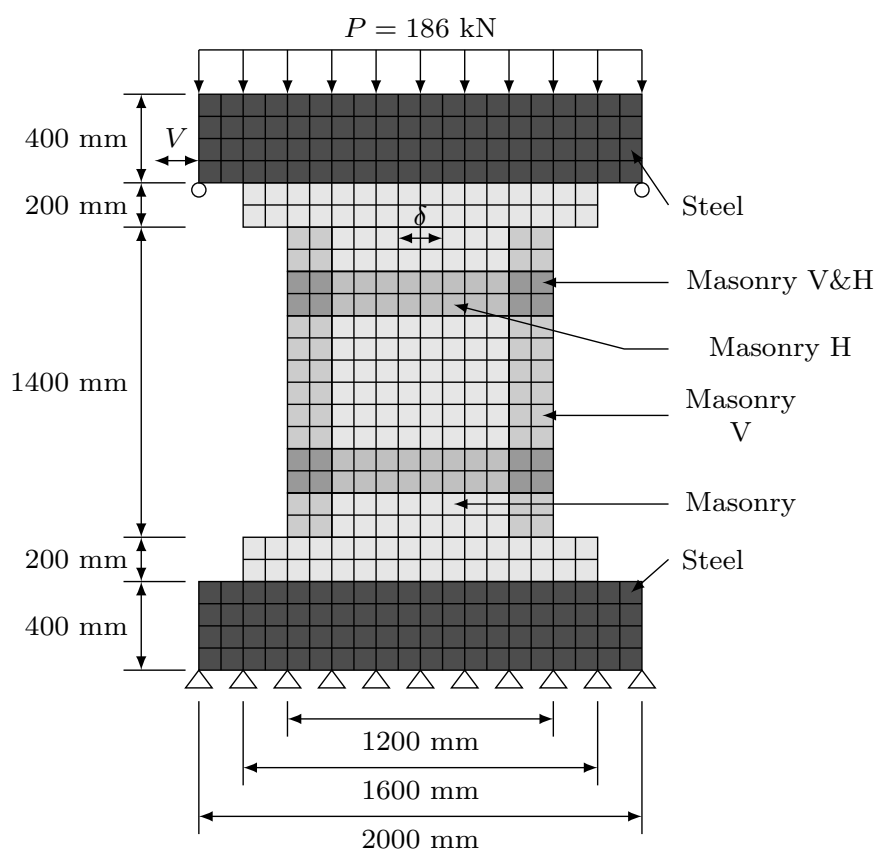

Finite Element Model Wall

HCBL-11-9 Shear Wall 
HCBL-11-9 Shear Wall (Chen et al., 1978) Model Options

\begin{tabular}{ll}
\hline Model & Selected Option \\
\hline Compression Pre-Peak & Hognestad \\
Compression Post-Peak & Pre-Peak Base Curve \\
Compression Softening & Vecchio \\
Tension Post-Peak & Vecchio \\
Cracking Criterion & Mohr-Coulomb \\
Slip & Walraven Reinforced \\
\hline
\end{tabular}

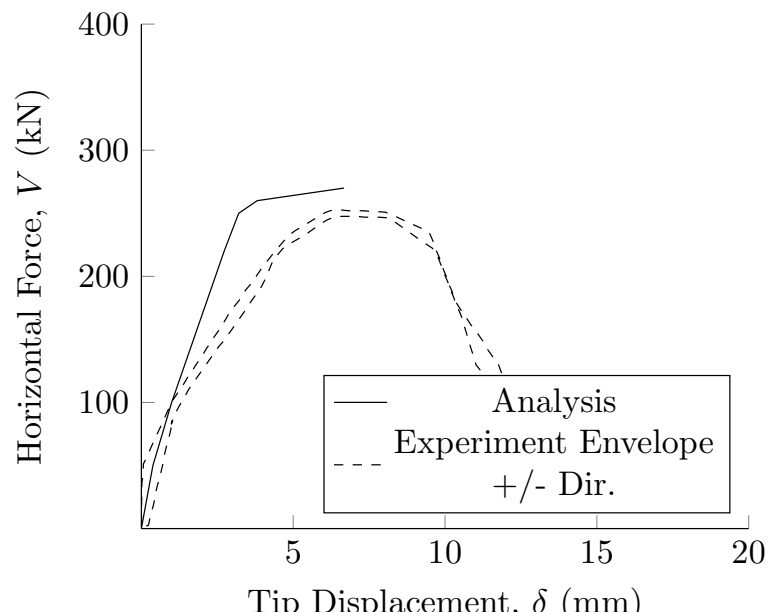

HCBL-11-9 Shear Wall (Chen et al., 1978) Horizontal Force vs Tip Displacement 
HCBL-11-9 Shear Wall (Chen et al., 1978) Material Properties

\begin{tabular}{|c|c|c|c|c|c|}
\hline \multirow{2}{*}{ Property } & \multicolumn{5}{|c|}{ Material } \\
\hline & Masonry & Masonry V & Masonry H & Masonry V\&H & Steel \\
\hline Thickness (mm) & 190 & 190 & 190 & 190 & 200 \\
\hline Grout Tensile Strength (MPa) & 1.90 & 1.90 & 1.90 & 1.90 & 350 \\
\hline \multicolumn{6}{|c|}{ Masonry Properties (Y-Direction) } \\
\hline Gross Peak Compression Strength (MPa) & 13.1 & 13.1 & 13.1 & 13.1 & 350 \\
\hline Peak Compressive Strain & 0.0026 & 0.0026 & 0.0026 & 0.0026 & 0.00175 \\
\hline Tensile Strength & 0 & 0 & 0 & 0 & 350 \\
\hline Tensile Strain & 0 & 0 & 0 & 0 & 0.00175 \\
\hline Initial Tangent Elastic Modulus (MPa) & 5200 & 5200 & 5200 & 5200 & 200000 \\
\hline Poisson's Ratio & 0 & 0 & 0 & 0 & 0 \\
\hline \multicolumn{6}{|c|}{ Masonry Properties (X-Direction) } \\
\hline Gross Peak Compression Strength (MPa) & 13.1 & 13.1 & 13.1 & 13.1 & 350 \\
\hline Peak Compressive Strain & 0.0026 & 0.0026 & 0.0026 & 0.0026 & 0.00175 \\
\hline Tensile Strength & 0 & 0 & 0 & 0 & 350 \\
\hline Tensile Strain & 0 & 0 & 0 & 0 & 0.00175 \\
\hline Initial Tangent Elastic Modulus (MPa) & 5200 & 5200 & 5200 & 5200 & 200000 \\
\hline Poisson's Ratio & 0 & 0 & 0 & 0 & 0 \\
\hline \multicolumn{6}{|c|}{ Masonry Properties (Block) } \\
\hline Length (mm) & 390 & 390 & 390 & 390 & 2000 \\
\hline Height (mm) & 190 & 190 & 190 & 190 & 400 \\
\hline Face Shell Thickness (mm) & 41 & 41 & 41 & 41 & 100 \\
\hline Percent Solid (\%) & 59 & 59 & 59 & 59 & 100 \\
\hline Tensile Strength & 1.51 & 1.51 & 1.51 & 1.51 & 350 \\
\hline \multicolumn{6}{|c|}{ Head Joint } \\
\hline Thickness (mm) & 10 & 10 & 10 & 10 & 10 \\
\hline Tensile Bond Strength (MPa) & 0.31 & 0.31 & 0.31 & 0.31 & 350 \\
\hline Joint Cohesion (MPa) & 4.95 & 4.95 & 4.95 & 4.95 & 350 \\
\hline Angle of Internal Friction (Deg) & 42 & 42 & 42 & 42 & 42 \\
\hline \multicolumn{6}{|c|}{ Bed Joint } \\
\hline Thickness (mm) & 10 & 10 & 10 & 10 & 10 \\
\hline Tensile Bond Strength (MPa) & 0.31 & 0.31 & 0.31 & 0.31 & 350 \\
\hline Joint Cohesion (MPa) & 4.95 & 4.95 & 4.95 & 4.95 & 350 \\
\hline Angle of Internal Friction (Deg) & 42 & 42 & 42 & 42 & 42 \\
\hline \multicolumn{6}{|c|}{ Reinforcement (Y-Direction) } \\
\hline Ratio (\%) & 0 & 0.43 & 0 & 0.43 & 100 \\
\hline Yield Strength (MPa) & 0 & 477 & 0 & 477 & 350 \\
\hline Yield Strain & 0 & 0.00239 & 0 & 0.00239 & 0.00175 \\
\hline Strain Hardening Strain & 0 & 0.02 & 0 & 0.02 & 0.02 \\
\hline Ultimate Strength (MPa) & 0 & 730 & 0 & 730 & 600 \\
\hline Ultimate Strain & 0 & 0.2 & 0 & 0.2 & 0.2 \\
\hline Prestrain & 0 & 0 & 0 & 0 & 0 \\
\hline \multicolumn{6}{|c|}{ Reinforcement (X-Direction) } \\
\hline Ratio (\%) & 0 & 0 & 0.15 & 0.15 & 100 \\
\hline Yield Strength (MPa) & 0 & 0 & 330 & 330 & 350 \\
\hline Yield Strain & 0 & 0 & 0.00165 & 0.00165 & 0.00175 \\
\hline Strain Hardening Strain & 0 & 0 & 0.02 & 0.02 & 0.02 \\
\hline Ultimate Strength (MPa) & 0 & 0 & 522 & 522 & 600 \\
\hline Ultimate Strain & 0 & 0 & 0.2 & 0.2 & 0.2 \\
\hline Prestrain & 0 & 0 & 0 & 0 & 0 \\
\hline
\end{tabular}

Numerical Modelling of Hollow Concrete Block Masonry Mechanics 

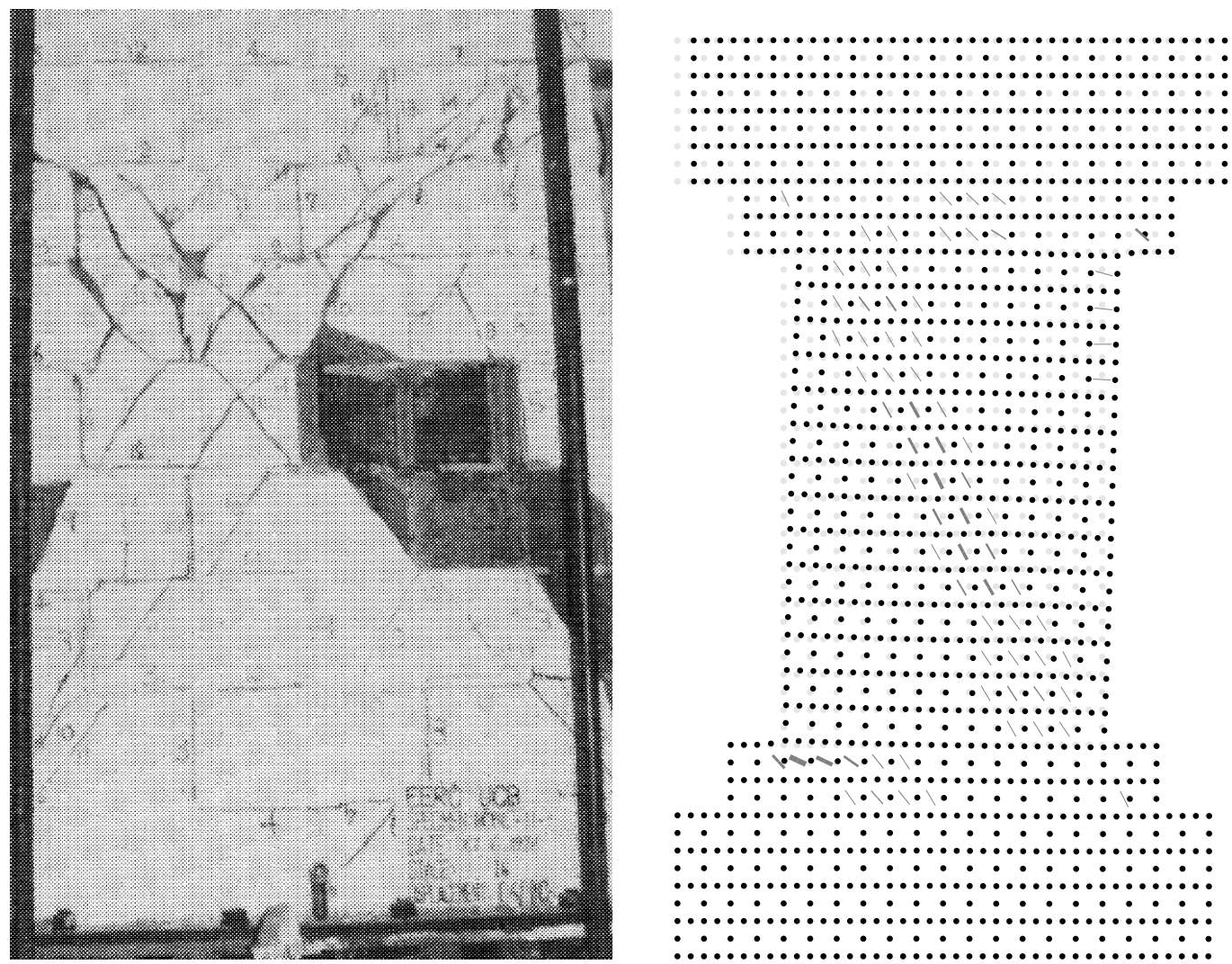

Experiment at Peak Horizontal Force (Chen Finite Element Model at Peak Horizontal Force et al., 1978) $\times 15$

HCBL-11-9 Shear Wall Displacement, Crack Pattern, and Crack Widths 


\section{HCBL-11-11 Shear Wall}

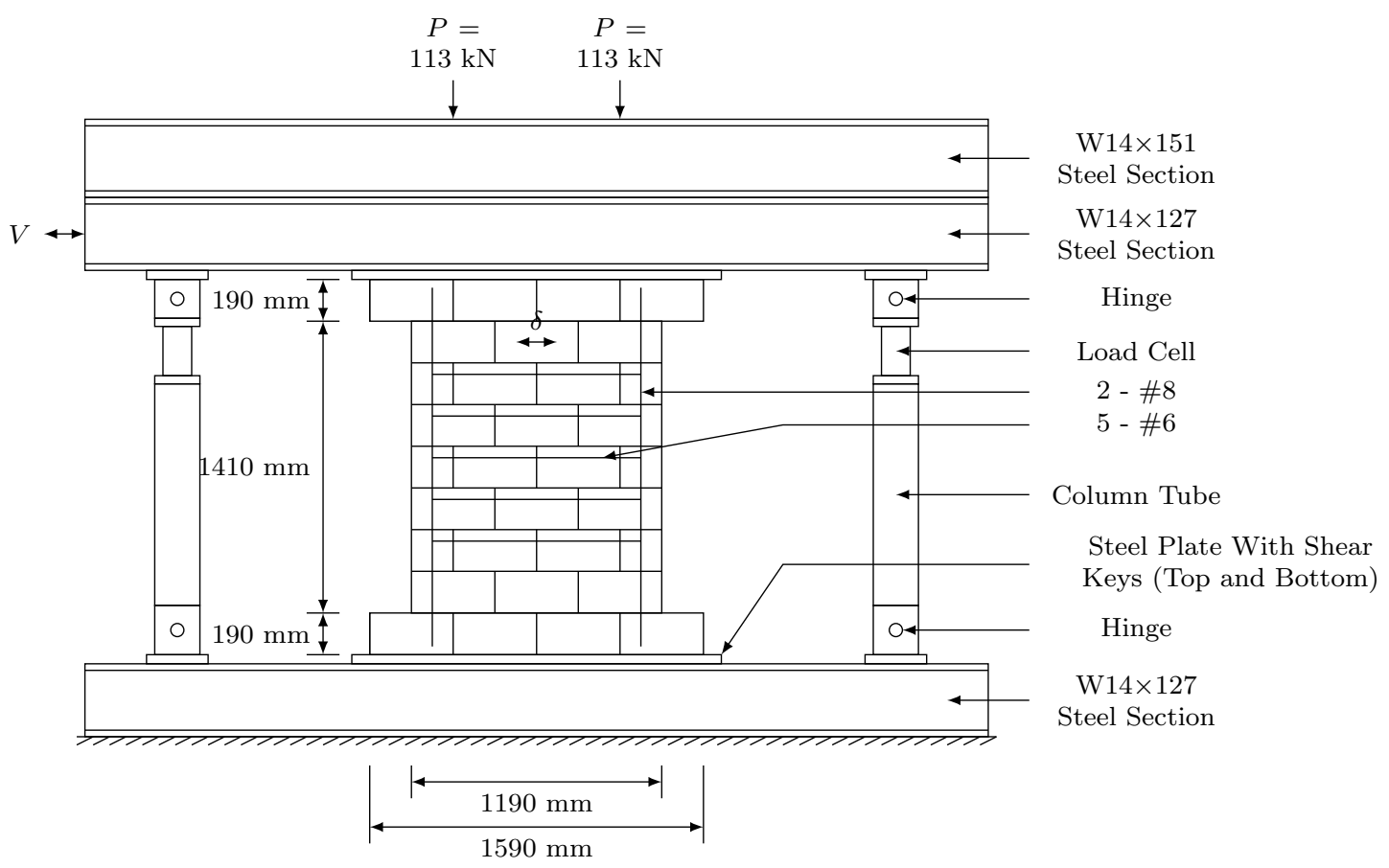

Experimental Wall (Chen et al., 1978)

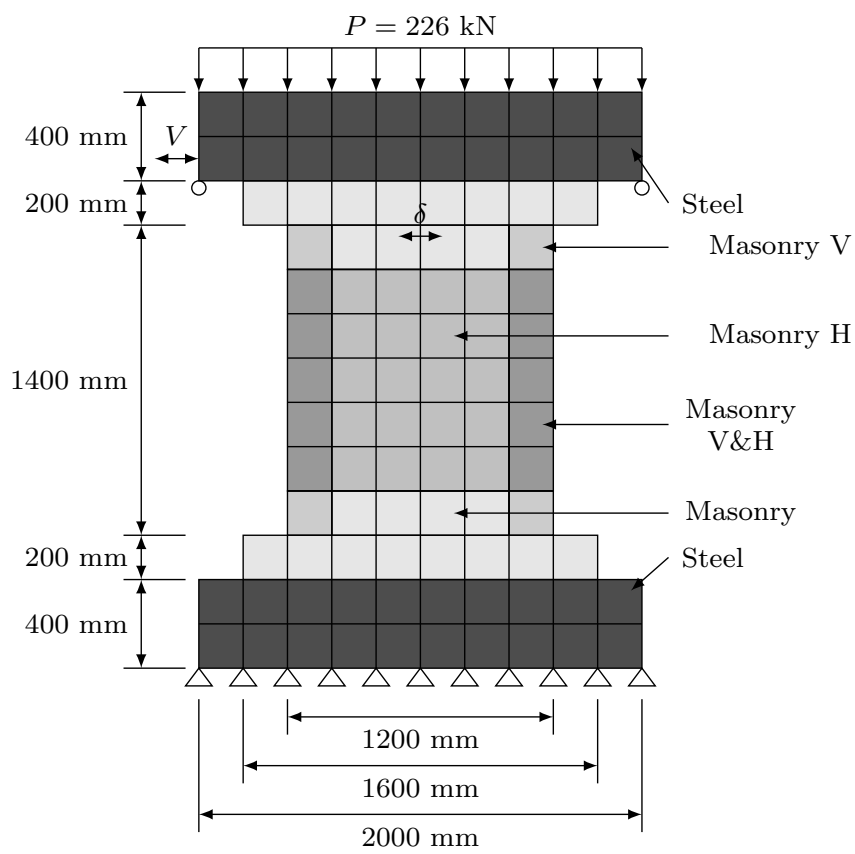

Finite Element Model Wall

HCBL-11-11 Shear Wall 
HCBL-11-11 Shear Wall (Chen et al., 1978) Model Options

\begin{tabular}{ll}
\hline Model & Selected Option \\
\hline Compression Pre-Peak & Hognestad \\
Compression Post-Peak & Pre-Peak Base Curve \\
Compression Softening & Vecchio \\
Tension Post-Peak & Vecchio \\
Cracking Criterion & Mohr-Coulomb \\
Slip & Walraven Reinforced \\
\hline
\end{tabular}

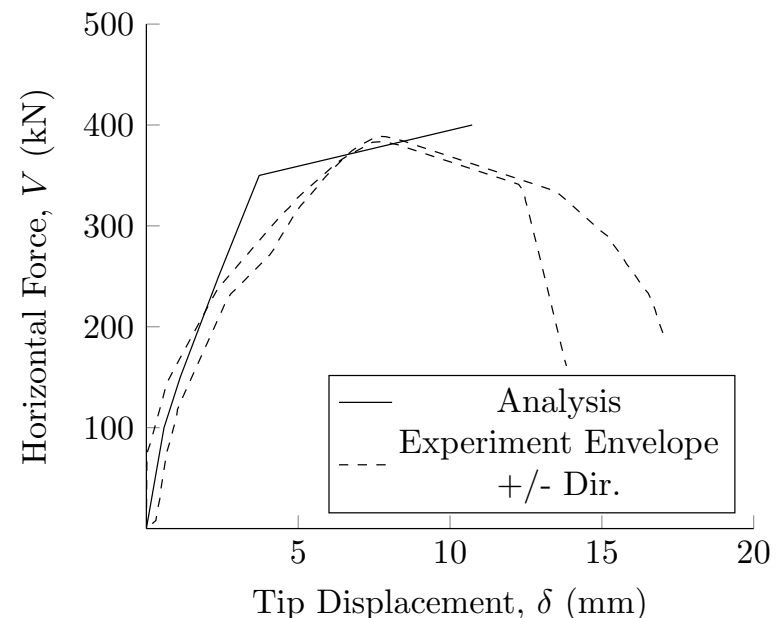

HCBL-11-11 Shear Wall (Chen et al., 1978) Horizontal Force vs Tip Displacement 
HCBL-11-11 Shear Wall (Chen et al., 1978) Material Properties

\begin{tabular}{|c|c|c|c|c|c|}
\hline \multirow{2}{*}{ Property } & \multicolumn{5}{|c|}{ Material } \\
\hline & Masonry & Masonry V & Masonry H & Masonry V\&H & Steel \\
\hline Thickness (mm) & 190 & 190 & 190 & 190 & 200 \\
\hline Grout Tensile Strength (MPa) & 1.90 & 1.90 & 1.90 & 1.90 & 350 \\
\hline \multicolumn{6}{|c|}{ Masonry Properties (Y-Direction) } \\
\hline Gross Peak Compression Strength (MPa) & 9.17 & 9.17 & 9.17 & 9.17 & 350 \\
\hline Peak Compressive Strain & 0.0024 & 0.0024 & 0.0024 & 0.0024 & 0.00175 \\
\hline Tensile Strength & 0 & 0 & 0 & 0 & 350 \\
\hline Tensile Strain & 0 & 0 & 0 & 0 & 0.00175 \\
\hline Initial Tangent Elastic Modulus (MPa) & 5150 & 5150 & 5150 & 5150 & 200000 \\
\hline Poisson's Ratio & 0 & 0 & 0 & 0 & 0 \\
\hline \multicolumn{6}{|c|}{ Masonry Properties (X-Direction) } \\
\hline Gross Peak Compression Strength (MPa) & 9.17 & 9.17 & 9.17 & 9.17 & 350 \\
\hline Peak Compressive Strain & 0.0024 & 0.0024 & 0.0024 & 0.0024 & 0.00175 \\
\hline Tensile Strength & 0 & 0 & 0 & 0 & 350 \\
\hline Tensile Strain & 0 & 0 & 0 & 0 & 0.00175 \\
\hline Initial Tangent Elastic Modulus (MPa) & 5150 & 5150 & 5150 & 5150 & 200000 \\
\hline Poisson's Ratio & 0 & 0 & 0 & 0 & 0 \\
\hline \multicolumn{6}{|c|}{ Masonry Properties (Block) } \\
\hline Length (mm) & 390 & 390 & 390 & 390 & 2000 \\
\hline Height (mm) & 190 & 190 & 190 & 190 & 400 \\
\hline Face Shell Thickness (mm) & 41 & 41 & 41 & 41 & 100 \\
\hline Percent Solid (\%) & 59 & 59 & 59 & 59 & 100 \\
\hline Tensile Strength & 1.51 & 1.51 & 1.51 & 1.51 & 350 \\
\hline \multicolumn{6}{|c|}{ Head Joint } \\
\hline Thickness (mm) & 10 & 10 & 10 & 10 & 10 \\
\hline Tensile Bond Strength (MPa) & 0.31 & 0.31 & 0.31 & 0.31 & 350 \\
\hline Joint Cohesion (MPa) & 4.89 & 4.89 & 4.89 & 4.89 & 350 \\
\hline Angle of Internal Friction (Deg) & 42 & 42 & 42 & 42 & 42 \\
\hline \multicolumn{6}{|c|}{ Bed Joint } \\
\hline Thickness (mm) & 10 & 10 & 10 & 10 & 10 \\
\hline Tensile Bond Strength (MPa) & 0.31 & 0.31 & 0.31 & 0.31 & 350 \\
\hline Joint Cohesion (MPa) & 4.89 & 4.89 & 4.89 & 4.89 & 350 \\
\hline Angle of Internal Friction (Deg) & 42 & 42 & 42 & 42 & 42 \\
\hline \multicolumn{6}{|c|}{ Reinforcement (Y-Direction) } \\
\hline Ratio (\%) & 0 & 0.43 & 0 & 0.43 & 100 \\
\hline Yield Strength (MPa) & 0 & 477 & 0 & 477 & 350 \\
\hline Yield Strain & 0 & 0.00239 & 0 & 0.00239 & 0.00175 \\
\hline Strain Hardening Strain & 0 & 0.02 & 0 & 0.02 & 0.02 \\
\hline Ultimate Strength $(\mathrm{MPa})$ & 0 & 730 & 0 & 730 & 600 \\
\hline Ultimate Strain & 0 & 0.2 & 0 & 0.2 & 0.2 \\
\hline Prestrain & 0 & 0 & 0 & 0 & 0 \\
\hline \multicolumn{6}{|c|}{ Reinforcement (X-Direction) } \\
\hline Ratio (\%) & 0 & 0 & 0.48 & 0.48 & 100 \\
\hline Yield Strength (MPa) & 0 & 0 & 509 & 509 & 350 \\
\hline Yield Strain & 0 & 0 & 0.00245 & 0.00245 & 0.00175 \\
\hline Strain Hardening Strain & 0 & 0 & 0.02 & 0.02 & 0.02 \\
\hline Ultimate Strength (MPa) & 0 & 0 & 705 & 705 & 600 \\
\hline Ultimate Strain & 0 & 0 & 0.2 & 0.2 & 0.2 \\
\hline Prestrain & 0 & 0 & 0 & 0 & 0 \\
\hline
\end{tabular}

Numerical Modelling of Hollow Concrete Block Masonry Mechanics 

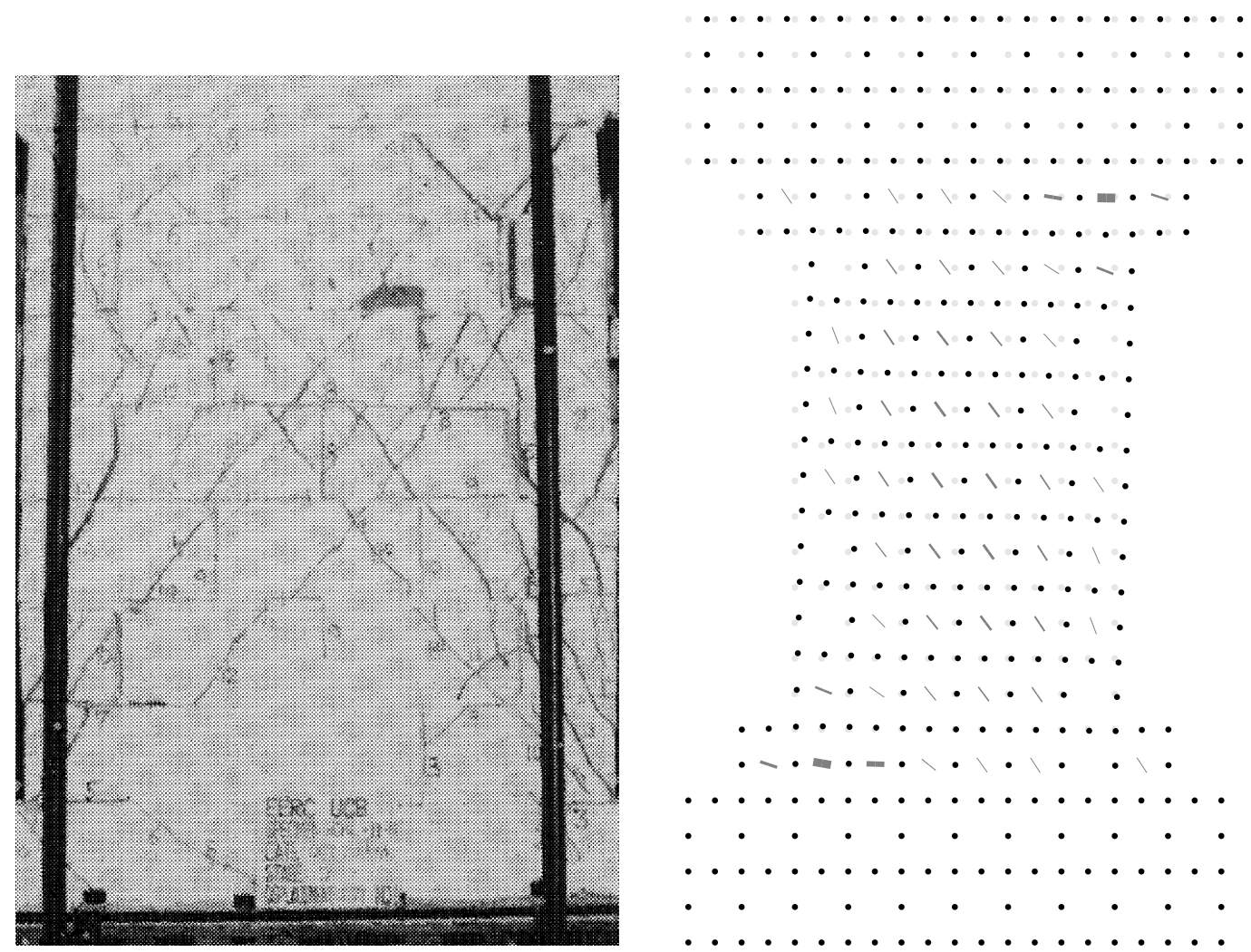

Experiment at Peak Horizontal Force (Chen Finite Element Model at Peak Horizontal Force et al., 1978) $\times 15$

HCBL-11-11 Shear Wall Displacement, Crack Pattern, and Crack Widths 
Hidalgo et al. (1979)

\section{HCBL-12-1 Shear Wall}

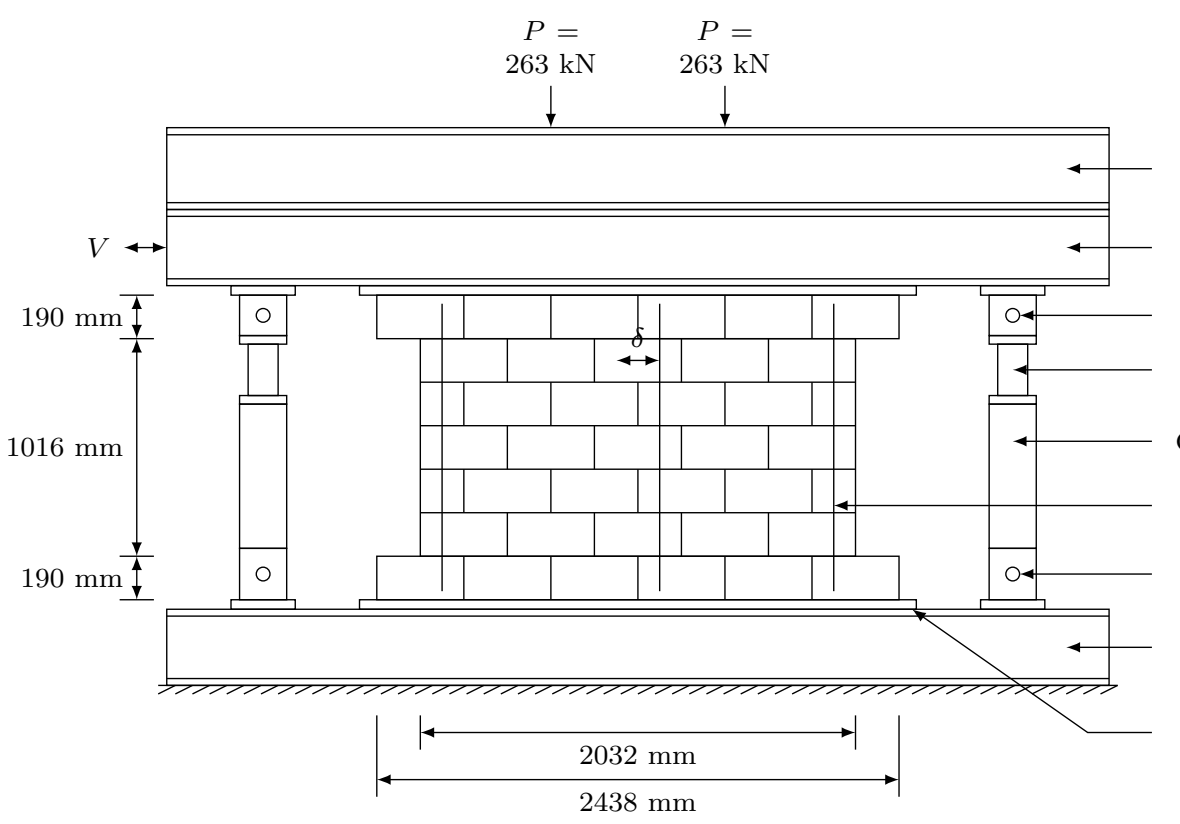

$\mathrm{W} 14 \times 151$

Steel Section

$\mathrm{W} 14 \times 127$

Steel Section

Hinge

Load Cell

Column Tube

$3-\# 7$

Hinge

$\mathrm{W} 14 \times 127$ Steel Section

Steel Plate With Shear Keys (Top and Bottom)

Experimental Wall (Hidalgo et al., 1979)

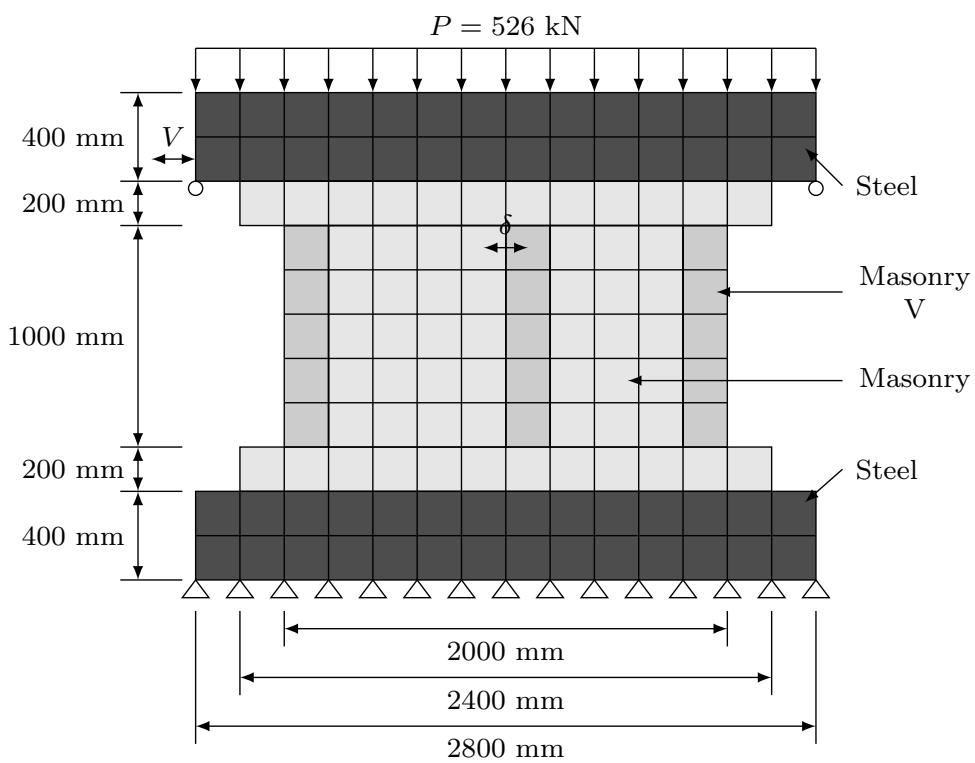

Finite Element Model Wall

HCBL-12-1 Shear Wall 
HCBL-12-1 Shear Wall (Hidalgo et al., 1979) Model Options

\begin{tabular}{ll}
\hline Model & Selected Option \\
\hline Compression Pre-Peak & Hognestad \\
Compression Post-Peak & Pre-Peak Base Curve \\
Compression Softening & Vecchio \\
Tension Post-Peak & Vecchio \\
Cracking Criterion & Mohr-Coulomb \\
Slip & Walraven Reinforced \\
\hline
\end{tabular}

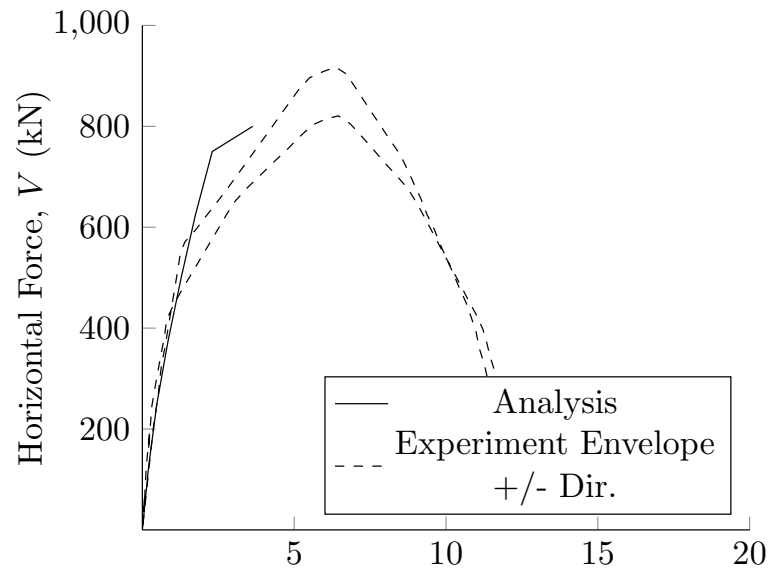

Tip Displacement, $\delta(\mathrm{mm})$

HCBL-12-1 Shear Wall (Hidalgo et al., 1979) Horizontal Force vs Tip Displacement 
HCBL-12-1 Shear Wall (Hidalgo et al., 1979) Material Properties

\begin{tabular}{|c|c|c|c|}
\hline \multirow{2}{*}{ Property } & \multicolumn{3}{|c|}{ Material } \\
\hline & Masonry & Masonry V & Steel \\
\hline Thickness (mm) & 190 & 190 & 200 \\
\hline Grout Tensile Strength (MPa) & 1.40 & 1.40 & 350 \\
\hline \multicolumn{4}{|c|}{ Masonry Properties (Y-Direction) } \\
\hline Gross Peak Compression Strength (MPa) & 20.6 & 20.6 & 350 \\
\hline Peak Compressive Strain & 0.004 & 0.004 & 0.00175 \\
\hline Tensile Strength & 0 & 0 & 350 \\
\hline Tensile Strain & 0 & 0 & 0.00175 \\
\hline Initial Tangent Elastic Modulus (MPa) & 5100 & 5100 & 200000 \\
\hline Poisson's Ratio & 0 & 0 & 0 \\
\hline \multicolumn{4}{|c|}{ Masonry Properties (X-Direction) } \\
\hline Gross Peak Compression Strength (MPa) & 20.6 & 20.6 & 350 \\
\hline Peak Compressive Strain & 0.004 & 0.004 & 0.00175 \\
\hline Tensile Strength & 0 & 0 & 350 \\
\hline Tensile Strain & 0 & 0 & 0.00175 \\
\hline Initial Tangent Elastic Modulus (MPa) & 5100 & 5100 & 200000 \\
\hline Poisson's Ratio & 0 & 0 & 0 \\
\hline \multicolumn{4}{|c|}{ Masonry Properties (Block) } \\
\hline Length (mm) & 390 & 390 & 2000 \\
\hline Height (mm) & 190 & 190 & 400 \\
\hline Face Shell Thickness (mm) & 41 & 41 & 100 \\
\hline Percent Solid (\%) & 59 & 59 & 100 \\
\hline Tensile Strength & 1.54 & 1.54 & 350 \\
\hline \multicolumn{4}{|c|}{ Head Joint } \\
\hline Thickness (mm) & 10 & 10 & 10 \\
\hline Tensile Bond Strength (MPa) & 0.46 & 0.46 & 350 \\
\hline Joint Cohesion (MPa) & 11.6 & 11.6 & 350 \\
\hline Angle of Internal Friction (Deg) & 42 & 42 & 42 \\
\hline \multicolumn{4}{|c|}{ Bed Joint } \\
\hline Thickness (mm) & 10 & 10 & 10 \\
\hline Tensile Bond Strength (MPa) & 0.46 & 0.46 & 350 \\
\hline Joint Cohesion (MPa) & 11.6 & 11.6 & 350 \\
\hline Angle of Internal Friction (Deg) & 42 & 42 & 42 \\
\hline \multicolumn{4}{|c|}{ Reinforcement (Y-Direction) } \\
\hline Ratio (\%) & 0 & 0.30 & 100 \\
\hline Yield Strength (MPa) & 0 & 544 & 350 \\
\hline Yield Strain & 0 & 0.00279 & 0.00175 \\
\hline Strain Hardening Strain & 0 & 0.02 & 0.02 \\
\hline Ultimate Strength (MPa) & 0 & 865 & 600 \\
\hline Ultimate Strain & 0 & 0.2 & 0.2 \\
\hline Prestrain & 0 & 0 & 0 \\
\hline \multicolumn{4}{|c|}{ Reinforcement (X-Direction) } \\
\hline Ratio (\%) & 0 & 0 & 100 \\
\hline Yield Strength (MPa) & 0 & 0 & 350 \\
\hline Yield Strain & 0 & 0 & 0.00175 \\
\hline Strain Hardening Strain & 0 & 0 & 0.02 \\
\hline Ultimate Strength $(\mathrm{MPa})$ & 0 & 0 & 600 \\
\hline Ultimate Strain & 0 & 0 & 0.2 \\
\hline Prestrain & 0 & 0 & 0 \\
\hline
\end{tabular}

Numerical Modelling of Hollow Concrete Block Masonry Mechanics 

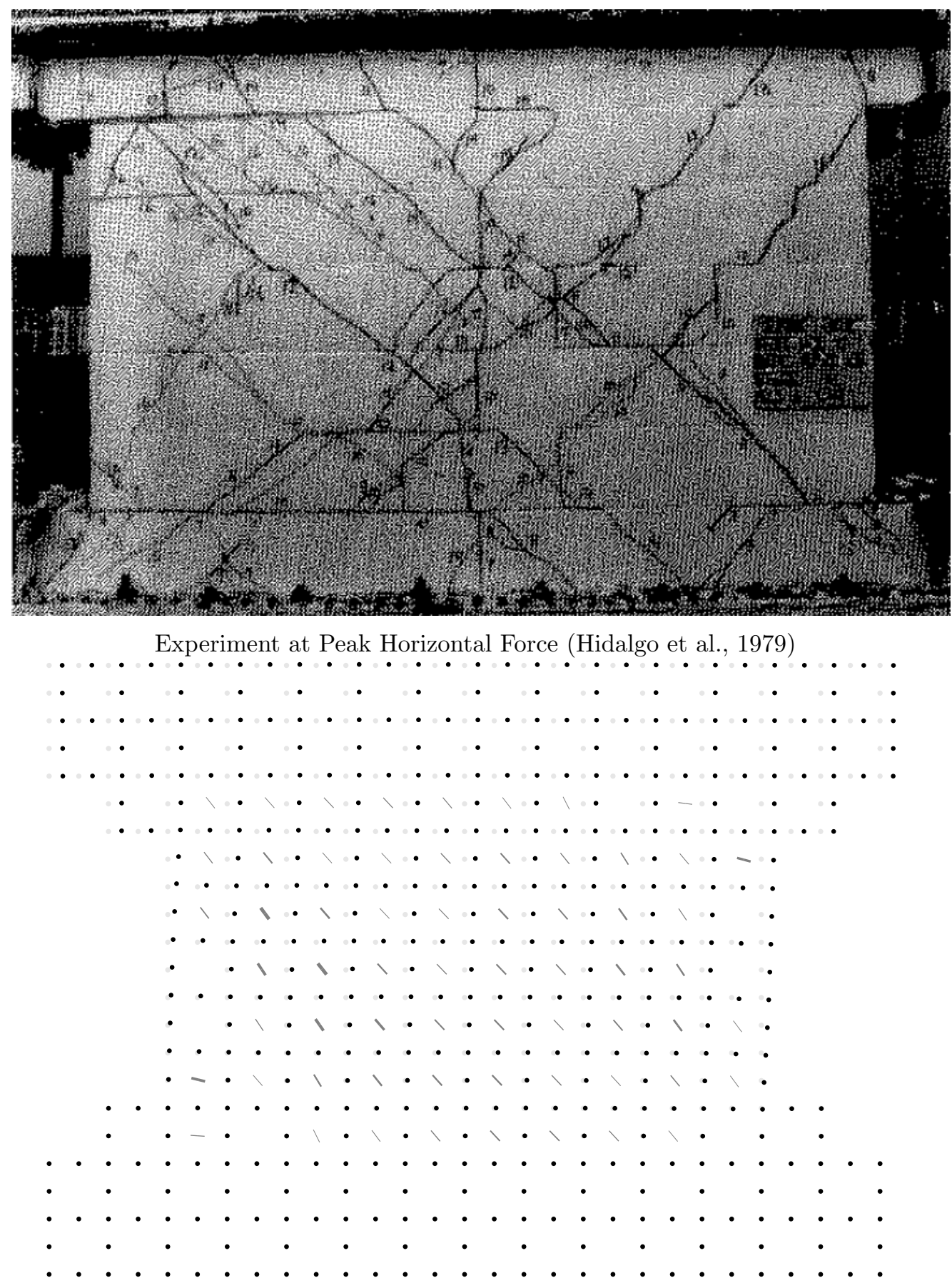

Finite Element Model at Peak Horizontal Force $\times 15$

HCBL-12-1 Shear Wall Displacement, Crack Pattern, and Crack Widths 


\section{HCBL-12-2 Shear Wall}

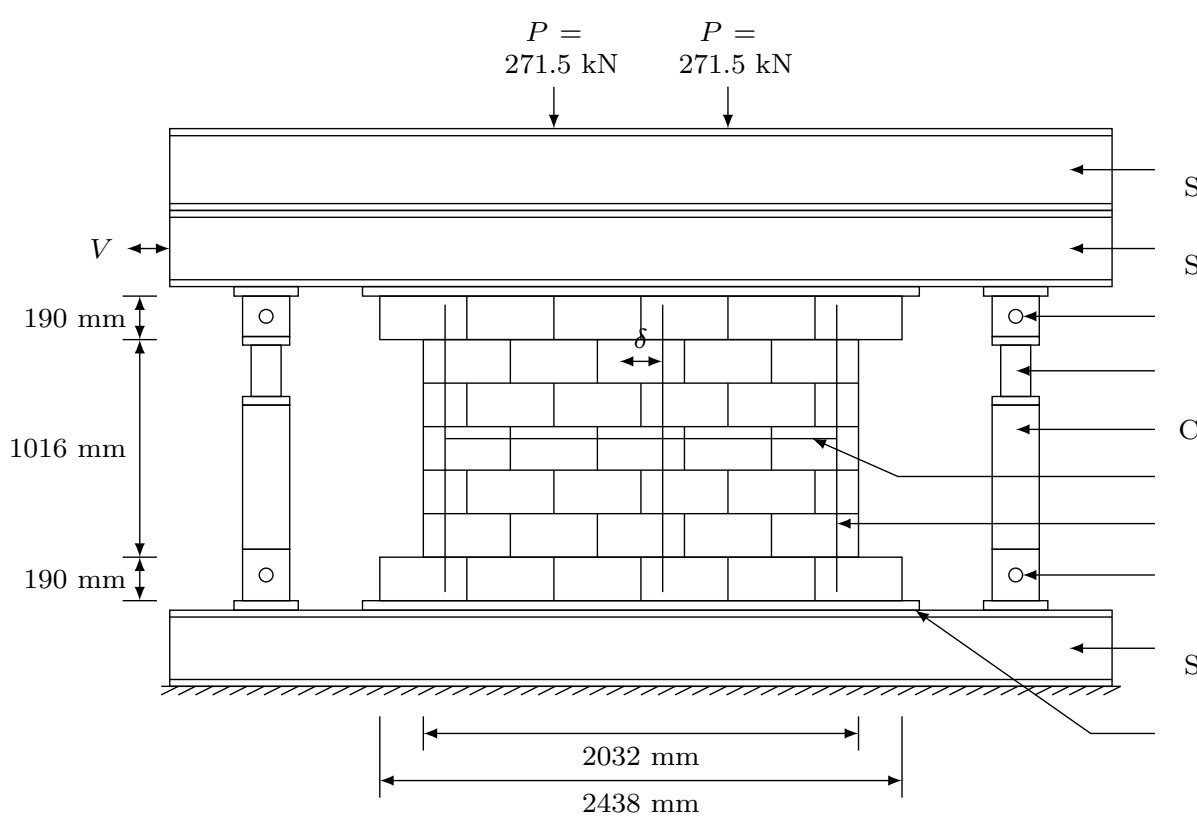

$\mathrm{W} 14 \times 151$

Steel Section

W14 $\times 127$

Steel Section

Hinge

Load Cell

Column Tube

\#5

$3-\# 7$

Hinge

$\mathrm{W} 14 \times 127$

Steel Section

Steel Plate With Shear

Keys (Top and Bottom)

Experimental Wall (Hidalgo et al., 1979)

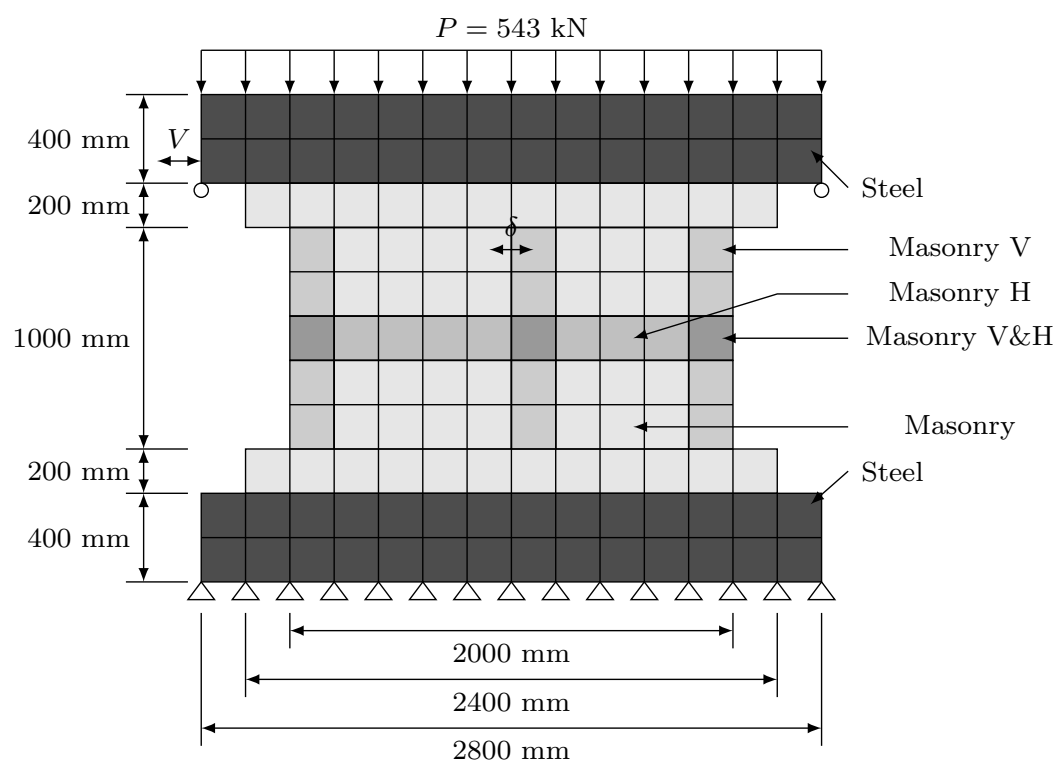

Finite Element Model Wall

HCBL-12-2 Shear Wall 
HCBL-12-2 Shear Wall (Hidalgo et al., 1979) Model Options

\begin{tabular}{ll}
\hline Model & Selected Option \\
\hline Compression Pre-Peak & Hognestad \\
Compression Post-Peak & Pre-Peak Base Curve \\
Compression Softening & Vecchio \\
Tension Post-Peak & Vecchio \\
Cracking Criterion & Mohr-Coulomb \\
Slip & Walraven Reinforced \\
\hline
\end{tabular}

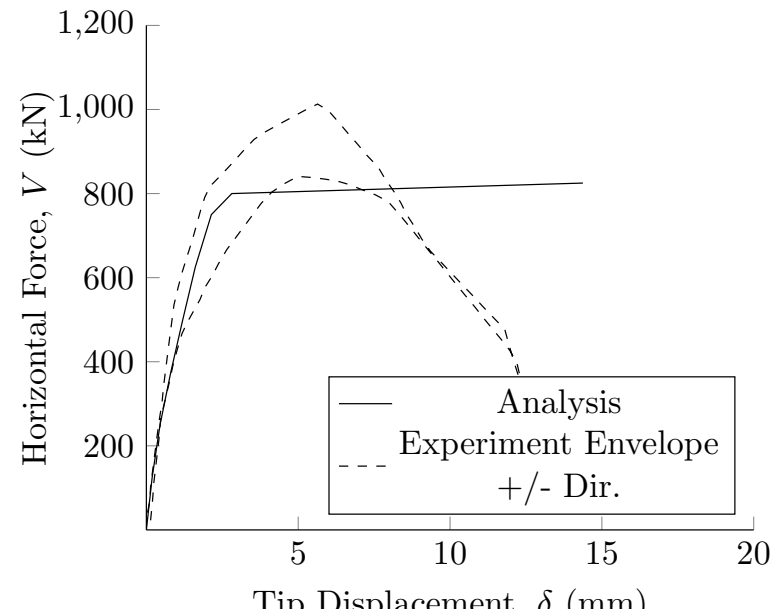

HCBL-12-2 Shear Wall (Hidalgo et al., 1979) Horizontal Force vs Tip Displacement 
HCBL-12-2 Shear Wall (Hidalgo et al., 1979) Material Properties

\begin{tabular}{|c|c|c|c|c|c|}
\hline \multirow{2}{*}{ Property } & \multicolumn{5}{|c|}{ Material } \\
\hline & Masonry & Masonry V & Masonry $\mathrm{H}$ & Masonry V\&H & Steel \\
\hline Thickness (mm) & 190 & 190 & 190 & 190 & 200 \\
\hline Grout Tensile Strength (MPa) & 1.40 & 1.40 & 1.40 & 1.40 & 350 \\
\hline \multicolumn{6}{|c|}{ Masonry Properties (Y-Direction) } \\
\hline Gross Peak Compression Strength (MPa) & 20.6 & 20.6 & 20.6 & 20.6 & 350 \\
\hline Peak Compressive Strain & 0.004 & 0.004 & 0.004 & 0.004 & 0.00175 \\
\hline Tensile Strength & 0 & 0 & 0 & 0 & 350 \\
\hline Tensile Strain & 0 & 0 & 0 & 0 & 0.00175 \\
\hline Initial Tangent Elastic Modulus (MPa) & 5500 & 5500 & 5500 & 5500 & 200000 \\
\hline Poisson's Ratio & 0 & 0 & 0 & 0 & 0 \\
\hline \multicolumn{6}{|c|}{ Masonry Properties (X-Direction) } \\
\hline Gross Peak Compression Strength (MPa) & 20.6 & 20.6 & 20.6 & 20.6 & 350 \\
\hline Peak Compressive Strain & 0.004 & 0.004 & 0.004 & 0.004 & 0.00175 \\
\hline Tensile Strength & 0 & 0 & 0 & 0 & 350 \\
\hline Tensile Strain & 0 & 0 & 0 & 0 & 0.00175 \\
\hline Initial Tangent Elastic Modulus (MPa) & 5500 & 5500 & 5500 & 5500 & 200000 \\
\hline Poisson's Ratio & 0 & 0 & 0 & 0 & 0 \\
\hline \multicolumn{6}{|c|}{ Masonry Properties (Block) } \\
\hline Length (mm) & 390 & 390 & 390 & 390 & 2000 \\
\hline Height (mm) & 190 & 190 & 190 & 190 & 400 \\
\hline Face Shell Thickness (mm) & 41 & 41 & 41 & 41 & 100 \\
\hline Percent Solid (\%) & 59 & 59 & 59 & 59 & 100 \\
\hline Tensile Strength & 1.54 & 1.54 & 1.54 & 1.54 & 350 \\
\hline \multicolumn{6}{|c|}{ Head Joint } \\
\hline Thickness (mm) & 10 & 10 & 10 & 10 & 10 \\
\hline Tensile Bond Strength (MPa) & 0.46 & 0.46 & 0.46 & 0.46 & 350 \\
\hline Joint Cohesion (MPa) & 11.6 & 11.6 & 11.6 & 11.6 & 350 \\
\hline Angle of Internal Friction (Deg) & 42 & 42 & 42 & 42 & 42 \\
\hline \multicolumn{6}{|c|}{ Bed Joint } \\
\hline Thickness (mm) & 10 & 10 & 10 & 10 & 10 \\
\hline Tensile Bond Strength (MPa) & 0.46 & 0.46 & 0.46 & 0.46 & 350 \\
\hline Joint Cohesion (MPa) & 11.6 & 11.6 & 11.6 & 11.6 & 350 \\
\hline Angle of Internal Friction (Deg) & 42 & 42 & 42 & 42 & 42 \\
\hline \multicolumn{6}{|c|}{ Reinforcement (Y-Direction) } \\
\hline Ratio (\%) & 0 & 0.30 & 0 & 0.30 & 100 \\
\hline Yield Strength (MPa) & 0 & 554 & 0 & 554 & 350 \\
\hline Yield Strain & 0 & 0.00279 & 0 & 0.00279 & 0.00175 \\
\hline Strain Hardening Strain & 0 & 0.02 & 0 & 0.02 & 0.02 \\
\hline Ultimate Strength (MPa) & 0 & 865 & 0 & 865 & 600 \\
\hline Ultimate Strain & 0 & 0.2 & 0 & 0.2 & 0.2 \\
\hline Prestrain & 0 & 0 & 0 & 0 & 0 \\
\hline \multicolumn{6}{|c|}{ Reinforcement (X-Direction) } \\
\hline Ratio (\%) & 0 & 0 & 0.05 & 0.05 & 100 \\
\hline Yield Strength (MPa) & 0 & 0 & 480 & 480 & 350 \\
\hline Yield Strain & 0 & 0 & 0.00243 & 0.00243 & 0.00175 \\
\hline Strain Hardening Strain & 0 & 0 & 0.02 & 0.02 & 0.02 \\
\hline Ultimate Strength (MPa) & 0 & 0 & 757 & 757 & 600 \\
\hline Ultimate Strain & 0 & 0 & 0.2 & 0.2 & 0.2 \\
\hline Prestrain & 0 & 0 & 0 & 0 & 0 \\
\hline
\end{tabular}

Numerical Modelling of Hollow Concrete Block Masonry Mechanics 


\section{HCBL-12-3 Shear Wall}

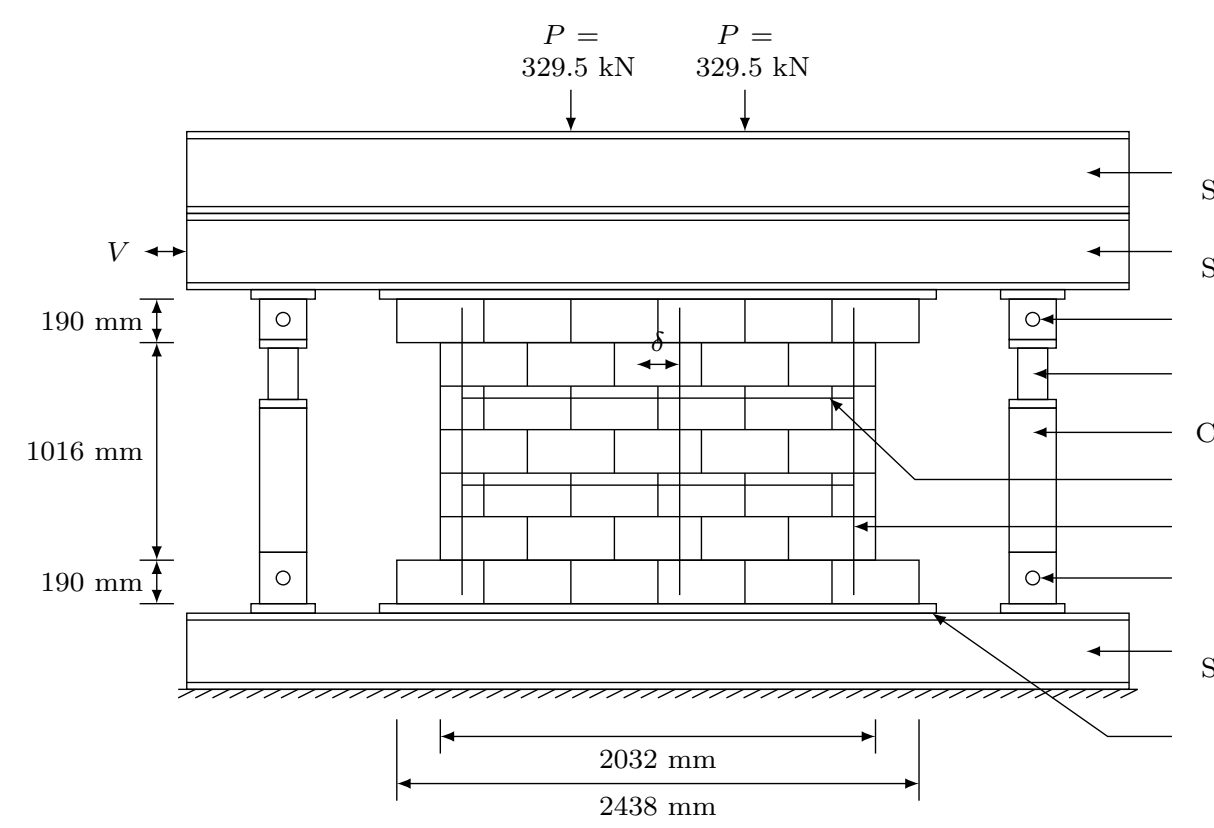

$\mathrm{W} 14 \times 151$

Steel Section

$\mathrm{W} 14 \times 127$

Steel Section

Hinge

Load Cell

Column Tube

$2-\# 5$

$3-\# 7$

Hinge

$\mathrm{W} 14 \times 127$

Steel Section

Steel Plate With Shear Keys (Top and Bottom)

Experimental Wall (Hidalgo et al., 1979)

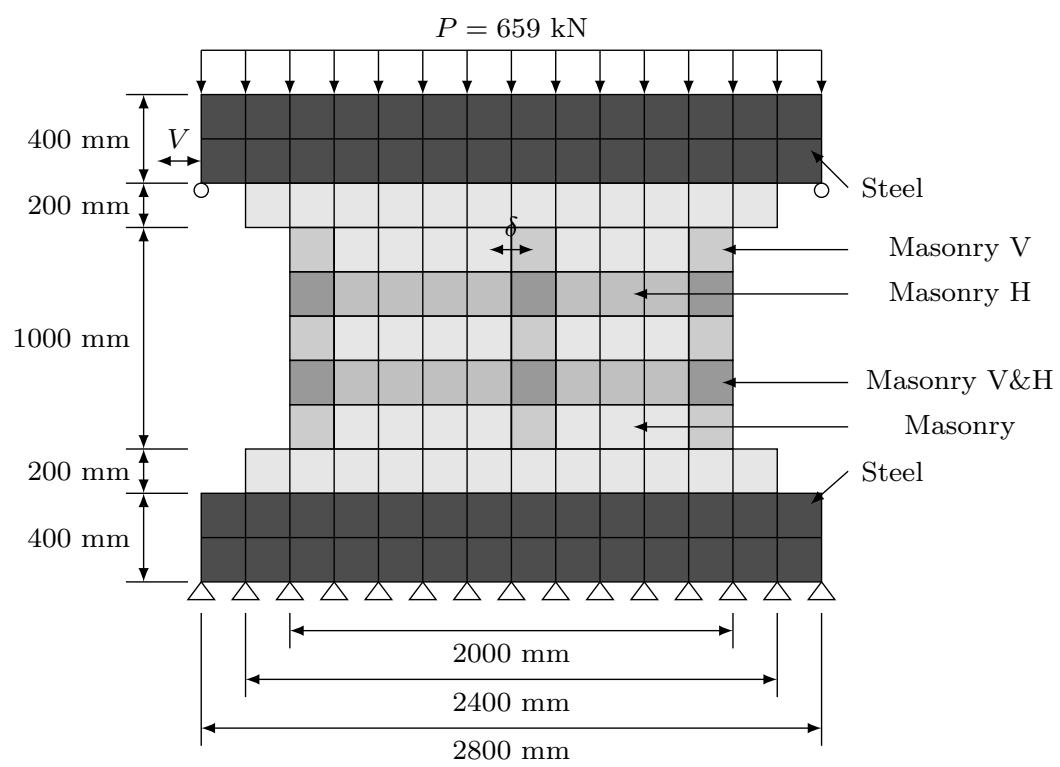

Finite Element Model Wall

HCBL-12-3 Shear Wall 
HCBL-12-3 Shear Wall (Hidalgo et al., 1979) Model Options

\begin{tabular}{ll}
\hline Model & Selected Option \\
\hline Compression Pre-Peak & Hognestad \\
Compression Post-Peak & Pre-Peak Base Curve \\
Compression Softening & Vecchio \\
Tension Post-Peak & Vecchio \\
Cracking Criterion & Mohr-Coulomb \\
Slip & Walraven Reinforced \\
\hline
\end{tabular}

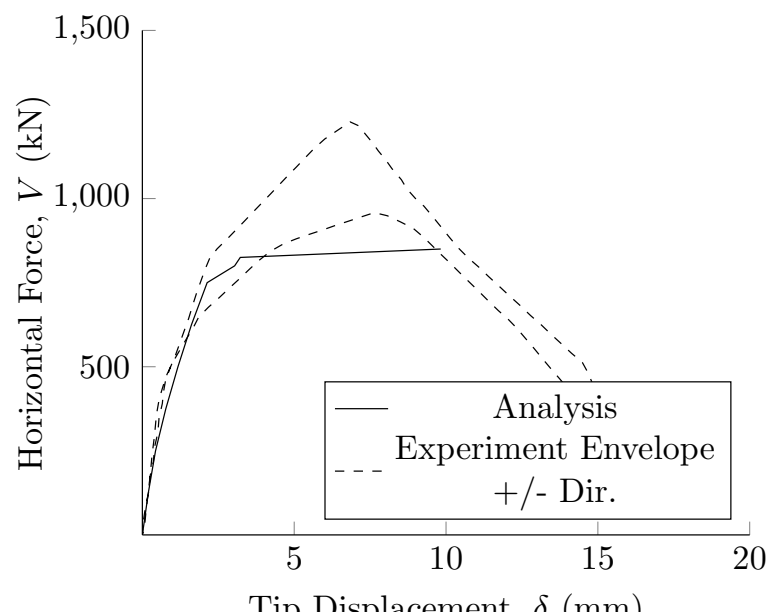

HCBL-12-3 Shear Wall (Hidalgo et al., 1979) Horizontal Force vs Tip Displacement 
HCBL-12-3 Shear Wall (Hidalgo et al., 1979) Material Properties

\begin{tabular}{|c|c|c|c|c|c|}
\hline \multirow{2}{*}{ Property } & \multicolumn{5}{|c|}{ Material } \\
\hline & Masonry & Masonry V & Masonry $\mathrm{H}$ & Masonry V\&H & Steel \\
\hline Thickness (mm) & 190 & 190 & 190 & 190 & 200 \\
\hline Grout Tensile Strength (MPa) & 1.40 & 1.40 & 1.40 & 1.40 & 350 \\
\hline \multicolumn{6}{|c|}{ Masonry Properties (Y-Direction) } \\
\hline Gross Peak Compression Strength (MPa) & 20.6 & 20.6 & 20.6 & 20.6 & 350 \\
\hline Peak Compressive Strain & 0.0042 & 0.0042 & 0.0042 & 0.0042 & 0.00175 \\
\hline Tensile Strength & 0 & 0 & 0 & 0 & 350 \\
\hline Tensile Strain & 0 & 0 & 0 & 0 & 0.00175 \\
\hline Initial Tangent Elastic Modulus (MPa) & 5500 & 5500 & 5500 & 5500 & 200000 \\
\hline Poisson's Ratio & 0 & 0 & 0 & 0 & 0 \\
\hline \multicolumn{6}{|c|}{ Masonry Properties (X-Direction) } \\
\hline Gross Peak Compression Strength (MPa) & 20.6 & 20.6 & 20.6 & 20.6 & 350 \\
\hline Peak Compressive Strain & 0.0042 & 0.0042 & 0.0042 & 0.0042 & 0.00175 \\
\hline Tensile Strength & 0 & 0 & 0 & 0 & 350 \\
\hline Tensile Strain & 0 & 0 & 0 & 0 & 0.00175 \\
\hline Initial Tangent Elastic Modulus (MPa) & 5500 & 5500 & 5500 & 5500 & 200000 \\
\hline Poisson's Ratio & 0 & 0 & 0 & 0 & 0 \\
\hline \multicolumn{6}{|c|}{ Masonry Properties (Block) } \\
\hline Length (mm) & 390 & 390 & 390 & 390 & 2000 \\
\hline Height (mm) & 190 & 190 & 190 & 190 & 400 \\
\hline Face Shell Thickness (mm) & 41 & 41 & 41 & 41 & 100 \\
\hline Percent Solid (\%) & 59 & 59 & 59 & 59 & 100 \\
\hline Tensile Strength & 1.54 & 1.54 & 1.54 & 1.54 & 350 \\
\hline \multicolumn{6}{|c|}{ Head Joint } \\
\hline Thickness (mm) & 10 & 10 & 10 & 10 & 10 \\
\hline Tensile Bond Strength (MPa) & 0.46 & 0.46 & 0.46 & 0.46 & 350 \\
\hline Joint Cohesion (MPa) & 11.6 & 11.6 & 11.6 & 11.6 & 350 \\
\hline Angle of Internal Friction (Deg) & 42 & 42 & 42 & 42 & 42 \\
\hline \multicolumn{6}{|c|}{ Bed Joint } \\
\hline Thickness (mm) & 10 & 10 & 10 & 10 & 10 \\
\hline Tensile Bond Strength (MPa) & 0.46 & 0.46 & 0.46 & 0.46 & 350 \\
\hline Joint Cohesion (MPa) & 11.6 & 11.6 & 11.6 & 11.6 & 350 \\
\hline Angle of Internal Friction (Deg) & 42 & 42 & 42 & 42 & 42 \\
\hline \multicolumn{6}{|c|}{ Reinforcement (Y-Direction) } \\
\hline Ratio (\%) & 0 & 0.30 & 0 & 0.30 & 100 \\
\hline Yield Strength (MPa) & 0 & 554 & 0 & 554 & 350 \\
\hline Yield Strain & 0 & 0.00279 & 0 & 0.00279 & 0.00175 \\
\hline Strain Hardening Strain & 0 & 0.02 & 0 & 0.02 & 0.02 \\
\hline Ultimate Strength (MPa) & 0 & 865 & 0 & 865 & 600 \\
\hline Ultimate Strain & 0 & 0.2 & 0 & 0.2 & 0.2 \\
\hline Prestrain & 0 & 0 & 0 & 0 & 0 \\
\hline \multicolumn{6}{|c|}{ Reinforcement (X-Direction) } \\
\hline Ratio (\%) & 0 & 0 & 0.10 & 0.10 & 100 \\
\hline Yield Strength (MPa) & 0 & 0 & 480 & 480 & 350 \\
\hline Yield Strain & 0 & 0 & 0.00243 & 0.00243 & 0.00175 \\
\hline Strain Hardening Strain & 0 & 0 & 0.02 & 0.02 & 0.02 \\
\hline Ultimate Strength (MPa) & 0 & 0 & 757 & 757 & 600 \\
\hline Ultimate Strain & 0 & 0 & 0.2 & 0.2 & 0.2 \\
\hline Prestrain & 0 & 0 & 0 & 0 & 0 \\
\hline
\end{tabular}

Numerical Modelling of Hollow Concrete Block Masonry Mechanics 


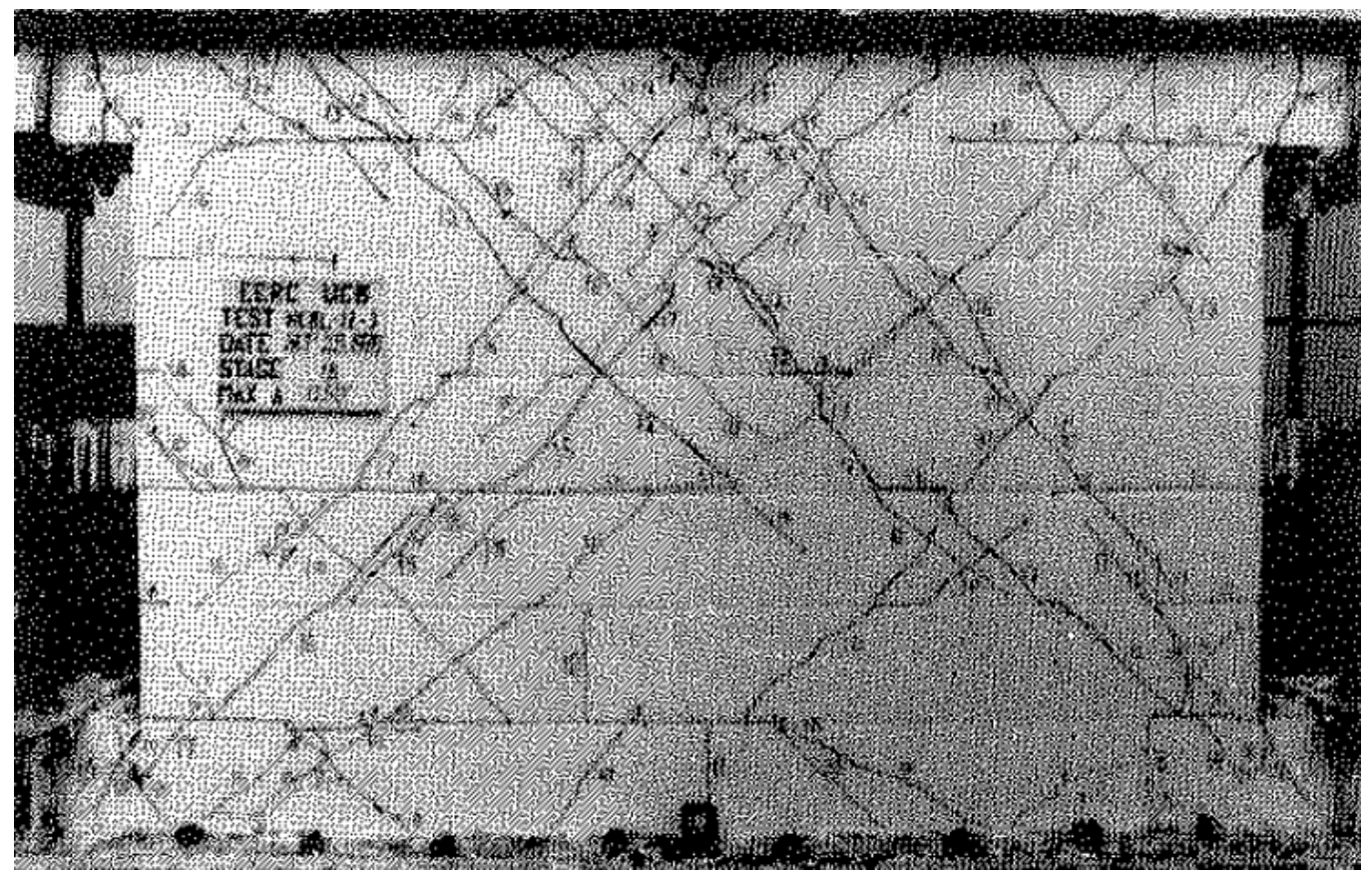

Experiment at Peak Horizontal Force (Hidalgo et al., 1979)

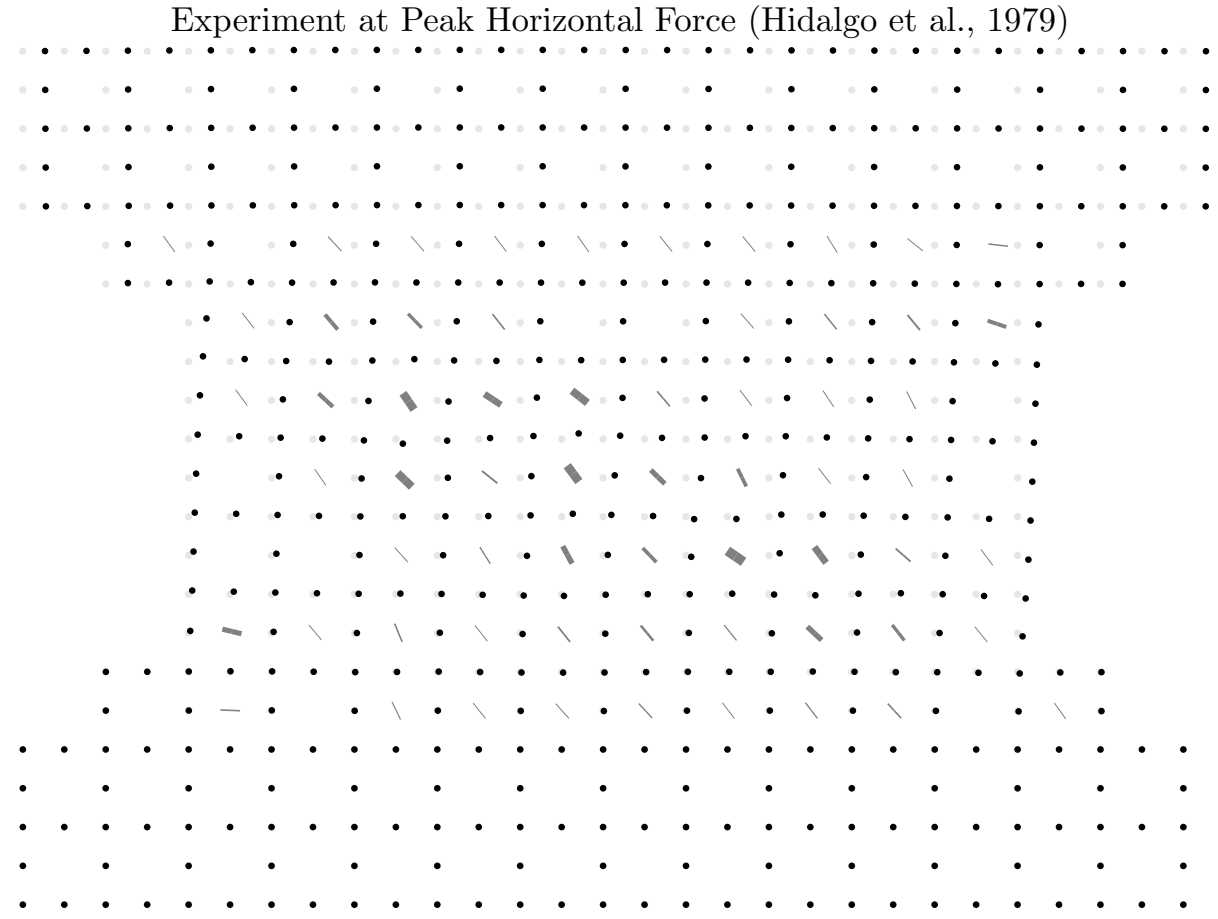

Finite Element Model at Peak Horizontal Force $\times 15$

HCBL-12-3 Shear Wall Displacement, Crack Pattern, and Crack Widths 


\section{HCBL-12-4 Shear Wall}

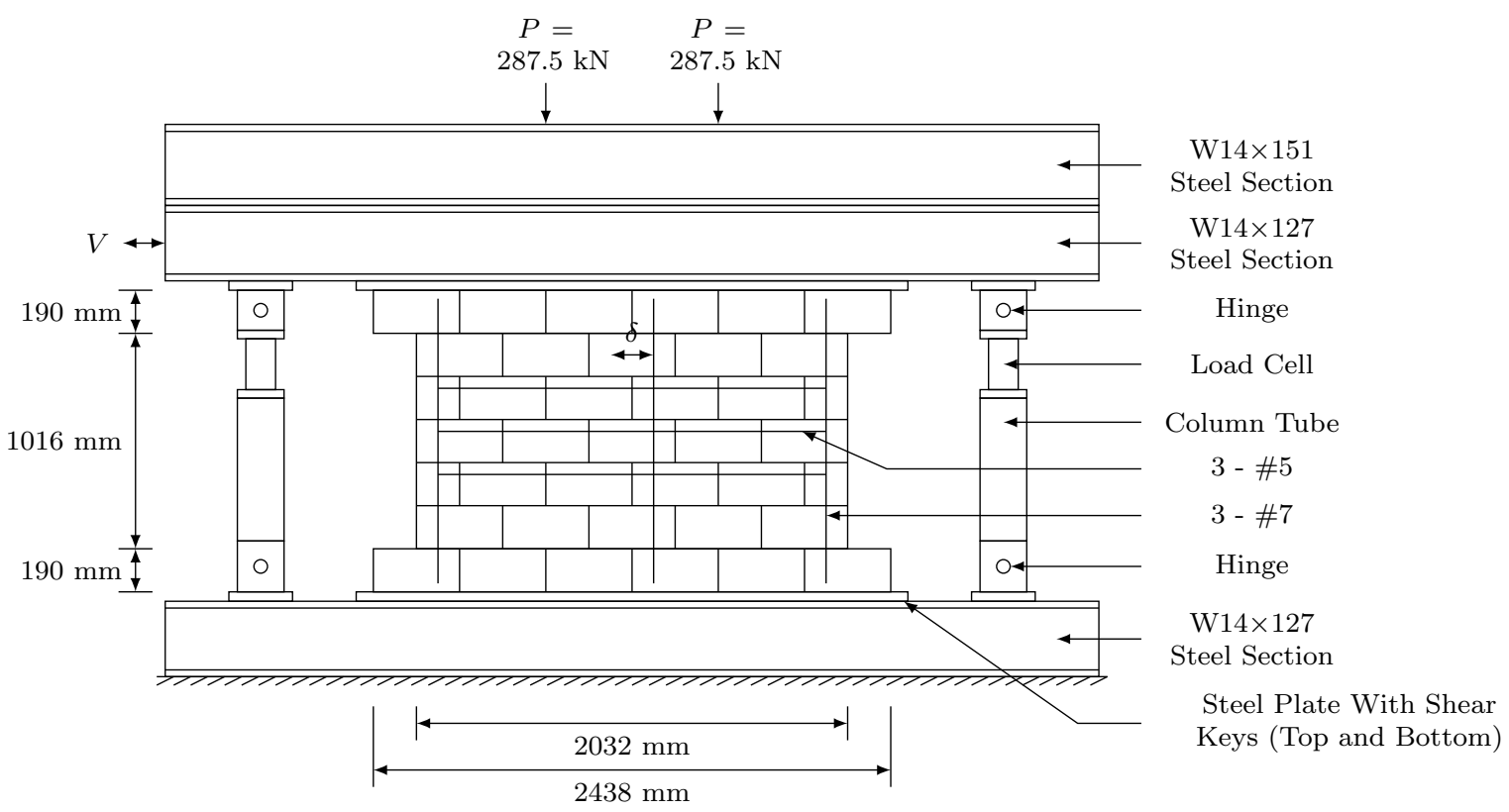

Experimental Wall (Hidalgo et al., 1979)

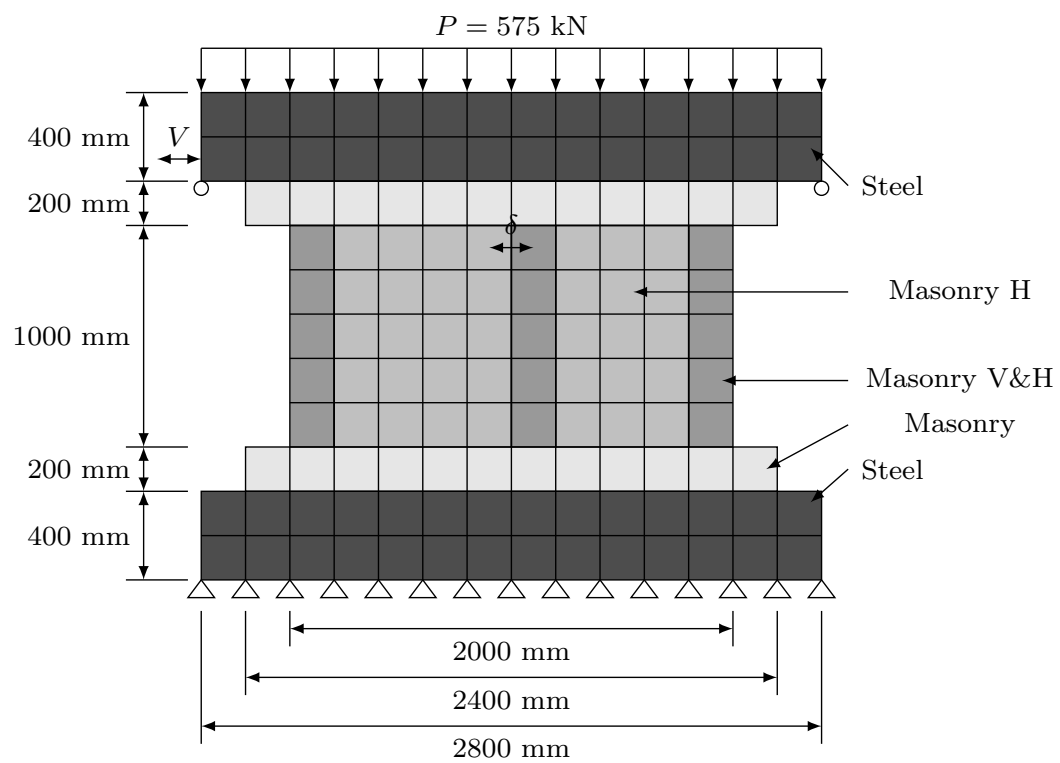

Finite Element Model Wall

HCBL-12-4 Shear Wall 
HCBL-12-4 Shear Wall (Hidalgo et al., 1979) Model Options

\begin{tabular}{ll}
\hline Model & Selected Option \\
\hline Compression Pre-Peak & Hognestad \\
Compression Post-Peak & Pre-Peak Base Curve \\
Compression Softening & Vecchio \\
Tension Post-Peak & Vecchio \\
Cracking Criterion & Mohr-Coulomb \\
Slip & Walraven Reinforced \\
\hline
\end{tabular}

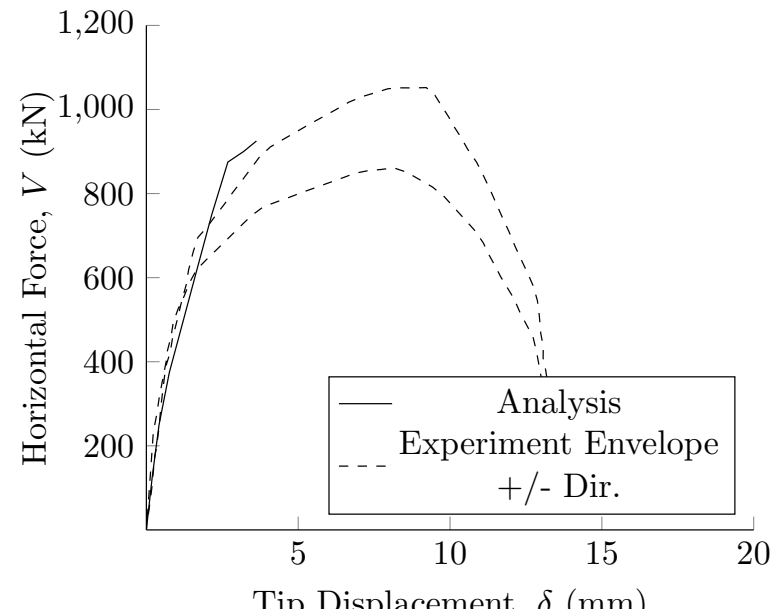

HCBL-12-4 Shear Wall (Hidalgo et al., 1979) Horizontal Force vs Tip Displacement 
HCBL-12-4 Shear Wall (Hidalgo et al., 1979) Material Properties

\begin{tabular}{|c|c|c|c|c|}
\hline \multirow{2}{*}{ Property } & \multicolumn{4}{|c|}{ Material } \\
\hline & Masonry & Masonry H & Masonry V\&H & Steel \\
\hline Thickness (mm) & 190 & 190 & 190 & 200 \\
\hline Grout Tensile Strength (MPa) & 1.40 & 1.40 & 1.40 & 350 \\
\hline \multicolumn{5}{|c|}{ Masonry Properties (Y-Direction) } \\
\hline Gross Peak Compression Strength (MPa) & 20.6 & 20.6 & 20.6 & 350 \\
\hline Peak Compressive Strain & 0.0042 & 0.0042 & 0.0042 & 0.00175 \\
\hline Tensile Strength & 0 & 0 & 0 & 350 \\
\hline Tensile Strain & 0 & 0 & 0 & 0.00175 \\
\hline Initial Tangent Elastic Modulus (MPa) & 5500 & 5500 & 5500 & 200000 \\
\hline Poisson's Ratio & 0 & 0 & 0 & 0 \\
\hline \multicolumn{5}{|c|}{ Masonry Properties (X-Direction) } \\
\hline Gross Peak Compression Strength (MPa) & 20.6 & 20.6 & 20.6 & 350 \\
\hline Peak Compressive Strain & 0.0042 & 0.0042 & 0.0042 & 0.00175 \\
\hline Tensile Strength & 0 & 0 & 0 & 350 \\
\hline Tensile Strain & 0 & 0 & 0 & 0.00175 \\
\hline Initial Tangent Elastic Modulus (MPa) & 5500 & 5500 & 5500 & 200000 \\
\hline Poisson's Ratio & 0 & 0 & 0 & 0 \\
\hline \multicolumn{5}{|c|}{ Masonry Properties (Block) } \\
\hline Length (mm) & 390 & 390 & 390 & 2000 \\
\hline Height (mm) & 190 & 190 & 190 & 400 \\
\hline Face Shell Thickness (mm) & 41 & 41 & 41 & 100 \\
\hline Percent Solid (\%) & 59 & 59 & 59 & 100 \\
\hline Tensile Strength & 1.54 & 1.54 & 1.54 & 350 \\
\hline \multicolumn{5}{|c|}{ Head Joint } \\
\hline Thickness $(\mathrm{mm})$ & 10 & 10 & 10 & 10 \\
\hline Tensile Bond Strength (MPa) & 0.46 & 0.46 & 0.46 & 350 \\
\hline Joint Cohesion (MPa) & 11.6 & 11.6 & 11.6 & 350 \\
\hline Angle of Internal Friction (Deg) & 42 & 42 & 42 & 42 \\
\hline \multicolumn{5}{|c|}{ Bed Joint } \\
\hline Thickness $(\mathrm{mm})$ & 10 & 10 & 10 & 10 \\
\hline Tensile Bond Strength (MPa) & 0.46 & 0.46 & 0.46 & 350 \\
\hline Joint Cohesion (MPa) & 11.6 & 11.6 & 11.6 & 350 \\
\hline Angle of Internal Friction (Deg) & 42 & 42 & 42 & 42 \\
\hline \multicolumn{5}{|c|}{ Reinforcement (Y-Direction) } \\
\hline Ratio (\%) & 0 & 0 & 0.30 & 100 \\
\hline Yield Strength (MPa) & 0 & 0 & 554 & 350 \\
\hline Yield Strain & 0 & 0 & 0.00279 & 0.00175 \\
\hline Strain Hardening Strain & 0 & 0 & 0.02 & 0.02 \\
\hline Ultimate Strength $(\mathrm{MPa})$ & 0 & 0 & 865 & 600 \\
\hline Ultimate Strain & 0 & 0 & 0.2 & 0.2 \\
\hline Prestrain & 0 & 0 & 0 & 0 \\
\hline \multicolumn{5}{|c|}{ Reinforcement (X-Direction) } \\
\hline Ratio (\%) & 0 & 0.15 & 0.15 & 100 \\
\hline Yield Strength (MPa) & 0 & 480 & 480 & 350 \\
\hline Yield Strain & 0 & 0.00243 & 0.00243 & 0.00175 \\
\hline Strain Hardening Strain & 0 & 0.02 & 0.02 & 0.02 \\
\hline Ultimate Strength (MPa) & 0 & 757 & 757 & 600 \\
\hline Ultimate Strain & 0 & 0.2 & 0.2 & 0.2 \\
\hline Prestrain & 0 & 0 & 0 & 0 \\
\hline
\end{tabular}

Numerical Modelling of Hollow Concrete Block Masonry Mechanics 


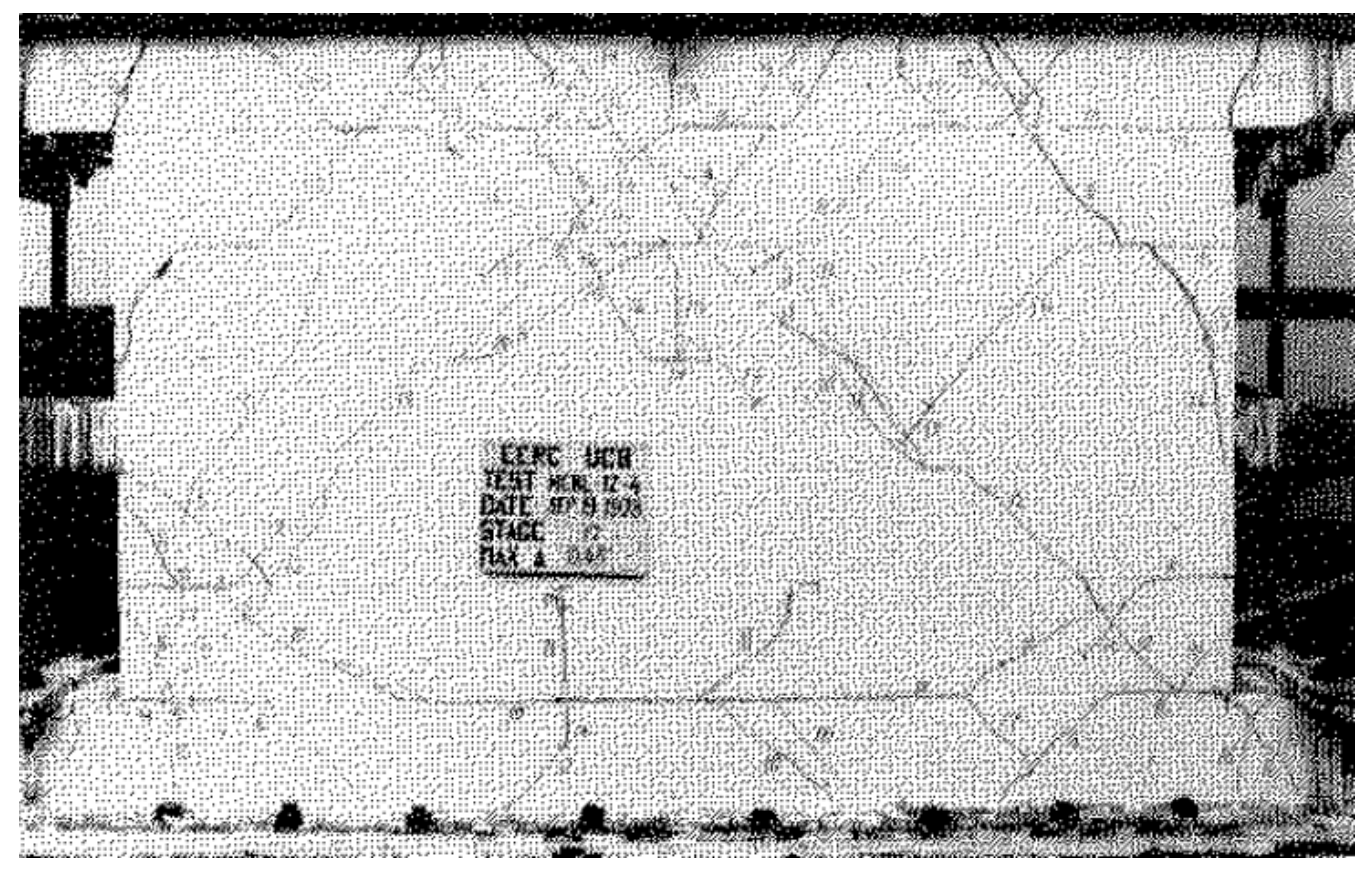

Experiment at Peak Horizontal Force (Hidalgo et al., 1979)

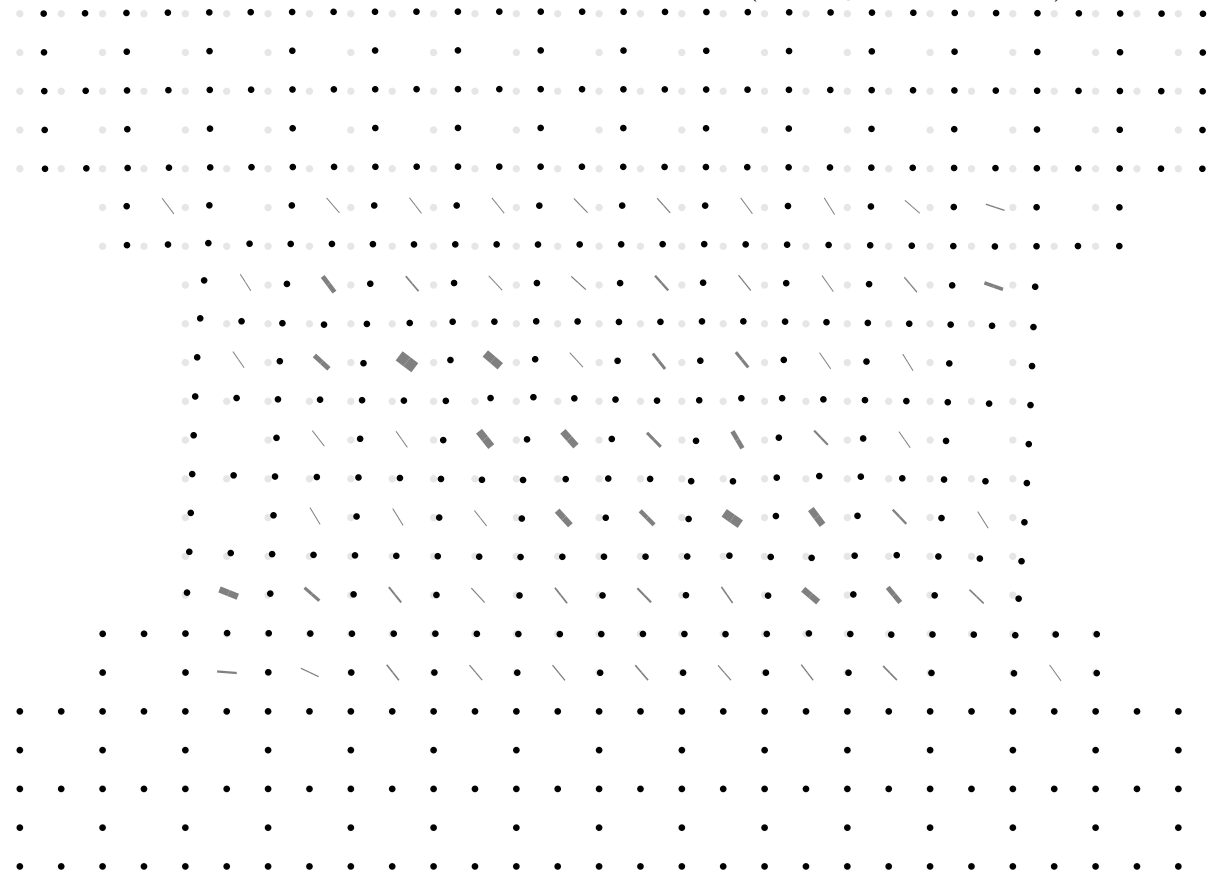

Finite Element Model at Peak Horizontal Force $\times 15$

HCBL-12-4 Shear Wall Displacement, Crack Pattern, and Crack Widths 


\section{HCBL-12-5 Shear Wall}

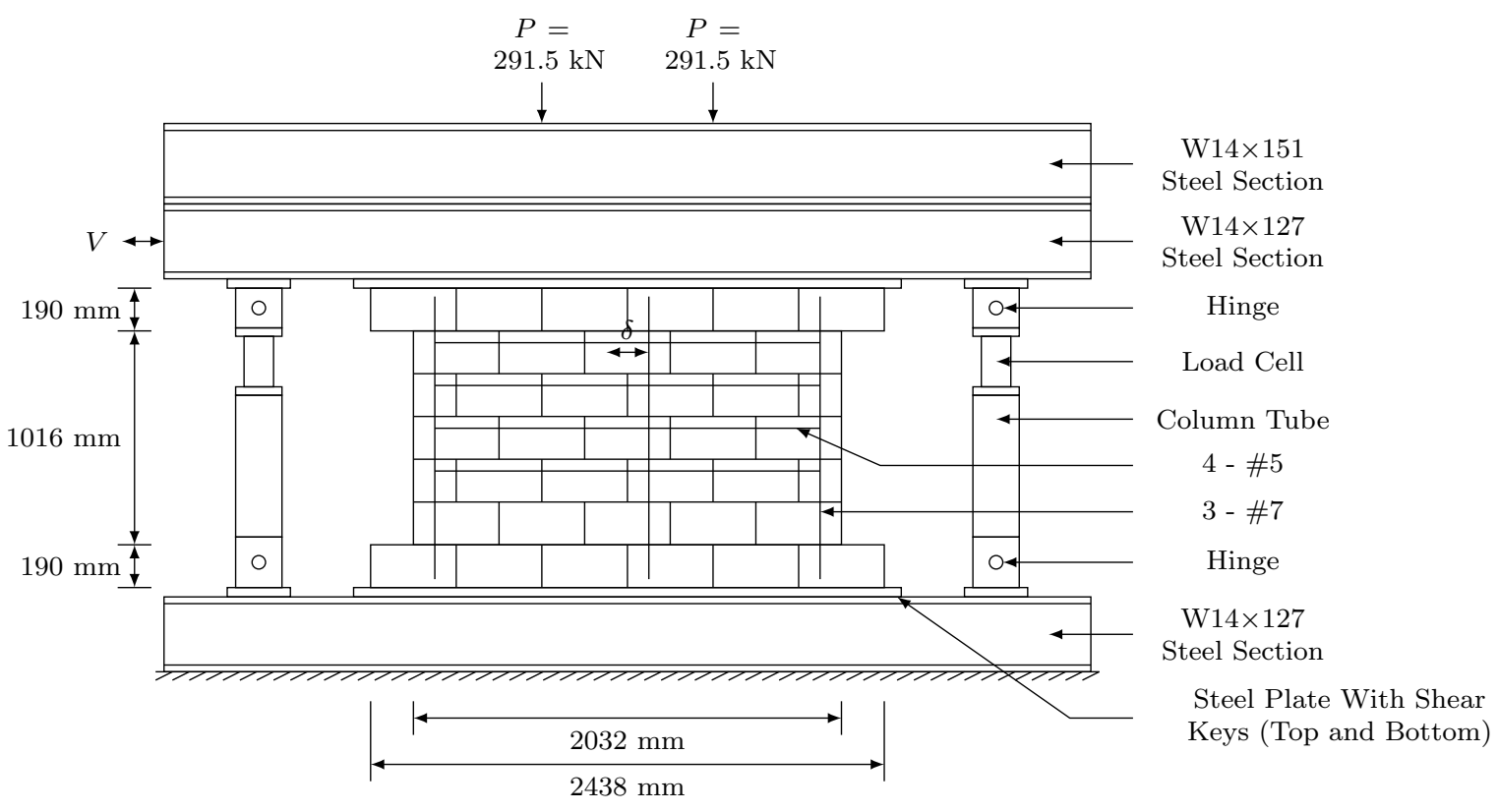

Experimental Wall (Hidalgo et al., 1979)

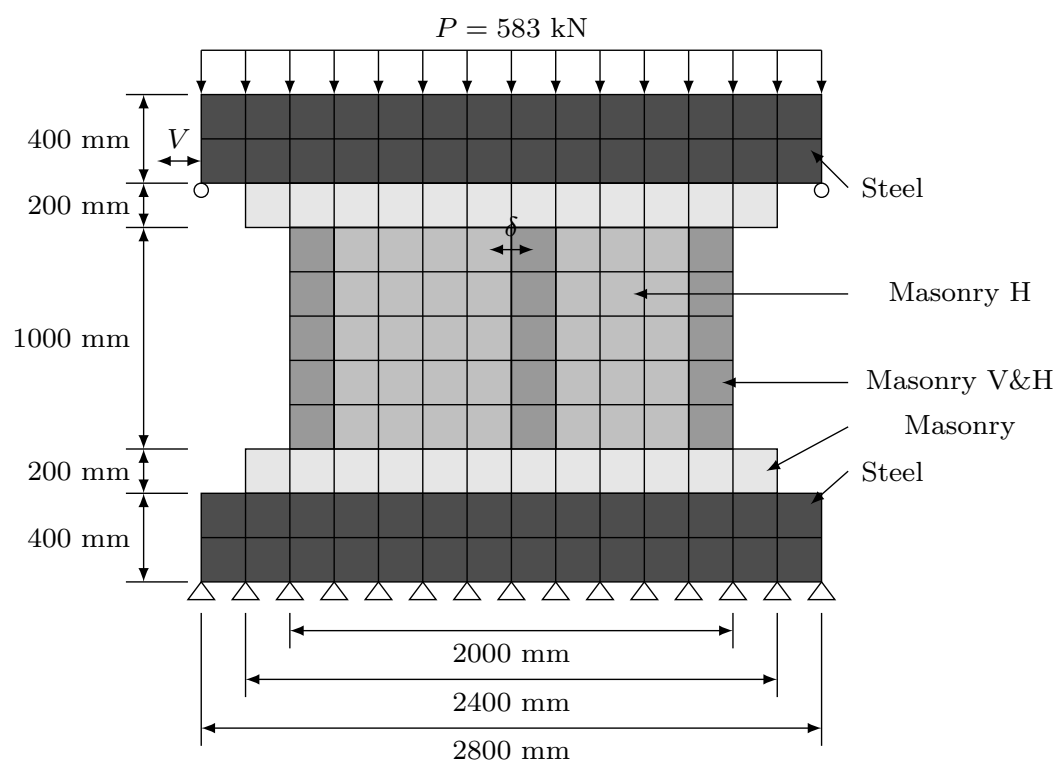

Finite Element Model Wall

HCBL-12-5 Shear Wall 
HCBL-12-5 Shear Wall (Hidalgo et al., 1979) Model Options

\begin{tabular}{ll}
\hline Model & Selected Option \\
\hline Compression Pre-Peak & Hognestad \\
Compression Post-Peak & Pre-Peak Base Curve \\
Compression Softening & Vecchio \\
Tension Post-Peak & Vecchio \\
Cracking Criterion & Mohr-Coulomb \\
Slip & Walraven Reinforced \\
\hline
\end{tabular}

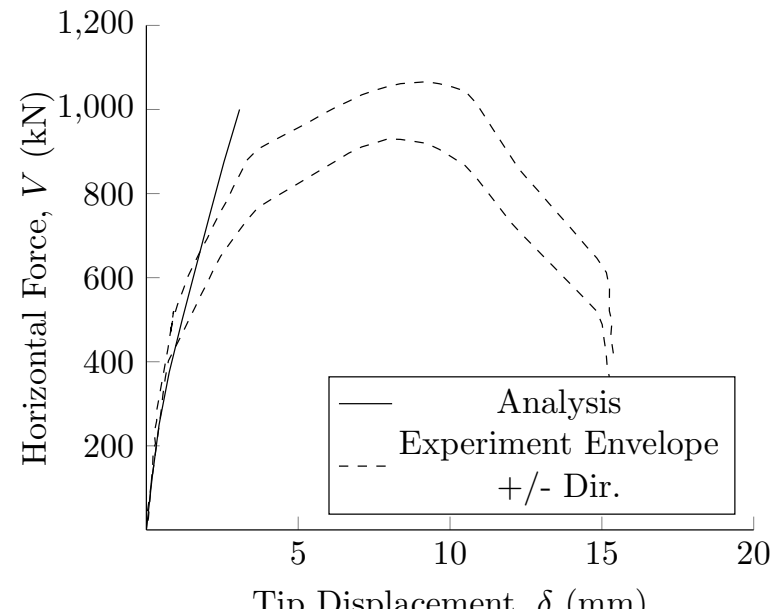

HCBL-12-5 Shear Wall (Hidalgo et al., 1979) Horizontal Force vs Tip Displacement 
HCBL-12-5 Shear Wall (Hidalgo et al., 1979) Material Properties

\begin{tabular}{|c|c|c|c|c|}
\hline \multirow{2}{*}{ Property } & \multicolumn{4}{|c|}{ Material } \\
\hline & Masonry & Masonry H & Masonry V\&H & Steel \\
\hline Thickness (mm) & 190 & 190 & 190 & 200 \\
\hline Grout Tensile Strength (MPa) & 1.40 & 1.40 & 1.40 & 350 \\
\hline \multicolumn{5}{|c|}{ Masonry Properties (Y-Direction) } \\
\hline Gross Peak Compression Strength (MPa) & 20.6 & 20.6 & 20.6 & 350 \\
\hline Peak Compressive Strain & 0.0042 & 0.0042 & 0.0042 & 0.00175 \\
\hline Tensile Strength & 0 & 0 & 0 & 350 \\
\hline Tensile Strain & 0 & 0 & 0 & 0.00175 \\
\hline Initial Tangent Elastic Modulus (MPa) & 5500 & 5500 & 5500 & 200000 \\
\hline Poisson's Ratio & 0 & 0 & 0 & 0 \\
\hline \multicolumn{5}{|c|}{ Masonry Properties (X-Direction) } \\
\hline Gross Peak Compression Strength (MPa) & 20.6 & 20.6 & 20.6 & 350 \\
\hline Peak Compressive Strain & 0.0042 & 0.0042 & 0.0042 & 0.00175 \\
\hline Tensile Strength & 0 & 0 & 0 & 350 \\
\hline Tensile Strain & 0 & 0 & 0 & 0.00175 \\
\hline Initial Tangent Elastic Modulus (MPa) & 5500 & 5500 & 5500 & 200000 \\
\hline Poisson's Ratio & 0 & 0 & 0 & 0 \\
\hline \multicolumn{5}{|c|}{ Masonry Properties (Block) } \\
\hline Length (mm) & 390 & 390 & 390 & 2000 \\
\hline Height (mm) & 190 & 190 & 190 & 400 \\
\hline Face Shell Thickness (mm) & 41 & 41 & 41 & 100 \\
\hline Percent Solid (\%) & 59 & 59 & 59 & 100 \\
\hline Tensile Strength & 1.54 & 1.54 & 1.54 & 350 \\
\hline \multicolumn{5}{|c|}{ Head Joint } \\
\hline Thickness $(\mathrm{mm})$ & 10 & 10 & 10 & 10 \\
\hline Tensile Bond Strength (MPa) & 0.46 & 0.46 & 0.46 & 350 \\
\hline Joint Cohesion (MPa) & 11.6 & 11.6 & 11.6 & 350 \\
\hline Angle of Internal Friction (Deg) & 42 & 42 & 42 & 42 \\
\hline \multicolumn{5}{|c|}{ Bed Joint } \\
\hline Thickness $(\mathrm{mm})$ & 10 & 10 & 10 & 10 \\
\hline Tensile Bond Strength (MPa) & 0.46 & 0.46 & 0.46 & 350 \\
\hline Joint Cohesion (MPa) & 11.6 & 11.6 & 11.6 & 350 \\
\hline Angle of Internal Friction (Deg) & 42 & 42 & 42 & 42 \\
\hline \multicolumn{5}{|c|}{ Reinforcement (Y-Direction) } \\
\hline Ratio (\%) & 0 & 0 & 0.30 & 100 \\
\hline Yield Strength (MPa) & 0 & 0 & 554 & 350 \\
\hline Yield Strain & 0 & 0 & 0.00279 & 0.00175 \\
\hline Strain Hardening Strain & 0 & 0 & 0.02 & 0.02 \\
\hline Ultimate Strength $(\mathrm{MPa})$ & 0 & 0 & 865 & 600 \\
\hline Ultimate Strain & 0 & 0 & 0.2 & 0.2 \\
\hline Prestrain & 0 & 0 & 0 & 0 \\
\hline \multicolumn{5}{|c|}{ Reinforcement (X-Direction) } \\
\hline Ratio (\%) & 0 & 0.20 & 0.20 & 100 \\
\hline Yield Strength (MPa) & 0 & 480 & 480 & 350 \\
\hline Yield Strain & 0 & 0.00243 & 0.00243 & 0.00175 \\
\hline Strain Hardening Strain & 0 & 0.02 & 0.02 & 0.02 \\
\hline Ultimate Strength (MPa) & 0 & 757 & 757 & 600 \\
\hline Ultimate Strain & 0 & 0.2 & 0.2 & 0.2 \\
\hline Prestrain & 0 & 0 & 0 & 0 \\
\hline
\end{tabular}

Numerical Modelling of Hollow Concrete Block Masonry Mechanics 


\section{HCBL-12-6 Shear Wall}

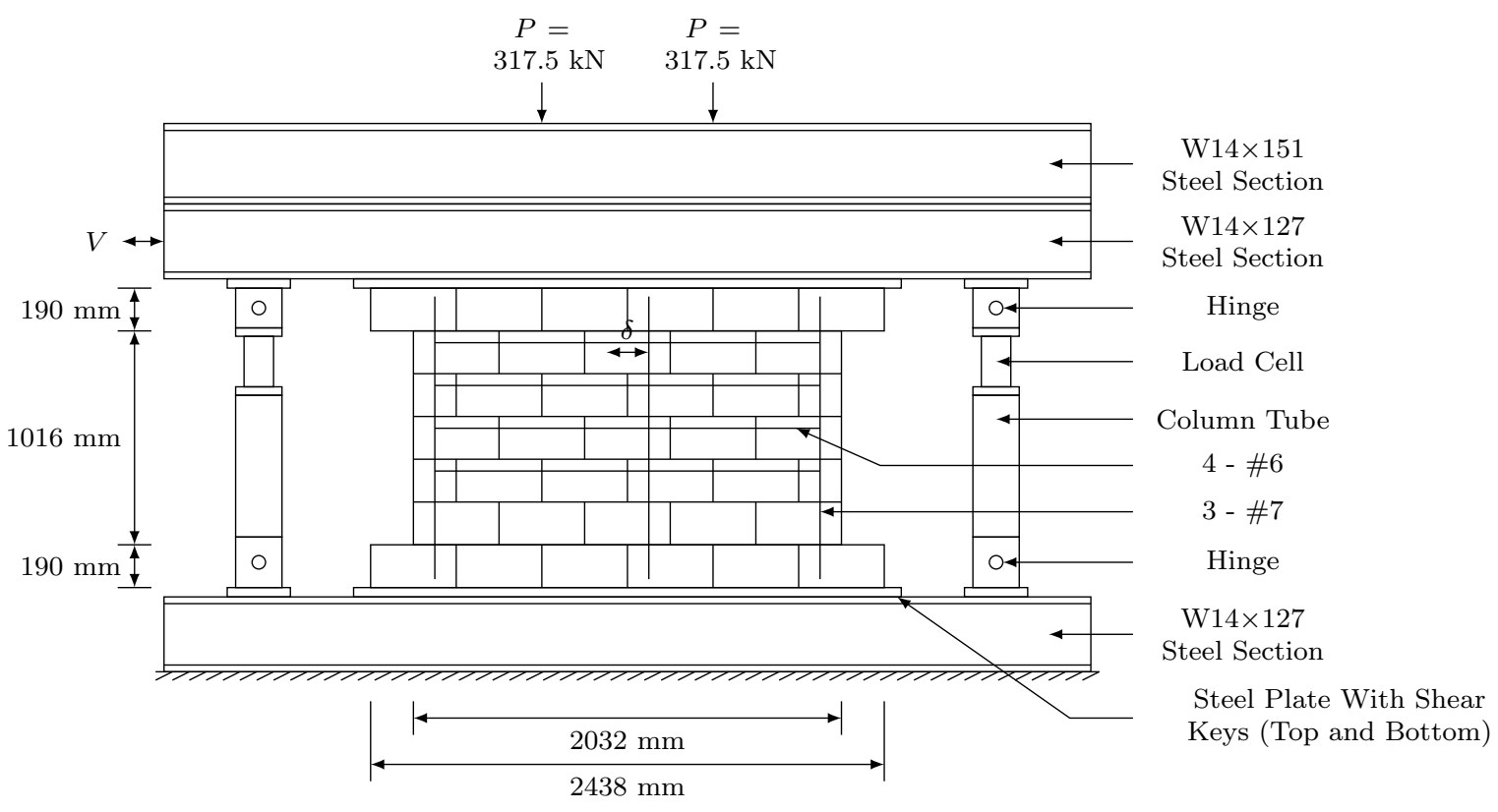

Experimental Wall (Hidalgo et al., 1979)

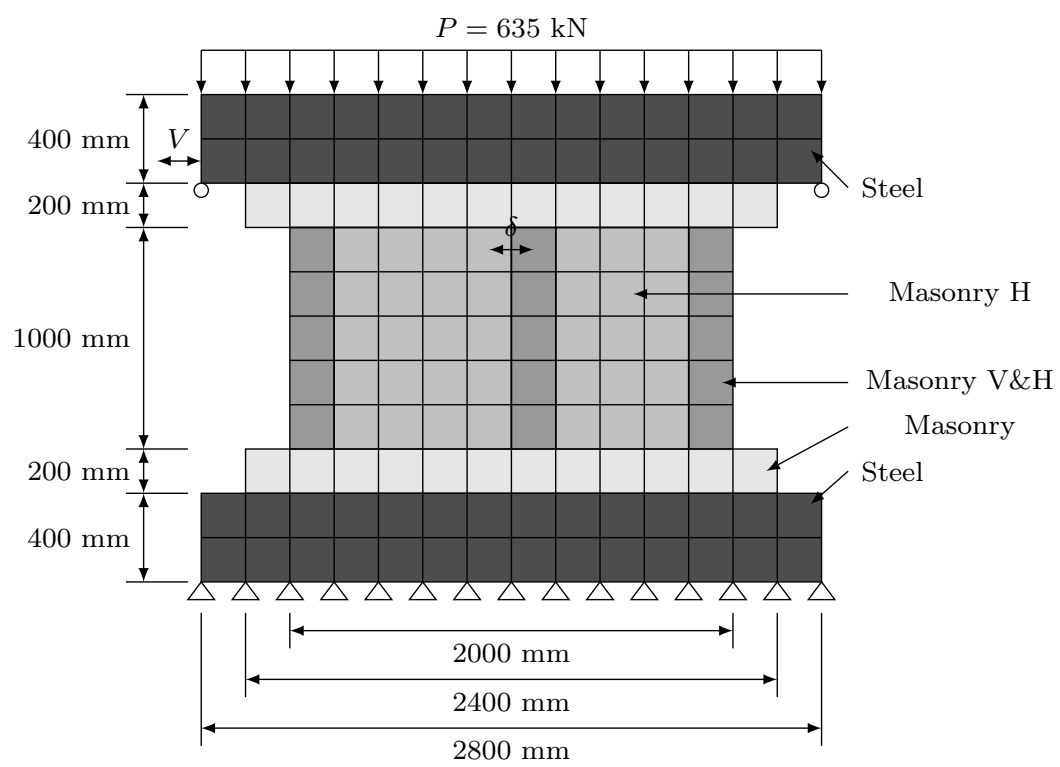

Finite Element Model Wall

HCBL-12-6 Shear Wall 
HCBL-12-6 Shear Wall (Hidalgo et al., 1979) Model Options

\begin{tabular}{ll}
\hline Model & Selected Option \\
\hline Compression Pre-Peak & Hognestad \\
Compression Post-Peak & Pre-Peak Base Curve \\
Compression Softening & Vecchio \\
Tension Post-Peak & Vecchio \\
Cracking Criterion & Mohr-Coulomb \\
Slip & Walraven Reinforced \\
\hline
\end{tabular}

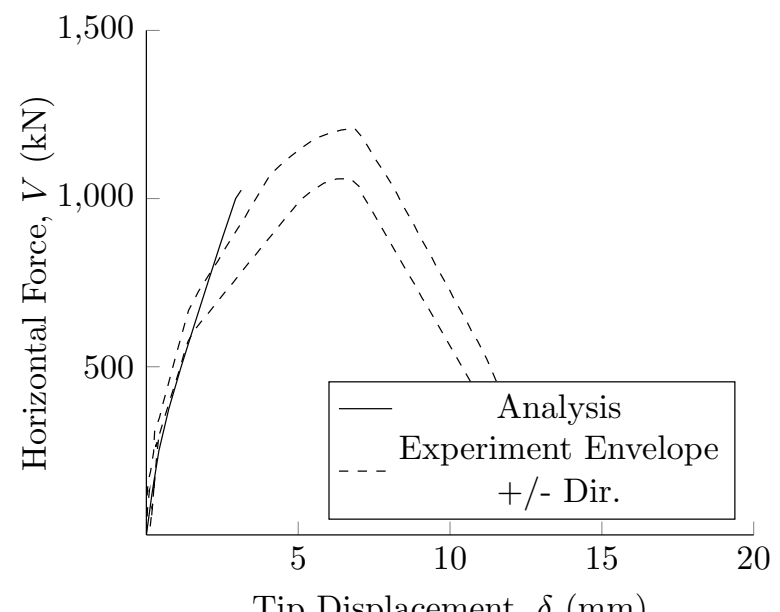

HCBL-12-6 Shear Wall (Hidalgo et al., 1979) Horizontal Force vs Tip Displacement 
HCBL-12-6 Shear Wall (Hidalgo et al., 1979) Material Properties

\begin{tabular}{|c|c|c|c|c|}
\hline \multirow{2}{*}{ Property } & \multicolumn{4}{|c|}{ Material } \\
\hline & Masonry & Masonry H & Masonry V\&H & Steel \\
\hline Thickness (mm) & 190 & 190 & 190 & 200 \\
\hline Grout Tensile Strength (MPa) & 1.40 & 1.40 & 1.40 & 350 \\
\hline \multicolumn{5}{|c|}{ Masonry Properties (Y-Direction) } \\
\hline Gross Peak Compression Strength (MPa) & 20.6 & 20.6 & 20.6 & 350 \\
\hline Peak Compressive Strain & 0.0042 & 0.0042 & 0.0042 & 0.00175 \\
\hline Tensile Strength & 0 & 0 & 0 & 350 \\
\hline Tensile Strain & 0 & 0 & 0 & 0.00175 \\
\hline Initial Tangent Elastic Modulus (MPa) & 5600 & 5600 & 5600 & 200000 \\
\hline Poisson's Ratio & 0 & 0 & 0 & 0 \\
\hline \multicolumn{5}{|c|}{ Masonry Properties (X-Direction) } \\
\hline Gross Peak Compression Strength (MPa) & 20.6 & 20.6 & 20.6 & 350 \\
\hline Peak Compressive Strain & 0.0042 & 0.0042 & 0.0042 & 0.00175 \\
\hline Tensile Strength & 0 & 0 & 0 & 350 \\
\hline Tensile Strain & 0 & 0 & 0 & 0.00175 \\
\hline Initial Tangent Elastic Modulus (MPa) & 5600 & 5600 & 5600 & 200000 \\
\hline Poisson's Ratio & 0 & 0 & 0 & 0 \\
\hline \multicolumn{5}{|c|}{ Masonry Properties (Block) } \\
\hline Length (mm) & 390 & 390 & 390 & 2000 \\
\hline Height (mm) & 190 & 190 & 190 & 400 \\
\hline Face Shell Thickness (mm) & 41 & 41 & 41 & 100 \\
\hline Percent Solid (\%) & 59 & 59 & 59 & 100 \\
\hline Tensile Strength & 1.54 & 1.54 & 1.54 & 350 \\
\hline \multicolumn{5}{|c|}{ Head Joint } \\
\hline Thickness $(\mathrm{mm})$ & 10 & 10 & 10 & 10 \\
\hline Tensile Bond Strength (MPa) & 0.46 & 0.46 & 0.46 & 350 \\
\hline Joint Cohesion (MPa) & 11.6 & 11.6 & 11.6 & 350 \\
\hline Angle of Internal Friction (Deg) & 42 & 42 & 42 & 42 \\
\hline \multicolumn{5}{|c|}{ Bed Joint } \\
\hline Thickness $(\mathrm{mm})$ & 10 & 10 & 10 & 10 \\
\hline Tensile Bond Strength (MPa) & 0.46 & 0.46 & 0.46 & 350 \\
\hline Joint Cohesion (MPa) & 11.6 & 11.6 & 11.6 & 350 \\
\hline Angle of Internal Friction (Deg) & 42 & 42 & 42 & 42 \\
\hline \multicolumn{5}{|c|}{ Reinforcement (Y-Direction) } \\
\hline Ratio (\%) & 0 & 0 & 0.30 & 100 \\
\hline Yield Strength (MPa) & 0 & 0 & 554 & 350 \\
\hline Yield Strain & 0 & 0 & 0.00279 & 0.00175 \\
\hline Strain Hardening Strain & 0 & 0 & 0.02 & 0.02 \\
\hline Ultimate Strength $(\mathrm{MPa})$ & 0 & 0 & 865 & 600 \\
\hline Ultimate Strain & 0 & 0 & 0.2 & 0.2 \\
\hline Prestrain & 0 & 0 & 0 & 0 \\
\hline \multicolumn{5}{|c|}{ Reinforcement (X-Direction) } \\
\hline Ratio (\%) & 0 & 0.29 & 0.29 & 100 \\
\hline Yield Strength (MPa) & 0 & 464 & 464 & 350 \\
\hline Yield Strain & 0 & 0.00234 & 0.00234 & 0.00175 \\
\hline Strain Hardening Strain & 0 & 0.02 & 0.02 & 0.02 \\
\hline Ultimate Strength (MPa) & 0 & 749 & 749 & 600 \\
\hline Ultimate Strain & 0 & 0.2 & 0.2 & 0.2 \\
\hline Prestrain & 0 & 0 & 0 & 0 \\
\hline
\end{tabular}

Numerical Modelling of Hollow Concrete Block Masonry Mechanics 


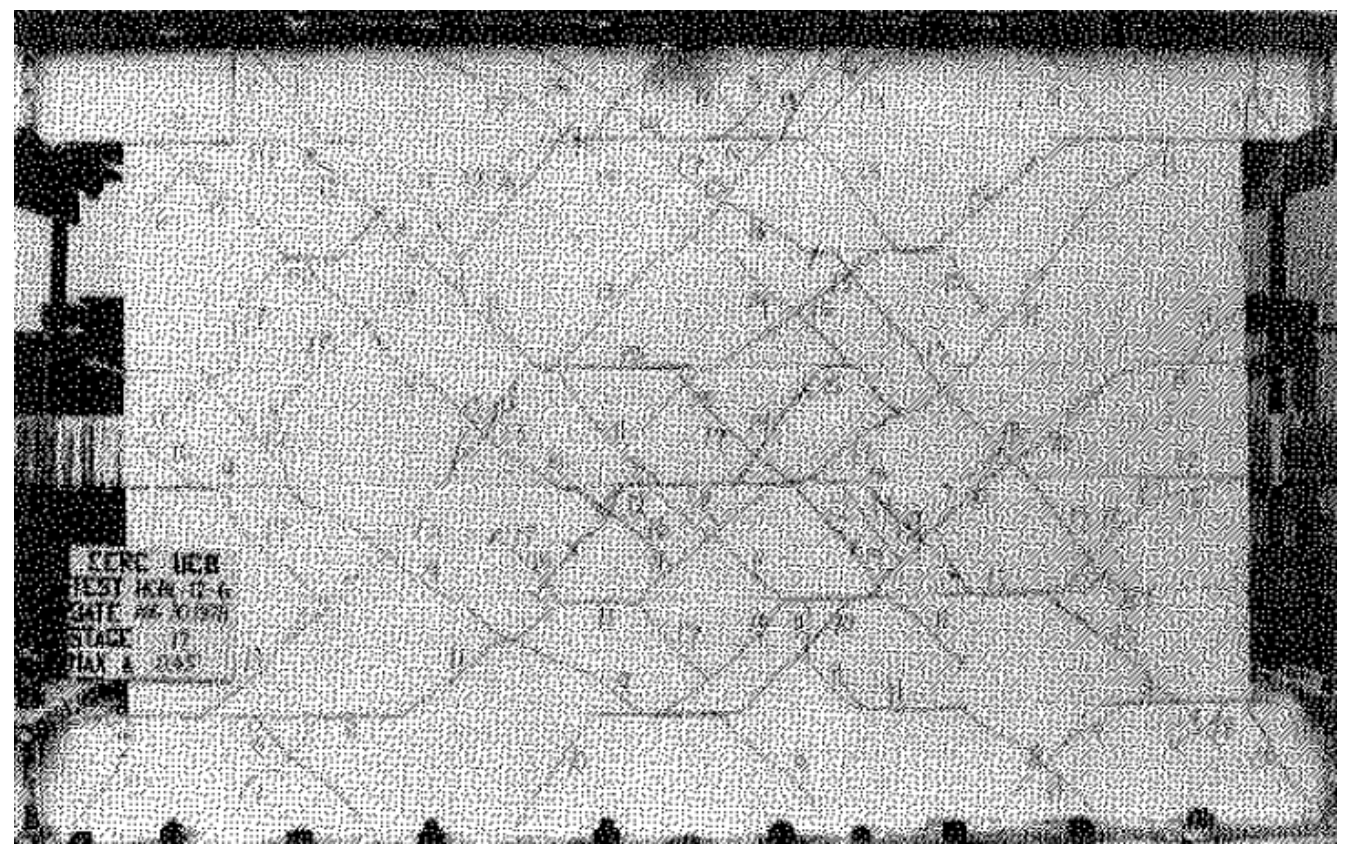

Experiment at Peak Horizontal Force (Hidalgo et al., 1979)

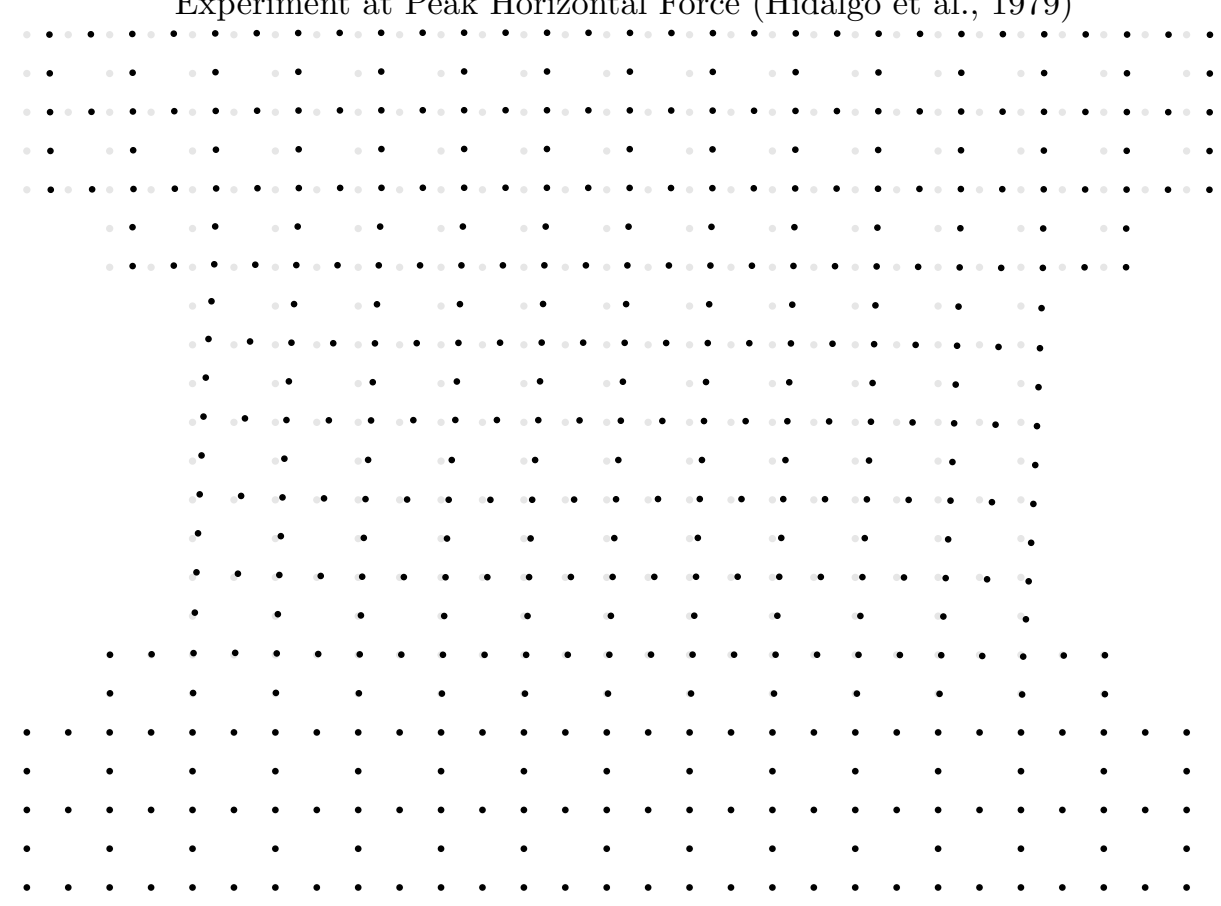

Finite Element Model at Peak Horizontal Force $\times 15$

HCBL-12-6 Shear Wall Displacement, Crack Pattern, and Crack Widths 


\section{Sveinsson et al. (1985)}

\section{HCBL-11-13 Shear Wall}

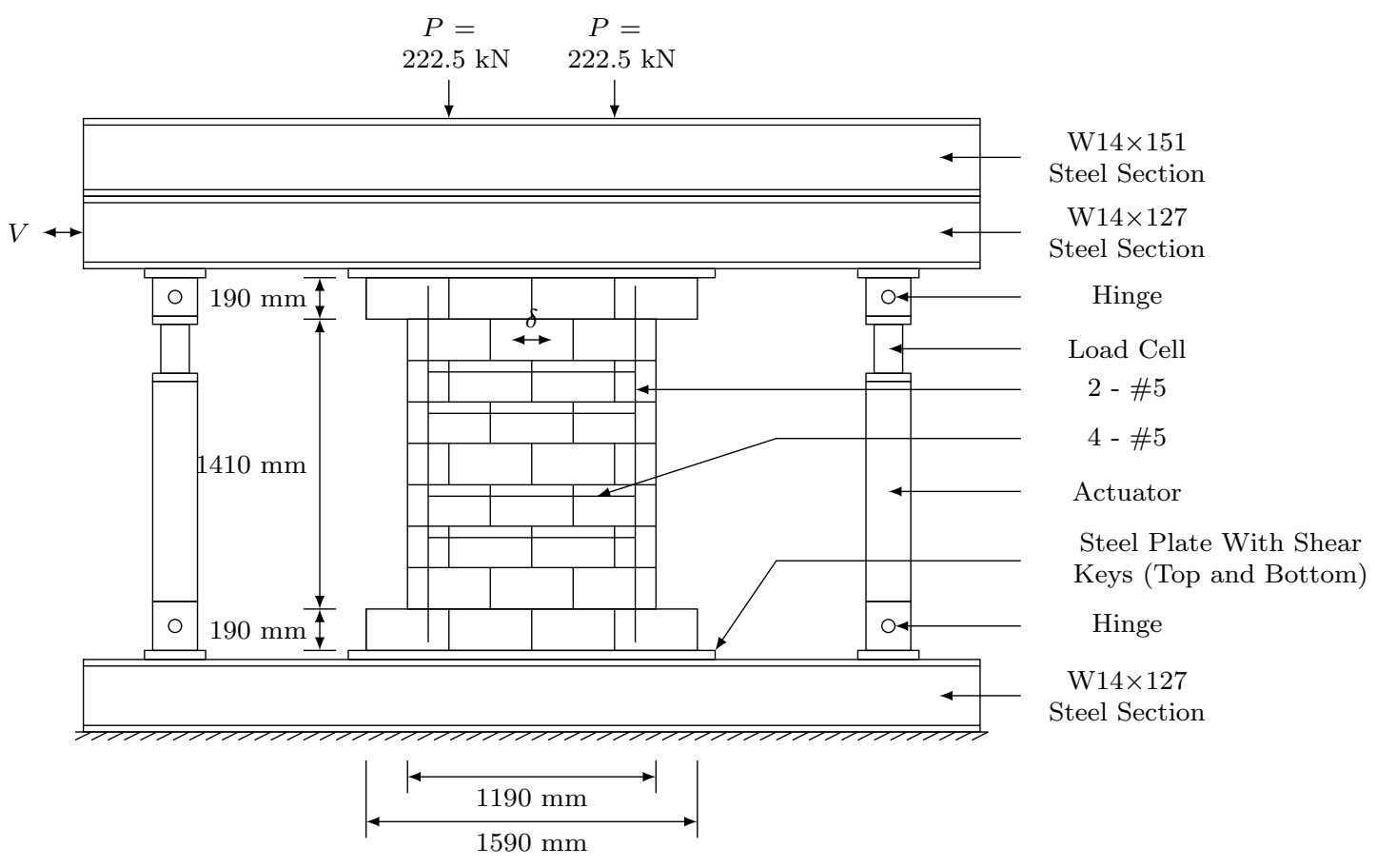

Experimental Wall (Sveinsson et al., 1985)

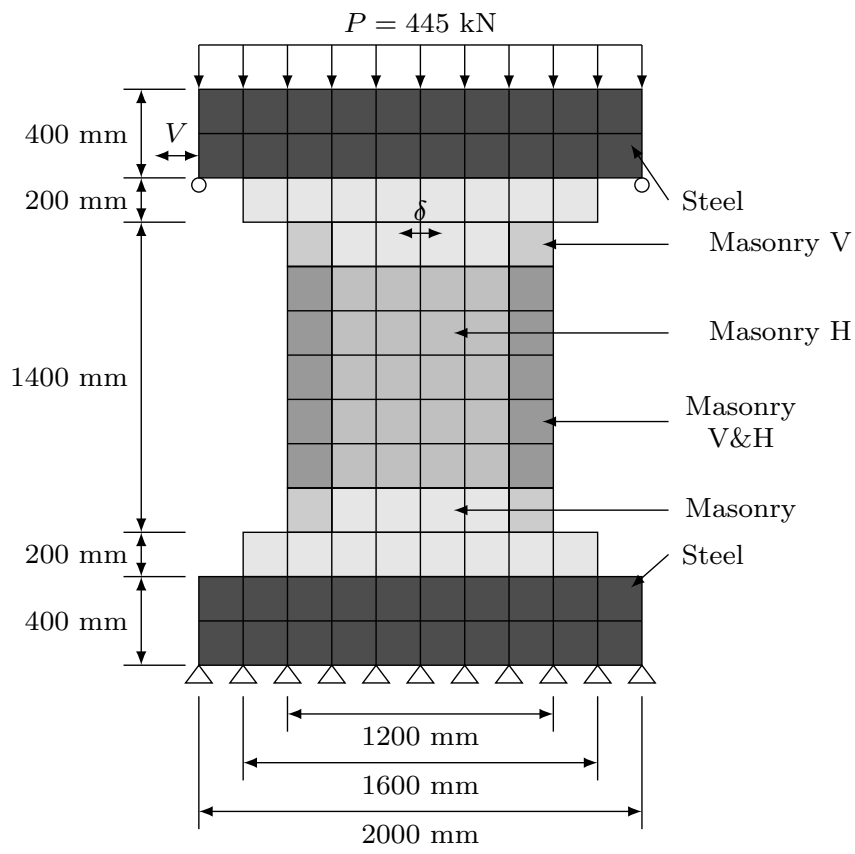

Finite Element Model Wall

HCBL-11-13 Shear Wall 
HCBL-11-13 Shear Wall (Sveinsson et al., 1985) Model Options

\begin{tabular}{ll}
\hline Model & Selected Option \\
\hline Compression Pre-Peak & Hognestad \\
Compression Post-Peak & Pre-Peak Base Curve \\
Compression Softening & Vecchio \\
Tension Post-Peak & Vecchio \\
Cracking Criterion & Mohr-Coulomb \\
Slip & Walraven Reinforced \\
\hline
\end{tabular}

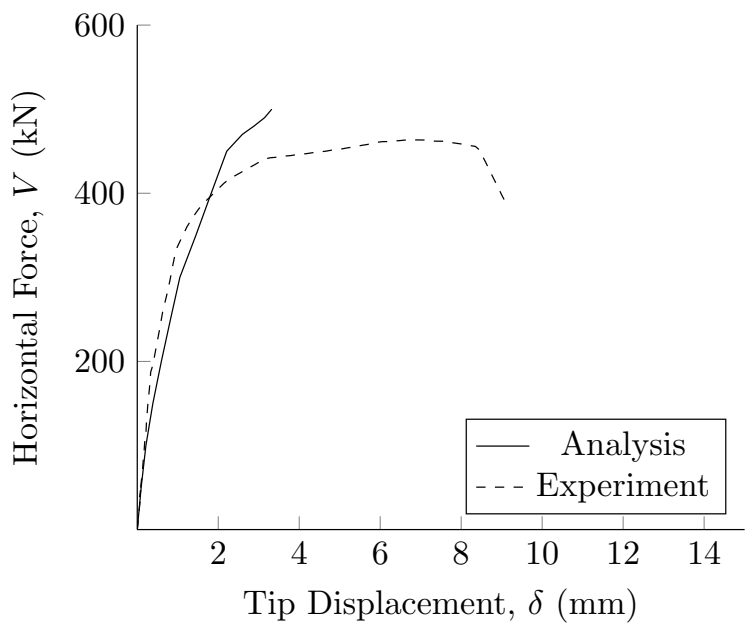

HCBL-11-13 Shear Wall (Sveinsson et al., 1985) Horizontal Force vs Tip Displacement 
HCBL-11-13 Shear Wall (Sveinsson et al., 1985) Material Properties

\begin{tabular}{|c|c|c|c|c|c|}
\hline \multirow{2}{*}{ Property } & \multicolumn{5}{|c|}{ Material } \\
\hline & Masonry & Masonry V & Masonry H & Masonry V\&H & Steel \\
\hline Thickness (mm) & 190 & 190 & 190 & 190 & 200 \\
\hline Grout Tensile Strength (MPa) & 1.40 & 1.40 & 1.40 & 1.40 & 350 \\
\hline \multicolumn{6}{|c|}{ Masonry Properties (Y-Direction) } \\
\hline Gross Peak Compression Strength (MPa) & 23.8 & 23.8 & 23.8 & 23.8 & 350 \\
\hline Peak Compressive Strain & 0.0025 & 0.0025 & 0.0025 & 0.0025 & 0.00175 \\
\hline Tensile Strength & 0 & 0 & 0 & 0 & 350 \\
\hline Tensile Strain & 0 & 0 & 0 & 0 & 0.00175 \\
\hline Initial Tangent Elastic Modulus (MPa) & 0 & 0 & 0 & 0 & 200000 \\
\hline Poisson's Ratio & 0 & 0 & 0 & 0 & 0 \\
\hline \multicolumn{6}{|c|}{ Masonry Properties (X-Direction) } \\
\hline Gross Peak Compression Strength (MPa) & 23.8 & 23.8 & 23.8 & 23.8 & 350 \\
\hline Peak Compressive Strain & 0.0025 & 0.0025 & 0.0025 & 0.0025 & 0.00175 \\
\hline Tensile Strength & 0 & 0 & 0 & 0 & 350 \\
\hline Tensile Strain & 0 & 0 & 0 & 0 & 0.00175 \\
\hline Initial Tangent Elastic Modulus (MPa) & 0 & 0 & 0 & 0 & 200000 \\
\hline Poisson's Ratio & 0 & 0 & 0 & 0 & 0 \\
\hline \multicolumn{6}{|c|}{ Masonry Properties (Block) } \\
\hline Length $(\mathrm{mm})$ & 390 & 390 & 390 & 390 & 2000 \\
\hline Height (mm) & 190 & 190 & 190 & 190 & 400 \\
\hline Face Shell Thickness (mm) & 41 & 41 & 41 & 41 & 100 \\
\hline Percent Solid (\%) & 59 & 59 & 59 & 59 & 100 \\
\hline Tensile Strength & 1.50 & 1.50 & 1.50 & 1.50 & 350 \\
\hline \multicolumn{6}{|c|}{ Head Joint } \\
\hline Thickness (mm) & 10 & 10 & 10 & 10 & 10 \\
\hline Tensile Bond Strength (MPa) & 0.46 & 0.46 & 0.46 & 0.46 & 350 \\
\hline Joint Cohesion (MPa) & 11.6 & 11.6 & 11.6 & 11.6 & 350 \\
\hline Angle of Internal Friction (Deg) & 42 & 42 & 42 & 42 & 42 \\
\hline \multicolumn{6}{|c|}{ Bed Joint } \\
\hline Thickness (mm) & 10 & 10 & 10 & 10 & 10 \\
\hline Tensile Bond Strength (MPa) & 0.46 & 0.46 & 0.46 & 0.46 & 350 \\
\hline Joint Cohesion (MPa) & 11.6 & 11.6 & 11.6 & 11.6 & 350 \\
\hline Angle of Internal Friction (Deg) & 42 & 42 & 42 & 42 & 42 \\
\hline \multicolumn{6}{|c|}{ Reinforcement (Y-Direction) } \\
\hline Ratio (\%) & 0 & 0.17 & 0 & 0.17 & 100 \\
\hline Yield Strength (MPa) & 0 & 465 & 0 & 465 & 350 \\
\hline Yield Strain & 0 & 0.0024 & 0 & 0.0024 & 0.00175 \\
\hline Strain Hardening Strain & 0 & 0.02 & 0 & 0.02 & 0.02 \\
\hline Ultimate Strength (MPa) & 0 & 741 & 0 & 741 & 600 \\
\hline Ultimate Strain & 0 & 0.2 & 0 & 0.2 & 0.2 \\
\hline Prestrain & 0 & 0 & 0 & 0 & 0 \\
\hline \multicolumn{6}{|c|}{ Reinforcement (X-Direction) } \\
\hline Ratio (\%) & 0 & 0 & 0.30 & 0.30 & 100 \\
\hline Yield Strength (MPa) & 0 & 0 & 407 & 407 & 350 \\
\hline Yield Strain & 0 & 0 & 0.0021 & 0.0021 & 0.00175 \\
\hline Strain Hardening Strain & 0 & 0 & 0.02 & 0.02 & 0.02 \\
\hline Ultimate Strength (MPa) & 0 & 0 & 607 & 607 & 600 \\
\hline Ultimate Strain & 0 & 0 & 0.2 & 0.2 & 0.2 \\
\hline Prestrain & 0 & 0 & 0 & 0 & 0 \\
\hline
\end{tabular}

Numerical Modelling of Hollow Concrete Block Masonry Mechanics 


\section{HCBL-11-15 Shear Wall}

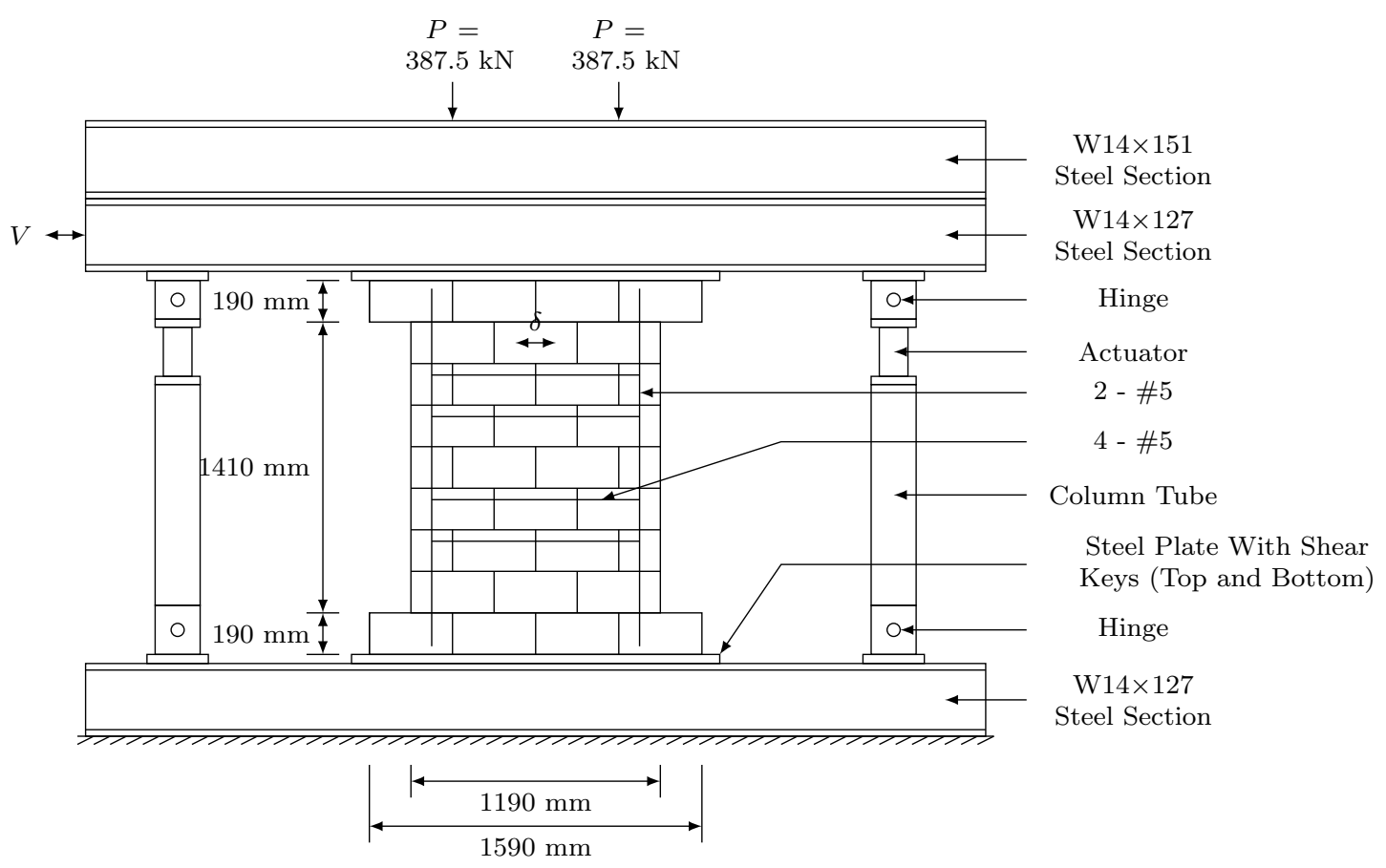

Experimental Wall (Sveinsson et al., 1985)

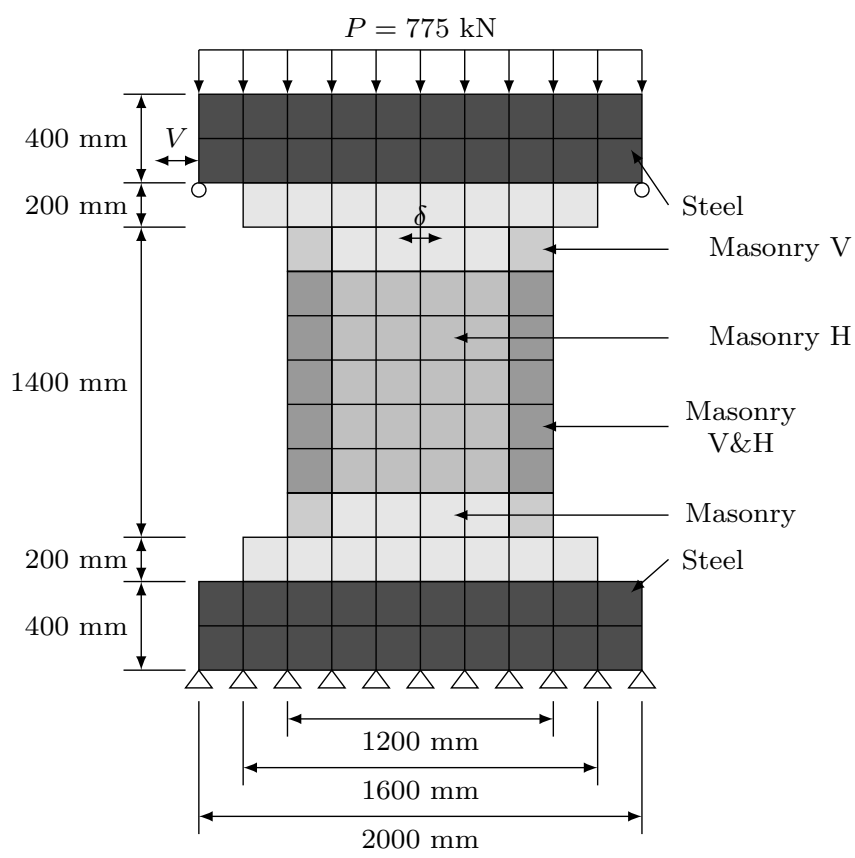

Finite Element Model Wall

HCBL-11-15 Shear Wall 
HCBL-11-15 Shear Wall (Sveinsson et al., 1985) Model Options

\begin{tabular}{ll}
\hline Model & Selected Option \\
\hline Compression Pre-Peak & Hognestad \\
Compression Post-Peak & Pre-Peak Base Curve \\
Compression Softening & Vecchio \\
Tension Post-Peak & Vecchio \\
Cracking Criterion & Mohr-Coulomb \\
Slip & Walraven Reinforced \\
\hline
\end{tabular}

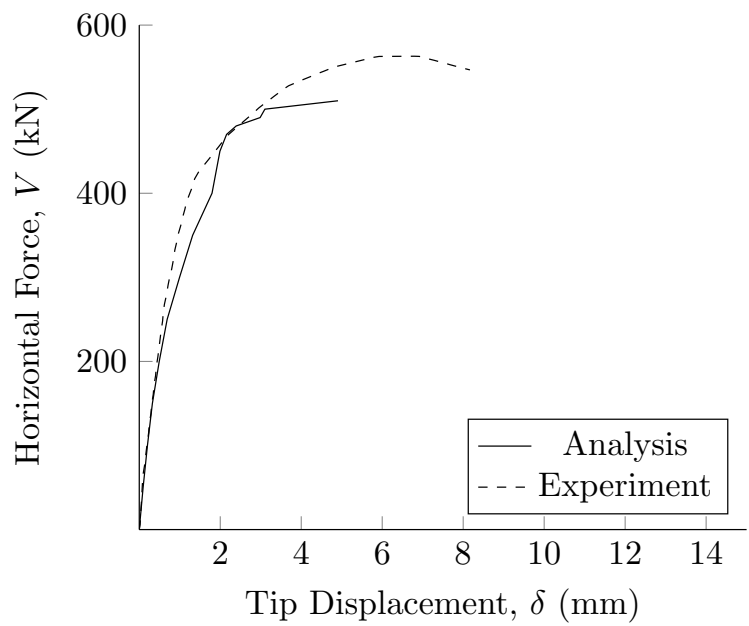

HCBL-11-15 Shear Wall (Sveinsson et al., 1985) Horizontal Force vs Tip Displacement 
HCBL-11-15 Shear Wall (Sveinsson et al., 1985) Material Properties

\begin{tabular}{|c|c|c|c|c|c|}
\hline \multirow{2}{*}{ Property } & \multicolumn{5}{|c|}{ Material } \\
\hline & Masonry & Masonry V & Masonry H & Masonry V\&H & Steel \\
\hline Thickness $(\mathrm{mm})$ & 190 & 190 & 190 & 190 & 200 \\
\hline Grout Tensile Strength (MPa) & 1.40 & 1.40 & 1.40 & 1.40 & 350 \\
\hline \multicolumn{6}{|c|}{ Masonry Properties (Y-Direction) } \\
\hline Gross Peak Compression Strength ( $\mathrm{MPa})$ & 23.8 & 23.8 & 23.8 & 23.8 & 350 \\
\hline Peak Compressive Strain & 0.0025 & 0.0025 & 0.0025 & 0.0025 & 0.00175 \\
\hline Tensile Strength & 0 & 0 & 0 & 0 & 350 \\
\hline Tensile Strain & 0 & 0 & 0 & 0 & 0.00175 \\
\hline Initial Tangent Elastic Modulus (MPa) & 0 & 0 & 0 & 0 & 200000 \\
\hline Poisson's Ratio & 0 & 0 & 0 & 0 & 0 \\
\hline \multicolumn{6}{|c|}{ Masonry Properties (X-Direction) } \\
\hline Gross Peak Compression Strength (MPa) & 23.8 & 23.8 & 23.8 & 23.8 & 350 \\
\hline Peak Compressive Strain & 0.0025 & 0.0025 & 0.0025 & 0.0025 & 0.00175 \\
\hline Tensile Strength & 0 & 0 & 0 & 0 & 350 \\
\hline Tensile Strain & 0 & 0 & 0 & 0 & 0.00175 \\
\hline Initial Tangent Elastic Modulus (MPa) & 0 & 0 & 0 & 0 & 200000 \\
\hline Poisson's Ratio & 0 & 0 & 0 & 0 & 0 \\
\hline \multicolumn{6}{|c|}{ Masonry Properties (Block) } \\
\hline Length $(\mathrm{mm})$ & 390 & 390 & 390 & 390 & 2000 \\
\hline Height (mm) & 190 & 190 & 190 & 190 & 400 \\
\hline Face Shell Thickness (mm) & 41 & 41 & 41 & 41 & 100 \\
\hline Percent Solid $(\%)$ & 59 & 59 & 59 & 59 & 100 \\
\hline Tensile Strength & 1.50 & 1.50 & 1.50 & 1.50 & 350 \\
\hline \multicolumn{6}{|c|}{ Head Joint } \\
\hline Thickness (mm) & 10 & 10 & 10 & 10 & 10 \\
\hline Tensile Bond Strength (MPa) & 0.46 & 0.46 & 0.46 & 0.46 & 350 \\
\hline Joint Cohesion (MPa) & 11.6 & 11.6 & 11.6 & 11.6 & 350 \\
\hline Angle of Internal Friction (Deg) & 42 & 42 & 42 & 42 & 42 \\
\hline \multicolumn{6}{|c|}{ Bed Joint } \\
\hline Thickness (mm) & 10 & 10 & 10 & 10 & 10 \\
\hline Tensile Bond Strength (MPa) & 0.46 & 0.46 & 0.46 & 0.46 & 350 \\
\hline Joint Cohesion (MPa) & 11.6 & 11.6 & 11.6 & 11.6 & 350 \\
\hline Angle of Internal Friction (Deg) & 42 & 42 & 42 & 42 & 42 \\
\hline \multicolumn{6}{|c|}{ Reinforcement (Y-Direction) } \\
\hline Ratio (\%) & 0 & 0.17 & 0 & 0.17 & 100 \\
\hline Yield Strength (MPa) & 0 & 465 & 0 & 465 & 350 \\
\hline Yield Strain & 0 & 0.0024 & 0 & 0.0024 & 0.00175 \\
\hline Strain Hardening Strain & 0 & 0.02 & 0 & 0.02 & 0.02 \\
\hline Ultimate Strength $(\mathrm{MPa})$ & 0 & 741 & 0 & 741 & 600 \\
\hline Ultimate Strain & 0 & 0.2 & 0 & 0.2 & 0.2 \\
\hline Prestrain & 0 & 0 & 0 & 0 & 0 \\
\hline \multicolumn{6}{|c|}{ Reinforcement (X-Direction) } \\
\hline Ratio (\%) & 0 & 0 & 0.30 & 0.30 & 100 \\
\hline Yield Strength (MPa) & 0 & 0 & 407 & 407 & 350 \\
\hline Yield Strain & 0 & 0 & 0.0021 & 0.0021 & 0.00175 \\
\hline Strain Hardening Strain & 0 & 0 & 0.02 & 0.02 & 0.02 \\
\hline Ultimate Strength (MPa) & 0 & 0 & 607 & 607 & 600 \\
\hline Ultimate Strain & 0 & 0 & 0.2 & 0.2 & 0.2 \\
\hline Prestrain & 0 & 0 & 0 & 0 & 0 \\
\hline
\end{tabular}

Numerical Modelling of Hollow Concrete Block Masonry Mechanics 


\section{Shing et al. (1991)}

\section{HCBL 1 Shear Wall}

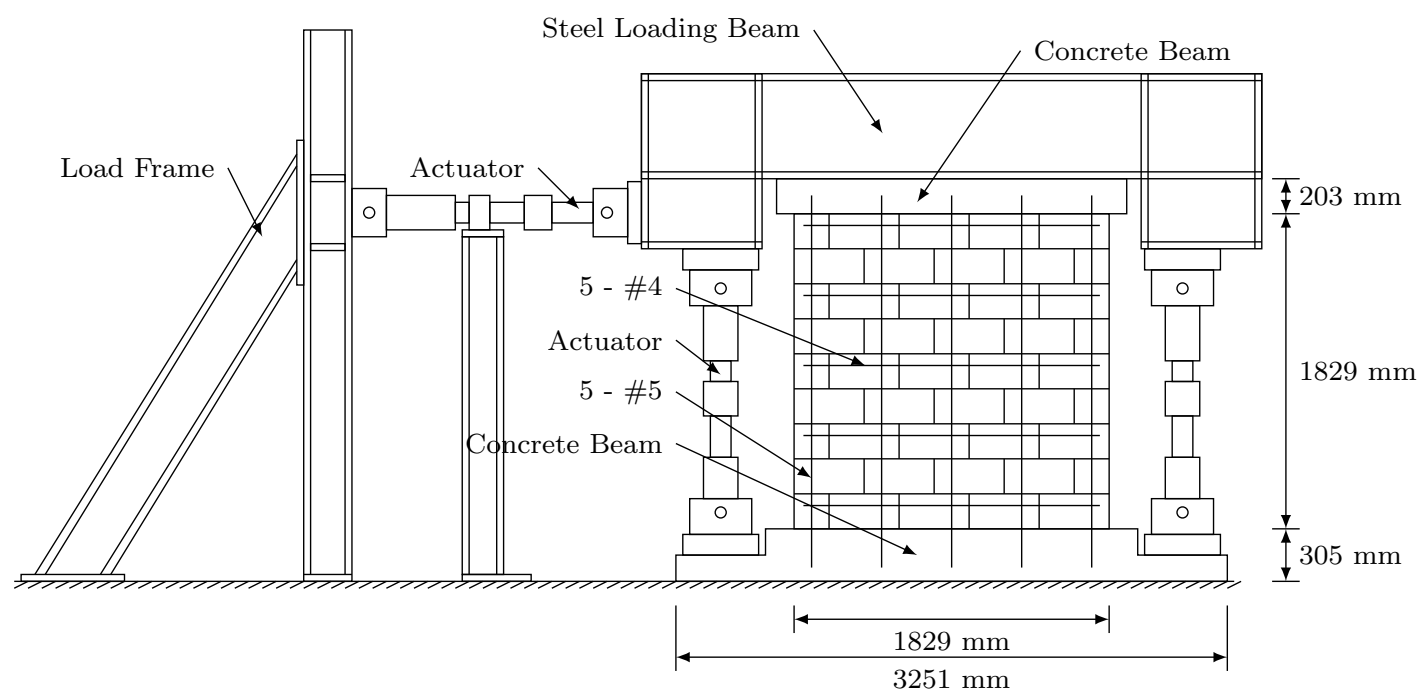

Experimental Wall Shing et al. (1991)

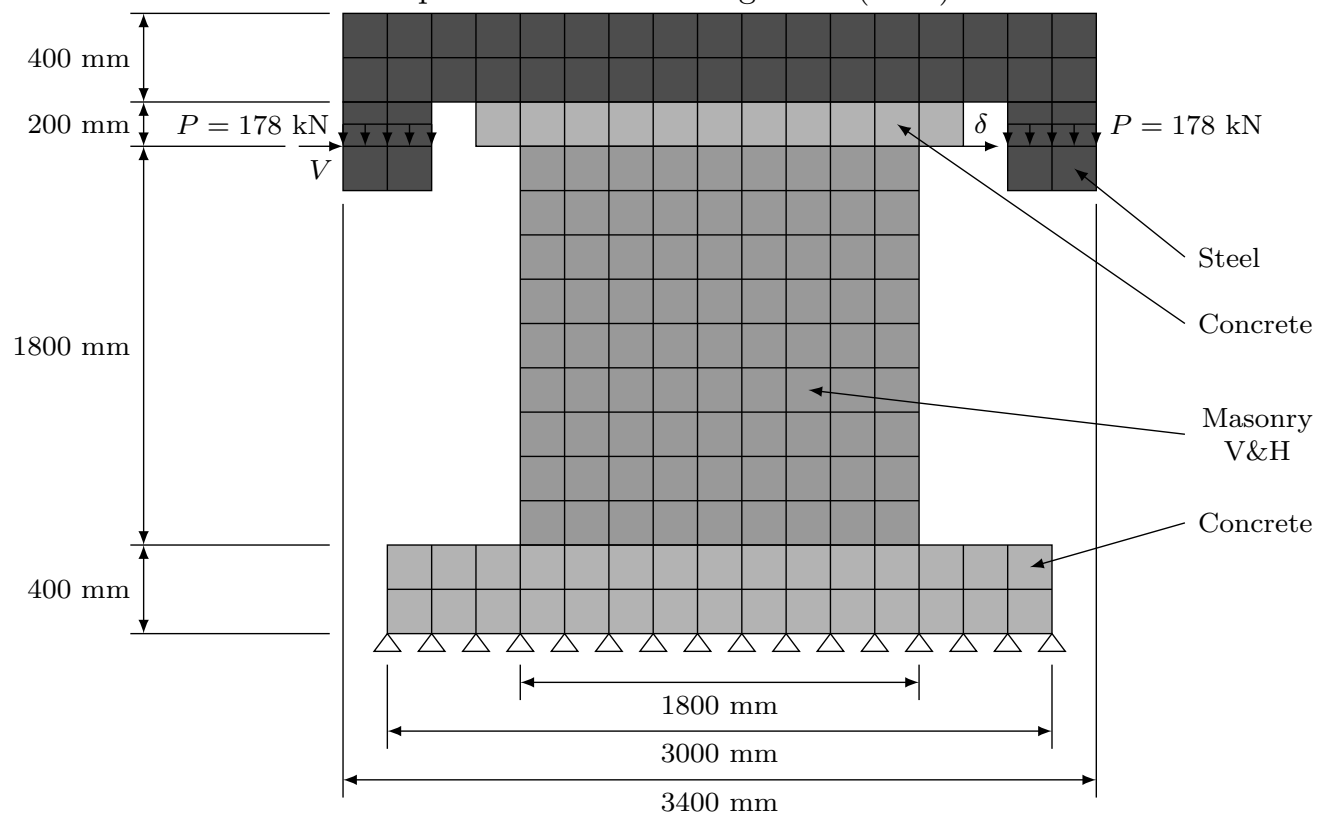

Finite Element Model Wall

HCBL 1 Shear Wall 
HCBL 1 Shear Wall Shing et al. (1991) Model Options

\begin{tabular}{ll}
\hline Model & Selected Option \\
\hline Compression Pre-Peak & Hognestad \\
Compression Post-Peak & Pre-Peak Base Curve \\
Compression Softening & Vecchio \\
Tension Post-Peak & Vecchio \\
Cracking Criterion & Mohr-Coulomb \\
Slip & Walraven Reinforced \\
\hline
\end{tabular}

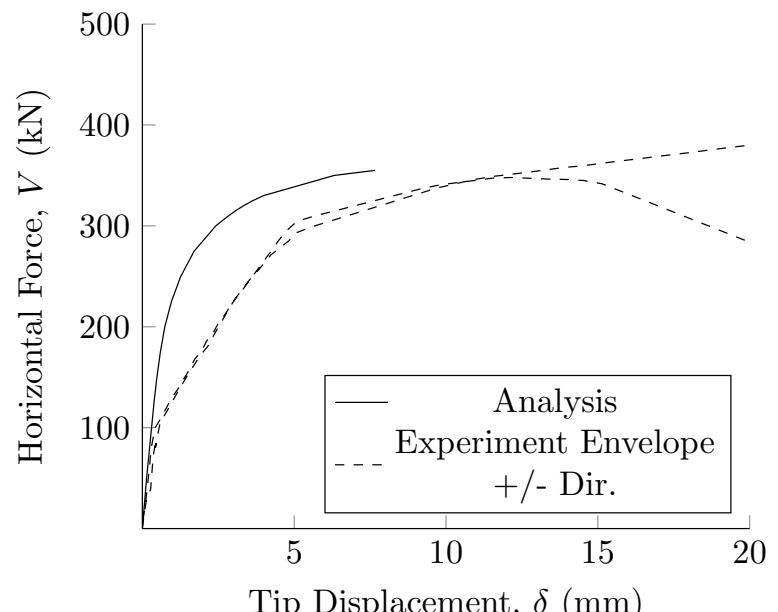

HCBL 1 Shear Wall Shing et al. (1991) Horizontal Force vs Tip Displacement 
HCBL 1 Shear Wall Shing et al. (1991) Material Properties

\begin{tabular}{|c|c|c|c|}
\hline \multirow{2}{*}{ Property } & \multicolumn{3}{|c|}{ Material } \\
\hline & Masonry V\&H & Concrete & Steel \\
\hline Thickness (mm) & 140 & 1220 & 400 \\
\hline Grout Tensile Strength (MPa) & 1.80 & 2.09 & 350 \\
\hline \multicolumn{4}{|c|}{ Masonry Properties (Y-Direction) } \\
\hline Gross Peak Compression Strength (MPa) & 20.1 & 40 & 350 \\
\hline Peak Compressive Strain & 0.0025 & 0.0021 & 0.00175 \\
\hline Tensile Strength & 0 & 2.09 & 350 \\
\hline Tensile Strain & 0 & 0 & 0.00175 \\
\hline Initial Tangent Elastic Modulus (MPa) & 0 & 34600 & 200000 \\
\hline Poisson's Ratio & 0 & 0 & 0 \\
\hline \multicolumn{4}{|c|}{ Masonry Properties (X-Direction) } \\
\hline Gross Peak Compression Strength (MPa) & 20.1 & 40 & 350 \\
\hline Peak Compressive Strain & 0.0025 & 0.0021 & 0.00175 \\
\hline Tensile Strength & 0 & 2.09 & 350 \\
\hline Tensile Strain & 0 & 0 & 0.00175 \\
\hline Initial Tangent Elastic Modulus (MPa) & 0 & 34600 & 200000 \\
\hline Poisson's Ratio & 0 & 0 & 0 \\
\hline \multicolumn{4}{|c|}{ Masonry Properties (Block) } \\
\hline Length (mm) & 390 & 2400 & 2000 \\
\hline Height (mm) & 190 & 400 & 400 \\
\hline Face Shell Thickness (mm) & 32 & 610 & 200 \\
\hline Percent Solid (\%) & 59 & 100 & 100 \\
\hline Tensile Strength & 1.75 & 2.09 & 350 \\
\hline \multicolumn{4}{|c|}{ Head Joint } \\
\hline Thickness (mm) & 10 & 10 & 10 \\
\hline Tensile Bond Strength (MPa) & 0.34 & 2.09 & 350 \\
\hline Joint Cohesion (MPa) & 6.13 & 2.09 & 350 \\
\hline Angle of Internal Friction (Deg) & 42 & 42 & 42 \\
\hline \multicolumn{4}{|c|}{ Bed Joint } \\
\hline Thickness (mm) & 10 & 10 & 10 \\
\hline Tensile Bond Strength (MPa) & 0.34 & 2.09 & 350 \\
\hline Joint Cohesion (MPa) & 6.13 & 2.09 & 350 \\
\hline Angle of Internal Friction (Deg) & 42 & 42 & 42 \\
\hline \multicolumn{4}{|c|}{ Reinforcement (Y-Direction) } \\
\hline Ratio (\%) & 0.38 & 1.38 & 100 \\
\hline Yield Strength (MPa) & 441 & 400 & 350 \\
\hline Yield Strain & 0.00221 & 0.002 & 0.00175 \\
\hline Strain Hardening Strain & 0.02 & 0.02 & 0.02 \\
\hline Ultimate Strength (MPa) & 710 & 600 & 600 \\
\hline Ultimate Strain & 0.2 & 0.2 & 0.2 \\
\hline Prestrain & 0 & 0 & 0 \\
\hline \multicolumn{4}{|c|}{ Reinforcement (X-Direction) } \\
\hline Ratio (\%) & 0.24 & 1.11 & 100 \\
\hline Yield Strength (MPa) & 462 & 400 & 350 \\
\hline Yield Strain & 0.00231 & 0.002 & 0.00175 \\
\hline Strain Hardening Strain & 0.02 & 0.02 & 0.02 \\
\hline Ultimate Strength (MPa) & 738 & 600 & 600 \\
\hline Ultimate Strain & 0.2 & 0.2 & 0.2 \\
\hline Prestrain & 0 & 0 & 0 \\
\hline
\end{tabular}

Numerical Modelling of Hollow Concrete Block Masonry Mechanics 


\section{HCBL 2 Shear Wall}

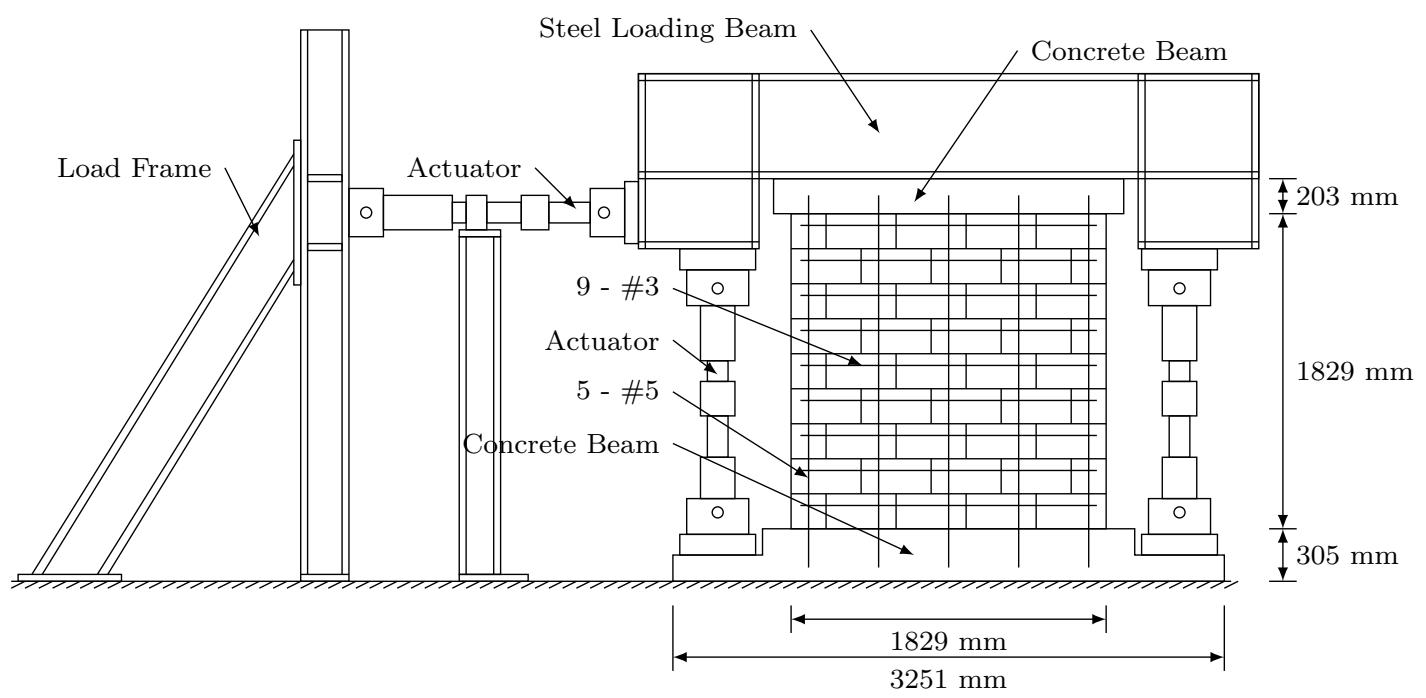

Experimental Wall Shing et al. (1991)

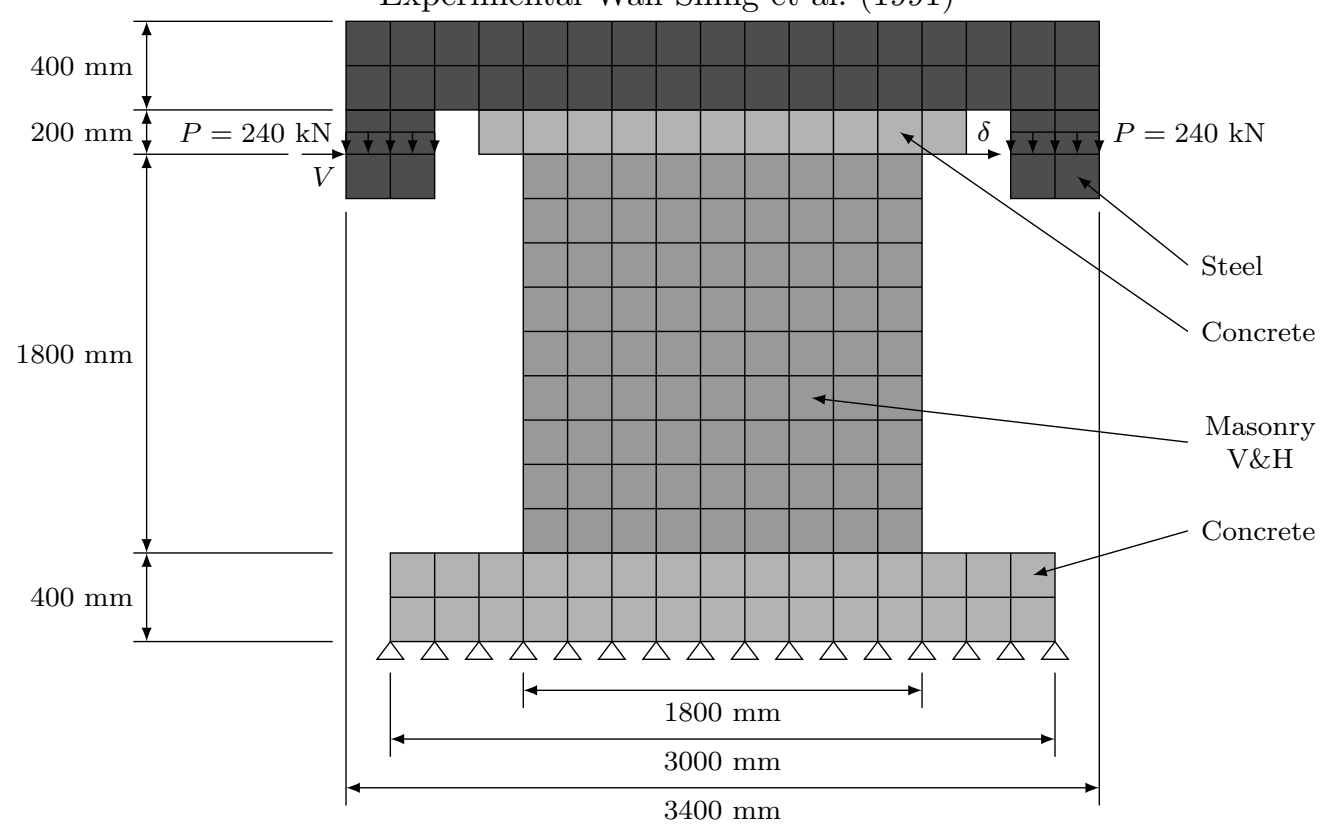

Finite Element Model Wall

HCBL 2 Shear Wall 
HCBL 2 Shear Wall Shing et al. (1991) Model Options

\begin{tabular}{ll}
\hline Model & Selected Option \\
\hline Compression Pre-Peak & Hognestad \\
Compression Post-Peak & Pre-Peak Base Curve \\
Compression Softening & Vecchio \\
Tension Post-Peak & Vecchio \\
Cracking Criterion & Mohr-Coulomb \\
Slip & Walraven Reinforced \\
\hline
\end{tabular}

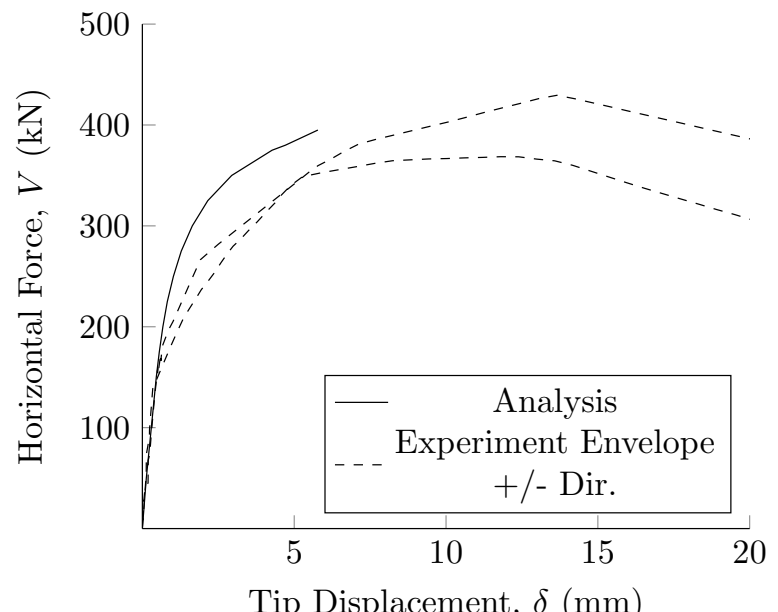

HCBL 2 Shear Wall Shing et al. (1991) Horizontal Force vs Tip Displacement 
HCBL 2 Shear Wall Shing et al. (1991) Material Properties

\begin{tabular}{|c|c|c|c|}
\hline \multirow{2}{*}{ Property } & \multicolumn{3}{|c|}{ Material } \\
\hline & Masonry V\&H & Concrete & Steel \\
\hline Thickness (mm) & 140 & 1220 & 400 \\
\hline Grout Tensile Strength (MPa) & 1.80 & 2.09 & 350 \\
\hline \multicolumn{4}{|c|}{ Masonry Properties (Y-Direction) } \\
\hline Gross Peak Compression Strength (MPa) & 20.1 & 40 & 350 \\
\hline Peak Compressive Strain & 0.0025 & 0.0021 & 0.00175 \\
\hline Tensile Strength & 0 & 2.09 & 350 \\
\hline Tensile Strain & 0 & 0 & 0.00175 \\
\hline Initial Tangent Elastic Modulus (MPa) & 0 & 34600 & 200000 \\
\hline Poisson's Ratio & 0 & 0 & 0 \\
\hline \multicolumn{4}{|c|}{ Masonry Properties (X-Direction) } \\
\hline Gross Peak Compression Strength (MPa) & 20.1 & 40 & 350 \\
\hline Peak Compressive Strain & 0.0025 & 0.0021 & 0.00175 \\
\hline Tensile Strength & 0 & 2.09 & 350 \\
\hline Tensile Strain & 0 & 0 & 0.00175 \\
\hline Initial Tangent Elastic Modulus (MPa) & 0 & 34600 & 200000 \\
\hline Poisson's Ratio & 0 & 0 & 0 \\
\hline \multicolumn{4}{|c|}{ Masonry Properties (Block) } \\
\hline Length (mm) & 390 & 2400 & 2000 \\
\hline Height (mm) & 190 & 400 & 400 \\
\hline Face Shell Thickness (mm) & 32 & 610 & 200 \\
\hline Percent Solid (\%) & 59 & 100 & 100 \\
\hline Tensile Strength & 1.75 & 2.09 & 350 \\
\hline \multicolumn{4}{|c|}{ Head Joint } \\
\hline Thickness (mm) & 10 & 10 & 10 \\
\hline Tensile Bond Strength (MPa) & 0.34 & 2.09 & 350 \\
\hline Joint Cohesion (MPa) & 6.13 & 2.09 & 350 \\
\hline Angle of Internal Friction (Deg) & 42 & 42 & 42 \\
\hline \multicolumn{4}{|c|}{ Bed Joint } \\
\hline Thickness (mm) & 10 & 10 & 10 \\
\hline Tensile Bond Strength (MPa) & 0.34 & 2.09 & 350 \\
\hline Joint Cohesion (MPa) & 6.13 & 2.09 & 350 \\
\hline Angle of Internal Friction (Deg) & 42 & 42 & 42 \\
\hline \multicolumn{4}{|c|}{ Reinforcement (Y-Direction) } \\
\hline Ratio (\%) & 0.38 & 1.38 & 100 \\
\hline Yield Strength (MPa) & 441 & 400 & 350 \\
\hline Yield Strain & 0.00221 & 0.002 & 0.00175 \\
\hline Strain Hardening Strain & 0.02 & 0.02 & 0.02 \\
\hline Ultimate Strength (MPa) & 710 & 600 & 600 \\
\hline Ultimate Strain & 0.2 & 0.2 & 0.2 \\
\hline Prestrain & 0 & 0 & 0 \\
\hline \multicolumn{4}{|c|}{ Reinforcement (X-Direction) } \\
\hline Ratio (\%) & 0.24 & 1.11 & 100 \\
\hline Yield Strength (MPa) & 462 & 400 & 350 \\
\hline Yield Strain & 0.00231 & 0.002 & 0.00175 \\
\hline Strain Hardening Strain & 0.02 & 0.02 & 0.02 \\
\hline Ultimate Strength (MPa) & 738 & 600 & 600 \\
\hline Ultimate Strain & 0.2 & 0.2 & 0.2 \\
\hline Prestrain & 0 & 0 & 0 \\
\hline
\end{tabular}

Numerical Modelling of Hollow Concrete Block Masonry Mechanics 


\section{HCBL 3 Shear Wall}

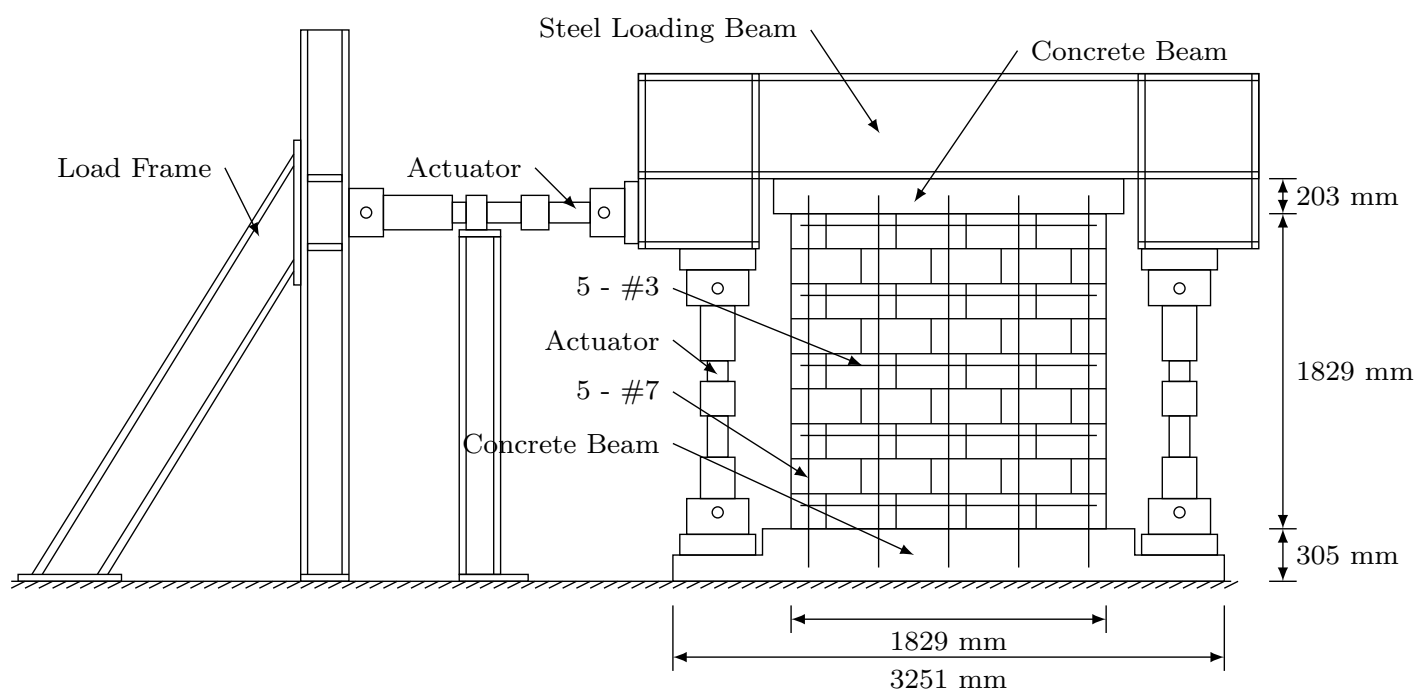

Experimental Wall Shing et al. (1991)

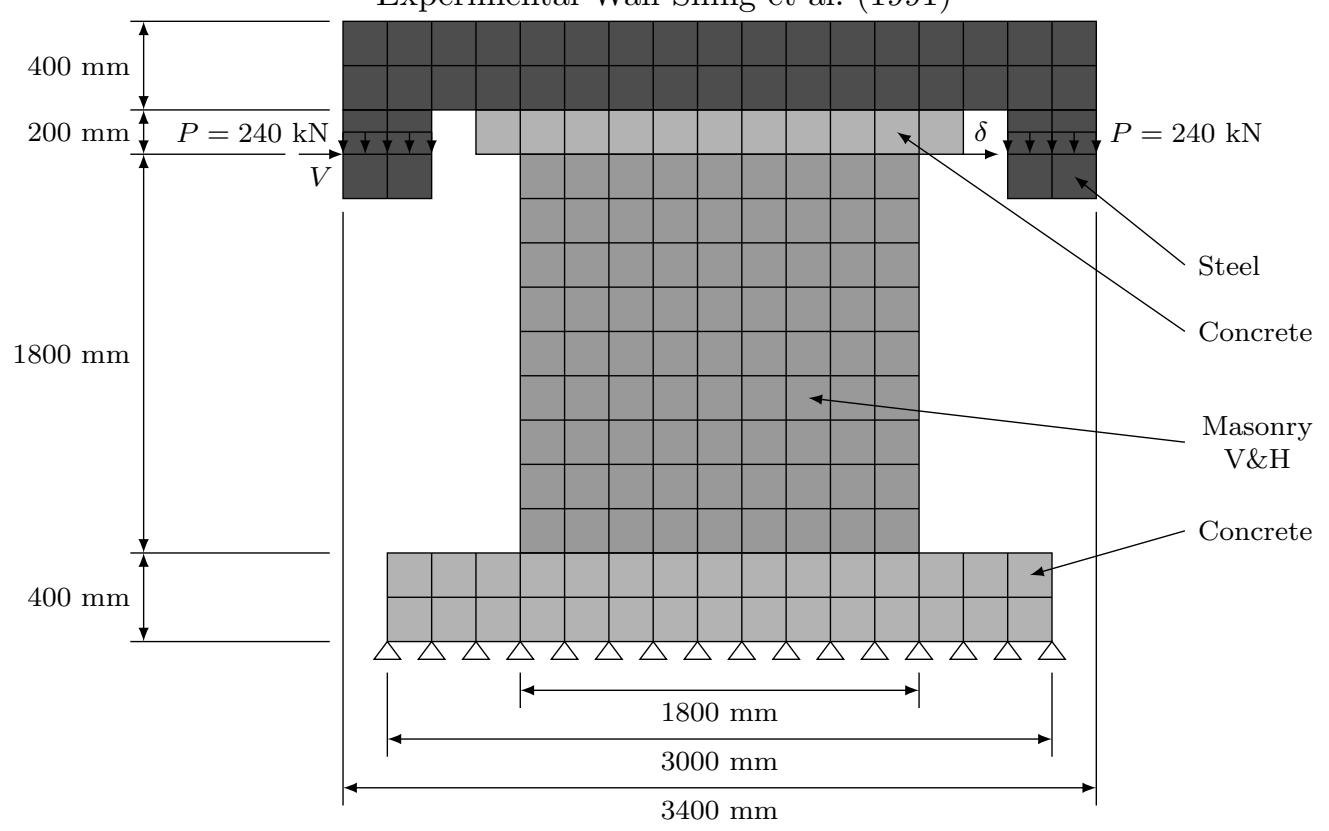

Finite Element Model Wall

HCBL 3 Shear Wall 
HCBL 3 Shear Wall Shing et al. (1991) Model Options

\begin{tabular}{ll}
\hline Model & Selected Option \\
\hline Compression Pre-Peak & Hognestad \\
Compression Post-Peak & Pre-Peak Base Curve \\
Compression Softening & Vecchio \\
Tension Post-Peak & Vecchio \\
Cracking Criterion & Mohr-Coulomb \\
Slip & Walraven Reinforced \\
\hline
\end{tabular}

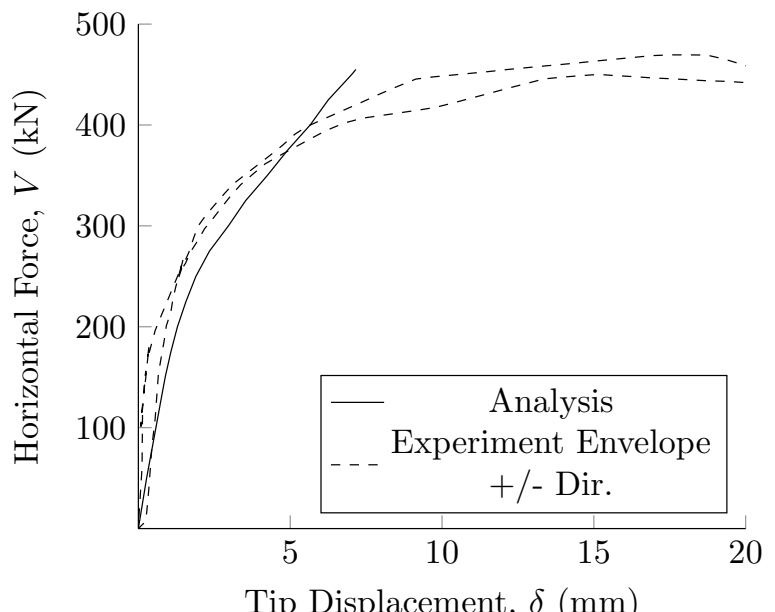

HCBL 3 Shear Wall Shing et al. (1991) Horizontal Force vs Tip Displacement 
HCBL 3 Shear Wall Shing et al. (1991) Material Properties

\begin{tabular}{|c|c|c|c|}
\hline \multirow{2}{*}{ Property } & \multicolumn{3}{|c|}{ Material } \\
\hline & Masonry V\&H & Concrete & Steel \\
\hline Thickness (mm) & 140 & 1220 & 400 \\
\hline Grout Tensile Strength (MPa) & 1.80 & 2.09 & 350 \\
\hline \multicolumn{4}{|c|}{ Masonry Properties (Y-Direction) } \\
\hline Gross Peak Compression Strength (MPa) & 20.4 & 40 & 350 \\
\hline Peak Compressive Strain & 0.0025 & 0.0021 & 0.00175 \\
\hline Tensile Strength & 0 & 2.09 & 350 \\
\hline Tensile Strain & 0 & 0 & 0.00175 \\
\hline Initial Tangent Elastic Modulus (MPa) & 7000 & 34600 & 200000 \\
\hline Poisson's Ratio & 0 & 0 & 0 \\
\hline \multicolumn{4}{|c|}{ Masonry Properties (X-Direction) } \\
\hline Gross Peak Compression Strength (MPa) & 20.4 & 40 & 350 \\
\hline Peak Compressive Strain & 0.0025 & 0.0021 & 0.00175 \\
\hline Tensile Strength & 0 & 2.09 & 350 \\
\hline Tensile Strain & 0 & 0 & 0.00175 \\
\hline Initial Tangent Elastic Modulus (MPa) & 7000 & 34600 & 200000 \\
\hline Poisson's Ratio & 0 & 0 & 0 \\
\hline \multicolumn{4}{|c|}{ Masonry Properties (Block) } \\
\hline Length (mm) & 390 & 2400 & 2000 \\
\hline Height (mm) & 190 & 400 & 400 \\
\hline Face Shell Thickness (mm) & 32 & 610 & 200 \\
\hline Percent Solid (\%) & 59 & 100 & 100 \\
\hline Tensile Strength & 1.75 & 2.09 & 350 \\
\hline \multicolumn{4}{|c|}{ Head Joint } \\
\hline Thickness (mm) & 10 & 10 & 10 \\
\hline Tensile Bond Strength (MPa) & 0.34 & 2.09 & 350 \\
\hline Joint Cohesion (MPa) & 6.13 & 2.09 & 350 \\
\hline Angle of Internal Friction (Deg) & 42 & 42 & 42 \\
\hline \multicolumn{4}{|c|}{ Bed Joint } \\
\hline Thickness (mm) & 10 & 10 & 10 \\
\hline Tensile Bond Strength (MPa) & 0.34 & 2.09 & 350 \\
\hline Joint Cohesion (MPa) & 6.13 & 2.09 & 350 \\
\hline Angle of Internal Friction (Deg) & 42 & 42 & 42 \\
\hline \multicolumn{4}{|c|}{ Reinforcement (Y-Direction) } \\
\hline Ratio (\%) & 0.74 & 1.38 & 100 \\
\hline Yield Strength (MPa) & 496 & 400 & 350 \\
\hline Yield Strain & 0.00248 & 0.002 & 0.00175 \\
\hline Strain Hardening Strain & 0.02 & 0.02 & 0.02 \\
\hline Ultimate Strength (MPa) & 710 & 600 & 600 \\
\hline Ultimate Strain & 0.2 & 0.2 & 0.2 \\
\hline Prestrain & 0 & 0 & 0 \\
\hline \multicolumn{4}{|c|}{ Reinforcement (X-Direction) } \\
\hline Ratio (\%) & 0.14 & 1.11 & 100 \\
\hline Yield Strength (MPa) & 386 & 400 & 350 \\
\hline Yield Strain & 0.00193 & 0.002 & 0.00175 \\
\hline Strain Hardening Strain & 0.02 & 0.02 & 0.02 \\
\hline Ultimate Strength (MPa) & 558 & 600 & 600 \\
\hline Ultimate Strain & 0.2 & 0.2 & 0.2 \\
\hline Prestrain & 0 & 0 & 0 \\
\hline
\end{tabular}

Numerical Modelling of Hollow Concrete Block Masonry Mechanics 


\section{HCBL 4 Shear Wall}

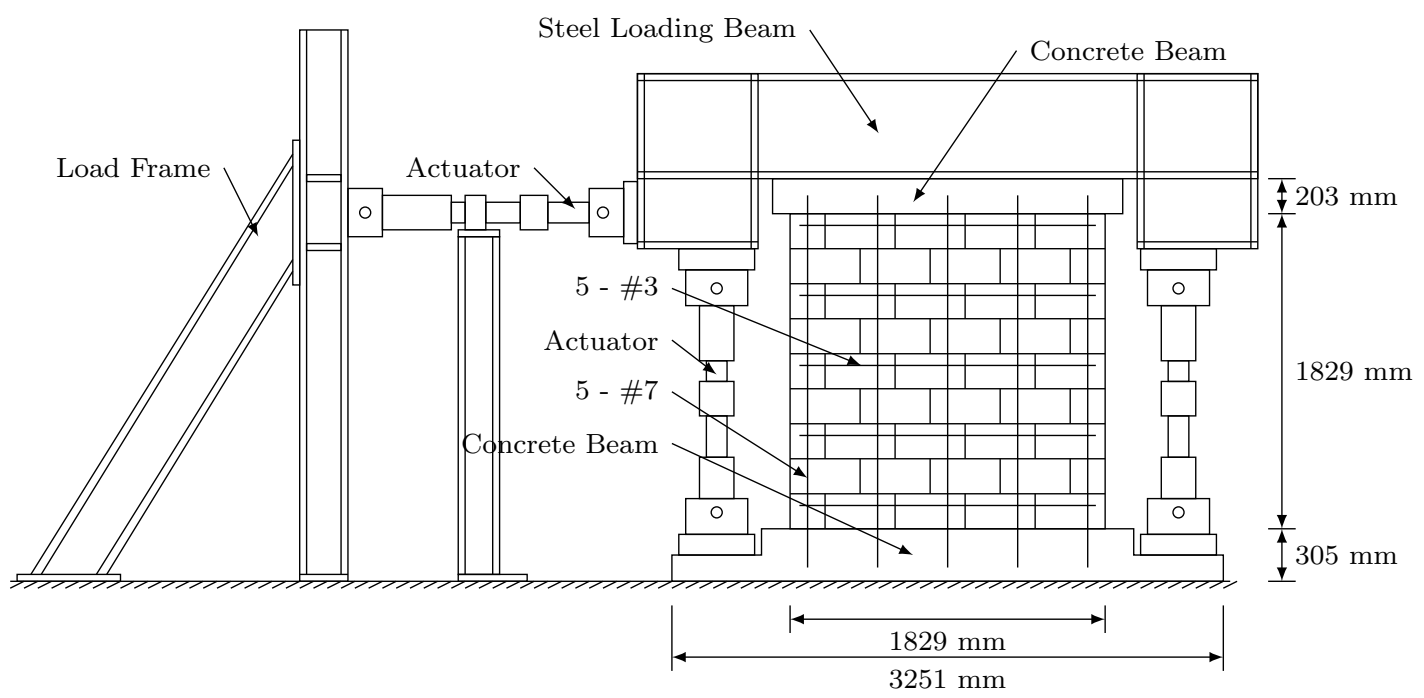

Experimental Wall Shing et al. (1991)

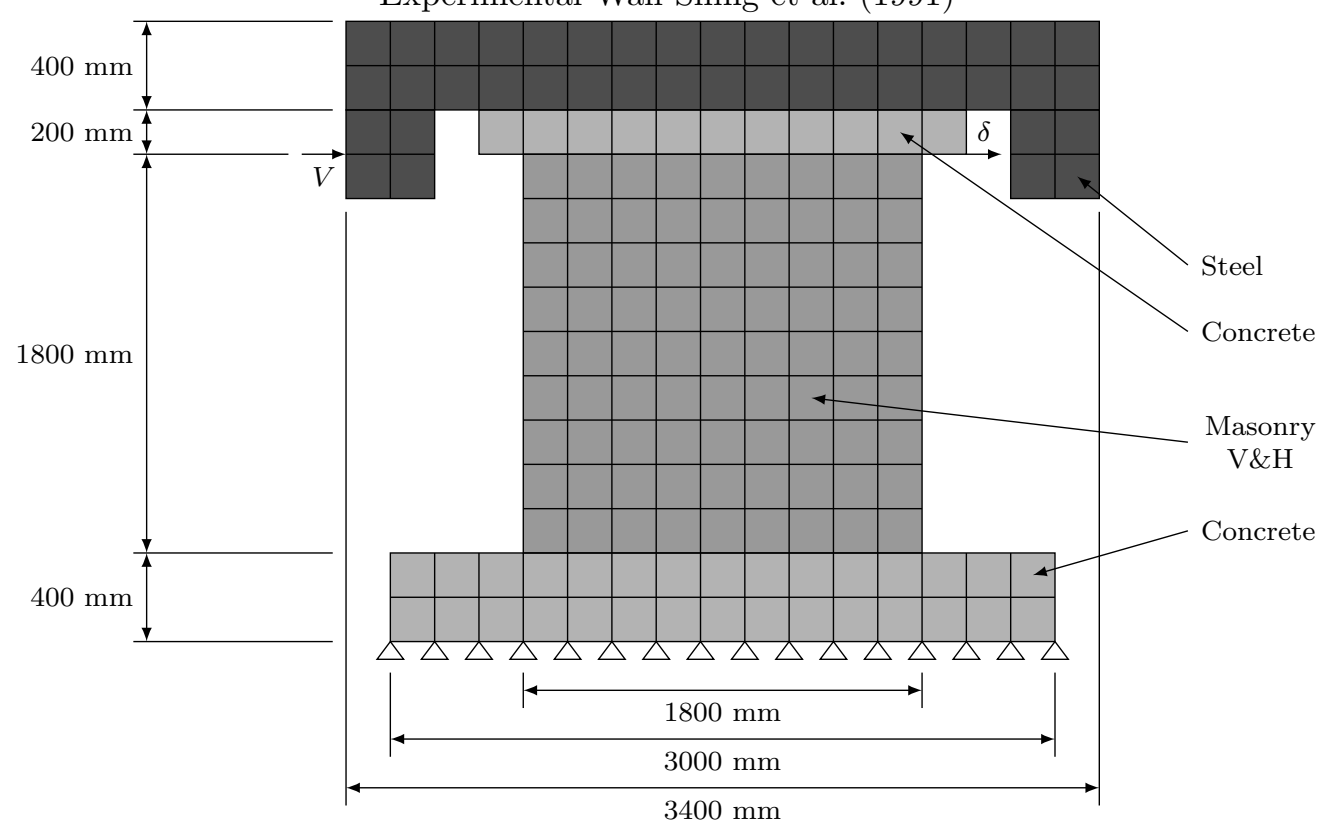

Finite Element Model Wall

HCBL 4 Shear Wall 
HCBL 4 Shear Wall Shing et al. (1991) Model Options

\begin{tabular}{ll}
\hline Model & Selected Option \\
\hline Compression Pre-Peak & Hognestad \\
Compression Post-Peak & Pre-Peak Base Curve \\
Compression Softening & Vecchio \\
Tension Post-Peak & Vecchio \\
Cracking Criterion & Mohr-Coulomb \\
Slip & Walraven Reinforced \\
\hline
\end{tabular}

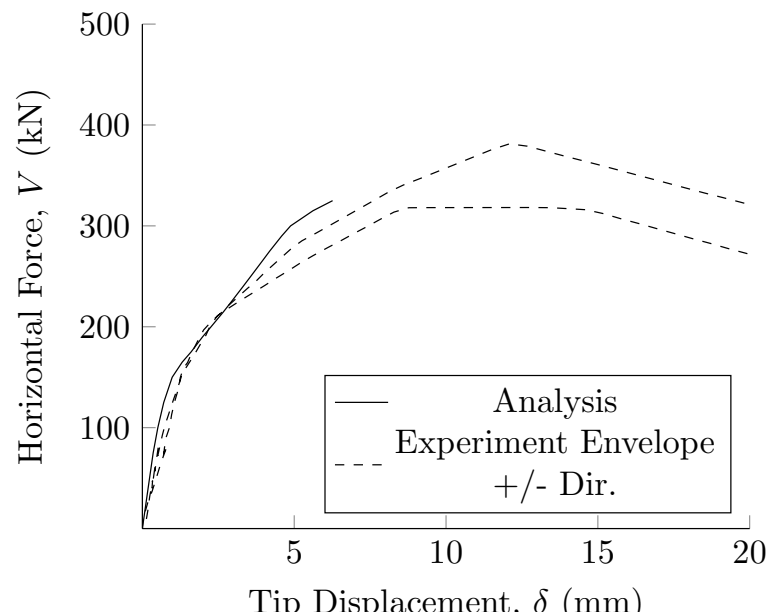

HCBL 4 Shear Wall Shing et al. (1991) Horizontal Force vs Tip Displacement 
HCBL 4 Shear Wall Shing et al. (1991) Material Properties

\begin{tabular}{|c|c|c|c|}
\hline \multirow{2}{*}{ Property } & \multicolumn{3}{|c|}{ Material } \\
\hline & Masonry V\&H & Concrete & Steel \\
\hline Thickness (mm) & 140 & 1220 & 400 \\
\hline Grout Tensile Strength (MPa) & 1.80 & 2.09 & 350 \\
\hline \multicolumn{4}{|c|}{ Masonry Properties (Y-Direction) } \\
\hline Gross Peak Compression Strength (MPa) & 18.2 & 40 & 350 \\
\hline Peak Compressive Strain & 0.0022 & 0.0021 & 0.00175 \\
\hline Tensile Strength & 0 & 2.09 & 350 \\
\hline Tensile Strain & 0 & 0 & 0.00175 \\
\hline Initial Tangent Elastic Modulus (MPa) & 9000 & 34600 & 200000 \\
\hline Poisson's Ratio & 0 & 0 & 0 \\
\hline \multicolumn{4}{|c|}{ Masonry Properties (X-Direction) } \\
\hline Gross Peak Compression Strength (MPa) & 18.2 & 40 & 350 \\
\hline Peak Compressive Strain & 0.0022 & 0.0021 & 0.00175 \\
\hline Tensile Strength & 0 & 2.09 & 350 \\
\hline Tensile Strain & 0 & 0 & 0.00175 \\
\hline Initial Tangent Elastic Modulus (MPa) & 9000 & 34600 & 200000 \\
\hline Poisson's Ratio & 0 & 0 & 0 \\
\hline \multicolumn{4}{|c|}{ Masonry Properties (Block) } \\
\hline Length (mm) & 390 & 2400 & 2000 \\
\hline Height (mm) & 190 & 400 & 400 \\
\hline Face Shell Thickness (mm) & 32 & 610 & 200 \\
\hline Percent Solid (\%) & 59 & 100 & 100 \\
\hline Tensile Strength & 1.75 & 2.09 & 350 \\
\hline \multicolumn{4}{|c|}{ Head Joint } \\
\hline Thickness (mm) & 10 & 10 & 10 \\
\hline Tensile Bond Strength (MPa) & 0.34 & 2.09 & 350 \\
\hline Joint Cohesion (MPa) & 6.13 & 2.09 & 350 \\
\hline Angle of Internal Friction (Deg) & 42 & 42 & 42 \\
\hline \multicolumn{4}{|c|}{ Bed Joint } \\
\hline Thickness (mm) & 10 & 10 & 10 \\
\hline Tensile Bond Strength (MPa) & 0.34 & 2.09 & 350 \\
\hline Joint Cohesion (MPa) & 6.13 & 2.09 & 350 \\
\hline Angle of Internal Friction (Deg) & 42 & 42 & 42 \\
\hline \multicolumn{4}{|c|}{ Reinforcement (Y-Direction) } \\
\hline Ratio (\%) & 0.74 & 1.38 & 100 \\
\hline Yield Strength (MPa) & 496 & 400 & 350 \\
\hline Yield Strain & 0.00248 & 0.002 & 0.00175 \\
\hline Strain Hardening Strain & 0.02 & 0.02 & 0.02 \\
\hline Ultimate Strength (MPa) & 710 & 600 & 600 \\
\hline Ultimate Strain & 0.2 & 0.2 & 0.2 \\
\hline Prestrain & 0 & 0 & 0 \\
\hline \multicolumn{4}{|c|}{ Reinforcement (X-Direction) } \\
\hline Ratio (\%) & 0.14 & 1.11 & 100 \\
\hline Yield Strength (MPa) & 386 & 400 & 350 \\
\hline Yield Strain & 0.00193 & 0.002 & 0.00175 \\
\hline Strain Hardening Strain & 0.02 & 0.02 & 0.02 \\
\hline Ultimate Strength (MPa) & 558 & 600 & 600 \\
\hline Ultimate Strain & 0.2 & 0.2 & 0.2 \\
\hline Prestrain & 0 & 0 & 0 \\
\hline
\end{tabular}

Numerical Modelling of Hollow Concrete Block Masonry Mechanics 


\section{HCBL 5 Shear Wall}

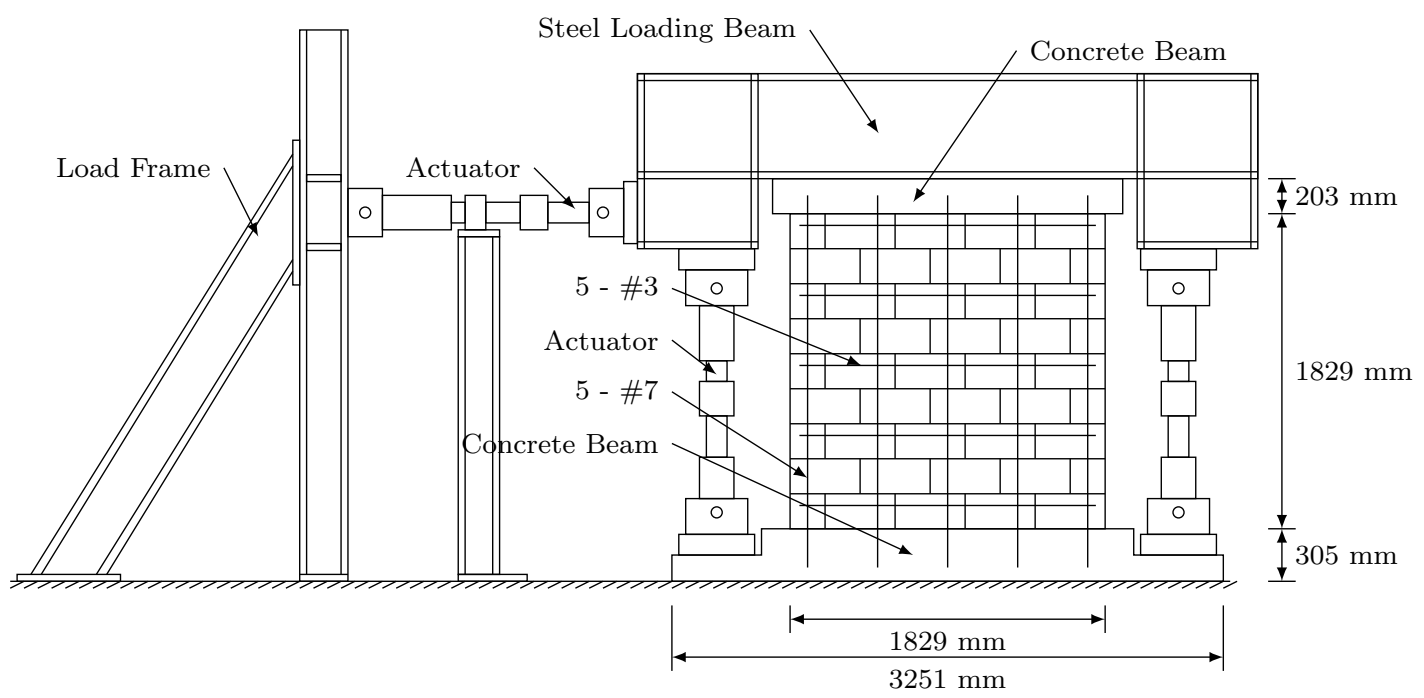

Experimental Wall Shing et al. (1991)

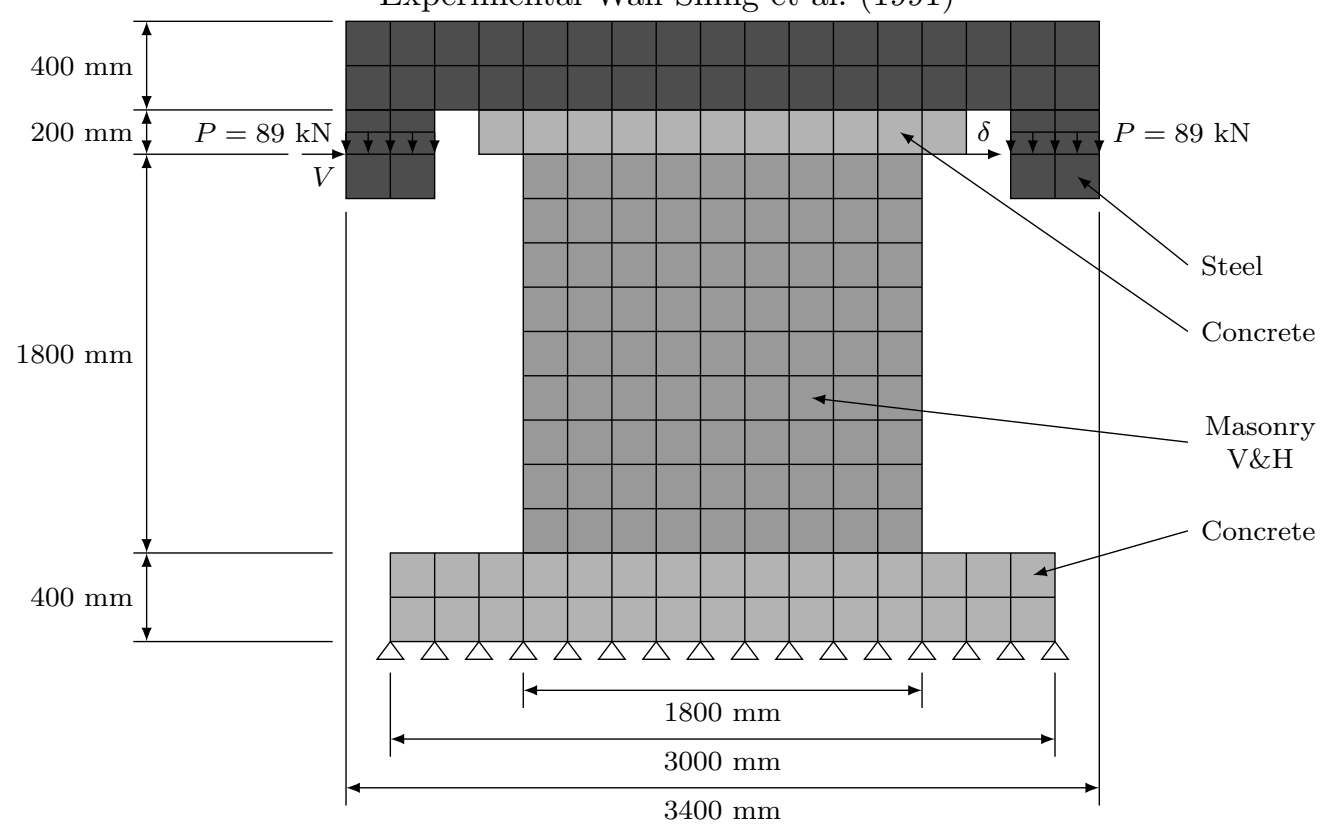

Finite Element Model Wall

HCBL 5 Shear Wall 
HCBL 5 Shear Wall Shing et al. (1991) Model Options

\begin{tabular}{ll}
\hline Model & Selected Option \\
\hline Compression Pre-Peak & Hognestad \\
Compression Post-Peak & Pre-Peak Base Curve \\
Compression Softening & Vecchio \\
Tension Post-Peak & Vecchio \\
Cracking Criterion & Mohr-Coulomb \\
Slip & Walraven Reinforced \\
\hline
\end{tabular}

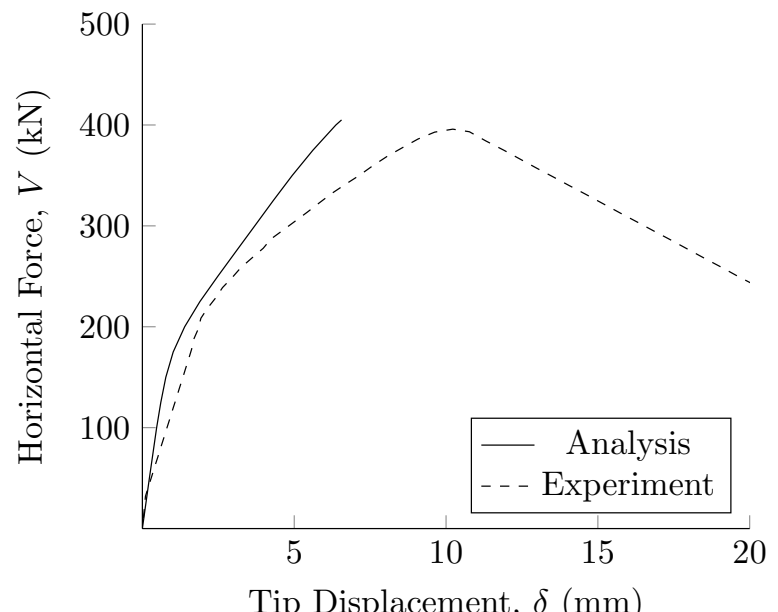

HCBL 5 Shear Wall Shing et al. (1991) Horizontal Force vs Tip Displacement 
HCBL 5 Shear Wall Shing et al. (1991) Material Properties

\begin{tabular}{|c|c|c|c|}
\hline \multirow{2}{*}{ Property } & \multicolumn{3}{|c|}{ Material } \\
\hline & Masonry V\&H & Concrete & Steel \\
\hline Thickness (mm) & 140 & 1220 & 400 \\
\hline Grout Tensile Strength (MPa) & 1.80 & 2.09 & 350 \\
\hline \multicolumn{4}{|c|}{ Masonry Properties (Y-Direction) } \\
\hline Gross Peak Compression Strength (MPa) & 18.2 & 40 & 350 \\
\hline Peak Compressive Strain & 0.0022 & 0.0021 & 0.00175 \\
\hline Tensile Strength & 0 & 2.09 & 350 \\
\hline Tensile Strain & 0 & 0 & 0.00175 \\
\hline Initial Tangent Elastic Modulus (MPa) & 9000 & 34600 & 200000 \\
\hline Poisson's Ratio & 0 & 0 & 0 \\
\hline \multicolumn{4}{|c|}{ Masonry Properties (X-Direction) } \\
\hline Gross Peak Compression Strength (MPa) & 18.2 & 40 & 350 \\
\hline Peak Compressive Strain & 0.0022 & 0.0021 & 0.00175 \\
\hline Tensile Strength & 0 & 2.09 & 350 \\
\hline Tensile Strain & 0 & 0 & 0.00175 \\
\hline Initial Tangent Elastic Modulus (MPa) & 9000 & 34600 & 200000 \\
\hline Poisson's Ratio & 0 & 0 & 0 \\
\hline \multicolumn{4}{|c|}{ Masonry Properties (Block) } \\
\hline Length (mm) & 390 & 2400 & 2000 \\
\hline Height (mm) & 190 & 400 & 400 \\
\hline Face Shell Thickness (mm) & 32 & 610 & 200 \\
\hline Percent Solid (\%) & 59 & 100 & 100 \\
\hline Tensile Strength & 1.75 & 2.09 & 350 \\
\hline \multicolumn{4}{|c|}{ Head Joint } \\
\hline Thickness (mm) & 10 & 10 & 10 \\
\hline Tensile Bond Strength (MPa) & 0.34 & 2.09 & 350 \\
\hline Joint Cohesion (MPa) & 6.13 & 2.09 & 350 \\
\hline Angle of Internal Friction (Deg) & 42 & 42 & 42 \\
\hline \multicolumn{4}{|c|}{ Bed Joint } \\
\hline Thickness (mm) & 10 & 10 & 10 \\
\hline Tensile Bond Strength (MPa) & 0.34 & 2.09 & 350 \\
\hline Joint Cohesion (MPa) & 6.13 & 2.09 & 350 \\
\hline Angle of Internal Friction (Deg) & 42 & 42 & 42 \\
\hline \multicolumn{4}{|c|}{ Reinforcement (Y-Direction) } \\
\hline Ratio (\%) & 0.74 & 1.38 & 100 \\
\hline Yield Strength (MPa) & 496 & 400 & 350 \\
\hline Yield Strain & 0.00248 & 0.002 & 0.00175 \\
\hline Strain Hardening Strain & 0.02 & 0.02 & 0.02 \\
\hline Ultimate Strength (MPa) & 710 & 600 & 600 \\
\hline Ultimate Strain & 0.2 & 0.2 & 0.2 \\
\hline Prestrain & 0 & 0 & 0 \\
\hline \multicolumn{4}{|c|}{ Reinforcement (X-Direction) } \\
\hline Ratio (\%) & 0.14 & 1.11 & 100 \\
\hline Yield Strength (MPa) & 386 & 400 & 350 \\
\hline Yield Strain & 0.00193 & 0.002 & 0.00175 \\
\hline Strain Hardening Strain & 0.02 & 0.02 & 0.02 \\
\hline Ultimate Strength (MPa) & 558 & 600 & 600 \\
\hline Ultimate Strain & 0.2 & 0.2 & 0.2 \\
\hline Prestrain & 0 & 0 & 0 \\
\hline
\end{tabular}

Numerical Modelling of Hollow Concrete Block Masonry Mechanics 


\section{HCBL 6 Shear Wall}

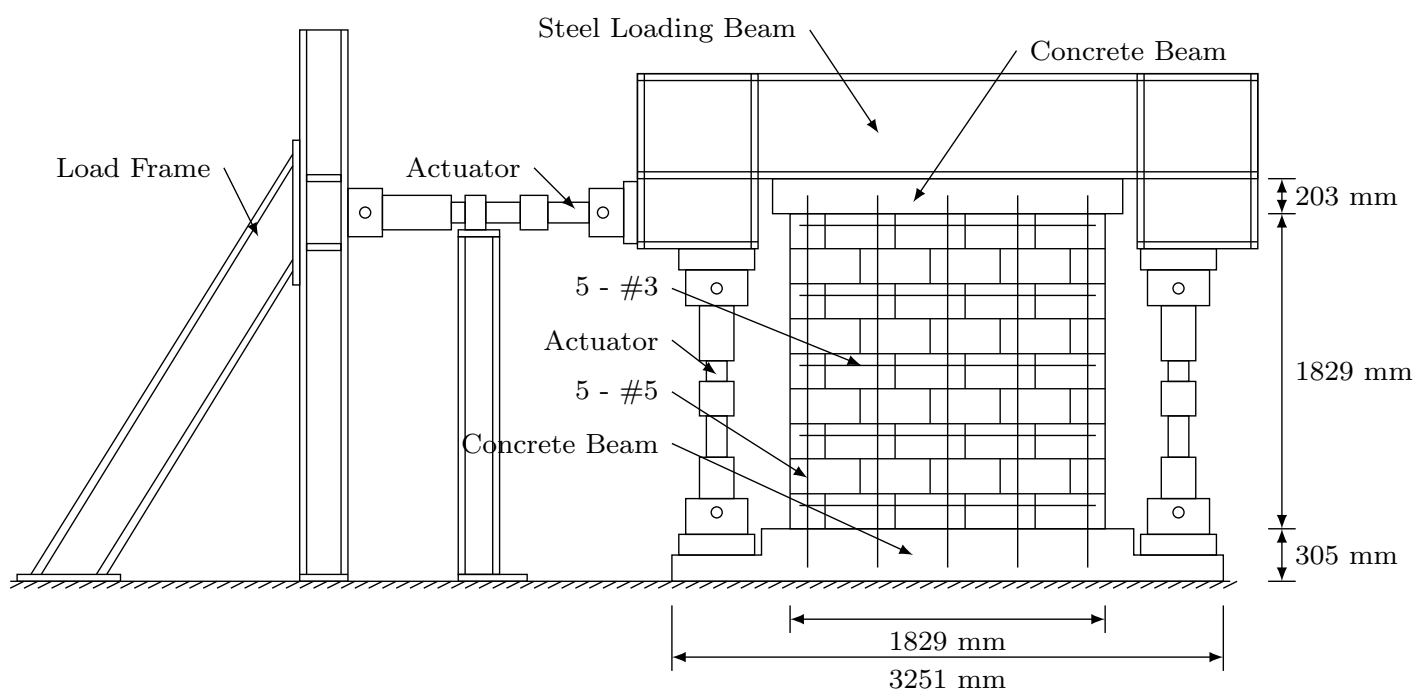

Experimental Wall Shing et al. (1991)

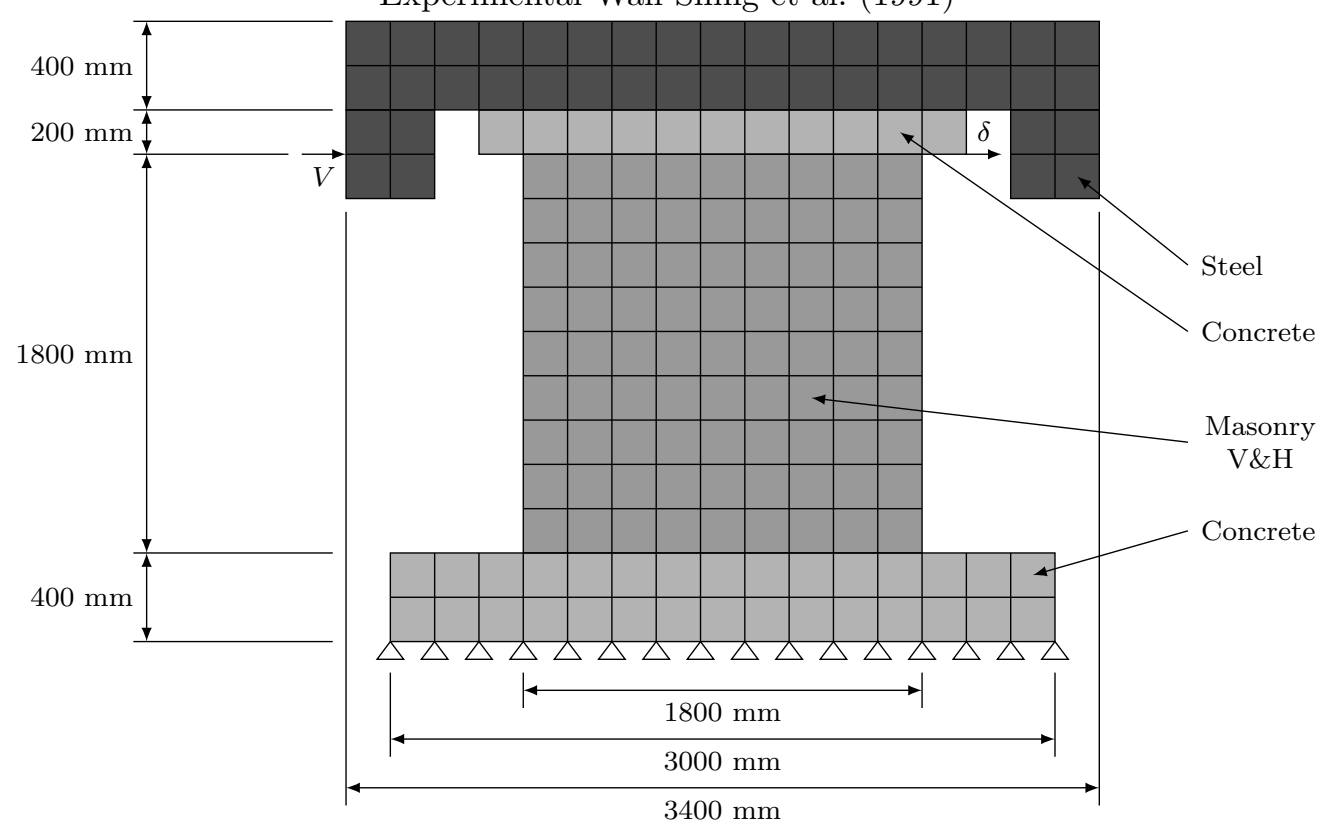

Finite Element Model Wall

HCBL 6 Shear Wall 
HCBL 6 Shear Wall Shing et al. (1991) Model Options

\begin{tabular}{ll}
\hline Model & Selected Option \\
\hline Compression Pre-Peak & Hognestad \\
Compression Post-Peak & Pre-Peak Base Curve \\
Compression Softening & Vecchio \\
Tension Post-Peak & Vecchio \\
Cracking Criterion & Mohr-Coulomb \\
Slip & Walraven Reinforced \\
\hline
\end{tabular}

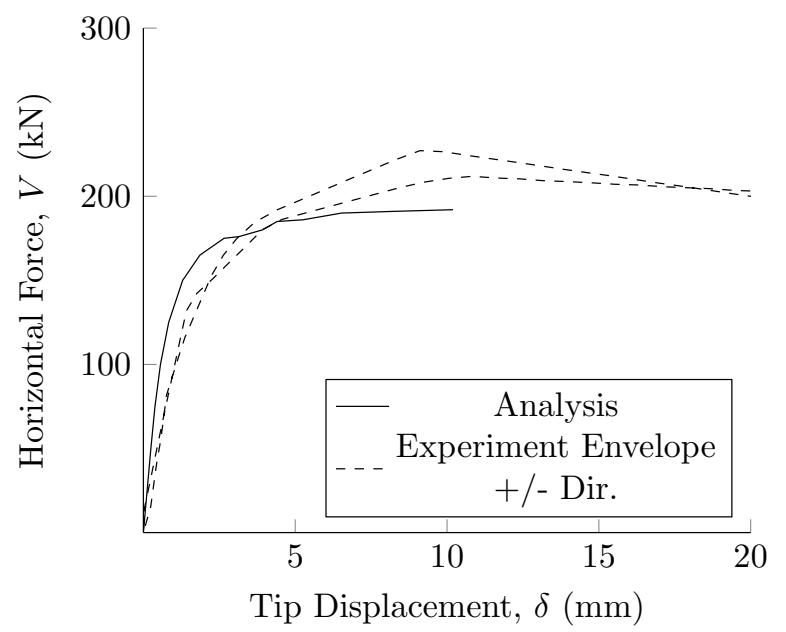

HCBL 6 Shear Wall Shing et al. (1991) Horizontal Force vs Tip Displacement 
HCBL 6 Shear Wall Shing et al. (1991) Material Properties

\begin{tabular}{|c|c|c|c|}
\hline \multirow{2}{*}{ Property } & \multicolumn{3}{|c|}{ Material } \\
\hline & Masonry V\&H & Concrete & Steel \\
\hline Thickness (mm) & 140 & 1220 & 400 \\
\hline Grout Tensile Strength (MPa) & 1.80 & 2.09 & 350 \\
\hline \multicolumn{4}{|c|}{ Masonry Properties (Y-Direction) } \\
\hline Gross Peak Compression Strength (MPa) & 18.2 & 40 & 350 \\
\hline Peak Compressive Strain & 0.0022 & 0.0021 & 0.00175 \\
\hline Tensile Strength & 0 & 2.09 & 350 \\
\hline Tensile Strain & 0 & 0 & 0.00175 \\
\hline Initial Tangent Elastic Modulus (MPa) & 9000 & 34600 & 200000 \\
\hline Poisson's Ratio & 0 & 0 & 0 \\
\hline \multicolumn{4}{|c|}{ Masonry Properties (X-Direction) } \\
\hline Gross Peak Compression Strength (MPa) & 18.2 & 40 & 350 \\
\hline Peak Compressive Strain & 0.0022 & 0.0021 & 0.00175 \\
\hline Tensile Strength & 0 & 2.09 & 350 \\
\hline Tensile Strain & 0 & 0 & 0.00175 \\
\hline Initial Tangent Elastic Modulus (MPa) & 9000 & 34600 & 200000 \\
\hline Poisson's Ratio & 0 & 0 & 0 \\
\hline \multicolumn{4}{|c|}{ Masonry Properties (Block) } \\
\hline Length (mm) & 390 & 2400 & 2000 \\
\hline Height (mm) & 190 & 400 & 400 \\
\hline Face Shell Thickness (mm) & 32 & 610 & 200 \\
\hline Percent Solid (\%) & 59 & 100 & 100 \\
\hline Tensile Strength & 1.75 & 2.09 & 350 \\
\hline \multicolumn{4}{|c|}{ Head Joint } \\
\hline Thickness (mm) & 10 & 10 & 10 \\
\hline Tensile Bond Strength (MPa) & 0.34 & 2.09 & 350 \\
\hline Joint Cohesion (MPa) & 6.13 & 2.09 & 350 \\
\hline Angle of Internal Friction (Deg) & 42 & 42 & 42 \\
\hline \multicolumn{4}{|c|}{ Bed Joint } \\
\hline Thickness (mm) & 10 & 10 & 10 \\
\hline Tensile Bond Strength (MPa) & 0.34 & 2.09 & 350 \\
\hline Joint Cohesion (MPa) & 6.13 & 2.09 & 350 \\
\hline Angle of Internal Friction (Deg) & 42 & 42 & 42 \\
\hline \multicolumn{4}{|c|}{ Reinforcement (Y-Direction) } \\
\hline Ratio (\%) & 0.38 & 1.38 & 100 \\
\hline Yield Strength (MPa) & 441 & 400 & 350 \\
\hline Yield Strain & 0.00221 & 0.002 & 0.00175 \\
\hline Strain Hardening Strain & 0.02 & 0.02 & 0.02 \\
\hline Ultimate Strength (MPa) & 710 & 600 & 600 \\
\hline Ultimate Strain & 0.2 & 0.2 & 0.2 \\
\hline Prestrain & 0 & 0 & 0 \\
\hline \multicolumn{4}{|c|}{ Reinforcement (X-Direction) } \\
\hline Ratio (\%) & 0.14 & 1.11 & 100 \\
\hline Yield Strength (MPa) & 386 & 400 & 350 \\
\hline Yield Strain & 0.00193 & 0.002 & 0.00175 \\
\hline Strain Hardening Strain & 0.02 & 0.02 & 0.02 \\
\hline Ultimate Strength (MPa) & 558 & 600 & 600 \\
\hline Ultimate Strain & 0.2 & 0.2 & 0.2 \\
\hline Prestrain & 0 & 0 & 0 \\
\hline
\end{tabular}

Numerical Modelling of Hollow Concrete Block Masonry Mechanics 


\section{HCBL 7 Shear Wall}

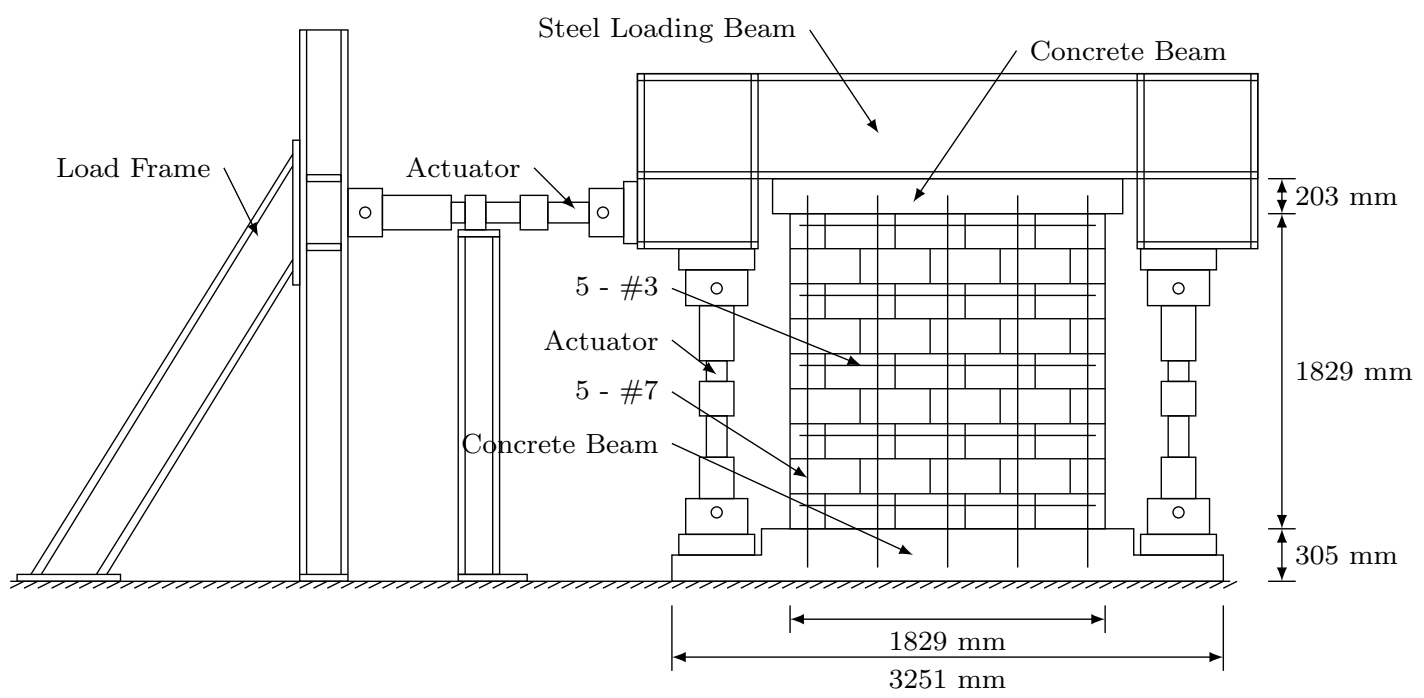

Experimental Wall Shing et al. (1991)

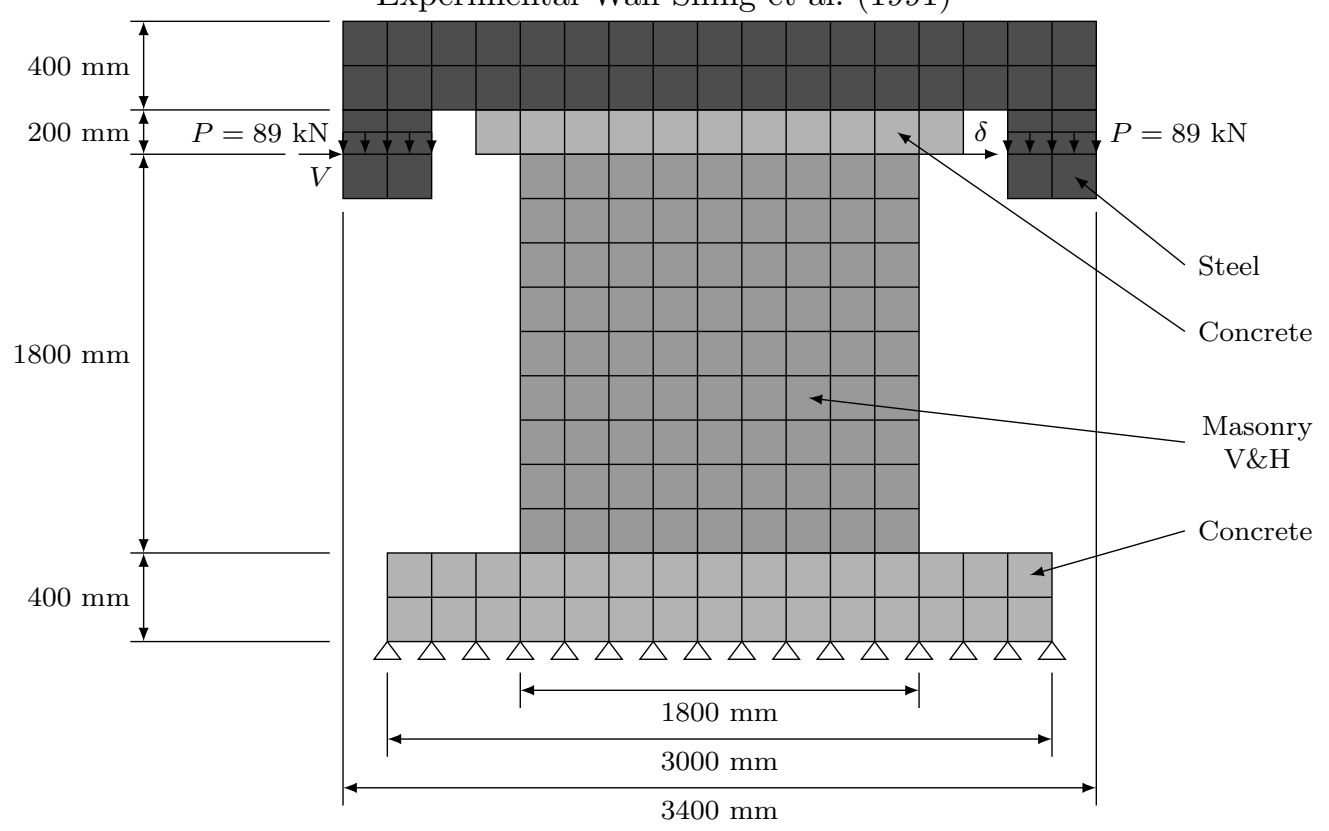

Finite Element Model Wall

HCBL 7 Shear Wall 
HCBL 7 Shear Wall Shing et al. (1991) Model Options

\begin{tabular}{ll}
\hline Model & Selected Option \\
\hline Compression Pre-Peak & Hognestad \\
Compression Post-Peak & Pre-Peak Base Curve \\
Compression Softening & Vecchio \\
Tension Post-Peak & Vecchio \\
Cracking Criterion & Mohr-Coulomb \\
Slip & Walraven Reinforced \\
\hline
\end{tabular}

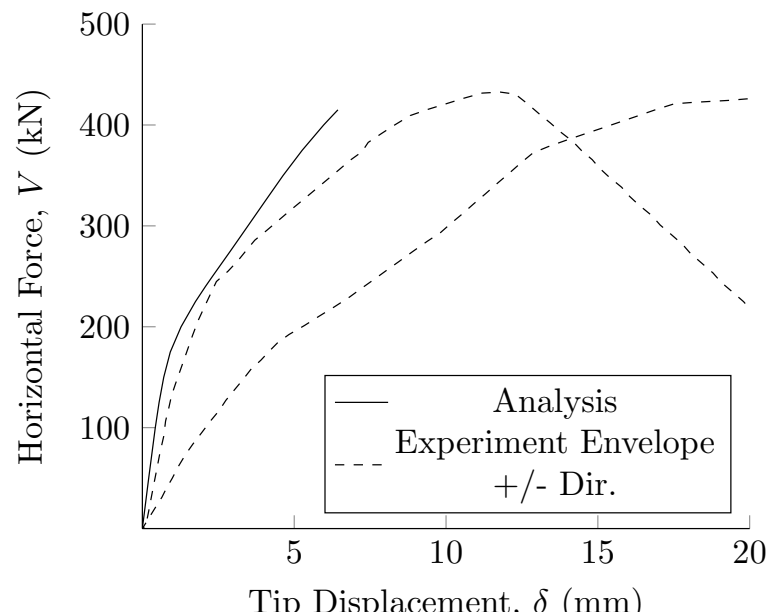

HCBL 7 Shear Wall Shing et al. (1991) Horizontal Force vs Tip Displacement 
HCBL 7 Shear Wall Shing et al. (1991) Material Properties

\begin{tabular}{|c|c|c|c|}
\hline \multirow{2}{*}{ Property } & \multicolumn{3}{|c|}{ Material } \\
\hline & Masonry V\&H & Concrete & Steel \\
\hline Thickness (mm) & 140 & 1220 & 400 \\
\hline Grout Tensile Strength (MPa) & 1.80 & 2.09 & 350 \\
\hline \multicolumn{4}{|c|}{ Masonry Properties (Y-Direction) } \\
\hline Gross Peak Compression Strength (MPa) & 22.4 & 40 & 350 \\
\hline Peak Compressive Strain & 0.0028 & 0.0021 & 0.00175 \\
\hline Tensile Strength & 0 & 2.09 & 350 \\
\hline Tensile Strain & 0 & 0 & 0.00175 \\
\hline Initial Tangent Elastic Modulus (MPa) & 10000 & 34600 & 200000 \\
\hline Poisson's Ratio & 0 & 0 & 0 \\
\hline \multicolumn{4}{|c|}{ Masonry Properties (X-Direction) } \\
\hline Gross Peak Compression Strength (MPa) & 22.4 & 40 & 350 \\
\hline Peak Compressive Strain & 0.0028 & 0.0021 & 0.00175 \\
\hline Tensile Strength & 0 & 2.09 & 350 \\
\hline Tensile Strain & 0 & 0 & 0.00175 \\
\hline Initial Tangent Elastic Modulus (MPa) & 10000 & 34600 & 200000 \\
\hline Poisson's Ratio & 0 & 0 & 0 \\
\hline \multicolumn{4}{|c|}{ Masonry Properties (Block) } \\
\hline Length (mm) & 390 & 2400 & 2000 \\
\hline Height (mm) & 190 & 400 & 400 \\
\hline Face Shell Thickness (mm) & 32 & 610 & 200 \\
\hline Percent Solid (\%) & 59 & 100 & 100 \\
\hline Tensile Strength & 1.75 & 2.09 & 350 \\
\hline \multicolumn{4}{|c|}{ Head Joint } \\
\hline Thickness (mm) & 10 & 10 & 10 \\
\hline Tensile Bond Strength (MPa) & 0.34 & 2.09 & 350 \\
\hline Joint Cohesion (MPa) & 6.13 & 2.09 & 350 \\
\hline Angle of Internal Friction (Deg) & 42 & 42 & 42 \\
\hline \multicolumn{4}{|c|}{ Bed Joint } \\
\hline Thickness (mm) & 10 & 10 & 10 \\
\hline Tensile Bond Strength (MPa) & 0.34 & 2.09 & 350 \\
\hline Joint Cohesion (MPa) & 6.13 & 2.09 & 350 \\
\hline Angle of Internal Friction (Deg) & 42 & 42 & 42 \\
\hline \multicolumn{4}{|c|}{ Reinforcement (Y-Direction) } \\
\hline Ratio (\%) & 0.74 & 1.38 & 100 \\
\hline Yield Strength (MPa) & 496 & 400 & 350 \\
\hline Yield Strain & 0.00248 & 0.002 & 0.00175 \\
\hline Strain Hardening Strain & 0.02 & 0.02 & 0.02 \\
\hline Ultimate Strength (MPa) & 710 & 600 & 600 \\
\hline Ultimate Strain & 0.2 & 0.2 & 0.2 \\
\hline Prestrain & 0 & 0 & 0 \\
\hline \multicolumn{4}{|c|}{ Reinforcement (X-Direction) } \\
\hline Ratio (\%) & 0.14 & 1.11 & 100 \\
\hline Yield Strength (MPa) & 386 & 400 & 350 \\
\hline Yield Strain & 0.00193 & 0.002 & 0.00175 \\
\hline Strain Hardening Strain & 0.02 & 0.02 & 0.02 \\
\hline Ultimate Strength (MPa) & 558 & 600 & 600 \\
\hline Ultimate Strain & 0.2 & 0.2 & 0.2 \\
\hline Prestrain & 0 & 0 & 0 \\
\hline
\end{tabular}

Numerical Modelling of Hollow Concrete Block Masonry Mechanics 


\section{HCBL 8 Shear Wall}

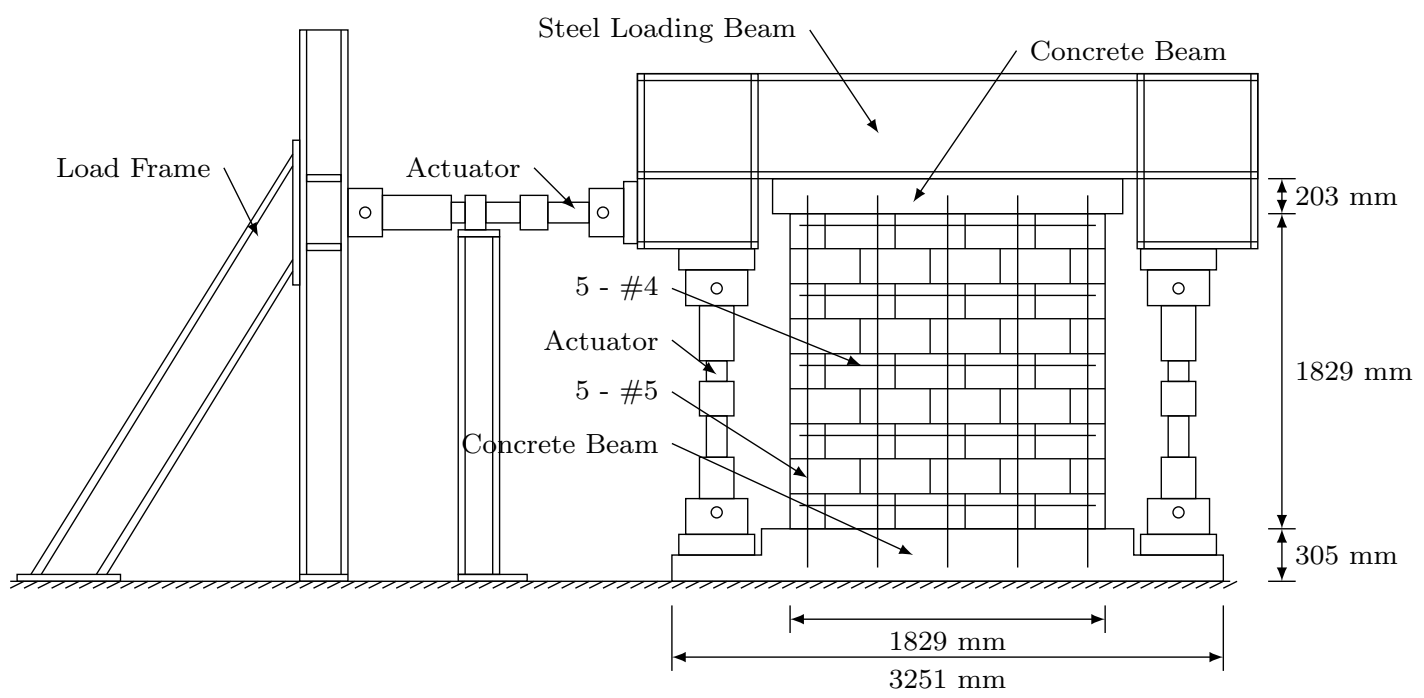

Experimental Wall Shing et al. (1991)

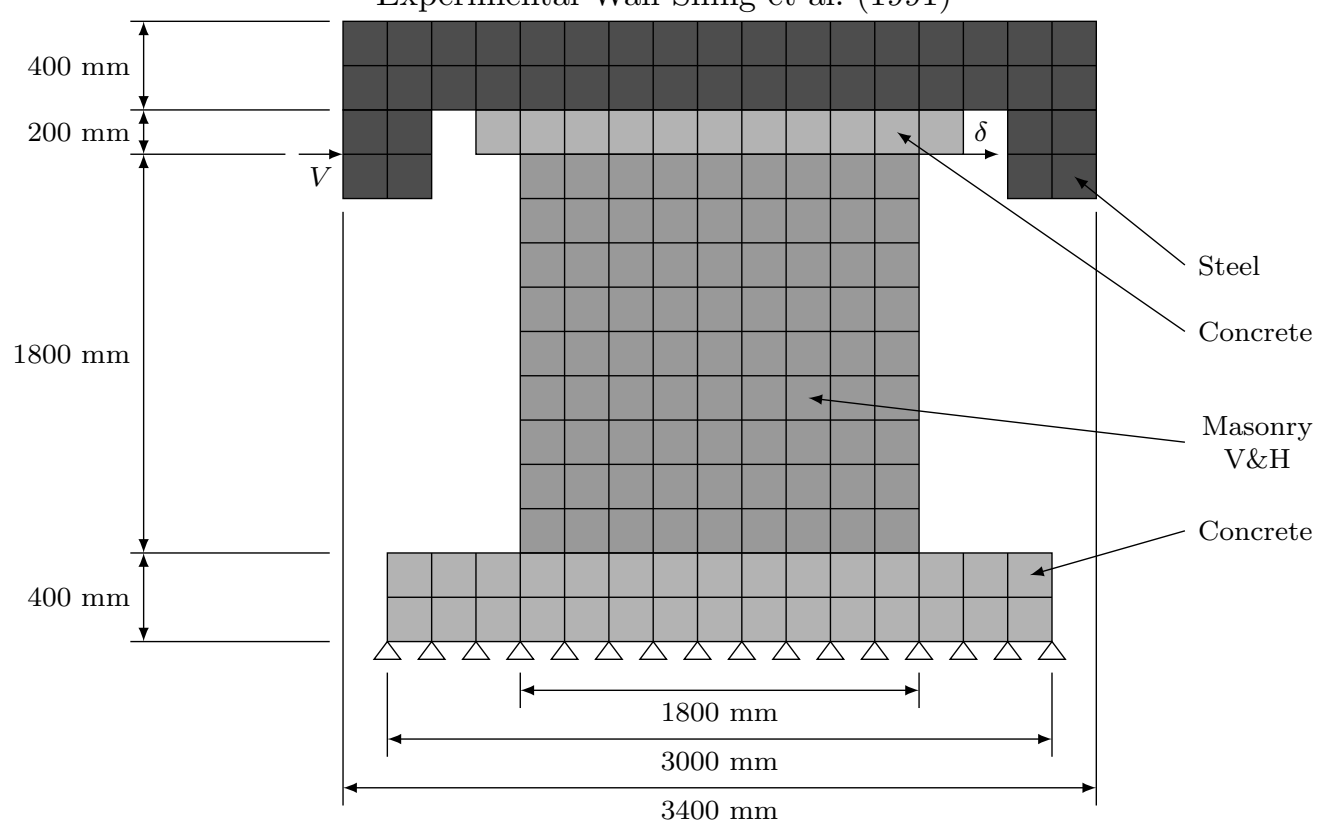

Finite Element Model Wall

HCBL 8 Shear Wall 
HCBL 8 Shear Wall Shing et al. (1991) Model Options

\begin{tabular}{ll}
\hline Model & Selected Option \\
\hline Compression Pre-Peak & Hognestad \\
Compression Post-Peak & Pre-Peak Base Curve \\
Compression Softening & Vecchio \\
Tension Post-Peak & Vecchio \\
Cracking Criterion & Mohr-Coulomb \\
Slip & Walraven Reinforced \\
\hline
\end{tabular}

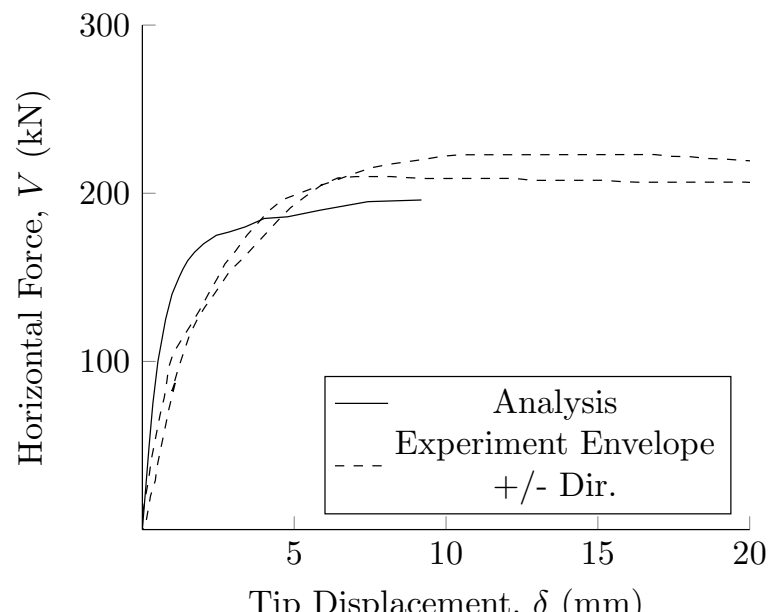

HCBL 8 Shear Wall Shing et al. (1991) Horizontal Force vs Tip Displacement 
HCBL 8 Shear Wall Shing et al. (1991) Material Properties

\begin{tabular}{|c|c|c|c|}
\hline \multirow{2}{*}{ Property } & \multicolumn{3}{|c|}{ Material } \\
\hline & Masonry V\&H & Concrete & Steel \\
\hline Thickness (mm) & 140 & 1220 & 400 \\
\hline Grout Tensile Strength (MPa) & 1.80 & 2.09 & 350 \\
\hline \multicolumn{4}{|c|}{ Masonry Properties (Y-Direction) } \\
\hline Gross Peak Compression Strength (MPa) & 22.4 & 40 & 350 \\
\hline Peak Compressive Strain & 0.0028 & 0.0021 & 0.00175 \\
\hline Tensile Strength & 0 & 2.09 & 350 \\
\hline Tensile Strain & 0 & 0 & 0.00175 \\
\hline Initial Tangent Elastic Modulus (MPa) & 10000 & 34600 & 200000 \\
\hline Poisson's Ratio & 0 & 0 & 0 \\
\hline \multicolumn{4}{|c|}{ Masonry Properties (X-Direction) } \\
\hline Gross Peak Compression Strength (MPa) & 22.4 & 40 & 350 \\
\hline Peak Compressive Strain & 0.0028 & 0.0021 & 0.00175 \\
\hline Tensile Strength & 0 & 2.09 & 350 \\
\hline Tensile Strain & 0 & 0 & 0.00175 \\
\hline Initial Tangent Elastic Modulus (MPa) & 10000 & 34600 & 200000 \\
\hline Poisson's Ratio & 0 & 0 & 0 \\
\hline \multicolumn{4}{|c|}{ Masonry Properties (Block) } \\
\hline Length (mm) & 390 & 2400 & 2000 \\
\hline Height (mm) & 190 & 400 & 400 \\
\hline Face Shell Thickness (mm) & 32 & 610 & 200 \\
\hline Percent Solid (\%) & 59 & 100 & 100 \\
\hline Tensile Strength & 1.75 & 2.09 & 350 \\
\hline \multicolumn{4}{|c|}{ Head Joint } \\
\hline Thickness (mm) & 10 & 10 & 10 \\
\hline Tensile Bond Strength (MPa) & 0.34 & 2.09 & 350 \\
\hline Joint Cohesion (MPa) & 6.13 & 2.09 & 350 \\
\hline Angle of Internal Friction (Deg) & 42 & 42 & 42 \\
\hline \multicolumn{4}{|c|}{ Bed Joint } \\
\hline Thickness (mm) & 10 & 10 & 10 \\
\hline Tensile Bond Strength (MPa) & 0.34 & 2.09 & 350 \\
\hline Joint Cohesion (MPa) & 6.13 & 2.09 & 350 \\
\hline Angle of Internal Friction (Deg) & 42 & 42 & 42 \\
\hline \multicolumn{4}{|c|}{ Reinforcement (Y-Direction) } \\
\hline Ratio (\%) & 0.38 & 1.38 & 100 \\
\hline Yield Strength (MPa) & 441 & 400 & 350 \\
\hline Yield Strain & 0.00221 & 0.002 & 0.00175 \\
\hline Strain Hardening Strain & 0.02 & 0.02 & 0.02 \\
\hline Ultimate Strength (MPa) & 710 & 600 & 600 \\
\hline Ultimate Strain & 0.2 & 0.2 & 0.2 \\
\hline Prestrain & 0 & 0 & 0 \\
\hline \multicolumn{4}{|c|}{ Reinforcement (X-Direction) } \\
\hline Ratio (\%) & 0.24 & 1.11 & 100 \\
\hline Yield Strength (MPa) & 462 & 400 & 350 \\
\hline Yield Strain & 0.00231 & 0.002 & 0.00175 \\
\hline Strain Hardening Strain & 0.02 & 0.02 & 0.02 \\
\hline Ultimate Strength (MPa) & 738 & 600 & 600 \\
\hline Ultimate Strain & 0.2 & 0.2 & 0.2 \\
\hline Prestrain & 0 & 0 & 0 \\
\hline
\end{tabular}

Numerical Modelling of Hollow Concrete Block Masonry Mechanics 


\section{HCBL 9 Shear Wall}

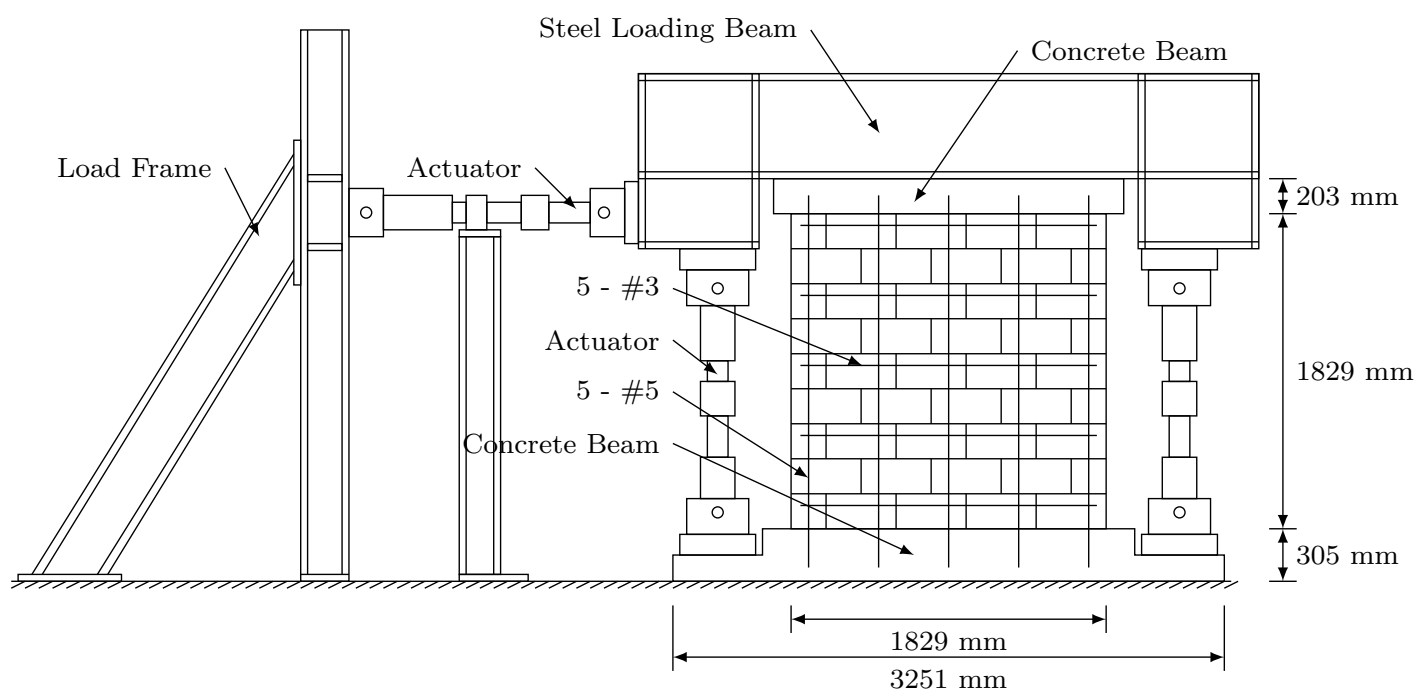

Experimental Wall Shing et al. (1991)

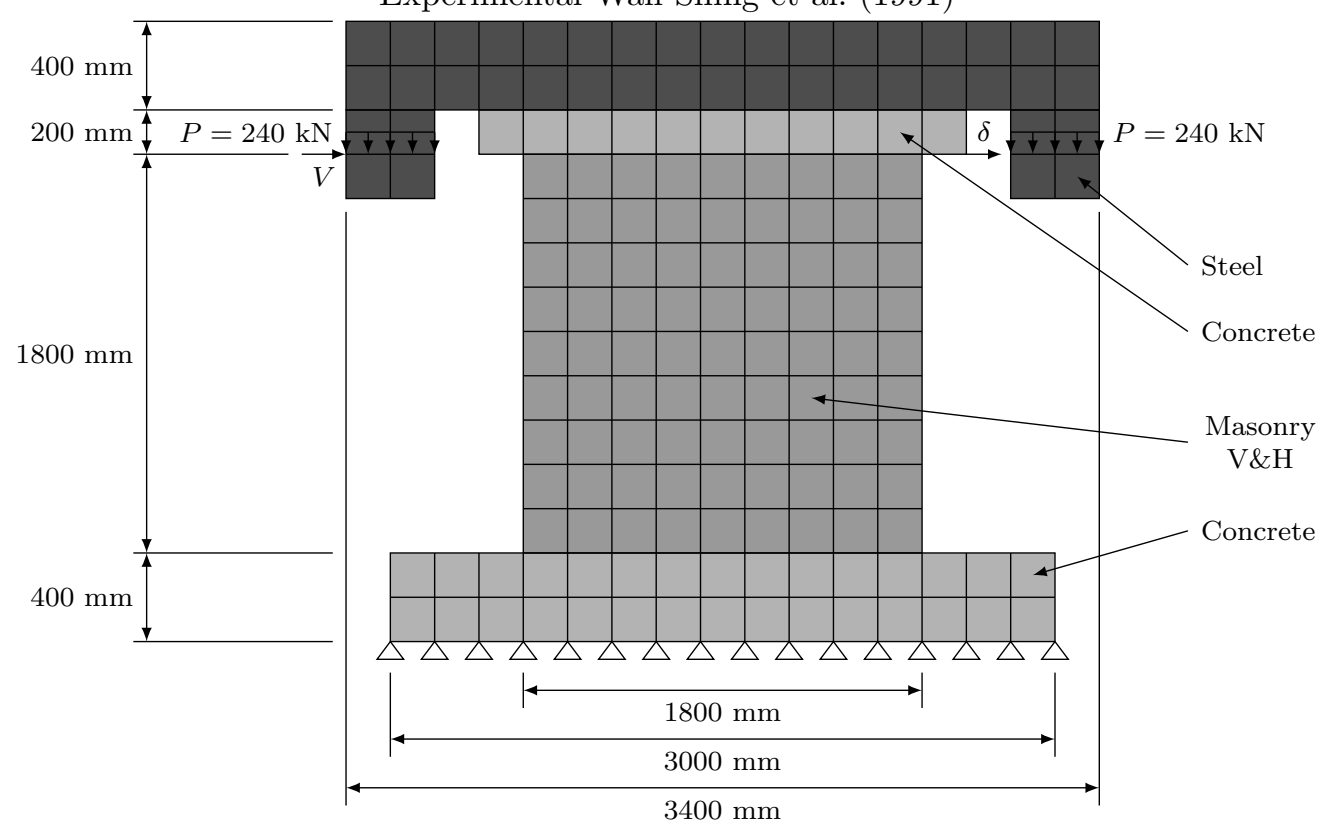

Finite Element Model Wall

HCBL 9 Shear Wall 
HCBL 9 Shear Wall Shing et al. (1991) Model Options

\begin{tabular}{ll}
\hline Model & Selected Option \\
\hline Compression Pre-Peak & Hognestad \\
Compression Post-Peak & Pre-Peak Base Curve \\
Compression Softening & Vecchio \\
Tension Post-Peak & Vecchio \\
Cracking Criterion & Mohr-Coulomb \\
Slip & Walraven Reinforced \\
\hline
\end{tabular}

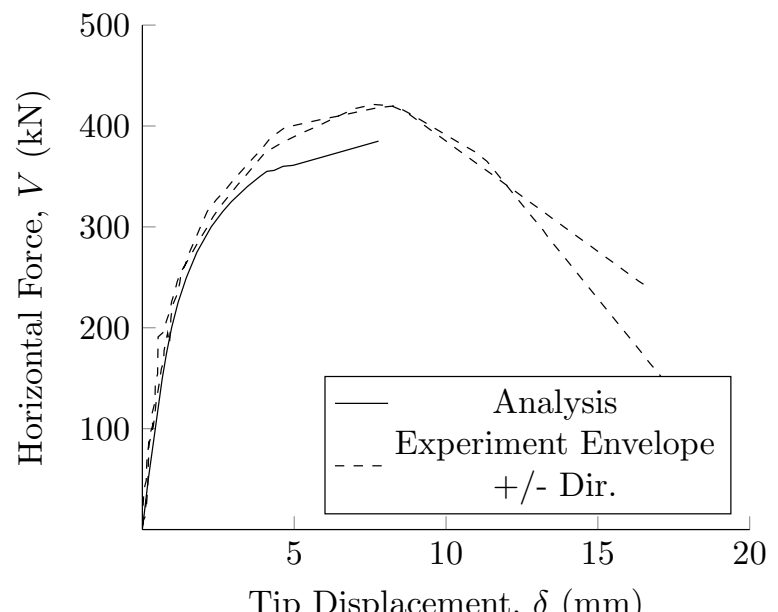

HCBL 9 Shear Wall Shing et al. (1991) Horizontal Force vs Tip Displacement 
HCBL 9 Shear Wall Shing et al. (1991) Material Properties

\begin{tabular}{|c|c|c|c|}
\hline \multirow{2}{*}{ Property } & \multicolumn{3}{|c|}{ Material } \\
\hline & Masonry V\&H & Concrete & Steel \\
\hline Thickness (mm) & 140 & 1220 & 400 \\
\hline Grout Tensile Strength (MPa) & 1.80 & 2.09 & 350 \\
\hline \multicolumn{4}{|c|}{ Masonry Properties (Y-Direction) } \\
\hline Gross Peak Compression Strength (MPa) & 22.4 & 40 & 350 \\
\hline Peak Compressive Strain & 0.0028 & 0.0021 & 0.00175 \\
\hline Tensile Strength & 0 & 2.09 & 350 \\
\hline Tensile Strain & 0 & 0 & 0.00175 \\
\hline Initial Tangent Elastic Modulus (MPa) & 10000 & 34600 & 200000 \\
\hline Poisson's Ratio & 0 & 0 & 0 \\
\hline \multicolumn{4}{|c|}{ Masonry Properties (X-Direction) } \\
\hline Gross Peak Compression Strength (MPa) & 22.4 & 40 & 350 \\
\hline Peak Compressive Strain & 0.0028 & 0.0021 & 0.00175 \\
\hline Tensile Strength & 0 & 2.09 & 350 \\
\hline Tensile Strain & 0 & 0 & 0.00175 \\
\hline Initial Tangent Elastic Modulus (MPa) & 10000 & 34600 & 200000 \\
\hline Poisson's Ratio & 0 & 0 & 0 \\
\hline \multicolumn{4}{|c|}{ Masonry Properties (Block) } \\
\hline Length (mm) & 390 & 2400 & 2000 \\
\hline Height (mm) & 190 & 400 & 400 \\
\hline Face Shell Thickness (mm) & 32 & 610 & 200 \\
\hline Percent Solid (\%) & 59 & 100 & 100 \\
\hline Tensile Strength & 1.75 & 2.09 & 350 \\
\hline \multicolumn{4}{|c|}{ Head Joint } \\
\hline Thickness (mm) & 10 & 10 & 10 \\
\hline Tensile Bond Strength (MPa) & 0.34 & 2.09 & 350 \\
\hline Joint Cohesion (MPa) & 6.13 & 2.09 & 350 \\
\hline Angle of Internal Friction (Deg) & 42 & 42 & 42 \\
\hline \multicolumn{4}{|c|}{ Bed Joint } \\
\hline Thickness (mm) & 10 & 10 & 10 \\
\hline Tensile Bond Strength (MPa) & 0.34 & 2.09 & 350 \\
\hline Joint Cohesion (MPa) & 6.13 & 2.09 & 350 \\
\hline Angle of Internal Friction (Deg) & 42 & 42 & 42 \\
\hline \multicolumn{4}{|c|}{ Reinforcement (Y-Direction) } \\
\hline Ratio (\%) & 0.38 & 1.38 & 100 \\
\hline Yield Strength (MPa) & 441 & 400 & 350 \\
\hline Yield Strain & 0.00221 & 0.002 & 0.00175 \\
\hline Strain Hardening Strain & 0.02 & 0.02 & 0.02 \\
\hline Ultimate Strength (MPa) & 710 & 600 & 600 \\
\hline Ultimate Strain & 0.2 & 0.2 & 0.2 \\
\hline Prestrain & 0 & 0 & 0 \\
\hline \multicolumn{4}{|c|}{ Reinforcement (X-Direction) } \\
\hline Ratio (\%) & 0.14 & 1.11 & 100 \\
\hline Yield Strength (MPa) & 386 & 400 & 350 \\
\hline Yield Strain & 0.00193 & 0.002 & 0.00175 \\
\hline Strain Hardening Strain & 0.02 & 0.02 & 0.02 \\
\hline Ultimate Strength (MPa) & 558 & 600 & 600 \\
\hline Ultimate Strain & 0.2 & 0.2 & 0.2 \\
\hline Prestrain & 0 & 0 & 0 \\
\hline
\end{tabular}

Numerical Modelling of Hollow Concrete Block Masonry Mechanics 


\section{HCBL 10 Shear Wall}

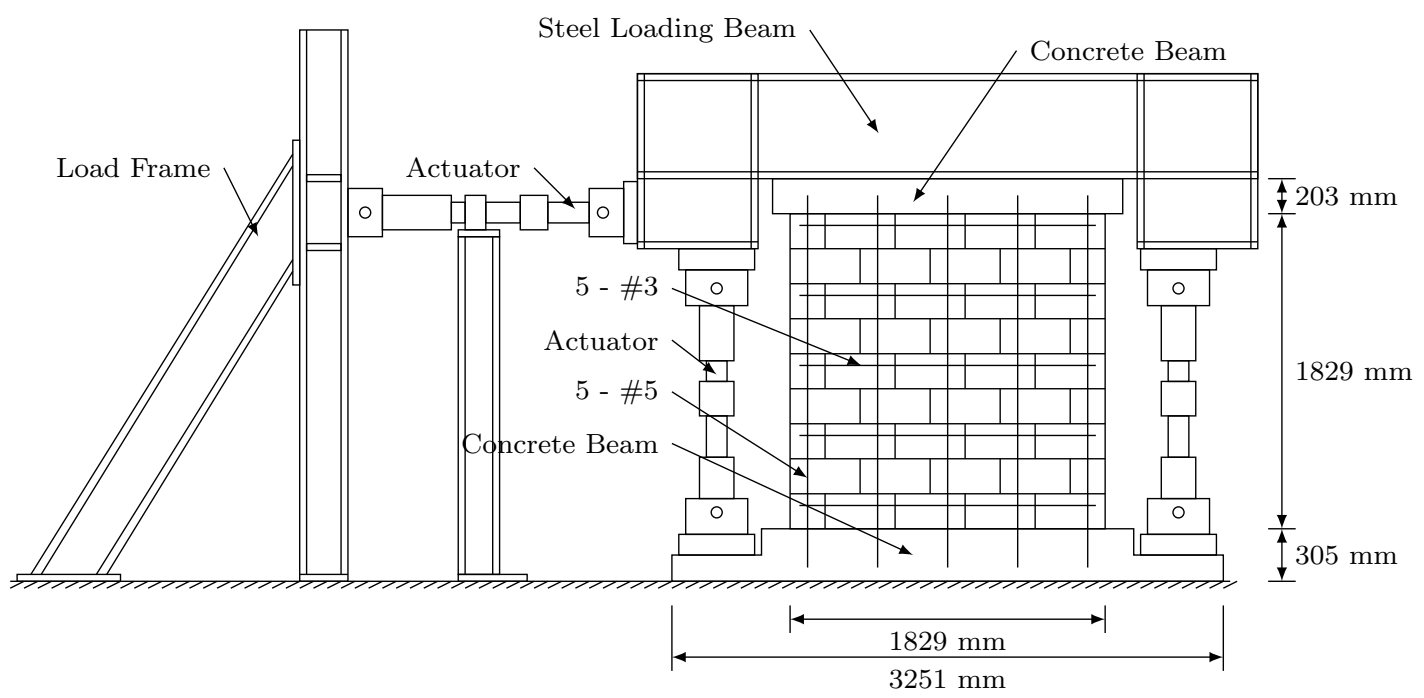

Experimental Wall Shing et al. (1991)

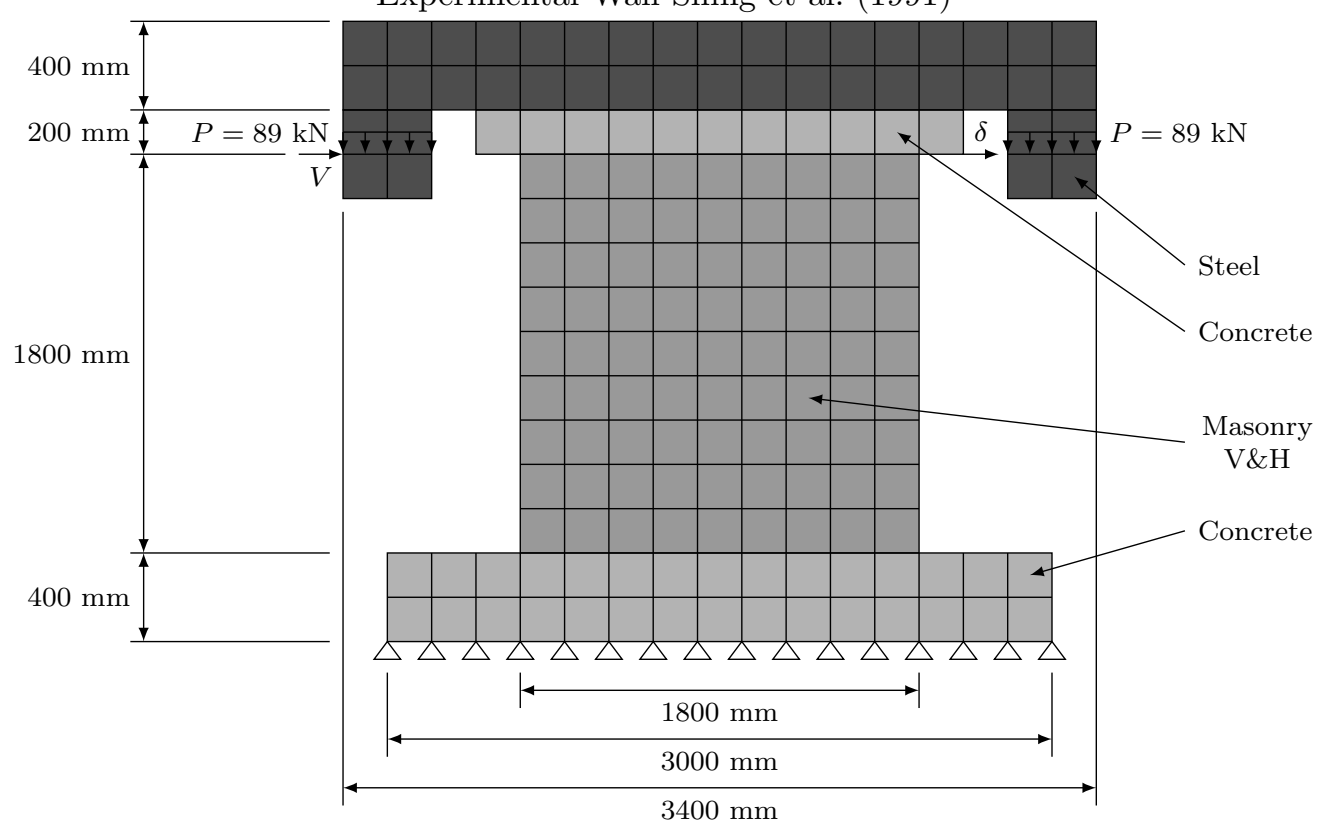

Finite Element Model Wall

HCBL 10 Shear Wall 
HCBL 10 Shear Wall Shing et al. (1991) Model Options

\begin{tabular}{ll}
\hline Model & Selected Option \\
\hline Compression Pre-Peak & Hognestad \\
Compression Post-Peak & Pre-Peak Base Curve \\
Compression Softening & Vecchio \\
Tension Post-Peak & Vecchio \\
Cracking Criterion & Mohr-Coulomb \\
Slip & Walraven Reinforced \\
\hline
\end{tabular}

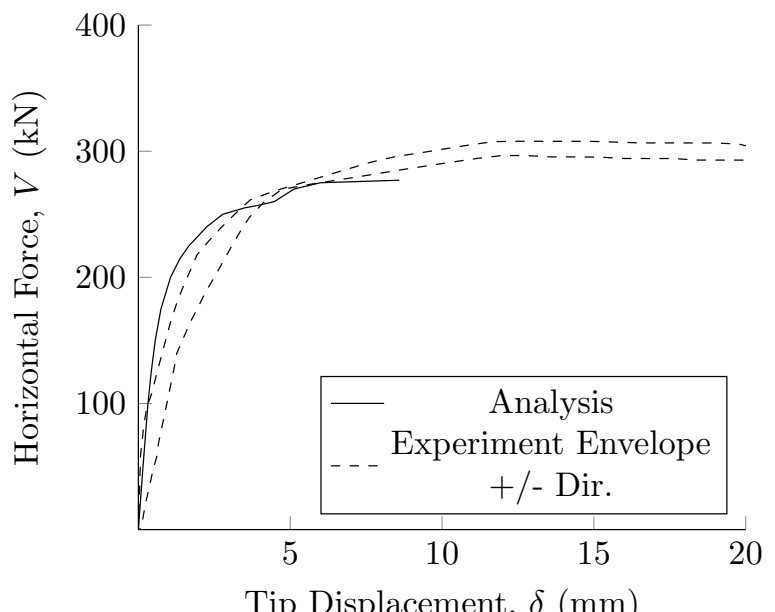

HCBL 10 Shear Wall Shing et al. (1991) Horizontal Force vs Tip Displacement 
HCBL 10 Shear Wall Shing et al. (1991) Material Properties

\begin{tabular}{|c|c|c|c|}
\hline \multirow{2}{*}{ Property } & \multicolumn{3}{|c|}{ Material } \\
\hline & Masonry V\&H & Concrete & Steel \\
\hline Thickness (mm) & 140 & 1220 & 400 \\
\hline Grout Tensile Strength (MPa) & 1.80 & 2.09 & 350 \\
\hline \multicolumn{4}{|c|}{ Masonry Properties (Y-Direction) } \\
\hline Gross Peak Compression Strength (MPa) & 22.3 & 40 & 350 \\
\hline Peak Compressive Strain & 0.0028 & 0.0021 & 0.00175 \\
\hline Tensile Strength & 0 & 2.09 & 350 \\
\hline Tensile Strain & 0 & 0 & 0.00175 \\
\hline Initial Tangent Elastic Modulus (MPa) & 14100 & 34600 & 200000 \\
\hline Poisson's Ratio & 0 & 0 & 0 \\
\hline \multicolumn{4}{|c|}{ Masonry Properties (X-Direction) } \\
\hline Gross Peak Compression Strength (MPa) & 22.3 & 40 & 350 \\
\hline Peak Compressive Strain & 0.0028 & 0.0021 & 0.00175 \\
\hline Tensile Strength & 0 & 2.09 & 350 \\
\hline Tensile Strain & 0 & 0 & 0.00175 \\
\hline Initial Tangent Elastic Modulus (MPa) & 14100 & 34600 & 200000 \\
\hline Poisson's Ratio & 0 & 0 & 0 \\
\hline \multicolumn{4}{|c|}{ Masonry Properties (Block) } \\
\hline Length (mm) & 390 & 2400 & 2000 \\
\hline Height (mm) & 190 & 400 & 400 \\
\hline Face Shell Thickness (mm) & 32 & 610 & 200 \\
\hline Percent Solid (\%) & 59 & 100 & 100 \\
\hline Tensile Strength & 1.75 & 2.09 & 350 \\
\hline \multicolumn{4}{|c|}{ Head Joint } \\
\hline Thickness (mm) & 10 & 10 & 10 \\
\hline Tensile Bond Strength (MPa) & 0.34 & 2.09 & 350 \\
\hline Joint Cohesion (MPa) & 6.13 & 2.09 & 350 \\
\hline Angle of Internal Friction (Deg) & 42 & 42 & 42 \\
\hline \multicolumn{4}{|c|}{ Bed Joint } \\
\hline Thickness (mm) & 10 & 10 & 10 \\
\hline Tensile Bond Strength (MPa) & 0.34 & 2.09 & 350 \\
\hline Joint Cohesion (MPa) & 6.13 & 2.09 & 350 \\
\hline Angle of Internal Friction (Deg) & 42 & 42 & 42 \\
\hline \multicolumn{4}{|c|}{ Reinforcement (Y-Direction) } \\
\hline Ratio (\%) & 0.38 & 1.38 & 100 \\
\hline Yield Strength (MPa) & 441 & 400 & 350 \\
\hline Yield Strain & 0.00221 & 0.002 & 0.00175 \\
\hline Strain Hardening Strain & 0.02 & 0.02 & 0.02 \\
\hline Ultimate Strength (MPa) & 710 & 600 & 600 \\
\hline Ultimate Strain & 0.2 & 0.2 & 0.2 \\
\hline Prestrain & 0 & 0 & 0 \\
\hline \multicolumn{4}{|c|}{ Reinforcement (X-Direction) } \\
\hline Ratio (\%) & 0.14 & 1.11 & 100 \\
\hline Yield Strength (MPa) & 386 & 400 & 350 \\
\hline Yield Strain & 0.00193 & 0.002 & 0.00175 \\
\hline Strain Hardening Strain & 0.02 & 0.02 & 0.02 \\
\hline Ultimate Strength (MPa) & 558 & 600 & 600 \\
\hline Ultimate Strain & 0.2 & 0.2 & 0.2 \\
\hline Prestrain & 0 & 0 & 0 \\
\hline
\end{tabular}

Numerical Modelling of Hollow Concrete Block Masonry Mechanics 


\section{HCBL 11 Shear Wall}

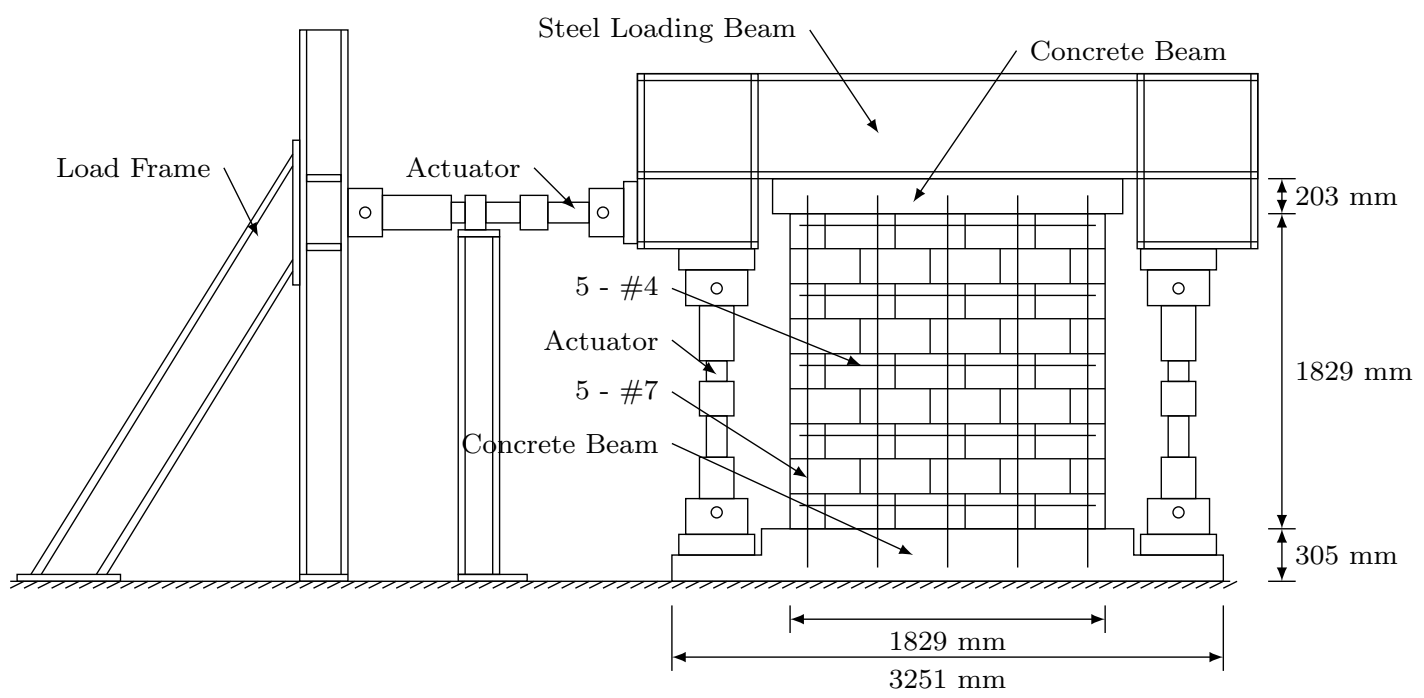

Experimental Wall Shing et al. (1991)

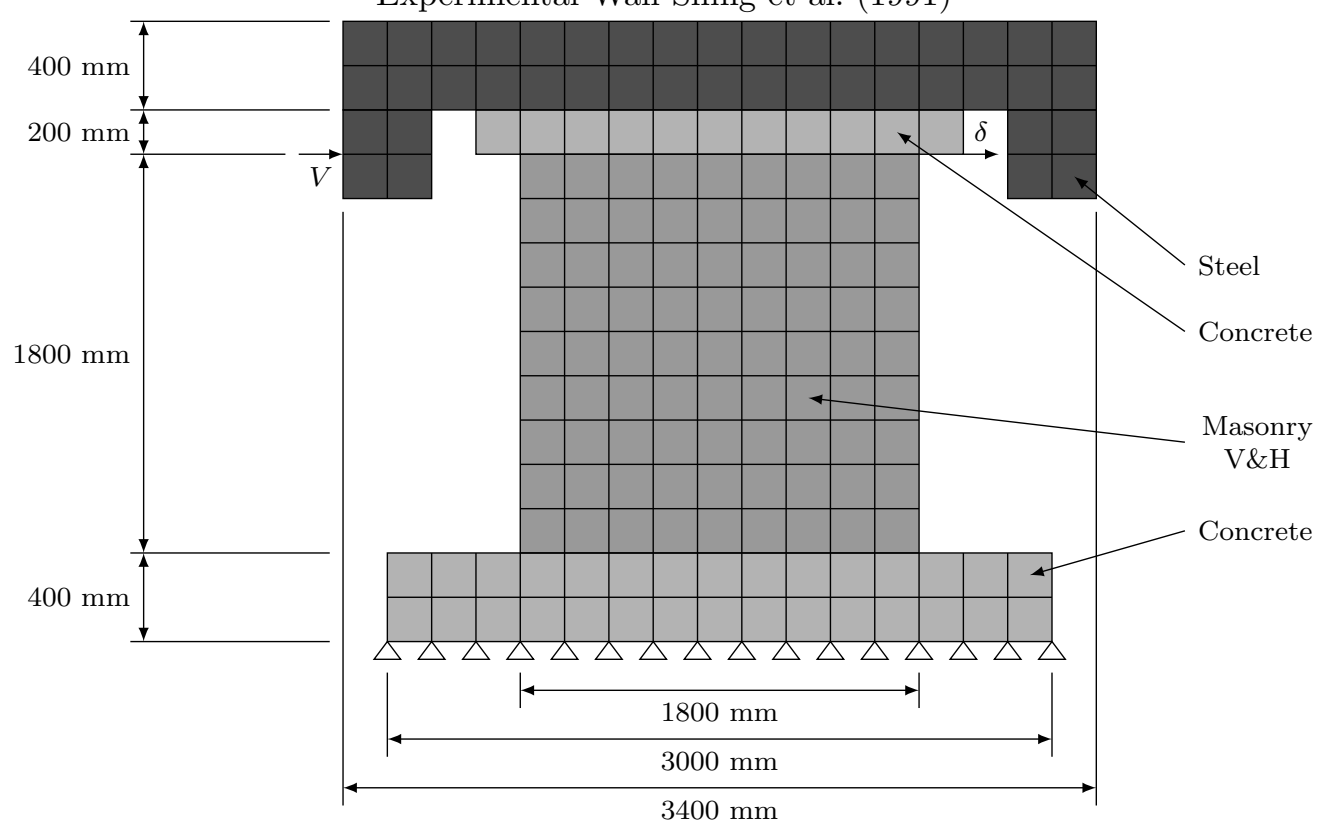

Finite Element Model Wall

HCBL 11 Shear Wall 
HCBL 11 Shear Wall Shing et al. (1991) Model Options

\begin{tabular}{ll}
\hline Model & Selected Option \\
\hline Compression Pre-Peak & Hognestad \\
Compression Post-Peak & Pre-Peak Base Curve \\
Compression Softening & Vecchio \\
Tension Post-Peak & Vecchio \\
Cracking Criterion & Mohr-Coulomb \\
Slip & Walraven Reinforced \\
\hline
\end{tabular}

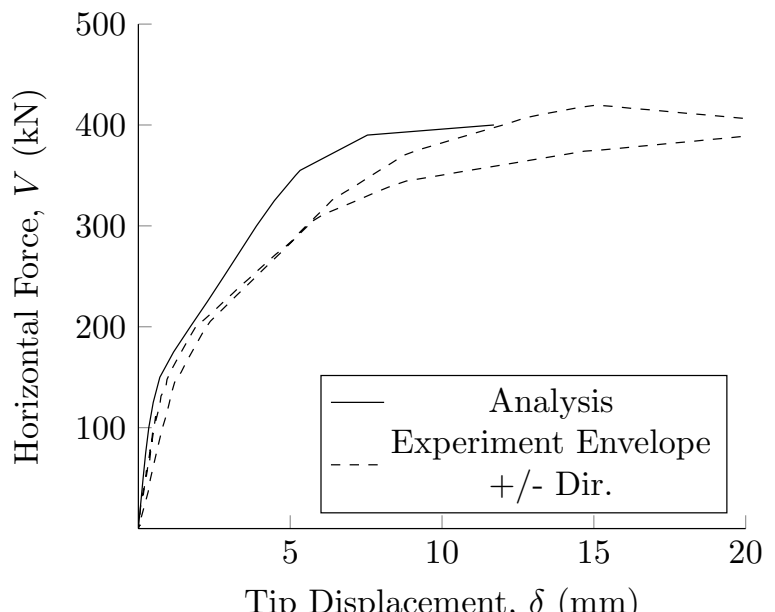

HCBL 11 Shear Wall Shing et al. (1991) Horizontal Force vs Tip Displacement 
HCBL 11 Shear Wall Shing et al. (1991) Material Properties

\begin{tabular}{|c|c|c|c|}
\hline \multirow{2}{*}{ Property } & \multicolumn{3}{|c|}{ Material } \\
\hline & Masonry V\&H & Concrete & Steel \\
\hline Thickness (mm) & 140 & 1220 & 400 \\
\hline Grout Tensile Strength (MPa) & 1.80 & 2.09 & 350 \\
\hline \multicolumn{4}{|c|}{ Masonry Properties (Y-Direction) } \\
\hline Gross Peak Compression Strength (MPa) & 22.3 & 40 & 350 \\
\hline Peak Compressive Strain & 0.0028 & 0.0021 & 0.00175 \\
\hline Tensile Strength & 0 & 2.09 & 350 \\
\hline Tensile Strain & 0 & 0 & 0.00175 \\
\hline Initial Tangent Elastic Modulus (MPa) & 14100 & 34600 & 200000 \\
\hline Poisson's Ratio & 0 & 0 & 0 \\
\hline \multicolumn{4}{|c|}{ Masonry Properties (X-Direction) } \\
\hline Gross Peak Compression Strength (MPa) & 22.3 & 40 & 350 \\
\hline Peak Compressive Strain & 0.0028 & 0.0021 & 0.00175 \\
\hline Tensile Strength & 0 & 2.09 & 350 \\
\hline Tensile Strain & 0 & 0 & 0.00175 \\
\hline Initial Tangent Elastic Modulus (MPa) & 14100 & 34600 & 200000 \\
\hline Poisson's Ratio & 0 & 0 & 0 \\
\hline \multicolumn{4}{|c|}{ Masonry Properties (Block) } \\
\hline Length (mm) & 390 & 2400 & 2000 \\
\hline Height (mm) & 190 & 400 & 400 \\
\hline Face Shell Thickness (mm) & 32 & 610 & 200 \\
\hline Percent Solid (\%) & 59 & 100 & 100 \\
\hline Tensile Strength & 1.75 & 2.09 & 350 \\
\hline \multicolumn{4}{|c|}{ Head Joint } \\
\hline Thickness (mm) & 10 & 10 & 10 \\
\hline Tensile Bond Strength (MPa) & 0.34 & 2.09 & 350 \\
\hline Joint Cohesion (MPa) & 6.13 & 2.09 & 350 \\
\hline Angle of Internal Friction (Deg) & 42 & 42 & 42 \\
\hline \multicolumn{4}{|c|}{ Bed Joint } \\
\hline Thickness (mm) & 10 & 10 & 10 \\
\hline Tensile Bond Strength (MPa) & 0.34 & 2.09 & 350 \\
\hline Joint Cohesion (MPa) & 6.13 & 2.09 & 350 \\
\hline Angle of Internal Friction (Deg) & 42 & 42 & 42 \\
\hline \multicolumn{4}{|c|}{ Reinforcement (Y-Direction) } \\
\hline Ratio (\%) & 0.74 & 1.38 & 100 \\
\hline Yield Strength (MPa) & 496 & 400 & 350 \\
\hline Yield Strain & 0.00248 & 0.002 & 0.00175 \\
\hline Strain Hardening Strain & 0.02 & 0.02 & 0.02 \\
\hline Ultimate Strength (MPa) & 710 & 600 & 600 \\
\hline Ultimate Strain & 0.2 & 0.2 & 0.2 \\
\hline Prestrain & 0 & 0 & 0 \\
\hline \multicolumn{4}{|c|}{ Reinforcement (X-Direction) } \\
\hline Ratio (\%) & 0.24 & 1.11 & 100 \\
\hline Yield Strength (MPa) & 462 & 400 & 350 \\
\hline Yield Strain & 0.00231 & 0.002 & 0.00175 \\
\hline Strain Hardening Strain & 0.02 & 0.02 & 0.02 \\
\hline Ultimate Strength (MPa) & 738 & 600 & 600 \\
\hline Ultimate Strain & 0.2 & 0.2 & 0.2 \\
\hline Prestrain & 0 & 0 & 0 \\
\hline
\end{tabular}

Numerical Modelling of Hollow Concrete Block Masonry Mechanics 


\section{HCBL 12 Shear Wall}

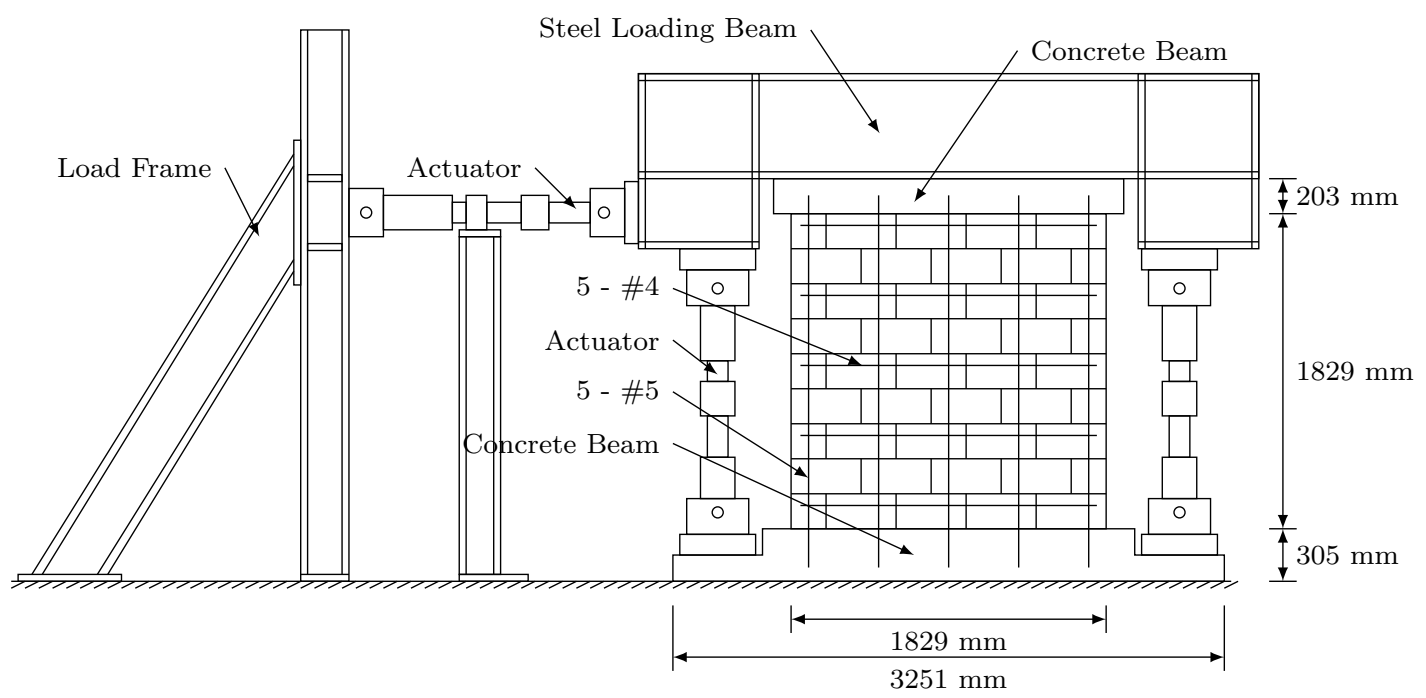

Experimental Wall Shing et al. (1991)

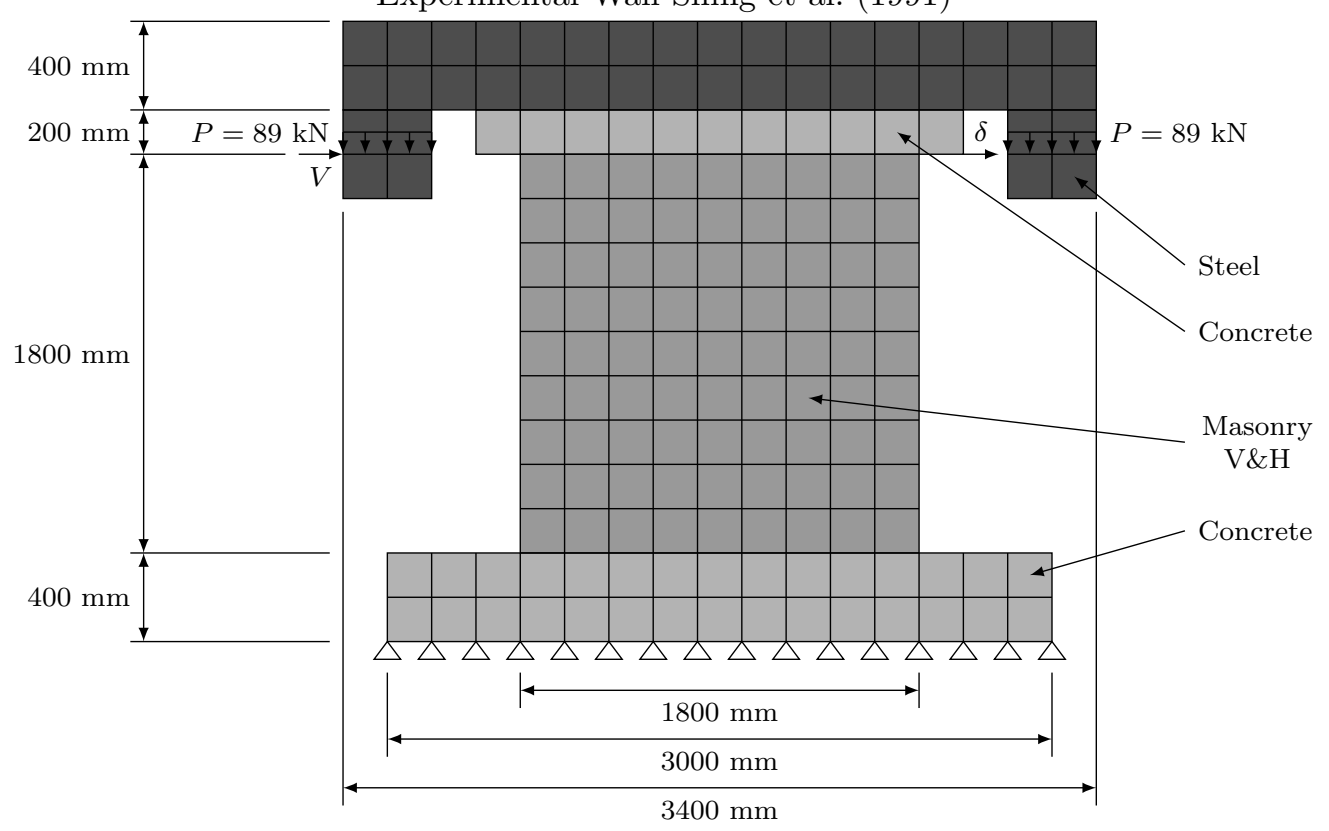

Finite Element Model Wall

HCBL 12 Shear Wall 
HCBL 12 Shear Wall Shing et al. (1991) Model Options

\begin{tabular}{ll}
\hline Model & Selected Option \\
\hline Compression Pre-Peak & Hognestad \\
Compression Post-Peak & Pre-Peak Base Curve \\
Compression Softening & Vecchio \\
Tension Post-Peak & Vecchio \\
Cracking Criterion & Mohr-Coulomb \\
Slip & Walraven Reinforced \\
\hline
\end{tabular}

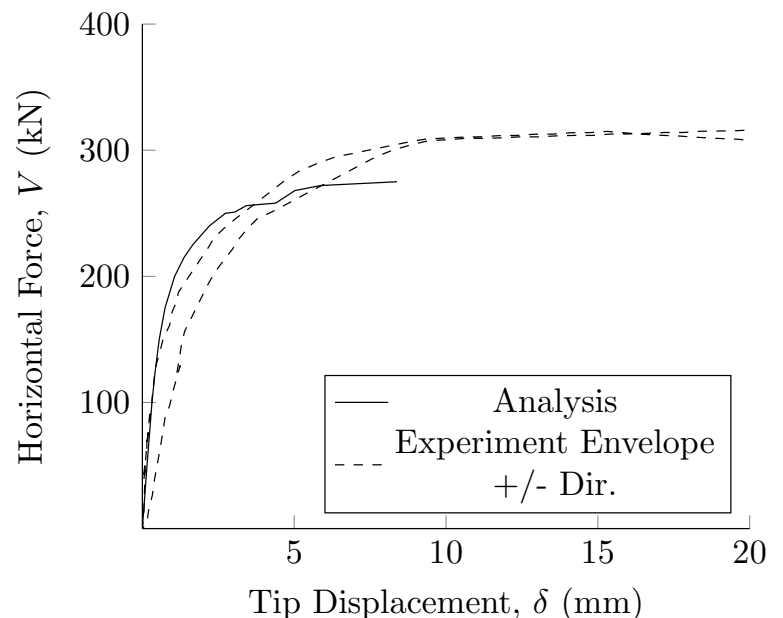

HCBL 12 Shear Wall Shing et al. (1991) Horizontal Force vs Tip Displacement 
HCBL 12 Shear Wall Shing et al. (1991) Material Properties

\begin{tabular}{|c|c|c|c|}
\hline \multirow{2}{*}{ Property } & \multicolumn{3}{|c|}{ Material } \\
\hline & Masonry V\&H & Concrete & Steel \\
\hline Thickness (mm) & 140 & 1220 & 400 \\
\hline Grout Tensile Strength (MPa) & 1.80 & 2.09 & 350 \\
\hline \multicolumn{4}{|c|}{ Masonry Properties (Y-Direction) } \\
\hline Gross Peak Compression Strength (MPa) & 22.3 & 40 & 350 \\
\hline Peak Compressive Strain & 0.0028 & 0.0021 & 0.00175 \\
\hline Tensile Strength & 0 & 2.09 & 350 \\
\hline Tensile Strain & 0 & 0 & 0.00175 \\
\hline Initial Tangent Elastic Modulus (MPa) & 14100 & 34600 & 200000 \\
\hline Poisson's Ratio & 0 & 0 & 0 \\
\hline \multicolumn{4}{|c|}{ Masonry Properties (X-Direction) } \\
\hline Gross Peak Compression Strength (MPa) & 22.3 & 40 & 350 \\
\hline Peak Compressive Strain & 0.0028 & 0.0021 & 0.00175 \\
\hline Tensile Strength & 0 & 2.09 & 350 \\
\hline Tensile Strain & 0 & 0 & 0.00175 \\
\hline Initial Tangent Elastic Modulus (MPa) & 14100 & 34600 & 200000 \\
\hline Poisson's Ratio & 0 & 0 & 0 \\
\hline \multicolumn{4}{|c|}{ Masonry Properties (Block) } \\
\hline Length (mm) & 390 & 2400 & 2000 \\
\hline Height (mm) & 190 & 400 & 400 \\
\hline Face Shell Thickness (mm) & 32 & 610 & 200 \\
\hline Percent Solid (\%) & 59 & 100 & 100 \\
\hline Tensile Strength & 1.75 & 2.09 & 350 \\
\hline \multicolumn{4}{|c|}{ Head Joint } \\
\hline Thickness (mm) & 10 & 10 & 10 \\
\hline Tensile Bond Strength (MPa) & 0.34 & 2.09 & 350 \\
\hline Joint Cohesion (MPa) & 6.13 & 2.09 & 350 \\
\hline Angle of Internal Friction (Deg) & 42 & 42 & 42 \\
\hline \multicolumn{4}{|c|}{ Bed Joint } \\
\hline Thickness (mm) & 10 & 10 & 10 \\
\hline Tensile Bond Strength (MPa) & 0.34 & 2.09 & 350 \\
\hline Joint Cohesion (MPa) & 6.13 & 2.09 & 350 \\
\hline Angle of Internal Friction (Deg) & 42 & 42 & 42 \\
\hline \multicolumn{4}{|c|}{ Reinforcement (Y-Direction) } \\
\hline Ratio (\%) & 0.38 & 1.38 & 100 \\
\hline Yield Strength (MPa) & 441 & 400 & 350 \\
\hline Yield Strain & 0.00221 & 0.002 & 0.00175 \\
\hline Strain Hardening Strain & 0.02 & 0.02 & 0.02 \\
\hline Ultimate Strength (MPa) & 710 & 600 & 600 \\
\hline Ultimate Strain & 0.2 & 0.2 & 0.2 \\
\hline Prestrain & 0 & 0 & 0 \\
\hline \multicolumn{4}{|c|}{ Reinforcement (X-Direction) } \\
\hline Ratio (\%) & 0.24 & 1.11 & 100 \\
\hline Yield Strength (MPa) & 462 & 400 & 350 \\
\hline Yield Strain & 0.00231 & 0.002 & 0.00175 \\
\hline Strain Hardening Strain & 0.02 & 0.02 & 0.02 \\
\hline Ultimate Strength (MPa) & 738 & 600 & 600 \\
\hline Ultimate Strain & 0.2 & 0.2 & 0.2 \\
\hline Prestrain & 0 & 0 & 0 \\
\hline
\end{tabular}

Numerical Modelling of Hollow Concrete Block Masonry Mechanics 


\section{HCBL 13 Shear Wall}

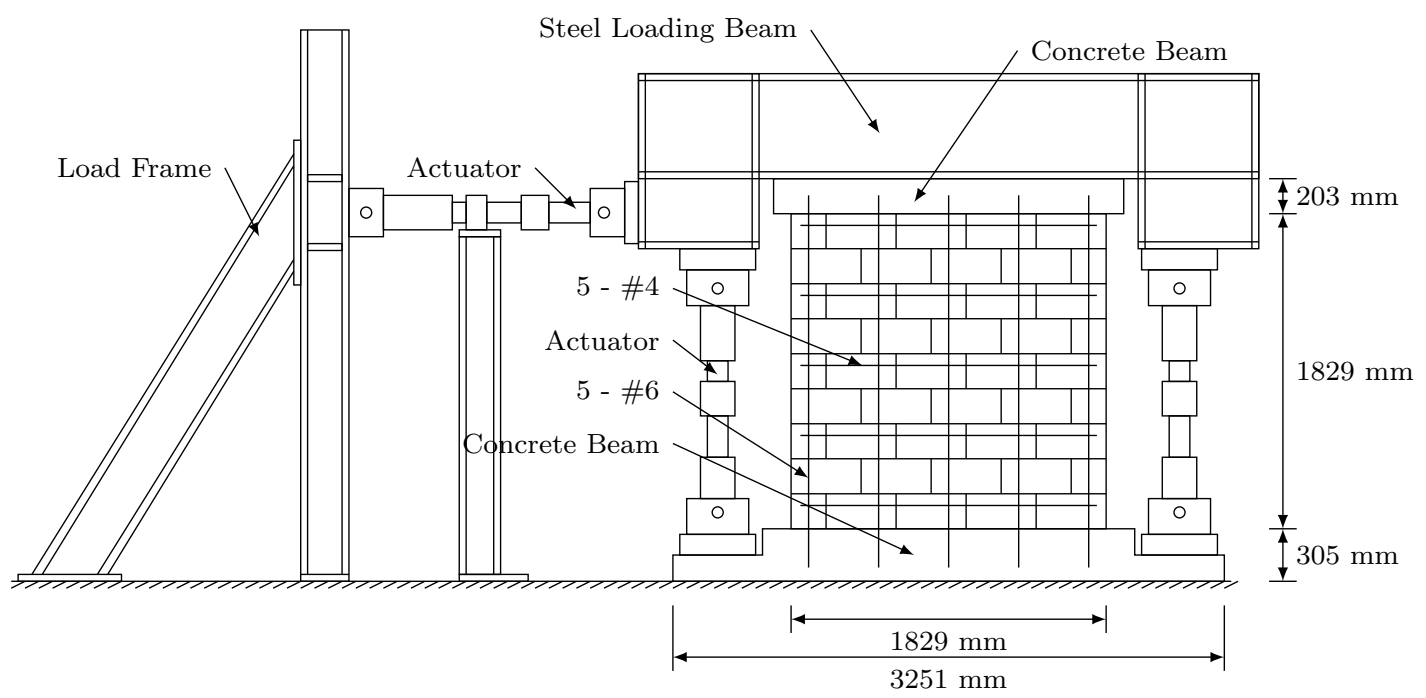

Experimental Wall Shing et al. (1991)

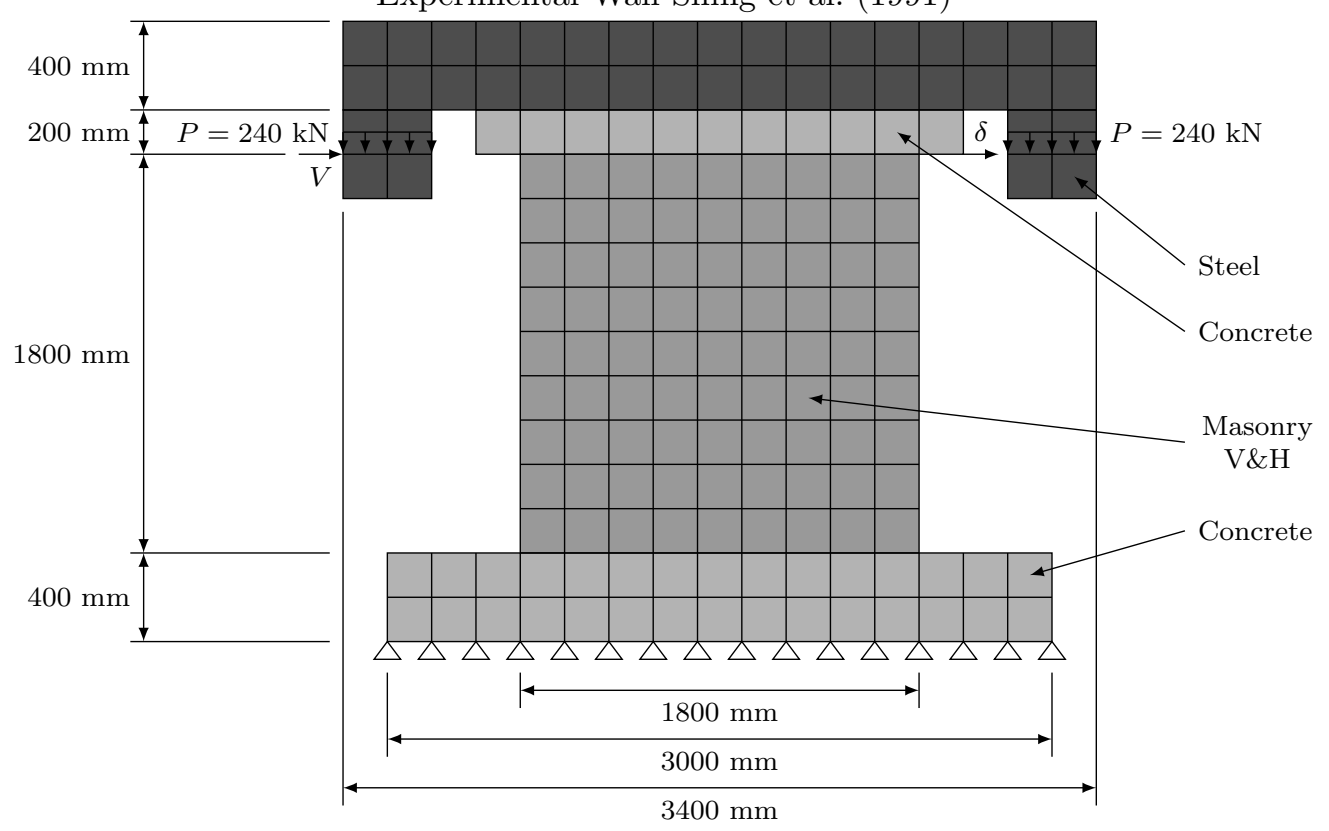

Finite Element Model Wall

HCBL 13 Shear Wall 
HCBL 13 Shear Wall Shing et al. (1991) Model Options

\begin{tabular}{ll}
\hline Model & Selected Option \\
\hline Compression Pre-Peak & Hognestad \\
Compression Post-Peak & Pre-Peak Base Curve \\
Compression Softening & Vecchio \\
Tension Post-Peak & Vecchio \\
Cracking Criterion & Mohr-Coulomb \\
Slip & Walraven Reinforced \\
\hline
\end{tabular}

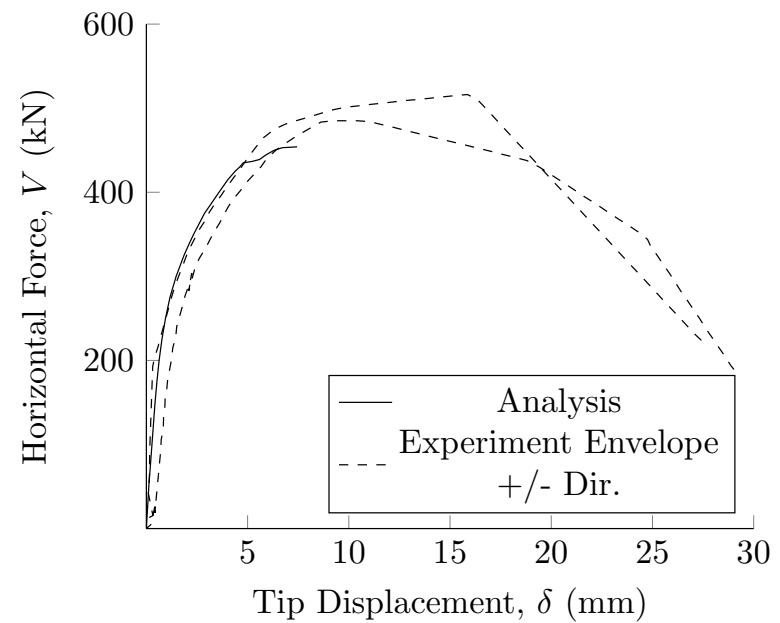

HCBL 13 Shear Wall Shing et al. (1991) Horizontal Force vs Tip Displacement 
HCBL 13 Shear Wall Shing et al. (1991) Material Properties

\begin{tabular}{|c|c|c|c|}
\hline \multirow{2}{*}{ Property } & \multicolumn{3}{|c|}{ Material } \\
\hline & Masonry V\&H & Concrete & Steel \\
\hline Thickness (mm) & 140 & 1220 & 400 \\
\hline Grout Tensile Strength (MPa) & 1.80 & 2.09 & 350 \\
\hline \multicolumn{4}{|c|}{ Masonry Properties (Y-Direction) } \\
\hline Gross Peak Compression Strength (MPa) & 22.8 & 40 & 350 \\
\hline Peak Compressive Strain & 0.0027 & 0.0021 & 0.00175 \\
\hline Tensile Strength & 0 & 2.09 & 350 \\
\hline Tensile Strain & 0 & 0 & 0.00175 \\
\hline Initial Tangent Elastic Modulus (MPa) & 0 & 34600 & 200000 \\
\hline Poisson's Ratio & 0 & 0 & 0 \\
\hline \multicolumn{4}{|c|}{ Masonry Properties (X-Direction) } \\
\hline Gross Peak Compression Strength (MPa) & 22.8 & 40 & 350 \\
\hline Peak Compressive Strain & 0.0027 & 0.0021 & 0.00175 \\
\hline Tensile Strength & 0 & 2.09 & 350 \\
\hline Tensile Strain & 0 & 0 & 0.00175 \\
\hline Initial Tangent Elastic Modulus (MPa) & 0 & 34600 & 200000 \\
\hline Poisson's Ratio & 0 & 0 & 0 \\
\hline \multicolumn{4}{|c|}{ Masonry Properties (Block) } \\
\hline Length (mm) & 390 & 2400 & 2000 \\
\hline Height (mm) & 190 & 400 & 400 \\
\hline Face Shell Thickness (mm) & 32 & 610 & 200 \\
\hline Percent Solid (\%) & 59 & 100 & 100 \\
\hline Tensile Strength & 1.75 & 2.09 & 350 \\
\hline \multicolumn{4}{|c|}{ Head Joint } \\
\hline Thickness (mm) & 10 & 10 & 10 \\
\hline Tensile Bond Strength (MPa) & 0.34 & 2.09 & 350 \\
\hline Joint Cohesion (MPa) & 6.13 & 2.09 & 350 \\
\hline Angle of Internal Friction (Deg) & 42 & 42 & 42 \\
\hline \multicolumn{4}{|c|}{ Bed Joint } \\
\hline Thickness (mm) & 10 & 10 & 10 \\
\hline Tensile Bond Strength (MPa) & 0.34 & 2.09 & 350 \\
\hline Joint Cohesion (MPa) & 6.13 & 2.09 & 350 \\
\hline Angle of Internal Friction (Deg) & 42 & 42 & 42 \\
\hline \multicolumn{4}{|c|}{ Reinforcement (Y-Direction) } \\
\hline Ratio (\%) & 0.54 & 1.38 & 100 \\
\hline Yield Strength (MPa) & 448 & 400 & 350 \\
\hline Yield Strain & 0.00224 & 0.002 & 0.00175 \\
\hline Strain Hardening Strain & 0.02 & 0.02 & 0.02 \\
\hline Ultimate Strength (MPa) & 738 & 600 & 600 \\
\hline Ultimate Strain & 0.2 & 0.2 & 0.2 \\
\hline Prestrain & 0 & 0 & 0 \\
\hline \multicolumn{4}{|c|}{ Reinforcement (X-Direction) } \\
\hline Ratio (\%) & 0.24 & 1.11 & 100 \\
\hline Yield Strength (MPa) & 462 & 400 & 350 \\
\hline Yield Strain & 0.00231 & 0.002 & 0.00175 \\
\hline Strain Hardening Strain & 0.02 & 0.02 & 0.02 \\
\hline Ultimate Strength (MPa) & 738 & 600 & 600 \\
\hline Ultimate Strain & 0.2 & 0.2 & 0.2 \\
\hline Prestrain & 0 & 0 & 0 \\
\hline
\end{tabular}

Numerical Modelling of Hollow Concrete Block Masonry Mechanics 


\section{HCBL 14 Shear Wall}

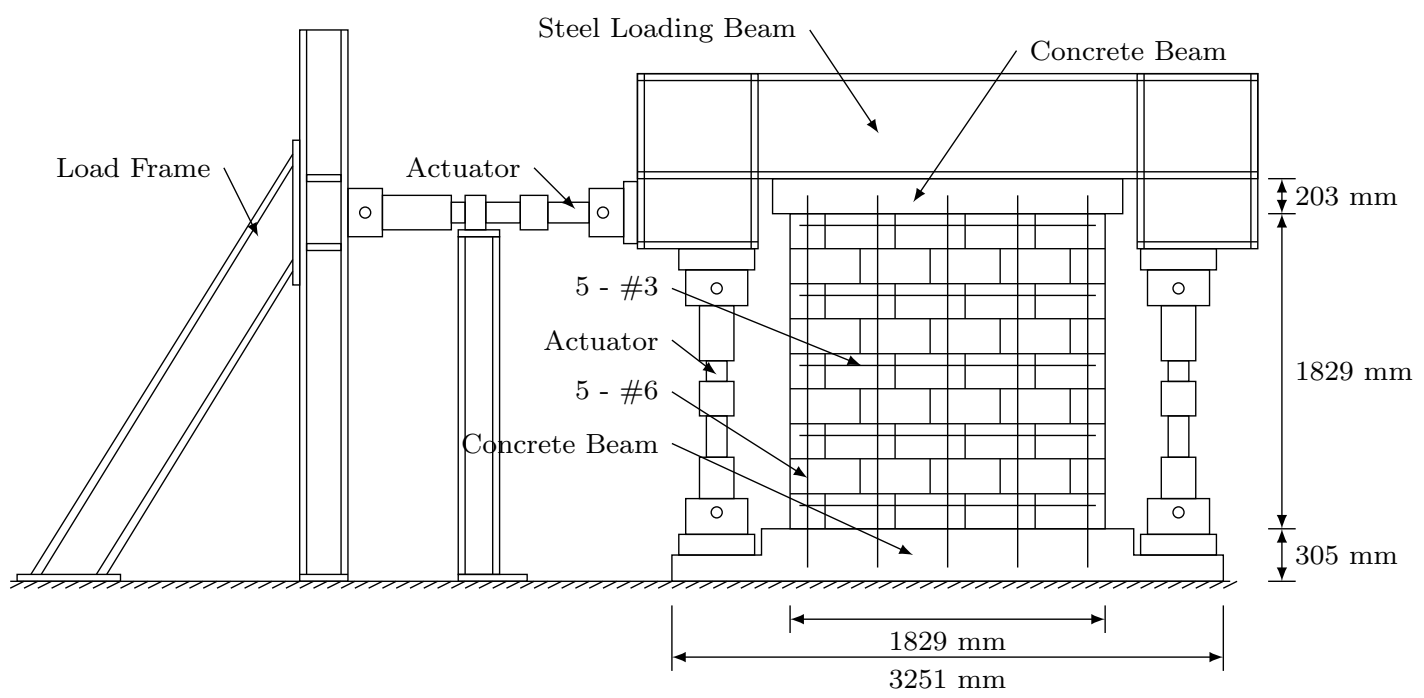

Experimental Wall Shing et al. (1991)

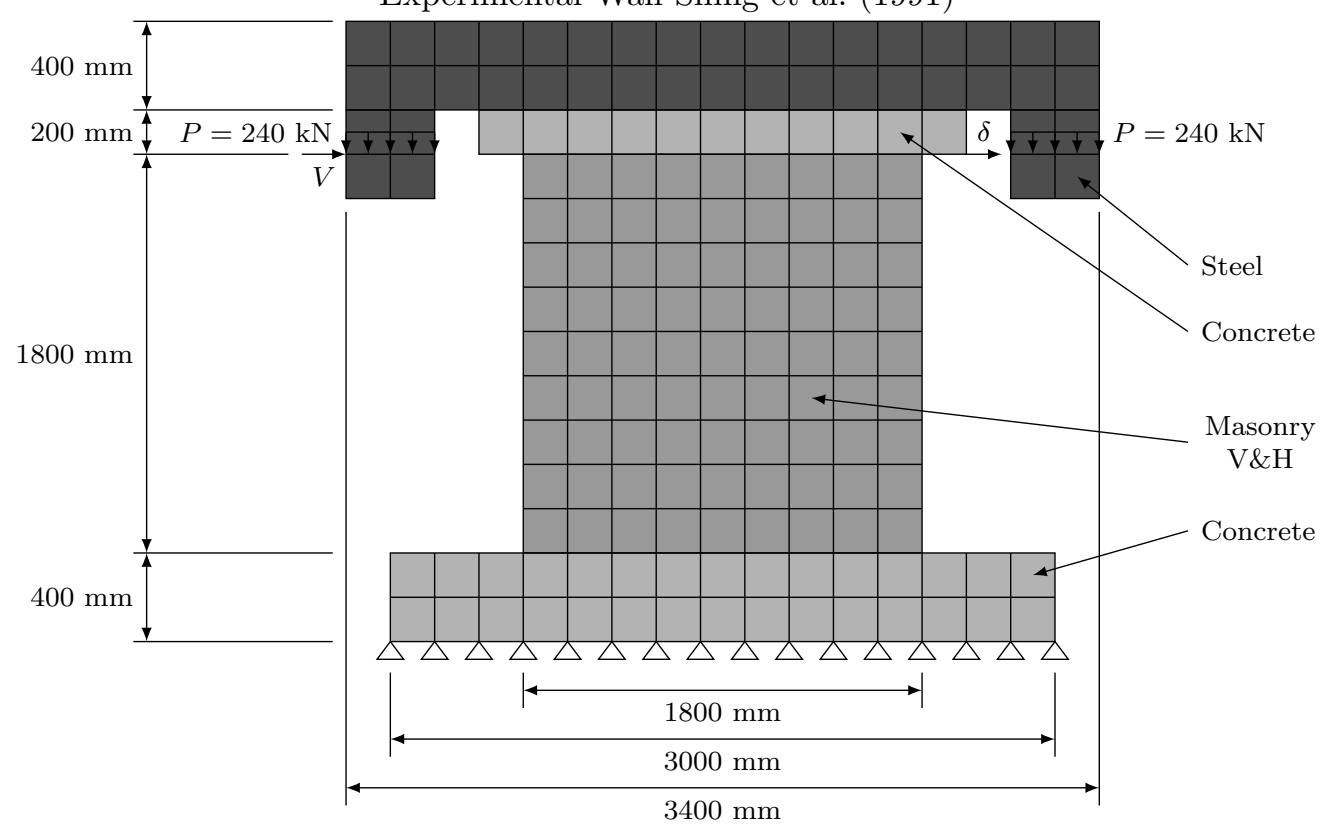

Finite Element Model Wall

HCBL 14 Shear Wall 
HCBL 14 Shear Wall Shing et al. (1991) Model Options

\begin{tabular}{ll}
\hline Model & Selected Option \\
\hline Compression Pre-Peak & Hognestad \\
Compression Post-Peak & Pre-Peak Base Curve \\
Compression Softening & Vecchio \\
Tension Post-Peak & Vecchio \\
Cracking Criterion & Mohr-Coulomb \\
Slip & Walraven Reinforced \\
\hline
\end{tabular}

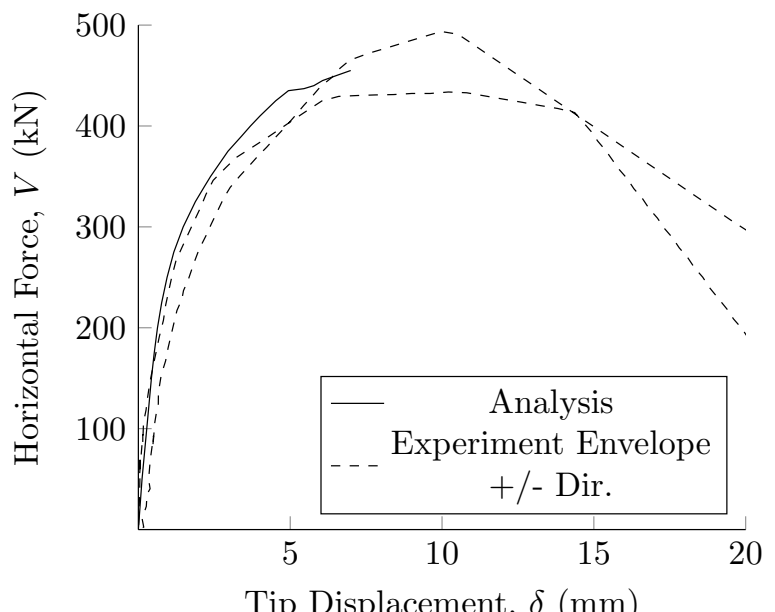

HCBL 14 Shear Wall Shing et al. (1991) Horizontal Force vs Tip Displacement 
HCBL 14 Shear Wall Shing et al. (1991) Material Properties

\begin{tabular}{|c|c|c|c|}
\hline \multirow{2}{*}{ Property } & \multicolumn{3}{|c|}{ Material } \\
\hline & Masonry V\&H & Concrete & Steel \\
\hline Thickness (mm) & 140 & 1220 & 400 \\
\hline Grout Tensile Strength (MPa) & 1.80 & 2.09 & 350 \\
\hline \multicolumn{4}{|c|}{ Masonry Properties (Y-Direction) } \\
\hline Gross Peak Compression Strength (MPa) & 22.8 & 40 & 350 \\
\hline Peak Compressive Strain & 0.0027 & 0.0021 & 0.00175 \\
\hline Tensile Strength & 0 & 2.09 & 350 \\
\hline Tensile Strain & 0 & 0 & 0.00175 \\
\hline Initial Tangent Elastic Modulus (MPa) & 0 & 34600 & 200000 \\
\hline Poisson's Ratio & 0 & 0 & 0 \\
\hline \multicolumn{4}{|c|}{ Masonry Properties (X-Direction) } \\
\hline Gross Peak Compression Strength (MPa) & 22.8 & 40 & 350 \\
\hline Peak Compressive Strain & 0.0027 & 0.0021 & 0.00175 \\
\hline Tensile Strength & 0 & 2.09 & 350 \\
\hline Tensile Strain & 0 & 0 & 0.00175 \\
\hline Initial Tangent Elastic Modulus (MPa) & 0 & 34600 & 200000 \\
\hline Poisson's Ratio & 0 & 0 & 0 \\
\hline \multicolumn{4}{|c|}{ Masonry Properties (Block) } \\
\hline Length (mm) & 390 & 2400 & 2000 \\
\hline Height (mm) & 190 & 400 & 400 \\
\hline Face Shell Thickness (mm) & 32 & 610 & 200 \\
\hline Percent Solid (\%) & 59 & 100 & 100 \\
\hline Tensile Strength & 1.75 & 2.09 & 350 \\
\hline \multicolumn{4}{|c|}{ Head Joint } \\
\hline Thickness (mm) & 10 & 10 & 10 \\
\hline Tensile Bond Strength (MPa) & 0.34 & 2.09 & 350 \\
\hline Joint Cohesion (MPa) & 6.13 & 2.09 & 350 \\
\hline Angle of Internal Friction (Deg) & 42 & 42 & 42 \\
\hline \multicolumn{4}{|c|}{ Bed Joint } \\
\hline Thickness (mm) & 10 & 10 & 10 \\
\hline Tensile Bond Strength (MPa) & 0.34 & 2.09 & 350 \\
\hline Joint Cohesion (MPa) & 6.13 & 2.09 & 350 \\
\hline Angle of Internal Friction (Deg) & 42 & 42 & 42 \\
\hline \multicolumn{4}{|c|}{ Reinforcement (Y-Direction) } \\
\hline Ratio (\%) & 0.54 & 1.38 & 100 \\
\hline Yield Strength (MPa) & 448 & 400 & 350 \\
\hline Yield Strain & 0.00224 & 0.002 & 0.00175 \\
\hline Strain Hardening Strain & 0.02 & 0.02 & 0.02 \\
\hline Ultimate Strength (MPa) & 738 & 600 & 600 \\
\hline Ultimate Strain & 0.2 & 0.2 & 0.2 \\
\hline Prestrain & 0 & 0 & 0 \\
\hline \multicolumn{4}{|c|}{ Reinforcement (X-Direction) } \\
\hline Ratio (\%) & 0.14 & 1.11 & 100 \\
\hline Yield Strength (MPa) & 386 & 400 & 350 \\
\hline Yield Strain & 0.00193 & 0.002 & 0.00175 \\
\hline Strain Hardening Strain & 0.02 & 0.02 & 0.02 \\
\hline Ultimate Strength (MPa) & 558 & 600 & 600 \\
\hline Ultimate Strain & 0.2 & 0.2 & 0.2 \\
\hline Prestrain & 0 & 0 & 0 \\
\hline
\end{tabular}

Numerical Modelling of Hollow Concrete Block Masonry Mechanics 


\section{HCBL 15 Shear Wall}

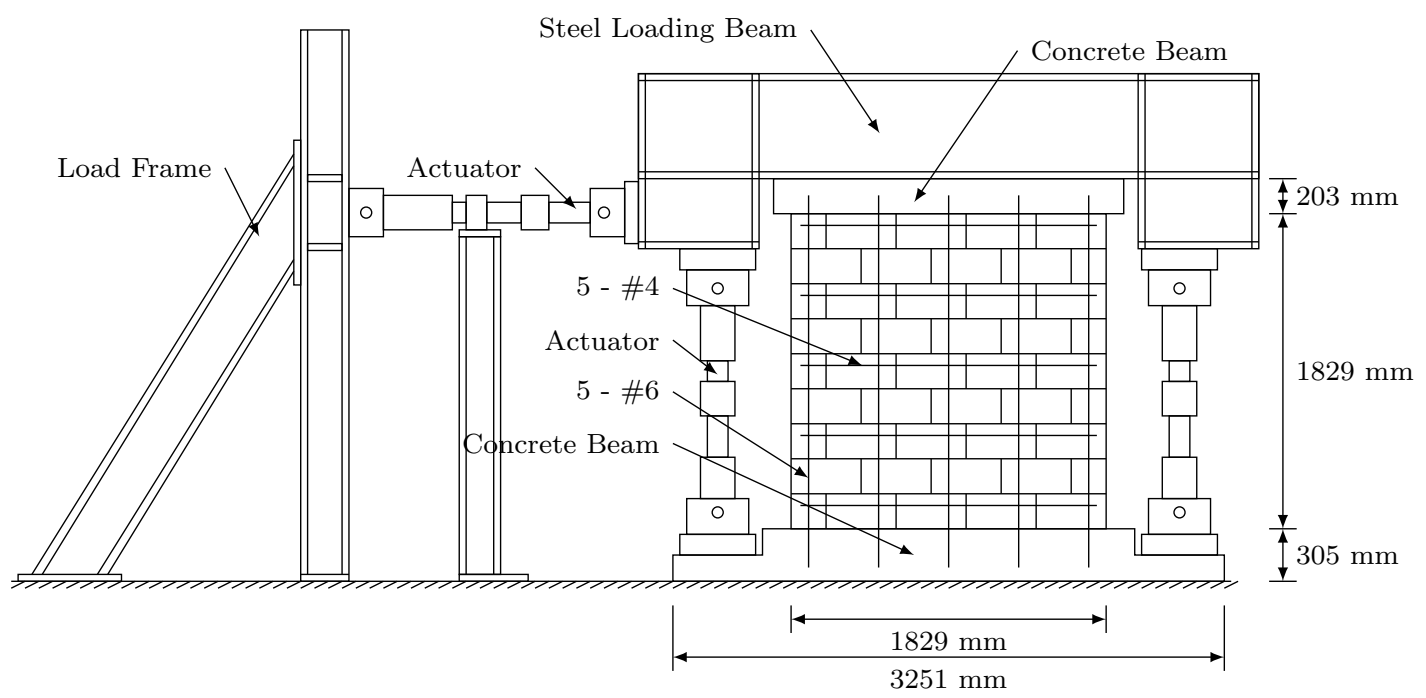

Experimental Wall Shing et al. (1991)

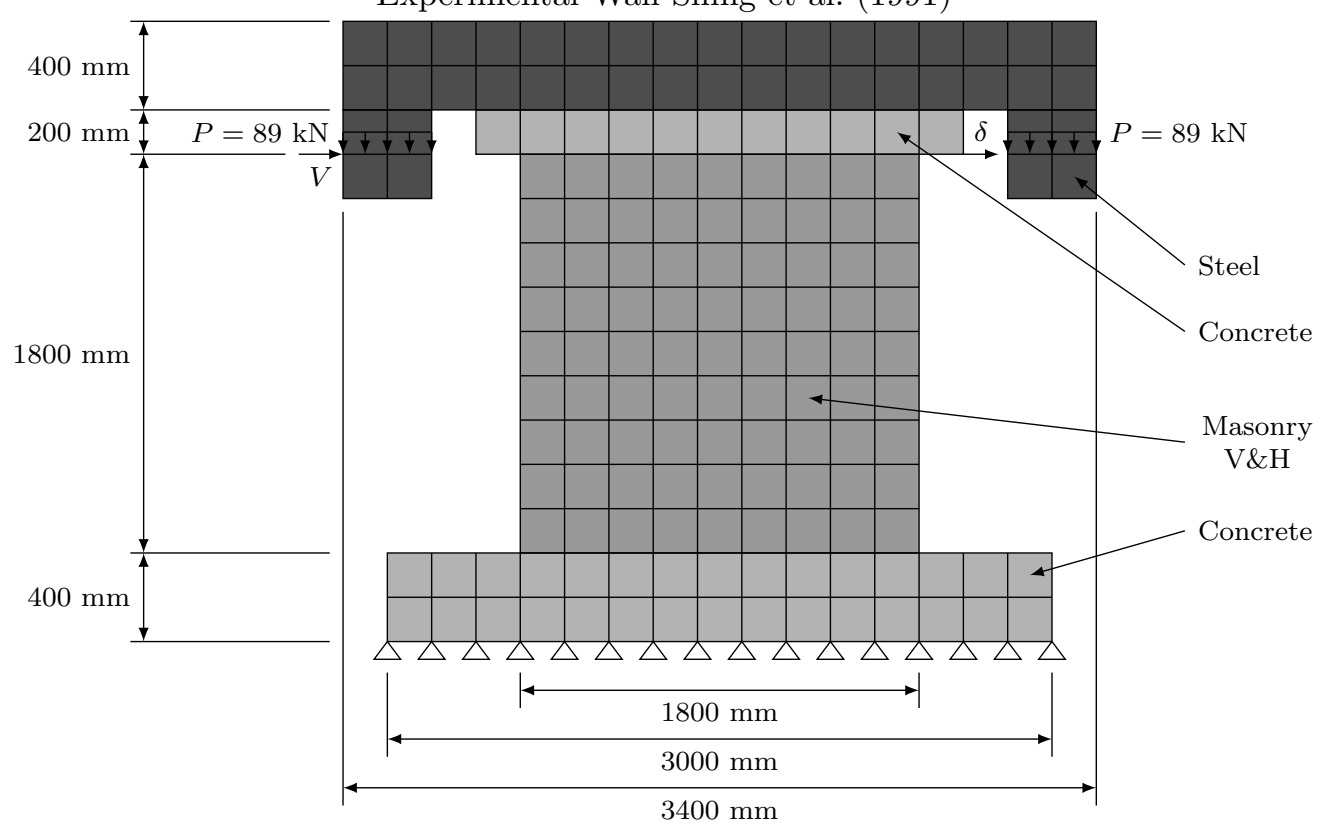

Finite Element Model Wall

HCBL 15 Shear Wall 
HCBL 15 Shear Wall Shing et al. (1991) Model Options

\begin{tabular}{ll}
\hline Model & Selected Option \\
\hline Compression Pre-Peak & Hognestad \\
Compression Post-Peak & Pre-Peak Base Curve \\
Compression Softening & Vecchio \\
Tension Post-Peak & Vecchio \\
Cracking Criterion & Mohr-Coulomb \\
Slip & Walraven Reinforced \\
\hline
\end{tabular}

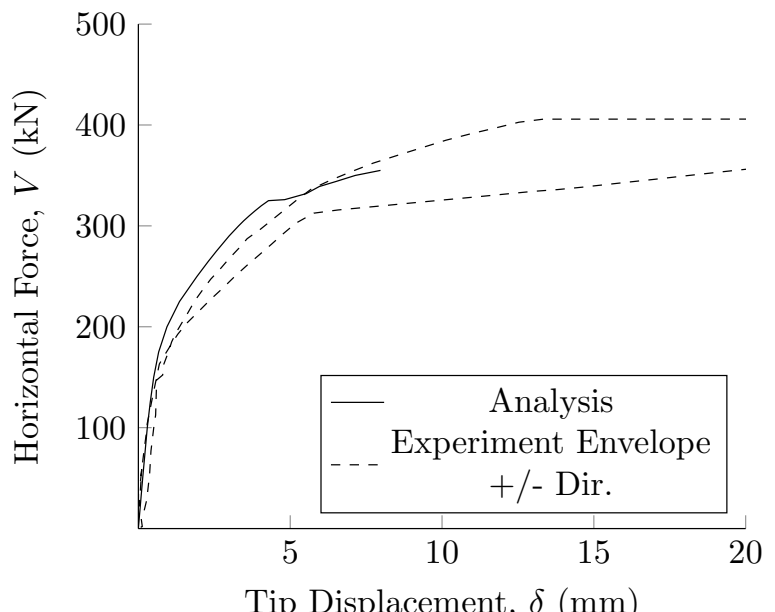

HCBL 15 Shear Wall Shing et al. (1991) Horizontal Force vs Tip Displacement 
HCBL 15 Shear Wall Shing et al. (1991) Material Properties

\begin{tabular}{|c|c|c|c|}
\hline \multirow{2}{*}{ Property } & \multicolumn{3}{|c|}{ Material } \\
\hline & Masonry V\&H & Concrete & Steel \\
\hline Thickness (mm) & 140 & 1220 & 400 \\
\hline Grout Tensile Strength (MPa) & 1.80 & 2.09 & 350 \\
\hline \multicolumn{4}{|c|}{ Masonry Properties (Y-Direction) } \\
\hline Gross Peak Compression Strength (MPa) & 22.8 & 40 & 350 \\
\hline Peak Compressive Strain & 0.0027 & 0.0021 & 0.00175 \\
\hline Tensile Strength & 0 & 2.09 & 350 \\
\hline Tensile Strain & 0 & 0 & 0.00175 \\
\hline Initial Tangent Elastic Modulus (MPa) & 0 & 34600 & 200000 \\
\hline Poisson's Ratio & 0 & 0 & 0 \\
\hline \multicolumn{4}{|c|}{ Masonry Properties (X-Direction) } \\
\hline Gross Peak Compression Strength (MPa) & 22.8 & 40 & 350 \\
\hline Peak Compressive Strain & 0.0027 & 0.0021 & 0.00175 \\
\hline Tensile Strength & 0 & 2.09 & 350 \\
\hline Tensile Strain & 0 & 0 & 0.00175 \\
\hline Initial Tangent Elastic Modulus (MPa) & 0 & 34600 & 200000 \\
\hline Poisson's Ratio & 0 & 0 & 0 \\
\hline \multicolumn{4}{|c|}{ Masonry Properties (Block) } \\
\hline Length (mm) & 390 & 2400 & 2000 \\
\hline Height (mm) & 190 & 400 & 400 \\
\hline Face Shell Thickness (mm) & 32 & 610 & 200 \\
\hline Percent Solid (\%) & 59 & 100 & 100 \\
\hline Tensile Strength & 1.75 & 2.09 & 350 \\
\hline \multicolumn{4}{|c|}{ Head Joint } \\
\hline Thickness (mm) & 10 & 10 & 10 \\
\hline Tensile Bond Strength (MPa) & 0.34 & 2.09 & 350 \\
\hline Joint Cohesion (MPa) & 6.13 & 2.09 & 350 \\
\hline Angle of Internal Friction (Deg) & 42 & 42 & 42 \\
\hline \multicolumn{4}{|c|}{ Bed Joint } \\
\hline Thickness (mm) & 10 & 10 & 10 \\
\hline Tensile Bond Strength (MPa) & 0.34 & 2.09 & 350 \\
\hline Joint Cohesion (MPa) & 6.13 & 2.09 & 350 \\
\hline Angle of Internal Friction (Deg) & 42 & 42 & 42 \\
\hline \multicolumn{4}{|c|}{ Reinforcement (Y-Direction) } \\
\hline Ratio (\%) & 0.54 & 1.38 & 100 \\
\hline Yield Strength (MPa) & 448 & 400 & 350 \\
\hline Yield Strain & 0.00224 & 0.002 & 0.00175 \\
\hline Strain Hardening Strain & 0.02 & 0.02 & 0.02 \\
\hline Ultimate Strength (MPa) & 738 & 600 & 600 \\
\hline Ultimate Strain & 0.2 & 0.2 & 0.2 \\
\hline Prestrain & 0 & 0 & 0 \\
\hline \multicolumn{4}{|c|}{ Reinforcement (X-Direction) } \\
\hline Ratio (\%) & 0.24 & 1.11 & 100 \\
\hline Yield Strength (MPa) & 462 & 400 & 350 \\
\hline Yield Strain & 0.00231 & 0.002 & 0.00175 \\
\hline Strain Hardening Strain & 0.02 & 0.02 & 0.02 \\
\hline Ultimate Strength (MPa) & 738 & 600 & 600 \\
\hline Ultimate Strain & 0.2 & 0.2 & 0.2 \\
\hline Prestrain & 0 & 0 & 0 \\
\hline
\end{tabular}

Numerical Modelling of Hollow Concrete Block Masonry Mechanics 


\section{HCBL 16 Shear Wall}

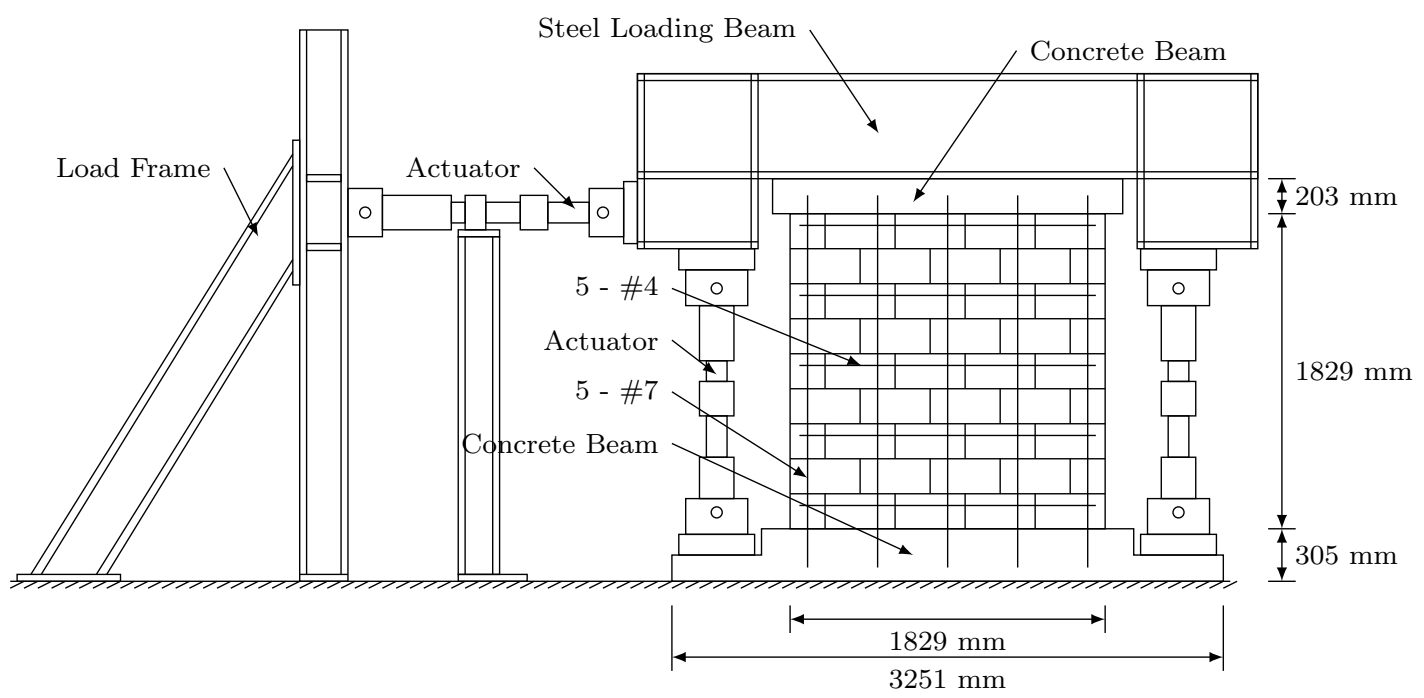

Experimental Wall Shing et al. (1991)

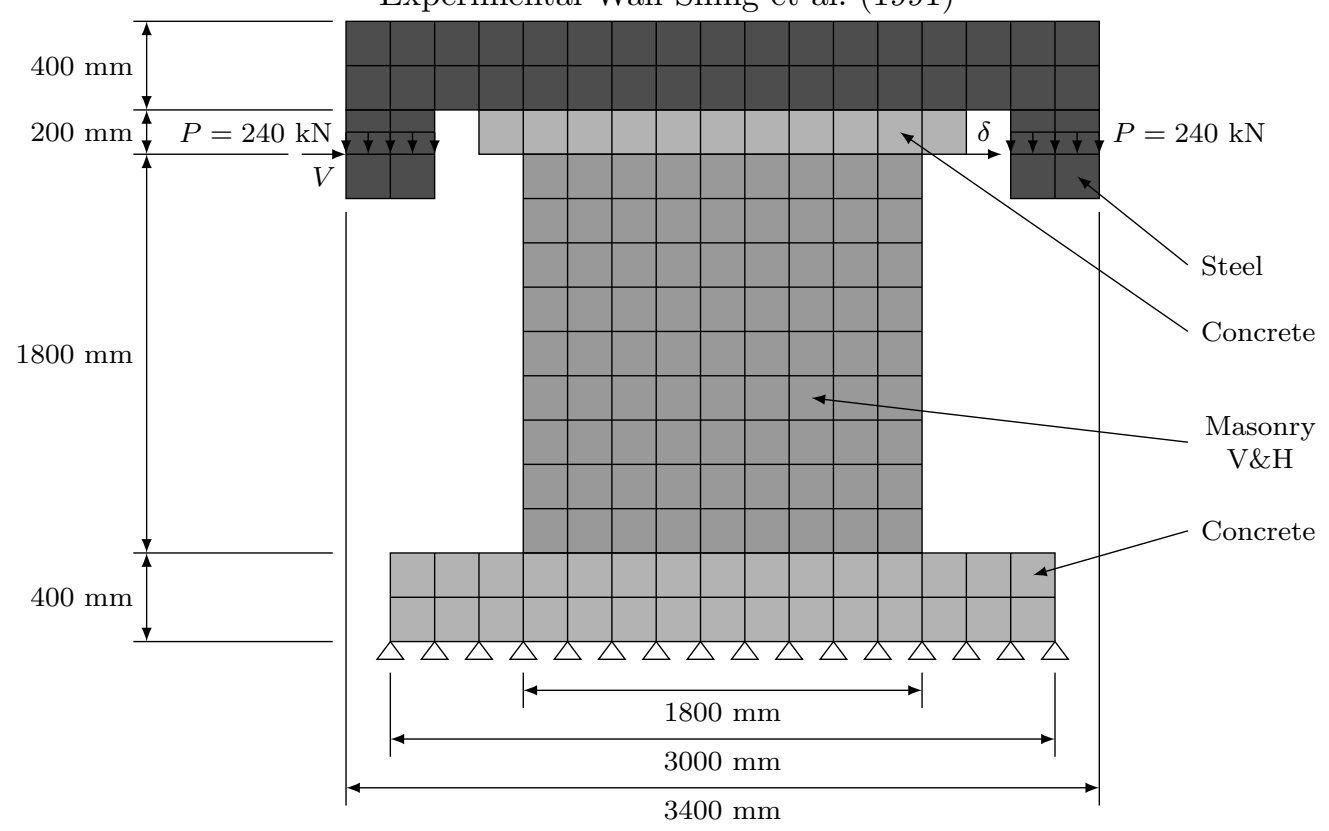

Finite Element Model Wall

HCBL 16 Shear Wall 
HCBL 16 Shear Wall Shing et al. (1991) Model Options

\begin{tabular}{ll}
\hline Model & Selected Option \\
\hline Compression Pre-Peak & Hognestad \\
Compression Post-Peak & Pre-Peak Base Curve \\
Compression Softening & Vecchio \\
Tension Post-Peak & Vecchio \\
Cracking Criterion & Mohr-Coulomb \\
Slip & Walraven Reinforced \\
\hline
\end{tabular}

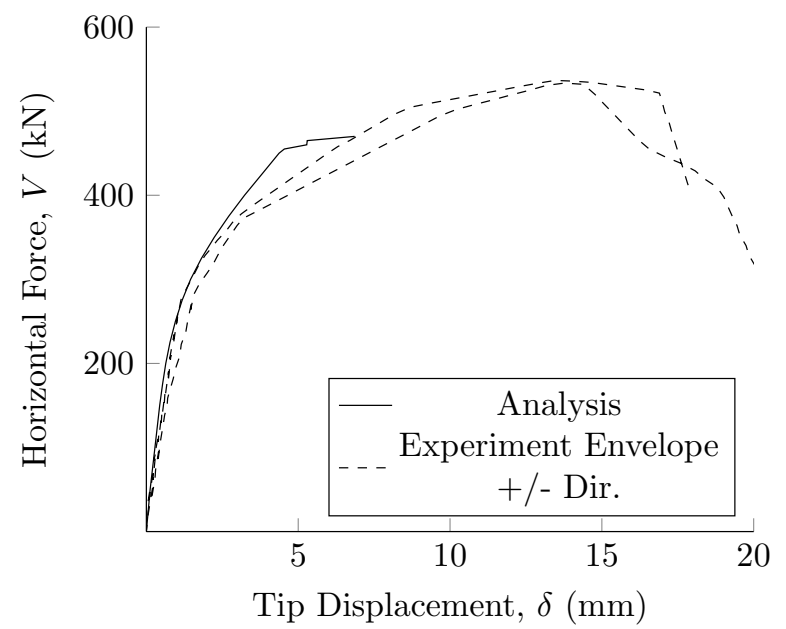

HCBL 16 Shear Wall Shing et al. (1991) Horizontal Force vs Tip Displacement 
HCBL 16 Shear Wall Shing et al. (1991) Material Properties

\begin{tabular}{|c|c|c|c|}
\hline \multirow{2}{*}{ Property } & \multicolumn{3}{|c|}{ Material } \\
\hline & Masonry V\&H & Concrete & Steel \\
\hline Thickness (mm) & 140 & 1220 & 400 \\
\hline Grout Tensile Strength (MPa) & 1.80 & 2.09 & 350 \\
\hline \multicolumn{4}{|c|}{ Masonry Properties (Y-Direction) } \\
\hline Gross Peak Compression Strength (MPa) & 17.1 & 40 & 350 \\
\hline Peak Compressive Strain & 0.0021 & 0.0021 & 0.00175 \\
\hline Tensile Strength & 0 & 2.09 & 350 \\
\hline Tensile Strain & 0 & 0 & 0.00175 \\
\hline Initial Tangent Elastic Modulus (MPa) & 0 & 34600 & 200000 \\
\hline Poisson's Ratio & 0 & 0 & 0 \\
\hline \multicolumn{4}{|c|}{ Masonry Properties (X-Direction) } \\
\hline Gross Peak Compression Strength (MPa) & 17.1 & 40 & 350 \\
\hline Peak Compressive Strain & 0.0021 & 0.0021 & 0.00175 \\
\hline Tensile Strength & 0 & 2.09 & 350 \\
\hline Tensile Strain & 0 & 0 & 0.00175 \\
\hline Initial Tangent Elastic Modulus (MPa) & 0 & 34600 & 200000 \\
\hline Poisson's Ratio & 0 & 0 & 0 \\
\hline \multicolumn{4}{|c|}{ Masonry Properties (Block) } \\
\hline Length (mm) & 390 & 2400 & 2000 \\
\hline Height (mm) & 190 & 400 & 400 \\
\hline Face Shell Thickness (mm) & 32 & 610 & 200 \\
\hline Percent Solid (\%) & 59 & 100 & 100 \\
\hline Tensile Strength & 1.75 & 2.09 & 350 \\
\hline \multicolumn{4}{|c|}{ Head Joint } \\
\hline Thickness (mm) & 10 & 10 & 10 \\
\hline Tensile Bond Strength (MPa) & 0.34 & 2.09 & 350 \\
\hline Joint Cohesion (MPa) & 6.13 & 2.09 & 350 \\
\hline Angle of Internal Friction (Deg) & 42 & 42 & 42 \\
\hline \multicolumn{4}{|c|}{ Bed Joint } \\
\hline Thickness (mm) & 10 & 10 & 10 \\
\hline Tensile Bond Strength (MPa) & 0.34 & 2.09 & 350 \\
\hline Joint Cohesion (MPa) & 6.13 & 2.09 & 350 \\
\hline Angle of Internal Friction (Deg) & 42 & 42 & 42 \\
\hline \multicolumn{4}{|c|}{ Reinforcement (Y-Direction) } \\
\hline Ratio (\%) & 0.74 & 1.38 & 100 \\
\hline Yield Strength (MPa) & 496 & 400 & 350 \\
\hline Yield Strain & 0.00248 & 0.002 & 0.00175 \\
\hline Strain Hardening Strain & 0.02 & 0.02 & 0.02 \\
\hline Ultimate Strength (MPa) & 710 & 600 & 600 \\
\hline Ultimate Strain & 0.2 & 0.2 & 0.2 \\
\hline Prestrain & 0 & 0 & 0 \\
\hline \multicolumn{4}{|c|}{ Reinforcement (X-Direction) } \\
\hline Ratio (\%) & 0.24 & 1.11 & 100 \\
\hline Yield Strength (MPa) & 462 & 400 & 350 \\
\hline Yield Strain & 0.00231 & 0.002 & 0.00175 \\
\hline Strain Hardening Strain & 0.02 & 0.02 & 0.02 \\
\hline Ultimate Strength (MPa) & 738 & 600 & 600 \\
\hline Ultimate Strain & 0.2 & 0.2 & 0.2 \\
\hline Prestrain & 0 & 0 & 0 \\
\hline
\end{tabular}

Numerical Modelling of Hollow Concrete Block Masonry Mechanics 


\section{HCBL 23 Shear Wall}

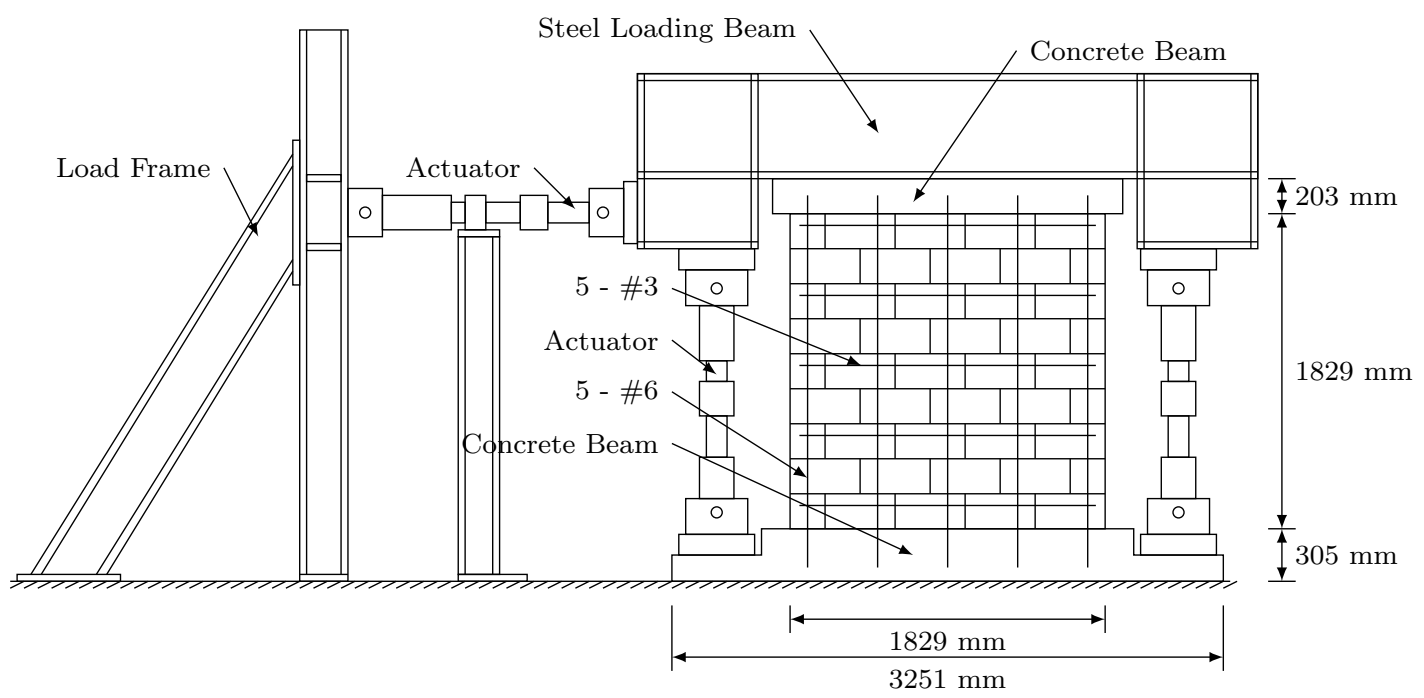

Experimental Wall Shing et al. (1991)

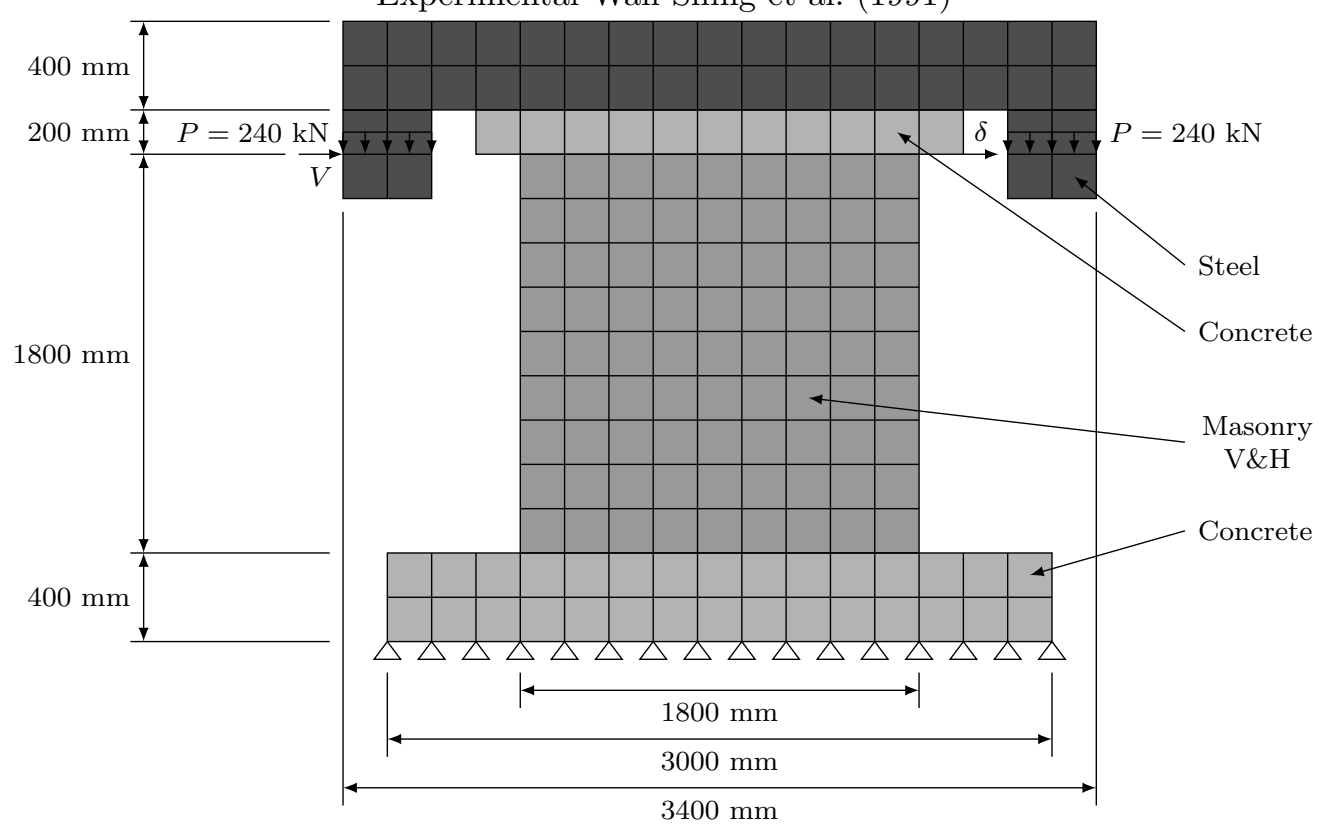

Finite Element Model Wall

HCBL 23 Shear Wall 
HCBL 23 Shear Wall Shing et al. (1991) Model Options

\begin{tabular}{ll}
\hline Model & Selected Option \\
\hline Compression Pre-Peak & Hognestad \\
Compression Post-Peak & Pre-Peak Base Curve \\
Compression Softening & Vecchio \\
Tension Post-Peak & Vecchio \\
Cracking Criterion & Mohr-Coulomb \\
Slip & Walraven Reinforced \\
\hline
\end{tabular}

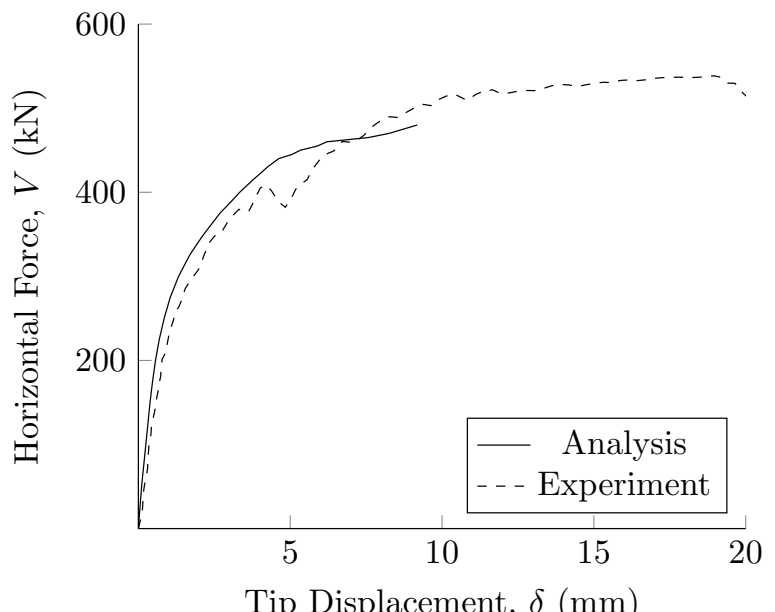

HCBL 23 Shear Wall Shing et al. (1991) Horizontal Force vs Tip Displacement 
HCBL 23 Shear Wall Shing et al. (1991) Material Properties

\begin{tabular}{|c|c|c|c|}
\hline \multirow{2}{*}{ Property } & \multicolumn{3}{|c|}{ Material } \\
\hline & Masonry V\&H & Concrete & Steel \\
\hline Thickness (mm) & 140 & 1220 & 400 \\
\hline Grout Tensile Strength (MPa) & 1.80 & 2.09 & 350 \\
\hline \multicolumn{4}{|c|}{ Masonry Properties (Y-Direction) } \\
\hline Gross Peak Compression Strength (MPa) & 28.3 & 40 & 350 \\
\hline Peak Compressive Strain & 0.003 & 0.0021 & 0.00175 \\
\hline Tensile Strength & 0 & 2.09 & 350 \\
\hline Tensile Strain & 0 & 0 & 0.00175 \\
\hline Initial Tangent Elastic Modulus (MPa) & 0 & 34600 & 200000 \\
\hline Poisson's Ratio & 0 & 0 & 0 \\
\hline \multicolumn{4}{|c|}{ Masonry Properties (X-Direction) } \\
\hline Gross Peak Compression Strength (MPa) & 28.3 & 40 & 350 \\
\hline Peak Compressive Strain & 0.003 & 0.0021 & 0.00175 \\
\hline Tensile Strength & 0 & 2.09 & 350 \\
\hline Tensile Strain & 0 & 0 & 0.00175 \\
\hline Initial Tangent Elastic Modulus (MPa) & 0 & 34600 & 200000 \\
\hline Poisson's Ratio & 0 & 0 & 0 \\
\hline \multicolumn{4}{|c|}{ Masonry Properties (Block) } \\
\hline Length (mm) & 390 & 2400 & 2000 \\
\hline Height (mm) & 190 & 400 & 400 \\
\hline Face Shell Thickness (mm) & 32 & 610 & 200 \\
\hline Percent Solid (\%) & 59 & 100 & 100 \\
\hline Tensile Strength & 1.75 & 2.09 & 350 \\
\hline \multicolumn{4}{|c|}{ Head Joint } \\
\hline Thickness (mm) & 10 & 10 & 10 \\
\hline Tensile Bond Strength (MPa) & 0.34 & 2.09 & 350 \\
\hline Joint Cohesion (MPa) & 6.13 & 2.09 & 350 \\
\hline Angle of Internal Friction (Deg) & 42 & 42 & 42 \\
\hline \multicolumn{4}{|c|}{ Bed Joint } \\
\hline Thickness (mm) & 10 & 10 & 10 \\
\hline Tensile Bond Strength (MPa) & 0.34 & 2.09 & 350 \\
\hline Joint Cohesion (MPa) & 6.13 & 2.09 & 350 \\
\hline Angle of Internal Friction (Deg) & 42 & 42 & 42 \\
\hline \multicolumn{4}{|c|}{ Reinforcement (Y-Direction) } \\
\hline Ratio (\%) & 0.54 & 1.38 & 100 \\
\hline Yield Strength (MPa) & 448 & 400 & 350 \\
\hline Yield Strain & 0.00224 & 0.002 & 0.00175 \\
\hline Strain Hardening Strain & 0.02 & 0.02 & 0.02 \\
\hline Ultimate Strength (MPa) & 738 & 600 & 600 \\
\hline Ultimate Strain & 0.2 & 0.2 & 0.2 \\
\hline Prestrain & 0 & 0 & 0 \\
\hline \multicolumn{4}{|c|}{ Reinforcement (X-Direction) } \\
\hline Ratio (\%) & 0.14 & 1.11 & 100 \\
\hline Yield Strength (MPa) & 386 & 400 & 350 \\
\hline Yield Strain & 0.00193 & 0.002 & 0.00175 \\
\hline Strain Hardening Strain & 0.02 & 0.02 & 0.02 \\
\hline Ultimate Strength (MPa) & 558 & 600 & 600 \\
\hline Ultimate Strain & 0.2 & 0.2 & 0.2 \\
\hline Prestrain & 0 & 0 & 0 \\
\hline
\end{tabular}

Numerical Modelling of Hollow Concrete Block Masonry Mechanics 


\section{HCBL 24 Shear Wall}

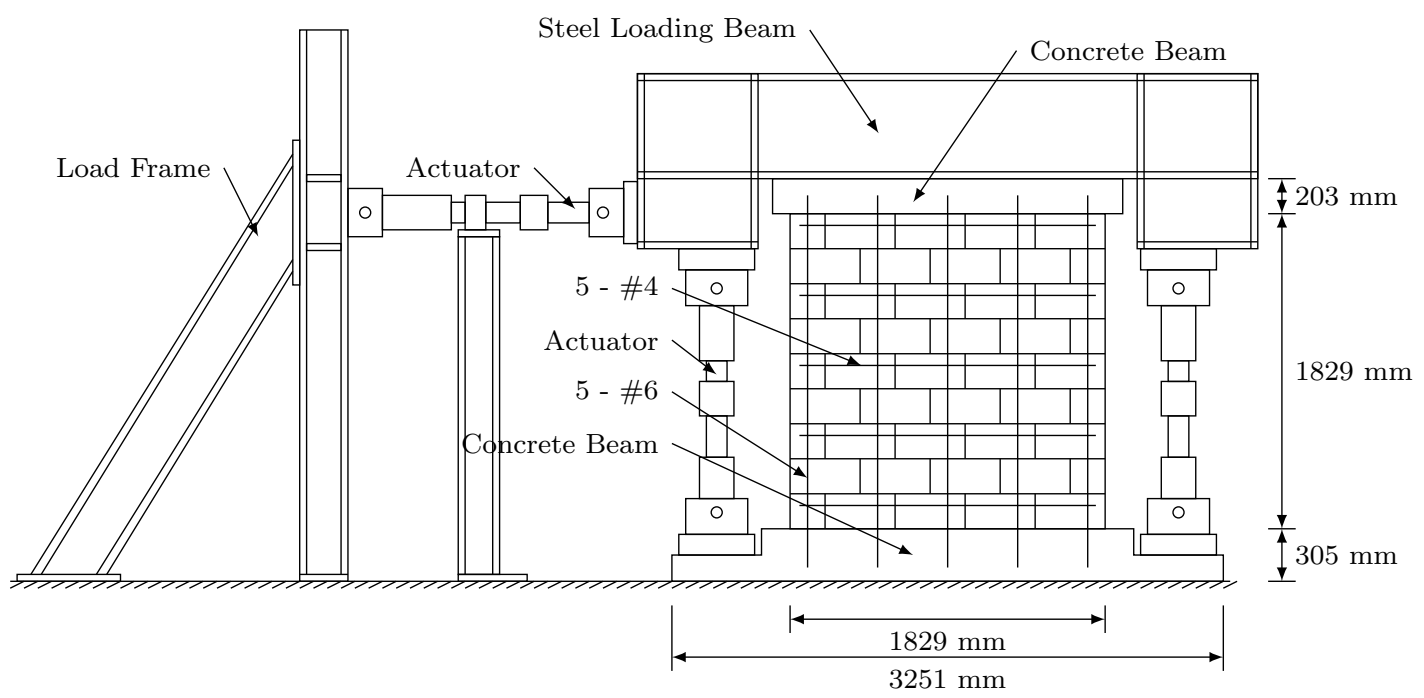

Experimental Wall Shing et al. (1991)

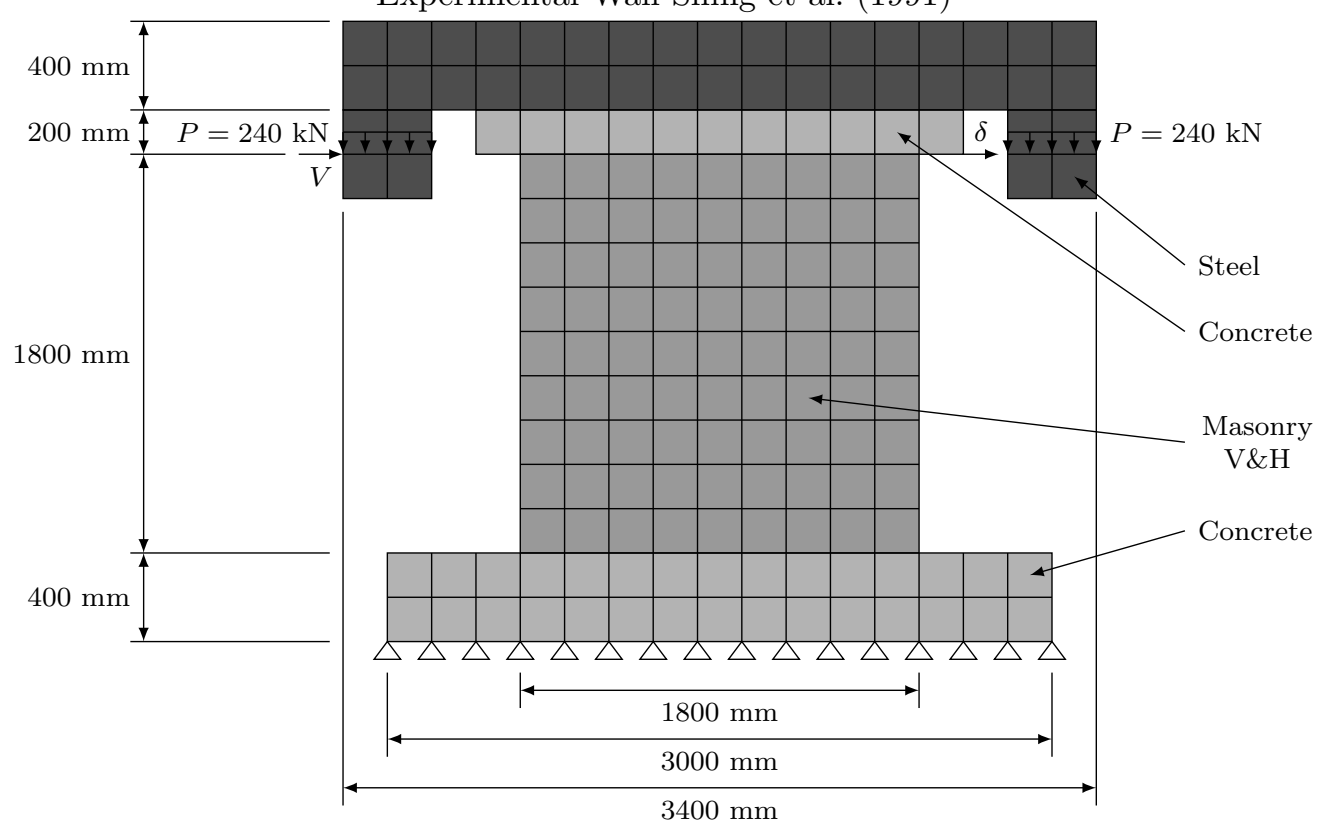

Finite Element Model Wall

HCBL 24 Shear Wall 
HCBL 24 Shear Wall Shing et al. (1991) Model Options

\begin{tabular}{ll}
\hline Model & Selected Option \\
\hline Compression Pre-Peak & Hognestad \\
Compression Post-Peak & Pre-Peak Base Curve \\
Compression Softening & Vecchio \\
Tension Post-Peak & Vecchio \\
Cracking Criterion & Mohr-Coulomb \\
Slip & Walraven Reinforced \\
\hline
\end{tabular}

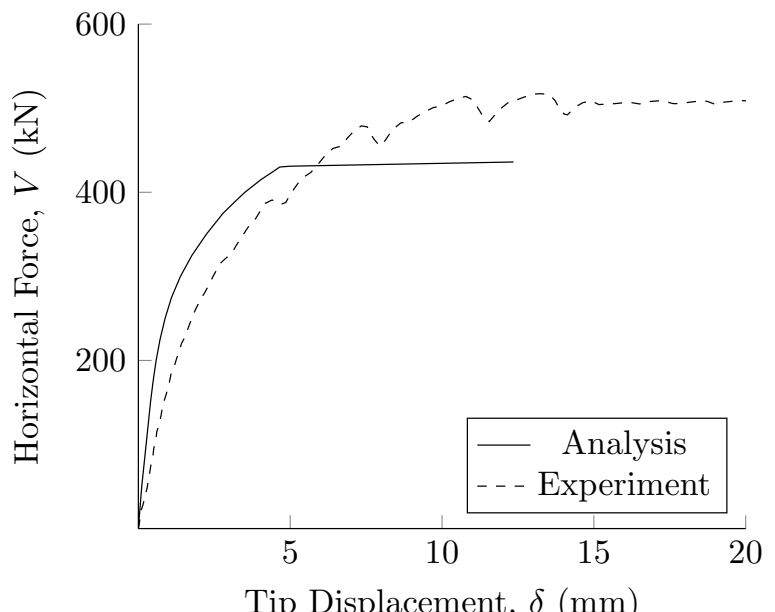

HCBL 24 Shear Wall Shing et al. (1991) Horizontal Force vs Tip Displacement 
HCBL 24 Shear Wall Shing et al. (1991) Material Properties

\begin{tabular}{|c|c|c|c|}
\hline \multirow{2}{*}{ Property } & \multicolumn{3}{|c|}{ Material } \\
\hline & Masonry V\&H & Concrete & Steel \\
\hline Thickness (mm) & 140 & 1220 & 400 \\
\hline Grout Tensile Strength (MPa) & 1.80 & 2.09 & 350 \\
\hline \multicolumn{4}{|c|}{ Masonry Properties (Y-Direction) } \\
\hline Gross Peak Compression Strength (MPa) & 19.1 & 40 & 350 \\
\hline Peak Compressive Strain & 0.0021 & 0.0021 & 0.00175 \\
\hline Tensile Strength & 0 & 2.09 & 350 \\
\hline Tensile Strain & 0 & 0 & 0.00175 \\
\hline Initial Tangent Elastic Modulus (MPa) & 0 & 34600 & 200000 \\
\hline Poisson's Ratio & 0 & 0 & 0 \\
\hline \multicolumn{4}{|c|}{ Masonry Properties (X-Direction) } \\
\hline Gross Peak Compression Strength (MPa) & 19.1 & 40 & 350 \\
\hline Peak Compressive Strain & 0.0021 & 0.0021 & 0.00175 \\
\hline Tensile Strength & 0 & 2.09 & 350 \\
\hline Tensile Strain & 0 & 0 & 0.00175 \\
\hline Initial Tangent Elastic Modulus (MPa) & 0 & 34600 & 200000 \\
\hline Poisson's Ratio & 0 & 0 & 0 \\
\hline \multicolumn{4}{|c|}{ Masonry Properties (Block) } \\
\hline Length (mm) & 390 & 2400 & 2000 \\
\hline Height (mm) & 190 & 400 & 400 \\
\hline Face Shell Thickness (mm) & 32 & 610 & 200 \\
\hline Percent Solid (\%) & 59 & 100 & 100 \\
\hline Tensile Strength & 1.75 & 2.09 & 350 \\
\hline \multicolumn{4}{|c|}{ Head Joint } \\
\hline Thickness (mm) & 10 & 10 & 10 \\
\hline Tensile Bond Strength (MPa) & 0.34 & 2.09 & 350 \\
\hline Joint Cohesion (MPa) & 6.13 & 2.09 & 350 \\
\hline Angle of Internal Friction (Deg) & 42 & 42 & 42 \\
\hline \multicolumn{4}{|c|}{ Bed Joint } \\
\hline Thickness (mm) & 10 & 10 & 10 \\
\hline Tensile Bond Strength (MPa) & 0.34 & 2.09 & 350 \\
\hline Joint Cohesion (MPa) & 6.13 & 2.09 & 350 \\
\hline Angle of Internal Friction (Deg) & 42 & 42 & 42 \\
\hline \multicolumn{4}{|c|}{ Reinforcement (Y-Direction) } \\
\hline Ratio (\%) & 0.54 & 1.38 & 100 \\
\hline Yield Strength (MPa) & 448 & 400 & 350 \\
\hline Yield Strain & 0.00224 & 0.002 & 0.00175 \\
\hline Strain Hardening Strain & 0.02 & 0.02 & 0.02 \\
\hline Ultimate Strength (MPa) & 738 & 600 & 600 \\
\hline Ultimate Strain & 0.2 & 0.2 & 0.2 \\
\hline Prestrain & 0 & 0 & 0 \\
\hline \multicolumn{4}{|c|}{ Reinforcement (X-Direction) } \\
\hline Ratio (\%) & 0.24 & 1.11 & 100 \\
\hline Yield Strength (MPa) & 462 & 400 & 350 \\
\hline Yield Strain & 0.00231 & 0.002 & 0.00175 \\
\hline Strain Hardening Strain & 0.02 & 0.02 & 0.02 \\
\hline Ultimate Strength (MPa) & 738 & 600 & 600 \\
\hline Ultimate Strain & 0.2 & 0.2 & 0.2 \\
\hline Prestrain & 0 & 0 & 0 \\
\hline
\end{tabular}

Numerical Modelling of Hollow Concrete Block Masonry Mechanics 


\section{Ibrahim (1995)}

\section{Wall 1 Shear Wall}

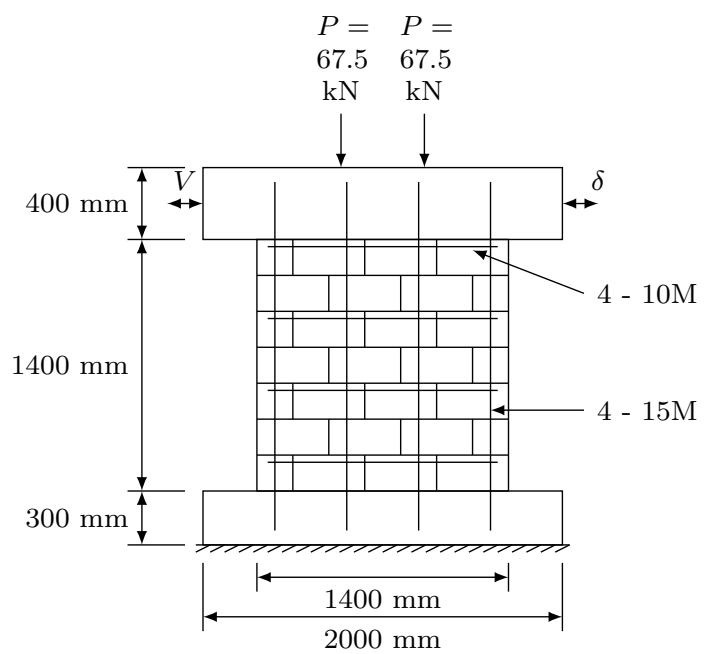

Experimental Wall Ibrahim (1995)

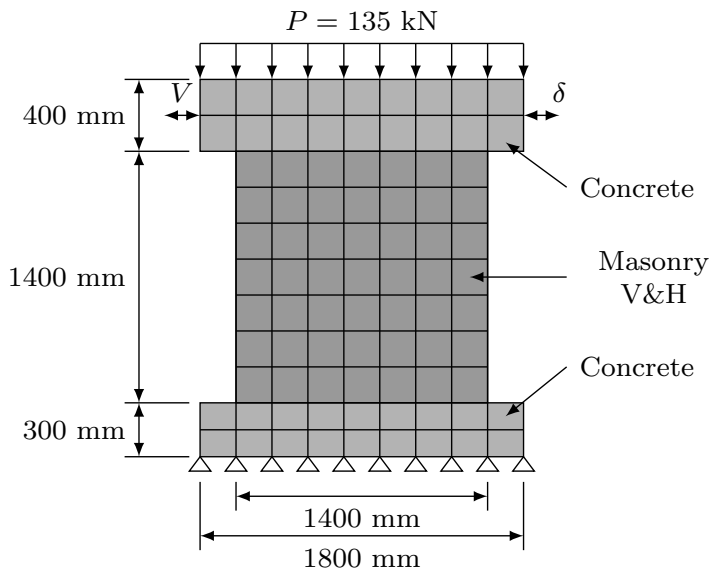

Finite Element Model Wall

Wall 1 Shear Wall

Wall 1 Shear Wall Ibrahim (1995) Model Options

\begin{tabular}{ll}
\hline Model & Selected Option \\
\hline Compression Pre-Peak & Hognestad \\
Compression Post-Peak & Pre-Peak Base Curve \\
Compression Softening & Vecchio \\
Tension Post-Peak & Vecchio \\
Cracking Criterion & Mohr-Coulomb \\
Slip & Walraven Reinforced \\
\hline
\end{tabular}




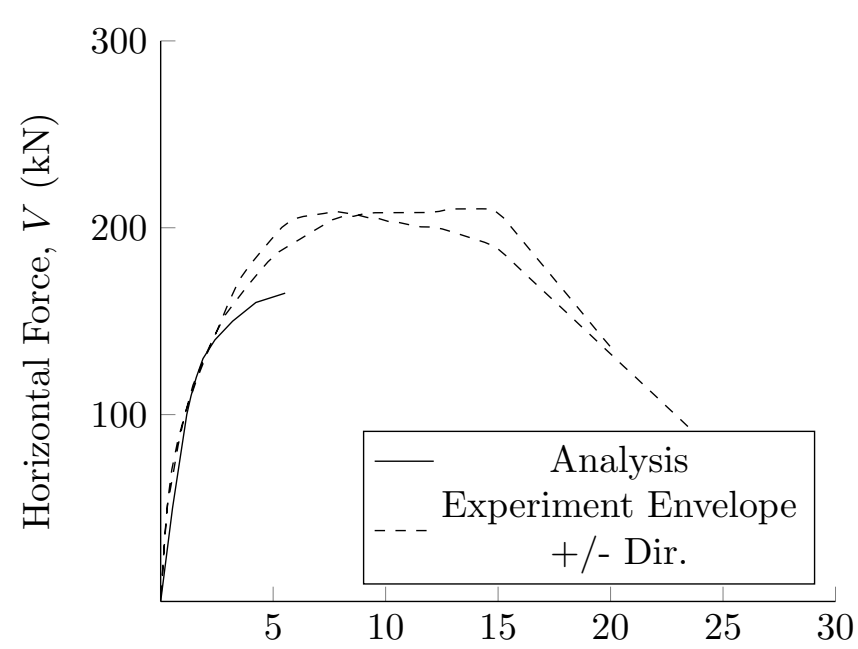

Tip Displacement, $\delta(\mathrm{mm})$

Wall 1 Shear Wall Ibrahim (1995) Horizontal Force vs Tip Displacement 
Wall 1 Shear Wall Ibrahim (1995) Material Properties

\begin{tabular}{|c|c|c|}
\hline \multirow{2}{*}{ Property } & \multicolumn{2}{|c|}{ Material } \\
\hline & Masonry V\&H & Concrete \\
\hline Thickness (mm) & 140 & 1100 \\
\hline Grout Tensile Strength (MPa) & 1.62 & 2.09 \\
\hline \multicolumn{3}{|c|}{ Masonry Properties (Y-Direction) } \\
\hline Gross Peak Compression Strength (MPa) & 12.1 & 40 \\
\hline Peak Compressive Strain & 0.002 & 0.0021 \\
\hline Tensile Strength & 0 & 2.09 \\
\hline Tensile Strain & 0 & 0 \\
\hline Initial Tangent Elastic Modulus (MPa) & 5300 & 34800 \\
\hline Poisson's Ratio & 0 & 0 \\
\hline \multicolumn{3}{|c|}{ Masonry Properties (X-Direction) } \\
\hline Gross Peak Compression Strength (MPa) & 12.1 & 40 \\
\hline Peak Compressive Strain & 0.002 & 0.0021 \\
\hline Tensile Strength & 0 & 2.09 \\
\hline Tensile Strain & 0 & 0 \\
\hline Initial Tangent Elastic Modulus (MPa) & 5300 & 34800 \\
\hline Poisson's Ratio & 0 & 0 \\
\hline \multicolumn{3}{|c|}{ Masonry Properties (Block) } \\
\hline Length (mm) & 390 & 1800 \\
\hline Height (mm) & 190 & 400 \\
\hline Face Shell Thickness (mm) & 45 & 550 \\
\hline Percent Solid (\%) & 60 & 100 \\
\hline Tensile Strength & 1.60 & 2.09 \\
\hline \multicolumn{3}{|l|}{ Head Joint } \\
\hline Thickness (mm) & 10 & 10 \\
\hline Tensile Bond Strength (MPa) & 0.28 & 2.09 \\
\hline Joint Cohesion (MPa) & 3.84 & 2.09 \\
\hline Angle of Internal Friction (Deg) & 42 & 42 \\
\hline \multicolumn{3}{|l|}{ Bed Joint } \\
\hline Thickness (mm) & 10 & 10 \\
\hline Tensile Bond Strength (MPa) & 0.28 & 2.09 \\
\hline Joint Cohesion (MPa) & 3.84 & 2.09 \\
\hline Angle of Internal Friction (Deg) & 42 & 42 \\
\hline \multicolumn{3}{|c|}{ Reinforcement (Y-Direction) } \\
\hline Ratio (\%) & 0.40 & 0.48 \\
\hline Yield Strength (MPa) & 440 & 440 \\
\hline Yield Strain & 0.0022 & 0.0022 \\
\hline Strain Hardening Strain & 0.02 & 0.02 \\
\hline Ultimate Strength (MPa) & 688 & 688 \\
\hline Ultimate Strain & 0.2 & 0.2 \\
\hline Prestrain & 0 & 0 \\
\hline \multicolumn{3}{|c|}{ Reinforcement (X-Direction) } \\
\hline Ratio (\%) & 0.20 & 1.64 \\
\hline Yield Strength (MPa) & 436 & 436 \\
\hline Yield Strain & 0.00218 & 0.00218 \\
\hline Strain Hardening Strain & 0.02 & 0.02 \\
\hline Ultimate Strength (MPa) & 654 & 654 \\
\hline Ultimate Strain & 0.2 & 0.2 \\
\hline Prestrain & 0 & 0 \\
\hline
\end{tabular}

Numerical Modelling of Hollow Concrete Block Masonry Mechanics 


\section{Wall 2 Shear Wall}

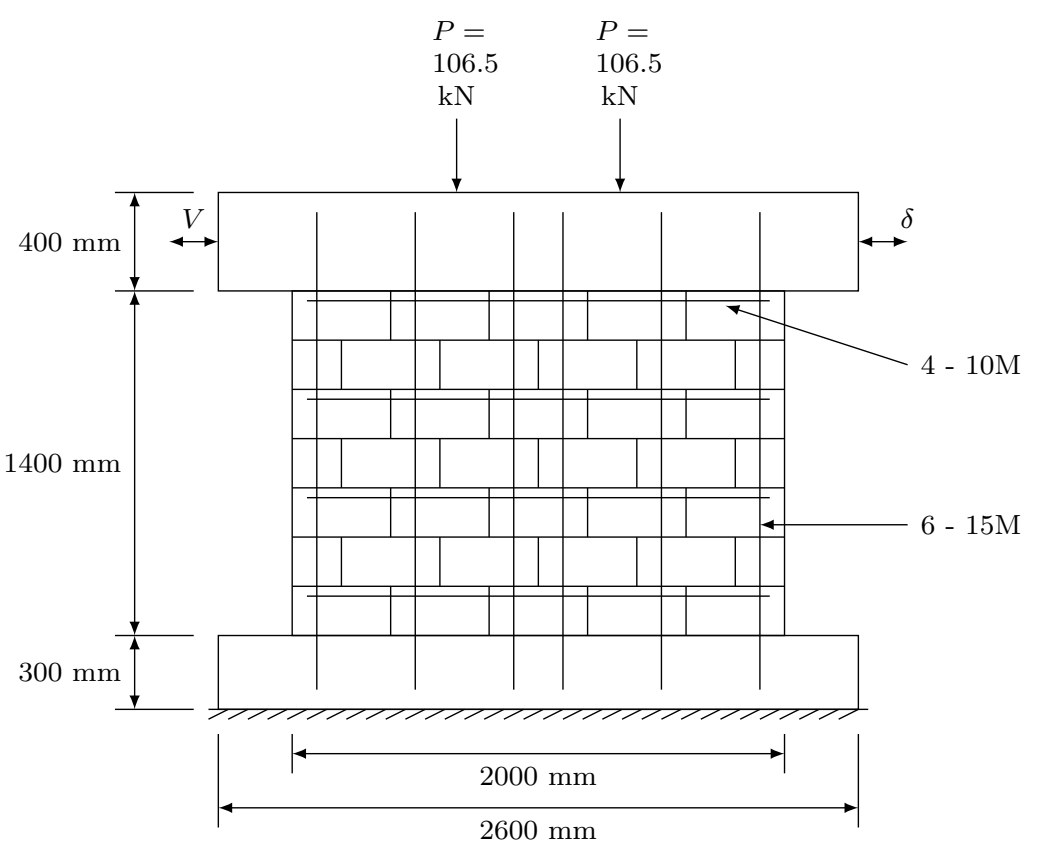

Experimental Wall Ibrahim (1995)

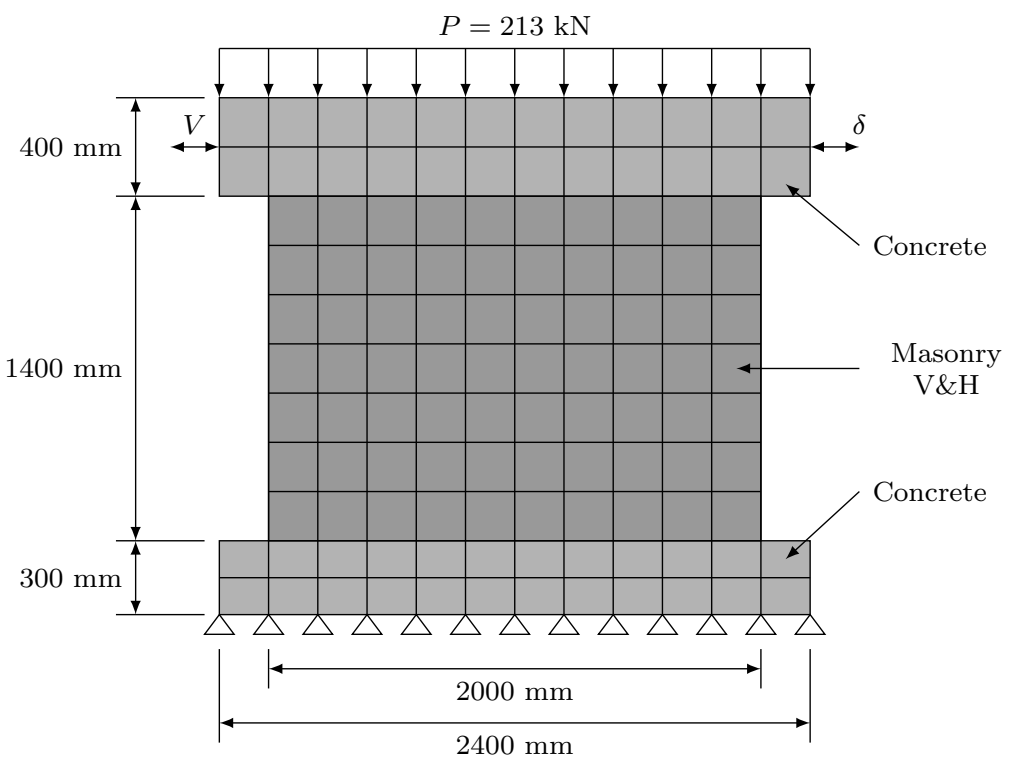

Finite Element Model Wall

Wall 2 Shear Wall 
Wall 2 Shear Wall Ibrahim (1995) Model Options

\begin{tabular}{ll}
\hline Model & Selected Option \\
\hline Compression Pre-Peak & Hognestad \\
Compression Post-Peak & Pre-Peak Base Curve \\
Compression Softening & Vecchio \\
Tension Post-Peak & Vecchio \\
Cracking Criterion & Mohr-Coulomb \\
Slip & Walraven Reinforced \\
\hline
\end{tabular}

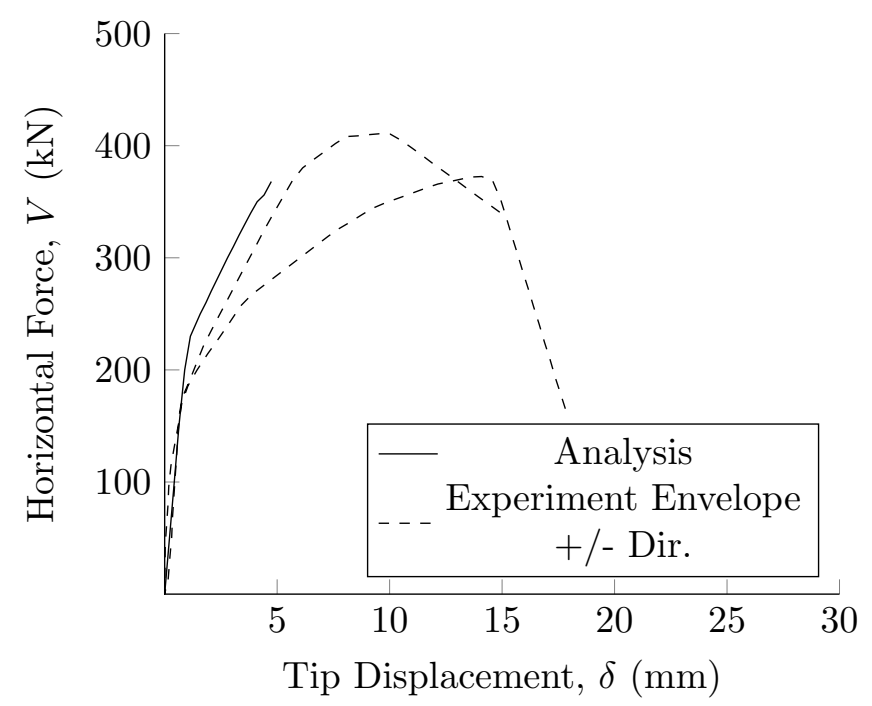

Wall 2 Shear Wall Ibrahim (1995) Horizontal Force vs Tip Displacement 
Wall 2 Shear Wall Ibrahim (1995) Material Properties

\begin{tabular}{|c|c|c|}
\hline \multirow{2}{*}{ Property } & \multicolumn{2}{|c|}{ Material } \\
\hline & Masonry V\&H & Concrete \\
\hline Thickness (mm) & 140 & 1100 \\
\hline Grout Tensile Strength (MPa) & 1.62 & 2.09 \\
\hline \multicolumn{3}{|c|}{ Masonry Properties (Y-Direction) } \\
\hline Gross Peak Compression Strength (MPa) & 12.1 & 40 \\
\hline Peak Compressive Strain & 0.002 & 0.0021 \\
\hline Tensile Strength & 0 & 2.09 \\
\hline Tensile Strain & 0 & 0 \\
\hline Initial Tangent Elastic Modulus (MPa) & 5000 & 34800 \\
\hline Poisson's Ratio & 0 & 0 \\
\hline \multicolumn{3}{|c|}{ Masonry Properties (X-Direction) } \\
\hline Gross Peak Compression Strength (MPa) & 12.1 & 40 \\
\hline Peak Compressive Strain & 0.002 & 0.0021 \\
\hline Tensile Strength & 0 & 2.09 \\
\hline Tensile Strain & 0 & 0 \\
\hline Initial Tangent Elastic Modulus (MPa) & 5000 & 34800 \\
\hline Poisson's Ratio & 0 & 0 \\
\hline \multicolumn{3}{|c|}{ Masonry Properties (Block) } \\
\hline Length (mm) & 390 & 1800 \\
\hline Height (mm) & 190 & 400 \\
\hline Face Shell Thickness (mm) & 45 & 550 \\
\hline Percent Solid (\%) & 60 & 100 \\
\hline Tensile Strength & 1.60 & 2.09 \\
\hline \multicolumn{3}{|l|}{ Head Joint } \\
\hline Thickness (mm) & 10 & 10 \\
\hline Tensile Bond Strength (MPa) & 0.28 & 2.09 \\
\hline Joint Cohesion (MPa) & 3.84 & 2.09 \\
\hline Angle of Internal Friction (Deg) & 42 & 42 \\
\hline \multicolumn{3}{|l|}{ Bed Joint } \\
\hline Thickness (mm) & 10 & 10 \\
\hline Tensile Bond Strength (MPa) & 0.28 & 2.09 \\
\hline Joint Cohesion (MPa) & 3.84 & 2.09 \\
\hline Angle of Internal Friction (Deg) & 42 & 42 \\
\hline \multicolumn{3}{|c|}{ Reinforcement (Y-Direction) } \\
\hline Ratio (\%) & 0.40 & 0.48 \\
\hline Yield Strength (MPa) & 440 & 440 \\
\hline Yield Strain & 0.0022 & 0.0022 \\
\hline Strain Hardening Strain & 0.02 & 0.02 \\
\hline Ultimate Strength (MPa) & 688 & 688 \\
\hline Ultimate Strain & 0.2 & 0.2 \\
\hline Prestrain & 0 & 0 \\
\hline \multicolumn{3}{|c|}{ Reinforcement (X-Direction) } \\
\hline Ratio (\%) & 0.20 & 1.64 \\
\hline Yield Strength (MPa) & 436 & 436 \\
\hline Yield Strain & 0.00218 & 0.00218 \\
\hline Strain Hardening Strain & 0.02 & 0.02 \\
\hline Ultimate Strength (MPa) & 654 & 654 \\
\hline Ultimate Strain & 0.2 & 0.2 \\
\hline Prestrain & 0 & 0 \\
\hline
\end{tabular}

Numerical Modelling of Hollow Concrete Block Masonry Mechanics 


\section{Wall 3 Shear Wall}

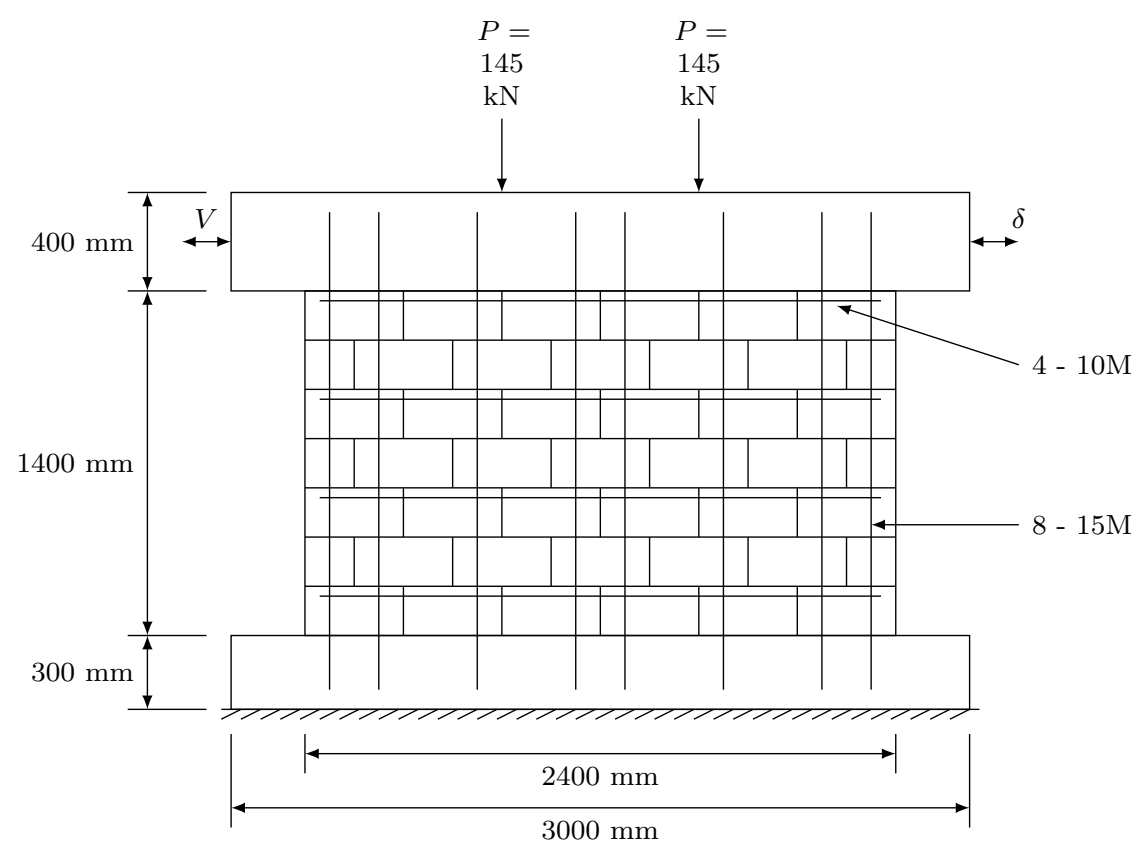

Experimental Wall Ibrahim (1995)

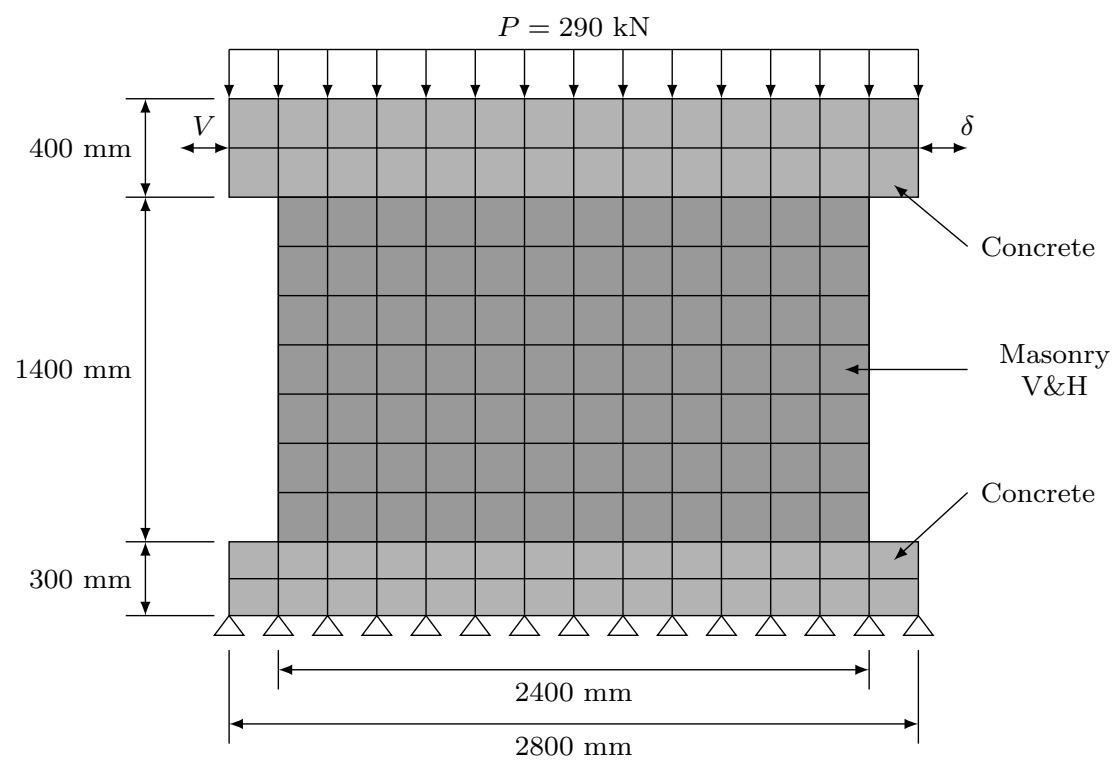

Finite Element Model Wall

Wall 3 Shear Wall 
Wall 3 Shear Wall Ibrahim (1995) Model Options

\begin{tabular}{ll}
\hline Model & Selected Option \\
\hline Compression Pre-Peak & Hognestad \\
Compression Post-Peak & Pre-Peak Base Curve \\
Compression Softening & Vecchio \\
Tension Post-Peak & Vecchio \\
Cracking Criterion & Mohr-Coulomb \\
Slip & Walraven Reinforced \\
\hline
\end{tabular}

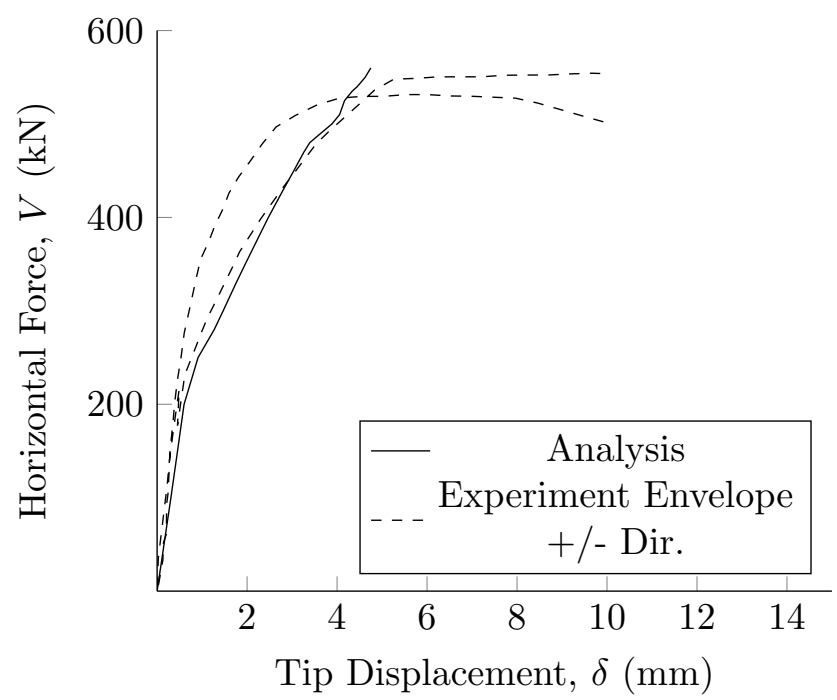

Wall 3 Shear Wall Ibrahim (1995) Horizontal Force vs Tip Displacement 
Wall 3 Shear Wall Ibrahim (1995) Material Properties

\begin{tabular}{|c|c|c|}
\hline \multirow{2}{*}{ Property } & \multicolumn{2}{|c|}{ Material } \\
\hline & Masonry V\&H & Concrete \\
\hline Thickness (mm) & 140 & 1100 \\
\hline Grout Tensile Strength (MPa) & 1.62 & 2.09 \\
\hline \multicolumn{3}{|c|}{ Masonry Properties (Y-Direction) } \\
\hline Gross Peak Compression Strength (MPa) & 12.1 & 40 \\
\hline Peak Compressive Strain & 0.002 & 0.0021 \\
\hline Tensile Strength & 0 & 2.09 \\
\hline Tensile Strain & 0 & 0 \\
\hline Initial Tangent Elastic Modulus (MPa) & 4000 & 34800 \\
\hline Poisson's Ratio & 0 & 0 \\
\hline \multicolumn{3}{|c|}{ Masonry Properties (X-Direction) } \\
\hline Gross Peak Compression Strength (MPa) & 12.1 & 40 \\
\hline Peak Compressive Strain & 0.002 & 0.0021 \\
\hline Tensile Strength & 0 & 2.09 \\
\hline Tensile Strain & 0 & 0 \\
\hline Initial Tangent Elastic Modulus (MPa) & 4000 & 34800 \\
\hline Poisson's Ratio & 0 & 0 \\
\hline \multicolumn{3}{|c|}{ Masonry Properties (Block) } \\
\hline Length (mm) & 390 & 1800 \\
\hline Height (mm) & 190 & 400 \\
\hline Face Shell Thickness (mm) & 45 & 550 \\
\hline Percent Solid (\%) & 60 & 100 \\
\hline Tensile Strength & 1.60 & 2.09 \\
\hline \multicolumn{3}{|l|}{ Head Joint } \\
\hline Thickness (mm) & 10 & 10 \\
\hline Tensile Bond Strength (MPa) & 0.28 & 2.09 \\
\hline Joint Cohesion (MPa) & 3.84 & 2.09 \\
\hline Angle of Internal Friction (Deg) & 42 & 42 \\
\hline \multicolumn{3}{|l|}{ Bed Joint } \\
\hline Thickness (mm) & 10 & 10 \\
\hline Tensile Bond Strength (MPa) & 0.28 & 2.09 \\
\hline Joint Cohesion (MPa) & 3.84 & 2.09 \\
\hline Angle of Internal Friction (Deg) & 42 & 42 \\
\hline \multicolumn{3}{|c|}{ Reinforcement (Y-Direction) } \\
\hline Ratio (\%) & 0.40 & 0.48 \\
\hline Yield Strength (MPa) & 440 & 440 \\
\hline Yield Strain & 0.0022 & 0.0022 \\
\hline Strain Hardening Strain & 0.02 & 0.02 \\
\hline Ultimate Strength (MPa) & 688 & 688 \\
\hline Ultimate Strain & 0.2 & 0.2 \\
\hline Prestrain & 0 & 0 \\
\hline \multicolumn{3}{|c|}{ Reinforcement (X-Direction) } \\
\hline Ratio (\%) & 0.20 & 1.64 \\
\hline Yield Strength (MPa) & 436 & 436 \\
\hline Yield Strain & 0.00218 & 0.00218 \\
\hline Strain Hardening Strain & 0.02 & 0.02 \\
\hline Ultimate Strength (MPa) & 654 & 654 \\
\hline Ultimate Strain & 0.2 & 0.2 \\
\hline Prestrain & 0 & 0 \\
\hline
\end{tabular}

Numerical Modelling of Hollow Concrete Block Masonry Mechanics 


\section{Wall 4 Shear Wall}

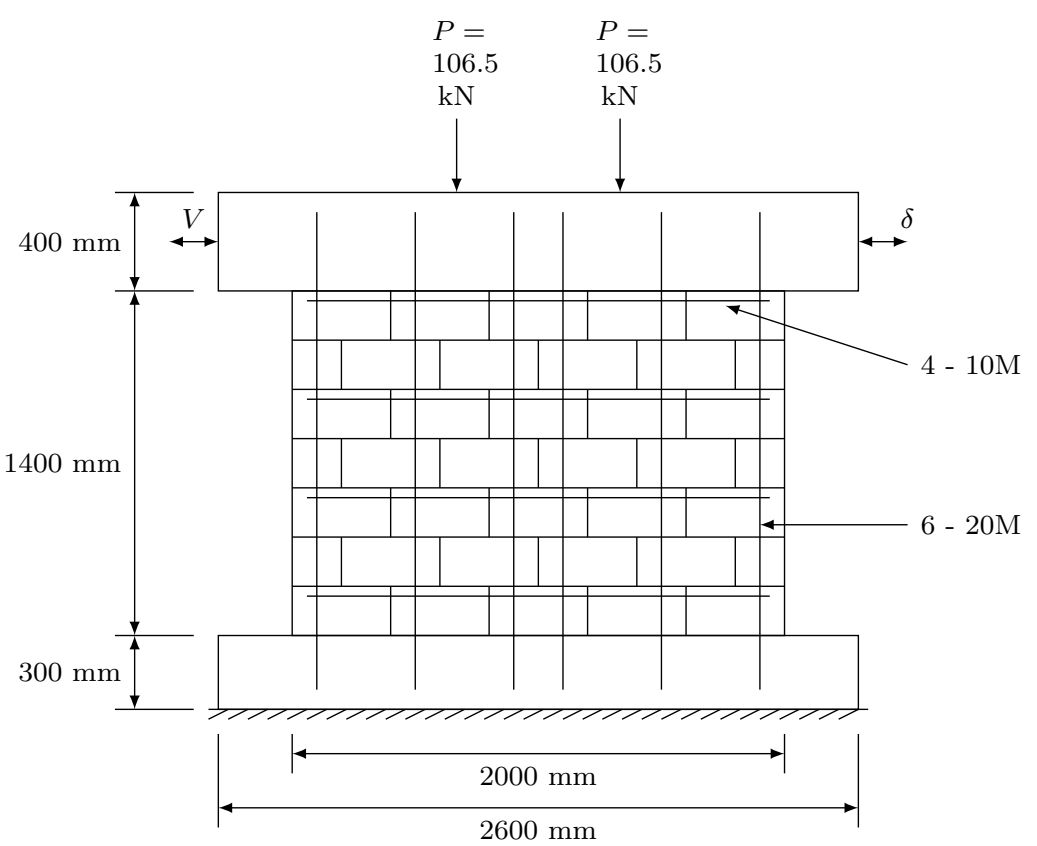

Experimental Wall Ibrahim (1995)

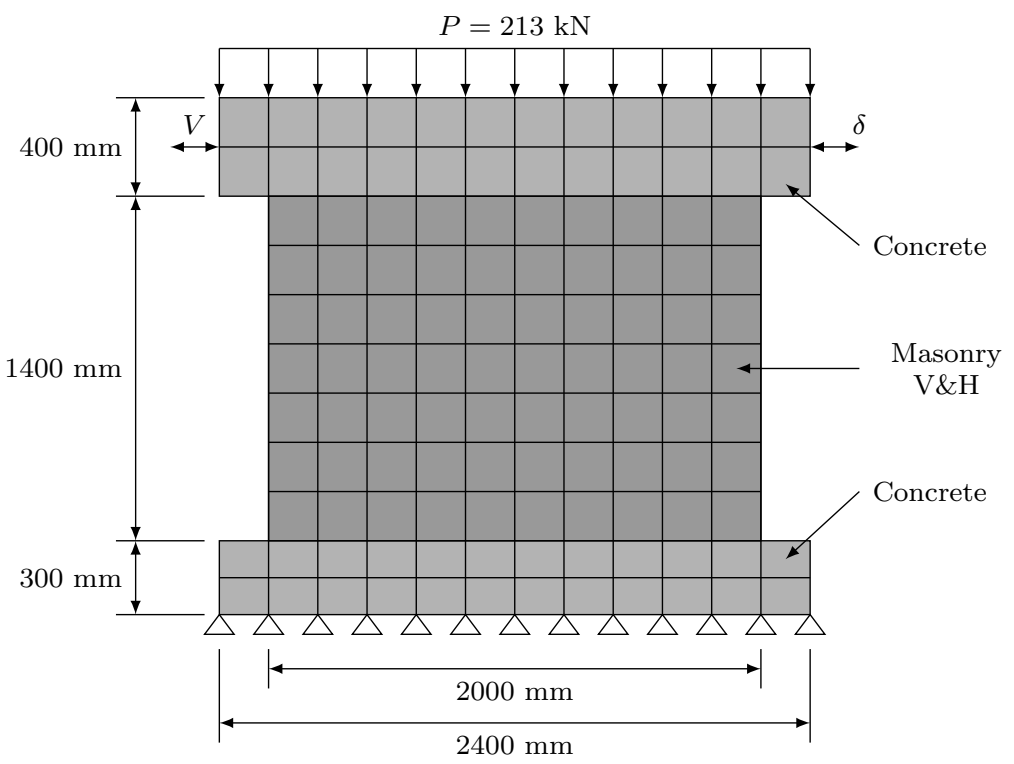

Finite Element Model Wall

Wall 4 Shear Wall 
Wall 4 Shear Wall Ibrahim (1995) Model Options

\begin{tabular}{ll}
\hline Model & Selected Option \\
\hline Compression Pre-Peak & Hognestad \\
Compression Post-Peak & Pre-Peak Base Curve \\
Compression Softening & Vecchio \\
Tension Post-Peak & Vecchio \\
Cracking Criterion & Mohr-Coulomb \\
Slip & Walraven Reinforced \\
\hline
\end{tabular}

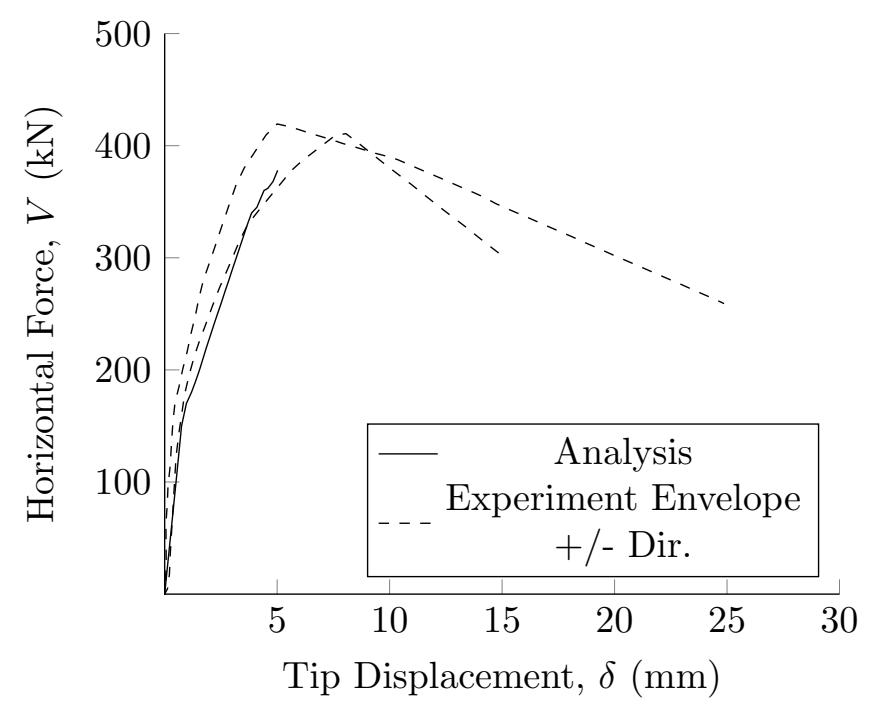

Wall 4 Shear Wall Ibrahim (1995) Horizontal Force vs Tip Displacement 
Wall 4 Shear Wall Ibrahim (1995) Material Properties

\begin{tabular}{|c|c|c|}
\hline \multirow{2}{*}{ Property } & \multicolumn{2}{|c|}{ Material } \\
\hline & Masonry V\&H & Concrete \\
\hline Thickness (mm) & 140 & 1100 \\
\hline Grout Tensile Strength (MPa) & 1.62 & 2.09 \\
\hline \multicolumn{3}{|c|}{ Masonry Properties (Y-Direction) } \\
\hline Gross Peak Compression Strength (MPa) & 12.1 & 40 \\
\hline Peak Compressive Strain & 0.002 & 0.0021 \\
\hline Tensile Strength & 0 & 2.09 \\
\hline Tensile Strain & 0 & 0 \\
\hline Initial Tangent Elastic Modulus (MPa) & 4000 & 34800 \\
\hline Poisson's Ratio & 0 & 0 \\
\hline \multicolumn{3}{|c|}{ Masonry Properties (X-Direction) } \\
\hline Gross Peak Compression Strength (MPa) & 12.1 & 40 \\
\hline Peak Compressive Strain & 0.002 & 0.0021 \\
\hline Tensile Strength & 0 & 2.09 \\
\hline Tensile Strain & 0 & 0 \\
\hline Initial Tangent Elastic Modulus (MPa) & 4000 & 34800 \\
\hline Poisson's Ratio & 0 & 0 \\
\hline \multicolumn{3}{|c|}{ Masonry Properties (Block) } \\
\hline Length (mm) & 390 & 1800 \\
\hline Height (mm) & 190 & 400 \\
\hline Face Shell Thickness (mm) & 45 & 550 \\
\hline Percent Solid (\%) & 60 & 100 \\
\hline Tensile Strength & 1.60 & 2.09 \\
\hline \multicolumn{3}{|l|}{ Head Joint } \\
\hline Thickness (mm) & 10 & 10 \\
\hline Tensile Bond Strength (MPa) & 0.28 & 2.09 \\
\hline Joint Cohesion (MPa) & 3.84 & 2.09 \\
\hline Angle of Internal Friction (Deg) & 42 & 42 \\
\hline \multicolumn{3}{|l|}{ Bed Joint } \\
\hline Thickness (mm) & 10 & 10 \\
\hline Tensile Bond Strength (MPa) & 0.28 & 2.09 \\
\hline Joint Cohesion (MPa) & 3.84 & 2.09 \\
\hline Angle of Internal Friction (Deg) & 42 & 42 \\
\hline \multicolumn{3}{|c|}{ Reinforcement (Y-Direction) } \\
\hline Ratio (\%) & 0.60 & 0.48 \\
\hline Yield Strength (MPa) & 438 & 440 \\
\hline Yield Strain & 0.00219 & 0.0022 \\
\hline Strain Hardening Strain & 0.02 & 0.02 \\
\hline Ultimate Strength (MPa) & 634 & 688 \\
\hline Ultimate Strain & 0.2 & 0.2 \\
\hline Prestrain & 0 & 0 \\
\hline \multicolumn{3}{|c|}{ Reinforcement (X-Direction) } \\
\hline Ratio (\%) & 0.20 & 1.64 \\
\hline Yield Strength (MPa) & 436 & 436 \\
\hline Yield Strain & 0.00218 & 0.00218 \\
\hline Strain Hardening Strain & 0.02 & 0.02 \\
\hline Ultimate Strength (MPa) & 654 & 654 \\
\hline Ultimate Strain & 0.2 & 0.2 \\
\hline Prestrain & 0 & 0 \\
\hline
\end{tabular}

Numerical Modelling of Hollow Concrete Block Masonry Mechanics 


\section{Wall 5 Shear Wall}

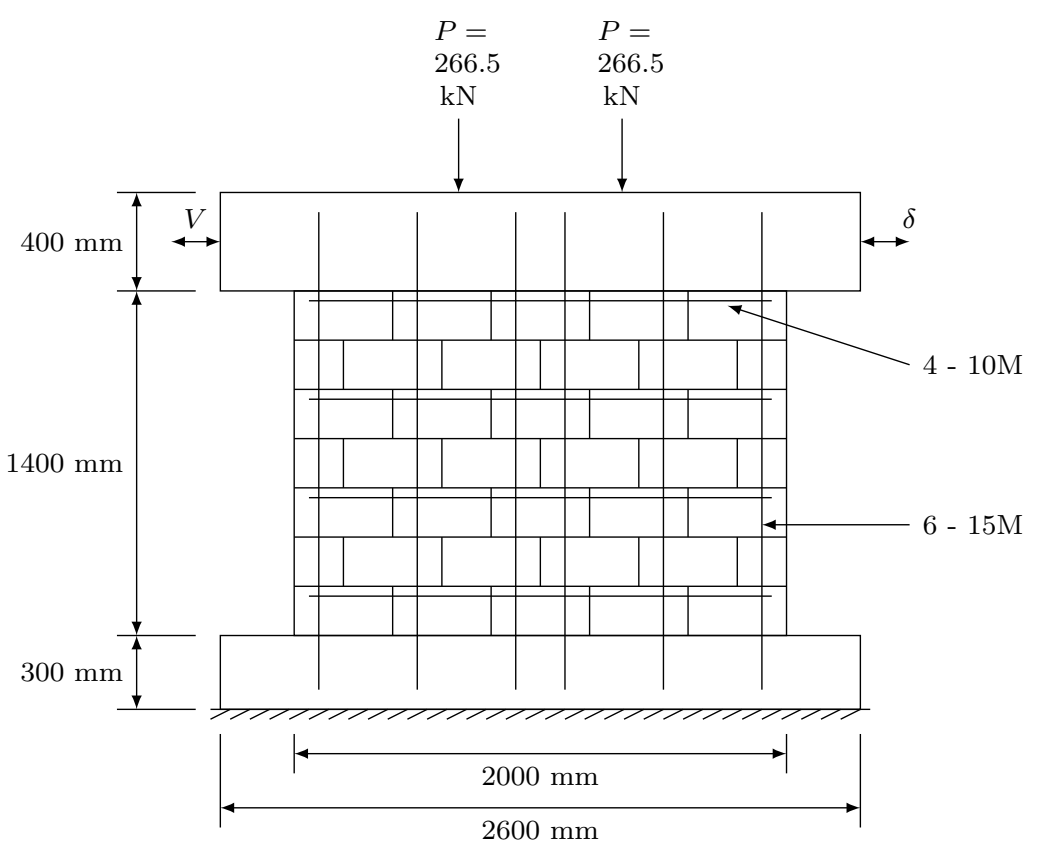

Experimental Wall Ibrahim (1995)

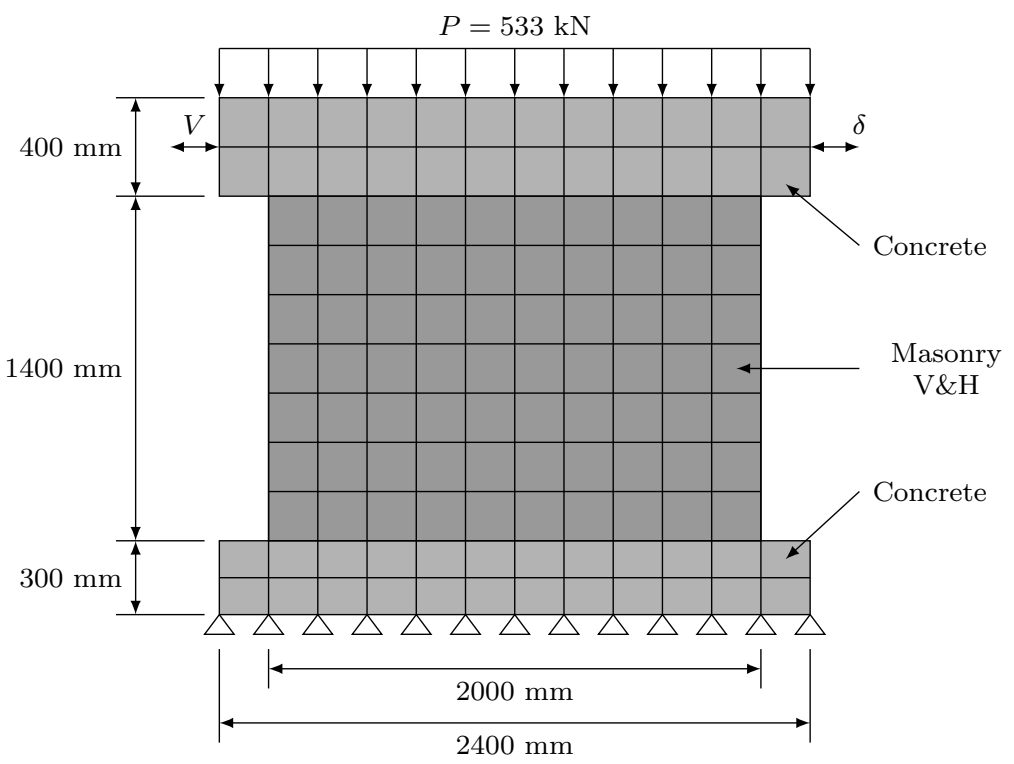

Finite Element Model Wall

Wall 5 Shear Wall 
Wall 5 Shear Wall Ibrahim (1995) Model Options

\begin{tabular}{ll}
\hline Model & Selected Option \\
\hline Compression Pre-Peak & Hognestad \\
Compression Post-Peak & Pre-Peak Base Curve \\
Compression Softening & Vecchio \\
Tension Post-Peak & Vecchio \\
Cracking Criterion & Mohr-Coulomb \\
Slip & Walraven Reinforced \\
\hline
\end{tabular}

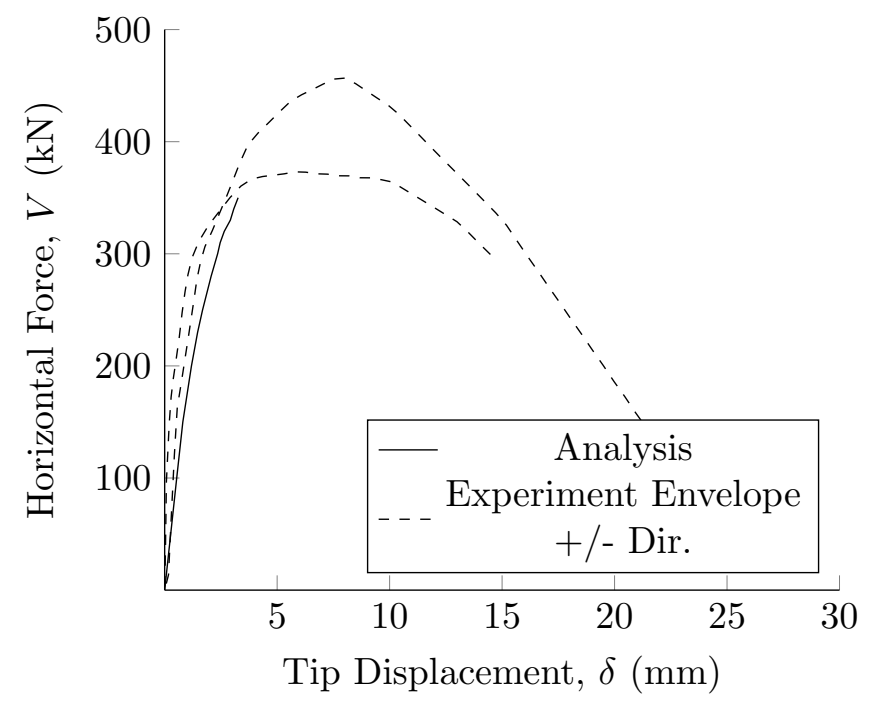

Wall 5 Shear Wall Ibrahim (1995) Horizontal Force vs Tip Displacement 
Wall 5 Shear Wall Ibrahim (1995) Material Properties

\begin{tabular}{|c|c|c|}
\hline \multirow{2}{*}{ Property } & \multicolumn{2}{|c|}{ Material } \\
\hline & Masonry V\&H & Concrete \\
\hline Thickness (mm) & 140 & 1100 \\
\hline Grout Tensile Strength (MPa) & 1.62 & 2.09 \\
\hline \multicolumn{3}{|c|}{ Masonry Properties (Y-Direction) } \\
\hline Gross Peak Compression Strength (MPa) & 12.1 & 40 \\
\hline Peak Compressive Strain & 0.002 & 0.0021 \\
\hline Tensile Strength & 0 & 2.09 \\
\hline Tensile Strain & 0 & 0 \\
\hline Initial Tangent Elastic Modulus (MPa) & 4000 & 34800 \\
\hline Poisson's Ratio & 0 & 0 \\
\hline \multicolumn{3}{|c|}{ Masonry Properties (X-Direction) } \\
\hline Gross Peak Compression Strength (MPa) & 12.1 & 40 \\
\hline Peak Compressive Strain & 0.002 & 0.0021 \\
\hline Tensile Strength & 0 & 2.09 \\
\hline Tensile Strain & 0 & 0 \\
\hline Initial Tangent Elastic Modulus (MPa) & 4000 & 34800 \\
\hline Poisson's Ratio & 0 & 0 \\
\hline \multicolumn{3}{|c|}{ Masonry Properties (Block) } \\
\hline Length (mm) & 390 & 1800 \\
\hline Height (mm) & 190 & 400 \\
\hline Face Shell Thickness (mm) & 45 & 550 \\
\hline Percent Solid (\%) & 60 & 100 \\
\hline Tensile Strength & 1.60 & 2.09 \\
\hline \multicolumn{3}{|l|}{ Head Joint } \\
\hline Thickness (mm) & 10 & 10 \\
\hline Tensile Bond Strength (MPa) & 0.28 & 2.09 \\
\hline Joint Cohesion (MPa) & 3.84 & 2.09 \\
\hline Angle of Internal Friction (Deg) & 42 & 42 \\
\hline \multicolumn{3}{|l|}{ Bed Joint } \\
\hline Thickness (mm) & 10 & 10 \\
\hline Tensile Bond Strength (MPa) & 0.28 & 2.09 \\
\hline Joint Cohesion (MPa) & 3.84 & 2.09 \\
\hline Angle of Internal Friction (Deg) & 42 & 42 \\
\hline \multicolumn{3}{|c|}{ Reinforcement (Y-Direction) } \\
\hline Ratio (\%) & 0.40 & 0.48 \\
\hline Yield Strength (MPa) & 440 & 440 \\
\hline Yield Strain & 0.0022 & 0.0022 \\
\hline Strain Hardening Strain & 0.02 & 0.02 \\
\hline Ultimate Strength (MPa) & 688 & 688 \\
\hline Ultimate Strain & 0.2 & 0.2 \\
\hline Prestrain & 0 & 0 \\
\hline \multicolumn{3}{|c|}{ Reinforcement (X-Direction) } \\
\hline Ratio (\%) & 0.20 & 1.64 \\
\hline Yield Strength (MPa) & 436 & 436 \\
\hline Yield Strain & 0.00218 & 0.00218 \\
\hline Strain Hardening Strain & 0.02 & 0.02 \\
\hline Ultimate Strength (MPa) & 654 & 654 \\
\hline Ultimate Strain & 0.2 & 0.2 \\
\hline Prestrain & 0 & 0 \\
\hline
\end{tabular}

Numerical Modelling of Hollow Concrete Block Masonry Mechanics 


\section{Voon (2007)}

\section{A1 Shear Wall}

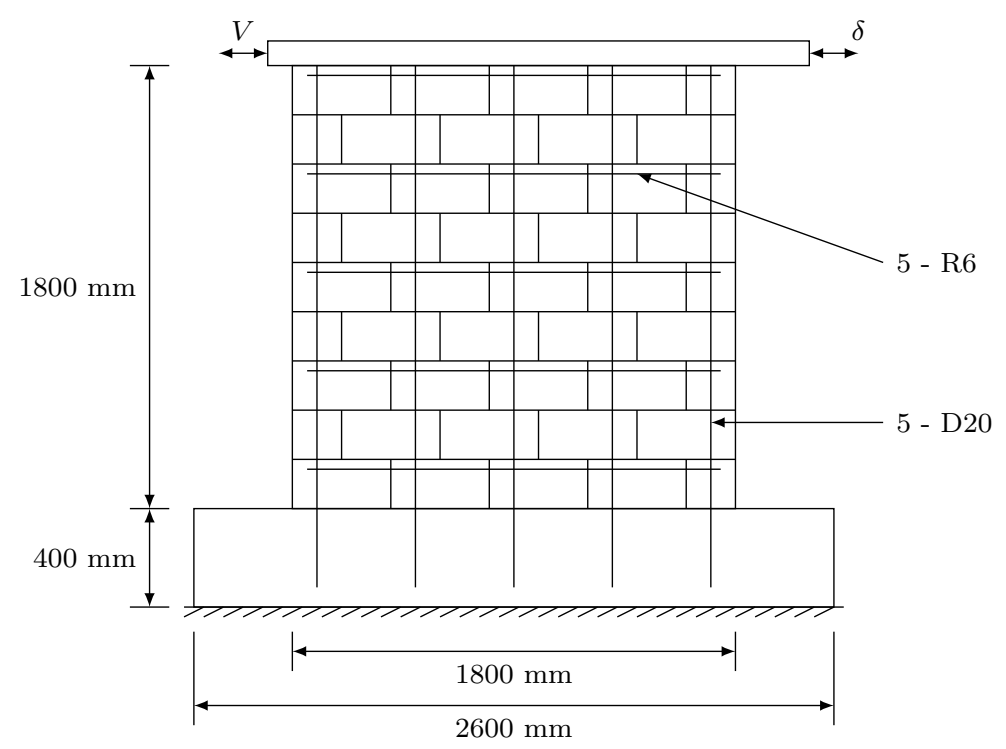

Experimental Wall Voon (2007)

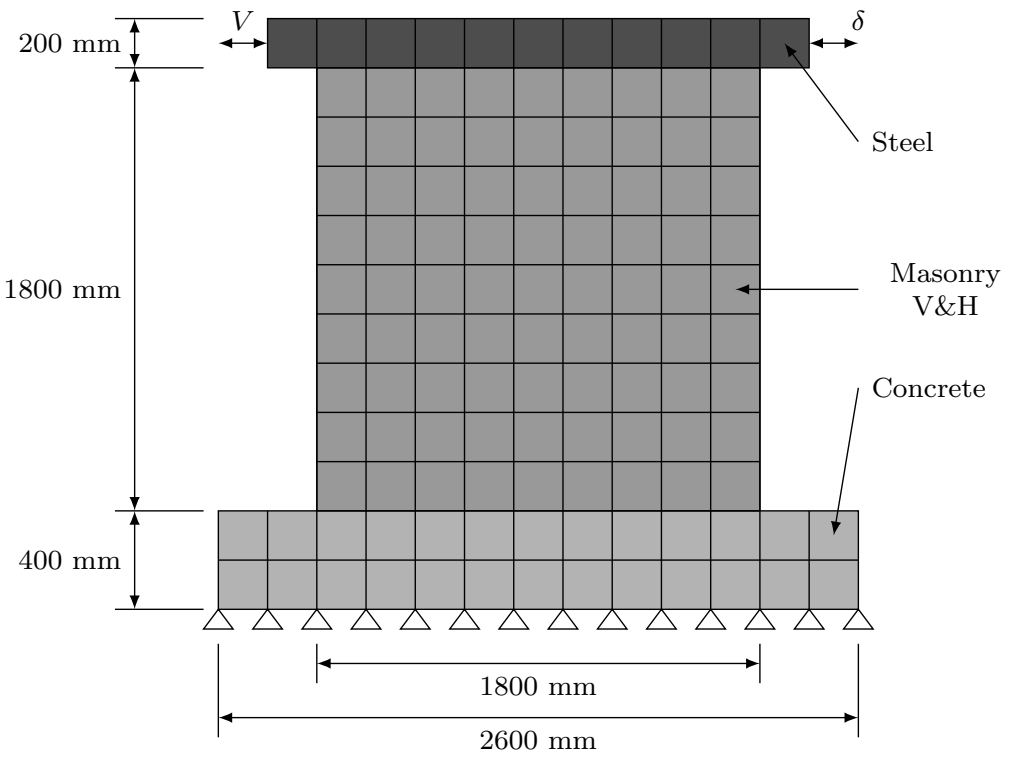

Finite Element Model Wall

A1 Shear Wall 
A1 Shear Wall Voon (2007) Material Properties

\begin{tabular}{|c|c|c|c|}
\hline \multirow{2}{*}{ Property } & \multicolumn{3}{|c|}{ Material } \\
\hline & Masonry & Concrete & Steel \\
\hline Thickness $(\mathrm{mm})$ & 140 & 600 & 400 \\
\hline Grout Tensile Strength (MPa) & 1.60 & 2.09 & 350 \\
\hline \multicolumn{4}{|c|}{ Masonry Properties (Y-Direction) } \\
\hline Gross Peak Compression Strength (MPa) & 17.6 & 40 & 350 \\
\hline Peak Compressive Strain & 0.0031 & 0.0021 & 0.00175 \\
\hline Tensile Strength & 0 & 2.09 & 350 \\
\hline Tensile Strain & 0 & 0 & 0 \\
\hline Initial Tangent Elastic Modulus (MPa) & 6500 & 34800 & 200000 \\
\hline Poisson's Ratio & 0 & 0.15 & 0 \\
\hline \multicolumn{4}{|c|}{ Masonry Properties (X-Direction) } \\
\hline Gross Peak Compression Strength (MPa) & 17.6 & 40 & 350 \\
\hline Peak Compressive Strain & 0.0031 & 0.0021 & 0.00175 \\
\hline Tensile Strength & 0 & 2.09 & 350 \\
\hline Tensile Strain & 0 & 0 & 0 \\
\hline Initial Tangent Elastic Modulus (MPa) & 6500 & 34800 & 200000 \\
\hline Poisson's Ratio & 0 & 0.15 & 0 \\
\hline \multicolumn{4}{|c|}{ Masonry Properties (Block) } \\
\hline Length (mm) & 390 & 2600 & 2000 \\
\hline Height (mm) & 190 & 400 & 200 \\
\hline Face Shell Thickness (mm) & 31 & 300 & 200 \\
\hline Percent Solid (\%) & 58 & 100 & 100 \\
\hline Tensile Strength & 1.50 & 2.09 & 350 \\
\hline \multicolumn{4}{|c|}{ Head Joint } \\
\hline Thickness $(\mathrm{mm})$ & 10 & 10 & 10 \\
\hline Tensile Bond Strength (MPa) & 0.30 & 2.09 & 350 \\
\hline Joint Cohesion $(\mathrm{MPa})$ & 4.50 & 2.09 & 350 \\
\hline Angle of Internal Friction (Deg) & 42 & 42 & 42 \\
\hline \multicolumn{4}{|l|}{ Bed Joint } \\
\hline Thickness (mm) & 10 & 10 & 10 \\
\hline Tensile Bond Strength (MPa) & 0.30 & 2.09 & 350 \\
\hline Joint Cohesion $(\mathrm{MPa})$ & 4.50 & 2.09 & 350 \\
\hline Angle of Internal Friction (Deg) & 42 & 42 & 42 \\
\hline \multicolumn{4}{|c|}{ Reinforcement (Y-Direction) } \\
\hline Ratio (\%) & 0.62 & 0.52 & 100 \\
\hline Yield Strength (MPa) & 318 & 318 & 350 \\
\hline Yield Strain & 0.00159 & 0.00159 & 0.00175 \\
\hline Strain Hardening Strain & 0.02 & 0.02 & 0.02 \\
\hline Ultimate Strength (MPa) & 450 & 450 & 600 \\
\hline Ultimate Strain & 0.18 & 0.18 & 0.2 \\
\hline Prestrain & 0 & 0 & 0 \\
\hline \multicolumn{4}{|c|}{ Reinforcement (X-Direction) } \\
\hline Ratio (\%) & 0.05 & 0.52 & 100 \\
\hline Yield Strength (MPa) & 325 & 325 & 350 \\
\hline Yield Strain & 0.00163 & 0.00163 & 0.00175 \\
\hline Strain Hardening Strain & 0.02 & 0.02 & 0.02 \\
\hline Ultimate Strength (MPa) & 450 & 450 & 600 \\
\hline Ultimate Strain & 0.2 & 0.2 & 0.2 \\
\hline Prestrain & 0 & 0 & 0 \\
\hline
\end{tabular}

Numerical Modelling of Hollow Concrete Block Masonry Mechanics 
A1 Shear Wall Voon (2007) Model Options

\begin{tabular}{ll}
\hline Model & Selected Option \\
\hline Compression Pre-Peak & Hognestad \\
Compression Post-Peak & Pre-Peak Base Curve \\
Compression Softening & Vecchio \\
Tension Post-Peak & Vecchio \\
Cracking Criterion & Mohr-Coulomb \\
Slip & Walraven Reinforced \\
\hline
\end{tabular}

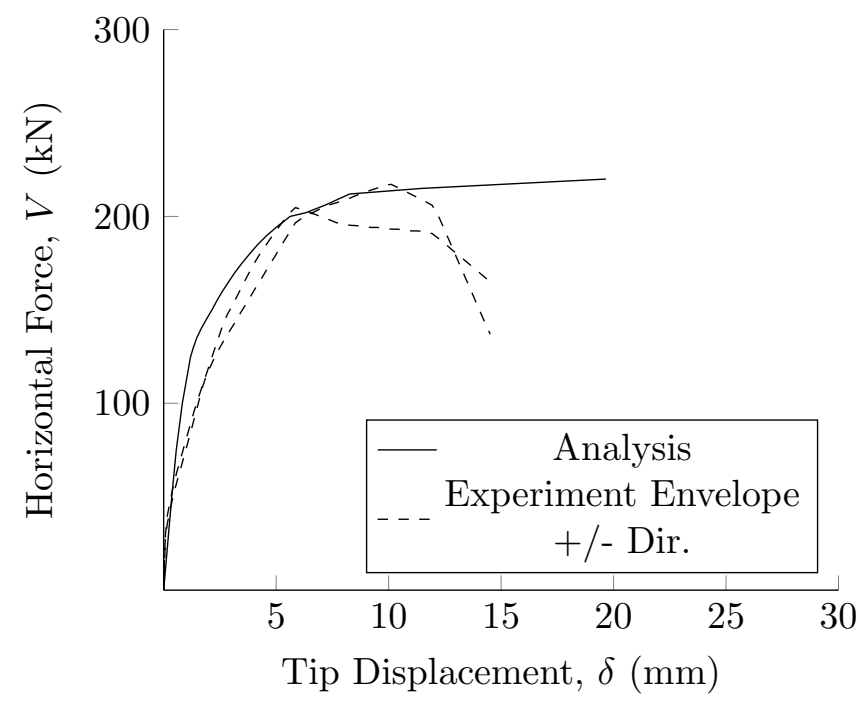

A1 Shear Wall Voon (2007) Horizontal Force vs Tip Displacement
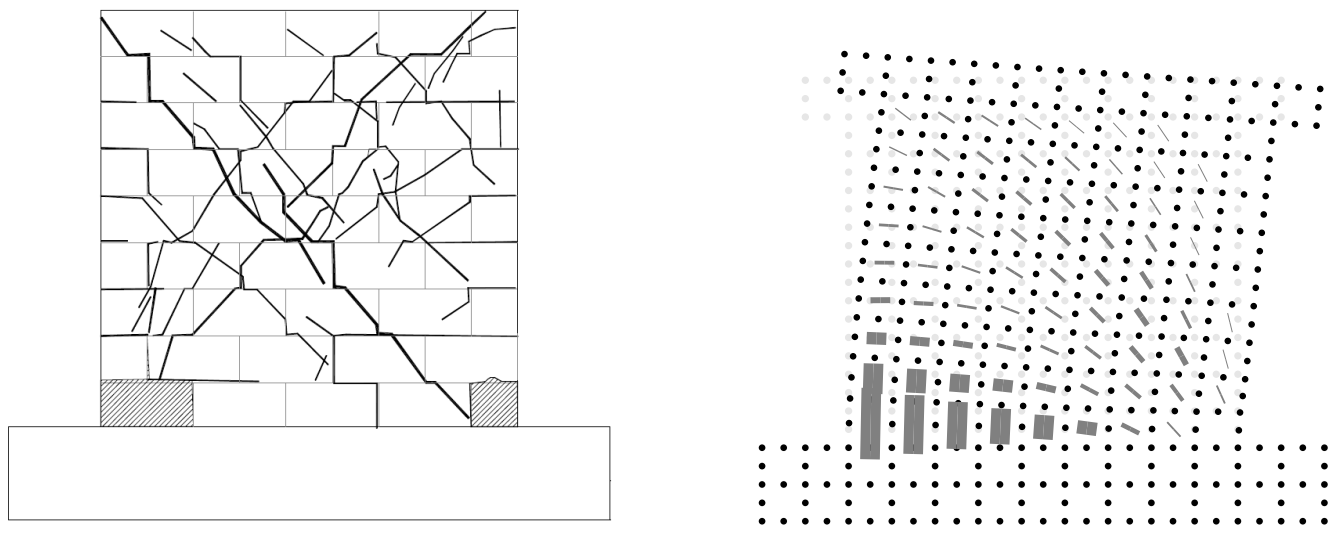

Experiment at Peak Horizontal Force (Voon, Finite Element Model at Peak Horizontal Force 2007) $\times 15$

A1 Shear Wall Displacement, Crack Pattern, and Crack Widths 


\section{A2 Shear Wall}

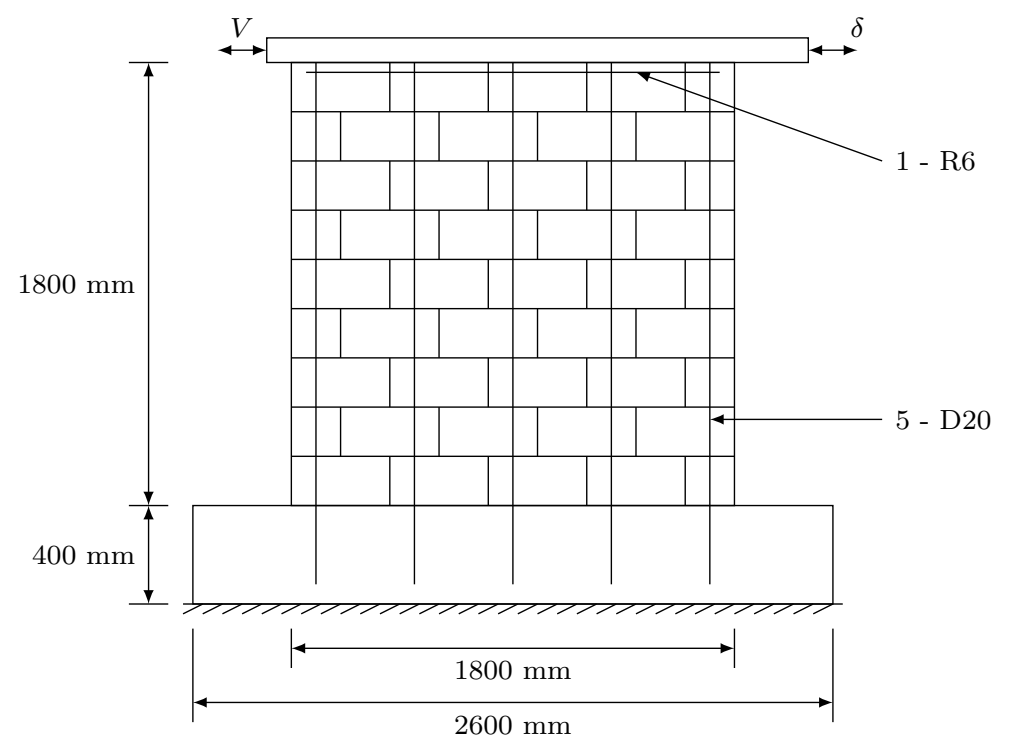

Experimental Wall Voon (2007)

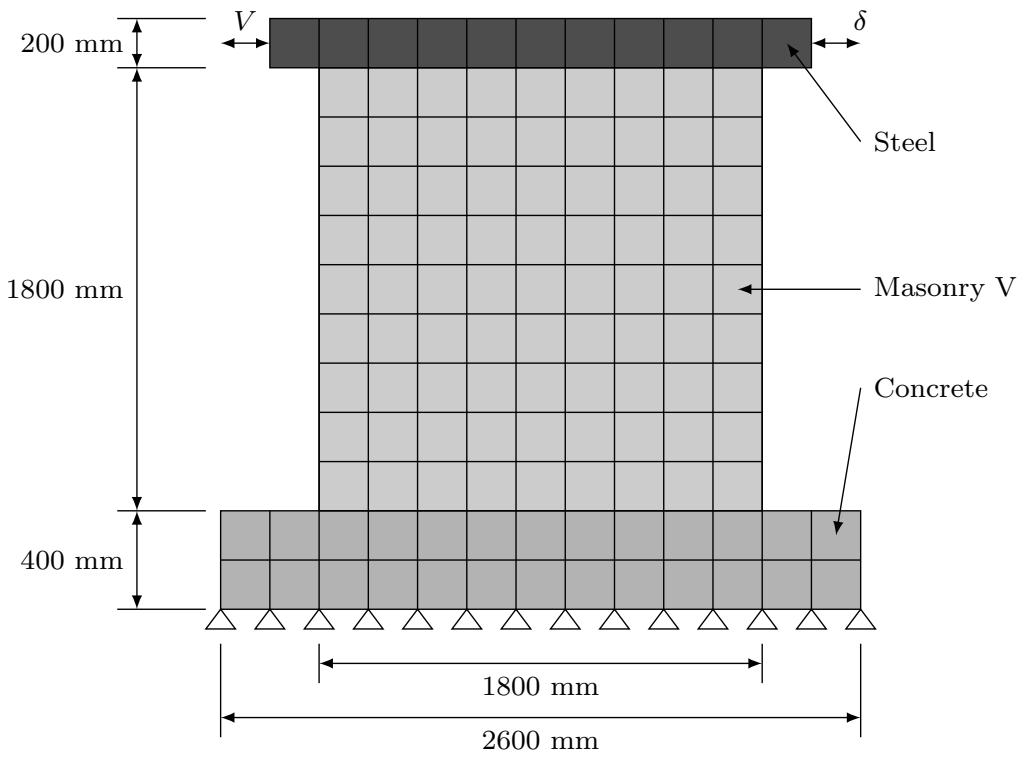

Finite Element Model Wall

A2 Shear Wall 


\section{A2 Shear Wall Voon (2007) Material Properties}

\begin{tabular}{|c|c|c|c|}
\hline \multirow{2}{*}{ Property } & \multicolumn{3}{|c|}{ Material } \\
\hline & Masonry V & Concrete & Steel \\
\hline Thickness (mm) & 140 & 600 & 400 \\
\hline Grout Tensile Strength (MPa) & 1.60 & 2.09 & 350 \\
\hline \multicolumn{4}{|c|}{ Masonry Properties (Y-Direction) } \\
\hline Gross Peak Compression Strength (MPa) & 17.6 & 40 & 350 \\
\hline Peak Compressive Strain & 0.0031 & 0.0021 & 0.00175 \\
\hline Tensile Strength & 0 & 2.09 & 350 \\
\hline Tensile Strain & 0 & 0 & 0 \\
\hline Initial Tangent Elastic Modulus (MPa) & 6500 & 34800 & 200000 \\
\hline Poisson's Ratio & 0 & 0.15 & 0 \\
\hline \multicolumn{4}{|c|}{ Masonry Properties (X-Direction) } \\
\hline Gross Peak Compression Strength (MPa) & 17.6 & 40 & 350 \\
\hline Peak Compressive Strain & 0.0031 & 0.0021 & 0.00175 \\
\hline Tensile Strength & 0 & 2.09 & 350 \\
\hline Tensile Strain & 0 & 0 & 0 \\
\hline Initial Tangent Elastic Modulus (MPa) & 6500 & 34800 & 200000 \\
\hline Poisson's Ratio & 0 & 0.15 & 0 \\
\hline \multicolumn{4}{|c|}{ Masonry Properties (Block) } \\
\hline Length (mm) & 390 & 2600 & 2000 \\
\hline Height (mm) & 190 & 400 & 200 \\
\hline Face Shell Thickness (mm) & 31 & 300 & 200 \\
\hline Percent Solid (\%) & 58 & 100 & 100 \\
\hline Tensile Strength & 1.50 & 2.09 & 350 \\
\hline \multicolumn{4}{|c|}{ Head Joint } \\
\hline Thickness $(\mathrm{mm})$ & 10 & 10 & 10 \\
\hline Tensile Bond Strength (MPa) & 0.30 & 2.09 & 350 \\
\hline Joint Cohesion (MPa) & 4.50 & 2.09 & 350 \\
\hline Angle of Internal Friction (Deg) & 42 & 42 & 42 \\
\hline \multicolumn{4}{|c|}{ Bed Joint } \\
\hline Thickness (mm) & 10 & 10 & 10 \\
\hline Tensile Bond Strength (MPa) & 0.30 & 2.09 & 350 \\
\hline Joint Cohesion (MPa) & 4.50 & 2.09 & 350 \\
\hline Angle of Internal Friction (Deg) & 42 & 42 & 42 \\
\hline \multicolumn{4}{|c|}{ Reinforcement (Y-Direction) } \\
\hline Ratio (\%) & 0.62 & 0.52 & 100 \\
\hline Yield Strength (MPa) & 318 & 318 & 350 \\
\hline Yield Strain & 0.00159 & 0.00159 & 0.00175 \\
\hline Strain Hardening Strain & 0.02 & 0.02 & 0.02 \\
\hline Ultimate Strength (MPa) & 450 & 450 & 600 \\
\hline Ultimate Strain & 0.18 & 0.18 & 0.2 \\
\hline Prestrain & 0 & 0 & 0 \\
\hline \multicolumn{4}{|c|}{ Reinforcement (X-Direction) } \\
\hline Ratio (\%) & 0 & 0.52 & 100 \\
\hline Yield Strength $(\mathrm{MPa})$ & 0 & 325 & 350 \\
\hline Yield Strain & 0 & 0.00163 & 0.00175 \\
\hline Strain Hardening Strain & 0 & 0.02 & 0.02 \\
\hline Ultimate Strength (MPa) & 0 & 450 & 600 \\
\hline Ultimate Strain & 0 & 0.2 & 0.2 \\
\hline Prestrain & 0 & 0 & 0 \\
\hline
\end{tabular}

Numerical Modelling of Hollow Concrete Block Masonry Mechanics 
A2 Shear Wall Voon (2007) Model Options

\begin{tabular}{ll}
\hline Model & Selected Option \\
\hline Compression Pre-Peak & Hognestad \\
Compression Post-Peak & Pre-Peak Base Curve \\
Compression Softening & Vecchio \\
Tension Post-Peak & Vecchio \\
Cracking Criterion & Mohr-Coulomb \\
Slip & Walraven Reinforced \\
\hline
\end{tabular}

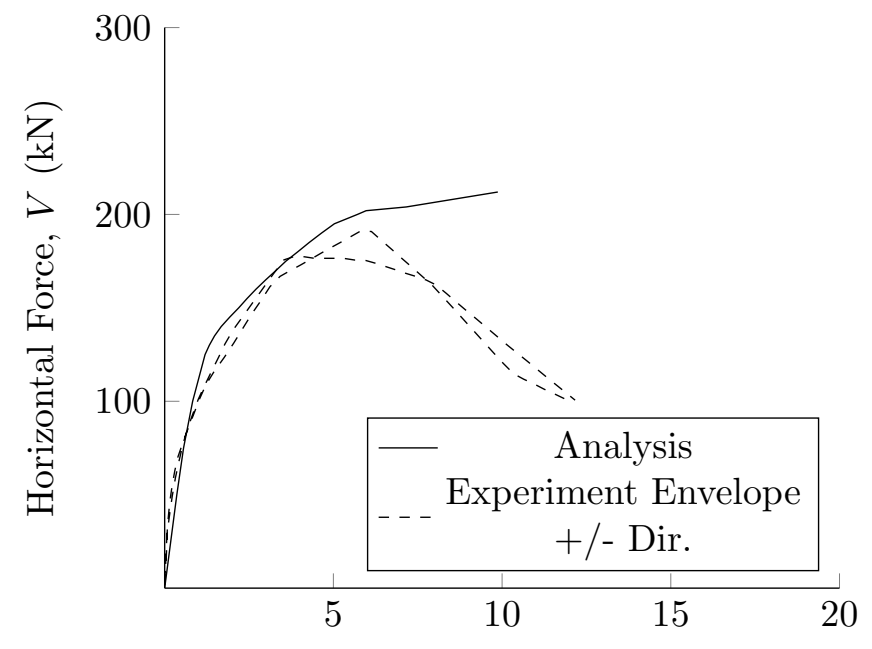

Tip Displacement, $\delta(\mathrm{mm})$

A2 Shear Wall Voon (2007) Horizontal Force vs Tip Displacement
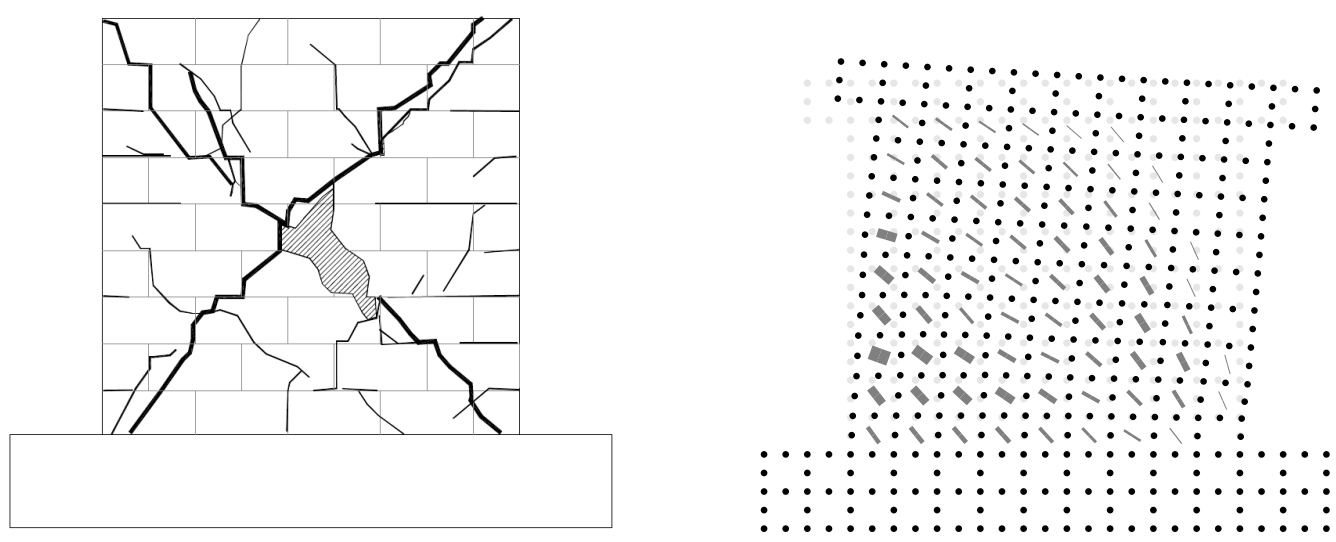

Experiment at Peak Horizontal Force (Voon, Finite Element Model at Peak Horizontal Force 2007) $\times 15$

A2 Shear Wall Displacement, Crack Pattern, and Crack Widths 


\section{A3 Shear Wall}

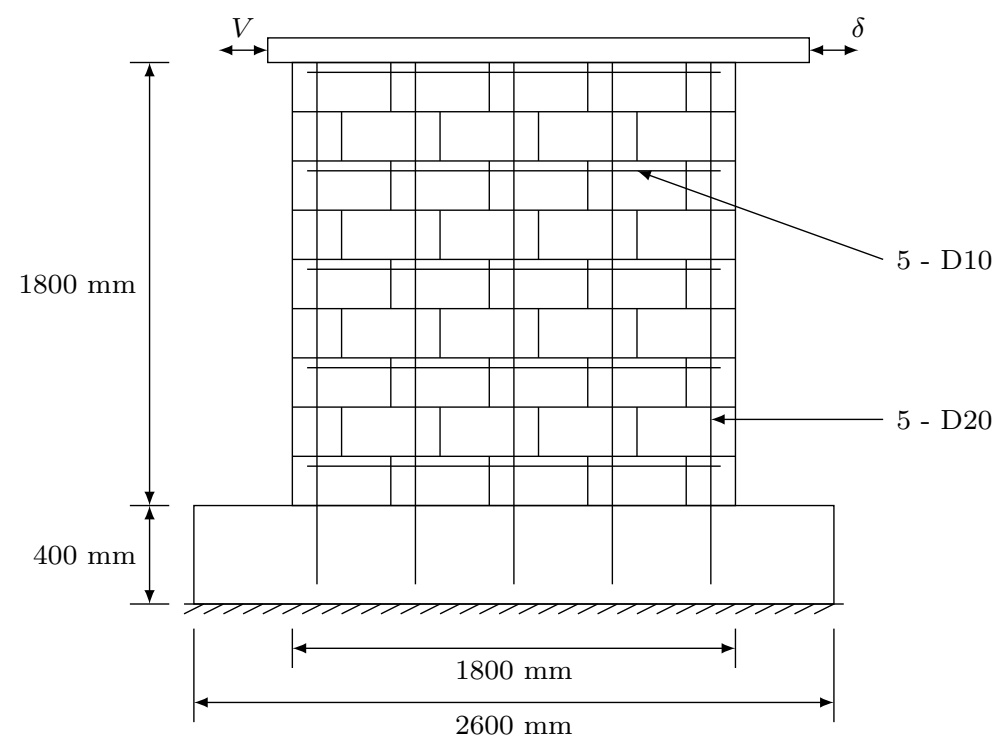

Experimental Wall Voon (2007)

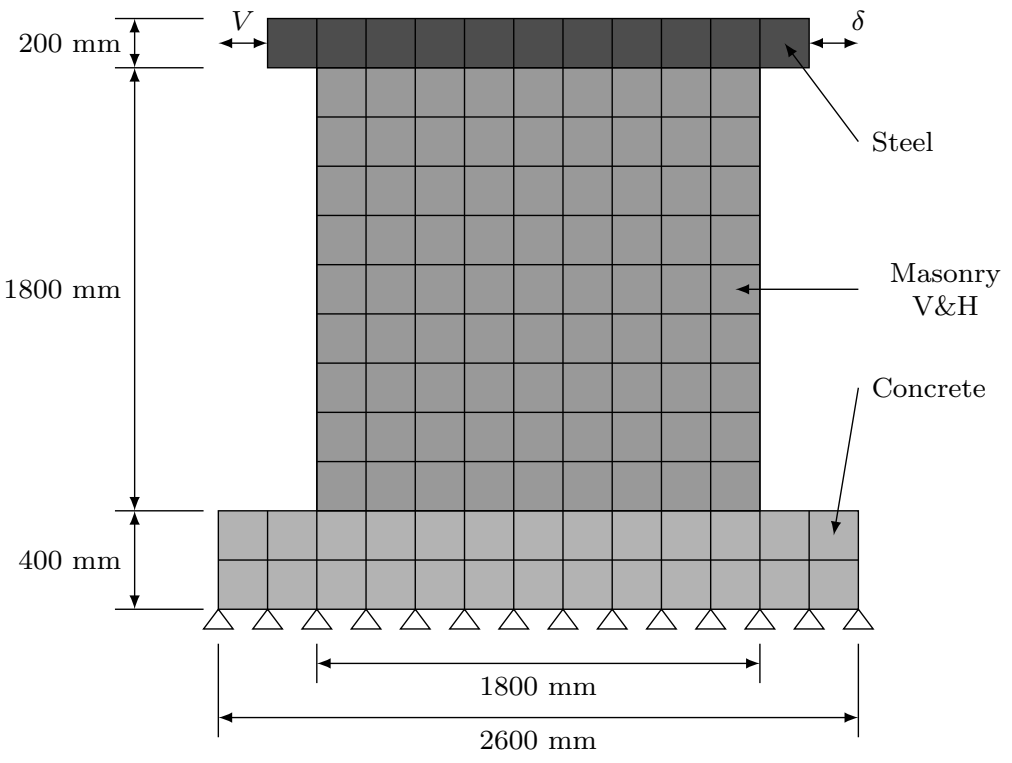

Finite Element Model Wall

A3 Shear Wall 
A3 Shear Wall Voon (2007) Material Properties

\begin{tabular}{|c|c|c|c|}
\hline \multirow{2}{*}{ Property } & \multicolumn{3}{|c|}{ Material } \\
\hline & Masonry & Concrete & Steel \\
\hline Thickness (mm) & 140 & 600 & 400 \\
\hline Grout Tensile Strength (MPa) & 1.60 & 2.09 & 350 \\
\hline \multicolumn{4}{|c|}{ Masonry Properties (Y-Direction) } \\
\hline Gross Peak Compression Strength (MPa) & 17 & 40 & 350 \\
\hline Peak Compressive Strain & 0.003 & 0.0021 & 0.00175 \\
\hline Tensile Strength & 0 & 2.09 & 350 \\
\hline Tensile Strain & 0 & 0 & 0 \\
\hline Initial Tangent Elastic Modulus (MPa) & 6500 & 34800 & 200000 \\
\hline Poisson's Ratio & 0 & 0.15 & 0 \\
\hline \multicolumn{4}{|c|}{ Masonry Properties (X-Direction) } \\
\hline Gross Peak Compression Strength (MPa) & 17 & 40 & 350 \\
\hline Peak Compressive Strain & 0.003 & 0.0021 & 0.00175 \\
\hline Tensile Strength & 0 & 2.09 & 350 \\
\hline Tensile Strain & 0 & 0 & 0 \\
\hline Initial Tangent Elastic Modulus (MPa) & 6500 & 34800 & 200000 \\
\hline Poisson's Ratio & 0 & 0.15 & 0 \\
\hline \multicolumn{4}{|c|}{ Masonry Properties (Block) } \\
\hline Length (mm) & 390 & 2600 & 2000 \\
\hline Height (mm) & 190 & 400 & 200 \\
\hline Face Shell Thickness (mm) & 31 & 300 & 200 \\
\hline Percent Solid (\%) & 58 & 100 & 100 \\
\hline Tensile Strength & 1.50 & 2.09 & 350 \\
\hline \multicolumn{4}{|c|}{ Head Joint } \\
\hline Thickness (mm) & 10 & 10 & 10 \\
\hline Tensile Bond Strength (MPa) & 0.30 & 2.09 & 350 \\
\hline Joint Cohesion (MPa) & 4.50 & 2.09 & 350 \\
\hline Angle of Internal Friction (Deg) & 42 & 42 & 42 \\
\hline \multicolumn{4}{|l|}{ Bed Joint } \\
\hline Thickness (mm) & 10 & 10 & 10 \\
\hline Tensile Bond Strength (MPa) & 0.30 & 2.09 & 350 \\
\hline Joint Cohesion (MPa) & 4.50 & 2.09 & 350 \\
\hline Angle of Internal Friction (Deg) & 42 & 42 & 42 \\
\hline \multicolumn{4}{|c|}{ Reinforcement (Y-Direction) } \\
\hline Ratio (\%) & 0.62 & 0.52 & 100 \\
\hline Yield Strength (MPa) & 318 & 318 & 350 \\
\hline Yield Strain & 0.00159 & 0.00159 & 0.00175 \\
\hline Strain Hardening Strain & 0.02 & 0.02 & 0.02 \\
\hline Ultimate Strength (MPa) & 450 & 450 & 600 \\
\hline Ultimate Strain & 0.18 & 0.18 & 0.2 \\
\hline Prestrain & 0 & 0 & 0 \\
\hline \multicolumn{4}{|c|}{ Reinforcement (X-Direction) } \\
\hline Ratio (\%) & 0.16 & 0.52 & 100 \\
\hline Yield Strength (MPa) & 320 & 325 & 350 \\
\hline Yield Strain & 0.00160 & 0.00163 & 0.00175 \\
\hline Strain Hardening Strain & 0.02 & 0.02 & 0.02 \\
\hline Ultimate Strength (MPa) & 450 & 450 & 600 \\
\hline Ultimate Strain & 0.2 & 0.2 & 0.2 \\
\hline Prestrain & 0 & 0 & 0 \\
\hline
\end{tabular}

Numerical Modelling of Hollow Concrete Block Masonry Mechanics 
A3 Shear Wall Voon (2007) Model Options

\begin{tabular}{ll}
\hline Model & Selected Option \\
\hline Compression Pre-Peak & Hognestad \\
Compression Post-Peak & Pre-Peak Base Curve \\
Compression Softening & Vecchio \\
Tension Post-Peak & Vecchio \\
Cracking Criterion & Mohr-Coulomb \\
Slip & Walraven Reinforced \\
\hline
\end{tabular}

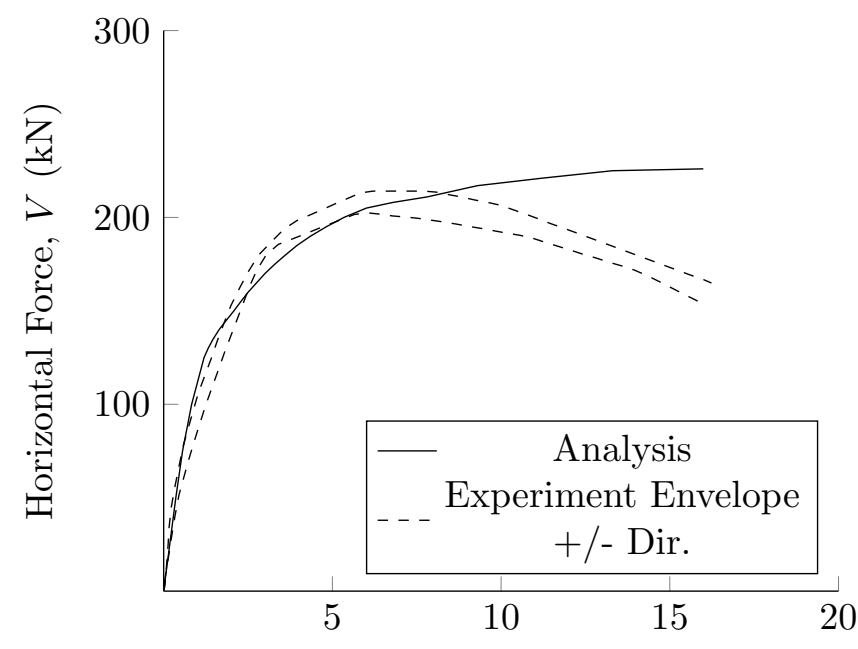

Tip Displacement, $\delta(\mathrm{mm})$

A3 Shear Wall Voon (2007) Horizontal Force vs Tip Displacement
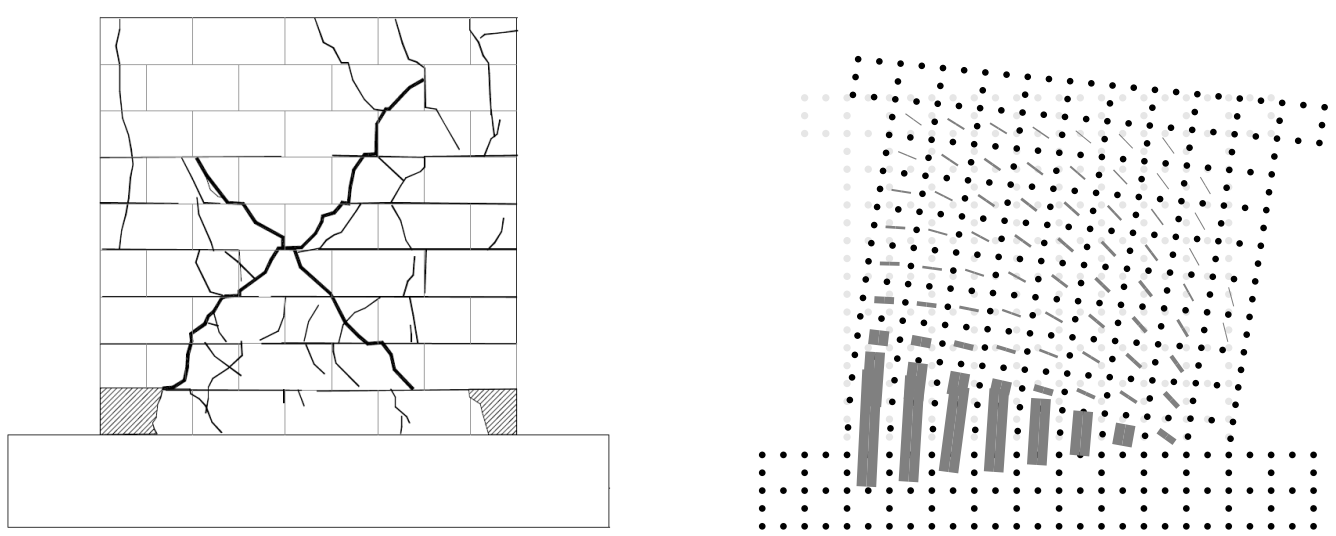

Experiment at Peak Horizontal Force (Voon, Finite Element Model at Peak Horizontal Force 2007) $\times 15$

A3 Shear Wall Displacement, Crack Pattern, and Crack Widths 


\section{A4 Shear Wall}

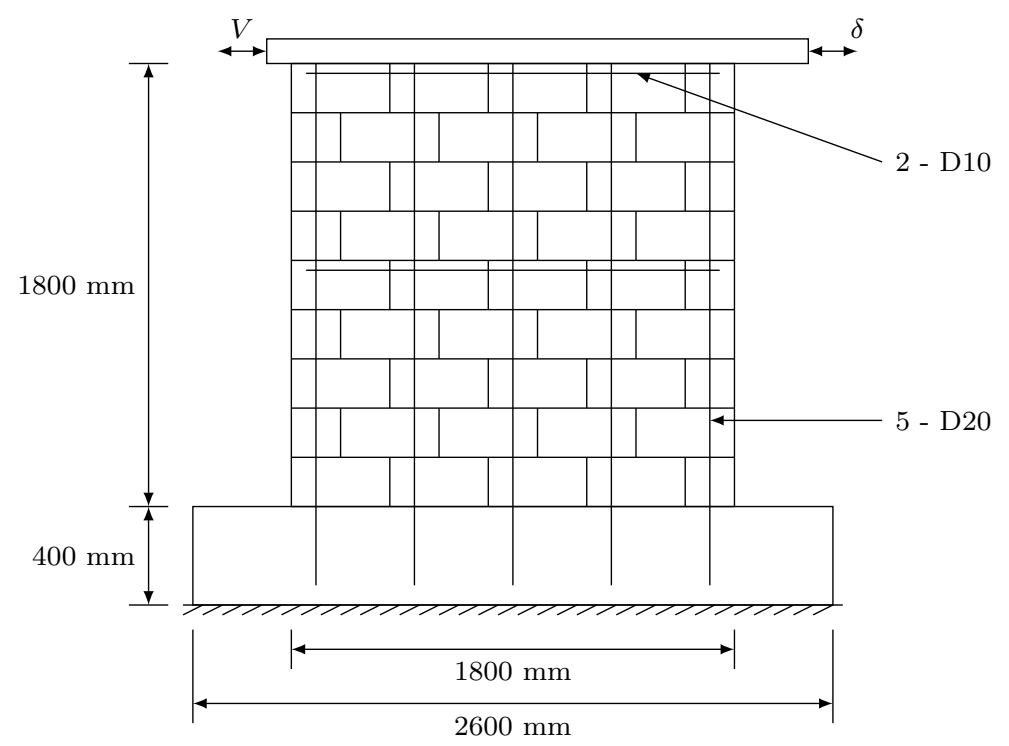

Experimental Wall Voon (2007)

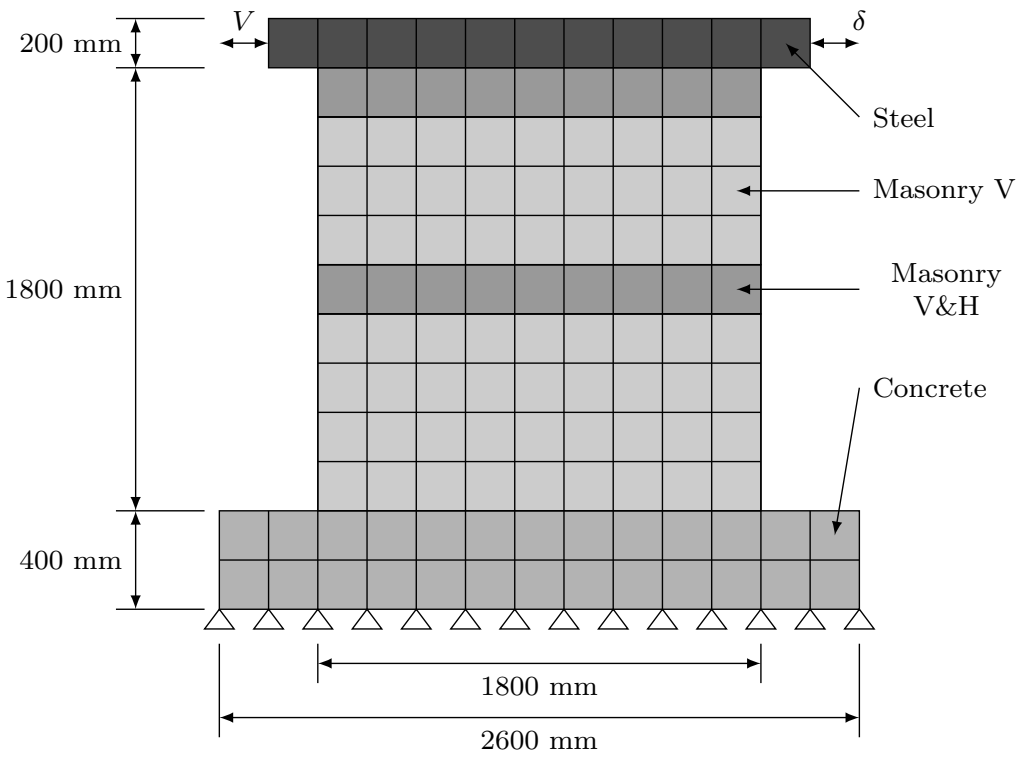

Finite Element Model Wall

A4 Shear Wall 
A3 Shear Wall Voon (2007) Material Properties

\begin{tabular}{|c|c|c|c|c|}
\hline \multirow{2}{*}{ Property } & \multicolumn{3}{|c|}{ Material } & \multirow[b]{2}{*}{ Steel } \\
\hline & Masonry V\&H & Masonry V & Concrete & \\
\hline Thickness (mm) & 140 & 140 & 600 & 400 \\
\hline Grout Tensile Strength $(\mathrm{MPa})$ & 1.60 & 1.60 & 2.09 & 350 \\
\hline \multicolumn{5}{|c|}{ Masonry Properties (Y-Direction) } \\
\hline Gross Peak Compression Strength (MPa) & 17 & 17 & 40 & 350 \\
\hline Peak Compressive Strain & 0.003 & 0.003 & 0.0021 & 0.00175 \\
\hline Tensile Strength & 0 & 0 & 2.09 & 350 \\
\hline Tensile Strain & 0 & 0 & 0 & 0 \\
\hline Initial Tangent Elastic Modulus (MPa) & 6500 & 6500 & 34800 & 200000 \\
\hline Poisson's Ratio & 0 & 0 & 0.15 & 0 \\
\hline \multicolumn{5}{|c|}{ Masonry Properties (X-Direction) } \\
\hline Gross Peak Compression Strength (MPa) & 17 & 17 & 40 & 350 \\
\hline Peak Compressive Strain & 0.003 & 0.003 & 0.0021 & 0.00175 \\
\hline Tensile Strength & 0 & 0 & 2.09 & 350 \\
\hline Tensile Strain & 0 & 0 & 0 & 0 \\
\hline Initial Tangent Elastic Modulus (MPa) & 6500 & 6500 & 34800 & 200000 \\
\hline Poisson's Ratio & 0 & 0 & 0.15 & 0 \\
\hline \multicolumn{5}{|c|}{ Masonry Properties (Block) } \\
\hline Length $(\mathrm{mm})$ & 390 & 390 & 2600 & 2000 \\
\hline Height $(\mathrm{mm})$ & 190 & 190 & 400 & 200 \\
\hline Face Shell Thickness (mm) & 31 & 31 & 300 & 200 \\
\hline Percent Solid (\%) & 58 & 58 & 100 & 100 \\
\hline Tensile Strength & 1.50 & 1.50 & 2.09 & 350 \\
\hline \multicolumn{5}{|c|}{ Head Joint } \\
\hline Thickness $(\mathrm{mm})$ & 10 & 10 & 10 & 10 \\
\hline Tensile Bond Strength (MPa) & 0.30 & 0.30 & 2.09 & 350 \\
\hline Joint Cohesion (MPa) & 4.50 & 4.50 & 2.09 & 350 \\
\hline Angle of Internal Friction (Deg) & 42 & 42 & 42 & 42 \\
\hline \multicolumn{5}{|c|}{ Bed Joint } \\
\hline Thickness (mm) & 10 & 10 & 10 & 10 \\
\hline Tensile Bond Strength (MPa) & 0.30 & 0.30 & 2.09 & 350 \\
\hline Joint Cohesion (MPa) & 4.50 & 4.50 & 2.09 & 350 \\
\hline Angle of Internal Friction (Deg) & 42 & 42 & 42 & 42 \\
\hline \multicolumn{5}{|c|}{ Reinforcement (Y-Direction) } \\
\hline Ratio (\%) & 0.62 & 0.62 & 0.52 & 100 \\
\hline Yield Strength (MPa) & 318 & 318 & 318 & 350 \\
\hline Yield Strain & 0.00159 & 0.00159 & 0.00159 & 0.00175 \\
\hline Strain Hardening Strain & 0.02 & 0.02 & 0.02 & 0.02 \\
\hline Ultimate Strength (MPa) & 450 & 450 & 450 & 600 \\
\hline Ultimate Strain & 0.18 & 0.18 & 0.18 & 0.2 \\
\hline Prestrain & 0 & 0 & 0 & 0 \\
\hline \multicolumn{5}{|c|}{ Reinforcement (X-Direction) } \\
\hline Ratio (\%) & 0.06 & 0 & 0.52 & 100 \\
\hline Yield Strength (MPa) & 320 & 0 & 325 & 350 \\
\hline Yield Strain & 0.00160 & 0 & 0.00163 & 0.00175 \\
\hline Strain Hardening Strain & 0.02 & 0 & 0.02 & 0.02 \\
\hline Ultimate Strength $(\mathrm{MPa})$ & 450 & 0 & 450 & 600 \\
\hline Ultimate Strain & 0.2 & 0 & 0.2 & 0.2 \\
\hline Prestrain & 0 & 0 & 0 & 0 \\
\hline
\end{tabular}

Numerical Modelling of Hollow Concrete Block Masonry Mechanics 
A4 Shear Wall Voon (2007) Model Options

\begin{tabular}{ll}
\hline Model & Selected Option \\
\hline Compression Pre-Peak & Hognestad \\
Compression Post-Peak & Pre-Peak Base Curve \\
Compression Softening & Vecchio \\
Tension Post-Peak & Vecchio \\
Cracking Criterion & Mohr-Coulomb \\
Slip & Walraven Reinforced \\
\hline
\end{tabular}

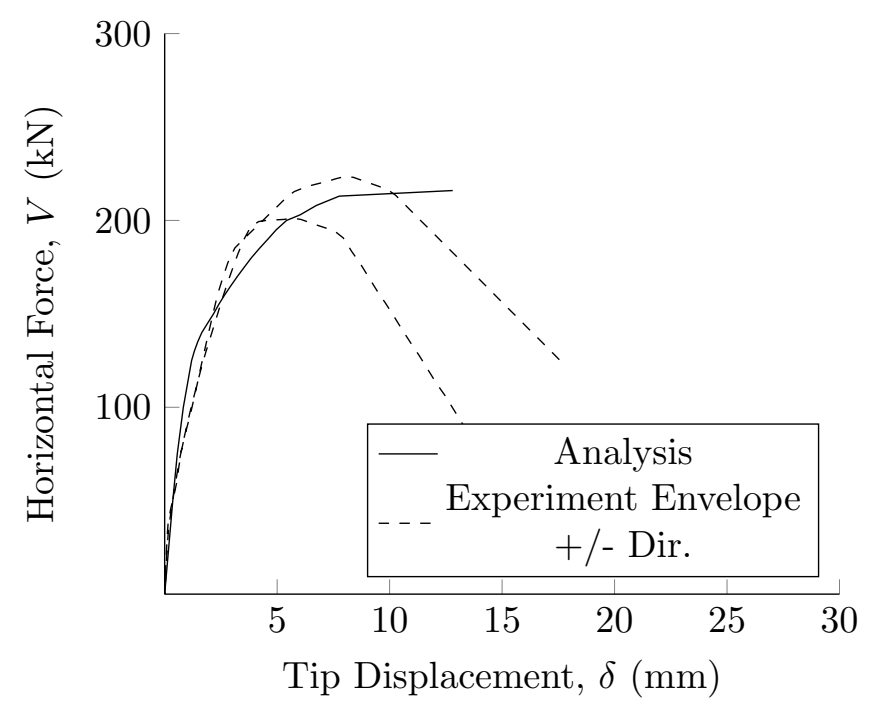

A4 Shear Wall Voon (2007) Horizontal Force vs Tip Displacement
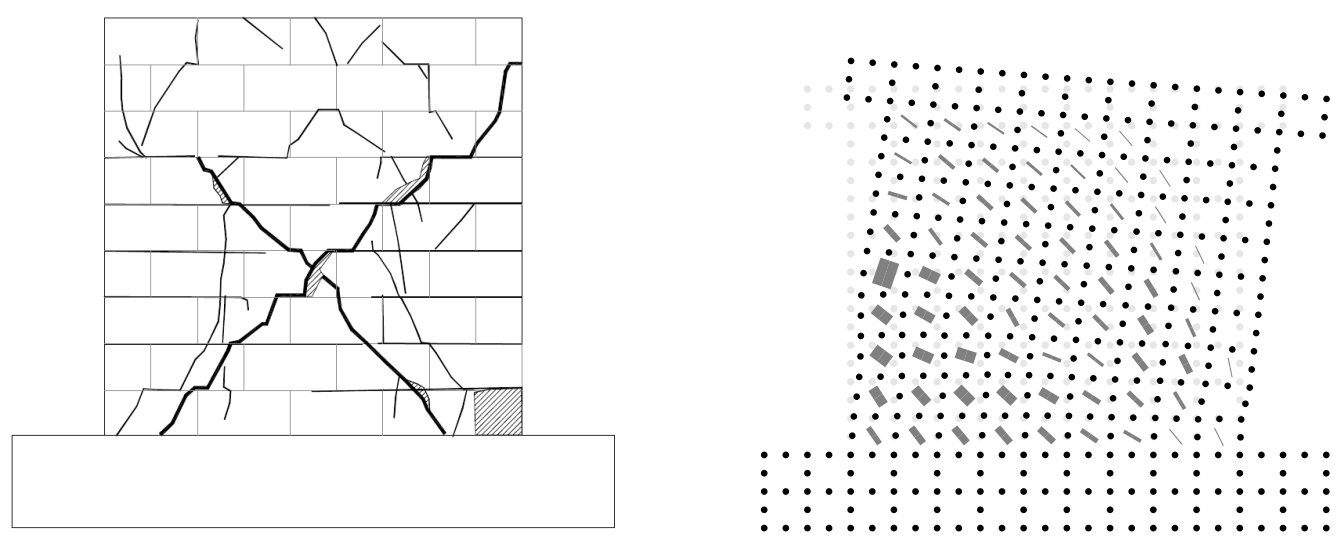

Experiment at Peak Horizontal Force (Voon, Finite Element Model at Peak Horizontal Force 2007) $\times 15$

A4 Shear Wall Displacement, Crack Pattern, and Crack Widths 


\section{A7 Shear Wall}

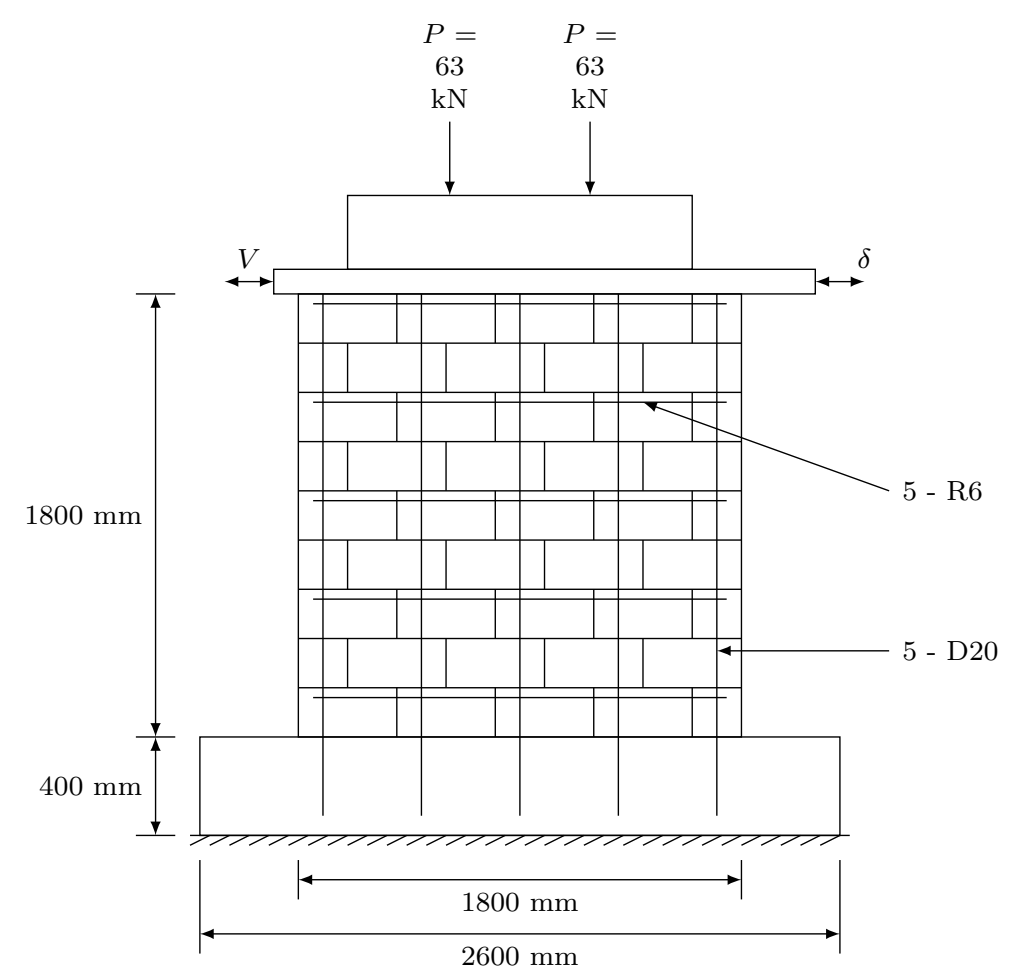

Experimental Wall Voon (2007)

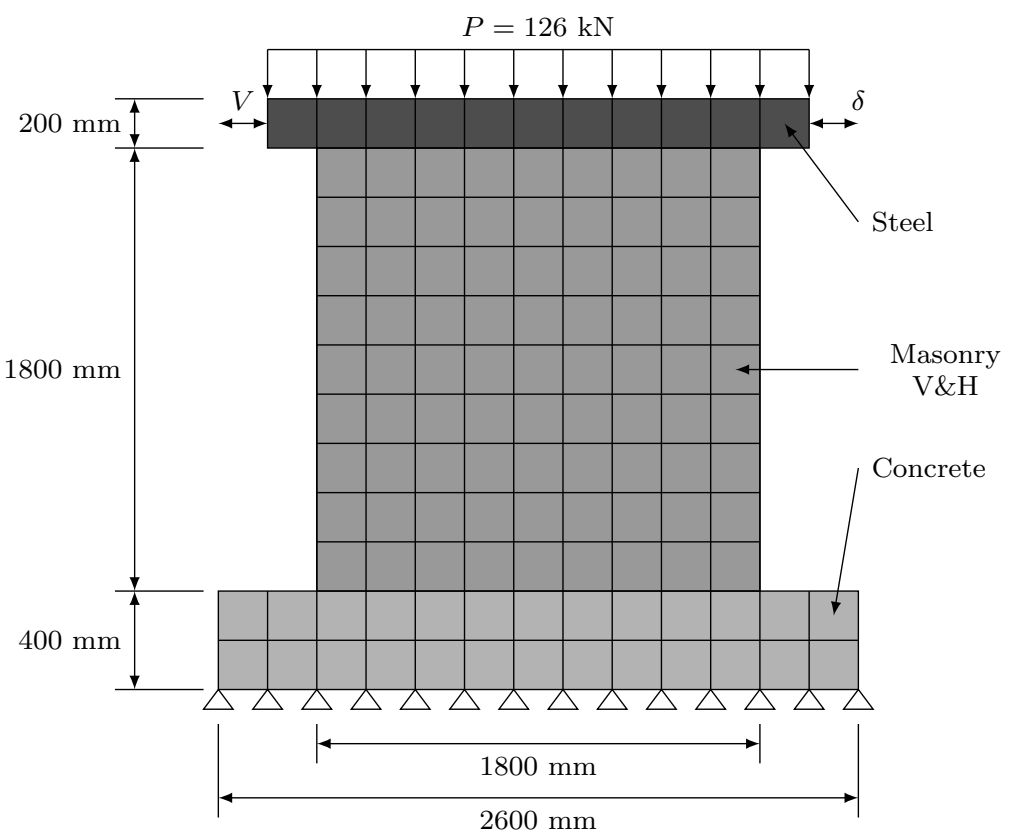

Finite Element Model Wall

A7 Shear Wall 
A7 Shear Wall Voon (2007) Material Properties

\begin{tabular}{|c|c|c|c|}
\hline \multirow{2}{*}{ Property } & \multicolumn{3}{|c|}{ Material } \\
\hline & Masonry & Concrete & Steel \\
\hline Thickness (mm) & 140 & 600 & 400 \\
\hline Grout Tensile Strength (MPa) & 1.60 & 2.09 & 350 \\
\hline \multicolumn{4}{|c|}{ Masonry Properties (Y-Direction) } \\
\hline Gross Peak Compression Strength (MPa) & 18.8 & 40 & 350 \\
\hline Peak Compressive Strain & 0.003 & 0.0021 & 0.00175 \\
\hline Tensile Strength & 0 & 2.09 & 350 \\
\hline Tensile Strain & 0 & 0 & 0 \\
\hline Initial Tangent Elastic Modulus (MPa) & 6700 & 34800 & 200000 \\
\hline Poisson's Ratio & 0 & 0.15 & 0 \\
\hline \multicolumn{4}{|c|}{ Masonry Properties (X-Direction) } \\
\hline Gross Peak Compression Strength (MPa) & 18.8 & 40 & 350 \\
\hline Peak Compressive Strain & 0.003 & 0.0021 & 0.00175 \\
\hline Tensile Strength & 0 & 2.09 & 350 \\
\hline Tensile Strain & 0 & 0 & 0 \\
\hline Initial Tangent Elastic Modulus (MPa) & 6700 & 34800 & 200000 \\
\hline Poisson's Ratio & 0 & 0.15 & 0 \\
\hline \multicolumn{4}{|c|}{ Masonry Properties (Block) } \\
\hline Length (mm) & 390 & 2600 & 2000 \\
\hline Height (mm) & 190 & 400 & 200 \\
\hline Face Shell Thickness (mm) & 31 & 300 & 200 \\
\hline Percent Solid (\%) & 58 & 100 & 100 \\
\hline Tensile Strength & 1.50 & 2.09 & 350 \\
\hline \multicolumn{4}{|c|}{ Head Joint } \\
\hline Thickness (mm) & 10 & 10 & 10 \\
\hline Tensile Bond Strength (MPa) & 0.30 & 2.09 & 350 \\
\hline Joint Cohesion (MPa) & 4.50 & 2.09 & 350 \\
\hline Angle of Internal Friction (Deg) & 42 & 42 & 42 \\
\hline \multicolumn{4}{|l|}{ Bed Joint } \\
\hline Thickness (mm) & 10 & 10 & 10 \\
\hline Tensile Bond Strength (MPa) & 0.30 & 2.09 & 350 \\
\hline Joint Cohesion (MPa) & 4.50 & 2.09 & 350 \\
\hline Angle of Internal Friction (Deg) & 42 & 42 & 42 \\
\hline \multicolumn{4}{|c|}{ Reinforcement (Y-Direction) } \\
\hline Ratio (\%) & 0.62 & 0.52 & 100 \\
\hline Yield Strength (MPa) & 318 & 318 & 350 \\
\hline Yield Strain & 0.00159 & 0.00159 & 0.00175 \\
\hline Strain Hardening Strain & 0.02 & 0.02 & 0.02 \\
\hline Ultimate Strength (MPa) & 450 & 450 & 600 \\
\hline Ultimate Strain & 0.18 & 0.18 & 0.2 \\
\hline Prestrain & 0 & 0 & 0 \\
\hline \multicolumn{4}{|c|}{ Reinforcement (X-Direction) } \\
\hline Ratio (\%) & 0.05 & 0.52 & 100 \\
\hline Yield Strength (MPa) & 325 & 325 & 350 \\
\hline Yield Strain & 0.00163 & 0.00163 & 0.00175 \\
\hline Strain Hardening Strain & 0.02 & 0.02 & 0.02 \\
\hline Ultimate Strength (MPa) & 450 & 450 & 600 \\
\hline Ultimate Strain & 0.2 & 0.2 & 0.2 \\
\hline Prestrain & 0 & 0 & 0 \\
\hline
\end{tabular}

Numerical Modelling of Hollow Concrete Block Masonry Mechanics 
A7 Shear Wall Voon (2007) Model Options

\begin{tabular}{ll}
\hline Model & Selected Option \\
\hline Compression Pre-Peak & Hognestad \\
Compression Post-Peak & Pre-Peak Base Curve \\
Compression Softening & Vecchio \\
Tension Post-Peak & Vecchio \\
Cracking Criterion & Mohr-Coulomb \\
Slip & Walraven Reinforced \\
\hline
\end{tabular}

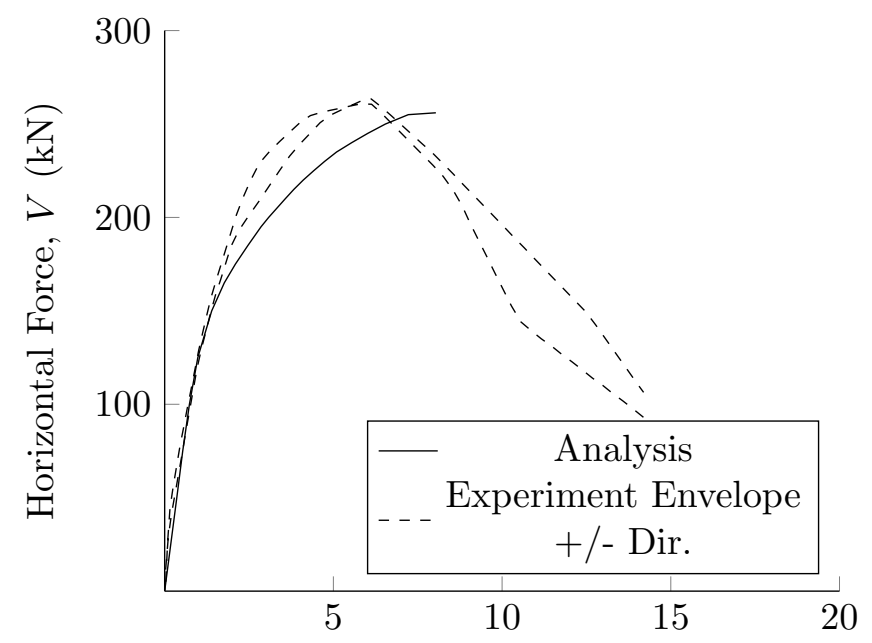

Tip Displacement, $\delta(\mathrm{mm})$

A7 Shear Wall Voon (2007) Horizontal Force vs Tip Displacement
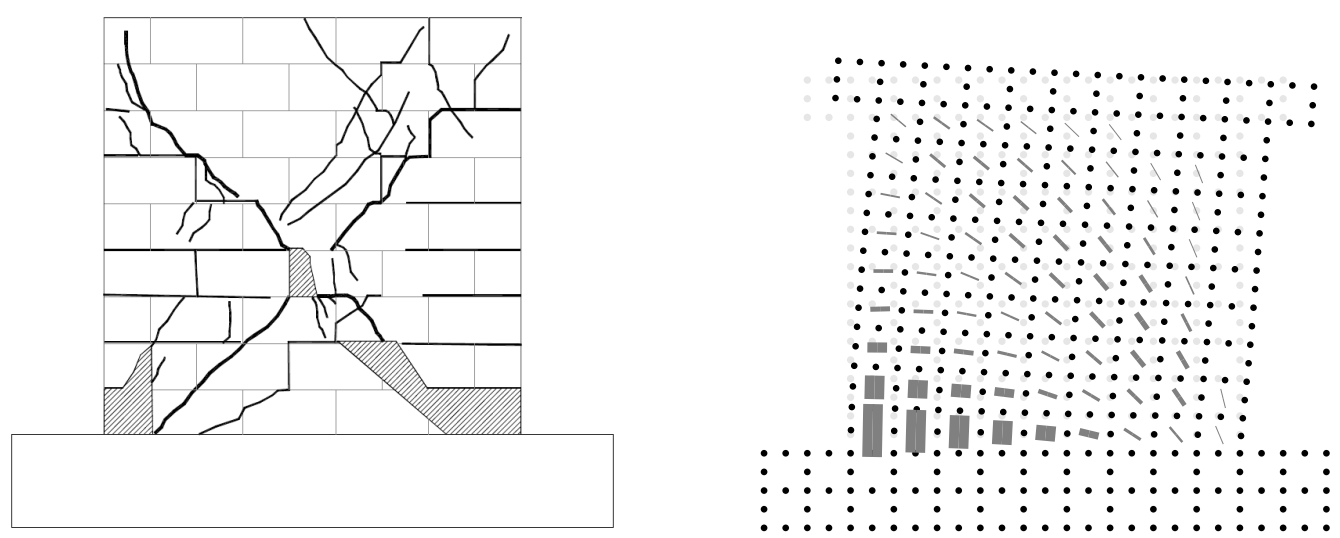

Experiment at Peak Horizontal Force (Voon, Finite Element Model at Peak Horizontal Force 2007) $\times 15$

A7 Shear Wall Displacement, Crack Pattern, and Crack Widths 


\section{A8 Shear Wall}

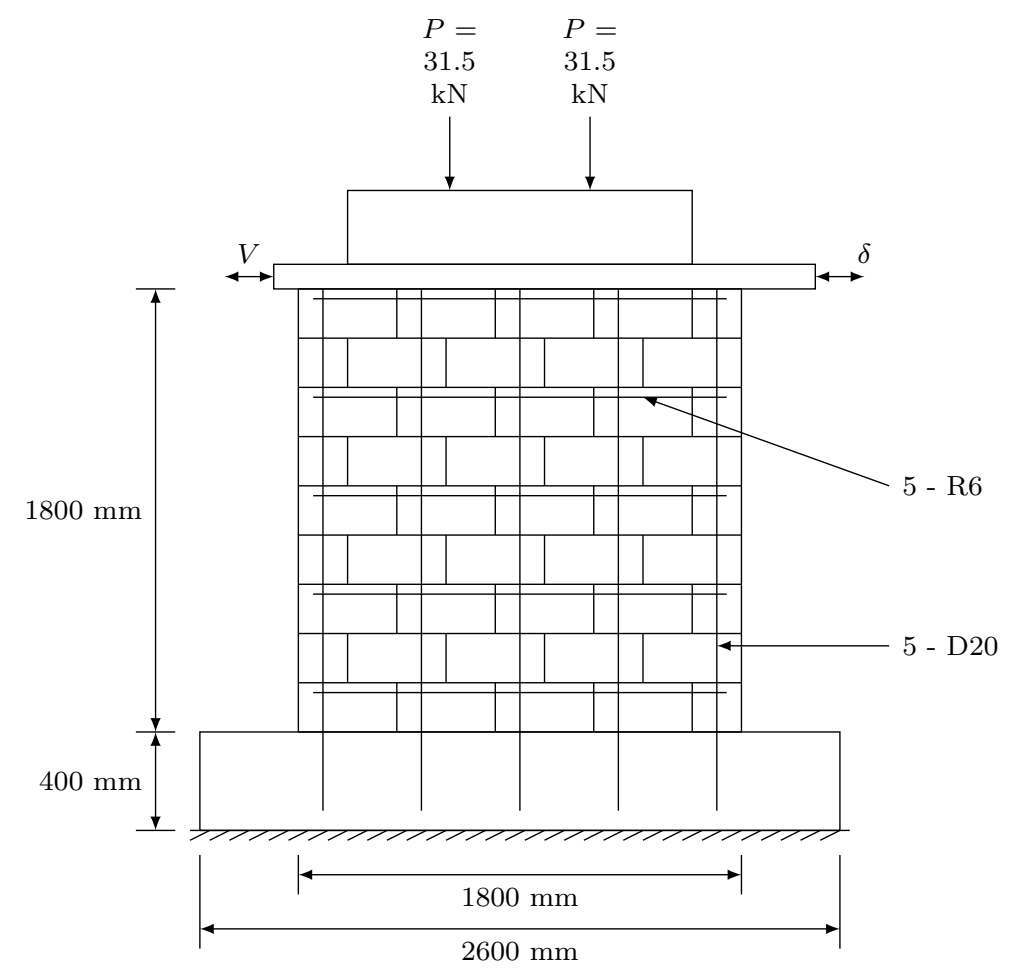

Experimental Wall Voon (2007)

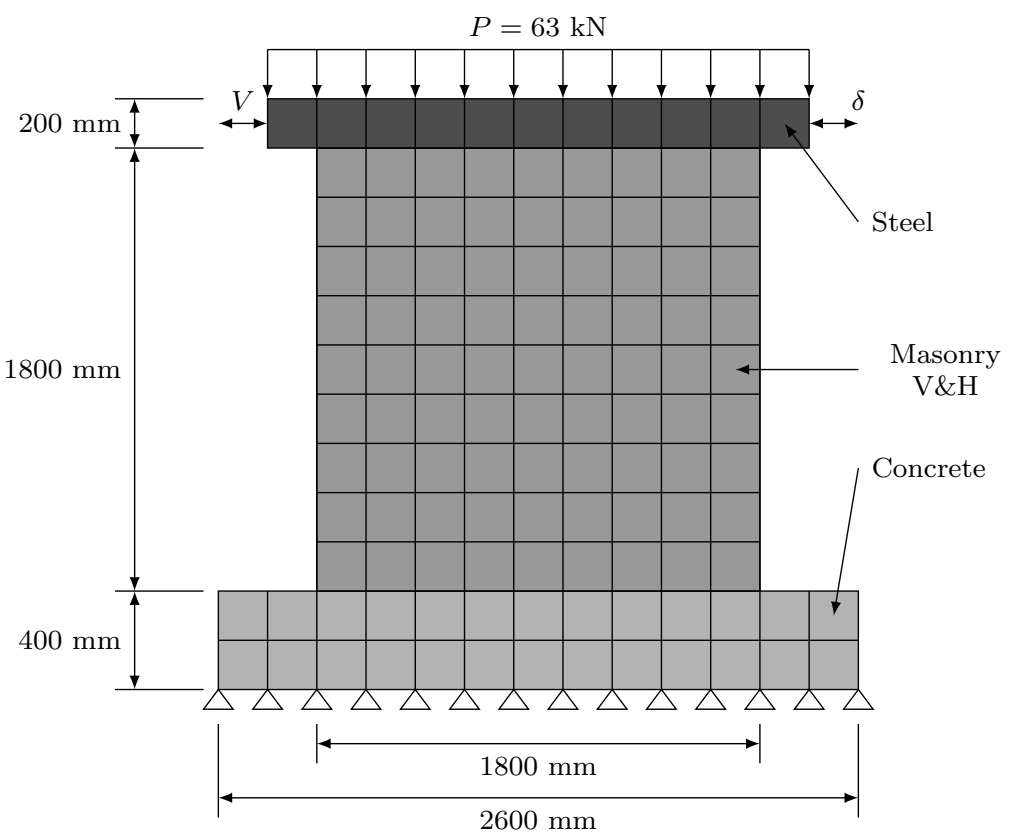

Finite Element Model Wall

A8 Shear Wall 
A8 Shear Wall Voon (2007) Material Properties

\begin{tabular}{|c|c|c|c|}
\hline \multirow{2}{*}{ Property } & \multicolumn{3}{|c|}{ Material } \\
\hline & Masonry & Concrete & Steel \\
\hline Thickness (mm) & 140 & 600 & 400 \\
\hline Grout Tensile Strength (MPa) & 1.60 & 2.09 & 350 \\
\hline \multicolumn{4}{|c|}{ Masonry Properties (Y-Direction) } \\
\hline Gross Peak Compression Strength (MPa) & 18.8 & 40 & 350 \\
\hline Peak Compressive Strain & 0.003 & 0.0021 & 0.00175 \\
\hline Tensile Strength & 0 & 2.09 & 350 \\
\hline Tensile Strain & 0 & 0 & 0 \\
\hline Initial Tangent Elastic Modulus (MPa) & 6700 & 34800 & 200000 \\
\hline Poisson's Ratio & 0 & 0.15 & 0 \\
\hline \multicolumn{4}{|c|}{ Masonry Properties (X-Direction) } \\
\hline Gross Peak Compression Strength (MPa) & 18.8 & 40 & 350 \\
\hline Peak Compressive Strain & 0.003 & 0.0021 & 0.00175 \\
\hline Tensile Strength & 0 & 2.09 & 350 \\
\hline Tensile Strain & 0 & 0 & 0 \\
\hline Initial Tangent Elastic Modulus (MPa) & 6700 & 34800 & 200000 \\
\hline Poisson's Ratio & 0 & 0.15 & 0 \\
\hline \multicolumn{4}{|c|}{ Masonry Properties (Block) } \\
\hline Length (mm) & 390 & 2600 & 2000 \\
\hline Height (mm) & 190 & 400 & 200 \\
\hline Face Shell Thickness (mm) & 31 & 300 & 200 \\
\hline Percent Solid (\%) & 58 & 100 & 100 \\
\hline Tensile Strength & 1.50 & 2.09 & 350 \\
\hline \multicolumn{4}{|c|}{ Head Joint } \\
\hline Thickness (mm) & 10 & 10 & 10 \\
\hline Tensile Bond Strength (MPa) & 0.30 & 2.09 & 350 \\
\hline Joint Cohesion (MPa) & 4.50 & 2.09 & 350 \\
\hline Angle of Internal Friction (Deg) & 42 & 42 & 42 \\
\hline \multicolumn{4}{|l|}{ Bed Joint } \\
\hline Thickness (mm) & 10 & 10 & 10 \\
\hline Tensile Bond Strength (MPa) & 0.30 & 2.09 & 350 \\
\hline Joint Cohesion (MPa) & 4.50 & 2.09 & 350 \\
\hline Angle of Internal Friction (Deg) & 42 & 42 & 42 \\
\hline \multicolumn{4}{|c|}{ Reinforcement (Y-Direction) } \\
\hline Ratio (\%) & 0.62 & 0.52 & 100 \\
\hline Yield Strength (MPa) & 318 & 318 & 350 \\
\hline Yield Strain & 0.00159 & 0.00159 & 0.00175 \\
\hline Strain Hardening Strain & 0.02 & 0.02 & 0.02 \\
\hline Ultimate Strength (MPa) & 450 & 450 & 600 \\
\hline Ultimate Strain & 0.18 & 0.18 & 0.2 \\
\hline Prestrain & 0 & 0 & 0 \\
\hline \multicolumn{4}{|c|}{ Reinforcement (X-Direction) } \\
\hline Ratio (\%) & 0.05 & 0.52 & 100 \\
\hline Yield Strength (MPa) & 325 & 325 & 350 \\
\hline Yield Strain & 0.00163 & 0.00163 & 0.00175 \\
\hline Strain Hardening Strain & 0.02 & 0.02 & 0.02 \\
\hline Ultimate Strength (MPa) & 450 & 450 & 600 \\
\hline Ultimate Strain & 0.2 & 0.2 & 0.2 \\
\hline Prestrain & 0 & 0 & 0 \\
\hline
\end{tabular}

Numerical Modelling of Hollow Concrete Block Masonry Mechanics 
A8 Shear Wall Voon (2007) Model Options

\begin{tabular}{ll}
\hline Model & Selected Option \\
\hline Compression Pre-Peak & Hognestad \\
Compression Post-Peak & Pre-Peak Base Curve \\
Compression Softening & Vecchio \\
Tension Post-Peak & Vecchio \\
Cracking Criterion & Mohr-Coulomb \\
Slip & Walraven Reinforced \\
\hline
\end{tabular}

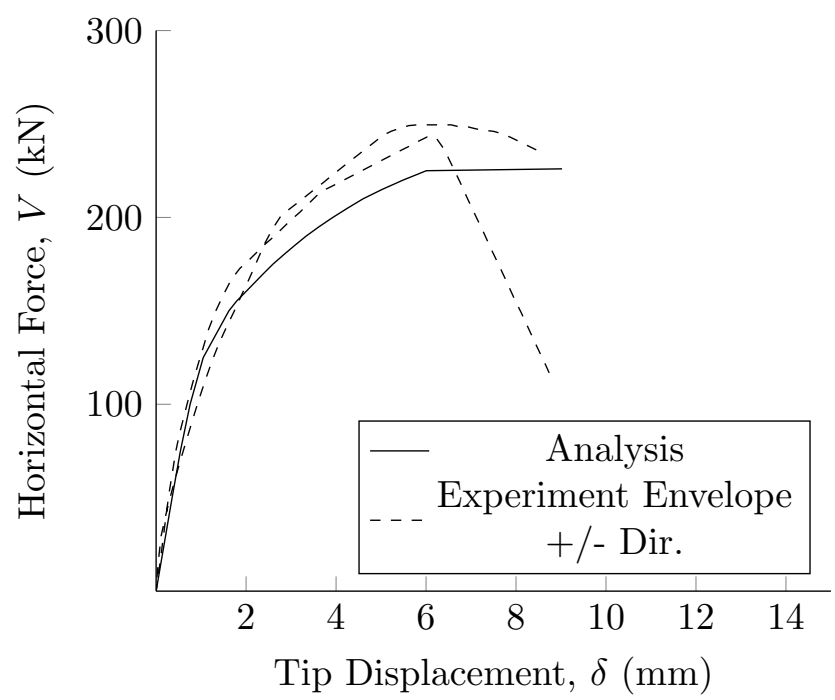

A8 Shear Wall Voon (2007) Horizontal Force vs Tip Displacement
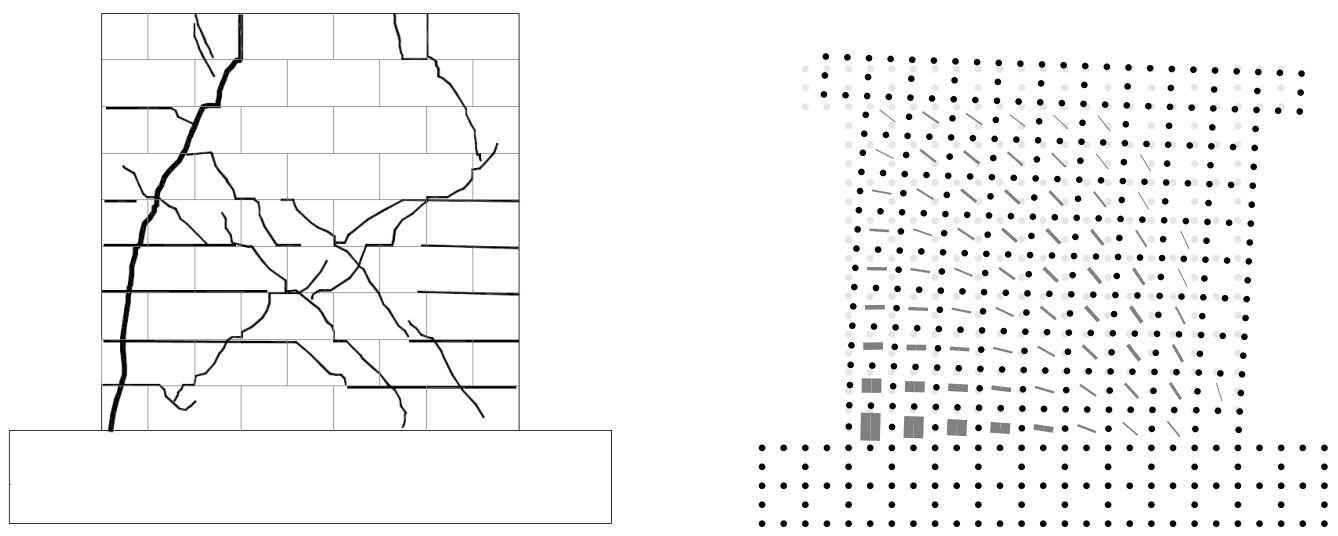

Experiment at Peak Horizontal Force (Voon, Finite Element Model at Peak Horizontal Force 2007) $\times 15$

A8 Shear Wall Displacement, Crack Pattern, and Crack Widths 


\section{A9 Shear Wall}

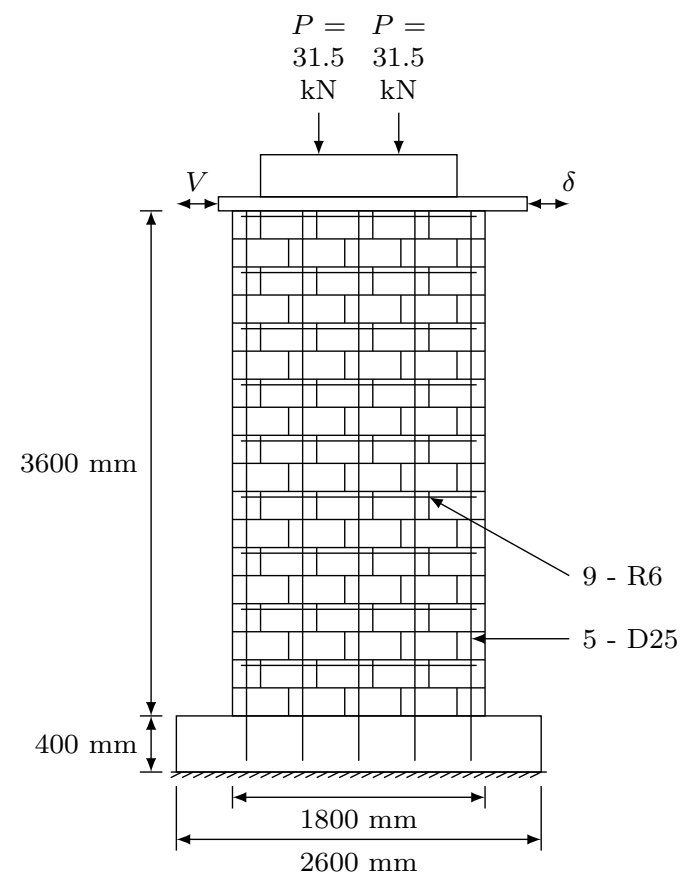

Experimental Wall Voon (2007)

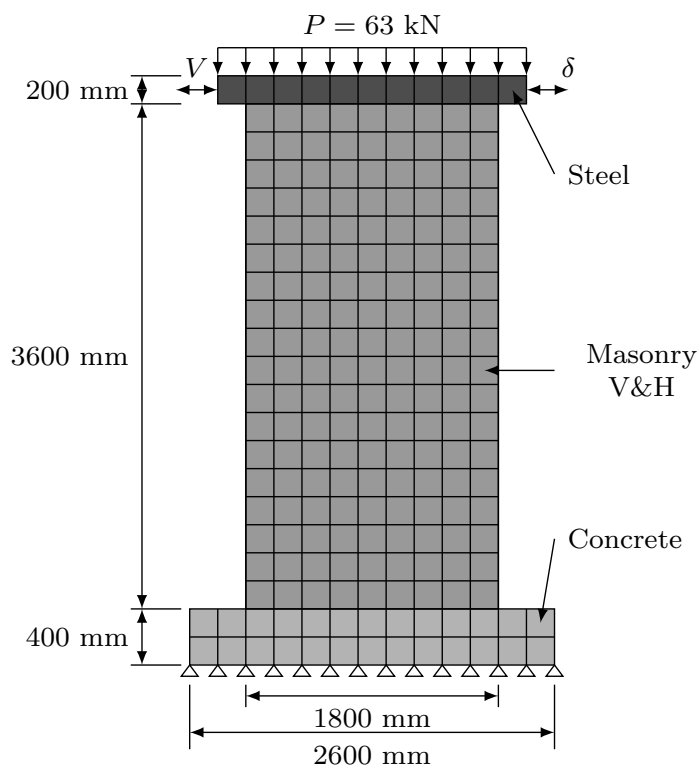

Finite Element Model Wall

A9 Shear Wall

A9 Shear Wall Voon (2007) Model Options

\begin{tabular}{ll}
\hline Model & Selected Option \\
\hline Compression Pre-Peak & Hognestad \\
Compression Post-Peak & Pre-Peak Base Curve \\
Compression Softening & Vecchio \\
Tension Post-Peak & Vecchio \\
Cracking Criterion & Mohr-Coulomb \\
Slip & Walraven Reinforced \\
\hline
\end{tabular}


A9 Shear Wall Voon (2007) Material Properties

\begin{tabular}{|c|c|c|c|}
\hline \multirow{2}{*}{ Property } & \multicolumn{3}{|c|}{ Material } \\
\hline & Masonry & Concrete & Steel \\
\hline Thickness $(\mathrm{mm})$ & 140 & 600 & 400 \\
\hline Grout Tensile Strength (MPa) & 1.90 & 2.09 & 350 \\
\hline \multicolumn{4}{|c|}{ Masonry Properties (Y-Direction) } \\
\hline Gross Peak Compression Strength (MPa) & 24.3 & 40 & 350 \\
\hline Peak Compressive Strain & 0.0032 & 0.0021 & 0.00175 \\
\hline Tensile Strength & 0 & 2.09 & 350 \\
\hline Tensile Strain & 0 & 0 & 0 \\
\hline Initial Tangent Elastic Modulus (MPa) & 0 & 34800 & 200000 \\
\hline Poisson's Ratio & 0 & 0.15 & 0 \\
\hline \multicolumn{4}{|c|}{ Masonry Properties (X-Direction) } \\
\hline Gross Peak Compression Strength (MPa) & 24.3 & 40 & 350 \\
\hline Peak Compressive Strain & 0.0032 & 0.0021 & 0.00175 \\
\hline Tensile Strength & 0 & 2.09 & 350 \\
\hline Tensile Strain & 0 & 0 & 0 \\
\hline Initial Tangent Elastic Modulus (MPa) & 0 & 34800 & 200000 \\
\hline Poisson's Ratio & 0 & 0.15 & 0 \\
\hline \multicolumn{4}{|c|}{ Masonry Properties (Block) } \\
\hline Length (mm) & 390 & 2600 & 2000 \\
\hline Height (mm) & 190 & 400 & 200 \\
\hline Face Shell Thickness (mm) & 31 & 300 & 200 \\
\hline Percent Solid (\%) & 58 & 100 & 100 \\
\hline Tensile Strength & 1.50 & 2.09 & 350 \\
\hline \multicolumn{4}{|c|}{ Head Joint } \\
\hline Thickness $(\mathrm{mm})$ & 10 & 10 & 10 \\
\hline Tensile Bond Strength (MPa) & 0.30 & 2.09 & 350 \\
\hline Joint Cohesion $(\mathrm{MPa})$ & 4.50 & 2.09 & 350 \\
\hline Angle of Internal Friction (Deg) & 42 & 42 & 42 \\
\hline \multicolumn{4}{|l|}{ Bed Joint } \\
\hline Thickness (mm) & 10 & 10 & 10 \\
\hline Tensile Bond Strength (MPa) & 0.30 & 2.09 & 350 \\
\hline Joint Cohesion $(\mathrm{MPa})$ & 4.50 & 2.09 & 350 \\
\hline Angle of Internal Friction (Deg) & 42 & 42 & 42 \\
\hline \multicolumn{4}{|c|}{ Reinforcement (Y-Direction) } \\
\hline Ratio (\%) & 0.97 & 0.52 & 100 \\
\hline Yield Strength (MPa) & 320 & 318 & 350 \\
\hline Yield Strain & 0.00159 & 0.00159 & 0.00175 \\
\hline Strain Hardening Strain & 0.02 & 0.02 & 0.02 \\
\hline Ultimate Strength (MPa) & 450 & 450 & 600 \\
\hline Ultimate Strain & 0.2 & 0.18 & 0.2 \\
\hline Prestrain & 0 & 0 & 0 \\
\hline \multicolumn{4}{|c|}{ Reinforcement (X-Direction) } \\
\hline Ratio (\%) & 0.05 & 0.52 & 100 \\
\hline Yield Strength (MPa) & 325 & 325 & 350 \\
\hline Yield Strain & 0.00163 & 0.00163 & 0.00175 \\
\hline Strain Hardening Strain & 0.02 & 0.02 & 0.02 \\
\hline Ultimate Strength (MPa) & 450 & 450 & 600 \\
\hline Ultimate Strain & 0.2 & 0.2 & 0.2 \\
\hline Prestrain & 0 & 0 & 0 \\
\hline
\end{tabular}

Numerical Modelling of Hollow Concrete Block Masonry Mechanics 


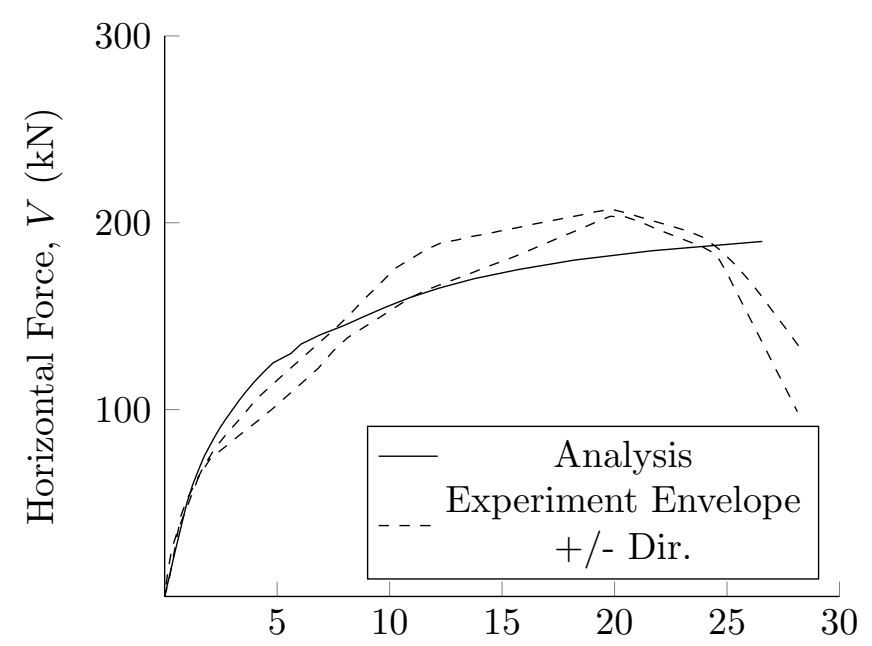

Tip Displacement, $\delta(\mathrm{mm})$

A9 Shear Wall Voon (2007) Horizontal Force vs Tip Displacement
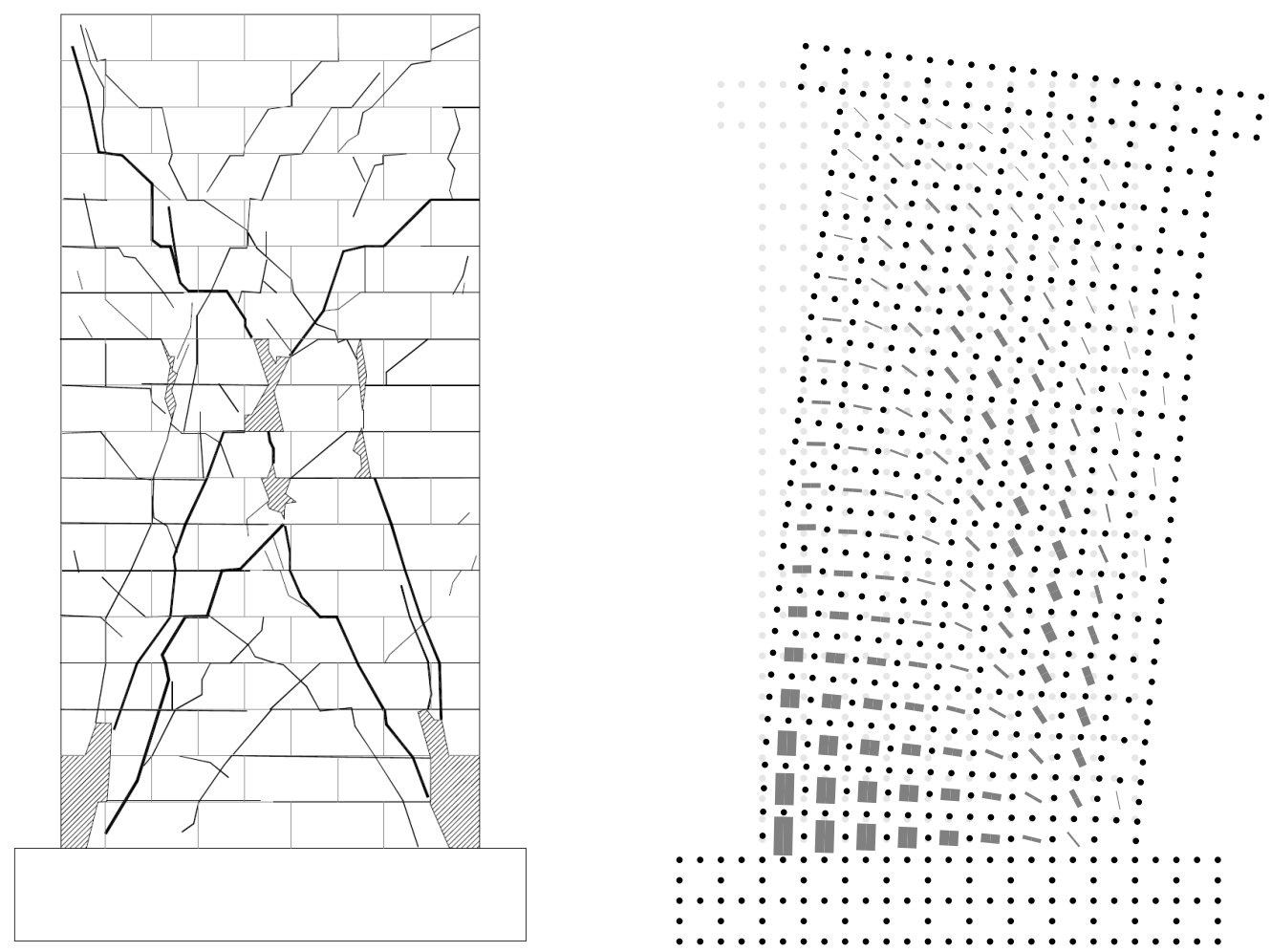

Experiment at Peak Horizontal Force (Voon, Finite Element Model at Peak Horizontal Force 2007) $\times 15$

A9 Shear Wall Displacement, Crack Pattern, and Crack Widths 


\section{A10 Shear Wall}

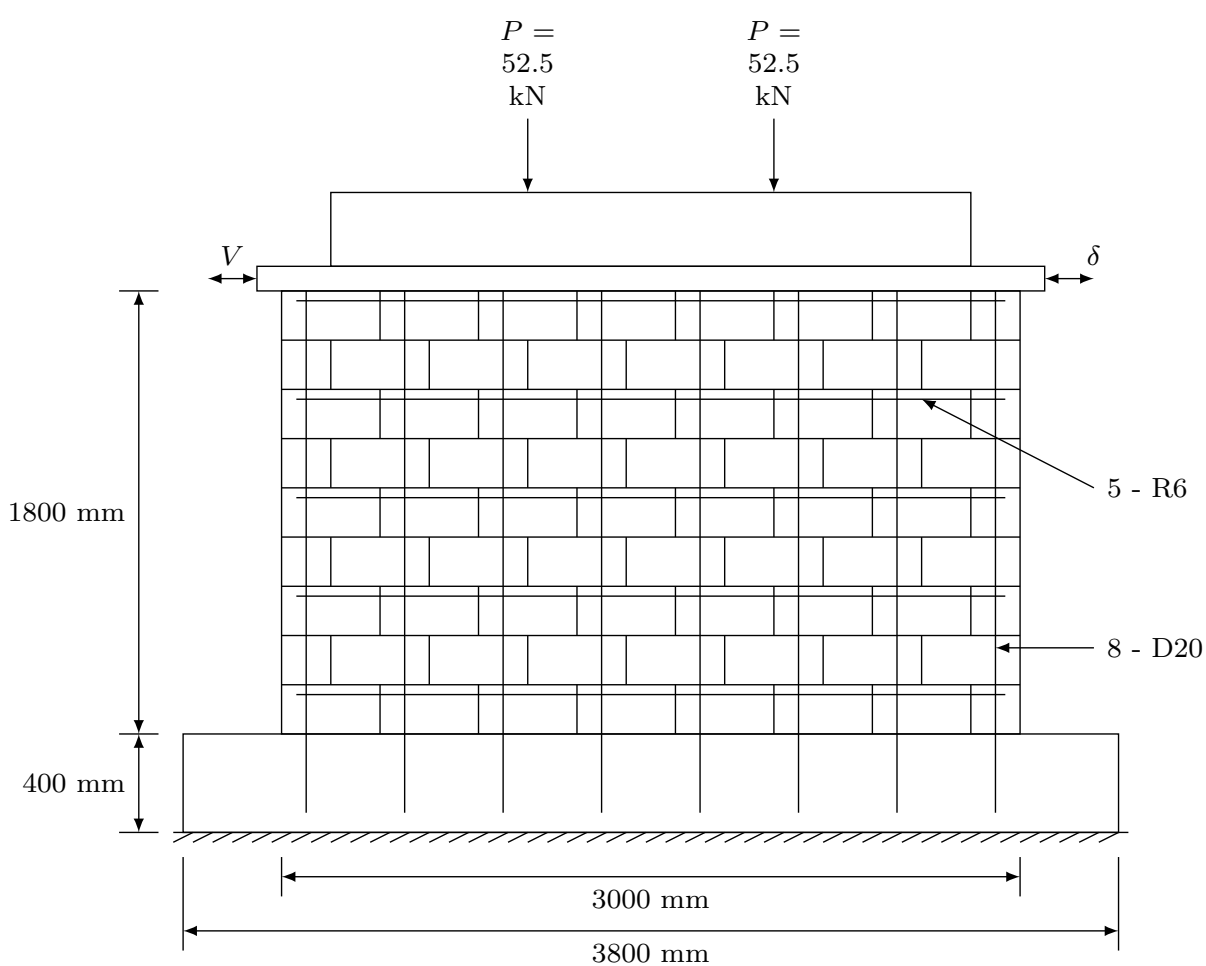

Experimental Wall Voon (2007)

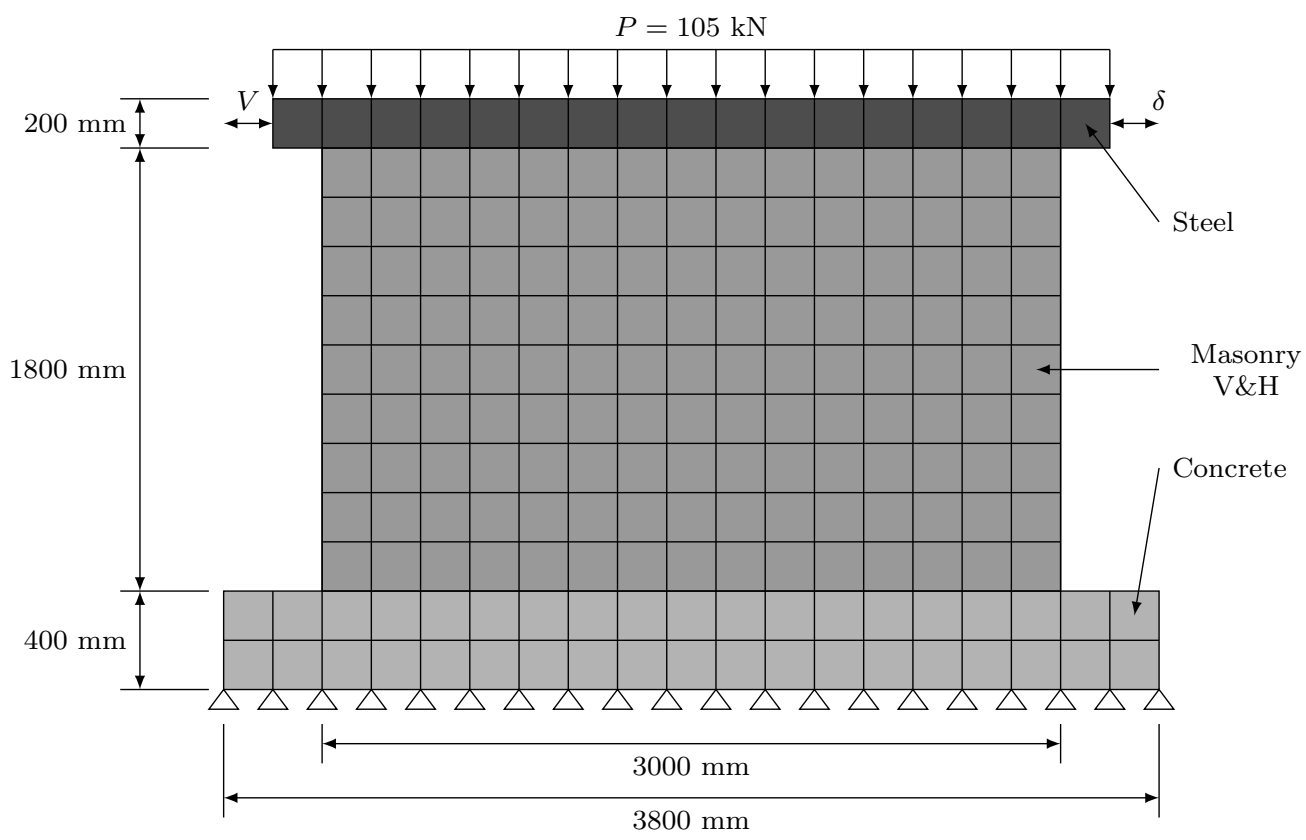

Finite Element Model Wall

A10 Shear Wall 
A10 Shear Wall Voon (2007) Material Properties

\begin{tabular}{|c|c|c|c|}
\hline \multirow{2}{*}{ Property } & \multicolumn{3}{|c|}{ Material } \\
\hline & Masonry & Concrete & Steel \\
\hline Thickness (mm) & 140 & 600 & 400 \\
\hline Grout Tensile Strength (MPa) & 1.90 & 2.09 & 350 \\
\hline \multicolumn{4}{|c|}{ Masonry Properties (Y-Direction) } \\
\hline Gross Peak Compression Strength (MPa) & 24.3 & 40 & 350 \\
\hline Peak Compressive Strain & 0.0032 & 0.0021 & 0.00175 \\
\hline Tensile Strength & 0 & 2.09 & 350 \\
\hline Tensile Strain & 0 & 0 & 0 \\
\hline Initial Tangent Elastic Modulus (MPa) & 0 & 34800 & 200000 \\
\hline Poisson's Ratio & 0 & 0.15 & 0 \\
\hline \multicolumn{4}{|c|}{ Masonry Properties (X-Direction) } \\
\hline Gross Peak Compression Strength $(\mathrm{MPa})$ & 24.3 & 40 & 350 \\
\hline Peak Compressive Strain & 0.0032 & 0.0021 & 0.00175 \\
\hline Tensile Strength & 0 & 2.09 & 350 \\
\hline Tensile Strain & 0 & 0 & 0 \\
\hline Initial Tangent Elastic Modulus (MPa) & 0 & 34800 & 200000 \\
\hline Poisson's Ratio & 0 & 0.15 & 0 \\
\hline \multicolumn{4}{|c|}{ Masonry Properties (Block) } \\
\hline Length (mm) & 390 & 2600 & 2000 \\
\hline Height (mm) & 190 & 400 & 200 \\
\hline Face Shell Thickness (mm) & 31 & 300 & 200 \\
\hline Percent Solid (\%) & 58 & 100 & 100 \\
\hline Tensile Strength & 1.50 & 2.09 & 350 \\
\hline \multicolumn{4}{|c|}{ Head Joint } \\
\hline Thickness (mm) & 10 & 10 & 10 \\
\hline Tensile Bond Strength (MPa) & 0.30 & 2.09 & 350 \\
\hline Joint Cohesion (MPa) & 4.50 & 2.09 & 350 \\
\hline Angle of Internal Friction (Deg) & 42 & 42 & 42 \\
\hline \multicolumn{4}{|c|}{ Bed Joint } \\
\hline Thickness (mm) & 10 & 10 & 10 \\
\hline Tensile Bond Strength (MPa) & 0.30 & 2.09 & 350 \\
\hline Joint Cohesion (MPa) & 4.50 & 2.09 & 350 \\
\hline Angle of Internal Friction (Deg) & 42 & 42 & 42 \\
\hline \multicolumn{4}{|c|}{ Reinforcement (Y-Direction) } \\
\hline Ratio (\%) & 0.60 & 0.52 & 100 \\
\hline Yield Strength (MPa) & 318 & 318 & 350 \\
\hline Yield Strain & 0.00159 & 0.00159 & 0.00175 \\
\hline Strain Hardening Strain & 0.02 & 0.02 & 0.02 \\
\hline Ultimate Strength (MPa) & 450 & 450 & 600 \\
\hline Ultimate Strain & 0.18 & 0.18 & 0.2 \\
\hline Prestrain & 0 & 0 & 0 \\
\hline \multicolumn{4}{|c|}{ Reinforcement (X-Direction) } \\
\hline Ratio (\%) & 0.05 & 0.52 & 100 \\
\hline Yield Strength (MPa) & 325 & 325 & 350 \\
\hline Yield Strain & 0.00163 & 0.00163 & 0.00175 \\
\hline Strain Hardening Strain & 0.02 & 0.02 & 0.02 \\
\hline Ultimate Strength (MPa) & 450 & 450 & 600 \\
\hline Ultimate Strain & 0.2 & 0.2 & 0.2 \\
\hline Prestrain & 0 & 0 & 0 \\
\hline
\end{tabular}

Numerical Modelling of Hollow Concrete Block Masonry Mechanics 
A10 Shear Wall Voon (2007) Model Options

\begin{tabular}{ll}
\hline Model & Selected Option \\
\hline Compression Pre-Peak & Hognestad \\
Compression Post-Peak & Pre-Peak Base Curve \\
Compression Softening & Vecchio \\
Tension Post-Peak & Vecchio \\
Cracking Criterion & Mohr-Coulomb \\
Slip & Walraven Reinforced \\
\hline
\end{tabular}

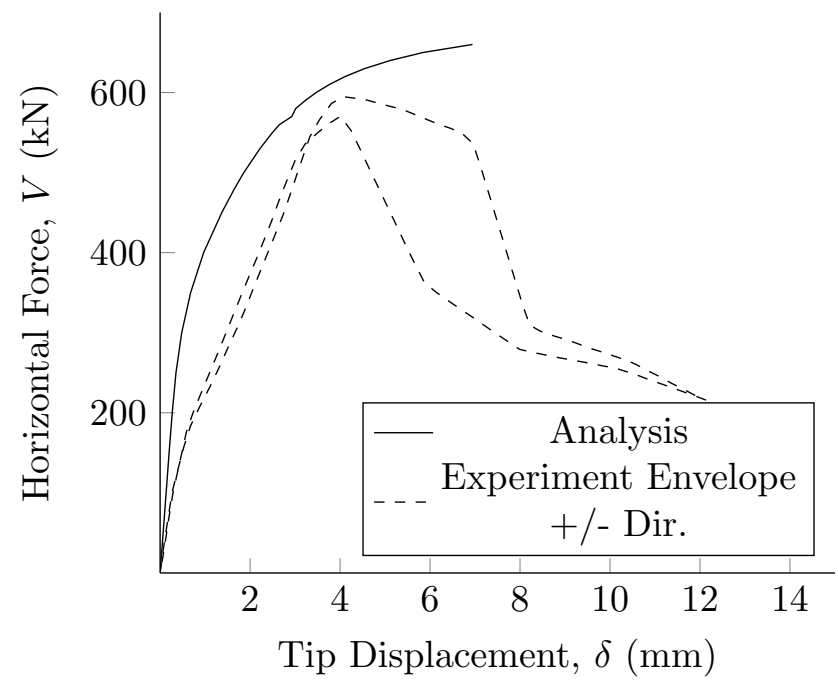

A10 Shear Wall Voon (2007) Horizontal Force vs Tip Displacement 


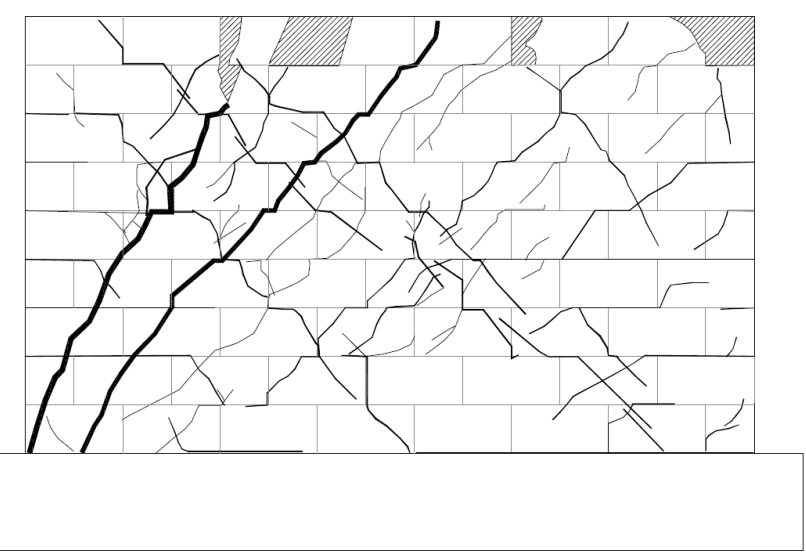

Experiment at Peak Horizontal Force (Voon, 2007)

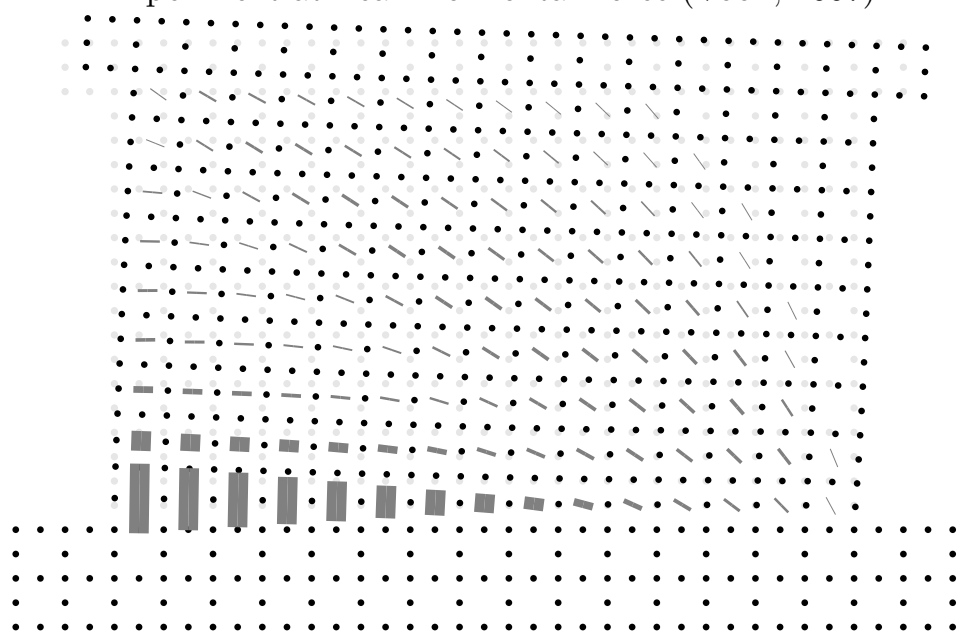

Finite Element Model at Peak Horizontal Force $\times 15$

A10 Shear Wall Displacement, Crack Pattern, and Crack Widths 


\section{Shedid et al. (2008)}

\section{Wall 1 Shear Wall}

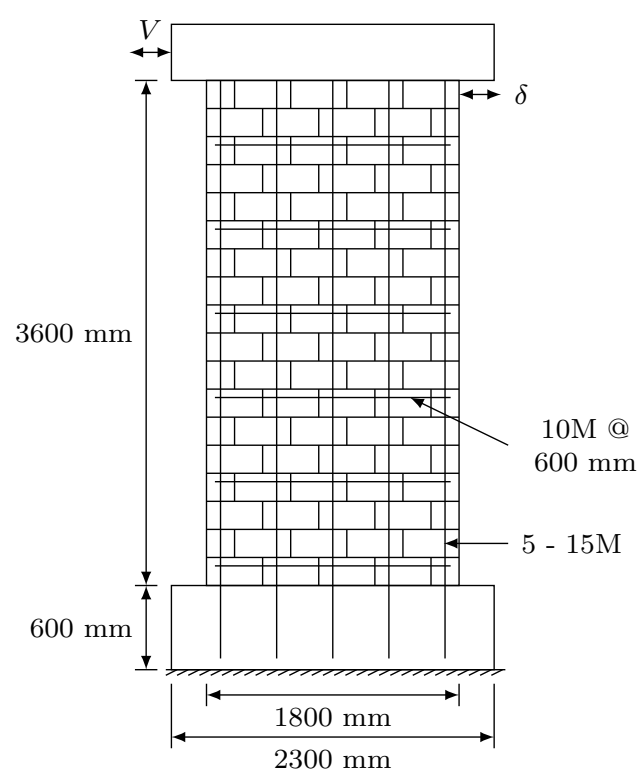

Experimental Wall Shedid et al. (2008)

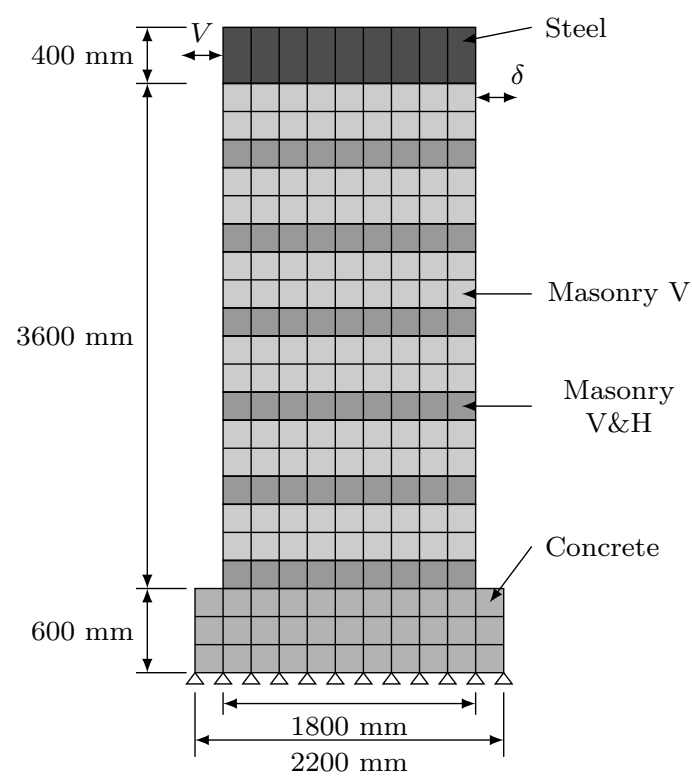

Finite Element Model Wall

Wall 1 Shear Wall

Wall 1 Shear Wall Shedid et al. (2008) Model Options

\begin{tabular}{ll}
\hline Model & Selected Option \\
\hline Compression Pre-Peak & Hognestad \\
Compression Post-Peak & Pre-Peak Base Curve \\
Compression Softening & Vecchio \\
Tension Post-Peak & Vecchio \\
Cracking Criterion & Mohr-Coulomb \\
Slip & Walraven Reinforced \\
\hline
\end{tabular}

Numerical Modelling of Hollow Concrete Block Masonry Mechanics 
Wall 1 Shear Wall Shedid et al. (2008) Material Properties

\begin{tabular}{|c|c|c|c|c|}
\hline \multirow{2}{*}{ Property } & \multicolumn{4}{|c|}{ Material } \\
\hline & Masonry V\&H & Masonry V & Concrete & Steel \\
\hline Thickness (mm) & 190 & 190 & 600 & 400 \\
\hline Grout Tensile Strength (MPa) & 1.87 & 1.87 & 2.08 & 350 \\
\hline \multicolumn{5}{|c|}{ Masonry Properties (Y-Direction) } \\
\hline Gross Peak Compression Strength (MPa) & 14.8 & 14.8 & 39.8 & 350 \\
\hline Peak Compressive Strain & 0.0027 & 0.0027 & 0.0021 & 0.00175 \\
\hline Tensile Strength & 0 & 0 & 2.08 & 350 \\
\hline Tensile Strain & 0 & 0 & 0 & 0.00175 \\
\hline Initial Tangent Elastic Modulus (MPa) & 0 & 0 & 34700 & 200000 \\
\hline Poisson's Ratio & 0 & 0 & 0.15 & 0 \\
\hline \multicolumn{5}{|c|}{ Masonry Properties (X-Direction) } \\
\hline Gross Peak Compression Strength (MPa) & 14.8 & 14.8 & 39.8 & 350 \\
\hline Peak Compressive Strain & 0.0027 & 0.0027 & 0.0021 & 0.00175 \\
\hline Tensile Strength & 0 & 0 & 2.08 & 350 \\
\hline Tensile Strain & 0 & 0 & 0 & 0.00175 \\
\hline Initial Tangent Elastic Modulus (MPa) & 0 & 0 & 34700 & 200000 \\
\hline Poisson's Ratio & 0 & 0 & 0.15 & 0 \\
\hline \multicolumn{5}{|c|}{ Masonry Properties (Block) } \\
\hline Length (mm) & 390 & 390 & 2200 & 1800 \\
\hline Height (mm) & 190 & 190 & 600 & 400 \\
\hline Face Shell Thickness (mm) & 32 & 32 & 300 & 200 \\
\hline Percent Solid (\%) & 52 & 52 & 100 & 100 \\
\hline Tensile Strength & 1.60 & 1.60 & 2.08 & 350 \\
\hline \multicolumn{5}{|c|}{ Head Joint } \\
\hline Thickness (mm) & 10 & 10 & 10 & 10 \\
\hline Tensile Bond Strength (MPa) & 0.36 & 0.36 & 2.08 & 350 \\
\hline Joint Cohesion (MPa) & 6.98 & 6.98 & 2.08 & 350 \\
\hline Angle of Internal Friction (Deg) & 42 & 42 & 42 & 42 \\
\hline \multicolumn{5}{|c|}{ Bed Joint } \\
\hline Thickness (mm) & 10 & 10 & 10 & 10 \\
\hline Tensile Bond Strength (MPa) & 0.36 & 0.36 & 2.08 & 350 \\
\hline Joint Cohesion (MPa) & 6.98 & 6.98 & 2.08 & 350 \\
\hline Angle of Internal Friction (Deg) & 42 & 42 & 42 & 42 \\
\hline \multicolumn{5}{|c|}{ Reinforcement (Y-Direction) } \\
\hline Ratio (\%) & 0.29 & 0.29 & 1 & 100 \\
\hline Yield Strength (MPa) & 502 & 502 & 502 & 350 \\
\hline Yield Strain & 0.00251 & 0.00251 & 0.00251 & 0.00175 \\
\hline Strain Hardening Strain & 0.02 & 0.02 & 0.02 & 0.02 \\
\hline Ultimate Strength $(\mathrm{MPa})$ & 650 & 650 & 650 & 600 \\
\hline Ultimate Strain & 0.2 & 0.2 & 0.2 & 0.2 \\
\hline Prestrain & 0 & 0 & 0 & 0 \\
\hline \multicolumn{5}{|c|}{ Reinforcement (X-Direction) } \\
\hline Ratio (\%) & 0.08 & 0 & 1 & 100 \\
\hline Yield Strength (MPa) & 491 & 0 & 491 & 350 \\
\hline Yield Strain & 0.00246 & 0 & 0.0246 & 0.00175 \\
\hline Strain Hardening Strain & 0.02 & 0 & 0.02 & 0.02 \\
\hline Ultimate Strength (MPa) & 650 & 0 & 650 & 600 \\
\hline Ultimate Strain & 0.2 & 0 & 0.2 & 0.2 \\
\hline Prestrain & 0 & 0 & 0 & 0 \\
\hline
\end{tabular}

Numerical Modelling of Hollow Concrete Block Masonry Mechanics 


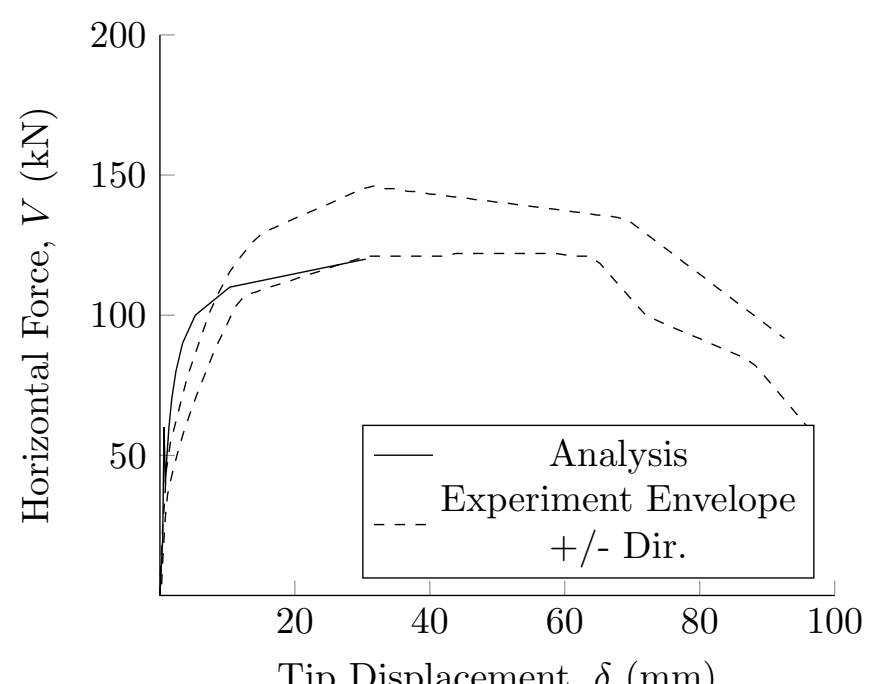

Wall 1 Shear Wall Shedid et al. (2008) Horizontal Force vs Tip Displacement 


\section{Wall 2 Shear Wall}

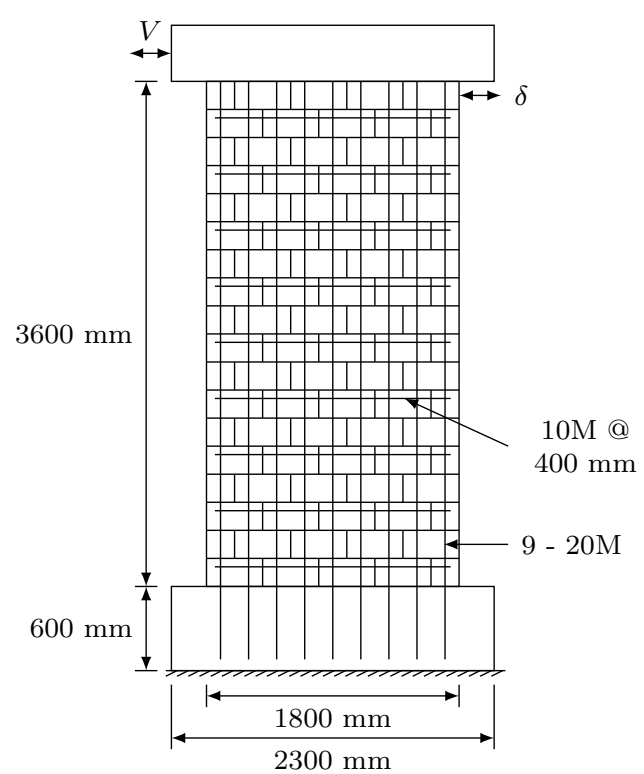

Experimental Wall Shedid et al. (2008)

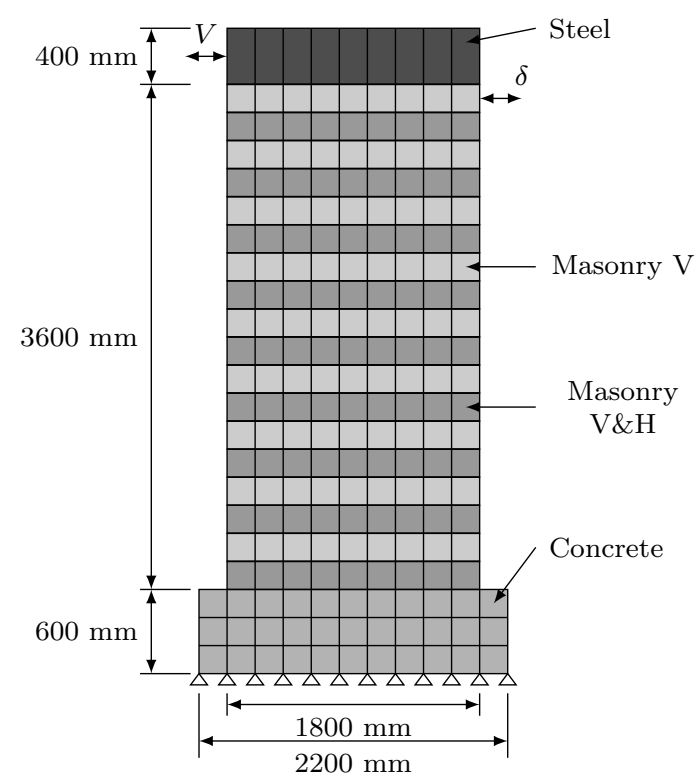

Finite Element Model Wall

Wall 2 Shear Wall

Wall 2 Shear Wall Shedid et al. (2008) Model Options

\begin{tabular}{ll}
\hline Model & Selected Option \\
\hline Compression Pre-Peak & Hognestad \\
Compression Post-Peak & Pre-Peak Base Curve \\
Compression Softening & Vecchio \\
Tension Post-Peak & Vecchio \\
Cracking Criterion & Mohr-Coulomb \\
Slip & Walraven Reinforced \\
\hline
\end{tabular}


Wall 2 Shear Wall Shedid et al. (2008) Material Properties

\begin{tabular}{|c|c|c|c|c|}
\hline \multirow{2}{*}{ Property } & \multicolumn{4}{|c|}{ Material } \\
\hline & Masonry V\&H & Masonry V & Concrete & Steel \\
\hline Thickness (mm) & 190 & 190 & 600 & 400 \\
\hline Grout Tensile Strength (MPa) & 1.87 & 1.87 & 2.08 & 350 \\
\hline \multicolumn{5}{|c|}{ Masonry Properties (Y-Direction) } \\
\hline Gross Peak Compression Strength (MPa) & 14.8 & 14.8 & 39.8 & 350 \\
\hline Peak Compressive Strain & 0.0027 & 0.0027 & 0.0021 & 0.00175 \\
\hline Tensile Strength & 0 & 0 & 2.08 & 350 \\
\hline Tensile Strain & 0 & 0 & 0 & 0.00175 \\
\hline Initial Tangent Elastic Modulus (MPa) & 0 & 0 & 34700 & 200000 \\
\hline Poisson's Ratio & 0 & 0 & 0.15 & 0 \\
\hline \multicolumn{5}{|c|}{ Masonry Properties (X-Direction) } \\
\hline Gross Peak Compression Strength (MPa) & 14.8 & 14.8 & 39.8 & 350 \\
\hline Peak Compressive Strain & 0.0027 & 0.0027 & 0.0021 & 0.00175 \\
\hline Tensile Strength & 0 & 0 & 2.08 & 350 \\
\hline Tensile Strain & 0 & 0 & 0 & 0.00175 \\
\hline Initial Tangent Elastic Modulus (MPa) & 0 & 0 & 34700 & 200000 \\
\hline Poisson's Ratio & 0 & 0 & 0.15 & 0 \\
\hline \multicolumn{5}{|c|}{ Masonry Properties (Block) } \\
\hline Length (mm) & 390 & 390 & 2200 & 1800 \\
\hline Height (mm) & 190 & 190 & 600 & 400 \\
\hline Face Shell Thickness (mm) & 32 & 32 & 300 & 200 \\
\hline Percent Solid (\%) & 52 & 52 & 100 & 100 \\
\hline Tensile Strength & 1.60 & 1.60 & 2.08 & 350 \\
\hline \multicolumn{5}{|c|}{ Head Joint } \\
\hline Thickness $(\mathrm{mm})$ & 10 & 10 & 10 & 10 \\
\hline Tensile Bond Strength (MPa) & 0.36 & 0.36 & 2.08 & 350 \\
\hline Joint Cohesion (MPa) & 6.98 & 6.98 & 2.08 & 350 \\
\hline Angle of Internal Friction (Deg) & 42 & 42 & 42 & 42 \\
\hline \multicolumn{5}{|c|}{ Bed Joint } \\
\hline Thickness (mm) & 10 & 10 & 10 & 10 \\
\hline Tensile Bond Strength (MPa) & 0.36 & 0.36 & 2.08 & 350 \\
\hline Joint Cohesion (MPa) & 6.98 & 6.98 & 2.08 & 350 \\
\hline Angle of Internal Friction (Deg) & 42 & 42 & 42 & 42 \\
\hline \multicolumn{5}{|c|}{ Reinforcement (Y-Direction) } \\
\hline Ratio (\%) & 0.78 & 0.78 & 1 & 100 \\
\hline Yield Strength (MPa) & 502 & 502 & 502 & 350 \\
\hline Yield Strain & 0.00251 & 0.00251 & 0.00251 & 0.00175 \\
\hline Strain Hardening Strain & 0.02 & 0.02 & 0.02 & 0.02 \\
\hline Ultimate Strength (MPa) & 650 & 650 & 650 & 600 \\
\hline Ultimate Strain & 0.2 & 0.2 & 0.2 & 0.2 \\
\hline Prestrain & 0 & 0 & 0 & 0 \\
\hline \multicolumn{5}{|c|}{ Reinforcement (X-Direction) } \\
\hline Ratio (\%) & 0.13 & 0 & 1 & 100 \\
\hline Yield Strength $(\mathrm{MPa})$ & 491 & 0 & 491 & 350 \\
\hline Yield Strain & 0.00246 & 0 & 0.0246 & 0.00175 \\
\hline Strain Hardening Strain & 0.02 & 0 & 0.02 & 0.02 \\
\hline Ultimate Strength (MPa) & 650 & 0 & 650 & 600 \\
\hline Ultimate Strain & 0.2 & 0 & 0.2 & 0.2 \\
\hline Prestrain & 0 & 0 & 0 & 0 \\
\hline
\end{tabular}

Numerical Modelling of Hollow Concrete Block Masonry Mechanics 


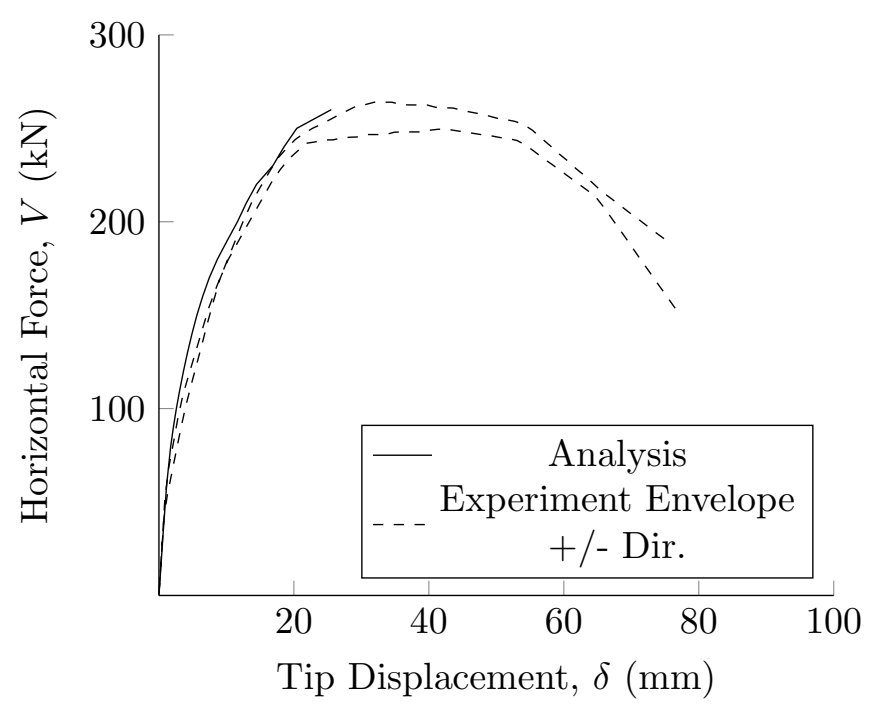

Wall 2 Shear Wall Shedid et al. (2008) Horizontal Force vs Tip Displacement 


\section{Wall 3 Shear Wall}

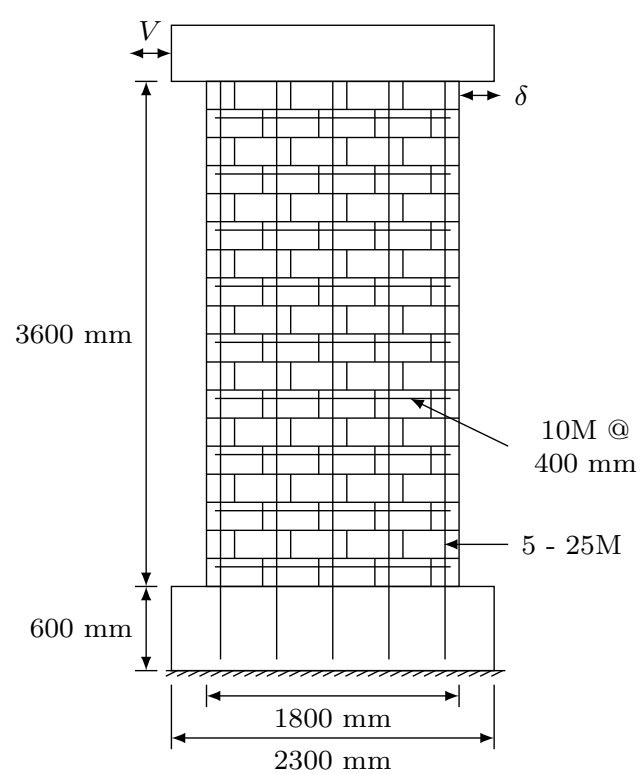

Experimental Wall Shedid et al. (2008)

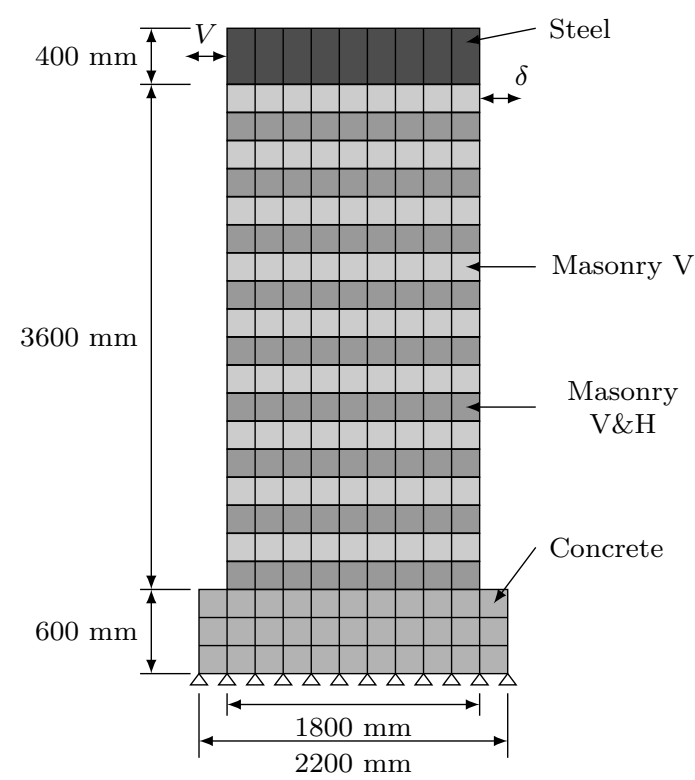

Finite Element Model Wall

Wall 3 Shear Wall

Wall 3 Shear Wall Shedid et al. (2008) Model Options

\begin{tabular}{ll}
\hline Model & Selected Option \\
\hline Compression Pre-Peak & Hognestad \\
Compression Post-Peak & Pre-Peak Base Curve \\
Compression Softening & Vecchio \\
Tension Post-Peak & Vecchio \\
Cracking Criterion & Mohr-Coulomb \\
Slip & Walraven Reinforced \\
\hline
\end{tabular}


Wall 3 Shear Wall Shedid et al. (2008) Material Properties

\begin{tabular}{|c|c|c|c|c|}
\hline \multirow{2}{*}{ Property } & \multicolumn{4}{|c|}{ Material } \\
\hline & Masonry V\&H & Masonry V & Concrete & Steel \\
\hline Thickness (mm) & 190 & 190 & 600 & 400 \\
\hline Grout Tensile Strength (MPa) & 1.87 & 1.87 & 2.08 & 350 \\
\hline \multicolumn{5}{|c|}{ Masonry Properties (Y-Direction) } \\
\hline Gross Peak Compression Strength (MPa) & 14.8 & 14.8 & 39.8 & 350 \\
\hline Peak Compressive Strain & 0.0027 & 0.0027 & 0.0021 & 0.00175 \\
\hline Tensile Strength & 0 & 0 & 2.08 & 350 \\
\hline Tensile Strain & 0 & 0 & 0 & 0.00175 \\
\hline Initial Tangent Elastic Modulus (MPa) & 0 & 0 & 34700 & 200000 \\
\hline Poisson's Ratio & 0 & 0 & 0.15 & 0 \\
\hline \multicolumn{5}{|c|}{ Masonry Properties (X-Direction) } \\
\hline Gross Peak Compression Strength (MPa) & 14.8 & 14.8 & 39.8 & 350 \\
\hline Peak Compressive Strain & 0.0027 & 0.0027 & 0.0021 & 0.00175 \\
\hline Tensile Strength & 0 & 0 & 2.08 & 350 \\
\hline Tensile Strain & 0 & 0 & 0 & 0.00175 \\
\hline Initial Tangent Elastic Modulus (MPa) & 0 & 0 & 34700 & 200000 \\
\hline Poisson's Ratio & 0 & 0 & 0.15 & 0 \\
\hline \multicolumn{5}{|c|}{ Masonry Properties (Block) } \\
\hline Length (mm) & 390 & 390 & 2200 & 1800 \\
\hline Height (mm) & 190 & 190 & 600 & 400 \\
\hline Face Shell Thickness (mm) & 32 & 32 & 300 & 200 \\
\hline Percent Solid (\%) & 52 & 52 & 100 & 100 \\
\hline Tensile Strength & 1.60 & 1.60 & 2.08 & 350 \\
\hline \multicolumn{5}{|c|}{ Head Joint } \\
\hline Thickness (mm) & 10 & 10 & 10 & 10 \\
\hline Tensile Bond Strength (MPa) & 0.36 & 0.36 & 2.08 & 350 \\
\hline Joint Cohesion (MPa) & 6.98 & 6.98 & 2.08 & 350 \\
\hline Angle of Internal Friction (Deg) & 42 & 42 & 42 & 42 \\
\hline \multicolumn{5}{|c|}{ Bed Joint } \\
\hline Thickness (mm) & 10 & 10 & 10 & 10 \\
\hline Tensile Bond Strength (MPa) & 0.36 & 0.36 & 2.08 & 350 \\
\hline Joint Cohesion (MPa) & 6.98 & 6.98 & 2.08 & 350 \\
\hline Angle of Internal Friction (Deg) & 42 & 42 & 42 & 42 \\
\hline \multicolumn{5}{|c|}{ Reinforcement (Y-Direction) } \\
\hline Ratio (\%) & 0.73 & 0.73 & 1 & 100 \\
\hline Yield Strength (MPa) & 502 & 502 & 502 & 350 \\
\hline Yield Strain & 0.00251 & 0.00251 & 0.00251 & 0.00175 \\
\hline Strain Hardening Strain & 0.02 & 0.02 & 0.02 & 0.02 \\
\hline Ultimate Strength $(\mathrm{MPa})$ & 650 & 650 & 650 & 600 \\
\hline Ultimate Strain & 0.2 & 0.2 & 0.2 & 0.2 \\
\hline Prestrain & 0 & 0 & 0 & 0 \\
\hline \multicolumn{5}{|c|}{ Reinforcement (X-Direction) } \\
\hline Ratio (\%) & 0.13 & 0 & 1 & 100 \\
\hline Yield Strength (MPa) & 491 & 0 & 491 & 350 \\
\hline Yield Strain & 0.00246 & 0 & 0.0246 & 0.00175 \\
\hline Strain Hardening Strain & 0.02 & 0 & 0.02 & 0.02 \\
\hline Ultimate Strength (MPa) & 650 & 0 & 650 & 600 \\
\hline Ultimate Strain & 0.2 & 0 & 0.2 & 0.2 \\
\hline Prestrain & 0 & 0 & 0 & 0 \\
\hline
\end{tabular}

Numerical Modelling of Hollow Concrete Block Masonry Mechanics 


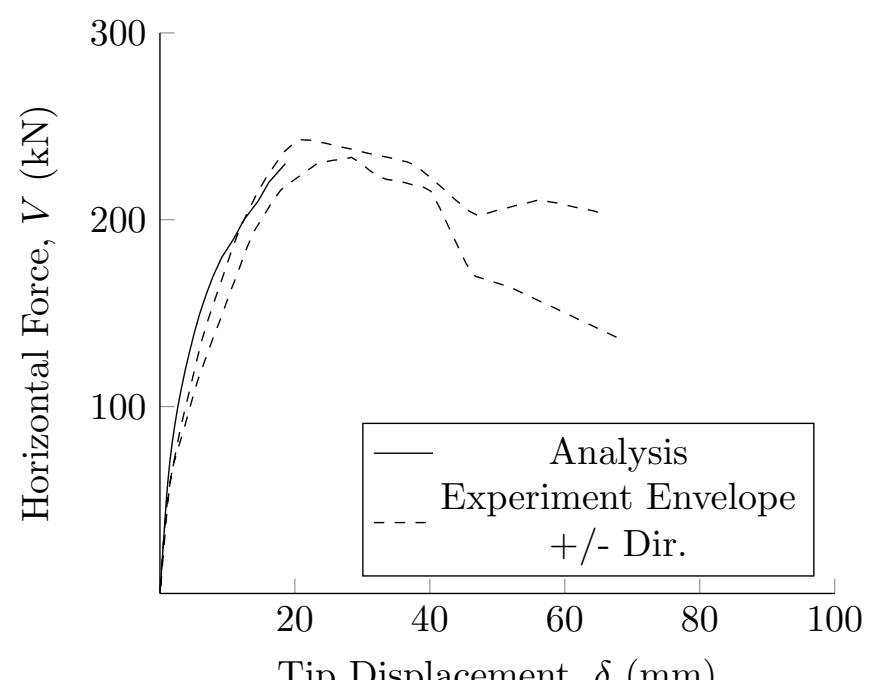

Wall 3 Shear Wall Shedid et al. (2008) Horizontal Force vs Tip Displacement 


\section{Wall 4 Shear Wall}

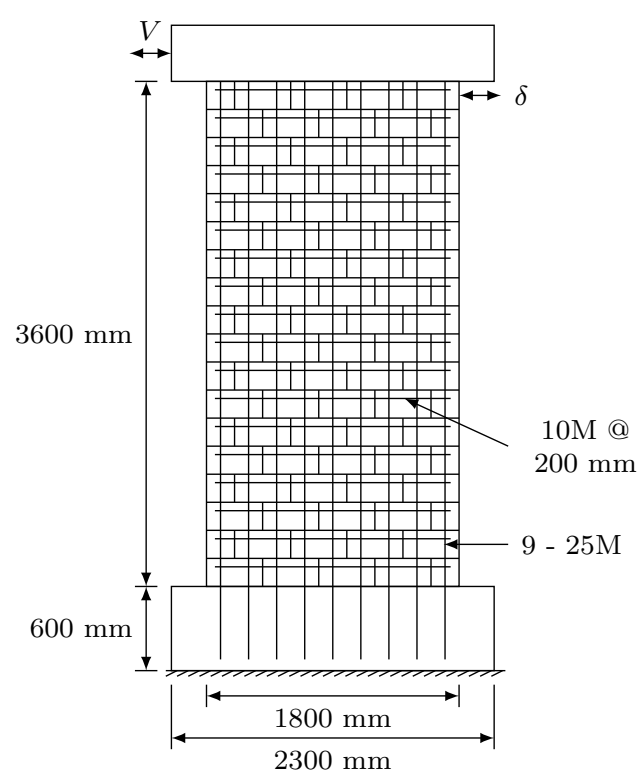

Experimental Wall Shedid et al. (2008)

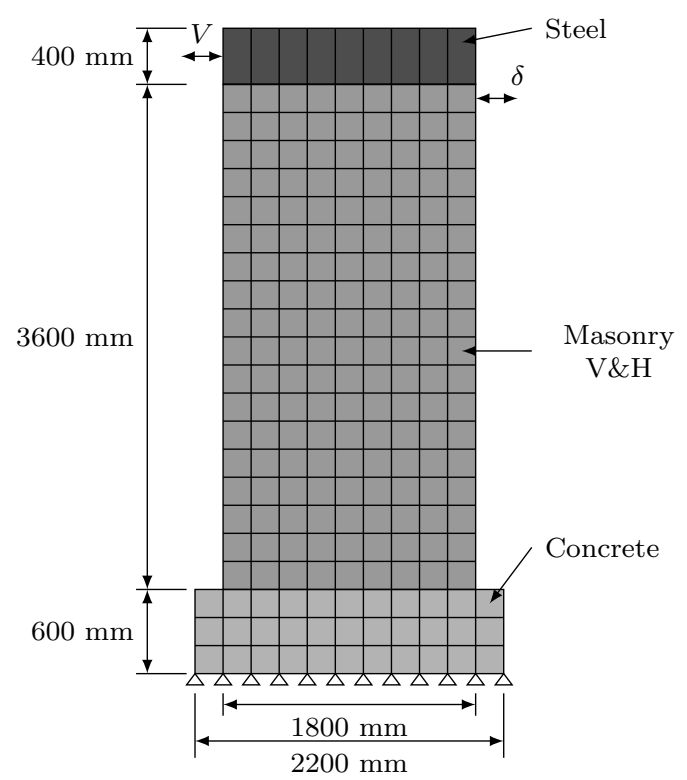

Finite Element Model Wall

Wall 4 Shear Wall

Wall 4 Shear Wall Shedid et al. (2008) Model Options

\begin{tabular}{ll}
\hline Model & Selected Option \\
\hline Compression Pre-Peak & Hognestad \\
Compression Post-Peak & Pre-Peak Base Curve \\
Compression Softening & Vecchio \\
Tension Post-Peak & Vecchio \\
Cracking Criterion & Mohr-Coulomb \\
Slip & Walraven Reinforced \\
\hline
\end{tabular}


Wall 4 Shear Wall Shedid et al. (2008) Material Properties

\begin{tabular}{|c|c|c|c|}
\hline \multirow{2}{*}{ Property } & \multicolumn{3}{|c|}{ Material } \\
\hline & Masonry V\&H & Concrete & Steel \\
\hline Thickness (mm) & 190 & 600 & 400 \\
\hline Grout Tensile Strength (MPa) & 1.87 & 2.08 & 350 \\
\hline \multicolumn{4}{|c|}{ Masonry Properties (Y-Direction) } \\
\hline Gross Peak Compression Strength (MPa) & 14.8 & 39.8 & 350 \\
\hline Peak Compressive Strain & 0.0027 & 0.0021 & 0.00175 \\
\hline Tensile Strength & 0 & 2.08 & 350 \\
\hline Tensile Strain & 0 & 0 & 0.00175 \\
\hline Initial Tangent Elastic Modulus (MPa) & 0 & 34700 & 200000 \\
\hline Poisson's Ratio & 0 & 0.15 & 0 \\
\hline \multicolumn{4}{|c|}{ Masonry Properties (X-Direction) } \\
\hline Gross Peak Compression Strength (MPa) & 14.8 & 39.8 & 350 \\
\hline Peak Compressive Strain & 0.0027 & 0.0021 & 0.00175 \\
\hline Tensile Strength & 0 & 2.08 & 350 \\
\hline Tensile Strain & 0 & 0 & 0.00175 \\
\hline Initial Tangent Elastic Modulus (MPa) & 0 & 34700 & 200000 \\
\hline Poisson's Ratio & 0 & 0.15 & 0 \\
\hline \multicolumn{4}{|c|}{ Masonry Properties (Block) } \\
\hline Length (mm) & 390 & 2200 & 1800 \\
\hline Height (mm) & 190 & 600 & 400 \\
\hline Face Shell Thickness (mm) & 32 & 300 & 200 \\
\hline Percent Solid (\%) & 52 & 100 & 100 \\
\hline Tensile Strength & 1.60 & 2.08 & 350 \\
\hline \multicolumn{4}{|c|}{ Head Joint } \\
\hline Thickness (mm) & 10 & 10 & 10 \\
\hline Tensile Bond Strength (MPa) & 0.36 & 2.08 & 350 \\
\hline Joint Cohesion (MPa) & 6.98 & 2.08 & 350 \\
\hline Angle of Internal Friction (Deg) & 42 & 42 & 42 \\
\hline \multicolumn{4}{|c|}{ Bed Joint } \\
\hline Thickness (mm) & 10 & 10 & 10 \\
\hline Tensile Bond Strength (MPa) & 0.36 & 2.08 & 350 \\
\hline Joint Cohesion (MPa) & 6.98 & 2.08 & 350 \\
\hline Angle of Internal Friction (Deg) & 42 & 42 & 42 \\
\hline \multicolumn{4}{|c|}{ Reinforcement (Y-Direction) } \\
\hline Ratio (\%) & 1.31 & 1 & 100 \\
\hline Yield Strength (MPa) & 502 & 502 & 350 \\
\hline Yield Strain & 0.00251 & 0.00251 & 0.00175 \\
\hline Strain Hardening Strain & 0.02 & 0.02 & 0.02 \\
\hline Ultimate Strength (MPa) & 650 & 650 & 600 \\
\hline Ultimate Strain & 0.2 & 0.2 & 0.2 \\
\hline Prestrain & 0 & 0 & 0 \\
\hline \multicolumn{4}{|c|}{ Reinforcement (X-Direction) } \\
\hline Ratio (\%) & 0.26 & 1 & 100 \\
\hline Yield Strength (MPa) & 491 & 491 & 350 \\
\hline Yield Strain & 0.00246 & 0.0246 & 0.00175 \\
\hline Strain Hardening Strain & 0.02 & 0.02 & 0.02 \\
\hline Ultimate Strength (MPa) & 650 & 650 & 600 \\
\hline Ultimate Strain & 0.2 & 0.2 & 0.2 \\
\hline Prestrain & 0 & 0 & 0 \\
\hline
\end{tabular}

Numerical Modelling of Hollow Concrete Block Masonry Mechanics 


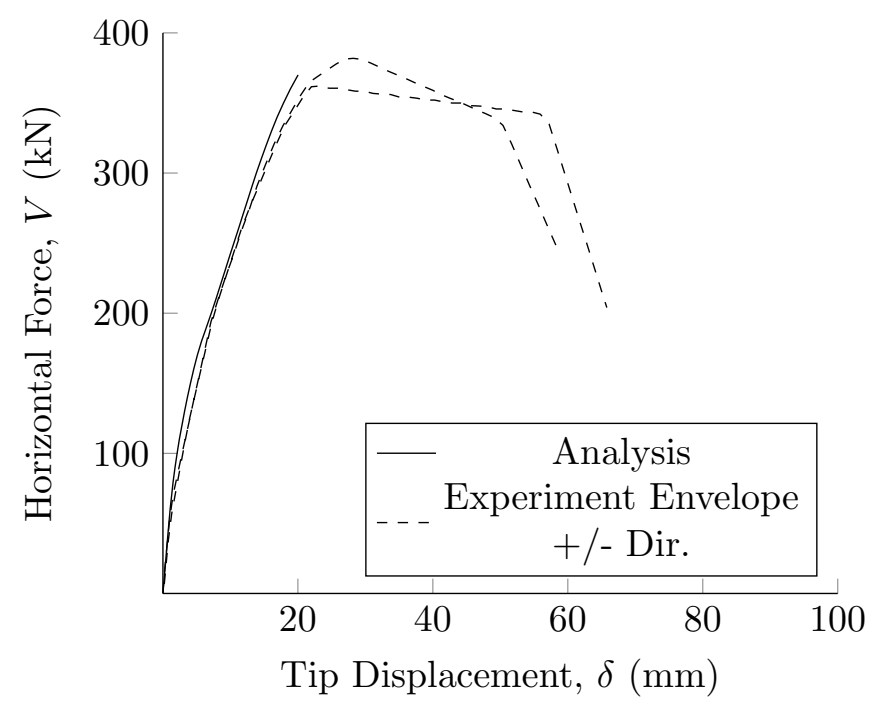

Wall 4 Shear Wall Shedid et al. (2008) Horizontal Force vs Tip Displacement 


\section{Wall 5 Shear Wall}

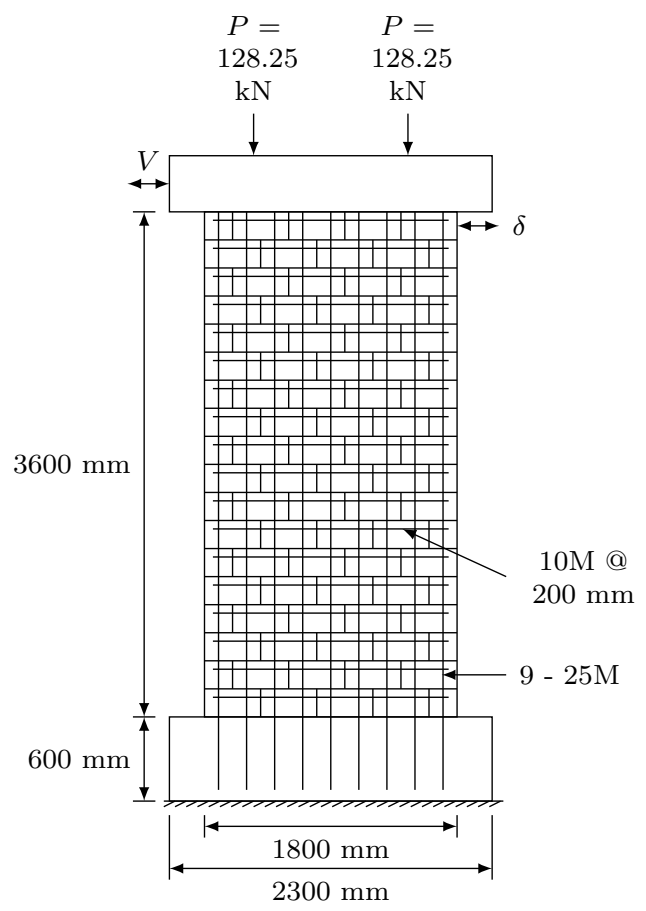

Experimental Wall Shedid et al. (2008)

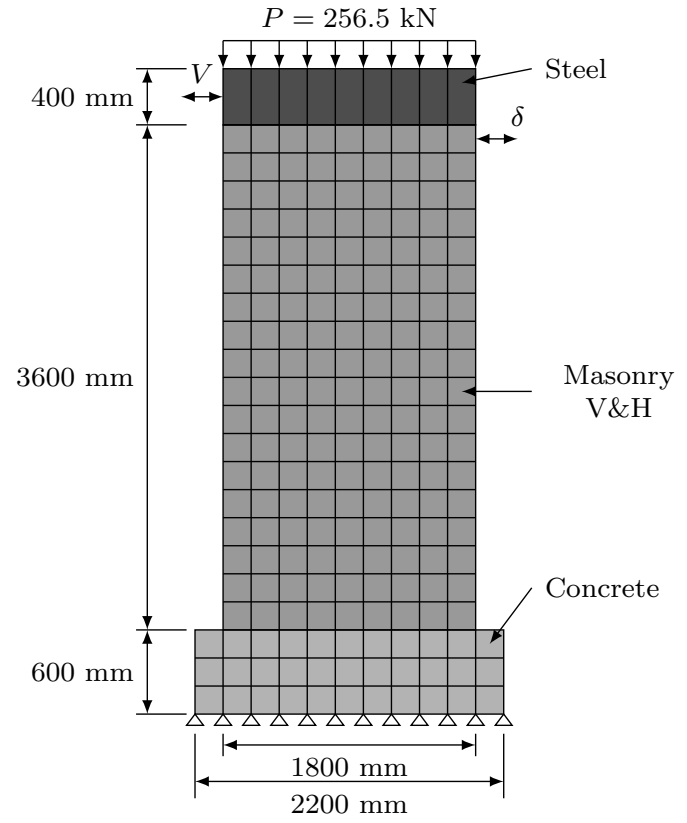

Finite Element Model Wall

Wall 5 Shear Wall

Wall 5 Shear Wall Shedid et al. (2008) Model Options

\begin{tabular}{ll}
\hline Model & Selected Option \\
\hline Compression Pre-Peak & Hognestad \\
Compression Post-Peak & Pre-Peak Base Curve \\
Compression Softening & Vecchio \\
Tension Post-Peak & Vecchio \\
Cracking Criterion & Mohr-Coulomb \\
Slip & Walraven Reinforced \\
\hline
\end{tabular}


Wall 5 Shear Wall Shedid et al. (2008) Material Properties

\begin{tabular}{|c|c|c|c|}
\hline \multirow{2}{*}{ Property } & \multicolumn{3}{|c|}{ Material } \\
\hline & Masonry V\&H & Concrete & Steel \\
\hline Thickness (mm) & 190 & 600 & 400 \\
\hline Grout Tensile Strength (MPa) & 1.87 & 2.08 & 350 \\
\hline \multicolumn{4}{|c|}{ Masonry Properties (Y-Direction) } \\
\hline Gross Peak Compression Strength (MPa) & 14.8 & 39.8 & 350 \\
\hline Peak Compressive Strain & 0.0027 & 0.0021 & 0.00175 \\
\hline Tensile Strength & 0 & 2.08 & 350 \\
\hline Tensile Strain & 0 & 0 & 0.00175 \\
\hline Initial Tangent Elastic Modulus (MPa) & 0 & 34700 & 200000 \\
\hline Poisson's Ratio & 0 & 0.15 & 0 \\
\hline \multicolumn{4}{|c|}{ Masonry Properties (X-Direction) } \\
\hline Gross Peak Compression Strength (MPa) & 14.8 & 39.8 & 350 \\
\hline Peak Compressive Strain & 0.0027 & 0.0021 & 0.00175 \\
\hline Tensile Strength & 0 & 2.08 & 350 \\
\hline Tensile Strain & 0 & 0 & 0.00175 \\
\hline Initial Tangent Elastic Modulus (MPa) & 0 & 34700 & 200000 \\
\hline Poisson's Ratio & 0 & 0.15 & 0 \\
\hline \multicolumn{4}{|c|}{ Masonry Properties (Block) } \\
\hline Length (mm) & 390 & 2200 & 1800 \\
\hline Height (mm) & 190 & 600 & 400 \\
\hline Face Shell Thickness (mm) & 32 & 300 & 200 \\
\hline Percent Solid (\%) & 52 & 100 & 100 \\
\hline Tensile Strength & 1.60 & 2.08 & 350 \\
\hline \multicolumn{4}{|c|}{ Head Joint } \\
\hline Thickness (mm) & 10 & 10 & 10 \\
\hline Tensile Bond Strength (MPa) & 0.36 & 2.08 & 350 \\
\hline Joint Cohesion (MPa) & 6.98 & 2.08 & 350 \\
\hline Angle of Internal Friction (Deg) & 42 & 42 & 42 \\
\hline \multicolumn{4}{|c|}{ Bed Joint } \\
\hline Thickness (mm) & 10 & 10 & 10 \\
\hline Tensile Bond Strength (MPa) & 0.36 & 2.08 & 350 \\
\hline Joint Cohesion (MPa) & 6.98 & 2.08 & 350 \\
\hline Angle of Internal Friction (Deg) & 42 & 42 & 42 \\
\hline \multicolumn{4}{|c|}{ Reinforcement (Y-Direction) } \\
\hline Ratio (\%) & 1.31 & 1 & 100 \\
\hline Yield Strength (MPa) & 502 & 502 & 350 \\
\hline Yield Strain & 0.00251 & 0.00251 & 0.00175 \\
\hline Strain Hardening Strain & 0.02 & 0.02 & 0.02 \\
\hline Ultimate Strength (MPa) & 650 & 650 & 600 \\
\hline Ultimate Strain & 0.2 & 0.2 & 0.2 \\
\hline Prestrain & 0 & 0 & 0 \\
\hline \multicolumn{4}{|c|}{ Reinforcement (X-Direction) } \\
\hline Ratio (\%) & 0.26 & 1 & 100 \\
\hline Yield Strength (MPa) & 491 & 491 & 350 \\
\hline Yield Strain & 0.00246 & 0.0246 & 0.00175 \\
\hline Strain Hardening Strain & 0.02 & 0.02 & 0.02 \\
\hline Ultimate Strength (MPa) & 650 & 650 & 600 \\
\hline Ultimate Strain & 0.2 & 0.2 & 0.2 \\
\hline Prestrain & 0 & 0 & 0 \\
\hline
\end{tabular}

Numerical Modelling of Hollow Concrete Block Masonry Mechanics 


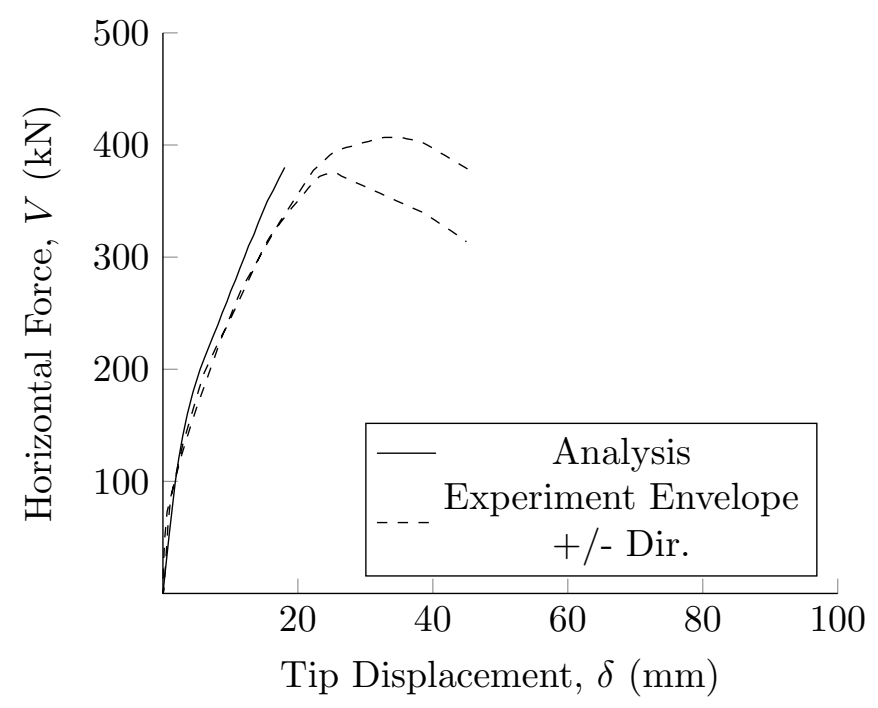

Wall 5 Shear Wall Shedid et al. (2008) Horizontal Force vs Tip Displacement 


\section{Wall 6 Shear Wall}

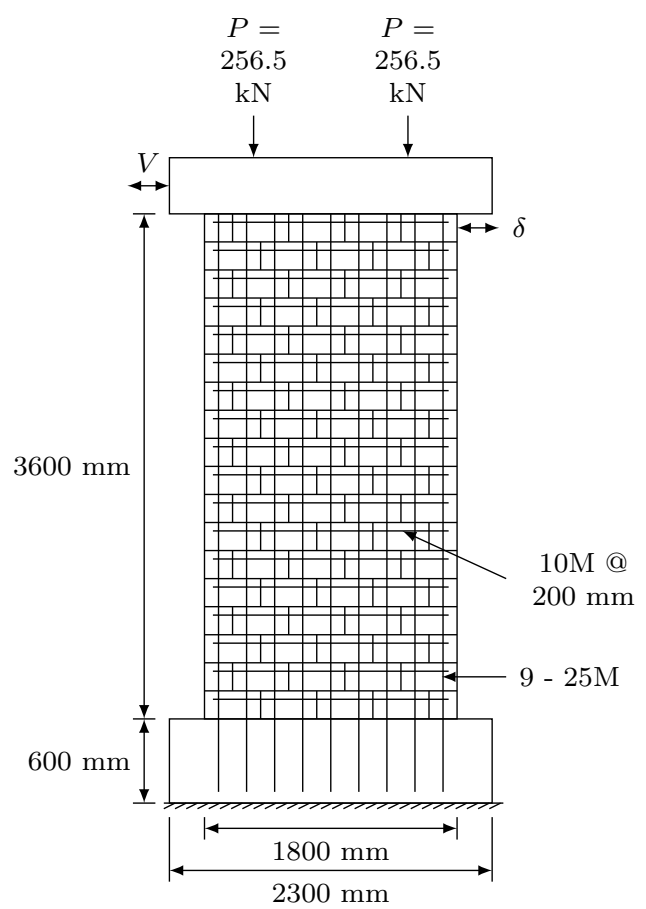

Experimental Wall Shedid et al. (2008)

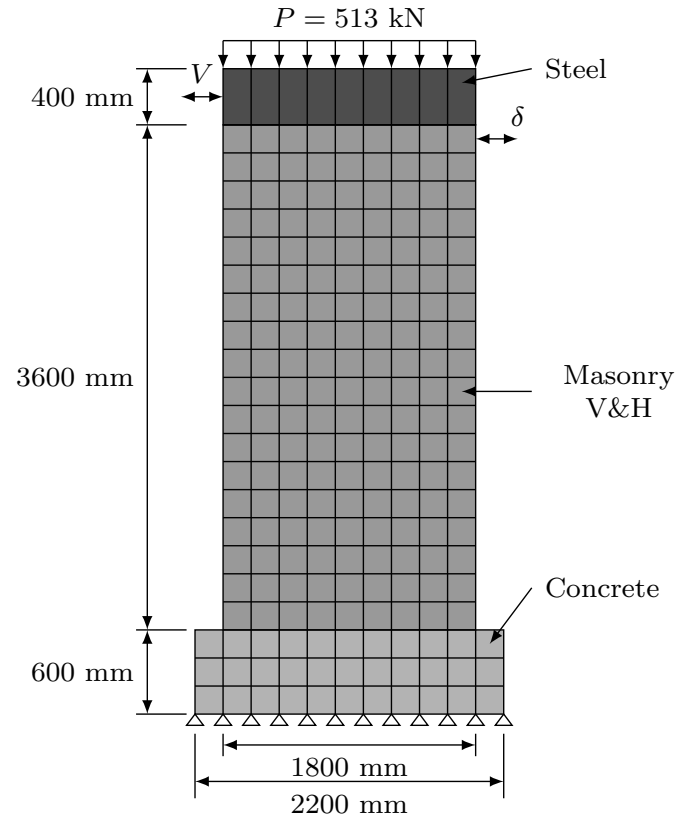

Finite Element Model Wall

Wall 6 Shear Wall

Wall 6 Shear Wall Shedid et al. (2008) Model Options

\begin{tabular}{ll}
\hline Model & Selected Option \\
\hline Compression Pre-Peak & Hognestad \\
Compression Post-Peak & Pre-Peak Base Curve \\
Compression Softening & Vecchio \\
Tension Post-Peak & Vecchio \\
Cracking Criterion & Mohr-Coulomb \\
Slip & Walraven Reinforced \\
\hline
\end{tabular}


Wall 6 Shear Wall Shedid et al. (2008) Material Properties

\begin{tabular}{|c|c|c|c|}
\hline \multirow{2}{*}{ Property } & \multicolumn{3}{|c|}{ Material } \\
\hline & Masonry V\&H & Concrete & Steel \\
\hline Thickness (mm) & 190 & 600 & 400 \\
\hline Grout Tensile Strength (MPa) & 1.87 & 2.08 & 350 \\
\hline \multicolumn{4}{|c|}{ Masonry Properties (Y-Direction) } \\
\hline Gross Peak Compression Strength (MPa) & 14.8 & 39.8 & 350 \\
\hline Peak Compressive Strain & 0.0027 & 0.0021 & 0.00175 \\
\hline Tensile Strength & 0 & 2.08 & 350 \\
\hline Tensile Strain & 0 & 0 & 0.00175 \\
\hline Initial Tangent Elastic Modulus (MPa) & 0 & 34700 & 200000 \\
\hline Poisson's Ratio & 0 & 0.15 & 0 \\
\hline \multicolumn{4}{|c|}{ Masonry Properties (X-Direction) } \\
\hline Gross Peak Compression Strength (MPa) & 14.8 & 39.8 & 350 \\
\hline Peak Compressive Strain & 0.0027 & 0.0021 & 0.00175 \\
\hline Tensile Strength & 0 & 2.08 & 350 \\
\hline Tensile Strain & 0 & 0 & 0.00175 \\
\hline Initial Tangent Elastic Modulus (MPa) & 0 & 34700 & 200000 \\
\hline Poisson's Ratio & 0 & 0.15 & 0 \\
\hline \multicolumn{4}{|c|}{ Masonry Properties (Block) } \\
\hline Length (mm) & 390 & 2200 & 1800 \\
\hline Height (mm) & 190 & 600 & 400 \\
\hline Face Shell Thickness (mm) & 32 & 300 & 200 \\
\hline Percent Solid (\%) & 52 & 100 & 100 \\
\hline Tensile Strength & 1.60 & 2.08 & 350 \\
\hline \multicolumn{4}{|c|}{ Head Joint } \\
\hline Thickness (mm) & 10 & 10 & 10 \\
\hline Tensile Bond Strength (MPa) & 0.36 & 2.08 & 350 \\
\hline Joint Cohesion (MPa) & 6.98 & 2.08 & 350 \\
\hline Angle of Internal Friction (Deg) & 42 & 42 & 42 \\
\hline \multicolumn{4}{|c|}{ Bed Joint } \\
\hline Thickness (mm) & 10 & 10 & 10 \\
\hline Tensile Bond Strength (MPa) & 0.36 & 2.08 & 350 \\
\hline Joint Cohesion (MPa) & 6.98 & 2.08 & 350 \\
\hline Angle of Internal Friction (Deg) & 42 & 42 & 42 \\
\hline \multicolumn{4}{|c|}{ Reinforcement (Y-Direction) } \\
\hline Ratio (\%) & 1.31 & 1 & 100 \\
\hline Yield Strength (MPa) & 502 & 502 & 350 \\
\hline Yield Strain & 0.00251 & 0.00251 & 0.00175 \\
\hline Strain Hardening Strain & 0.02 & 0.02 & 0.02 \\
\hline Ultimate Strength (MPa) & 650 & 650 & 600 \\
\hline Ultimate Strain & 0.2 & 0.2 & 0.2 \\
\hline Prestrain & 0 & 0 & 0 \\
\hline \multicolumn{4}{|c|}{ Reinforcement (X-Direction) } \\
\hline Ratio (\%) & 0.26 & 1 & 100 \\
\hline Yield Strength (MPa) & 491 & 491 & 350 \\
\hline Yield Strain & 0.00246 & 0.0246 & 0.00175 \\
\hline Strain Hardening Strain & 0.02 & 0.02 & 0.02 \\
\hline Ultimate Strength (MPa) & 650 & 650 & 600 \\
\hline Ultimate Strain & 0.2 & 0.2 & 0.2 \\
\hline Prestrain & 0 & 0 & 0 \\
\hline
\end{tabular}

Numerical Modelling of Hollow Concrete Block Masonry Mechanics 


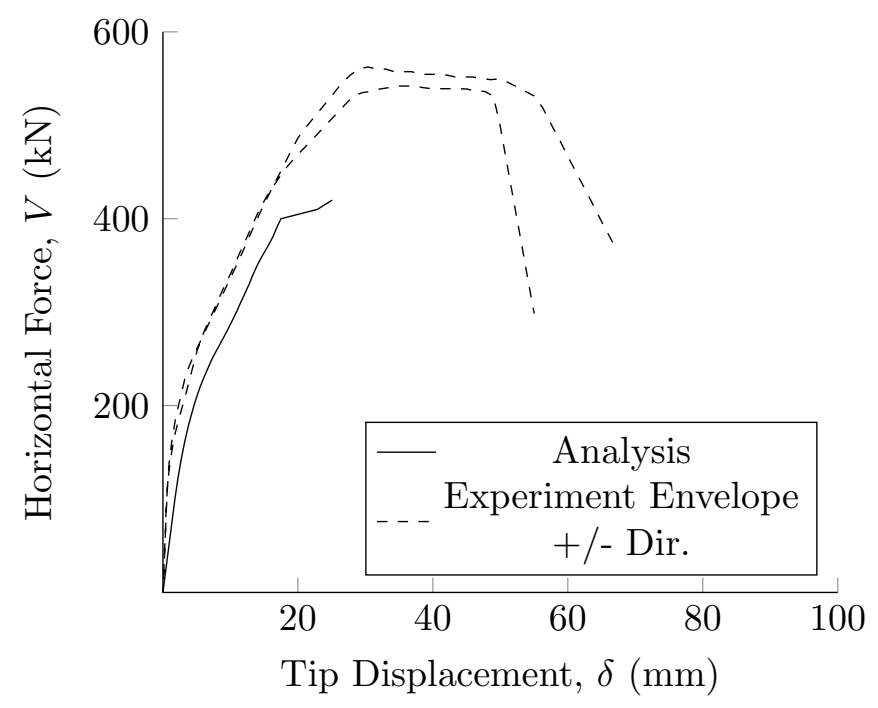

Wall 6 Shear Wall Shedid et al. (2008) Horizontal Force vs Tip Displacement 


\section{Seif ElDin (2016)}

\section{W-Ref Shear Wall}

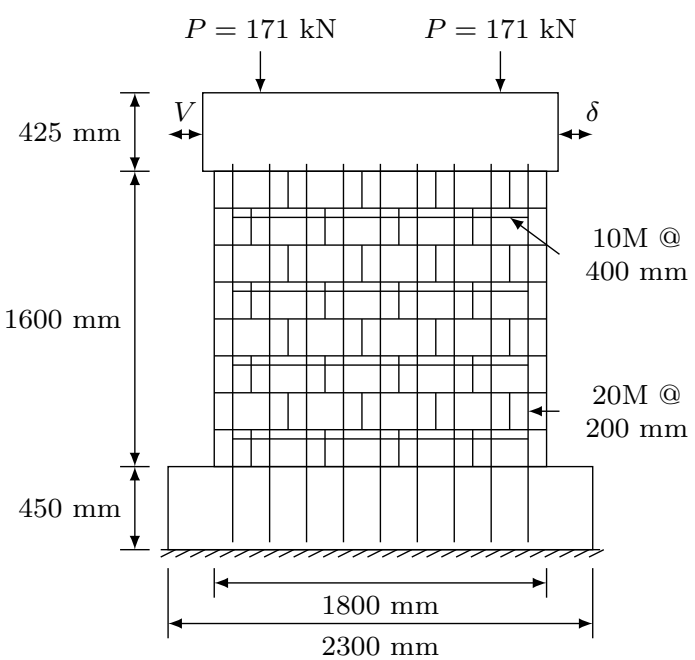

Experimental Wall (Seif ElDin, 2016)

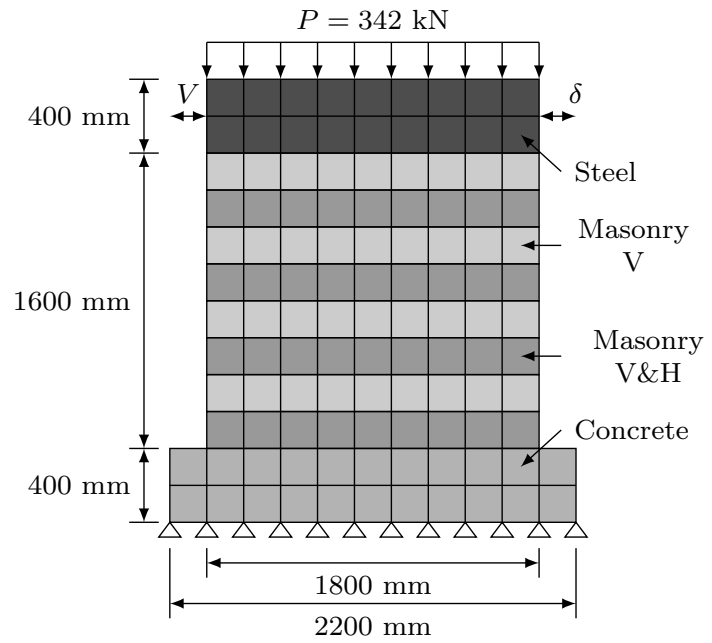

Finite Element Model Wall

W-Ref Shear Wall

W-Ref Shear Wall (Seif ElDin, 2016) Model Options

\begin{tabular}{ll}
\hline Model & Selected Option \\
\hline Compression Pre-Peak & Hognestad \\
Compression Post-Peak & Pre-Peak Base Curve \\
Compression Softening & Vecchio \\
Tension Post-Peak & Vecchio \\
Cracking Criterion & Mohr-Coulomb \\
Slip & Walraven Reinforced \\
\hline
\end{tabular}

Numerical Modelling of Hollow Concrete Block Masonry Mechanics 
W-Ref Shear Wall (Seif ElDin, 2016) Material Properties

\begin{tabular}{|c|c|c|c|c|}
\hline \multirow{2}{*}{ Property } & \multicolumn{4}{|c|}{ Material } \\
\hline & Masonry V\&H & Masonry V & Concrete & Steel \\
\hline Thickness (mm) & 190 & 190 & 640 & 400 \\
\hline Grout Tensile Strength (MPa) & 1.99 & 1.99 & 2.07 & 350 \\
\hline \multicolumn{5}{|c|}{ Masonry Properties (Y-Direction) } \\
\hline Gross Peak Compression Strength (MPa) & 13.1 & 13.1 & 39.5 & 350 \\
\hline Peak Compressive Strain & 0.0025 & 0.0025 & 0.0021 & 0.00175 \\
\hline Tensile Strength & 0 & 0 & 2.07 & 350 \\
\hline Tensile Strain & 0 & 0 & 0 & 0.00175 \\
\hline Initial Tangent Elastic Modulus (MPa) & 3750 & 3750 & 34600 & 200000 \\
\hline Poisson's Ratio & 0 & 0 & 0.15 & 0 \\
\hline \multicolumn{5}{|c|}{ Masonry Properties (X-Direction) } \\
\hline Gross Peak Compression Strength (MPa) & 13.1 & 13.1 & 39.5 & 350 \\
\hline Peak Compressive Strain & 0.0025 & 0.0025 & 0.0021 & 0.00175 \\
\hline Tensile Strength & 2.07 & 0 & 0 & 350 \\
\hline Tensile Strain & 0 & 0 & 0 & 0.00175 \\
\hline Initial Tangent Elastic Modulus (MPa) & 3750 & 3750 & 34600 & 200000 \\
\hline Poisson's Ratio & 0 & 0 & 0.15 & 0 \\
\hline \multicolumn{5}{|c|}{ Masonry Properties (Block) } \\
\hline Length (mm) & 390 & 390 & 2200 & 1800 \\
\hline Height (mm) & 190 & 190 & 400 & 400 \\
\hline Face Shell Thickness (mm) & 35 & 35 & 320 & 200 \\
\hline Percent Solid (\%) & 52 & 52 & 100 & 100 \\
\hline Tensile Strength & 1.40 & 1.40 & 2.07 & 350 \\
\hline \multicolumn{5}{|c|}{ Head Joint } \\
\hline Thickness (mm) & 10 & 10 & 10 & 10 \\
\hline Tensile Bond Strength (MPa) & 0.27 & 0.27 & 2.07 & 350 \\
\hline Joint Cohesion (MPa) & 3.48 & 3.48 & 2.07 & 350 \\
\hline Angle of Internal Friction (Deg) & 42 & 42 & 42 & 42 \\
\hline \multicolumn{5}{|c|}{ Bed Joint } \\
\hline Thickness (mm) & 10 & 10 & 10 & 10 \\
\hline Tensile Bond Strength (MPa) & 0.27 & 0.27 & 2.07 & 350 \\
\hline Joint Cohesion (MPa) & 3.48 & 3.48 & 2.07 & 350 \\
\hline Angle of Internal Friction (Deg) & 42 & 42 & 42 & 42 \\
\hline \multicolumn{5}{|c|}{ Reinforcement (Y-Direction) } \\
\hline Ratio (\%) & 0.79 & 0.79 & 0.42 & 100 \\
\hline Yield Strength (MPa) & 430 & 430 & 430 & 350 \\
\hline Yield Strain & 0.00219 & 0.00219 & 0.00219 & 0.00175 \\
\hline Strain Hardening Strain & 0.02 & 0.02 & 0.02 & 0.02 \\
\hline Ultimate Strength (MPa) & 542 & 542 & 542 & 600 \\
\hline Ultimate Strain & 0.198 & 0.198 & 0.198 & 0.2 \\
\hline Prestrain & 0 & 0 & 0 & 0 \\
\hline \multicolumn{5}{|c|}{ Reinforcement (X-Direction) } \\
\hline Ratio (\%) & 0.13 & 0 & 0.87 & 100 \\
\hline Yield Strength (MPa) & 430 & 0 & 430 & 350 \\
\hline Yield Strain & 0.00219 & 0 & 0.00219 & 0.00175 \\
\hline Strain Hardening Strain & 0.02 & 0 & 0.02 & 0.02 \\
\hline Ultimate Strength (MPa) & 540 & 0 & 542 & 600 \\
\hline Ultimate Strain & 0.205 & 0 & 0.198 & 0.2 \\
\hline Prestrain & 0 & 0 & 0 & 0 \\
\hline
\end{tabular}

Numerical Modelling of Hollow Concrete Block Masonry Mechanics 


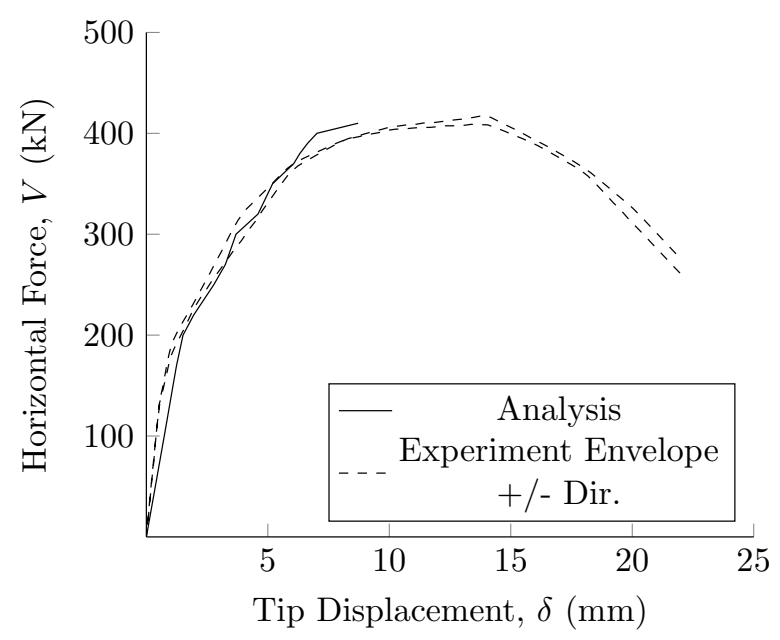

W-Ref Shear Wall (Seif ElDin, 2016) Horizontal Force vs Tip Displacement
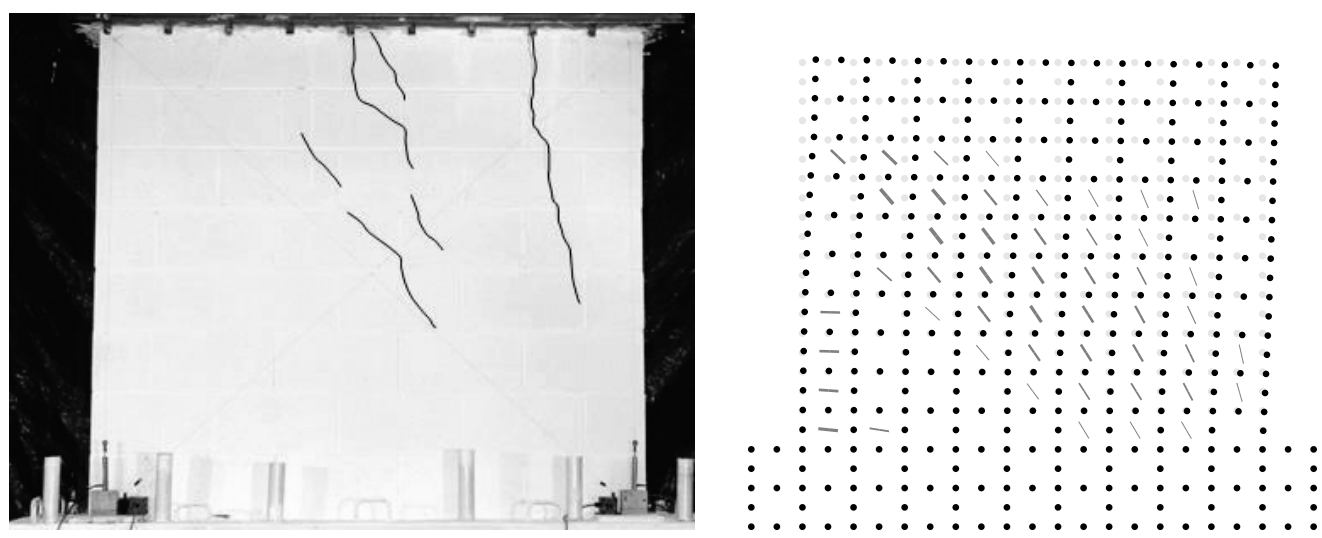

Experiment at $3.2 \mathrm{~mm}$ Tip Displacement Finite Element Model at $3.2 \mathrm{~mm}$ Tip Displace(Seif ElDin, 2016)

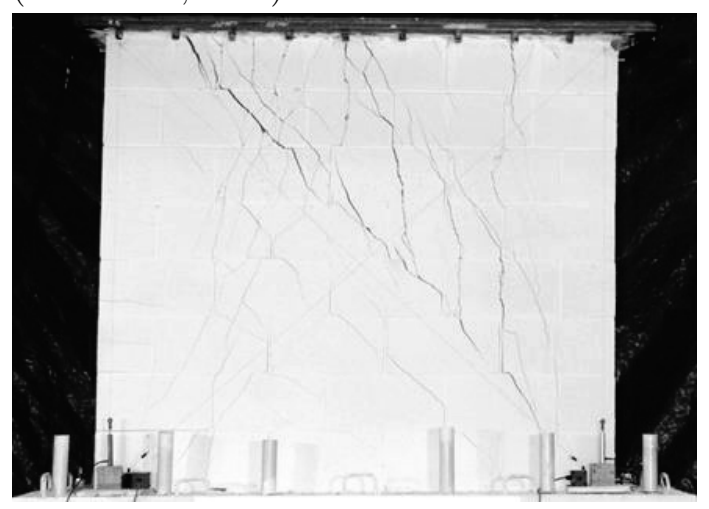
ment $\times 15$

Experiment at Peak Horizontal Force (Seif El- Finite Element Model at Peak Horizontal Force Din, 2016) $\times 15$

W-Ref Shear Wall Displacement, Crack Pattern, and Crack Widths 


\section{W- $\rho_{h} 0$ Shear Wall}

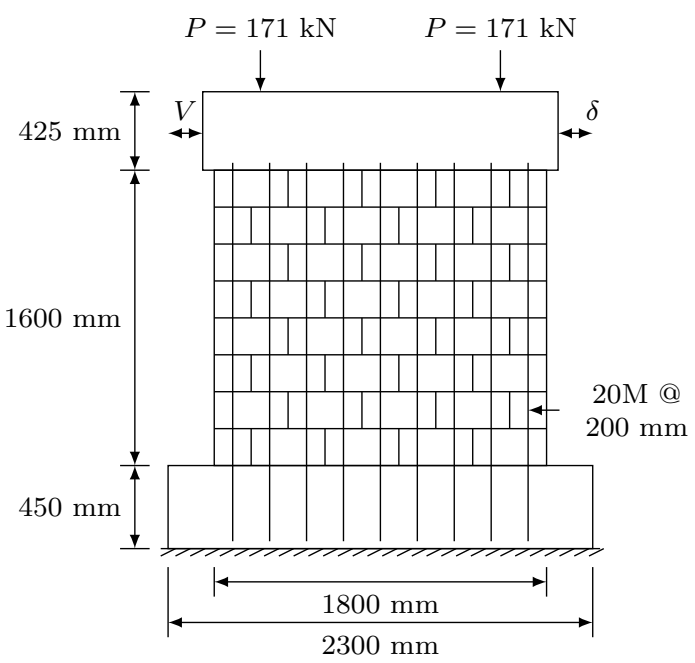

Experimental Wall (Seif ElDin, 2016)

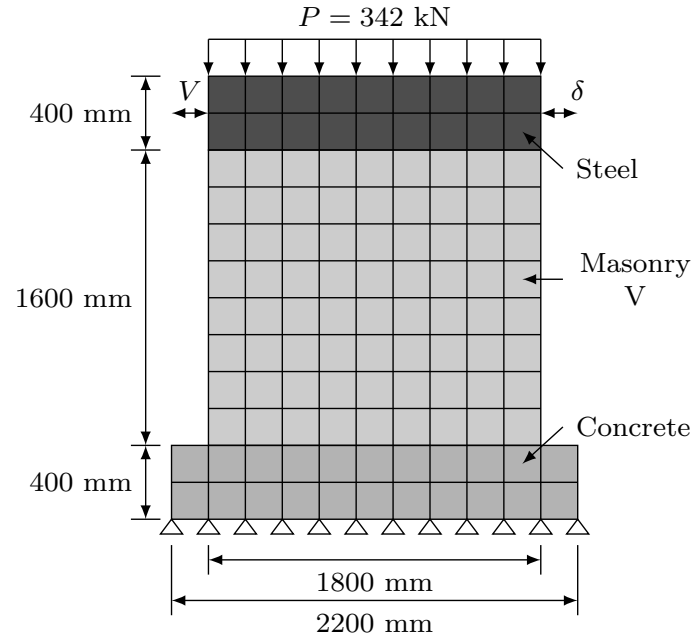

Finite Element Model Wall

$$
\mathrm{W}-\rho_{h} 0 \text { Shear Wall }
$$

W- $\rho_{h} 0$ Shear Wall (Seif ElDin, 2016) Model Options

\begin{tabular}{ll}
\hline Model & Selected Option \\
\hline Compression Pre-Peak & Hognestad \\
Compression Post-Peak & Pre-Peak Base Curve \\
Compression Softening & Vecchio \\
Tension Post-Peak & Vecchio \\
Cracking Criterion & Mohr-Coulomb \\
Slip & Walraven Reinforced \\
\hline
\end{tabular}


W- $\rho_{h} 0$ Shear Wall (Seif ElDin, 2016) Material Properties

\begin{tabular}{|c|c|c|c|}
\hline \multirow{2}{*}{ Property } & \multicolumn{3}{|c|}{ Material } \\
\hline & Masonry V & Concrete & Steel \\
\hline Thickness (mm) & 190 & 640 & 400 \\
\hline Grout Tensile Strength (MPa) & 1.99 & 2.07 & 350 \\
\hline \multicolumn{4}{|c|}{ Masonry Properties (Y-Direction) } \\
\hline Gross Peak Compression Strength (MPa) & 13.1 & 39.5 & 350 \\
\hline Peak Compressive Strain & 0.0025 & 0.0021 & 0.00175 \\
\hline Tensile Strength & 0 & 2.07 & 350 \\
\hline Tensile Strain & 0 & 0 & 0.00175 \\
\hline Initial Tangent Elastic Modulus (MPa) & 4200 & 34600 & 200000 \\
\hline Poisson's Ratio & 0 & 0.15 & 0 \\
\hline \multicolumn{4}{|c|}{ Masonry Properties (X-Direction) } \\
\hline Gross Peak Compression Strength (MPa) & 13.1 & 39.5 & 350 \\
\hline Peak Compressive Strain & 0.0025 & 0.0021 & 0.00175 \\
\hline Tensile Strength & 0 & 2.07 & 350 \\
\hline Tensile Strain & 0 & 0 & 0.00175 \\
\hline Initial Tangent Elastic Modulus (MPa) & 4200 & 34600 & 200000 \\
\hline Poisson's Ratio & 0 & 0.15 & 0 \\
\hline \multicolumn{4}{|c|}{ Masonry Properties (Block) } \\
\hline Length (mm) & 390 & 2200 & 1800 \\
\hline Height (mm) & 190 & 400 & 400 \\
\hline Face Shell Thickness (mm) & 35 & 320 & 200 \\
\hline Percent Solid (\%) & 52 & 100 & 100 \\
\hline Tensile Strength & 1.40 & 2.07 & 350 \\
\hline \multicolumn{4}{|c|}{ Head Joint } \\
\hline Thickness (mm) & 10 & 10 & 10 \\
\hline Tensile Bond Strength (MPa) 0.27 & 2.07 & 350 & \\
\hline Joint Cohesion (MPa) & 3.48 & 2.07 & 350 \\
\hline Angle of Internal Friction (Deg) & 42 & 42 & 42 \\
\hline \multicolumn{4}{|c|}{ Bed Joint } \\
\hline Thickness (mm) & 10 & 10 & 10 \\
\hline Tensile Bond Strength (MPa) & 0.27 & 2.07 & 350 \\
\hline Joint Cohesion (MPa) & 3.48 & 2.07 & 350 \\
\hline Angle of Internal Friction (Deg) & 42 & 42 & 42 \\
\hline \multicolumn{4}{|c|}{ Reinforcement (Y-Direction) } \\
\hline Ratio (\%) & 0.79 & 0.42 & 100 \\
\hline Yield Strength (MPa) & 430 & 430 & 350 \\
\hline Yield Strain & 0.00219 & 0.00219 & 0.00175 \\
\hline Strain Hardening Strain & 0.02 & 0.02 & 0.02 \\
\hline Ultimate Strength (MPa) & 542 & 542 & 600 \\
\hline Ultimate Strain & 0.198 & 0.198 & 0.2 \\
\hline Prestrain & 0 & 0 & 0 \\
\hline \multicolumn{4}{|c|}{ Reinforcement (X-Direction) } \\
\hline Ratio (\%) & 0 & 0.87 & 100 \\
\hline Yield Strength (MPa) & 0 & 430 & 350 \\
\hline Yield Strain & 0 & 0.00219 & 0.00175 \\
\hline Strain Hardening Strain & 0 & 0.02 & 0.02 \\
\hline Ultimate Strength (MPa) & 0 & 542 & 600 \\
\hline Ultimate Strain & 0 & 0.198 & 0.2 \\
\hline Prestrain & 0 & 0 & 0 \\
\hline
\end{tabular}

Numerical Modelling of Hollow Concrete Block Masonry Mechanics 


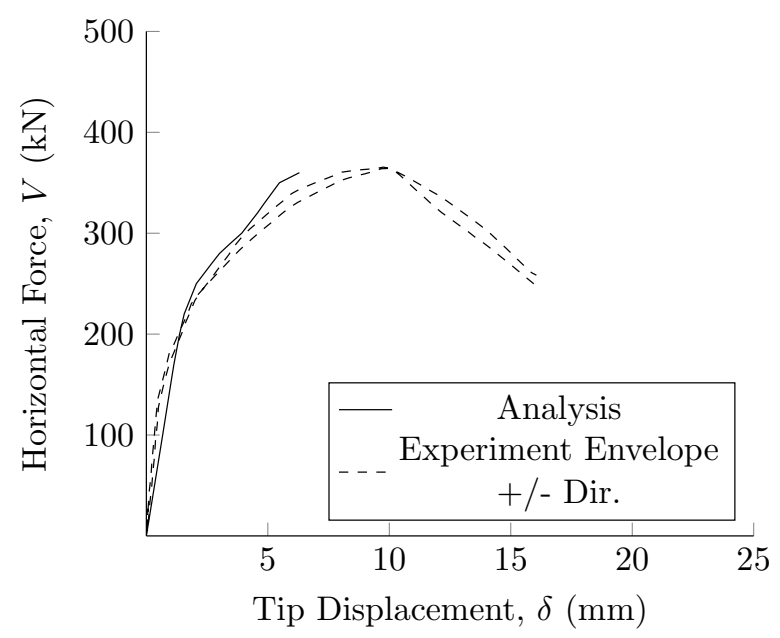

W- $\rho_{h} 0$ Shear Wall (Seif ElDin, 2016) Horizontal Force vs Tip Displacement
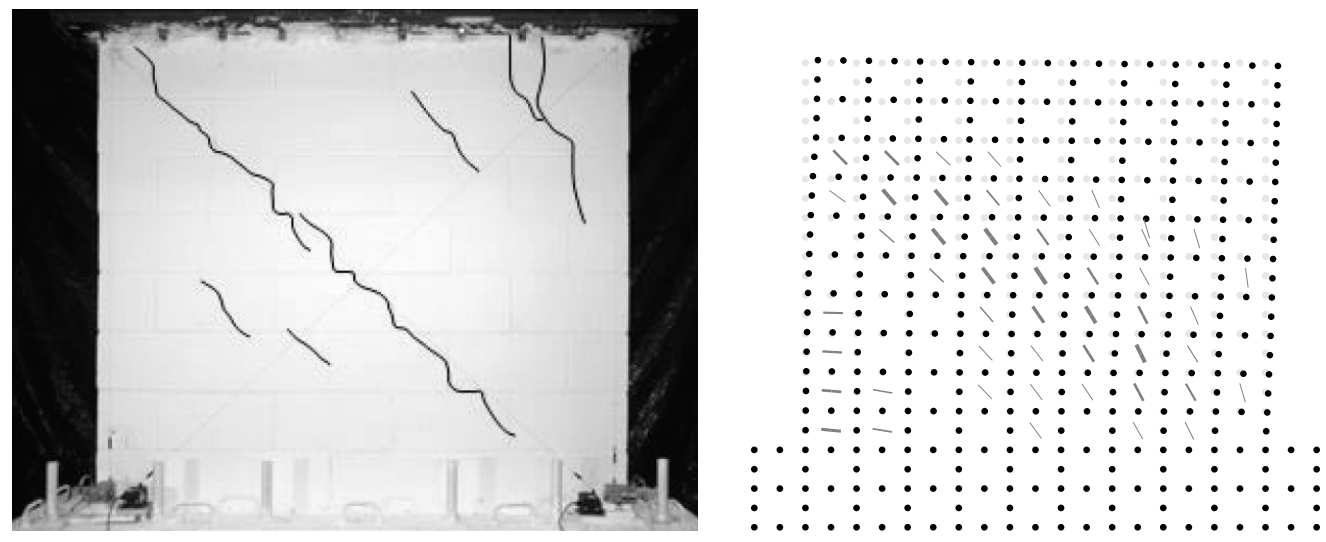

Experiment at $3.1 \mathrm{~mm}$ Tip Displacement Finite Element Model at $3.1 \mathrm{~mm}$ Tip Displace(Seif ElDin, 2016)

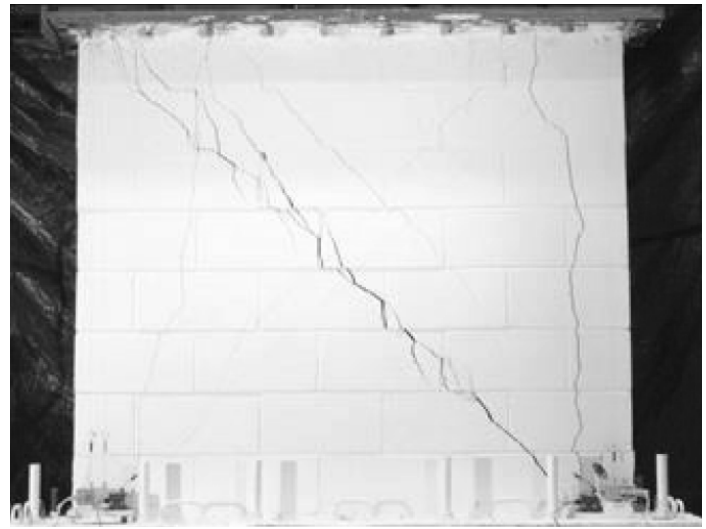
ment $\times 15$

Experiment at Peak Horizontal Force (Seif El- Finite Element Model at Peak Horizontal Force Din, 2016) $\times 15$

W- $\rho_{h} 0$ Shear Wall Displacement, Crack Pattern, and Crack Widths 


\section{W- $\sigma_{n} 0$ Shear Wall}

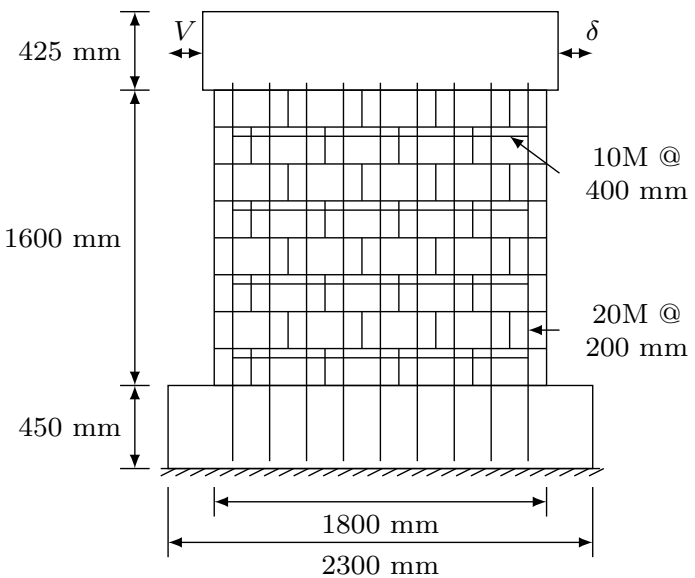

Experimental Wall (Seif ElDin, 2016)

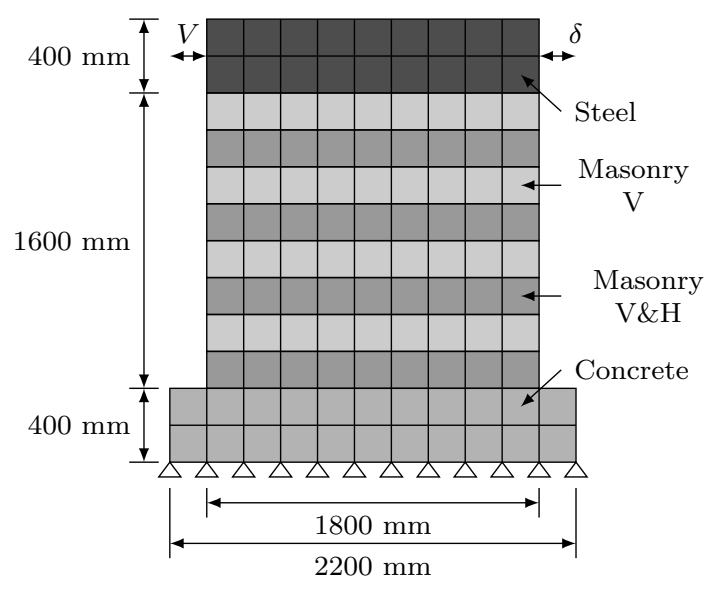

Finite Element Model Wall

$$
\mathrm{W}-\sigma_{n} 0 \text { Shear Wall }
$$

W- $\sigma_{n} 0$ Shear Wall (Seif ElDin, 2016) Model Options

\begin{tabular}{ll}
\hline Model & Selected Option \\
\hline Compression Pre-Peak & Hognestad \\
Compression Post-Peak & Pre-Peak Base Curve \\
Compression Softening & Vecchio \\
Tension Post-Peak & Vecchio \\
Cracking Criterion & Mohr-Coulomb \\
Slip & Walraven Reinforced \\
\hline
\end{tabular}


W- $\sigma_{n} 0$ Shear Wall (Seif ElDin, 2016) Material Properties

\begin{tabular}{|c|c|c|c|c|}
\hline \multirow{2}{*}{ Property } & \multicolumn{4}{|c|}{ Material } \\
\hline & Masonry V\&H & Masonry V & Concrete & Steel \\
\hline Thickness (mm) & 190 & 190 & 640 & 400 \\
\hline Grout Tensile Strength (MPa) & 1.99 & 1.99 & 2.07 & 350 \\
\hline \multicolumn{5}{|c|}{ Masonry Properties (Y-Direction) } \\
\hline Gross Peak Compression Strength (MPa) & 13.1 & 13.1 & 39.5 & 350 \\
\hline Peak Compressive Strain & 0.0025 & 0.0025 & 0.0021 & 0.00175 \\
\hline Tensile Strength & 0 & 0 & 2.07 & 350 \\
\hline Tensile Strain & 0 & 0 & 0 & 0.00175 \\
\hline Initial Tangent Elastic Modulus (MPa) & 3750 & 3750 & 34600 & 200000 \\
\hline Poisson's Ratio & 0 & 0 & 0.15 & 0 \\
\hline \multicolumn{5}{|c|}{ Masonry Properties (X-Direction) } \\
\hline Gross Peak Compression Strength (MPa) & 13.1 & 13.1 & 39.5 & 350 \\
\hline Peak Compressive Strain & 0.0025 & 0.0025 & 0.0021 & 0.00175 \\
\hline Tensile Strength & 2.07 & 0 & 0 & 350 \\
\hline Tensile Strain & 0 & 0 & 0 & 0.00175 \\
\hline Initial Tangent Elastic Modulus (MPa) & 3750 & 3750 & 34600 & 200000 \\
\hline Poisson's Ratio & 0 & 0 & 0.15 & 0 \\
\hline \multicolumn{5}{|c|}{ Masonry Properties (Block) } \\
\hline Length (mm) & 390 & 390 & 2200 & 1800 \\
\hline Height (mm) & 190 & 190 & 400 & 400 \\
\hline Face Shell Thickness (mm) & 35 & 35 & 320 & 200 \\
\hline Percent Solid (\%) & 52 & 52 & 100 & 100 \\
\hline Tensile Strength & 1.40 & 1.40 & 2.07 & 350 \\
\hline \multicolumn{5}{|c|}{ Head Joint } \\
\hline Thickness (mm) & 10 & 10 & 10 & 10 \\
\hline Tensile Bond Strength (MPa) & 0.27 & 0.27 & 2.07 & 350 \\
\hline Joint Cohesion (MPa) & 3.48 & 3.48 & 2.07 & 350 \\
\hline Angle of Internal Friction (Deg) & 42 & 42 & 42 & 42 \\
\hline \multicolumn{5}{|c|}{ Bed Joint } \\
\hline Thickness (mm) & 10 & 10 & 10 & 10 \\
\hline Tensile Bond Strength (MPa) & 0.27 & 0.27 & 2.07 & 350 \\
\hline Joint Cohesion (MPa) & 3.48 & 3.48 & 2.07 & 350 \\
\hline Angle of Internal Friction (Deg) & 42 & 42 & 42 & 42 \\
\hline \multicolumn{5}{|c|}{ Reinforcement (Y-Direction) } \\
\hline Ratio (\%) & 0.79 & 0.79 & 0.42 & 100 \\
\hline Yield Strength (MPa) & 430 & 430 & 430 & 350 \\
\hline Yield Strain & 0.00219 & 0.00219 & 0.00219 & 0.00175 \\
\hline Strain Hardening Strain & 0.02 & 0.02 & 0.02 & 0.02 \\
\hline Ultimate Strength (MPa) & 542 & 542 & 542 & 600 \\
\hline Ultimate Strain & 0.198 & 0.198 & 0.198 & 0.2 \\
\hline Prestrain & 0 & 0 & 0 & 0 \\
\hline \multicolumn{5}{|c|}{ Reinforcement (X-Direction) } \\
\hline Ratio (\%) & 0.13 & 0 & 0.87 & 100 \\
\hline Yield Strength (MPa) & 430 & 0 & 430 & 350 \\
\hline Yield Strain & 0.00219 & 0 & 0.00219 & 0.00175 \\
\hline Strain Hardening Strain & 0.02 & 0 & 0.02 & 0.02 \\
\hline Ultimate Strength (MPa) & 540 & 0 & 542 & 600 \\
\hline Ultimate Strain & 0.205 & 0 & 0.198 & 0.2 \\
\hline Prestrain & 0 & 0 & 0 & 0 \\
\hline
\end{tabular}

Numerical Modelling of Hollow Concrete Block Masonry Mechanics 


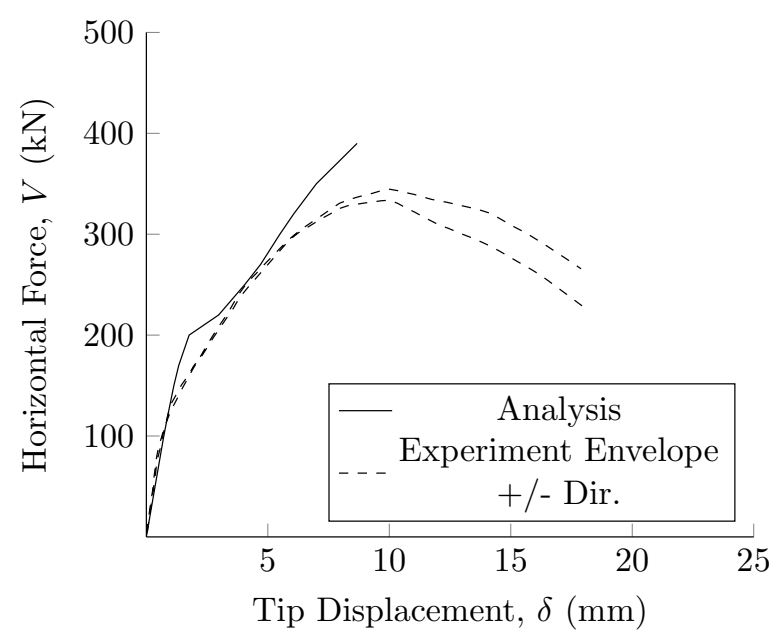

$\mathrm{W}-\sigma_{n} 0$ Shear (Seif ElDin, 2016)
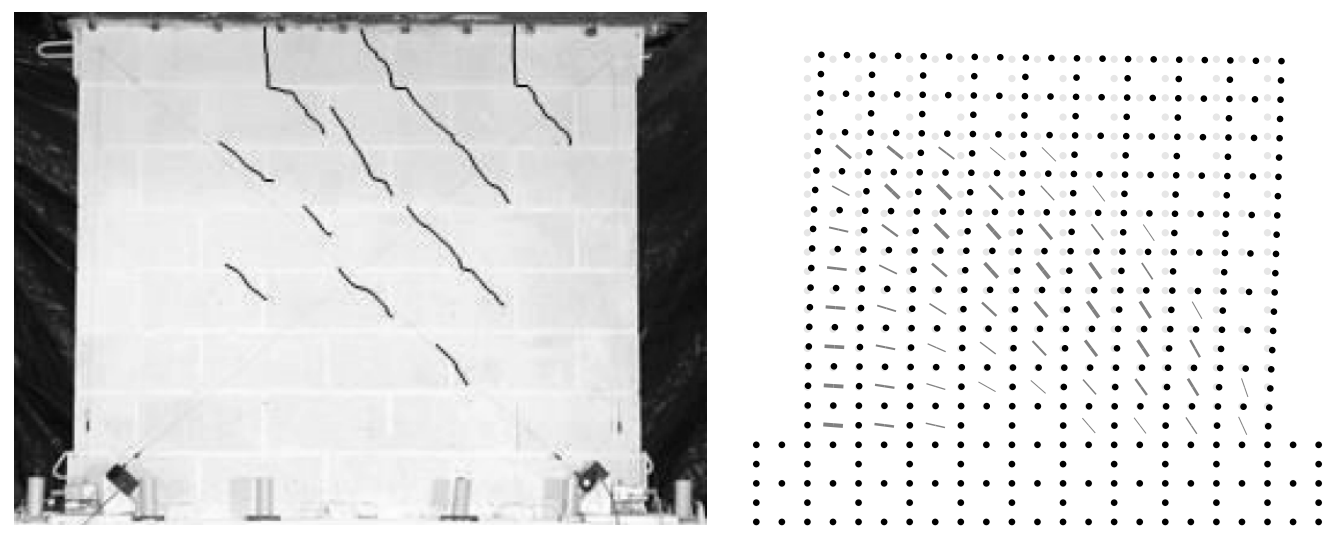

Experiment at $3.4 \mathrm{~mm}$ Tip Displacement Finite Element Model at $3.4 \mathrm{~mm}$ Tip Displace(Seif ElDin, 2016)

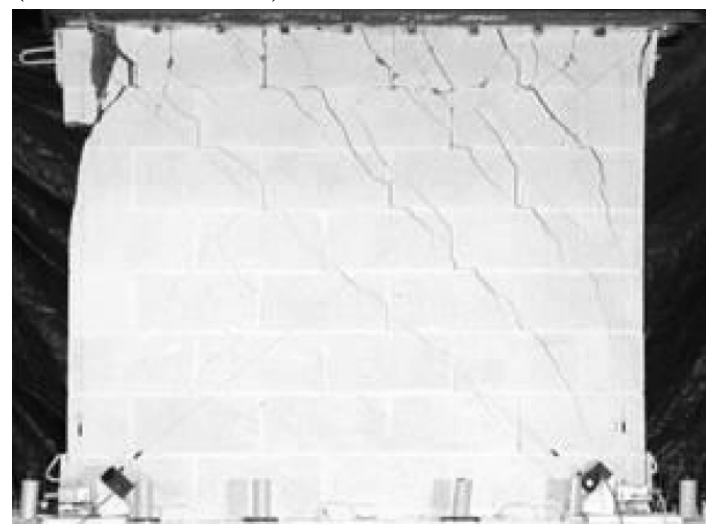
ment $\times 15$

Experiment at Peak Horizontal Force (Seif El- Finite Element Model at Peak Horizontal Force Din, 2016) $\times 15$

W- $\sigma_{n} 0$ Shear Wall Displacement, Crack Pattern, and Crack Widths 


\section{W- $\sigma_{n} 1.5$ Shear Wall}

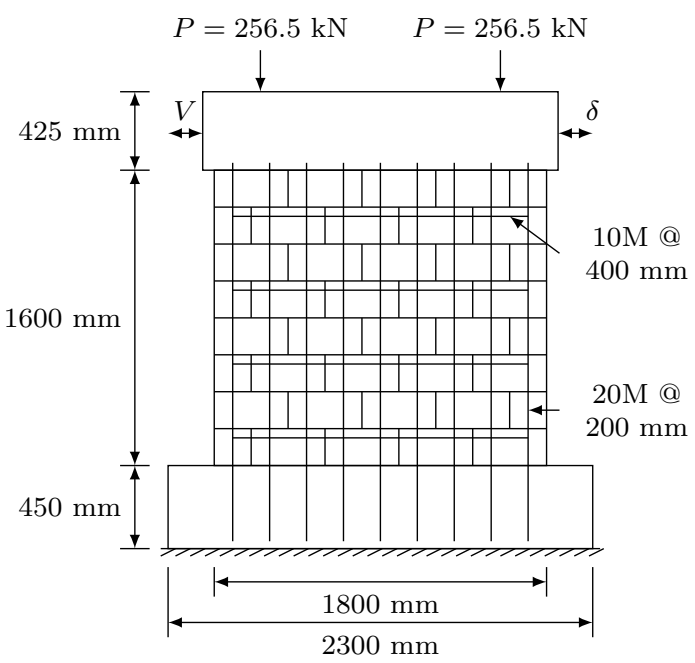

Experimental Wall (Seif ElDin, 2016)

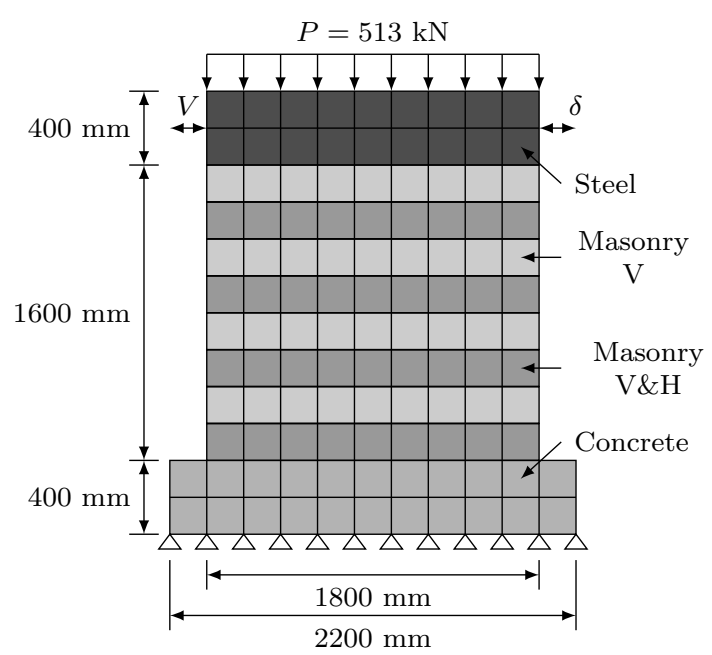

Finite Element Model Wall

$\mathrm{W}-\sigma_{n} 1.5$ Shear Wall

W- $\sigma_{n} 1.5$ Shear Wall (Seif ElDin, 2016) Model Options

\begin{tabular}{ll}
\hline Model & Selected Option \\
\hline Compression Pre-Peak & Hognestad \\
Compression Post-Peak & Pre-Peak Base Curve \\
Compression Softening & Vecchio \\
Tension Post-Peak & Vecchio \\
Cracking Criterion & Mohr-Coulomb \\
Slip & Walraven Reinforced \\
\hline
\end{tabular}

Numerical Modelling of Hollow Concrete Block Masonry Mechanics 
W- $\sigma_{n} 1.5$ Shear Wall (Seif ElDin, 2016) Material Properties

\begin{tabular}{|c|c|c|c|c|}
\hline \multirow{2}{*}{ Property } & \multicolumn{4}{|c|}{ Material } \\
\hline & Masonry V\&H & Masonry V & Concrete & Steel \\
\hline Thickness (mm) & 190 & 190 & 640 & 400 \\
\hline Grout Tensile Strength (MPa) & 1.99 & 1.99 & 2.07 & 350 \\
\hline \multicolumn{5}{|c|}{ Masonry Properties (Y-Direction) } \\
\hline Gross Peak Compression Strength (MPa) & 13.1 & 13.1 & 39.5 & 350 \\
\hline Peak Compressive Strain & 0.0025 & 0.0025 & 0.0021 & 0.00175 \\
\hline Tensile Strength & 0 & 0 & 2.07 & 350 \\
\hline Tensile Strain & 0 & 0 & 0 & 0.00175 \\
\hline Initial Tangent Elastic Modulus (MPa) & 3750 & 3750 & 34600 & 200000 \\
\hline Poisson's Ratio & 0 & 0 & 0.15 & 0 \\
\hline \multicolumn{5}{|c|}{ Masonry Properties (X-Direction) } \\
\hline Gross Peak Compression Strength (MPa) & 13.1 & 13.1 & 39.5 & 350 \\
\hline Peak Compressive Strain & 0.0025 & 0.0025 & 0.0021 & 0.00175 \\
\hline Tensile Strength & 2.07 & 0 & 0 & 350 \\
\hline Tensile Strain & 0 & 0 & 0 & 0.00175 \\
\hline Initial Tangent Elastic Modulus (MPa) & 3750 & 3750 & 34600 & 200000 \\
\hline Poisson's Ratio & 0 & 0 & 0.15 & 0 \\
\hline \multicolumn{5}{|c|}{ Masonry Properties (Block) } \\
\hline Length (mm) & 390 & 390 & 2200 & 1800 \\
\hline Height (mm) & 190 & 190 & 400 & 400 \\
\hline Face Shell Thickness (mm) & 35 & 35 & 320 & 200 \\
\hline Percent Solid (\%) & 52 & 52 & 100 & 100 \\
\hline Tensile Strength & 1.40 & 1.40 & 2.07 & 350 \\
\hline \multicolumn{5}{|c|}{ Head Joint } \\
\hline Thickness (mm) & 10 & 10 & 10 & 10 \\
\hline Tensile Bond Strength (MPa) & 0.27 & 0.27 & 2.07 & 350 \\
\hline Joint Cohesion (MPa) & 3.48 & 3.48 & 2.07 & 350 \\
\hline Angle of Internal Friction (Deg) & 42 & 42 & 42 & 42 \\
\hline \multicolumn{5}{|c|}{ Bed Joint } \\
\hline Thickness (mm) & 10 & 10 & 10 & 10 \\
\hline Tensile Bond Strength (MPa) & 0.27 & 0.27 & 2.07 & 350 \\
\hline Joint Cohesion (MPa) & 3.48 & 3.48 & 2.07 & 350 \\
\hline Angle of Internal Friction (Deg) & 42 & 42 & 42 & 42 \\
\hline \multicolumn{5}{|c|}{ Reinforcement (Y-Direction) } \\
\hline Ratio (\%) & 0.79 & 0.79 & 0.42 & 100 \\
\hline Yield Strength (MPa) & 430 & 430 & 430 & 350 \\
\hline Yield Strain & 0.00219 & 0.00219 & 0.00219 & 0.00175 \\
\hline Strain Hardening Strain & 0.02 & 0.02 & 0.02 & 0.02 \\
\hline Ultimate Strength (MPa) & 542 & 542 & 542 & 600 \\
\hline Ultimate Strain & 0.198 & 0.198 & 0.198 & 0.2 \\
\hline Prestrain & 0 & 0 & 0 & 0 \\
\hline \multicolumn{5}{|c|}{ Reinforcement (X-Direction) } \\
\hline Ratio (\%) & 0.13 & 0 & 0.87 & 100 \\
\hline Yield Strength (MPa) & 430 & 0 & 430 & 350 \\
\hline Yield Strain & 0.00219 & 0 & 0.00219 & 0.00175 \\
\hline Strain Hardening Strain & 0.02 & 0 & 0.02 & 0.02 \\
\hline Ultimate Strength (MPa) & 540 & 0 & 542 & 600 \\
\hline Ultimate Strain & 0.205 & 0 & 0.198 & 0.2 \\
\hline Prestrain & 0 & 0 & 0 & 0 \\
\hline
\end{tabular}

Numerical Modelling of Hollow Concrete Block Masonry Mechanics 


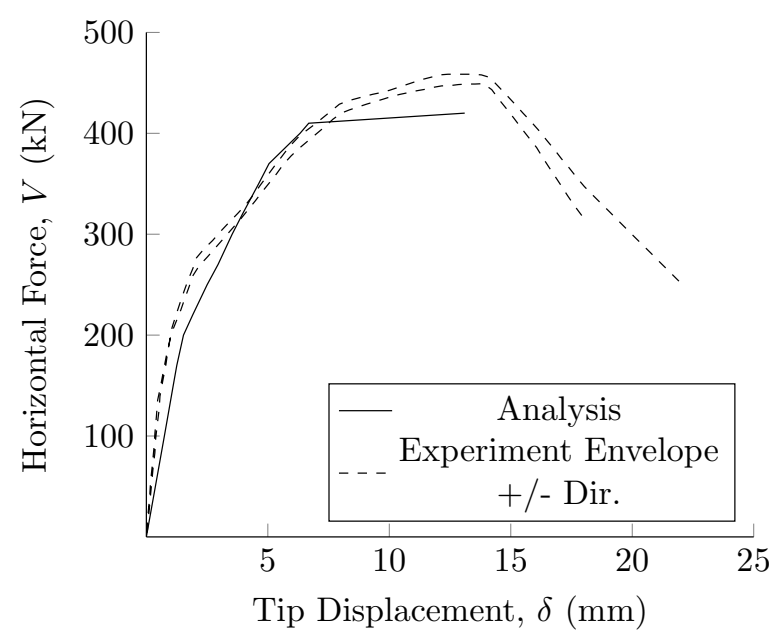

$\mathrm{W}-\sigma_{n} 1.5$ Shear Wall (Seif ElDin, 2016)
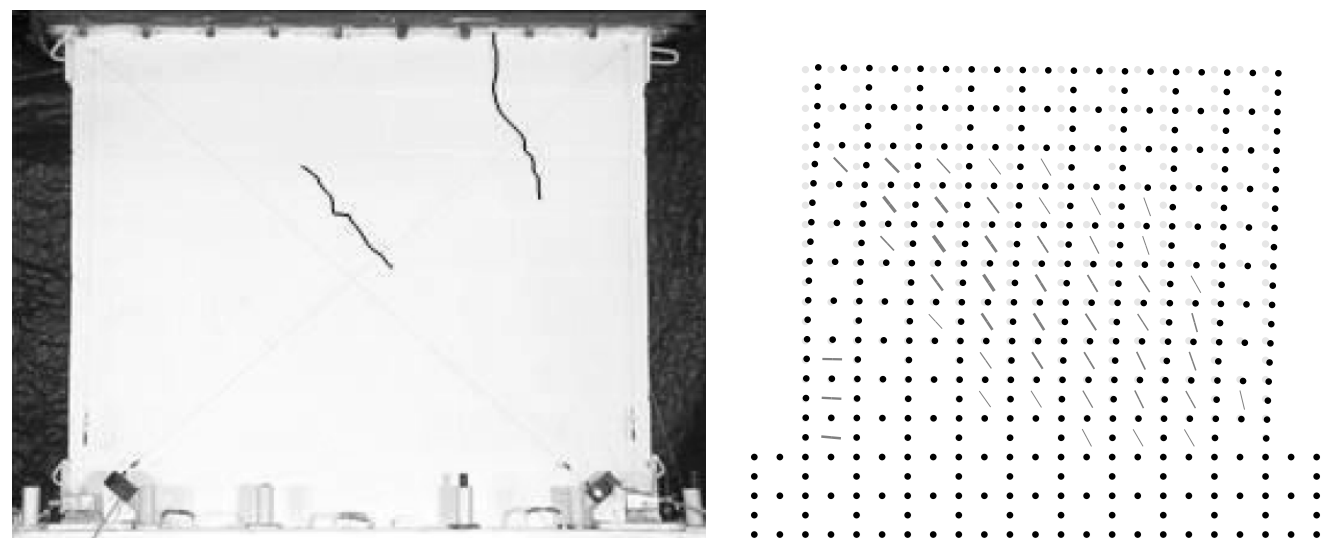

Experiment at $3.1 \mathrm{~mm}$ Tip Displacement Finite Element Model at $3.1 \mathrm{~mm}$ Tip Displace(Seif ElDin, 2016)

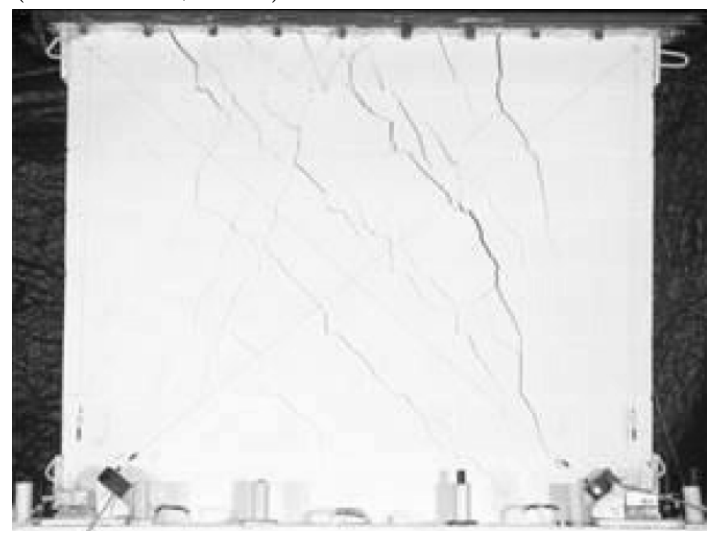
ment $\times 15$

Experiment at Peak Horizontal Force (Seif El- Finite Element Model at Peak Horizontal Force Din, 2016) $\times 15$

W- $\sigma_{n} 1.5$ Shear Wall Displacement, Crack Pattern, and Crack Widths 


\section{W- $M / V_{d} 1.8$ Shear Wall}

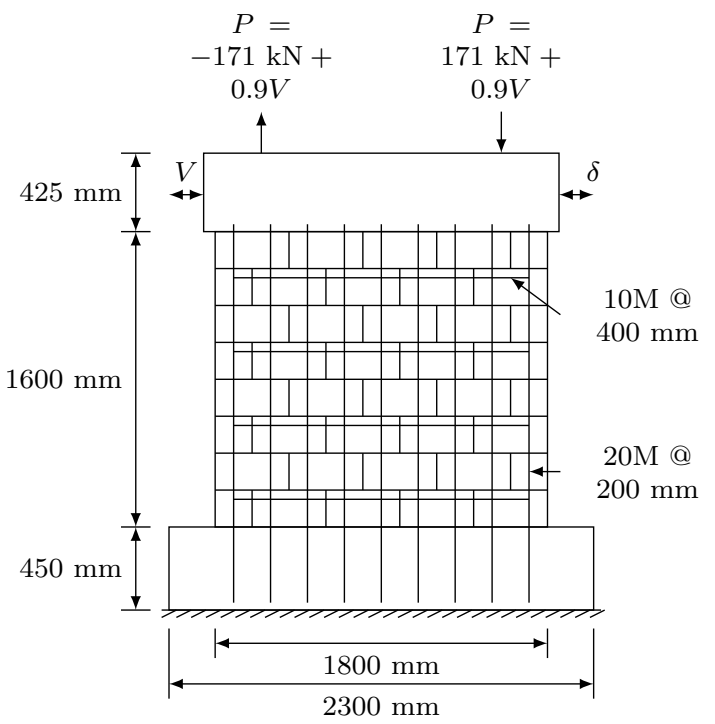

Experimental Wall (Seif ElDin, 2016)

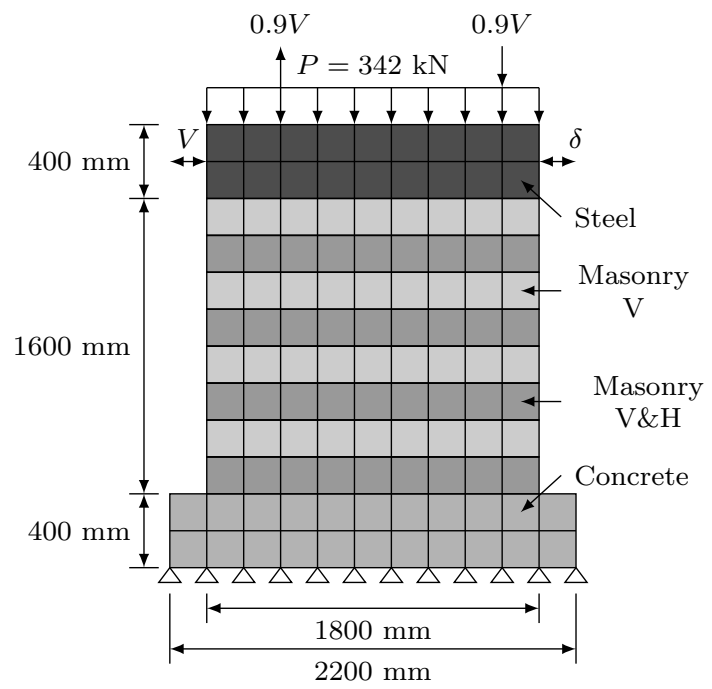

Finite Element Model Wall

$\mathrm{W}-M / V_{d} 1.8$ Shear Wall

W- $M / V_{d} 1.8$ Shear Wall (Seif ElDin, 2016) Model Options

\begin{tabular}{ll}
\hline Model & Selected Option \\
\hline Compression Pre-Peak & Hognestad \\
Compression Post-Peak & Pre-Peak Base Curve \\
Compression Softening & Vecchio \\
Tension Post-Peak & Vecchio \\
Cracking Criterion & Mohr-Coulomb \\
Slip & Walraven Reinforced \\
\hline
\end{tabular}

Numerical Modelling of Hollow Concrete Block Masonry Mechanics 
W- $M / V_{d} 1.8$ Shear Wall (Seif ElDin, 2016) Material Properties

\begin{tabular}{|c|c|c|c|c|}
\hline \multirow{2}{*}{ Property } & \multicolumn{4}{|c|}{ Material } \\
\hline & Masonry V\&H & Masonry V & Concrete & Steel \\
\hline Thickness (mm) & 190 & 190 & 640 & 400 \\
\hline Grout Tensile Strength (MPa) & 1.99 & 1.99 & 2.07 & 350 \\
\hline \multicolumn{5}{|c|}{ Masonry Properties (Y-Direction) } \\
\hline Gross Peak Compression Strength (MPa) & 13.1 & 13.1 & 39.5 & 350 \\
\hline Peak Compressive Strain & 0.0025 & 0.0025 & 0.0021 & 0.00175 \\
\hline Tensile Strength & 0 & 0 & 2.07 & 350 \\
\hline Tensile Strain & 0 & 0 & 0 & 0.00175 \\
\hline Initial Tangent Elastic Modulus (MPa) & 3750 & 3750 & 34600 & 200000 \\
\hline Poisson's Ratio & 0 & 0 & 0.15 & 0 \\
\hline \multicolumn{5}{|c|}{ Masonry Properties (X-Direction) } \\
\hline Gross Peak Compression Strength (MPa) & 13.1 & 13.1 & 39.5 & 350 \\
\hline Peak Compressive Strain & 0.0025 & 0.0025 & 0.0021 & 0.00175 \\
\hline Tensile Strength & 2.07 & 0 & 0 & 350 \\
\hline Tensile Strain & 0 & 0 & 0 & 0.00175 \\
\hline Initial Tangent Elastic Modulus (MPa) & 3750 & 3750 & 34600 & 200000 \\
\hline Poisson's Ratio & 0 & 0 & 0.15 & 0 \\
\hline \multicolumn{5}{|c|}{ Masonry Properties (Block) } \\
\hline Length (mm) & 390 & 390 & 2200 & 1800 \\
\hline Height (mm) & 190 & 190 & 400 & 400 \\
\hline Face Shell Thickness (mm) & 35 & 35 & 320 & 200 \\
\hline Percent Solid (\%) & 52 & 52 & 100 & 100 \\
\hline Tensile Strength & 1.40 & 1.40 & 2.07 & 350 \\
\hline \multicolumn{5}{|c|}{ Head Joint } \\
\hline Thickness (mm) & 10 & 10 & 10 & 10 \\
\hline Tensile Bond Strength (MPa) & 0.27 & 0.27 & 2.07 & 350 \\
\hline Joint Cohesion (MPa) & 3.48 & 3.48 & 2.07 & 350 \\
\hline Angle of Internal Friction (Deg) & 42 & 42 & 42 & 42 \\
\hline \multicolumn{5}{|c|}{ Bed Joint } \\
\hline Thickness (mm) & 10 & 10 & 10 & 10 \\
\hline Tensile Bond Strength (MPa) & 0.27 & 0.27 & 2.07 & 350 \\
\hline Joint Cohesion (MPa) & 3.48 & 3.48 & 2.07 & 350 \\
\hline Angle of Internal Friction (Deg) & 42 & 42 & 42 & 42 \\
\hline \multicolumn{5}{|c|}{ Reinforcement (Y-Direction) } \\
\hline Ratio (\%) & 0.79 & 0.79 & 0.42 & 100 \\
\hline Yield Strength (MPa) & 430 & 430 & 430 & 350 \\
\hline Yield Strain & 0.00219 & 0.00219 & 0.00219 & 0.00175 \\
\hline Strain Hardening Strain & 0.02 & 0.02 & 0.02 & 0.02 \\
\hline Ultimate Strength (MPa) & 542 & 542 & 542 & 600 \\
\hline Ultimate Strain & 0.198 & 0.198 & 0.198 & 0.2 \\
\hline Prestrain & 0 & 0 & 0 & 0 \\
\hline \multicolumn{5}{|c|}{ Reinforcement (X-Direction) } \\
\hline Ratio (\%) & 0.13 & 0 & 0.87 & 100 \\
\hline Yield Strength (MPa) & 430 & 0 & 430 & 350 \\
\hline Yield Strain & 0.00219 & 0 & 0.00219 & 0.00175 \\
\hline Strain Hardening Strain & 0.02 & 0 & 0.02 & 0.02 \\
\hline Ultimate Strength (MPa) & 540 & 0 & 542 & 600 \\
\hline Ultimate Strain & 0.205 & 0 & 0.198 & 0.2 \\
\hline Prestrain & 0 & 0 & 0 & 0 \\
\hline
\end{tabular}

Numerical Modelling of Hollow Concrete Block Masonry Mechanics 


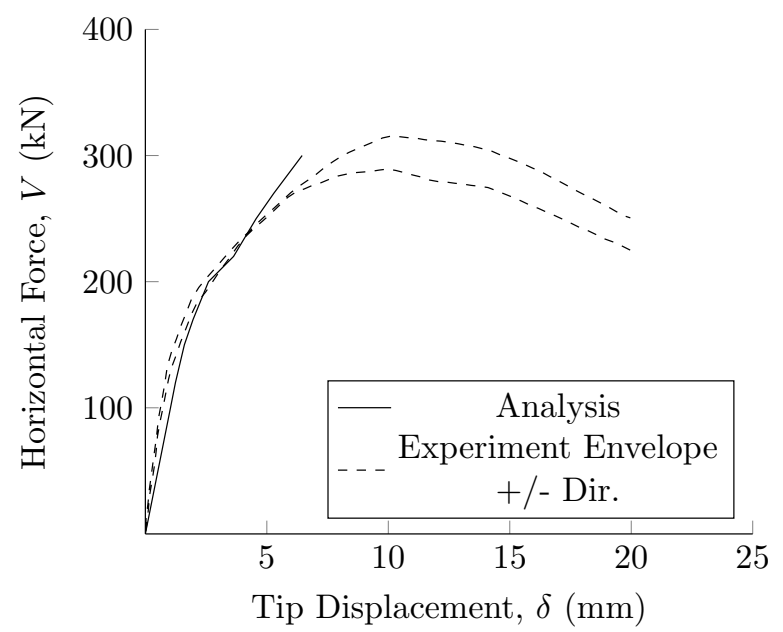

W- $M / V_{d} 1.8$ Shear Wall Seif ElDin (2016)
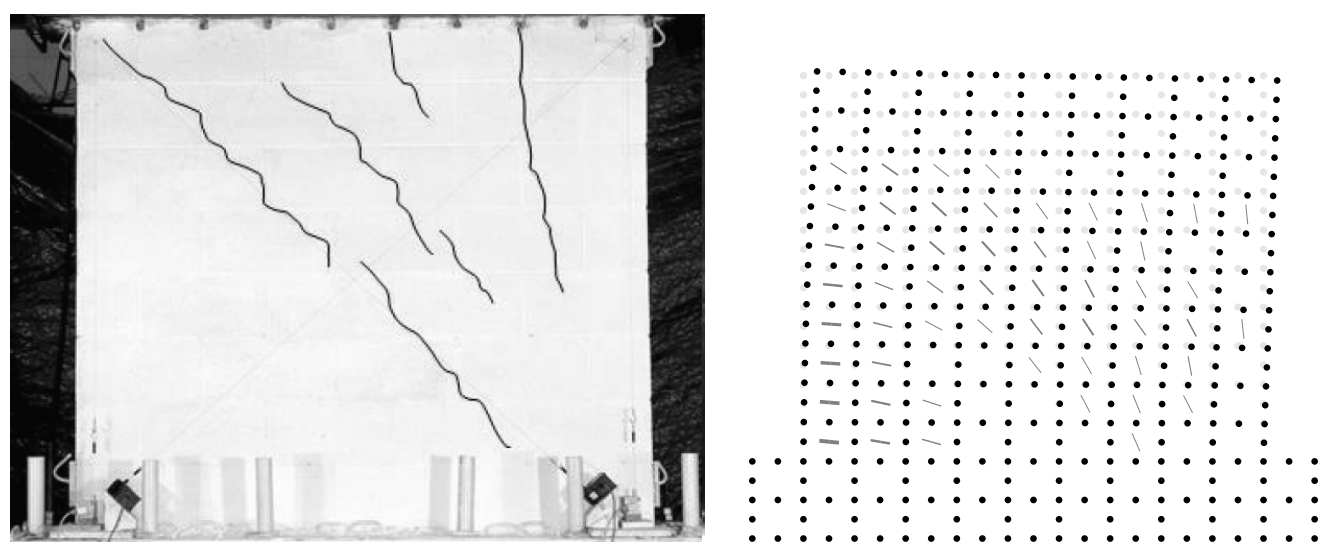

Experiment at $3.2 \mathrm{~mm}$ Tip Displacement Finite Element Model at $3.2 \mathrm{~mm}$ Tip Displace(Seif ElDin, 2016)

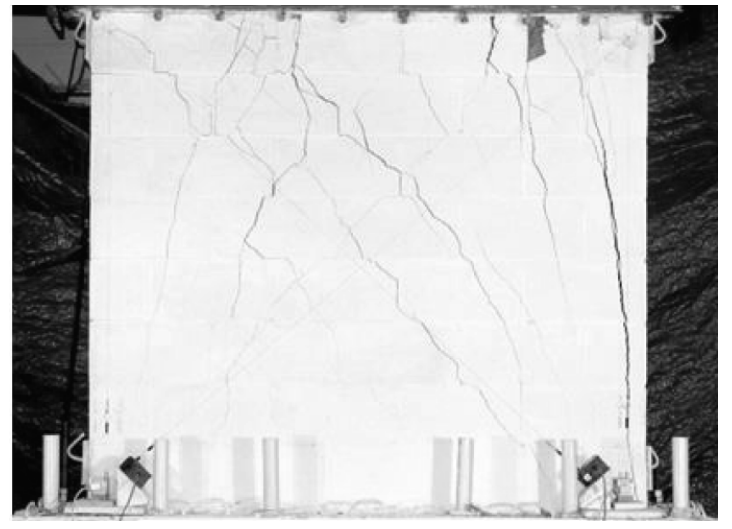
ment $\times 15$

Experiment at Peak Horizontal Force (Seif El- Finite Element Model at Peak Horizontal Force Din, 2016) $\times 15$

W- $M / V_{d} 1.8$ Shear Wall Displacement, Crack Pattern, and Crack Widths 


\section{W- $S_{v} 800$ Shear Wall}

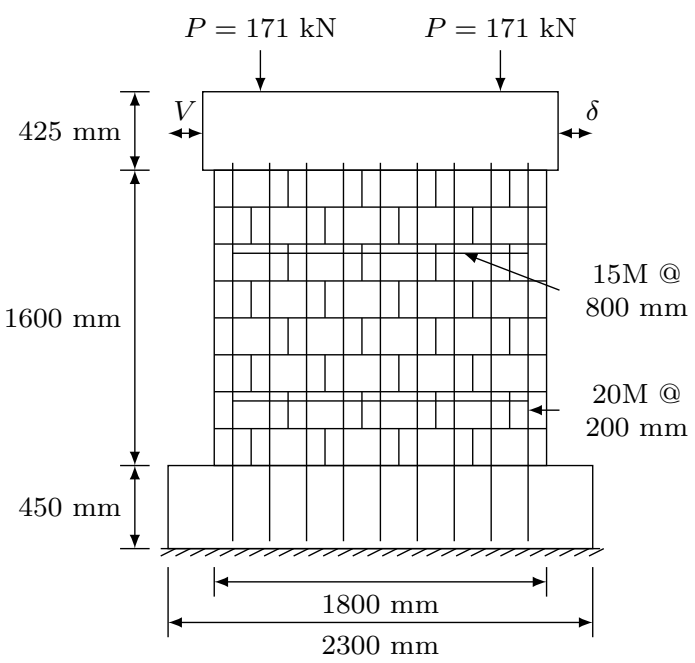

Experimental Wall (Seif ElDin, 2016)

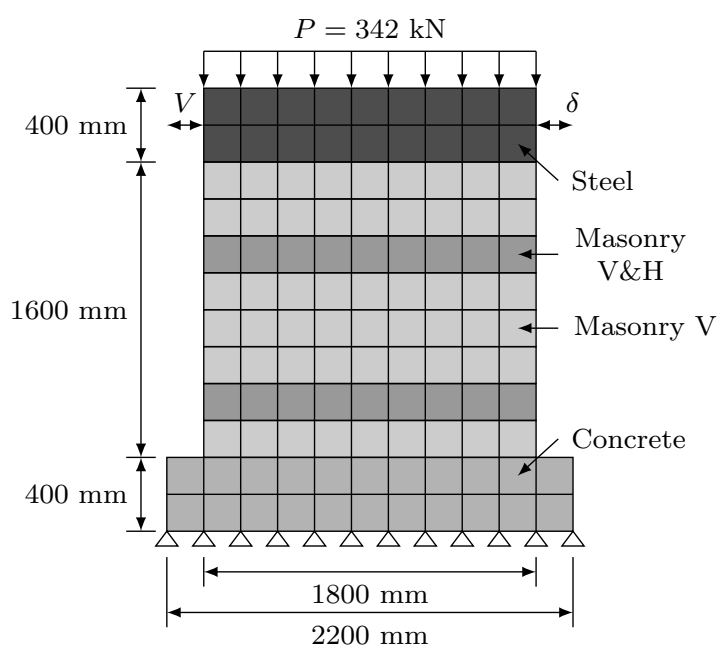

Finite Element Model Wall

$\mathrm{W}-S_{v} 800$ Shear Wall

W-S $S_{v} 800$ Shear Wall (Seif ElDin, 2016) Model Options

\begin{tabular}{ll}
\hline Model & Selected Option \\
\hline Compression Pre-Peak & Hognestad \\
Compression Post-Peak & Pre-Peak Base Curve \\
Compression Softening & Vecchio \\
Tension Post-Peak & Vecchio \\
Cracking Criterion & Mohr-Coulomb \\
Slip & Walraven Reinforced \\
\hline
\end{tabular}

Numerical Modelling of Hollow Concrete Block Masonry Mechanics 
W- $S_{v} 800$ Shear Wall (Seif ElDin, 2016) Material Properties

\begin{tabular}{|c|c|c|c|c|}
\hline \multirow{2}{*}{ Property } & \multicolumn{4}{|c|}{ Material } \\
\hline & Masonry V\&H & Masonry V & Concrete & Steel \\
\hline Thickness (mm) & 190 & 190 & 640 & 400 \\
\hline Grout Tensile Strength (MPa) & 1.99 & 1.99 & 2.07 & 350 \\
\hline \multicolumn{5}{|c|}{ Masonry Properties (Y-Direction) } \\
\hline Gross Peak Compression Strength (MPa) & 13.1 & 13.1 & 39.5 & 350 \\
\hline Peak Compressive Strain & 0.0025 & 0.0025 & 0.0021 & 0.00175 \\
\hline Tensile Strength & 0 & 0 & 2.07 & 350 \\
\hline Tensile Strain & 0 & 0 & 0 & 0.00175 \\
\hline Initial Tangent Elastic Modulus (MPa) & 3750 & 3750 & 34600 & 200000 \\
\hline Poisson's Ratio & 0 & 0 & 0.15 & 0 \\
\hline \multicolumn{5}{|c|}{ Masonry Properties (X-Direction) } \\
\hline Gross Peak Compression Strength (MPa) & 13.1 & 13.1 & 39.5 & 350 \\
\hline Peak Compressive Strain & 0.0025 & 0.0025 & 0.0021 & 0.00175 \\
\hline Tensile Strength & 2.07 & 0 & 0 & 350 \\
\hline Tensile Strain & 0 & 0 & 0 & 0.00175 \\
\hline Initial Tangent Elastic Modulus (MPa) & 3750 & 3750 & 34600 & 200000 \\
\hline Poisson's Ratio & 0 & 0 & 0.15 & 0 \\
\hline \multicolumn{5}{|c|}{ Masonry Properties (Block) } \\
\hline Length (mm) & 390 & 390 & 2200 & 1800 \\
\hline Height $(\mathrm{mm})$ & 190 & 190 & 400 & 400 \\
\hline Face Shell Thickness (mm) & 35 & 35 & 320 & 200 \\
\hline Percent Solid (\%) & 52 & 52 & 100 & 100 \\
\hline Tensile Strength & 1.40 & 1.40 & 2.07 & 350 \\
\hline \multicolumn{5}{|c|}{ Head Joint } \\
\hline Thickness (mm) & 10 & 10 & 10 & 10 \\
\hline Tensile Bond Strength (MPa) & 0.27 & 0.27 & 2.07 & 350 \\
\hline Joint Cohesion (MPa) & 3.48 & 3.48 & 2.07 & 350 \\
\hline Angle of Internal Friction (Deg) & 42 & 42 & 42 & 42 \\
\hline \multicolumn{5}{|c|}{ Bed Joint } \\
\hline Thickness (mm) & 10 & 10 & 10 & 10 \\
\hline Tensile Bond Strength (MPa) & 0.27 & 0.27 & 2.07 & 350 \\
\hline Joint Cohesion (MPa) & 3.48 & 3.48 & 2.07 & 350 \\
\hline Angle of Internal Friction (Deg) & 42 & 42 & 42 & 42 \\
\hline \multicolumn{5}{|c|}{ Reinforcement (Y-Direction) } \\
\hline Ratio (\%) & 0.79 & 0.79 & 0.42 & 100 \\
\hline Yield Strength (MPa) & 430 & 430 & 430 & 350 \\
\hline Yield Strain & 0.00219 & 0.00219 & 0.00219 & 0.00175 \\
\hline Strain Hardening Strain & 0.02 & 0.02 & 0.02 & 0.02 \\
\hline Ultimate Strength (MPa) & 542 & 542 & 542 & 600 \\
\hline Ultimate Strain & 0.198 & 0.198 & 0.198 & 0.2 \\
\hline Prestrain & 0 & 0 & 0 & 0 \\
\hline \multicolumn{5}{|c|}{ Reinforcement (X-Direction) } \\
\hline Ratio (\%) & 0.13 & 0 & 0.87 & 100 \\
\hline Yield Strength (MPa) & 430 & 0 & 430 & 350 \\
\hline Yield Strain & 0.00219 & 0 & 0.00219 & 0.00175 \\
\hline Strain Hardening Strain & 0.02 & 0 & 0.02 & 0.02 \\
\hline Ultimate Strength (MPa) & 540 & 0 & 542 & 600 \\
\hline Ultimate Strain & 0.205 & 0 & 0.198 & 0.2 \\
\hline Prestrain & 0 & 0 & 0 & 0 \\
\hline
\end{tabular}

Numerical Modelling of Hollow Concrete Block Masonry Mechanics 


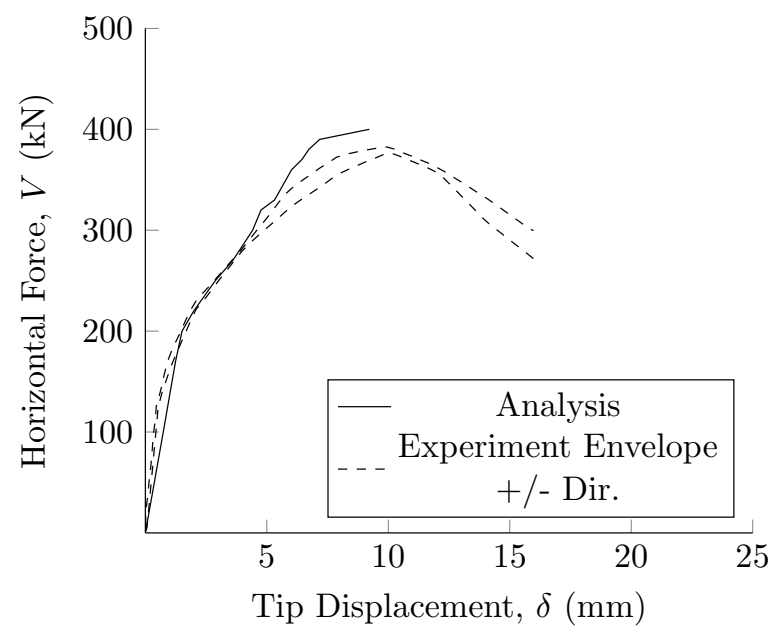

W- $S_{v} 800$ Shear Wall Seif ElDin (2016)
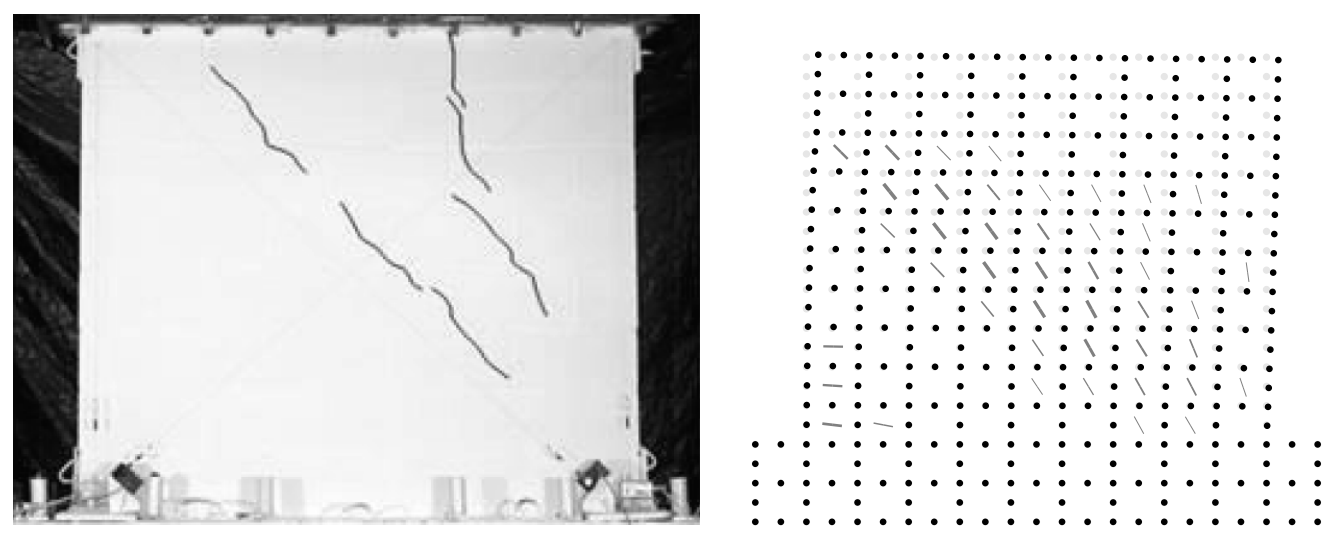

Experiment at $2.8 \mathrm{~mm}$ Tip Displacement Finite Element Model at $2.8 \mathrm{~mm}$ Tip Displace(Seif ElDin, 2016)

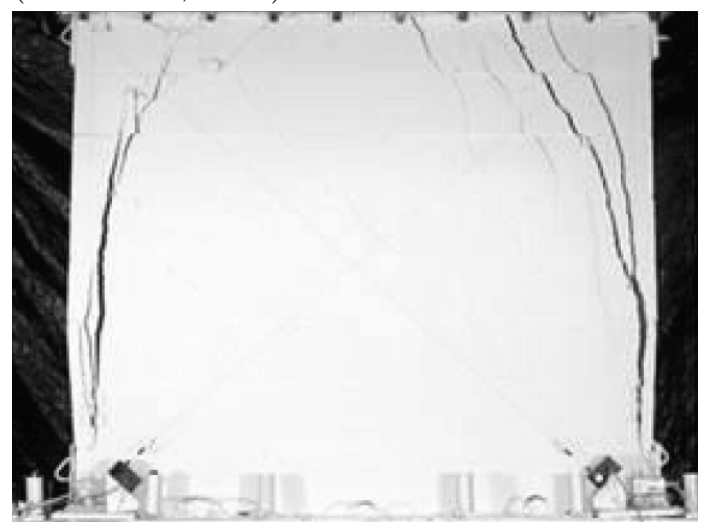
ment $\times 15$

Experiment at Peak Horizontal Force (Seif El- Finite Element Model at Peak Horizontal Force Din, 2016) $\times 15$

W- $S_{v} 800$ Shear Wall Displacement, Crack Pattern, and Crack Widths 


\section{W- $S_{h} 800$ Shear Wall}

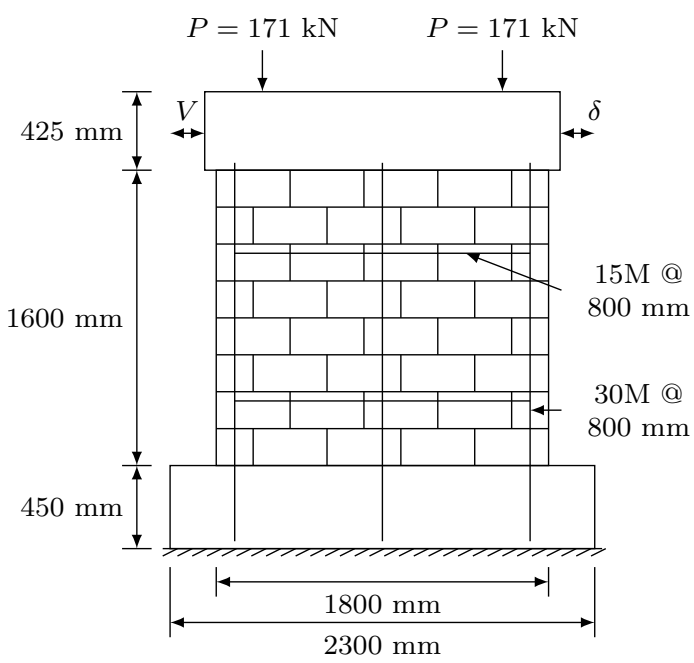

Experimental Wall (Seif ElDin, 2016)

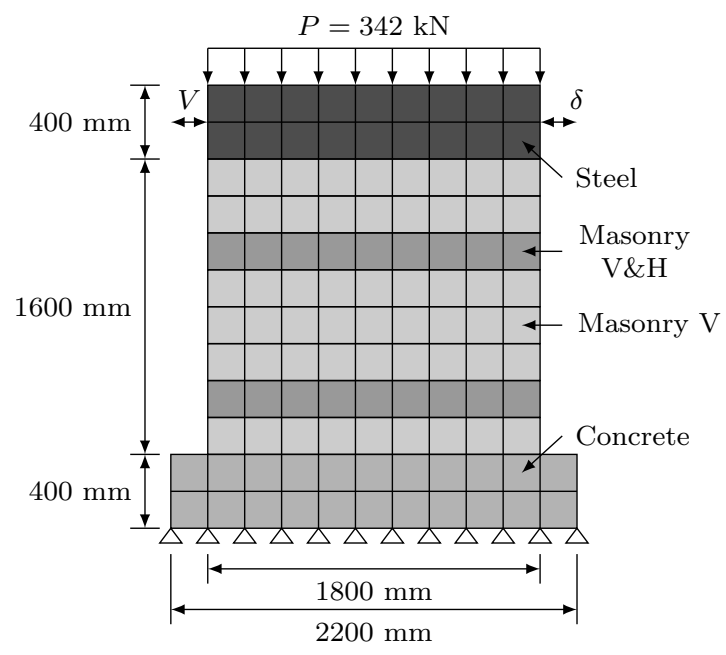

Finite Element Model Wall

W- $S_{h} 800$ Shear Wall

W- $S_{h} 800$ Shear Wall (Seif ElDin, 2016) Model Options

\begin{tabular}{ll}
\hline Model & Selected Option \\
\hline Compression Pre-Peak & Hognestad \\
Compression Post-Peak & Pre-Peak Base Curve \\
Compression Softening & Vecchio \\
Tension Post-Peak & Vecchio \\
Cracking Criterion & Mohr-Coulomb \\
Slip & Walraven Reinforced \\
\hline
\end{tabular}

Numerical Modelling of Hollow Concrete Block Masonry Mechanics 
W- $S_{h} 800$ Shear Wall (Seif ElDin, 2016) Material Properties

\begin{tabular}{|c|c|c|c|c|}
\hline \multirow{2}{*}{ Property } & \multicolumn{4}{|c|}{ Material } \\
\hline & Masonry V\&H & Masonry V & Concrete & Steel \\
\hline Thickness (mm) & 190 & 190 & 640 & 400 \\
\hline Grout Tensile Strength $(\mathrm{MPa})$ & 1.99 & 1.99 & 2.07 & 350 \\
\hline \multicolumn{5}{|c|}{ Masonry Properties (Y-Direction) } \\
\hline Gross Peak Compression Strength (MPa) & 13.1 & 13.1 & 39.5 & 350 \\
\hline Peak Compressive Strain & 0.0025 & 0.0025 & 0.0021 & 0.00175 \\
\hline Tensile Strength & 0 & 0 & 2.07 & 350 \\
\hline Tensile Strain & 0 & 0 & 0 & 0.00175 \\
\hline Initial Tangent Elastic Modulus (MPa) & 3750 & 3750 & 34600 & 200000 \\
\hline Poisson's Ratio & 0 & 0 & 0.15 & 0 \\
\hline \multicolumn{5}{|c|}{ Masonry Properties (X-Direction) } \\
\hline Gross Peak Compression Strength (MPa) & 13.1 & 13.1 & 39.5 & 350 \\
\hline Peak Compressive Strain & 0.0025 & 0.0025 & 0.0021 & 0.00175 \\
\hline Tensile Strength & 2.07 & 0 & 0 & 350 \\
\hline Tensile Strain & 0 & 0 & 0 & 0.00175 \\
\hline Initial Tangent Elastic Modulus (MPa) & 3750 & 3750 & 34600 & 200000 \\
\hline Poisson's Ratio & 0 & 0 & 0.15 & 0 \\
\hline \multicolumn{5}{|c|}{ Masonry Properties (Block) } \\
\hline Length $(\mathrm{mm})$ & 390 & 390 & 2200 & 1800 \\
\hline Height (mm) & 190 & 190 & 400 & 400 \\
\hline Face Shell Thickness (mm) & 35 & 35 & 320 & 200 \\
\hline Percent Solid (\%) & 52 & 52 & 100 & 100 \\
\hline Tensile Strength & 1.40 & 1.40 & 2.07 & 350 \\
\hline \multicolumn{5}{|c|}{ Head Joint } \\
\hline Thickness $(\mathrm{mm})$ & 10 & 10 & 10 & 10 \\
\hline Tensile Bond Strength (MPa) & 0.27 & 0.27 & 2.07 & 350 \\
\hline Joint Cohesion (MPa) & 3.48 & 3.48 & 2.07 & 350 \\
\hline Angle of Internal Friction (Deg) & 42 & 42 & 42 & 42 \\
\hline \multicolumn{5}{|c|}{ Bed Joint } \\
\hline Thickness (mm) & 10 & 10 & 10 & 10 \\
\hline Tensile Bond Strength (MPa) & 0.27 & 0.27 & 2.07 & 350 \\
\hline Joint Cohesion (MPa) & 3.48 & 3.48 & 2.07 & 350 \\
\hline Angle of Internal Friction (Deg) & 42 & 42 & 42 & 42 \\
\hline \multicolumn{5}{|c|}{ Reinforcement (Y-Direction) } \\
\hline Ratio (\%) & 0.61 & 0.61 & 0.42 & 100 \\
\hline Yield Strength (MPa) & 430 & 430 & 430 & 350 \\
\hline Yield Strain & 0.00219 & 0.00219 & 0.00219 & 0.00175 \\
\hline Strain Hardening Strain & 0.02 & 0.02 & 0.02 & 0.02 \\
\hline Ultimate Strength (MPa) & 542 & 542 & 542 & 600 \\
\hline Ultimate Strain & 0.198 & 0.198 & 0.198 & 0.2 \\
\hline Prestrain & 0 & 0 & 0 & 0 \\
\hline \multicolumn{5}{|c|}{ Reinforcement (X-Direction) } \\
\hline Ratio (\%) & 0.13 & 0 & 0.87 & 100 \\
\hline Yield Strength (MPa) & 430 & 0 & 430 & 350 \\
\hline Yield Strain & 0.00219 & 0 & 0.00219 & 0.00175 \\
\hline Strain Hardening Strain & 0.02 & 0 & 0.02 & 0.02 \\
\hline Ultimate Strength (MPa) & 540 & 0 & 542 & 600 \\
\hline Ultimate Strain & 0.205 & 0 & 0.198 & 0.2 \\
\hline Prestrain & 0 & 0 & 0 & 0 \\
\hline
\end{tabular}

Numerical Modelling of Hollow Concrete Block Masonry Mechanics 


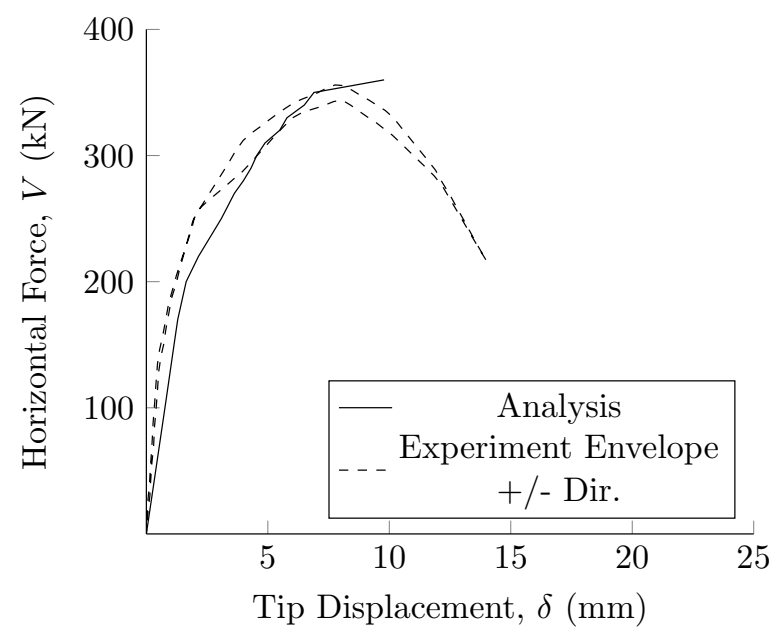

W- $S_{h} 800$ Shear Wall Seif ElDin (2016)
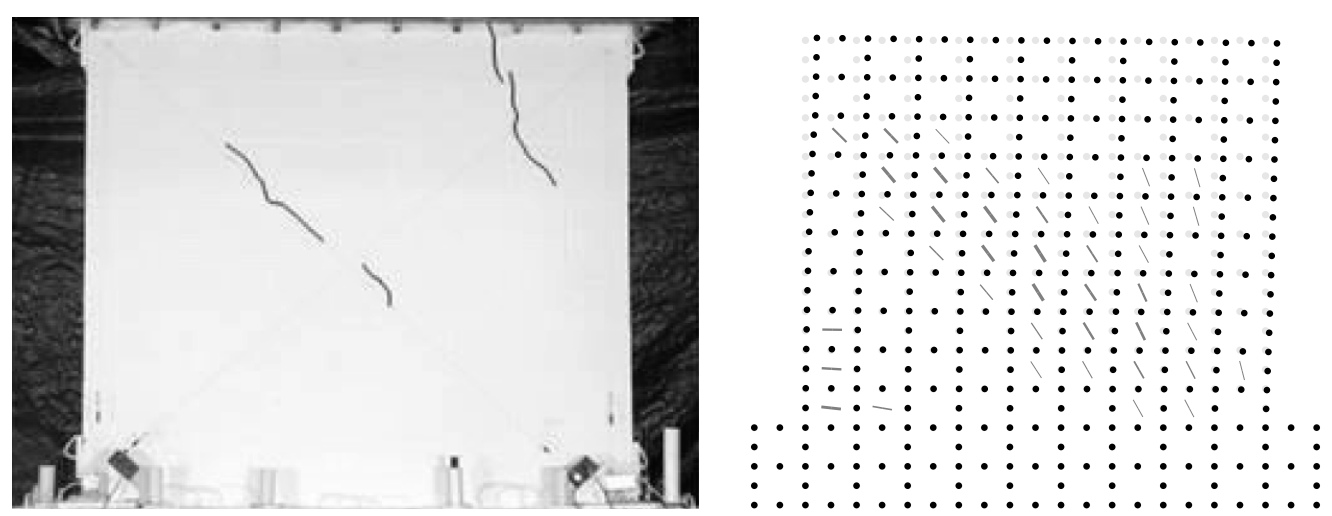

Experiment at $2.6 \mathrm{~mm}$ Tip Displacement Finite Element Model at $2.6 \mathrm{~mm}$ Tip Displace(Seif ElDin, 2016)

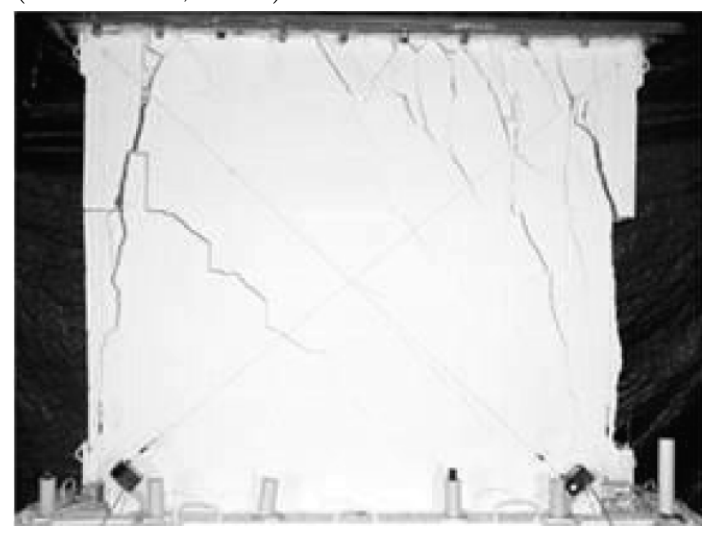
ment $\times 15$

Experiment at Peak Horizontal Force (Seif El- Finite Element Model at Peak Horizontal Force Din, 2016) $\times 15$

W- $S_{h} 800$ Shear Wall Displacement, Crack Pattern, and Crack Widths 


\section{SINGLE PIEAR UNGROUTED SHEAR WALL SMEARED AREA FINITE ELEMENT MODEL RESULTS}

\section{Woodward and Rankin (1985)}

\section{HL160-5L01 Shear Wall}

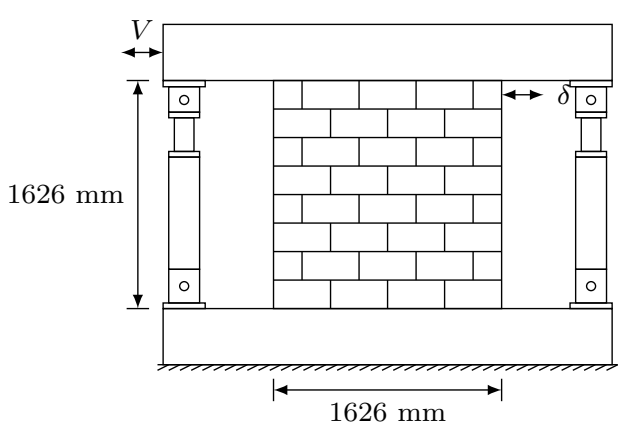

Experimental Wall Woodward and Rankin (1985)

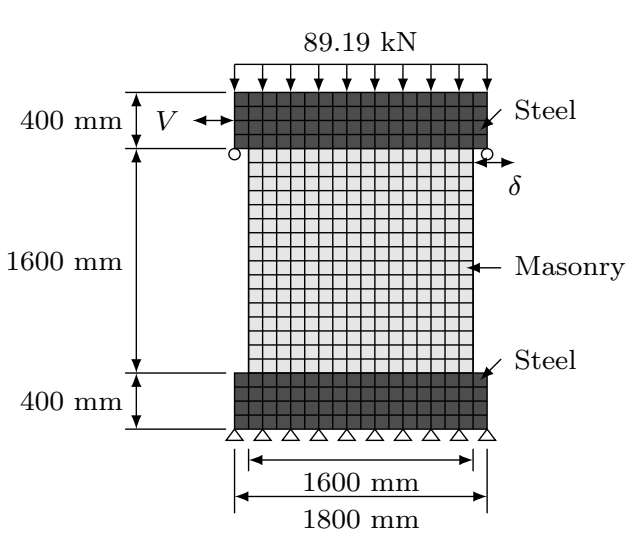

Finite Element Model Wall

64HL160-5L01 Shear Wall

64HL160-5L01 Shear Wall Woodward and Rankin (1985) Model Options

\begin{tabular}{ll}
\hline Model & Selected Option \\
\hline Compression Pre-Peak & Hognestad \\
Compression Post-Peak & Pre-Peak Base Curve \\
Compression Softening & Vecchio \\
Tension Post-Peak & Hordijk \\
Cracking Criterion & Mohr-Coulomb \\
Slip & Constant Rotation Lag \\
\hline
\end{tabular}


64HL160-5L01 Shear Wall Woodward and Rankin (1985) Material Properties

\begin{tabular}{|c|c|c|}
\hline \multirow{2}{*}{ Property } & \multicolumn{2}{|c|}{ Material } \\
\hline & Masonry & Steel \\
\hline Thickness (mm) & 194 & 200 \\
\hline Grout Tensile Strength (MPa) & 0 & 350 \\
\hline \multicolumn{3}{|c|}{ Masonry Properties (Y-Direction) } \\
\hline Gross Peak Compression Strength (MPa) & 5.79 & 350 \\
\hline Peak Compressive Strain & 0.0025 & 0.00175 \\
\hline Tensile Strength & 0 & 350 \\
\hline Tensile Strain & 0 & 0.00175 \\
\hline Initial Tangent Elastic Modulus (MPa) & 0 & 200000 \\
\hline Poisson's Ratio & 0 & 0 \\
\hline \multicolumn{3}{|c|}{ Masonry Properties (X-Direction) } \\
\hline Gross Peak Compression Strength (MPa) & 5.64 & 350 \\
\hline Peak Compressive Strain & 0.0025 & 0.00175 \\
\hline Tensile Strength & 0 & 350 \\
\hline Tensile Strain & 0 & 0.00175 \\
\hline Initial Tangent Elastic Modulus (MPa) & 0 & 200000 \\
\hline Poisson's Ratio & 0 & 0 \\
\hline \multicolumn{3}{|l|}{ Masonry Properties (Block) } \\
\hline Length $(\mathrm{mm})$ & 397 & 1800 \\
\hline Height (mm) & 194 & 400 \\
\hline Face Shell Thickness (mm) & 33 & 100 \\
\hline Percent Solid (\%) & 52 & 100 \\
\hline Tensile Strength & 1.62 & 350 \\
\hline \multicolumn{3}{|l|}{ Head Joint } \\
\hline Thickness (mm) & 10 & 10 \\
\hline Tensile Bond Strength (MPa) & 0.10 & 350 \\
\hline Joint Cohesion (MPa) & 2.50 & 350 \\
\hline Angle of Internal Friction (Deg) & 42 & 42 \\
\hline \multicolumn{3}{|l|}{ Bed Joint } \\
\hline Thickness (mm) & 10 & 10 \\
\hline Tensile Bond Strength (MPa) & 0.10 & 350 \\
\hline Joint Cohesion $(\mathrm{MPa})$ & 2.50 & 350 \\
\hline Angle of Internal Friction (Deg) & 42 & 42 \\
\hline \multicolumn{3}{|l|}{ Reinforcement (Y-Direction) } \\
\hline Ratio (\%) & 0 & 100 \\
\hline Yield Strength (MPa) & 0 & 350 \\
\hline Yield Strain & 0 & 0.00175 \\
\hline Strain Hardening Strain & 0 & 0.02 \\
\hline Ultimate Strength (MPa) & 0 & 600 \\
\hline Ultimate Strain & 0 & 0.2 \\
\hline Prestrain & 0 & 0 \\
\hline \multicolumn{3}{|c|}{ Reinforcement (X-Direction) } \\
\hline Ratio (\%) & 0 & 100 \\
\hline Yield Strength (MPa) & 0 & 350 \\
\hline Yield Strain & 0 & 0.00175 \\
\hline Strain Hardening Strain & 0 & 0.02 \\
\hline Ultimate Strength (MPa) & 0 & 600 \\
\hline Ultimate Strain & 0 & 0.2 \\
\hline Prestrain & 0 & 0 \\
\hline
\end{tabular}

Numerical Modelling of Hollow Concrete Block Masonry Mechanics 


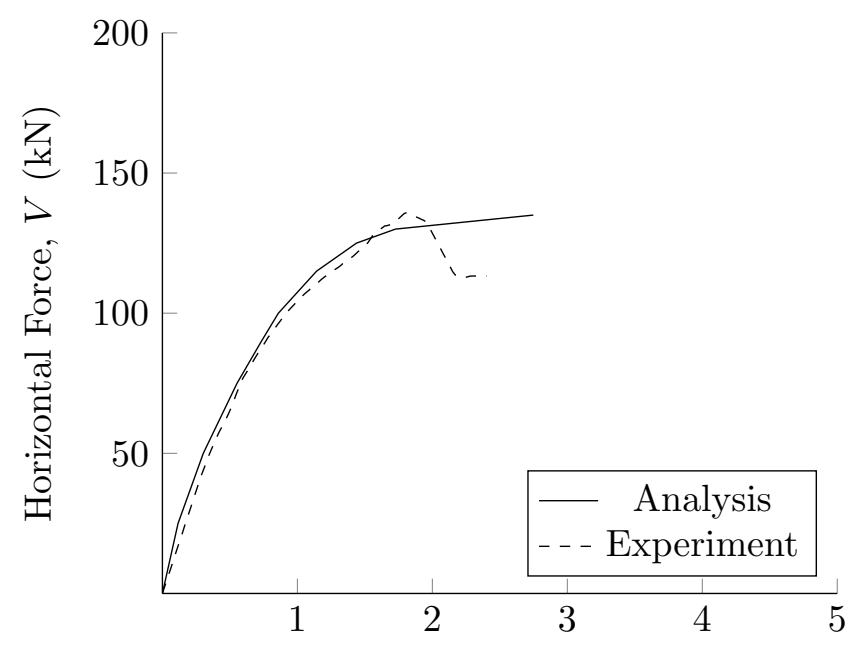

Tip Displacement, $\delta(\mathrm{mm})$

64HL160-5L01 Shear Wall Woodward and Rankin (1985) Horizontal Force vs Tip Displacement
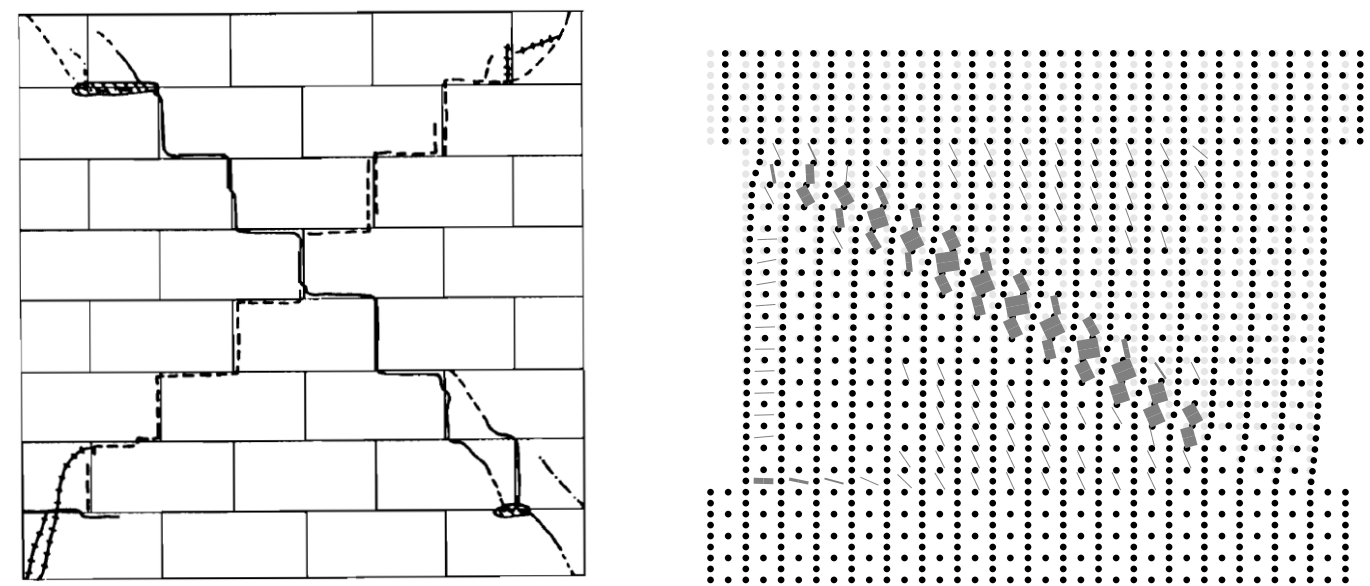

Experiment at Peak Horizontal Force (Wood- Finite Element Model at Peak Horizontal Force ward and Rankin, 1985) $\times 15$

64HL160-5L01 Shear Wall Displacement, Crack Pattern, and Crack Widths 


\section{HL400-5L04 Shear Wall}

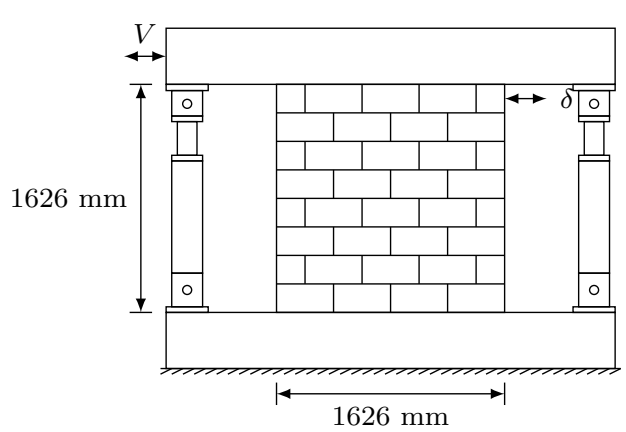

Experimental Wall Woodward and Rankin (1985)

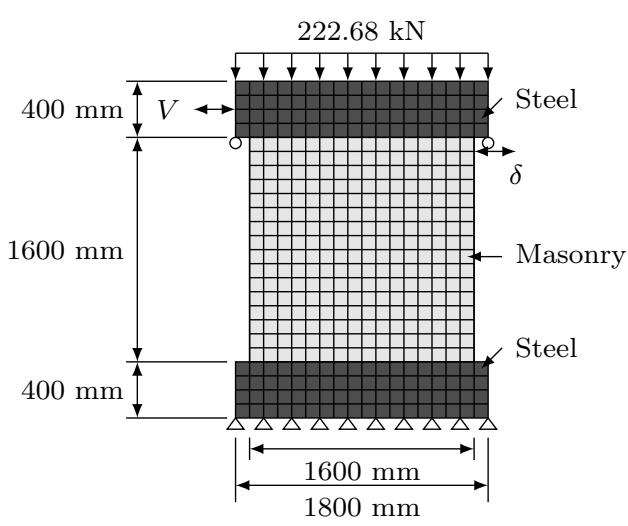

Finite Element Model Wall

64HL400-5L04 Shear Wall

64HL400-5L04 Shear Wall Woodward and Rankin (1985) Model Options

\begin{tabular}{ll}
\hline Model & Selected Option \\
\hline Compression Pre-Peak & Hognestad \\
Compression Post-Peak & Pre-Peak Base Curve \\
Compression Softening & Vecchio \\
Tension Post-Peak & Hordijk \\
Cracking Criterion & Mohr-Coulomb \\
Slip & Constant Rotation Lag \\
\hline
\end{tabular}

Numerical Modelling of Hollow Concrete Block Masonry Mechanics 
64HL400-5L04 Shear Wall Woodward and Rankin (1985) Material Properties

\begin{tabular}{|c|c|c|}
\hline \multirow{2}{*}{ Property } & \multicolumn{2}{|c|}{ Material } \\
\hline & Masonry & Steel \\
\hline Thickness (mm) & 194 & 200 \\
\hline Grout Tensile Strength (MPa) & 0 & 350 \\
\hline \multicolumn{3}{|c|}{ Masonry Properties (Y-Direction) } \\
\hline Gross Peak Compression Strength (MPa) & 5.83 & 350 \\
\hline Peak Compressive Strain & 0.002 & 0.00175 \\
\hline Tensile Strength & 0 & 350 \\
\hline Tensile Strain & 0 & 0.00175 \\
\hline Initial Tangent Elastic Modulus (MPa) & 0 & 200000 \\
\hline Poisson's Ratio & 0 & 0 \\
\hline \multicolumn{3}{|c|}{ Masonry Properties (X-Direction) } \\
\hline Gross Peak Compression Strength (MPa) & 5.65 & 350 \\
\hline Peak Compressive Strain & 0.002 & 0.00175 \\
\hline Tensile Strength & 0 & 350 \\
\hline Tensile Strain & 0 & 0.00175 \\
\hline Initial Tangent Elastic Modulus (MPa) & 0 & 200000 \\
\hline Poisson's Ratio & 0 & 0 \\
\hline \multicolumn{3}{|l|}{ Masonry Properties (Block) } \\
\hline Length $(\mathrm{mm})$ & 397 & 1800 \\
\hline Height (mm) & 194 & 400 \\
\hline Face Shell Thickness (mm) & 33 & 100 \\
\hline Percent Solid (\%) & 52 & 100 \\
\hline Tensile Strength & 1.62 & 350 \\
\hline \multicolumn{3}{|l|}{ Head Joint } \\
\hline Thickness (mm) & 10 & 10 \\
\hline Tensile Bond Strength (MPa) & 0.18 & 350 \\
\hline Joint Cohesion (MPa) & 2.50 & 350 \\
\hline Angle of Internal Friction (Deg) & 42 & 42 \\
\hline \multicolumn{3}{|l|}{ Bed Joint } \\
\hline Thickness (mm) & 10 & 10 \\
\hline Tensile Bond Strength (MPa) & 0.18 & 350 \\
\hline Joint Cohesion $(\mathrm{MPa})$ & 2.50 & 350 \\
\hline Angle of Internal Friction (Deg) & 42 & 42 \\
\hline \multicolumn{3}{|l|}{ Reinforcement (Y-Direction) } \\
\hline Ratio (\%) & 0 & 100 \\
\hline Yield Strength (MPa) & 0 & 350 \\
\hline Yield Strain & 0 & 0.00175 \\
\hline Strain Hardening Strain & 0 & 0.02 \\
\hline Ultimate Strength (MPa) & 0 & 600 \\
\hline Ultimate Strain & 0 & 0.2 \\
\hline Prestrain & 0 & 0 \\
\hline \multicolumn{3}{|c|}{ Reinforcement (X-Direction) } \\
\hline Ratio (\%) & 0 & 100 \\
\hline Yield Strength (MPa) & 0 & 350 \\
\hline Yield Strain & 0 & 0.00175 \\
\hline Strain Hardening Strain & 0 & 0.02 \\
\hline Ultimate Strength (MPa) & 0 & 600 \\
\hline Ultimate Strain & 0 & 0.2 \\
\hline Prestrain & 0 & 0 \\
\hline
\end{tabular}

Numerical Modelling of Hollow Concrete Block Masonry Mechanics 


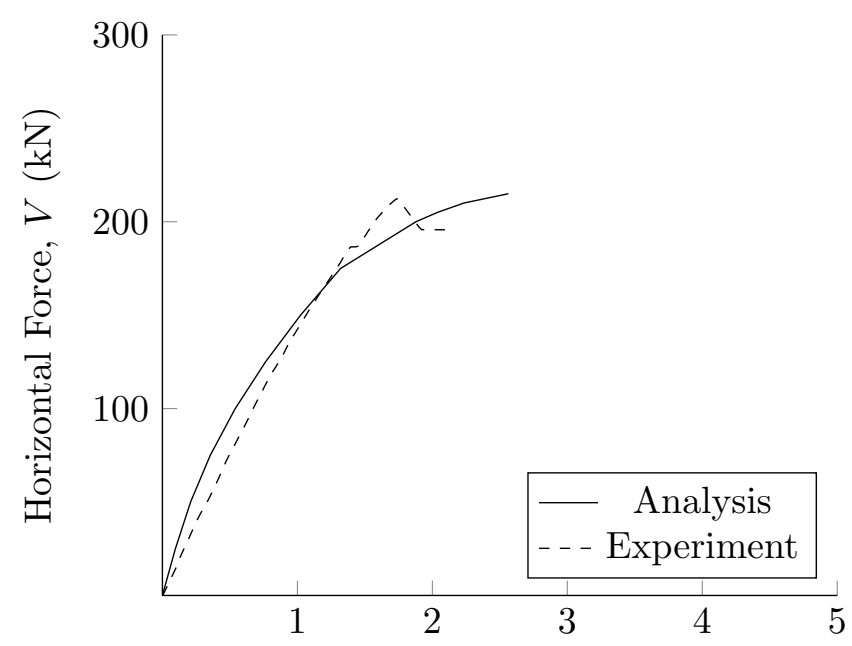

Tip Displacement, $\delta(\mathrm{mm})$

64HL400-5L04 Shear Wall Woodward and Rankin (1985) Horizontal Force vs Tip Displacement
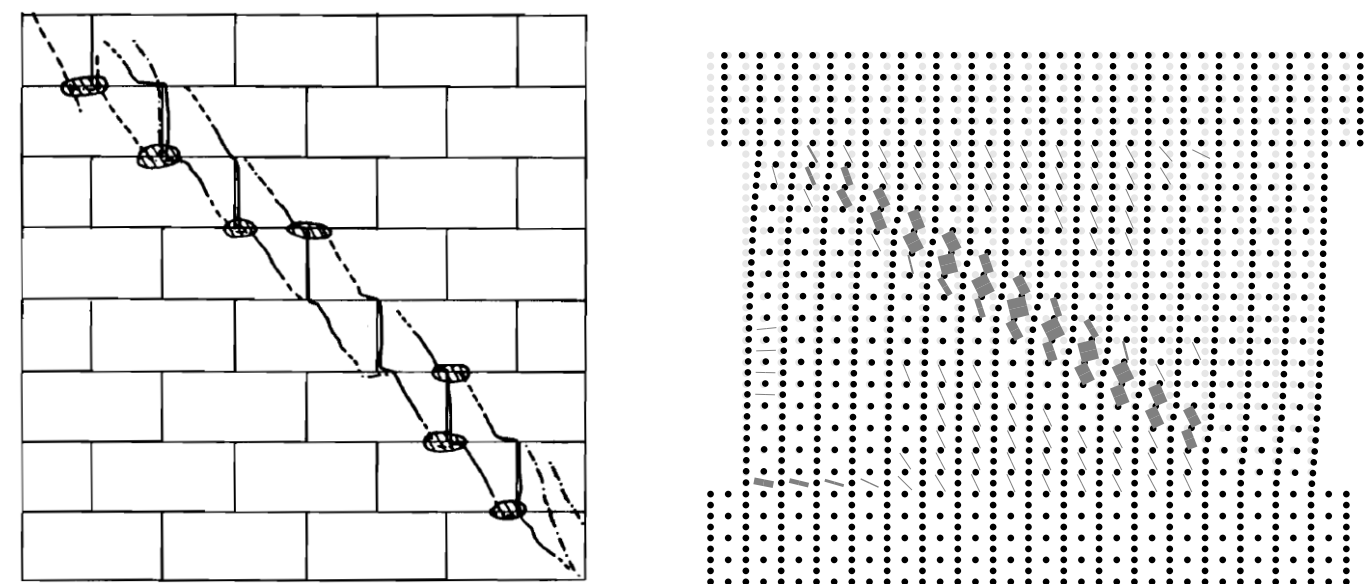

Experiment at Peak Horizontal Force (Wood- Finite Element Model at Peak Horizontal Force ward and Rankin, 1985) $\times 15$

64HL400-5L04 Shear Wall Displacement, Crack Pattern, and Crack Widths 


\section{LL170-6L07 Shear Wall}

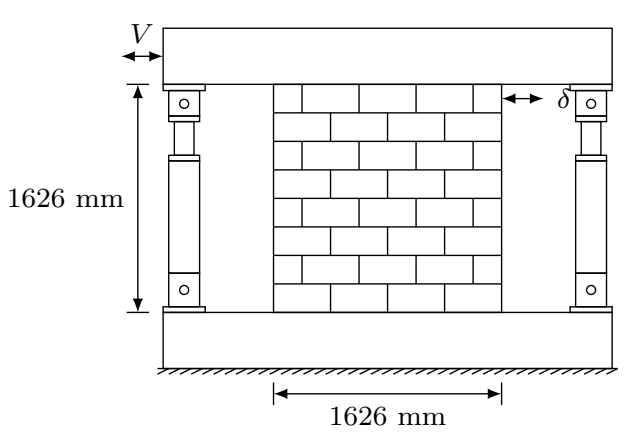

Experimental Wall Woodward and Rankin (1985)

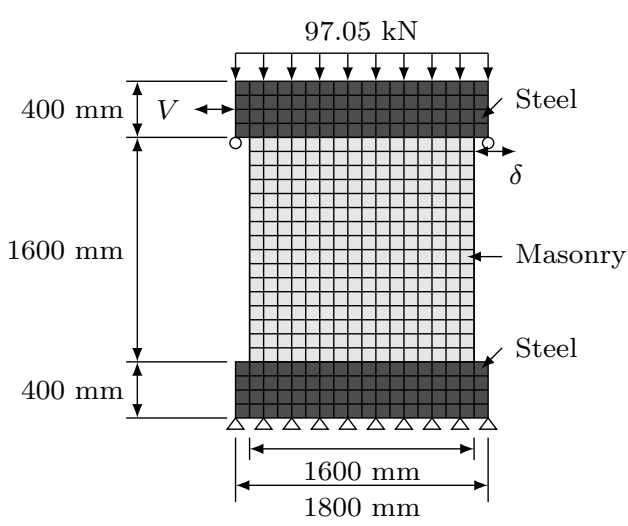

Finite Element Model Wall

64LL170-6L07 Shear Wall

64LL170-6L07 Shear Wall Woodward and Rankin (1985) Model Options

\begin{tabular}{ll}
\hline Model & Selected Option \\
\hline Compression Pre-Peak & Hognestad \\
Compression Post-Peak & Pre-Peak Base Curve \\
Compression Softening & Vecchio \\
Tension Post-Peak & Hordijk \\
Cracking Criterion & Mohr-Coulomb \\
Slip & Constant Rotation Lag \\
\hline
\end{tabular}


64LL170-6L07 Shear Wall Woodward and Rankin (1985) Material Properties

\begin{tabular}{|c|c|c|}
\hline \multirow{2}{*}{ Property } & \multicolumn{2}{|c|}{ Material } \\
\hline & Masonry & Steel \\
\hline Thickness (mm) & 194 & 200 \\
\hline Grout Tensile Strength (MPa) & 0 & 350 \\
\hline \multicolumn{3}{|c|}{ Masonry Properties (Y-Direction) } \\
\hline Gross Peak Compression Strength (MPa) & 4.32 & 350 \\
\hline Peak Compressive Strain & 0.002 & 0.00175 \\
\hline Tensile Strength & 0 & 350 \\
\hline Tensile Strain & 0 & 0.00175 \\
\hline Initial Tangent Elastic Modulus (MPa) & 0 & 200000 \\
\hline Poisson's Ratio & 0 & 0 \\
\hline \multicolumn{3}{|c|}{ Masonry Properties (X-Direction) } \\
\hline Gross Peak Compression Strength (MPa) & 4.10 & 350 \\
\hline Peak Compressive Strain & 0.002 & 0.00175 \\
\hline Tensile Strength & 0 & 350 \\
\hline Tensile Strain & 0 & 0.00175 \\
\hline Initial Tangent Elastic Modulus (MPa) & 0 & 200000 \\
\hline Poisson's Ratio & 0 & 0 \\
\hline \multicolumn{3}{|c|}{ Masonry Properties (Block) } \\
\hline Length (mm) & 397 & 1800 \\
\hline Height (mm) & 194 & 400 \\
\hline Face Shell Thickness (mm) & 33 & 100 \\
\hline Percent Solid (\%) & 50 & 100 \\
\hline Tensile Strength & 1.39 & 350 \\
\hline \multicolumn{3}{|l|}{ Head Joint } \\
\hline Thickness (mm) & 10 & 10 \\
\hline Tensile Bond Strength (MPa) & 0.15 & 350 \\
\hline Joint Cohesion (MPa) & 2.50 & 350 \\
\hline Angle of Internal Friction (Deg) & 42 & 42 \\
\hline \multicolumn{3}{|l|}{ Bed Joint } \\
\hline Thickness (mm) & 10 & 10 \\
\hline Tensile Bond Strength (MPa) & 0.15 & 350 \\
\hline Joint Cohesion (MPa) & 2.50 & 350 \\
\hline Angle of Internal Friction (Deg) & 42 & 42 \\
\hline \multicolumn{3}{|c|}{ Reinforcement (Y-Direction) } \\
\hline Ratio (\%) & 0 & 100 \\
\hline Yield Strength (MPa) & 0 & 350 \\
\hline Yield Strain & 0 & 0.00175 \\
\hline Strain Hardening Strain & 0 & 0.02 \\
\hline Ultimate Strength (MPa) & 0 & 600 \\
\hline Ultimate Strain & 0 & 0.2 \\
\hline Prestrain & 0 & 0 \\
\hline \multicolumn{3}{|c|}{ Reinforcement (X-Direction) } \\
\hline Ratio (\%) & 0 & 100 \\
\hline Yield Strength (MPa) & 0 & 350 \\
\hline Yield Strain & 0 & 0.00175 \\
\hline Strain Hardening Strain & 0 & 0.02 \\
\hline Ultimate Strength (MPa) & 0 & 600 \\
\hline Ultimate Strain & 0 & 0.2 \\
\hline Prestrain & 0 & 0 \\
\hline
\end{tabular}

Numerical Modelling of Hollow Concrete Block Masonry Mechanics 


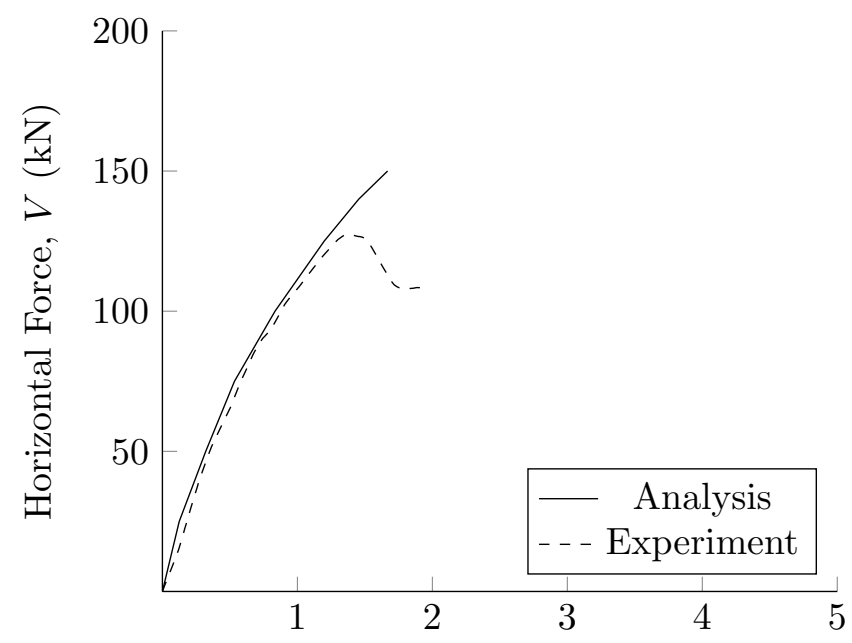

Tip Displacement, $\delta(\mathrm{mm})$

64LL170-6L07 Shear Wall Woodward and Rankin (1985) Horizontal Force vs Tip Displacement
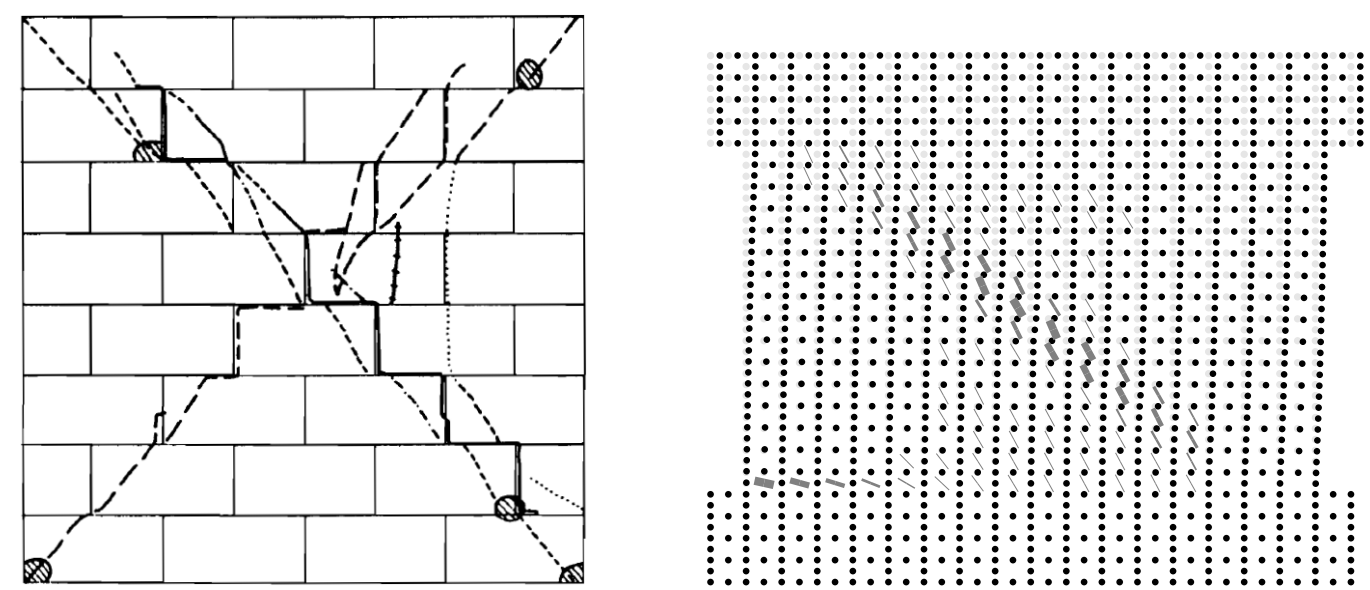

Experiment at Peak Horizontal Force (Wood- Finite Element Model at Peak Horizontal Force ward and Rankin, 1985) $\times 15$

64LL170-6L07 Shear Wall Displacement, Crack Pattern, and Crack Widths 


\section{LL250-6L06 Shear Wall}

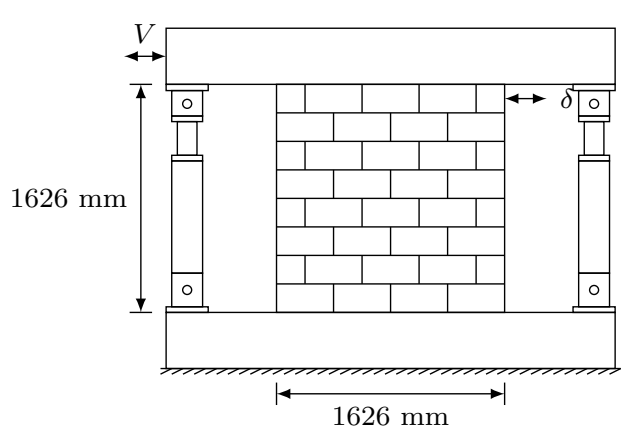

Experimental Wall Woodward and Rankin (1985)

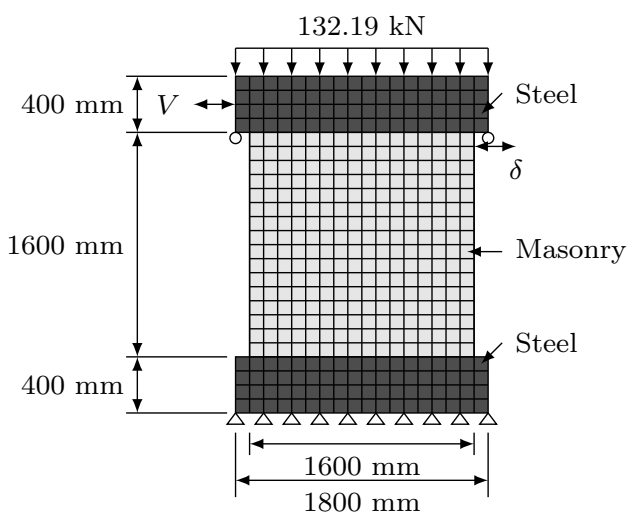

Finite Element Model Wall

64LL250-6L06 Shear Wall

64LL250-6L06 Shear Wall Woodward and Rankin (1985) Model Options

\begin{tabular}{ll}
\hline Model & Selected Option \\
\hline Compression Pre-Peak & Hognestad \\
Compression Post-Peak & Pre-Peak Base Curve \\
Compression Softening & Vecchio \\
Tension Post-Peak & Hordijk \\
Cracking Criterion & Mohr-Coulomb \\
Slip & Constant Rotation Lag \\
\hline
\end{tabular}

Numerical Modelling of Hollow Concrete Block Masonry Mechanics 
64LL250-6L06 Shear Wall Woodward and Rankin (1985) Material Properties

\begin{tabular}{|c|c|c|}
\hline \multirow{2}{*}{ Property } & \multicolumn{2}{|c|}{ Material } \\
\hline & Masonry & Steel \\
\hline Thickness (mm) & 194 & 200 \\
\hline Grout Tensile Strength (MPa) & 0 & 350 \\
\hline \multicolumn{3}{|c|}{ Masonry Properties (Y-Direction) } \\
\hline Gross Peak Compression Strength (MPa) & 4.49 & 350 \\
\hline Peak Compressive Strain & 0.0015 & 0.00175 \\
\hline Tensile Strength & 0 & 350 \\
\hline Tensile Strain & 0 & 0.00175 \\
\hline Initial Tangent Elastic Modulus (MPa) & 0 & 200000 \\
\hline Poisson's Ratio & 0 & 0 \\
\hline \multicolumn{3}{|c|}{ Masonry Properties (X-Direction) } \\
\hline Gross Peak Compression Strength (MPa) & 4.15 & 350 \\
\hline Peak Compressive Strain & 0.0015 & 0.00175 \\
\hline Tensile Strength & 0 & 350 \\
\hline Tensile Strain & 0 & 0.00175 \\
\hline Initial Tangent Elastic Modulus (MPa) & 0 & 200000 \\
\hline Poisson's Ratio & 0 & 0 \\
\hline \multicolumn{3}{|l|}{ Masonry Properties (Block) } \\
\hline Length $(\mathrm{mm})$ & 397 & 1800 \\
\hline Height (mm) & 194 & 400 \\
\hline Face Shell Thickness (mm) & 33 & 100 \\
\hline Percent Solid (\%) & 50 & 100 \\
\hline Tensile Strength & 1.39 & 350 \\
\hline \multicolumn{3}{|l|}{ Head Joint } \\
\hline Thickness (mm) & 10 & 10 \\
\hline Tensile Bond Strength (MPa) & 0.15 & 350 \\
\hline Joint Cohesion (MPa) & 2.00 & 350 \\
\hline Angle of Internal Friction (Deg) & 42 & 42 \\
\hline \multicolumn{3}{|l|}{ Bed Joint } \\
\hline Thickness (mm) & 10 & 10 \\
\hline Tensile Bond Strength (MPa) & 0.15 & 350 \\
\hline Joint Cohesion $(\mathrm{MPa})$ & 2.00 & 350 \\
\hline Angle of Internal Friction (Deg) & 42 & 42 \\
\hline \multicolumn{3}{|l|}{ Reinforcement (Y-Direction) } \\
\hline Ratio (\%) & 0 & 100 \\
\hline Yield Strength (MPa) & 0 & 350 \\
\hline Yield Strain & 0 & 0.00175 \\
\hline Strain Hardening Strain & 0 & 0.02 \\
\hline Ultimate Strength (MPa) & 0 & 600 \\
\hline Ultimate Strain & 0 & 0.2 \\
\hline Prestrain & 0 & 0 \\
\hline \multicolumn{3}{|c|}{ Reinforcement (X-Direction) } \\
\hline Ratio (\%) & 0 & 100 \\
\hline Yield Strength (MPa) & 0 & 350 \\
\hline Yield Strain & 0 & 0.00175 \\
\hline Strain Hardening Strain & 0 & 0.02 \\
\hline Ultimate Strength (MPa) & 0 & 600 \\
\hline Ultimate Strain & 0 & 0.2 \\
\hline Prestrain & 0 & 0 \\
\hline
\end{tabular}

Numerical Modelling of Hollow Concrete Block Masonry Mechanics 


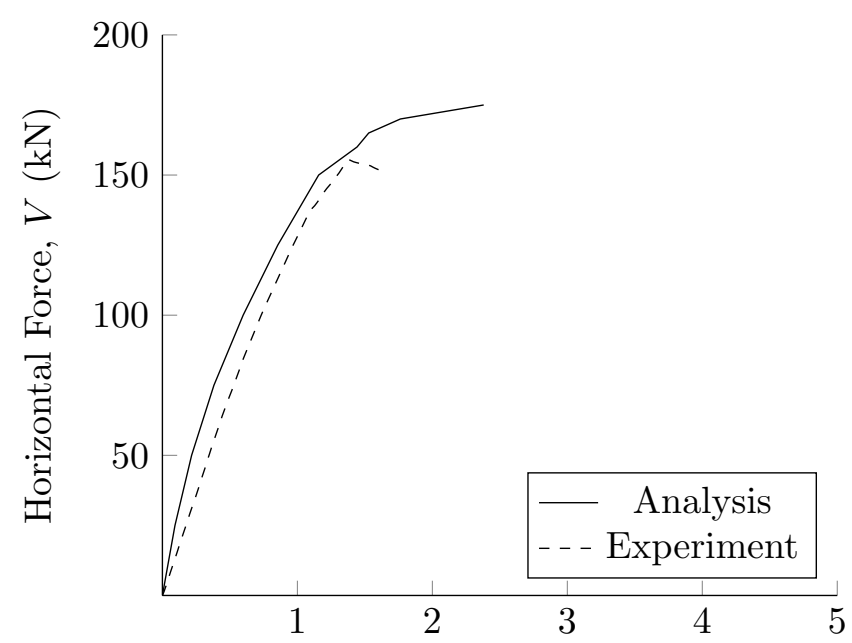

Tip Displacement, $\delta(\mathrm{mm})$

64LL250-6L06 Shear Wall Woodward and Rankin (1985) Horizontal Force vs Tip Displacement
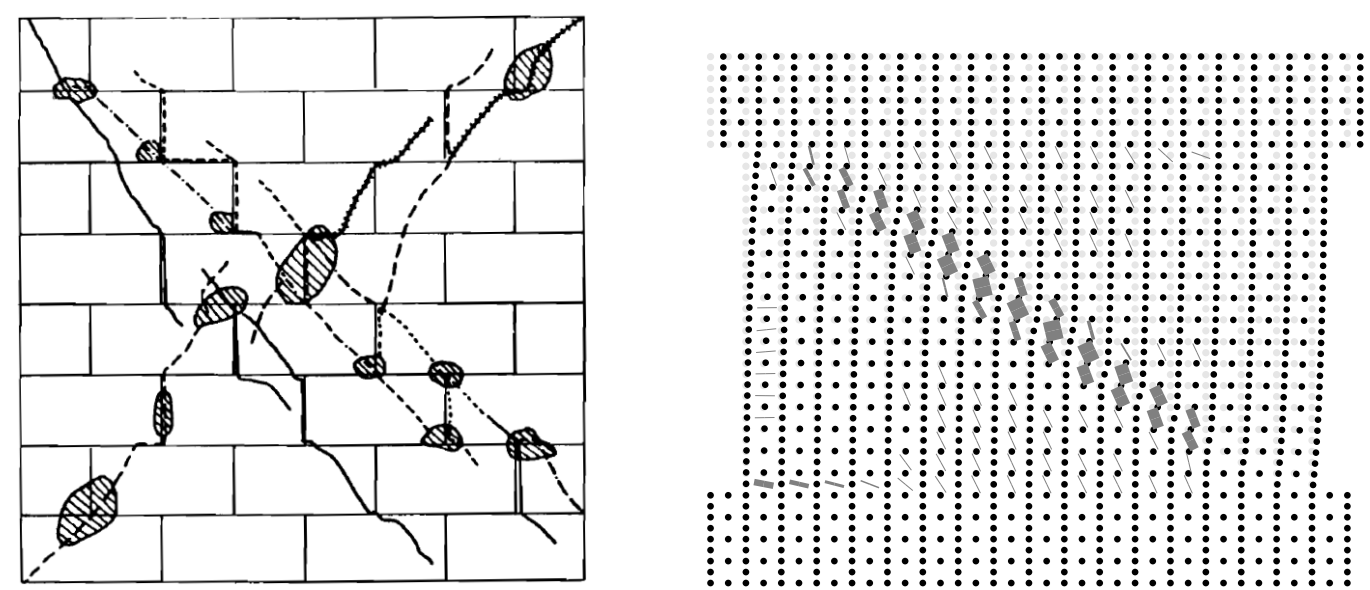

Experiment at Peak Horizontal Force (Wood- Finite Element Model at Peak Horizontal Force ward and Rankin, 1985) $\times 15$

64LL250-6L06 Shear Wall Displacement, Crack Pattern, and Crack Widths 


\section{LL340-6L08 Shear Wall}

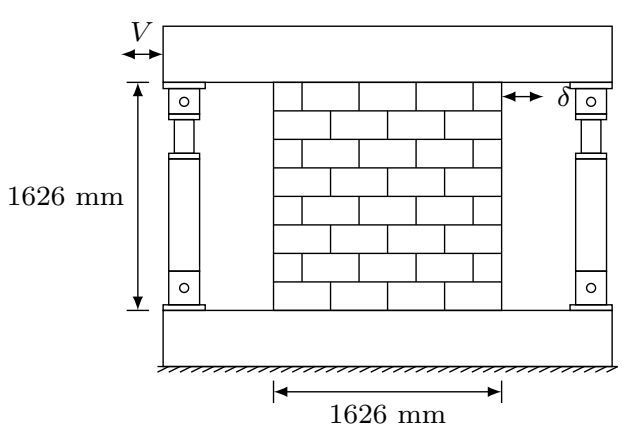

Experimental Wall Woodward and Rankin (1985)

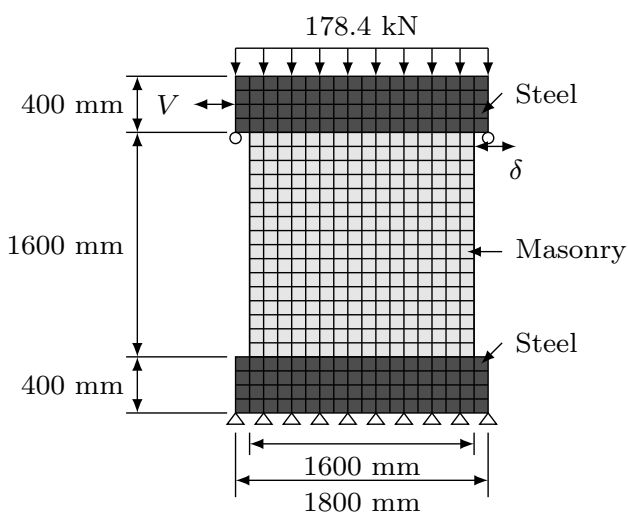

Finite Element Model Wall

64LL340-6L08 Shear Wall

64LL340-6L08 Shear Wall Woodward and Rankin (1985) Model Options

\begin{tabular}{ll}
\hline Model & Selected Option \\
\hline Compression Pre-Peak & Hognestad \\
Compression Post-Peak & Pre-Peak Base Curve \\
Compression Softening & Vecchio \\
Tension Post-Peak & Hordijk \\
Cracking Criterion & Mohr-Coulomb \\
Slip & Constant Rotation Lag \\
\hline
\end{tabular}

Numerical Modelling of Hollow Concrete Block Masonry Mechanics 
64LL340-6L08 Shear Wall Woodward and Rankin (1985) Material Properties

\begin{tabular}{|c|c|c|}
\hline \multirow{2}{*}{ Property } & \multicolumn{2}{|c|}{ Material } \\
\hline & Masonry & Steel \\
\hline Thickness (mm) & 194 & 200 \\
\hline Grout Tensile Strength (MPa) & 0 & 350 \\
\hline \multicolumn{3}{|c|}{ Masonry Properties (Y-Direction) } \\
\hline Gross Peak Compression Strength (MPa) & 4.60 & 350 \\
\hline Peak Compressive Strain & 0.0015 & 0.00175 \\
\hline Tensile Strength & 0 & 350 \\
\hline Tensile Strain & 0 & 0.00175 \\
\hline Initial Tangent Elastic Modulus (MPa) & 0 & 200000 \\
\hline Poisson's Ratio & 0 & 0 \\
\hline \multicolumn{3}{|c|}{ Masonry Properties (X-Direction) } \\
\hline Gross Peak Compression Strength (MPa) & 4.18 & 350 \\
\hline Peak Compressive Strain & 0.0015 & 0.00175 \\
\hline Tensile Strength & 0 & 350 \\
\hline Tensile Strain & 0 & 0.00175 \\
\hline Initial Tangent Elastic Modulus (MPa) & 0 & 200000 \\
\hline Poisson's Ratio & 0 & 0 \\
\hline \multicolumn{3}{|c|}{ Masonry Properties (Block) } \\
\hline Length (mm) & 397 & 1800 \\
\hline Height (mm) & 194 & 400 \\
\hline Face Shell Thickness (mm) & 33 & 100 \\
\hline Percent Solid (\%) & 50 & 100 \\
\hline Tensile Strength & 1.39 & 350 \\
\hline \multicolumn{3}{|l|}{ Head Joint } \\
\hline Thickness (mm) & 10 & 10 \\
\hline Tensile Bond Strength (MPa) & 0.17 & 350 \\
\hline Joint Cohesion (MPa) & 2.50 & 350 \\
\hline Angle of Internal Friction (Deg) & 42 & 42 \\
\hline \multicolumn{3}{|l|}{ Bed Joint } \\
\hline Thickness (mm) & 10 & 10 \\
\hline Tensile Bond Strength (MPa) & 0.17 & 350 \\
\hline Joint Cohesion (MPa) & 2.50 & 350 \\
\hline Angle of Internal Friction (Deg) & 42 & 42 \\
\hline \multicolumn{3}{|c|}{ Reinforcement (Y-Direction) } \\
\hline Ratio (\%) & 0 & 100 \\
\hline Yield Strength (MPa) & 0 & 350 \\
\hline Yield Strain & 0 & 0.00175 \\
\hline Strain Hardening Strain & 0 & 0.02 \\
\hline Ultimate Strength (MPa) & 0 & 600 \\
\hline Ultimate Strain & 0 & 0.2 \\
\hline Prestrain & 0 & 0 \\
\hline \multicolumn{3}{|c|}{ Reinforcement (X-Direction) } \\
\hline Ratio (\%) & 0 & 100 \\
\hline Yield Strength (MPa) & 0 & 350 \\
\hline Yield Strain & 0 & 0.00175 \\
\hline Strain Hardening Strain & 0 & 0.02 \\
\hline Ultimate Strength (MPa) & 0 & 600 \\
\hline Ultimate Strain & 0 & 0.2 \\
\hline Prestrain & 0 & 0 \\
\hline
\end{tabular}

Numerical Modelling of Hollow Concrete Block Masonry Mechanics 


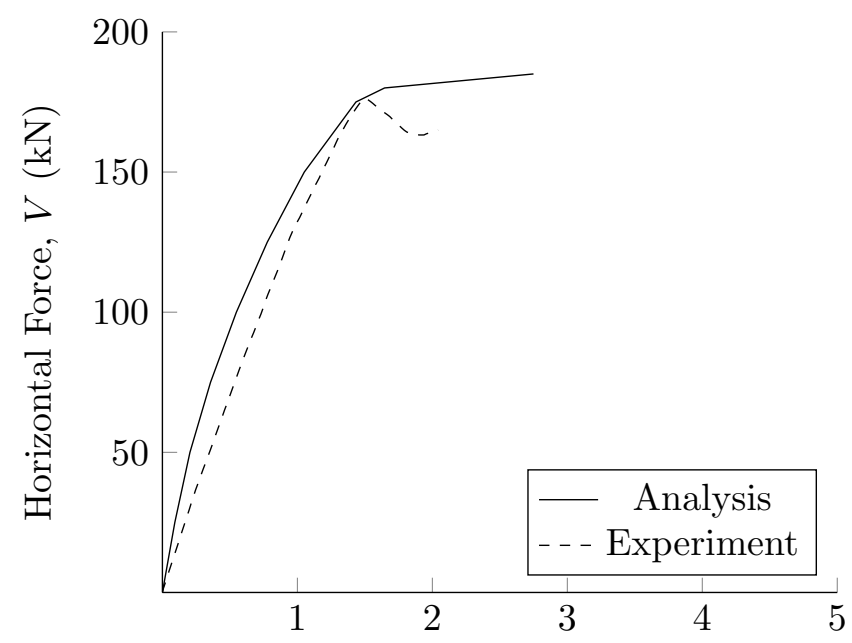

Tip Displacement, $\delta(\mathrm{mm})$

64LL340-6L08 Shear Wall Woodward and Rankin (1985) Horizontal Force vs Tip Displacement
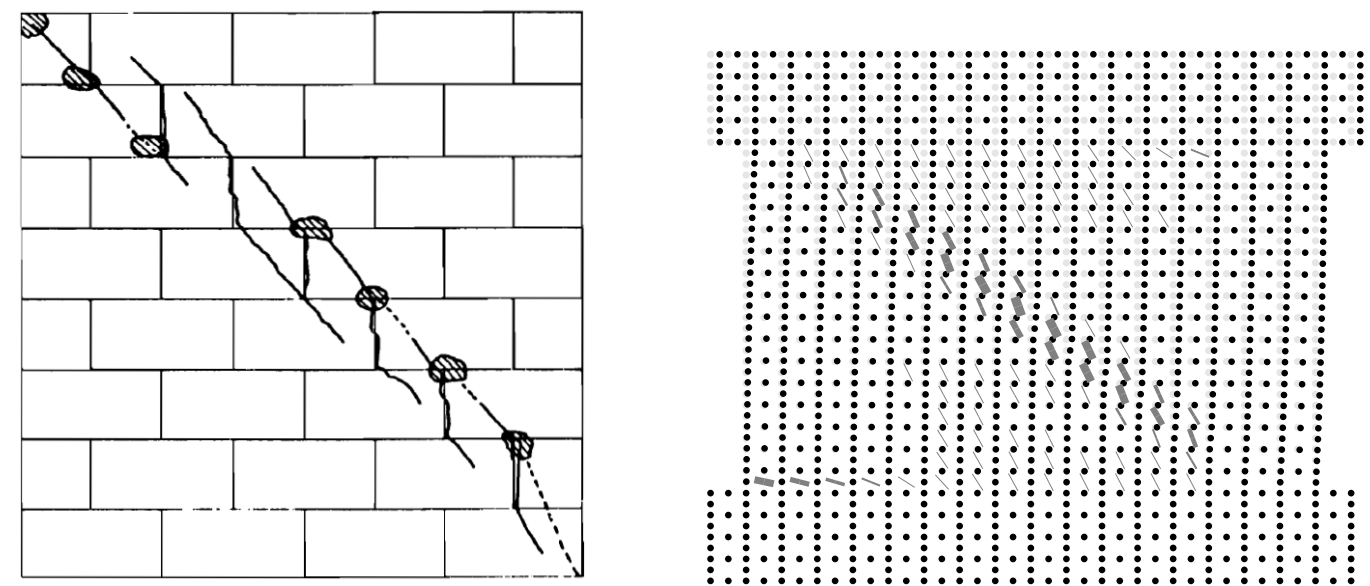

Experiment at Peak Horizontal Force (Wood- Finite Element Model at Peak Horizontal Force ward and Rankin, 1985) $\times 15$

64LL340-6L08 Shear Wall Displacement, Crack Pattern, and Crack Widths 


\section{LL420-6L09 Shear Wall}

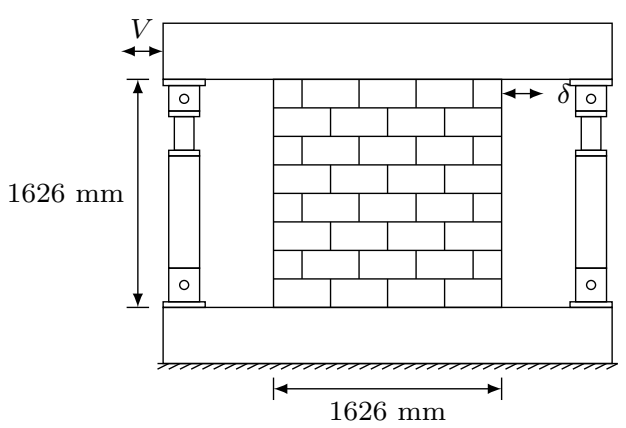

Experimental Wall Woodward and Rankin (1985)

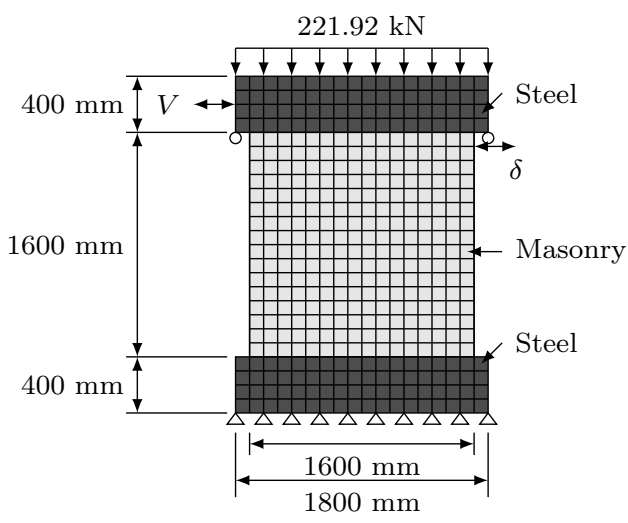

Finite Element Model Wall

64LL420-6L09 Shear Wall

64LL420-6L09 Shear Wall Woodward and Rankin (1985) Model Options

\begin{tabular}{ll}
\hline Model & Selected Option \\
\hline Compression Pre-Peak & Hognestad \\
Compression Post-Peak & Pre-Peak Base Curve \\
Compression Softening & Vecchio \\
Tension Post-Peak & Hordijk \\
Cracking Criterion & Mohr-Coulomb \\
Slip & Constant Rotation Lag \\
\hline
\end{tabular}


64LL420-6L09 Shear Wall Woodward and Rankin (1985) Material Properties

\begin{tabular}{|c|c|c|}
\hline \multirow{2}{*}{ Property } & \multicolumn{2}{|c|}{ Material } \\
\hline & Masonry & Steel \\
\hline Thickness (mm) & 194 & 200 \\
\hline Grout Tensile Strength (MPa) & 0 & 350 \\
\hline \multicolumn{3}{|c|}{ Masonry Properties (Y-Direction) } \\
\hline Gross Peak Compression Strength (MPa) & 4.51 & 350 \\
\hline Peak Compressive Strain & 0.0015 & 0.00175 \\
\hline Tensile Strength & 0 & 350 \\
\hline Tensile Strain & 0 & 0.00175 \\
\hline Initial Tangent Elastic Modulus (MPa) & 0 & 200000 \\
\hline Poisson's Ratio & 0 & 0 \\
\hline \multicolumn{3}{|c|}{ Masonry Properties (X-Direction) } \\
\hline Gross Peak Compression Strength (MPa) & 4.15 & 350 \\
\hline Peak Compressive Strain & 0.0015 & 0.00175 \\
\hline Tensile Strength & 0 & 350 \\
\hline Tensile Strain & 0 & 0.00175 \\
\hline Initial Tangent Elastic Modulus (MPa) & 0 & 200000 \\
\hline Poisson's Ratio & 0 & 0 \\
\hline \multicolumn{3}{|l|}{ Masonry Properties (Block) } \\
\hline Length $(\mathrm{mm})$ & 397 & 1800 \\
\hline Height (mm) & 194 & 400 \\
\hline Face Shell Thickness (mm) & 33 & 100 \\
\hline Percent Solid (\%) & 50 & 100 \\
\hline Tensile Strength & 1.39 & 350 \\
\hline \multicolumn{3}{|l|}{ Head Joint } \\
\hline Thickness (mm) & 10 & 10 \\
\hline Tensile Bond Strength (MPa) & 0.17 & 350 \\
\hline Joint Cohesion (MPa) & 2.50 & 350 \\
\hline Angle of Internal Friction (Deg) & 42 & 42 \\
\hline \multicolumn{3}{|l|}{ Bed Joint } \\
\hline Thickness (mm) & 10 & 10 \\
\hline Tensile Bond Strength (MPa) & 0.17 & 350 \\
\hline Joint Cohesion $(\mathrm{MPa})$ & 2.50 & 350 \\
\hline Angle of Internal Friction (Deg) & 42 & 42 \\
\hline \multicolumn{3}{|l|}{ Reinforcement (Y-Direction) } \\
\hline Ratio (\%) & 0 & 100 \\
\hline Yield Strength (MPa) & 0 & 350 \\
\hline Yield Strain & 0 & 0.00175 \\
\hline Strain Hardening Strain & 0 & 0.02 \\
\hline Ultimate Strength (MPa) & 0 & 600 \\
\hline Ultimate Strain & 0 & 0.2 \\
\hline Prestrain & 0 & 0 \\
\hline \multicolumn{3}{|c|}{ Reinforcement (X-Direction) } \\
\hline Ratio (\%) & 0 & 100 \\
\hline Yield Strength (MPa) & 0 & 350 \\
\hline Yield Strain & 0 & 0.00175 \\
\hline Strain Hardening Strain & 0 & 0.02 \\
\hline Ultimate Strength (MPa) & 0 & 600 \\
\hline Ultimate Strain & 0 & 0.2 \\
\hline Prestrain & 0 & 0 \\
\hline
\end{tabular}

Numerical Modelling of Hollow Concrete Block Masonry Mechanics 


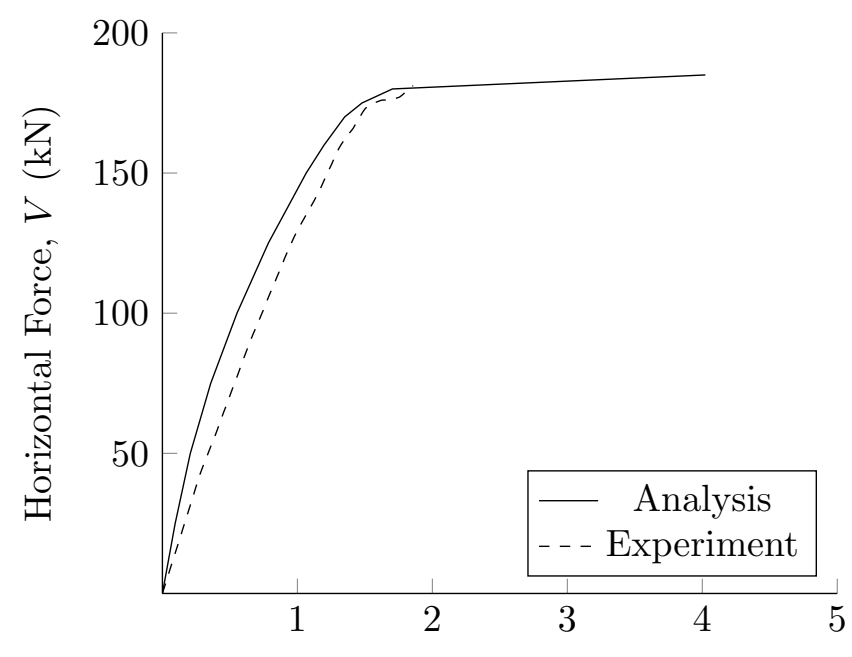

Tip Displacement, $\delta(\mathrm{mm})$

64LL420-6L09 Shear Wall Woodward and Rankin (1985) Horizontal Force vs Tip Displacement
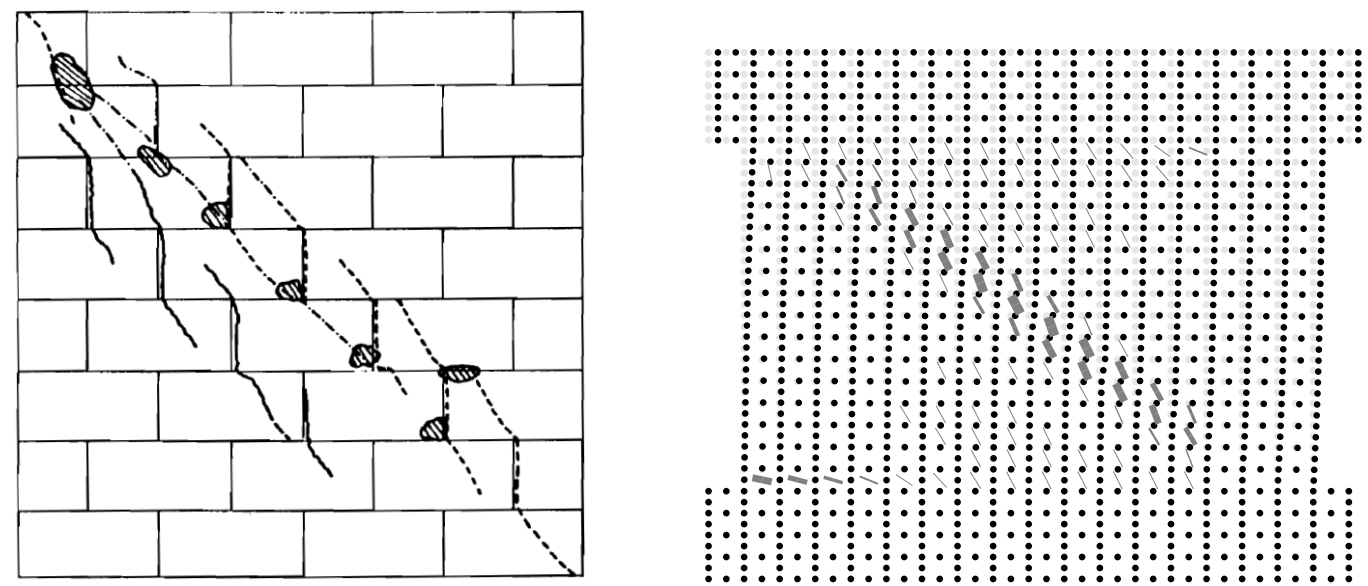

Experiment at Peak Horizontal Force (Wood- Finite Element Model at Peak Horizontal Force ward and Rankin, 1985) $\times 15$

64LL420-6L09 Shear Wall Displacement, Crack Pattern, and Crack Widths 


\section{LH105-6L01 Shear Wall}

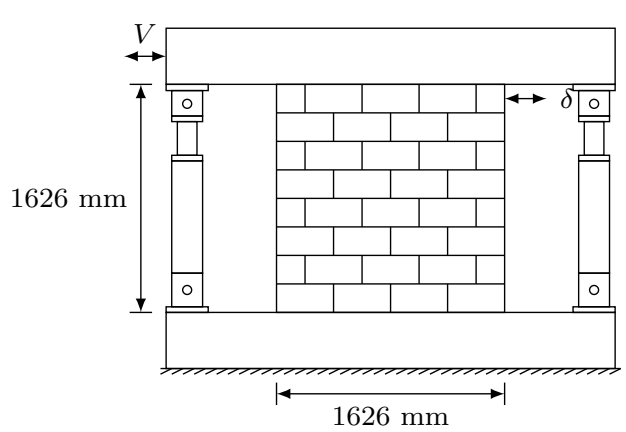

Experimental Wall Woodward and Rankin (1985)

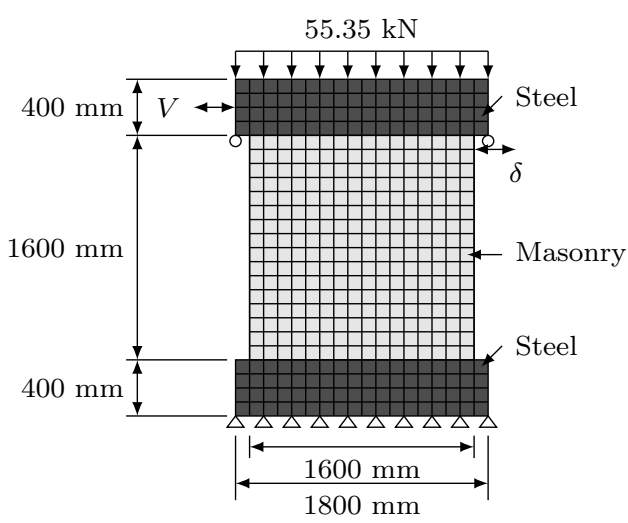

Finite Element Model Wall

64LH105-6L01 Shear Wall

64LH105-6L01 Shear Wall Woodward and Rankin (1985) Model Options

\begin{tabular}{ll}
\hline Model & Selected Option \\
\hline Compression Pre-Peak & Hognestad \\
Compression Post-Peak & Pre-Peak Base Curve \\
Compression Softening & Vecchio \\
Tension Post-Peak & Hordijk \\
Cracking Criterion & Mohr-Coulomb \\
Slip & Constant Rotation Lag \\
\hline
\end{tabular}


64LH105-6L01 Shear Wall Woodward and Rankin (1985) Material Properties

\begin{tabular}{|c|c|c|}
\hline \multirow{2}{*}{ Property } & \multicolumn{2}{|c|}{ Material } \\
\hline & Masonry & Steel \\
\hline Thickness (mm) & 194 & 200 \\
\hline Grout Tensile Strength (MPa) & 0 & 350 \\
\hline \multicolumn{3}{|c|}{ Masonry Properties (Y-Direction) } \\
\hline Gross Peak Compression Strength (MPa) & 6.01 & 350 \\
\hline Peak Compressive Strain & 0.0025 & 0.00175 \\
\hline Tensile Strength & 0 & 350 \\
\hline Tensile Strain & 0 & 0.00175 \\
\hline Initial Tangent Elastic Modulus (MPa) & 0 & 200000 \\
\hline Poisson's Ratio & 0 & 0 \\
\hline \multicolumn{3}{|c|}{ Masonry Properties (X-Direction) } \\
\hline Gross Peak Compression Strength (MPa) & 4.46 & 350 \\
\hline Peak Compressive Strain & 0.0025 & 0.00175 \\
\hline Tensile Strength & 0 & 350 \\
\hline Tensile Strain & 0 & 0.00175 \\
\hline Initial Tangent Elastic Modulus (MPa) & 0 & 200000 \\
\hline Poisson's Ratio & 0 & 0 \\
\hline \multicolumn{3}{|l|}{ Masonry Properties (Block) } \\
\hline Length $(\mathrm{mm})$ & 397 & 1800 \\
\hline Height (mm) & 194 & 400 \\
\hline Face Shell Thickness (mm) & 33 & 100 \\
\hline Percent Solid (\%) & 50 & 100 \\
\hline Tensile Strength & 1.39 & 350 \\
\hline \multicolumn{3}{|l|}{ Head Joint } \\
\hline Thickness (mm) & 10 & 10 \\
\hline Tensile Bond Strength (MPa) & 0.08 & 350 \\
\hline Joint Cohesion (MPa) & 1.50 & 350 \\
\hline Angle of Internal Friction (Deg) & 42 & 42 \\
\hline \multicolumn{3}{|l|}{ Bed Joint } \\
\hline Thickness (mm) & 10 & 10 \\
\hline Tensile Bond Strength (MPa) & 0.08 & 350 \\
\hline Joint Cohesion $(\mathrm{MPa})$ & 1.50 & 350 \\
\hline Angle of Internal Friction (Deg) & 42 & 42 \\
\hline \multicolumn{3}{|l|}{ Reinforcement (Y-Direction) } \\
\hline Ratio (\%) & 0 & 100 \\
\hline Yield Strength (MPa) & 0 & 350 \\
\hline Yield Strain & 0 & 0.00175 \\
\hline Strain Hardening Strain & 0 & 0.02 \\
\hline Ultimate Strength (MPa) & 0 & 600 \\
\hline Ultimate Strain & 0 & 0.2 \\
\hline Prestrain & 0 & 0 \\
\hline \multicolumn{3}{|c|}{ Reinforcement (X-Direction) } \\
\hline Ratio (\%) & 0 & 100 \\
\hline Yield Strength (MPa) & 0 & 350 \\
\hline Yield Strain & 0 & 0.00175 \\
\hline Strain Hardening Strain & 0 & 0.02 \\
\hline Ultimate Strength (MPa) & 0 & 600 \\
\hline Ultimate Strain & 0 & 0.2 \\
\hline Prestrain & 0 & 0 \\
\hline
\end{tabular}

Numerical Modelling of Hollow Concrete Block Masonry Mechanics 


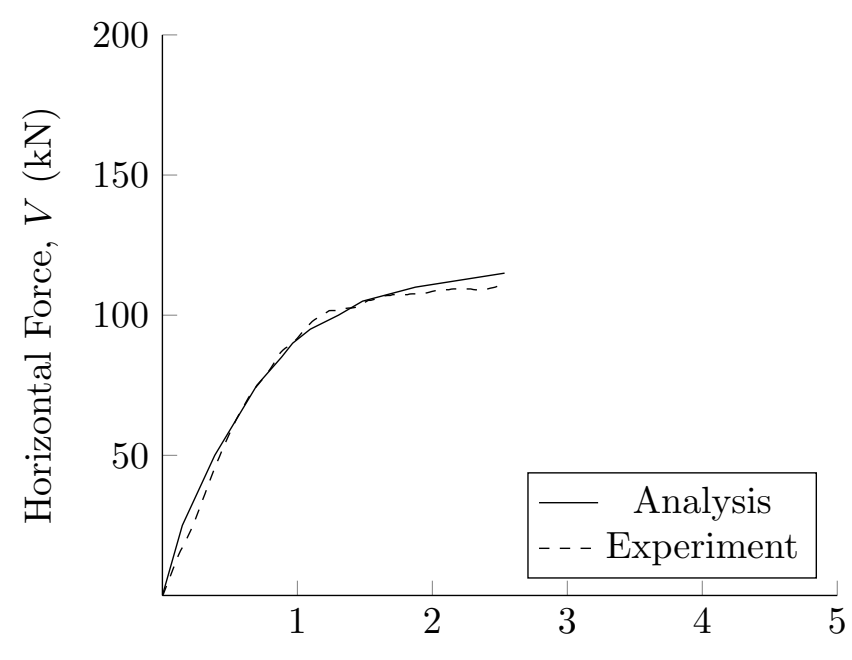

Tip Displacement, $\delta(\mathrm{mm})$

64LH105-6L01 Shear Wall Woodward and Rankin (1985) Horizontal Force vs Tip Displacement
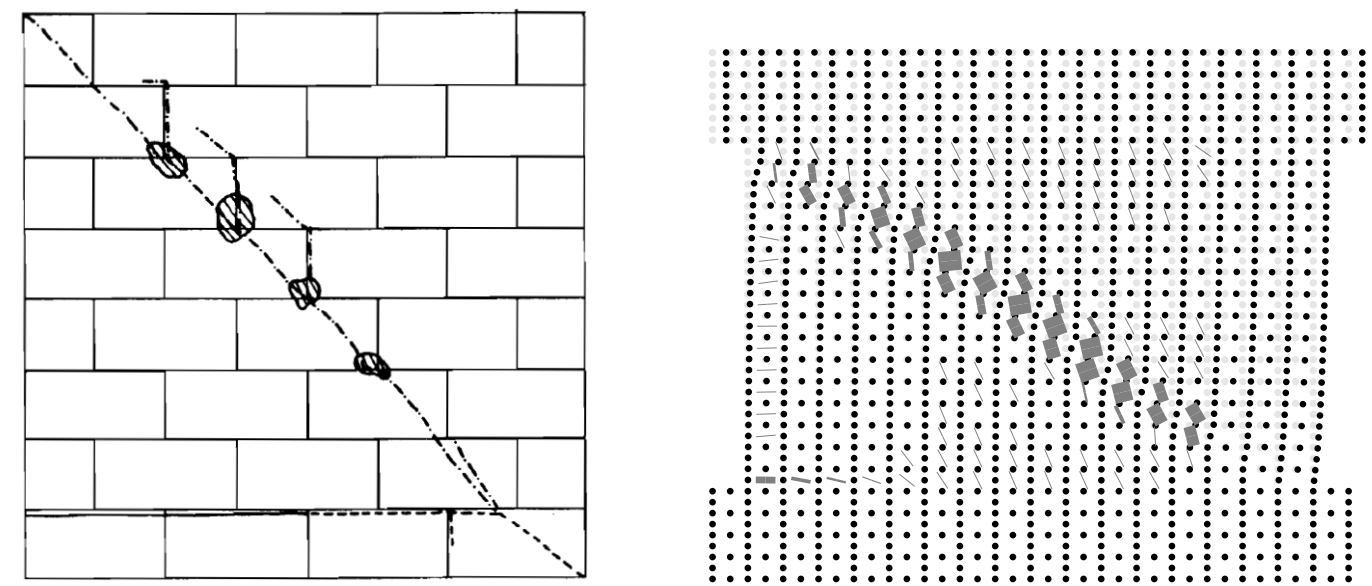

Experiment at Peak Horizontal Force (Wood- Finite Element Model at Peak Horizontal Force ward and Rankin, 1985) $\times 15$

64LH105-6L01 Shear Wall Displacement, Crack Pattern, and Crack Widths 


\section{LH170-6L02 Shear Wall}

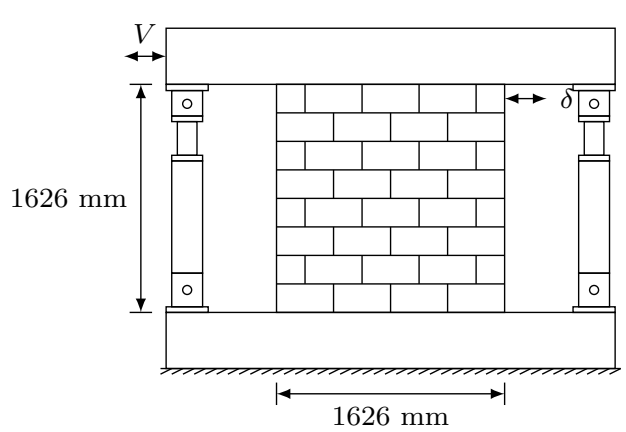

Experimental Wall Woodward and Rankin (1985)

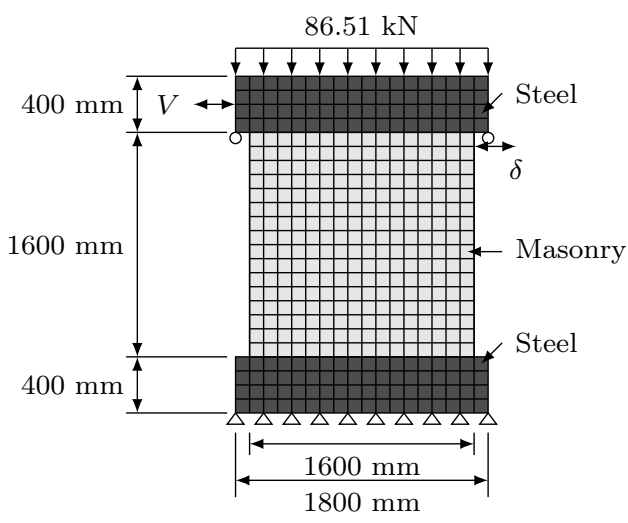

Finite Element Model Wall

64LH170-6L02 Shear Wall

64LH170-6L02 Shear Wall Woodward and Rankin (1985) Model Options

\begin{tabular}{ll}
\hline Model & Selected Option \\
\hline Compression Pre-Peak & Hognestad \\
Compression Post-Peak & Pre-Peak Base Curve \\
Compression Softening & Vecchio \\
Tension Post-Peak & Hordijk \\
Cracking Criterion & Mohr-Coulomb \\
Slip & Constant Rotation Lag \\
\hline
\end{tabular}

Numerical Modelling of Hollow Concrete Block Masonry Mechanics 
64LH170-6L02 Shear Wall Woodward and Rankin (1985) Material Properties

\begin{tabular}{|c|c|c|}
\hline \multirow{2}{*}{ Property } & \multicolumn{2}{|c|}{ Material } \\
\hline & Masonry & Steel \\
\hline Thickness (mm) & 194 & 200 \\
\hline Grout Tensile Strength (MPa) & 0 & 350 \\
\hline \multicolumn{3}{|c|}{ Masonry Properties (Y-Direction) } \\
\hline Gross Peak Compression Strength (MPa) & 5.66 & 350 \\
\hline Peak Compressive Strain & 0.0025 & 0.00175 \\
\hline Tensile Strength & 0 & 350 \\
\hline Tensile Strain & 0 & 0.00175 \\
\hline Initial Tangent Elastic Modulus (MPa) & 0 & 200000 \\
\hline Poisson's Ratio & 0 & 0 \\
\hline \multicolumn{3}{|c|}{ Masonry Properties (X-Direction) } \\
\hline Gross Peak Compression Strength (MPa) & 4.47 & 350 \\
\hline Peak Compressive Strain & 0.0025 & 0.00175 \\
\hline Tensile Strength & 0 & 350 \\
\hline Tensile Strain & 0 & 0.00175 \\
\hline Initial Tangent Elastic Modulus (MPa) & 0 & 200000 \\
\hline Poisson's Ratio & 0 & 0 \\
\hline \multicolumn{3}{|c|}{ Masonry Properties (Block) } \\
\hline Length (mm) & 397 & 1800 \\
\hline Height (mm) & 194 & 400 \\
\hline Face Shell Thickness (mm) & 33 & 100 \\
\hline Percent Solid (\%) & 50 & 100 \\
\hline Tensile Strength & 1.39 & 350 \\
\hline \multicolumn{3}{|l|}{ Head Joint } \\
\hline Thickness (mm) & 10 & 10 \\
\hline Tensile Bond Strength (MPa) & 0.13 & 350 \\
\hline Joint Cohesion (MPa) & 1.50 & 350 \\
\hline Angle of Internal Friction (Deg) & 42 & 42 \\
\hline \multicolumn{3}{|l|}{ Bed Joint } \\
\hline Thickness (mm) & 10 & 10 \\
\hline Tensile Bond Strength (MPa) & 0.13 & 350 \\
\hline Joint Cohesion (MPa) & 1.50 & 350 \\
\hline Angle of Internal Friction (Deg) & 42 & 42 \\
\hline \multicolumn{3}{|c|}{ Reinforcement (Y-Direction) } \\
\hline Ratio $(\%)$ & 0 & 100 \\
\hline Yield Strength (MPa) & 0 & 350 \\
\hline Yield Strain & 0 & 0.00175 \\
\hline Strain Hardening Strain & 0 & 0.02 \\
\hline Ultimate Strength (MPa) & 0 & 600 \\
\hline Ultimate Strain & 0 & 0.2 \\
\hline Prestrain & 0 & 0 \\
\hline \multicolumn{3}{|c|}{ Reinforcement (X-Direction) } \\
\hline Ratio (\%) & 0 & 100 \\
\hline Yield Strength (MPa) & 0 & 350 \\
\hline Yield Strain & 0 & 0.00175 \\
\hline Strain Hardening Strain & 0 & 0.02 \\
\hline Ultimate Strength (MPa) & 0 & 600 \\
\hline Ultimate Strain & 0 & 0.2 \\
\hline Prestrain & 0 & 0 \\
\hline
\end{tabular}

Numerical Modelling of Hollow Concrete Block Masonry Mechanics 


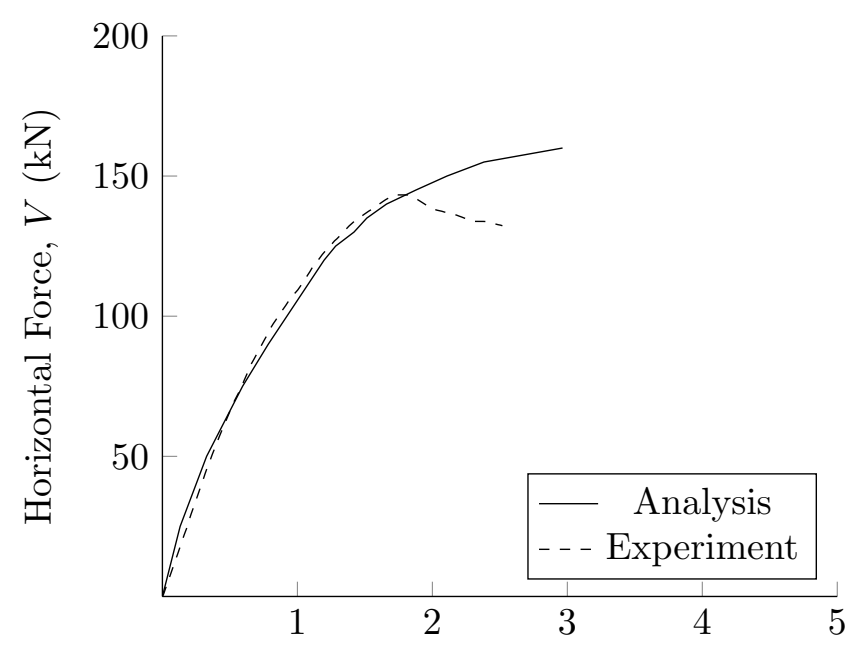

Tip Displacement, $\delta(\mathrm{mm})$

64LH170-6L02 Shear Wall Woodward and Rankin (1985) Horizontal Force vs Tip Displacement
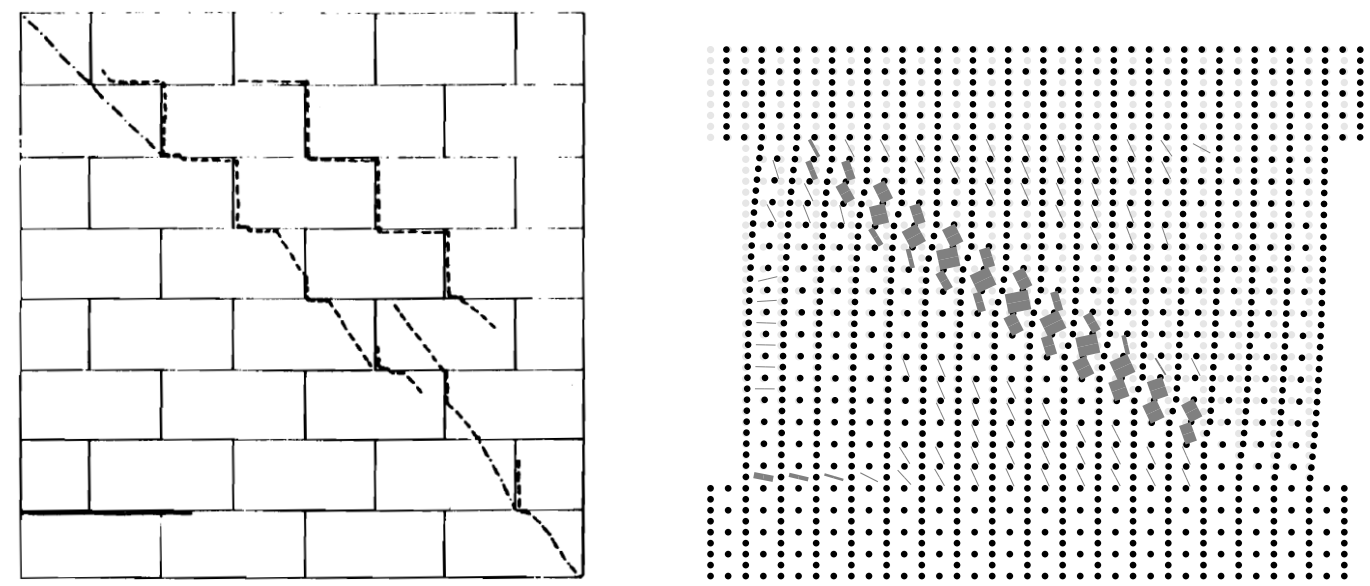

Experiment at Peak Horizontal Force (Wood- Finite Element Model at Peak Horizontal Force ward and Rankin, 1985) $\times 15$

64LH170-6L02 Shear Wall Displacement, Crack Pattern, and Crack Widths 


\section{LH250-6L03 Shear Wall}

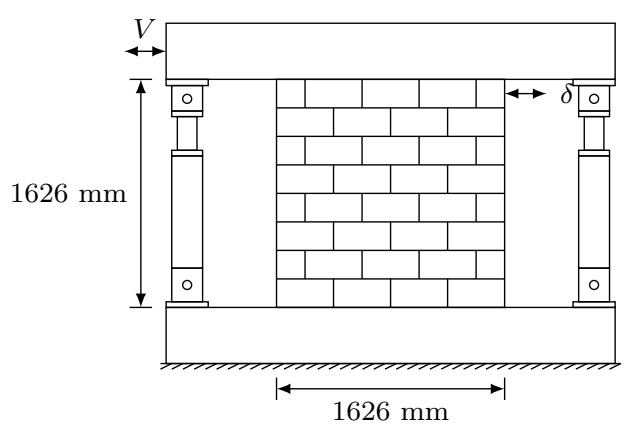

Experimental Wall Woodward and Rankin (1985)

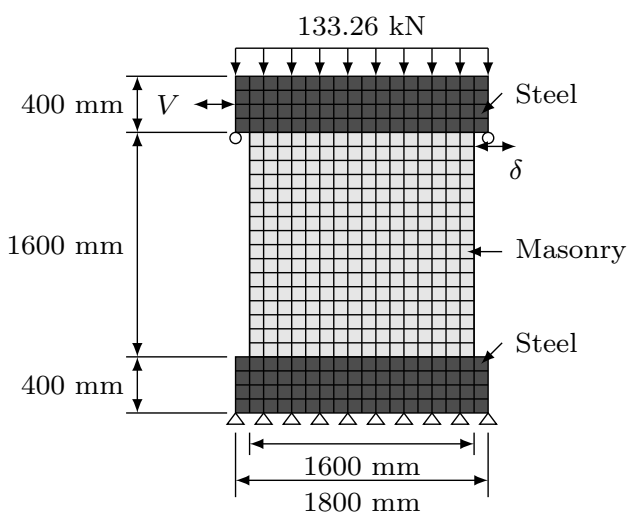

Finite Element Model Wall

64LH250-6L03 Shear Wall

64LH250-6L03 Shear Wall Woodward and Rankin (1985) Model Options

\begin{tabular}{ll}
\hline Model & Selected Option \\
\hline Compression Pre-Peak & Hognestad \\
Compression Post-Peak & Pre-Peak Base Curve \\
Compression Softening & Vecchio \\
Tension Post-Peak & Hordijk \\
Cracking Criterion & Mohr-Coulomb \\
Slip & Constant Rotation Lag \\
\hline
\end{tabular}

Numerical Modelling of Hollow Concrete Block Masonry Mechanics 
64LH250-6L03 Shear Wall Woodward and Rankin (1985) Material Properties

\begin{tabular}{|c|c|c|}
\hline \multirow{2}{*}{ Property } & \multicolumn{2}{|c|}{ Material } \\
\hline & Masonry & Steel \\
\hline Thickness (mm) & 194 & 200 \\
\hline Grout Tensile Strength (MPa) & 0 & 350 \\
\hline \multicolumn{3}{|c|}{ Masonry Properties (Y-Direction) } \\
\hline Gross Peak Compression Strength (MPa) & 5.24 & 350 \\
\hline Peak Compressive Strain & 0.002 & 0.00175 \\
\hline Tensile Strength & 0 & 350 \\
\hline Tensile Strain & 0 & 0.00175 \\
\hline Initial Tangent Elastic Modulus (MPa) & 0 & 200000 \\
\hline Poisson's Ratio & 0 & 0 \\
\hline \multicolumn{3}{|c|}{ Masonry Properties (X-Direction) } \\
\hline Gross Peak Compression Strength (MPa) & 4.34 & 350 \\
\hline Peak Compressive Strain & 0.002 & 0.00175 \\
\hline Tensile Strength & 0 & 350 \\
\hline Tensile Strain & 0 & 0.00175 \\
\hline Initial Tangent Elastic Modulus (MPa) & 0 & 200000 \\
\hline Poisson's Ratio & 0 & 0 \\
\hline \multicolumn{3}{|l|}{ Masonry Properties (Block) } \\
\hline Length $(\mathrm{mm})$ & 397 & 1800 \\
\hline Height (mm) & 194 & 400 \\
\hline Face Shell Thickness (mm) & 33 & 100 \\
\hline Percent Solid (\%) & 50 & 100 \\
\hline Tensile Strength & 1.39 & 350 \\
\hline \multicolumn{3}{|l|}{ Head Joint } \\
\hline Thickness (mm) & 10 & 10 \\
\hline Tensile Bond Strength (MPa) & 0.15 & 350 \\
\hline Joint Cohesion (MPa) & 2.00 & 350 \\
\hline Angle of Internal Friction (Deg) & 42 & 42 \\
\hline \multicolumn{3}{|l|}{ Bed Joint } \\
\hline Thickness (mm) & 10 & 10 \\
\hline Tensile Bond Strength (MPa) & 0.15 & 350 \\
\hline Joint Cohesion $(\mathrm{MPa})$ & 2.00 & 350 \\
\hline Angle of Internal Friction (Deg) & 42 & 42 \\
\hline \multicolumn{3}{|l|}{ Reinforcement (Y-Direction) } \\
\hline Ratio (\%) & 0 & 100 \\
\hline Yield Strength (MPa) & 0 & 350 \\
\hline Yield Strain & 0 & 0.00175 \\
\hline Strain Hardening Strain & 0 & 0.02 \\
\hline Ultimate Strength (MPa) & 0 & 600 \\
\hline Ultimate Strain & 0 & 0.2 \\
\hline Prestrain & 0 & 0 \\
\hline \multicolumn{3}{|c|}{ Reinforcement (X-Direction) } \\
\hline Ratio (\%) & 0 & 100 \\
\hline Yield Strength (MPa) & 0 & 350 \\
\hline Yield Strain & 0 & 0.00175 \\
\hline Strain Hardening Strain & 0 & 0.02 \\
\hline Ultimate Strength (MPa) & 0 & 600 \\
\hline Ultimate Strain & 0 & 0.2 \\
\hline Prestrain & 0 & 0 \\
\hline
\end{tabular}

Numerical Modelling of Hollow Concrete Block Masonry Mechanics 


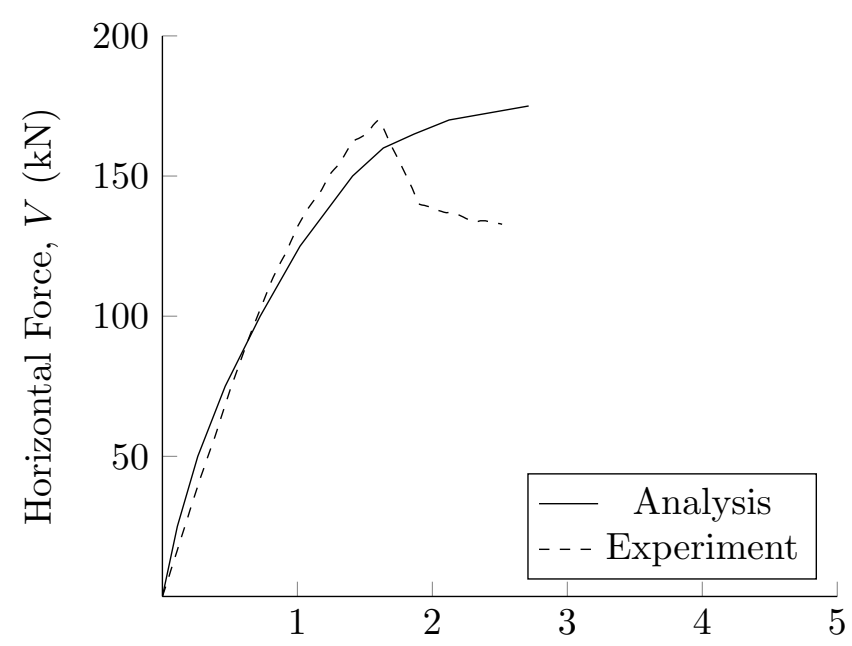

Tip Displacement, $\delta(\mathrm{mm})$

64LH250-6L03 Shear Wall Woodward and Rankin (1985) Horizontal Force vs Tip Displacement
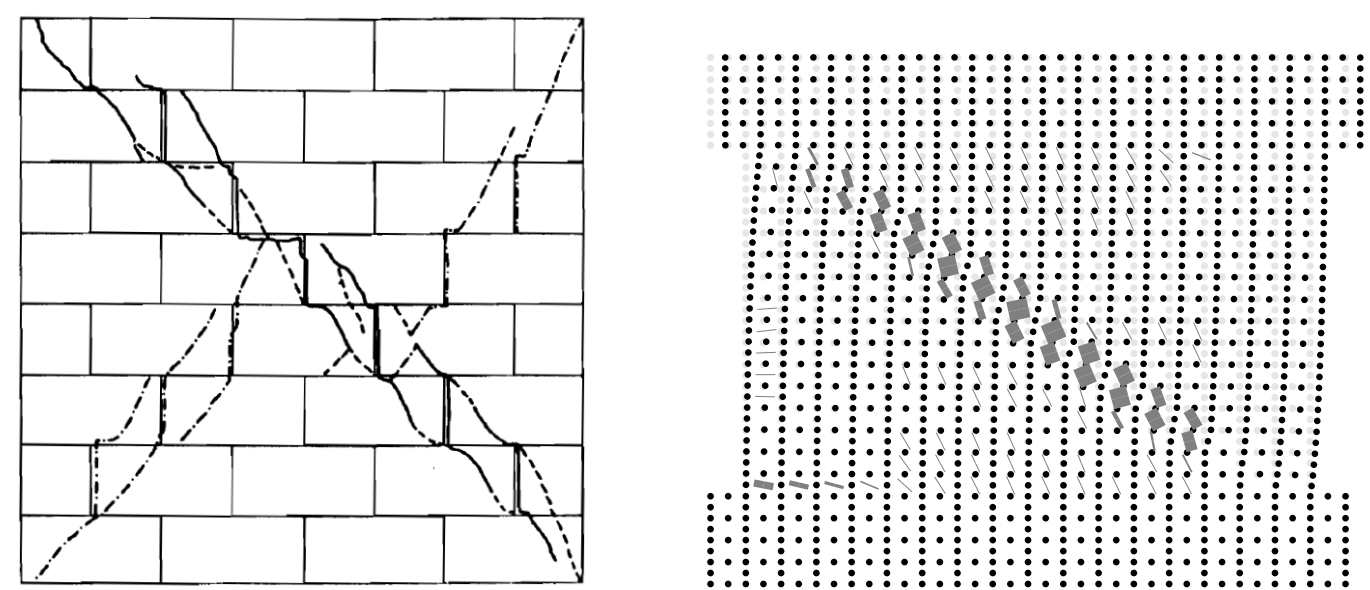

Experiment at Peak Horizontal Force (Wood- Finite Element Model at Peak Horizontal Force ward and Rankin, 1985) $\times 15$

64LH250-6L03 Shear Wall Displacement, Crack Pattern, and Crack Widths 


\section{LH340-6L04 Shear Wall}

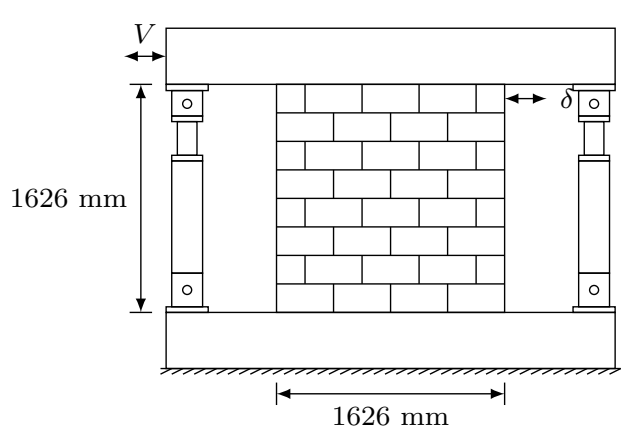

Experimental Wall Woodward and Rankin (1985)

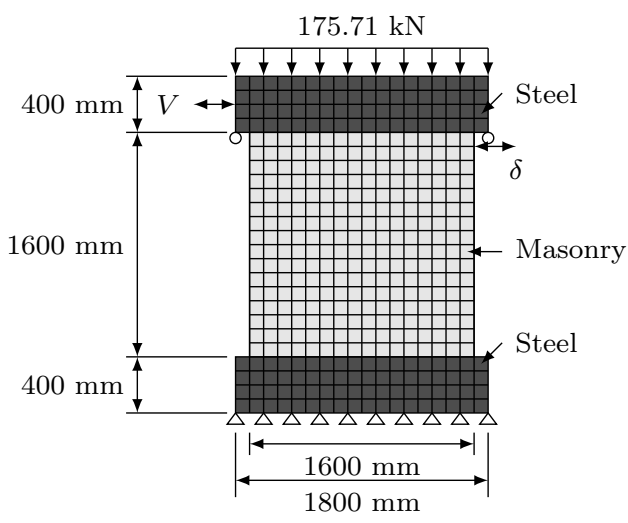

Finite Element Model Wall

64LH340-6L04 Shear Wall

64LH340-6L04 Shear Wall Woodward and Rankin (1985) Model Options

\begin{tabular}{ll}
\hline Model & Selected Option \\
\hline Compression Pre-Peak & Hognestad \\
Compression Post-Peak & Pre-Peak Base Curve \\
Compression Softening & Vecchio \\
Tension Post-Peak & Hordijk \\
Cracking Criterion & Mohr-Coulomb \\
Slip & Constant Rotation Lag \\
\hline
\end{tabular}

Numerical Modelling of Hollow Concrete Block Masonry Mechanics 
64LH340-6L04 Shear Wall Woodward and Rankin (1985) Material Properties

\begin{tabular}{|c|c|c|}
\hline \multirow{2}{*}{ Property } & \multicolumn{2}{|c|}{ Material } \\
\hline & Masonry & Steel \\
\hline Thickness (mm) & 194 & 200 \\
\hline Grout Tensile Strength (MPa) & 0 & 350 \\
\hline \multicolumn{3}{|c|}{ Masonry Properties (Y-Direction) } \\
\hline Gross Peak Compression Strength (MPa) & 7.05 & 350 \\
\hline Peak Compressive Strain & 0.002 & 0.00175 \\
\hline Tensile Strength & 0 & 350 \\
\hline Tensile Strain & 0 & 0.00175 \\
\hline Initial Tangent Elastic Modulus (MPa) & 0 & 200000 \\
\hline Poisson's Ratio & 0 & 0 \\
\hline \multicolumn{3}{|c|}{ Masonry Properties (X-Direction) } \\
\hline Gross Peak Compression Strength (MPa) & 4.67 & 350 \\
\hline Peak Compressive Strain & 0.002 & 0.00175 \\
\hline Tensile Strength & 0 & 350 \\
\hline Tensile Strain & 0 & 0.00175 \\
\hline Initial Tangent Elastic Modulus (MPa) & 0 & 200000 \\
\hline Poisson's Ratio & 0 & 0 \\
\hline \multicolumn{3}{|l|}{ Masonry Properties (Block) } \\
\hline Length $(\mathrm{mm})$ & 397 & 1800 \\
\hline Height (mm) & 194 & 400 \\
\hline Face Shell Thickness (mm) & 33 & 100 \\
\hline Percent Solid (\%) & 50 & 100 \\
\hline Tensile Strength & 1.39 & 350 \\
\hline \multicolumn{3}{|l|}{ Head Joint } \\
\hline Thickness (mm) & 10 & 10 \\
\hline Tensile Bond Strength (MPa) & 0.15 & 350 \\
\hline Joint Cohesion (MPa) & 2.00 & 350 \\
\hline Angle of Internal Friction (Deg) & 42 & 42 \\
\hline \multicolumn{3}{|l|}{ Bed Joint } \\
\hline Thickness (mm) & 10 & 10 \\
\hline Tensile Bond Strength (MPa) & 0.15 & 350 \\
\hline Joint Cohesion $(\mathrm{MPa})$ & 2.00 & 350 \\
\hline Angle of Internal Friction (Deg) & 42 & 42 \\
\hline \multicolumn{3}{|l|}{ Reinforcement (Y-Direction) } \\
\hline Ratio (\%) & 0 & 100 \\
\hline Yield Strength (MPa) & 0 & 350 \\
\hline Yield Strain & 0 & 0.00175 \\
\hline Strain Hardening Strain & 0 & 0.02 \\
\hline Ultimate Strength (MPa) & 0 & 600 \\
\hline Ultimate Strain & 0 & 0.2 \\
\hline Prestrain & 0 & 0 \\
\hline \multicolumn{3}{|c|}{ Reinforcement (X-Direction) } \\
\hline Ratio (\%) & 0 & 100 \\
\hline Yield Strength (MPa) & 0 & 350 \\
\hline Yield Strain & 0 & 0.00175 \\
\hline Strain Hardening Strain & 0 & 0.02 \\
\hline Ultimate Strength (MPa) & 0 & 600 \\
\hline Ultimate Strain & 0 & 0.2 \\
\hline Prestrain & 0 & 0 \\
\hline
\end{tabular}

Numerical Modelling of Hollow Concrete Block Masonry Mechanics 


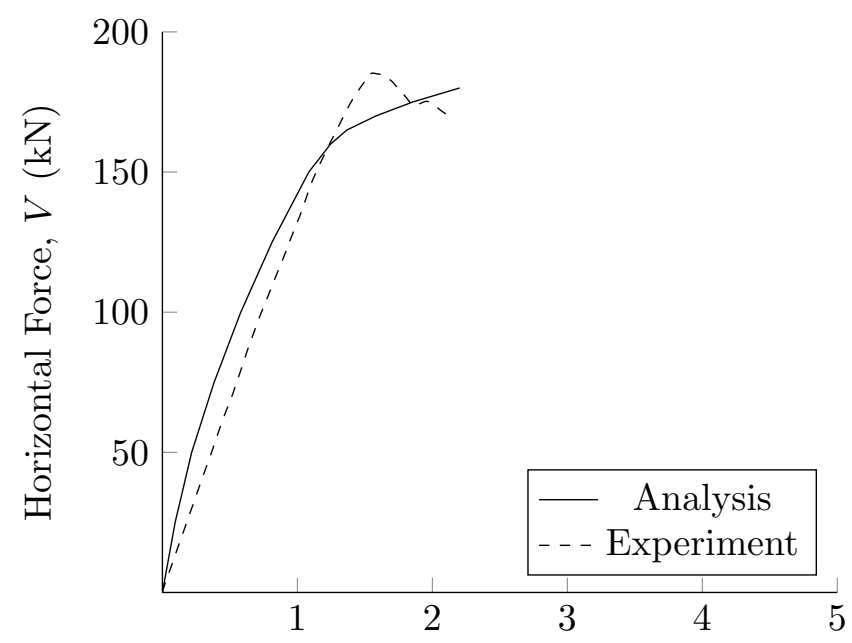

Tip Displacement, $\delta(\mathrm{mm})$

64LH340-6L04 Shear Wall Woodward and Rankin (1985) Horizontal Force vs Tip Displacement
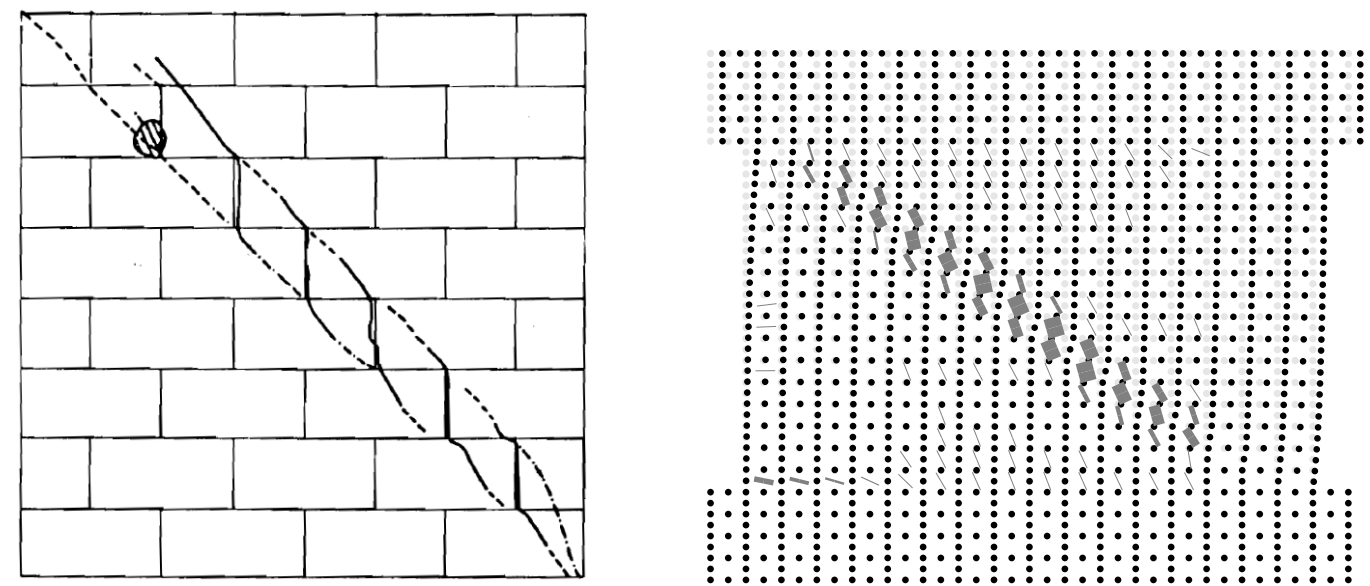

Experiment at Peak Horizontal Force (Wood- Finite Element Model at Peak Horizontal Force ward and Rankin, 1985) $\times 15$

64LH340-6L04 Shear Wall Displacement, Crack Pattern, and Crack Widths 


\section{LH170-6L10 Shear Wall}

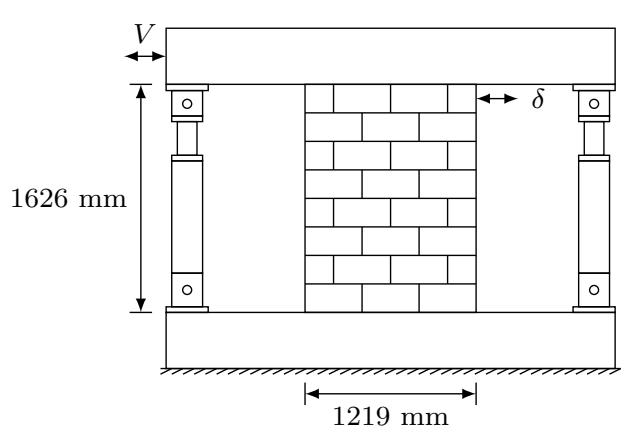

Experimental Wall Woodward and Rankin (1985)

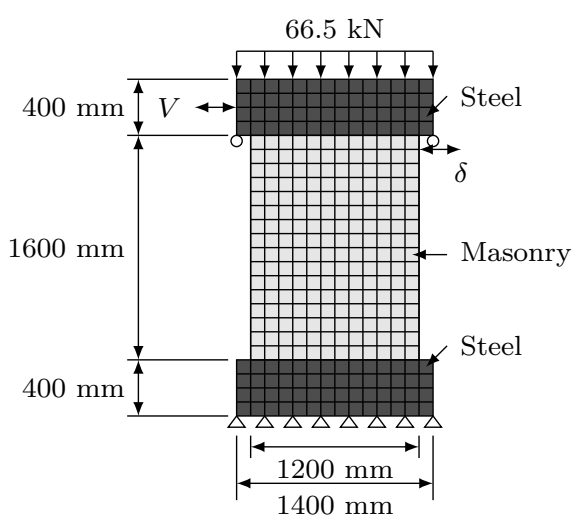

Finite Element Model Wall

48LH170-6L10 Shear Wall

48LH170-6L10 Shear Wall Woodward and Rankin (1985) Model Options

\begin{tabular}{ll}
\hline Model & Selected Option \\
\hline Compression Pre-Peak & Hognestad \\
Compression Post-Peak & Pre-Peak Base Curve \\
Compression Softening & Vecchio \\
Tension Post-Peak & Hordijk \\
Cracking Criterion & Mohr-Coulomb \\
Slip & Constant Rotation Lag \\
\hline
\end{tabular}

Numerical Modelling of Hollow Concrete Block Masonry Mechanics 
48LH170-6L10 Shear Wall Woodward and Rankin (1985) Material Properties

\begin{tabular}{|c|c|c|}
\hline \multirow{2}{*}{ Property } & \multicolumn{2}{|c|}{ Material } \\
\hline & Masonry & Steel \\
\hline Thickness (mm) & 194 & 200 \\
\hline Grout Tensile Strength (MPa) & 0 & 350 \\
\hline \multicolumn{3}{|c|}{ Masonry Properties (Y-Direction) } \\
\hline Gross Peak Compression Strength (MPa) & 6.21 & 350 \\
\hline Peak Compressive Strain & 0.002 & 0.00175 \\
\hline Tensile Strength & 0 & 350 \\
\hline Tensile Strain & 0 & 0.00175 \\
\hline Initial Tangent Elastic Modulus (MPa) & 0 & 200000 \\
\hline Poisson's Ratio & 0 & 0 \\
\hline \multicolumn{3}{|c|}{ Masonry Properties (X-Direction) } \\
\hline Gross Peak Compression Strength (MPa) & 4.47 & 350 \\
\hline Peak Compressive Strain & 0.002 & 0.00175 \\
\hline Tensile Strength & 0 & 350 \\
\hline Tensile Strain & 0 & 0.00175 \\
\hline Initial Tangent Elastic Modulus (MPa) & 0 & 200000 \\
\hline Poisson's Ratio & 0 & 0 \\
\hline \multicolumn{3}{|l|}{ Masonry Properties (Block) } \\
\hline Length $(\mathrm{mm})$ & 397 & 1800 \\
\hline Height (mm) & 194 & 400 \\
\hline Face Shell Thickness (mm) & 33 & 100 \\
\hline Percent Solid (\%) & 50 & 100 \\
\hline Tensile Strength & 1.39 & 350 \\
\hline \multicolumn{3}{|l|}{ Head Joint } \\
\hline Thickness (mm) & 10 & 10 \\
\hline Tensile Bond Strength (MPa) & 0.12 & 350 \\
\hline Joint Cohesion (MPa) & 2.00 & 350 \\
\hline Angle of Internal Friction (Deg) & 42 & 42 \\
\hline \multicolumn{3}{|l|}{ Bed Joint } \\
\hline Thickness (mm) & 10 & 10 \\
\hline Tensile Bond Strength (MPa) & 0.12 & 350 \\
\hline Joint Cohesion $(\mathrm{MPa})$ & 2.00 & 350 \\
\hline Angle of Internal Friction (Deg) & 42 & 42 \\
\hline \multicolumn{3}{|l|}{ Reinforcement (Y-Direction) } \\
\hline Ratio (\%) & 0 & 100 \\
\hline Yield Strength (MPa) & 0 & 350 \\
\hline Yield Strain & 0 & 0.00175 \\
\hline Strain Hardening Strain & 0 & 0.02 \\
\hline Ultimate Strength (MPa) & 0 & 600 \\
\hline Ultimate Strain & 0 & 0.2 \\
\hline Prestrain & 0 & 0 \\
\hline \multicolumn{3}{|c|}{ Reinforcement (X-Direction) } \\
\hline Ratio (\%) & 0 & 100 \\
\hline Yield Strength (MPa) & 0 & 350 \\
\hline Yield Strain & 0 & 0.00175 \\
\hline Strain Hardening Strain & 0 & 0.02 \\
\hline Ultimate Strength (MPa) & 0 & 600 \\
\hline Ultimate Strain & 0 & 0.2 \\
\hline Prestrain & 0 & 0 \\
\hline
\end{tabular}

Numerical Modelling of Hollow Concrete Block Masonry Mechanics 


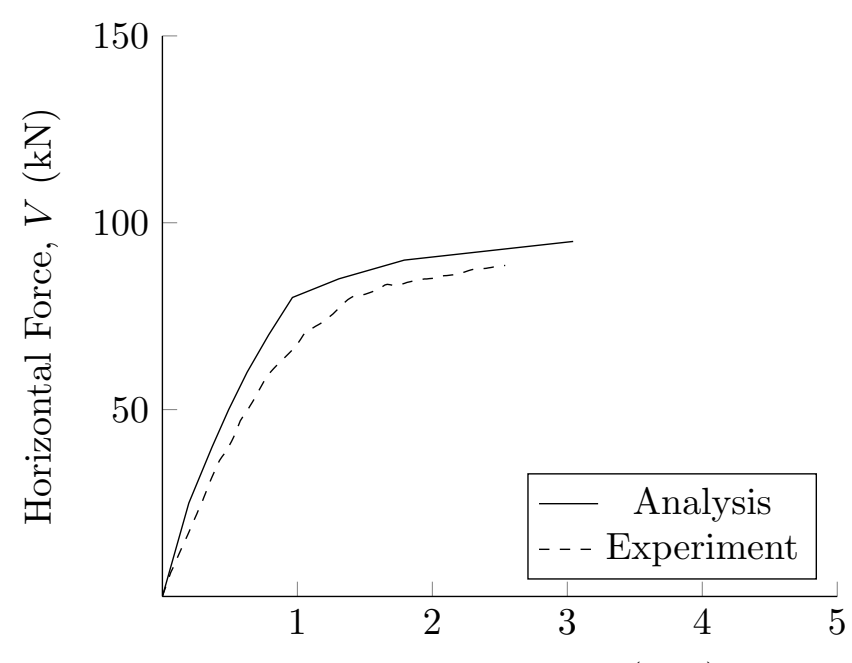

Tip Displacement, $\delta(\mathrm{mm})$

48LH170-6L10 Shear Wall Woodward and Rankin (1985) Horizontal Force vs Tip Displacement
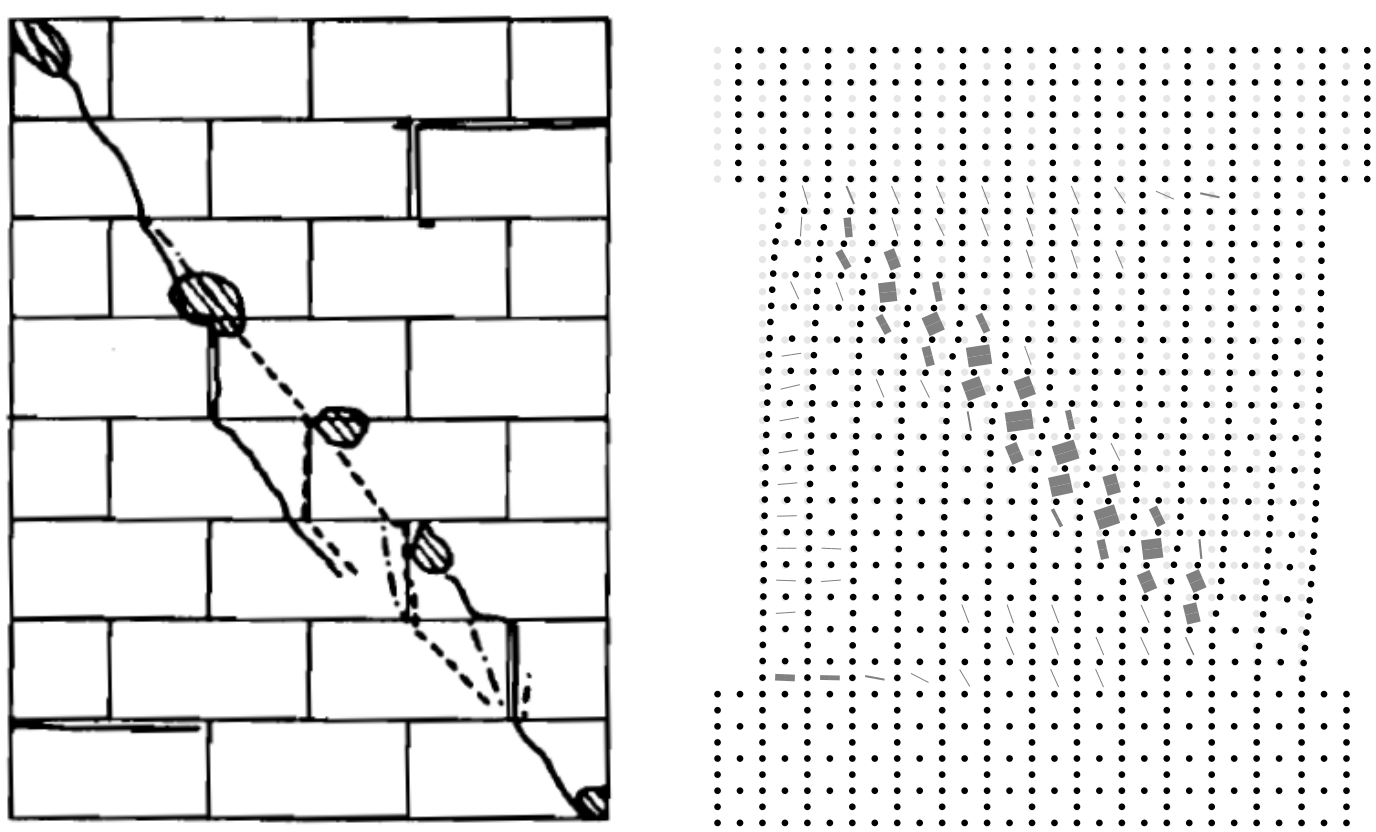

Experiment at Peak Horizontal Force (Wood- Finite Element Model at Peak Horizontal Force ward and Rankin, 1985) $\times 15$

48LH170-6L10 Shear Wall Displacement, Crack Pattern, and Crack Widths 


\section{LH450-6L11 Shear Wall}

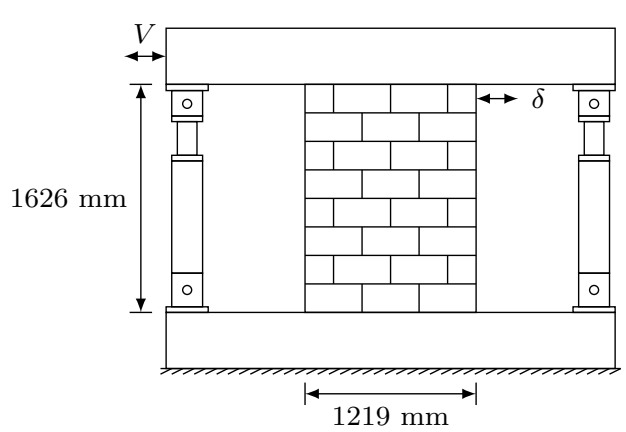

Experimental Wall Woodward and Rankin (1985)

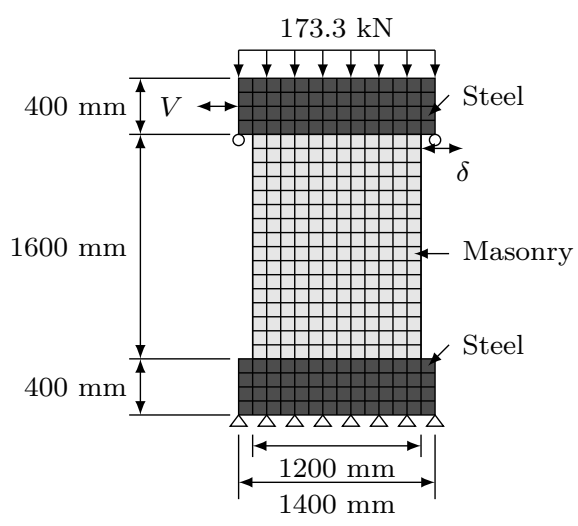

Finite Element Model Wall

48LH450-6L11 Shear Wall

48LH450-6L11 Shear Wall Woodward and Rankin (1985) Model Options

\begin{tabular}{ll}
\hline Model & Selected Option \\
\hline Compression Pre-Peak & Hognestad \\
Compression Post-Peak & Pre-Peak Base Curve \\
Compression Softening & Vecchio \\
Tension Post-Peak & Hordijk \\
Cracking Criterion & Mohr-Coulomb \\
Slip & Constant Rotation Lag \\
\hline
\end{tabular}

Numerical Modelling of Hollow Concrete Block Masonry Mechanics 
48LH450-6L11 Shear Wall Woodward and Rankin (1985) Material Properties

\begin{tabular}{|c|c|c|}
\hline \multirow{2}{*}{ Property } & \multicolumn{2}{|c|}{ Material } \\
\hline & Masonry & Steel \\
\hline Thickness (mm) & 194 & 200 \\
\hline Grout Tensile Strength (MPa) & 0 & 350 \\
\hline \multicolumn{3}{|c|}{ Masonry Properties (Y-Direction) } \\
\hline Gross Peak Compression Strength (MPa) & 6.79 & 350 \\
\hline Peak Compressive Strain & 0.0017 & 0.00175 \\
\hline Tensile Strength & 0 & 350 \\
\hline Tensile Strain & 0 & 0.00175 \\
\hline Initial Tangent Elastic Modulus (MPa) & 0 & 200000 \\
\hline Poisson's Ratio & 0 & 0 \\
\hline \multicolumn{3}{|c|}{ Masonry Properties (X-Direction) } \\
\hline Gross Peak Compression Strength (MPa) & 4.49 & 350 \\
\hline Peak Compressive Strain & 0.0017 & 0.00175 \\
\hline Tensile Strength & 0 & 350 \\
\hline Tensile Strain & 0 & 0.00175 \\
\hline Initial Tangent Elastic Modulus (MPa) & 0 & 200000 \\
\hline Poisson's Ratio & 0 & 0 \\
\hline \multicolumn{3}{|l|}{ Masonry Properties (Block) } \\
\hline Length $(\mathrm{mm})$ & 397 & 1800 \\
\hline Height (mm) & 194 & 400 \\
\hline Face Shell Thickness (mm) & 33 & 100 \\
\hline Percent Solid (\%) & 50 & 100 \\
\hline Tensile Strength & 1.39 & 350 \\
\hline \multicolumn{3}{|l|}{ Head Joint } \\
\hline Thickness (mm) & 10 & 10 \\
\hline Tensile Bond Strength (MPa) & 0.20 & 350 \\
\hline Joint Cohesion (MPa) & 2.00 & 350 \\
\hline Angle of Internal Friction (Deg) & 42 & 42 \\
\hline \multicolumn{3}{|l|}{ Bed Joint } \\
\hline Thickness (mm) & 10 & 10 \\
\hline Tensile Bond Strength (MPa) & 0.20 & 350 \\
\hline Joint Cohesion $(\mathrm{MPa})$ & 2.00 & 350 \\
\hline Angle of Internal Friction (Deg) & 42 & 42 \\
\hline \multicolumn{3}{|l|}{ Reinforcement (Y-Direction) } \\
\hline Ratio (\%) & 0 & 100 \\
\hline Yield Strength (MPa) & 0 & 350 \\
\hline Yield Strain & 0 & 0.00175 \\
\hline Strain Hardening Strain & 0 & 0.02 \\
\hline Ultimate Strength (MPa) & 0 & 600 \\
\hline Ultimate Strain & 0 & 0.2 \\
\hline Prestrain & 0 & 0 \\
\hline \multicolumn{3}{|c|}{ Reinforcement (X-Direction) } \\
\hline Ratio (\%) & 0 & 100 \\
\hline Yield Strength (MPa) & 0 & 350 \\
\hline Yield Strain & 0 & 0.00175 \\
\hline Strain Hardening Strain & 0 & 0.02 \\
\hline Ultimate Strength (MPa) & 0 & 600 \\
\hline Ultimate Strain & 0 & 0.2 \\
\hline Prestrain & 0 & 0 \\
\hline
\end{tabular}

Numerical Modelling of Hollow Concrete Block Masonry Mechanics 


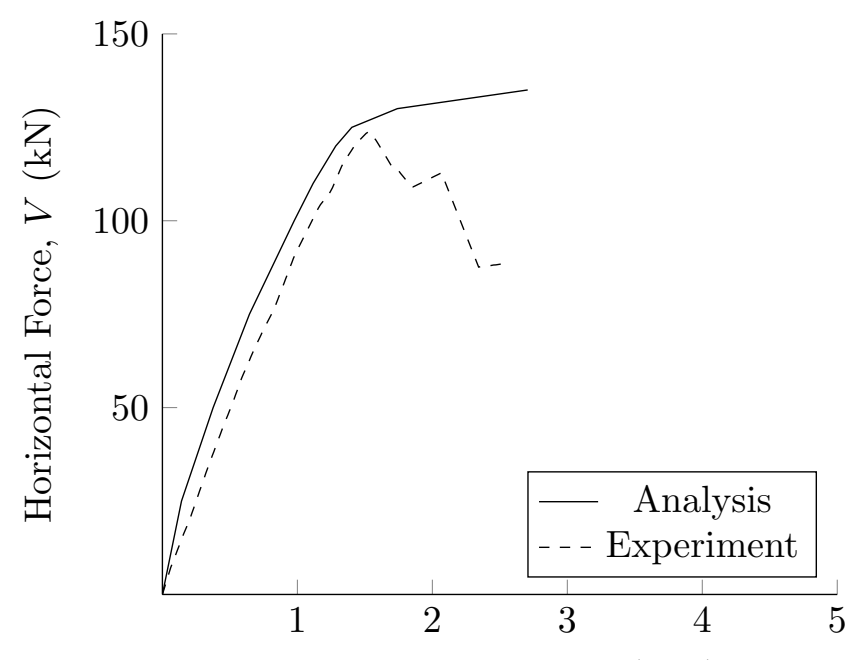

Tip Displacement, $\delta(\mathrm{mm})$

48LH450-6L11 Shear Wall Woodward and Rankin (1985) Horizontal Force vs Tip Displacement
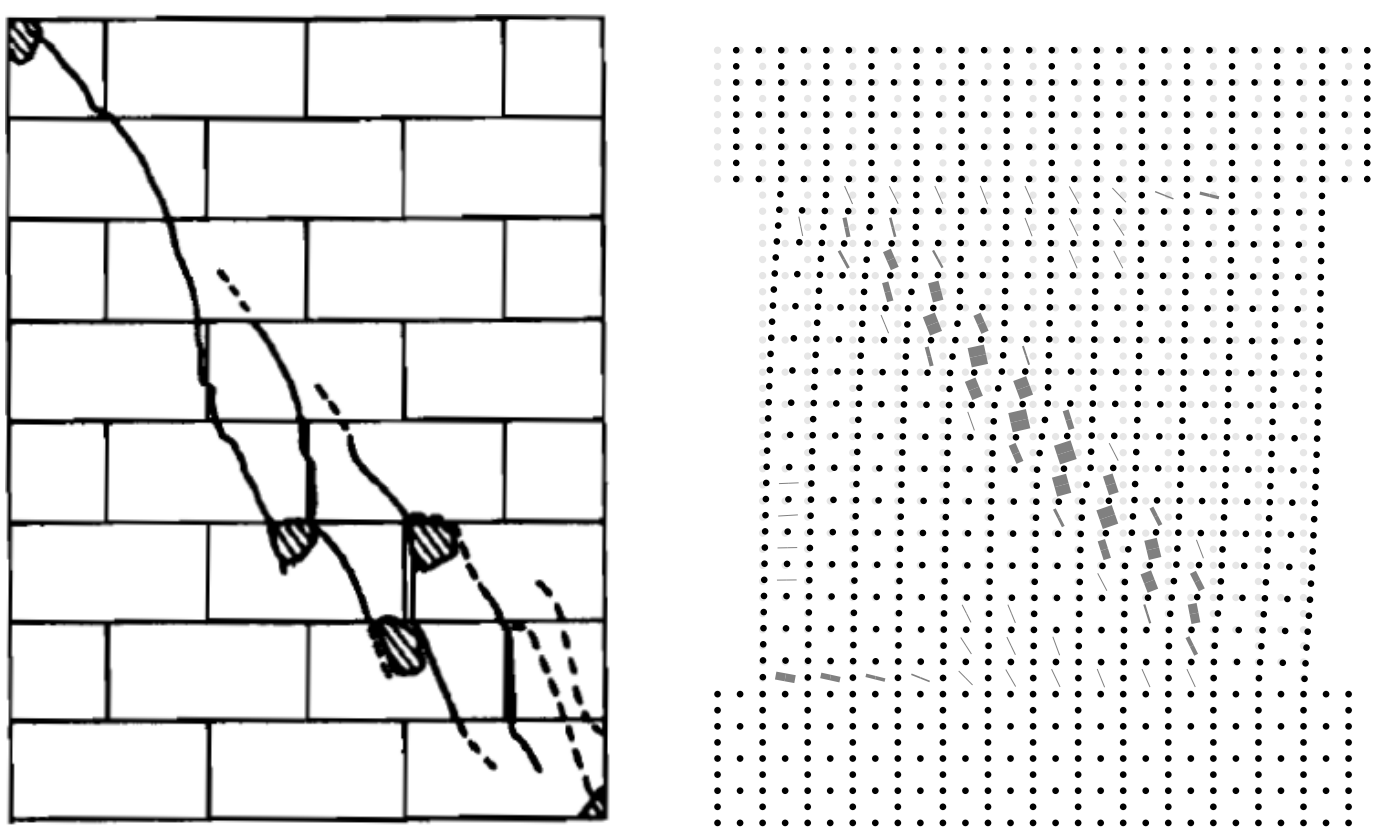

Experiment at Peak Horizontal Force (Wood- Finite Element Model at Peak Horizontal Force ward and Rankin, 1985) $\times 15$

48LH450-6L11 Shear Wall Displacement, Crack Pattern, and Crack Widths 


\section{LH220-6L12 Shear Wall}

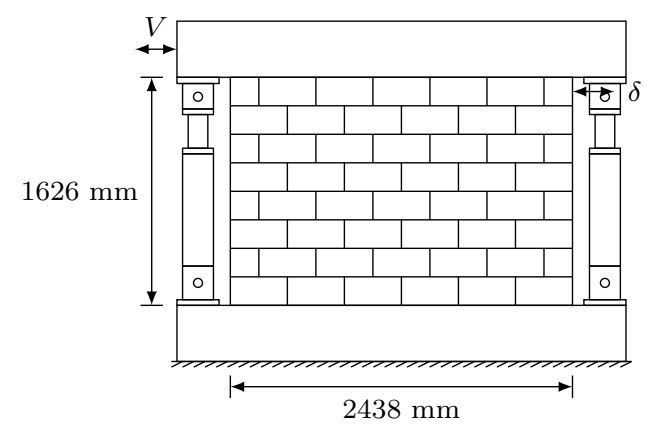

Experimental Wall Woodward and Rankin (1985)

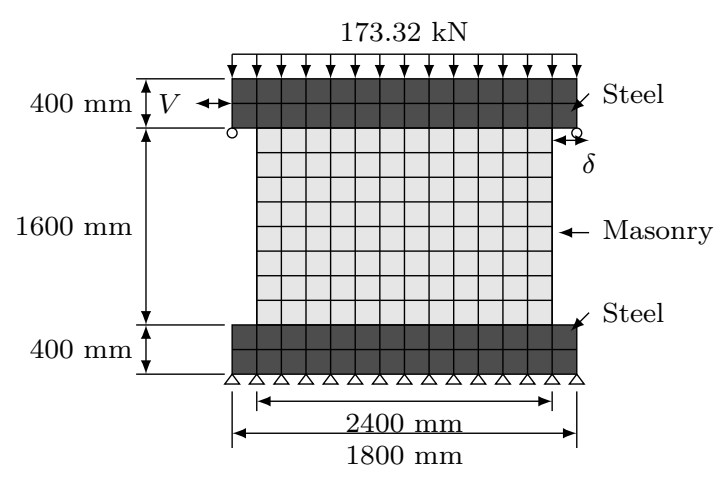

Finite Element Model Wall

96LH220-6L12 Shear Wall

96LH220-6L12 Shear Wall Woodward and Rankin (1985) Model Options

\begin{tabular}{ll}
\hline Model & Selected Option \\
\hline Compression Pre-Peak & Hognestad \\
Compression Post-Peak & Pre-Peak Base Curve \\
Compression Softening & Vecchio \\
Tension Post-Peak & Hordijk \\
Cracking Criterion & Mohr-Coulomb \\
Slip & Constant Rotation Lag \\
\hline
\end{tabular}

Numerical Modelling of Hollow Concrete Block Masonry Mechanics 
96LH220-6L12 Shear Wall Woodward and Rankin (1985) Material Properties

\begin{tabular}{|c|c|c|}
\hline \multirow{2}{*}{ Property } & \multicolumn{2}{|c|}{ Material } \\
\hline & Masonry & Steel \\
\hline Thickness (mm) & 194 & 200 \\
\hline Grout Tensile Strength (MPa) & 0 & 350 \\
\hline \multicolumn{3}{|c|}{ Masonry Properties (Y-Direction) } \\
\hline Gross Peak Compression Strength (MPa) & 5.62 & 350 \\
\hline Peak Compressive Strain & 0.002 & 0.00175 \\
\hline Tensile Strength & 0 & 350 \\
\hline Tensile Strain & 0 & 0.00175 \\
\hline Initial Tangent Elastic Modulus (MPa) & 0 & 200000 \\
\hline Poisson's Ratio & 0 & 0 \\
\hline \multicolumn{3}{|c|}{ Masonry Properties (X-Direction) } \\
\hline Gross Peak Compression Strength (MPa) & 4.41 & 350 \\
\hline Peak Compressive Strain & 0.002 & 0.00175 \\
\hline Tensile Strength & 0 & 350 \\
\hline Tensile Strain & 0 & 0.00175 \\
\hline Initial Tangent Elastic Modulus (MPa) & 0 & 200000 \\
\hline Poisson's Ratio & 0 & 0 \\
\hline \multicolumn{3}{|c|}{ Masonry Properties (Block) } \\
\hline Length (mm) & 397 & 1800 \\
\hline Height (mm) & 194 & 400 \\
\hline Face Shell Thickness (mm) & 33 & 100 \\
\hline Percent Solid (\%) & 50 & 100 \\
\hline Tensile Strength & 1.39 & 350 \\
\hline \multicolumn{3}{|l|}{ Head Joint } \\
\hline Thickness (mm) & 10 & 10 \\
\hline Tensile Bond Strength (MPa) & 0.05 & 350 \\
\hline Joint Cohesion (MPa) & 1.00 & 350 \\
\hline Angle of Internal Friction (Deg) & 42 & 42 \\
\hline \multicolumn{3}{|l|}{ Bed Joint } \\
\hline Thickness (mm) & 10 & 10 \\
\hline Tensile Bond Strength (MPa) & 0.05 & 350 \\
\hline Joint Cohesion (MPa) & 1.00 & 350 \\
\hline Angle of Internal Friction (Deg) & 42 & 42 \\
\hline \multicolumn{3}{|c|}{ Reinforcement (Y-Direction) } \\
\hline Ratio (\%) & 0 & 100 \\
\hline Yield Strength (MPa) & 0 & 350 \\
\hline Yield Strain & 0 & 0.00175 \\
\hline Strain Hardening Strain & 0 & 0.02 \\
\hline Ultimate Strength (MPa) & 0 & 600 \\
\hline Ultimate Strain & 0 & 0.2 \\
\hline Prestrain & 0 & 0 \\
\hline \multicolumn{3}{|c|}{ Reinforcement (X-Direction) } \\
\hline Ratio (\%) & 0 & 100 \\
\hline Yield Strength (MPa) & 0 & 350 \\
\hline Yield Strain & 0 & 0.00175 \\
\hline Strain Hardening Strain & 0 & 0.02 \\
\hline Ultimate Strength (MPa) & 0 & 600 \\
\hline Ultimate Strain & 0 & 0.2 \\
\hline Prestrain & 0 & 0 \\
\hline
\end{tabular}

Numerical Modelling of Hollow Concrete Block Masonry Mechanics 


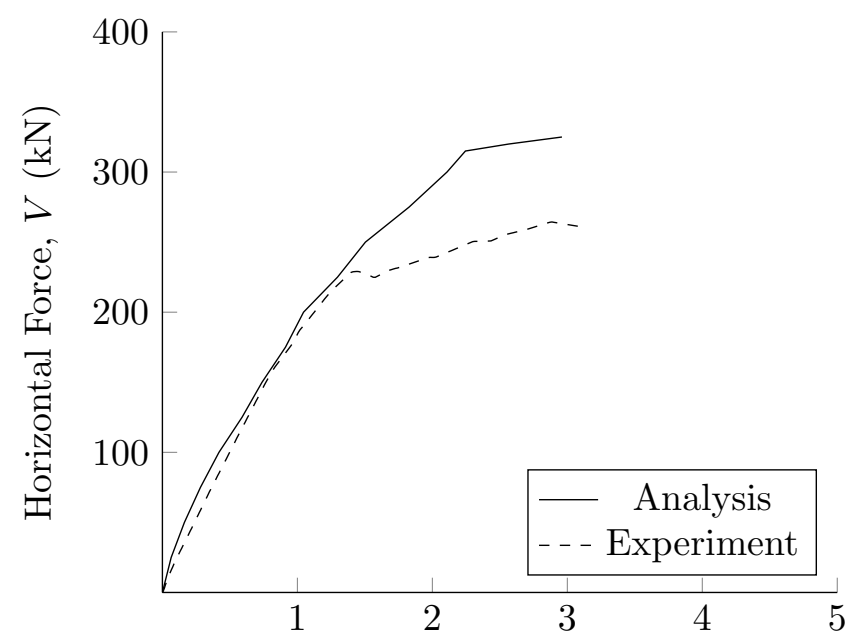

Tip Displacement, $\delta(\mathrm{mm})$

96LH220-6L12 Shear Wall Woodward and Rankin (1985) Horizontal Force vs Tip Displacement
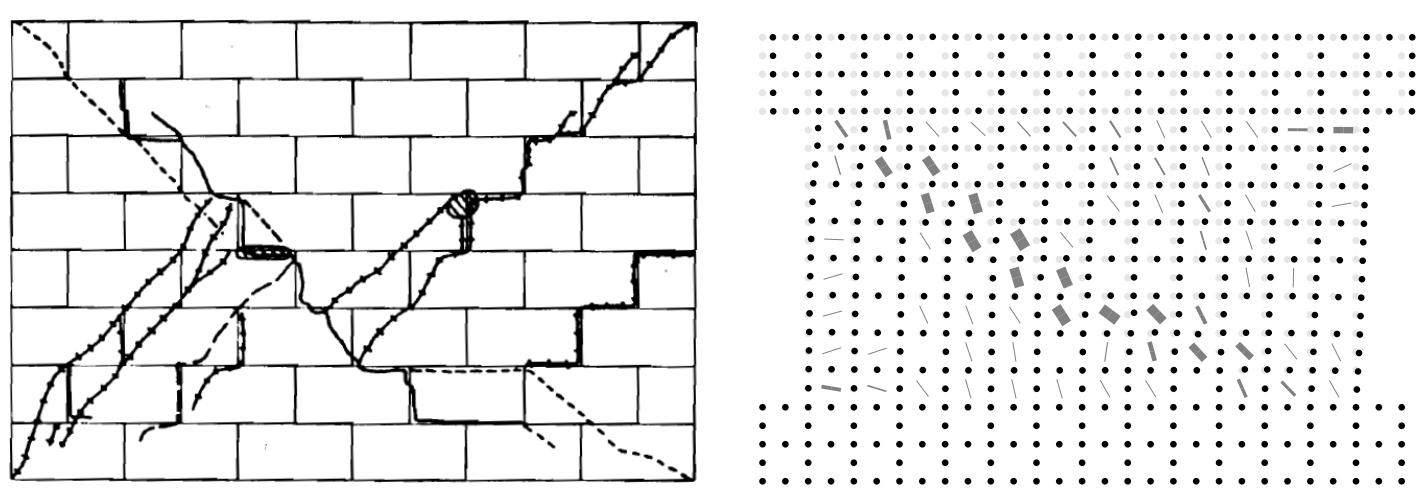

Experiment at Peak Horizontal Force (Wood- Finite Element Model at Peak Horizontal Force ward and Rankin, 1985)

96LH220-6L12 Shear Wall Displacement, Crack Pattern, and Crack Widths 


\section{LH320-6L13 Shear Wall}

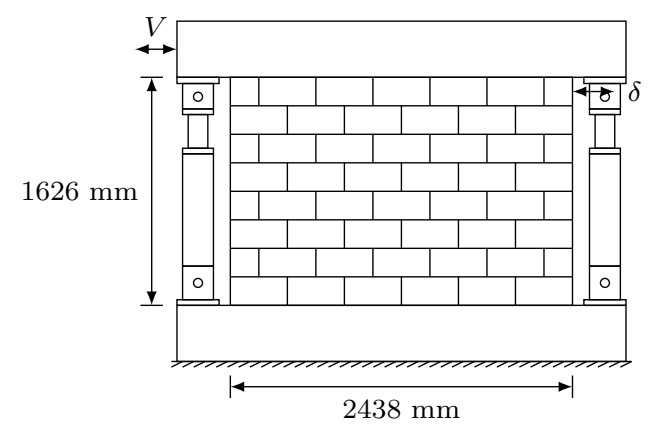

Experimental Wall Woodward and Rankin (1985)

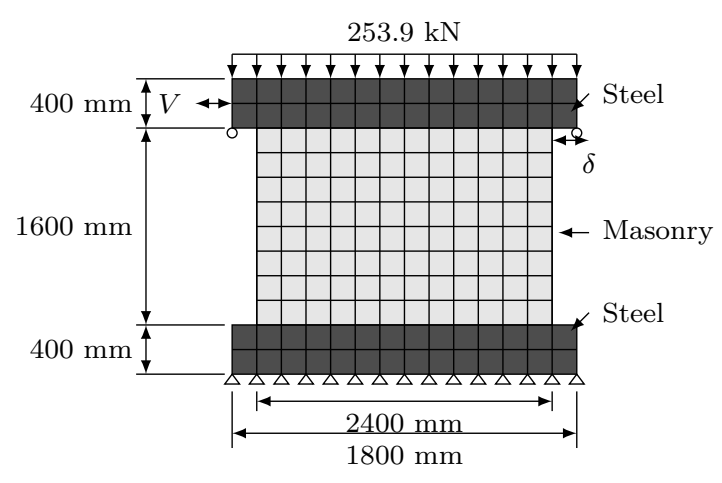

Finite Element Model Wall

96LH320-6L13 Shear Wall

96LH320-6L13 Shear Wall Woodward and Rankin (1985) Model Options

\begin{tabular}{ll}
\hline Model & Selected Option \\
\hline Compression Pre-Peak & Hognestad \\
Compression Post-Peak & Pre-Peak Base Curve \\
Compression Softening & Vecchio \\
Tension Post-Peak & Hordijk \\
Cracking Criterion & Mohr-Coulomb \\
Slip & Constant Rotation Lag \\
\hline
\end{tabular}

Numerical Modelling of Hollow Concrete Block Masonry Mechanics 
96LH320-6L13 Shear Wall Woodward and Rankin (1985) Material Properties

\begin{tabular}{|c|c|c|}
\hline \multirow{2}{*}{ Property } & \multicolumn{2}{|c|}{ Material } \\
\hline & Masonry & Steel \\
\hline Thickness (mm) & 194 & 200 \\
\hline Grout Tensile Strength (MPa) & 0 & 350 \\
\hline \multicolumn{3}{|c|}{ Masonry Properties (Y-Direction) } \\
\hline Gross Peak Compression Strength (MPa) & 5.38 & 350 \\
\hline Peak Compressive Strain & 0.003 & 0.00175 \\
\hline Tensile Strength & 0 & 350 \\
\hline Tensile Strain & 0 & 0.00175 \\
\hline Initial Tangent Elastic Modulus (MPa) & 0 & 200000 \\
\hline Poisson's Ratio & 0 & 0 \\
\hline \multicolumn{3}{|c|}{ Masonry Properties (X-Direction) } \\
\hline Gross Peak Compression Strength (MPa) & 4.37 & 350 \\
\hline Peak Compressive Strain & 0.003 & 0.00175 \\
\hline Tensile Strength & 0 & 350 \\
\hline Tensile Strain & 0 & 0.00175 \\
\hline Initial Tangent Elastic Modulus (MPa) & 0 & 200000 \\
\hline Poisson's Ratio & 0 & 0 \\
\hline \multicolumn{3}{|l|}{ Masonry Properties (Block) } \\
\hline Length $(\mathrm{mm})$ & 397 & 1800 \\
\hline Height (mm) & 194 & 400 \\
\hline Face Shell Thickness (mm) & 33 & 100 \\
\hline Percent Solid (\%) & 50 & 100 \\
\hline Tensile Strength & 1.39 & 350 \\
\hline \multicolumn{3}{|l|}{ Head Joint } \\
\hline Thickness (mm) & 10 & 10 \\
\hline Tensile Bond Strength (MPa) & 0.12 & 350 \\
\hline Joint Cohesion (MPa) & 1.20 & 350 \\
\hline Angle of Internal Friction (Deg) & 42 & 42 \\
\hline \multicolumn{3}{|l|}{ Bed Joint } \\
\hline Thickness (mm) & 10 & 10 \\
\hline Tensile Bond Strength (MPa) & 0.12 & 350 \\
\hline Joint Cohesion $(\mathrm{MPa})$ & 1.20 & 350 \\
\hline Angle of Internal Friction (Deg) & 42 & 42 \\
\hline \multicolumn{3}{|l|}{ Reinforcement (Y-Direction) } \\
\hline Ratio (\%) & 0 & 100 \\
\hline Yield Strength (MPa) & 0 & 350 \\
\hline Yield Strain & 0 & 0.00175 \\
\hline Strain Hardening Strain & 0 & 0.02 \\
\hline Ultimate Strength (MPa) & 0 & 600 \\
\hline Ultimate Strain & 0 & 0.2 \\
\hline Prestrain & 0 & 0 \\
\hline \multicolumn{3}{|c|}{ Reinforcement (X-Direction) } \\
\hline Ratio (\%) & 0 & 100 \\
\hline Yield Strength (MPa) & 0 & 350 \\
\hline Yield Strain & 0 & 0.00175 \\
\hline Strain Hardening Strain & 0 & 0.02 \\
\hline Ultimate Strength (MPa) & 0 & 600 \\
\hline Ultimate Strain & 0 & 0.2 \\
\hline Prestrain & 0 & 0 \\
\hline
\end{tabular}

Numerical Modelling of Hollow Concrete Block Masonry Mechanics 


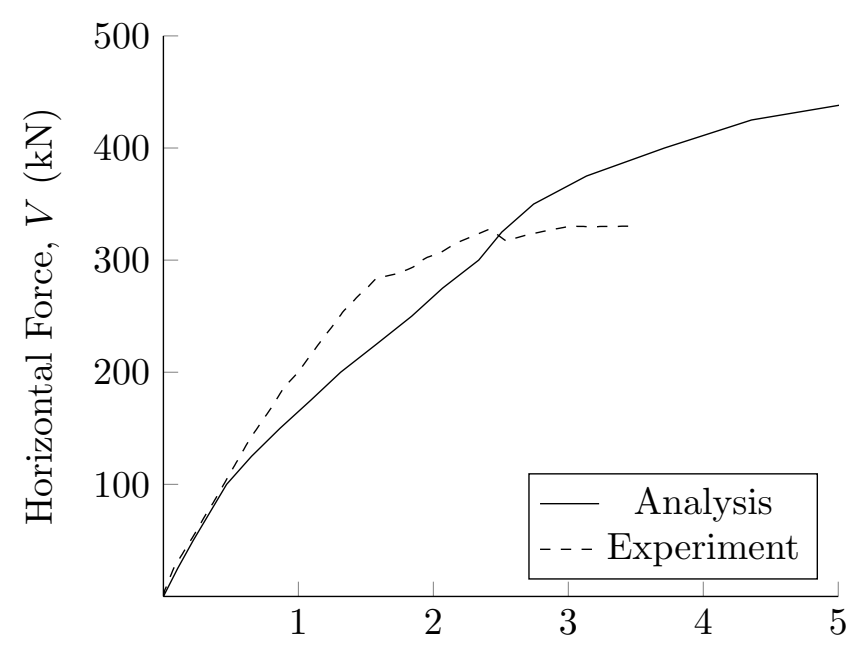

Tip Displacement, $\delta(\mathrm{mm})$

96LH320-6L13 Shear Wall Woodward and Rankin (1985) Horizontal Force vs Tip Displacement
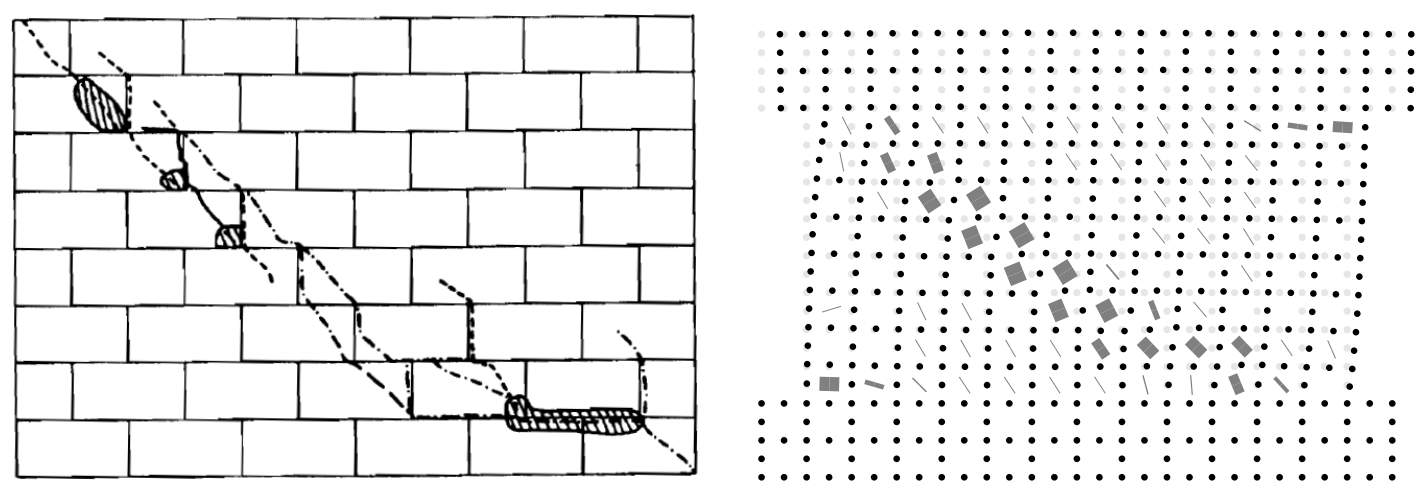

Experiment at Peak Horizontal Force (Wood- Finite Element Model at Peak Horizontal Force ward and Rankin, 1985) $\times 15$

96LH220-6L12 Shear Wall Displacement, Crack Pattern, and Crack Widths 


\section{APPENDIX F}

\section{REINFORCED BEAM SMEARED AREA FINITE ELE- MENT MODEL RESULTS}

Ring (2009)

B4-6(100) Beams

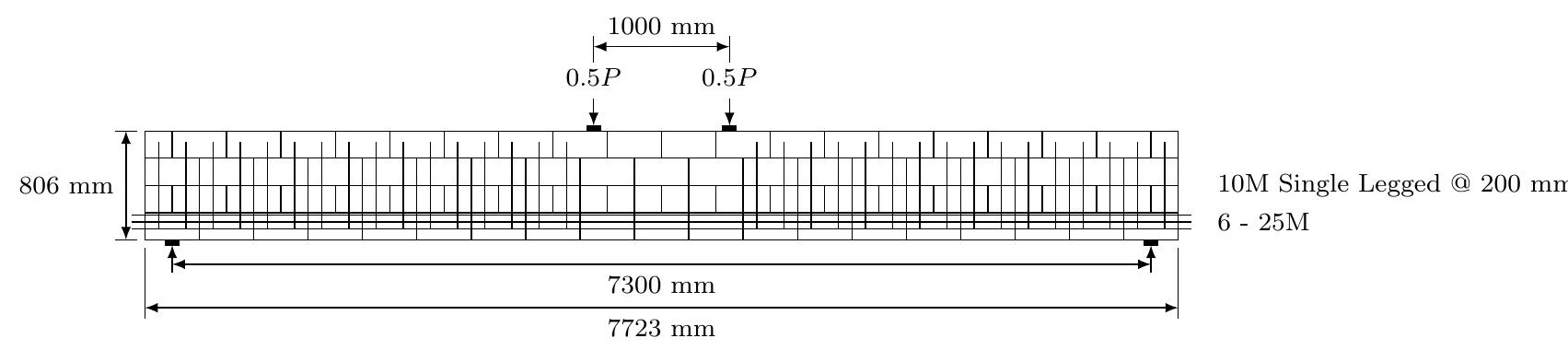

Experimental Beams Ring (2009)

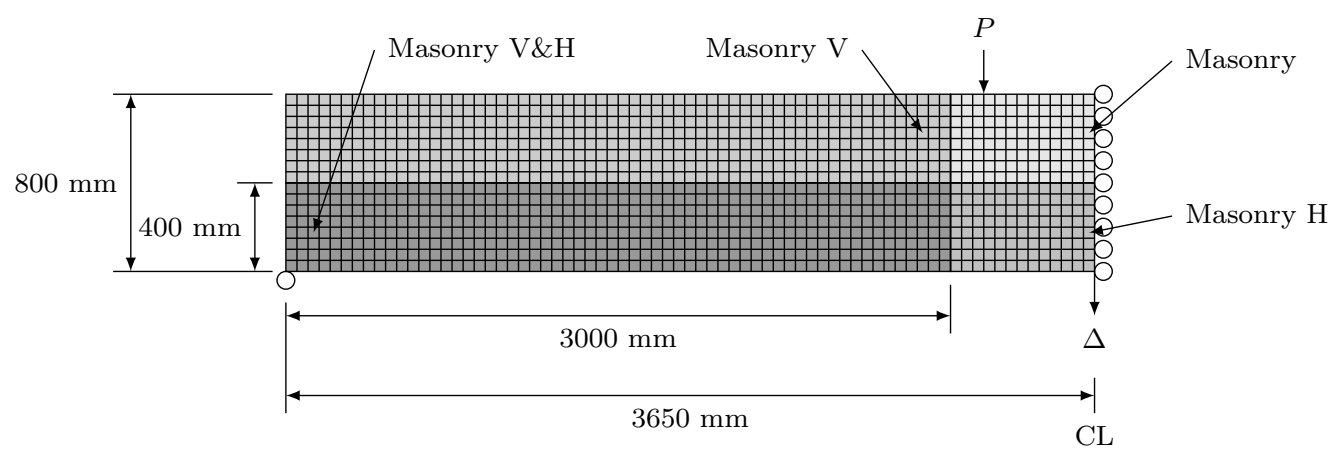

Finite Element Model Beams

B4-6(100) Beams 
B4-6(100) Beams (Ring, 2009) Model Options

\begin{tabular}{ll}
\hline Model & Selected Option \\
\hline Compression Pre-Peak & Hognestad \\
Compression Post-Peak & Pre-Peak Base Curve \\
Compression Softening & Vecchio \\
Tension Post-Peak & Vecchio \\
Cracking Criterion & Mohr-Coulomb \\
Slip & Walraven Reinforced \\
\hline
\end{tabular}

Numerical Modelling of Hollow Concrete Block Masonry Mechanics 
B4-6(100) Beams (Ring, 2009) Material Properties

\begin{tabular}{|c|c|c|c|c|}
\hline \multirow{2}{*}{ Property } & \multicolumn{4}{|c|}{ Material } \\
\hline & Masonry V\&H & Masonry $\mathrm{H}$ & Masonry V & Masonry \\
\hline Thickness (mm) & 194 & 194 & 194 & 194 \\
\hline Grout Tensile Strength (MPa) & 1.77 & 1.77 & 1.77 & 1.77 \\
\hline \multicolumn{5}{|c|}{ Masonry Properties (Y-Direction) } \\
\hline Gross Peak Compression Strength (MPa) & 22.6 & 22.6 & 22.6 & 22.6 \\
\hline Peak Compressive Strain & 0.0025 & 0.0025 & 0.0025 & 0.0025 \\
\hline Tensile Strength & 0 & 0 & 0 & 0 \\
\hline Tensile Strain & 0 & 0 & 0 & 0 \\
\hline Initial Tangent Elastic Modulus (MPa) & 0 & 0 & 0 & 0 \\
\hline Poisson's Ratio & 0 & 0 & 0 & 0 \\
\hline \multicolumn{5}{|c|}{ Masonry Properties (X-Direction) } \\
\hline Gross Peak Compression Strength (MPa) & 22.6 & 22.6 & 22.6 & 22.6 \\
\hline Peak Compressive Strain & 0.0025 & 0.0025 & 0.0025 & 0.0025 \\
\hline Tensile Strength & 0 & 0 & 0 & 0 \\
\hline Tensile Strain & 0 & 0 & 0 & 0 \\
\hline Initial Tangent Elastic Modulus (MPa) & 0 & 0 & 0 & 0 \\
\hline Poisson's Ratio & 0 & 0 & 0 & 0 \\
\hline \multicolumn{5}{|c|}{ Masonry Properties (Block) } \\
\hline Length (mm) & 397 & 397 & 397 & 397 \\
\hline Height (mm) & 194 & 194 & 194 & 194 \\
\hline Face Shell Thickness (mm) & 35 & 35 & 35 & 35 \\
\hline Percent Solid (\%) & 56 & 56 & 56 & 56 \\
\hline Tensile Strength & 1.83 & 1.83 & 1.83 & 1.83 \\
\hline \multicolumn{5}{|c|}{ Head Joint } \\
\hline Thickness (mm) & 10 & 10 & 10 & 10 \\
\hline Tensile Bond Strength (MPa) & 0.32 & 0.32 & 0.32 & 0.32 \\
\hline Joint Cohesion (MPa) & 5.10 & 5.10 & 5.10 & 5.10 \\
\hline Angle of Internal Friction (Deg) & 42 & 42 & 42 & 42 \\
\hline \multicolumn{5}{|c|}{ Bed Joint } \\
\hline Thickness (mm) & 10 & 10 & 10 & 10 \\
\hline Tensile Bond Strength (MPa) & 0.32 & 0.32 & 0.32 & 0.32 \\
\hline Joint Cohesion (MPa) & 5.10 & 5.10 & 5.10 & 5.10 \\
\hline Angle of Internal Friction (Deg) & 42 & 42 & 42 & 42 \\
\hline \multicolumn{5}{|c|}{ Reinforcement (Y-Direction) } \\
\hline Ratio (\%) & 0.26 & 0 & 0.26 & 0 \\
\hline Yield Strength (MPa) & 400 & 0 & 400 & 0 \\
\hline Yield Strain & 0.002 & 0 & 0.002 & 0 \\
\hline Strain Hardening Strain & 0.01 & 0 & 0.01 & 0 \\
\hline Ultimate Strength (MPa) & 650 & 0 & 650 & 0 \\
\hline Ultimate Strain & 0.2 & 0 & 0.2 & 0 \\
\hline Prestrain & 0 & 0 & 0 & 0 \\
\hline \multicolumn{5}{|c|}{ Reinforcement (X-Direction) } \\
\hline Ratio (\%) & 4 & 4 & 0 & 0 \\
\hline Yield Strength (MPa) & 547 & 547 & 0 & 0 \\
\hline Yield Strain & 0.0025 & 0.0025 & 0 & 0 \\
\hline Strain Hardening Strain & 0.01 & 0.01 & 0 & 0 \\
\hline Ultimate Strength (MPa) & 632 & 632 & 0 & 0 \\
\hline Ultimate Strain & 0.2 & 0.2 & 0 & 0 \\
\hline Prestrain & 0 & 0 & 0 & 0 \\
\hline
\end{tabular}

Numerical Modelling of Hollow Concrete Block Masonry Mechanics 


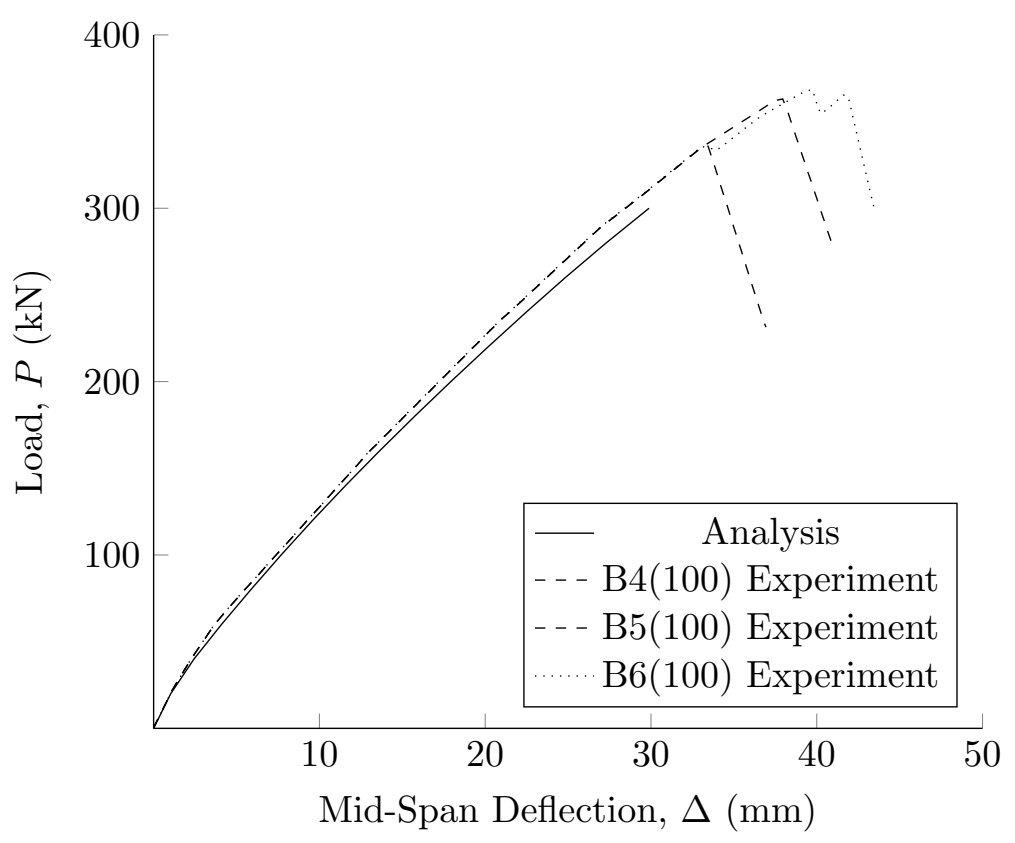

B4-6(100) Beams Ring (2009) Load vs Mid-Span Deflection 


\section{B7-9(47) Beams}

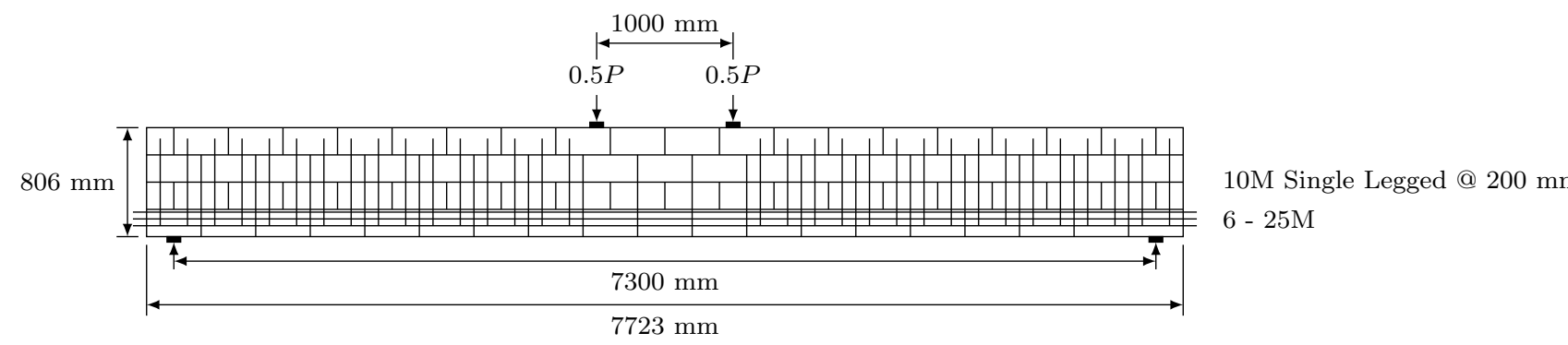

Experimental Beams Ring (2009)

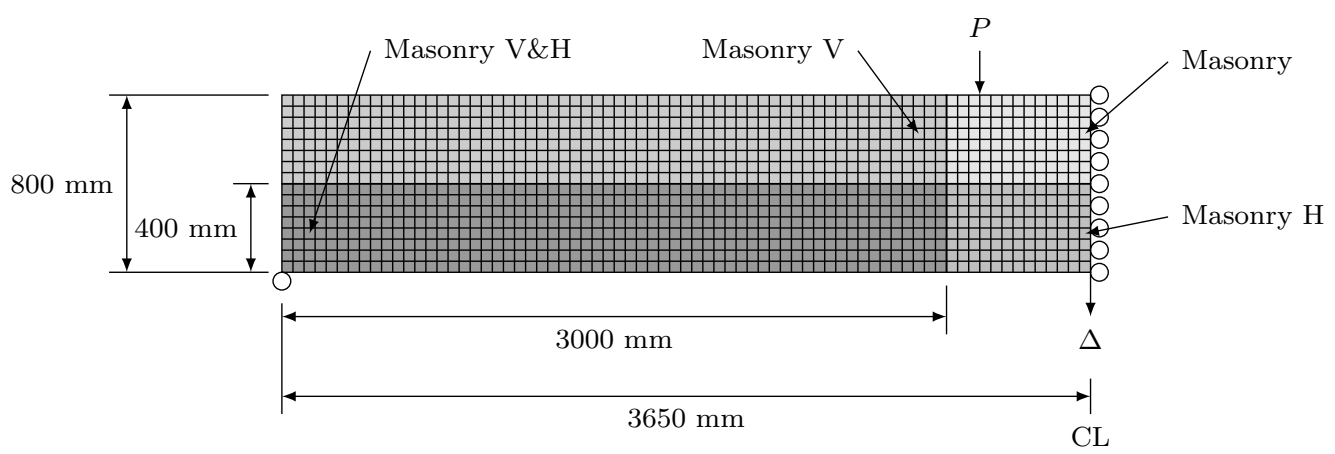

Finite Element Model Beams

B7-9(47) Beams

B7-9(47) Beams (Ring, 2009) Model Options

\begin{tabular}{ll}
\hline Model & Selected Option \\
\hline Compression Pre-Peak & Hognestad \\
Compression Post-Peak & Pre-Peak Base Curve \\
Compression Softening & Vecchio \\
Tension Post-Peak & Vecchio \\
Cracking Criterion & Mohr-Coulomb \\
Slip & Walraven Reinforced \\
\hline
\end{tabular}

Numerical Modelling of Hollow Concrete Block Masonry Mechanics 
B7-9(47) Beams (Ring, 2009) Material Properties

\begin{tabular}{|c|c|c|c|c|}
\hline \multirow{2}{*}{ Property } & \multicolumn{4}{|c|}{ Material } \\
\hline & Masonry V\&H & Masonry H & Masonry V & Masonry \\
\hline Thickness (mm) & 194 & 194 & 194 & 194 \\
\hline Grout Tensile Strength (MPa) & 1.77 & 1.77 & 1.77 & 1.77 \\
\hline \multicolumn{5}{|c|}{ Masonry Properties (Y-Direction) } \\
\hline Gross Peak Compression Strength (MPa) & 21.3 & 21.3 & 21.3 & 21.3 \\
\hline Peak Compressive Strain & 0.0028 & 0.0028 & 0.0028 & 0.0028 \\
\hline Tensile Strength & 0 & 0 & 0 & 0 \\
\hline Tensile Strain & 0 & 0 & 0 & 0 \\
\hline Initial Tangent Elastic Modulus (MPa) & 0 & 0 & 0 & 0 \\
\hline Poisson's Ratio & 0 & 0 & 0 & 0 \\
\hline \multicolumn{5}{|c|}{ Masonry Properties (X-Direction) } \\
\hline Gross Peak Compression Strength (MPa) & 21.3 & 21.3 & 21.3 & 21.3 \\
\hline Peak Compressive Strain & 0.0028 & 0.0028 & 0.0028 & 0.0028 \\
\hline Tensile Strength & 0 & 0 & 0 & 0 \\
\hline Tensile Strain & 0 & 0 & 0 & 0 \\
\hline Initial Tangent Elastic Modulus (MPa) & 0 & 0 & 0 & 0 \\
\hline Poisson's Ratio & 0 & 0 & 0 & 0 \\
\hline \multicolumn{5}{|c|}{ Masonry Properties (Block) } \\
\hline Length (mm) & 397 & 397 & 397 & 397 \\
\hline Height (mm) & 194 & 194 & 194 & 194 \\
\hline Face Shell Thickness (mm) & 35 & 35 & 35 & 35 \\
\hline Percent Solid (\%) & 56 & 56 & 56 & 56 \\
\hline Tensile Strength & 1.83 & 1.83 & 1.83 & 1.83 \\
\hline \multicolumn{5}{|c|}{ Head Joint } \\
\hline Thickness (mm) & 10 & 10 & 10 & 10 \\
\hline Tensile Bond Strength (MPa) & 0.30 & 0.30 & 0.30 & 0.30 \\
\hline Joint Cohesion (MPa) & 4.42 & 4.42 & 4.42 & 4.42 \\
\hline Angle of Internal Friction (Deg) & 42 & 42 & 42 & 42 \\
\hline \multicolumn{5}{|c|}{ Bed Joint } \\
\hline Thickness (mm) & 10 & 10 & 10 & 10 \\
\hline Tensile Bond Strength (MPa) & 0.30 & 0.30 & 0.30 & 0.30 \\
\hline Joint Cohesion (MPa) & 4.42 & 4.42 & 4.42 & 4.42 \\
\hline Angle of Internal Friction (Deg) & 42 & 42 & 42 & 42 \\
\hline \multicolumn{5}{|c|}{ Reinforcement (Y-Direction) } \\
\hline Ratio (\%) & 0.26 & 0 & 0.26 & 0 \\
\hline Yield Strength (MPa) & 400 & 0 & 400 & 0 \\
\hline Yield Strain & 0.002 & 0 & 0.002 & 0 \\
\hline Strain Hardening Strain & 0.01 & 0 & 0.01 & 0 \\
\hline Ultimate Strength (MPa) & 650 & 0 & 650 & 0 \\
\hline Ultimate Strain & 0.2 & 0 & 0.2 & 0 \\
\hline Prestrain & 0 & 0 & 0 & 0 \\
\hline \multicolumn{5}{|c|}{ Reinforcement (X-Direction) } \\
\hline Ratio (\%) & 4 & 4 & 0 & 0 \\
\hline Yield Strength (MPa) & 547 & 547 & 0 & 0 \\
\hline Yield Strain & 0.0025 & 0.0025 & 0 & 0 \\
\hline Strain Hardening Strain & 0.01 & 0.01 & 0 & 0 \\
\hline Ultimate Strength (MPa) & 632 & 632 & 0 & 0 \\
\hline Ultimate Strain & 0.2 & 0.2 & 0 & 0 \\
\hline Prestrain & 0 & 0 & 0 & 0 \\
\hline
\end{tabular}

Numerical Modelling of Hollow Concrete Block Masonry Mechanics 


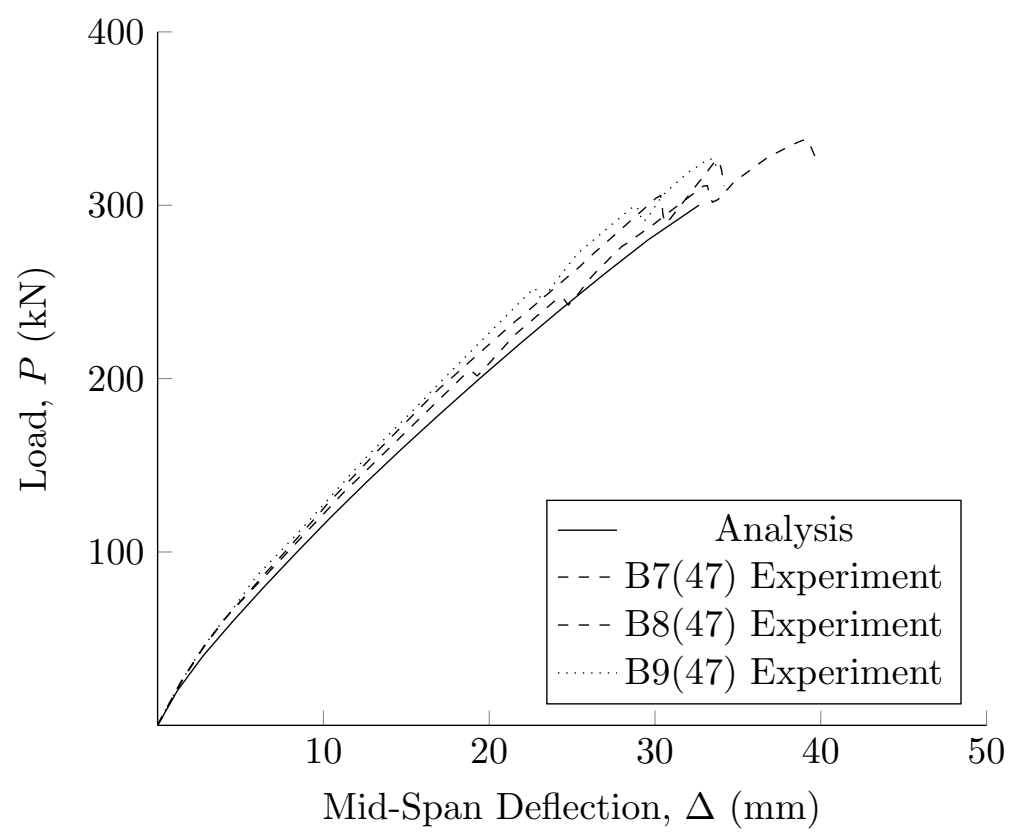

B7-9(47) Beams Ring (2009) Load vs Mid-Span Deflection 


\section{B10-12(32) Beams}

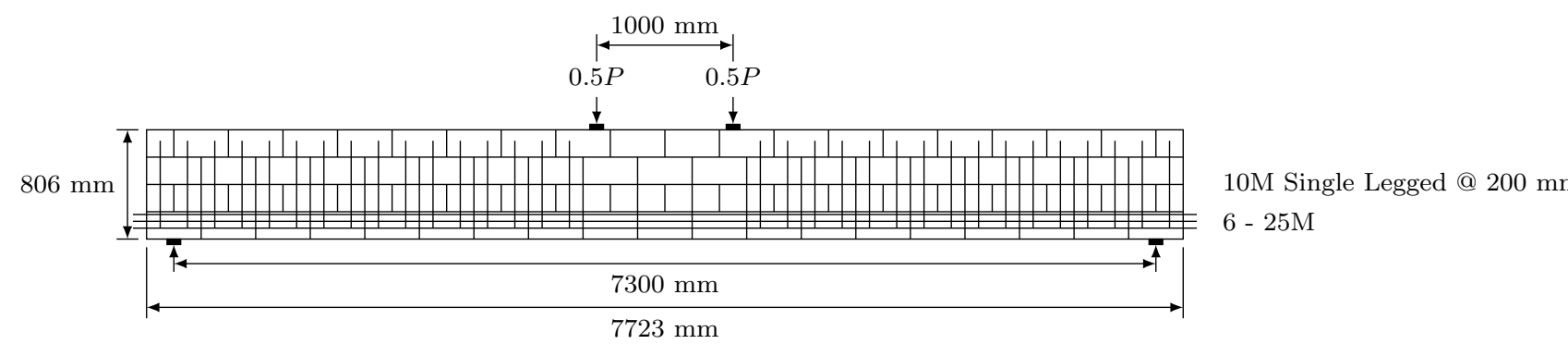

Experimental Beams Ring (2009)

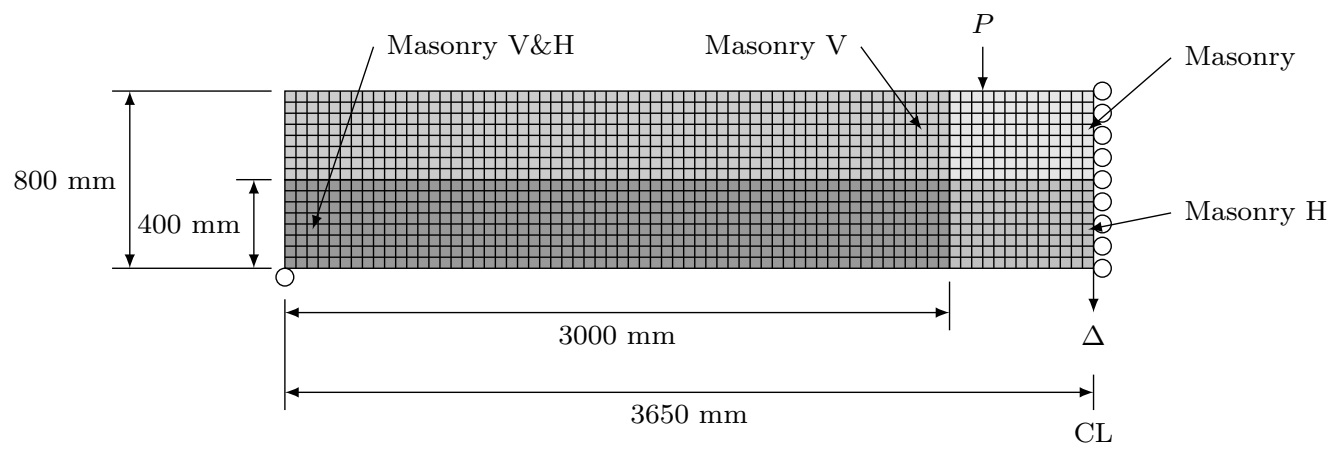

Finite Element Model Beams

B10-12(32) Beams

B10-12(32) Beams (Ring, 2009) Model Options

\begin{tabular}{ll}
\hline Model & Selected Option \\
\hline Compression Pre-Peak & Hognestad \\
Compression Post-Peak & Pre-Peak Base Curve \\
Compression Softening & Vecchio \\
Tension Post-Peak & Vecchio \\
Cracking Criterion & Mohr-Coulomb \\
Slip & Walraven Reinforced \\
\hline
\end{tabular}

Numerical Modelling of Hollow Concrete Block Masonry Mechanics 
B10-12(32) Beams (Ring, 2009) Material Properties

\begin{tabular}{|c|c|c|c|c|}
\hline \multirow{2}{*}{ Property } & \multicolumn{4}{|c|}{ Material } \\
\hline & Masonry V\&H & Masonry H & Masonry V & Masonry \\
\hline Thickness (mm) & 194 & 194 & 194 & 194 \\
\hline Grout Tensile Strength (MPa) & 1.77 & 1.77 & 1.77 & 1.77 \\
\hline \multicolumn{5}{|c|}{ Masonry Properties (Y-Direction) } \\
\hline Gross Peak Compression Strength (MPa) & 25.7 & 25.7 & 25.7 & 25.7 \\
\hline Peak Compressive Strain & 0.0026 & 0.0026 & 0.0026 & 0.0026 \\
\hline Tensile Strength & 0 & 0 & 0 & 0 \\
\hline Tensile Strain & 0 & 0 & 0 & 0 \\
\hline Initial Tangent Elastic Modulus (MPa) & 0 & 0 & 0 & 0 \\
\hline Poisson's Ratio & 0 & 0 & 0 & 0 \\
\hline \multicolumn{5}{|c|}{ Masonry Properties (X-Direction) } \\
\hline Gross Peak Compression Strength (MPa) & 25.7 & 25.7 & 25.7 & 25.7 \\
\hline Peak Compressive Strain & 0.0026 & 0.0026 & 0.0026 & 0.0026 \\
\hline Tensile Strength & 0 & 0 & 0 & 0 \\
\hline Tensile Strain & 0 & 0 & 0 & 0 \\
\hline Initial Tangent Elastic Modulus (MPa) & 0 & 0 & 0 & 0 \\
\hline Poisson's Ratio & 0 & 0 & 0 & 0 \\
\hline \multicolumn{5}{|c|}{ Masonry Properties (Block) } \\
\hline Length $(\mathrm{mm})$ & 397 & 397 & 397 & 397 \\
\hline Height (mm) & 194 & 194 & 194 & 194 \\
\hline Face Shell Thickness (mm) & 35 & 35 & 35 & 35 \\
\hline Percent Solid (\%) & 56 & 56 & 56 & 56 \\
\hline Tensile Strength & 1.83 & 1.83 & 1.83 & 1.83 \\
\hline \multicolumn{5}{|c|}{ Head Joint } \\
\hline Thickness (mm) & 10 & 10 & 10 & 10 \\
\hline Tensile Bond Strength (MPa) & 0.34 & 0.34 & 0.34 & 0.34 \\
\hline Joint Cohesion $(\mathrm{MPa})$ & 5.93 & 5.93 & 5.93 & 5.93 \\
\hline Angle of Internal Friction (Deg) & 42 & 42 & 42 & 42 \\
\hline \multicolumn{5}{|c|}{ Bed Joint } \\
\hline Thickness (mm) & 10 & 10 & 10 & 10 \\
\hline Tensile Bond Strength (MPa) & 0.34 & 0.34 & 0.34 & 0.34 \\
\hline Joint Cohesion (MPa) & 5.93 & 5.93 & 5.93 & 5.93 \\
\hline Angle of Internal Friction (Deg) & 42 & 42 & 42 & 42 \\
\hline \multicolumn{5}{|c|}{ Reinforcement (Y-Direction) } \\
\hline Ratio (\%) & 0.26 & 0 & 0.26 & 0 \\
\hline Yield Strength (MPa) & 400 & 0 & 400 & 0 \\
\hline Yield Strain & 0.002 & 0 & 0.002 & 0 \\
\hline Strain Hardening Strain & 0.01 & 0 & 0.01 & 0 \\
\hline Ultimate Strength (MPa) & 650 & 0 & 650 & 0 \\
\hline Ultimate Strain & 0.2 & 0 & 0.2 & 0 \\
\hline Prestrain & 0 & 0 & 0 & 0 \\
\hline \multicolumn{5}{|c|}{ Reinforcement (X-Direction) } \\
\hline Ratio (\%) & 4 & 4 & 0 & 0 \\
\hline Yield Strength (MPa) & 547 & 547 & 0 & 0 \\
\hline Yield Strain & 0.0025 & 0.0025 & 0 & 0 \\
\hline Strain Hardening Strain & 0.01 & 0.01 & 0 & 0 \\
\hline Ultimate Strength (MPa) & 632 & 632 & 0 & 0 \\
\hline Ultimate Strain & 0.2 & 0.2 & 0 & 0 \\
\hline Prestrain & 0 & 0 & 0 & 0 \\
\hline
\end{tabular}

Numerical Modelling of Hollow Concrete Block Masonry Mechanics 


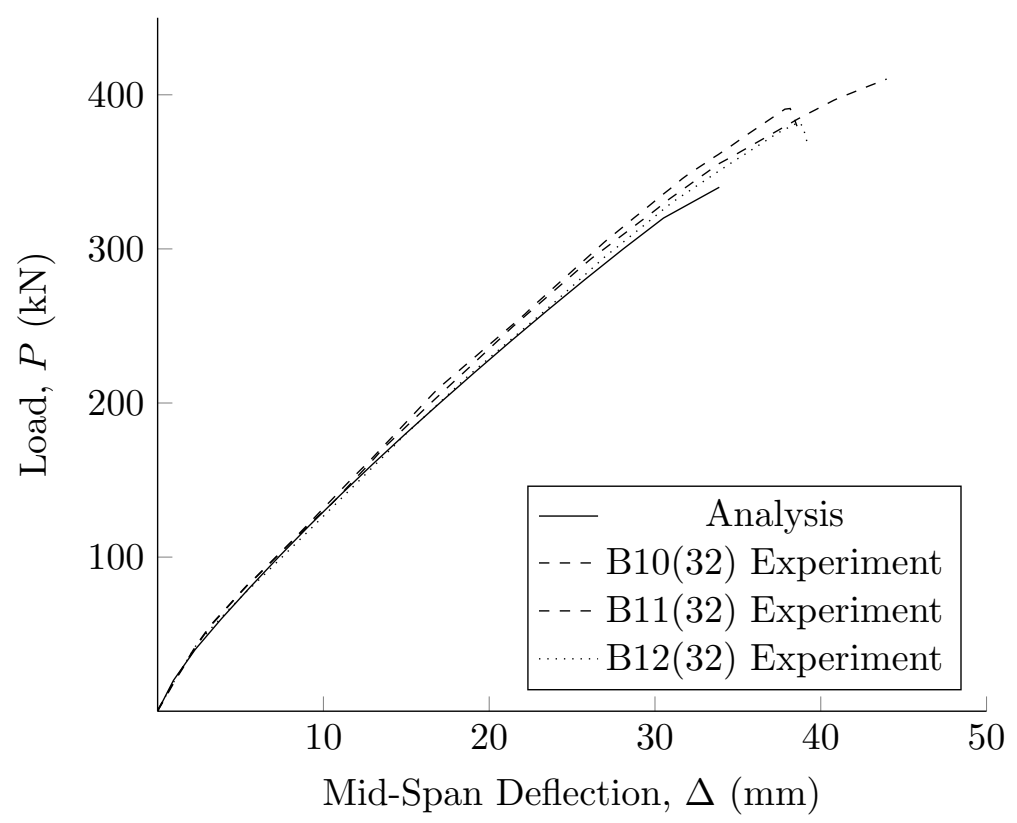

B10-12(32) Beams Ring (2009) Load vs Mid-Span Deflection 


\section{Sarhat (2016)}

\section{SS1 Beam}

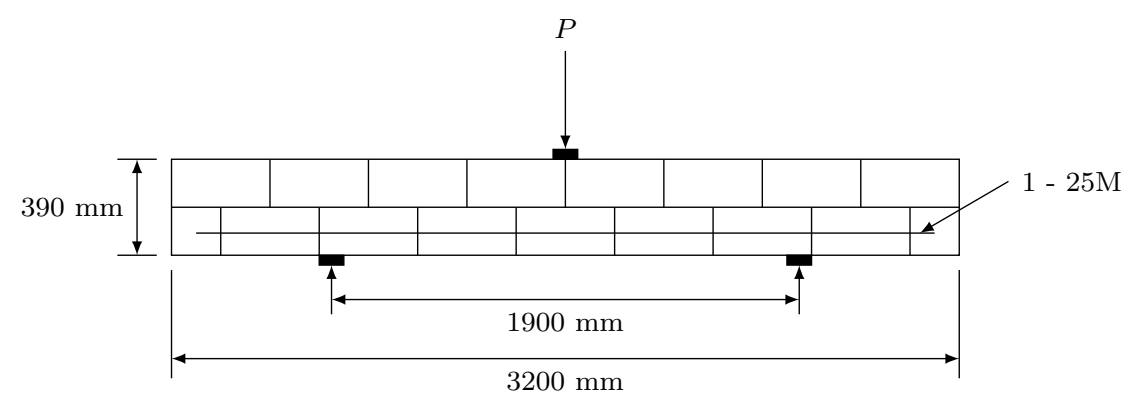

Experimental Beam Sarhat (2016)

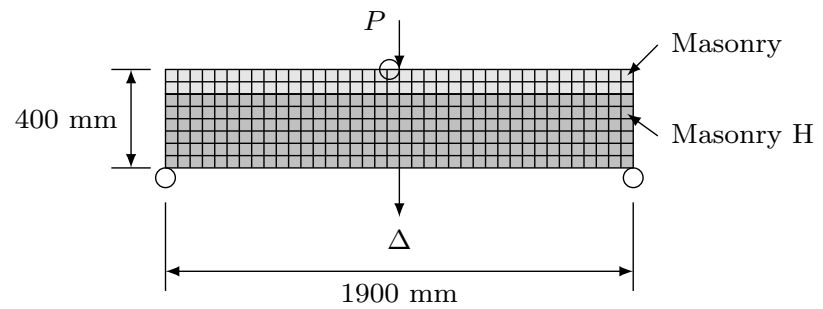

Finite Element Model Beam

SS1 Beam

SS1 Beam (Sarhat, 2016) Model Options

\begin{tabular}{ll}
\hline Model & Selected Option \\
\hline Compression Pre-Peak & Hognestad \\
Compression Post-Peak & Pre-Peak Base Curve \\
Compression Softening & Vecchio \\
Tension Post-Peak & Vecchio \\
Cracking Criterion & Mohr-Coulomb \\
Slip & Walraven Reinforced \\
\hline
\end{tabular}


SS1 Beam (Sarhat, 2016) Material Properties

\begin{tabular}{|c|c|c|}
\hline \multirow{2}{*}{ Property } & \multicolumn{2}{|c|}{ Material } \\
\hline & Masonry & Masonry $\mathrm{H}$ \\
\hline Thickness (mm) & 190 & 190 \\
\hline Grout Tensile Strength (MPa) & 1.64 & 1.64 \\
\hline \multicolumn{3}{|c|}{ Masonry Properties (Y-Direction) } \\
\hline Gross Peak Compression Strength (MPa) & 17.5 & 17.5 \\
\hline Peak Compressive Strain & 0.0014 & 0.0014 \\
\hline Tensile Strength & 0 & 0 \\
\hline Tensile Strain & 0 & 0 \\
\hline Initial Tangent Elastic Modulus (MPa) & 0 & 0 \\
\hline Poisson's Ratio & 0 & 0 \\
\hline \multicolumn{3}{|c|}{ Masonry Properties (X-Direction) } \\
\hline Gross Peak Compression Strength (MPa) & 17.5 & 17.5 \\
\hline Peak Compressive Strain & 0.0014 & 0.0014 \\
\hline Tensile Strength & 0 & 0 \\
\hline Tensile Strain & 0 & 0 \\
\hline Initial Tangent Elastic Modulus (MPa) & 0 & 0 \\
\hline Poisson's Ratio & 0 & 0 \\
\hline \multicolumn{3}{|c|}{ Masonry Properties (Block) } \\
\hline Length (mm) & 390 & 390 \\
\hline Height (mm) & 190 & 190 \\
\hline Face Shell Thickness (mm) & 35 & 35 \\
\hline Percent Solid (\%) & 56 & 56 \\
\hline Tensile Strength & 2.01 & 2.01 \\
\hline \multicolumn{3}{|l|}{ Head Joint } \\
\hline Thickness (mm) & 10 & 10 \\
\hline Tensile Bond Strength (MPa) & 0.31 & 0.31 \\
\hline Joint Cohesion (MPa) & 4.77 & 4.77 \\
\hline Angle of Internal Friction (Deg) & 42 & 42 \\
\hline \multicolumn{3}{|l|}{ Bed Joint } \\
\hline Thickness (mm) & 10 & 10 \\
\hline Tensile Bond Strength (MPa) & 0.31 & 0.31 \\
\hline Joint Cohesion (MPa) & 4.77 & 4.77 \\
\hline Angle of Internal Friction (Deg) & 42 & 42 \\
\hline \multicolumn{3}{|c|}{ Reinforcement (Y-Direction) } \\
\hline Ratio (\%) & 0 & 0 \\
\hline Yield Strength (MPa) & 0 & 0 \\
\hline Yield Strain & 0 & 0 \\
\hline Strain Hardening Strain & 0 & 0 \\
\hline Ultimate Strength (MPa) & 0 & 0 \\
\hline Ultimate Strain & 0 & 0 \\
\hline Prestrain & 0 & 0 \\
\hline \multicolumn{3}{|c|}{ Reinforcement (X-Direction) } \\
\hline Ratio (\%) & 0 & 0.95 \\
\hline Yield Strength (MPa) & 0 & 405 \\
\hline Yield Strain & 0 & 0.0025 \\
\hline Strain Hardening Strain & 0 & 0.01 \\
\hline Ultimate Strength (MPa) & 0 & 650 \\
\hline Ultimate Strain & 0 & 0.2 \\
\hline Prestrain & 0 & 0 \\
\hline
\end{tabular}

Numerical Modelling of Hollow Concrete Block Masonry Mechanics 


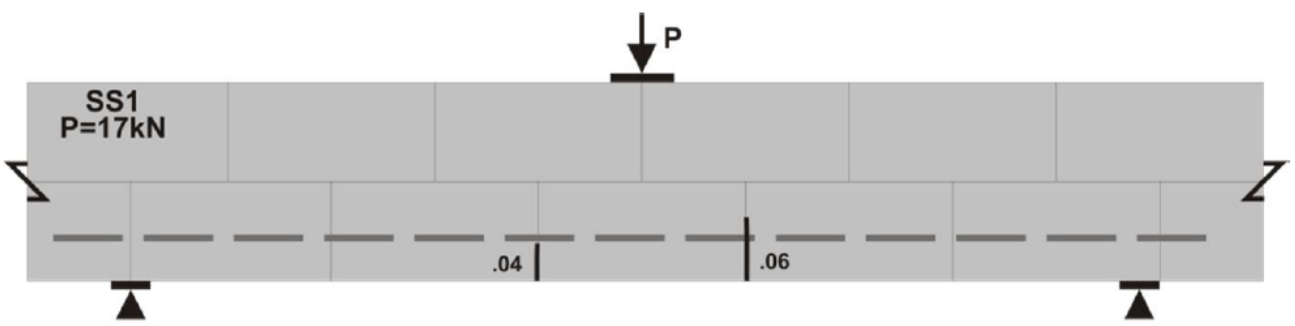

Experiment at $17 \mathrm{kN}$ Load (Sarhat, 2016)

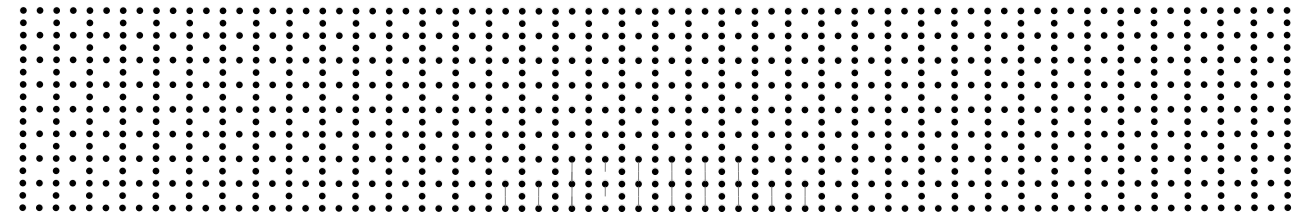

Finite Element Model at $17 \mathrm{kN} \times 15$

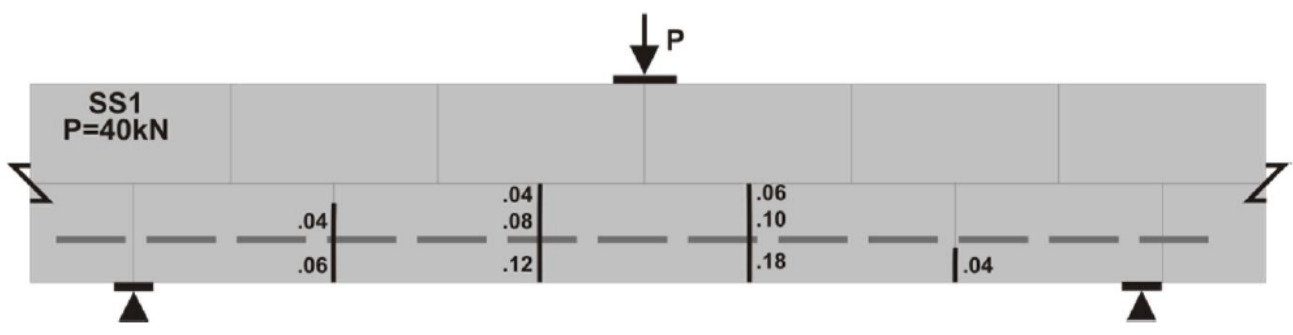

Experiment at $40 \mathrm{kN}$ Load (Sarhat, 2016)

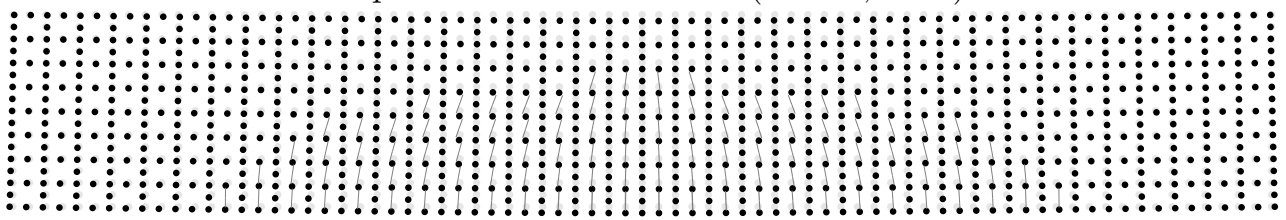

Finite Element Model at $40 \mathrm{kN} \times 15$

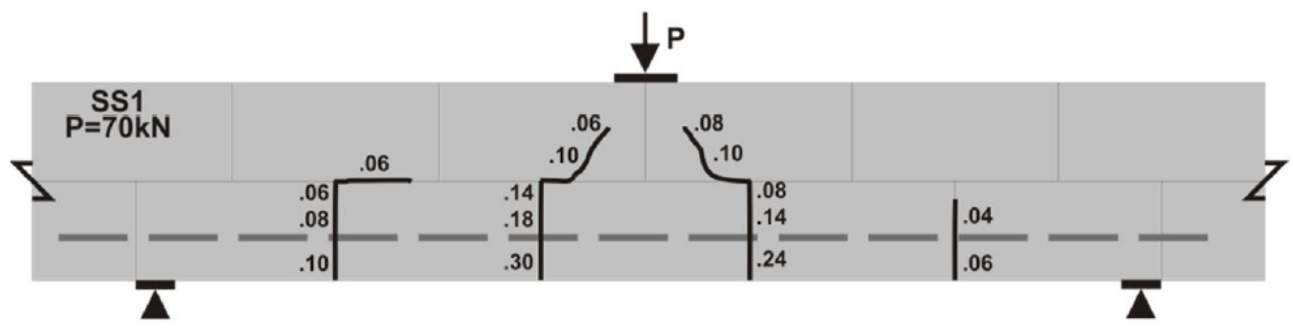

Experiment at $70 \mathrm{kN}$ Load (Sarhat, 2016)

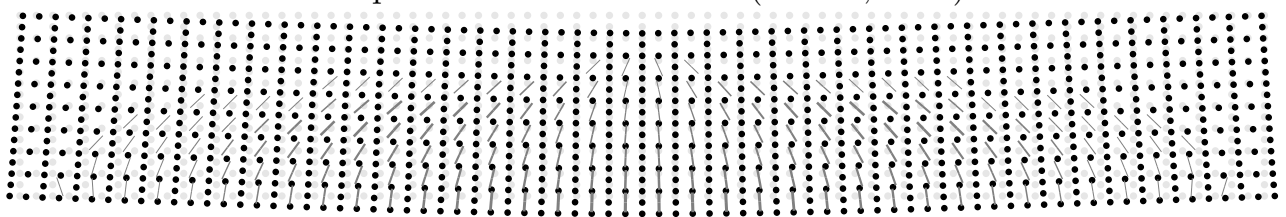

Finite Element Model at $70 \mathrm{kN} \times 15$

SS1 Beam Displacement, Crack Pattern, and Crack Widths 


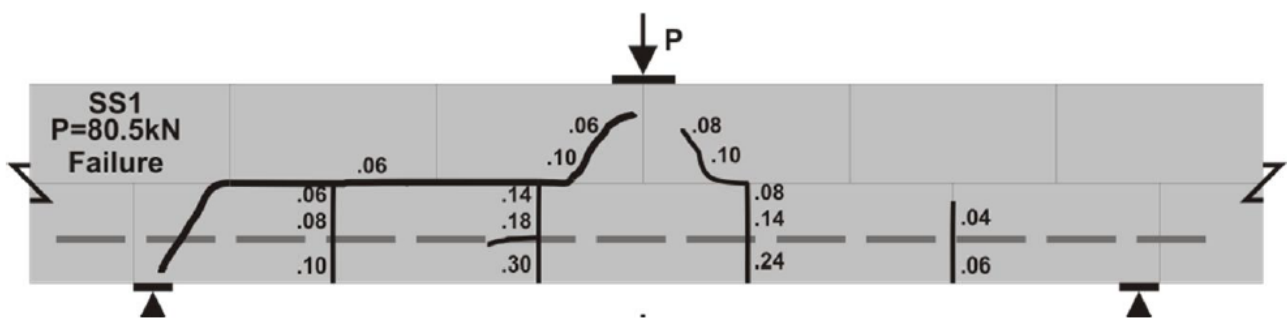

Experiment at Failure (Sarhat, 2016)

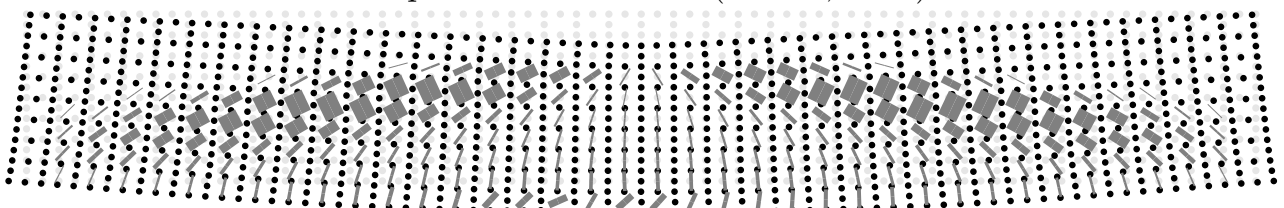

Finite Element Model at Failure $\times 15$

SS1 Beam Displacement, Crack Pattern, and Crack Widths at Failure

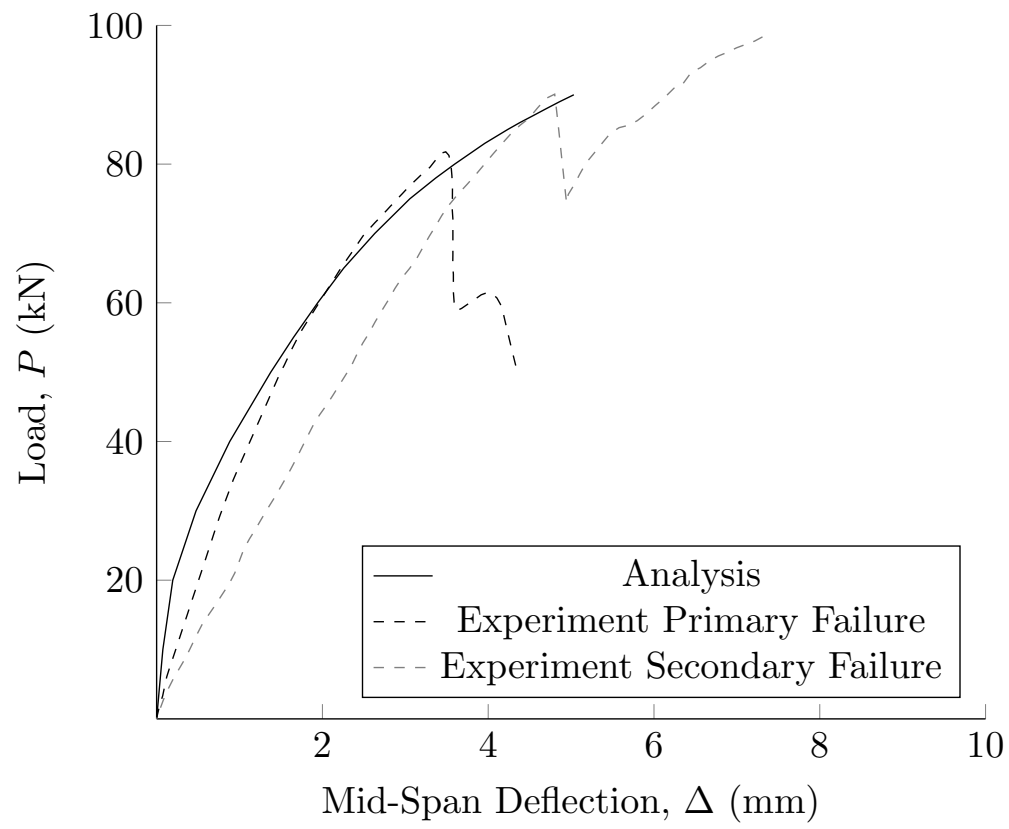

SS1 Beam Sarhat (2016) Load vs Mid-Span Deflection 


\section{SS2 Beam}

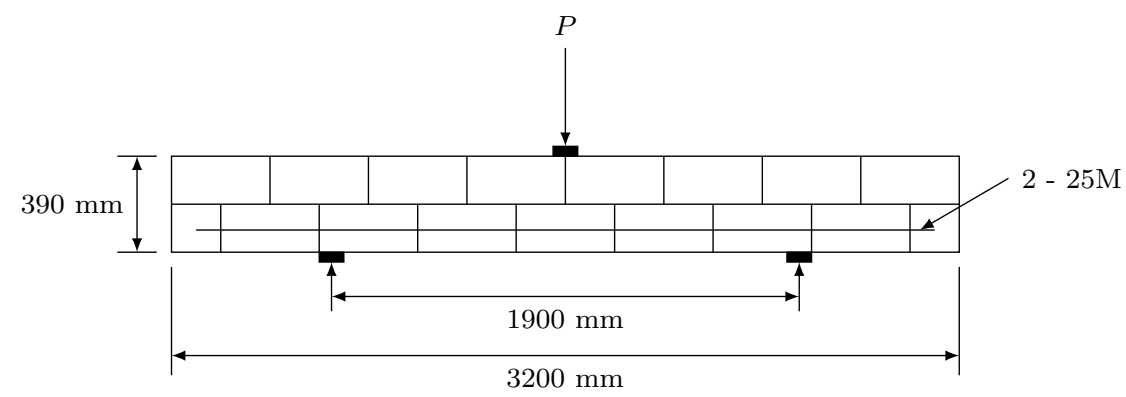

Experimental Beam Sarhat (2016)

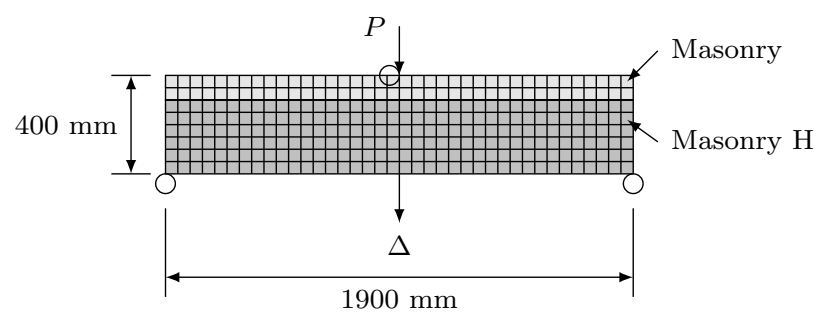

Finite Element Model Beam

SS2 Beam

SS2 Beam (Sarhat, 2016) Model Options

\begin{tabular}{ll}
\hline Model & Selected Option \\
\hline Compression Pre-Peak & Hognestad \\
Compression Post-Peak & Pre-Peak Base Curve \\
Compression Softening & Vecchio \\
Tension Post-Peak & Vecchio \\
Cracking Criterion & Mohr-Coulomb \\
Slip & Walraven Reinforced \\
\hline
\end{tabular}


SS2 Beam (Sarhat, 2016) Material Properties

\begin{tabular}{|c|c|c|}
\hline \multirow{2}{*}{ Property } & \multicolumn{2}{|c|}{ Material } \\
\hline & Masonry & Masonry $\mathrm{H}$ \\
\hline Thickness (mm) & 190 & 190 \\
\hline Grout Tensile Strength (MPa) & 1.64 & 1.64 \\
\hline \multicolumn{3}{|c|}{ Masonry Properties (Y-Direction) } \\
\hline Gross Peak Compression Strength (MPa) & 17.5 & 17.5 \\
\hline Peak Compressive Strain & 0.0014 & 0.0014 \\
\hline Tensile Strength & 0 & 0 \\
\hline Tensile Strain & 0 & 0 \\
\hline Initial Tangent Elastic Modulus (MPa) & 0 & 0 \\
\hline Poisson's Ratio & 0 & 0 \\
\hline \multicolumn{3}{|c|}{ Masonry Properties (X-Direction) } \\
\hline Gross Peak Compression Strength (MPa) & 17.5 & 17.5 \\
\hline Peak Compressive Strain & 0.0014 & 0.0014 \\
\hline Tensile Strength & 0 & 0 \\
\hline Tensile Strain & 0 & 0 \\
\hline Initial Tangent Elastic Modulus (MPa) & 0 & 0 \\
\hline Poisson's Ratio & 0 & 0 \\
\hline \multicolumn{3}{|c|}{ Masonry Properties (Block) } \\
\hline Length (mm) & 390 & 390 \\
\hline Height (mm) & 190 & 190 \\
\hline Face Shell Thickness (mm) & 35 & 35 \\
\hline Percent Solid (\%) & 56 & 56 \\
\hline Tensile Strength & 2.01 & 2.01 \\
\hline \multicolumn{3}{|l|}{ Head Joint } \\
\hline Thickness (mm) & 10 & 10 \\
\hline Tensile Bond Strength (MPa) & 0.31 & 0.31 \\
\hline Joint Cohesion (MPa) & 4.77 & 4.77 \\
\hline Angle of Internal Friction (Deg) & 42 & 42 \\
\hline \multicolumn{3}{|l|}{ Bed Joint } \\
\hline Thickness (mm) & 10 & 10 \\
\hline Tensile Bond Strength (MPa) & 0.31 & 0.31 \\
\hline Joint Cohesion (MPa) & 4.77 & 4.77 \\
\hline Angle of Internal Friction (Deg) & 42 & 42 \\
\hline \multicolumn{3}{|c|}{ Reinforcement (Y-Direction) } \\
\hline Ratio (\%) & 0 & 0 \\
\hline Yield Strength (MPa) & 0 & 0 \\
\hline Yield Strain & 0 & 0 \\
\hline Strain Hardening Strain & 0 & 0 \\
\hline Ultimate Strength (MPa) & 0 & 0 \\
\hline Ultimate Strain & 0 & 0 \\
\hline Prestrain & 0 & 0 \\
\hline \multicolumn{3}{|c|}{ Reinforcement (X-Direction) } \\
\hline Ratio (\%) & 0 & 1.90 \\
\hline Yield Strength (MPa) & 0 & 405 \\
\hline Yield Strain & 0 & 0.0025 \\
\hline Strain Hardening Strain & 0 & 0.01 \\
\hline Ultimate Strength (MPa) & 0 & 650 \\
\hline Ultimate Strain & 0 & 0.2 \\
\hline Prestrain & 0 & 0 \\
\hline
\end{tabular}

Numerical Modelling of Hollow Concrete Block Masonry Mechanics 


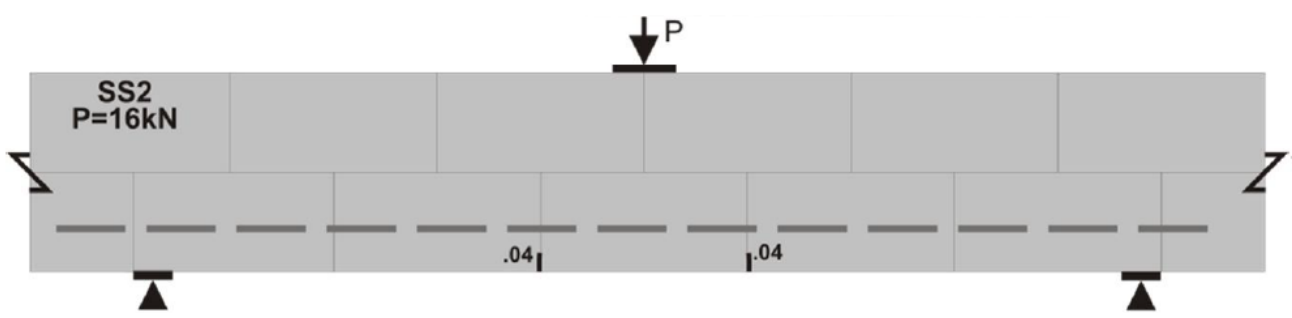

Experiment at $16 \mathrm{kN}$ Load (Sarhat, 2016)

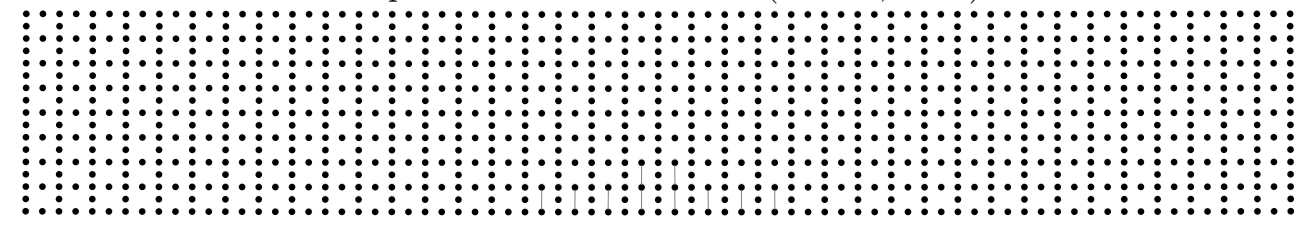

Finite Element Model at $16 \mathrm{kN} \times 15$

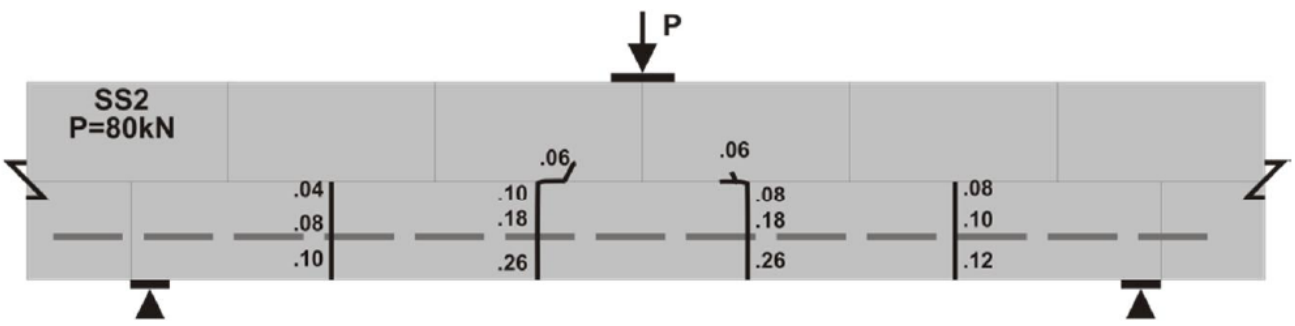

Experiment at $80 \mathrm{kN}$ Load (Sarhat, 2016)

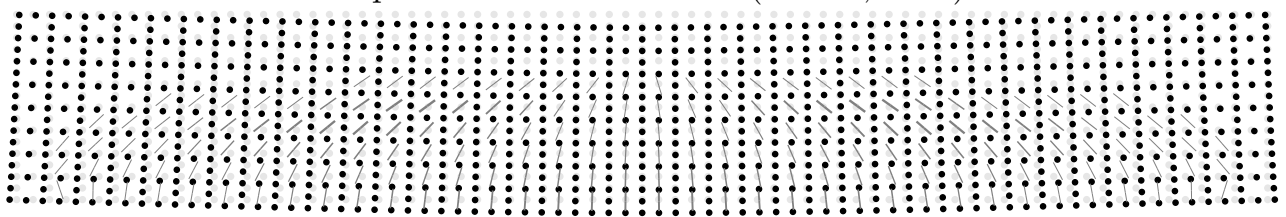

Finite Element Model at $80 \mathrm{kN} \times 15$

SS2 Beam Displacement, Crack Pattern, and Crack Widths 


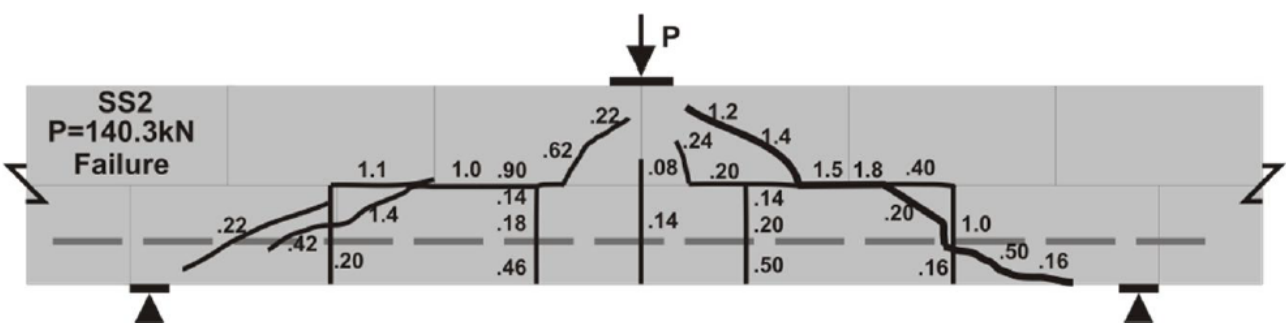

Experiment at Failure (Sarhat, 2016)

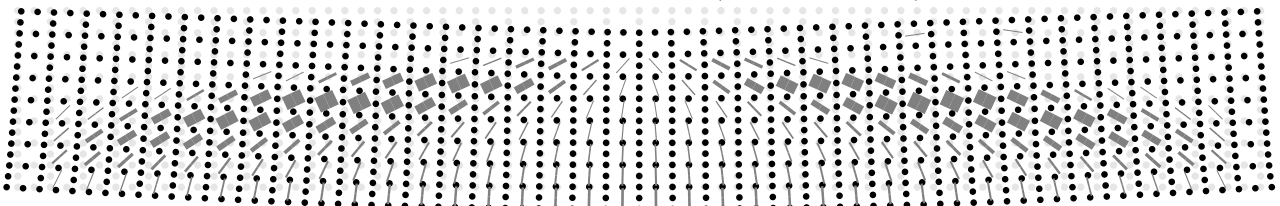

Finite Element Model at Failure $\times 15$

SS2 Beam Displacement, Crack Pattern, and Crack Widths at Failure

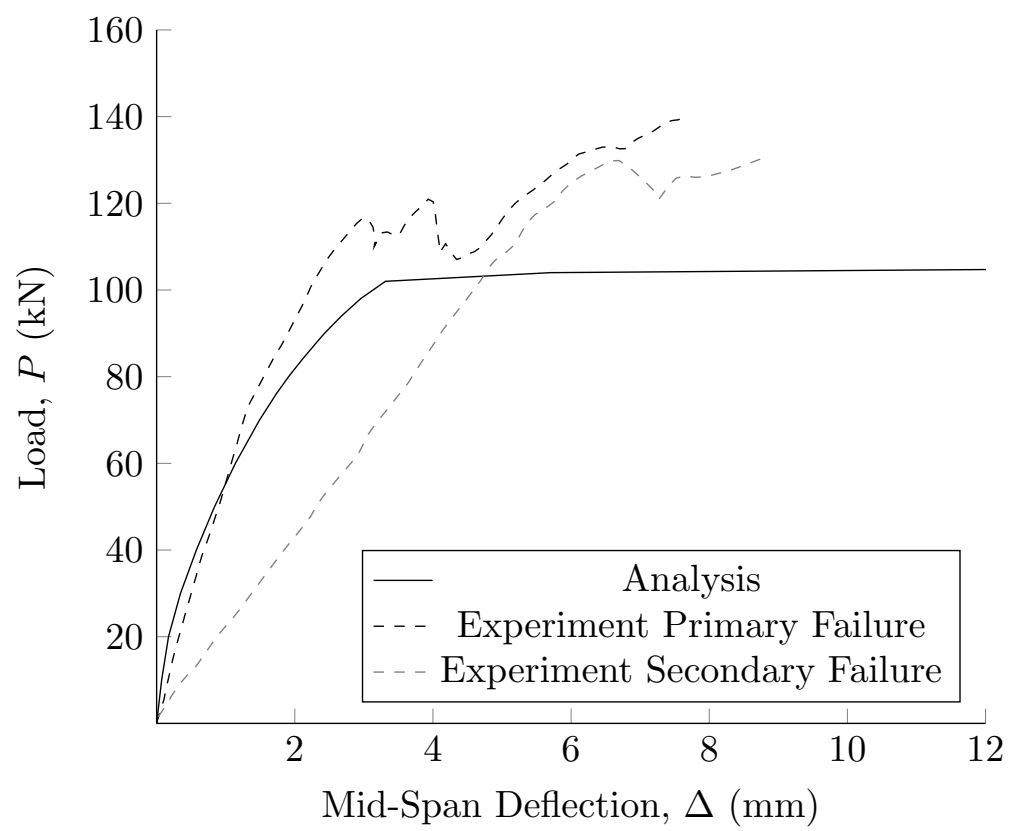

SS2 Beam Sarhat (2016) Load vs Mid-Span Deflection 


\section{SS3 Beam}

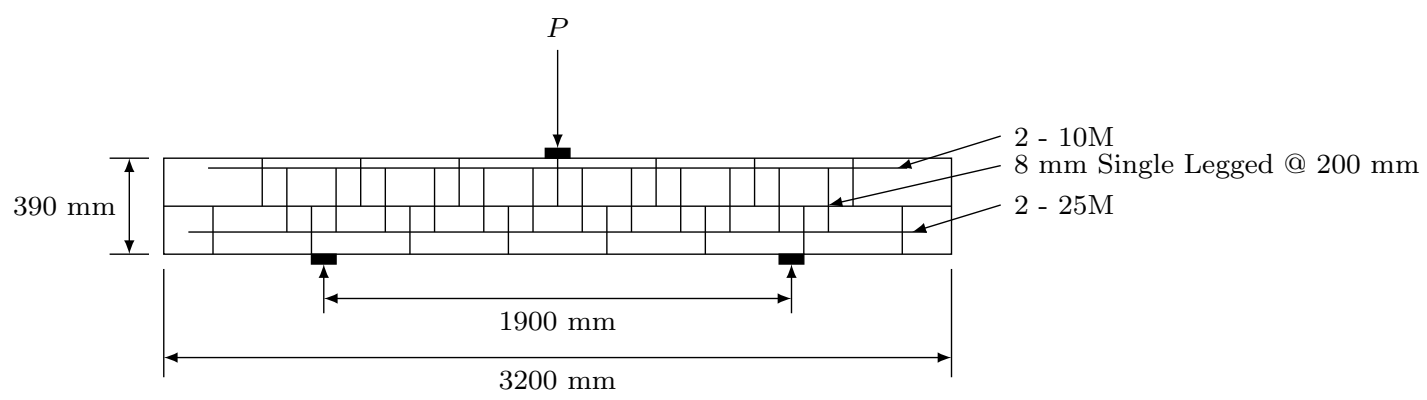

Experimental Beam Sarhat (2016)

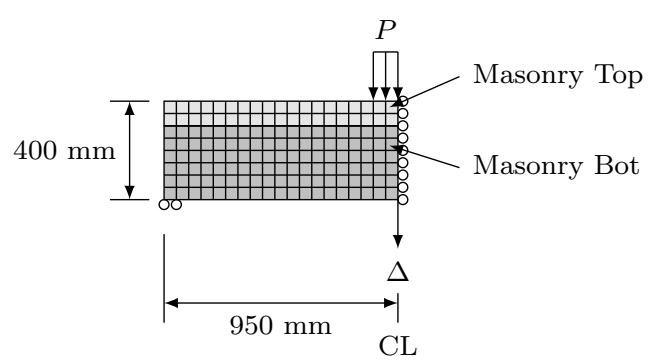

Finite Element Model Beam

SS3 Beam

SS3 Beam (Sarhat, 2016) Model Options

\begin{tabular}{ll}
\hline Model & Selected Option \\
\hline Compression Pre-Peak & Hognestad \\
Compression Post-Peak & Pre-Peak Base Curve \\
Compression Softening & Vecchio \\
Tension Post-Peak & Vecchio \\
Cracking Criterion & Mohr-Coulomb \\
Slip & Walraven Reinforced \\
\hline
\end{tabular}

Numerical Modelling of Hollow Concrete Block Masonry Mechanics 
SS3 Beam (Sarhat, 2016) Material Properties

\begin{tabular}{|c|c|c|}
\hline \multirow{2}{*}{ Property } & \multicolumn{2}{|c|}{ Material } \\
\hline & Masonry Bot & Masonry Top \\
\hline Thickness (mm) & 190 & 190 \\
\hline Grout Tensile Strength (MPa) & 1.64 & 1.64 \\
\hline \multicolumn{3}{|c|}{ Masonry Properties (Y-Direction) } \\
\hline Gross Peak Compression Strength (MPa) & 17.5 & 17.5 \\
\hline Peak Compressive Strain & 0.0014 & 0.0014 \\
\hline Tensile Strength & 0 & 0 \\
\hline Tensile Strain & 0 & 0 \\
\hline Initial Tangent Elastic Modulus (MPa) & 0 & 0 \\
\hline Poisson's Ratio & 0 & 0 \\
\hline \multicolumn{3}{|c|}{ Masonry Properties (X-Direction) } \\
\hline Gross Peak Compression Strength (MPa) & 17.5 & 17.5 \\
\hline Peak Compressive Strain & 0.0014 & 0.0014 \\
\hline Tensile Strength & 0 & 0 \\
\hline Tensile Strain & 0 & 0 \\
\hline Initial Tangent Elastic Modulus (MPa) & 0 & 0 \\
\hline Poisson's Ratio & 0 & 0 \\
\hline \multicolumn{3}{|c|}{ Masonry Properties (Block) } \\
\hline Length (mm) & 390 & 390 \\
\hline Height (mm) & 190 & 190 \\
\hline Face Shell Thickness (mm) & 35 & 35 \\
\hline Percent Solid (\%) & 56 & 56 \\
\hline Tensile Strength & 2.01 & 2.01 \\
\hline \multicolumn{3}{|l|}{ Head Joint } \\
\hline Thickness (mm) & 10 & 10 \\
\hline Tensile Bond Strength (MPa) & 0.31 & 0.31 \\
\hline Joint Cohesion (MPa) & 4.77 & 4.77 \\
\hline Angle of Internal Friction (Deg) & 42 & 42 \\
\hline \multicolumn{3}{|l|}{ Bed Joint } \\
\hline Thickness (mm) & 10 & 10 \\
\hline Tensile Bond Strength (MPa) & 0.31 & 0.31 \\
\hline Joint Cohesion (MPa) & 4.77 & 4.77 \\
\hline Angle of Internal Friction (Deg) & 42 & 42 \\
\hline \multicolumn{3}{|c|}{ Reinforcement (Y-Direction) } \\
\hline Ratio (\%) & 0.13 & 0.13 \\
\hline Yield Strength (MPa) & 525 & 525 \\
\hline Yield Strain & 0.0025 & 0.0025 \\
\hline Strain Hardening Strain & 0.01 & 0.01 \\
\hline Ultimate Strength $(\mathrm{MPa})$ & 650 & 650 \\
\hline Ultimate Strain & 0.02 & 0.02 \\
\hline Prestrain & 0 & 0 \\
\hline \multicolumn{3}{|c|}{ Reinforcement (X-Direction) } \\
\hline Ratio (\%) & 1.40 & 1.90 \\
\hline Yield Strength (MPa) & 425 & 405 \\
\hline Yield Strain & 0.0025 & 0.0025 \\
\hline Strain Hardening Strain & 0.01 & 0.01 \\
\hline Ultimate Strength (MPa) & 650 & 650 \\
\hline Ultimate Strain & 0.2 & 0.2 \\
\hline Prestrain & 0 & 0 \\
\hline
\end{tabular}

Numerical Modelling of Hollow Concrete Block Masonry Mechanics 


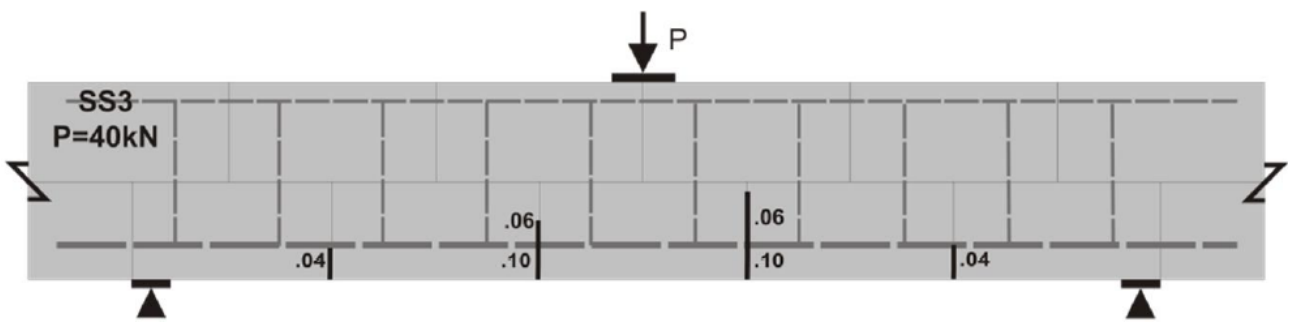

Experiment at $40 \mathrm{kN}$ Load (Sarhat, 2016)

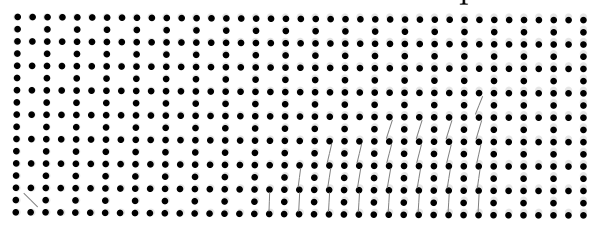

Finite Element Model at $40 \mathrm{kN} \times 15$

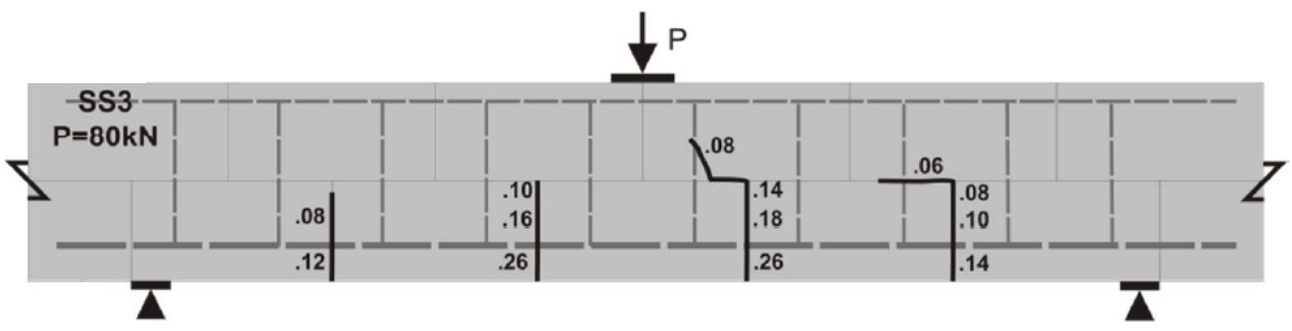

Experiment at $80 \mathrm{kN}$ Load (Sarhat, 2016)

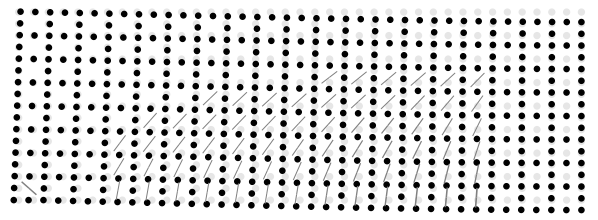

Finite Element Model at $80 \mathrm{kN} \times 15$

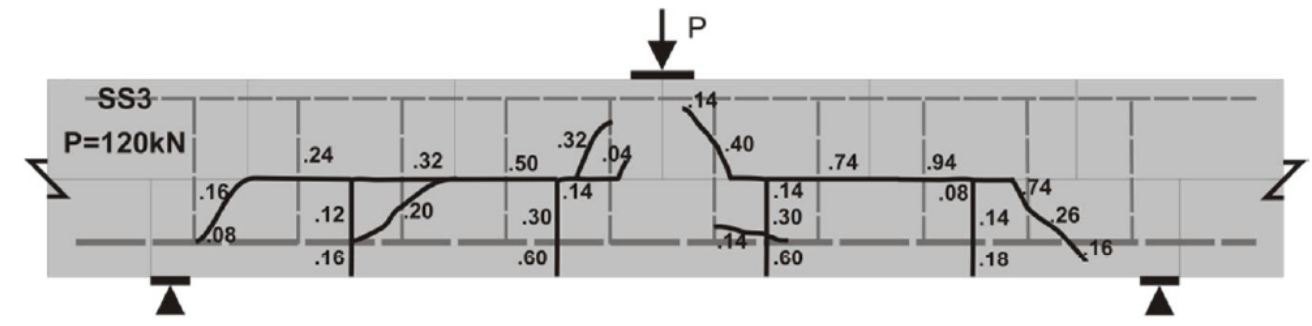

Experiment at $120 \mathrm{kN}$ Load (Sarhat, 2016)

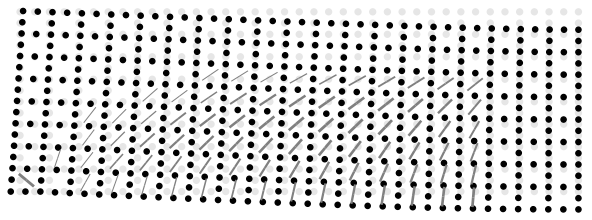

Finite Element Model at $120 \mathrm{kN} \times 15$

SS3 Beam Displacement, Crack Pattern, and Crack Widths 


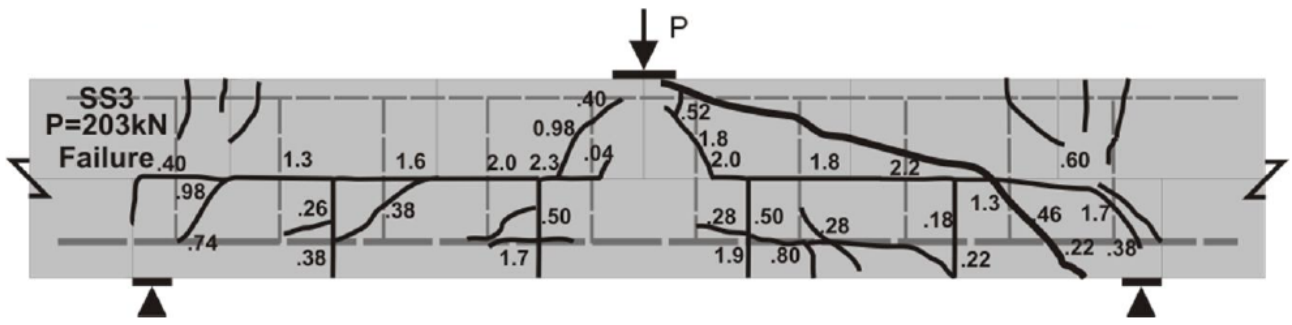

Experiment at Failure (Sarhat, 2016)

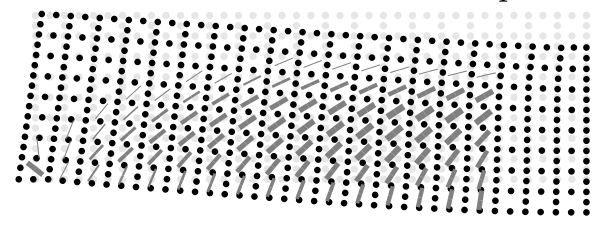

Finite Element Model at Failure $\times 15$

SS3 Beam Displacement, Crack Pattern, and Crack Widths at Failure

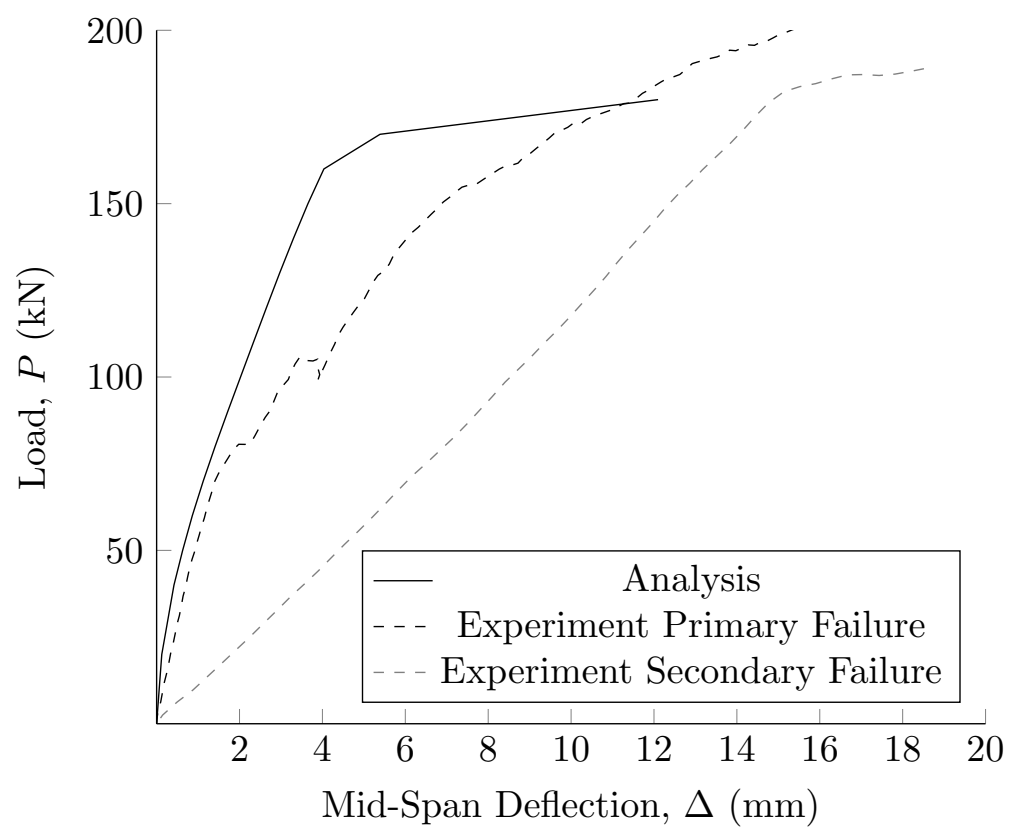

SS3 Beam Sarhat (2016) Load vs Mid-Span Deflection 


\section{SS6 Beam}

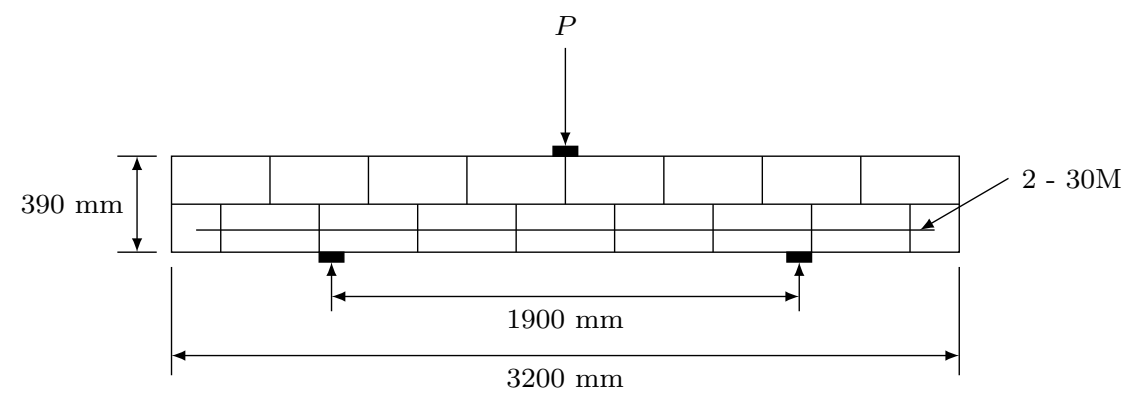

Experimental Beam Sarhat (2016)

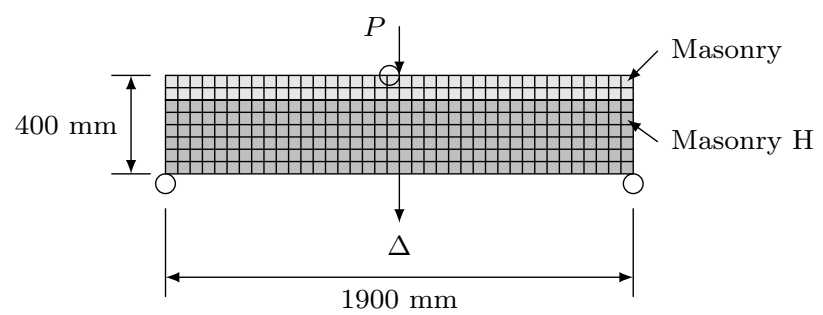

Finite Element Model Beam

SS6 Beam

SS6 Beam (Sarhat, 2016) Model Options

\begin{tabular}{ll}
\hline Model & Selected Option \\
\hline Compression Pre-Peak & Hognestad \\
Compression Post-Peak & Pre-Peak Base Curve \\
Compression Softening & Vecchio \\
Tension Post-Peak & Vecchio \\
Cracking Criterion & Mohr-Coulomb \\
Slip & Walraven Reinforced \\
\hline
\end{tabular}


SS6 Beam (Sarhat, 2016) Material Properties

\begin{tabular}{|c|c|c|}
\hline \multirow{2}{*}{ Property } & \multicolumn{2}{|c|}{ Material } \\
\hline & Masonry & Masonry $\mathrm{H}$ \\
\hline Thickness (mm) & 190 & 190 \\
\hline Grout Tensile Strength (MPa) & 1.69 & 1.69 \\
\hline \multicolumn{3}{|c|}{ Masonry Properties (Y-Direction) } \\
\hline Gross Peak Compression Strength (MPa) & 18.0 & 18.0 \\
\hline Peak Compressive Strain & 0.0012 & 0.0012 \\
\hline Tensile Strength & 0 & 0 \\
\hline Tensile Strain & 0 & 0 \\
\hline Initial Tangent Elastic Modulus (MPa) & 0 & 0 \\
\hline Poisson's Ratio & 0 & 0 \\
\hline \multicolumn{3}{|c|}{ Masonry Properties (X-Direction) } \\
\hline Gross Peak Compression Strength (MPa) & 18.0 & 18.0 \\
\hline Peak Compressive Strain & 0.0012 & 0.0012 \\
\hline Tensile Strength & 0 & 0 \\
\hline Tensile Strain & 0 & 0 \\
\hline Initial Tangent Elastic Modulus (MPa) & 0 & 0 \\
\hline Poisson's Ratio & 0 & 0 \\
\hline \multicolumn{3}{|c|}{ Masonry Properties (Block) } \\
\hline Length (mm) & 390 & 390 \\
\hline Height (mm) & 190 & 190 \\
\hline Face Shell Thickness (mm) & 35 & 35 \\
\hline Percent Solid (\%) & 56 & 56 \\
\hline Tensile Strength & 2.02 & 2.02 \\
\hline \multicolumn{3}{|l|}{ Head Joint } \\
\hline Thickness (mm) & 10 & 10 \\
\hline Tensile Bond Strength (MPa) & 0.36 & 0.36 \\
\hline Joint Cohesion (MPa) & 6.65 & 6.65 \\
\hline Angle of Internal Friction (Deg) & 42 & 42 \\
\hline \multicolumn{3}{|l|}{ Bed Joint } \\
\hline Thickness (mm) & 10 & 10 \\
\hline Tensile Bond Strength (MPa) & 0.36 & 0.36 \\
\hline Joint Cohesion (MPa) & 6.65 & 6.65 \\
\hline Angle of Internal Friction (Deg) & 42 & 42 \\
\hline \multicolumn{3}{|c|}{ Reinforcement (Y-Direction) } \\
\hline Ratio (\%) & 0 & 0 \\
\hline Yield Strength (MPa) & 0 & 0 \\
\hline Yield Strain & 0 & 0 \\
\hline Strain Hardening Strain & 0 & 0 \\
\hline Ultimate Strength (MPa) & 0 & 0 \\
\hline Ultimate Strain & 0 & 0 \\
\hline Prestrain & 0 & 0 \\
\hline \multicolumn{3}{|c|}{ Reinforcement (X-Direction) } \\
\hline Ratio (\%) & 0 & 2.32 \\
\hline Yield Strength (MPa) & 0 & 438 \\
\hline Yield Strain & 0 & 0.0025 \\
\hline Strain Hardening Strain & 0 & 0.01 \\
\hline Ultimate Strength (MPa) & 0 & 727 \\
\hline Ultimate Strain & 0 & 0.2 \\
\hline Prestrain & 0 & 0 \\
\hline
\end{tabular}

Numerical Modelling of Hollow Concrete Block Masonry Mechanics 


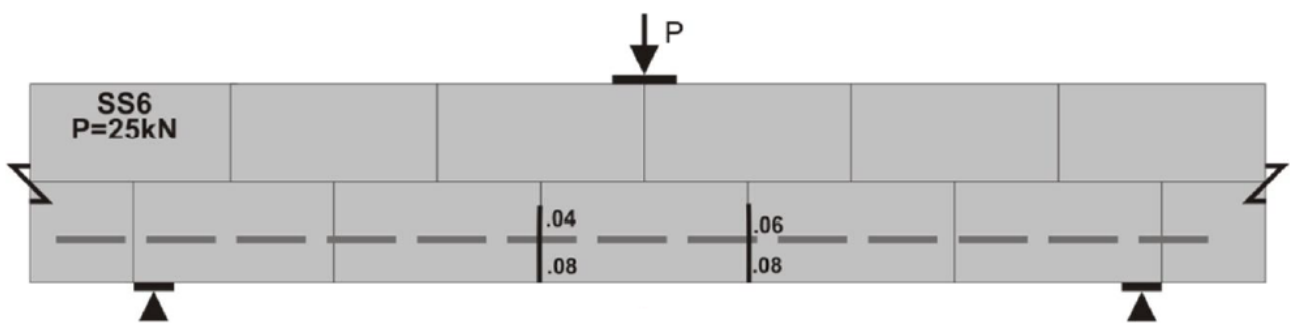

Experiment at $25 \mathrm{kN}$ Load (Sarhat, 2016)

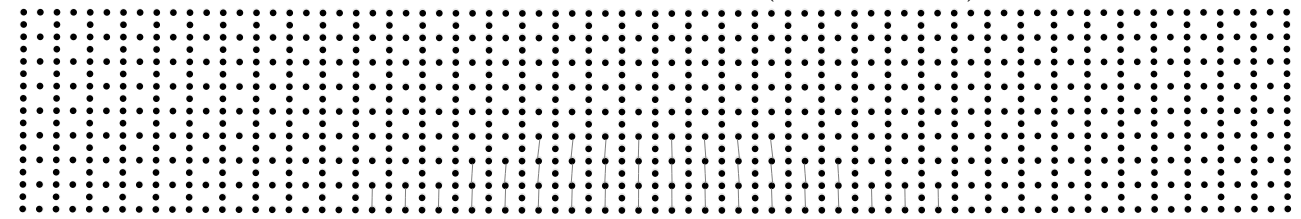

Finite Element Model at $25 \mathrm{kN} \times 15$

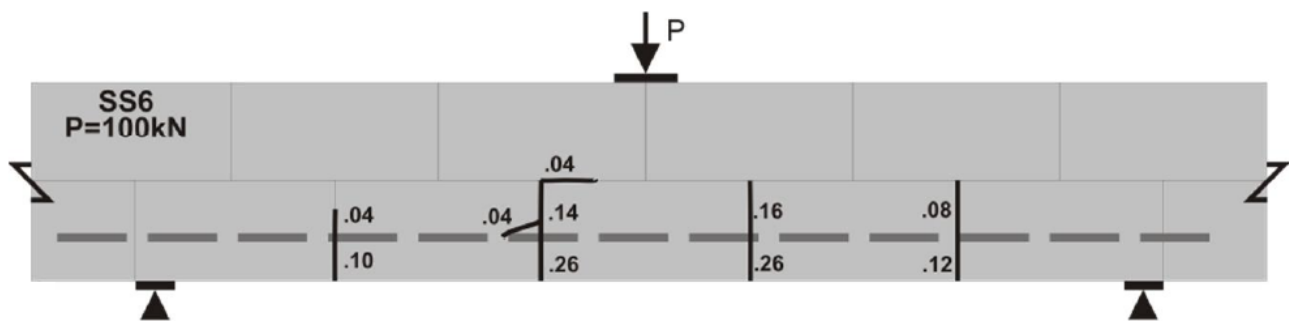

Experiment at $100 \mathrm{kN}$ Load (Sarhat, 2016)

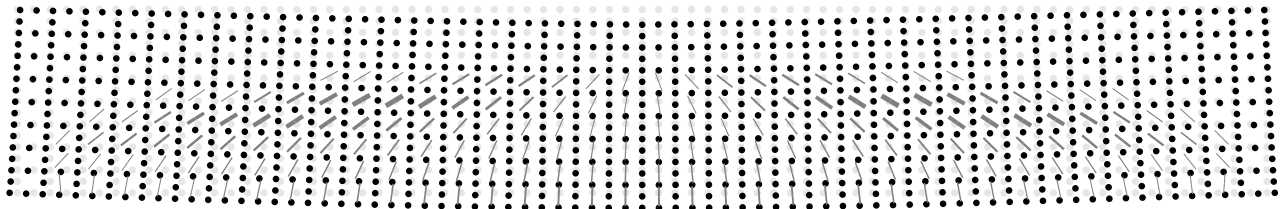

Finite Element Model at $100 \mathrm{kN} \times 15$

SS6 Beam Displacement, Crack Pattern, and Crack Widths 


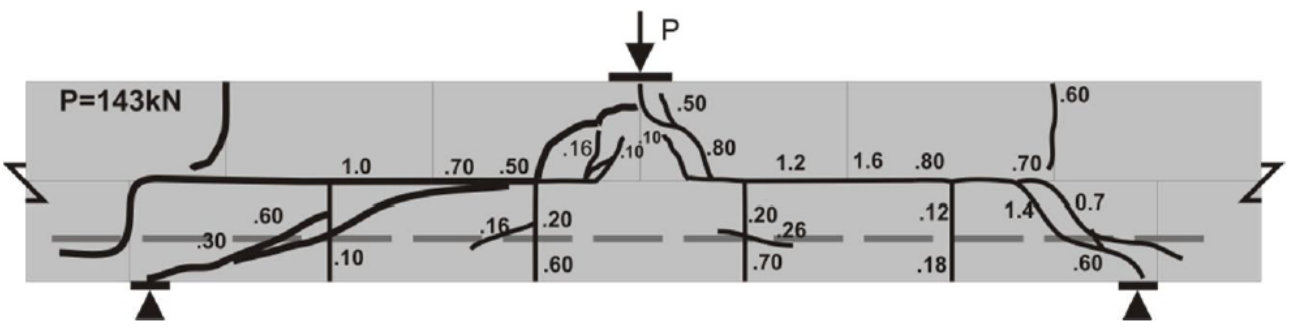

Experiment at Failure (Sarhat, 2016)

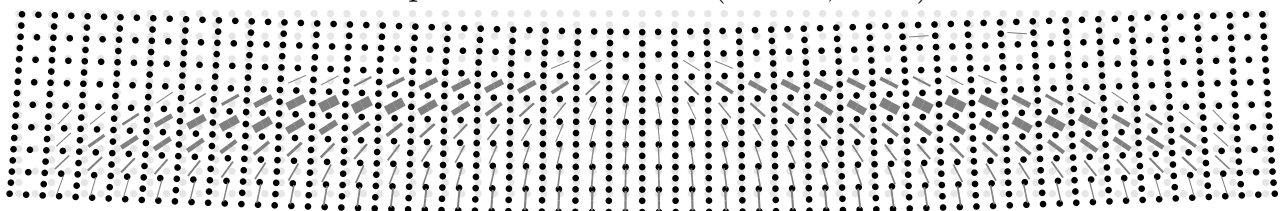

Finite Element Model at Failure $\times 15$

SS6 Beam Displacement, Crack Pattern, and Crack Widths at Failure

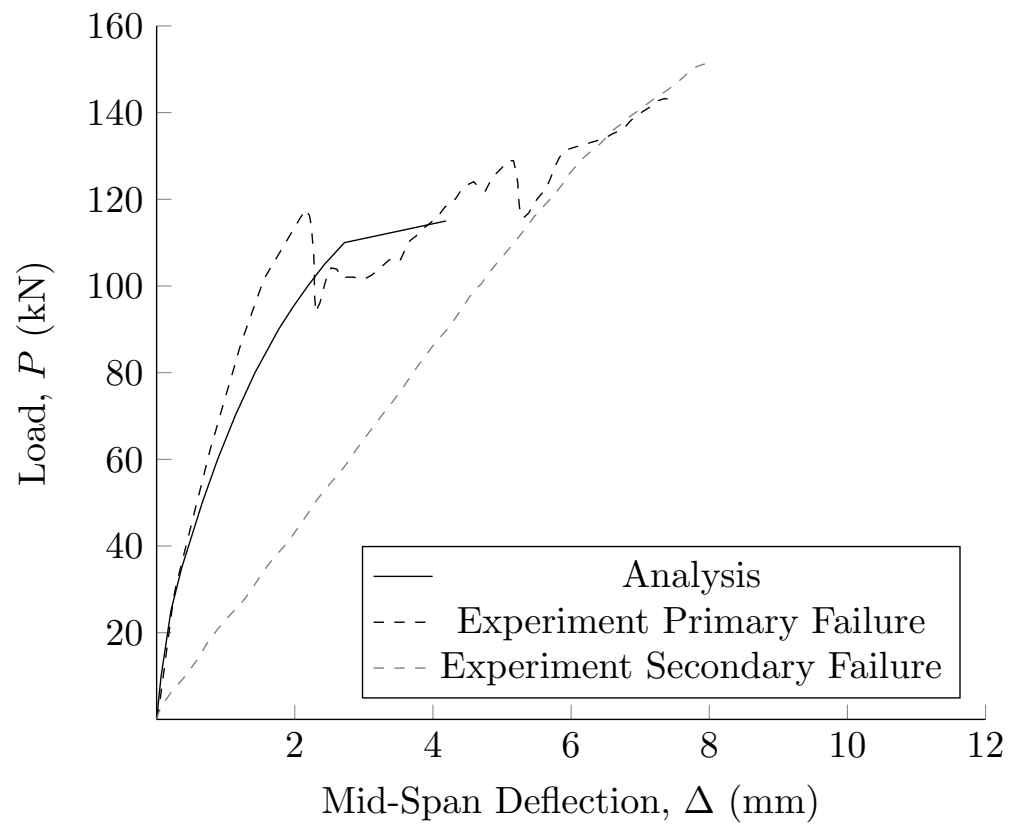

SS6 Beam Sarhat (2016) Load vs Mid-Span Deflection 


\section{SM1 Beam}

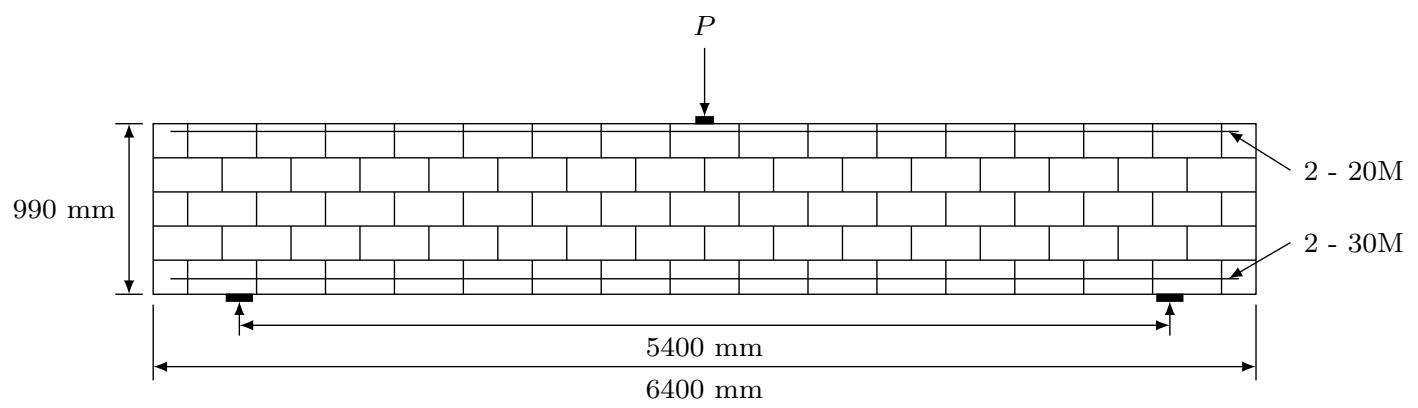

Experimental Beam Sarhat (2016)

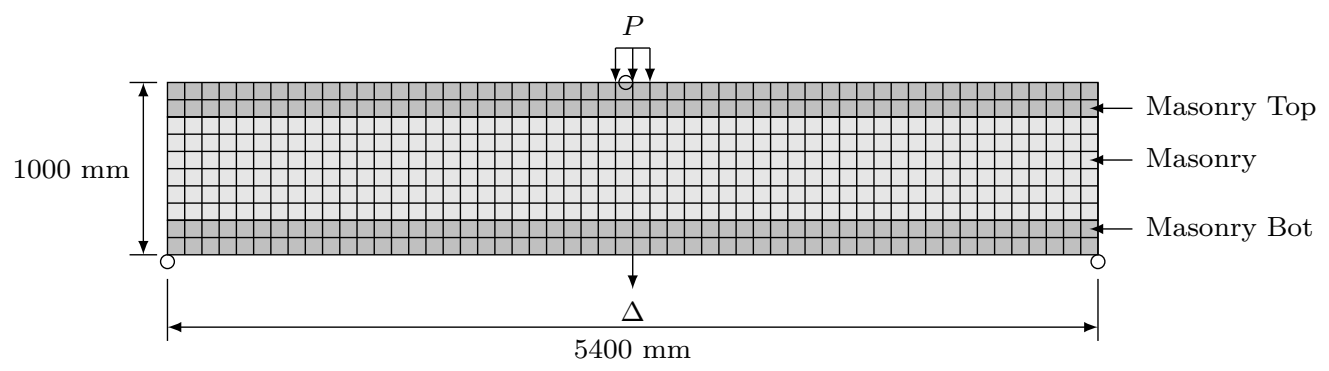

Finite Element Model Beam

SM1 Beam

SM1 Beam (Sarhat, 2016) Model Options

\begin{tabular}{ll}
\hline Model & Selected Option \\
\hline Compression Pre-Peak & Hognestad \\
Compression Post-Peak & Pre-Peak Base Curve \\
Compression Softening & Vecchio \\
Tension Post-Peak & Vecchio \\
Cracking Criterion & Mohr-Coulomb \\
Slip & Walraven Reinforced \\
\hline
\end{tabular}




\section{SM1 Beam (Sarhat, 2016) Material Properties}

\begin{tabular}{|c|c|c|c|}
\hline \multirow{2}{*}{ Property } & \multicolumn{3}{|c|}{ Material } \\
\hline & Masonry Bot & Masonry & Masonry Top \\
\hline Thickness (mm) & 190 & 190 & 190 \\
\hline Grout Tensile Strength (MPa) & 1.74 & 1.74 & 1.74 \\
\hline \multicolumn{4}{|c|}{ Masonry Properties (Y-Direction) } \\
\hline Gross Peak Compression Strength (MPa) & 17.9 & 17.9 & 17.9 \\
\hline Peak Compressive Strain & 0.0013 & 0.0013 & 0.0013 \\
\hline Tensile Strength & 0 & 0 & 0 \\
\hline Tensile Strain & 0 & 0 & 0 \\
\hline Initial Tangent Elastic Modulus (MPa) & 0 & 0 & 0 \\
\hline Poisson's Ratio & 0 & 0 & 0 \\
\hline \multicolumn{4}{|c|}{ Masonry Properties (X-Direction) } \\
\hline Gross Peak Compression Strength (MPa) & 17.9 & 17.9 & 17.9 \\
\hline Peak Compressive Strain & 0.0013 & 0.0013 & 0.0013 \\
\hline Tensile Strength & 0 & 0 & 0 \\
\hline Tensile Strain & 0 & 0 & 0 \\
\hline Initial Tangent Elastic Modulus (MPa) & 0 & 0 & 0 \\
\hline Poisson's Ratio & 0 & 0 & 0 \\
\hline \multicolumn{4}{|c|}{ Masonry Properties (Block) } \\
\hline Length (mm) & 390 & 390 & 390 \\
\hline Height $(\mathrm{mm})$ & 190 & 190 & 190 \\
\hline Face Shell Thickness (mm) & 35 & 35 & 35 \\
\hline Percent Solid (\%) & 56 & 56 & 56 \\
\hline Tensile Strength & 1.99 & 1.99 & 1.99 \\
\hline \multicolumn{4}{|c|}{ Head Joint } \\
\hline Thickness (mm) & 10 & 10 & 10 \\
\hline Tensile Bond Strength (MPa) & 0.35 & 0.35 & 0.35 \\
\hline Joint Cohesion (MPa) & 6.29 & 6.29 & 6.29 \\
\hline Angle of Internal Friction (Deg) & 42 & 42 & 42 \\
\hline \multicolumn{4}{|c|}{ Bed Joint } \\
\hline Thickness (mm) & 10 & 10 & 10 \\
\hline Tensile Bond Strength (MPa) & 0.35 & 0.35 & 0.35 \\
\hline Joint Cohesion (MPa) & 6.29 & 6.29 & 6.29 \\
\hline Angle of Internal Friction (Deg) & 42 & 42 & 42 \\
\hline \multicolumn{4}{|c|}{ Reinforcement (Y-Direction) } \\
\hline Ratio (\%) & 0 & 0 & 0 \\
\hline Yield Strength (MPa) & 0 & 0 & 0 \\
\hline Yield Strain & 0 & 0 & 0 \\
\hline Strain Hardening Strain & 0 & 0 & 0 \\
\hline Ultimate Strength (MPa) & 0 & 0 & 0 \\
\hline Ultimate Strain & 0 & 0 & 0 \\
\hline Prestrain & 0 & 0 & 0 \\
\hline \multicolumn{4}{|c|}{ Reinforcement (X-Direction) } \\
\hline Ratio (\%) & 2.23 & 0 & 1.62 \\
\hline Yield Strength (MPa) & 438 & 0 & 398 \\
\hline Yield Strain & 0.0025 & 0 & 0.002 \\
\hline Strain Hardening Strain & 0.01 & 0 & 0.01 \\
\hline Ultimate Strength (MPa) & 727 & 0 & 639 \\
\hline Ultimate Strain & 0.2 & 0 & 0.2 \\
\hline Prestrain & 0 & 0 & 0 \\
\hline
\end{tabular}

Numerical Modelling of Hollow Concrete Block Masonry Mechanics 


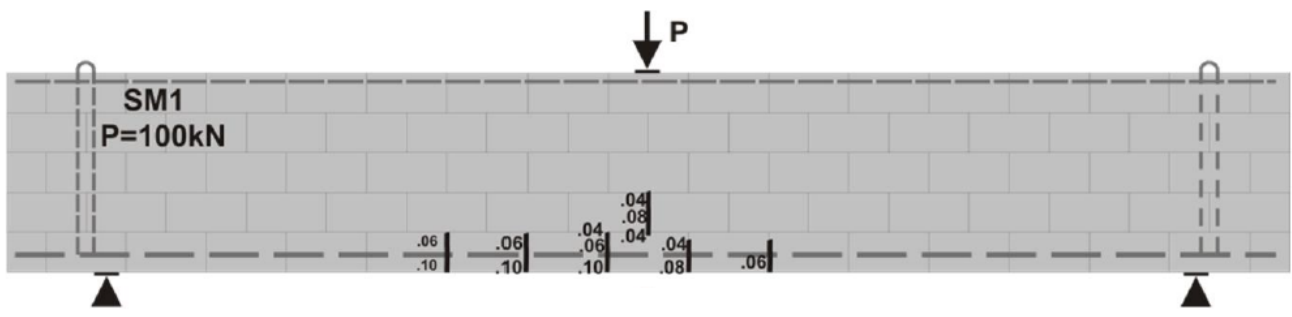

Experiment at $100 \mathrm{kN}$ Load (Sarhat, 2016)

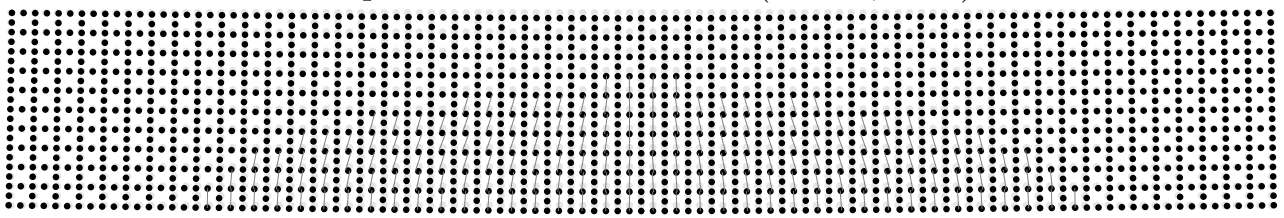

Finite Element Model at $100 \mathrm{kN} \times 15$

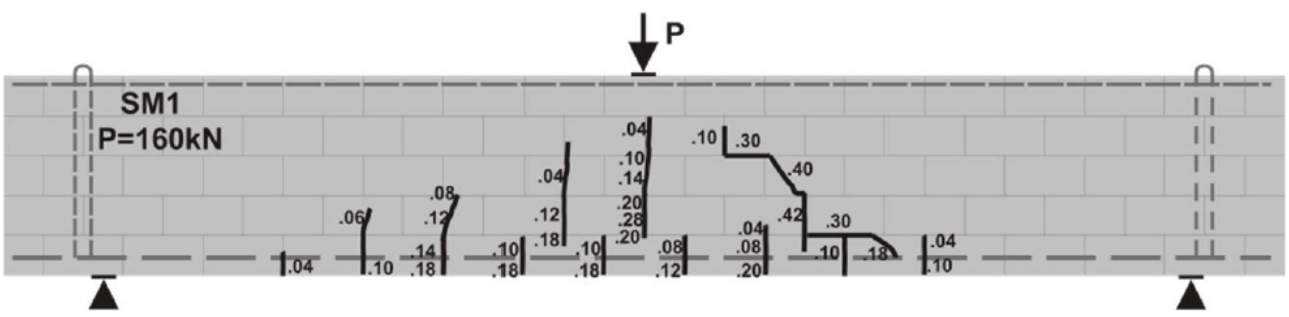

Experiment at $160 \mathrm{kN}$ Load (Sarhat, 2016)

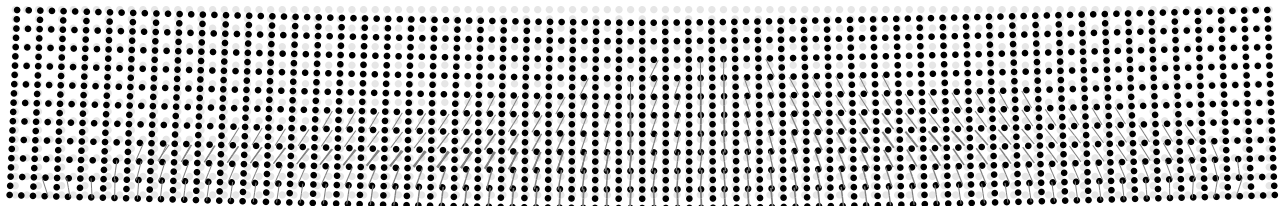

Finite Element Model at $160 \mathrm{kN} \times 15$

SM1 Beam Displacement, Crack Pattern, and Crack Widths 


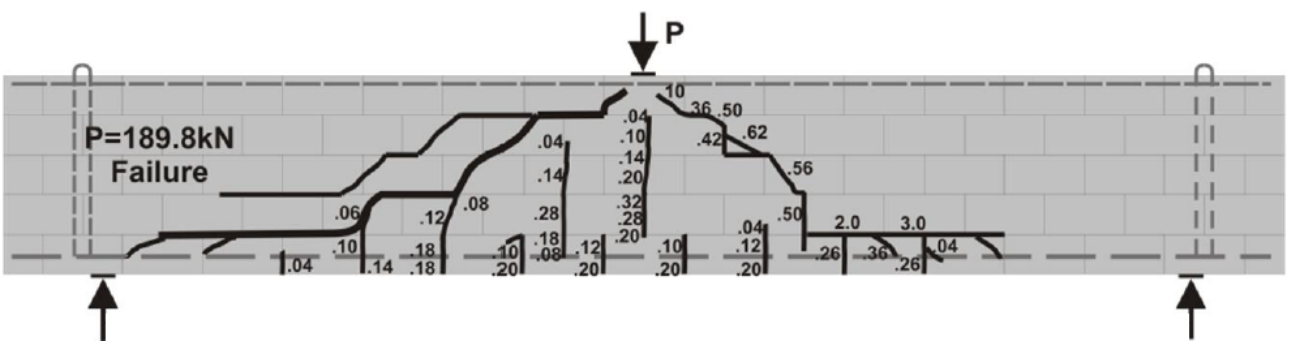

Experiment at Failure (Sarhat, 2016)

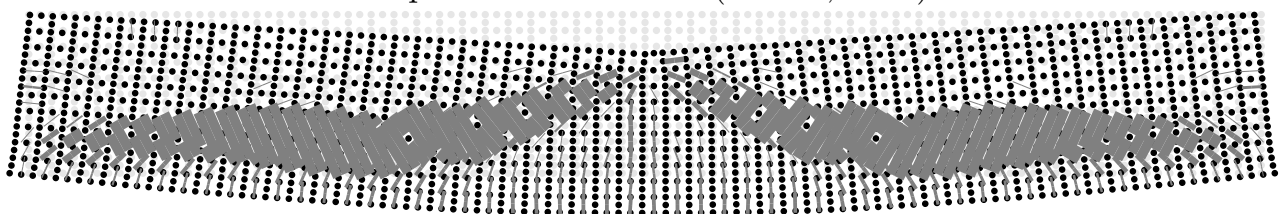

Finite Element Model at Failure $\times 15$

SM1 Beam Displacement, Crack Pattern, and Crack Widths at Failure

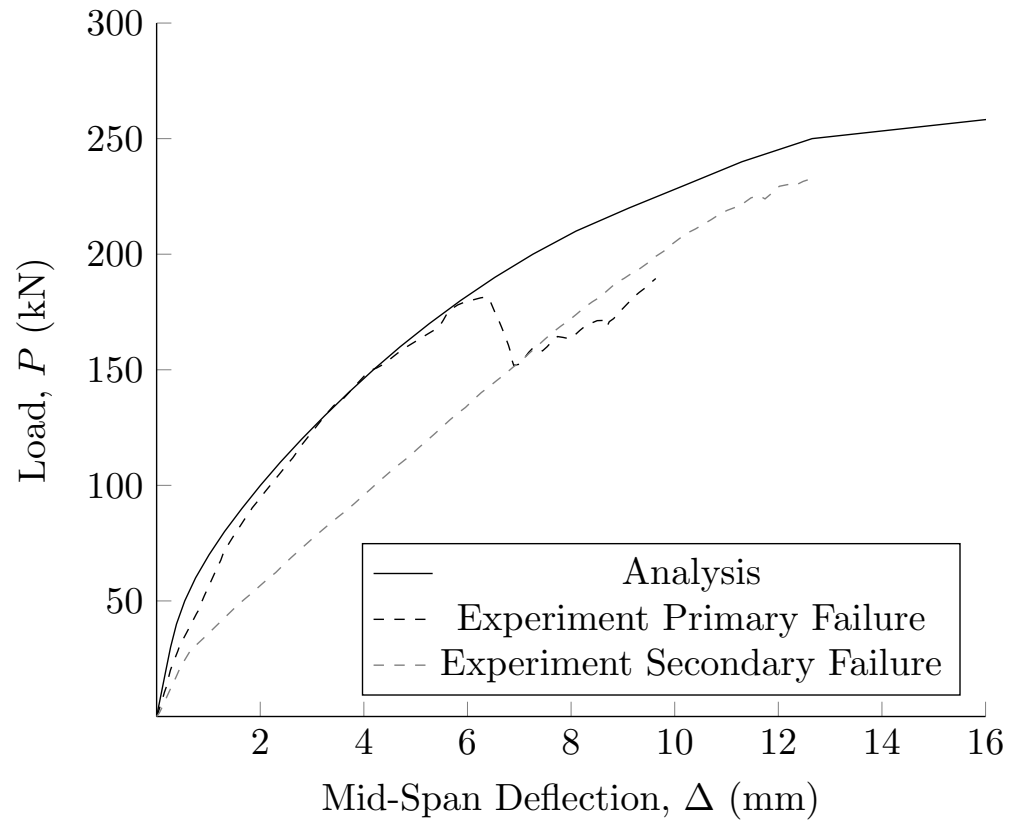

SM1 Beam Sarhat (2016) Load vs Mid-Span Deflection 


\section{SM2 Beam}
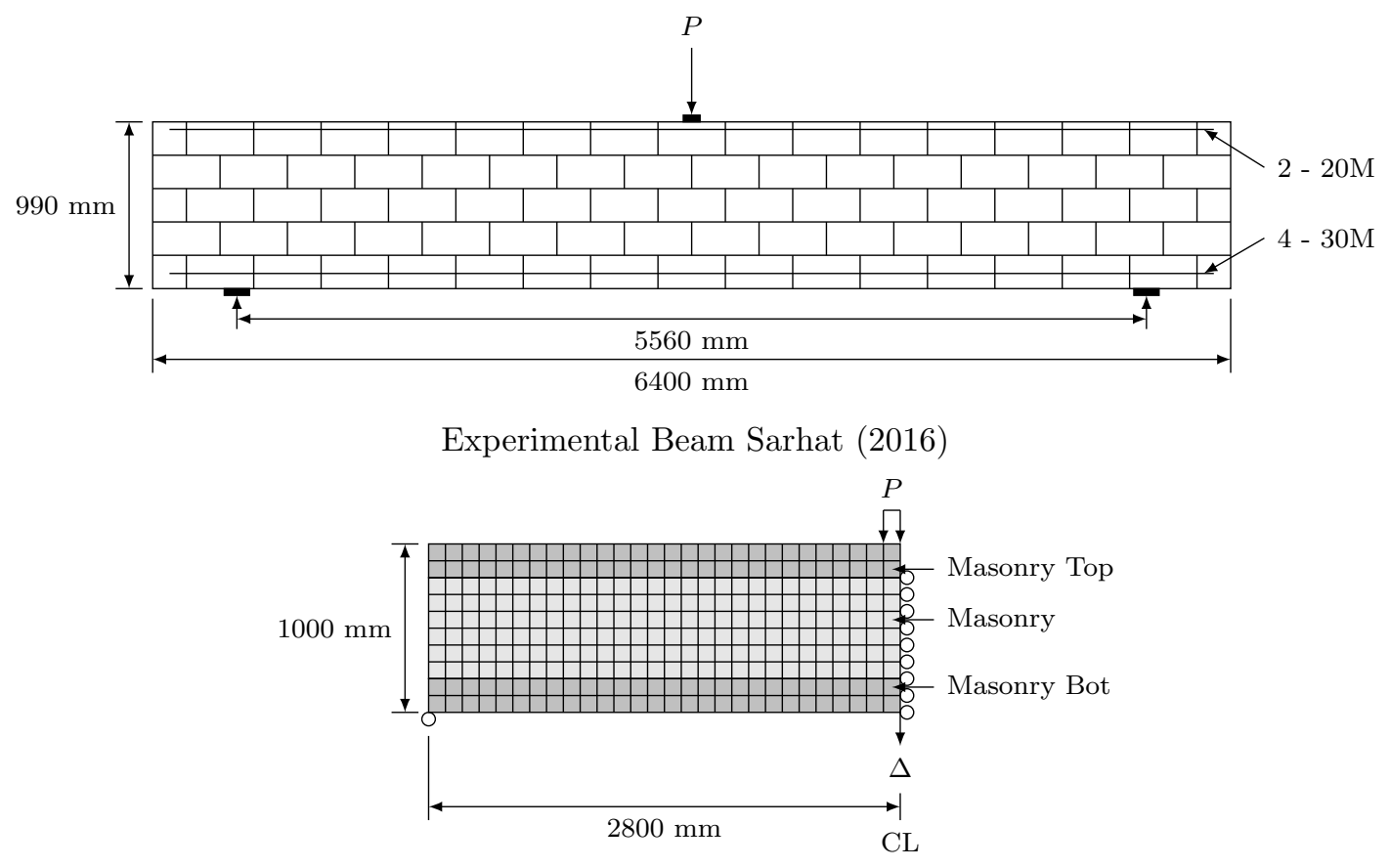

Finite Element Model Beam

SM2 Beam

SM2 Beam (Sarhat, 2016) Model Options

\begin{tabular}{ll}
\hline Model & Selected Option \\
\hline Compression Pre-Peak & Hognestad \\
Compression Post-Peak & Pre-Peak Base Curve \\
Compression Softening & Vecchio \\
Tension Post-Peak & Vecchio \\
Cracking Criterion & Mohr-Coulomb \\
Slip & Walraven Reinforced \\
\hline
\end{tabular}


SM2 Beam (Sarhat, 2016) Material Properties

\begin{tabular}{|c|c|c|c|}
\hline \multirow{2}{*}{ Property } & \multicolumn{3}{|c|}{ Material } \\
\hline & Masonry Bot & Masonry & Masonry Top \\
\hline Thickness (mm) & 190 & 190 & 190 \\
\hline Grout Tensile Strength (MPa) & 1.73 & 1.73 & 1.73 \\
\hline \multicolumn{4}{|c|}{ Masonry Properties (Y-Direction) } \\
\hline Gross Peak Compression Strength (MPa) & 17.9 & 17.9 & 17.9 \\
\hline Peak Compressive Strain & 0.0012 & 0.0012 & 0.0012 \\
\hline Tensile Strength & 0 & 0 & 0 \\
\hline Tensile Strain & 0 & 0 & 0 \\
\hline Initial Tangent Elastic Modulus (MPa) & 0 & 0 & 0 \\
\hline Poisson's Ratio & 0 & 0 & 0 \\
\hline \multicolumn{4}{|c|}{ Masonry Properties (X-Direction) } \\
\hline Gross Peak Compression Strength (MPa) & 17.9 & 17.9 & 17.9 \\
\hline Peak Compressive Strain & 0.0012 & 0.0012 & 0.0012 \\
\hline Tensile Strength & 0 & 0 & 0 \\
\hline Tensile Strain & 0 & 0 & 0 \\
\hline Initial Tangent Elastic Modulus (MPa) & 0 & 0 & 0 \\
\hline Poisson's Ratio & 0 & 0 & 0 \\
\hline \multicolumn{4}{|c|}{ Masonry Properties (Block) } \\
\hline Length $(\mathrm{mm})$ & 390 & 390 & 390 \\
\hline Height (mm) & 190 & 190 & 190 \\
\hline Face Shell Thickness (mm) & 35 & 35 & 35 \\
\hline Percent Solid (\%) & 56 & 56 & 56 \\
\hline Tensile Strength & 1.99 & 1.99 & 1.99 \\
\hline \multicolumn{4}{|c|}{ Head Joint } \\
\hline Thickness (mm) & 10 & 10 & 10 \\
\hline Tensile Bond Strength (MPa) & 0.36 & 0.36 & 0.36 \\
\hline Joint Cohesion (MPa) & 5.87 & 5.87 & 5.87 \\
\hline Angle of Internal Friction (Deg) & 42 & 42 & 42 \\
\hline \multicolumn{4}{|c|}{ Bed Joint } \\
\hline Thickness (mm) & 10 & 10 & 10 \\
\hline Tensile Bond Strength (MPa) & 0.36 & 0.36 & 0.36 \\
\hline Joint Cohesion (MPa) & 5.87 & 5.87 & 5.87 \\
\hline Angle of Internal Friction (Deg) & 42 & 42 & 42 \\
\hline \multicolumn{4}{|c|}{ Reinforcement (Y-Direction) } \\
\hline Ratio (\%) & 0 & 0 & 0 \\
\hline Yield Strength (MPa) & 0 & 0 & 0 \\
\hline Yield Strain & 0 & 0 & 0 \\
\hline Strain Hardening Strain & 0 & 0 & 0 \\
\hline Ultimate Strength (MPa) & 0 & 0 & 0 \\
\hline Ultimate Strain & 0 & 0 & 0 \\
\hline Prestrain & 0 & 0 & 0 \\
\hline \multicolumn{4}{|c|}{ Reinforcement (X-Direction) } \\
\hline Ratio (\%) & 4.33 & 0 & 1.62 \\
\hline Yield Strength (MPa) & 438 & 0 & 398 \\
\hline Yield Strain & 0.0025 & 0 & 0.002 \\
\hline Strain Hardening Strain & 0.01 & 0 & 0.01 \\
\hline Ultimate Strength (MPa) & 727 & 0 & 639 \\
\hline Ultimate Strain & 0.2 & 0 & 0.2 \\
\hline Prestrain & 0 & 0 & 0 \\
\hline
\end{tabular}

Numerical Modelling of Hollow Concrete Block Masonry Mechanics 


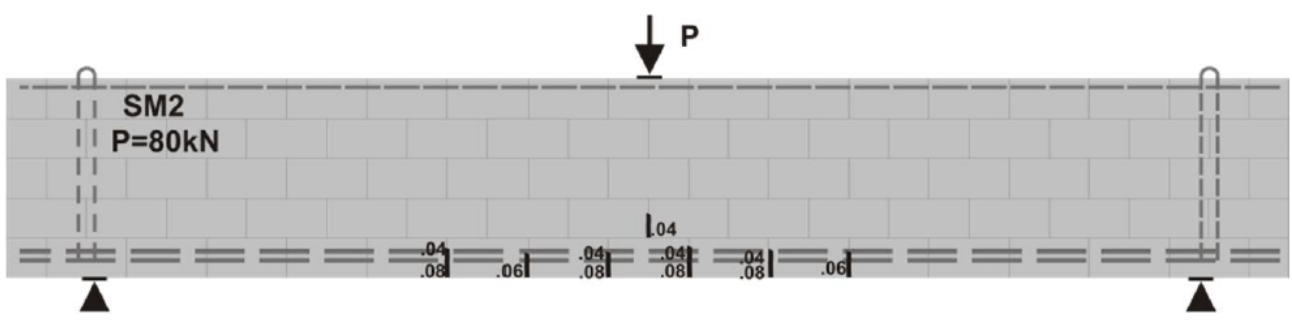

Experiment at $80 \mathrm{kN}$ Load (Sarhat, 2016)

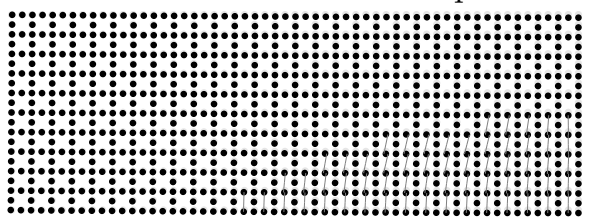

Finite Element Model at $80 \mathrm{kN} \times 15$

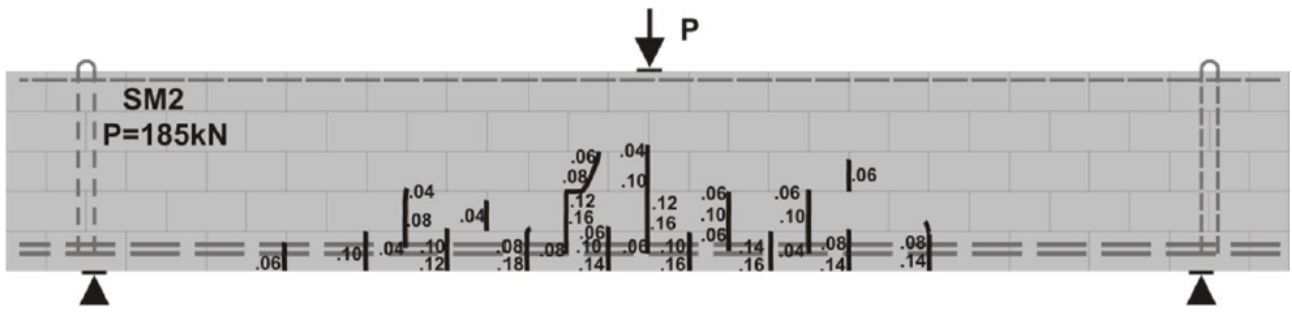

Experiment at $185 \mathrm{kN}$ Load (Sarhat, 2016)

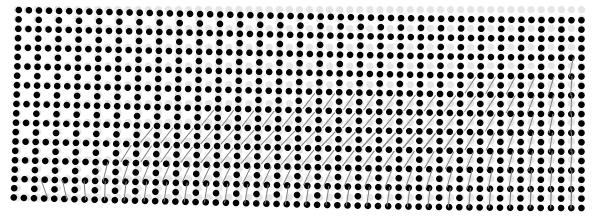

Finite Element Model at $180 \mathrm{kN} \times 15$

SM2 Beam Displacement, Crack Pattern, and Crack Widths 


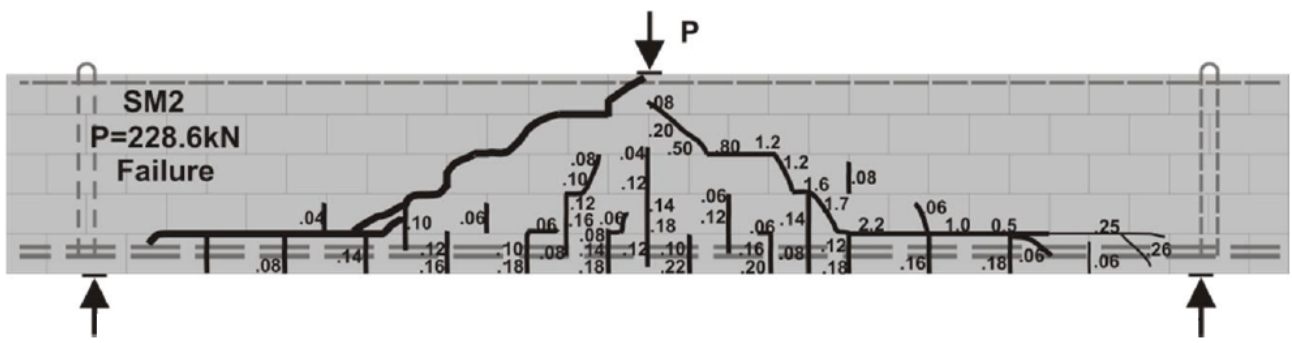

Experiment at Failure (Sarhat, 2016)

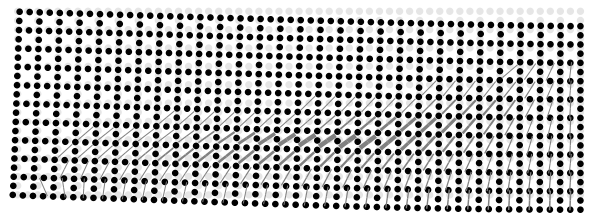

Finite Element Model at Failure $\times 15$

SM2 Beam Displacement, Crack Pattern, and Crack Widths at Failure

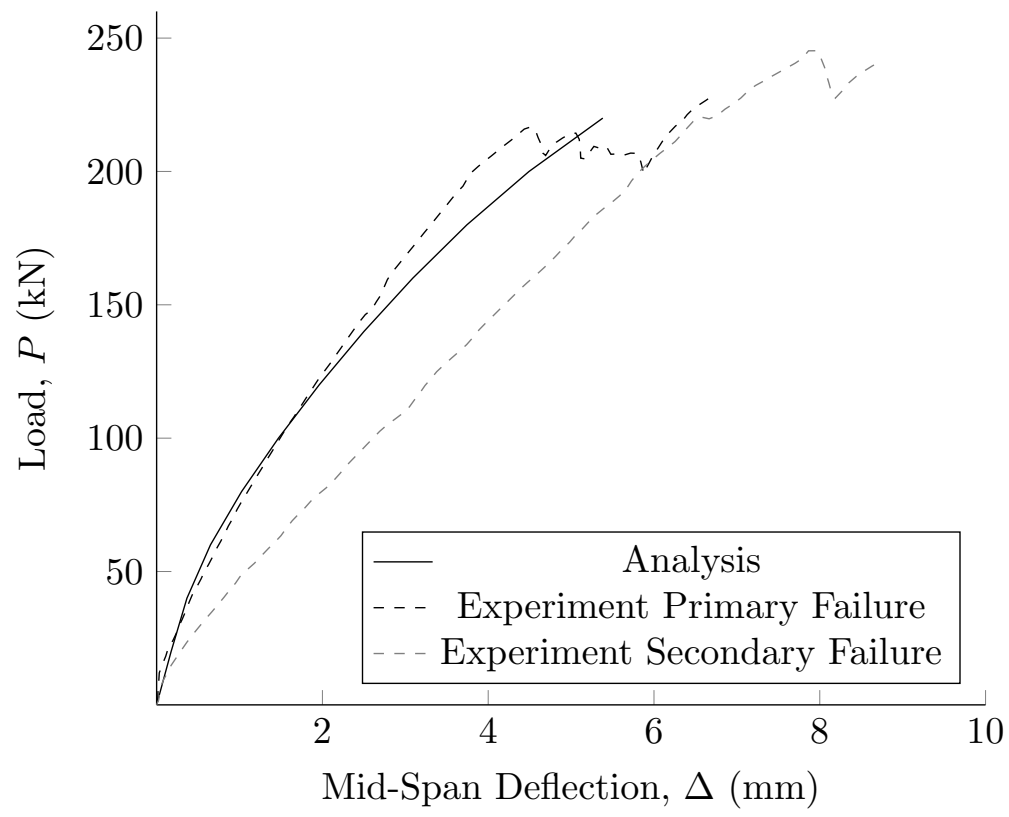

SM2 Beam Sarhat (2016) Load vs Mid-Span Deflection 


\section{SM3 Beam}
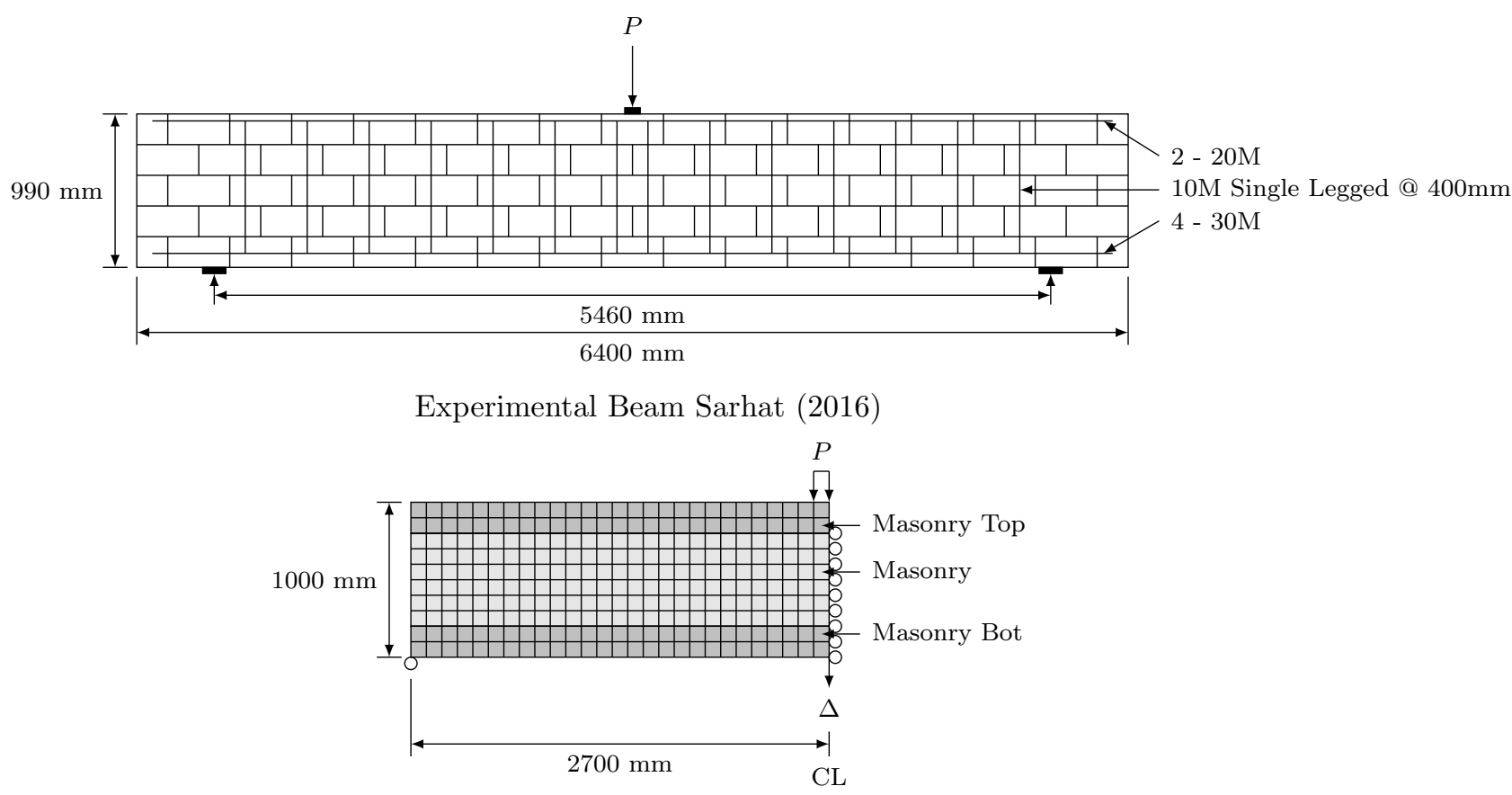

Finite Element Model Beam

SM3 Beam

SM3 Beam (Sarhat, 2016) Model Options

\begin{tabular}{ll}
\hline Model & Selected Option \\
\hline Compression Pre-Peak & Hognestad \\
Compression Post-Peak & Pre-Peak Base Curve \\
Compression Softening & Vecchio \\
Tension Post-Peak & Vecchio \\
Cracking Criterion & Mohr-Coulomb \\
Slip & Walraven Reinforced \\
\hline
\end{tabular}


SM3 Beam (Sarhat, 2016) Material Properties

\begin{tabular}{|c|c|c|c|}
\hline \multirow{2}{*}{ Property } & \multicolumn{3}{|c|}{ Material } \\
\hline & Masonry Bot & Masonry & Masonry Top \\
\hline Thickness (mm) & 190 & 190 & 190 \\
\hline Grout Tensile Strength (MPa) & 1.79 & 1.79 & 1.79 \\
\hline \multicolumn{4}{|c|}{ Masonry Properties (Y-Direction) } \\
\hline Gross Peak Compression Strength (MPa) & 17.9 & 17.9 & 17.9 \\
\hline Peak Compressive Strain & 0.0012 & 0.0012 & 0.0012 \\
\hline Tensile Strength & 0 & 0 & 0 \\
\hline Tensile Strain & 0 & 0 & 0 \\
\hline Initial Tangent Elastic Modulus (MPa) & 0 & 0 & 0 \\
\hline Poisson's Ratio & 0 & 0 & 0 \\
\hline \multicolumn{4}{|c|}{ Masonry Properties (X-Direction) } \\
\hline Gross Peak Compression Strength (MPa) & 17.9 & 17.9 & 17.9 \\
\hline Peak Compressive Strain & 0.0012 & 0.0012 & 0.0012 \\
\hline Tensile Strength & 0 & 0 & 0 \\
\hline Tensile Strain & 0 & 0 & 0 \\
\hline Initial Tangent Elastic Modulus (MPa) & 0 & 0 & 0 \\
\hline Poisson's Ratio & 0 & 0 & 0 \\
\hline \multicolumn{4}{|c|}{ Masonry Properties (Block) } \\
\hline Length $(\mathrm{mm})$ & 390 & 390 & 390 \\
\hline Height (mm) & 190 & 190 & 190 \\
\hline Face Shell Thickness (mm) & 35 & 35 & 35 \\
\hline Percent Solid (\%) & 56 & 56 & 56 \\
\hline Tensile Strength & 2.02 & 2.02 & 2.02 \\
\hline \multicolumn{4}{|c|}{ Head Joint } \\
\hline Thickness (mm) & 10 & 10 & 10 \\
\hline Tensile Bond Strength (MPa) & 0.45 & 0.45 & 0.45 \\
\hline Joint Cohesion (MPa) & 7.54 & 7.54 & 7.54 \\
\hline Angle of Internal Friction (Deg) & 42 & 42 & 42 \\
\hline \multicolumn{4}{|c|}{ Bed Joint } \\
\hline Thickness $(\mathrm{mm})$ & 10 & 10 & 10 \\
\hline Tensile Bond Strength (MPa) & 0.45 & 0.45 & 0.45 \\
\hline Joint Cohesion (MPa) & 7.54 & 7.54 & 7.54 \\
\hline Angle of Internal Friction (Deg) & 42 & 42 & 42 \\
\hline \multicolumn{4}{|c|}{ Reinforcement (Y-Direction) } \\
\hline Ratio (\%) & 0.13 & 0.13 & 0.13 \\
\hline Yield Strength (MPa) & 425 & 425 & 425 \\
\hline Yield Strain & 0.0025 & 0.0025 & 0.0025 \\
\hline Strain Hardening Strain & 0.01 & 0.01 & 0.01 \\
\hline Ultimate Strength (MPa) & 650 & 650 & 650 \\
\hline Ultimate Strain & 0.2 & 0.2 & 0.2 \\
\hline Prestrain & 0 & 0 & 0 \\
\hline \multicolumn{4}{|c|}{ Reinforcement (X-Direction) } \\
\hline Ratio (\%) & 4.33 & 0 & 1.62 \\
\hline Yield Strength (MPa) & 438 & 0 & 398 \\
\hline Yield Strain & 0.0025 & 0 & 0.002 \\
\hline Strain Hardening Strain & 0.01 & 0 & 0.01 \\
\hline Ultimate Strength (MPa) & 727 & 0 & 639 \\
\hline Ultimate Strain & 0.2 & 0 & 0.2 \\
\hline Prestrain & 0 & 0 & 0 \\
\hline
\end{tabular}

Numerical Modelling of Hollow Concrete Block Masonry Mechanics 


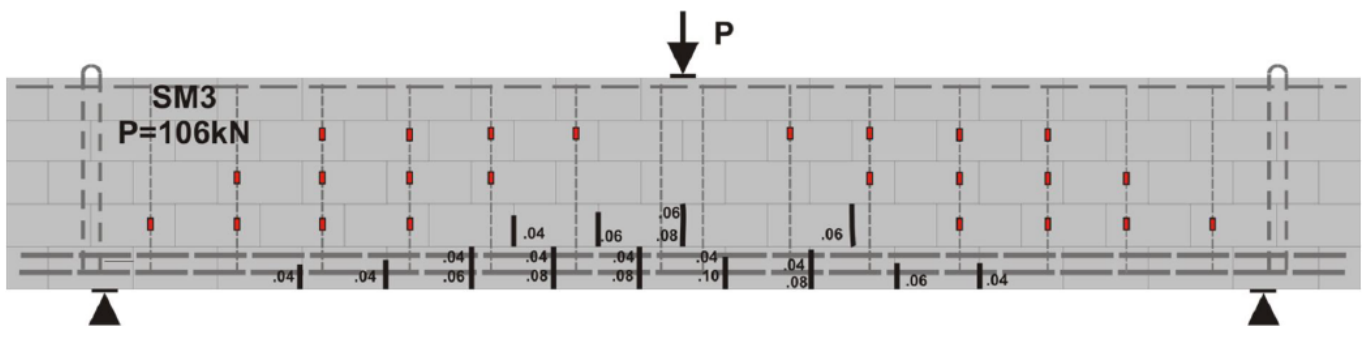

Experiment at $106 \mathrm{kN}$ Load (Sarhat, 2016)

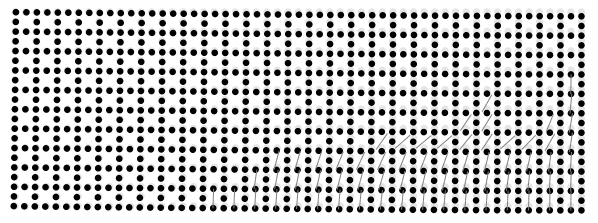

Finite Element Model at $100 \mathrm{kN} \times 15$

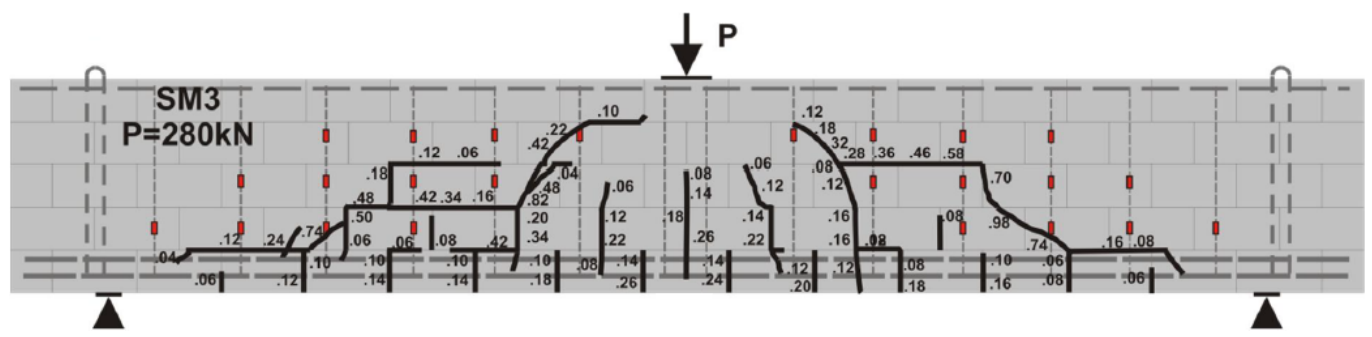

Experiment at $280 \mathrm{kN}$ Load (Sarhat, 2016)

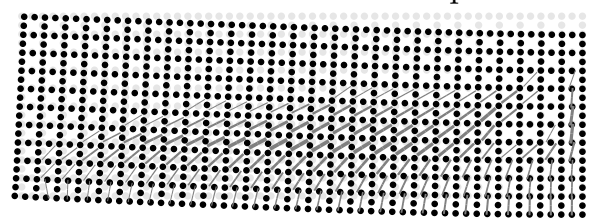

Finite Element Model at $280 \mathrm{kN} \times 15$

SM3 Beam Displacement, Crack Pattern, and Crack Widths 


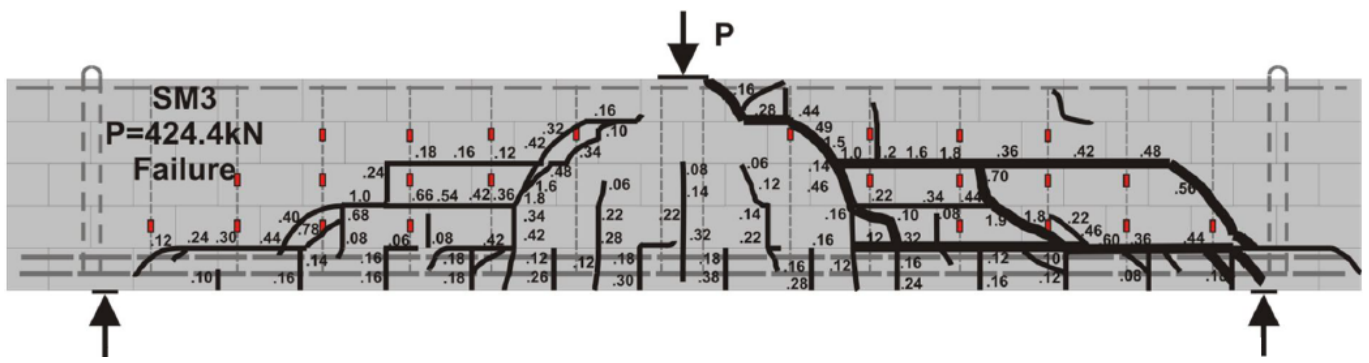

Experiment at Failure (Sarhat, 2016)

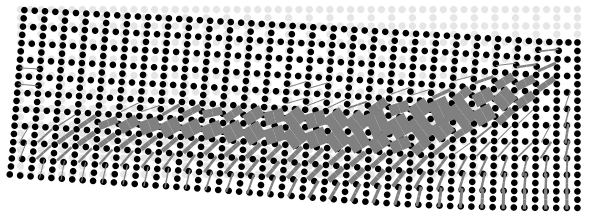

Finite Element Model at Failure $\times 15$

SM3 Beam Displacement, Crack Pattern, and Crack Widths at Failure

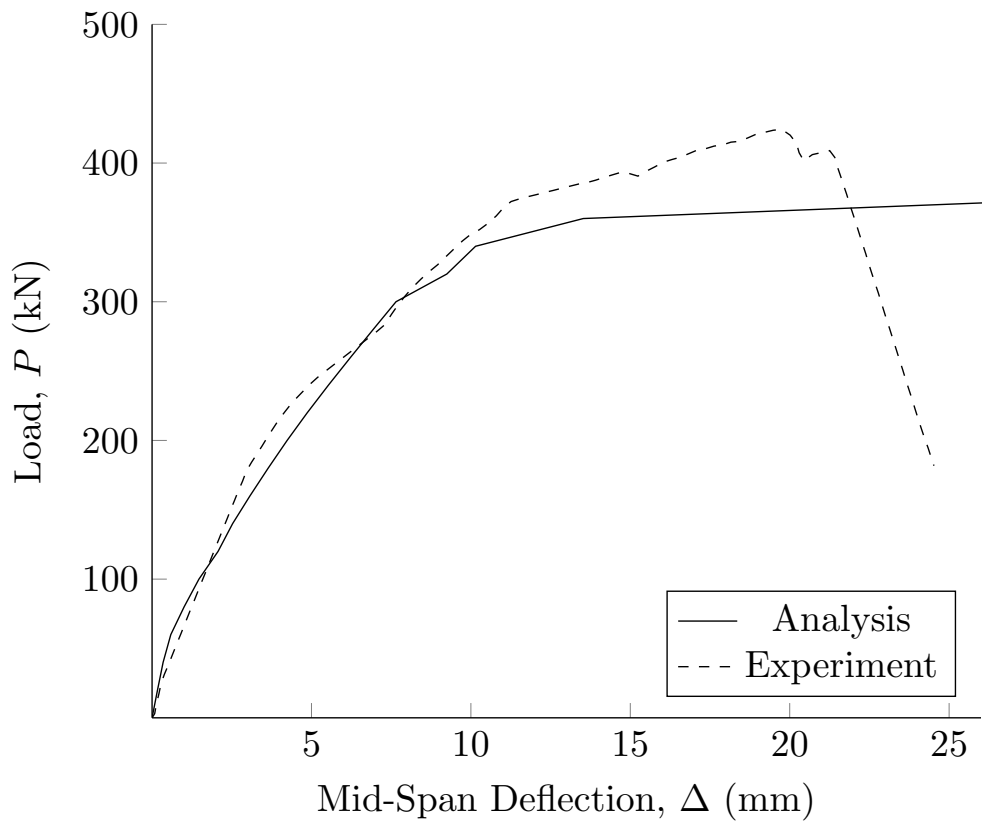

SM3 Beam Sarhat (2016) Load vs Mid-Span Deflection 


\section{SM6 Beam}
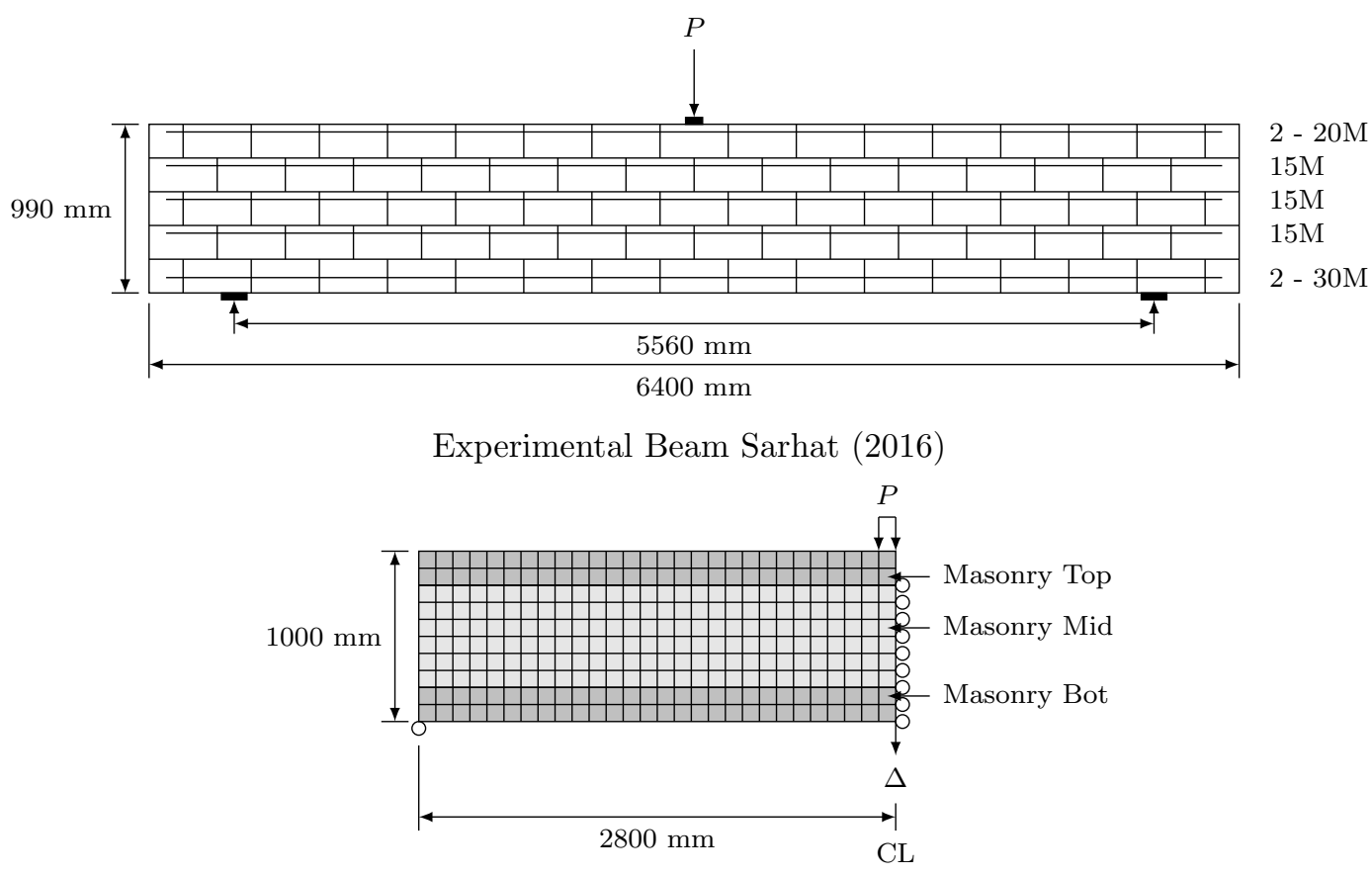

Finite Element Model Beam

SM6 Beam

SM6 Beam (Sarhat, 2016) Model Options

\begin{tabular}{ll}
\hline Model & Selected Option \\
\hline Compression Pre-Peak & Hognestad \\
Compression Post-Peak & Pre-Peak Base Curve \\
Compression Softening & Vecchio \\
Tension Post-Peak & Vecchio \\
Cracking Criterion & Mohr-Coulomb \\
Slip & Walraven Reinforced \\
\hline
\end{tabular}


SM6 Beam (Sarhat, 2016) Material Properties

\begin{tabular}{|c|c|c|c|}
\hline \multirow{2}{*}{ Property } & \multicolumn{3}{|c|}{ Material } \\
\hline & Masonry Bot & Masonry Mid & Masonry Top \\
\hline Thickness (mm) & 190 & 190 & 190 \\
\hline Grout Tensile Strength $(\mathrm{MPa})$ & 1.63 & 1.63 & 1.63 \\
\hline \multicolumn{4}{|c|}{ Masonry Properties (Y-Direction) } \\
\hline Gross Peak Compression Strength (MPa) & 18.0 & 18.0 & 18.0 \\
\hline Peak Compressive Strain & 0.0012 & 0.0012 & 0.0012 \\
\hline Tensile Strength & 0 & 0 & 0 \\
\hline Tensile Strain & 0 & 0 & 0 \\
\hline Initial Tangent Elastic Modulus (MPa) & 0 & 0 & 0 \\
\hline Poisson's Ratio & 0 & 0 & 0 \\
\hline \multicolumn{4}{|c|}{ Masonry Properties (X-Direction) } \\
\hline Gross Peak Compression Strength (MPa) & 18.0 & 18.0 & 18.0 \\
\hline Peak Compressive Strain & 0.0012 & 0.0012 & 0.0012 \\
\hline Tensile Strength & 0 & 0 & 0 \\
\hline Tensile Strain & 0 & 0 & 0 \\
\hline Initial Tangent Elastic Modulus (MPa) & 0 & 0 & 0 \\
\hline Poisson's Ratio & 0 & 0 & 0 \\
\hline \multicolumn{4}{|c|}{ Masonry Properties (Block) } \\
\hline Length $(\mathrm{mm})$ & 390 & 390 & 390 \\
\hline Height (mm) & 190 & 190 & 190 \\
\hline Face Shell Thickness (mm) & 35 & 35 & 35 \\
\hline Percent Solid $(\%)$ & 56 & 56 & 56 \\
\hline Tensile Strength & 2.02 & 2.02 & 2.02 \\
\hline \multicolumn{4}{|c|}{ Head Joint } \\
\hline Thickness (mm) & 10 & 10 & 10 \\
\hline Tensile Bond Strength (MPa) & 0.30 & 0.30 & 0.30 \\
\hline Joint Cohesion (MPa) & 5.89 & 5.89 & 5.89 \\
\hline Angle of Internal Friction (Deg) & 42 & 42 & 42 \\
\hline \multicolumn{4}{|c|}{ Bed Joint } \\
\hline Thickness (mm) & 10 & 10 & 10 \\
\hline Tensile Bond Strength (MPa) & 0.30 & 0.30 & 0.30 \\
\hline Joint Cohesion $(\mathrm{MPa})$ & 5.89 & 5.89 & 5.89 \\
\hline Angle of Internal Friction (Deg) & 42 & 42 & 42 \\
\hline \multicolumn{4}{|c|}{ Reinforcement (Y-Direction) } \\
\hline Ratio (\%) & 0 & 0 & 0 \\
\hline Yield Strength (MPa) & 0 & 0 & 0 \\
\hline Yield Strain & 0 & 0 & 0 \\
\hline Strain Hardening Strain & 0 & 0 & 0 \\
\hline Ultimate Strength $(\mathrm{MPa})$ & 0 & 0 & 0 \\
\hline Ultimate Strain & 0 & 0 & 0 \\
\hline Prestrain & 0 & 0 & 0 \\
\hline \multicolumn{4}{|c|}{ Reinforcement (X-Direction) } \\
\hline Ratio (\%) & 2.20 & 0.58 & 1.62 \\
\hline Yield Strength (MPa) & 438 & 425 & 398 \\
\hline Yield Strain & 0.0025 & 0.0025 & 0.002 \\
\hline Strain Hardening Strain & 0.01 & 0.01 & 0.01 \\
\hline Ultimate Strength $(\mathrm{MPa})$ & 727 & 650 & 639 \\
\hline Ultimate Strain & 0.2 & 0.2 & 0.2 \\
\hline Prestrain & 0 & 0 & 0 \\
\hline
\end{tabular}

Numerical Modelling of Hollow Concrete Block Masonry Mechanics 


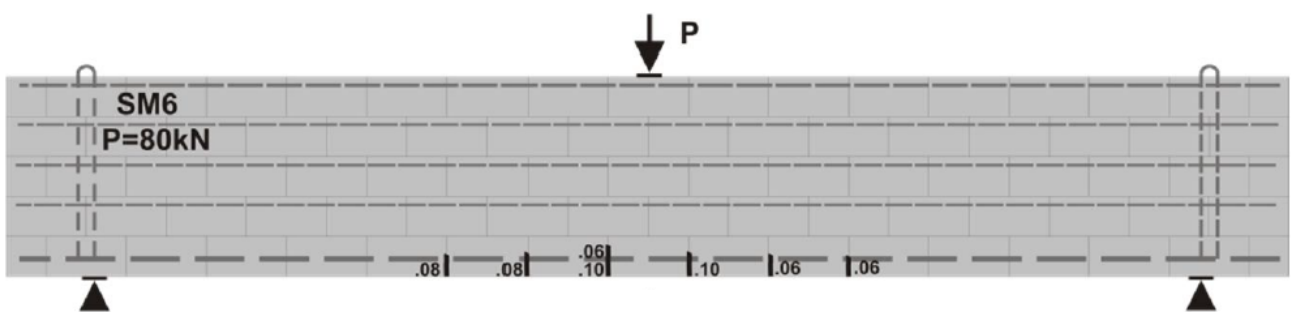

Experiment at $80 \mathrm{kN}$ Load (Sarhat, 2016)

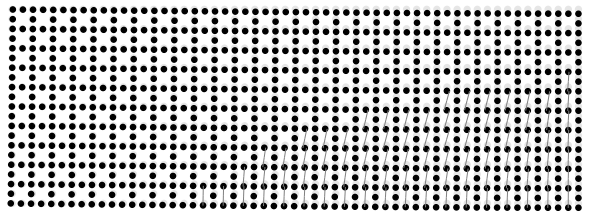

Finite Element Model at $80 \mathrm{kN} \times 15$

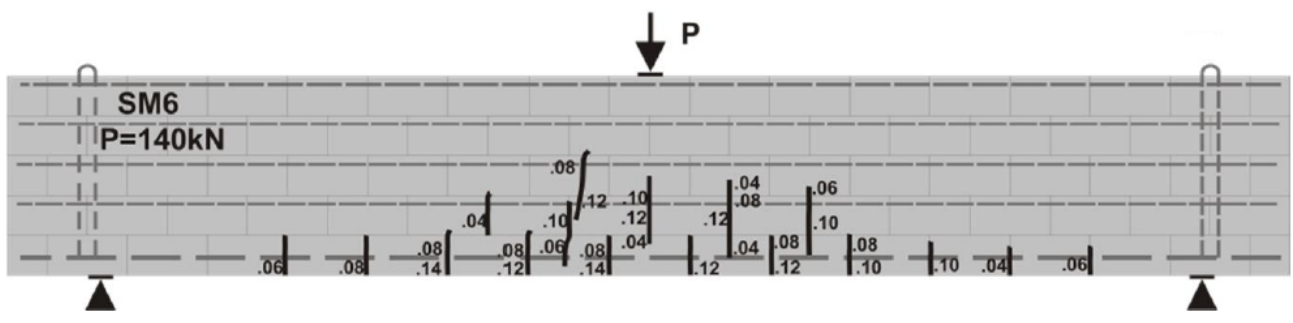

Experiment at $140 \mathrm{kN}$ Load (Sarhat, 2016)

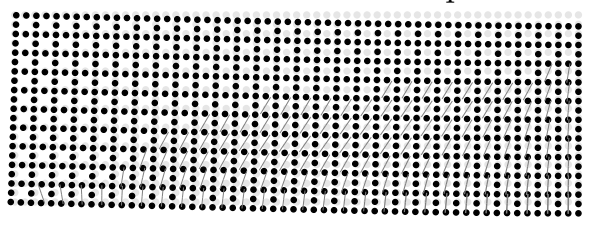

Finite Element Model at $140 \mathrm{kN} \times 15$

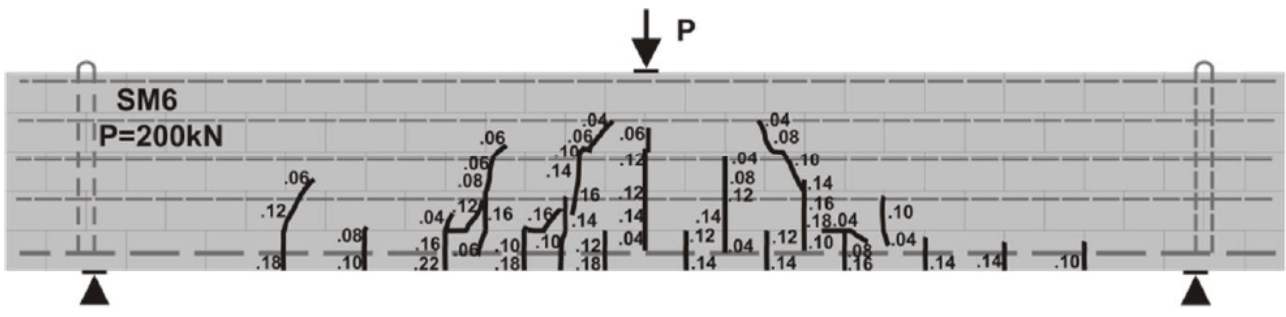

Experiment at $200 \mathrm{kN}$ Load (Sarhat, 2016)

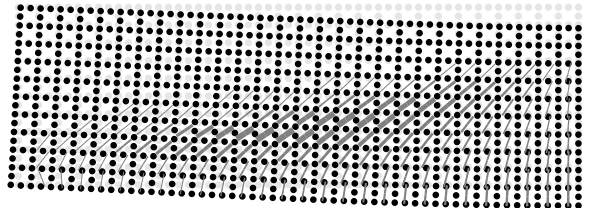

Finite Element Model at $200 \mathrm{kN} \times 15$

SM6 Beam Displacement, Crack Pattern, and Crack Widths 


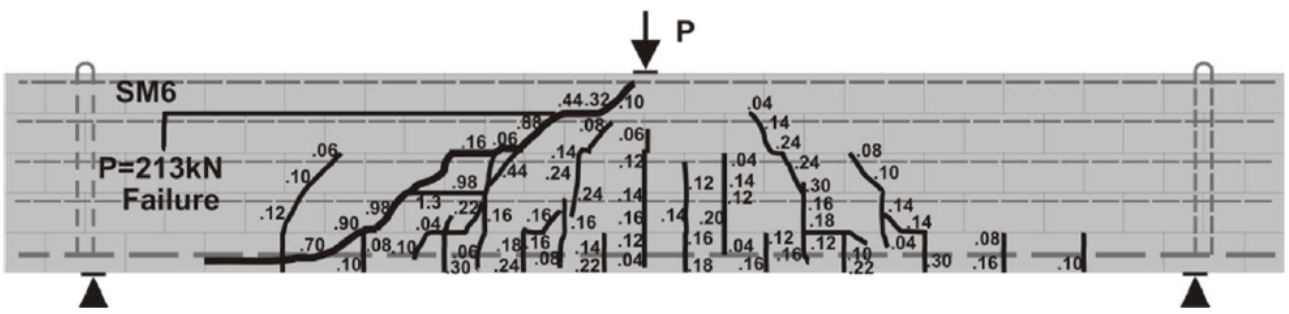

Experiment at Failure (Sarhat, 2016)

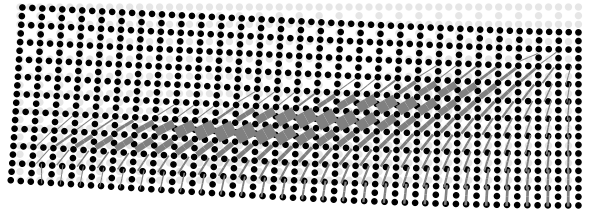

Finite Element Model at Failure $\times 15$

SM6 Beam Displacement, Crack Pattern, and Crack Widths at Failure

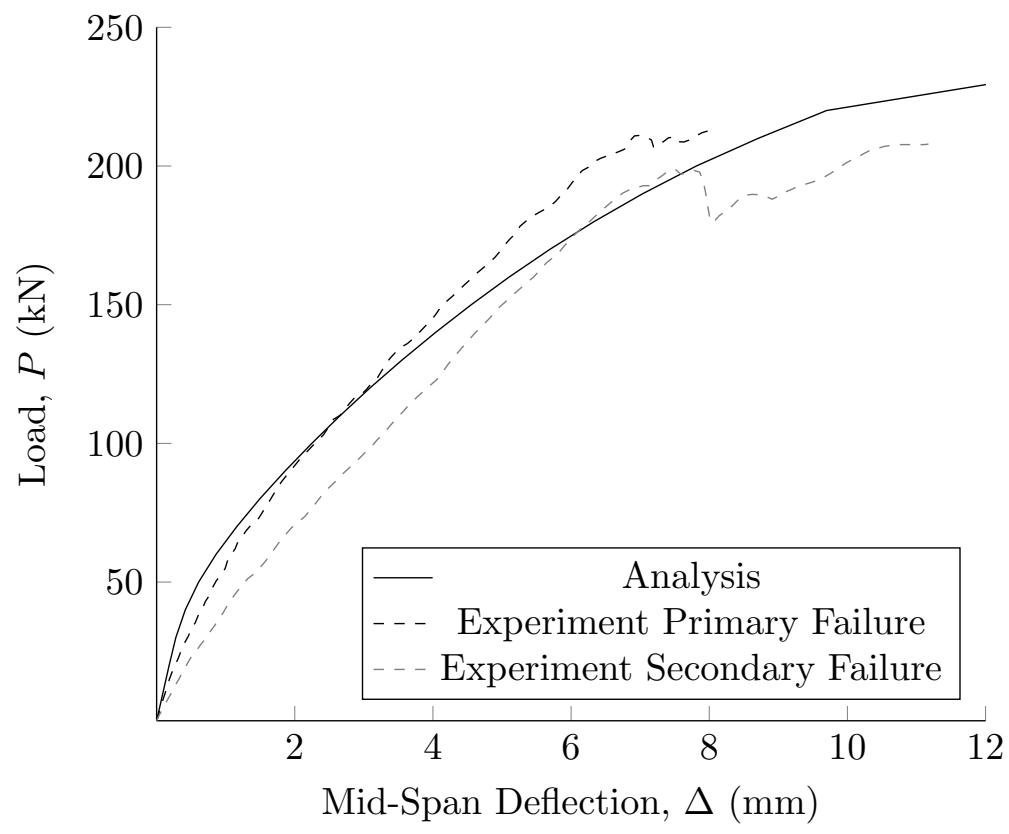

SM6 Beam Sarhat (2016) Load vs Mid-Span Deflection 


\section{SL1 Beam}

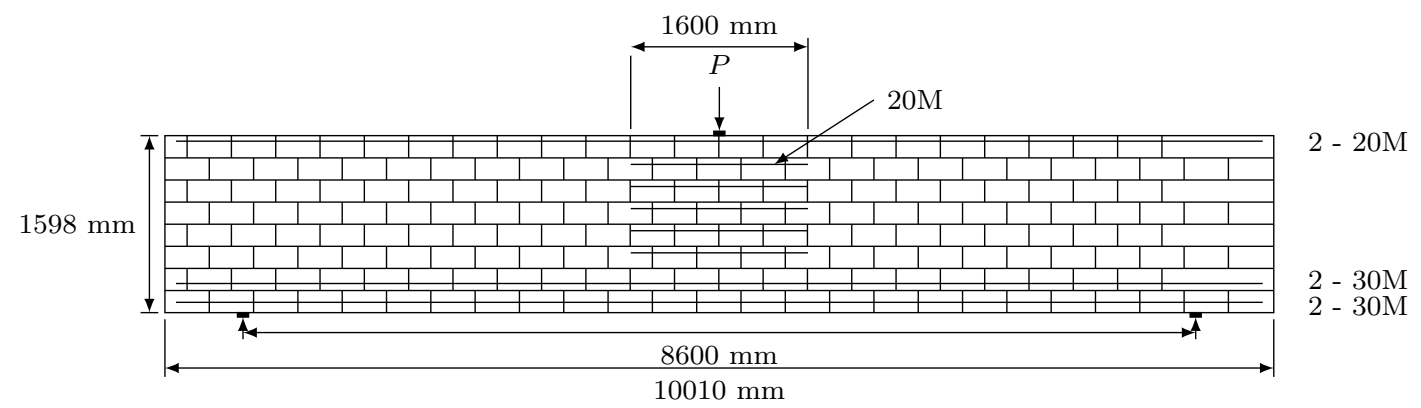

Experimental Beam Sarhat (2016)

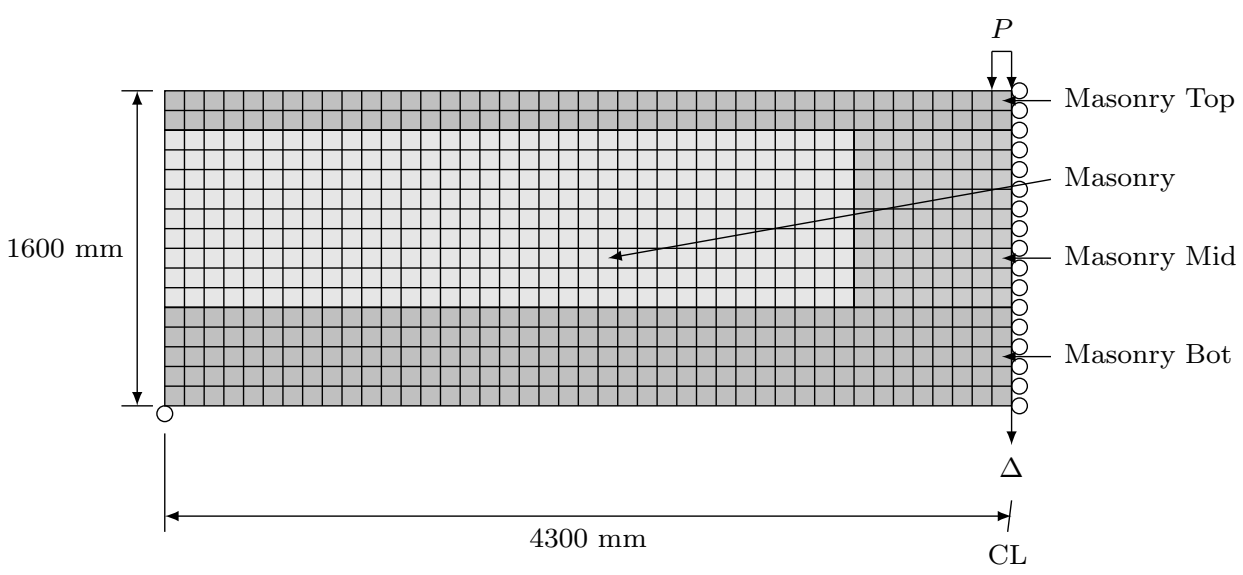

Finite Element Model Beam

SL1 Beam

SL1 Beam (Sarhat, 2016) Model Options

\begin{tabular}{ll}
\hline Model & Selected Option \\
\hline Compression Pre-Peak & Hognestad \\
Compression Post-Peak & Pre-Peak Base Curve \\
Compression Softening & Vecchio \\
Tension Post-Peak & Vecchio \\
Cracking Criterion & Mohr-Coulomb \\
Slip & Walraven Reinforced \\
\hline
\end{tabular}


SL1 Beam (Sarhat, 2016) Material Properties

\begin{tabular}{|c|c|c|c|c|}
\hline \multirow{2}{*}{ Property } & \multicolumn{4}{|c|}{ Material } \\
\hline & Masonry Bot & Masonry & Masonry Mid & Masonry Top \\
\hline Thickness (mm) & 190 & 190 & 190 & 190 \\
\hline Grout Tensile Strength (MPa) & 1.83 & 1.83 & 1.83 & 1.83 \\
\hline \multicolumn{5}{|c|}{ Masonry Properties (Y-Direction) } \\
\hline Gross Peak Compression Strength (MPa) & 18.9 & 18.9 & 18.9 & 18.9 \\
\hline Peak Compressive Strain & 0.0014 & 0.0014 & 0.0014 & 0.0014 \\
\hline Tensile Strength & 0 & 0 & 0 & 0 \\
\hline Tensile Strain & 0 & 0 & 0 & 0 \\
\hline Initial Tangent Elastic Modulus (MPa) & 9800 & 9800 & 9800 & \\
\hline Poisson's Ratio & 0 & 0 & 0 & 0 \\
\hline \multicolumn{5}{|c|}{ Masonry Properties (X-Direction) } \\
\hline Gross Peak Compression Strength (MPa) & 18.9 & 18.9 & 18.9 & 18.9 \\
\hline Peak Compressive Strain & 0.0014 & 0.0014 & 0.0014 & 0.0014 \\
\hline Tensile Strength & 0 & 0 & 0 & 0 \\
\hline Tensile Strain & 0 & 0 & 0 & 0 \\
\hline Initial Tangent Elastic Modulus (MPa) & 9800 & 9800 & 9800 & \\
\hline Poisson's Ratio & 0 & 0 & 0 & 0 \\
\hline \multicolumn{5}{|c|}{ Masonry Properties (Block) } \\
\hline Length (mm) & 390 & 390 & 390 & 390 \\
\hline Height (mm) & 190 & 190 & 190 & 190 \\
\hline Face Shell Thickness (mm) & 35 & 35 & 35 & 35 \\
\hline Percent Solid (\%) & 56 & 56 & 56 & 56 \\
\hline Tensile Strength & 1.80 & 1.80 & 1.80 & 1.80 \\
\hline \multicolumn{5}{|c|}{ Head Joint } \\
\hline Thickness $(\mathrm{mm})$ & 10 & 10 & 10 & 10 \\
\hline Tensile Bond Strength (MPa) & 0.37 & 0.37 & 0.37 & 0.37 \\
\hline Joint Cohesion $(\mathrm{MPa})$ & 5.90 & 5.90 & 5.90 & 5.90 \\
\hline Angle of Internal Friction (Deg) & 42 & 42 & 42 & 42 \\
\hline \multicolumn{5}{|c|}{ Bed Joint } \\
\hline Thickness $(\mathrm{mm})$ & 10 & 10 & 10 & 10 \\
\hline Tensile Bond Strength (MPa) & 0.37 & 0.37 & 0.37 & 0.37 \\
\hline Joint Cohesion (MPa) & 5.90 & 5.90 & 5.90 & 5.90 \\
\hline Angle of Internal Friction (Deg) & 42 & 42 & 42 & 42 \\
\hline \multicolumn{5}{|c|}{ Reinforcement (Y-Direction) } \\
\hline Ratio (\%) & 0 & 0 & 0 & 0 \\
\hline Yield Strength (MPa) & 0 & 0 & 0 & 0 \\
\hline Yield Strain & 0 & 0 & 0 & 0 \\
\hline Strain Hardening Strain & 0 & 0 & 0 & 0 \\
\hline Ultimate Strength (MPa) & 0 & 0 & 0 & 0 \\
\hline Ultimate Strain & 0 & 0 & 0 & 0 \\
\hline Prestrain & 0 & 0 & 0 & 0 \\
\hline \multicolumn{5}{|c|}{ Reinforcement (X-Direction) } \\
\hline Ratio (\%) & 2.39 & 0 & 0.63 & 1.25 \\
\hline Yield Strength (MPa) & 438 & 0 & 398 & 398 \\
\hline Yield Strain & 0.0025 & 0 & 0.002 & 0.002 \\
\hline Strain Hardening Strain & 0.01 & 0 & 0.01 & 0.01 \\
\hline Ultimate Strength $(\mathrm{MPa})$ & 727 & 0 & 639 & 639 \\
\hline Ultimate Strain & 0.2 & 0 & 0.2 & 0.2 \\
\hline Prestrain & 0 & 0 & 0 & 0 \\
\hline
\end{tabular}

Numerical Modelling of Hollow Concrete Block Masonry Mechanics 


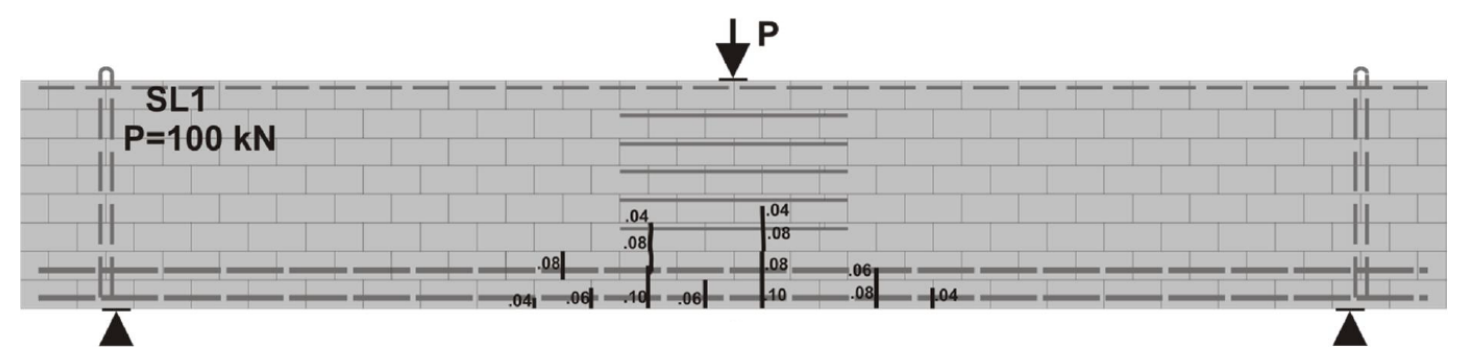

Experiment at $100 \mathrm{kN}$ Load (Sarhat, 2016)

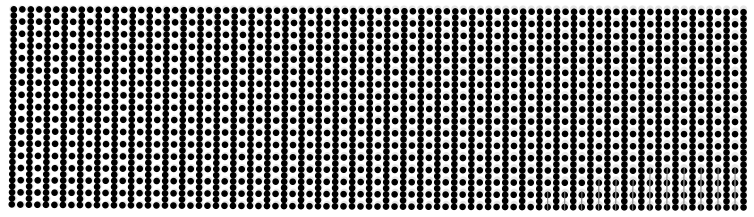

Finite Element Model at $100 \mathrm{kN} \times 15$

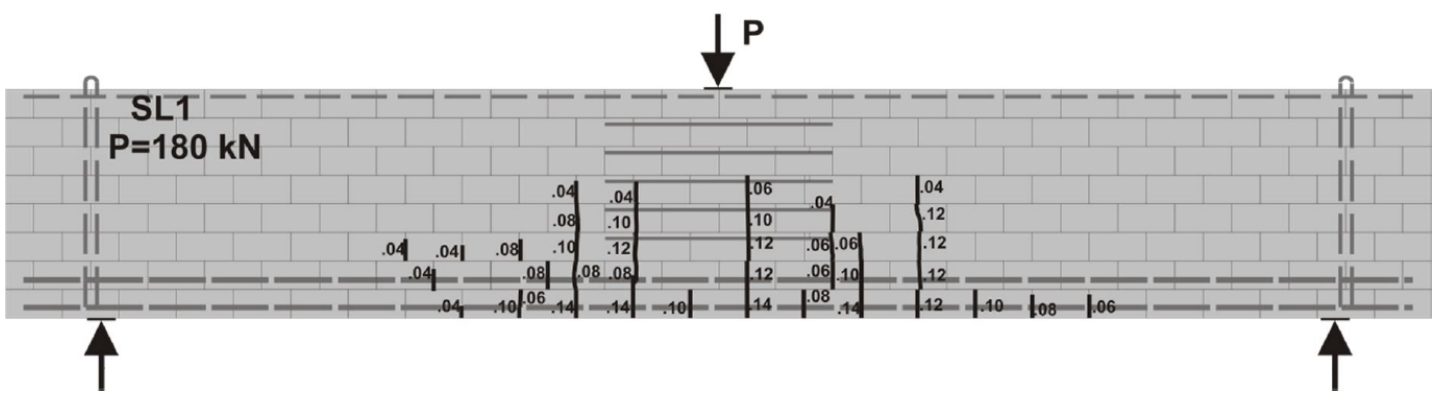

Experiment at $180 \mathrm{kN}$ Load (Sarhat, 2016)

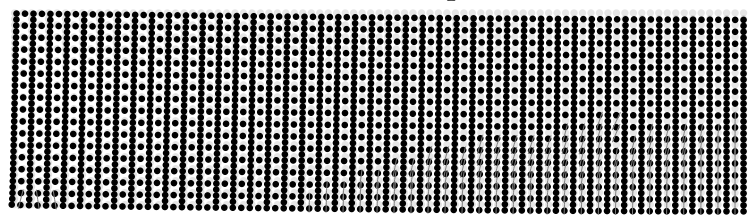

Finite Element Model at $180 \mathrm{kN} \times 15$

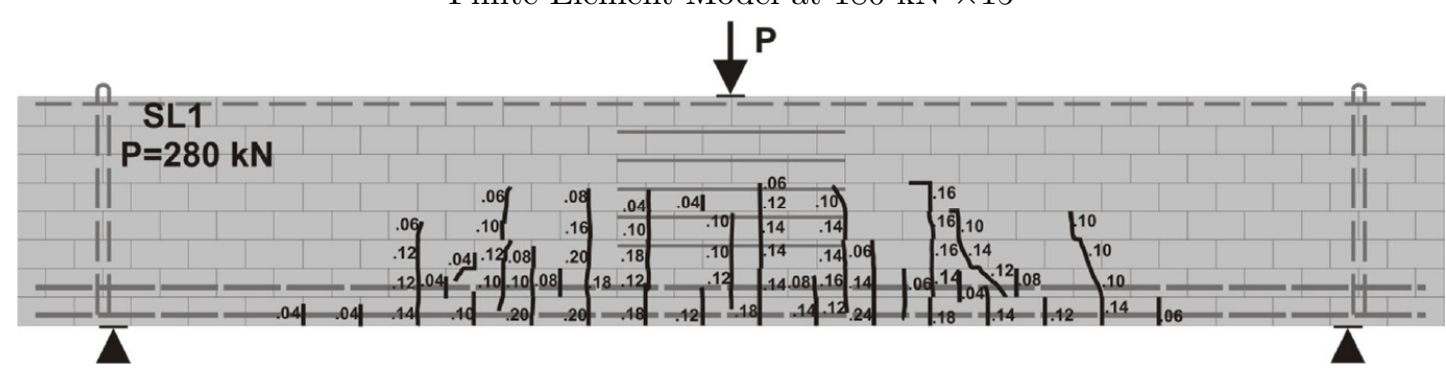

Experiment at $280 \mathrm{kN}$ Load (Sarhat, 2016)

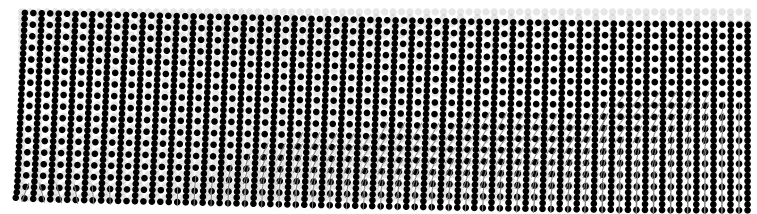

Finite Element Model at $280 \mathrm{kN} \times 15$

SL1 Beam Displacement, Crack Pattern, and Crack Widths 


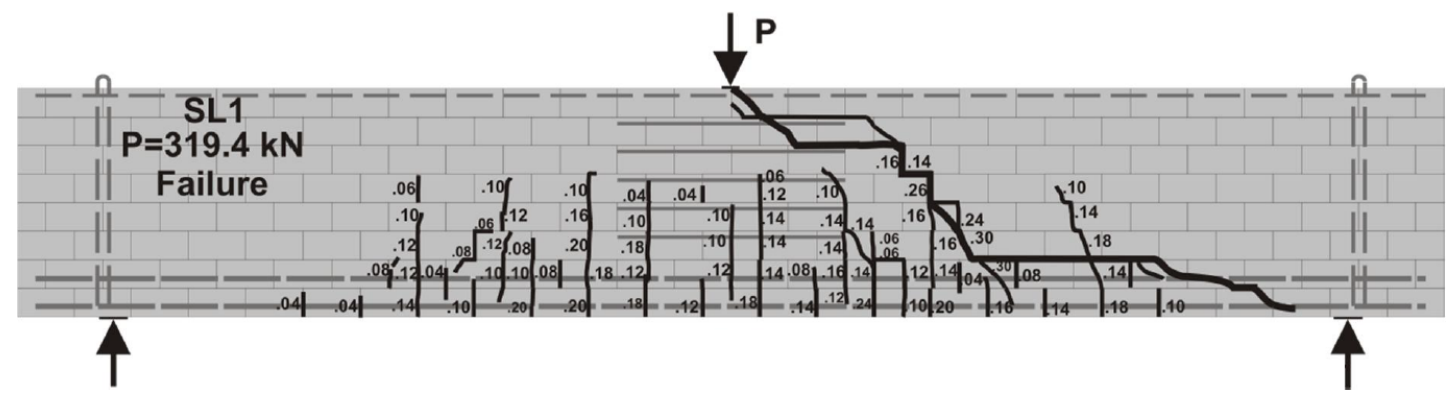

Experiment at Failure (Sarhat, 2016)

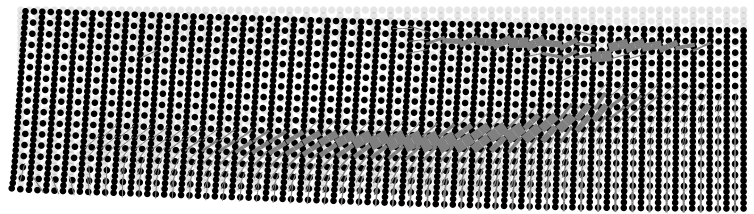

Finite Element Model at Failure $\times 15$

SL1 Beam Displacement, Crack Pattern, and Crack Widths at Failure

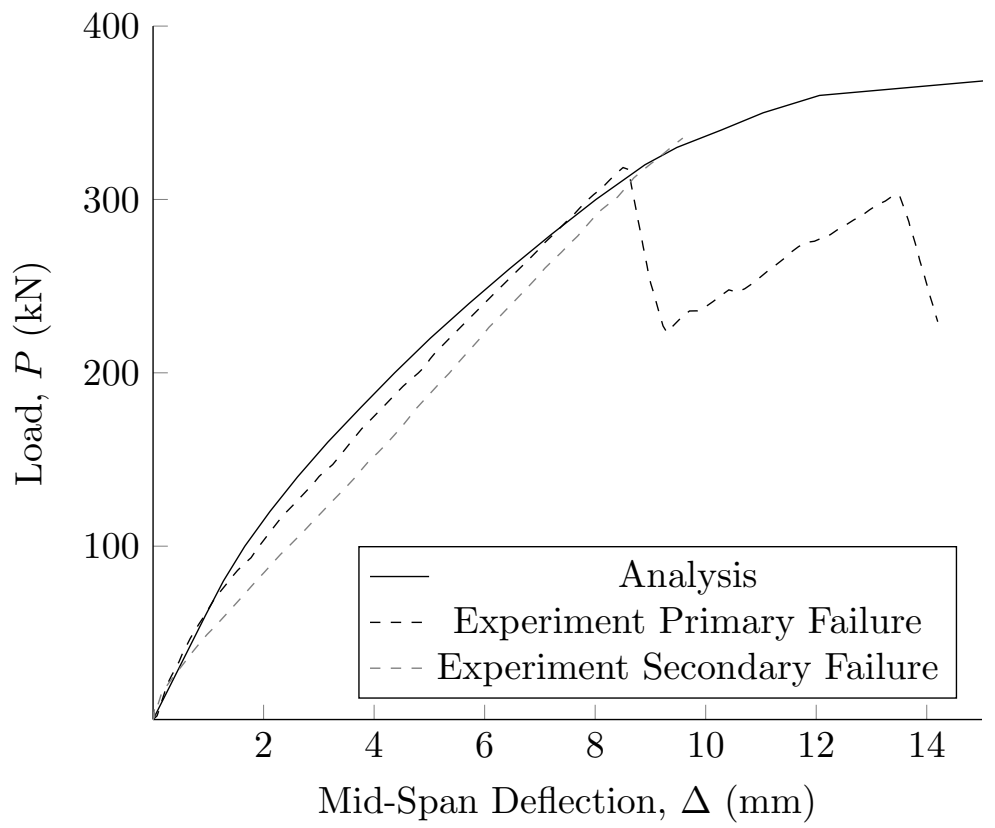

SL1 Beam Sarhat (2016) Load vs Mid-Span Deflection 


\section{SL2 Beam}

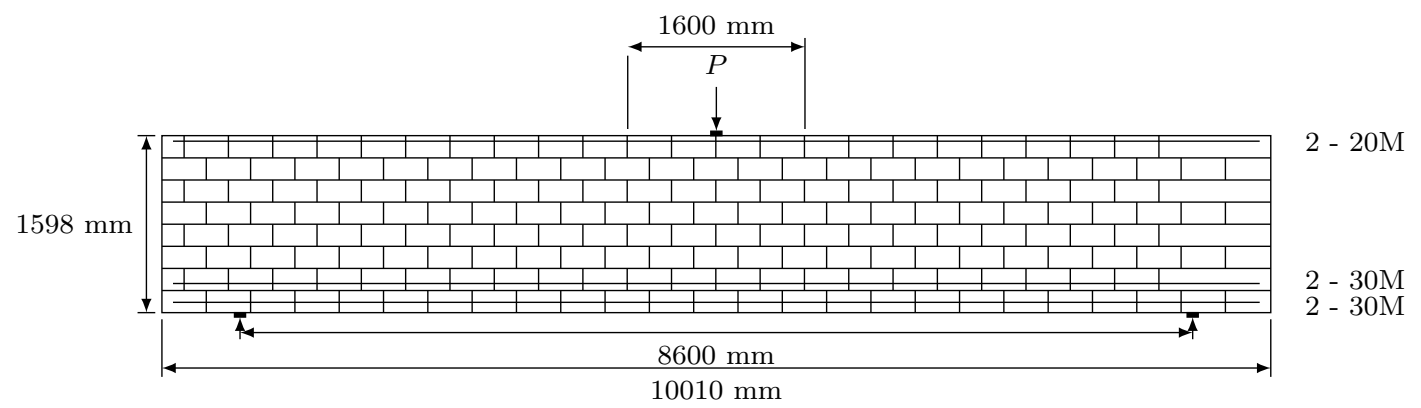

Experimental Beam Sarhat (2016)

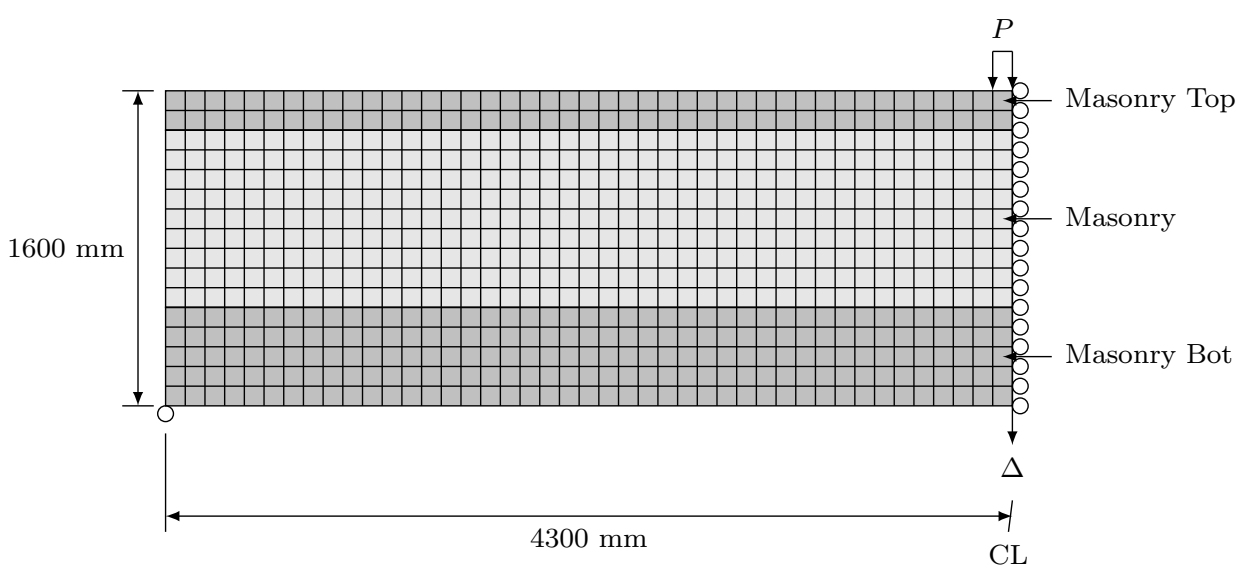

Finite Element Model Beam

SL2 Beam

SL2 Beam (Sarhat, 2016) Model Options

\begin{tabular}{ll}
\hline Model & Selected Option \\
\hline Compression Pre-Peak & Hognestad \\
Compression Post-Peak & Pre-Peak Base Curve \\
Compression Softening & Vecchio \\
Tension Post-Peak & Vecchio \\
Cracking Criterion & Mohr-Coulomb \\
Slip & Walraven Reinforced \\
\hline
\end{tabular}


SL1 Beam (Sarhat, 2016) Material Properties

\begin{tabular}{|c|c|c|c|}
\hline \multirow{2}{*}{ Property } & \multicolumn{3}{|c|}{ Material } \\
\hline & Masonry Bot & Masonry & Masonry Top \\
\hline Thickness (mm) & 190 & 190 & 190 \\
\hline Grout Tensile Strength (MPa) & 1.78 & 1.78 & 1.78 \\
\hline \multicolumn{4}{|c|}{ Masonry Properties (Y-Direction) } \\
\hline Gross Peak Compression Strength (MPa) & 18.9 & 18.9 & 18.9 \\
\hline Peak Compressive Strain & 0.0014 & 0.0014 & 0.0014 \\
\hline Tensile Strength & 0 & 0 & 0 \\
\hline Tensile Strain & 0 & 0 & 0 \\
\hline Initial Tangent Elastic Modulus (MPa) & 9800 & 9800 & 9800 \\
\hline Poisson's Ratio & 0 & 0 & 0 \\
\hline \multicolumn{4}{|c|}{ Masonry Properties (X-Direction) } \\
\hline Gross Peak Compression Strength (MPa) & 18.9 & 18.9 & 18.9 \\
\hline Peak Compressive Strain & 0.0014 & 0.0014 & 0.0014 \\
\hline Tensile Strength & 0 & 0 & 0 \\
\hline Tensile Strain & 0 & 0 & 0 \\
\hline Initial Tangent Elastic Modulus (MPa) & 9800 & 9800 & 9800 \\
\hline Poisson's Ratio & 0 & 0 & 0 \\
\hline \multicolumn{4}{|c|}{ Masonry Properties (Block) } \\
\hline Length (mm) & 390 & 390 & 390 \\
\hline Height (mm) & 190 & 190 & 190 \\
\hline Face Shell Thickness (mm) & 35 & 35 & 35 \\
\hline Percent Solid (\%) & 56 & 56 & 56 \\
\hline Tensile Strength & 1.80 & 1.80 & 1.80 \\
\hline \multicolumn{4}{|c|}{ Head Joint } \\
\hline Thickness (mm) & 10 & 10 & 10 \\
\hline Tensile Bond Strength (MPa) & 0.37 & 0.37 & 0.37 \\
\hline Joint Cohesion (MPa) & 5.90 & 5.90 & 5.90 \\
\hline Angle of Internal Friction (Deg) & 42 & 42 & 42 \\
\hline \multicolumn{4}{|c|}{ Bed Joint } \\
\hline Thickness (mm) & 10 & 10 & 10 \\
\hline Tensile Bond Strength (MPa) & 0.37 & 0.37 & 0.37 \\
\hline Joint Cohesion (MPa) & 5.90 & 5.90 & 5.90 \\
\hline Angle of Internal Friction (Deg) & 42 & 42 & 42 \\
\hline \multicolumn{4}{|c|}{ Reinforcement (Y-Direction) } \\
\hline Ratio (\%) & 0 & 0 & 0 \\
\hline Yield Strength (MPa) & 0 & 0 & 0 \\
\hline Yield Strain & 0 & 0 & 0 \\
\hline Strain Hardening Strain & 0 & 0 & 0 \\
\hline Ultimate Strength (MPa) & 0 & 0 & 0 \\
\hline Ultimate Strain & 0 & 0 & 0 \\
\hline Prestrain & 0 & 0 & 0 \\
\hline \multicolumn{4}{|c|}{ Reinforcement (X-Direction) } \\
\hline Ratio (\%) & 2.39 & 0 & 1.25 \\
\hline Yield Strength (MPa) & 438 & 0 & 398 \\
\hline Yield Strain & 0.0025 & 0 & 0.002 \\
\hline Strain Hardening Strain & 0.01 & 0 & 0.01 \\
\hline Ultimate Strength (MPa) & 727 & 0 & 639 \\
\hline Ultimate Strain & 0.2 & 0 & 0.2 \\
\hline Prestrain & 0 & 0 & 0 \\
\hline
\end{tabular}

Numerical Modelling of Hollow Concrete Block Masonry Mechanics 


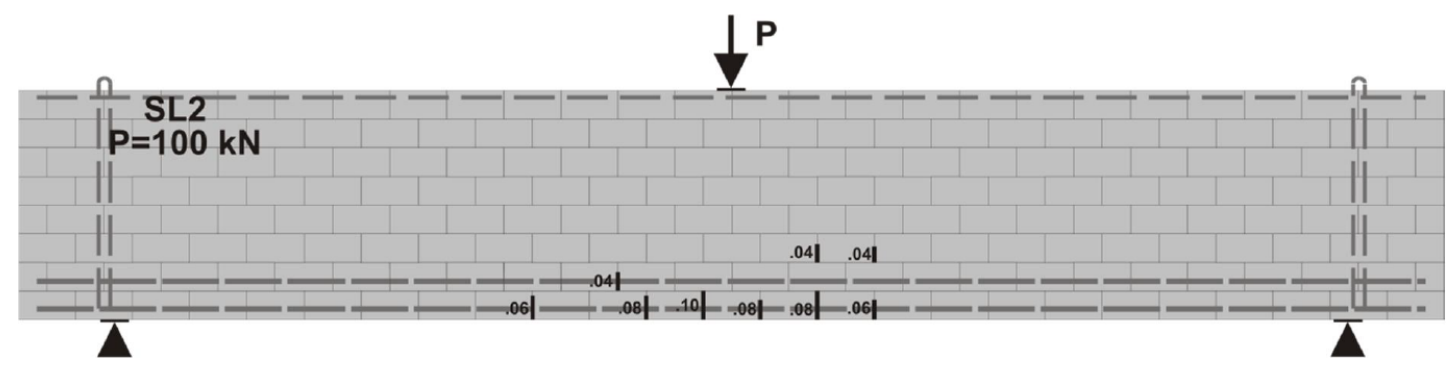

Experiment at $100 \mathrm{kN}$ Load (Sarhat, 2016)

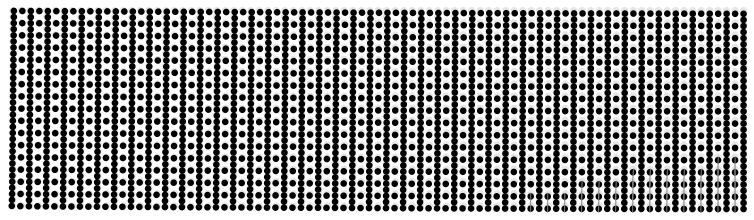

Finite Element Model at $100 \mathrm{kN} \times 15$

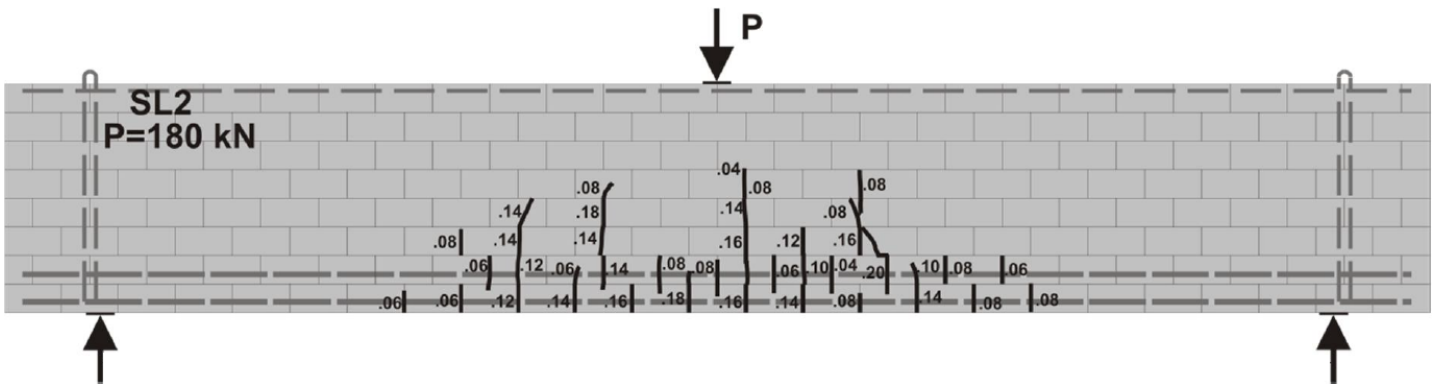

Experiment at $180 \mathrm{kN}$ Load (Sarhat, 2016)

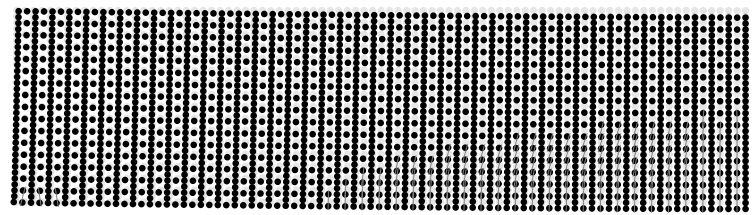

Finite Element Model at $180 \mathrm{kN} \times 15$

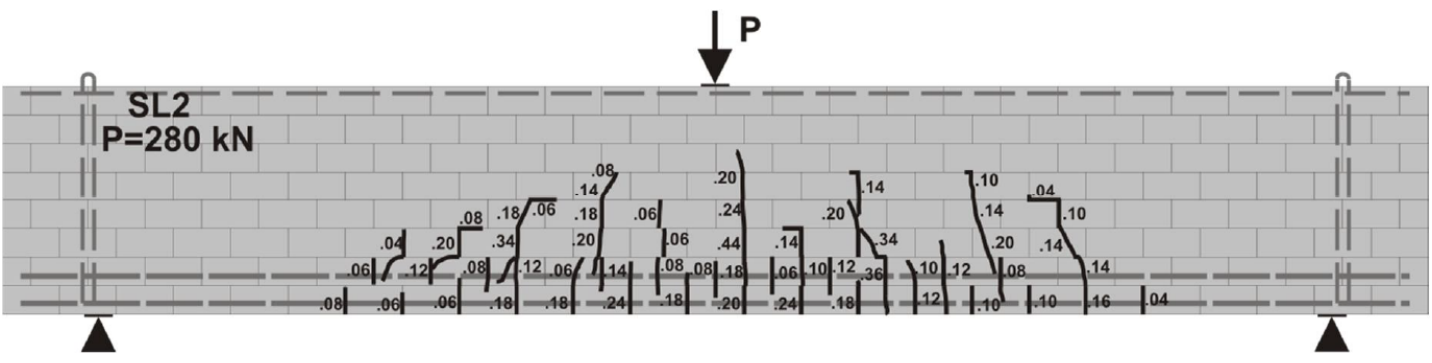

Experiment at $280 \mathrm{kN}$ Load (Sarhat, 2016)

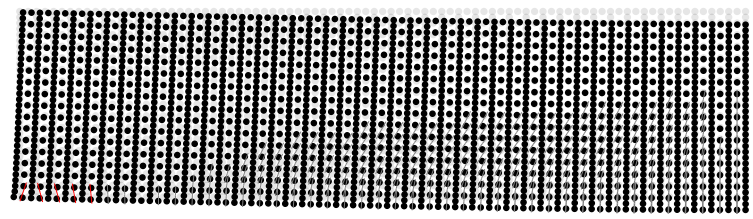

Finite Element Model at $280 \mathrm{kN} \times 15$

SL2 Beam Displacement, Crack Pattern, and Crack Widths

Numerical Modelling of Hollow Concrete Block Masonry Mechanics 


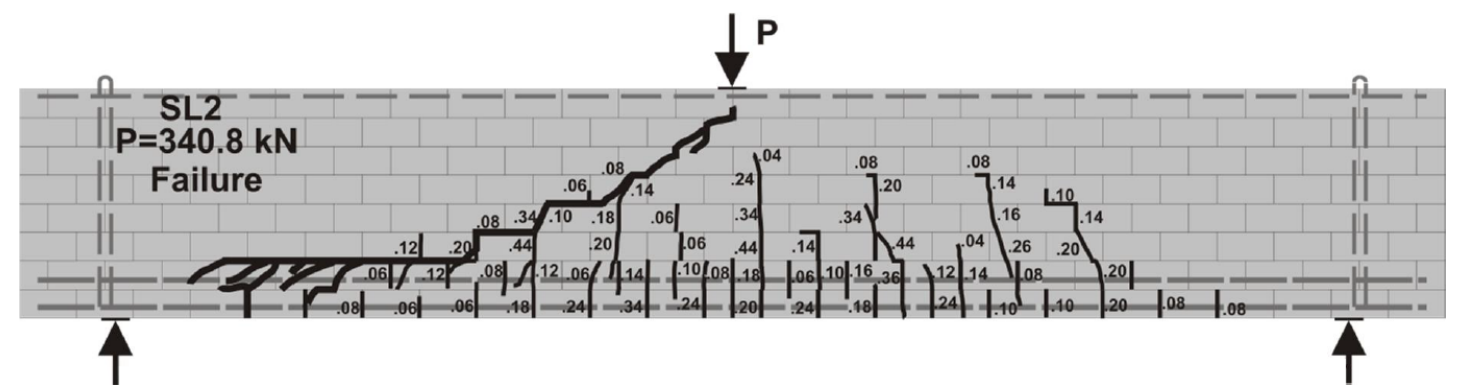

Experiment at Failure (Sarhat, 2016)

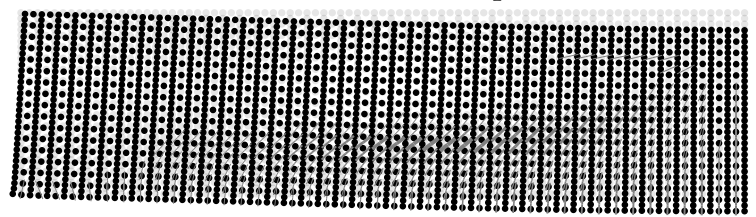

Finite Element Model at Failure $\times 15$

SL2 Beam Displacement, Crack Pattern, and Crack Widths at Failure

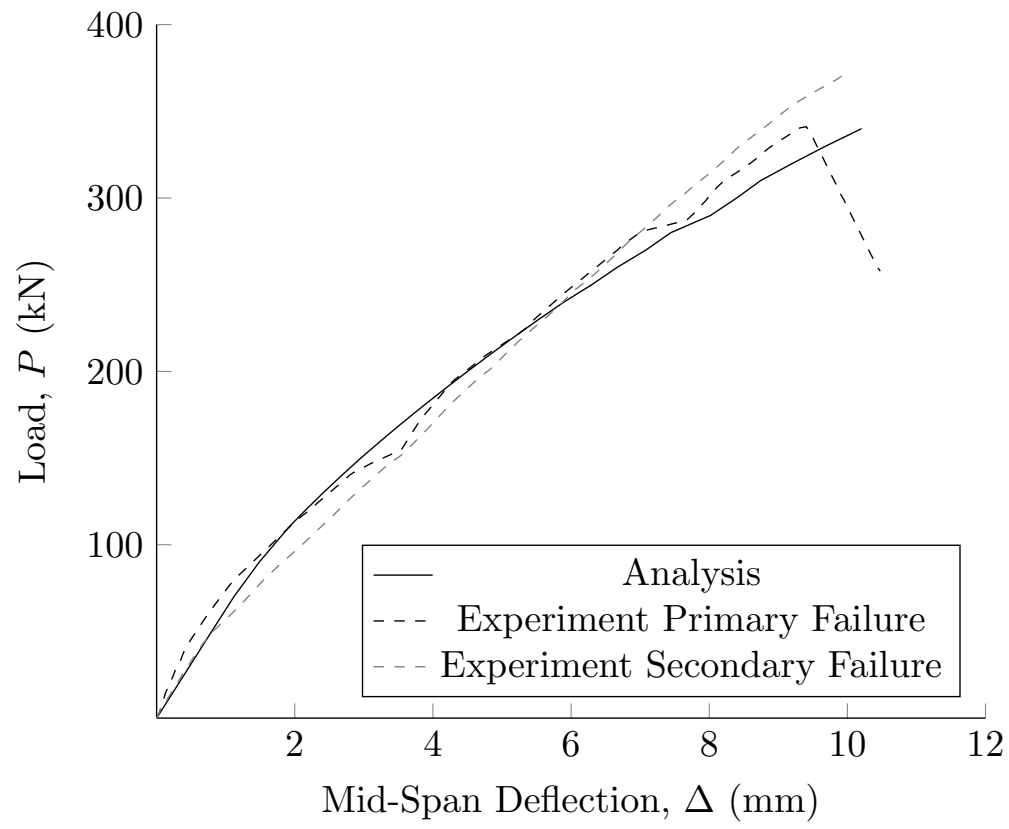

SL2 Beam Sarhat (2016) Load vs Mid-Span Deflection 


\section{THREE PARAMETER KINEMATIC MODEL RESULTS}

\section{Priestley (1977)}

\section{A1 Shear Wall}

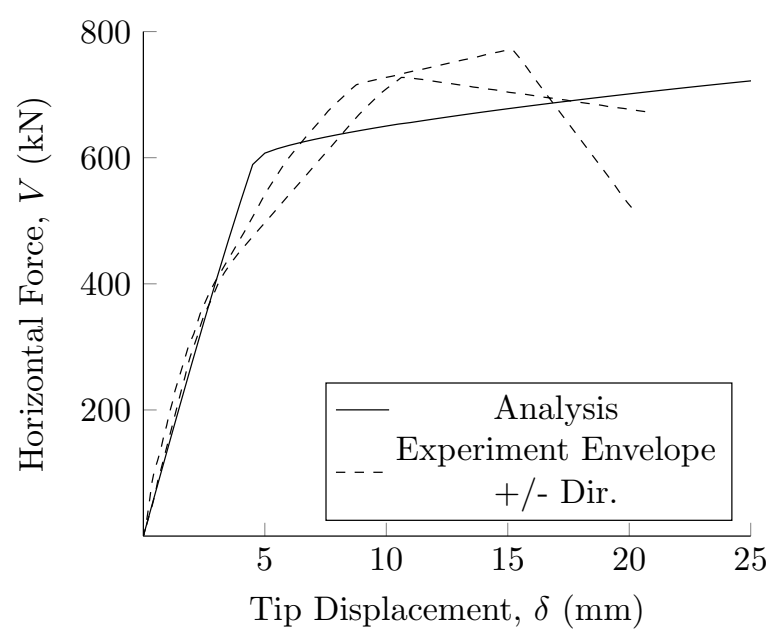

A1 Shear Wall (Priestley, 1977) Horizontal Force vs Tip Displacement

A1 Shear Wall (Priestley, 1977) Model Options

\begin{tabular}{ll}
\hline Model Option & Value \\
\hline Axial Compression Load (N) & 0 \\
Total Lateral Displacement & 50 \\
Number of Load Stages & 100 \\
Convergence Limit & 0.01 \\
Maximum Iterations & 100 \\
\hline
\end{tabular}


A1 Shear Wall (Priestley, 1977) Material Properties

\begin{tabular}{|c|c|}
\hline Property & Value \\
\hline \multicolumn{2}{|l|}{ Masonry Properties } \\
\hline Y-Direction Peak Compression Strength (MPa) & 19.3 \\
\hline X-Direction Peak Compression Strength ( $\mathrm{MPa})$ & 19.3 \\
\hline Maximum Aggregate Size (mm) & 5 \\
\hline \multicolumn{2}{|l|}{ Wall Dimensions } \\
\hline Wall Span (mm) & 1854 \\
\hline Wall Height (mm) & 1651 \\
\hline Wall Length (mm) & 2429 \\
\hline \multicolumn{2}{|l|}{ Wall Dimensions } \\
\hline Block Height (mm) & 190 \\
\hline Block Thickness (mm) & 143 \\
\hline Bed Joint Thickness (mm) & 10 \\
\hline \multicolumn{2}{|l|}{ Longitudinal Reinforcement Properties } \\
\hline Longitudinal Reinforcement Ratio (\%) & 0.45 \\
\hline Area in Flexural Tension $\left(\mathrm{mm}^{2}\right)$ & 1140 \\
\hline Bar Diameter (mm) & 19.05 \\
\hline Distance to Furthest Tension Bar (mm) & 2327 \\
\hline Effective Distance (mm) & 1870 \\
\hline Modulus of Elasticity (MPa) & 200000 \\
\hline Yield Strength (MPa) & 528 \\
\hline Ultimate Strength (MPa) & 871 \\
\hline Ultimate Stain & 0.2 \\
\hline \multicolumn{2}{|l|}{ Transverse Reinforcement Properties } \\
\hline Transverse Reinforcement Ratio (\%) & 0.97 \\
\hline Modulus of Elasticity (MPa) & 200000 \\
\hline Yield Strength (MPa) & 505 \\
\hline Ultimate Strength (MPa) & 841 \\
\hline Ultimate Stain & 0.2 \\
\hline \multicolumn{2}{|l|}{ Critical Loading Zone Properties } \\
\hline Area of Longitudinal Reinforcement $\left(\mathrm{mm}^{2}\right)$ & 285 \\
\hline Longitudinal Reinforcement Center-to-Center Spacing (mm) & 200 \\
\hline Longitudinal Reinforcement Clear Spacing (mm) & 180 \\
\hline Area of Tie $\left(\mathrm{mm}^{2}\right)$ & 0 \\
\hline Tie Clear Spacing (mm) & 180 \\
\hline Tie Depth $(\mathrm{mm})$ & 120 \\
\hline Tie Width (mm) & 190 \\
\hline
\end{tabular}




\section{A2 Shear Wall}

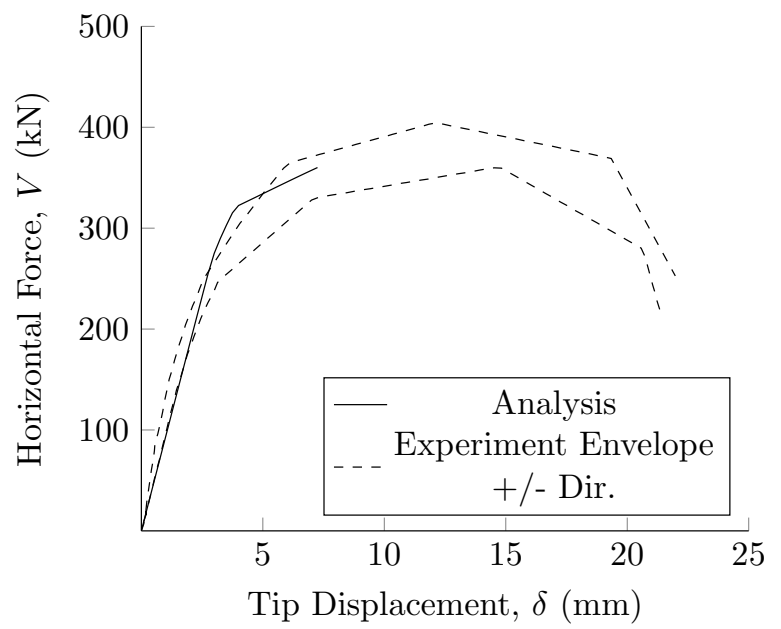

A2 Shear Wall (Priestley, 1977) Horizontal Force vs Tip Displacement

A2 Shear Wall (Priestley, 1977) Model Options

\begin{tabular}{ll}
\hline Model Option & Value \\
\hline Axial Compression Load (N) & 0 \\
Total Lateral Displacement & 25 \\
Number of Load Stages & 100 \\
Convergence Limit & 0.01 \\
Maximum Iterations & 100 \\
\hline
\end{tabular}


A2 Shear Wall (Priestley, 1977) Material Properties

\begin{tabular}{|c|c|}
\hline Property & Value \\
\hline \multicolumn{2}{|l|}{ Masonry Properties } \\
\hline Y-Direction Peak Compression Strength (MPa) & 18.3 \\
\hline X-Direction Peak Compression Strength ( $\mathrm{MPa})$ & 18.3 \\
\hline Maximum Aggregate Size (mm) & 5 \\
\hline \multicolumn{2}{|l|}{ Wall Dimensions } \\
\hline Wall Span (mm) & 1854 \\
\hline Wall Height (mm) & 1651 \\
\hline Wall Length (mm) & 2429 \\
\hline \multicolumn{2}{|l|}{ Wall Dimensions } \\
\hline Block Height (mm) & 190 \\
\hline Block Thickness (mm) & 143 \\
\hline Bed Joint Thickness (mm) & 10 \\
\hline \multicolumn{2}{|l|}{ Longitudinal Reinforcement Properties } \\
\hline Longitudinal Reinforcement Ratio (\%) & 0.34 \\
\hline Area in Flexural Tension $\left(\mathrm{mm}^{2}\right)$ & 596 \\
\hline Bar Diameter (mm) & 15.9 \\
\hline Distance to Furthest Tension Bar (mm) & 2327 \\
\hline Effective Distance (mm) & 1923 \\
\hline Modulus of Elasticity (MPa) & 200000 \\
\hline Yield Strength (MPa) & 432 \\
\hline Ultimate Strength (MPa) & 711 \\
\hline Ultimate Stain & 0.2 \\
\hline \multicolumn{2}{|l|}{ Transverse Reinforcement Properties } \\
\hline Transverse Reinforcement Ratio (\%) & 0.67 \\
\hline Modulus of Elasticity (MPa) & 200000 \\
\hline Yield Strength (MPa) & 421 \\
\hline Ultimate Strength (MPa) & 664 \\
\hline Ultimate Stain & 0.2 \\
\hline \multicolumn{2}{|l|}{ Critical Loading Zone Properties } \\
\hline Area of Longitudinal Reinforcement $\left(\mathrm{mm}^{2}\right)$ & 199 \\
\hline Longitudinal Reinforcement Center-to-Center Spacing (mm) & 400 \\
\hline Longitudinal Reinforcement Clear Spacing (mm) & 384 \\
\hline Area of Tie $\left(\mathrm{mm}^{2}\right)$ & 0 \\
\hline Tie Clear Spacing (mm) & 184 \\
\hline Tie Depth $(\mathrm{mm})$ & 120 \\
\hline Tie Width (mm) & 190 \\
\hline
\end{tabular}




\section{A5 Shear Wall}

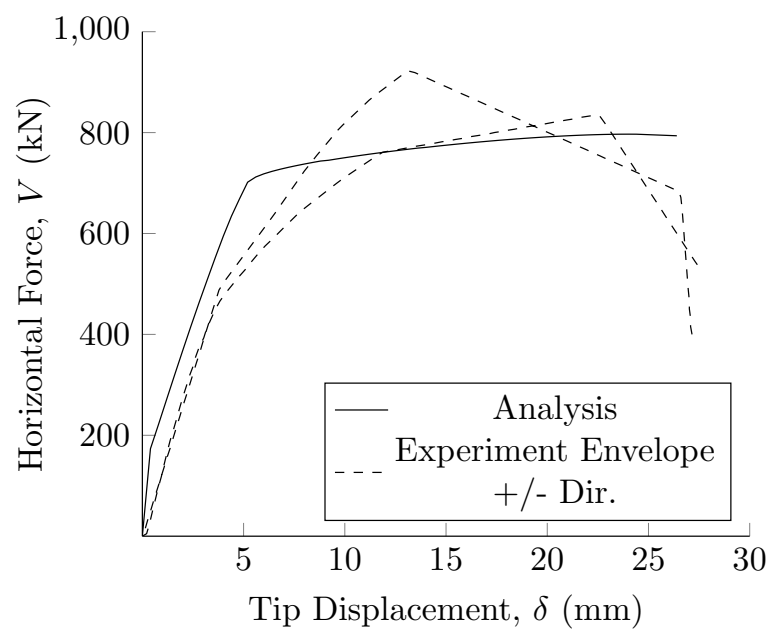

A5 Shear Wall (Priestley, 1977) Horizontal Force vs Tip Displacement

A5 Shear Wall (Priestley, 1977) Model Options

\begin{tabular}{ll}
\hline Model Option & Value \\
\hline Axial Compression Load (N) & 240000 \\
Total Lateral Displacement & 40 \\
Number of Load Stages & 100 \\
Convergence Limit & 0.01 \\
Maximum Iterations & 100 \\
\hline
\end{tabular}


A5 Shear Wall (Priestley, 1977) Material Properties

\begin{tabular}{|c|c|}
\hline Property & Value \\
\hline \multicolumn{2}{|l|}{ Masonry Properties } \\
\hline Y-Direction Peak Compression Strength (MPa) & 18.3 \\
\hline X-Direction Peak Compression Strength ( $\mathrm{MPa})$ & 18.3 \\
\hline Maximum Aggregate Size (mm) & 5 \\
\hline \multicolumn{2}{|l|}{ Wall Dimensions } \\
\hline Wall Span (mm) & 1854 \\
\hline Wall Height (mm) & 1651 \\
\hline Wall Length (mm) & 2429 \\
\hline \multicolumn{2}{|l|}{ Wall Dimensions } \\
\hline Block Height (mm) & 190 \\
\hline Block Thickness (mm) & 143 \\
\hline Bed Joint Thickness (mm) & 10 \\
\hline \multicolumn{2}{|l|}{ Longitudinal Reinforcement Properties } \\
\hline Longitudinal Reinforcement Ratio (\%) & 0.45 \\
\hline Area in Flexural Tension $\left(\mathrm{mm}^{2}\right)$ & 1140 \\
\hline Bar Diameter (mm) & 19.05 \\
\hline Distance to Furthest Tension Bar (mm) & 2327 \\
\hline Effective Distance (mm) & 1870 \\
\hline Modulus of Elasticity (MPa) & 200000 \\
\hline Yield Strength (MPa) & 488 \\
\hline Ultimate Strength (MPa) & 802 \\
\hline Ultimate Stain & 0.2 \\
\hline \multicolumn{2}{|l|}{ Transverse Reinforcement Properties } \\
\hline Transverse Reinforcement Ratio (\%) & 0.97 \\
\hline Modulus of Elasticity (MPa) & 200000 \\
\hline Yield Strength (MPa) & 489 \\
\hline Ultimate Strength (MPa) & 664 \\
\hline Ultimate Stain & 0.2 \\
\hline \multicolumn{2}{|l|}{ Critical Loading Zone Properties } \\
\hline Area of Longitudinal Reinforcement $\left(\mathrm{mm}^{2}\right)$ & 285 \\
\hline Longitudinal Reinforcement Center-to-Center Spacing (mm) & 200 \\
\hline Longitudinal Reinforcement Clear Spacing (mm) & 180 \\
\hline Area of Tie $\left(\mathrm{mm}^{2}\right)$ & 0 \\
\hline Tie Clear Spacing (mm) & 180 \\
\hline Tie Depth $(\mathrm{mm})$ & 120 \\
\hline Tie Width (mm) & 190 \\
\hline
\end{tabular}




\section{Shing et al. (1991)}

\section{HCBL 1 Shear Wall}

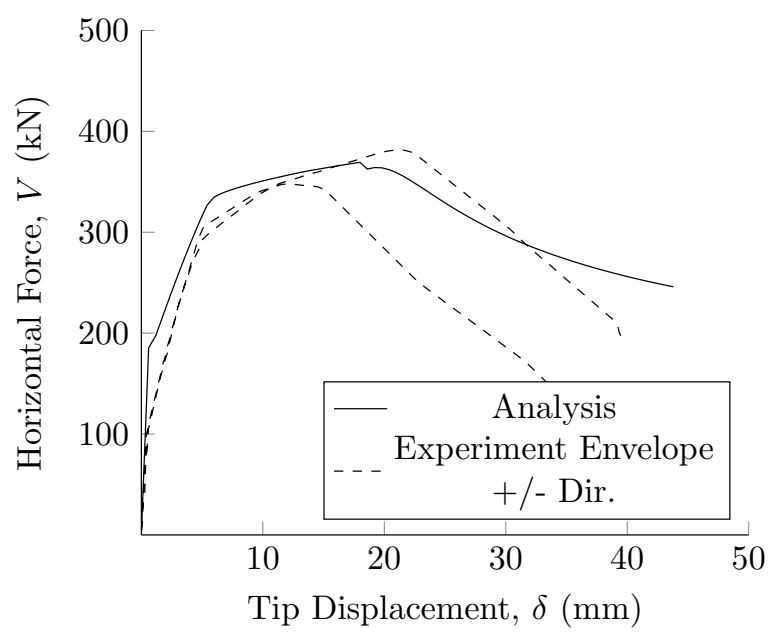

HCBL 1 Shear Wall Shing et al. (1991) Horizontal Force vs Tip Displacement

HCBL 1 Shear Wall Shing et al. (1991) Model Options

\begin{tabular}{ll}
\hline Model Option & Value \\
\hline Axial Compression Load (N) & 356000 \\
Total Lateral Displacement & 60 \\
Number of Load Stages & 100 \\
Convergence Limit & 0.01 \\
Maximum Iterations & 100 \\
\hline
\end{tabular}


HCBL 1 Shear Wall Shing et al. (1991) Material Properties

\begin{tabular}{|c|c|}
\hline Property & Value \\
\hline \multicolumn{2}{|l|}{ Masonry Properties } \\
\hline Y-Direction Peak Compression Strength (MPa) & 20.1 \\
\hline X-Direction Peak Compression Strength (MPa) & 20.1 \\
\hline Maximum Aggregate Size (mm) & 5 \\
\hline \multicolumn{2}{|l|}{ Wall Dimensions } \\
\hline Wall Span (mm) & 1829 \\
\hline Wall Height (mm) & 1829 \\
\hline Wall Length (mm) & 1829 \\
\hline \multicolumn{2}{|l|}{ Wall Dimensions } \\
\hline Block Height (mm) & 190 \\
\hline Block Thickness (mm) & 140 \\
\hline Bed Joint Thickness (mm) & 10 \\
\hline \multicolumn{2}{|l|}{ Longitudinal Reinforcement Properties } \\
\hline Longitudinal Reinforcement Ratio (\%) & 0.25 \\
\hline Area in Flexural Tension $\left(\mathrm{mm}^{2}\right)$ & 498 \\
\hline Bar Diameter (mm) & 15.9 \\
\hline Distance to Furthest Tension Bar (mm) & 1727 \\
\hline Effective Distance (mm) & 1404 \\
\hline Modulus of Elasticity (MPa) & 200000 \\
\hline Yield Strength (MPa) & 441 \\
\hline Ultimate Strength (MPa) & 710 \\
\hline Ultimate Stain & 0.2 \\
\hline \multicolumn{2}{|l|}{ Transverse Reinforcement Properties } \\
\hline Transverse Reinforcement Ratio (\%) & 0.24 \\
\hline Modulus of Elasticity (MPa) & 200000 \\
\hline Yield Strength (MPa) & 462 \\
\hline Ultimate Strength (MPa) & 738 \\
\hline Ultimate Stain & 0.2 \\
\hline \multicolumn{2}{|l|}{ Critical Loading Zone Properties } \\
\hline Area of Longitudinal Reinforcement $\left(\mathrm{mm}^{2}\right)$ & 199 \\
\hline Longitudinal Reinforcement Center-to-Center Spacing (mm) & 400 \\
\hline Longitudinal Reinforcement Clear Spacing (mm) & 384 \\
\hline Area of Tie $\left(\mathrm{mm}^{2}\right)$ & 0 \\
\hline Tie Clear Spacing (mm) & 387 \\
\hline Tie Depth (mm) & 120 \\
\hline Tie Width (mm) & 190 \\
\hline
\end{tabular}




\section{HCBL 2 Shear Wall}

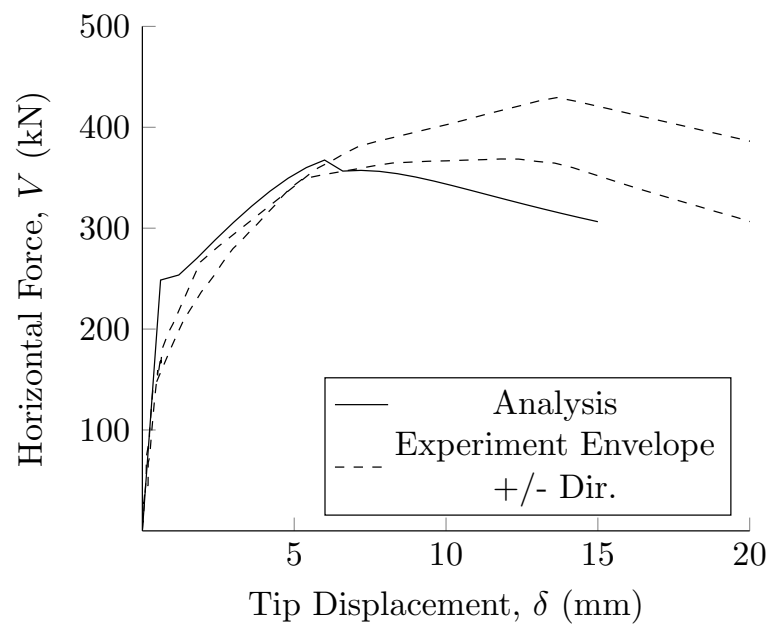

HCBL 2 Shear Wall Shing et al. (1991) Horizontal Force vs Tip Displacement

HCBL 2 Shear Wall Shing et al. (1991) Model Options

\begin{tabular}{ll}
\hline Model Option & Value \\
\hline Axial Compression Load (N) & 480000 \\
Total Lateral Displacement & 60 \\
Number of Load Stages & 100 \\
Convergence Limit & 0.01 \\
Maximum Iterations & 100 \\
\hline
\end{tabular}


HCBL 2 Shear Wall Shing et al. (1991) Material Properties

\begin{tabular}{|c|c|}
\hline Property & Value \\
\hline \multicolumn{2}{|l|}{ Masonry Properties } \\
\hline Y-Direction Peak Compression Strength (MPa) & 20.1 \\
\hline X-Direction Peak Compression Strength ( $\mathrm{MPa})$ & 20.1 \\
\hline Maximum Aggregate Size (mm) & 5 \\
\hline \multicolumn{2}{|l|}{ Wall Dimensions } \\
\hline Wall Span (mm) & 1829 \\
\hline Wall Height (mm) & 1829 \\
\hline Wall Length (mm) & 1829 \\
\hline \multicolumn{2}{|l|}{ Wall Dimensions } \\
\hline Block Height (mm) & 190 \\
\hline Block Thickness (mm) & 140 \\
\hline Bed Joint Thickness (mm) & 10 \\
\hline \multicolumn{2}{|l|}{ Longitudinal Reinforcement Properties } \\
\hline Longitudinal Reinforcement Ratio (\%) & 0.25 \\
\hline Area in Flexural Tension $\left(\mathrm{mm}^{2}\right)$ & 498 \\
\hline Bar Diameter (mm) & 15.9 \\
\hline Distance to Furthest Tension Bar (mm) & 1727 \\
\hline Effective Distance (mm) & 1404 \\
\hline Modulus of Elasticity (MPa) & 200000 \\
\hline Yield Strength (MPa) & 441 \\
\hline Ultimate Strength (MPa) & 710 \\
\hline Ultimate Stain & 0.2 \\
\hline \multicolumn{2}{|l|}{ Transverse Reinforcement Properties } \\
\hline Transverse Reinforcement Ratio (\%) & 0.24 \\
\hline Modulus of Elasticity (MPa) & 200000 \\
\hline Yield Strength (MPa) & 379 \\
\hline Ultimate Strength (MPa) & 558 \\
\hline Ultimate Stain & 0.2 \\
\hline \multicolumn{2}{|l|}{ Critical Loading Zone Properties } \\
\hline Area of Longitudinal Reinforcement $\left(\mathrm{mm}^{2}\right)$ & 199 \\
\hline Longitudinal Reinforcement Center-to-Center Spacing (mm) & 400 \\
\hline Longitudinal Reinforcement Clear Spacing (mm) & 384 \\
\hline Area of Tie $\left(\mathrm{mm}^{2}\right)$ & 0 \\
\hline Tie Clear Spacing (mm) & 387 \\
\hline Tie Depth (mm) & 120 \\
\hline Tie Width (mm) & 190 \\
\hline
\end{tabular}




\section{HCBL 3 Shear Wall}

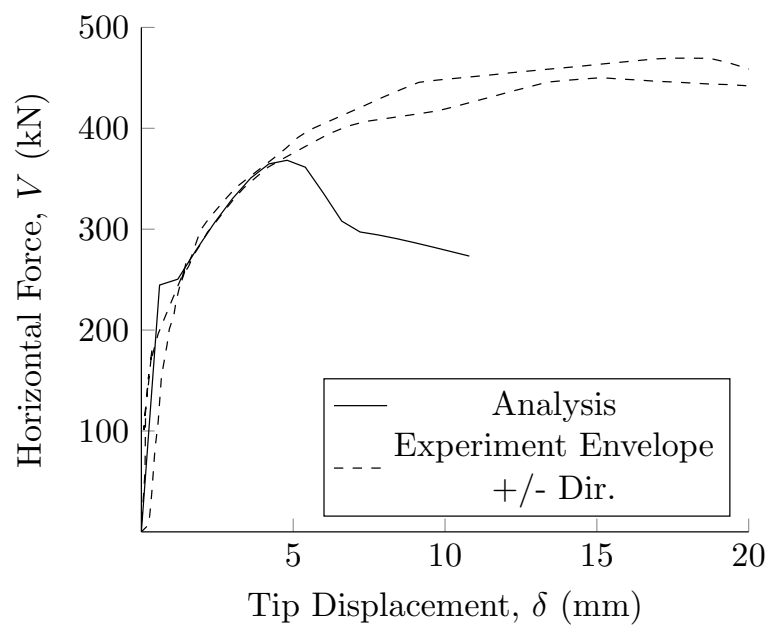

HCBL 3 Shear Wall Shing et al. (1991) Horizontal Force vs Tip Displacement

HCBL 3 Shear Wall Shing et al. (1991) Model Options

\begin{tabular}{ll}
\hline Model Option & Value \\
\hline Axial Compression Load (N) & 480000 \\
Total Lateral Displacement & 60 \\
Number of Load Stages & 100 \\
Convergence Limit & 0.01 \\
Maximum Iterations & 100 \\
\hline
\end{tabular}


HCBL 3 Shear Wall Shing et al. (1991) Material Properties

\begin{tabular}{|c|c|}
\hline Property & Value \\
\hline \multicolumn{2}{|l|}{ Masonry Properties } \\
\hline Y-Direction Peak Compression Strength (MPa) & 20.4 \\
\hline X-Direction Peak Compression Strength (MPa) & 20.4 \\
\hline Maximum Aggregate Size (mm) & 5 \\
\hline \multicolumn{2}{|l|}{ Wall Dimensions } \\
\hline Wall Span (mm) & 1829 \\
\hline Wall Height (mm) & 1829 \\
\hline Wall Length (mm) & 1829 \\
\hline \multicolumn{2}{|l|}{ Wall Dimensions } \\
\hline Block Height (mm) & 190 \\
\hline Block Thickness (mm) & 140 \\
\hline Bed Joint Thickness (mm) & 10 \\
\hline \multicolumn{2}{|l|}{ Longitudinal Reinforcement Properties } \\
\hline Longitudinal Reinforcement Ratio (\%) & 0.49 \\
\hline Area in Flexural Tension $\left(\mathrm{mm}^{2}\right)$ & 970 \\
\hline Bar Diameter (mm) & 22.2 \\
\hline Distance to Furthest Tension Bar (mm) & 1727 \\
\hline Effective Distance (mm) & 1404 \\
\hline Modulus of Elasticity (MPa) & 200000 \\
\hline Yield Strength (MPa) & 496 \\
\hline Ultimate Strength (MPa) & 710 \\
\hline Ultimate Stain & 0.2 \\
\hline \multicolumn{2}{|l|}{ Transverse Reinforcement Properties } \\
\hline Transverse Reinforcement Ratio (\%) & 0.14 \\
\hline Modulus of Elasticity (MPa) & 200000 \\
\hline Yield Strength (MPa) & 386 \\
\hline Ultimate Strength (MPa) & 558 \\
\hline Ultimate Stain & 0.2 \\
\hline \multicolumn{2}{|l|}{ Critical Loading Zone Properties } \\
\hline Area of Longitudinal Reinforcement $\left(\mathrm{mm}^{2}\right)$ & 388 \\
\hline Longitudinal Reinforcement Center-to-Center Spacing (mm) & 400 \\
\hline Longitudinal Reinforcement Clear Spacing (mm) & 378 \\
\hline Area of Tie $\left(\mathrm{mm}^{2}\right)$ & 0 \\
\hline Tie Clear Spacing (mm) & 390 \\
\hline Tie Depth (mm) & 120 \\
\hline Tie Width (mm) & 190 \\
\hline
\end{tabular}




\section{HCBL 4 Shear Wall}

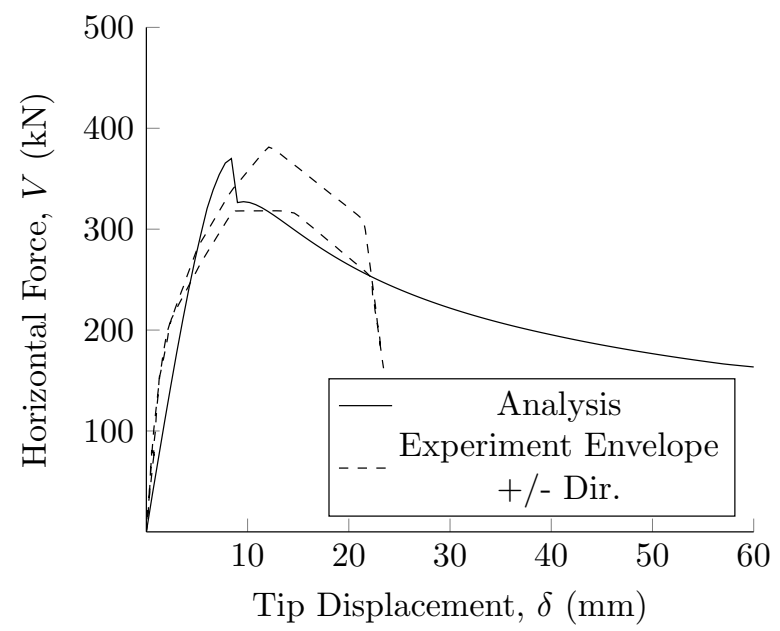

HCBL 4 Shear Wall Shing et al. (1991) Horizontal Force vs Tip Displacement

HCBL 4 Shear Wall Shing et al. (1991) Model Options

\begin{tabular}{ll}
\hline Model Option & Value \\
\hline Axial Compression Load $(\mathrm{N})$ & 0 \\
Total Lateral Displacement & 60 \\
Number of Load Stages & 100 \\
Convergence Limit & 0.01 \\
Maximum Iterations & 100 \\
\hline
\end{tabular}


HCBL 4 Shear Wall Shing et al. (1991) Material Properties

\begin{tabular}{|c|c|}
\hline Property & Value \\
\hline \multicolumn{2}{|l|}{ Masonry Properties } \\
\hline Y-Direction Peak Compression Strength (MPa) & 18.2 \\
\hline X-Direction Peak Compression Strength (MPa) & 18.2 \\
\hline Maximum Aggregate Size (mm) & 5 \\
\hline \multicolumn{2}{|l|}{ Wall Dimensions } \\
\hline Wall Span (mm) & 1829 \\
\hline Wall Height (mm) & 1829 \\
\hline Wall Length (mm) & 1829 \\
\hline \multicolumn{2}{|l|}{ Wall Dimensions } \\
\hline Block Height (mm) & 190 \\
\hline Block Thickness (mm) & 140 \\
\hline Bed Joint Thickness (mm) & 10 \\
\hline \multicolumn{2}{|l|}{ Longitudinal Reinforcement Properties } \\
\hline Longitudinal Reinforcement Ratio (\%) & 0.49 \\
\hline Area in Flexural Tension $\left(\mathrm{mm}^{2}\right)$ & 970 \\
\hline Bar Diameter (mm) & 22.2 \\
\hline Distance to Furthest Tension Bar (mm) & 1727 \\
\hline Effective Distance (mm) & 1404 \\
\hline Modulus of Elasticity (MPa) & 200000 \\
\hline Yield Strength (MPa) & 496 \\
\hline Ultimate Strength (MPa) & 710 \\
\hline Ultimate Stain & 0.2 \\
\hline \multicolumn{2}{|l|}{ Transverse Reinforcement Properties } \\
\hline Transverse Reinforcement Ratio (\%) & 0.14 \\
\hline Modulus of Elasticity (MPa) & 200000 \\
\hline Yield Strength (MPa) & 386 \\
\hline Ultimate Strength (MPa) & 558 \\
\hline Ultimate Stain & 0.2 \\
\hline \multicolumn{2}{|l|}{ Critical Loading Zone Properties } \\
\hline Area of Longitudinal Reinforcement $\left(\mathrm{mm}^{2}\right)$ & 388 \\
\hline Longitudinal Reinforcement Center-to-Center Spacing (mm) & 400 \\
\hline Longitudinal Reinforcement Clear Spacing (mm) & 378 \\
\hline Area of Tie $\left(\mathrm{mm}^{2}\right)$ & 0 \\
\hline Tie Clear Spacing (mm) & 390 \\
\hline Tie Depth (mm) & 120 \\
\hline Tie Width (mm) & 190 \\
\hline
\end{tabular}




\section{HCBL 5 Shear Wall}

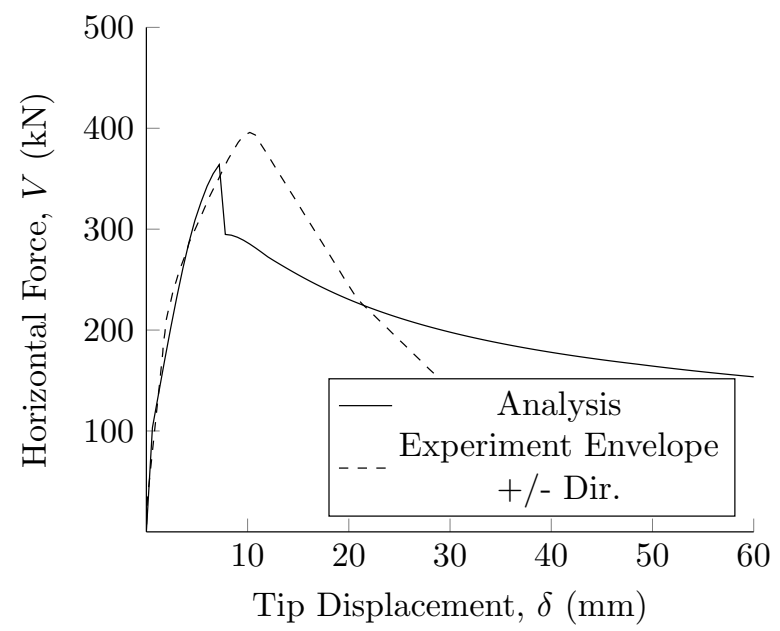

HCBL 5 Shear Wall Shing et al. (1991) Horizontal Force vs Tip Displacement

HCBL 5 Shear Wall Shing et al. (1991) Model Options

\begin{tabular}{ll}
\hline Model Option & Value \\
\hline Axial Compression Load (N) & 178000 \\
Total Lateral Displacement & 60 \\
Number of Load Stages & 100 \\
Convergence Limit & 0.01 \\
Maximum Iterations & 100 \\
\hline
\end{tabular}


HCBL 5 Shear Wall Shing et al. (1991) Material Properties

\begin{tabular}{|c|c|}
\hline Property & Value \\
\hline \multicolumn{2}{|l|}{ Masonry Properties } \\
\hline Y-Direction Peak Compression Strength (MPa) & 18.2 \\
\hline X-Direction Peak Compression Strength (MPa) & 18.2 \\
\hline Maximum Aggregate Size (mm) & 5 \\
\hline \multicolumn{2}{|l|}{ Wall Dimensions } \\
\hline Wall Span (mm) & 1829 \\
\hline Wall Height (mm) & 1829 \\
\hline Wall Length (mm) & 1829 \\
\hline \multicolumn{2}{|l|}{ Wall Dimensions } \\
\hline Block Height (mm) & 190 \\
\hline Block Thickness (mm) & 140 \\
\hline Bed Joint Thickness (mm) & 10 \\
\hline \multicolumn{2}{|l|}{ Longitudinal Reinforcement Properties } \\
\hline Longitudinal Reinforcement Ratio (\%) & 0.49 \\
\hline Area in Flexural Tension $\left(\mathrm{mm}^{2}\right)$ & 970 \\
\hline Bar Diameter (mm) & 22.2 \\
\hline Distance to Furthest Tension Bar (mm) & 1727 \\
\hline Effective Distance (mm) & 1404 \\
\hline Modulus of Elasticity (MPa) & 200000 \\
\hline Yield Strength (MPa) & 496 \\
\hline Ultimate Strength (MPa) & 710 \\
\hline Ultimate Stain & 0.2 \\
\hline \multicolumn{2}{|l|}{ Transverse Reinforcement Properties } \\
\hline Transverse Reinforcement Ratio (\%) & 0.14 \\
\hline Modulus of Elasticity (MPa) & 200000 \\
\hline Yield Strength (MPa) & 386 \\
\hline Ultimate Strength (MPa) & 558 \\
\hline Ultimate Stain & 0.2 \\
\hline \multicolumn{2}{|l|}{ Critical Loading Zone Properties } \\
\hline Area of Longitudinal Reinforcement $\left(\mathrm{mm}^{2}\right)$ & 388 \\
\hline Longitudinal Reinforcement Center-to-Center Spacing (mm) & 400 \\
\hline Longitudinal Reinforcement Clear Spacing (mm) & 378 \\
\hline Area of Tie $\left(\mathrm{mm}^{2}\right)$ & 0 \\
\hline Tie Clear Spacing (mm) & 390 \\
\hline Tie Depth (mm) & 120 \\
\hline Tie Width (mm) & 190 \\
\hline
\end{tabular}




\section{HCBL 6 Shear Wall}

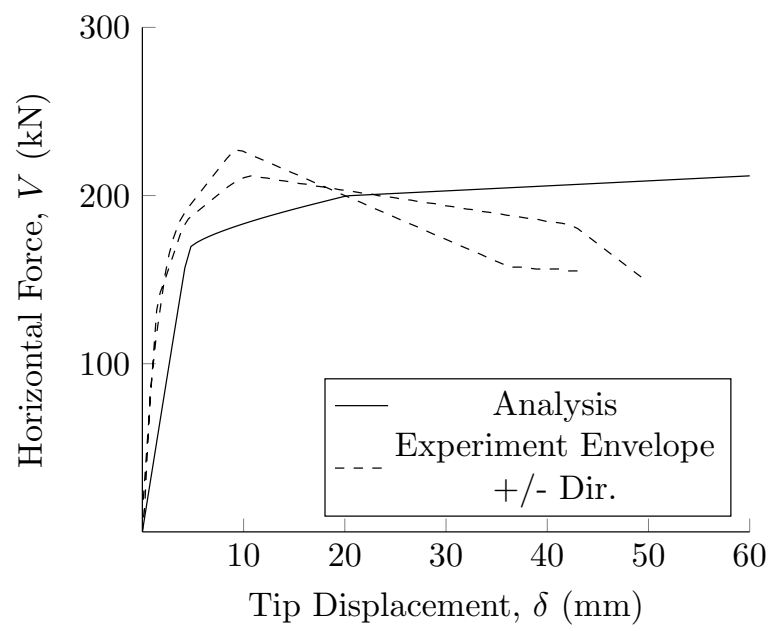

HCBL 6 Shear Wall Shing et al. (1991) Horizontal Force vs Tip Displacement

HCBL 6 Shear Wall Shing et al. (1991) Model Options

\begin{tabular}{ll}
\hline Model Option & Value \\
\hline Axial Compression Load (N) & 0 \\
Total Lateral Displacement & 60 \\
Number of Load Stages & 100 \\
Convergence Limit & 0.01 \\
Maximum Iterations & 100 \\
\hline
\end{tabular}


HCBL 6 Shear Wall Shing et al. (1991) Material Properties

\begin{tabular}{|c|c|}
\hline Property & Value \\
\hline \multicolumn{2}{|l|}{ Masonry Properties } \\
\hline Y-Direction Peak Compression Strength (MPa) & 18.2 \\
\hline X-Direction Peak Compression Strength (MPa) & 18.2 \\
\hline Maximum Aggregate Size (mm) & 5 \\
\hline \multicolumn{2}{|l|}{ Wall Dimensions } \\
\hline Wall Span (mm) & 1829 \\
\hline Wall Height (mm) & 1829 \\
\hline Wall Length (mm) & 1829 \\
\hline \multicolumn{2}{|l|}{ Wall Dimensions } \\
\hline Block Height (mm) & 190 \\
\hline Block Thickness (mm) & 140 \\
\hline Bed Joint Thickness (mm) & 10 \\
\hline \multicolumn{2}{|l|}{ Longitudinal Reinforcement Properties } \\
\hline Longitudinal Reinforcement Ratio (\%) & 0.25 \\
\hline Area in Flexural Tension $\left(\mathrm{mm}^{2}\right)$ & 498 \\
\hline Bar Diameter (mm) & 15.9 \\
\hline Distance to Furthest Tension Bar (mm) & 1727 \\
\hline Effective Distance (mm) & 1404 \\
\hline Modulus of Elasticity (MPa) & 200000 \\
\hline Yield Strength (MPa) & 441 \\
\hline Ultimate Strength (MPa) & 710 \\
\hline Ultimate Stain & 0.2 \\
\hline \multicolumn{2}{|l|}{ Transverse Reinforcement Properties } \\
\hline Transverse Reinforcement Ratio (\%) & 0.14 \\
\hline Modulus of Elasticity (MPa) & 200000 \\
\hline Yield Strength (MPa) & 386 \\
\hline Ultimate Strength (MPa) & 558 \\
\hline Ultimate Stain & 0.2 \\
\hline \multicolumn{2}{|l|}{ Critical Loading Zone Properties } \\
\hline Area of Longitudinal Reinforcement $\left(\mathrm{mm}^{2}\right)$ & 199 \\
\hline Longitudinal Reinforcement Center-to-Center Spacing (mm) & 400 \\
\hline Longitudinal Reinforcement Clear Spacing (mm) & 384 \\
\hline Area of Tie $\left(\mathrm{mm}^{2}\right)$ & 0 \\
\hline Tie Clear Spacing (mm) & 390 \\
\hline Tie Depth (mm) & 120 \\
\hline Tie Width (mm) & 190 \\
\hline
\end{tabular}




\section{HCBL 7 Shear Wall}

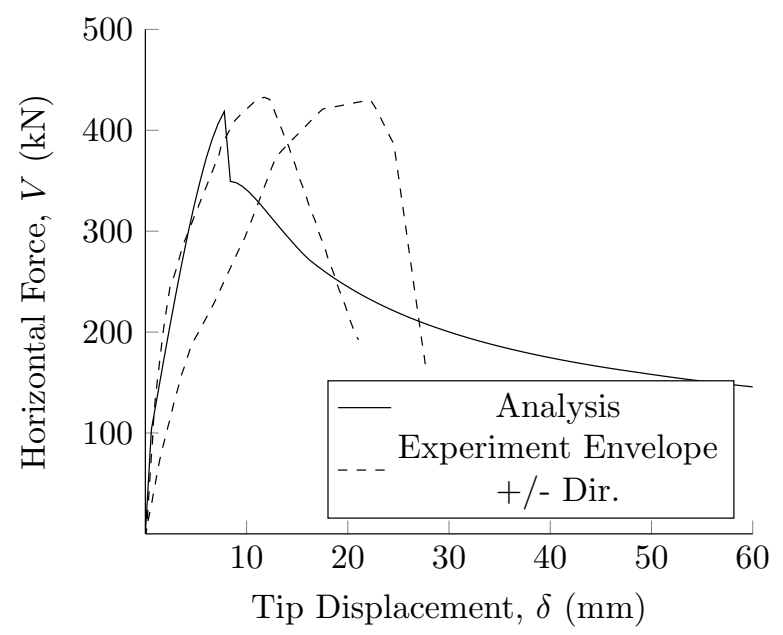

HCBL 7 Shear Wall Shing et al. (1991) Horizontal Force vs Tip Displacement

HCBL 7 Shear Wall Shing et al. (1991) Model Options

\begin{tabular}{ll}
\hline Model Option & Value \\
\hline Axial Compression Load (N) & 178000 \\
Total Lateral Displacement & 60 \\
Number of Load Stages & 100 \\
Convergence Limit & 0.01 \\
Maximum Iterations & 100 \\
\hline
\end{tabular}


HCBL 7 Shear Wall Shing et al. (1991) Material Properties

\begin{tabular}{|c|c|}
\hline Property & Value \\
\hline \multicolumn{2}{|l|}{ Masonry Properties } \\
\hline Y-Direction Peak Compression Strength (MPa) & 22.4 \\
\hline X-Direction Peak Compression Strength (MPa) & 22.4 \\
\hline Maximum Aggregate Size (mm) & 5 \\
\hline \multicolumn{2}{|l|}{ Wall Dimensions } \\
\hline Wall Span (mm) & 1829 \\
\hline Wall Height (mm) & 1829 \\
\hline Wall Length (mm) & 1829 \\
\hline \multicolumn{2}{|l|}{ Wall Dimensions } \\
\hline Block Height (mm) & 190 \\
\hline Block Thickness (mm) & 140 \\
\hline Bed Joint Thickness (mm) & 10 \\
\hline \multicolumn{2}{|l|}{ Longitudinal Reinforcement Properties } \\
\hline Longitudinal Reinforcement Ratio (\%) & 0.49 \\
\hline Area in Flexural Tension $\left(\mathrm{mm}^{2}\right)$ & 970 \\
\hline Bar Diameter (mm) & 22.2 \\
\hline Distance to Furthest Tension Bar (mm) & 1727 \\
\hline Effective Distance (mm) & 1404 \\
\hline Modulus of Elasticity (MPa) & 200000 \\
\hline Yield Strength (MPa) & 496 \\
\hline Ultimate Strength (MPa) & 710 \\
\hline Ultimate Stain & 0.2 \\
\hline \multicolumn{2}{|l|}{ Transverse Reinforcement Properties } \\
\hline Transverse Reinforcement Ratio (\%) & 0.14 \\
\hline Modulus of Elasticity (MPa) & 200000 \\
\hline Yield Strength (MPa) & 386 \\
\hline Ultimate Strength (MPa) & 558 \\
\hline Ultimate Stain & 0.2 \\
\hline \multicolumn{2}{|l|}{ Critical Loading Zone Properties } \\
\hline Area of Longitudinal Reinforcement $\left(\mathrm{mm}^{2}\right)$ & 388 \\
\hline Longitudinal Reinforcement Center-to-Center Spacing (mm) & 400 \\
\hline Longitudinal Reinforcement Clear Spacing (mm) & 378 \\
\hline Area of Tie $\left(\mathrm{mm}^{2}\right)$ & 0 \\
\hline Tie Clear Spacing (mm) & 390 \\
\hline Tie Depth (mm) & 120 \\
\hline Tie Width (mm) & 190 \\
\hline
\end{tabular}




\section{HCBL 8 Shear Wall}

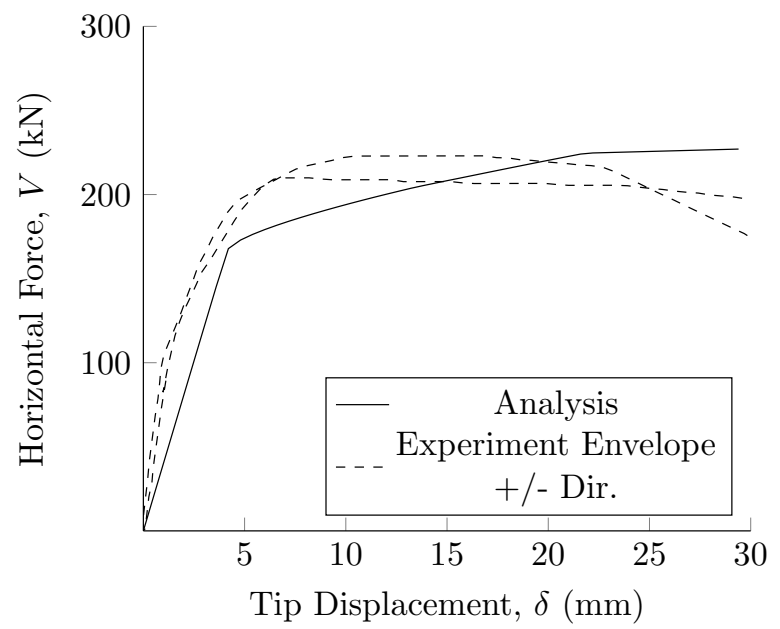

HCBL 8 Shear Wall Shing et al. (1991) Horizontal Force vs Tip Displacement

HCBL 8 Shear Wall Shing et al. (1991) Model Options

\begin{tabular}{ll}
\hline Model Option & Value \\
\hline Axial Compression Load (N) & 0 \\
Total Lateral Displacement & 60 \\
Number of Load Stages & 100 \\
Convergence Limit & 0.01 \\
Maximum Iterations & 100 \\
\hline
\end{tabular}


HCBL 8 Shear Wall Shing et al. (1991) Material Properties

\begin{tabular}{|c|c|}
\hline Property & Value \\
\hline \multicolumn{2}{|l|}{ Masonry Properties } \\
\hline Y-Direction Peak Compression Strength (MPa) & 22.4 \\
\hline X-Direction Peak Compression Strength (MPa) & 22.4 \\
\hline Maximum Aggregate Size (mm) & 5 \\
\hline \multicolumn{2}{|l|}{ Wall Dimensions } \\
\hline Wall Span (mm) & 1829 \\
\hline Wall Height (mm) & 1829 \\
\hline Wall Length (mm) & 1829 \\
\hline \multicolumn{2}{|l|}{ Wall Dimensions } \\
\hline Block Height (mm) & 190 \\
\hline Block Thickness (mm) & 140 \\
\hline Bed Joint Thickness (mm) & 10 \\
\hline \multicolumn{2}{|l|}{ Longitudinal Reinforcement Properties } \\
\hline Longitudinal Reinforcement Ratio (\%) & 0.25 \\
\hline Area in Flexural Tension $\left(\mathrm{mm}^{2}\right)$ & 498 \\
\hline Bar Diameter (mm) & 15.9 \\
\hline Distance to Furthest Tension Bar (mm) & 1727 \\
\hline Effective Distance (mm) & 1404 \\
\hline Modulus of Elasticity (MPa) & 200000 \\
\hline Yield Strength (MPa) & 441 \\
\hline Ultimate Strength (MPa) & 710 \\
\hline Ultimate Stain & 0.2 \\
\hline \multicolumn{2}{|l|}{ Transverse Reinforcement Properties } \\
\hline Transverse Reinforcement Ratio (\%) & 0.24 \\
\hline Modulus of Elasticity (MPa) & 200000 \\
\hline Yield Strength (MPa) & 462 \\
\hline Ultimate Strength (MPa) & 738 \\
\hline Ultimate Stain & 0.2 \\
\hline \multicolumn{2}{|l|}{ Critical Loading Zone Properties } \\
\hline Area of Longitudinal Reinforcement $\left(\mathrm{mm}^{2}\right)$ & 199 \\
\hline Longitudinal Reinforcement Center-to-Center Spacing (mm) & 400 \\
\hline Longitudinal Reinforcement Clear Spacing (mm) & 384 \\
\hline Area of Tie $\left(\mathrm{mm}^{2}\right)$ & 0 \\
\hline Tie Clear Spacing (mm) & 388 \\
\hline Tie Depth (mm) & 120 \\
\hline Tie Width (mm) & 190 \\
\hline
\end{tabular}




\section{HCBL 9 Shear Wall}

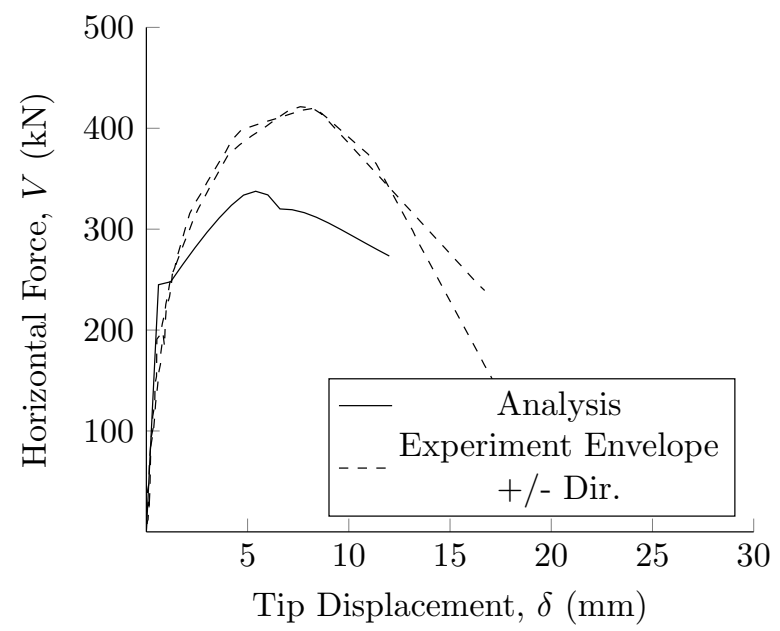

HCBL 9 Shear Wall Shing et al. (1991) Horizontal Force vs Tip Displacement

HCBL 9 Shear Wall Shing et al. (1991) Model Options

\begin{tabular}{ll}
\hline Model Option & Value \\
\hline Axial Compression Load (N) & 480000 \\
Total Lateral Displacement & 60 \\
Number of Load Stages & 100 \\
Convergence Limit & 0.01 \\
Maximum Iterations & 100 \\
\hline
\end{tabular}


HCBL 9 Shear Wall Shing et al. (1991) Material Properties

\begin{tabular}{|c|c|}
\hline Property & Value \\
\hline \multicolumn{2}{|l|}{ Masonry Properties } \\
\hline Y-Direction Peak Compression Strength (MPa) & 22.4 \\
\hline X-Direction Peak Compression Strength (MPa) & 22.4 \\
\hline Maximum Aggregate Size (mm) & 5 \\
\hline \multicolumn{2}{|l|}{ Wall Dimensions } \\
\hline Wall Span (mm) & 1829 \\
\hline Wall Height (mm) & 1829 \\
\hline Wall Length (mm) & 1829 \\
\hline \multicolumn{2}{|l|}{ Wall Dimensions } \\
\hline Block Height (mm) & 190 \\
\hline Block Thickness (mm) & 140 \\
\hline Bed Joint Thickness (mm) & 10 \\
\hline \multicolumn{2}{|l|}{ Longitudinal Reinforcement Properties } \\
\hline Longitudinal Reinforcement Ratio (\%) & 0.25 \\
\hline Area in Flexural Tension $\left(\mathrm{mm}^{2}\right)$ & 498 \\
\hline Bar Diameter (mm) & 15.9 \\
\hline Distance to Furthest Tension Bar (mm) & 1727 \\
\hline Effective Distance (mm) & 1404 \\
\hline Modulus of Elasticity (MPa) & 200000 \\
\hline Yield Strength (MPa) & 441 \\
\hline Ultimate Strength (MPa) & 710 \\
\hline Ultimate Stain & 0.2 \\
\hline \multicolumn{2}{|l|}{ Transverse Reinforcement Properties } \\
\hline Transverse Reinforcement Ratio (\%) & 0.14 \\
\hline Modulus of Elasticity (MPa) & 200000 \\
\hline Yield Strength (MPa) & 386 \\
\hline Ultimate Strength (MPa) & 558 \\
\hline Ultimate Stain & 0.2 \\
\hline \multicolumn{2}{|l|}{ Critical Loading Zone Properties } \\
\hline Area of Longitudinal Reinforcement $\left(\mathrm{mm}^{2}\right)$ & 199 \\
\hline Longitudinal Reinforcement Center-to-Center Spacing (mm) & 400 \\
\hline Longitudinal Reinforcement Clear Spacing (mm) & 384 \\
\hline Area of Tie $\left(\mathrm{mm}^{2}\right)$ & 0 \\
\hline Tie Clear Spacing (mm) & 390 \\
\hline Tie Depth (mm) & 120 \\
\hline Tie Width (mm) & 190 \\
\hline
\end{tabular}




\section{HCBL 10 Shear Wall}

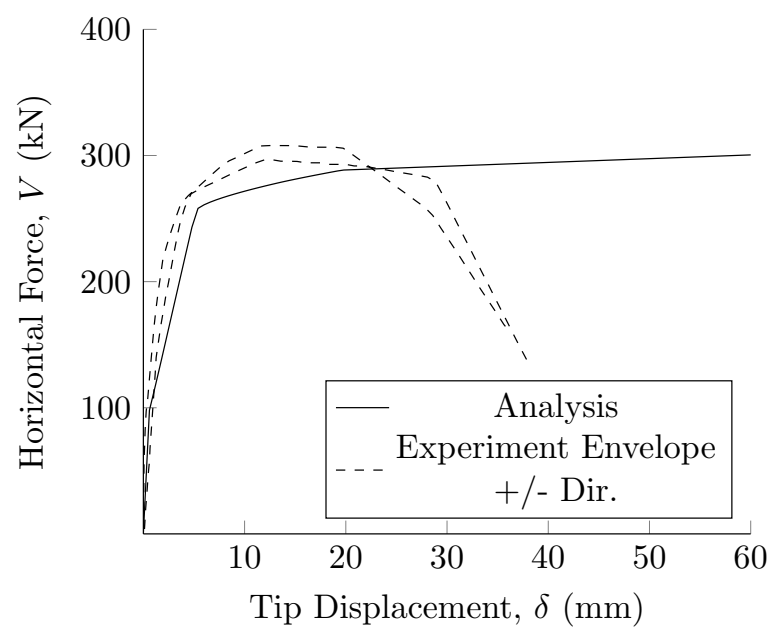

HCBL 10 Shear Wall Shing et al. (1991) Horizontal Force vs Tip Displacement

HCBL 10 Shear Wall Shing et al. (1991) Model Options

\begin{tabular}{ll}
\hline Model Option & Value \\
\hline Axial Compression Load (N) & 178000 \\
Total Lateral Displacement & 60 \\
Number of Load Stages & 100 \\
Convergence Limit & 0.01 \\
Maximum Iterations & 100 \\
\hline
\end{tabular}


HCBL 10 Shear Wall Shing et al. (1991) Material Properties

\begin{tabular}{|c|c|}
\hline Property & Value \\
\hline \multicolumn{2}{|l|}{ Masonry Properties } \\
\hline Y-Direction Peak Compression Strength (MPa) & 22.3 \\
\hline X-Direction Peak Compression Strength ( $\mathrm{MPa})$ & 22.3 \\
\hline Maximum Aggregate Size (mm) & 5 \\
\hline \multicolumn{2}{|l|}{ Wall Dimensions } \\
\hline Wall Span (mm) & 1829 \\
\hline Wall Height (mm) & 1829 \\
\hline Wall Length (mm) & 1829 \\
\hline \multicolumn{2}{|l|}{ Wall Dimensions } \\
\hline Block Height (mm) & 190 \\
\hline Block Thickness (mm) & 140 \\
\hline Bed Joint Thickness (mm) & 10 \\
\hline \multicolumn{2}{|l|}{ Longitudinal Reinforcement Properties } \\
\hline Longitudinal Reinforcement Ratio (\%) & 0.25 \\
\hline Area in Flexural Tension $\left(\mathrm{mm}^{2}\right)$ & 498 \\
\hline Bar Diameter (mm) & 15.9 \\
\hline Distance to Furthest Tension Bar (mm) & 1727 \\
\hline Effective Distance (mm) & 1404 \\
\hline Modulus of Elasticity (MPa) & 200000 \\
\hline Yield Strength (MPa) & 441 \\
\hline Ultimate Strength (MPa) & 710 \\
\hline Ultimate Stain & 0.2 \\
\hline \multicolumn{2}{|l|}{ Transverse Reinforcement Properties } \\
\hline Transverse Reinforcement Ratio (\%) & 0.14 \\
\hline Modulus of Elasticity (MPa) & 200000 \\
\hline Yield Strength (MPa) & 386 \\
\hline Ultimate Strength (MPa) & 558 \\
\hline Ultimate Stain & 0.2 \\
\hline \multicolumn{2}{|l|}{ Critical Loading Zone Properties } \\
\hline Area of Longitudinal Reinforcement $\left(\mathrm{mm}^{2}\right)$ & 199 \\
\hline Longitudinal Reinforcement Center-to-Center Spacing (mm) & 400 \\
\hline Longitudinal Reinforcement Clear Spacing (mm) & 384 \\
\hline Area of Tie $\left(\mathrm{mm}^{2}\right)$ & 0 \\
\hline Tie Clear Spacing (mm) & 390 \\
\hline Tie Depth (mm) & 120 \\
\hline Tie Width (mm) & 190 \\
\hline
\end{tabular}




\section{HCBL 11 Shear Wall}

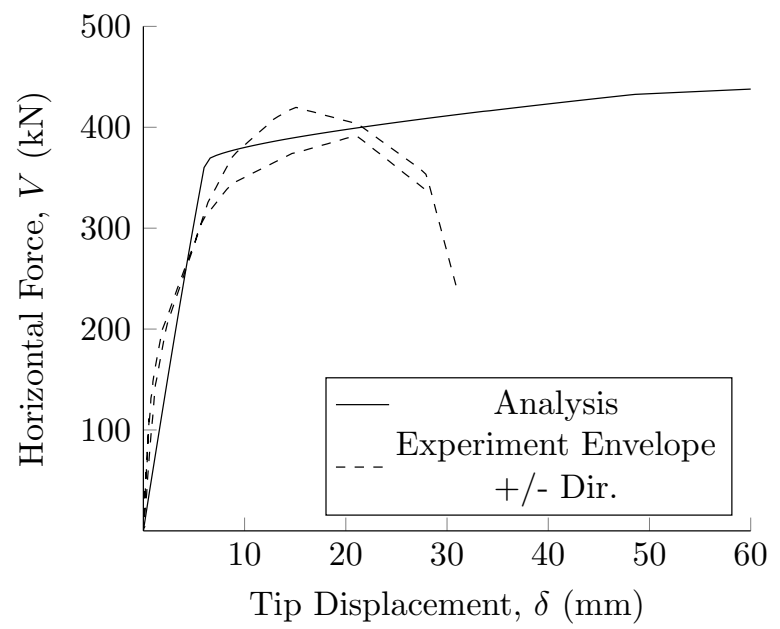

HCBL 11 Shear Wall Shing et al. (1991) Horizontal Force vs Tip Displacement

HCBL 11 Shear Wall Shing et al. (1991) Model Options

\begin{tabular}{ll}
\hline Model Option & Value \\
\hline Axial Compression Load (N) & 0 \\
Total Lateral Displacement & 60 \\
Number of Load Stages & 100 \\
Convergence Limit & 0.01 \\
Maximum Iterations & 100 \\
\hline
\end{tabular}


HCBL 11 Shear Wall Shing et al. (1991) Material Properties

\begin{tabular}{|c|c|}
\hline Property & Value \\
\hline \multicolumn{2}{|l|}{ Masonry Properties } \\
\hline Y-Direction Peak Compression Strength (MPa) & 22.3 \\
\hline X-Direction Peak Compression Strength ( $\mathrm{MPa})$ & 22.3 \\
\hline Maximum Aggregate Size (mm) & 5 \\
\hline \multicolumn{2}{|l|}{ Wall Dimensions } \\
\hline Wall Span (mm) & 1829 \\
\hline Wall Height (mm) & 1829 \\
\hline Wall Length (mm) & 1829 \\
\hline \multicolumn{2}{|l|}{ Wall Dimensions } \\
\hline Block Height (mm) & 190 \\
\hline Block Thickness (mm) & 140 \\
\hline Bed Joint Thickness (mm) & 10 \\
\hline \multicolumn{2}{|l|}{ Longitudinal Reinforcement Properties } \\
\hline Longitudinal Reinforcement Ratio (\%) & 0.49 \\
\hline Area in Flexural Tension $\left(\mathrm{mm}^{2}\right)$ & 970 \\
\hline Bar Diameter (mm) & 22.2 \\
\hline Distance to Furthest Tension Bar (mm) & 1727 \\
\hline Effective Distance (mm) & 1404 \\
\hline Modulus of Elasticity (MPa) & 200000 \\
\hline Yield Strength (MPa) & 496 \\
\hline Ultimate Strength (MPa) & 710 \\
\hline Ultimate Stain & 0.2 \\
\hline \multicolumn{2}{|l|}{ Transverse Reinforcement Properties } \\
\hline Transverse Reinforcement Ratio (\%) & 0.24 \\
\hline Modulus of Elasticity (MPa) & 200000 \\
\hline Yield Strength (MPa) & 462 \\
\hline Ultimate Strength (MPa) & 738 \\
\hline Ultimate Stain & 0.2 \\
\hline \multicolumn{2}{|l|}{ Critical Loading Zone Properties } \\
\hline Area of Longitudinal Reinforcement $\left(\mathrm{mm}^{2}\right)$ & 388 \\
\hline Longitudinal Reinforcement Center-to-Center Spacing (mm) & 400 \\
\hline Longitudinal Reinforcement Clear Spacing (mm) & 378 \\
\hline Area of Tie $\left(\mathrm{mm}^{2}\right)$ & 0 \\
\hline Tie Clear Spacing (mm) & 388 \\
\hline Tie Depth (mm) & 120 \\
\hline Tie Width (mm) & 190 \\
\hline
\end{tabular}




\section{HCBL 12 Shear Wall}

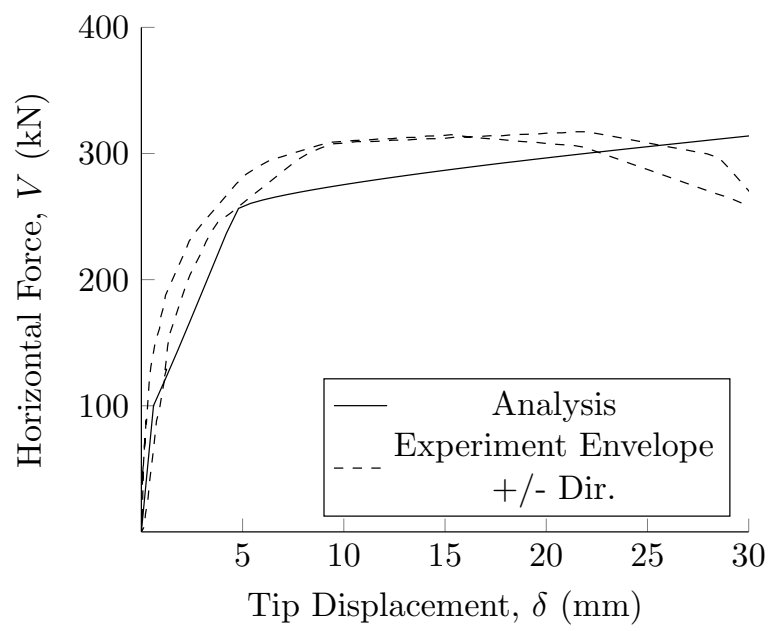

HCBL 12 Shear Wall Shing et al. (1991) Horizontal Force vs Tip Displacement

HCBL 12 Shear Wall Shing et al. (1991) Model Options

\begin{tabular}{ll}
\hline Model Option & Value \\
\hline Axial Compression Load (N) & 178000 \\
Total Lateral Displacement & 60 \\
Number of Load Stages & 100 \\
Convergence Limit & 0.01 \\
Maximum Iterations & 100 \\
\hline
\end{tabular}


HCBL 12 Shear Wall Shing et al. (1991) Material Properties

\begin{tabular}{|c|c|}
\hline Property & Value \\
\hline \multicolumn{2}{|l|}{ Masonry Properties } \\
\hline Y-Direction Peak Compression Strength (MPa) & 22.3 \\
\hline X-Direction Peak Compression Strength ( $\mathrm{MPa})$ & 22.3 \\
\hline Maximum Aggregate Size (mm) & 5 \\
\hline \multicolumn{2}{|l|}{ Wall Dimensions } \\
\hline Wall Span (mm) & 1829 \\
\hline Wall Height (mm) & 1829 \\
\hline Wall Length (mm) & 1829 \\
\hline \multicolumn{2}{|l|}{ Wall Dimensions } \\
\hline Block Height (mm) & 190 \\
\hline Block Thickness (mm) & 140 \\
\hline Bed Joint Thickness (mm) & 10 \\
\hline \multicolumn{2}{|l|}{ Longitudinal Reinforcement Properties } \\
\hline Longitudinal Reinforcement Ratio (\%) & 0.25 \\
\hline Area in Flexural Tension $\left(\mathrm{mm}^{2}\right)$ & 498 \\
\hline Bar Diameter (mm) & 15.9 \\
\hline Distance to Furthest Tension Bar (mm) & 1727 \\
\hline Effective Distance (mm) & 1404 \\
\hline Modulus of Elasticity (MPa) & 200000 \\
\hline Yield Strength (MPa) & 441 \\
\hline Ultimate Strength (MPa) & 710 \\
\hline Ultimate Stain & 0.2 \\
\hline \multicolumn{2}{|l|}{ Transverse Reinforcement Properties } \\
\hline Transverse Reinforcement Ratio (\%) & 0.24 \\
\hline Modulus of Elasticity (MPa) & 200000 \\
\hline Yield Strength (MPa) & 462 \\
\hline Ultimate Strength (MPa) & 738 \\
\hline Ultimate Stain & 0.2 \\
\hline \multicolumn{2}{|l|}{ Critical Loading Zone Properties } \\
\hline Area of Longitudinal Reinforcement $\left(\mathrm{mm}^{2}\right)$ & 199 \\
\hline Longitudinal Reinforcement Center-to-Center Spacing (mm) & 400 \\
\hline Longitudinal Reinforcement Clear Spacing (mm) & 384 \\
\hline Area of Tie $\left(\mathrm{mm}^{2}\right)$ & 0 \\
\hline Tie Clear Spacing (mm) & 388 \\
\hline Tie Depth (mm) & 120 \\
\hline Tie Width (mm) & 190 \\
\hline
\end{tabular}




\section{HCBL 13 Shear Wall}

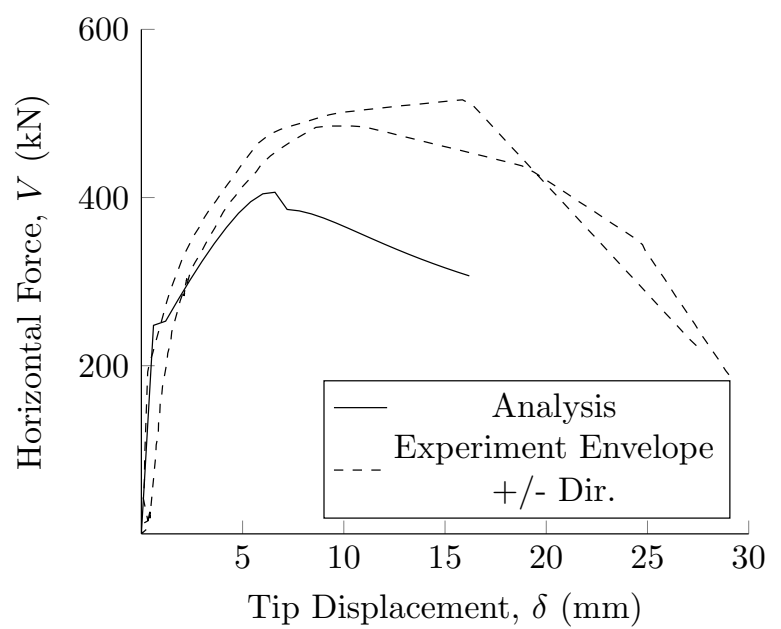

HCBL 13 Shear Wall Shing et al. (1991) Horizontal Force vs Tip Displacement

HCBL 13 Shear Wall Shing et al. (1991) Model Options

\begin{tabular}{ll}
\hline Model Option & Value \\
\hline Axial Compression Load (N) & 480000 \\
Total Lateral Displacement & 60 \\
Number of Load Stages & 100 \\
Convergence Limit & 0.01 \\
Maximum Iterations & 100 \\
\hline
\end{tabular}


HCBL 13 Shear Wall Shing et al. (1991) Material Properties

\begin{tabular}{|c|c|}
\hline Property & Value \\
\hline \multicolumn{2}{|l|}{ Masonry Properties } \\
\hline Y-Direction Peak Compression Strength (MPa) & 22.8 \\
\hline X-Direction Peak Compression Strength ( $\mathrm{MPa})$ & 22.8 \\
\hline Maximum Aggregate Size (mm) & 5 \\
\hline \multicolumn{2}{|l|}{ Wall Dimensions } \\
\hline Wall Span (mm) & 1829 \\
\hline Wall Height (mm) & 1829 \\
\hline Wall Length (mm) & 1829 \\
\hline \multicolumn{2}{|l|}{ Wall Dimensions } \\
\hline Block Height (mm) & 190 \\
\hline Block Thickness (mm) & 140 \\
\hline Bed Joint Thickness (mm) & 10 \\
\hline \multicolumn{2}{|l|}{ Longitudinal Reinforcement Properties } \\
\hline Longitudinal Reinforcement Ratio (\%) & 0.36 \\
\hline Area in Flexural Tension $\left(\mathrm{mm}^{2}\right)$ & 713 \\
\hline Bar Diameter (mm) & 19.05 \\
\hline Distance to Furthest Tension Bar (mm) & 1727 \\
\hline Effective Distance (mm) & 1404 \\
\hline Modulus of Elasticity (MPa) & 200000 \\
\hline Yield Strength (MPa) & 448 \\
\hline Ultimate Strength (MPa) & 738 \\
\hline Ultimate Stain & 0.2 \\
\hline \multicolumn{2}{|l|}{ Transverse Reinforcement Properties } \\
\hline Transverse Reinforcement Ratio (\%) & 0.24 \\
\hline Modulus of Elasticity (MPa) & 200000 \\
\hline Yield Strength (MPa) & 462 \\
\hline Ultimate Strength (MPa) & 738 \\
\hline Ultimate Stain & 0.2 \\
\hline \multicolumn{2}{|l|}{ Critical Loading Zone Properties } \\
\hline Area of Longitudinal Reinforcement $\left(\mathrm{mm}^{2}\right)$ & 285 \\
\hline Longitudinal Reinforcement Center-to-Center Spacing (mm) & 400 \\
\hline Longitudinal Reinforcement Clear Spacing (mm) & 381 \\
\hline Area of Tie $\left(\mathrm{mm}^{2}\right)$ & 0 \\
\hline Tie Clear Spacing (mm) & 388 \\
\hline Tie Depth (mm) & 120 \\
\hline Tie Width (mm) & 190 \\
\hline
\end{tabular}




\section{HCBL 14 Shear Wall}

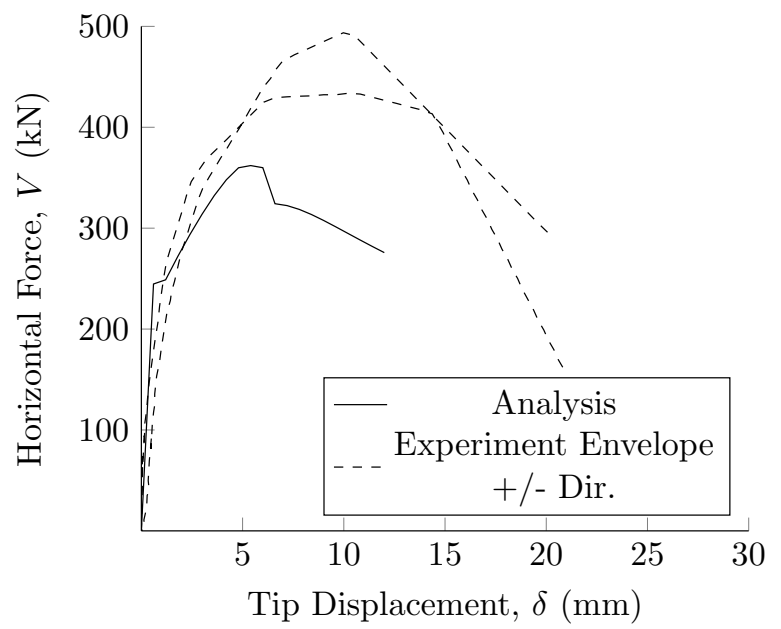

HCBL 14 Shear Wall Shing et al. (1991) Horizontal Force vs Tip Displacement

HCBL 14 Shear Wall Shing et al. (1991) Model Options

\begin{tabular}{ll}
\hline Model Option & Value \\
\hline Axial Compression Load (N) & 480000 \\
Total Lateral Displacement & 60 \\
Number of Load Stages & 100 \\
Convergence Limit & 0.01 \\
Maximum Iterations & 100 \\
\hline
\end{tabular}


HCBL 14 Shear Wall Shing et al. (1991) Material Properties

\begin{tabular}{|c|c|}
\hline Property & Value \\
\hline \multicolumn{2}{|l|}{ Masonry Properties } \\
\hline Y-Direction Peak Compression Strength (MPa) & 22.8 \\
\hline X-Direction Peak Compression Strength ( $\mathrm{MPa})$ & 22.8 \\
\hline Maximum Aggregate Size (mm) & 5 \\
\hline \multicolumn{2}{|l|}{ Wall Dimensions } \\
\hline Wall Span (mm) & 1829 \\
\hline Wall Height (mm) & 1829 \\
\hline Wall Length (mm) & 1829 \\
\hline \multicolumn{2}{|l|}{ Wall Dimensions } \\
\hline Block Height (mm) & 190 \\
\hline Block Thickness (mm) & 140 \\
\hline Bed Joint Thickness (mm) & 10 \\
\hline \multicolumn{2}{|l|}{ Longitudinal Reinforcement Properties } \\
\hline Longitudinal Reinforcement Ratio (\%) & 0.36 \\
\hline Area in Flexural Tension $\left(\mathrm{mm}^{2}\right)$ & 713 \\
\hline Bar Diameter (mm) & 19.05 \\
\hline Distance to Furthest Tension Bar (mm) & 1727 \\
\hline Effective Distance (mm) & 1404 \\
\hline Modulus of Elasticity (MPa) & 200000 \\
\hline Yield Strength (MPa) & 448 \\
\hline Ultimate Strength (MPa) & 738 \\
\hline Ultimate Stain & 0.2 \\
\hline \multicolumn{2}{|l|}{ Transverse Reinforcement Properties } \\
\hline Transverse Reinforcement Ratio (\%) & 0.14 \\
\hline Modulus of Elasticity (MPa) & 200000 \\
\hline Yield Strength (MPa) & 386 \\
\hline Ultimate Strength (MPa) & 558 \\
\hline Ultimate Stain & 0.2 \\
\hline \multicolumn{2}{|l|}{ Critical Loading Zone Properties } \\
\hline Area of Longitudinal Reinforcement $\left(\mathrm{mm}^{2}\right)$ & 285 \\
\hline Longitudinal Reinforcement Center-to-Center Spacing (mm) & 400 \\
\hline Longitudinal Reinforcement Clear Spacing (mm) & 381 \\
\hline Area of Tie $\left(\mathrm{mm}^{2}\right)$ & 0 \\
\hline Tie Clear Spacing (mm) & 390 \\
\hline Tie Depth (mm) & 120 \\
\hline Tie Width (mm) & 190 \\
\hline
\end{tabular}




\section{HCBL 15 Shear Wall}

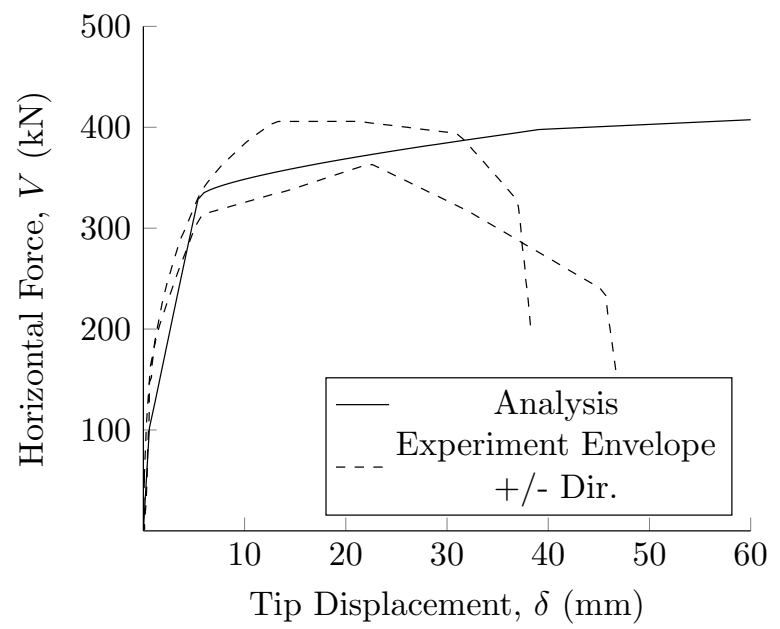

HCBL 15 Shear Wall Shing et al. (1991) Horizontal Force vs Tip Displacement

HCBL 15 Shear Wall Shing et al. (1991) Model Options

\begin{tabular}{ll}
\hline Model Option & Value \\
\hline Axial Compression Load (N) & 178000 \\
Total Lateral Displacement & 60 \\
Number of Load Stages & 100 \\
Convergence Limit & 0.01 \\
Maximum Iterations & 100 \\
\hline
\end{tabular}


HCBL 15 Shear Wall Shing et al. (1991) Material Properties

\begin{tabular}{|c|c|}
\hline Property & Value \\
\hline \multicolumn{2}{|l|}{ Masonry Properties } \\
\hline Y-Direction Peak Compression Strength (MPa) & 22.8 \\
\hline X-Direction Peak Compression Strength ( $\mathrm{MPa})$ & 22.8 \\
\hline Maximum Aggregate Size (mm) & 5 \\
\hline \multicolumn{2}{|l|}{ Wall Dimensions } \\
\hline Wall Span (mm) & 1829 \\
\hline Wall Height (mm) & 1829 \\
\hline Wall Length (mm) & 1829 \\
\hline \multicolumn{2}{|l|}{ Wall Dimensions } \\
\hline Block Height (mm) & 190 \\
\hline Block Thickness (mm) & 140 \\
\hline Bed Joint Thickness (mm) & 10 \\
\hline \multicolumn{2}{|l|}{ Longitudinal Reinforcement Properties } \\
\hline Longitudinal Reinforcement Ratio (\%) & 0.36 \\
\hline Area in Flexural Tension $\left(\mathrm{mm}^{2}\right)$ & 713 \\
\hline Bar Diameter (mm) & 19.05 \\
\hline Distance to Furthest Tension Bar (mm) & 1727 \\
\hline Effective Distance (mm) & 1404 \\
\hline Modulus of Elasticity (MPa) & 200000 \\
\hline Yield Strength (MPa) & 448 \\
\hline Ultimate Strength (MPa) & 738 \\
\hline Ultimate Stain & 0.2 \\
\hline \multicolumn{2}{|l|}{ Transverse Reinforcement Properties } \\
\hline Transverse Reinforcement Ratio (\%) & 0.24 \\
\hline Modulus of Elasticity (MPa) & 200000 \\
\hline Yield Strength (MPa) & 462 \\
\hline Ultimate Strength (MPa) & 738 \\
\hline Ultimate Stain & 0.2 \\
\hline \multicolumn{2}{|l|}{ Critical Loading Zone Properties } \\
\hline Area of Longitudinal Reinforcement $\left(\mathrm{mm}^{2}\right)$ & 285 \\
\hline Longitudinal Reinforcement Center-to-Center Spacing (mm) & 400 \\
\hline Longitudinal Reinforcement Clear Spacing (mm) & 381 \\
\hline Area of Tie $\left(\mathrm{mm}^{2}\right)$ & 0 \\
\hline Tie Clear Spacing (mm) & 388 \\
\hline Tie Depth (mm) & 120 \\
\hline Tie Width (mm) & 190 \\
\hline
\end{tabular}




\section{HCBL 16 Shear Wall}

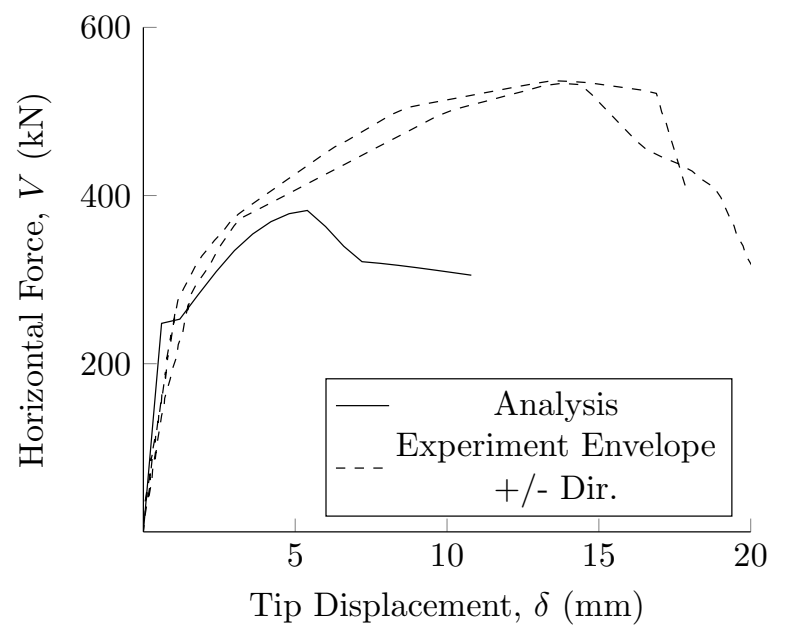

HCBL 16 Shear Wall Shing et al. (1991) Horizontal Force vs Tip Displacement

HCBL 16 Shear Wall Shing et al. (1991) Model Options

\begin{tabular}{ll}
\hline Model Option & Value \\
\hline Axial Compression Load (N) & 480000 \\
Total Lateral Displacement & 60 \\
Number of Load Stages & 100 \\
Convergence Limit & 0.01 \\
Maximum Iterations & 100 \\
\hline
\end{tabular}


HCBL 16 Shear Wall Shing et al. (1991) Material Properties

\begin{tabular}{|c|c|}
\hline Property & Value \\
\hline \multicolumn{2}{|l|}{ Masonry Properties } \\
\hline Y-Direction Peak Compression Strength (MPa) & 17.1 \\
\hline X-Direction Peak Compression Strength ( $\mathrm{MPa})$ & 17.1 \\
\hline Maximum Aggregate Size (mm) & 5 \\
\hline \multicolumn{2}{|l|}{ Wall Dimensions } \\
\hline Wall Span (mm) & 1829 \\
\hline Wall Height (mm) & 1829 \\
\hline Wall Length (mm) & 1829 \\
\hline \multicolumn{2}{|l|}{ Wall Dimensions } \\
\hline Block Height (mm) & 190 \\
\hline Block Thickness (mm) & 140 \\
\hline Bed Joint Thickness (mm) & 10 \\
\hline \multicolumn{2}{|l|}{ Longitudinal Reinforcement Properties } \\
\hline Longitudinal Reinforcement Ratio (\%) & 0.49 \\
\hline Area in Flexural Tension $\left(\mathrm{mm}^{2}\right)$ & 970 \\
\hline Bar Diameter (mm) & 22.2 \\
\hline Distance to Furthest Tension Bar (mm) & 1727 \\
\hline Effective Distance (mm) & 1404 \\
\hline Modulus of Elasticity (MPa) & 200000 \\
\hline Yield Strength (MPa) & 496 \\
\hline Ultimate Strength (MPa) & 710 \\
\hline Ultimate Stain & 0.2 \\
\hline \multicolumn{2}{|l|}{ Transverse Reinforcement Properties } \\
\hline Transverse Reinforcement Ratio (\%) & 0.24 \\
\hline Modulus of Elasticity (MPa) & 200000 \\
\hline Yield Strength (MPa) & 462 \\
\hline Ultimate Strength (MPa) & 738 \\
\hline Ultimate Stain & 0.2 \\
\hline \multicolumn{2}{|l|}{ Critical Loading Zone Properties } \\
\hline Area of Longitudinal Reinforcement $\left(\mathrm{mm}^{2}\right)$ & 388 \\
\hline Longitudinal Reinforcement Center-to-Center Spacing (mm) & 400 \\
\hline Longitudinal Reinforcement Clear Spacing (mm) & 378 \\
\hline Area of Tie $\left(\mathrm{mm}^{2}\right)$ & 0 \\
\hline Tie Clear Spacing (mm) & 388 \\
\hline Tie Depth (mm) & 120 \\
\hline Tie Width (mm) & 190 \\
\hline
\end{tabular}




\section{HCBL 23 Shear Wall}

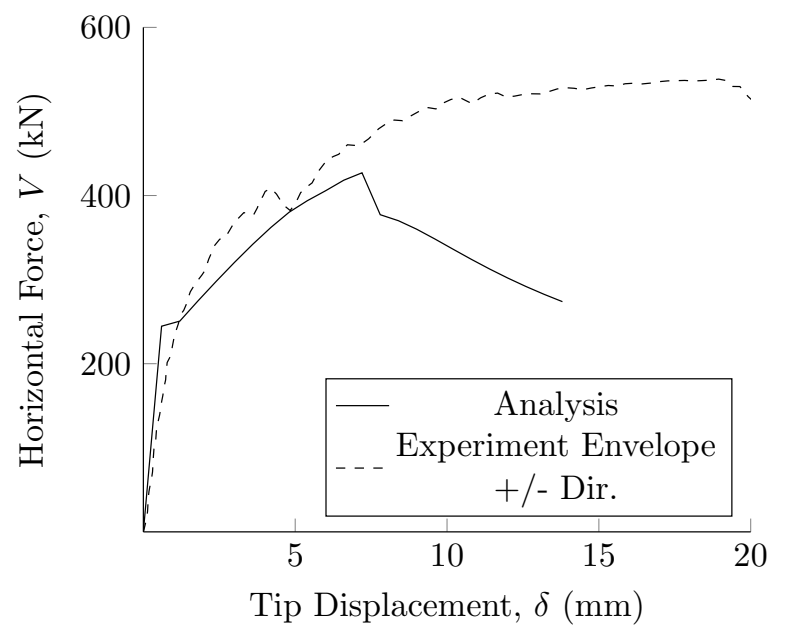

HCBL 23 Shear Wall Shing et al. (1991) Horizontal Force vs Tip Displacement

HCBL 23 Shear Wall Shing et al. (1991) Model Options

\begin{tabular}{ll}
\hline Model Option & Value \\
\hline Axial Compression Load (N) & 480000 \\
Total Lateral Displacement & 60 \\
Number of Load Stages & 100 \\
Convergence Limit & 0.01 \\
Maximum Iterations & 100 \\
\hline
\end{tabular}


HCBL 23 Shear Wall Shing et al. (1991) Material Properties

\begin{tabular}{|c|c|}
\hline Property & Value \\
\hline \multicolumn{2}{|l|}{ Masonry Properties } \\
\hline Y-Direction Peak Compression Strength (MPa) & 28.3 \\
\hline X-Direction Peak Compression Strength ( $\mathrm{MPa})$ & 28.3 \\
\hline Maximum Aggregate Size (mm) & 5 \\
\hline \multicolumn{2}{|l|}{ Wall Dimensions } \\
\hline Wall Span (mm) & 1829 \\
\hline Wall Height (mm) & 1829 \\
\hline Wall Length (mm) & 1829 \\
\hline \multicolumn{2}{|l|}{ Wall Dimensions } \\
\hline Block Height (mm) & 190 \\
\hline Block Thickness (mm) & 140 \\
\hline Bed Joint Thickness (mm) & 10 \\
\hline \multicolumn{2}{|l|}{ Longitudinal Reinforcement Properties } \\
\hline Longitudinal Reinforcement Ratio (\%) & 0.36 \\
\hline Area in Flexural Tension $\left(\mathrm{mm}^{2}\right)$ & 713 \\
\hline Bar Diameter (mm) & 19.05 \\
\hline Distance to Furthest Tension Bar (mm) & 1727 \\
\hline Effective Distance (mm) & 1404 \\
\hline Modulus of Elasticity (MPa) & 200000 \\
\hline Yield Strength (MPa) & 448 \\
\hline Ultimate Strength (MPa) & 738 \\
\hline Ultimate Stain & 0.2 \\
\hline \multicolumn{2}{|l|}{ Transverse Reinforcement Properties } \\
\hline Transverse Reinforcement Ratio (\%) & 0.14 \\
\hline Modulus of Elasticity (MPa) & 200000 \\
\hline Yield Strength (MPa) & 386 \\
\hline Ultimate Strength (MPa) & 558 \\
\hline Ultimate Stain & 0.2 \\
\hline \multicolumn{2}{|l|}{ Critical Loading Zone Properties } \\
\hline Area of Longitudinal Reinforcement $\left(\mathrm{mm}^{2}\right)$ & 285 \\
\hline Longitudinal Reinforcement Center-to-Center Spacing (mm) & 400 \\
\hline Longitudinal Reinforcement Clear Spacing (mm) & 381 \\
\hline Area of Tie $\left(\mathrm{mm}^{2}\right)$ & 0 \\
\hline Tie Clear Spacing (mm) & 390 \\
\hline Tie Depth (mm) & 120 \\
\hline Tie Width (mm) & 190 \\
\hline
\end{tabular}




\section{HCBL 24 Shear Wall}

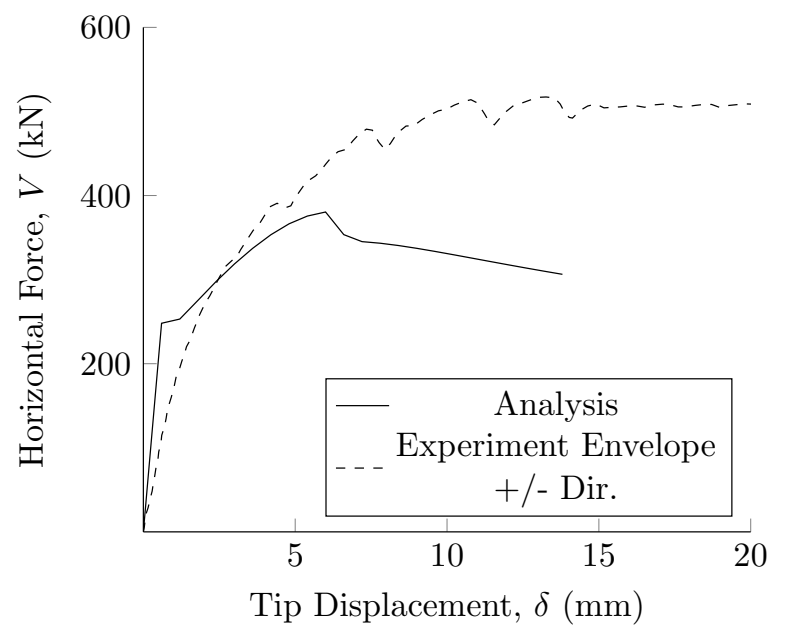

HCBL 24 Shear Wall Shing et al. (1991) Horizontal Force vs Tip Displacement

HCBL 24 Shear Wall Shing et al. (1991) Model Options

\begin{tabular}{ll}
\hline Model Option & Value \\
\hline Axial Compression Load (N) & 480000 \\
Total Lateral Displacement & 60 \\
Number of Load Stages & 100 \\
Convergence Limit & 0.01 \\
Maximum Iterations & 100 \\
\hline
\end{tabular}


HCBL 24 Shear Wall Shing et al. (1991) Material Properties

\begin{tabular}{|c|c|}
\hline Property & Value \\
\hline \multicolumn{2}{|l|}{ Masonry Properties } \\
\hline Y-Direction Peak Compression Strength (MPa) & 28.3 \\
\hline X-Direction Peak Compression Strength ( $\mathrm{MPa})$ & 28.3 \\
\hline Maximum Aggregate Size (mm) & 5 \\
\hline \multicolumn{2}{|l|}{ Wall Dimensions } \\
\hline Wall Span (mm) & 1829 \\
\hline Wall Height (mm) & 1829 \\
\hline Wall Length (mm) & 1829 \\
\hline \multicolumn{2}{|l|}{ Wall Dimensions } \\
\hline Block Height (mm) & 190 \\
\hline Block Thickness (mm) & 140 \\
\hline Bed Joint Thickness (mm) & 10 \\
\hline \multicolumn{2}{|l|}{ Longitudinal Reinforcement Properties } \\
\hline Longitudinal Reinforcement Ratio (\%) & 0.36 \\
\hline Area in Flexural Tension $\left(\mathrm{mm}^{2}\right)$ & 713 \\
\hline Bar Diameter (mm) & 19.05 \\
\hline Distance to Furthest Tension Bar (mm) & 1727 \\
\hline Effective Distance (mm) & 1404 \\
\hline Modulus of Elasticity (MPa) & 200000 \\
\hline Yield Strength (MPa) & 448 \\
\hline Ultimate Strength (MPa) & 738 \\
\hline Ultimate Stain & 0.2 \\
\hline \multicolumn{2}{|l|}{ Transverse Reinforcement Properties } \\
\hline Transverse Reinforcement Ratio (\%) & 0.24 \\
\hline Modulus of Elasticity (MPa) & 200000 \\
\hline Yield Strength (MPa) & 462 \\
\hline Ultimate Strength (MPa) & 738 \\
\hline Ultimate Stain & 0.2 \\
\hline \multicolumn{2}{|l|}{ Critical Loading Zone Properties } \\
\hline Area of Longitudinal Reinforcement $\left(\mathrm{mm}^{2}\right)$ & 285 \\
\hline Longitudinal Reinforcement Center-to-Center Spacing (mm) & 400 \\
\hline Longitudinal Reinforcement Clear Spacing (mm) & 381 \\
\hline Area of Tie $\left(\mathrm{mm}^{2}\right)$ & 0 \\
\hline Tie Clear Spacing (mm) & 390 \\
\hline Tie Depth (mm) & 120 \\
\hline Tie Width (mm) & 190 \\
\hline
\end{tabular}




\section{Ibrahim (1995)}

\section{Wall 1 Shear Wall}

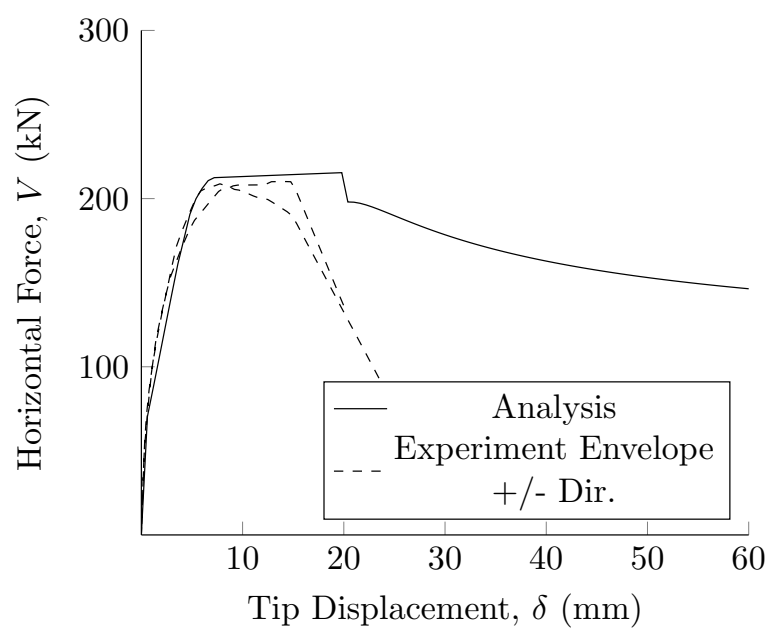

Wall 1 Shear Wall Ibrahim (1995) Horizontal Force vs Tip Displacement

Wall 1 Shear Wall Ibrahim (1995) Model Options

\begin{tabular}{ll}
\hline Model Option & Value \\
\hline Axial Compression Load (N) & 135000 \\
Total Lateral Displacement & 60 \\
Number of Load Stages & 100 \\
Convergence Limit & 0.01 \\
Maximum Iterations & 100 \\
\hline
\end{tabular}


Wall 1 Shear Wall Ibrahim (1995) Material Properties

\begin{tabular}{|c|c|}
\hline Property & Value \\
\hline \multicolumn{2}{|l|}{ Masonry Properties } \\
\hline Y-Direction Peak Compression Strength (MPa) & 12.1 \\
\hline X-Direction Peak Compression Strength (MPa) & 12.1 \\
\hline Maximum Aggregate Size (mm) & 5 \\
\hline \multicolumn{2}{|l|}{ Wall Dimensions } \\
\hline Wall Span (mm) & 1600 \\
\hline Wall Height (mm) & 1400 \\
\hline Wall Length (mm) & 1400 \\
\hline \multicolumn{2}{|l|}{ Wall Dimensions } \\
\hline Block Height (mm) & 190 \\
\hline Block Thickness (mm) & 140 \\
\hline Bed Joint Thickness (mm) & 10 \\
\hline \multicolumn{2}{|l|}{ Longitudinal Reinforcement Properties } \\
\hline Longitudinal Reinforcement Ratio (\%) & 0.24 \\
\hline Area in Flexural Tension $\left(\mathrm{mm}^{2}\right)$ & 400 \\
\hline Bar Diameter $(\mathrm{mm})$ & 16 \\
\hline Distance to Furthest Tension Bar (mm) & 1300 \\
\hline Effective Distance (mm) & 1100 \\
\hline Modulus of Elasticity (MPa) & 200000 \\
\hline Yield Strength (MPa) & 440 \\
\hline Ultimate Strength (MPa) & 688 \\
\hline Ultimate Stain & 0.2 \\
\hline \multicolumn{2}{|l|}{ Transverse Reinforcement Properties } \\
\hline Transverse Reinforcement Ratio (\%) & 0.20 \\
\hline Modulus of Elasticity (MPa) & 200000 \\
\hline Yield Strength (MPa) & 463 \\
\hline Ultimate Strength (MPa) & 654 \\
\hline Ultimate Stain & 0.2 \\
\hline \multicolumn{2}{|l|}{ Critical Loading Zone Properties } \\
\hline Area of Longitudinal Reinforcement $\left(\mathrm{mm}^{2}\right)$ & 200 \\
\hline Longitudinal Reinforcement Center-to-Center Spacing (mm) & 400 \\
\hline Longitudinal Reinforcement Clear Spacing (mm) & 384 \\
\hline Area of Tie $\left(\mathrm{mm}^{2}\right)$ & 0 \\
\hline Tie Clear Spacing (mm) & 390 \\
\hline Tie Depth $(\mathrm{mm})$ & 120 \\
\hline Tie Width (mm) & 190 \\
\hline
\end{tabular}




\section{Wall 2 Shear Wall}

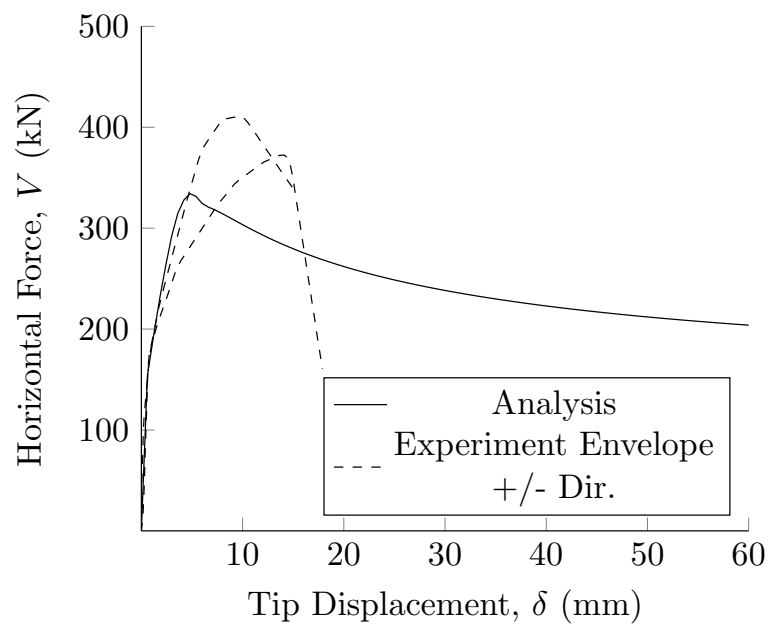

Wall 2 Shear Wall Ibrahim (1995) Horizontal Force vs Tip Displacement

Wall 2 Shear Wall Ibrahim (1995) Model Options

\begin{tabular}{ll}
\hline Model Option & Value \\
\hline Axial Compression Load (N) & 213000 \\
Total Lateral Displacement & 60 \\
Number of Load Stages & 100 \\
Convergence Limit & 0.01 \\
Maximum Iterations & 100 \\
\hline
\end{tabular}


Wall 2 Shear Wall Ibrahim (1995) Material Properties

\begin{tabular}{|c|c|}
\hline Property & Value \\
\hline \multicolumn{2}{|l|}{ Masonry Properties } \\
\hline Y-Direction Peak Compression Strength (MPa) & 12.1 \\
\hline X-Direction Peak Compression Strength (MPa) & 12.1 \\
\hline Maximum Aggregate Size (mm) & 5 \\
\hline \multicolumn{2}{|l|}{ Wall Dimensions } \\
\hline Wall Span (mm) & 1600 \\
\hline Wall Height (mm) & 1400 \\
\hline Wall Length (mm) & 2000 \\
\hline \multicolumn{2}{|l|}{ Wall Dimensions } \\
\hline Block Height (mm) & 190 \\
\hline Block Thickness (mm) & 140 \\
\hline Bed Joint Thickness (mm) & 10 \\
\hline \multicolumn{2}{|l|}{ Longitudinal Reinforcement Properties } \\
\hline Longitudinal Reinforcement Ratio (\%) & 0.29 \\
\hline Area in Flexural Tension $\left(\mathrm{mm}^{2}\right)$ & 600 \\
\hline Bar Diameter (mm) & 16 \\
\hline Distance to Furthest Tension Bar (mm) & 1900 \\
\hline Effective Distance (mm) & 1500 \\
\hline Modulus of Elasticity (MPa) & 200000 \\
\hline Yield Strength (MPa) & 440 \\
\hline Ultimate Strength (MPa) & 688 \\
\hline Ultimate Stain & 0.2 \\
\hline \multicolumn{2}{|l|}{ Transverse Reinforcement Properties } \\
\hline Transverse Reinforcement Ratio (\%) & 0.20 \\
\hline Modulus of Elasticity (MPa) & 200000 \\
\hline Yield Strength (MPa) & 463 \\
\hline Ultimate Strength (MPa) & 654 \\
\hline Ultimate Stain & 0.2 \\
\hline \multicolumn{2}{|l|}{ Critical Loading Zone Properties } \\
\hline Area of Longitudinal Reinforcement $\left(\mathrm{mm}^{2}\right)$ & 200 \\
\hline Longitudinal Reinforcement Center-to-Center Spacing (mm) & 400 \\
\hline Longitudinal Reinforcement Clear Spacing (mm) & 384 \\
\hline Area of Tie $\left(\mathrm{mm}^{2}\right)$ & 0 \\
\hline Tie Clear Spacing (mm) & 390 \\
\hline Tie Depth $(\mathrm{mm})$ & 120 \\
\hline Tie Width (mm) & 190 \\
\hline
\end{tabular}




\section{Wall 3 Shear Wall}

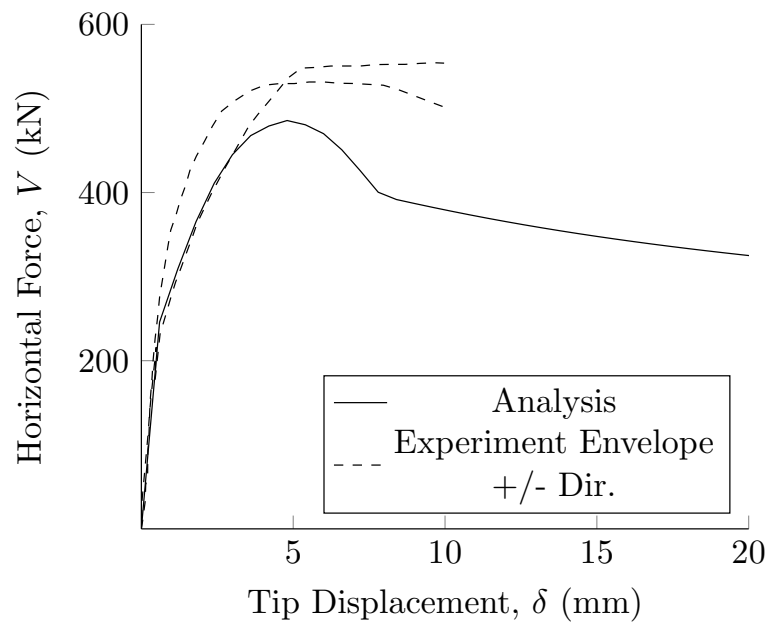

Wall 3 Shear Wall Ibrahim (1995) Horizontal Force vs Tip Displacement

Wall 3 Shear Wall Ibrahim (1995) Model Options

\begin{tabular}{ll}
\hline Model Option & Value \\
\hline Axial Compression Load (N) & 290000 \\
Total Lateral Displacement & 60 \\
Number of Load Stages & 100 \\
Convergence Limit & 0.01 \\
Maximum Iterations & 100 \\
\hline
\end{tabular}


Wall 3 Shear Wall Ibrahim (1995) Material Properties

\begin{tabular}{|c|c|}
\hline Property & Value \\
\hline \multicolumn{2}{|l|}{ Masonry Properties } \\
\hline Y-Direction Peak Compression Strength (MPa) & 12.1 \\
\hline X-Direction Peak Compression Strength (MPa) & 12.1 \\
\hline Maximum Aggregate Size (mm) & 5 \\
\hline \multicolumn{2}{|l|}{ Wall Dimensions } \\
\hline Wall Span (mm) & 1600 \\
\hline Wall Height (mm) & 1400 \\
\hline Wall Length (mm) & 2400 \\
\hline \multicolumn{2}{|l|}{ Wall Dimensions } \\
\hline Block Height (mm) & 190 \\
\hline Block Thickness (mm) & 140 \\
\hline Bed Joint Thickness (mm) & 10 \\
\hline \multicolumn{2}{|l|}{ Longitudinal Reinforcement Properties } \\
\hline Longitudinal Reinforcement Ratio (\%) & 0.31 \\
\hline Area in Flexural Tension $\left(\mathrm{mm}^{2}\right)$ & 800 \\
\hline Bar Diameter $(\mathrm{mm})$ & 16 \\
\hline Distance to Furthest Tension Bar (mm) & 2300 \\
\hline Effective Distance (mm) & 1850 \\
\hline Modulus of Elasticity (MPa) & 200000 \\
\hline Yield Strength (MPa) & 440 \\
\hline Ultimate Strength (MPa) & 688 \\
\hline Ultimate Stain & 0.2 \\
\hline \multicolumn{2}{|l|}{ Transverse Reinforcement Properties } \\
\hline Transverse Reinforcement Ratio (\%) & 0.20 \\
\hline Modulus of Elasticity (MPa) & 200000 \\
\hline Yield Strength (MPa) & 463 \\
\hline Ultimate Strength (MPa) & 654 \\
\hline Ultimate Stain & 0.2 \\
\hline \multicolumn{2}{|l|}{ Critical Loading Zone Properties } \\
\hline Area of Longitudinal Reinforcement $\left(\mathrm{mm}^{2}\right)$ & 200 \\
\hline Longitudinal Reinforcement Center-to-Center Spacing (mm) & 400 \\
\hline Longitudinal Reinforcement Clear Spacing (mm) & 384 \\
\hline Area of Tie $\left(\mathrm{mm}^{2}\right)$ & 0 \\
\hline Tie Clear Spacing (mm) & 390 \\
\hline Tie Depth $(\mathrm{mm})$ & 120 \\
\hline Tie Width (mm) & 190 \\
\hline
\end{tabular}




\section{Wall 4 Shear Wall}

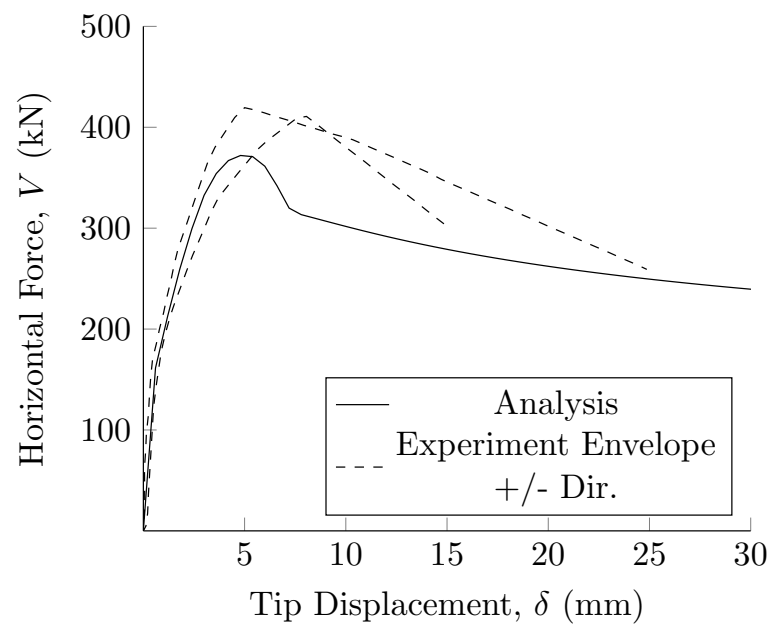

Wall 4 Shear Wall Ibrahim (1995) Horizontal Force vs Tip Displacement

Wall 4 Shear Wall Ibrahim (1995) Model Options

\begin{tabular}{ll}
\hline Model Option & Value \\
\hline Axial Compression Load (N) & 213000 \\
Total Lateral Displacement & 60 \\
Number of Load Stages & 100 \\
Convergence Limit & 0.01 \\
Maximum Iterations & 100 \\
\hline
\end{tabular}


Wall 4 Shear Wall Ibrahim (1995) Material Properties

\begin{tabular}{|c|c|}
\hline Property & Value \\
\hline \multicolumn{2}{|l|}{ Masonry Properties } \\
\hline Y-Direction Peak Compression Strength (MPa) & 12.1 \\
\hline X-Direction Peak Compression Strength (MPa) & 12.1 \\
\hline Maximum Aggregate Size (mm) & 5 \\
\hline \multicolumn{2}{|l|}{ Wall Dimensions } \\
\hline Wall Span (mm) & 1600 \\
\hline Wall Height (mm) & 1400 \\
\hline Wall Length (mm) & 2000 \\
\hline \multicolumn{2}{|l|}{ Wall Dimensions } \\
\hline Block Height (mm) & 190 \\
\hline Block Thickness (mm) & 140 \\
\hline Bed Joint Thickness (mm) & 10 \\
\hline \multicolumn{2}{|l|}{ Longitudinal Reinforcement Properties } \\
\hline Longitudinal Reinforcement Ratio (\%) & 0.43 \\
\hline Area in Flexural Tension $\left(\mathrm{mm}^{2}\right)$ & 900 \\
\hline Bar Diameter (mm) & 19.5 \\
\hline Distance to Furthest Tension Bar (mm) & 1900 \\
\hline Effective Distance (mm) & 1500 \\
\hline Modulus of Elasticity (MPa) & 200000 \\
\hline Yield Strength (MPa) & 438 \\
\hline Ultimate Strength (MPa) & 634 \\
\hline Ultimate Stain & 0.2 \\
\hline \multicolumn{2}{|l|}{ Transverse Reinforcement Properties } \\
\hline Transverse Reinforcement Ratio (\%) & 0.20 \\
\hline Modulus of Elasticity (MPa) & 200000 \\
\hline Yield Strength (MPa) & 463 \\
\hline Ultimate Strength (MPa) & 654 \\
\hline Ultimate Stain & 0.2 \\
\hline \multicolumn{2}{|l|}{ Critical Loading Zone Properties } \\
\hline Area of Longitudinal Reinforcement $\left(\mathrm{mm}^{2}\right)$ & 300 \\
\hline Longitudinal Reinforcement Center-to-Center Spacing (mm) & 400 \\
\hline Longitudinal Reinforcement Clear Spacing (mm) & 380 \\
\hline Area of Tie $\left(\mathrm{mm}^{2}\right)$ & 0 \\
\hline Tie Clear Spacing (mm) & 390 \\
\hline Tie Depth $(\mathrm{mm})$ & 120 \\
\hline Tie Width (mm) & 190 \\
\hline
\end{tabular}




\section{Wall 5 Shear Wall}

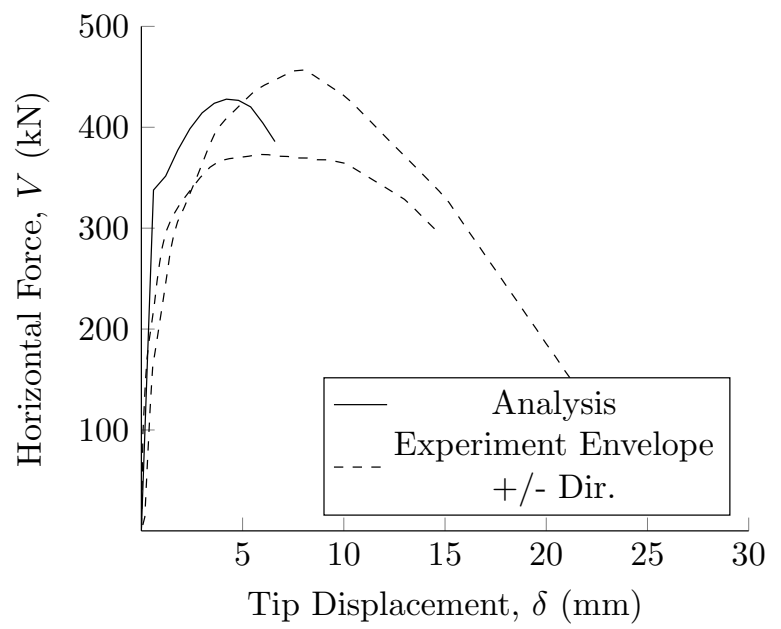

Wall 5 Shear Wall Ibrahim (1995) Horizontal Force vs Tip Displacement

Wall 5 Shear Wall Ibrahim (1995) Model Options

\begin{tabular}{ll}
\hline Model Option & Value \\
\hline Axial Compression Load (N) & 533000 \\
Total Lateral Displacement & 60 \\
Number of Load Stages & 100 \\
Convergence Limit & 0.01 \\
Maximum Iterations & 100 \\
\hline
\end{tabular}


Wall 5 Shear Wall Ibrahim (1995) Material Properties

\begin{tabular}{|c|c|}
\hline Property & Value \\
\hline \multicolumn{2}{|l|}{ Masonry Properties } \\
\hline Y-Direction Peak Compression Strength (MPa) & 12.1 \\
\hline X-Direction Peak Compression Strength (MPa) & 12.1 \\
\hline Maximum Aggregate Size (mm) & 5 \\
\hline \multicolumn{2}{|l|}{ Wall Dimensions } \\
\hline Wall Span (mm) & 1600 \\
\hline Wall Height (mm) & 1400 \\
\hline Wall Length (mm) & 2000 \\
\hline \multicolumn{2}{|l|}{ Wall Dimensions } \\
\hline Block Height (mm) & 190 \\
\hline Block Thickness (mm) & 140 \\
\hline Bed Joint Thickness (mm) & 10 \\
\hline \multicolumn{2}{|l|}{ Longitudinal Reinforcement Properties } \\
\hline Longitudinal Reinforcement Ratio (\%) & 0.29 \\
\hline Area in Flexural Tension $\left(\mathrm{mm}^{2}\right)$ & 600 \\
\hline Bar Diameter $(\mathrm{mm})$ & 16 \\
\hline Distance to Furthest Tension Bar (mm) & 1900 \\
\hline Effective Distance (mm) & 1500 \\
\hline Modulus of Elasticity (MPa) & 200000 \\
\hline Yield Strength (MPa) & 440 \\
\hline Ultimate Strength (MPa) & 688 \\
\hline Ultimate Stain & 0.2 \\
\hline \multicolumn{2}{|l|}{ Transverse Reinforcement Properties } \\
\hline Transverse Reinforcement Ratio (\%) & 0.20 \\
\hline Modulus of Elasticity (MPa) & 200000 \\
\hline Yield Strength (MPa) & 463 \\
\hline Ultimate Strength (MPa) & 654 \\
\hline Ultimate Stain & 0.2 \\
\hline \multicolumn{2}{|l|}{ Critical Loading Zone Properties } \\
\hline Area of Longitudinal Reinforcement $\left(\mathrm{mm}^{2}\right)$ & 200 \\
\hline Longitudinal Reinforcement Center-to-Center Spacing (mm) & 400 \\
\hline Longitudinal Reinforcement Clear Spacing (mm) & 384 \\
\hline Area of Tie $\left(\mathrm{mm}^{2}\right)$ & 0 \\
\hline Tie Clear Spacing (mm) & 390 \\
\hline Tie Depth $(\mathrm{mm})$ & 120 \\
\hline Tie Width (mm) & 190 \\
\hline
\end{tabular}




\section{Voon (2007)}

\section{A1 Shear Wall}

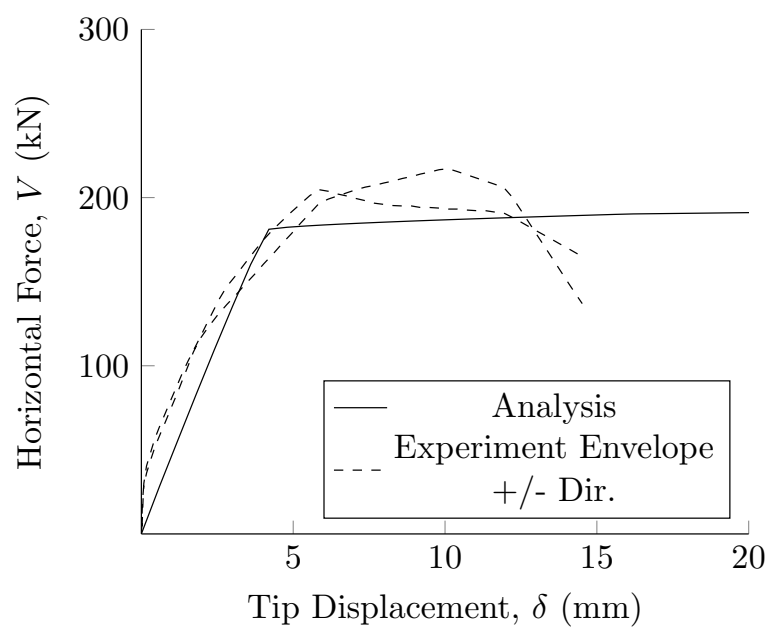

Wall A1 Shear Wall Voon (2007) Horizontal Force vs Tip Displacement

Wall A1 Shear Wall Voon (2007) Model Options

\begin{tabular}{ll}
\hline Model Option & Value \\
\hline Axial Compression Load (N) & 0 \\
Total Lateral Displacement & 60 \\
Number of Load Stages & 100 \\
Convergence Limit & 0.01 \\
Maximum Iterations & 100 \\
\hline
\end{tabular}


Wall A1 Shear Wall Voon (2007) Material Properties

\begin{tabular}{|c|c|}
\hline Property & Value \\
\hline \multicolumn{2}{|l|}{ Masonry Properties } \\
\hline Y-Direction Peak Compression Strength (MPa) & 17.6 \\
\hline X-Direction Peak Compression Strength (MPa) & 17.6 \\
\hline Maximum Aggregate Size (mm) & 5 \\
\hline \multicolumn{2}{|l|}{ Wall Dimensions } \\
\hline Wall Span (mm) & 1900 \\
\hline Wall Height (mm) & 1800 \\
\hline Wall Length (mm) & 1800 \\
\hline \multicolumn{2}{|l|}{ Wall Dimensions } \\
\hline Block Height (mm) & 190 \\
\hline Block Thickness (mm) & 140 \\
\hline Bed Joint Thickness (mm) & 10 \\
\hline \multicolumn{2}{|l|}{ Longitudinal Reinforcement Properties } \\
\hline Longitudinal Reinforcement Ratio (\%) & 0.41 \\
\hline Area in Flexural Tension $\left(\mathrm{mm}^{2}\right)$ & 785 \\
\hline Bar Diameter (mm) & 20 \\
\hline Distance to Furthest Tension Bar (mm) & 1700 \\
\hline Effective Distance (mm) & 1380 \\
\hline Modulus of Elasticity (MPa) & 200000 \\
\hline Yield Strength (MPa) & 318 \\
\hline Ultimate Strength (MPa) & 450 \\
\hline Ultimate Stain & 0.18 \\
\hline \multicolumn{2}{|l|}{ Transverse Reinforcement Properties } \\
\hline Transverse Reinforcement Ratio (\%) & 0.05 \\
\hline Modulus of Elasticity (MPa) & 200000 \\
\hline Yield Strength (MPa) & 325 \\
\hline Ultimate Strength (MPa) & 750 \\
\hline Ultimate Stain & 0.2 \\
\hline \multicolumn{2}{|l|}{ Critical Loading Zone Properties } \\
\hline Area of Longitudinal Reinforcement $\left(\mathrm{mm}^{2}\right)$ & 314 \\
\hline Longitudinal Reinforcement Center-to-Center Spacing (mm) & 400 \\
\hline Longitudinal Reinforcement Clear Spacing (mm) & 380 \\
\hline Area of Tie $\left(\mathrm{mm}^{2}\right)$ & 0 \\
\hline Tie Clear Spacing (mm) & 388 \\
\hline Tie Depth $(\mathrm{mm})$ & 120 \\
\hline Tie Width (mm) & 190 \\
\hline
\end{tabular}




\section{A2 Shear Wall}

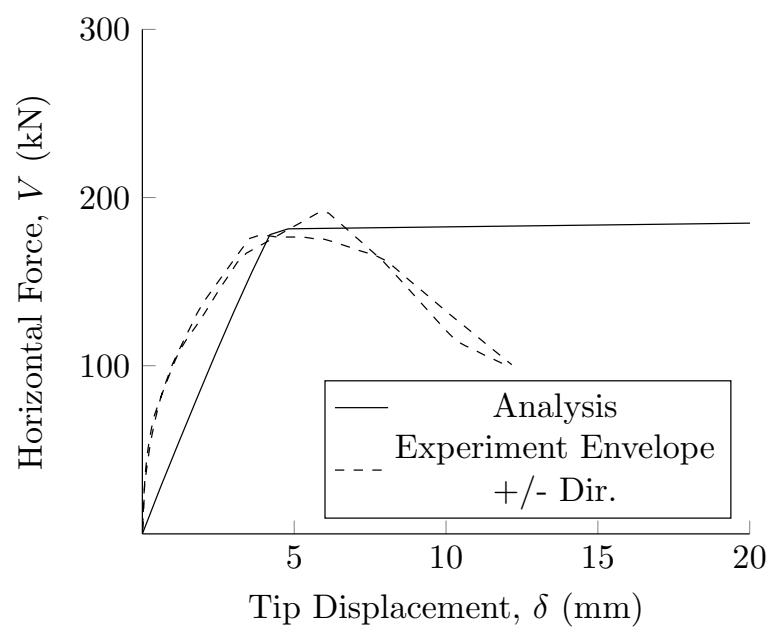

Wall A2 Shear Wall Voon (2007) Horizontal Force vs Tip Displacement

Wall A2 Shear Wall Voon (2007) Model Options

\begin{tabular}{ll}
\hline Model Option & Value \\
\hline Axial Compression Load (N) & 0 \\
Total Lateral Displacement & 60 \\
Number of Load Stages & 100 \\
Convergence Limit & 0.01 \\
Maximum Iterations & 100 \\
\hline
\end{tabular}


Wall A2 Shear Wall Voon (2007) Material Properties

\begin{tabular}{|c|c|}
\hline Property & Value \\
\hline \multicolumn{2}{|l|}{ Masonry Properties } \\
\hline Y-Direction Peak Compression Strength (MPa) & 17.6 \\
\hline X-Direction Peak Compression Strength (MPa) & 17.6 \\
\hline Maximum Aggregate Size (mm) & 5 \\
\hline \multicolumn{2}{|l|}{ Wall Dimensions } \\
\hline Wall Span (mm) & 1900 \\
\hline Wall Height (mm) & 1800 \\
\hline Wall Length (mm) & 1800 \\
\hline \multicolumn{2}{|l|}{ Wall Dimensions } \\
\hline Block Height (mm) & 190 \\
\hline Block Thickness (mm) & 140 \\
\hline Bed Joint Thickness (mm) & 10 \\
\hline \multicolumn{2}{|l|}{ Longitudinal Reinforcement Properties } \\
\hline Longitudinal Reinforcement Ratio (\%) & 0.41 \\
\hline Area in Flexural Tension $\left(\mathrm{mm}^{2}\right)$ & 785 \\
\hline Bar Diameter (mm) & 20 \\
\hline Distance to Furthest Tension Bar (mm) & 1700 \\
\hline Effective Distance (mm) & 1380 \\
\hline Modulus of Elasticity (MPa) & 200000 \\
\hline Yield Strength (MPa) & 318 \\
\hline Ultimate Strength (MPa) & 450 \\
\hline Ultimate Stain & 0.18 \\
\hline \multicolumn{2}{|l|}{ Transverse Reinforcement Properties } \\
\hline Transverse Reinforcement Ratio (\%) & 0 \\
\hline Modulus of Elasticity (MPa) & 0 \\
\hline Yield Strength (MPa) & 0 \\
\hline Ultimate Strength (MPa) & 0 \\
\hline Ultimate Stain & 0.2 \\
\hline \multicolumn{2}{|l|}{ Critical Loading Zone Properties } \\
\hline Area of Longitudinal Reinforcement $\left(\mathrm{mm}^{2}\right)$ & 314 \\
\hline Longitudinal Reinforcement Center-to-Center Spacing (mm) & 400 \\
\hline Longitudinal Reinforcement Clear Spacing (mm) & 380 \\
\hline Area of Tie $\left(\mathrm{mm}^{2}\right)$ & 0 \\
\hline Tie Clear Spacing (mm) & 0 \\
\hline Tie Depth (mm) & 0 \\
\hline Tie Width (mm) & 0 \\
\hline
\end{tabular}




\section{A3 Shear Wall}

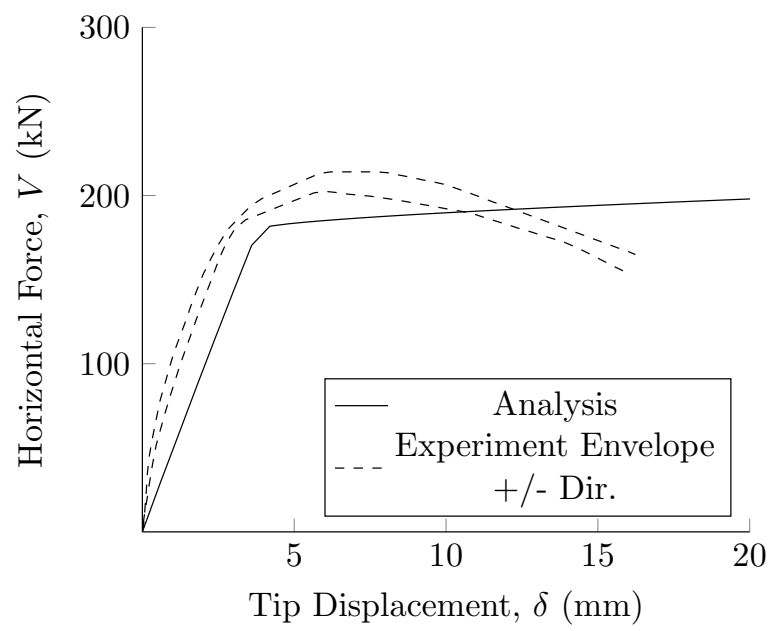

Wall A3 Shear Wall Voon (2007) Horizontal Force vs Tip Displacement

Wall A3 Shear Wall Voon (2007) Model Options

\begin{tabular}{ll}
\hline Model Option & Value \\
\hline Axial Compression Load $(\mathrm{N})$ & 0 \\
Total Lateral Displacement & 60 \\
Number of Load Stages & 100 \\
Convergence Limit & 0.01 \\
Maximum Iterations & 100 \\
\hline
\end{tabular}


Wall A3 Shear Wall Voon (2007) Material Properties

\begin{tabular}{|c|c|}
\hline Property & Value \\
\hline \multicolumn{2}{|l|}{ Masonry Properties } \\
\hline Y-Direction Peak Compression Strength (MPa) & 17 \\
\hline X-Direction Peak Compression Strength (MPa) & 17 \\
\hline Maximum Aggregate Size (mm) & 5 \\
\hline \multicolumn{2}{|l|}{ Wall Dimensions } \\
\hline Wall Span (mm) & 1900 \\
\hline Wall Height (mm) & 1800 \\
\hline Wall Length (mm) & 1800 \\
\hline \multicolumn{2}{|l|}{ Wall Dimensions } \\
\hline Block Height (mm) & 190 \\
\hline Block Thickness (mm) & 140 \\
\hline Bed Joint Thickness (mm) & 10 \\
\hline \multicolumn{2}{|l|}{ Longitudinal Reinforcement Properties } \\
\hline Longitudinal Reinforcement Ratio (\%) & 0.41 \\
\hline Area in Flexural Tension $\left(\mathrm{mm}^{2}\right)$ & 785 \\
\hline Bar Diameter (mm) & 20 \\
\hline Distance to Furthest Tension Bar (mm) & 1700 \\
\hline Effective Distance (mm) & 1380 \\
\hline Modulus of Elasticity (MPa) & 200000 \\
\hline Yield Strength (MPa) & 318 \\
\hline Ultimate Strength (MPa) & 450 \\
\hline Ultimate Stain & 0.18 \\
\hline \multicolumn{2}{|l|}{ Transverse Reinforcement Properties } \\
\hline Transverse Reinforcement Ratio (\%) & 0.16 \\
\hline Modulus of Elasticity (MPa) & 200000 \\
\hline Yield Strength (MPa) & 320 \\
\hline Ultimate Strength (MPa) & 450 \\
\hline Ultimate Stain & 0.2 \\
\hline \multicolumn{2}{|l|}{ Critical Loading Zone Properties } \\
\hline Area of Longitudinal Reinforcement $\left(\mathrm{mm}^{2}\right)$ & 314 \\
\hline Longitudinal Reinforcement Center-to-Center Spacing (mm) & 400 \\
\hline Longitudinal Reinforcement Clear Spacing (mm) & 380 \\
\hline Area of Tie $\left(\mathrm{mm}^{2}\right)$ & 0 \\
\hline Tie Clear Spacing (mm) & 390 \\
\hline Tie Depth $(\mathrm{mm})$ & 120 \\
\hline Tie Width (mm) & 190 \\
\hline
\end{tabular}




\section{A4 Shear Wall}

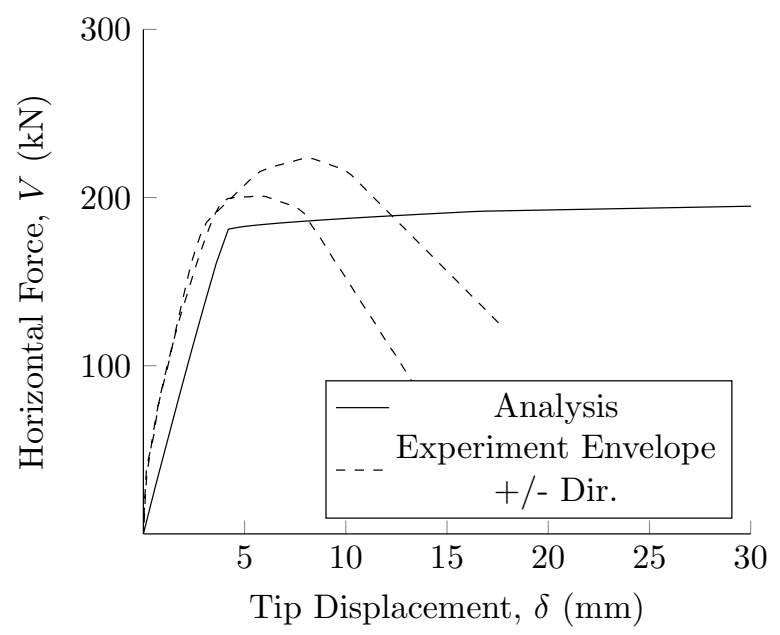

Wall A4 Shear Wall Voon (2007) Horizontal Force vs Tip Displacement

Wall A4 Shear Wall Voon (2007) Model Options

\begin{tabular}{ll}
\hline Model Option & Value \\
\hline Axial Compression Load (N) & 0 \\
Total Lateral Displacement & 60 \\
Number of Load Stages & 100 \\
Convergence Limit & 0.01 \\
Maximum Iterations & 100 \\
\hline
\end{tabular}


Wall A4 Shear Wall Voon (2007) Material Properties

\begin{tabular}{|c|c|}
\hline Property & Value \\
\hline \multicolumn{2}{|l|}{ Masonry Properties } \\
\hline Y-Direction Peak Compression Strength (MPa) & 17 \\
\hline X-Direction Peak Compression Strength (MPa) & 17 \\
\hline Maximum Aggregate Size (mm) & 5 \\
\hline \multicolumn{2}{|l|}{ Wall Dimensions } \\
\hline Wall Span (mm) & 1900 \\
\hline Wall Height (mm) & 1800 \\
\hline Wall Length (mm) & 1800 \\
\hline \multicolumn{2}{|l|}{ Wall Dimensions } \\
\hline Block Height (mm) & 190 \\
\hline Block Thickness (mm) & 140 \\
\hline Bed Joint Thickness (mm) & 10 \\
\hline \multicolumn{2}{|l|}{ Longitudinal Reinforcement Properties } \\
\hline Longitudinal Reinforcement Ratio (\%) & 0.41 \\
\hline Area in Flexural Tension $\left(\mathrm{mm}^{2}\right)$ & 785 \\
\hline Bar Diameter (mm) & 20 \\
\hline Distance to Furthest Tension Bar (mm) & 1700 \\
\hline Effective Distance (mm) & 1380 \\
\hline Modulus of Elasticity (MPa) & 200000 \\
\hline Yield Strength (MPa) & 318 \\
\hline Ultimate Strength (MPa) & 450 \\
\hline Ultimate Stain & 0.18 \\
\hline \multicolumn{2}{|l|}{ Transverse Reinforcement Properties } \\
\hline Transverse Reinforcement Ratio (\%) & 0.06 \\
\hline Modulus of Elasticity (MPa) & 200000 \\
\hline Yield Strength (MPa) & 320 \\
\hline Ultimate Strength (MPa) & 450 \\
\hline Ultimate Stain & 0.2 \\
\hline \multicolumn{2}{|l|}{ Critical Loading Zone Properties } \\
\hline Area of Longitudinal Reinforcement $\left(\mathrm{mm}^{2}\right)$ & 314 \\
\hline Longitudinal Reinforcement Center-to-Center Spacing (mm) & 400 \\
\hline Longitudinal Reinforcement Clear Spacing (mm) & 380 \\
\hline Area of Tie $\left(\mathrm{mm}^{2}\right)$ & 0 \\
\hline Tie Clear Spacing (mm) & 0 \\
\hline Tie Depth $(\mathrm{mm})$ & 120 \\
\hline Tie Width (mm) & 190 \\
\hline
\end{tabular}


A7 Shear Wall

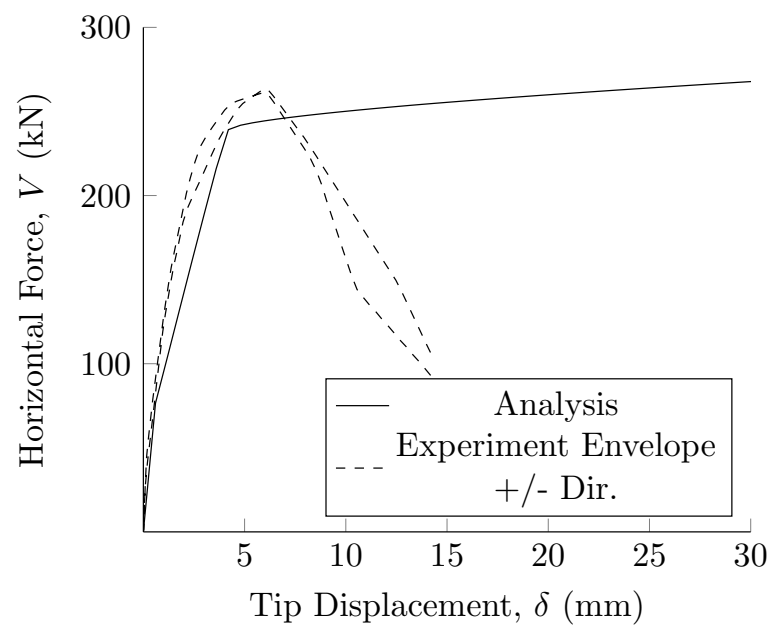

Wall A7 Shear Wall Voon (2007) Horizontal Force vs Tip Displacement

Wall A7 Shear Wall Voon (2007) Model Options

\begin{tabular}{ll}
\hline Model Option & Value \\
\hline Axial Compression Load (N) & 126000 \\
Total Lateral Displacement & 60 \\
Number of Load Stages & 100 \\
Convergence Limit & 0.01 \\
Maximum Iterations & 100 \\
\hline
\end{tabular}


Wall A7 Shear Wall Voon (2007) Material Properties

\begin{tabular}{|c|c|}
\hline Property & Value \\
\hline \multicolumn{2}{|l|}{ Masonry Properties } \\
\hline Y-Direction Peak Compression Strength (MPa) & 18.8 \\
\hline X-Direction Peak Compression Strength (MPa) & 18.8 \\
\hline Maximum Aggregate Size (mm) & 5 \\
\hline \multicolumn{2}{|l|}{ Wall Dimensions } \\
\hline Wall Span (mm) & 1900 \\
\hline Wall Height (mm) & 1800 \\
\hline Wall Length (mm) & 1800 \\
\hline \multicolumn{2}{|l|}{ Wall Dimensions } \\
\hline Block Height (mm) & 190 \\
\hline Block Thickness (mm) & 140 \\
\hline Bed Joint Thickness (mm) & 10 \\
\hline \multicolumn{2}{|l|}{ Longitudinal Reinforcement Properties } \\
\hline Longitudinal Reinforcement Ratio (\%) & 0.41 \\
\hline Area in Flexural Tension $\left(\mathrm{mm}^{2}\right)$ & 785 \\
\hline Bar Diameter (mm) & 20 \\
\hline Distance to Furthest Tension Bar (mm) & 1700 \\
\hline Effective Distance (mm) & 1380 \\
\hline Modulus of Elasticity (MPa) & 200000 \\
\hline Yield Strength (MPa) & 318 \\
\hline Ultimate Strength (MPa) & 450 \\
\hline Ultimate Stain & 0.18 \\
\hline \multicolumn{2}{|l|}{ Transverse Reinforcement Properties } \\
\hline Transverse Reinforcement Ratio (\%) & 0.22 \\
\hline Modulus of Elasticity (MPa) & 200000 \\
\hline Yield Strength (MPa) & 325 \\
\hline Ultimate Strength (MPa) & 450 \\
\hline Ultimate Stain & 0.2 \\
\hline \multicolumn{2}{|l|}{ Critical Loading Zone Properties } \\
\hline Area of Longitudinal Reinforcement $\left(\mathrm{mm}^{2}\right)$ & 314 \\
\hline Longitudinal Reinforcement Center-to-Center Spacing (mm) & 400 \\
\hline Longitudinal Reinforcement Clear Spacing (mm) & 380 \\
\hline Area of Tie $\left(\mathrm{mm}^{2}\right)$ & 0 \\
\hline Tie Clear Spacing (mm) & 388 \\
\hline Tie Depth (mm) & 120 \\
\hline Tie Width (mm) & 190 \\
\hline
\end{tabular}




\section{A8 Shear Wall}

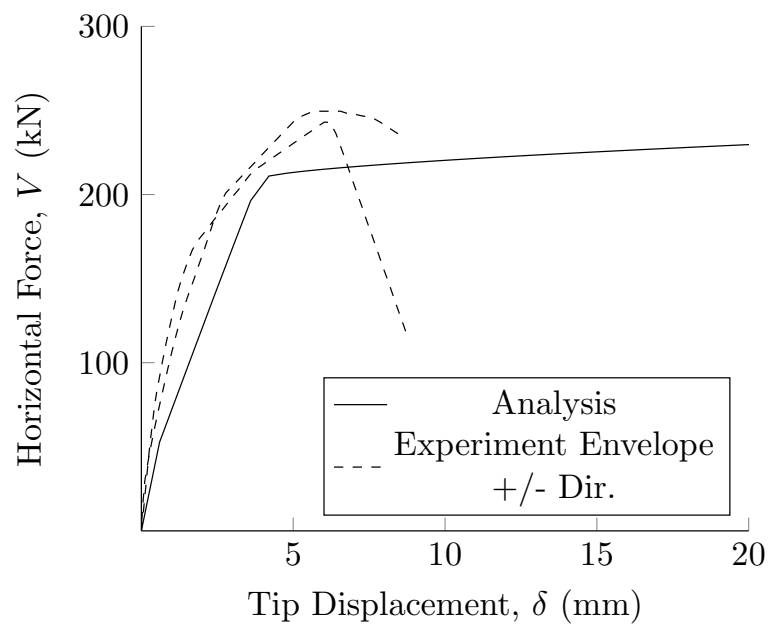

Wall A8 Shear Wall Voon (2007) Horizontal Force vs Tip Displacement

Wall A8 Shear Wall Voon (2007) Model Options

\begin{tabular}{ll}
\hline Model Option & Value \\
\hline Axial Compression Load (N) & 63000 \\
Total Lateral Displacement & 60 \\
Number of Load Stages & 100 \\
Convergence Limit & 0.01 \\
Maximum Iterations & 100 \\
\hline
\end{tabular}


Wall A8 Shear Wall Voon (2007) Material Properties

\begin{tabular}{|c|c|}
\hline Property & Value \\
\hline \multicolumn{2}{|l|}{ Masonry Properties } \\
\hline Y-Direction Peak Compression Strength (MPa) & 18.8 \\
\hline X-Direction Peak Compression Strength (MPa) & 18.8 \\
\hline Maximum Aggregate Size (mm) & 5 \\
\hline \multicolumn{2}{|l|}{ Wall Dimensions } \\
\hline Wall Span (mm) & 1900 \\
\hline Wall Height (mm) & 1800 \\
\hline Wall Length (mm) & 1800 \\
\hline \multicolumn{2}{|l|}{ Wall Dimensions } \\
\hline Block Height (mm) & 190 \\
\hline Block Thickness (mm) & 140 \\
\hline Bed Joint Thickness (mm) & 10 \\
\hline \multicolumn{2}{|l|}{ Longitudinal Reinforcement Properties } \\
\hline Longitudinal Reinforcement Ratio (\%) & 0.41 \\
\hline Area in Flexural Tension $\left(\mathrm{mm}^{2}\right)$ & 785 \\
\hline Bar Diameter (mm) & 20 \\
\hline Distance to Furthest Tension Bar (mm) & 1700 \\
\hline Effective Distance (mm) & 1380 \\
\hline Modulus of Elasticity (MPa) & 200000 \\
\hline Yield Strength (MPa) & 318 \\
\hline Ultimate Strength (MPa) & 450 \\
\hline Ultimate Stain & 0.18 \\
\hline \multicolumn{2}{|l|}{ Transverse Reinforcement Properties } \\
\hline Transverse Reinforcement Ratio (\%) & 0.22 \\
\hline Modulus of Elasticity (MPa) & 200000 \\
\hline Yield Strength (MPa) & 325 \\
\hline Ultimate Strength (MPa) & 450 \\
\hline Ultimate Stain & 0.2 \\
\hline \multicolumn{2}{|l|}{ Critical Loading Zone Properties } \\
\hline Area of Longitudinal Reinforcement $\left(\mathrm{mm}^{2}\right)$ & 314 \\
\hline Longitudinal Reinforcement Center-to-Center Spacing (mm) & 400 \\
\hline Longitudinal Reinforcement Clear Spacing (mm) & 380 \\
\hline Area of Tie $\left(\mathrm{mm}^{2}\right)$ & 0 \\
\hline Tie Clear Spacing (mm) & 388 \\
\hline Tie Depth (mm) & 120 \\
\hline Tie Width (mm) & 190 \\
\hline
\end{tabular}




\section{A9 Shear Wall}

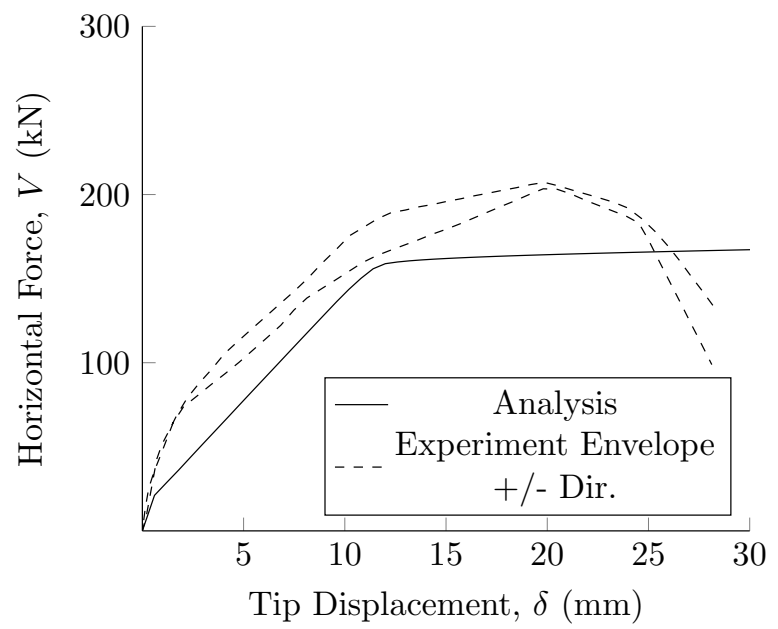

Wall A9 Shear Wall Voon (2007) Horizontal Force vs Tip Displacement

Wall A9 Shear Wall Voon (2007) Model Options

\begin{tabular}{ll}
\hline Model Option & Value \\
\hline Axial Compression Load (N) & 63000 \\
Total Lateral Displacement & 60 \\
Number of Load Stages & 100 \\
Convergence Limit & 0.01 \\
Maximum Iterations & 100 \\
\hline
\end{tabular}


Wall A9 Shear Wall Voon (2007) Material Properties

\begin{tabular}{|c|c|}
\hline Property & Value \\
\hline \multicolumn{2}{|l|}{ Masonry Properties } \\
\hline Y-Direction Peak Compression Strength (MPa) & 24.3 \\
\hline X-Direction Peak Compression Strength (MPa) & 24.3 \\
\hline Maximum Aggregate Size (mm) & 5 \\
\hline \multicolumn{2}{|l|}{ Wall Dimensions } \\
\hline Wall Span (mm) & 3700 \\
\hline Wall Height (mm) & 3600 \\
\hline Wall Length (mm) & 1800 \\
\hline \multicolumn{2}{|l|}{ Wall Dimensions } \\
\hline Block Height (mm) & 190 \\
\hline Block Thickness (mm) & 140 \\
\hline Bed Joint Thickness (mm) & 10 \\
\hline \multicolumn{2}{|l|}{ Longitudinal Reinforcement Properties } \\
\hline Longitudinal Reinforcement Ratio (\%) & 0.64 \\
\hline Area in Flexural Tension $\left(\mathrm{mm}^{2}\right)$ & 1227 \\
\hline Bar Diameter (mm) & 25 \\
\hline Distance to Furthest Tension Bar (mm) & 1700 \\
\hline Effective Distance (mm) & 1380 \\
\hline Modulus of Elasticity (MPa) & 200000 \\
\hline Yield Strength (MPa) & 320 \\
\hline Ultimate Strength (MPa) & 450 \\
\hline Ultimate Stain & 0.2 \\
\hline \multicolumn{2}{|l|}{ Transverse Reinforcement Properties } \\
\hline Transverse Reinforcement Ratio (\%) & 0.2 \\
\hline Modulus of Elasticity (MPa) & 200000 \\
\hline Yield Strength (MPa) & 325 \\
\hline Ultimate Strength (MPa) & 450 \\
\hline Ultimate Stain & 0.2 \\
\hline \multicolumn{2}{|l|}{ Critical Loading Zone Properties } \\
\hline Area of Longitudinal Reinforcement $\left(\mathrm{mm}^{2}\right)$ & 491 \\
\hline Longitudinal Reinforcement Center-to-Center Spacing (mm) & 400 \\
\hline Longitudinal Reinforcement Clear Spacing (mm) & 375 \\
\hline Area of Tie $\left(\mathrm{mm}^{2}\right)$ & 0 \\
\hline Tie Clear Spacing (mm) & 388 \\
\hline Tie Depth (mm) & 120 \\
\hline Tie Width (mm) & 190 \\
\hline
\end{tabular}


A10 Shear Wall

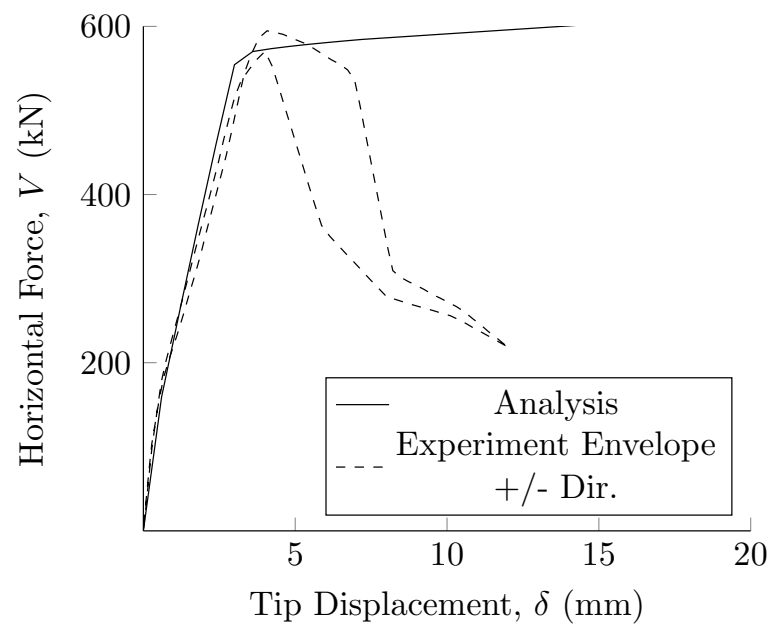

Wall A10 Shear Wall Voon (2007) Horizontal Force vs Tip Displacement

Wall A10 Shear Wall Voon (2007) Model Options

\begin{tabular}{ll}
\hline Model Option & Value \\
\hline Axial Compression Load (N) & 105000 \\
Total Lateral Displacement & 60 \\
Number of Load Stages & 100 \\
Convergence Limit & 0.01 \\
Maximum Iterations & 100 \\
\hline
\end{tabular}


Wall A10 Shear Wall Voon (2007) Material Properties

\begin{tabular}{|c|c|}
\hline Property & Value \\
\hline \multicolumn{2}{|l|}{ Masonry Properties } \\
\hline Y-Direction Peak Compression Strength (MPa) & 24.3 \\
\hline X-Direction Peak Compression Strength (MPa) & 24.3 \\
\hline Maximum Aggregate Size (mm) & 5 \\
\hline \multicolumn{2}{|l|}{ Wall Dimensions } \\
\hline Wall Span (mm) & 1900 \\
\hline Wall Height (mm) & 1800 \\
\hline Wall Length (mm) & 3000 \\
\hline \multicolumn{2}{|l|}{ Wall Dimensions } \\
\hline Block Height (mm) & 190 \\
\hline Block Thickness (mm) & 140 \\
\hline Bed Joint Thickness (mm) & 10 \\
\hline \multicolumn{2}{|l|}{ Longitudinal Reinforcement Properties } \\
\hline Longitudinal Reinforcement Ratio (\%) & 0.39 \\
\hline Area in Flexural Tension $\left(\mathrm{mm}^{2}\right)$ & 1257 \\
\hline Bar Diameter (mm) & 20 \\
\hline Distance to Furthest Tension Bar (mm) & 2900 \\
\hline Effective Distance (mm) & 2300 \\
\hline Modulus of Elasticity (MPa) & 200000 \\
\hline Yield Strength (MPa) & 318 \\
\hline Ultimate Strength (MPa) & 450 \\
\hline Ultimate Stain & 0.18 \\
\hline \multicolumn{2}{|l|}{ Transverse Reinforcement Properties } \\
\hline Transverse Reinforcement Ratio (\%) & 0.22 \\
\hline Modulus of Elasticity (MPa) & 200000 \\
\hline Yield Strength (MPa) & 325 \\
\hline Ultimate Strength (MPa) & 450 \\
\hline Ultimate Stain & 0.2 \\
\hline \multicolumn{2}{|l|}{ Critical Loading Zone Properties } \\
\hline Area of Longitudinal Reinforcement $\left(\mathrm{mm}^{2}\right)$ & 491 \\
\hline Longitudinal Reinforcement Center-to-Center Spacing (mm) & 400 \\
\hline Longitudinal Reinforcement Clear Spacing (mm) & 380 \\
\hline Area of Tie $\left(\mathrm{mm}^{2}\right)$ & 0 \\
\hline Tie Clear Spacing (mm) & 388 \\
\hline Tie Depth (mm) & 120 \\
\hline Tie Width (mm) & 190 \\
\hline
\end{tabular}




\section{Shedid et al. (2008)}

\section{Wall 1 Shear Wall}

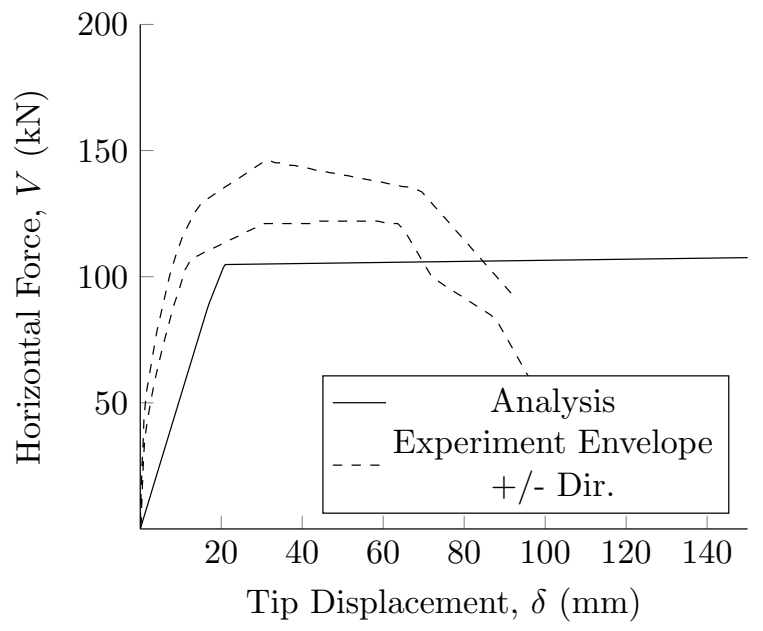

Wall 1 Shear Wall Shedid et al. (2008) Horizontal Force vs Tip Displacement

Wall 1 Shear Wall Shedid et al. (2008) Model Options

\begin{tabular}{ll}
\hline Model Option & Value \\
\hline Axial Compression Load (N) & 0 \\
Total Lateral Displacement & 150 \\
Number of Load Stages & 300 \\
Convergence Limit & 0.01 \\
Maximum Iterations & 100 \\
\hline
\end{tabular}


Wall 1 Shear Wall Shedid et al. (2008) Material Properties

\begin{tabular}{|c|c|}
\hline Property & Value \\
\hline \multicolumn{2}{|l|}{ Masonry Properties } \\
\hline Y-Direction Peak Compression Strength (MPa) & 14.8 \\
\hline X-Direction Peak Compression Strength (MPa) & 14.8 \\
\hline Maximum Aggregate Size (mm) & 5 \\
\hline \multicolumn{2}{|l|}{ Wall Dimensions } \\
\hline Wall Span (mm) & 3800 \\
\hline Wall Height (mm) & 3600 \\
\hline Wall Length (mm) & 1800 \\
\hline \multicolumn{2}{|l|}{ Wall Dimensions } \\
\hline Block Height (mm) & 190 \\
\hline Block Thickness (mm) & 190 \\
\hline Bed Joint Thickness (mm) & 10 \\
\hline \multicolumn{2}{|l|}{ Longitudinal Reinforcement Properties } \\
\hline Longitudinal Reinforcement Ratio (\%) & 0.19 \\
\hline Area in Flexural Tension $\left(\mathrm{mm}^{2}\right)$ & 500 \\
\hline Bar Diameter (mm) & 15 \\
\hline Distance to Furthest Tension Bar (mm) & 1700 \\
\hline Effective Distance (mm) & 1380 \\
\hline Modulus of Elasticity (MPa) & 200000 \\
\hline Yield Strength (MPa) & 502 \\
\hline Ultimate Strength (MPa) & 650 \\
\hline Ultimate Stain & 0.2 \\
\hline \multicolumn{2}{|l|}{ Transverse Reinforcement Properties } \\
\hline Transverse Reinforcement Ratio (\%) & 0.09 \\
\hline Modulus of Elasticity (MPa) & 200000 \\
\hline Yield Strength (MPa) & 491 \\
\hline Ultimate Strength (MPa) & 650 \\
\hline Ultimate Stain & 0.2 \\
\hline \multicolumn{2}{|l|}{ Critical Loading Zone Properties } \\
\hline Area of Longitudinal Reinforcement $\left(\mathrm{mm}^{2}\right)$ & 200 \\
\hline Longitudinal Reinforcement Center-to-Center Spacing (mm) & 400 \\
\hline Longitudinal Reinforcement Clear Spacing (mm) & 375 \\
\hline Area of Tie $\left(\mathrm{mm}^{2}\right)$ & 0 \\
\hline Tie Clear Spacing (mm) & 590 \\
\hline Tie Depth (mm) & 120 \\
\hline Tie Width (mm) & 190 \\
\hline
\end{tabular}




\section{Wall 2 Shear Wall}

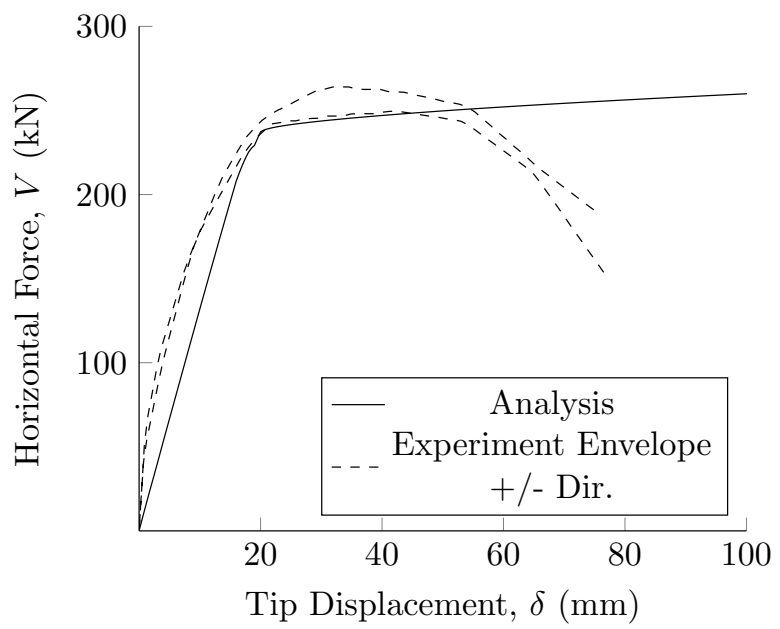

Wall 2 Shear Wall Shedid et al. (2008) Horizontal Force vs Tip Displacement

Wall 2 Shear Wall Shedid et al. (2008) Model Options

\begin{tabular}{ll}
\hline Model Option & Value \\
\hline Axial Compression Load (N) & 0 \\
Total Lateral Displacement & 150 \\
Number of Load Stages & 300 \\
Convergence Limit & 0.01 \\
Maximum Iterations & 100 \\
\hline
\end{tabular}


Wall 2 Shear Wall Shedid et al. (2008) Material Properties

\begin{tabular}{|c|c|}
\hline Property & Value \\
\hline \multicolumn{2}{|l|}{ Masonry Properties } \\
\hline Y-Direction Peak Compression Strength (MPa) & 14.8 \\
\hline X-Direction Peak Compression Strength (MPa) & 14.8 \\
\hline Maximum Aggregate Size (mm) & 5 \\
\hline \multicolumn{2}{|l|}{ Wall Dimensions } \\
\hline Wall Span (mm) & 3800 \\
\hline Wall Height (mm) & 3600 \\
\hline Wall Length (mm) & 1800 \\
\hline \multicolumn{2}{|l|}{ Wall Dimensions } \\
\hline Block Height (mm) & 190 \\
\hline Block Thickness (mm) & 190 \\
\hline Bed Joint Thickness (mm) & 10 \\
\hline \multicolumn{2}{|l|}{ Longitudinal Reinforcement Properties } \\
\hline Longitudinal Reinforcement Ratio (\%) & 0.53 \\
\hline Area in Flexural Tension $\left(\mathrm{mm}^{2}\right)$ & 1350 \\
\hline Bar Diameter (mm) & 20 \\
\hline Distance to Furthest Tension Bar (mm) & 1700 \\
\hline Effective Distance (mm) & 1344 \\
\hline Modulus of Elasticity (MPa) & 200000 \\
\hline Yield Strength (MPa) & 502 \\
\hline Ultimate Strength (MPa) & 650 \\
\hline Ultimate Stain & 0.2 \\
\hline \multicolumn{2}{|l|}{ Transverse Reinforcement Properties } \\
\hline Transverse Reinforcement Ratio (\%) & 0.13 \\
\hline Modulus of Elasticity (MPa) & 200000 \\
\hline Yield Strength (MPa) & 491 \\
\hline Ultimate Strength (MPa) & 650 \\
\hline Ultimate Stain & 0.2 \\
\hline \multicolumn{2}{|l|}{ Critical Loading Zone Properties } \\
\hline Area of Longitudinal Reinforcement $\left(\mathrm{mm}^{2}\right)$ & 600 \\
\hline Longitudinal Reinforcement Center-to-Center Spacing (mm) & 200 \\
\hline Longitudinal Reinforcement Clear Spacing (mm) & 180 \\
\hline Area of Tie $\left(\mathrm{mm}^{2}\right)$ & 0 \\
\hline Tie Clear Spacing (mm) & 390 \\
\hline Tie Depth $(\mathrm{mm})$ & 120 \\
\hline Tie Width (mm) & 190 \\
\hline
\end{tabular}




\section{Wall 3 Shear Wall}

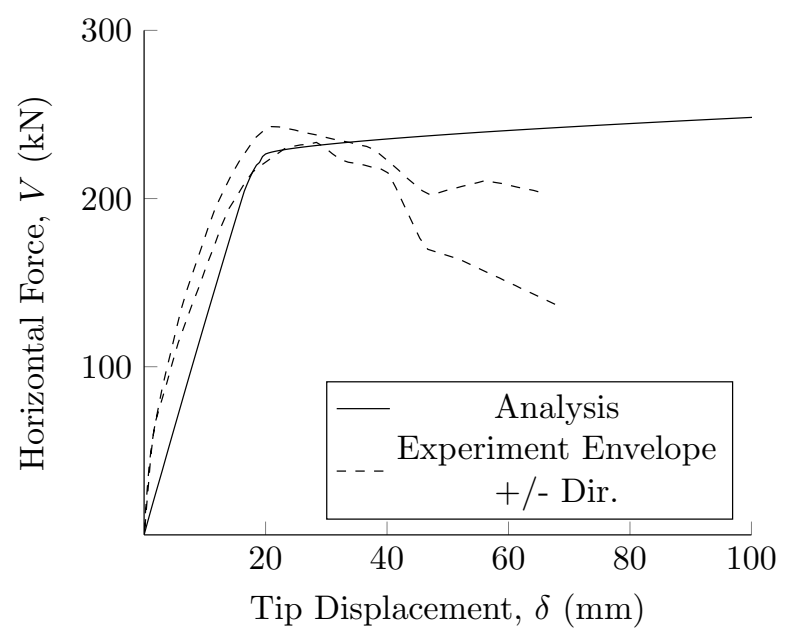

Wall 3 Shear Wall Shedid et al. (2008) Horizontal Force vs Tip Displacement

Wall 3 Shear Wall Shedid et al. (2008) Model Options

\begin{tabular}{ll}
\hline Model Option & Value \\
\hline Axial Compression Load $(\mathrm{N})$ & 0 \\
Total Lateral Displacement & 150 \\
Number of Load Stages & 300 \\
Convergence Limit & 0.01 \\
Maximum Iterations & 100 \\
\hline
\end{tabular}


Wall 3 Shear Wall Shedid et al. (2008) Material Properties

\begin{tabular}{|c|c|}
\hline Property & Value \\
\hline \multicolumn{2}{|l|}{ Masonry Properties } \\
\hline Y-Direction Peak Compression Strength (MPa) & 14.8 \\
\hline X-Direction Peak Compression Strength (MPa) & 14.8 \\
\hline Maximum Aggregate Size (mm) & 5 \\
\hline \multicolumn{2}{|l|}{ Wall Dimensions } \\
\hline Wall Span (mm) & 3800 \\
\hline Wall Height (mm) & 3600 \\
\hline Wall Length (mm) & 1800 \\
\hline \multicolumn{2}{|l|}{ Wall Dimensions } \\
\hline Block Height (mm) & 190 \\
\hline Block Thickness (mm) & 190 \\
\hline Bed Joint Thickness (mm) & 10 \\
\hline \multicolumn{2}{|l|}{ Longitudinal Reinforcement Properties } \\
\hline Longitudinal Reinforcement Ratio (\%) & 0.48 \\
\hline Area in Flexural Tension $\left(\mathrm{mm}^{2}\right)$ & 1250 \\
\hline Bar Diameter (mm) & 25 \\
\hline Distance to Furthest Tension Bar (mm) & 1700 \\
\hline Effective Distance (mm) & 1380 \\
\hline Modulus of Elasticity (MPa) & 200000 \\
\hline Yield Strength (MPa) & 502 \\
\hline Ultimate Strength (MPa) & 650 \\
\hline Ultimate Stain & 0.2 \\
\hline \multicolumn{2}{|l|}{ Transverse Reinforcement Properties } \\
\hline Transverse Reinforcement Ratio (\%) & 0.13 \\
\hline Modulus of Elasticity (MPa) & 200000 \\
\hline Yield Strength (MPa) & 491 \\
\hline Ultimate Strength (MPa) & 650 \\
\hline Ultimate Stain & 0.2 \\
\hline \multicolumn{2}{|l|}{ Critical Loading Zone Properties } \\
\hline Area of Longitudinal Reinforcement $\left(\mathrm{mm}^{2}\right)$ & 500 \\
\hline Longitudinal Reinforcement Center-to-Center Spacing (mm) & 400 \\
\hline Longitudinal Reinforcement Clear Spacing (mm) & 375 \\
\hline Area of Tie $\left(\mathrm{mm}^{2}\right)$ & 0 \\
\hline Tie Clear Spacing (mm) & 390 \\
\hline Tie Depth (mm) & 120 \\
\hline Tie Width (mm) & 190 \\
\hline
\end{tabular}




\section{Wall 4 Shear Wall}

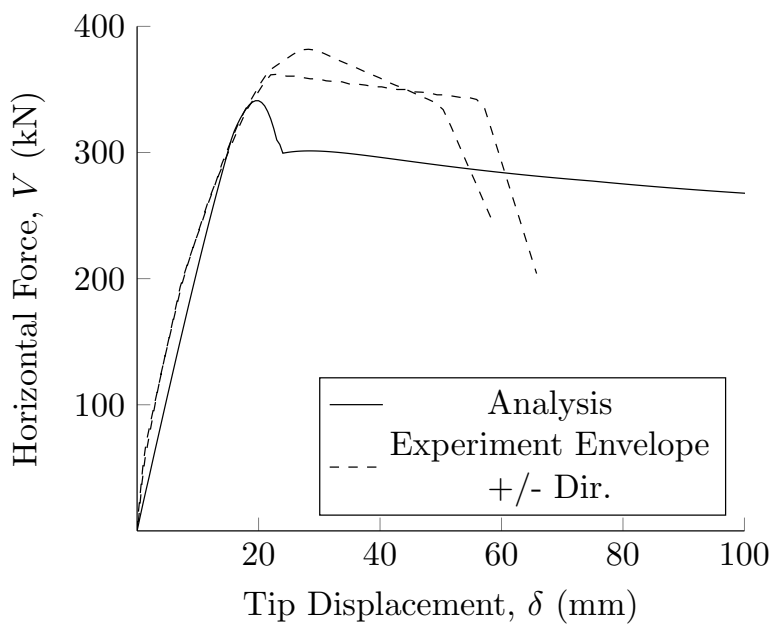

Wall 4 Shear Wall Shedid et al. (2008) Horizontal Force vs Tip Displacement

Wall 4 Shear Wall Shedid et al. (2008) Model Options

\begin{tabular}{ll}
\hline Model Option & Value \\
\hline Axial Compression Load (N) & 0 \\
Total Lateral Displacement & 150 \\
Number of Load Stages & 300 \\
Convergence Limit & 0.01 \\
Maximum Iterations & 100 \\
\hline
\end{tabular}


Wall 4 Shear Wall Shedid et al. (2008) Material Properties

\begin{tabular}{|c|c|}
\hline Property & Value \\
\hline \multicolumn{2}{|l|}{ Masonry Properties } \\
\hline Y-Direction Peak Compression Strength (MPa) & 14.8 \\
\hline X-Direction Peak Compression Strength (MPa) & 14.8 \\
\hline Maximum Aggregate Size (mm) & 5 \\
\hline \multicolumn{2}{|l|}{ Wall Dimensions } \\
\hline Wall Span (mm) & 3800 \\
\hline Wall Height (mm) & 3600 \\
\hline Wall Length (mm) & 1800 \\
\hline \multicolumn{2}{|l|}{ Wall Dimensions } \\
\hline Block Height (mm) & 190 \\
\hline Block Thickness (mm) & 190 \\
\hline Bed Joint Thickness (mm) & 10 \\
\hline \multicolumn{2}{|l|}{ Longitudinal Reinforcement Properties } \\
\hline Longitudinal Reinforcement Ratio (\%) & 0.88 \\
\hline Area in Flexural Tension $\left(\mathrm{mm}^{2}\right)$ & 2250 \\
\hline Bar Diameter (mm) & 25 \\
\hline Distance to Furthest Tension Bar (mm) & 1700 \\
\hline Effective Distance (mm) & 1344 \\
\hline Modulus of Elasticity (MPa) & 200000 \\
\hline Yield Strength (MPa) & 502 \\
\hline Ultimate Strength (MPa) & 650 \\
\hline Ultimate Stain & 0.2 \\
\hline \multicolumn{2}{|l|}{ Transverse Reinforcement Properties } \\
\hline Transverse Reinforcement Ratio (\%) & 0.26 \\
\hline Modulus of Elasticity (MPa) & 200000 \\
\hline Yield Strength (MPa) & 491 \\
\hline Ultimate Strength (MPa) & 650 \\
\hline Ultimate Stain & 0.2 \\
\hline \multicolumn{2}{|l|}{ Critical Loading Zone Properties } \\
\hline Area of Longitudinal Reinforcement $\left(\mathrm{mm}^{2}\right)$ & 1000 \\
\hline Longitudinal Reinforcement Center-to-Center Spacing (mm) & 200 \\
\hline Longitudinal Reinforcement Clear Spacing (mm) & 175 \\
\hline Area of Tie $\left(\mathrm{mm}^{2}\right)$ & 0 \\
\hline Tie Clear Spacing (mm) & 190 \\
\hline Tie Depth (mm) & 120 \\
\hline Tie Width (mm) & 190 \\
\hline
\end{tabular}




\section{Wall 5 Shear Wall}

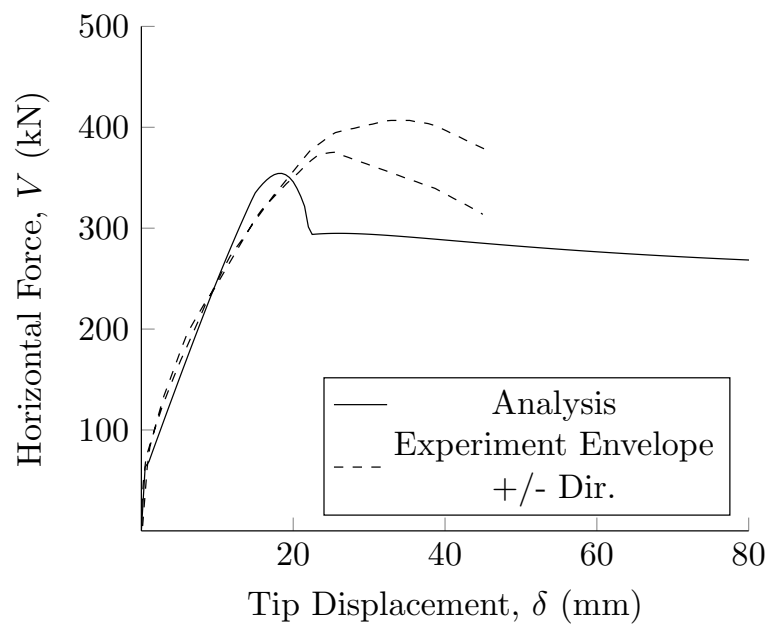

Wall 5 Shear Wall Shedid et al. (2008) Horizontal Force vs Tip Displacement

Wall 5 Shear Wall Shedid et al. (2008) Model Options

\begin{tabular}{ll}
\hline Model Option & Value \\
\hline Axial Compression Load (N) & 256500 \\
Total Lateral Displacement & 150 \\
Number of Load Stages & 300 \\
Convergence Limit & 0.01 \\
Maximum Iterations & 100 \\
\hline
\end{tabular}


Wall 5 Shear Wall Shedid et al. (2008) Material Properties

\begin{tabular}{|c|c|}
\hline Property & Value \\
\hline \multicolumn{2}{|l|}{ Masonry Properties } \\
\hline Y-Direction Peak Compression Strength (MPa) & 14.8 \\
\hline X-Direction Peak Compression Strength (MPa) & 14.8 \\
\hline Maximum Aggregate Size (mm) & 5 \\
\hline \multicolumn{2}{|l|}{ Wall Dimensions } \\
\hline Wall Span (mm) & 3800 \\
\hline Wall Height (mm) & 3600 \\
\hline Wall Length (mm) & 1800 \\
\hline \multicolumn{2}{|l|}{ Wall Dimensions } \\
\hline Block Height (mm) & 190 \\
\hline Block Thickness (mm) & 190 \\
\hline Bed Joint Thickness (mm) & 10 \\
\hline \multicolumn{2}{|l|}{ Longitudinal Reinforcement Properties } \\
\hline Longitudinal Reinforcement Ratio (\%) & 0.88 \\
\hline Area in Flexural Tension $\left(\mathrm{mm}^{2}\right)$ & 2250 \\
\hline Bar Diameter (mm) & 25 \\
\hline Distance to Furthest Tension Bar (mm) & 1700 \\
\hline Effective Distance (mm) & 1344 \\
\hline Modulus of Elasticity (MPa) & 200000 \\
\hline Yield Strength (MPa) & 502 \\
\hline Ultimate Strength (MPa) & 650 \\
\hline Ultimate Stain & 0.2 \\
\hline \multicolumn{2}{|l|}{ Transverse Reinforcement Properties } \\
\hline Transverse Reinforcement Ratio (\%) & 0.26 \\
\hline Modulus of Elasticity (MPa) & 200000 \\
\hline Yield Strength (MPa) & 491 \\
\hline Ultimate Strength (MPa) & 650 \\
\hline Ultimate Stain & 0.2 \\
\hline \multicolumn{2}{|l|}{ Critical Loading Zone Properties } \\
\hline Area of Longitudinal Reinforcement $\left(\mathrm{mm}^{2}\right)$ & 1000 \\
\hline Longitudinal Reinforcement Center-to-Center Spacing (mm) & 200 \\
\hline Longitudinal Reinforcement Clear Spacing (mm) & 175 \\
\hline Area of Tie $\left(\mathrm{mm}^{2}\right)$ & 0 \\
\hline Tie Clear Spacing (mm) & 190 \\
\hline Tie Depth (mm) & 120 \\
\hline Tie Width (mm) & 190 \\
\hline
\end{tabular}




\section{Wall 6 Shear Wall}

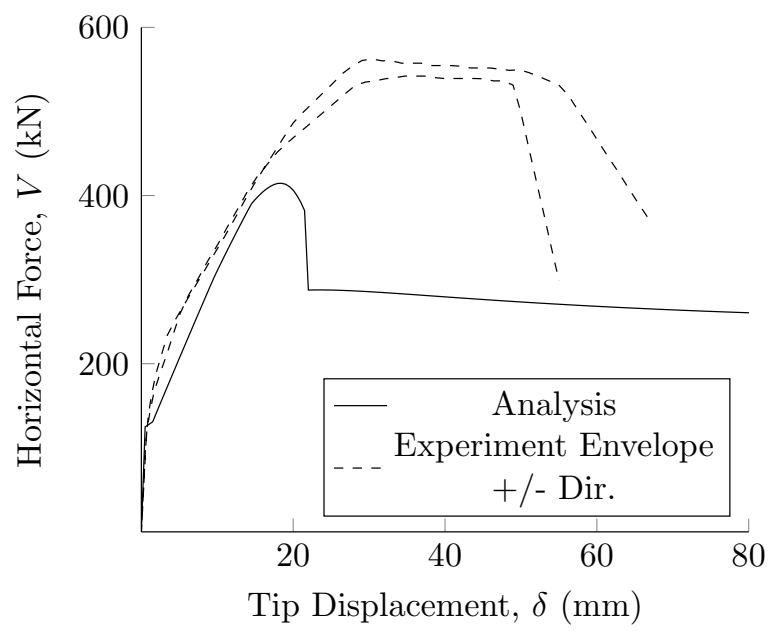

Wall 6 Shear Wall Shedid et al. (2008) Horizontal Force vs Tip Displacement

Wall 6 Shear Wall Shedid et al. (2008) Model Options

\begin{tabular}{ll}
\hline Model Option & Value \\
\hline Axial Compression Load (N) & 513000 \\
Total Lateral Displacement & 150 \\
Number of Load Stages & 300 \\
Convergence Limit & 0.01 \\
Maximum Iterations & 100 \\
\hline
\end{tabular}


Wall 6 Shear Wall Shedid et al. (2008) Material Properties

\begin{tabular}{|c|c|}
\hline Property & Value \\
\hline \multicolumn{2}{|l|}{ Masonry Properties } \\
\hline Y-Direction Peak Compression Strength (MPa) & 14.8 \\
\hline X-Direction Peak Compression Strength (MPa) & 14.8 \\
\hline Maximum Aggregate Size (mm) & 5 \\
\hline \multicolumn{2}{|l|}{ Wall Dimensions } \\
\hline Wall Span (mm) & 3800 \\
\hline Wall Height (mm) & 3600 \\
\hline Wall Length (mm) & 1800 \\
\hline \multicolumn{2}{|l|}{ Wall Dimensions } \\
\hline Block Height (mm) & 190 \\
\hline Block Thickness (mm) & 190 \\
\hline Bed Joint Thickness (mm) & 10 \\
\hline \multicolumn{2}{|l|}{ Longitudinal Reinforcement Properties } \\
\hline Longitudinal Reinforcement Ratio (\%) & 0.88 \\
\hline Area in Flexural Tension $\left(\mathrm{mm}^{2}\right)$ & 2250 \\
\hline Bar Diameter (mm) & 25 \\
\hline Distance to Furthest Tension Bar (mm) & 1700 \\
\hline Effective Distance (mm) & 1344 \\
\hline Modulus of Elasticity (MPa) & 200000 \\
\hline Yield Strength (MPa) & 502 \\
\hline Ultimate Strength (MPa) & 650 \\
\hline Ultimate Stain & 0.2 \\
\hline \multicolumn{2}{|l|}{ Transverse Reinforcement Properties } \\
\hline Transverse Reinforcement Ratio (\%) & 0.26 \\
\hline Modulus of Elasticity (MPa) & 200000 \\
\hline Yield Strength (MPa) & 491 \\
\hline Ultimate Strength (MPa) & 650 \\
\hline Ultimate Stain & 0.2 \\
\hline \multicolumn{2}{|l|}{ Critical Loading Zone Properties } \\
\hline Area of Longitudinal Reinforcement $\left(\mathrm{mm}^{2}\right)$ & 1000 \\
\hline Longitudinal Reinforcement Center-to-Center Spacing (mm) & 200 \\
\hline Longitudinal Reinforcement Clear Spacing (mm) & 175 \\
\hline Area of Tie $\left(\mathrm{mm}^{2}\right)$ & 0 \\
\hline Tie Clear Spacing (mm) & 190 \\
\hline Tie Depth (mm) & 120 \\
\hline Tie Width (mm) & 190 \\
\hline
\end{tabular}

